$=08$

(1) $13 \%$

करते " "00"

100 - क्या दो

Rent" = 0
0
0
0
0
0
0
0
0
0

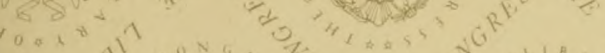

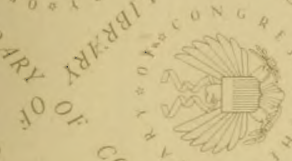

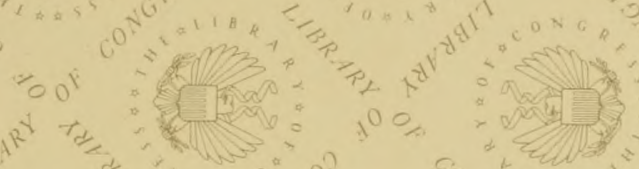
(1) oo

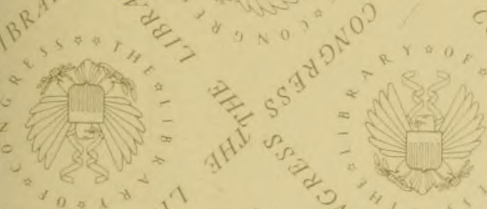

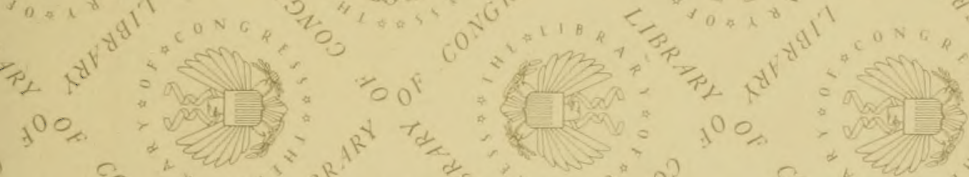

ond "of (8) 100 O

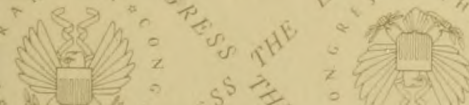
$x^{5}=2$

\section{साओ}

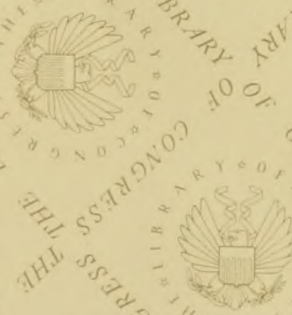
100
18
18 होil (i) $x_{x \rightarrow 1}$ $300^{\circ}$ $\left(x^{2} t_{t}\right.$ 100 Rा ? 100 wat 





\title{
The Diseases of the Genital Organs of Domestic Animals
}

\author{
BY

\section{W. L. WILLIAMS} \\ PROFESSOR OF OBSTETRICS AND RESEARCH PROFESSOR \\ IN THE DISEASES OF BREEDING CATTLE \\ IN THE
}

NEW YORK STATE VETERINARY COLLEGE AT CORNELL UNIVERSITY

WITH THE COLLABORATION OF

W. W. WILLIAMS, B.A., D.V.M.

SPRINGFIELD, MASS.

Published BY the Author

ITHACA, N. Y.

1921 


$$
\begin{gathered}
5+871 \\
\sqrt{45}
\end{gathered}
$$

\section{COPYRIGHT BY}

W. L. WILLIAMS

1921 


\section{PREFACE}

No attempt has been made heretofore to describe systematically the genital diseases of domestic animals. The pathologic processes acting within the genital organs and interfering with the ideal production of young are chiefly hidden from view. Only certain phenomena caused by them become apparent. Of these the most striking phenomenon is the observed expulsion of a fetal cadaver, which is designated abortion. The observer is generally forewarned of impending death of a born animal because it is commonly preceded by visible injury or illness, but abortion produces a profound impression because the death of a potentially valuable unborn animal has occurred unseen and is revealed only when the cadaver is seen to be expelled. This tends to draw a veil of mystery about the event. If the spermatozöon, unfertilized or fertilized ovum, or the small embryo perishes, the dead cell or body is not observed and therefore excites little or no comment.

In the early history of medicine, certain striking phenomena were regarded and described as diseases. When the science of pathology became established, a rearrangement of medical literature became necessary and the phenomena became grouped about their causes, so far as known. It followed that various phenomena which had been regarded as distinct diseases were in some cases due to a common cause. In other cases a phenomenon classed as a disease has been split up because the phenomenon was inconstant in its cause. In primitive veterinary literature glanders and farcy were described as distinct affections, but later it became known that the two supposed diseases were merely separate phenomena resulting from one cause. Nasal gleet occupied a prominent place in primitive veterinary literature, but as veterinary science advanced the phenomenon of nasal discharge was split up, assigned in each case to the disease of which it constituted one symptom, and nasal gleet disappeared from the pages of veterinary literature. 
The pathologic processes occurring in the genitalia of animals have been almost wholly described under the primitive method, each striking phenomenon being elevated to the dignity of a disease. Consequently a certain infection, by causing a wide variety of outstanding phenomena, injected into veterinary literature numerous alleged "diseases" each due to a single infection. It was unavoidable also that a given phenomenon, such as fetal death, which may be due to numerous different infecting agents, should be known as one disease. Thus abortion, metritis, retained fetal membranes, epididymitis, semino-vesiculitis, and dysentery of the new-born, all of which in a given series of cases may be due to an identical infection, have been described as six distinct diseases and scattered from end to end of veterinary literature.

The re-casting of the genital diseases of animals into a systematic treatise is a formidable task, the first effort at which must be very imperfect. In 1909 I published "VETERINARY OBSTETRICS; INCLUding THE Diseases OF BREedING ANIMALS AND OF THE NEW BorN", in which many of the diseases of the genital organs were included under the primitive plan. It was abandoned at the exhaustion of the first edition. In 1917 I published "VETERINARY OBSTETRICS" and began the preparation of the present volume. The two treatises have been designated "companion volumes" because they are closely allied in subject matter and may be profitably studied together.

The present treatise appears at a critical period in the history of the diseases of the genital organs of animals, when an old, firmly entrenched belief is slowly crumbling and a modern one is struggling for recognition. Abortion has long been regarded as a specific infectious disease, due in a given species of animals to one bacterium and to one only. If an exception arose and a given abortion or group of abortions was apparently due to an infection other than that specified for the species of animal concerned, it was not infectious abortion but merely abortion due to infection.

The belief in a specific infectious abortion has occupied 
the central position in the literature upon genital diseases in animals so long, that anyone attempting to write a systematic treatise in this field must either make infectious abortion the cornerstone or reject it wholly. While in this treatise it is held that all abortions in animals (except those induced surgically) are due to infection, it is denied that such infection is specific, as that term is commonly understood in medicine. It is denied as a principle in pathology that conception, parturition, birth, or other physiologic act draws across the path of life an impenetrable barrier to disease. It is held that any infection competent to invade a female may likewise invade a male; if able to invade a pregnant animal, it may also invade the same animal when nonpregnant; if it can gain a habitat in the pregnant female, it may persist after parturition; if it can invade and imperil the life of an embryo or fetus, it may continue through birth and affect the new-born, or may attack the new-born from external sources. Any infection which can invade and injure or kill a post-natal animal, may cause the same injury to it while intra-uterine, provided that contact is made. If tuberculosis or syphilis exists in the pregnant uterus, the infection may invade the embryo. There is no evidence to show, and no reason to believe, that any acute infection may not attack and destroy the embryo if the virus is brought into contact with it. It is true that acute infections, when attacking a pregnant female, rarely if ever pass the intact placental filter and reach the embryo. It does not follow that the embryo is immune to the infection, but merely that it is in a hermetically sealed sac, so isolated that the virus fails to acquire contact.

Although the placental isolation of the embryo is efficient against filterable viruses, the supporters of the "specific infectious abortion" theory hold that certain comparatively large bacteria defy this important law, invade the pregnant female, pass directly to the existing embryo, and cause its death and expulsion. But in invading the pregnant female, presumably through the mouth, the infection is alleged to reach the endometrium and embryo without leaving behind 
any lesion in its path or causing any disease or sign of disease.

This treatise is built upon the belief that abortion is only an incident in the ravages of infections existing within the pregnant uterus at the date of conception, or deposited with the semen at coitus. The infection is not specific, is not limited to one species of bacterium, invades organs other than the uterus, and involves both sexes and all ages. This general infection causes a long list of phenomena or lesions. The basic conflict of view with the majority of investigators will inevitably draw much unfavorable criticism. In order to justify the views stated, controversial matter has been included. This is regretted because it increases the size of the volume, and because some highly esteemed colleagues may erroneously regard such statements as personal reflections.

The conflicting views have served to delay publication. A belief so long and thoroughly entrenched as that of specific infectious abortion can not be overthrown quickly. Neither would the overthrow of what I believe an error be a gain unless a foundation were ready upon which a more valuable structure could be erected. I made the first open attack upon the specific infectious abortion theory in 1912, and that has been followed periodically from various angles. But external attack has not been as effective as internal disintegrating forces. The champions of a specific infectious abortion have admitted that at least several different species of bacteria may and do cause abortion in a given species of animal, and that the lesions caused by the several bacterial forms are not differentiable clinically or macroscopically. A most serious drawback to the theory has been its failure in practical application. It was announced twenty-five years ago that, since the cause of infections abortion had been proven, it could be controlled. The twenty-five years which have elapsed have seen no progress in the control of abortion based upon the specific theory. The prevalence of abortion is greater today than at any prior date in history. During the past decade there has been developing gradu- 
ally another view which appears more logical, is in harmony with the general principles of pathology, and is in full accord with all data recorded by the believers in specific infectious abortion. That portion of the volume designated "The General Genital Infections" must be regarded as the cornerstone; if the principles it has been attempted to establish there break down, it fails as a systematic treatise. The principles which it is attempted to establish place the common and most destructive genital infections upon a plane analogous to wound infection. Various bacteria exist in the genitalia of both sexes of all ages and species. The virulence and variety vary at different times and in different individuals. They are modified by general health, coitus, pregnancy, parturition and other critical incidents in life.

The belief in a specific infectious abortion logically demands for the control of the disease the isolation of the pregnant female from all possible carriers of infections; in this treatise it is held that physiologic reproduction is based upon coitus between two sexually healthy animals. The first is a hypothesis of despair, confirmed by lamentable failure over a period of twenty-five years: the latter a doctrine of hope, supported by success. Before publishing this treatise it was regarded as essential that the principles should have been tried thoroughly and proven successful in the crucible of practical clinical application. This has been a difficult task requiring unbounded and infinite patience under great discouragements and against opposition.

The treatise is inevitably very imperfect in every part, and those who are inclined to criticise will have excellent opportunity. But the volume was not made to cause or to avoid criticism. It is a first effort in a new field which, it is hoped, may be helpful to some and which may serve to awaken a new interest in the diseases interfering with reproduction in animals. The call of the hour to veterinarian and breeder is for increased efficiency of each domestic animal. The room for animal numbers is decreasing, while the demand for service in the production of milk, meat, labor or other products is increasing. Most of the great animal 
plagues which imperil the lives of adults have been brought under measurable control, but those infections involving the safety of the embryo are constantly gathering force. Yet it must be remembered that the sexual health of those animals desired for reproduction is the first basic need in animal husbandry. Reproductive efficiency in purebred cattle, swine, and horses, so far as can be estimated, has dropped below 60 per cent. of the ideal and continues to descend at a discouraging pace. The condition can be relieved only through conscientious and skillful work by veterinarians and breeders. The problem is difficult and the duration of the task infinite. It calls for the adoption of a permanent policy of guarding sexual health in animals-not of "curing" established lesions. The advancement of reproductive efficiency in the best animals is at present the greatest economic problem in veterinary medicine.

In a higher realm the study of genital diseases in domestic animals may be made of incalculable value in the elucidation of the basic principles of genital diseases in man. Aside from the specific venereal diseases of man, the genital infections are probably analogous to, and possibly identical with, the general genital infections of animals. What is true of the intra-uterine infections of the embryo in domestic animals is presumably equally true in principle of the human embryo. The veterinarian has opportunities for the study of the infections of the genital organs and embryos of animals wholly denied to practitioners of human medicine. The veterinarian has freely available in the abattoir the genitalia in health and in disease of thousands and millions of freshly slaughtered cattle, sheep, and swine of all ages and of both sexes. The females are pregnant and non-pregnant and the embryos are of all ages, diseased and healthy. Clinically the veterinarian can palpate the genital organs of the cow and mare freely per rectum and read the anatomical lesions of ovaries, oviducts and uterus as the blind read braille. The fetus can be manipulated, its movements in response to mechanical stimulation studied, and its physiologic function of swallowing its amniotic fluid recognized. 
The new-born animal can be studied freely regarding the infections carried from the uterus, can be fed experimentally, and may be destroyed at will for examination. If the veterinary profession is fully to live up to its opportunities and discharge its obligations to the state, it must contribute its share to the advancement of the knowledge of disease. Nowhere else can it contribute more, nor as much, of value, as in the basic principles of the physiology, pathology and hygiene of reproduction.

Purely a clinician, I have been dependent upon others for bacteriologic and histologic facts. In this field Drs. W. A. Hagan and C. M. Carpenter have rendered invaluable aid. Working first in my department and later in the department of pathology and bacteriology, they have extended every possible assistance. Similar help has been given by Dr. Herbert L. Gilman, at present in my department. The reader should undertsand the difficulty of securing knowledge regarding genital infections in animals. In general bacteriologists have not studied the genitalia of animals for infections, but for one specific infection, and have left unrecorded and unstudied all others. If the bacillus thought by the investigator to be the cause of infectious abortion was recognized, that was deemed final proof of it, and no other, caused the disease. Drs. Hagan, Carpenter, and Gilman have so far as practicable studied all bacteria found in the genital organs and their significance in the problem of reproduction.

My collaborator, Dr. W. W. Williams, has contributed greatly to the value of the treatise. Having devoted his entire energy for a number of years to the handling of genital diseases and the advancement of reproductive efficiency in purebred cattle, he has demonstrated with unusual clearness the value of the scientific handling of this group of infections. He has contributed especially the sections upon the surgery of the cervix and the genital infections of bulls.

Cornell University

W. L. Williams.

Ithaca, N. Y.

April, 1921 



\title{
TABLE OF CONTENTS
}

\author{
PAR'T I
}

\section{DISEASES AND DEFEC'TS NOT A'TRIBUTABLE TO INFEC'TION}

\section{CHAPTER I}

THE ANATOMY AND PHYSIOLOGY OF THE GENITALIA

THE GENITAL ORGANS OF THE MALE

The Testicles and Their Excretory Duct

'The Vesicula Seminalis, Prostate, and Cowper's Glands ... Io

The Penis . . . . . . . . . . . . . I4

The Sheath and Prepuce . . . . . . . . . 15

The Genital ORgans of the FeMale ........ is

The Ovaries ................... Is

The Muellerian Ducts . . . . . . . . . . . . . 22

The Oviducts ............. . . . . 25

The Uterus . . . . . . . . . . . . 26

The Cervix ............ . . . . . . . 33

The Vagina . . . . . . . . . . . . . . . . 36

Gärtner's Ducts . . . . . . . . . . . . . 39

The Vulva . . . . . . . . . . . . . . . 4 I

The Physiology of the Genitalia . . . . . . . . . 44

The Ripening of the Ovisac and Ovum . . . . . . . . 44

Estrum . . . . . . . . . . . . . 47

Orulation . . . . . . . . . . . . . . . . 49

Menstruation ................ . . 5 I

The Corpus Luteum of Estrum . . . . . . . . . 52

Copulation ... .............. 53

The Ejaculation of Semen ........... . . 53

The Migration of Spermatozoa . . . . . . . . 54

Fertilization .............. 55

The Migration and Implantation of the Orum . . . . . 55

The Corpus Luteum of Pregnancy . . . . . . . . 56

The Fetal Membranes . . . . . . . . . . . $5_{5} 8$

\section{CHAPTER II}

THE CLINICAL EXAMINATION OF THE GENITALIA . . . 73

The Clinical, Examination of THE Male Genitalia * . 73

The Testicles . . . . . . . . . . . 74

The Epididymis . . . . . . . . . . . 75

The Seminal Vesicles, Prostate, and Cowper's Glands . . . 76

The Semen and Spermatozoa . . . . . . . . . 77 
The Clinical, Examination of the Female Genitalia a . 79

The Examination of Vulva and Vagina ...... . 84

Rectal Palpation................ 89

The Examination of the Cervical Canal and Douching of the

Uterus ..................... 99

The Diagnosis of ESTRUM ............ . . . III

The Diagnosis of Pregnancy . . . . . . . . . . . II 3

CHAPTER III

ARTIFICIAL INTERFERENCES WITH REPRODUCTIVE

FUNCTIONS ...................... I40

THE INDUCTION OF ESTRUM . . . . . . . . . . . I4O

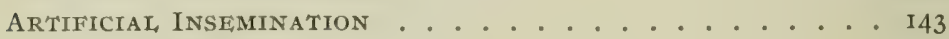

ARTIFICIAL ABORTION . . . . . . . . . . . . I46

CHAPTER IV

ARRESTS AND ABERRATIONS IN GENITAL DEVELOPMENT I5I

ASEXUAL OR BISEXUAL, ABERRATIONS . . . . . . I5I

Neuters or Freemartins .......... I5I

Hermaphroditism . . . . . . . . . . . I57

ARRESTS IN THE DEVELOPMENT OF THE MALE GENITALIA . . I6O

Arrests and Aberrations in the Development of the Testes . . I60

Cryptorchidy . . . . . . . . . . . . r6o

Aberration in Position of Scrotal Testes . . . . . . I6I

Arrest in Functional Development of Scrotal Testes, Fetal

Testes ............... I6I

Aberrations in the Development of the Penis . . . . . I63

CONGENITAL, DEFECTS OF THE FEMIALE GENITALIA . . . . . I63

Uterus Unicornis. . . . . . . . . . . 165

Double Uterus, Double Cervix . . . . . . . . I65

Persistence of Median Walls of Muellerian Ducts in Vaginal

Area, Double Vagina . . . . . . . . . . . 167

Imperforate Hymen, Persistent Hymen . . . . . . . I69

CHAPTER V

MISCEIIAANEOUS DEFECTS AND DISEASES INTERFERING

WITH FERTILITY . . . . . . . . . . . I72

Umbilic Hernia . . . . . . . . . . . 172

Ventral Hernia .............. I74

Horizontal Vulva ................ 174

Stricture of Vagina and Vulva . . . . . . . 175

Diseases of the Feet and Limbs . . . . . . . . 177

Paralysis, Plumbism . . . . . . . . . . 179

Torsion of the Testicle . . . . . . . . . . ISO

Tranmatic Orchitis .............. I8I

Tubal Pregnancy ...................... 185

Vaginal Hernia . . . . . . . . . . 185

Torsion of the Uterus . . . . . . . . . . . 189

Pelvic Tumors and Calluses ........... I90 
CHAPTER VI

SYSTEMIC DISEASES AND DERANGEMENTS INTERFERING WITH REPRODUC'TION . . . . . . . . . . . . . 192

Physical Overwork ................... I92

Starvation ........................... 193

Obesity ....................... I94

Idleness and Overfeeding ................ I96

Sexual Excess : . . . . . . . . . . 200

Onanism or Masturbation ........... 203

CHAPTER VII

COITAL INJURIES . . . . . . . . . . . . 205

Kicks and Fractures . . . . . . . . . 206

False Copulation, Rectal Injuries . . . . . . . 2IO

Laceration and Rupture of the Vagina ........ . 214

Coital Rupture of the Uterus . . . . . . . . . 216

Coital Rupture of the Urinary Bladder . . . . . . . . 217

Vaginal Hemorrhage following Coitus ........ 218

Urethral Hemorrhage in Stallion .......... 2 2 S

Contusions of Neck and Withers .......... . 219

Penial Injuries .............. . . 2 I9

Rupture of Prepuce ............. . . 223

Strangulated Hernia . . . . . . . . . . . 225

CHAPTER VIII

PARTURIENT INJURIES ............ . . $22 \mathrm{.}$

Lacerations of Perineum . . . . . . . . . 228

Recto-Vaginal Fistula . . . . . . . . . 229

Lacerations of Cervic . . . . . . . . 229

CHAP'TER IX

SODOMY AND SADISM

\section{PAR'T II}

\section{TUMORS OF THE GENITALIA}

\section{CHAPTER X}

MALIGNANT NEOPLASMS . . . . . . . . . . 239

Malignant Tumors of the Male Genitalia . . . . . . . 239

Amputation of the Penis . . . . . . . . . . 245

Malignant New-Growths of the Female Genitalia . . . . 250

CHAPTER XI

BENIGN TUMORS OF THE GENITALIA . . . . . . . . 253

Benign Tumors of the Male Genitalia . . . . . . . . . 253

Benign Tumors of the Female Genitalia . . . . . . . . 257

Ovariotomy ...................... 263 


\title{
INFEC'TIONS OF 'THE GENITAL ORGANS
}

\author{
SECTION I. THE GENITAL INFECTIONS OF CATTLE \\ CHAP'TER XII
}

THE SPECIFIC VENEREAL DISEASES . . . . . . 278

The Vesicular Venereal Disease of Cattle . . . . . 278

The Nodular Venereal Disease ......... 283

CHAPTER XIII

YON-VENEREAL INFECTIONS WHICH INVADE THE GENITAL

ORGANS .......................... 317

Genital Actinomycosis . . . . . . . . 317

Genital Tuberculosis . . . . . . . . . . 319

\section{CHAPTER XIV}

THE GENERAI, INFECTIONS OF THE GENITAL ORGANS . . 356

I. INFECTIONS OF THE GENITAL ORGANS OF BULLS . 358

ORCHITIS . . . . . . . . . . . . . 375

Degenerative Orchitis. Destruction of Spermatogenetic Epithelium in Calves . . . . . . . . . . 375

Orchitis in Adult Bulls. Abscessation and Necrosis of Testicles 379

Epididymitis . . . . . . . . . . . . . 385

Degenerative Epididymitis of Calves . . . . . . 385

Arrest in Development . . . . . . . . . . . 385

Chronic Indurated Epididymal Abscesses of Calves . . . 386

Epididymitis of Adult Bulls . . . . . . . . . . 388

INFECTIONS OF THE GLANDS OF THE PELVIC URETHRA . . . . 389

Spermato-Cystitis . . . . . . . . . . 389

Diseases of Prostate and Cowper's Glands . . . . . . . 393

Balantitis. Balano-Posthitis. "ThE GRanUlar Venereal,

DISEASE" . . . . . . . . . . . . 393

II. GENERAL INFECTIONS OF THE GENITAL ORGANS OF HEIFERS AND COWS

DISEASES OF THE OVARIES. OVARITIS . . . . . . . . 394

Atretic Follicles . . . . . . . . . . . . . 395

Cystic Degeneration of the Ovaries, Nymphomania . . . 395

Intra-Follicular Hemorrhage . . . . . . . . . . . 409

Post-Ovulation Hemorrhage into the Follicular Crater . . . 4 II

Hemorrhagic Corpus Luteum . . . . . . . . . 4I2

Cystic Degeneration of the Corpus Luteum . . . . . 4 43

Hypertrophy of the Corpus Luteum . . . . . . . . 418

Persistent Corpus Luteum . . . . . . . . . 42 I

Central or Embedded Corpus I,uteum. The Corpus Luteum of

Pyometra and of Retained Fetal Cadaver . . . . . . 422 
Abscess of Ovary ...................... 424

Corpora Nigra . . . . . . . . . . . . . . . 425

Par-Ovarian Cysts . . . . . . . . . . . 425

'IUbal, INFECTIONS . . . . . . . . . . . . . . 426

Salpingitis, Pavilionitis, Adherent Ovary ...... 428

Pyosalpinx. Tubo-Ovarian Abscesses . . . . . . . 445

Hydrosalpinx. Dropsy of the Pavilion . . . . . . . 448

Cysts in the Mesosalpinx . . . . . . . . . . . 452

UTERINE INFECTIONS . . . . . . . . . . . . . 453

The Diseases of the Uterus of the Heifer Calf and Virgin Heifer 453

Intra-Uterine Infection and Endometritis . . . . . . 453

Pyometra . . . . . . . . . . . . . 458

THE INFECTIONS OF THE GRAVID UTERUS . . . . . . . . 462

Apical Endometritis with Necrosis of the Fetal Sac . . . . 462

Diffuse Findometritis, with Atony of Uterine Walls. Dystocia 464

Cervical Endometritis . . . . . . . . . . . 465

Uterine Gangrene . . . . . . . . . . . . . 467

Placentitis ................. . . . 469

Peripheral Placentitis with Necrosis and Dehiscence at the

Periphery of the Cotyledons ... . . . . . . . . . 469

Inter-Placental Hemorrhage with Desiccation of the Fetus.

IIummification . . . . . . . . . . . 470

Incarcerating Placentitis . . . . . . . . . 474

Necrosis of Cotyledons. Necrotic Placentitis . . . . . 474

Adventitions Placenta . . . . . . . . . 475

Calcification of Placenta . . . . . . . . . 476

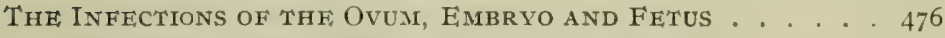

The Death of the Fertilized Ovum . . . . . . . 476

The Death of the Embryo, with Survival of the Embryonic Sac.

Cystic Mole . . . . . . . . . . . 477

Death and Maceration of the Embryo or Fetus. . . . . 480

The Pyometra of Fetal Decomposition ...... 480

Abscessation of the Gravid Uterus ...... . . $4 \mathrm{~S}_{4}$

Emphysema of the Fetus . . . . . . . . . 487

The Observed Expulsion of the Fetal Cadaver. Abortion . . 487

The History of Abortion . . . . . . . . . . 488

The Prevalence and Frequency of Abortion ..... . 49r

"Accidental Abortion" . . . . . . . . . . 495

"Food Abortion" . . . . . . . . . . . . . 496

The Biology of Abortion .......... . . 498

The Colon-Like Bacillus and the Micrococcus of Nocard 498

The Nodular Venereal Disease of Isepponi . . . . 499

The Bacterium Abortus of Bang . . . . . . . . 499

The Paratyphoid Bacillus of Moussu ..... . 5 I I

The Spirillum of Smith and others ... . . . . 5II

Miscellaneous Bacteria........ 5 I I 
The Nature of Abortion .......... . 5 II

The Symptoms and Diagnosis of Abortion . . . . 517

The Date of Invasion .......... 518

The Control of Abortion ......... . . . . 519

Diseases of the Puerperal Uterus . . . . . . . . 538

Uterine Hemorrhage. Uterine Hematoma ..... . 539

Endometritis . . . . . . . . . 546

Septic Metritis ............ 554

Placentitis, Cotyledonitis. Retained Fetal Membranes . 560

Puerperal Tetanus . . . . . . . . . . 584

Puerperal Laminitis . . . . . . . . . . 585

Puerperal Meningitis ........... 585

Post-Puerperal Uterine Infections . . . . . . . 586

Endometritis ........... . . . 589

Pyometra ............. . . 594

Uterine Abscess .............. . . 601

Abscessation of Uterine Submucosa . . . . . . . . 604

Sclerotic Metritis ... ......... 605

Pelvic Adhesions. Parametritis . . . . . . . . 606

Pyemia, Pyemic Arthritis . . . . . . . . 609

Pyemic Abscesses . . . . . . . . . . . . 609

Cystic Degeneration of Uterine Walls with Hydrometra . 6I I

Infections of the Cervix .........6 6I4

Cervicitis ............. 6 6 64

Retention of Menstrual Debris, Cystic Uterus . . . . 640

Retention of Fetus from Cervical Adhesions . . . . . 64I

Retention Cysts of Cervix ... . . . . . . . 642

Cervical Prolapse ........... . . . 642

Infections of the Vagina . . . . . . . . . 645

Vaginitis, The Nodular Venereal Disease . . . . 645

Gangrene . . . . . . . . . . . . 647

Perivaginal Phlegmon . . . . . . . . . 648

Cysts and Abscesses of Gärtner's Ducts . . . . . . . 649

The Infections of the Vulva . . . . . . . . 650

III. CONGENITAL, INFECTIONS OF CALVES

Dysentery Neonatorum, Cal, F SCOURS, White Scours, Calf

Pneumonia, Arthritis, Pyemic Abscesses . . . . . . . 65I

THE PROBLEM OF THE GENITAL, INFECTIONS OF CATTLE AS A

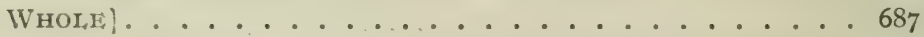

The Assembling of Herds and the Addition of Cattle to

Existing Herds . . . . . . . . . 688

Equipment ............... . . 694

The Systematic Handling of Herds for the Control of

Genital Infections . . . . . . . . . . . 697

The Genital Infections of Cattle as an Economic Problem 708 
The Genital INFections of CatTle in their RELAtion to

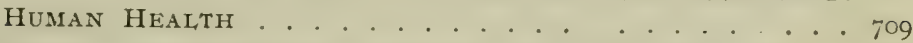

SECTION II. THE GENITAL INFECTIONS OF SHEEP AND GOATS CHAPTER XV

THE SPECIFIC VENEREAL DISEASES . . . . . . . 7I3

The Nodular Venereal Disease ......... . . 7 I 3

CHAPTER XVI

NON-YENEREAL INFECTIONS IVHICH INVADE THE GENITAL

ORGANS ......................... 713

Necrotic Disease of the Genital Organs Associated with Lipand-Leg Ulceration ................ $7 \mathrm{I} 3$

CHAPTER XVII

THE GENERAL, GENITAL INFECTIONS OF SHEEP AND GOATS 717

Abortion Associated with a Vibrio or Spirillum ... . . . 717

Retained Placenta in Ewes.... . . . . . . . 7r7

SECTION III. GENITAL INFECTIONS OF SWINE,

CHAP'TER XVIII

SPECIFIC VENEREAL INFEC'TIONS . . . . . . . . 736

The Nodular Venereal Disease........ 736

CHAPTER XIX

NON-VENEREAL SPECIFIC INFECTIONS INVADING THE

GENITALIA OF SWINE . . . . . . . . . . . . 736

Tuberculosis ................ . . 736

CHAPTER XX

THE GENERAL INFECTIONS OF THE GENITALIA OF SWINE . 738

Metritis, Death and Maceration of Embryos, Abortion . . 738

SECTION IV. GENITAL INFECTIONS OF HORSES $75^{2}$ CHAPTER XXI

THE SPECIFIC VENEREAL DISEASES OF HORSES . . . . 752

DOURINE . . . . . . . . . . . 752

GENITAL HORSE POX . . . . . . . . . 766

\section{CHAPTER XXII}

NON-VENEREAL SPECIFIC DISEASES WHICH INVADE THE

GENITALIA OR AROUSE INFECTIONS ALREADY EXISTING

WITHIN THE GENITAL, TRACT . . . . . . . . . 770

INFRCTJOUS CELLULITIS. "PINK EYE." . . . . . . . 770

BURSATTEE . . . . . . . . . . . 775

CHAPTER XXIII

THE GENERAL, INFECTIONS OF THE GENITAI, TRAC'T. "CON-

TAGIOUS ABORTION." ........... 778

INFECTIONS OF THE GENITAL ORGANS OF STALLIONS . . $7 \mathrm{~S}_{2}$

ORCHITIS AND EPIDIDYMiTIS .......... . $78_{2}$

Diseases of Seminal Vesicles, Semino-Vesiculitis . . . . 785 
II. GENERAI, INFECTIONS OF THE GENITAI, ORGANS OF MARES DISEASES OF THE OVARIES. OVARITIS . . . . . 788

Atretic Follicles . . . . . . . . . . $78 s$

Cystic Degeneration. Nymphomania . . . . . . . 788

UTERINE INFECTIONS . . . . . . . . . . . . . 793

Infections of the Gravid Uterus, Ovum, Embryo or Fetus.

Abortion .............. . . 793

Diseases of the Puerperal Uterus, Retained Afterbirth, Retention of Non-Gravid Horn of Chorion, Endometritis, Puerperal Laminitis . . . . . . . . . . 799

Metritis. Metro-peritonitis .......... 803

Post-Puerperal Infections . . . . . . . . . . . 805

Endometritis ........... . . . So5

Uterine Abscess . . . . . . . . . . . . . 806

Pyometra .............. . . . 807

Cervicitis ............ 8 . . . . . .

Vaginitis . . . . . . . . . . . SII

Vulvar Gangrene . . . . . . . . . . . 813

III. CONGENITAL INFECTIONS OF FOALS SI4

Septicemia of the Foal............ SI4

Retention of the Meconium ......... 8I4

Dysentery............... . 8I5

Arthritis. Pyemia ............. 815

Re-Opening of Urachus ......... 820

Rupture of Tendons ........... 824

SECTION V. THE GENITAL INFECTIONS OF CARNIVORA 828 CHAPTER XXIV

SPECIFIC VENEREAL DISEASES

The Venereal Tumors of Dogs ........ . 828

CHAPTER XXV

THE GENERAL INFECTIONS OF THE GENITALIA

Prostatitis of Dogs. Abortion, etc........ 83 I

CHAPTER XXVI

VENEREAL DISEASE OF RABBITS .......... . . 838 



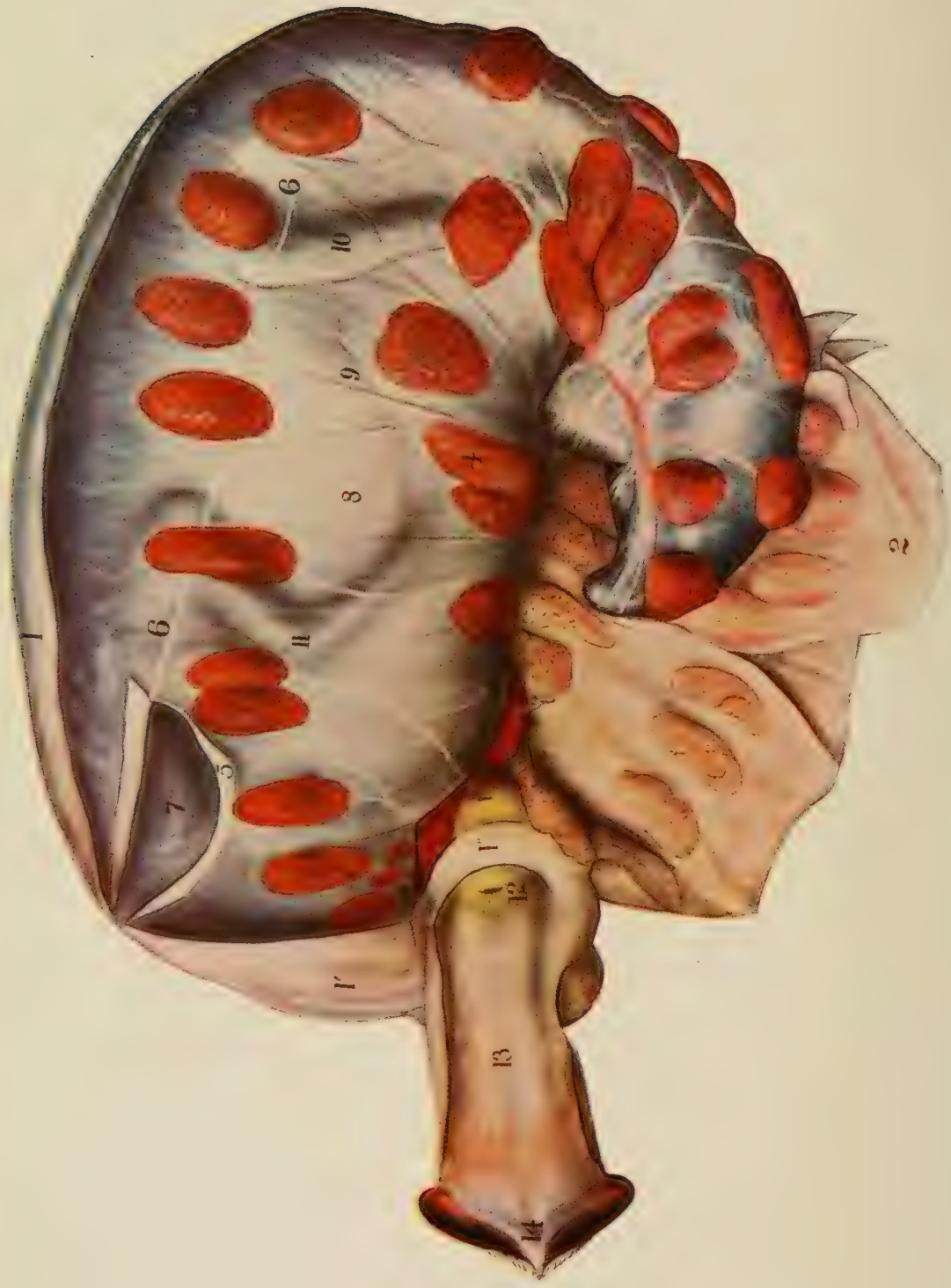




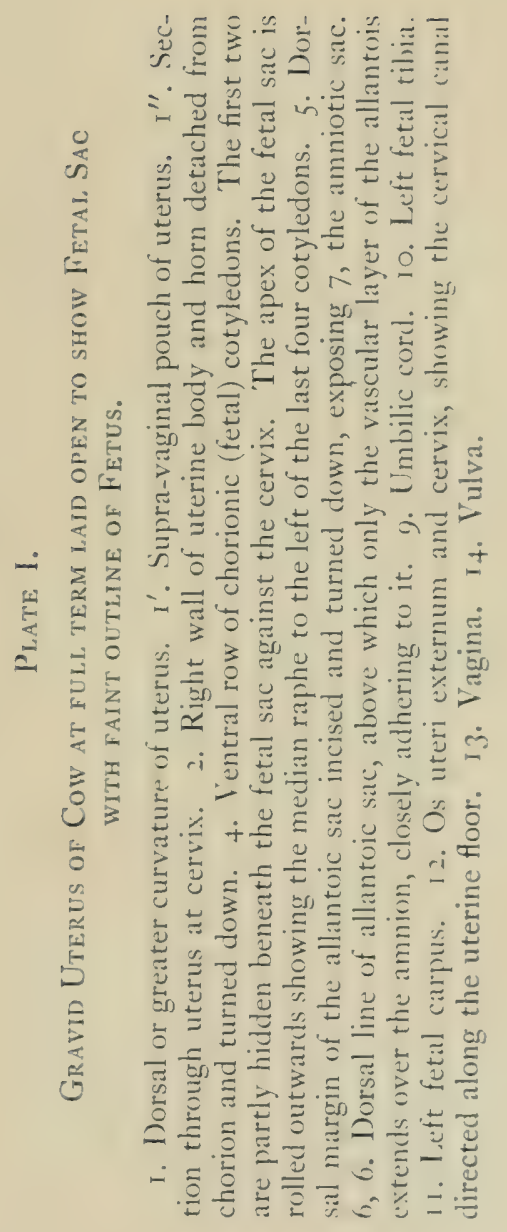





\title{
DISEASES OF THE GENITAL ORGANS NOT ATTRIBUTED TO INFECTION
}

\author{
CHAPTER I
}

\section{THE ANATOMY AND PHYSIOLOGY OF THE GENITAL ORGANS}

\section{A. The Male Genitalia}

The male genital organs consist of the testicles, epididymes, vasa deferentia, vesiculae seminales, prostate and Cowper's glands, and the penis with its sheath and prepuce.

Prior to the disappearance of the Wolffian bodies in the embryo, there appear near their anterior end at their median side, two parallel thickenings in the peritoneum designated the genital ridges, which by an elaboration of their epithelium ultimately form the genital glands. At first they cannot be differentiated, but they quickly assume definite sex characters and can be recognized as male (testicles) or female (ovaries) glands.

\section{The Testicles AND Their Excretory DuCts}

a. The testicles at first lie against the vertebral column, in close proximity to each other, between the anterior ends of the Wolffian bodies. The essential structures of the testicles, the tubuli seminiferi and the specialized cells elaborating the spermatozoa, are formed from the peritoneal cells of the genital ridges. The contiguous peritoneal areas draw over the genital ridges, perpetuating the general peritoneal surface while the glandular mass of the testicle lies outside or above the peritoneal cavity.

The Wolffian bodies soon disappear as independent structures. The anterior end of each Wolffian body later forms the head of the corresponding epididymis, becoming continuous with the proximal end of the testicle, upon its lateral 
side. The Wolffian duct, after the virtual elimination of the Wolffian body, undergoes elaboration to constitute the body and tail of the epididymis and the vas deferens.

The artery of the testicle derived from the posterior aorta, is at first very short, but with the descent of the gland into the scrotum, it becomes one of the longest unbranched arteries in the body. The artery with its satellite vein, nerves, and lymphatics, and the investing peritoneum, constitutes the vascular or spermatic cord, one of the three fundamental connections of the testicle with the body walls.

A second attachment of the testicle to the body wall consists of the fibrous, cord-like gubernaculum testis. It is formed at about the same date as the genital ridge and extends from that part of the external integument, which is to constitute the fundus of the scrotum through the inguinal space, internal inguinal ring, and thence outside the peritoneum, upward, forward, and inward across the tail of the epididymis to the posterior end of the testicle to which it is attached.

As the testicle increases in volume and weight, it drops down into the peritoneal cavity towards the internal inguinal ring. In its descent the three attachments of the testicle, the vascular or spermatic cord (artery, vein, nerves, and lymphatics), vas deferens, and gubernaculum testis, each drags with it the peritoneum which furnishes to each structure a double fold, thus maintaining the continuity of the visceral with the parietal peritoneum, resulting in three double peritoneal folds, as shown in Fig. 1 . When the testicle descends into the scrotum, the gubernaculum shortens throughout its course. In solipeds the tension of the gubernaculum, prior to the complete descent of the testicle, exerts a degree of traction upon its scrotal attachment sufficient to cause a depression or dimple in the scrotum. The approach of the testicle to the internal inguinal ring is accompanied by an evagination of peritoneum through it anterior to the gubernaculum, to constitute the processus vaginalis, which eventually forms the parietal peritoneum of the scrotum. When the testicle descends, it and the spermatic cord carry 
down their peritoneal fold which extends from the gubernaculum below the origin of the spermatic artery in the lumbar region. It is attached anteriorly to the spermatic cord and posteriorly to the scrotal wall, to form the mesorchium which constitutes a membranous median partition in the posterior part of each scrotal sac from the fundus to the internal inguinal ring, as shown in Figs. 2, 3, and 4. The processus vaginalis, in everting, pushes before it on the lateral side the as yet invisible cremaster which quickly develops when the testicle descends into a bright red, highly functioning muscle. The scrotal sac thus consists of two distinct groups of tissues, the general integument composed of the skin and dartos and the intra-abdominal portion consisting of the parietal peritoneum and the cremaster muscle with the intervening fasciae. The two groups of structures remain easily separable throughout life. The surgeon in performing the "covered operation," when castrating animals with scrotal hernia, avails himself of this distinction

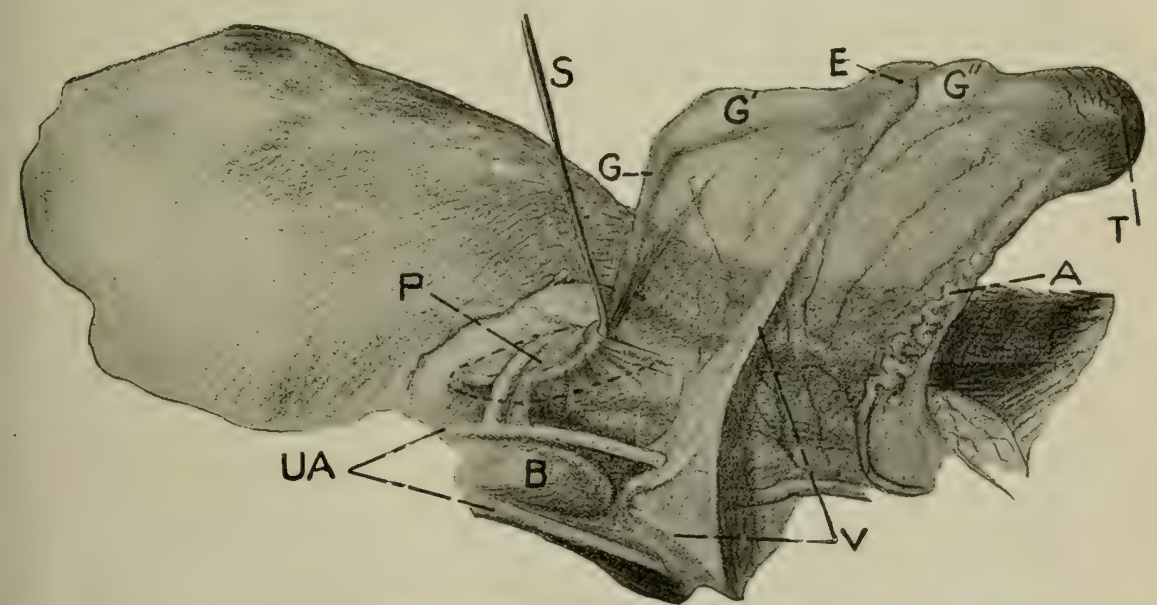

FIG. I. - The Testicle and its Attachments.

Right inguinal region and testicle of $24 \mathrm{hrs}$. colt. $P$, Processus vaginalis surrounded by a dotted line and containing a curved sound, $S ; G$, first portion of gubernaclum testis; $G^{\prime}$, second portion of gubernaculum testis extending to the epididymis, $E$; $E$, epididymis ; $G^{\prime \prime}$, gubernaculum extending from epididy mis (globus minor) to the testicle; $T$, testicle; $A$, testicular artery $V, V$, vasa deferentia; $B$, urinary bladder; $U A$, umbilical arteries. 
and readily separates the two groups between the dartos and cremaster so that he may ligate the structures of intraabdominal origin just at, or near to, the internal inguinal ring.

In domestic animals, except the elephant, the descent of the testicles into the scrotum is a prerequisite to fertility; otherwise, spermatozoa are not formed.

In the descent of the testicle, the gubernaculum shortens, the processus vaginalis develops, and the tail of the epididymis with the caudal end of the testicle traverses the inguinal ring followed by the body and head of the epididymis and cephalic end of the testicle.

The descent of the epididymis and testicle of ruminants is directly downward. The gland comes to rest with the tail of the epididymis and that portion of the testicle which was, in the embryo, the posterior end directed downward. The long axis of the testicle is perpendicular to the spinal column. The epididymis thus rests upon the postero-lateral face of the testicle, its head directed toward the internal inguinal ring, its tail at the distal end of the testicle. The vas deferens passes inward across the lower margin of the mesorchium and turns upward on the postero-median surface of the testicle closely applied to it. The epididymis and vas deferens are thus parallel and separated from each other by the mesorchium or peritoneal fold connecting the visceral and parietal layers. It is important to remember this arrangement of structures when examining clinically the testicles for evidences of disease.

In solipeds the cutaneous attachment of the gubernaculum testis is farther posterior and higher than in ruminants. This draws the tail of the epididymis upward and backward causing its head and the corresponding end of the testicle to tilt forward. The gland revolves through 90 degrees on its short axis and comes to rest with its long axis parallel to the spinal column. The vas deferens, instead of doubling back along the postero-median surface of the testis as in the ruminant, leaves the horizontal testicle immediately at the tail of the epididymis and passes obliquely upward and forward, to come in contact with the vascular cord in the inguinal canal. 


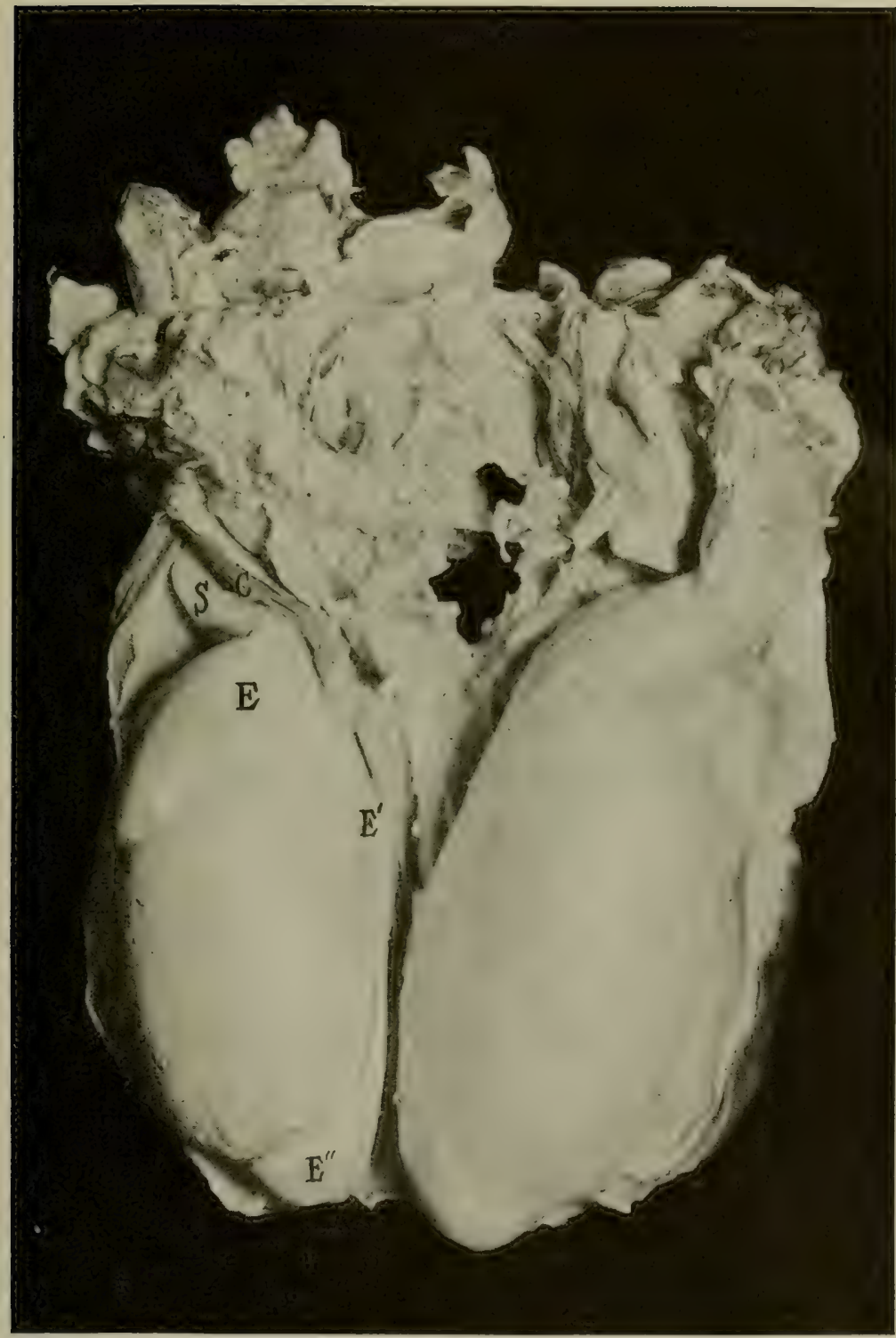

FIG. 2-Testicles of Bull.

Section through the posterior scrotal wall of the bull. The left testicle is fully exposed, the right is covered by the cremaster muscle and fascia and by the scrotal peritoneum. C. Cremaster muscle divided longitudinally ; $S$, spermatic cord ; $E$, head of epididymis ; $E^{\prime}$, body ditto ; $E^{\prime \prime}$, tail ditto. 
The gubernaculum of carnivora has its cutaneous attachment higher and more posteriorly than that of solipeds, the testicles are drawn further backward and upward so that they rest between the posterior portions of the thighs, and the glands are tilted so that their long axes are obliquely downward and forward from the tail of the epididymis. Finally, in swine, the cutaneous origin of the gubernaculum testis is located high in the perineal region just beneath the anus, and the tail of the epididymis with the lesser extremity of the testicle is caught and held at that point with the head of the epididymis and greater end of the testicle presenting almost perpendicularly downward. Its direction in the scrotum is almost the reverse of the testicle of the ruminant.

b. The epididymis, Figs. 2, 3, 4, is formed in part from the tubules of the Wolffian body and partly from the Wolffian duct. That portion of the epididymis derived from the tubules of the Wolffian body leaves the anterior or cephalic end of the embryonic testicle as a series of greatly coiled tubules which constitute an enlarged mass designated the head, or globus major, of the epididymis. The tubules all converge to become the single tubule representing the Wolffian duct. The latter, greatly coiled, forms the enlongated body of the epididymis which is equal in length to about three-fourths of the long diameter of the testicle. At the caudal or tail end of the testicle the single tube becomes greatly coiled to constitute a second enlargment, the tail, or globus minor. The tube then becomes less coiled and finally straight to constitute the vas deferens.

The head of the epididymis, through the tubules, the vasa deferentia, is continuous with the tubular structure of the testicle, and through these the spermatozoa escape from the gland and reach the single tube of the body and tail of the epididymis which in turn is continued by the vas deferens. Only the head of the epididymis, therefore, is continuous with the tissues of the testicle, while the body and tail lie closely moulded against the gland and attached to it through the agency of peritoneum and connective tissue. The tail and 
body of the epididymis are, therefore, only loosely attached to the testicle and these permit, under some circumstances, of wide separation from the gland. Thus in cryptorchidy in the horse, the gubernaculum, as shown in Fig. 1, crosses the tail of the epididymis and is closely attached to it. In entering the inguinal ring, the tail of the epididymis, with the gubernaculum, must lead and physiologically the tail of the testicle must accompany it. But sometimes the caudal end of the testicle becomes caught at the ring, the tail of the

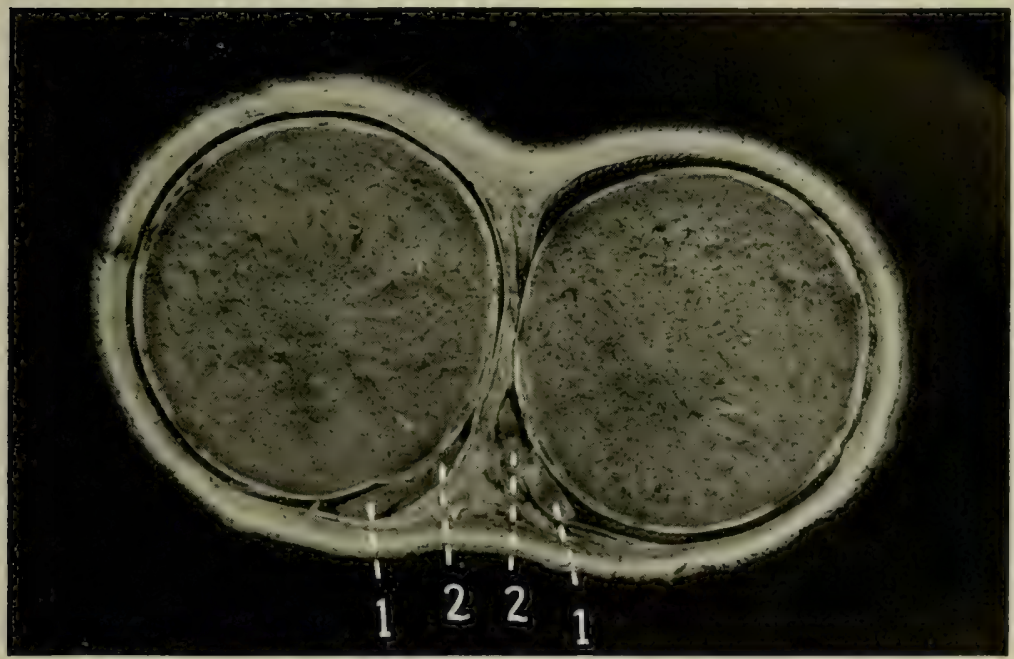

Fig. 3-Cross Section through Scrotum and Testicles of Bull Calf. $I, I$, Body of epididymis; 2,2 , vas deferens on the anterior side of the mesorchium.

epididymis, followed by the body, and even the head, passes through the ring, the testicle turns upon its transverse axis, and its head becomes incarcerated in the inguinal ring, its caudal end remaining within the abdomen. The inexperienced surgeon attempts to castrate the cryptorchid, encounters the epididymis in the peritoneal pouch and amputates it from the testicle under the impression that he is castrating the animal. Later a second operation is necessary and within the abdomen there is found a "third testicle." 
A study of Fig. 1 will show that in the descent of the testicle and attached structures, a broad peritoneal sheet, the mesorchium, is formed, extending from the spermatic artery, A, to the gubernaculum, G. Midway in this a second peritoneal duplicature leaves the broad expanse to envelop the vas deferens, $V$. The ruminant testicle descends directly and in doing so, the first section of the gubernaculum, $G$, passes through the inguinal ring followed by the tail

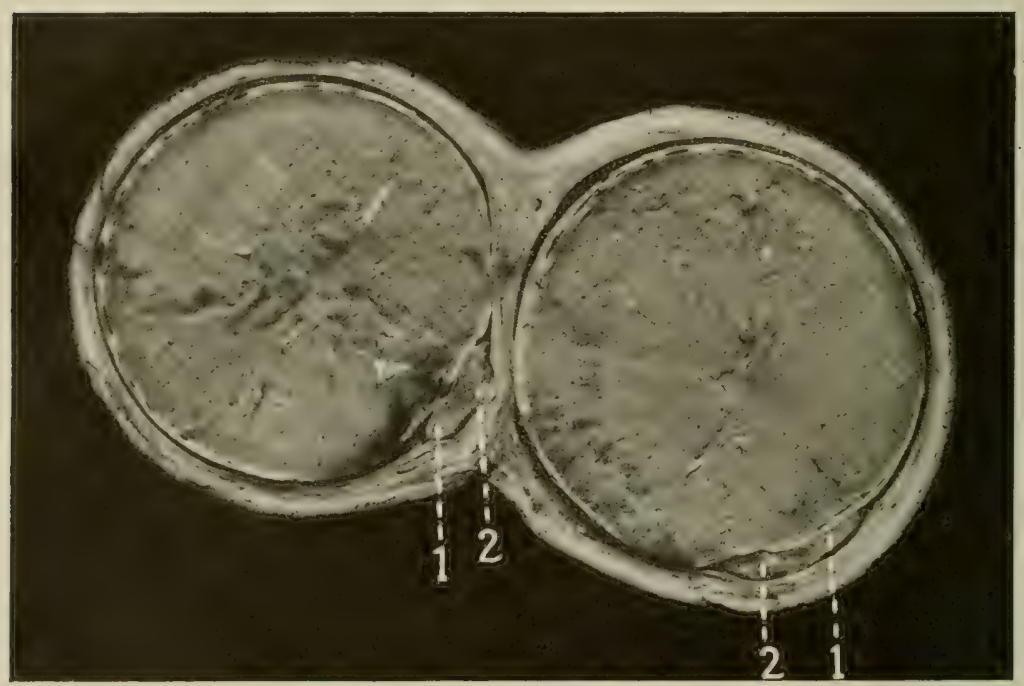

Fig. 4--Cross Section through Scrotum of Young Bull viewed from Posterior Side.

$I, I$, Body of epididymis ; 2,2 , vas deferens. The right testicle has revolved on its long axis so that the epididymis and vas diferens with the mesorchium between them rest upon the postero-external surface of the gland.

of the epididymis, E, with the caudal end of the testicle, and when this point reaches the fundus of the scrotum and the basal point of the attachment of $\mathrm{G}$ to the dartos, the descent is completed. The testicle of the bull descends in a direct line and when it comes to rest upon the bottom of the scrotum by its caudal end, it remains upright, with the epididymis lying against its median side on the outer side of the mesorchium, and the vas deferens upon its median side. The spermatic artery, A, instead of leaving the gland per- 
pendicular to the long axis, shifts its course and leaves the head end of the testicle in a direct line parallel to the long axis of the gland. This causes the vas deferens to turn abruptly at $\mathrm{E}$ and follow the mesorchium on its median surface along the testicle until at the cephalic end of the latter, it joins the artery to contribute to the spermatic cord. In the bull the gubernaculum is anchored in the median wall of the scrotum with the result that both the epididymis and vas deferens are normally applied against the postero-median side of the testicle, as shown in Figs. 2-4, the epididymis behind, and the vas deferens in front of the mesorchium. The head of the epididymis of the bull begins superiorly upon the lateral surface of the cephalic end of the testicle, passes across the posterior surface upward, medianward, and then downward, in the form of an arc. It is flattened against the surface of the testicle and is not materially enlarged to form a "globus major" as in the horse. The tail of the epididymis, or globus minor, on the other hand, is large and prominent. It projects definitely beyond the caudal end of the testicle and lies chiefly on the postero-median surface where it is readily palpable and visible through the scrotum. It is the dominant seat of infection in the epididymis, with or without abscessation, and as such merits special attention.

The descent of the testicle of the horse is at first fundamentally the same as that of the bull but upon reaching the scrotum, the head end tilts forward and finally comes to rest with its long axis parallel to the spinal axis. The epididymis consequently lies upon the dorsal surface of the testicle, lateral to the mesorchium, and the vas deferens, instead of turning forward along the median side of the mesorchium, is free from the gland and upon leaving the tail of the epididymis, passes directly upward and forward to join the artery near the external abdominal ring. This results in a very marked difference in relation and subjects the testicle of the horse to the possibility of torsion.

After the vas deferens has joined the spermatic artery, its behavior is essentially alike in all species. It accompanies 
the artery through the inguinal ring, parts there from it and, curving upward, inward, and backward, finally approaches its fellow from the opposite side, passes above the neck of the urinary bladder between or beneath the seminal vesicles, and enters the urethra from above. It is reaily palable per rectum in the horse and bull.

\section{The Vesiculae Seminales, Prostate, and COWPER'S GLANDS}

The subsidiary sex glands and appendages to the sex organs of domestic animals show wide variations. Each vesicula seminalis is formed as an outgrowth from the vas deferens. In most animals the vesicle consists of a long, intricately folded, coecal tubule which, when incised, appears like an acinous gland. In solipeds the structure assumes the form of a vesicle similar in outline to the urinary bladder. The two vesicles in the horse are oblong, about six to eight inches long by two inches in diameter, their anterior ends round. The posterior ends taper sharply to their outlet. They lie side by side above the neck of the urinary bladder and the termination of the vas deferens. Their walls are thick and their mucosa richly supplied with glands. The vesiculae secrete a thin mucoid fluid, presumed to dilute the semen at the time of ejaculation. Ordinarily the vesicle contains a small amount of this secretion, and occasionally a few spermatozoa make their way into the cavity, but the organ does not serve as a seminal reservoir in any domestic animal. In the gelding the vesiculae sometimes suffer from catarrhal accumulation. When attempting to catheterize geldings, the catheter sometimes passes into the dilated orifice of the vesicle and lodges, rendering it difficult, and sometimes impossible, for the operator to pass the catheter into the urinary bladder.

In ruminants the vesiculae seminales are composed of greatly elongated tubules so coiled as to give to the structure the appearance of an acinous gland. In the bull (See Figs. 5, 6, 7) the seminal vesicles are two oblong, nodular bodies varying with the size of the animal from two to three 


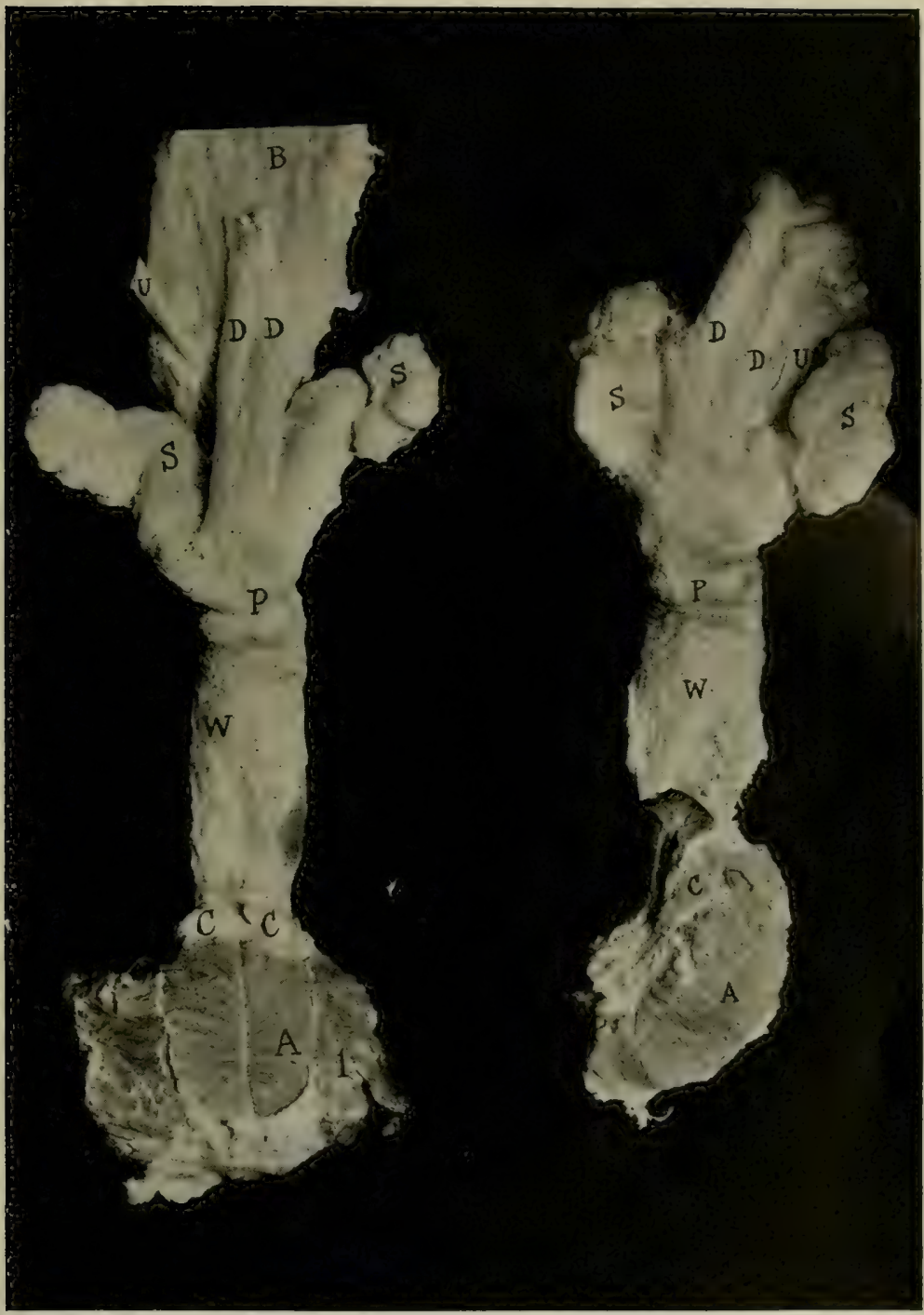

FIG. 5

Fig. 6

Glands Attached to Pelvic Urethra of Bull viewed from above.

Fig. 5. $A$, Accelerator urinae muscle sectioned horizontally ; $C, C$, Cowper's glands : $W$, Wilson's muscle ; $P$, body of prostrate ; $S, S$, seminal vesicles ; $D, D$, vasa deferentia ; $U$, ureter ; $B$, urinary bladder ; $I$, ischio-cavernosus muscle.

FIG. 6. A transverse section has been made through the posterior part "of IVilson's muscle and the urethra and a median section through the accelerator urinae muscle and Gowper's gland. $A$, Accelerator urinae muscle; $C$, Cowper's gland just above the urethra. Other lettering same as in Fig. 5. 
inches in length, one to one and a quarter inches in width, and approximately one half inch in diameter from above to below. They are readily palpated per rectum, by which means they are recognized as oblong bodies meeting posteriorly on the median line where their ducts empty into the urethra, while anteriorly they diverge and drop down upon a level with the urethra. They can be partially picked up and give to the examiner a sensation of a nodular, acinous gland. In swine the seminal vesicles are very large and similar in character to those of the bull. The structures are absent in the dog.

Physiologically they appear to be of fundamental importance in those animals where present since they apparently furnish a thin, mucoid secretion which contributes the greater volume of the seminal fluid. In those animals where it is not present, its place appears to be taken by an increased volume and activity of the prostate gland.

The prostate glands, lying across the urethra just behind the posterior ends of the seminal vesicles, are small in the horse.

The prostate of the bull is of about the same volume as either of the seminal vesicles. The body of the prostate is a small, yellowish, transverse band lying across the urethra just behind the termination of the seminal vesicles and in front of the anterior end of Wilson's muscle, as shown in Fig. 6. Posteriorly, it extends to the vicinity of Cowper's glands. It surrounds, but lies chiefly above, the urethra. On cross section it shows a U-shaped structure with the urethral canal lying in the bottom of the U. Below and at the sides, it is heavily covered by Wilson's muscle, and above, the aponeurosis of the muscle stretches across from side to side so that this, the more voluminous and important portion of the gland, is completely hidden from sight and touch. Only the body, or that portion not covered by Wilson's muscle, is palpable per rectum.

The gland is of moderate size in the boar. In carnivora the prostate glands are large and apparently perform the functions of the absent vesiculae seminales. It is virtually 


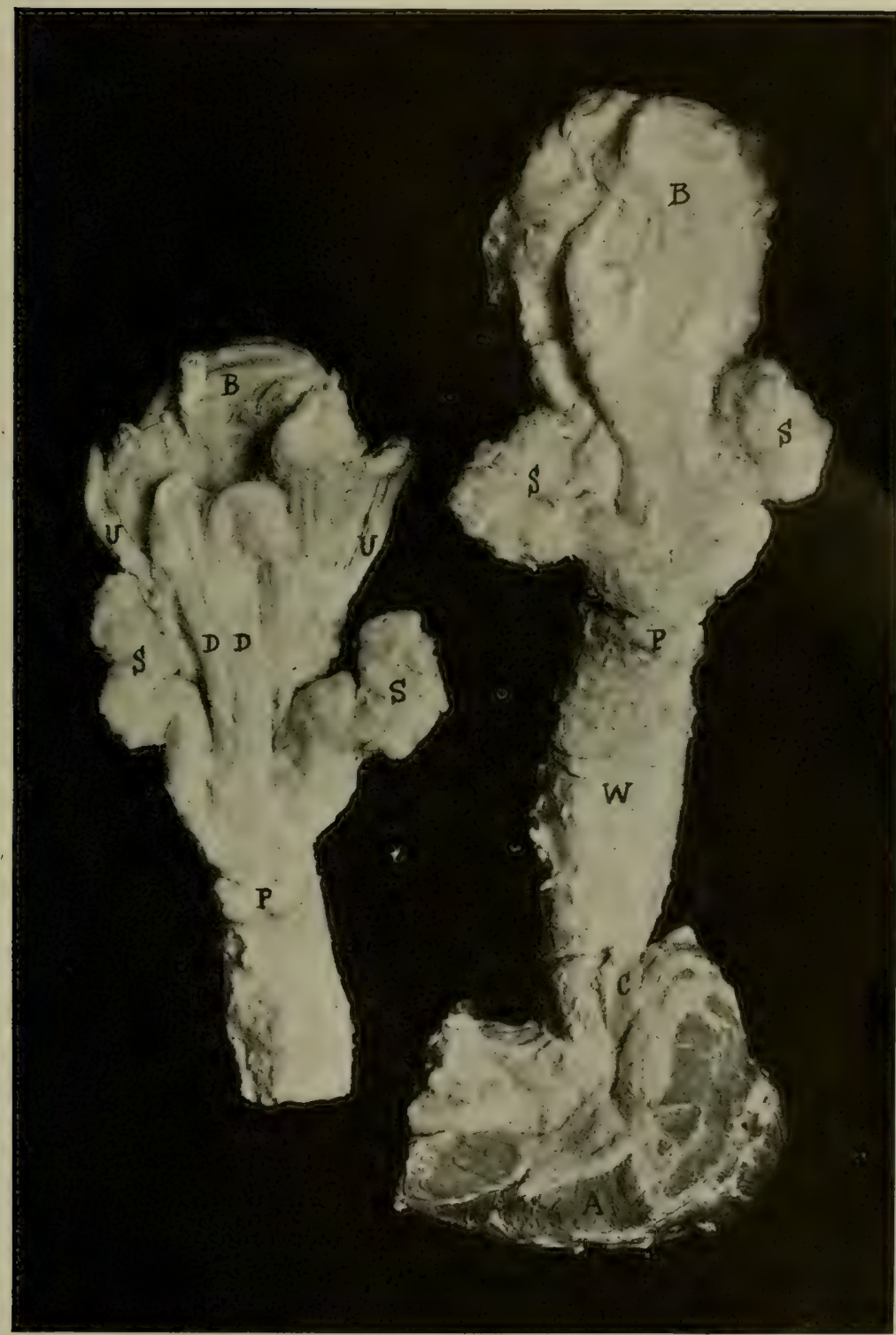

Fig. 7. Same as Figs. 5 and 6 with same lettering. The left hand figure is from a bull calf. In the right figure a median section through the posterior part of the urethra has been made exposing to clear view Cowper's gland, $C$. 
only in the dog, amongst domestic animals, that the prostate gland assumes pathologic importance.

Cowper's glands are present in solipeds, ruminants, and the cat. They lie on either side of the urethra in the perineal region above the ischial arch. They have little or no known pathologic importance. The glands are absent in the dog.

\section{The Penis}

The penis of domestic animals is normally completely hidden, when at rest, within the sheath, and the glans penis has a second covering, the prepuce. The soliped and dog ordinarily protrude the penis out of both prepuce and sheath during urination. The protrusion of the penis dur-

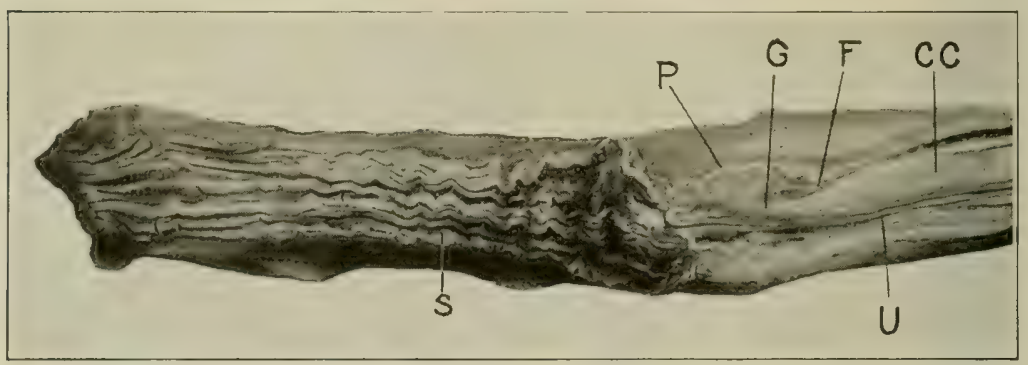

FIG. 8-Penis of Calf with Preputial Sac Unopened.

$S$, Sheath; $U$, urethra ; $P$, prepuce, not yet free from the glans : $G$, glans. Between the prepuce and the glans is soft embryonic tissue. $F$, fornix; $C, C$, corpus caveruosum.

ing coitus or sexual excitement is brought about by its erection and by the relaxation of the retractor penis muscle. In solipeds and carnivora, the retractor muscle is closely applied throughout its length to the ventral side of the penis, so that the length of the retractor muscle from the tuber ischii to its penial insertion is identical with the length of the penis from the ischial notch to the muscular insertion. In ruminants and swine, the muscle is not closely applied to the ventrum of the penis but passes directly across the postero-inferior mouth of the curvature of that organ without following its course so that the length of the muscle is only about one-half that of the penis from the ischial notch to the muscular insertion. 
Thus it occurs that the protrusion and retraction of the penis are brought about in a different manner in the horse from that observed in ruminants and swine. The penis of the soliped is protruded chiefly through an elongation of the organ itself in the process of erection; the penis of the ruminant is protruded largely through the elimination of the S-curve and temporary lengthening of the retractor penis muscle.

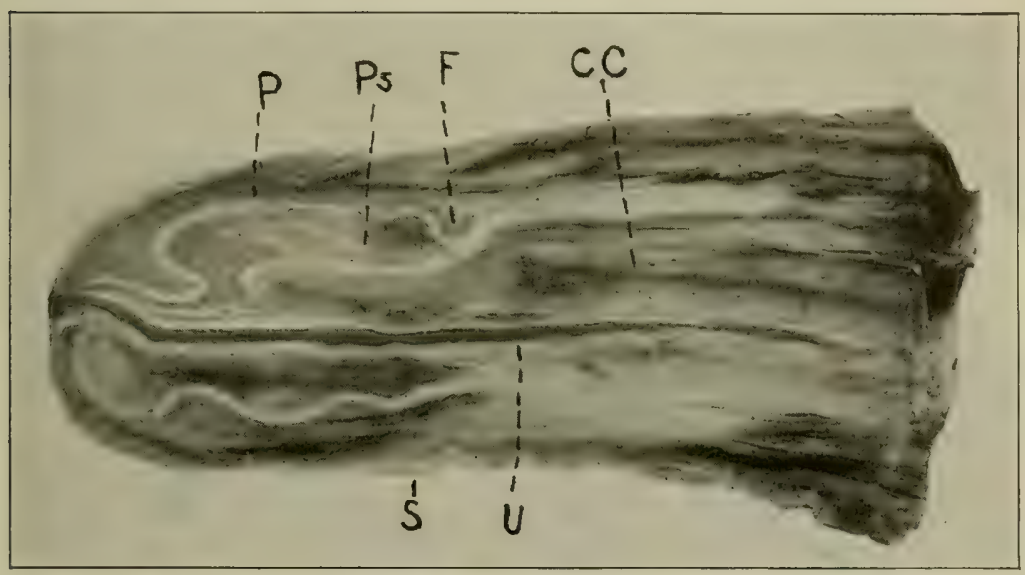

FIG. 9-Penis of Calf with Sheath forcibly Everted showing Unopened Preputial Sac. $\mathbf{x} 3$.

$P s$, Preputial sac, Other lettering same as in Fig. 8.

\section{The Sheath and Prepuce}

Veterinary anatomists commonly use the words "sheath" and "prepuce" as synonomous terms. When studying the diseases of the genital organs, it is preferable to differentiate the sheath and prepuce as two separate structures of different embryologic history. It will simplify the study of the pathology of these structures if the prepuce is regarded as that sac which reaches from the fornix, or base of the glans, to its apex when the organ is at rest. The sheath begins at the apex of the penis, when the organ is at rest, and extends forward to the exterior opening where it becomes continuous with the general integument. The pre- 
puce is wholly free from hairs; the sheath at its external opening contains numerous hairs. In the bull these are greatly developed to constitute the preputial tuft. The preputial sac of solipeds forms and opens early in embryonic life, but ruminants and swine are born without this structure. The new-born male soliped can readily protrude the penis and commonly does so when urinating. The young ruminant and porcine male cannot protrude the penis, as is shown in Figs. 8 and 9, and nccessarily urinate in the sheath. With the advent of puberty, the frail layer of embryonic tissue, serving to bind the prepuce to the glans,

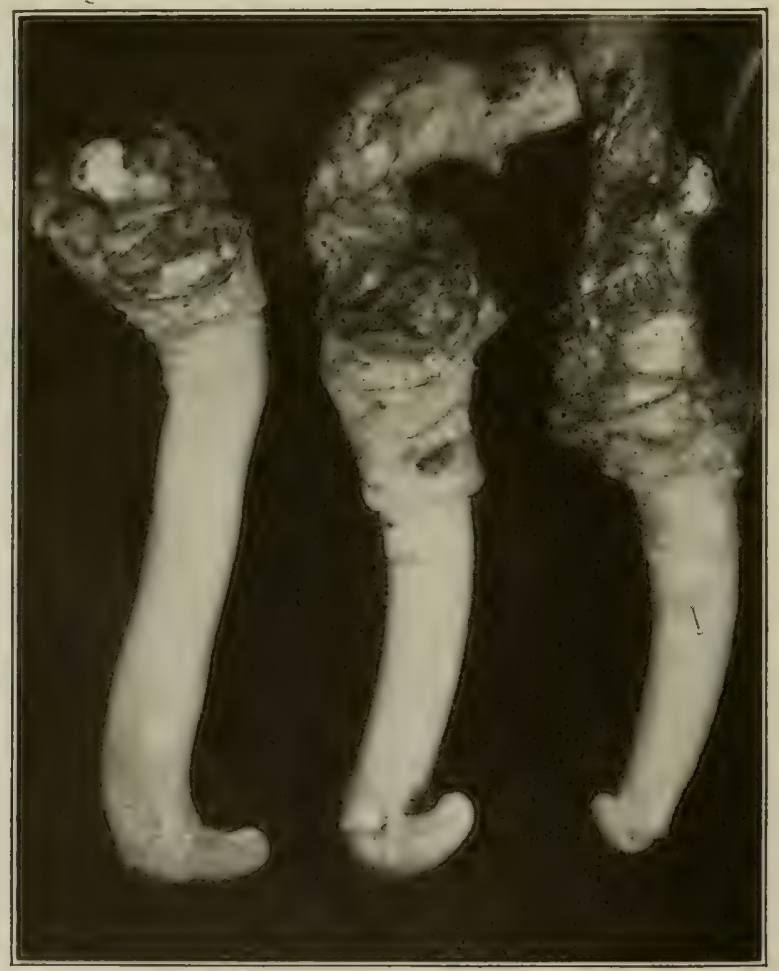

FIG. 9a-Penises of Steers showing various degrees in the development of the Prepuce.

The left hand figure shows in the glans a few elevations from the granular venereal disease. 
slowly yields and the preputial sac opens, after which the penis may be protruded. If the young animal is castrated early, the formation of the prepuce is held in abeyance at varying stages. The animal must then urinate within the sheath throughout life and is subject to special diseases not observed in entire males. In the young lamb, the urethral orifice at the tip of the vermiform appendix of the penis opens into the fundus of the sheath, the tip of the vermiform appendix accordingly indicating the line of demarcation between the sheath and the eventual preputial sac.

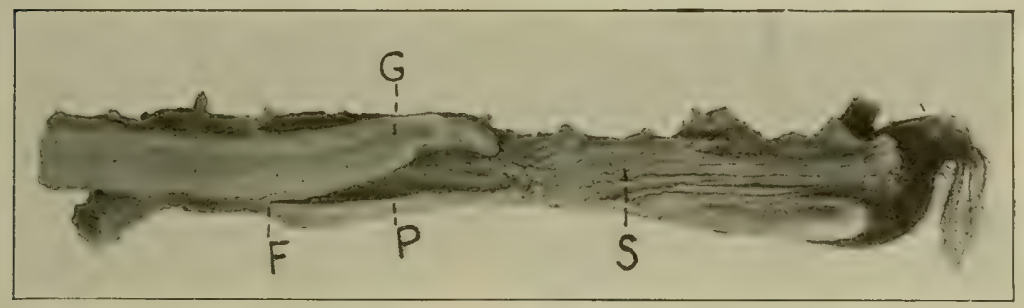

FIG. IO-Sheath, Prepuce and Retracted Penis of Bull. $F$. Fornix ; $G$, glans; $P$, prepuce; $S$, sheath.

The epithelial coverings of the glans penis and the prepuce of solipeds are corneous and highly protective. They produce a moist sebum in the sinus about the urethral opening, but over the rest of its surface the sebum is normally dry and forms gross masses of crusts which are readily detached. In ruminants, swine, and carnivora, on the contrary, the preputial sac and glans penis are covered by a very delicate mucous membrane which normally secretes sufficient mucus to keep the parts constantly moist. The prepuce of ruminants and of swine is accordingly more vulnerable to abrasions and infections than that of solipeds, and affects quite profoundly the type of lesions observed in the two classes. The preputial secretions are not as a rule markedly odoriferous.

The sheath of all domestic animals secretes a smegma having a specific odor. The boar has two culs-de-sac in the superior wall of the sheath which may contain as much as a 
pint of highly fetid urine and smegma. In the barrow these cavities are much smaller but contain a similar fetid material.

When the penis is protruded as in copulation, the glans penis pushes its way out of the prepuce and that membrane becomes everted to constitute the covering of the penis from the fornix backward for a distance approximately equal to

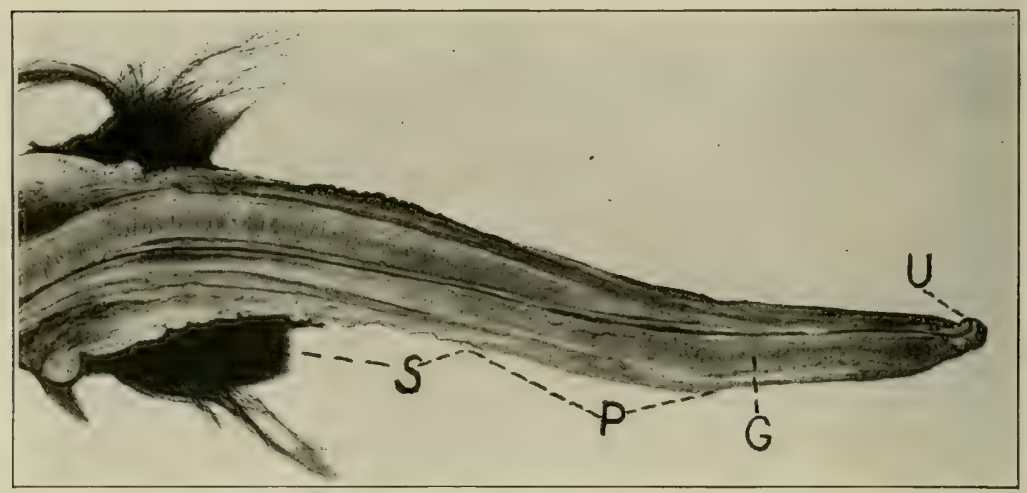

FIg. I I-Sheath, Prepuce and Protruded Penis of Bull. Lettering same as in Fig. Io.

the length of the glans. The further protrusion of the penis requires the eversion of the sheath by which it becomes the visceral covering of the base of the penis. It thus transpires that the prepuce and sheath, the epithelial surfaces of which face inwardly while the penis is at rest with a cavity between them and the penis, become reversed when the organ is erected and constitute its immediate covering with its epithelium facing outward. The general plan is indicated in Figs. 8-11.

\section{B. The Female Generative Organs}

The genital system of the mammalian female consists of the ovaries, oviducts, uterus, cervix, vagina, and vulva.

\section{THE OVARIES}

The ovaries are the essential reproductive glands of the female. They perform the fundamental generative func- 
tion by elaborating the ovum, or egg, which, after being discharged from the ovary and fertilized by the spermatozoon, becomes a distinct, but in mammalia not immediately an independent, being. The fertilized ovum is still dependent for a time upon the oviducts for its transportation to the uterus, upon the uterus for its nutrition and protection during pregnancy and its expulsion at fetal maturity, and upon the milk from the udder of the mother as food immediately after birth.

The size, form, and location of the ovaries of domestic animals differ greatly according to species, breed, age, and individual; even the two glands in the same animal are not ordinarily alike and may vary greatly in size and form. The ovary of the mare is much the largest seen in the domestic animals, reniform, very dense owing to its thick tunica albuginea, with a smooth surface which is elevated here and there in many cases by the presence near the surface of cysts varying from less than 0.25 inch to 2 or more inches in diameter. The gland attains its maximum size when the animal has reached the age of three or four years, and begins to atrophy at ten to fifteen years, to become very small and fibrous as age advances. The ovary of the young mare is usually $31 / 2$ to 4 inches in its greatest diameter and weighs about 4 ounces, while in the aged it may shrink to $11 / 2$ inch in its greatest diameter and in weight to $1 / 2$ ounce. The ovary of the cow is much smaller than that of the mare, varying in its greatest diameter between 0.25 and 2 inches and weighing about 0.25 to 0.5 ounce; it is oblong and generally regular in outline; its tunica albuginea is only moderately dense. The ovary of the sow is comparatively very large. The numerous ovisacs appear prominently on its surface in such a manner that the most superficial of them stand out entirely beyond the general ovarian surface attached to the body of the organ by a somewhat constricted neck. The ovary of the cat is very oblong, with pointed extremities, and of a bright scarlet color, with the ovisacs standing out thickly over the surface in a way suggesting a mulberry. 
The ovary of the bitch is very small, livid in color, even in outline, and, unlike those of other domestic animals, is completely enveloped in the pavilion of the oviduct, except for a minute oblong opening of so small size that the gland can not be readily exposed to plain view through it.

The location of the ovary in the adult varies much with species. The size of the gland, when normal, has little or no influence upon its position. It rests upon the superior surface of the anterior border of the broad ligament of the uterus, naked in most animals but closely invested in the bitch by the pavilion of the oviduct. The pavilion of the oviduct in the mare is attached by one of its fimbria to the ovary near its hilus, and its connection with the uterus is further maintained by the utero-ovarian ligament, composed largely of connective tissue and pale muscle fibers. In the cow one of the fimbriae of the pavilion is adherent to the outer or lateral end of the ovary. The ovary is also influenced in its position by the round ligament, which is analogous to the gubernaculum testis of the male. Arising like the latter from the skin and dartos at the point where the fundus of the scrotum of the male normally appears in the given species, it extends up through the inguinal ring behind the peritoneum, to become attached to the uterine cornu or oviduct, not far from the ovary. Its attachments tend to cause the ovary to follow a line of descent comparable to that of the testicle, but normally it becomes arrested in its migration at some point between its place of origin and the internal inguinal ring. In the bitch, it remains closely applied to the sub-lumbar region, immediately behind the kidney, virtually at its point of origin. In the mare, its movement is arrested early and it remains rather firmly suspended not far behind the kidney and above the middle of the posterior part of the abdominal cavity. The ovary of the ruminant lies loosely in the pelvis, alongside the cervix uteri or the base of the cornu, behind the internal inguinal ring, or just in front of the pubic brim, somewhat below it, resting upon the abdominal floor.. The ovary of the sow floats quite freely in the peritoneal cavity 
and abnormally at times passes out through the inguinal ring and comes to rest in that part of the perineal region corresponding to the scrotum of the boar. The same displacement of the ovary occurs rarely in bitches. It is possible in any species. In general it may be said that in the elongated uteri of multiparous animals the ovaries are situated further forward than in the uniparous or biparous genera. The location of the ovary is further modified by the pregnant state. The gravid uterus, in its descent, drags the gland downward and forward. It is also dragged downward and forward by any increase in weight due to disease of the uterus, oviducts, or ovary, such as pyometra, tumors of the uterus, oviducts and ovaries.

The functional activity of the ovary modifies its size and form. During the breeding life of the animal the cortex, or peripheral area, of the ovary is largely composed of egg columns, or masses, consisting of germinal cells arranged in the form of a hollow sphere. Resting upon an intruding mass of genital cells is a specially developed cell, the ovum. The spheroidal hollow mass of cells contains a fluid, the follicular liquid. When an ovum, or ova, matures and an ovisac ripens, the female is in estrum or "heat." The ovisac becomes distended with follicular fluid, presses aside the superposed ovarian tissues, and attenuates or destroys the tunica albuginea at the involved point. In most animals the active ovisac pushes its way above the general ovarian surface as a hemispherical cyst with very thin walls. In the mare it regularly protrudes at the hilus of the gland, where it is not readily apparent. The ripe ovisac -or ovisacs-ruptures at the close of estrum, producing a lesion, which usually behaves differently according to whether the discharged ovum becomes fertilized and undergoes development into a fetus or, failing of fecundation, perishes. The crater resulting from the rupture of the sac becomes filled with lymph, blood, or other products of the disturbances of the tissues. Should the ovum perish, the lesion tends to heal rapidly, the blood and lymph in the crater are resorbed, and from its walls there forms a characteristic tissue, known as the corpus luteum of estrum. 
In the cow and in most other animals it is identical in form, consistence, and volume with the corpus luteum of pregnancy, but its color differs. In the non-pregnant cow it is chocolate-colored. Prior to the next estrum it atrophies.

Should the ovum become fecundated and undergo normal development, the corpus luteum forming in the crater is almost always markedly yellow, lemon, or orange. It projects beyond the ovarian surface. It persists regularly up to the time of parturition or abortion and for a varying length of time thereafter. It is known as a true corpus luteum. Pathologically, it sometimes atrophies during pregnancy and is one of the causes of estrum in pregnant animals. While in unipara the corpus luteum regularly atrophies soon after the termination of pregnancy, the atrophy is ordinarily dependent upon the expulsion of the uterine contents. If the fetus perishes, but is retained and desiccates or macerates, the corpus luteum remains. In the sow (and presumably in other multipara) the number of corpora lutea almost always exceeds the number of fetuses, both living and dead, registering the fact that not all ova discharged at a given estrum successfully mature. When very few pigs are born in a litter, it indicates that numerous ova or embryos have perished, rather than that few ova were discharged. The size and form of the ovary are accordingly modified by the presence of a ripe ovisac or ovisacs or of a corpus luteum. In the cow the corpus luteum is frequently larger than the remainder of the ovary-approximately $5 / 8$ to $3 / 4$ inch.

\section{The Muellerian Ducts}

The beginning of the genital canal in the embryo consists of two parallel rods, which later become excavated to form tubes-the ducts of Mueller-extending from near the posterior body opening, upward and forward to the region of the ovary. Eventually the two ducts fuse at their posterior ends, producing a single tube. The ducts become differentiated, through specialization in their development, into four essentially separate segments, each having its distinctive function. The anterior segment constitutes the 
oviduct, or Fallopian tube, dilated at its anterior end to form the ampulla, or pavilion. The oviduct constitutes a conduit through which the spermatozoa of the male may pass from the vagina and uterus to the ampulla, there to meet and fertilize the freshly discharged ovum. It serves later to transport the ovum, fertilized or unfertilized, to the uterus. The second, or middle portion of the genital tube constitutes the uterine cavity, including the cornua, in which the fetus may find lodgment, attachment, nutrition, and protection during its development. The third segment consists of the cervix, which acts as a barrier between the uterus and the vagina. The fourth, or posterior segment-the vagina-extending from the uterus to the vulva, serves first as an essential copulative organ and later as a passage for the fetus at the time of birth.

The degree of the fusion of the Muellerian ducts and the extent of the specialization of the different areas modify greatly the form and relations of the various segments. The entire genital tube, having a common embryonic origin, has a similar structure, characterized chiefly by three coats -peritoneal, muscular, and mucous. The peritoneal layer, which invests the genital tract almost completely, is derived from the peritoneum of the body wall, behind which the ducts of Mueller originate. At its anterior extremity the genital tract opens, through the pavilion of the oviduct, into the peritoneal cavity. The peritoneum stops at the margin of the pavilion, to be succeeded by the mucous membrane of the oviducts

The genital tract is supported by the broad ligaments, which consist of two peritoneal layers resulting from the departure of the genital tube from its seat of origin behind the peritoneum. Along the line of their attachment to the genital tube, the peritoneal investment is interrupted to the extent of the area between the two peritoneal folds occupied by the vessels, nerves, pale muscle, and connective tissue fibres constituting the body of the ligament. At the posterior extremity of the genital tract, where it opens into the cloaca of the embryo, the peritoneum is reflected upon 
neighboring organs and the genital tube is surrounded by the intra-pelvic connective tissue, so that eventually the peritoneum covers, in addition to the oviducts and uterus, only the more anterior portions of the vagina. The fusion of the two Muellerian ducts, to form the vagina and uterus, destroys the contiguous median walls of the tubes as far as they coalesce, and the adjacent tubes become a single canal. The broad ligament of the uterus varies greatly in its form and amplitude acording to species and the functional activity of the genital tract. In the bitch, cat, and sow, in which the anterior extremities of the uterine cornua remain throughout the life of the animal about as far forward as the posterior border of the kidney, the broad ligament maintains its anterior attachment to the abdominal wall at or near the point of origin of the ovary just behind the kidney; in the ruminant, where the ovary and the anterior ends of the uterine cornua are turned backward to the immediate vicinity of the internal inguinal ring, the parietal attachment of the anterior border of the ligament likewise moves backward for a considerable distance so that the middle of the recurved cornu tends to project forward beyond the anterior margin of the ligamentous attachment. When the uterus of a quadrupedal mammal becomes gravid, the weight of the fetus drags the occupied portion downward and forward until the organ comes to rest upon the abdominal floor in front of the anterior point of fixation of the ligament to the abdominal wall. The genital tube is further maintained in its position by its attachment posteriorly to the vulva and also by the round ligament of the uterus.

The muscular walls of the genital tube are composed of circular and longitudinal layers of pale fibres, varying greatly in the different sections of the tract and also according to whether the animal be pregnant or not.

The mucous coat of the genital tract offers the greatest possible variations and assumes widely differing and highly important functions, according to the particular area concerned. 
a. The oviducts, formed from the anterior or ovarian extremities of the Muellerian ducts, are two long, tortuous tubes, varying in length and other characters according to species. Their length is much greater than the distance from the anterior extremity of the uterine cornu to the ovary. This distance is fixed by the utero-ovarian ligament. In some animals the oviducts are naked and clearly visible. In the bitch they are hidden in the abundant fat of the broad ligament. The utero-ovarian ligament in the dog and cat is very short, so that the anterior end of the uterine cornu is virtually in contact with the ovary, while the oviduct, 3 to 4 inches in length, is thrown into numerous folds, to terminate in the immediate region of its origin, so that casual observation, without dissection, might lead to the assumption that the oviduct was well-nigh absent, whereas it is very similar in actual length to that of other species. When divested of any concealing coverings of peritoneum, fat, or other tissues, the oviduct appears as a very convoluted white cord about 0.1 inch in diameter. It is very firm to the touch and gives a sensation much like its analogue in the male, the vas deferens. After it is dissected out from the surrounding tissues and its numerous abrupt curves are carefully eliminated, a very fine sound, as one of the tail hairs of a horse, may be inserted at one opening and passed through its entire length. While technically the oviducts complete a communication between the peritoneal cavity and the exterior, the tubes are ideally impassable, except to ova and spermatozoa. They do not under usual conditions permit the forcing of liquids through their canal when intrauterine injections are made under comparatively high pressure. Clinically, they also offer valuable resistance to the passage of bacteria. Frequently, pathogenic organisms, such as those involved in abortion and retained placenta in cows and other animals, travel along the oviducts, causing disease of them or, reaching the ovary, induce abcess or other disease, causing sterility.

The intimacy of contact between the peritoneal covering of the oviduct and the muscular walls varies according to 
species. The investment is very close in ruminants and the sow, so that the ducts are readily seen without dissection. In the mare the duct is surrounded by much connective tissue, which serves to conceal it from view until the peritoneal and fibrous coverings are dissected away. In the bitch the concealment is further accentuated by large amounts of adipose tissue.

The oviduct opens anteriorly through the ostium abdominale into the pavilion of the tube and posteriorly into the uterine cornu through the ostium uterinum, which usually projects somewhat as a small eminence into the cavity of the cornu.

The muscular coat of the oviducts is characterized chiefly by its density, which gives to it an almost cartilaginous consistency, and its paleness, which amounts almost to translucency. The superficial layer of the mucosa of the oviducts consists of ciliated columnar epithelium with the cilia vibrating toward the uterus. The oviduct is the most rigid and undilatable portion of the genital tube. It provides passage for the migration of the spermatozoa of the male, which, in spite of the movements of the cilia in the contrary direction, advance from the uterus toward the ovary, in virtue of their inherent power of movement, meet the ovum in the pavilion, and fecundate it. Later the oviduct conveys the ovum, fecundated or not, from the ovary to the uterus. In this function the cilia play an essential part. In rare cases the fecundated ovum lodges in the duct and undergoes partial development, to constitute tubal pregnancy, but the undilatability of the duct serves, as a rule, to cause a rupture of its walls and leads to the escape of the fetus from the duct into the peritoneal cavity, either to cause sudden death of the mother from hemorrhage or to constitute extrauterine, or abdominal, pregnancy.

b. The uterus, see Figs. 12, 13, a bifurcated, musculomembranous sac, designed for the reception, nutrition, and protection of the ovum, finally takes the initial part in the expulsion of the fetus at the time of birth. The uterus varies greatly in form and disposition, dependent partly 
upon the degree of fusion which has occurred between the two tubes. There are recognized in domestic animals a uterine body and two cornua, each having essentially like functions in varying degrees. This is in sharp contrast to the uterus of woman, where the uterine body constitutes essentially the entire functional organ and the cornua are unimportant appendages. In the rabbit there is no uterine body, but two distinct tubular uteri opening separately into the vagina. In the bitch, cat, and sow the uterine body is limited in extent and physiologically unimportant, rarely containing placental attachments and only a small portion of one fetus except while in transit at the time of birth. The two cornua are extensive, and in them develop the fetuses, so that physiologically the horns represent the two separate uteri of the rabbit. In ruminants the uterine body is larger and assumes higher importance physiologically, while the cornua remain relatively large. The one, two, or, very rarely, more fetuses are habitually located almost equally in the body and the cornua, so that they represent a middle stage in the transition between the double uterus of the rabbit and the single uterus of woman, physiologically devoid of cornua. In the mare the uterine body becomes relatively more important than in the ruminant.

The relative sizes of the cornua and of the uterine body bear a close relation to the number of young brought forth at a given birth. In multiparous animals, there are two uteri, as in the rabbit, or the two extensive cornua with functionally unimportant uterine body, as in the bitch, cat, and sow. In ruminants, which are largely biparous, the uterine body and cornua become approximately equal in extent and function. In the normally uniparous mare, the cornua are smaller than the body.

The uterus and its cornua, within the above limitations, are uniform in their plan of structure and function. They consist of three coats: mucous, muscular and peritoneal.

The mucous coat constitutes the essential physiologic basis of the organ. The mucous epithelium is very elaborate, consisting superficially of columnar cells, embedded deeply with- 
in which are numerous tubular structures of a glandular character, the utricular glands, believed to secrete the socalled uterine milk, which is presumed to play an important role in the nutrition of the ovum pending the formation of the embryo and the establishment of its intricate connection

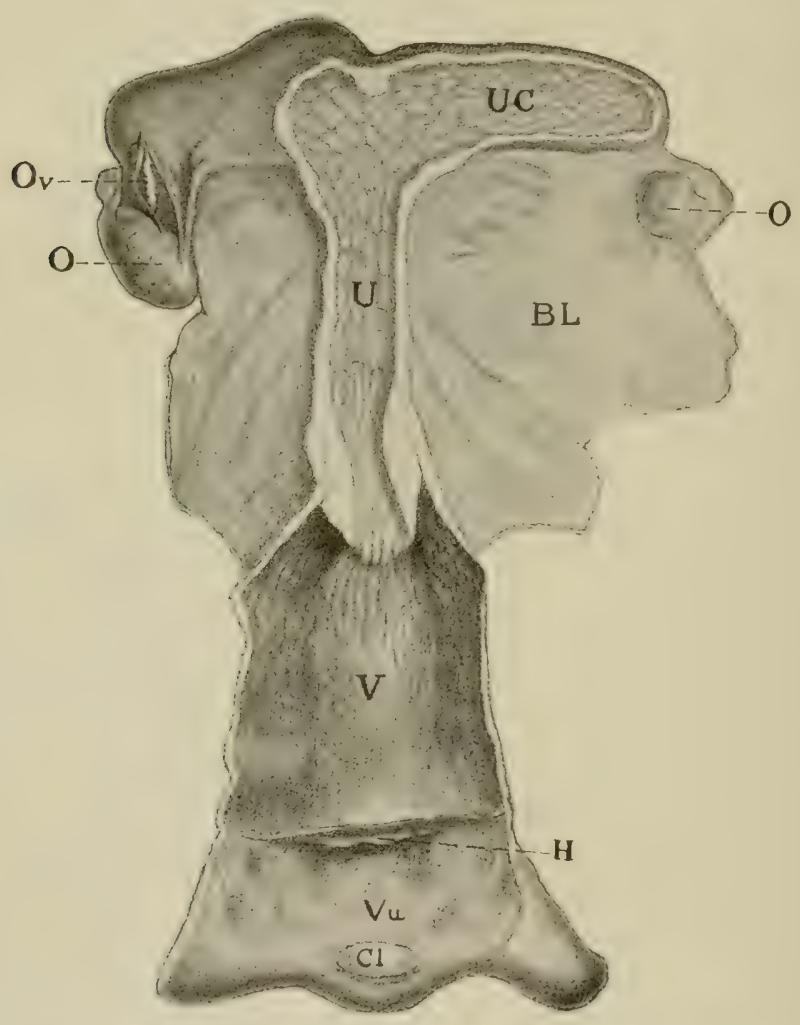

HIG. I2-Non-gravid Uterus of Mare viewed from the Dorsal Surface. $O, O$. Ovary; Ov, oviduct; $U$, uterus ; $U C$, uterine cornu ; $B L$, broad ligament ; $V$, vagina ; $I /$, vulva; $C l$, clitoris ; $H$, hymen.

with the mucous membrane through the chorion. Yet more important, it is through a special elaboration of the uterine mucosa that the highly intricate and essential maternal placenta is formed, to constitute a physical and physiologic 
bond between the mother and fetus during the span of pregnancy. The uterine mucosa is presumed to exert important bactericidal powers, but a majority of uteri of cows, both pregnant and non-pregnant, contain bacteria, several of which appear competent to induce important disease (sterility, abortion). In the non-gravid uterus, as is common with distensible hollow organs, the mucous membrane is thrown into numerous longitudinal folds, which facilitate prompt and extensive dilation of the cavity without violence.

The muscular coat consists of two somewhat differentiable groups of longitudinal and circular pale muscle fibers which increase during pregnancy in size, activity, and, it is believed, to some extent in number. The proportion of the longitudinal to the circular fibres varies.

The peritoneal layer of the uterus, which is derived from that of the abdominal walls, envelops the organ completely except at the points of continuity with the oviducts and vagina and the interstice between the two peritoneal sheets of the broad ligament at their points of uterine attachment.

The uterus is retained in position by its broad ligaments and by its continuity anteriorly with the oviducts and posteriorly with the cervix. The round ligament of the uterus, arising from the skin, or dartos, in the region normally constituting the fundus of the scrotum in the male, and passing up through the inguinal canal and abdominal ring and thence to the cornu, or oviduct, is functionally of scant interest as a ligament of fixation. The uterus is located immediately beneath the rectum, with its two cornua passing obliquely outward and forward on either side.

The uterus of the mare, with its cornua, constitutes a somewhat crucial organ: the horns leave the body laterally at right angles or somewhat recurved. The uterine body is oblong, flattened somewhat from above to below, varying from 5 to 8 inches in length and $11 / 2$ to $21 / 2$ inches in width. Anteriorly, the cavity of the uterine body of the mare is continuous with that of the two cornua, without a distinct line of demarcation beyond an abrupt turn at right angles or slight recurvation to its long axis. Each cornu is much 
like the body. The cavity of each is essentially equal to that of the body, and the combined length of the two is greater than that of the body. They end obtusely not far from the ovary. Each presents at its apex a conical projection in which there is an opening, the os uterinum, or uterine orifice of the oviducts.

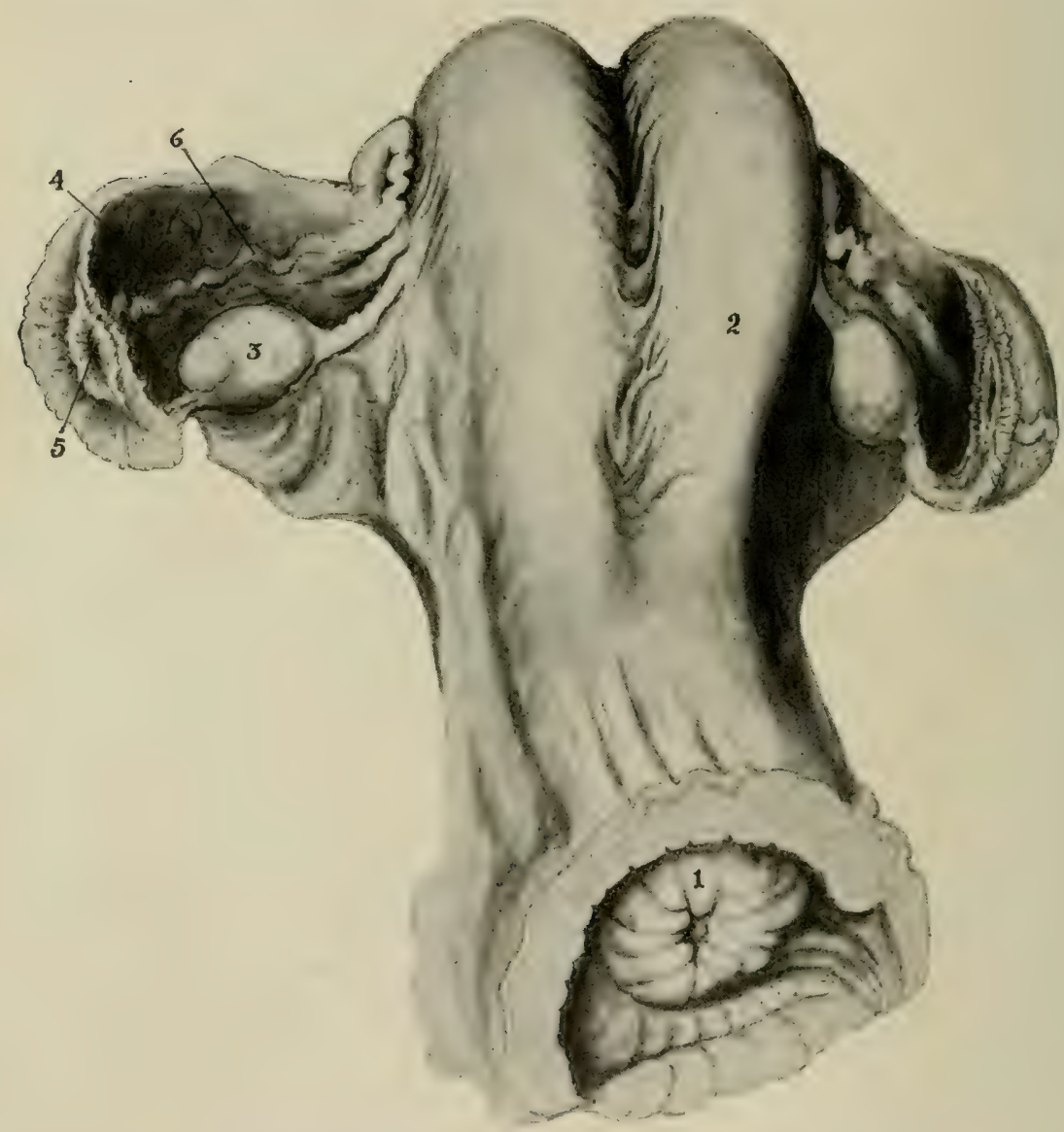

FIG. I3-Ovaries, Oviducts, Uterus, and Cervix of Cow.

$I$, Os uteri externum ; 2 , right cornu; 3 , ovary ; 4 , ovarian ventricle ; 5 , ostium abdominale of the oviduct, 6 . 
The peculiar crucial form of the uterus of the mare-the two ample horns with the bases of their cavities directly opposite, so that they have a common long axis-renders

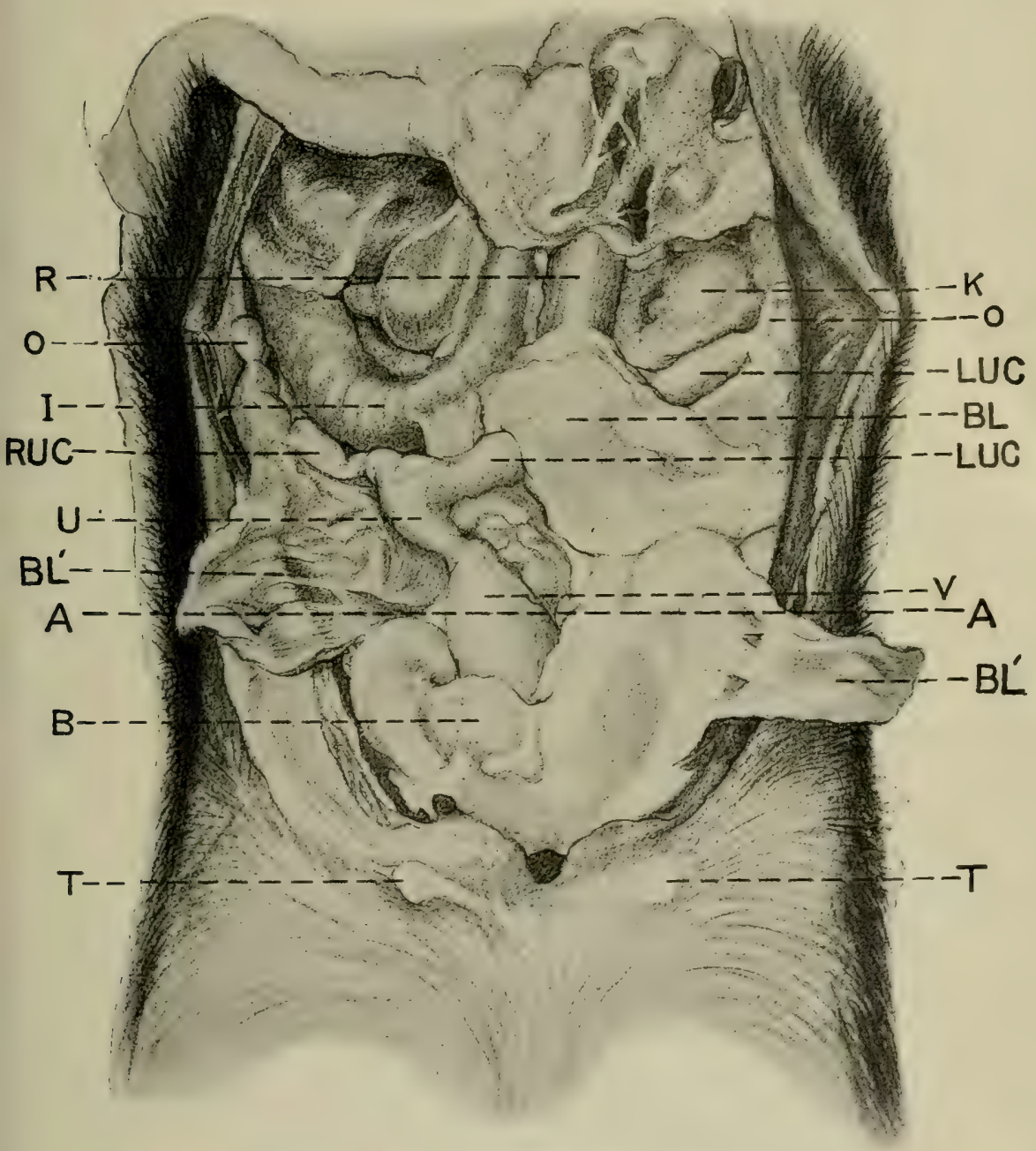

Fig. I4-Genital Organs of the Bitch.

$T, T$, Posterior teat ; $B$, urinary bladder ; $I$, vagina ; $U$, uterus ; $L U C$, left uterine cornu with a portion of the broad ligament, $B L$, lying across it; $R U C$, right uterine cornu with its broad ligament, $B L^{\prime}$, turned outward exposing the full length of the cornu ; $O$, $O$, ovary ; $R$, rectum ; $\dot{K}$, left kidney. 
bicornual, or transverse pregnancy so easy that it occurs not infrequently, resulting in a variety of transverse presentations and causing some of the most serious dystocias known to the veterinary practitioner.

In ruminants the uterine body is less pronounced in size, when viewed exteriorly, and even less upon section, while the cornua are much longer, quite tapering, and comparatively more ample than in the mare. The two cornua separate at a very acute angle, for a time extend forward almost parallel to each other, and then, becoming somewhat more divergent, curve downward, outward, backward, and finally upward, to end alongside the cervix within the pelvis. The uterine body and cornua are much denser, narrower, and more cylindrical than in the mare. The cornua taper gradually from their bases at the uterine body to their apices, where they pass almost insensibly into the oviducts.

The broad ligaments of the uterus of the mare arise from the abdominal walls in the sublumbar region, beginning not far posterior to the kidneys and extending from this point obliquely downward, backward, and medianward into the pelvic cavity, where the two layers of peritoneum become reflected from the vagina upon the bladder, rectum, and pelvic walls. Compared with the broad ligaments of other domestic animals, they are limited in extent, especially transversely, causing a comparatively rigid fixation of the uterus, and, since their anterior attachment is approximately in a direct line with the long axis of the cornua, the entire organ is held well forward in the abdominal cavity.

The broad ligaments of ruminants, as compared with those of the mare, are much more ample transversely, while antero-posteriorly they are much less extensive. Their anterior point of attachment to the abdominal parietes is much farther posterior than in the mare. This variation in disposition and relations of the broad ligament produces two well-marked clinical differences. In the non-gravid uterus of the cow, the anterior parietal attachment of its broad ligament is already posterior to the anterior curvature of the uterine cornua, which in the gravid uterus be- 
comes sharply accentuated, so that almost the entire organ rests anterior to its ligamentous attachments to the abdominal parietes. In the cow and ewe, the broad ligaments become largely powerless in preventing the pregnant organ from revolving upon its long axis, so that torsion of the uterus becomes quite common, while in the mare the more rigidly fixed organ, with the anterior parietal attachment of the ligament much farther forward, serves to render the displacement comparatively rare. In ruminants, the comparative amplitude of the ligaments, with their anterior point of parietal attachment but little anterior to the pelvic inlet, permits more readily than in other animals inversion and prolapse of the uterus and vagina.

In multiparous animals the broad ligaments are necessarily very extensive and uniformly have their anterior point of attachment to the abdominal walls far forward in the post-renal region. In the bitch, the ligament at its anterior border is very short, so that the ovary and ovarian end of the cornu are closely fixed in the sublumbar region, just posterior to the kidney, tending to stretch the cornu between this anterior, sublumbar attachment and the vagina. The ligaments are exceedingly ample, except at the anterior border, and are much broader than the distance from the parietal attachments to the position of the cornu, resulting in a large antero-posterior fold in the ligament, which drops down on the lateral side of the cornu and covers it in a double fold of broad ligament (Fig. 14). Unlike other domestic animals, the broad ligaments of the bitch are uniformly the seat of extensive deposits of fat, which cause them to resemble the gastric omentum in general appearance.

c. The cervix, or neck of the uterus, consists of a powerful, sphincter-like segment of the genital tract, serving to separate anatomically and physiologically the uterus from the vagina. It is continuous anteriorly with the uterine body and posteriorly with the vagina. It is a tubular organ having walls very much thicker than those of the uterus or vagina and very dense and rigid. In the cow especially the 
walls are intensely rigid and hard like cartilage. In rigidity the cervix is closely analogous to the oviducts. In function, too, they are analogous: the one acts as a powerful guardian of the anterior, or peritoneal opening of the uterus; the other of the posterior, or vaginal portal. The cervix varies in size in the different genera and in individuals. The posterior end of the cervix projects into the vagina as an obtuse cone, $11 / 2$ to $21 / 2$ inches in the mare and

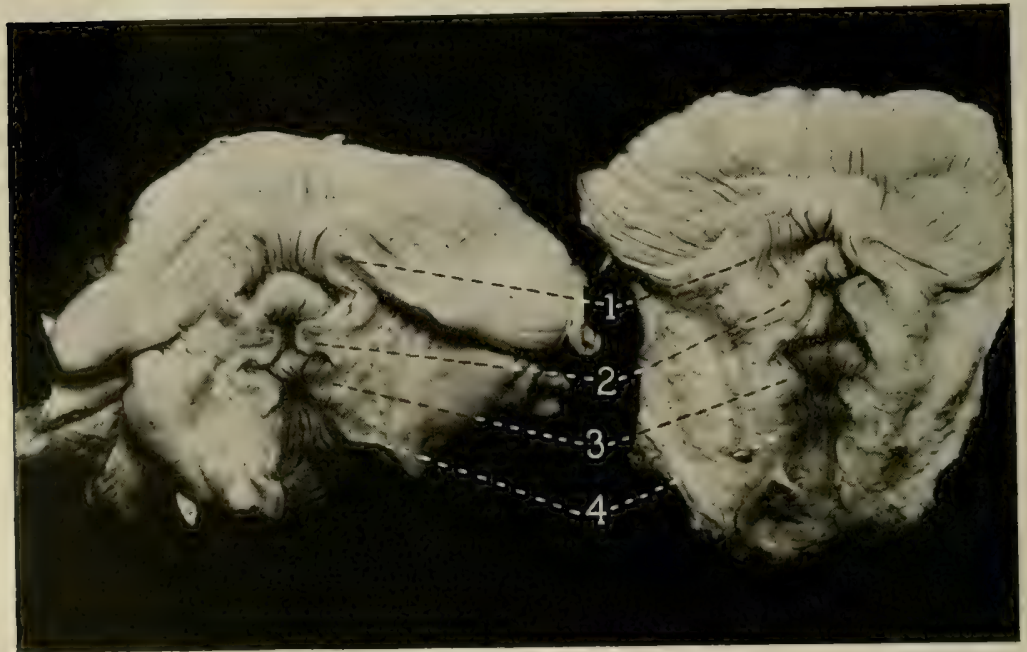

FIG. I5-Cervices of Virgin Heifers opened from above on the median line exposing the Annular Folds of the Mucosa.

$I$, First or viginal fold; $2,3,7$, the second, third and fourth folds respectively.

the cow. The cow has the longest and most powerful cervix: it is 3 to 5 inches antero-posteriorly and 2 to 3 inches transversely. Its muscular walls are so rigid, and its circular muscle fibres so contracted that it is exceedingly difficult to dilate the canal mechanically, but it dilates freely under the influence of estrum and parturition. The cervix of the mare is shorter than that of the cow and the walls far thinner and more pliable, rendering its canal readily dilatable. 
The cervical mucosa is very complex. It is thrown into deep and ample longitudinal folds, which upon cross section (See Figs. 16 and 17) are seen to radiate centralward from the basal mucosa, the summits of the rugae converging to meet at the central axis of the cervical canal. The cervical mucosa of the ruminant is also thrown into very ample cir-

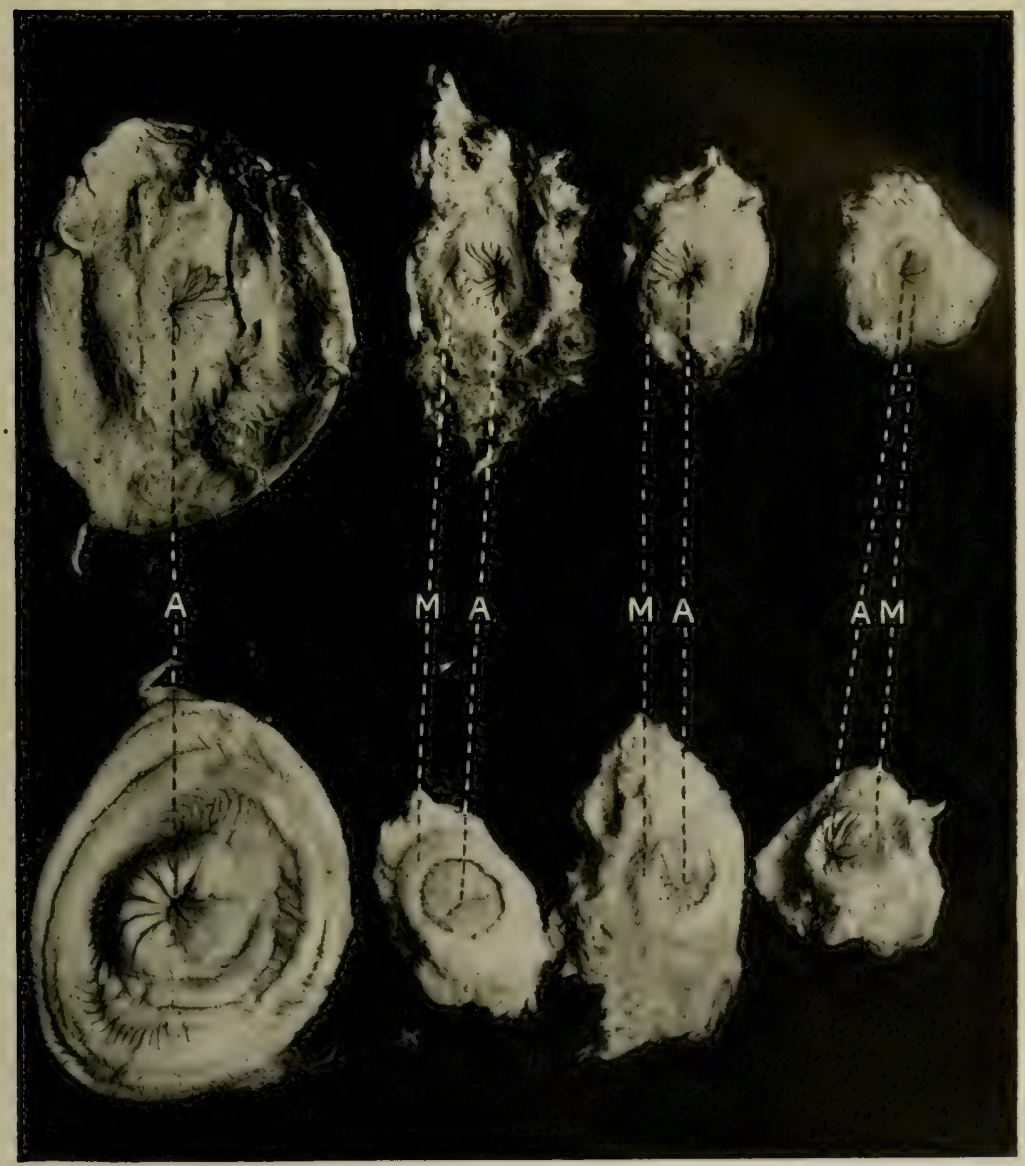

Fig. I6 (Above) AND Fig. I7 ( Below).

Transverse section of the cervix between the Annular Folds.

FIG. 16--Viewed from the uterine end. From left to right they represent the first, second, third, and fourth annular rings respectively. $A$, Cervical canal; $M$. muscular wall of cervix.

FIG. 17-Annular folds viewed from the vaginal end. 
cular folds, with their summits, or free surfaces, directed vaginalward. In the cow these circular folds measure onehalf to three-fourths of an inch from the base to the free summit, and in disease may become elongated to several inches and protrude into the vagina to constitute large polypoid masses. The cervical mucosa is rich in special mucous glands, which during pregnancy elaborate the uterine seal-a very tough, firm, adhesive structure completely sealing the cervical canal from the uterus to the vagina and constituting a highly elaborate and efficient barrier against invasion of the pregnant uterus by infection from the vagina. The cervical canal, elaborately barricaded by mucous folds and by the firm contraction of the circular muscles, is extremely narrow, and in the ruminant is besides exceedingly crooked (See Figs. 40, 41). This disposition of the cervical mucosa has given rise in the past to the fiction of "closure of the womb" and led to the belief in "opening the womb" as a panacea in sterility. Anteriorly, the cervical canal opens into the uterine cavity by the os uteri internum; posteriorly, at the summit of the conical projection into the vagina by the os uteri externum.

During estrum the muscular walls of the cervix relax and the cervical canal dilates somewhat. When parturition ensues, the cervix, as such, becomes temporarily obliterated, and the canal dilates sufficiently for the passage of the fetus - that is, until its cavity is co-extensive with the pelvic cavity and with the cavities of the uterus and vagina. At this time it becomes virtually non-recognizable; all the posterior segments of the genital tract-uterus, cervix, and vagina -become one continuous tube of virtually uniform calibre. In that type of cystic disease of the ovaries causing nymphomania, the cervical canal undergoes pathologic dilation. In the sow the cervix is not so well defined and no elaborate uterine seal forms. Instead, the vagina and cervix extend as an irregular tube up to the bifurcation of the essentially double uterus.

d. The vagina is a musculo-membranous canal, formed from the fusion of the posterior ends of Mueller's ducts and 


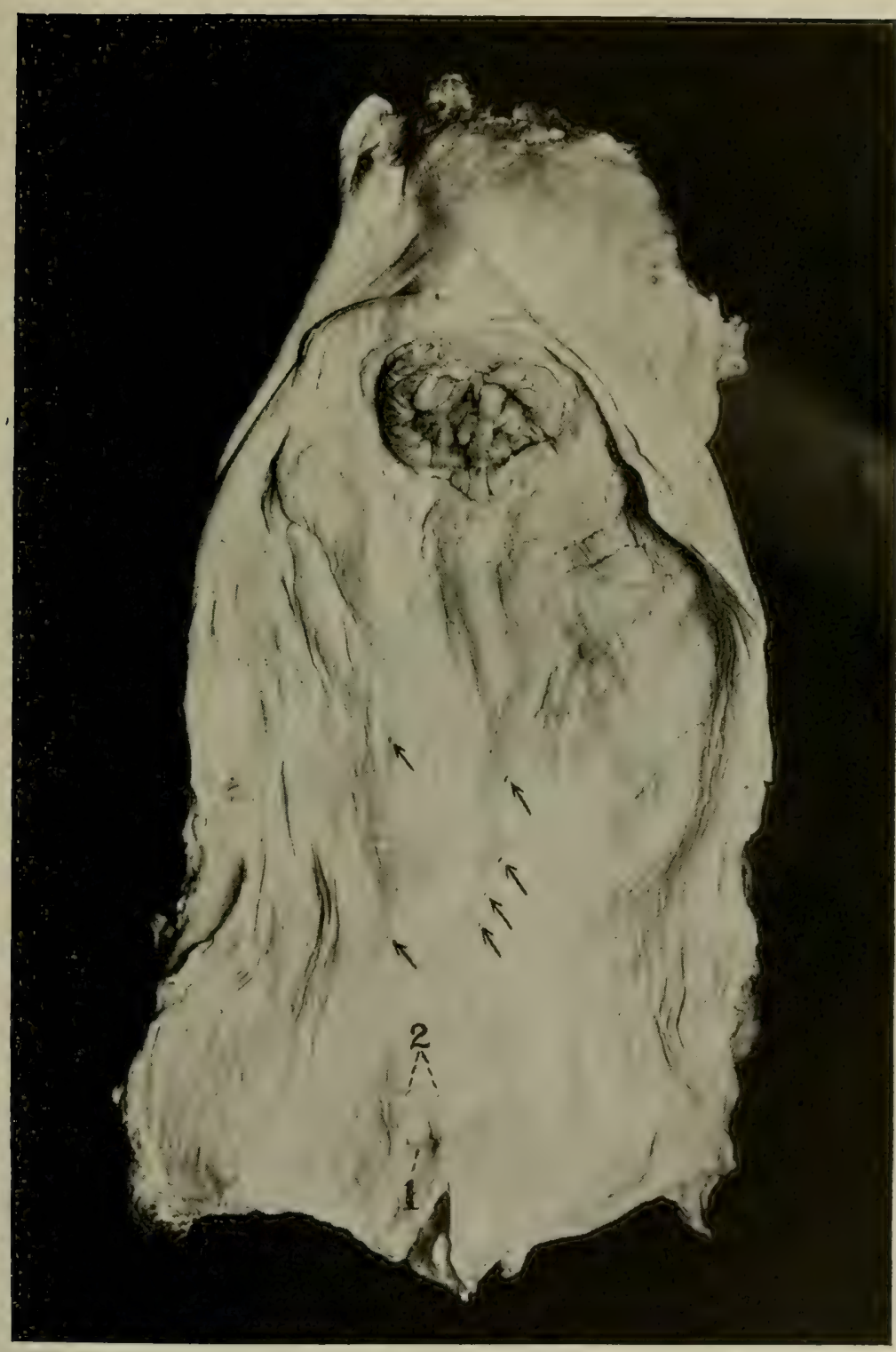

FIG. IS. Vulva and Vagina of Cow showing Gärtner's Ducts. $A$, Os uteri externum; 1 , meatus urinarius ; 2 , the outiets of the two ducts of Gärtner. The arrows point to small openings from the duct into the vagina, apparently from ruptures of small cysts or abscesses, 
extending from the cervix to the vulva. It begins at the os uteri externum and ends posteriorly at the position of the hymen, just anterior to the meatus urinarius, where the vulva succeeds it. In the cow, incomplete fusion of the Muellerian ducts in the vaginal segment is evidenced occasionally by a muscular column of varying size stretching from the roof to the floor of the vagina on the central line, just against, but free from the vaginal end of the cervix.

The vagina of the mare is eight to twelve inches in length and capable of lateral distension to the full size of the pelvic cavity. It is lined with squamous epithelium. Its mucosa is thrown into longitudinal folds, which, when at rest, lie in contact with each other.

The mucous membrane of the vagina has in its deeper portions numerous mucous glands which serve to keep the surface at all times moist and which become especially active during sexual excitement and at the close of pregnancy. The muscular coat does not differ fundamentally in arrangement from that of the uterus, though less in volume.

In the mare, the peritoneal covering extends backward from the anterior extremity three to five inches, where it becomes reflected upon the rectum, bladder, and pelvic walls. In the posterior portion of its course, the vagina is surrounded by the loose pelvic connective tissue, which permits comparatively free movement.

The function of the vagina is chiefly copulative, receiving the penis of the male during coition. During parturition, it affords a passage for the fetus from the uterus to the vulva.

In the mare and the cow the vagina frequently becomes ballooned during manual exploration of the organ, owing to an inflow of air at the moment of inspiration. During exploration the vulva is propped open by the hand or arm of the operator, permitting the air to flow in, distending the vagina so that it fills the pelvic cavity completely from side to side and from roof to floor, presenting a vast cavity with smooth, rigid walls, which are in close contact laterally with the bony or ligamentous pelvic walls, superiorly with the 
sacrum except in the area where the rectum intervenes, and inferiorly with the pubis except for the urinary bladder. The ballooning of the vagina of the mare or the cow is readily induced by intra-vaginal manipulation, by the injection into the organ of bland, tepid fluids, or by various

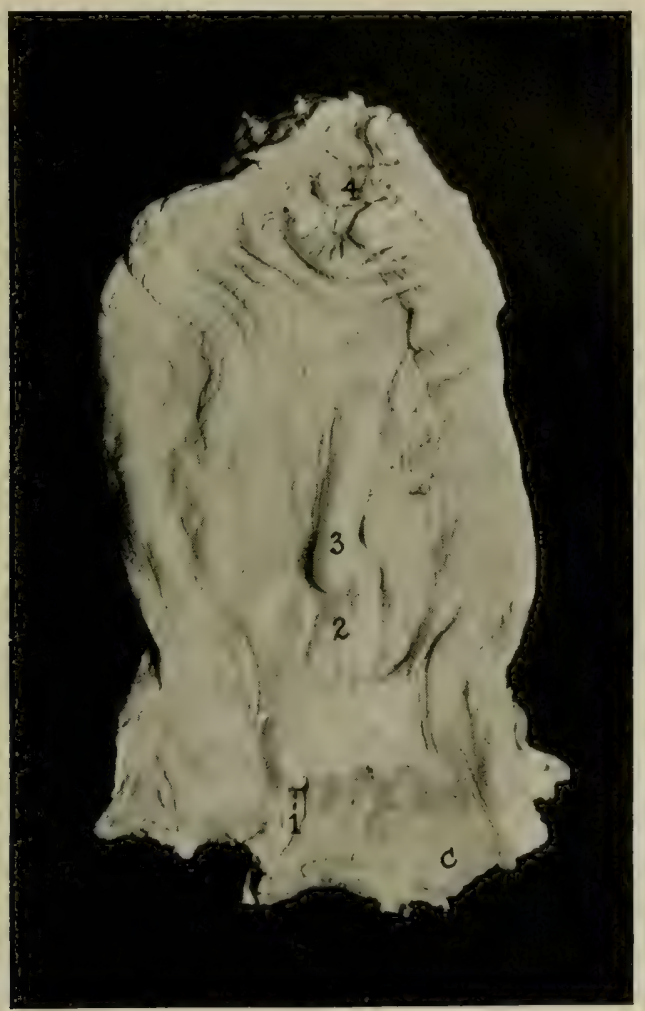

FIC. I9. Vulva and Vagina of Cow Showing Gärtner's Ducts and Bartholin's Glands.

$I$, Meatus urinarius ; 2, 3, cystic areas in the right duct of Gärtner; 4, cervical canal laid open ; $C$, small cyst in Bartholin's gland.

other means. When the moist hand is introduced into the organ at the time of estrum, marked inflation generally occurs at once.

e. Gärtner's Ducts. The Wolffian ducts of the embryo do not wholly disappear in the female but persist in varying 
degrees as essentially functionless structures. The caudal ends of the ducts persist as recognizable structures along the floor of the vagina in the cow. They end posteriorly just in front of the meatus urinarius where they lie near together. They consist of very fine tubes which diverge

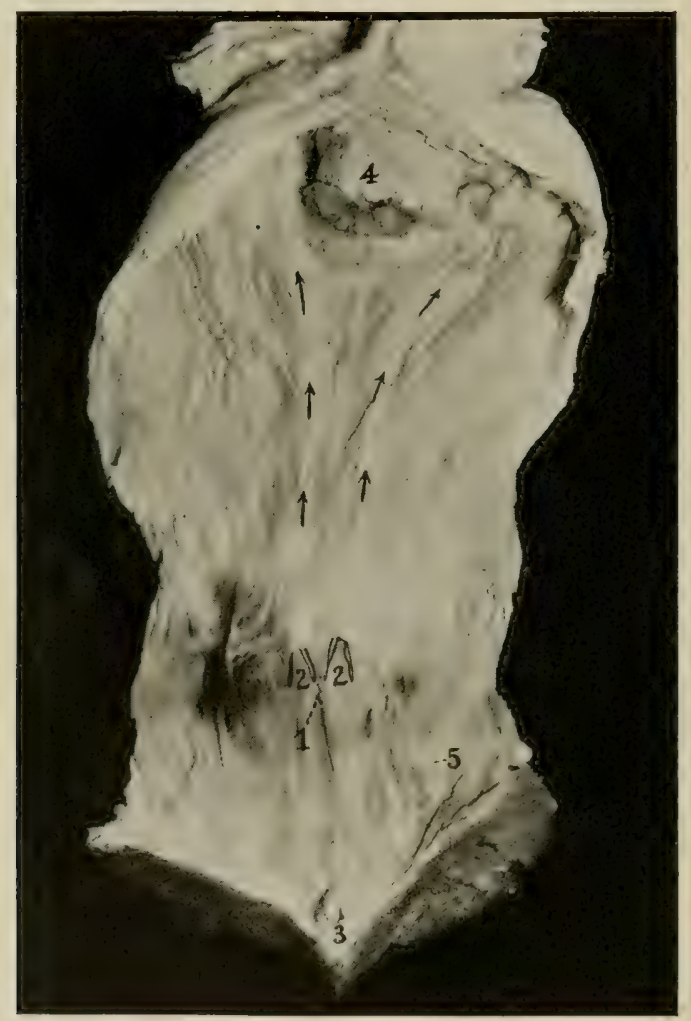

FIG. 20. Vulva and Vagina of Cow.

$I$, Meatus urinarius ; 2, 2, enlarged openings of Gärtner's duct in which a wire loop has been inserted; 3 , opening of Bartholin's gland; 4, cervix. The conrse of Gärtner's ducts is indicated by arrows upon slight ridges.

slightly as they pass forward to disappear to the right and left of the cervix. As a result of vaginitis, their orifices occasionally become occluded and the structures assume the character of cylindrical or spindle-shaped retention cysts. (See Figs. 18, 19, 20, 21.) 


\section{The Vulva}

The vulva, located immediately beneath the anus, constitutes the posterior termination of the genital canal. Instead of being derived from the mesodermic Muellerian ducts like the preceding organs, the vulvar epithelium originates from the ectoderm of the embryo.

Anteriorly, the boundary between the vagina and the vulva is marked by the hymen, a transverse membranous expanse stretching across the genital canal, which represents the partition between the termination of the hind gut and the proctodeum of the embryo, in the lower, or genito-urinary, division of the cloaca. Generally it atrophies and almost or wholly disappears in domestic animals before birth, but at times it persists, either as vertical bands stretching across from the roof to the floor of the vagina or as a circular expanse largely closing the genital canal except in its center. Rarely in domestic animals the hymen persists as an impervious membrane.

The vulva opens externally by means of a vertically elongated slit, bounded upon either side by the labiae vulvae, which meet above and below to form the superior and inferior vulvar commissures.

The vulvar labiae are covered externally by a very fine skin. In the mare the growth of hairs is inconspicuous; in the cow, there is a prominent tuft of hairs about the inferior commissure. The parts are so scantily haired that their color, which as a rule is simply that of the adjacent skin, becomes very conspicuous. In dark-skinned mares with white faces, eyes, and feet, the cutaneous covering of the vulva tends also to be devoid of pigment. The prominence of the pigmentation of the skin of this part serves as an aid in the clinical diagnosis of the venereal diseases of horses, in which important discolorations occur.

The muscles of the vulva, which are chiefly circular, are divided into two groups-the posterior and anterior constrictors. The posterior constrictor, situated within the vulvar lips, constitutes a true sphincter, analogous to those 
about other body openings. Above, its fibers become lost in the perineum and the sphincter ani; below, some go to the base of the clitoris and some pass downward, to become lost in the skin and other tissues of the thighs. Their contraction brings about the closure of the vulvar opening. The anterior group of muscle fibers invests the vulva in the

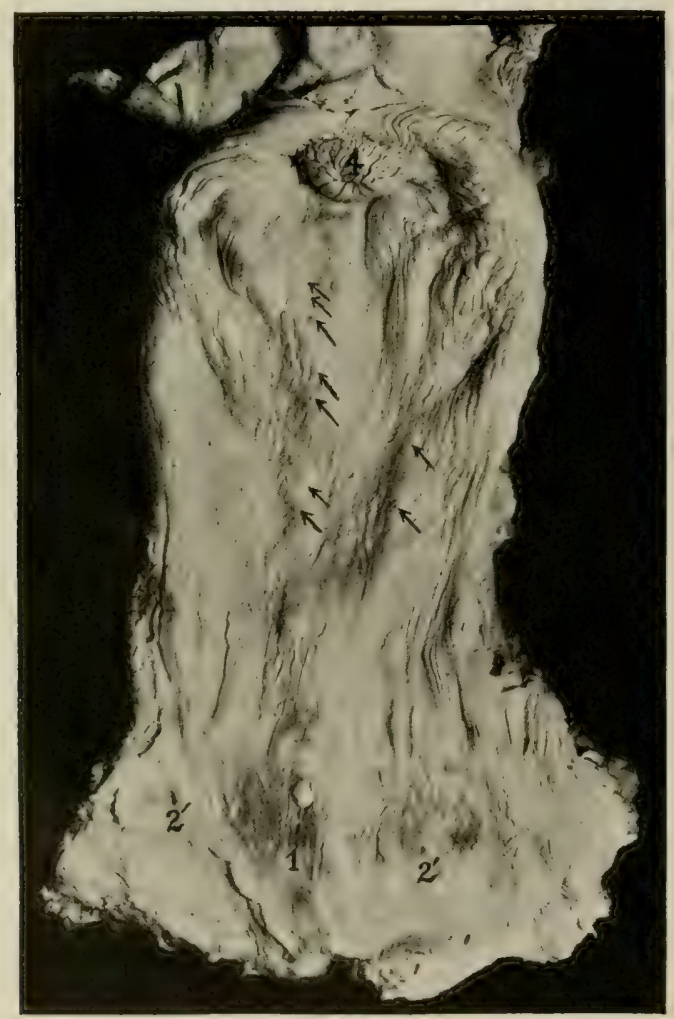

FIG. 2I. Vulva and Vagina of Cow.

$r$, Meatus urinarius; 2,2 , mouths of Bartholin's glands. The arrows point to very small cystic swellings in Gärtner's ducts.

region of the hymen, just anterior to the meatus urinarius, where, by their contraction, they produce a constriction on the vulvo-vaginal border line.

Through the medium of the surrounding aponeuroses, the vulvar muscles acquire continuity with the ischium, sacrum, 
and postero-superior border of the sacro-sciatic ligament, thus affording the vulva a secure attachment to the posterior opening of the pelvis and furnishing a fixed base upon which the entire genital canal may act in parturition. This fixation enables the uterus, when contracting, to force the fetus back toward and through the cervical canal, the vagina, and the vulva. It is this fixed base of attachment which enables the contracting uterus at the time of parturition to dilate and virtually efface the cervical canal, obliterating all its mucous folds and increasing its calibre sufficiently to afford room for the passage of the fetus.

The vulvar mucous membrane, continuous with that of the urinary bladder and the vagina, is covered with squamous epithelium and contains numerous mucous glands, which are largely displaced near the labial margins and about the clitoris by sebaceous follicles, the secretions from which are odoriferous, especially during estrual periods, when the odor becomes very marked in a manner characteristic of the species.

Within the vulvar cavity are to be noted the meatus urinarius, clitoris, Bartholin's glands, and vaginal bulb. In the mare the meatus urinarius, or terminal opening of the urethra, is located along the floor of the vulva at a distance of three to four inches from the external opening. The urethral canal passes obliquely downward and forward through the vaginal floor to the urinary bladder. In most animals the meatus urinarius is comparatively small and inextensible. In the mare it is large and freely dilatable. With patience, one, two or more fingers may be pressed gradually through it into the bladder, and it is not rare to find the opening, in heavy, coarse animals, sufficiently large to admit without great difficulty the entire hand. The amplitude of the meatus urinarius in the mare is of special significance clinically, as it favors eversion and prolapse of the bladder, an accident not particularly rare in this animal, though extremely so in others. In the cow the narrow meatus urinarius has beneath its vulvar opening a small sac, or infundibulum. The supero-anterior wall of the 
sinus constitutes the inferior terminal wall of the ureter causing it to act essentially as a valve, rendering catheterization difficult.

Bartholin's glands, (See Figs. 18, 21) like Gärtner's ducts, are vestigial structures presumed to represent the bulbo-urethral, or Cowper's glands, of the male. In the cow they consist of small, glandular structures located behind the vulvo-vaginal or hymeneal border, about midway from floor to roof of the copulatory canal, backward, upward and outward from the meatus urinarius. Their interest lies largely in the fact that, like Gärtner's ducts, their mouths become occluded as a result of infection causing, in the cow especially, retention cysts in the vaginal walls.

The clitoris is composed chiefly of erectile tissue like that of the corpus cavernosum of the penis. The clitoris of the bitch, like the penis of the dog, contains a small bone. The functions of the clitoris are not important, although it is alleged to exert an influence upon sexual excitement. In observations upon a large number of sows from which the clitoris had been removed by an empiric, the operation had no influence upon estrum or fecundation and all bred normally.

\section{The Physiology of the Genitalia}

The specific function of the generative organs is the procreation of the species, including conception, the intrauterine nutrition and development of the fetus, and its expulsion after a certain degree of development. As the two sexes in mammalia are wholly distinct, before conception can take place a conjunction of the male and femalecoition, or copulation - must occur. In each individual there are more or less apparent vestiges of each part of the genital apparatus of the opposite sex, but these naturally become arrested in their development in the embryonic stage and remain wholly functionless.

Before reproduction becomes possible, the breeding animal must have reached the period of puberty, or sexual maturity, which is marked by the advent of sexual desire. When this period has been reached, ova mature in the ovary 
of the female and spermatozoa in the testicle of the male. Up to this time, the reproductive organs are dormant in so far as their specific functions are concerned. Puberty, or sexual maturity, occurs at varying ages in different species, breeds, and individuals, depending upon the food supply and rapidity of growth. It occurs quite uniformly in both sexes prior to the completion of growth.

Reproduction is a complex physiologic process, accompanied by or associated with phenomena which bear an important relation to each other. In approximately the following order, there are observed the maturation of the ovisac-or Graafian follicle-estrum, copulation, rupture of the ovisac, fecundation, and possibly menstruation. The chain of phenomena is finally completed by pregnancy, parturition, and the nutrition of the new-born.

The ovaries consist at first of a mere thickening of the peritoneum, due to an elaboration of its epithelial cells. The external cell layer becomes columnar. The cells of the deeper strata assume a more or less cuboidal form, to constitute the germinal cells, and certain of their number become distinctly larger than the others, to form the primitive ova. The deeper epithelial layers are broken into irregular columnar masses, or $e g g$ columns, through the growth among them from below of blood vessels and connective tissue. Prior to birth, in those young which are born in a well developed state, and shortly after birth in the immature young like that of the rabbit, some of the primitive ova become materially changed, to constitute permanent ova. In the cell masses the permanent ova, developed from the primitive ova, become much larger than the others, while the nucleus, or germinal vesicle enlarges and its enveloping membrane becomes distinct. The contents of the nucleus become massed at one point and form a distinct reticulum, in which one or more nodal points enlarge to constitute the nucleolus, or germinal spot. The neighboring germinal cells become arranged about the ovum in such a manner as to enclose it completely in a follicle, which has at first a single layer of cells. Later a second layer of cells 
forms within the first, closely investing the ovum and continuous at one point with the cellular layer of the wall. This mass of cells, bearing within it the permanent ovum, constitutes the discus proligerus.

The cells of the follicular walls multiply rapidly. The external enveloping layer extends more rapidly than the inner discus proligerus causing a separation between the two, except at the point of attachment of the latter. A section through the discus proligerus and follicle reveals a crescent-shaped cavity filled with fluid. Fully developed, this constitutes the ovisac, or Graafian follicle, which consists of the outer layer of follicular cells, or tunica granulosa, and the inner granular cells, the discus proligerus, embedded within which lies the ovum. The cavity of the follicle between the two masses of cells is occupied by the follicular fluid. The immature egg sac usually lies deep within the ovary. As it matures and the volume of follicular fluid increases, it approaches the surface of the ovary. In the cow about one-half of the ovisac protrudes finally beyond the general ovarian surface as a hemisphere, -. 'ile the other half remains below the general level of the gland. The ripe ovisac of the cow is about one-half to fiveeighths of an inch in diameter. In the sow the ovisac grows completely beyond the ovarian surface and appears as a pedunculated cyst one-fourth inch in diameter. In pushing toward and beyond the ovarian surface, the tunica albuginea, or ovarian capsule, atrophies and disappears at the summit of the distended sac, and the peritoneum is pushed outward. The follicular wall becomes very thin. During rectal palpation in the cow, the ovisac often ruptures under very slight pressure. During the maturation of the ovisac, the ovum undergoes important changes. Before the ovisac ruptures, the nucleus of the ovum passes from the center of the egg toward its periphery; a definite vitelline membrane is formed within the zona radiata immediately about the nucleus of the ovum; the nucleus becomes indistinct; and, while the yolk retracts slightly from the vitelline membrane at one point, the first polar body, a small mass apparently derived from an unequal division of the nucleus, is assumed to be extruded. 
When the egg has fully ripened and the ovisac has completed its growth and is fully distended with fluid, estrum, or "heat", occurs.

\section{Estrum}

Preliminary to reproduction, an irresistible sexual desire occurs, leading to coition.

Estrum finds expression in various ways in different species of animals. In general there is a nervous excitability, the external genitals are swollen and vascular, and there is an increased secretion of mucus from the vulva and the

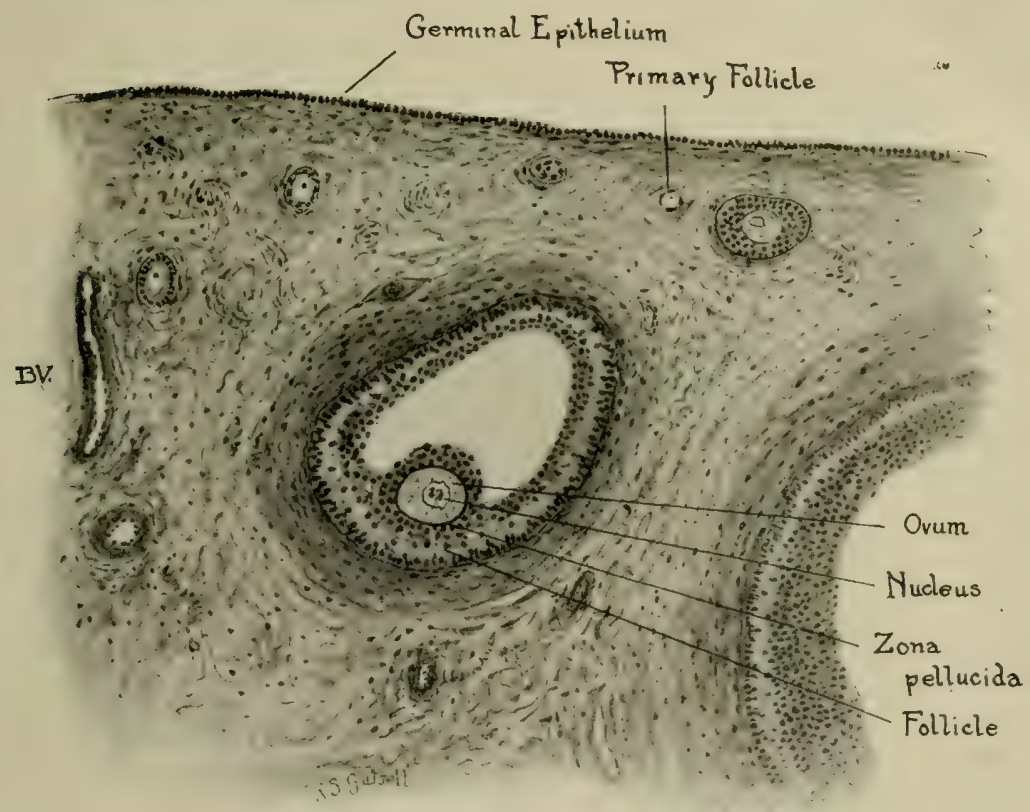

IVIG. 22-Section of ovary of cow showing a follicle, $0.6 \mathrm{~mm}$., with ovum.

vagina. In the mare, when other horses-especially stallions-are present, there are frequent emissions of urine in small quantities, and the vulvar lips are frequently opened and the erected clitoris protruded. In the cow and the sow, the female imitates the copulatory act of the male by mount- 
ing, or standing to be mounted by, other animals of its species. The ewe ordinarily shows little or no evidence of estrum except in the presence of the male. In certain pathologic cases (nymphomania) the cow especially does not confine her expressions of sexual desire to her own kind, but may attempt to mount other species of animals.

In all animals there is a tendency during estrum for the female to wander from home. This is most noticeable in the bitch and the cat, which, if not securely confined, disappear, tending to wander long distances and to remain away during a large part or all of the estrual period. The sexual wanderings of domestic animals constitute an interesting chapter in the dissemination of infectious diseases.

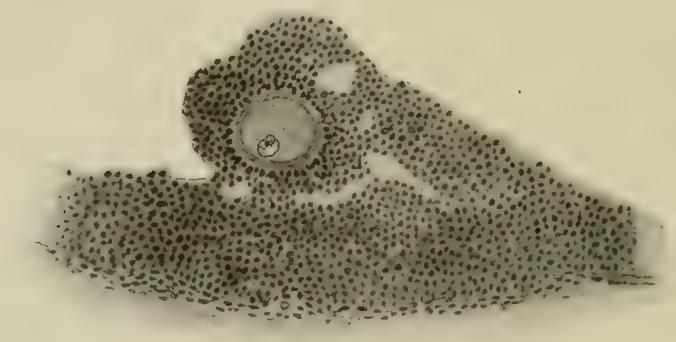

FIG. 23-Section of ovary of cow showing a follicle ot $2 \mathrm{~mm}$., with ovum.

The female exhibits sexual desire only for a comparatively brief time, during the existence of a ripe ovisac or ovisacs in the ovary. The duration of the estrual period varies widely, according to species. It is shortest in the cow, ranging ordinarily between eighteen and twenty-four hours, or a trifle longer. The mare is usually in estrum for several days. The bitch is in estrum for ten to fifteen days. In healthy females, the estrual cycle is quite uniform. In the cow it is twenty to twenty-two days, usually twenty-one; in the mare 3 to 4 weeks; in the bitch ordinarily every six months. The frequency, intensity, and duration of estrum are profoundly modified by the state of nutrition, diseases of the genital organs, and by serious systemic diseases. 


\section{Ovulation}

At about the close of estrum, in those animals where the phenomena have been studied, as in the cow, the distended ovisac ruptures and discharges the ovum. Prior to the rupture of the ovisac, preparation for the detachment of the ovum from the enveloping and supporting cells of the discus proligerus has been made by the formation of numerous vacuoles in the mound, as shown in Fig. 24. The attachment of the ovum is rendered exceedingly frail, subject to detachment upon the slightest disturbance. The ovum is apparently maintained in situ chiefly, if not wholly, by the

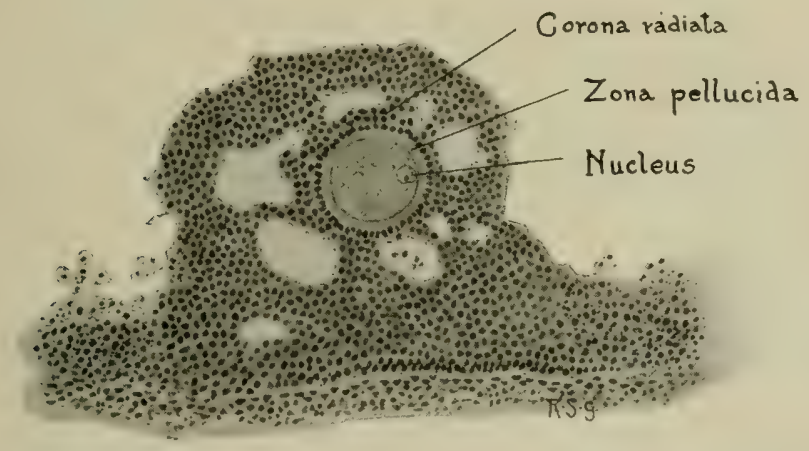

FIG. 24-Section of ovary of cow with a nearly ripe ovum in an ovisac, $5 \times 7 \mathrm{~mm}$., showing vacuoles in the cumulus preparatory to ovulation.

sustaining pressure of the follicular liquid. When the ovisac ruptures, the ovum, the follicular fluid, and some of the granular cells of the discus proligerus in which the ovum was embedded are discharged into the pavilion of the oviduct, which is at this epoch erected and closely applied to the ovary at that point where the ovisac is about to rupture. Here, if successful copulation has occurred, spermatozoa are already present, meet the ovum and a single spermatozoon fuses with each, to constitute fertilization.

The precise period at which ovulation occurs, as related to estrum, has not been determined for all domestic animals. In the rabbit, which has given birth to young, copulation 
occurs immediately following parturition. Ovulation follows about twelve hours later-an interval sufficiently great for the migration of spermatozoa from the vagina to the ovary. In the cow, estrum persists ordinarily for but twelve to twenty-four hours, and ovulation occurs at about the close of this period. If copulation occurs early in estrum, better opportunity is afforded for the arrival of spermatozoa at the pavilion of the oviduct prior to the rupture of the ovisac and discharge of the ovum. In swine, carnivora, and to some degree in solipeds, the estrual period is prolonged, extending over several days. In these there are no accurate data of the epoch of ovulation. Probably ovulation occurs at a time corresponding to that observed in the cow-that is, at about the close of estrum.

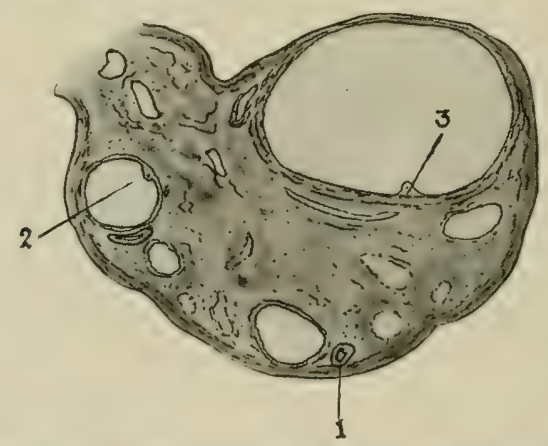

FIG. 25-Diagram of ovary of cow showing comparative sizes of the follicles shown in Figs. 22, 23, 24

The number of ovisacs rupturing at a given estrual period corresponds as a rule with the maximum number of possible fetuses. It is said that rarely two ova are contained in one ovisac. I have been unable to verify this statement, and have in all cases of twins in the cow observed two corpora lutea, sometimes both in one ovary but most frequently one in each. Sometimes a single fertilized ovum divides to constitute two embryos, which form identical twins in man, but this is not known to occur in domestic animals, though it probably does so. In multipara, it is assumed that there are at least as many ova discharged, from as many ovisacs, as there are embryos. 
Physiologically, estrum and ovulation are inhibited by pregnancy; abnormally, they occur while the animal is pregnant and the fetus alive and well.

\section{Menstruation}

If a heifer or a cow fails to conceive, when estrum, copulation, and ovulation have occurred, or if copulation has failed, she regularly menstruates. If she conceives, she does not as a rule menstruate. Evidently the engorgement of the uterus during estrum is designed to fit the genital tract for the protection and nutrition of the fertilized ovum. Should fertilization fail, capillary hemorrhage from the placental areas follows. The volume of hemorrhage is sufficient that the blood is expelled through the vagina and vulva to stain the exterior vulvar region and adjacent parts. Upon the killing floor of the abattoir, heifers or cows having in their ovaries ripe ovisacs have engorged uteri with special engorgement of the placental areas. If the ripe ovisac has ruptured, and presumably fertilization has failed, the placental areas are covered over with thin coagula of bright red blood. Later this is expelled, to constitute the menstrual fluid. The exact significance of menstruation is not fully determined. By my observations I have been led to the conclusion that menstruation constitutes reliable evidence of a failure to conceive, so far as the estrum just past is concerned. Apparently there are exceptions to the rule.

There is much controversy amongst embryologists and human obstetrists regarding the relationship between menstruation, ovulation, and conception. There is no outstanding estrual period in woman, and scant opportunity for the clinical study of ovulation. The cow offers the best available animal for such study. Her estrual period is sharp, clear, and brief; the ovary is conveniently palpated per rectum; the ripe ovisac is clearly recognizable by palpation; and the crater of the recently ruptured sac is clearly palpable. The order of the phenomena is clearly and indisputablymaturation of the ovisac, estrum (with copulation, if opportunity exists), ovulation, and fertilization, or, if fertilization 
fails, menstruation. The cow is probably representative for mammalia in general.

\section{The Corpus Luteum of Estrum}

Ovulation leaves behind a crater having the same diameter as the ovisac which ruptured. Into this crater, physiologically, a small amount of blood escapes at once and quickly clots. The walls of the crater rapidly approach each other and within a few hours there remains a small, deep cavity, about one-eighth to three-sixteenths of an inch, filled with a blood clot. Ovulation has necessarily caused a lesion, comparable in some respects to a wound, in which ordinarily the wound cavity is to a greater or less extent filled with coagulated blood, and like the wound in the body covering, the lesion offers an open avenue for invasion by any bacteria existing in the peritoneal cavity or within the oviduct. Such infection frequently interrupts the orderly physiologic processes and leads to a variety of pathologic lesions.

Soon after the rupture of the ovisac and discharge of the ovum, there is elaborated from the walls of the crater a distinctive tissue designated the corpus luteum (yellow body) because its prevailing color in pregnant animals is yellow. The corpus luteum consists of a network of capillaries, some connective tissue, and essentially of special lutein cells emanating from the membrana granulosa and theca interna of the ovisac. The method of growth causes the corpus luteum to repeat at first the form of the crater from the sides of which it originates. Originally it is a cup-shaped structure opening upon the surface and filled in its center by the small blood coagulum which formed in the crater of the ruptured ovisac immediately after ovulation. The corpus luteum pushes its way out somewhat through the rupture in the ovarian tunic and projects (in the cow) somewhat above the ovarian surface as a cylindrical or conical mass of granulations, one-eighth to onehalf inch in diameter. The cavity in the center of the corpus luteum of the cow is gradually eliminated by the growth of the lutein tissue and at the same time the epithelium of 
the ovary draws over the naked granulating surface until at seven to ten days, the structure, if physiologic, has lost its central cavity, the blood coagulum has disappeared and the epithelium of the ovary has recovered its continuity. The corpus luteum of the cow is then five-eighths to threequarters of an inch in diameter, usually spherical or ovoid, but subject to numerous variations, has an even contour and is firm in consistence. It is readily recognized in the cow by rectal palpation and usually easily dislodged by digital compression. If the ovum discharged is not fertilized, or if the fertilized ovum perishes quickly, the corpus luteum of the cow is not yellow but with great uniformity is chocolate or liver-colored. Its size, contour, and consistence are essentially the same as in pregnancy. The volume remains virtually constant after its growth has been completed at seven to ten days until at a period of about three or four days prior to the maturation of the next ovisac, when it commences to atrophy rapidly, and when about one-fourth inch in diameter, a new ovisac ripens, generally in the opposite ovary, the animal is again in estrum and ovulates. The old yellow body has permanently ceased, so far as known, to exert any influence upon the reproductive processes. It continues to atrophy slowly. There are frequently visible in a single section in the ovary of the cow, three to five old corpora lutea, one-eighth inch or less in diameter, with white strands of connective tissue scattered amongst the nests of lemon or orange-colored lutein cells.

\section{Copulation}

The male ordinarily exhibits little or no sexual desire except in the presence of a female in estrum, but the testes function constantly. The sexual desire may be activated at any time when a female in estrum approaches sufficiently near to be recognized, and copulation occurs when opportunity offers.

During the act of copulation, the semen from the male is ejaculated into the vagina of the female, possibly in part into the cervical canal. The semen has as its essential con- 
stituent the spermatozoa, or sperm cells, elaborated by the testicles. Prior to copulation the spermatozoa lie chiefly in the epididymis and vas deferens, being exceedingly concentrated in these tubules. At the moment of ejaculation, there is thrown into the pelvic urethra the liquid secretions from the vesiculae seminales, prostate and Cowper's glands which serve to dilute greatly the very dense mass of spermatozoa and facilitate the forcible ejaculation of the semen. Chief amongst these glands in the bull, are the seminal vesicles. If these are removed, or are so diseased that their function is impaired or in abeyance, the bull copulates with difficulty apparently owing to the absence of the diluent fluid from these structures. Some think that most or all of the semen is ejaculated directly through the cervical canal into the body of the uterus and that the urethral opening of the male comes in direct contact with or enters the os uteri. Harms, quoting Duranton (Journal de Lyon, 1888), cites a case of alleged rupture in the uterine cornu of a cow during copulation, owing to the entrance of the penis of the bull through the cervical canal, but this must have been an error in clinical history. A study of the cervical canal of the cow, as delineated in figures $15,16,17$, suffices to refute any allegation of the entrance of the penis into it. It is an anatomical impossibility. In the mare, with a far more dilatable cervical canal, copulatory injuries from an unusually long penis occur in the vagina-not in the uterus. Male animals, after the amputation of a considerable portion of the penis, are quite as fertile as before, although the stump of the organ can not reach the os uteri. Clinically, a large part of the semen is ejected from the vulva of the female immediately after copulation, and quantities of semen are readily obtained from the vagina at this time.

The condition essential to fecundation, so far as the male is concerned, is that physiologically perfect spermatozoa shall gain the cervical canal, traverse the uterus and oviducts, and meet the ovum in the pavilion of the oviduct. Of the countless myriads of sperm cells in one discharge of semen, but one can take part in the fertilization of the ovum.

The migration of the spermatozoa from the vagina to the 
pavilion of the oviduct involves a period of brief duration, probably varying in different species. Marshall estimates that in the rabbit, the migration of the spermatozoa requires but fifteen minutes to two hours. Copulation occurs eight or more hours prior to ovulation so that the spermatozoa ordinarily arrive at the pavilion of the tube six or more hours prior to ovulation and lie in wait preparatory to immediate fertilization. According to clinical observations in cows, ovulation occurs at approximately eight to twelve hours after the cessation of estrum. Ovulation is apparently hastened (and estrum abbreviated) by copulation. The influence of copulation upon ovulation in higher animals appears somewhat analogous to insemination in the frog, where the sexual embrace of the male apparently favors the ovulation. It appears from available data that the spermatozoa of the bull traverse the twenty to twenty-five or more inches of the genital tube (cervix, uterus, and oviducts) in two or three hours and are present in the pavilion of the oviduct some time prior to ovulation. In this manner the single ovum meets at once many spermatozoa so that its prompt fertilization is luxuriantly provided for.

\section{Fertilization}

Fertilization, fecundation, or conception follows when a healthy female copulates naturally with a healthy male. Since copulation, in those animals in which the sequence is known, precedes ovulation, spermatozoa have already migrated through the genital tube and have reached the pavilion of the oviduct, where they await the discharge of the ovum. One spermatozoon penetrates each egg and traverses the zona radiata to the nucleus. The nucleus of the spermatozoon fuses with that of the ovum. A beginning has been made for the development of a new individual. The animal has conceived.

\section{The Migration of the Fertilized Ovum}

After the ovum has been fertilized in the pavilion of the oviduct, it must traverse the length of the tube, reach a 
suitable location in the uterus, and become implanted there before it can advantageously receive ample nutriment for needed growth and development. The migration through the oviduct must occur with sufficient celerity that the ovum shall enter the uterine cavity prior to any noteworthy growth in volume for the reason that the lumen of the tube is so small that important increase in the size of the egg would imperil its passage. It is probably owing to pathologic decrease in its lumen that the ovum occasionally lodges in the oviduct of woman (tubal pregnancy), destroying the life of the embryo and greatly imperilling the life of the mother. The migration of the ovum, unlike that of the spermatozoa, is passive and is brought about by the action of the cilia of the tubal epithelium which wave toward the uterus, and probably by muscular action in the walls of the oviduct. The time required in domestic animals is not known but in the cow it is probably eight to fifteen days.

\section{The Corpus Luteum of Pregnancy}

The formation of the corpus luteum of pregnancy is fundamentally like that of estrum as described above. Its source of origin, its method of growth and its ultimate volume is the same. It presents two notable characters which serve to distinguish it.

The color of the corpus luteum of pregnancy in the cow is regularly of a bright lemon color, in contrast with the chestnut or muscle-colored structure of estrum. The color distinction is not universally present. Amongst approximately 2000 pregnant cows and heifers, I have observed two corpora nigra, or black bodies, one normal in size, the other about double the normal volume. The rule of chestnut color for the corpus luteum of estrum and lemon or orange color for the corpus luteum of pregnancy appears at times to be reversed. Some have claimed that the corpus luteum of estrum is often lemon or orange-colored but I believe most of these reports are referable to error. That is, observers not familiar with their ground see in the abattoir an empty 


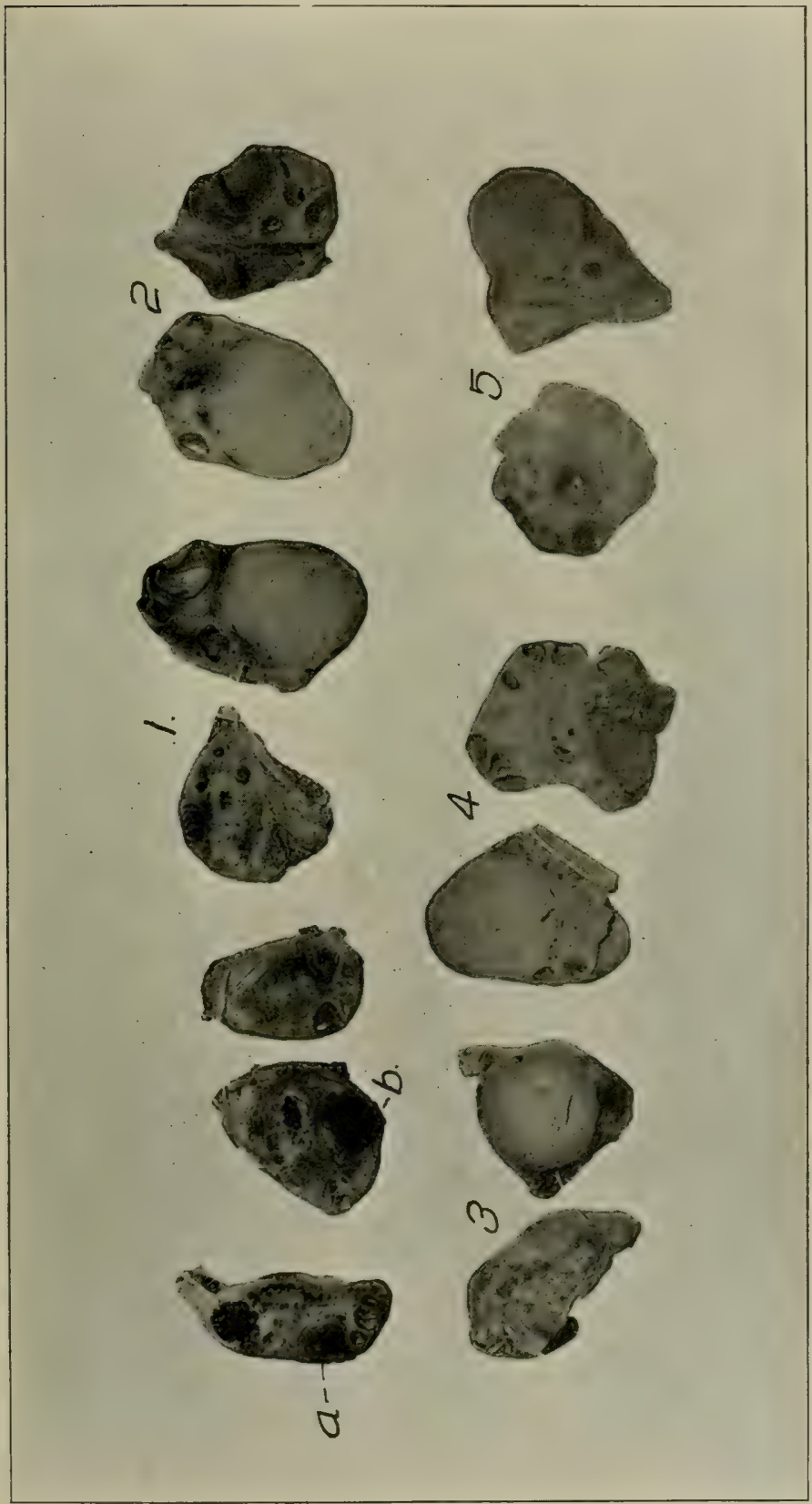

苞

ن

당

踏

क्ष०

คำ ปัँ

प्山े

으르

ป

훙현

긍

里

웅

0 记

व. क

부을

굴

늘

i.

江落

을

Uु

$\approx$

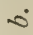

8 
uterus and an orange-colored corpus luteum and consider the structure that of estrum. But there has been pregnancy and the corpus luteum has not yet disappeared.

The duration, or life, of the corpus luteum of pregnancy presents a strong contrast in most domestic animals to the corpus luteum of estrum. The corpus luteum of pregnancy has as its minimum physiologic life the duration of pregnancy. The simplest cycle in ovulation, pregnancy, and atrophy of the corpus luteum is in the rabbit where the duration of the corpus luteum and of pregnancy are identical. The doe conceives, the corpus luteum forms, in four weeks she gives birth to young, the corpora lutea have atrophied, new ovisacs have matured and immediately after parturition she copulates with the male and conceives.

In the cow, the corpus luteum forms promptly after conception, attains its growth in seven to ten days and physiologically remains static until thirty to ninety days post partum, or for a period of ten to twelve calendar months. Pathologically (retained corpus luteum, pyometra, macerated or desiccated fetus, etc.), the corpus luteum of pregnancy may remain static indefinitely and inhibit estrum and ovulation. In the mare it appears that the corpus luteum of pregnancy atrophies promptly after parturition so that she is ordinarily in estrum at seven to nine days post partum, thus giving it a physiologic duration of about three hundred fifty-five days. In those animals which have short periods of pregnancy and are normally in estrum semi-annually, like the bitch, the corpus luteum appears to persist for a comparatively long period after parturition or estrum.

\section{The Fetal Membranes}

After fertilization has occurred, the ovum passes through the oviduct, propelled largely by the cilia of the tubal epithelium, and soon reaches the uterine cavity. During its tubal migration, the ovum has undergone segmentation. The stored food material in the ovum is insignificant, so that it must be dependent upon the uterus for any nutrient material for the maintenance of life and development. The nutrition of the ovum, embryo, and fetus is throughout in- 


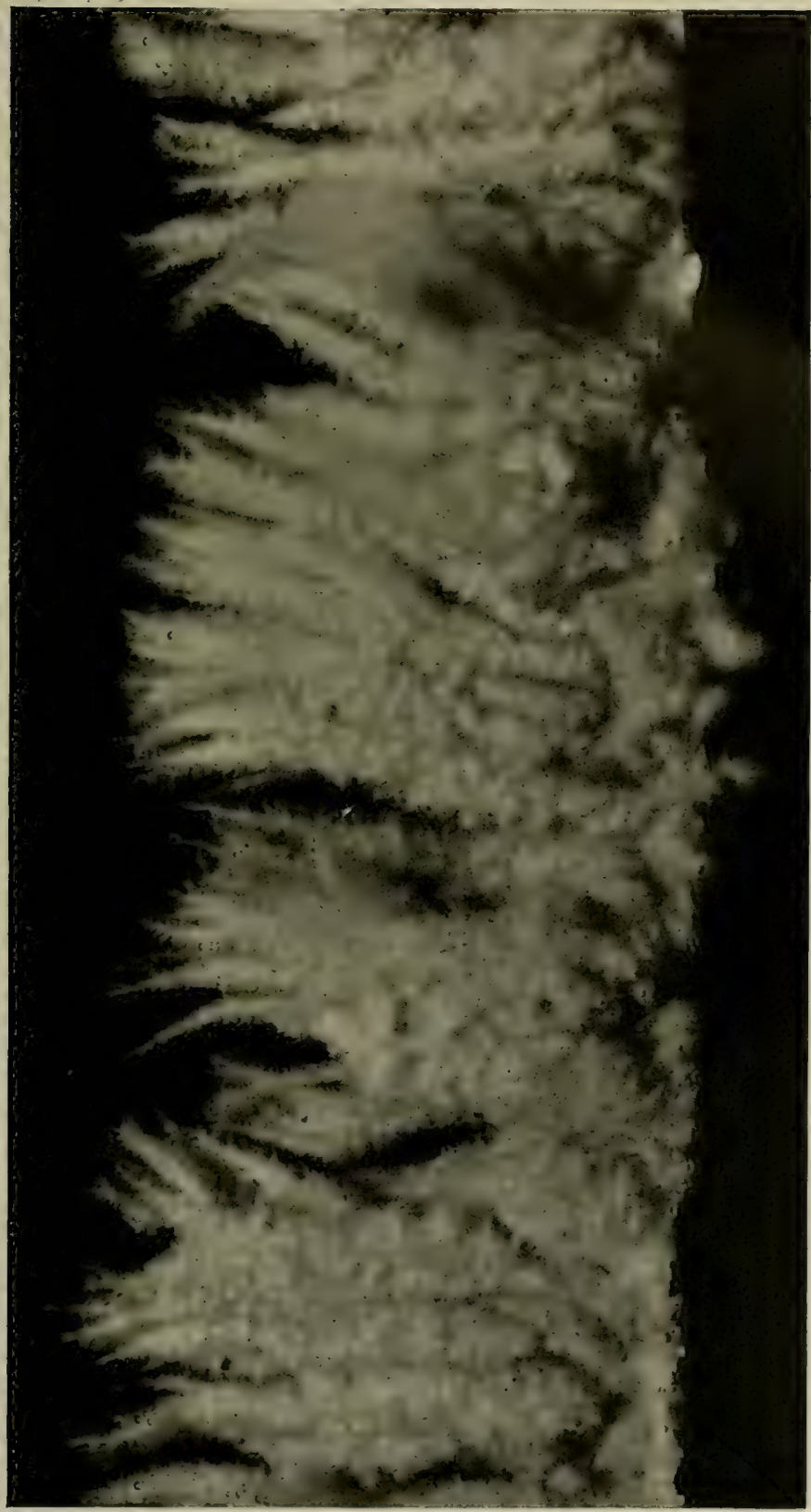



ํㅕㄹ

.

क्ष

ठํํㅇ

4

娄

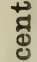

$\stackrel{\Xi}{0}$

평

영

80

둔

(1)

过 
direct: that is, when the ovum is discharged from the ovisac, its direct connection with the tissues of the mother is finally and permanently severed. The fertilized ovum at first lies free upon the uterine mucosa while nutrient fluids poured out by the endometrium are absorbed by the ovum to provide for its development. Thus far, the life of the

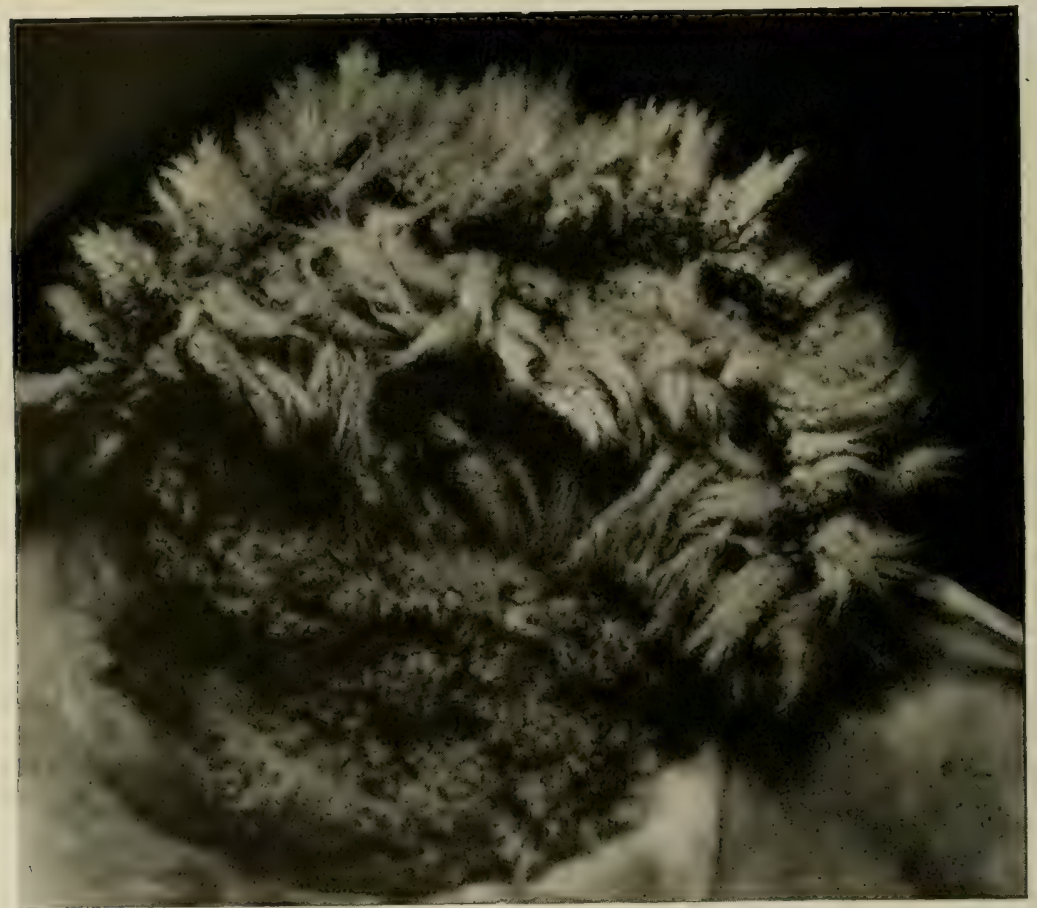

FIG. 2S-Fetal Cotyledon of Cow showing primary and adventitious Placentae. Photograph.

ovum has been supremely precarious, relying wholly upon a delicately balanced physiologic state of the oviduct and uterus and their contents. It has no known inherited power to resist the attacks of any bacteria contained within the tubo-uterine cavity and has no known power to maintain a given location. Clinical observation leads readily to the belief that the mortality of the fertilized ova during this period 
is the greatest occurring at any epoch in life. The death is unseen, and is necessarily designated sterility. The newly created individual needs greater fixidity in its place, more accurate protection against invasion, and especially a safe and abundant food supply. While the ovum lies free in the uterine cavity, any liquid nutriment available is in constant danger of being contaminated by any bacteria or other deleterious substances present in the uterus. Such toxic sub-

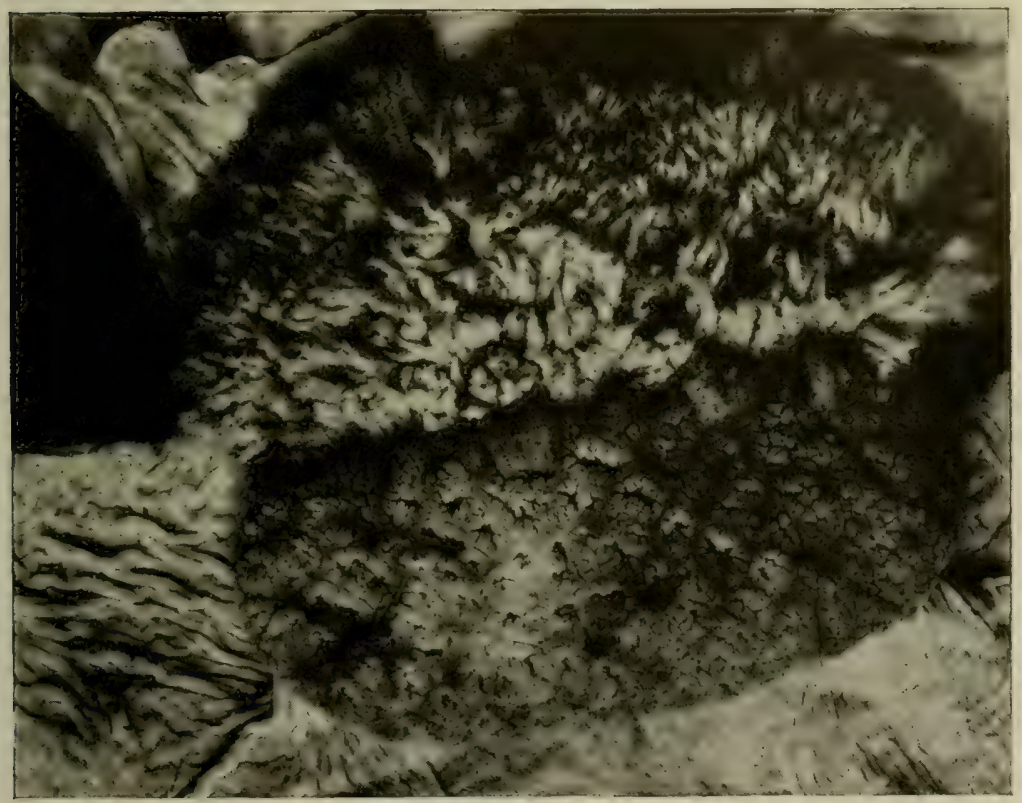

FIG. 29-Maternal (bottom) and Fetal (top) Cotyledons of Cow partly detached. A portion of the uterine wall seen at bottom and of choriom at top.

stances constitute a menace by threatening to invade the new individual mixed with nutritive substances, through a common portal, the external layer of the morula. The nutrition needs to be more dependable, abundant, and immediate, and guarded as far as possible against contamination from every source. The uterine seal hermetically closes the cervical canal to direct invasion from the vagina. Since the ovarian end of the genital tube, though opening into the 
peritoneal cavity, is very narrow and the peritoneal cavity is assumed to be physiologically sterile, the embryo lying in the genital tube between the tubal and cervical openings, once the uterine seal is physiologically formed, enjoys a high degree of safety in these directions. Two vital dangers

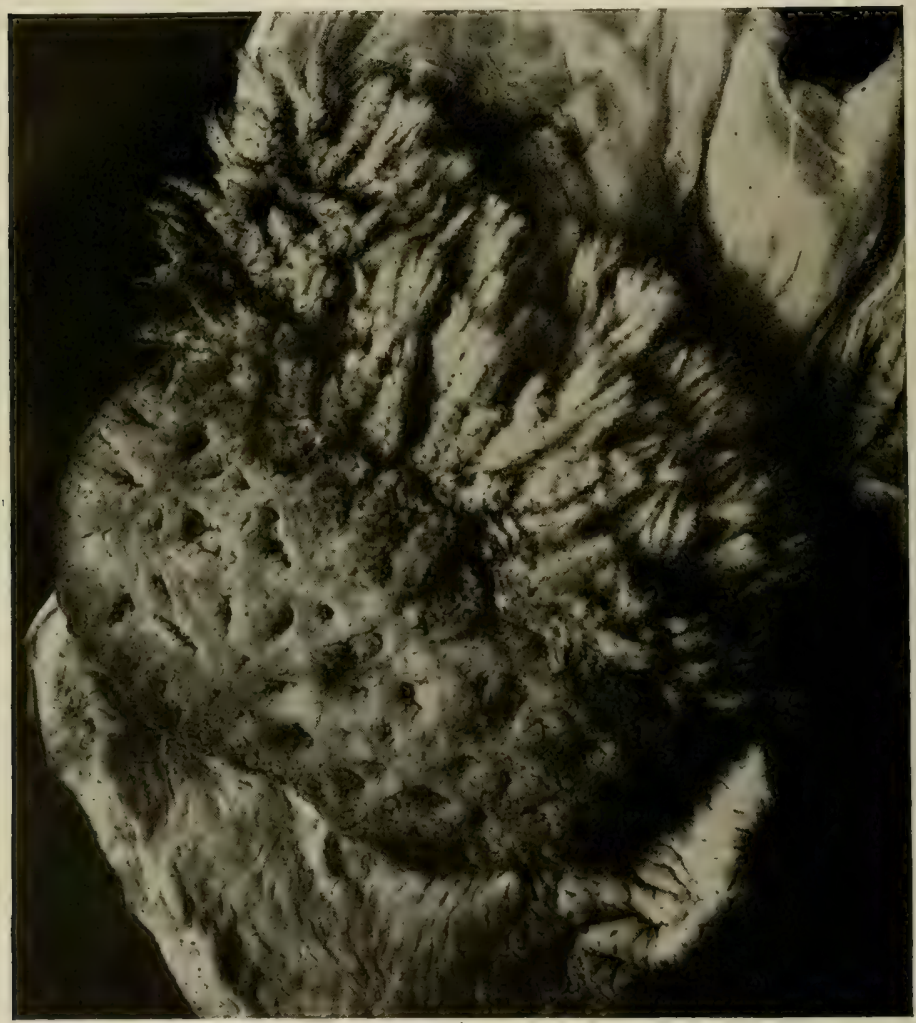

FIG. 30-Same as Fig. 29, showing a different type of Placental Crypts with thickened, fibrous partitions possibly due to prior disease.

remain. There is constant peril to the embryo from bacteria, already old habitants of the utero-cervical cavity, and there is a further threat of unknown potentiality from micro-organisms floating in the blood of the mother, which may invade the uterus from the blood stream. These dan- 
gers are obviated in part by the formation of the placenta.

The placenta consists of two complementary parts-one due to a special development of the whole or certain areas of the uterine mucosa, or endometrium, of the mother, and the other to an analogous development from the chorion of the fetus. The embryo or embryos early become arrested and detained for the rest of intra-uterine life in a definite and comparatively fixed location. In unipara the rule is

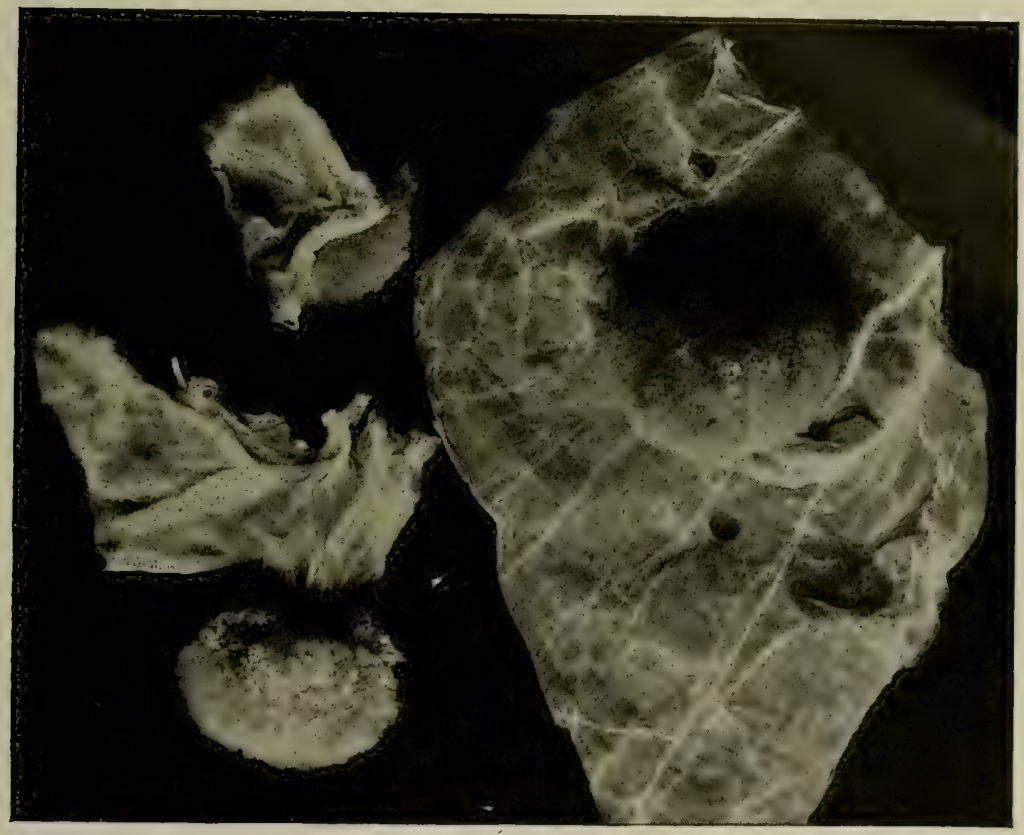

Fig. 3I-Chorionic Placenta of Ewe.

The right hand figure includes a portion of the Chorion.

that the embryo becomes located partly within the cavity of the uterine body, with the remainder of its body lying in that horn of the uterus from the corresponding ovary of which the ovum was derived. Pathologically, the fetus of a soliped, instead of growing into the uterine body, may extend across the ovarian end of the uterine body cavity into the opposite horn, to constitute bicornual pregnancy. In multipara the embryos become halted at approximately 
equidistant points in the cornu corresponding to the ovary from which the ova emanated. If but one embryo is present in a horn, it is ordinarily near its base, and very rarely may become, as in the mare, bicornual. When near full term, the fetuses may be very irregularly located, owing to the fact that one or several of them has perished and later has been absorbed.

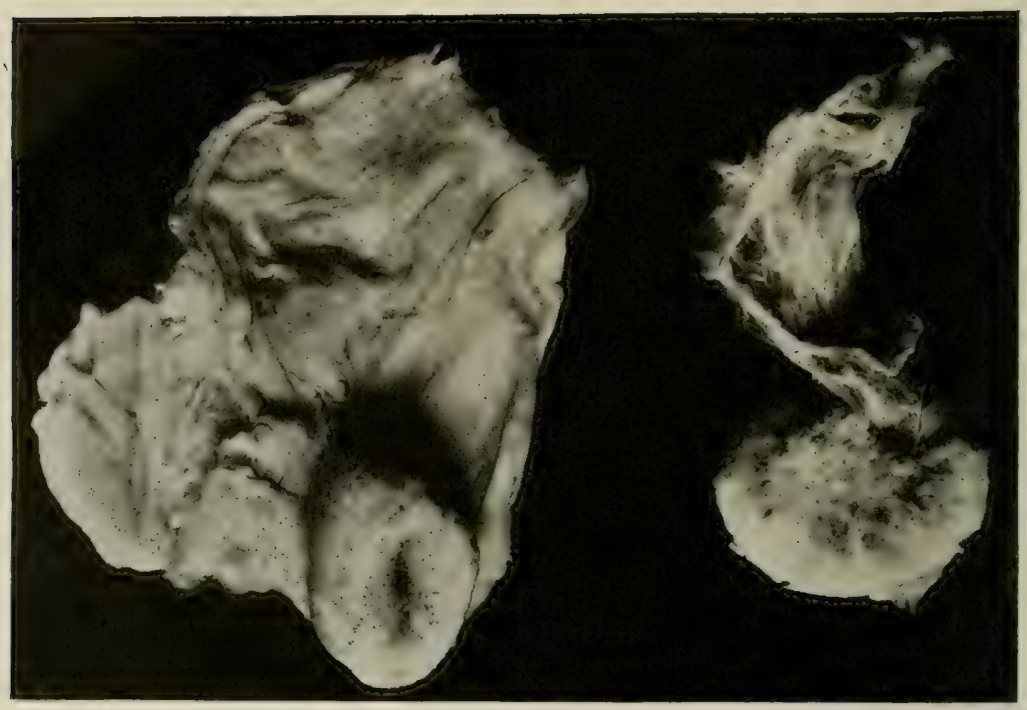

FIG. 32-Left. Uterine Cotyledon of Ewe,

from which Fetal Cotyledon has been removed, leaving a cavity.

Right. Median section of Maternal and Fetal Cotyledon of Ewe, with portion of Chorion attached.

At the point where a fertilized ovum becomes located within the uterus, the adjacent uterine walls promptly assume increased functional activity, become increased in thickness, and show heightened vascularity. The vital changes occur in the endometrium, and particularly in the placental areas. In ruminants with multiple cotyledonal placentae, the placental areas are already visible in the uterus of the female fetus as definite elevations in the uterine mucosa. When the existing placental areas or caruncles of 
the-ruminant have been destroyed by disease, the former non-placental areas develop adventitious placental structures, but in such cases want their multiple cotyledonal character and take on the simpler structure of a diffuse placenta.

As soon as the embryo begins to form, its enveloping membranes commence to develop, first the amnion, followed closely by the allantois. The external allantoic wall fuses with the external amniotic layer (false amnnion) to con-

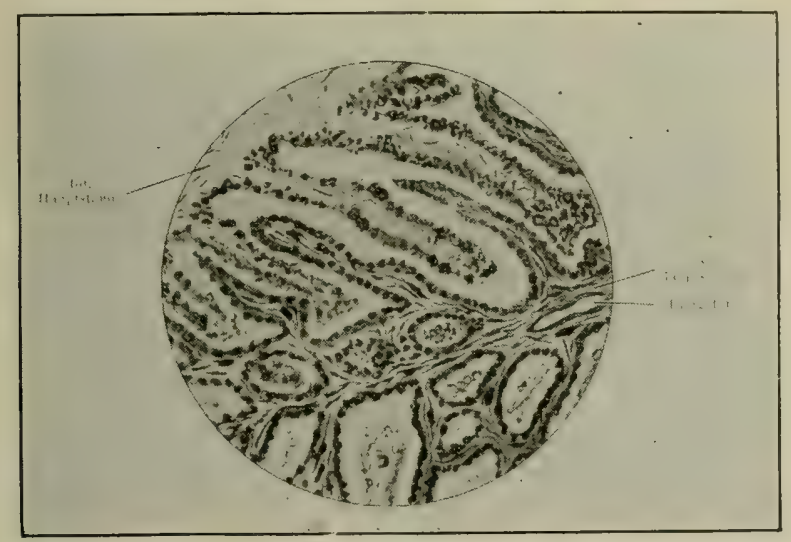

Fig. 33-Fetal and Maternal Placenta of Cow.

The chorionic or fetal placental tufts are shown above and to the left, projecting downward, and to the right, into the placental crypts of the uterus. $x_{3}$ ro (Pomayer).

stitute the chorion. In all domestic animals the amnion and allantois each constitutes a sac containing considerable volumes of amniotic and allantoic fluids respectively. In unipara, the allantoic sac normally pushes out to the apices of both uterine horns and to the os uteri internum, thus acquiring contact with the uterine mucosa throughout. In ruminants and swine, however, the tips of the allantoic sac early become necrotic in varying degree, as shown in Figs. 35 and 36 . The necrotic portion retracts at times from the apex of the cornual cavity, leaving the lumen filled with pus and debris. In extreme cases, the entire non-gravid horn of the allantois of the cow becomes necrotic as shown in Plate 
III. Sometimes, also, as shown in Plate II, the chorion becomes necrotic where it passes across the os uteri internum. The common, small necrotic tips, shown in Figs. 35 and 36, are designated as "normal" by embryologists but these pass by imperceptible gradations into the degree of necrosis shown in Plate III which is clearly and grossly pathologic. The allantoic sac, fully distended with fluid, fills the uterine cavity in such a manner that violent movements of the mother cannot readily cause any material displacement between the uterine wall and the chorion, but both are moved

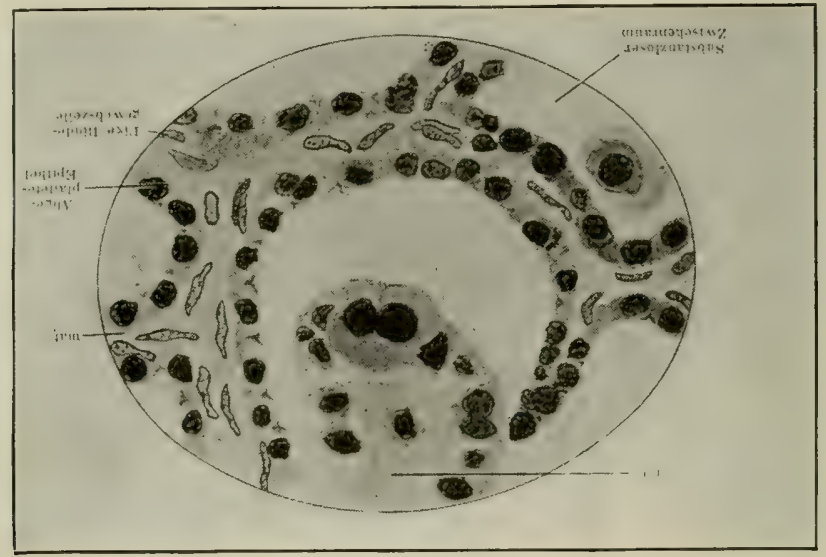

FIG. 34. Same as Fig. 33. x 900. (Pomayer).

as a unit, obviating largely any dragging upon the placenta which might threaten to cause injury thereto. The fetus floats within the amniotic fluid and the amniotic sac, in turn, floats within the allantoic fluid. In solipeds, swine and carnivora, the amniotic sac floats freely within the allantoic fluid, except for the allantoic segment of the umbilic cord; in ruminants, the dorsum of the amniotic sac is intimately fused with the chorion, obliterating the allantoic cavity in this area. The fetuses of solipeds, swine, and carnivora may be expelled from the uterus through a rent in the chorion, with the more or less intact amnion enveloping them, but in ruminants the adhesion of the amnion 


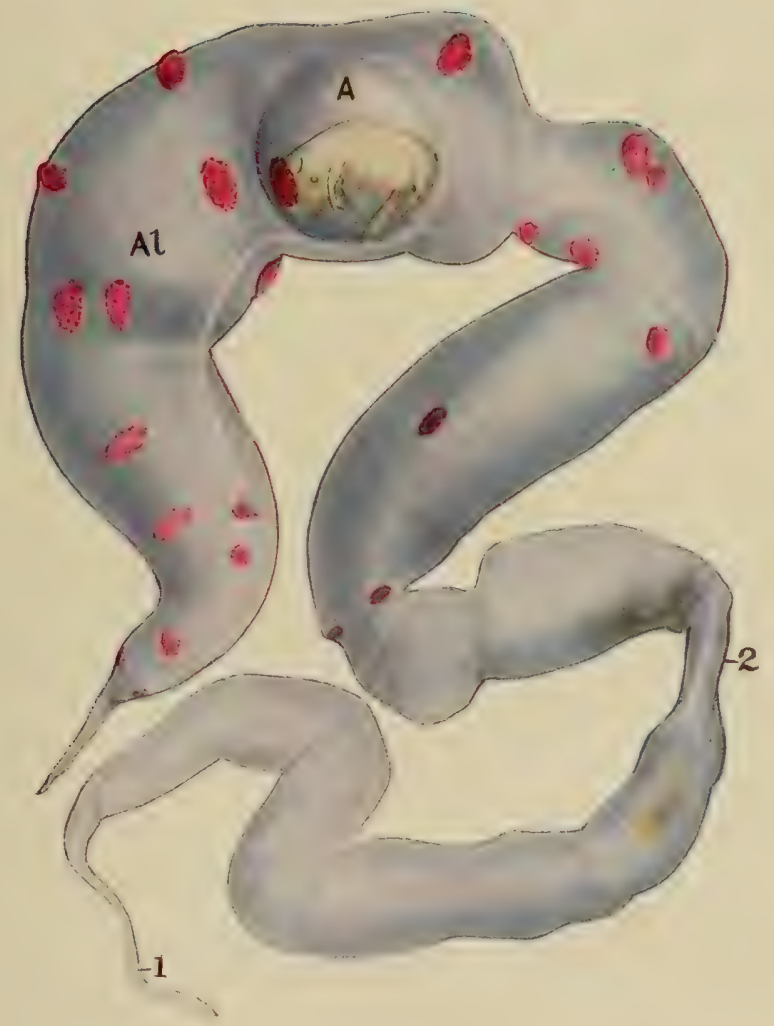

Plate II

Fetal Sac of Cow at About ioo Days

t. Ammion.

A1. Allantois.

1. Necrotic tip of non-gravid horn.

2 Necrotic isthmus between gravid and non-gravid horn. 



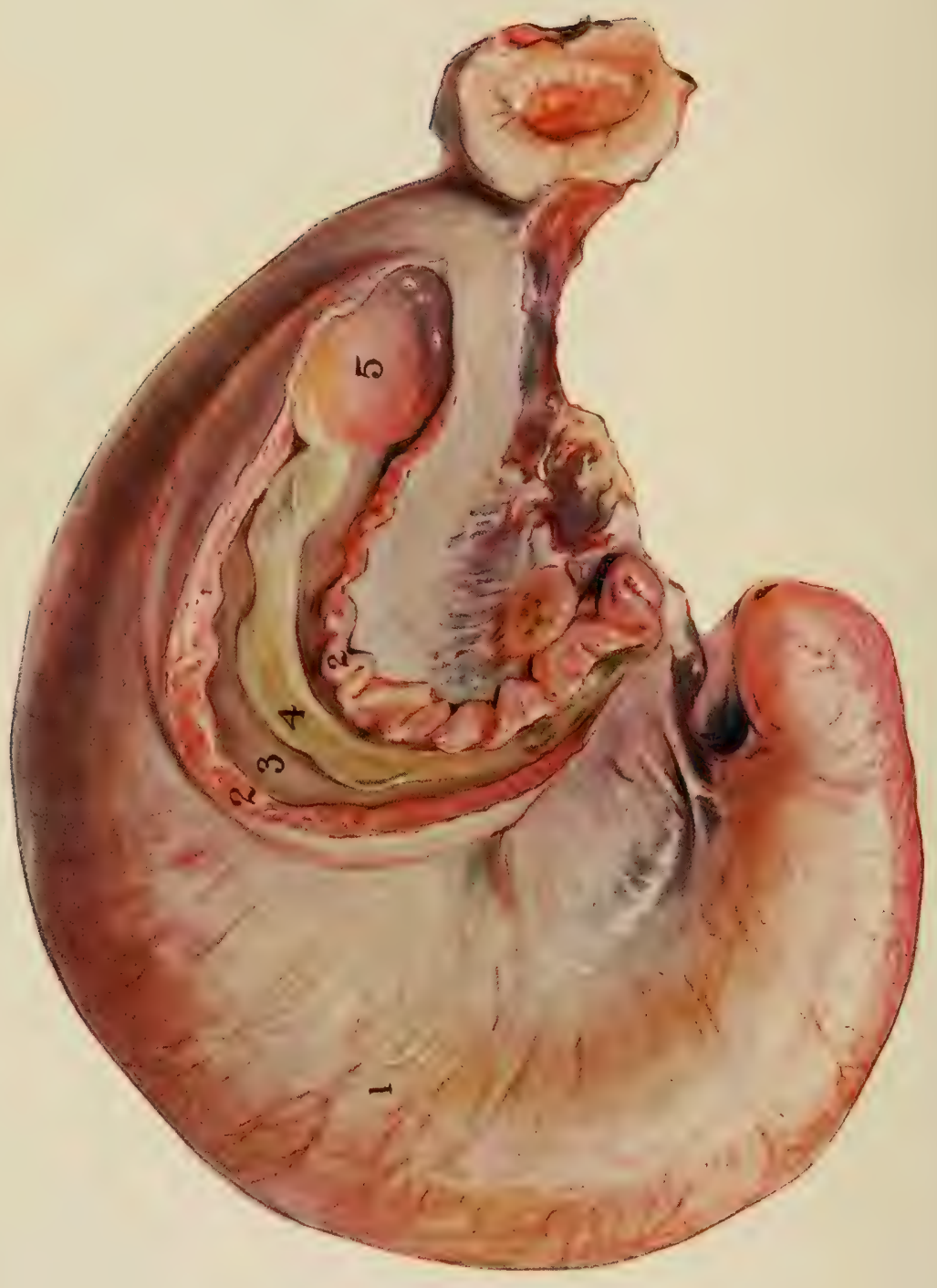




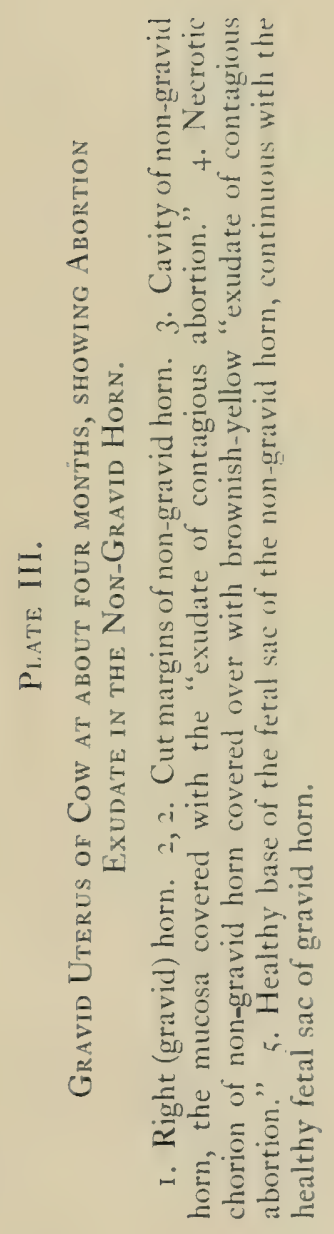



to the allantois detains the former within the chorion, leaving it an essential part of the afterbirth which may only escape with the chorion. The ruminant is, therefore, born naked.

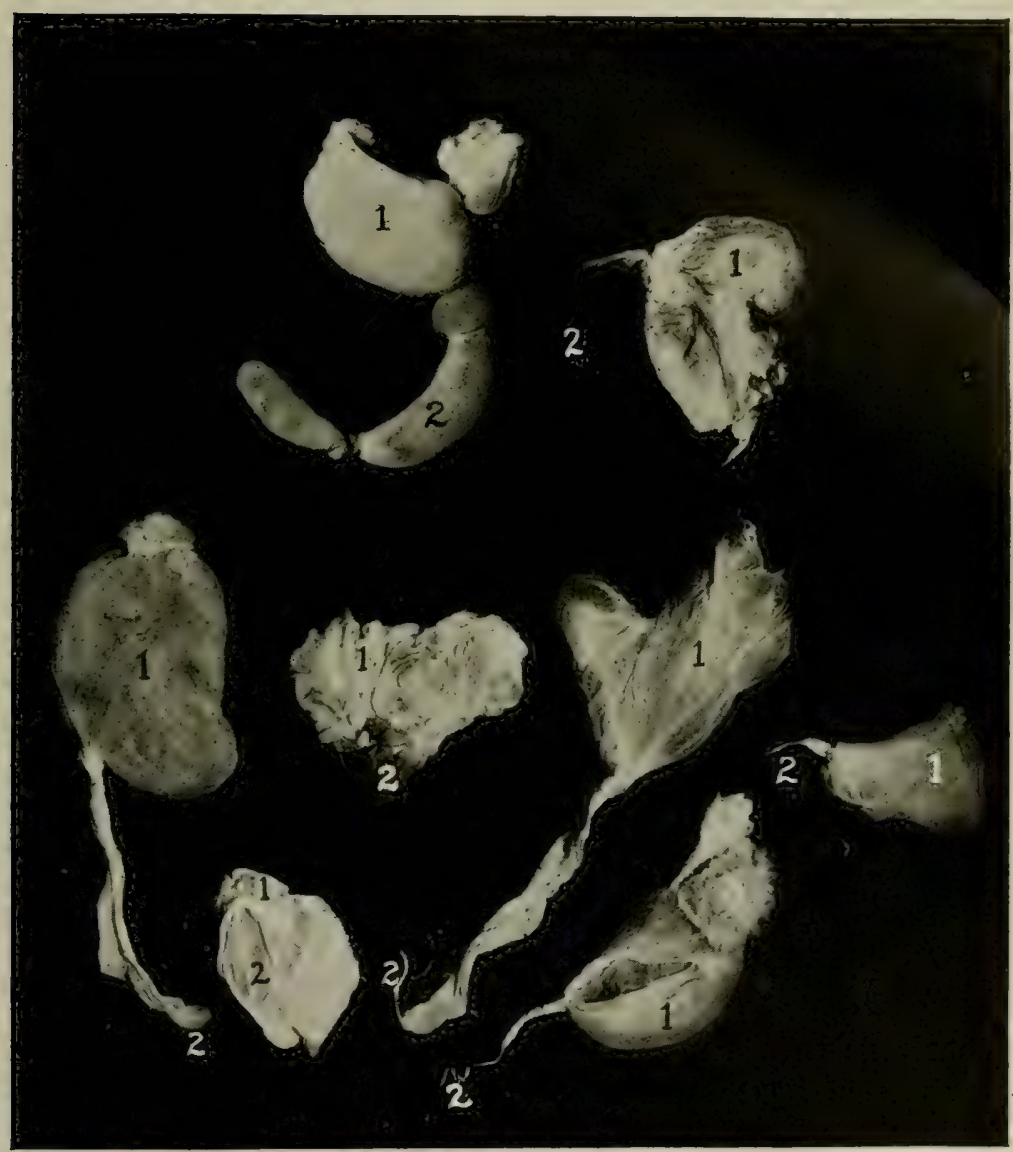

Fig. 35-Necrotic Tips of Fetal Sac, Cow.

$I, I, I$, Living allantois ; $2,2,2$, necrotic tips ; 3 , chorionic placenta.

The fetal placental structures, originating as outgrowths from the allantois-chorion, consist fundamentally of branching, arborescent capillary tufts continuous with the great vascular network of the allantois. Regardless of placental 
type, the fundamental structure is essentially the same in all species. In the cotyledonal placentae of ruminants, the tufts, longer and far more elaborately branched, sink correspondingly deeply into the placental crypts of the endometrium. The chorionic tufts have in detail their counterpart in the crypts into which they project. The ultimate

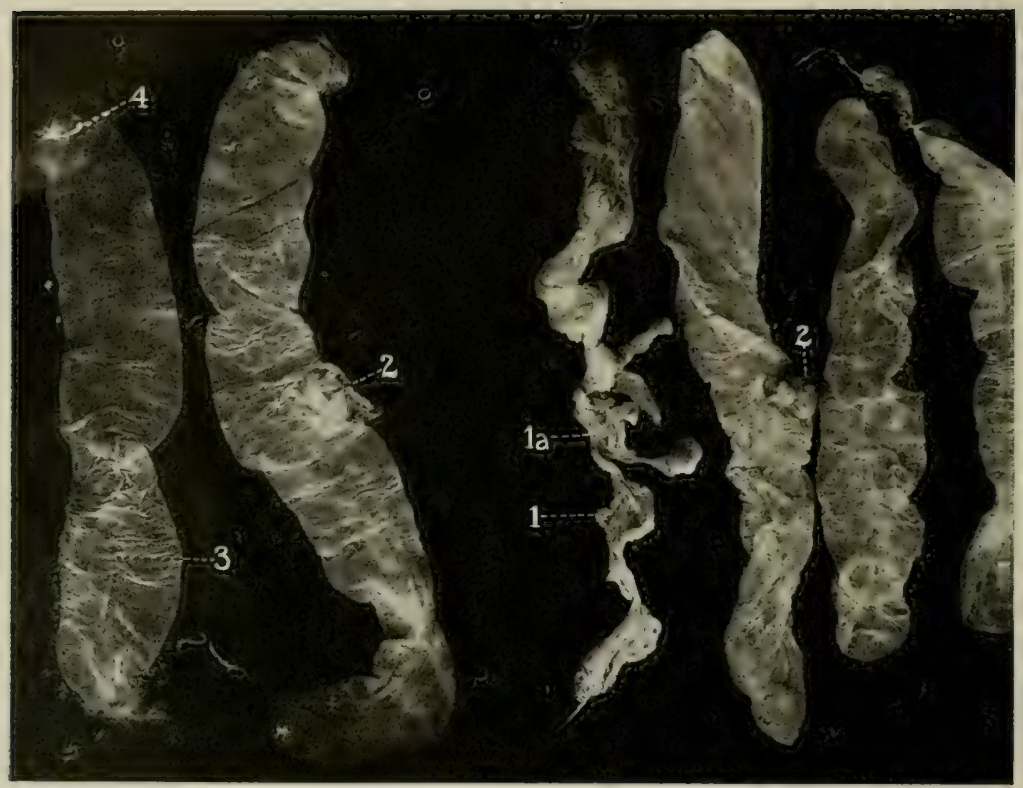

FIG. 36-Necrotic Tips of Fetal Sacs, Swine.

$I, I a$, Necrotic embryos in totally necrosed fetal sacs; 2,2 , normal embryos within amnion, the allantois having been opened ;

4, necrotic tip of allantois.

chorionic tufts consist of capillary walls with a single epithelial layer, while the corresponding maternal crypt consists also of capillary loops with a single, delicate, epithelial layer. The fetal and maternal capillaries remain permanently separate and the two delicate epithelial layers permanently separate the maternal from the fetal blood. All nutriment and waste products exchanged between the mother and the fetus must pass through these two epithelial barriers, which together constitute the placental filter. Through 

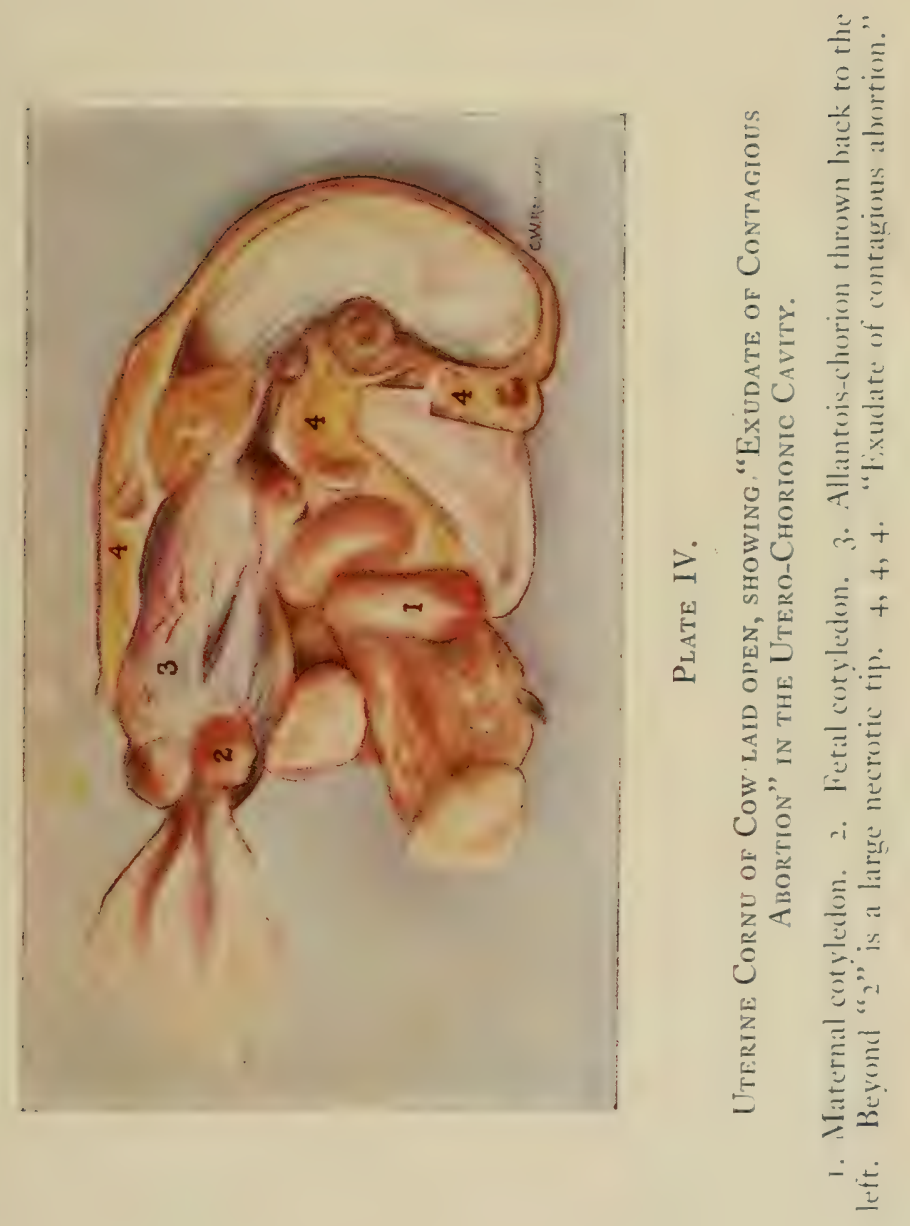

this filter, so long as it is healthy, no solids or other visible substances ordinarily pass. Bacteria are not known to pass this filter while it is healthy. Researches show further that rarely or never do the hypothetical antibodies pass this filter, so that a mother may be highly infected with a given bacterium and her blood react strongly by agglutination or complement-fixation to such organism, but the blood of her new-born fails to react. The contact between the fetal and the maternal epithelium is so intimate, that no infection nor foreign substance may readily exist between them. Hence in the placental area, so long as the structures are healthy, so far as known, infection can not pass from the mother to the fetus through the placental filter, nor can infection invade the fetus from the uterine cavity by passing between the two fetal structures, and thence gain the fetal circulation, without having first attacked and severely damaged the placental structures themselves.

The Utero-Chorionic Space. There is, however, a more vulnerable area through which bacterial invasion of the embryonic sac may occur. There are about one hundred functioning cotyledons in the healthy gravid uterus of the cow. Each of these cotyledons, in the slaughtered animal (they are doubtless much thicker in the living animal while they are distended with blood) is one to one and one-half inches in its perpendicular diameter while the peduncle of the cotyledon measures an inch or more, thus tending to push the chorion away from the non-placental endometrium for a distance of two inches. The one hundred cotyledons with their stalks serve as a great colonnade producing a vast space between the non-placental areas of the uterus and chorion, designated the utero-chorionic space. At full term of pregnancy, the uterine mucosa offers an area of approximately fifteen square feet. In this space, as in the nongravid uterus, bacteria commonly exist and need only to invade successfully the thin non-placental chorion and adherent amnion to gain the amniotic cavity and fluid. With the fluid, the fetus swallows any bacteria present. But the bacteria have other interesting portals of entry, the importance 
of which is not at present known. At the apex of each cornu, the tip of the chorion is necrotic and is commonly surrounded by exudate or pus. It would appear that such an area would offer but little resistance to invasion and once the bacteria have reached the allantoic fluid, the very thin amnion offers scant barrier.

Placental Hemorrhage. At the cervical end of the uterus there also exist conditions which apparently favor invasion of the fetal sac. Nearly always placental hemorrhages exist at the os uteri internum and some of the cotyledons have separated. In this manner the placental areas, denuded of protective epithelium, and with important volumes of blood resting upon their surfaces, offer an inviting field for invasion.

Uterine Sand. Such placental hemorrhages must be of very frequent occurrence. It would be supposed that such hemorrhage would be resorbed in a few days so that the regular meeting with them would seem to indicate that they occur almost constantly. The frequency of these is further suggested by the existence in the utero-chorionic space in essentially all cows in advanced pregnancy, of bodies I have designated uterine sand. These are free, spherical bodies generally about one-sixteenth inch, translucent and ambercolored. They are very hard, are insoluble in water and alcohol. They vary greatly in numbers and volume. By detaching the fetal membranes from the uterus, washing both surfaces in a vessel of water, and then decanting it off, there remains behind a mass of uterine sand varying from half a dram to one ounce, feeling much like sand when grasped between the fingers. Its exact character and origin are unknown but it appears most probably due to disintegration of red blood cells, and if so, indicates frequent and important placental hemorrhages.

In ruminants, therefore, with the immense utero-chorionic space, the inter-cotyledonal area of the chorion offers the avenue of least resistance to the invasion of the fetus. In the soliped, where no non-placental area exists, any vulnerable field must be created by the invasion of the placental 
structures. After such infection has destroyed the placental power of resistance, it is free to invade the fetus.

The development of the placentae is a cumulative process from the time of the formation of the fetal envelopes until the pregnancy terminates. It has been stated that as parturition approaches, the placenta undergoes fatty degeneration in preparation for dehiscence. That is untrue. The embryonic sac at first lies free in the uterine or cornual cavity without visible placental structures. Gradually the placental structures become macroscopically apparent but the embryonic sac can still be lifted out of the opened uterus without resistance. Gradually the chorionic tufts lengthen, become more branched, and sink deeper and deeper into the maternal crypts. In the abattoir, it becomes necessary, in separating the placenta, to use gradually increasing care and force as the duration of pregnancy advances. Early in pregnancy the chorionic tufts of the cow appear very much the same as the short, simple structures of the soliped, but they gradually become more complex as the close of pregnancy nears. This is essential to fetal development, which gathers in force and rapidity from the time of fertilization up to the end of pregnancy.

The force which maintains the contact between the fetal and the maternal placenta is not readily defined. It might be termed physiologic magnetism. This force continues so long as the fetus lives and the umbilic cord is intact. When birth occurs and the navel cord ruptures, the magnetism ceases. The blood in the fetal placental capillaries escapes through the broken umbilic veins. The capillaries in the chorionic tufts collapse, automatically fall away from the crypt walls and drop out. Thus, in preparing histologic sections of healthy placental structures one does not see the chorionic tufts lying in contact with the crypt walls, but quite detached therefrom, with the fetal and maternal epithelial layers widely separated, as shown in Figs. 33, 34. If, however, disease has invaded the placental tissues, the picture promptly changes; the damaged fetal and maternal epithelia or their debris lie in contact, and placentitis with 
retained or adherent placenta is present. The tendency to retention of the fetal membranes, when placentitis is present, logically increases as the size and complexity of the chorionic tufts advance. Thus retention of the fetal membranes is commonest and most severe in the cow, and increases in severity with the advancement in pregnancy. It rarely occurs during the first four months of pregnancy, but becomes increasingly common as the duration of pregnancy increases. The simple, short, chorionic tufts of solipeds tend to obviate retention of fetal membranes. 


\section{ChAPTER II}

\section{THE CLINICAL EXAMINATION OF THE GENITAL ORGANS}

\section{A. The Clinical Examination of the Male Genital Organs}

The clinical examination of the genital organs of the stallion and the bull is, as a rule, best made with the animal in the standing position after necessary measures have been taken for the safety of the examiner. Most stallions stand well but exceptions are sufficiently numerous to dictate that the animal be securely confined. A few are safe when carefully held by bridle or halter; some are controlled by the application of the twitch or by holding up a fore foot; some are safe only when side lines or breeding hobbles are applied. Even with these the examiner must be on his guard. The side line interferes with examination from that side. If the examination is made from behind, the animal may forcibly lift his hind foot and give the examiner a bad blow with the summit of the os calcis. The stocks make an ideal method for confining, safe alike for examiner and patient. Very refractory animals may need to be cast or placed upon the operating table.

The bull is to be confined preferably in the stanchion, resorting to the use of the leading or nose ring in lieu of the twitch. Sufficient precautions should be taken against crowding and trampling upon the examiner. Kicking with one foot may be conveniently prevented by securing a rope upon one metatarsus, carrying it once around the other metatarsus, and having a man hold the free end. The animal can be promptly released if he falls.

When the animal is properly secured, the testicles of both stallion and bull are best examined from behind because both glands can be viewed simultaneously or palpated from the same point. The smaller ruminants are readily examined standing; carnivora need to be securely controlled against scratching or biting. 


\section{The Testicles}

The testicles are to be examined with reference to location, position, volume, consistence, clearness of outline, and evidences of heat, pain, and swelling.

It is a well established clinical principle that, in order to be fertile, there must be one testicle in the scrotum. It is desirable that both glands be in the scrotum because when one is retained, owing to arrest in development, the progeny are liable to inherit the defect. The testicles of the foal and the calf should be in the scrotum at birth. The position of the testicle apparently matters but little; at least occasionally the testicle of the stallion is observed to be rotated on its transverse axis so that the tail of the epididymis presents forward, without apparent detriment. In one bull, the tail of the testicle was caught well up in the posterior part of the scrotal sac and the large end of the gland tipped forward to come to rest in the position of the stallion testicle.

In volume, the two testicles of the horse are rarely alike. Usually the right gland is clearly the larger. In ruminants the two glands are more nearly alike. The size varies with the size of the animal, the breed, the age, and the individual. It is only when the glands depart widely from the average volume that their size becomes significant. Even then the size needs to be considered with the consistence. They become enlarged in orchitis, abscessation, etc. When arrest in development has occurred, they are usually abnormally small.

The consistence of the testicle is highly important. Physiologically the gland is very firm and tense and its contour is clear and definite. The testicle may, from inflammation, become hard and unyielding, or in advanced abscessation, soft and fluctuating. In arrest in development the testicle is generally small and flaccid. This is commonly so in the abdominal testicle but is observed also in arrest in development of scrotal testicles. Such testes do not form spermotozoa but do (commonly, at least) induce sex reflex. If a healthy male of similar age and size is available for comparison, the examination may thus be rendered more accurate. 


\section{The EPIDIDYMis}

The epididymis and scrotal section of the vas deferens are also palpable. The commonest point for the lodgment of chronic infections is in the tail of the epididymis which should be examined accordingly with special care.

In the stallion, bull, and perhaps other males, there is a tendency towards the obliteration of the peritoneal sac of the scrotum due to adhesions between the parietal and visceral layers of peritoneum. This lessens or destroys the mobility of the gland within its peritoneal sac but the very loose areolar fascia between the cremaster and the dartos and skin leave the testicle, with the adherent parietal peritoneum and the closely applied cremaster, movable within the dartos. The adhesions between the two peritoneal layers are not very readily diagnosed and accordingly careful search should be made.

The size of the penis is not generally of clinical importance, but it is occasionally so large in the stallion that it imperils the vagina of the mare. This can be best determined by bringing a mare into the presence of the stallion and causing complete erection, when the examiner can make a reasonable estimate of the safety or danger when mated with a mare of a certain size. In some cases urethral hemorrhage follows coitus so that it is essential to cause the animal to copulate in order to render the examination effective.

An examination of the relaxed penis of the horse may be made by passing the hand up through the sheath into the prepuce and grasping the glans firmly behind the corona. By exerting moderate traction, sometimes for several minutes, the retractor muscle becomes fatigued and the penis can be completely exposed and examined. If the organ is moist and not readily grasped with the hand, the hold may be rendered secure by wrapping the penis in a dry towel or a piece of gauze.

The S-curved penis of ruminants cannot ordinarily be examined satisfactorily while the patient is standing. Copulation is virtually instantaneous so that only a hurried glance can be had at such times. The sheath is so narrow that the 
glans cannot be reached and grasped with the hand. The animal is to be confined in lateral recumbency, with the anterior limbs extended forward and the posterior limbs, backward, thus completely exposing the ventral surface of the body and giving the operator free and safe access to the parts. The bull is best extended by placing him between two strong posts or other solid points for attachment and stretching the limbs by means of two stout ropes, one extending from the hind, and the other from the fore feet to the corresponding posts. The penis is then to be exposed by eliminating the S-curve and pushing the sheath backward. The elimination of the sigmoid flexure is accomplished by grasping the distal curve at the point of insertion of the retractor muscle and, pushing firmly upward and forward, fatigue the muscle until it finally yields and the glans penis moves forward. The tissues about the sheath opening are then to be pressed backward toward the scrotum, thus shortening the sheath; the two movements combined serve to expose the glans. A piece of dry gauze wrapped about the glans enables the operator to grasp it firmly, and by the exertion of moderate and prudent traction the retractor muscle may be kept in a state of fatigue and the penis fully exposed. It can then be examined leisurely and carefully. The complete exposure of the penis also exposes fully the mucosa of the prepuce and sheath which, as shown in Fig. 9, now constitutes the covering of the body of the penis from the glans backward.

\section{The Seminal Vesicles, Prostate, and Cowper's}

\section{GLANDS}

The abdominal vas deferens, the vesicula seminalis, and the prostate gland may be palpated per rectum in the stallion and the bull. The prostate may be palpated digitally per anum in the dog. In the stallion and the bull the vas deferens may be palpated as it emerges from the internal inguinal ring and passes upward, forward, and inward to reach the superior surface of the neck of the urinary bladder, where it passes backward to disappear beneath the corresponding vesicula seminalis. 


\section{The Semen and Spermatozoa}

The satisfactory examination of the male genitalia ordinarily involves an examination of the semen. This is best accomplished by causing the male to copulate with a female and removing immediately some of the semen from the vagina. This may be conveniently attained by introducing a sterile swab, like that used in diphtheria, deeply into the vagina. Then withdraw the swab and from it make a smear on an ordinary slide, apply a cover glass and examine under moderate magnification. If the examination is made in a warm room and the preparation is kept moist by adding a few drops of physiologic salt solution, healthy spermatozoa continue to move vigorously during a period ample for study of the living cells. If the semen is kept warm and moist, it may be carried some distance and examined after several hours. It is best, however, to examine the semen as to motility soon after ejaculation.

The quantity of semen ejaculated is likewise of fundamental importance in judging the fertility of a sire. While technically but one spermatozoon is necessary for the fertilization of an egg, there are physiologically many millions of spermatozoa for each ovum. The luxurious numbers serve as a safeguard against their total destruction during the hurried migration from the vagina to the ovary. Not only is it important that great numbers of spermatozoa shall be present in order to make it certain that at least one vigorous cell will reach the ovum, but great numbers constitute, under certain limitations, an indication of sexual health and vigor. The amount of semen needs to be determined by some form of approximate measurement. In horses and cattle the vagina of the female may be well washed out with sterile salt solution or with a $0.25 \%$ chlorazene solution which dissolves the vaginal mucus, followed by the salt solution, and by inserting the well disinfected hand, the animal may be caused to eject all fluid or it may be ladled out with the hand. If the male is then caused to copulate with her, the contents of her vagina may be at once ladled out of the 
vagina, measured, and a fair approximation of the volume of semen ejaculated obtained. If the vagina has not been cleaned out immediately prior to coitus, no accurate idea can be had of the volume of the semen because there may be several ounces of thin mucus or other fluid lying in the vagina. Studied under this plan, the bull ejaculates as a rule 6 to 8 mils of semen at a copulation. The semen consists largely of the secretions from the seminal vesicles and prostate gland in which the spermatozoa swim freely. If the spermatozoa are dead immediately after ejaculation, that is conclusive evidence of sterility but does not reveal the cause of the sperm death. Living spermatozoa, however abundant, do not afford final proof of fertility. Spermatozoa may be suffering from disease and their elements undergoing disintegration and death. Or there may be bacteria ejaculated in the semen capable of causing the death of most or all spermatozoa. It is accordingly essential to accurate diagnosis that the spermatozoa shall be so prepared by staining and otherwise that their structural details may be carefully and effectively studied. The various parts need to be rendered clear so that any commencing degeneration may be detected.

The degeneration of the spermatozoa and their capacity to fertilize is not the end of the question. There is striking clinical evidence that stallions, apparently in good general health, transmit venereally to mares the infection of epizootic cellulitis or "pink eye" and at the same time prohibit fertilization. Some sterile bulls apparently render sterile the females which they have unsuccessfully served.

It is important that the copulatory act be closely observed so as to determine whether it is normal-that there is the normal sexual desire and ejaculation, followed by normal relaxation. Any hesitancy in copulation should be carefully noted and its cause determined if possible. Imperfect coitus may arise from physical difficulty in mounting the female due to painful diseases of the hind feet or limbs, or to paralysis. The diffculty may be due to mechanical interferences with the protrusion of the penis. In other instances there is 
such pain in protruding the penis or in ejaculating semen, that the animal fails to copulate properly. The examiner must reach a proper diagnosis regarding the group of causes under which a given case falls and search in detail for a definite solution of the problem.

\section{B. The Clinical Examination of the Female Genital Organs}

The clinical examination of the genital organs of the mare and the cow reveals to the veterinarian data of great economic and scientific interest. The freedom with which the veterinarian may palpate the entire genital system per rectum in these animals gives to him a marked advantage over his confrere in human medicine in the examination of woman. In the small ruminants, with a rectum too small to admit the operator's hand and a tense abdominal tunic which well-nigh shuts out abdominal taxis, the veterinarian is placed at a serious disadvantage. The same holds true of the sow, with the thick skin and dense, abundant, subcutaneous fat. The bitch and cat, with thin flaccid abdominal walls, offer a moderate opportunity for palpation of the genital organs, but far inferior to that enjoyed in the mare and cow.

By means of clinical examination of the genitalia, especially of the cow and the mare, the veterinarian is enabled to diagnose estrum, ovulation, pregnancy, and innumerable genital diseases.

Most mares and cows will stand quietly for both rectal and vaginal examination, but the prudent veterinarian will take no unnecessary risks. The danger, usually greatest at the commencement of the examination, is far greater in the mare than in the cow. The chief danger is, of course, from kicks. The greatest resistance is generally offered when the hand is introduced through the vulva. As a rule of practice, in examining the mare, one assistant should hold the head securely and well elevated, another should hold up one fore foot, and a third hold the tail out of the examiner's 
way. Should the mare be restless, a twitch should be applied, and upon the least show of viciousness, breeding hobbles should be used and drawn up tight. Even then, the veterinarian should have care that she does not give him a blow with one or both os calces. The operator should look carefully to having abundant room, especially that the mare may not crowd him against a wall, and also that, when his arm is in the rectum, she shall not be able to move violently to the right or left and, by pressing her buttocks close to a post, crush his arm.

The cow does not often kick during rectal or vaginal examinations, but I know to my cost that exceptionally she kicks fiendishly and very dangerously. Next to the worst kick in my career was from a cow while I was attempting to examine her genital organs. Usually the cow can be controlled by a strong stableman grasping her muzzle firmly, or still better by applying a leading ring or pincers to her muzzle and pulling firmly. However, a few vicious cows need to be shackled by tying a rope or strap about one metatarsus, giving it a turn about the other, and then having a strong, alert man hold the end of the rope firmly, ready in event of a fall to release her. I have encountered a number of heifers which were exceedingly resistant to the instrumental examination of the cervico-uterine cavity, and have found it necessary to lay them down and secure them. It is inadvisable to waste time on these obstinate patients, because the longer one works the more resistant they become. Finally they exhaust the operator and then, if ever, he is liable under fatigue to injure the patient seriously. Therefore it is best, when the animal cannot be controlled in a manner to render both patient and operator safe and to avoid fatigue, that the animal be secured in lateral recumbency. She may be cast in a variety of ways, but for convenience of securing for the operation, the best method is that of stretching her between two posts. The head should be tied securely to one, with a second post or other fixed point for securing a rope about twenty feet or more behind her. A stout rope is then tied in a running noose about one 
metatarsus, and a half hitch taken about the other. Two or three strong men may then draw regularly and firmly upon the rope, gradually stretching the animal until she finally falls upon her side. The process is rendered easier by applying a second rope to the anterior feet and having a man stretch these forward, but the cow may then fall harder and should be guarded against injury by an ample bed. The ropes drawing upon the metatarsi or metacarpi may cause pressure injuries unless the precaution is taken to pad the parts beneath the rope, which is conveniently done usually by wrapping the part with a grain sack before applying the rope. Finally the rope on the hind feet is to be secured to the rear post and that on the fore feet to the front post. The operator may now proceed deliberately, conveniently, and safely with his examination. The examination of the recumbent heifer thus secured is easier and safer. In the wellbehaved heifer the advantage of securing in lateral recumbency is more than counterbalanced by the time and labor of securing and the peril of casting injuries. I have found it necessary to secure a heifer in lateral recumbency three or four times in succession for examination or treatment, until finally she yields and stands without resistance.

The preparation of a special stall or stanchion into which all cows in an establishment may be led for examination is a great convenience. In some herds a sort of movable stocks, somewhat like a shipping crate, is prepared, which can be placed anywhere according to light or other convenience, or moved from one stable to another.

The ready introduction of the hand into the vulva or rectum requires some form of lubrication. As a rule, the best is a warm salt solution of approximately 0.7 to 1 per cent. Many veterinarians resort to soap as a lubricant, but this is undesirable because even very weak soapsuds almost inevitably irritates both the rectal and the vaginal mucosa and causes tenesmus-the worst foe with which the examiner must contend. The addition of antiseptics to the water is much worse than the use of soap. No antiseptic can be used of such strength as to produce even very feeble disinfection 
without causing much irritation and straining. The operator may use antiseptics on his hands and arms to cleanse them, and may use moderately strong antiseptic solutions, if desired, about the exterior of the anus and vulva, but these should not, as a rule, be carried on the hand, even in small amount, into the rectum or vagina. Oils and fats make good lubricants, but when used they interfere very seriously with the sense of touch in the vagina, especially when one wishes to palpate for the uterine seal or to determine the character of the mucus within the vagina. Sometimes, however, lubrication of the hand with oil or fat is virtually necessary, especially when introducing it through a very narrow vulva. Powdered elm bark suspended in warm water has a lubricant value essentially equal to that of fats, and has the advantage that it is readily washed away with water without the use of soap. Vaseline and other heavy fats make very bad lubricants because of the difficulty of getting rid of them after using. The value of any examination of the genital organs rests absolutely upon the knowledge, skill, and care in every detail. The veterinarian needs a thorough knowledge of the anatomy of the entire genital system. This cannot be had from books or lectures, nor in the anatomical laboratory of a veterinary college. These help form the foundation, but in order to get an accurate sense of the form, size, density, and other attributes of the living organs, the veterinarian needs to examine them promptly after death. The abattoir offers an ideal place for the study of the genitalia of cattle, sheep, and swine, and no veterinarian interested in the diseases of their genital organs should neglect to avail himself of this ideal opportunity for study. Here he can see and handle nongravid uteri and uteri in every stage of pregnancy, and by approximate measurements may learn to recognize with fair accuracy the duration of pregnancy by the transverse diameter of the distended uterus. In the abattoir, the uterine seal, the corpus luteum, cysts, ripe and freshly ruptured ovisacs may be recognized and their character studied by sight and touch. No such opportunity for fundamental 
knowledge exists elsewhere. By availing himself of the freshly slaughtered animal, the veterinarian makes the closest possible approach to the next logical step, the clinical examination.

\section{STERILITY-ABORTION \\ ORIGINAL EXAMINATION}

Owner

Address.

Name or Herd Number of Animal

Date of Birth

Has animal been pregnant?

If she has bred, state number of calves produced

Date of termination of last pregnancy.

Duration of last pregniancy in days.

Was calf alive or dead?

If living, was it healthy? Did cow have retained afterbirth?

or discharge from uterus?

If a heifer which is not known to have been pregnant, insert in chart the date or dates of service or in case of estrum or "heat" and not served write date in parenthesis (). In animals which have been pregnant, indicate the dates of service since calving or aborting and dates of estrum when not served, as in the heifers.

\begin{tabular}{|r|c|c|c|c|c|c|c|c|c|c|c|c|}
\hline YEAR & JAN. & FEB. & MAR. & APR. & MAY & JUNE & JULY & AUG. & SEPr. & OCT. & NOV. & DRC. \\
\hline & & & & & & & & & & \\
\hline & & & & & & & & & & \\
\hline
\end{tabular}

Character of estrum, regular or irregular.

General condition

State of lactatiou.

\section{Date of Examination}

Right broad ligament

Left broad ligament.

Vulva

Vagina

Cervix

Uterus.

\section{Right ovary}

Right oviduct

Left ovary

Left oviduct

Diagnosis.

Prognosis

Treatment applied

Treatment advised

REMARKS :

FIG. 37-Examination Blank for Recording Findings in the Examination of the Genitalia of Cows. Reduced. 
There is no field of veterinary practice where system and detailed permanent records are so essential to efficiency. The precise order of examination needs to be varied sometimes according to the individual, but generally a definite order may be followed from beginning to end. I have found the examination blank, shown in Fig. 37, convenient in making a systematic and complete examination. After the identity and the history of the animal have been satisfactorily recorded, any evidences of abnormality in the broad, or sacro-sciatic ligaments, such as their sunken condition in nymphomania, with external evidences of vulvar disease are noted.

\section{EXAMination of VUlva AND VAgina}

It is generally advisable next to insert the hand into the vagina and carefully palpate and record findings in the vulva, vagina, and os uteri externum. Unless palpation reveals counter-indications, the twenty-inch uterine forceps should be introduced alongside the hand already within the vagina and the cervix grasped. This is best accomplished by pushing the index finger against or into the os uteri externum. With the forceps slightly open, push the one jaw into the os far enough to secure a firm hold upon its lip and then close and lock the forceps. In some heifers, the vaginal portion of the cervix forms such a sharp cone, and the cervical canal is so narrow, that the tip of the forceps jaw cannot be inserted sufficiently to grasp the cervix. In such cases the forceps should be opened wide enough to grasp the cervical cone at the side near its base.

Moderate traction upon the forceps draws the cervix, uterus, and ovaries backward four to ten inches, stretches the uterus in its ligaments, renders its outline clearer, and brings the ovaries into a more accessible position. When an ovary is unusually large and the abdomen pendulous, the gland frequently drops forward and downward where the examiner cannot reach it or can do so only with great difficulty not devoid of peril to the rectal walls. The traction 
with the forceps usually draws the uterus, the apices of the cornua, and the ovaries up into the pelvis or against the pubic brim, where they are readily palpated. At about the 90 th to the 120th day of pregnancy, the fetus is sometimes far forward in the gravid cornu and the uterus has fallen down in a pendulous abdomen, where it is difficult to palpate, making error in diagnosis easy. Here again the traction upon the cervix tenses the genital tube so that it may be readily followed and brings up within reach the gravid portion of the horn, where its volume may be recognized and the approximate duration of pregnancy determined.

There are some important exceptions to this order of procedure. In heifers with narrow vulvae, and perhaps with considerable hymeneal stricture, the force necessary in order to introduce the hand in attaching the forceps may arouse so great an irritation as to induce severe and obstinate straining, which may render rectal palpation very tiresome, frequently inaccurate, and sometimes impossible, so far as any reliable findings go. With heifers, therefore, I generally prefer to postpone the vulvo-vaginal palpation and omit the application of the forceps to the cervix until after the rectal examination has been attempted. The very frequent intense straining may thus be avoided. The traction upon the cervix is by no means so important in the heifer as in the cow. The uterus of the non-pregnant heifer, unless long sterile, is of good tone, firm, small, and lies in the pelvis within easy reach. If the heifer is pregnant, her uterus virtually always remains firm and retains its position with a portion of the distended organ within, or just in front of, the pelvis, where it is readily palpated and its volume recognized. In a large proportion of pregnant heifers, reliable and satisfactory diagnosis can be made readily by rectal palpation without applying forceps to the cervix or inserting the hand into the vagina, thus rendering these superfluous.

If the heifer is not pregnant and after several copulations her sterility is deemed serious or important, the vulvo-vaginal and cervical canals and the uterine cavity need to be ex- 
amined in order to arrive at a reliable diagnosis. The vulva and vagina then need to be forcibly dilated. This is best done with the hand. After lubricating with warm oil, fat, or emulsion of elm bark, the fingers should be formed in a cone and, under gradual and firm pressure, the vulva slowly dilated. In extreme cases the dilation may require an hour or two, or may demand repeated efforts on successive days. Any intelligent helper may, under proper instruction and caution, undertake the work, but it must not be unduly rushed. In some extreme cases I have inserted the uterine forceps into the vagina without inserting my hand, directing it on the median line, from side to side, inclined very slightly

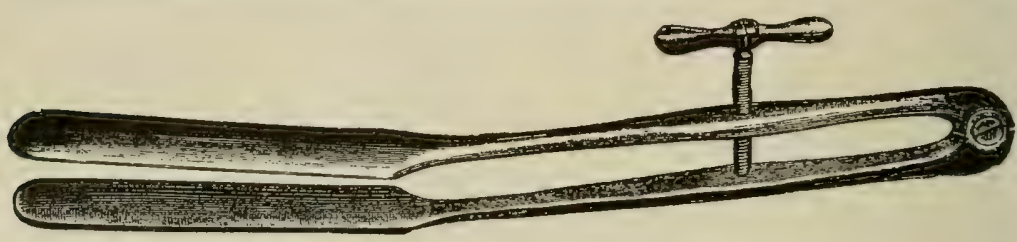

Fig. 38-Bischoff's Vaginal Dilator.

downward, to the end of the vagina. Opening the forceps moderately and pressing them forward against the cervical end of the vagina, closing and locking them, and then applying gentle traction, I have occasionally been able to draw the vaginal portion of the cervix back to the vulva, where it could be palpated by inserting one or two fingers and eventually secured by guiding the second pair of forceps to the part by palpation. With both forceps applied to the cervix, the vagina may be visually inspected and the cervical canal and uterine cavity examined instrumentally.

In some heifers, the vulva is constricted by the presence of a small band of connective tissue or aponeurosis, located about one-half inch from the margin of the lips, just beneath the vulvar mucosa. When vulvar dilation is attempted, the veterinarian encounters this firm, small, cord-like structure, which under the force employed cuts into his hand. I have observed this in one family of Holstein heifers, the members of which were unusually large and rugged, where the vulvae 
were so constricted by this cord-like structure that a small hand could not be inserted. The difficulty was overcome by severing the cord at one or two points. I encountered one sterile Jersey heifer of breeding age, with so small a vulva that I could not insert my hand at all and so small an anus that I could not palpate her genitalia, per rectum, though the heifer herself was not extremely small. Vulvo-vaginal strictures occur from disease, rendering palpation of the anterior end of the vagina impossible. These will be considered later.

Albrechtsen makes extensive use of the vaginal speculum in studying the vagina and cervix, but I have not found it of great value. When one pair of the uterine forceps is attached to the right and the other pair to the left lip of the os uteri externum, traction exerted, and the forceps pressed apart, the vulvar opening is widely dilated and in almost all animals all parts of the vaginal mucosa and the vaginal portion of the cervix are brought into view more clearly than with the speculum. The visual examination of the vaginal end of the cervix may be further facilitated in cases of need by applying a third pair of forceps to the superior margin of the cervical border. In a few instances of a very narlow vulva or vagina, a speculum of sufficiently small size may be of great aid. The best speculum in my experience for this purpose is that of Bischoff's Vaginal Dilator, shown in Fig. 38.

In the manual examination of the vulva and vagina, the examiner should take note of all abnormal contents and of abnormalities of the vulvo-vaginal mucosae and of the perivaginal tissues and organs. Physiologically, the vagina of the cow at estrum and when nearing parturition contains an abundance of clear, rather fluid, highly lubricant mucus. The vagina of the non-pregnant cow in the interval between estrual periods contains a small amount of lubricant mucus. The vagina of the pregnant cow, except when nearing parturition, contains a highly adhesive mucus. These physiologic variations in quantity and quality of vaginal mucus need to be learned by actual clinical observation before the various pathologic contents may be readily recognized. 
The walls of the vagina are physiologically soft, pliable, and thin, but this is largely comparative which the examiner must learn to appreciate by clinical study. In severe granular venereal disease and in other chronic inflammatory conditions, the mucosa and entire walls become thickened and hard. In the acute vesicular venereal disease the vulva and vagina become highly sensitive and exceedingly painful to the touch.

In order that these variations in the vaginal walls may be recognized by palpation, it is essential that the organ be flaccid. It is impossible to determine clearly the state of the vaginal walls while the patient is straining violently. A still greater impediment to palpation of the vaginal walls is the ballooning or inflation of the vagina with air. During inspiration, if the vulva, the anus, or the cervical canal is open, air may be drawn into the vagina, rectum, or uterine cavity respectively, and the involved organ become greatly inflated like a balloon. These inflations appear to be wholly involuntary, but the tendency for them to occur varies greatly in individuals. Once the vagina becomes inflated, the ballooned organ completely fills the pelvis; the walls are hard and firm; and, except for the rectum above, lie in immediate contact everywhere with the pelvis. The condition of the vaginal walls and of the surrounding tissues or organs cannot be determined by palpation through this tightly stretched, firm, unyielding structure. Sometimes, by moving the hand rapidly and vigorously from side to side and slapping the tense walls, the cow or mare may be induced to contract the vagina and expel the air. If the cervix is seized with forceps and drawn backward, the vaginal cavity is largely eliminated and the air forced out. The air may be forced out of the vagina by exerting manual pressure upon the ballooned organ from the rectum. In many cases the evacuation of the air from the vagina is none too readily accomplished, but it must be done before the palpation of the vagina and perivaginal structures can be accomplished. The veterinarian must, therefore, be patient and persevere until his object has been attained. Through the flaccid vaginal 
walls, the examiner may recognize perivaginal abscesses of lymph glands, tumors, pelvic fractures, diseases of the urinary bladder, ureters, and other organs. In advanced pregnancy in the cow, the head of the fetus commonly lies in the pelvis above the vagina, as shown in Plate I, possibly misleading the unwary veterinarian and causing him to suspect extra-uterine pregnancy.

\section{Rectal Palpation}

The palpation of the genitalia of the cow per rectum is on the whole the most important part of the clinical examination, because it reveals such an extended list of data. The essential preliminaries to rectal palpation of the genitalia are that the rectum shall be empty of feces, that it shall not be ballooned with drawn-in air, and that straining or tenesmus shall not be present. Some have advised emptying the rectum by means of an enema, but the liquid is rarely all evacuated until after the operator inserts his hand, at which time the liquid feces are often forced out, causing unpleasant soiling. Hess recommends inserting the hand into the anus in the form of a cone, spreading the fingers apart and dilating the anal ring enough to admit some air which is commonly followed by defecation. But one must wait for a time and although defecation finally occurs, quantities of feces usually still remain in the rectum which must be invaded for successful palpation. It is essential that the rectum be emptied as far as the operator wishes to insert his hand and, in the end, this must be accomplished largely by the manual removal of the feces.

The ballooning of the rectum is an annoying impediment to rectal palpation, holding the examination completely in abeyance so long as it persists. Rapid movements of the hand, as suggested for ballooning of the vagina, frequently suffice, causing the rectum to contract and expel the air. The ballooning generally involves but twelve to sixteen inches of the rectum at the anal end, and the operator may reach beyond into an empty section of the gut, cautiously engage his fingers in the intestinal folds, and, drawing the 
anterior segment of the rectum analward, force the air out of the ballooned portion. Patience must be exercised until the ballooning is completely overcome. It is useless to attempt palpation while it exists, because the rectal walls are so stretched, hard, and firm that reliable operation is simply impossible.

Tenesmus, or straining, also inhibits, so long as it lasts, any reliable palpation of the genital organs. It has already been remarked that soapsuds, antiseptics, etc., must not be introduced into the rectum on the hand, arm or otherwise, since they may cause very annoying straining. It has also been noted that if force has been used in dilating the vulvovaginal tract of a heifer just prior to rectal exploration, the pain already caused in the genital tract will result in violent, and frequently uncontrollable straining during rectal palpation. These causes must be avoided. But when the hand can be readily introduced into the vagina and the forceps fixed in the cervix, any moderate traction exerted tends to prevent rather than to cause straining. Gentleness and patience are the chief factors in controlling straining. During straining the operator should always let his hand lie perfectly passive in the rectum. He must not even use force to retain a position already reached. If his fingers are in a saccule of the rectum and he holds his hand rigidly in position, the cow will rupture her rectum against his finger tips. Even with the greatest gentleness and patience, one sometimes encounters an animal which strains persistently and violently. One cow strained constantly during efforts at rectal palpation by my colleagues and myself, and although repeated attempts were made at different times, accurate palpation failed. Peri-anal injections of apothesene and moderate chloral enemas both failed. Not only accurate work, but the safety and life of the patient, are dependent upon the gentleness and prudence of the operator.

It is virtually impossible sometimes to avoid injuries sufficient to cause hemorrhage from the rectal mucosa. In small heifers the anal opening seems unduly constricted and at some point the mucosa may tear and slight hemorrhage 
result, but this is extremely rare. Injuries to the rectal mucosa from the palpation are far more common. Veterinarians with very large hands and arms should not undertake rectal palpation of the genitalia per rectum in small heifers. As a rule, however, bleeding from the rectal mucosa is not due to a big hand, but to roughness, inexperience, or untrimmed finger nails. The nails are to be trimmed to the quick and carefully filed until they are as smooth as possible, softened in warm water, and then all possible care and gentleness must be used. Under no conditions is the rectum to be dragged upon violently. While it is ballooned or the cow is straining, no reliable palpation can be accomplished, but severe or fatal injury is always impending.

Even if the injury is not serious, hemorrhage is a discredit to the examiner. When he removes his hand, covered with blood, it looks bad to the owner, and to one who knows the subject it shows that the examiner has failed to attain his object, which is a reliable palpation, but has instead injured the rectum. The prudent veterinarian will, therefore, have care to reduce to a minimum the frequency and amount of hemorrhage from the rectal mucosa, and will always feel that he has erred whenever his hand, when withdrawn, is stained with blood. After other precautions to prevent straining have failed, it may sometimes be controlled by pinching the skin of the back, pressing over the spine with a rough stick, pinching the muzzle with the fingers and thumb in the nostrils, or applying a leading-ring to the nose.

When the foregoing preliminary precautions against injury to the intestines have been made and other preparations for rectal palpation satisfactorily attained, the examiner is ready to proceed with the palpation of the genital organs per rectum. If forceps have been applied and slight traction is exerted, the cervix of the non-pregnant, adult cow may usually be drawn back almost or quite to the vulva.

There are occasional exceptions. In Shorthorn cows, the vagina is quite long and narrow and the uterus is held far forward by its ligaments so that in many individuals it is very difficult, and sometimes impossible, to draw the cervix. 
far enough back to permit of visual examination or of desired manipulations.

When the operator inserts his hand into the rectum, the logical starting point for the palpation is the cervix. With the forceps applied to the vaginal end, the operator may recognize the structure by following the instrument to its point of attachment. When the forceps have not been applied, as in heifers, the cervix must be sought and recognized by its location, size, consistence, and attachments. Without traction with the forceps, the vaginal end of the cervix lies eight to twelve or more inches anterior to the vulva. In the non-pregnant heifer, it ordinarily lies not more than eight inches from the vulva; in old, pregnant cows, it may be dragged far forward out of the pelvic, into the abdominal, cavity. Similar displacement may exist in pyometra, hydrometra, large ovarian and uterine tumors, and other pathologic conditions. Not infrequently, portions of the small intestines are pushed back in the recto-vaginal pouch, and must be dislodged forward before the cervix, uterus, or ovaries may be palpated. Ordinarily it is a very easy task to dislodge these by applying very slight intermittent pressure downward and forward.

When the urinary bladder is empty, the cervix may lie upon the empty viscus, but it is generally slightly deviated to the right and rests upon the pelvic floor. When the urinary bladder is distended, the cervix (and uterus) is almost always deflected to the right and lies in the furrow between the distended viscus on its left, and the right pelvic wall. Rarely the cervix and uterus lie upon the distended urinary bladder or, yet more rarely, may be displaced to the left. Sometimes the greatly distended urinary bladder, containing four or more quarts of urine, almost fills the pelvis, the cervix and uterus fall far down between the bladder and pelvis, and the bladder rolls or extends over to the right, virtually covering the cervix and uterus. The distended bladder then needs to be pushed to the left in order to palpate the cervix and uterus. Rarely I find it desirable, before satisfactory palpation may be made, to catheterize the bladder and evacu- 
ate the urine. The small or extra-small Albrechtsen uterine catheter answers the purpose satisfactorily.

Sometimes great care is necessary in order to avoid confusion on account of the bladder. It may give the impression at first of the distended pregnant uterus, and the examiner, off his guard, may fall into a very embarrassing error. His safety is assured only by the clear recognition of the cervix and uterus. When the urinary bladder is empty, it forms a flattened, pear-shaped body, palpable beneath the cervix, about three to four inches long, rather firm in consistency, and finely irregular, due to the wrinkling of the mucosa and contracted muscle bands. Then follows every gradation in size, up to four or more quarts of contents, when the distended organ may fall over the pubic brim and hang down in the abdomen.

The cervix is recognizable by palpation as a very firm, almost cartilaginous, cylindrical organ, three to six inches long, and one to three or four inches in diameter. In heifers it is very small and firm, usually about three to three and one-half inches long, and one to one and one-half inches in diameter. With age, and yet more with disease, it increases in all its diameters. In health, owing to the amplitude of its broad ligaments, the organ may be picked up per rectum, and virtually its entire circumference freely palpated. In disease, it may be adherent in varying degrees and difficult of clear identification. Behind, the cervix is continued by the flaccid, empty vagina. Sometimes the inexperienced practitioner may be confused on account of the ballooned vagina, which has already been mentioned. The cervix is then pushed forward by the ballooning and the operator feels beneath the rectum, filling more or less completely the pelvic cavity, a moderately tense, inflated sac, which he may at first mistake for the urinary bladder or be otherwise misled. Traction upon the cervix with the forceps pushes the air out through the vulva, or it may be readily evacuated by gentle pressure from the rectum.

a. The Uterus. Forward, the cervix ends in the usually somewhat broader and markedly softer uterine body. In 
severe cervicitis, the cervix often has a far greater transverse diameter than the body of the uterus.

Just in front of the cervix, the body of the bovine uterus is small and inconspicuous when compared with the size of the uterus as a whole. Externally it is ordinarily two to three inches long, but the originally separate Muellerian ducts are still faintly evident upon palpation, in the form of a slight antero-posterior depression on the dorsal surface. This depression gradually deepens into a distinct furrow, soon the bifurcation is reached and the two distinct cornua follow, constituting the chief volume of the uterus. Each uterine horn has about sixty per cent of the transverse diameter of the body.

The examiner recognizes the non-pregnant uterus by its continuity with the cervix anteriorly, its bicornual character, and its firmness and evenness to the touch, each cornu forming the segment of a circle with its convex border presenting dorso-anteriorly. In the healthy uterus of the heifer, the uterine body and horns are very firm, smooth, regular, thus serving to differentiate them clearly from all other pelvic or abdominal viscera. The cornu of the heifer is onehalf to three-fourths of an inch in diameter at the base, and tapers gradually to a fine point at the ovarian end, where it passes almost imperceptibly into the oviduct. The examiner should trace each cornu separately, as it extends obliquely forward to the right or left, where it soon turns downward, thence backward and upward by a regular curved line, to end, the right cornu on the right side, the left cornu to the left, in close proximity to its base, thus describing an almost complete circle. The healthy cornu of the heifer is eight to ten inches long on its convex border, thus forming an incomplete circle two and one-half to three inches in diameter. The entire organ may, therefore, be picked up through the rectal floor and held in the hollow of the hand. The uterus of the cow is larger, is slightly uneven owing to longitudinal wrinkles, and may be slightly sinuous because the cornua are longer than their broad ligaments. That is, the uterus, once pregnant, never wholly returns to the original form, outline 
and volume of the uterus of the heifer. Estrum, pregnancy, and disease alter profoundly the volume, form, position, and consistency. Whatever the changes may be, the basic principles in recognition are the continuity with the vagina and cervix and the fundamental position, attachments, and relations.

b. The Oviduct. The healthy oviducts are difficult of recognition by rectal palpation, but under favorable conditions are recognizable. They are less than one-eighth inch in diameter, sinuous, very smooth and firm. Arising almost imperceptibly from the apex of the cornu, they pass lateralward, in front of and below the ovaries embedded in the ovarian ventricle or pocket of the broad ligament, as shown in Fig. 13.

Passing beyond the outer or lateral extremity of the ovary, it bends inward, or medianward, so that the pavilion of the tube presents toward the antero-lateral surface of that gland, with one of the fimbriae of the pavilion attached to the lateral end of the ovary. When either pyosalpinx or hydrosalpinx is present, the tube becomes distinctly enlarged and usually readily palpable. The distension of its lumen or the thickening of its walls increases not only its transverse diameter but also its length. It then becomes readily recognizable by palpation per rectum in almost every case. Generally the infection involves the pavilion and also the ovarian pocket, causing them to become adherent to the ovary in varying degrees.

c. The Ovary. The palpation of the ovaries per rectum logically follows the examination of the oviducts. The glands, which in the cow physiologically lie approximately in the position indicated in Fig. 13, are to be recognized by their size, form, consistence, and attachments. In the healthy, non-pregnant heifer or cow, the ovary usually lies lateral to and somewhat below the dorsal border of the base of the uterine cornu, between it and the ascending, lateral portion of the pubis. Usually it lies upon the pubic floor, near the brim, or just beyond in the abdominal cavity, at or slightly below the brim of the pubis. Pregnancy and dis- 
ease greatly modify the position of the ovaries. Anything which contributes a weight in excess of the physiologic, nongravid uterus, oviducts, or ovaries causes the latter to drop downward and forward. The extent of the dislocation is further influenced by the degree of pendulousness of the abdomen.

The ovary swings freely in its attachments, may be picked up per rectum and ordinarily moved freely for a distance of four to six inches or more. The healthy ovary sometimes lies hidden in the ovarian pocket (mesosalpinx), or drops over in front of, and down beneath, the broad ligament, so that manipulation is required in order to free it and render accurate palpation possible. Once it is disentangled from the pocket or other accidental covering, the outline of the gland is very free and the examiner may recognize clearly ripe ovisacs, the crater following the recent rupture of an ovisac, the corpus luteum in its varying stages of growth and decline, its abnormalities and diseases, its adhesions with the pavilion of the tube and diseases of the ovary, with concurrent diseases of both ovary and oviducts.

The ripe ovisac of the cow is a thin-walled, yielding, and readily ruptured cyst of three-eighths to one-half inch in diameter, standing well above the ovarian surface, usually upon the convex or greater border of the gland. The crater resulting from the rupture of an ovisac is a deep pit with somewhat irregular edges, three-eighths to one-fourth inch across its mouth. The fresh, growing corpus luteum is very soft to the touch. Its dome, which may project one-fourth inch or more above the general ovarian surface, is about onefourth inch wide. Upon palpation, it feels like a small, atonic granulations tumor. Gradually it increases in diameter, becomes firm, and after about ten days has reached its full size of three-quarters to seven-eighths of an inch. The contiguous covering of the ovary grows over the corpus luteum. The latter is usually spheroidal, sometimes ovoid, with its base implanted in the body of the ovary. The yellow body may be somewhat dumb-bell shaped owing apparently to a constriction about its center, due to a narrow, firm open- 
ing of the crater when the ovisac ruptured. The two ovaries are rarely symmetrical; the left is usually the smaller-not rarely, minute,-no larger than a small pea. From such a minimum, the healthy ovary may be of any size, up to two, or even three inches in some large cattle.

The corpus luteum modifies greatly the gross volume of the ovary. In the very small ovary, the corpus luteum, fully developed, may increase the volume of the organ ten- to twentyfold, while the development of a seven-eighths inch corpus luteum in an ovary of two inches, though actually adding the same volume, does not cause the great comparative increase in size.

The healthy corpus luteum of the non-pregnant heifer or cow retains its maximum volume until about the sixteenth to eighteenth day after ovulation, when it atrophies rapidly for two or three days, and at twenty to twenty-two days, when it has decreased to one-fourth or three-eighths of an inch in diameter, estrum and ovulation recur. After the corpus luteum has formed as a solid mass, physiologically it remains solid throughout its history. It is solid, firm, and somewhat elastic to the touch.

The corpus luteum of pregnancy does not differ (except in color, which is of no concern here) from the corpus luteum of estrum in its general characters, but in duration it physiologically continues unchanged throughout pregnancy and for thirty to sixty days thereafter, when it atrophies and estrum and ovulation recur. Pathologically, the corpus luteum may be grossly enlarged to even two or three inches in diameter, may undergo cystic degeneration, and otherwise depart from the normal volume, consistence, and character. It may undergo rapid degeneration, with abnormally short intervals between estrual periods, may atrophy during pregnancy and cause ovulation and estrum, may shift its position and sink deeply into the body of the gland, and may persist at full size for month after month, even to years, inhibiting estrum and ovulation. The ovary is subject to such a wide variety of pathologic changes that the veterinarian needs to study it carefully and abundantly both in the abat- 
toir and clinically by palpation in order to be able to read every change in the gland with his finger-tips as unerringly as a blind man reads braille.

The rectal palpation of the uterus, oviducts and ovaries of cows and of some heifers, is greatly facilitated by the application of the forceps to the vaginal end of the cervix and the exertion of moderate traction thereon. The traction draws the uterus, oviducts and ovaries backward and upward, tenses the uterine horns and renders their outline more distinct, and enables the operator to study all parts of the genital tract at a far greater advantage. The plan avoids many errors in diagnosis. Some veterinarians fear that the application of the forceps to the cervix of a pregnant cow and the exertion of traction is dangerous, but I have failed to observe any untoward results. Without the aid of forceps, I have seen colleagues mistake gross pyometra for pregnancy and make other regrettable blunders. As a rule of practice, therefore, rectal palpation of the genital organs should regularly be aided by traction upon the cervix with forceps. Certain exceptions arise. It has already been noted that the genital organs of a heifer with a small vulva may be advantageously palpated per rectum without the application of the forceps. In pregnancy of 30 to 90 days, one may generally make a clear diagnosis without applying forceps. But in an old cow with a pendulous belly, the uterus in early pregnancy may be dropped too far forward and be entirely too flaccid to admit of safe recognition. To add to the difficulty, the embryo in such cows may be located in the apex of a horn, quite out of reliable reach. Then the operator paws about unconsciously in an effort to drag the uterus back per rectum which is infinitely more dangerous and far less efficient than traction with forceps.

The vaginal and rectal palpation, in addition to revealing an endless category of pathologic lesions, serves almost always to determine the presence or absence of pregnancy. If pregnancy is diagnosed, the examination is ordinarily complete. If the animal is non-pregnant, instrumental search of the cervical canal and uterine cavity is necessary for the 
complete assembling of available data for diagnosis and prognosis. During vaginal palpation, the operator logically palpates the os uteri externum for the uterine seal as an indication of pregnancy. When a healthy heifer is pregnant 30 days or more, if the examiner will press the tip of his index finger, without oil, vaseline, or other lubricant, against the os uteri externum for a short interval, and then carefully withdraw it, he is able to recognize in the os a small, highly adhesive seal. The seal grows and expands, becoming more and more recognizable throughout pregnancy. Its presence is a warning against the invasion of the cervical canal until ample evidence is at hand that the seal is spurious.

\section{The Examination of the Cervical Canal and DOUCHING THE UTERUS}

Having applied the uterine forceps either to the lips or to the sides of the cervix, and satisfactorily determined that the animal is not pregnant, the operator may exert traction and draw the vaginal end of the cervix back to the vulva or as near thereto as is safe. Continuing a safe degree of traction and drawing the handles of the two pairs of forceps apart, he brings the vagiral end of the cervix into view. If there is good light from a window behind the cow, the parts may be satisfactorily examined visually or in the absence of efficient natural light, one may secure excellent illumination by using a reflecting electric lamp on an extension cord, or an ordinary flash light. The examiner should note the state of the mucous membrane at the os uteri externum. In the healthy heifer, the vaginal end of the cervix terminates in a sharp cone, at the apex of which opens the very narrow os uteri externum with its mucosa everywhere in contact. The index finger cannot be inserted without violence. Usually it will admit for a short distance, without resorting to force, a smooth sound, one-fourth inch in diameter. The vaginal mucosa envelops completely the entire cone of the vaginal portion of the cervix and invaginates slightly into the os uteri externum. 
After a cow has been pregnant, increasing with each pregnancy, the relations of the cervical tissues become modified. The second annular fold of the cervical mucosa becomes enlarged, both elongated and thickened, and the antero-poste-

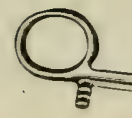

1
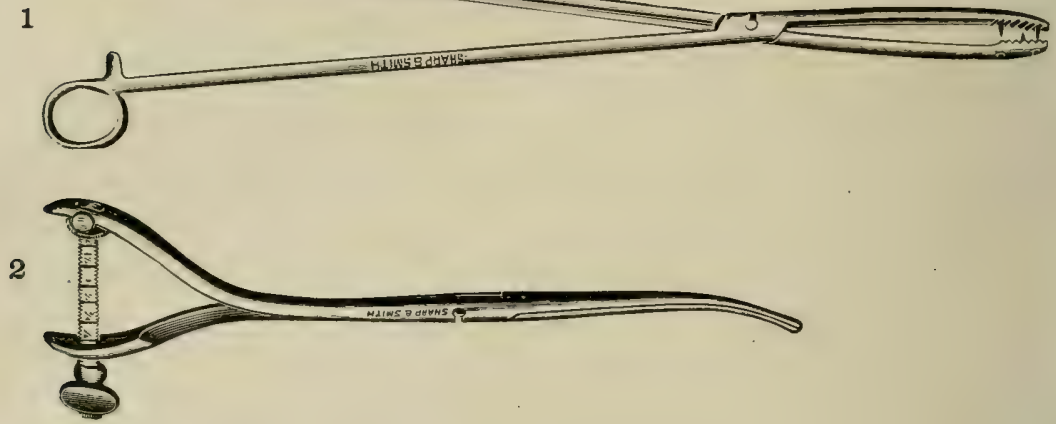

3

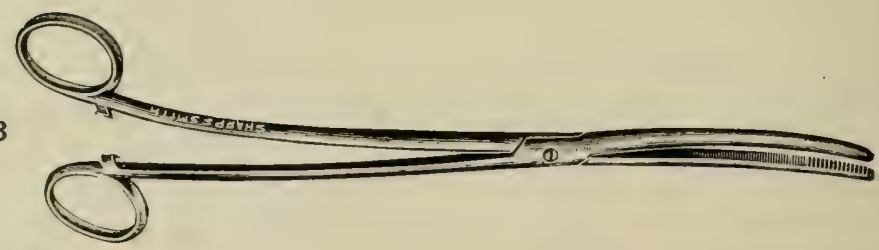

4

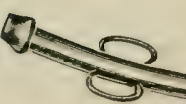

Fig. 39-Instruments for the Examination of the Genitalia of Cows. $I, 2 O^{\prime \prime}$ uterine forceps ; 2 , Palmer's uterine dilator, modified; 3 , Bozeman's uterine dressing forceps ; 4 , small uterine catheter.

rior grooves become comparatively deeper, cutting the ring into a series of deep, pedunculated lobes. Eventually these push their way out through the vaginal ring or os uteri externum into the vagina. The vaginal ring now serves as a band surrounding the protruding second ring. The second ring then becomes the most posterior portion of the cervix, 
and in disease may project three to four inches beyond the original os uteri externum toward the vulva. In severe disease the third annular fold may also force its way out through the vaginal ring. In disease these everted, swollen rings of cervical mucosa constitute large, cauliflower-like

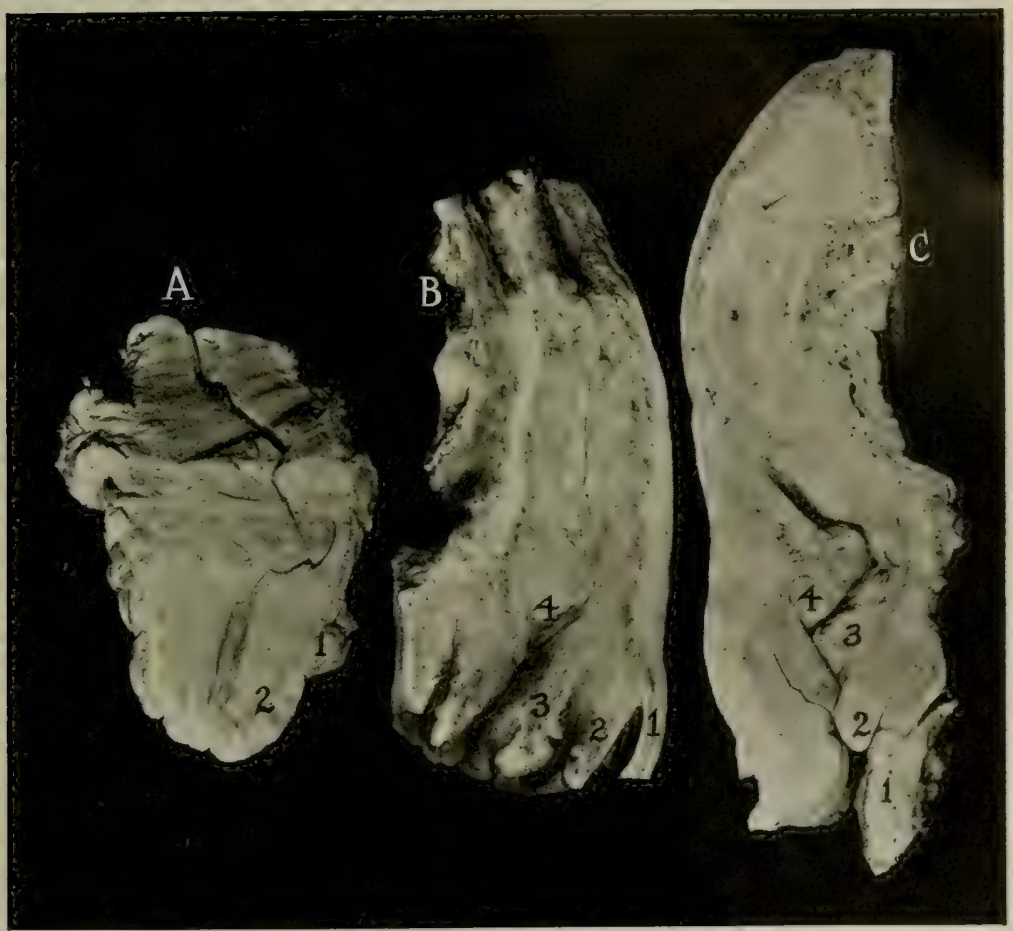

FIG. 40-Median Longitudinal Sections of Cervices of Cows showing the Windings of the Cervical Canal.

$I$, The vagina ; 2 , lip of the os uteri externum; 3 , first annular mucous fold; 4 , second annular mucous fold.

tumors. The healthy mucosa of the cervix is smooth, of a pinkish hue, paler than the vaginal mucosa. Its surface is moist, but should reveal no signs of pus. During estrum the cervical canal is dilated and filled with clear, highly lubricant mucus.

The deeper portions of the cervical canal may be examined 


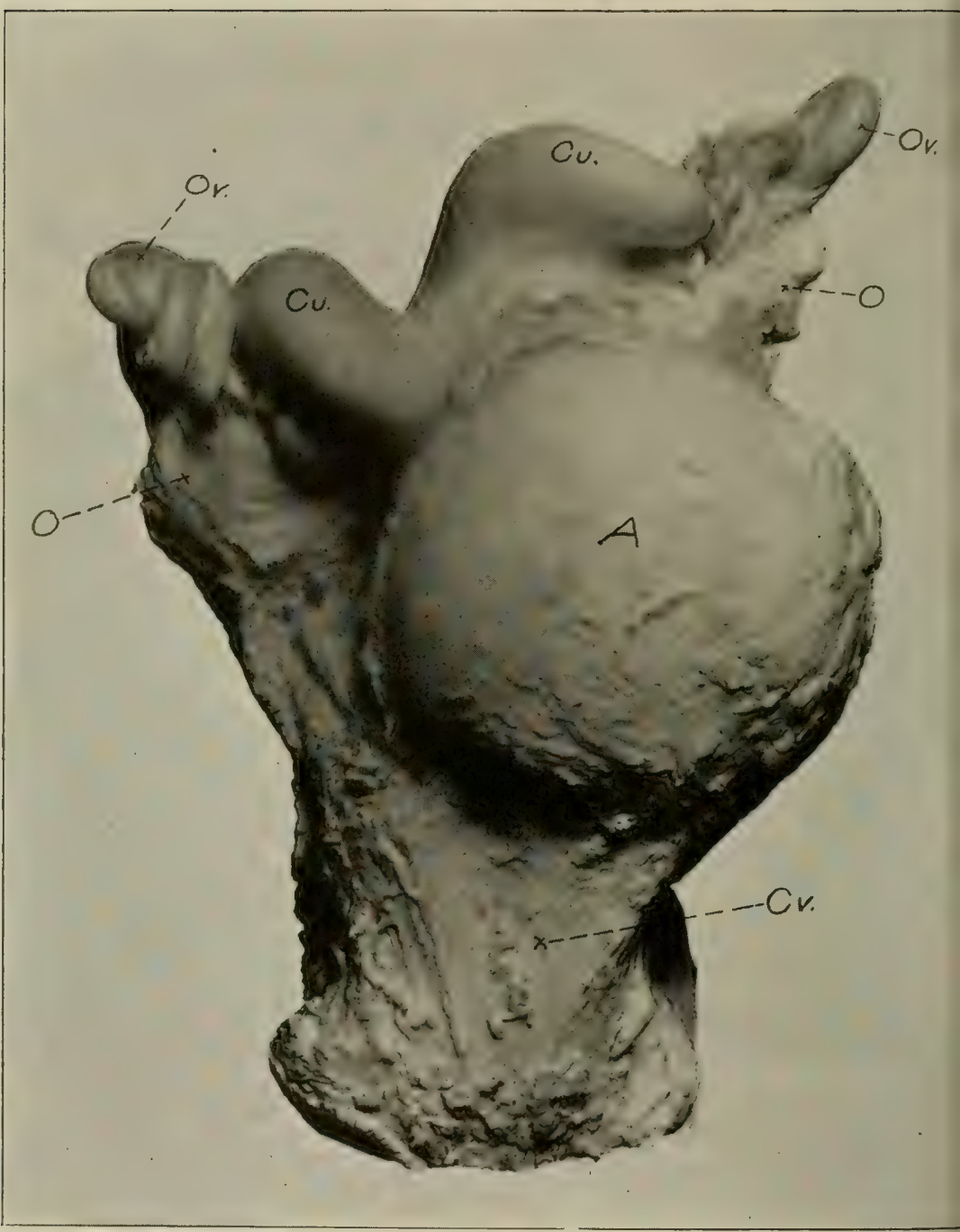

FIG. 42. Rupture of Cervix by an Amateur in an effort to "Open the Mouth of the womb" followed by Chronic Abscess of Cervix.

$C v$., Cervix; $A$, abscess in inferior wall of cervix; $O . O$, approximate location of ovaries lying above the uterine ligaments; $O v, O v$, cystic oviducts (hydrosalpinx) which were causing the sterility; Cu., Cu., uterine cornua. 
by means of dilator, catheter, or sound. The dilator (Fig. 39 ) is usually preferable. As will be seen in Figs. 40, 41, the cervical canal is extremely narrow, sinuous, and at times angular, and the free margins of its annular mucous folds, directed toward the vagina, offer serious obstacles to the prompt passage of an instrument into the uterus. The canal usually extends sharply downward and forward, very slightly to the right, for one to one and one-half inch; then bends sharply upward and forward, and thence approximately forward, with slight divergences, to the cavity of the uterus. The windings of the canal are difficult to follow and the free margins of the annular folds, directed toward the vagina, constitute deep culs-de-sac, into which the dilator tends to pass and its progress to be thus arrested. The operator is, therefore, to take these conditions into account and, without violence or force, retreat and advance at various angles, in his effort to follow the tortuous canal and evade the barriers formed by the mucous folds. Sometimes the free edges of the mucous folds appear to become pushed forward by the instrument into the lumen of the canal and occlude it, preventing for the time the passage of the instrument. In disease, the annular folds often become so hypertrophied and their bases so sclerotic as to cause acute bendings of the canal and to close quite firmly its lumen, making it exceedingly difficult and rarely impossible at a given time to pass an instrument through the canal into the uterus.

The operator needs to be patient here, as well as skilful. Any undue force may rupture the cervical wall and penetrate the surrounding connective tissue, causing pelvic abscessation, as shown in Fig. 42, with extreme peril to the physical and breeding life of the animal. If the operation cannot be made to succeed at first effort, it should be suspended for the time and undertaken later. I have worked for an hour or two at a time, four or five times in succession, before finally succeeding in reaching the uterine cavity. However, it is always infinitely better to try repeatedly, and finally fail, than to use force and ruin the animal, not so much because of the breeding value of such an animal, since 
that is always seriously impaired already, but because the rupture of the cervical walls, with the unpleasant consequences attending, leaves an ugly stain on the veterinarian's reputation. If the passage of the canal is very difficult, one should not, as a rule, work at it for more than an hour or an hour and a half, since by that time he will tend to injure the

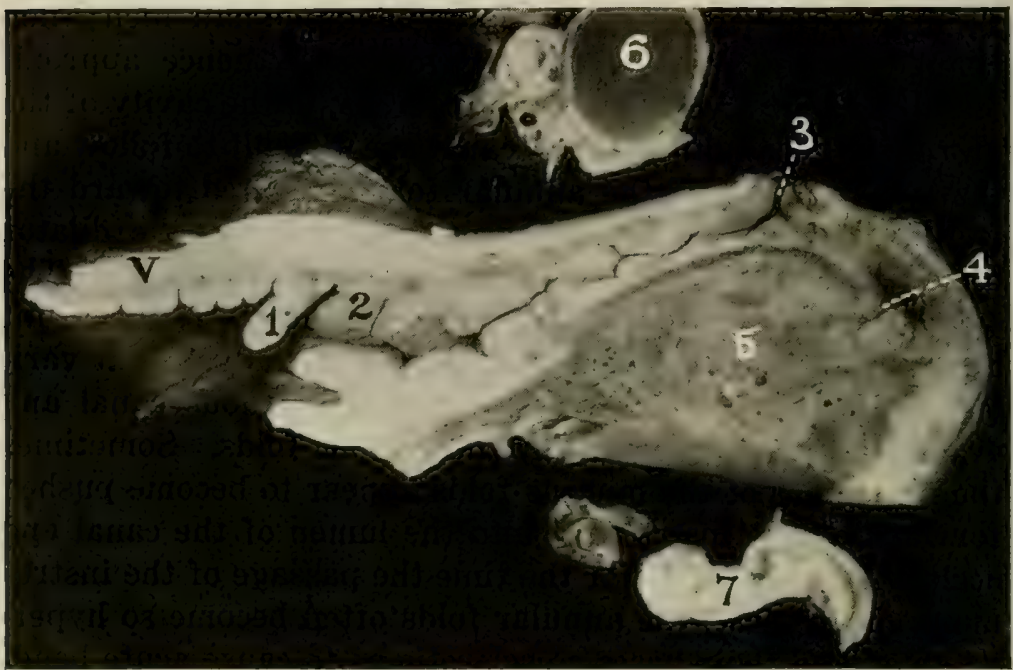

FIG. 43. Rupture of the Uterus with Uterine Catheter. Cow.

$V$., Vagina; $r$, lip of os uteri externum; 2 , first annular inucous fold; 3 , instrumental rupture in the roof of the horn; 4, instrumental rupture of the floor of the uterine horn ; 5 , mesometrium enormously thickened and inflamed; 6 , cystic corpus luteum with adherent cystic oviduct to the left ; 7 , median section through cystic oviduct. The veterinarian had killed the cow douching the uterus when the sterility was caused by the inoperable hydrosalpinx.

tissues unwarrantably if he continues his efforts longer, and, still more important, he becomes fatigued. A fatigued operator is always a dangerous operator. If prudent, he will not accept the attendant risks which he inevitably faces when fatigued.

When the examiner has succeeded in passing the uterine dilator, the catheter, or other instrument into the uterine cavity, he faces the danger of puncturing the uterine wall, 
as shown in Fig. 43, and invading the peritoneal cavity. In order to avoid wounding the uterus, the operator needs to judge well the approximate length of the cervix and when the end of the instrument reaches the region of the os uteri internum, it should be directed slightly downward. That is, using a curved instrument, as is always most convenient and safest, the operator should at this time turn the instrument so that the concave side of the curve is directed downward. Ordinarily no metal instrument should pass far beyond the os uteri internum. In the ordinary empty, non-pregnant uterus, the downward curvature of the uterus is so marked that there is great peril of puncturing the walls if the instrument is pushed more than one to two inches into the uterine cavity. In addition to this danger, if the uterus is flaccid or if the pushing of the instrument through the cervical canal requires some degree of pressure, the uterus bends sharply and the instrument catches in this and punctures the wall.

If chronic cervicitis is present, it inevitably causes sclerosis of the annular mucous folds of the cervix, and the hypertrophy of the sclerotic areas presses across against the opposite side of the cervical canal, bending it out of its course and narrowing its lumen. The sharp bendings of the canal, with the intruding sclerotic and hypertrophied masses, so impede the passage of the instrument that once it has entered the uterine cavity, the resistance of the uterine wall is not clearly recognizable if the instrument is pushed into or through it. When using the dilators, therefore, the advancement of the instrument should cease when the uterine cavity is reached. The length of the cervix, if doubt arises, may be learned by rectal palpation. The same means may be used to learn when the dilator has passed the cervical canal. In all cases where the cervical canal is not freely open and sufficiently direct to permit the easy passage of the uterine catheter, it is prudent and advisable to use first the uterine dilator. I have found the Palmer uterine dilator of the human gynecologist, modified as shown in Fig. 39, the best instrument for this purpose. It is small and the curvature of its blades corresponds fairly well with the average 
bendings of the cervical canal. The dilation of the cervical canal is essentially alike for diagnosis and therapy. When the canal is very narrow and is pressed upon by sclerotic enlargements in the mucosa, the passage of a catheter sufficiently large to permit of satisfactory douching either for diagnosis or treatment, is both difficult and dangerous. Even if it is accomplished, the cervical disease cannot be satisfactorily handled until the canal has been freely dilated.

Once the instrument has reached the os uteri internum, the gradual dilation of the canal should be undertaken by forcing the forceps jaws apart with the thumb screw. The opening of the jaws should be very gradual and prudence used in the amount of force applied. In heifers especially which have suffered severely from cervicitis, and there is consequently well advanced sclerosis, the tissues may fail to relax under the strain but rupture instead. The instrument must on no account be pushed toward the uterus while the jaws are opened as there is great danger of pushing them through the uterine or cervical wall. There is great danger, while dilation is proceeding, if the operator pushes toward the uterus when the patient strains. At such times the instrument must be permitted to move backward with the cervix. The process of dilation should be continued for a sufficient period of time to render the cervical canal of sufficient size to admit of the ready passage of a small uterine catheter, uterine dressing forceps, or other desired instruments. Once the forceps blades are forced apart, the operator may cautiously exert traction upon them, eventually drawing the forceps out with the jaws widely separated. This presses the free borders of the annular mucous folds outward against the cervical walls and tends to cause them to remain out of the way temporarily while the uterine catheter may be introduced. It is best in case of a very narrow cervical canal to open and close the dilator alternately and while closed to revolve the instrument in varying degrees so that the direct pressure of the blades shall be exerted on all parts of the cervical walls. With patience, the pale muscles of the cervix gradually become exhausted and finally the canal at- 
tains a sufficient lumen so that a moderately large uterine catheter may be passed through it without difficulty or danger. This having been attained, catheterization may be undertaken. The catheter used should always be of as large caliber as the cervical canal will readily and safely admit, because the greater the caliber of the catheter, the more efficient the douching of the uterus. If the operator has carefully observed the windings of the cervical canal while using the dilator, he will better understand the direction which the catheter must take. The catheter selected as of proper dimensions should be introduced into the cervical canal very gently and advanced with great care. Force is at all times to be avoided. If the catheter will not pass without force, it should be laid aside and the dilators again used and this plan repeated until the dilation suffices.

The uterine catheter, like the dilator, should not be pressed deeply into the uterus but merely far enough that the fenestrum of the instrument is free in the cavity of the uterus so that douching will be practicable. At the moment of entering the uterus, the concave side of the catheter should be directed ventralward and so maintained throughout douching. Occasionally blood escapes through the catheter, indicating mechailical injury to the uterine mucosa, and this should cause the operator to beware of impending trouble. Once I misjudged the length of the cervical canal, pushed the uterine catheter too far, and caused an extensive antero-posterior laceration in the uterine mucosa along the dorsal surface with copious hemorrhage. I had been careful and could not identify the character of the injury and it was only later when she was slaughtered as a tuberculin reactor, that the cicatrix in the organ revealed the character of the lesion. In this instance the blood did not escape through the catheter but was forced out after the withdrawal of the instrument. The cow suffered greatly, kicked at her belly, lay down and rose frequently, trembled, sweat, and breathed rapidly. The hemorrhage was not of sufficient volume to cause the symptoms directly from the loss of blood nor could the lesion in itself cause so much pain. Apparently the distress was due to uterine colic, that is, the small, empty 
uterus, not recently gravid, rapidly filled with blood and the cervix being firm, it could not readily escape but, being retained, aroused violent contractions like those seen when a volume of fluid is inadvertently left behind when douching. The difficulty passed in an hour or two and she continued apparently well. Whenever there is any question as to the course of the catheter or other instrument, the operator should cautiously palpate per rectum and determine if all is well.

Not rarely, when the catheter enters the uterine cavity there is a sudden inrush of air by which the uterus becomes greatly ballooned, as has already been noted of the vagina and rectum under similar conditions. The inflation of the uterus sometimes proves annoying. It is difficult and sometimes impssible to douche the inflated organ until a very large amount of fluid has been introduced because the liquid drops downward and forward to the ovarian end of the uterus while the air occupies the cervical end of the cavity which is highest. If left in the uterus, it may cause colic. It can be pressed out through the catheter by careful rectal manipulation. (Gynecologists apparently encounter the same condition in woman and some writers describe it as "acute dilation of the uterus".)

When the catheter is accidentally pushed through the uterine wall and the peritoneal cavity is entered, there is generally an inrush of air through the instrument. In this one regard the inflation of the uterine cavity through the catheter and the flow of air into the peritoneal cavity are much alike but in general the rate and volume of air drawn in are greater in the latter.

When an antiseptic fluid, like a two per cent Lugol's solution, is introduced into the peritoneal cavity through the catheter, it immediately causes marked depression and uneasiness, with rapid breathing, trembling, sometimes lying down, and other evidences of distress. The cow tenses her abdominal walls, arches her back and visually appears to be bloated. If the uterus is clean and little or no infection is forced into the peritoneal cavity, lymph is soon thrown 
out, which dilutes the Lugol's solution. Rapid absorption may follow and iodism result. The distress from the introduction of Lugol's solution into the peritoneal cavity is of short duration and within one-half to two hours she seems as well as before. The uterine wound heals promptly and little or no harm follows. When coal tar disinfectants and others of a highly irritant character, especially those not freely soluble in the lymph, are introduced, the irritation is more severe and prolonged, with great danger of a severe peritonitis with serious peril to life. If death is avoided, the peritonitis may lead to extensive adhesions destroying the value of the patient. Some veterinarians have counseled the use of bicarbonate or chloride of sodium solution as a douche, but if this or other neutral fluid is used and a puncture of the uterus occurs with the fluids entering the peritoneal cavity, the danger is extreme because usually infection is borne from the uterus and sets up a violent and fatal peritonitis. In Fig. 43, is shown a uterus punctured by pushing a catheter much too far, a fatal peritonitis following douching with, it was claimed, soda bicarbonate solution. Whether the infection was due to a dirty catheter, whether it bore infection from the cervix or uterus into the peritoneal cavity, or whether the soda solution itself was septic, does not materially alter the case. No antiseptic was present to destroy the infection.

When pus is present in the uterus in large quantities and of high virulence, as is most frequently seen in the puerperal animal, a gross rupture of the organ is followed at once by very alarming symptoms which frequently prove fatal within two or three hours. The shock is especially profound when it is attempted to douche a large paretic uterus containing a great volume of virulent material and a great amount of water is introduced to add to the mass of liquid. A large rupture of the uterus releasing the great mass of infected liquid, which immediately reaches the total peritoneal surface, produces prompt collapse. The abdominal walls at once become tense and fixed, the breathing being thoracic, rapid and shallow. The patient appears tympani- 
tic, shows intense suffering and, as a rule, quickly succumbs. Some of them, however, after remaining in an extremely critical state for some days slowly improve but as a rule never regain condition and must eventually be destroyed as useless.

Consequently in the instrumental examination of the uterus, as in that of the cervix, ample care is always to be taken to avoid rupture. Such care must be based upon accurate anatomic knowledge, surgical skill, patience, and the application of the invariable rule that, if the instrument cannot be passed without the use of force, the effort must be suspended. The cervical canal dilates during estrum, at which time an instrument may far more readily be passed into the uterus. If the operator desires, he may dislodge the corpus luteum and return after three days with reasonable assurance that he will find the patient in estrum and her cervical canal dilated. If he will then use the dilators prudently, he will leave the canal dilated and the angles modified in a manner to render catheterization of the uterus less difficult if again undertaken in a few days. Aside from the diagnosis of estrum and the advantage of the dilated cervix facilitating the catheterization of the uterus, estrum is a very poor time to examine the genitalia, because vagina, cervix, and uterus are so filled with mucus, and the uterus so enlarged and engorged that many pathologic conditions may be so completely screened as to be wholly unrecognizable.

When the catheter has entered the uterine cavity, the examination is to be completed by douching. For this purpose I prefer a one to two per cent. Lugol's solution, rather than sterile salt solution or other neutral liquid, because, in my judgment, the disinfectant renders the operation safer, especially when any minor injury to the cervical or uterine mucosa has occurred. The faintly brownish and clear fluid serves to form a contrast with pus or muco-pus rendering them more apparent. Physiologically the douche should return clear and uniform except that in estrum there should first escape some clear mucus, and during the one or two days following estrum, some partly clotted menstrual blood. 
Admitting fully the dangers incurred in the manual and instrumental examination of the genital organs of mares and cows, it nevertheless remains that (1) such examination is not in the ordinary sense necessarily dangerous and (2) the examination is absolutely essential to a safe diagnosis. Accidental injuries to the genital organs of cows and mares in the course of examination are happily rare in the hands of competent veterinarians. At present there is much activity in the handling of sterility in cows and many veterinarians, some of whom have always blundered at each critical step, have plunged headlong into this field with neither the chart of anatomical knowledge nor the compass of ordinary prudence. Naturally they make many and serious errors and bring undeserved discredit upon one of the most valued fields of veterinary service. The veterinarian who will not give long and conscientious study to the subject, has no moral right to attempt the work.

The physical examination of the genitalia not only serves the necessary purpose of diagnosing disease but also serves to identify estrum, the period of sexual rest between estrual periods, and pregnancy.

\section{The Diagnosis of Estrum}

The diagnosis of estrum is of great practical importance because in numerous instances the ordinary clinical signs fail unless the veterinarian is able to apply a more technical and accurate method of determination. Without technical examination, the breeder may fail to recognize the condition and hence omit breeding. The cow or heifer commonly reveals the presence of estrum by bellowing and restlessness, whether in stable or field. In the field or paddock with other cattle, the animal in estrum repeatedly mounts other cattle or stands to be mounted by them. Often there is a discharge of thin mucus from the vulva. In many cases cattle are not out in paddock or field daily so that the tendency to mount other cattle is not apparent. Some individuals show little excitement and do not bellow enough to attract attention in the stable. Even when turned daily into the paddock 
or field, as is commonly done in winter for only a brief interval, a cow may pass through her active estrual period during the interval between two periods in the paddock. Not infrequently such unrecognized estrual periods result in expensive delay in breeding.

The chief evidences of estrum revealed upon physical examination are:

(1) The vagina contains much thin, lubricant, clear mucus. In metritis, cervicitis, and vaginitis the mucus may contain floccules of muco-pus. The flow of the mucus of estrum appears in the vagina twenty-four hours or more prior to the establishment of sexual desire and terminates about twenty-four hours after the cessation of estrum. If fertilization fails, the discharge of estrual mucus is displaced by the menstrual flow.

(2) The cervix is relaxed and the canal more dilated and dilatable than in the inter-estrual period of rest.

(3) The uterus is engorged. Prior to the full development of estrum, the uterus may be normal to the touch at first, but under palpation becomes engorged or erected. When estrum is at its height, the engorgement is constant. The diameter of the engorged uterus of estrum is increased about one-half, is much firmer than during the inter-estrual pause, and is smooth, even, and very firm. It is about the same size as the pregnant uterus at thirty to forty days, but is distinguishable by its firm, engorged character, in contrast with the fluctuant, tense uterus of pregnancy. It is less hard, and smaller than the uterus of chronic sclerotic metritis with destruction of the uterine mucosa.

(4) The corpus luteum of the preceding estrum is atrophied to about one-half its ordinary diameter, or about threeeighths of an inch.

(5) There is present, generally upon the opposite ovary, a mature ovisac, a thin-walled cyst one-half inch or a trifle more in diameter. (See Figs. 22-25.)

If it is desired to breed the animal during the existing estrum, the examiner needs to be very cautious in palpating the ripe ovisac since under very slight pressure it ruptures, 
the ovum escapes into the peritoneal cavity, and conception is barred for that estrual period.

Recent estrum is recognized:

(1) By menstrual blood in the vagina or the soiling of the tail and buttocks with blood, about twenty-four hours after estrum has ceased;

(2) By engorgement of the uterus which continues during menstruation;

(3) By the rapid atrophy of the corpus luteum of the preceding estrum;

(4) By the palpation of the crater of the ruptured ovisac.

The diagnosis of estrum may be rendered more certain by concentrating the observations to a brief period. Thus, when the soiling of the tail and buttocks with menstrual blood is observed, if the breeder will watch the animal closely from the eighteenth to the twentieth day thereafter and take occasion to place the cow in paddock or field with the bull or other cattle, estrum will in all probability be recognized. The time during which close watch is to be kept may be even further concentrated by dislodging the corpus luteum when it is well formed and watching the patient from the third to the fifth day thereafter.

\section{The Diagnosis of Pregnancy}

The accurate diagnosis of pregnancy in the cow is a fundamental necessity in the handling of diseases of the genital organs. The gravest and most embarrassing errors made in dealing with the genital organs are the failure to diagnose an existing pregnancy and the erroneous diagnosis of pregnancy when the animal is sterile. The failure to recognize an existing pregnancy frequently leads to instrumental abortion or to the slaughter of a valuable pregnant animal which is wrongly believed to be sterile. The diagnosis of pregnancy, when the animal is sterile, leads often to months of expensive delay in breeding. The examination for pregnancy of valuable animals which are for sale is gradually assuming scientific and economic importance. In some large herds placed in dispersal sales, all the cattle are ex- 
amined in detail and certificates of pregnancy or non-pregnancy by a skilled veterinarian issued for each female. Eventually, probably before most veterinary practitioners are ready to render highly efficient service, buyers of expensive breeding cattle will demand reliable veterinary examinations as to sexual soundness. In the female assumed to be pregnant, the prudent buyer will place first reliance upon the judgment of an expert veterinarian.

The signs of pregnancy are many. Each has its value and each is subject to certain limitations:

\section{The Failure of Menstruation After Copulation}

When conception follows coition, estrum is rarely followed by menstruation. If menstruation follows coition after 24 to 48 hours, the cow is probably non-pregnant.

\section{The Cessation of Estrum}

If a cow or heifer has been regular in her estrual periods, and estrum fails to recur at the normal date, the animal is quite probably pregnant. The probability is accentuated if menstruation following coitus failed. The sign is of little value in irregular estrum. The estrual period may be comparatively short or long, but in order to be of value in judging conception, the periods need to be alike. That is, one animal may have an estrual cycle of eighteen, and another of twenty-two, days but an animal having once an interval of eighteen days and at another time a cycle of twenty-two days offers suspicion of ovarian disease. The failure of an estrual period in such an animal is not a good index of conception. In healthy cows and heifers there is little variation in the estrual cycle in the individual; the variation rarely exceeds twenty-four hours. It is important alike for the breeder and the veterinarian that accurate records be kept for each heifer and cow.

Estrum does not always cease with conception. In my judgment, one to two per cent. of pregnant cows and heifers show estrum. In some of these, the estrum recurs at varying intervals up to the seventh or eighth month, but as a rule 
it ceases to recur after the third or fourth month of pregnancy. Sometimes the recurrence of estrum is regular, repeating the cycle prevailing in the individual prior to conception, but usually the cycle is irregular. So far as I have observed, the estrum of pregnancy is not followed by menstruation. In fact, menstruation is not ordinarily possible, owing to the uterine seal blocking the cervical canal and preventing exit.

Estrum not infrequently fails to recur after copulation although conception did not follow. Sometimes the failure of estrum to recur is due to retained corpus luteum, sometimes pyometra (without discharge) follows estrum and coitus, inhibits estrum and misleads the caretaker. Poverty serves to prevent alike ovulation and estrum. The failure of estrum to recur in non-pregnant, and the recurrence of estrum in pregnant animals render it clear that the presence or absence of estrum does not furnish secure ground for diagnosis relative to pregnancy. The evidence must be supported by other facts in order to be reliable.

The estrum of pregnancy has been little studied. In one case which I had the good fortune to examine while the animal was in estrum, the corpus luteum of pregnancy had atrophied and a ripe ovisac was present on the non-gravid side. I think it more than possible that the estrum of pregnancy is generally due to this cause. The corpus luteum of pregnancy, for some unknown reason, atrophies, loses its inhibitory power upon the maturation of ovisacs, and estrum, so far as the ovaries are concerned, appears in the ordinary manner.

It is of scientific interest to note here also that the estrum of pregnancy, with the atrophy of the corpus luteum and the ripening of an ovisac upon the opposite ovary, is the possible foundation for numerous errors regarding the migration of the ovum. It has been claimed that finding a fetus in one horn and the corpus luteum in the alternate ovary is proof of the migration of the ovum, and that the fertilized ovum has dropped into the peritoneal cavity, been transported to the pavilion of the opposite oviduct and penetrated it, or that 
the ovum has passed down the horn corresponding to the ovary from which it emanated, thence across the uterine body cavity and up the opposite horn. My observation makes it perfectly clear that the alternating of the fetus and the corpus luteum is not proof of migration.

The abnormality of estrum (and coitus) appears to have no serious peril for either the pregnant animal or the fetus. It is clear evidence of a pathologic state, but many cows and heifers show frequent estrum and copulate freely without showing any marked tendency to abort or for the pregnancy to terminate unfavorably in any marked respect. Some of them abort, it is true, but I have been unable to learn that their tendency to abort is greater than in pregnant cows which do not show estrum. A few cases have occurred under conditions which have led some to believe that coitus caused abortion almost immediately. Thus I recall one cow which came in estrum in the sixth month of pregnancy, copulated, and the next day aborted. In other instances, a cow carrying a desiccated fetus develops estrum, copulates, and a few hours later expels the mummy. I think it reasonably certain that the facts have been misinterpreted, and that, instead of the coitus causing the expulsion of the fetus, the corpus luteum has atrophied, an ovisac has ripened, estrum and coitus have occurred, and because of the atrophy of the corpus luteum, not because of coitus, the uterus contracts and expels the fetus. That is, instead of the estrum and copulation causing the abortion, the disease has brought about the changes in the ovary, the estrum, the copulation, and the expulsion of the fetus. The problem merits further study.

Menstruation following estrum in pregnancy is incompatible with the life of the fetus, because the menstrual blood consists of a hemorrhage emanating from the placental areas (cotyledons) of the uterus. Menstruation would, therefore, result first of all in a hemorrhage between the maternal and fetal structures which would force the two structures asunder (compare "Desiccation of the Fetus"). 


\section{The Uterine SEAL}

If the vagina and cervix are healthy, the cervical canal is well sealed at from thirty to forty days after conception and it may be felt clearly upon palpation per vaginam. In order to recognize this, the finger tip must be pressed carefully and gently against the external os uteri and then cautiously withdrawn; if the seal is present, it is readily revealed by its adhesiveness. In the earlier stages the seal is very small, only one-eighth to one-fourth inch across, and does not pro-

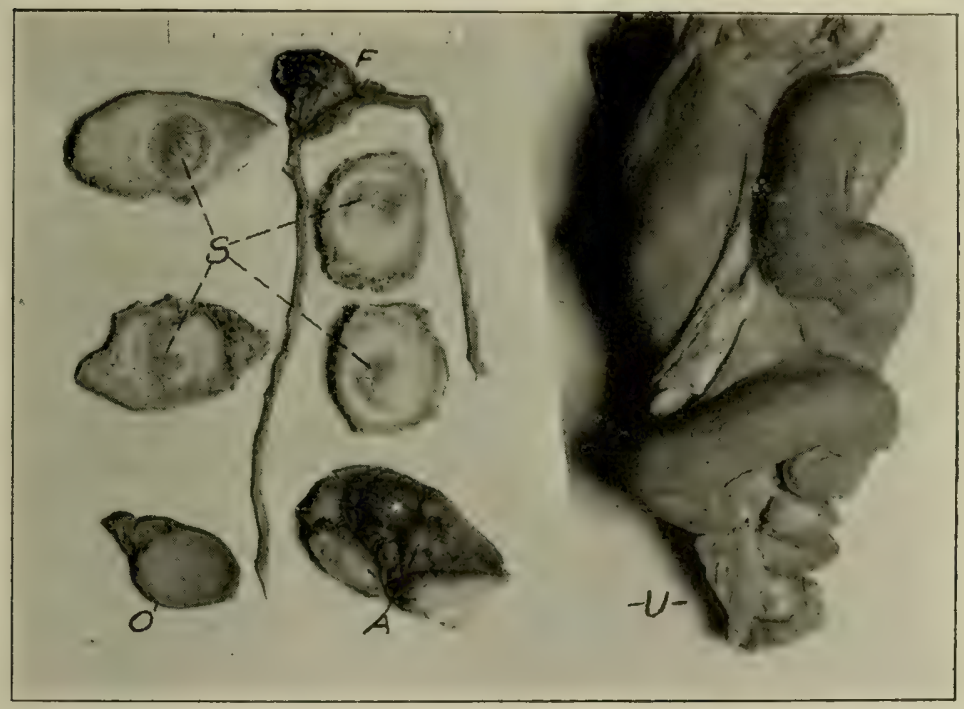

FIG. 44-The Uterine Seal in Early Pregnancy in Heifer.

$F$, Embryo $1 / 4$ inch long in the embryonic sac; $S$, uterine seal in cross section; $O$, ovary with corpus lutem of pregnancy; $A$, uterine seal protruding from the vaginal end of the cervix.

ject into the vagina. It is distinct, however, and adheres clearly to the finger tips. It continues to grow and soon bulges out into the vagina. The uterine seal involves the cervical mucosa only. As stated earlier, however, the annular folds of the cervical mucosa push out into the vagina in the cow which has calved and especially when she has suffered from cervicitis. The protruding portions of the cervical 
mucosa function as though they remained in the canal so that in such animals the uterine seal proceeds from, and covers over, the mucosa protruding into the vagina. In the mare, in advanced pregnancy, the uterine seal projects into

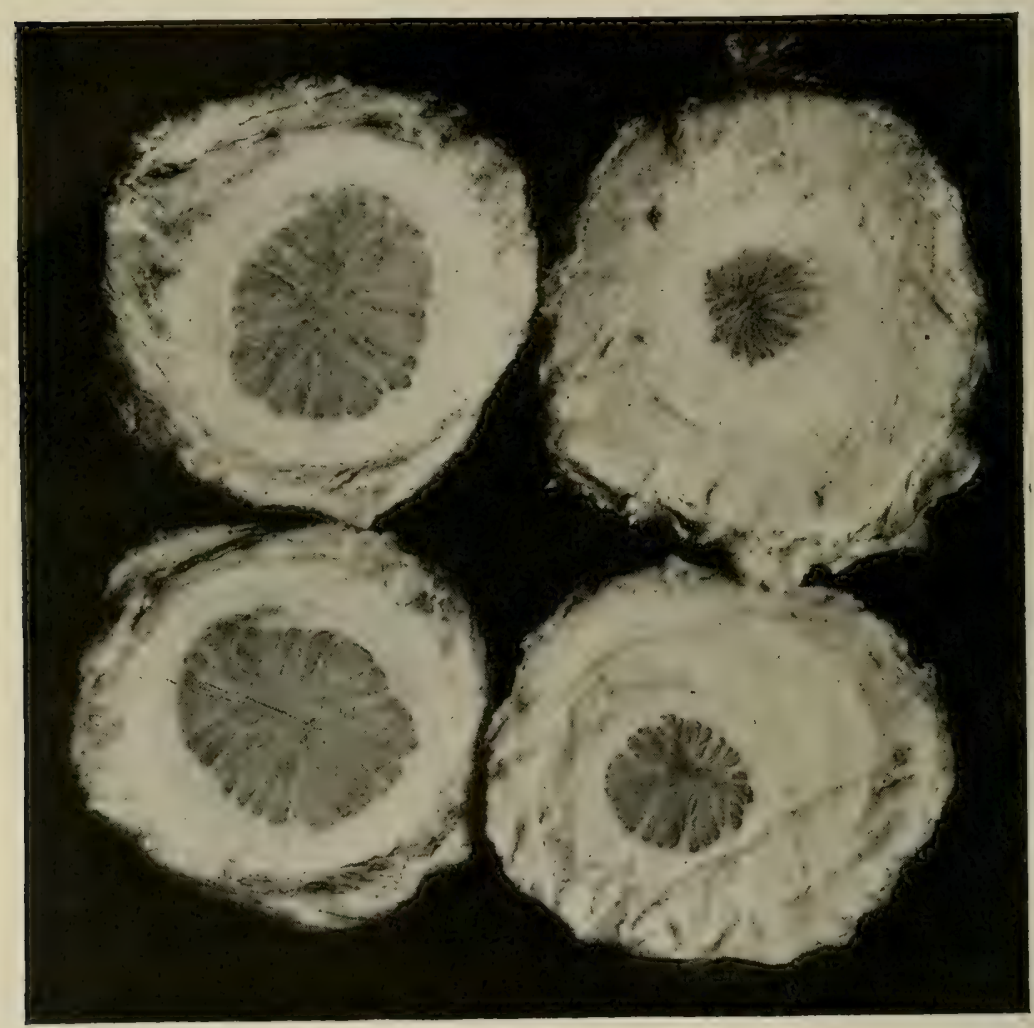

FIG. 45-The Uterine Seal of an Adult Cow in Advanced Pregnancy. Cross sections through the cervix at various points. The larger seals are near the vaginal end.

the vagina as an adhesive, somewhat cylindrical body, one, two, or more inches in length. Its size and protrusion increase until just before the close of pregnancy, when it softens, changes to lubricant mucus, and strings from the vulva. During pregnancy the mucus secreted by the vagina becomes exceedingly sticky, having much the same character as the uterine seal. 
Sometimes a false uterine seal forms, as a result of disease, as in retained corpus luteum and in cervicitis. The uterine seal persists when the fetus dies and desiccates, and when the embryo dies and the embryonic sac remains to constitute a "mole".

The uterine seal may fail to form or it may break down when the cervix is badly diseased and abortion is imminent. Consequently the presence of the typical seal not only gives reliable evidence of pregnancy, but adds to that a certain feeling of security, suggesting not only that the animal is

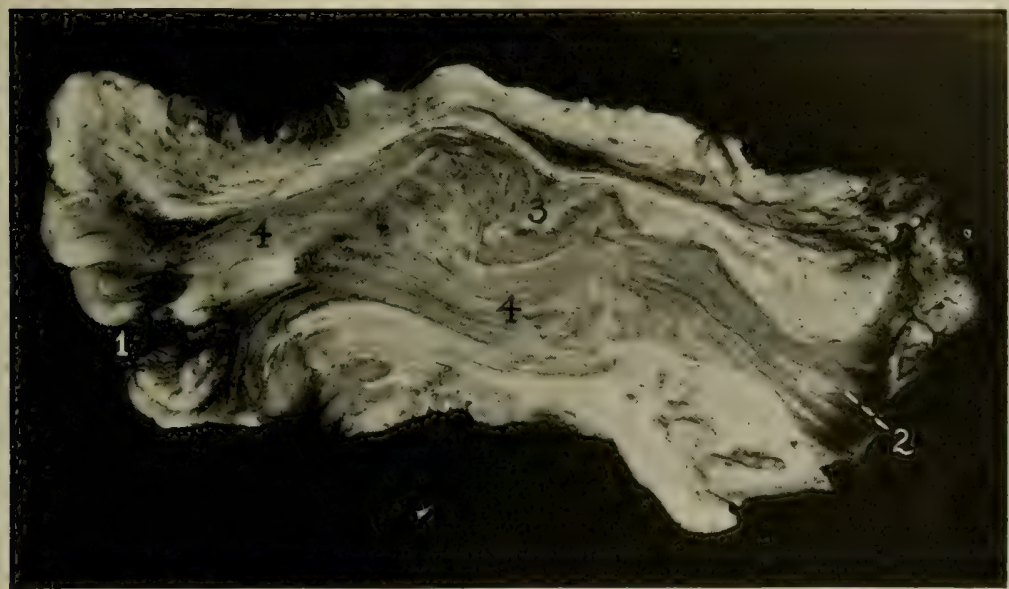

FIG. 45a-The Uterine Seal in Longitudinal Section. Cow. $I$, Os uteri externum ; 2 , os uteri internum; 3 , base of second annular cervical fold; 4 , uterine seal extending throughout the length of the canal.

pregnant, but perhaps safely so. In severe purulent vaginitis or purulent cervicitis of the vaginal portion, the uterine seal may exist in the uterine end of the cervical canal and be displaced by pus in the vaginal portion. The seal may then be recognized by palpating it with a metallic sound, uterine catheter, or dilator introduced cautiously along the cervical canal. While the uterine seal is one of the most important evidences of pregnancy, it has its limitations in diagnosis and, like most signs, needs to be considered in conjunction with other findings. 


\section{The Corpus Luteum of Pregnancy}

(See Fig. 26)

Physiologically, a corpus luteum develops after each estrum, and is of the same form, size, and consistency, whether the animal be pregnant or not. In sterility, however, the corpus luteum is one of the most common sufferers from pathologic changes. The bovine corpus luteum of pregnancy is normally five-eighths to three-quarters of an inch in diameter, somewhat oval in form, usually even in contour, and firm in consistency. Forming, as it does, within the crater left behind when the ovisac ruptures, it is at first naked. That is, it is not covered by the tunic of the ovary. and it protrudes somewhat beyond the surface of the gland. If the animal conceives, the tunic of the ovary soon draws over the surface of the yellow body and leaves it smooth and firm. The wound is healed. Sometimes a considerable projection remains, even though the tunic has drawn over the yellow body. In sterility, however, the wound in the ovisac frequently fails to heal and the yellow body remains for a long period of time protruding and naked. The protruding dome feels soft upon palpation, like the exuberant granulation of an unhealthy ulcer. The corpus luteum of sterility frequently undergoes cystic degeneration in its center. It becomes soft and more or less fluctuant, according to the degree of degeneration. Finally, as the degeneration extends, the lutein tissue disappears and a cyst remains. When one can recognize these conditions in the ovary, he can with very rare exceptions rest assured that pregnancy has not occurred through fertilization of the ovum which was discharged from the ovisac in which the diseased yellow body develops. He must not be deceived, however, because of the presence of a cyst or cysts in an ovary. The typical corpus luteum of pregnancy almost always exists in a pregnant animal, but cysts may co-exist. Accordingly, while the absence of a corpus luteum of a normal type is fairly conclusive evidence that the animal is not pregnant, the coexistence of a large cyst in the same or other ovary is not final proof, though it is strong evidence that the animal is 
non-pregnant. When a typical corpus luteum of pregnancy is present and other findings are in accord, the evidence it affords is of the greatest possible value. Amongst thousands of ovaries which I have examined, I have seen but one corpus luteum of pregnancy which departed markedly in volume from the normal. The one exception was more than one inch in diameter. In cows which, though pregnant, nevertheless show estrum, the corpus luteum disappears, at least sometimes. Hence a cow may be pregnant and no corpus luteum present.

The corpus luteum of pregnancy persists throughout pregnancy and for thirty to sixty days after its close. The corpus luteum of estrum is a temporary structure, which begins to disappear rapidly at about eighteen days after estrum, so that at the average period of twenty-one days it has become so far resorbed that it no longer inhibits the maturing of a fresh ovisac.

It is at this period that the corpus luteum becomes of special significance in the diagnosis of very early pregnancy. If estrum has been regular and the animal is not pregnant, it is very certain that at about twenty-one days the animal will again be in estrum and that the corpus luteum of the previous estrum will have decreased to a small size. At the same time, if estrum is near, the uterus is engorged, the cervical canal is dilated, and the vagina contains the lubricant mucus of estrum. Hence, if the practitioner examines a cow at about eighteen to twenty-one days after breeding, if she is pregnant, there is almost invariably a typical corpus luteum; if she is not pregnant, the corpus luteum is definitely atrophied. This is one of the best times for making a diagnosis, and a highly important date, because if the animal is not pregnant, immediate measures may be taken, such as disinfecting the uterine cavity, preparatory to breeding when the approaching estrum has become established.

\section{The Uterine Arteries}

The uterine arteries afford definite evidences of pregnancy. As soon as the fertilized ovum has become implanted 
in the uterus, all vessels leading to that organ awaken to a wholly new activity. There are three pairs of arteries concerned, the utero-ovarian, the uterine, and the vaginal. In the healthy non-pregnant cow or mare each of these is clearly palpable per rectum, approximately one-eighth inch in diameter and, being but lightly covered, yields a clear, though not strong, pulsation.

The utero-ovarian artery, analogue of the chief artery of the testicle in the male, arises from the aorta near the origin of the small mesenteric, and, passing downward, backward and inward between the peritoneal layers of the broad ligament, divides near the ovary into two branches, one going to the ovary, the other supplying the oviduct and the apex of the uterine horn. Readily palpable in the non-gravid animal, it is soon dragged so far forward that rectal palpation is rendered inconvenient. The two other pairs, which are readily reached, suffice in virtually all cases, and consequently the utero-ovarian artery is commonly ignored. Its palpation becomes of great importance in the diagnosis of ovarian tumors.

The middle or chief uterine artery arises usually from the posterior aorta near its division into the iliacs, or from one of these, and is easily recognized as it leaves the arterial trunk, at almost right angles, in the region of the anterior border of the shaft of the ilium. It curves backward, medianward, and finally, in the form of an arc, forward, to disappear in the uterine walls at or near to the base of the uterine horn. During this course it lies between the two peritoneal layers of the broad ligament of the uterus, is much longer than the broad ligament and is thrown into numerous S-shaped coils. It is readily picked up per rectum and palpated in the non-pregnant animal and in all stages of pregnancy. With the advent of pregnancy, the artery quickly enlarges and the pulsation becomes strong and bounding. The arterial stream feels as if impeded and there is a sensation of grating not encountered in other healthy arteries. The artery is about one-quarter of an inch in diameter at 120 to 150 days; at eight months it is one-half 
inch or over; and in a large cow closely approaching full term it may be nearly three-quarters inch, full and bounding.

The posterior uterine or vaginal artery is given off from the internal pudic, approximately opposite the apex of the angle between the sacro-sciatic ligament and its point of attachment to the sacrum, and passes across the pelvic cavity in the broad ligament of the vagina to the vagina and cervix. In the healthy non-pregnant cow it is almost as large as the main uterine artery. It is easily picked up per rectum and palpated. Like the middie uterine artery, it enlarges very rapidly as soon as pregnancy is established but attains no such great dimensions as the former. It reaches ordinarily the size of a lead pencil.

The examiner, in the course of his work, should acquaint himself with these various changes by studying animals pregnant for a known period. In this manner he becomes familiar with the clinical findings and is able to reach a closer approximation of the date of pregnancy of females whose history of conception is wanting.

\section{Palpation of the Uterus per Rectum}

The palpation of the uterus of the cow and mare per rectum affords the most valuable means which we possess for the diagnosis of pregnancy. After conception the uterus undergoes prompt and marked changes in volume, form, consistence, and location. When estrum occurs, there is a marked engorgement. The organ is hard, tense, and smooth. The condition is most notable in heifers. If conception occurs, the engorged, undulatory character continues for a time and in heifers the organ remains almost static in size for fifteen to twenty days, after which it gradually enlarges. In cows which have calved previously, these symptoms do not become so apparent and the enlargement is not pronounced until thirty to sixty, or even seventy. days. Then the uterus becomes larger, most markedly in the gravid horn.

Owing to the great variations of the size of the uterus in adult cows and mares, due partly to individual peculiarities and partly to the health or disease of the organ, one has 
sometimes to await a quite definite increase in volume before this becomes of distinct value in determining pregnancy. The same may be true of heifers which have been sterile for a long period and in which the uterus has undergone enlargement because of chronic endometritis.

As a general rule, however, heifers which have conceived at all promptly show distinct and characteristic enlargement of the uterus within twenty to thirty days after conception, and cows at any time from thirty to sixty or seventy days. The enlargement of the uterus as a result of conception is very characteristic. The organ may be enlarged from a great variety of causes, but the enlargement due to other influences than pregnancy differs materially and clearly in almost every case. In pregnancy the uterus is smooth and even in outline, firm, tense, and fluctuating. Except in case of twin pregnancy, the pregnant horn is much larger than the other and regularly corresponds with the corpus luteum of pregnancy. While the corpus luteum is regularly palpable in early pregnancy in the cow, it is not so in the mare because it is hidden deeply in the hilus of her ovary. At the region of the internal os uteri, the superior uterine wall of the pregnant cow rises up suddenly like a terrace instead of sloping forward gradually as is observed when pus or lymph distends the organ. The firmness of the uterine wall is in marked contrast also to its character when the organ is distended with pus or lymph. In pregnancy the uterus is distinctly firm and exhibits to the touch a sense of vigor and life. When lymph distends the uterus, the walls may be thin as in pregnancy but they are almost always soft and flaccid and the horns are usually somewhat equally filled. In rare instances, owing to compression of the cervical canal due to sclerotic swellings in the cervical walls associated with cervicitis, menstrual debris is retained, distending the uterus quite firmly and giving a tension much like that of pregnancy, but the two horns are alike and there is but one corpus luteum. Usually in such cases no uterine seal is present. When the organ is distended with pus, the contents do not move as freely as the fetal liquids. The uterine walls 
are soft and flabby with a moderate amount of thickening, or they are tense and at some place an abscess may point, or the organ is very dense, with thick, hard walls.

As pregnancy advances, additional evidences appear. The uterus becomes very. greatly enlarged, the walls are tense, and the fetal fluids are quite readily recognized as such. Later, cotyledons are palpable through the uterine wall and when pregnancy has reached four to five months one will occasionally recognize the fetus itself floating in its fluids. The fetus becomes increasingly easy of recognition as pregnancy advances. In some cases, however, both fetus and uterus fall forward into the abdomen and may be beyond the reach of the examiner. In such cases there is still good evidence of pregnancy. The vagina is drawn far forward, the cervix has been dragged anterior to the pubis, and the vaginal end of the uterus constitutes a large, thick, firmly stretched band passing downward and forward beyond the examiner's reach. This of itself does not indicate pregnancy with absolute certainty, because the same displacement of the uterus also occurs from the presence of large uterine or ovarian tumors, from extensive pyometra, or from other pathologic conditions which would cause an increased weight of the organ. The application of the uterine forceps to the cervix, followed by traction on these, thus drawing the uterus upward and backward, to or into the pelvis, as already advised, is of supreme importance in making an accurate diagnosis in these cases. No other means alone can afford so great assistance and security. In this emergency, however, other signs appear to aid the examiner in making his diagnosis. As a general rule, the diagnosis can be verified by the presence of the uterine seal, by abdominal ballottement, or by palpation of the enormously enlarged uterine arteries. In some instances it is practicable to diagnose the physical well-being of the pregnancy. It is easy to diagnose desiccation of the fetus from the time when the interplacental hemorrhage occurs. When the metritis of pregnancy is so far advanced that abortion is not very distant, it may be diagnosed by vaginal and rectal 
palpation, even though the fetus be yet alive, but this is not as easy in the cow as in the mare. In the latter I have been able to pass my finger through the wide, short cervical canal, from which the uterine seal had disappeared, palpate the suppurating, necrotic area of the chorion about the os uteri internum, and, beyond, touch the active fetus. In the cow this is impracticable, but one may recognize by vaginal examination the disease or destruction of the uterine seal or portions of necrotic chorion in the cervical canal or protruding into the vagina. By rectal examination, one may recognize clearly the paretic uterus of metritis, its want of tone, its flaccidity at the cervical end, and the thickened wall. By these means I have been able to make the diagnosis "Pregnant, but unsafe" and, to the astonishment of the breeder, have my diagnosis verified in a few weeks by the cow aborting. The dead and macerating or emphysematous fetus and such displacements as torsion of the uterus without clinical symptoms are clearly diagnosed by rectal palpation. The palpation of the pregnant uterus per rectum has, therefore, a very wide range of usefulness and is capable of important advancement by further study.

Much fiction has been indulged in regarding the dangers from palpation of the gravid uterus per rectum. While some persons are bungling enough to rupture the rectum in this procedure, that is not the fault of the operation, but merely the ignorance, stupidity, or carelessness of the examiner. The idea that abortion may be caused by a prudent rectal palpation is no better founded, if as well, than the unavoidability of rupturing the rectum. A stupid examiner might rupture the gravid uterus, or possibly roll it over and cause torsion, but that is not abortion, and does not lead to abortion. I might illustrate the safety of the examination by a typical incident to which reference will be made later. I was examining for pregnancy a cow which had been bred 145 days previously. The findings were exceedingly confusing. I palpated the uterus for one-half hour before I finally recognized the presence of torsion. The cow was left for about twenty hours, then was cast, 
rolled over several times, released and caused to get up, then thrown and rolled again. In all, she was rolled completely over six times, thrown twice, and the gravid uterus palpated with considerable vigor. She calved easily and without assistance, at 280 days. Both mother and calf were healthy. Thus, she endured severe uterine torsion, violent rolling, and prolonged palpation per rectum, without visible disturbance of the course of pregnancy.

Some veterinarians are afraid to apply forceps to the cervix of a pregnant cow or mare and exert traction. The proper application of the uterine forceps to the cervix does not cause any material disturbance to the uterine seal and causes only the most trivial wound of the cervix. The traction applied to the cervix by any veterinarian of very ordinary prudence is not so great as the tension caused upon the same parts when the animal is lying down or getting up.

Generally life can be recognized in the fetus by rectal exploration long before it can be by auscultation of the heart or by other means. When the cow has been pregnant four or five months, the fetus, eight to twelve inches long, when touched, generally reacts to the impact and moves in a clearly recognizable manner.

\section{The Diagnosis of Twin Pregnancy}

The diagnosis of twin pregnancy can be made only by rectal examination and is especially easy in the cow at 90 to 120 days, when the entire uterus may generally be palpated, as well as the ovaries. At this time, if the twins are bicornual, as is the rule, the diagnosis is very simple. The two horns are equal in size, and, as discussed in the following paragraph, there is a corpus luteum of pregnancy in each ovary. With all cardinal symptoms of pregnancy present, the two elements mentioned make the diagnosis very reliable. Later, when the uterus becomes greatly enlarged and passes far forward in the abdomen, the diagnosis is more difficult and less secure. The corpora lutea cannot then be felt, as the ovaries are out of reach and the uterine horns cannot be well compared. The diagnosis of twins must then 
rest upon the palpation of two fetuses, and there is no certainty whether they are to present alike or alternately. Generally they alternate; one presents cephalically and the other caudally. In campylorrhachis and schistocormus reflexus, however, we meet with a single fetus presenting both by its cephalic and caudal ends. Double monsters may also be met, confusing the examiner. In unicornual twins, the examiner faces great difficulty. Almost, if not always, they lie end to end-either head to head or tail to tail-causing an enormous elongation of the gravid horn which reaches, at the close of pregnancy, double the length of the antero-posterior diameter of the abdominal cavity, necessitating the doubling of the uterus upon itself, as in the bitch. Then the fetuses lie side by side, with two uterine walls between them and their contact ends lying against the diaphragm of the mother. Their free ends-two heads or two tails-lying side by side, are directed toward the pelvis. The basal fetus presents on a line with the long axis of the cervix; the fetus occupying the ovarian end of the horn lies lateral to the basal fetus, on the side of the ovary of pregnancy. That is, if unicornual twins are in the right horn, the basal fetus presents with its head or tail towards the cervix, and on the right of this lies the other fetus, presenting by the same part but lying to the right of the cervix. The ovary may now be pushed toward the pelvis and its two corpora lutea should be palpable. On the left the nongravid horn would be palpable. In one cow about 60 days pregnant, unicornual twins were quite securely diagnosable by the presence of two typical corpora lutea in the ovary on the pregnant side. That does not make twin birth necessary. One embryo may perish and be absorbed but the diagnosis is still important because the other embryo may be a "freemartin", or neuter, though born singly. If a good diagnosis of twin pregnancy has been made, a single birth follows, and the offspring is apparently a female, its sex may well be doubted. 


\section{Diagnosis of the Duration of Pregnancy}

It is highly important that the veterinarian shall be able to diagnose with reasonable accuracy the duration of an existing pregnancy. As already related, the heifer or cow sometimes comes in estrum and is re-bred perhaps repeatedly after conception. It is then desirable to be able to state, approximately, the stage of pregnancy in order that it may be determined at about what period parturition may be anticipated. When heifers or cows are bred in pasture so that the date of conception is practically unknown, the determination of the duration of pregnancy is at times sought by the owner. In pedigreed cattle, when pregnant females come in estrum, the bull is sometimes changed and confusion of pedigree results, which may often be satisfactorily solved by physical examination to determine the duration. Sometimes conception occurs unexpectedly and a determination of the stage of pregnancy aids in determining how it occurred. Thus in one instance, in a cow supposed to be non-pregnant which showed no estrum, examination led me to say that she was pregnant four months. The owner was certain she had not been served, but it was finally recalled that four months previously a bull, supposed to be too young to copulate, had been with her at pasture. In another instance a pure-bred Holstein cow purchased a year previously as pregnant six months, had not calved, had not been in estrum so far as known, and, according to history, had at no time been where a bull could copulate with her unknown to the custodian. In addition, her vagina contained a quart or more of highly fetid pus. In spite of such a history, I pronounced her pregnant six months and the fetus alive. She calved true to diagnosis. When the custodian saw clearly that the cow was to calve soon, he recalled that she had, at the date indicated, escaped to the premises of a Shorthorn breeder, though it was not known that she had copulated with the Shorthorn bull.

During the first five or six months, the stage of pregnancy in cattle is to be determined by the transverse diameter of the pregnant horn of the uterus. During the latter 
stages, the duration is to be determined by the volume of the palpable part or parts of the fetus, the volume of the uterine arteries, and to some extent by the volume of the uterine seal, the dilation of the cervical canal, the enlargement of the udder, and the sinking of the pelvic ligaments. In a heifer of medium size, pregnant thirty days, the gravid horn is about one to one and one-quarter inches in diameter. When forty-five days pregnant, the gravid horn has increased to one and one-quarter or one and one-half inches. At sixty days, it has reached about one and three-quarters inches; at ninety days, two to two and one-half inches; at one hundred to one hundred and twenty days, three to three and one-half inches. At one hundred and fifty days, the gravid horn is four and one-half to six inches, and at one hundred eighty days, seven to eight inches. In the cow, during the first sixty days, the uterus is somewhat larger than in the heifer, and later is not quite as tense as in the latter, but there is no recognizable difference in size.

At about one hundred fifty to one hundred eighty days, the fetus is generally palpable. Frequently it is palpable at a much earlier date even when only six to eight inches long, but recognition of the fetus at this time is not at all certain in any given case. Even when the fetus is recognized at this early date, it is of little or no advantage, as a means for determining the stage of pregnancy, over the transverse diameter of the gravid horn.

Once the fetus is palpable, its age is to be judged by the volume of the extremity palpated. The length of the fetus cannot be determined. The parts usually palpated are the head or the anterior feet when in the anterior presentation, or buttocks when in posterior presentation. The size of these will vary with the size and age of the pregnant animal and with breed. The Holstein fetus is the largest commonly encountered and has large head and feet. The Jersey presents the other extreme. The examiner is to bear in mind individual variations in size of fetuses also. The determination of the stage of pregnancy by physical examination is evidently only approximation but, with the breeding history, 
may be rendered very exact. Thus when a cow or mare has been bred but twice with an interval of two to four months between and the two dates definitely known, the veterinarian can then positively state by which service the animal is pregnant.

Depending upon the value of the animal and the skill of the veterinarian, the rectal palpation of pregnant animals may be made of wider use to breeders. The trained human obstetrist examines his patient at intervals during her pregnancy in order to determine if all is well and, if possible, to recognize any impending danger to mother or fetus. So in animals there is an interesting and useful opportunity for service. Thus in the cow with torsion of the uterus, to which reference was made above, the statement is quite justified that this examination of an apparently well cow saved the lives of herself and fetus. In mares and cows the posterior presentation of the fetus is as a rule a definite peril. In many instances definite assistance is required during parturition. If the presentation were known in advance, close watch might be kept and veterinary aid more promptly summoned. Perhaps one of the most striking instances where the diagnosis of the presentation of the fetus would prove of tremendous value to the breeder, is in the transverse or bicornual development of the fetus in the uterus of the mare. In the commonest of these, the ventral transverse presentation, virtually every foal is lost, since the sacrifice of its life in the performance of embryotomy is essential to any hope for the life of the mare. Nevertheless, the mortality in mares from this presentation is appalling. When the condition which I have described in Veterinary Obstetrics as "compound rotated bicornual pregnancy" is encountered, no foal or mare, so far as recorded, has emerged from the ordeal alive. It seems to me that the most hopeful outlook for such cases would be to diagnose them as early as midterm, and either bring about artificial delivery while the fetus is yet small, or resort to Caesarian section some time during the eleventh month. 
There lies before the veterinarian an interesting, and as yet wholly undeveloped field of usefulness in watching pregnant animals and guarding them against important perils.

\section{Palpation of the Fetus per Vaginam}

Palpation of the fetus per vaginam generally succeeds only during the last months of pregnancy. At this time, in many cases, some portions of the fetal body, usually the head and two anterior feet, rest upon the vagina posterior to the os uteri externum, occasionally giving the inexperienced examiner the impression of extra-uterine pregnancy.

\section{Abdominal Ballottement}

After the sixth month of pregnancy, in the cow, the buttocks or other portions of the fetus usually lie in close contact with the abdominal floor of the lower right flank region. If the hand is placed firmly against the abdominal wall, a somewhat vigorous thrust made upward, and then suddenly the force released, but the hand kept in contact with the parts, the adjacent portion of the fetus is pushed upward in its fluids, floats for a moment, and then drops back against the examiner's hand with a recognizable impact. This is a good sign of pregnancy, but not always reliable. Sometimes no portion of the fetus chances to lie in sufficiently close contact with the abdominal wall to render the test efficient. On the other hand, a tumor or a very large and heavy intestinal calculus might mislead the examiner when the animal is actually sterile.

\section{Auscultation of the fetal Heart}

In the advanced stages of pregnancy it is frequently possible, by careful auscultation of the abdominal walls over the region of the fetus, to detect the beat of the fetal heart, which is usually at least twice as rapid as the beat of the maternal heart.

The abdominal ballottement and the auscultation of the fetal heart are of minor value to the skilled veterinarian, because when these can be successfully applied the other signs 
which have been enumerated should have decided the question long before.

\section{The Abderhalden Reaction}

Abderhalden ( ${ }^{1}$ ) announced in 1912 the discovery of a highly interesting method for diagnosing pregnancy by a laboratory test. A test fluid is made from placental tissues, to which is added serum from the animal to be tested, and the mixture is placed in a dialyzing tube. If the animal is pregnant, portions of the ferments existing within the blood serum dialyze into surrounding distilled water, in which they may be recognized by a delicate purple coloring, upon the addition of ninhydrin followed by heat. It is claimed that the placental ferments appear in the blood as early as eight days after conception and disappear within fourteen to twenty-one days after the expulsion of the placenta. In the larger domestic animals it is more than eight days before any placental tissue commences to form. It is not easy to understand how a test for placental elements can act until placental tissues exist. The statement that a reaction may occur as early as at eight days is probably based upon researches upon some small rodent, where the placenta develops much earlier.

Since the preparation of the test material and the test itself are very complex, requiring delicate laboratory apparatus and highly technical skill, the test is expensive as compared to its value in the veterinary field. Even in human obstetrics and gynecology, where the cost is not as great an element and where an accurate physical examination is not nearly as practicable as in the mare and cow, the test is not regarded by many of the foremost authorities as efficient. J. Whitridge Williams(") states, "While I consider that the method should be regarded as one of the important recent contributions to medicine, I do not believe that it is destined to come into extensive practical use for several reasons: First, its technic is so complicated that it can be

${ }^{1}$ Die Serodiagnostik der Schwangerschaft, Deıtsche med. Wochenschr., I9I2, No. 46.

2 Obsteirics, 1917. 
satisfactorily employed only by especially trained laboratory workers. Second, even its most enthusiastic advocates admit that the results fail to correspond with the clinical findings in 5 to 10 per cent. of cases, and thirdly, that it is available only in normal pregnancy, as it gives uncertain results in inflammatory conditions and uterine myomata, or when the pregnancy is complicated by nephritis or toxaemia."

Graves $\left({ }^{1}\right)$, speaking of this test, states, "Opinions differ as to the present value of the Abderhalden test for pregnancy, some regarding it as too inaccurate to be of much clinical value, while others consider it reliable and practical (McCord). The test requires much skill and care, there being numerous chances for error. For that reason it has not yet come into general practical use."

Thus for woman, where accurate diagnosis is most important, leading authors either condemn the test as unreliable or seriously question its value in the present state of knowledge concerning it.

Zell (') concludes that the test is very reliable, but when questioned admits that the serum of a cow may react for twenty or more days after abortion, that many tests are valueless because of slight errors in drawing the blood or decanting the serum, and that various diseases nullify the value of the test. Finally, he admits that the test is not at all sufficient or complete within itself and must always be considered in conjunction with-not as a substitute forphysical examination. In 78 clinical cases summarized, the test was erroneous in 5 animals-or 6.4 per cent. Any fairly good diagnostician can be more accurate by physical examination. Some have claimed a place for the Abderhalden test on the ground that, in the cow and the mare, physical diagnosis is impracticable in the first half of pregnancy and in the second half physical examination imperils the pregnancy. Neither of these assumptions is true Should the test become simplified and its accuracy be ad-

${ }^{1}$ Gynecology, Graves, Wm. P., 1917.

${ }^{2}$ C. A. Zell, The Abderhalden Test for Pregnancy in Animals, Jour. Am. Vet. Med. Assu., Oct., 1917. New Series Vol. 5, No. 1, page 39. 
vanced, it may prove valuable in sheep, goats, swine, and carnivora, where rectal palpation is not practicable, but in these a diagnosis is rarely of sufficient importance to warrant the cost.

It is claimed that the test is highly valuable in cases of sale and warranty, but at the same time it is admitted that the blood of a cow which has recently calved, and presumably also of one which has recently aborted, gives a positive reaction for a somewhat indefinite period. Accordingly, even though a test is positive and no error in technic has been made, the Abderhalden test can at best show only an alternative result-that is, the animal either is or has recently been pregnant. Just how recently, we do not know. In this respect it is inferior to physical diagnosis, because the latter shows that the animal is actually pregnant at the time of examination and in many cases gives valuable information as to the safety of the pregnancy.

In another direction the Abderhalden test is very defective. In examining cows for sterility, the examiner must decide upon the spot whether a cow is pregnant. In many cases the whole value of his work in relation to sterility depends upon a prompt and accurate diagnosis without resort to laboratory methods, with the accompanying delays and difficulties. For example, when called to examine a cow which has proven sterile, at about the time for a return of the estrual period, the practitioner needs to make a prompt and accurate diagnosis and apply the proper handling immediately. If he cannot make a diagnosis and take prompt action, if the cow is nearing estrum, he must lose one period without having made any material progress. At present it seems highly improbable that the Abderhalden test for pregnancy is destined to attain a prominent place in veterinary medicine.

The physical diagnosis of pregnancy is not dependent upon any one sign, but rather upon a summary of all signs, all of which are in close accord and are available to the careful examiner. We have dealt with these individually. They should always be considered individually, and later 
collectively. For example, if an antimal has been bred for thirty days, if estrum has not recurred, if the uterine seal is present and definite, if one of the horns is larger than the other, and on the same side with the enlarged horn there is a typical corpus luteum of pregnancy, the cow is pregnant, and the diagnosis is as accurate and secure as any diagnosis which we can make. Other signs exist which may aid us and add to the security when any one of these fundamental signs is absent or in some manner clouded. With experience and care, any veterinarian can learn to make a safe and accurate diagnosis of pregnancy by physical examination.

Certain other signs of pregnancy frequently relied upon have a minor value. One of the commonest physical signs of pregnancy is a change in the volume and form of the abdomen. However, this is not in itself dependable. Many non-pregnant animals have greatly enlarged abdomens, and many in advanced pregnancy show but little enlargement of the abdomen. In a heifer, gaunt from advanced tuberculosis and believed non-pregnant, I diagnosed twins, to which she gave birth a few weeks later.

The enlargement of the mammae is generally a reliable sign of advanced pregnancy, but some cows do not show marked enlargement. In other cases sterile heifers lactate freely. I saw one, which was not and had not been pregnant, yield twenty pounds of milk daily.

\section{Confusing Findings in Examination for Pregnancy}

While engaged in rectal palpation, one sometimes encounters conditions which confuse the examiner, at least temporarily.

a. The left kidney, in all cows, is displaced somewhat to the right and appears quite pendulous. It is much larger in the living than in the dead animal. It contains a great volume of blood. In some cases, it hangs very pendant, and an inexperienced veterinarian, coming in contact with it for the first time, may for a moment think it a tumor. 

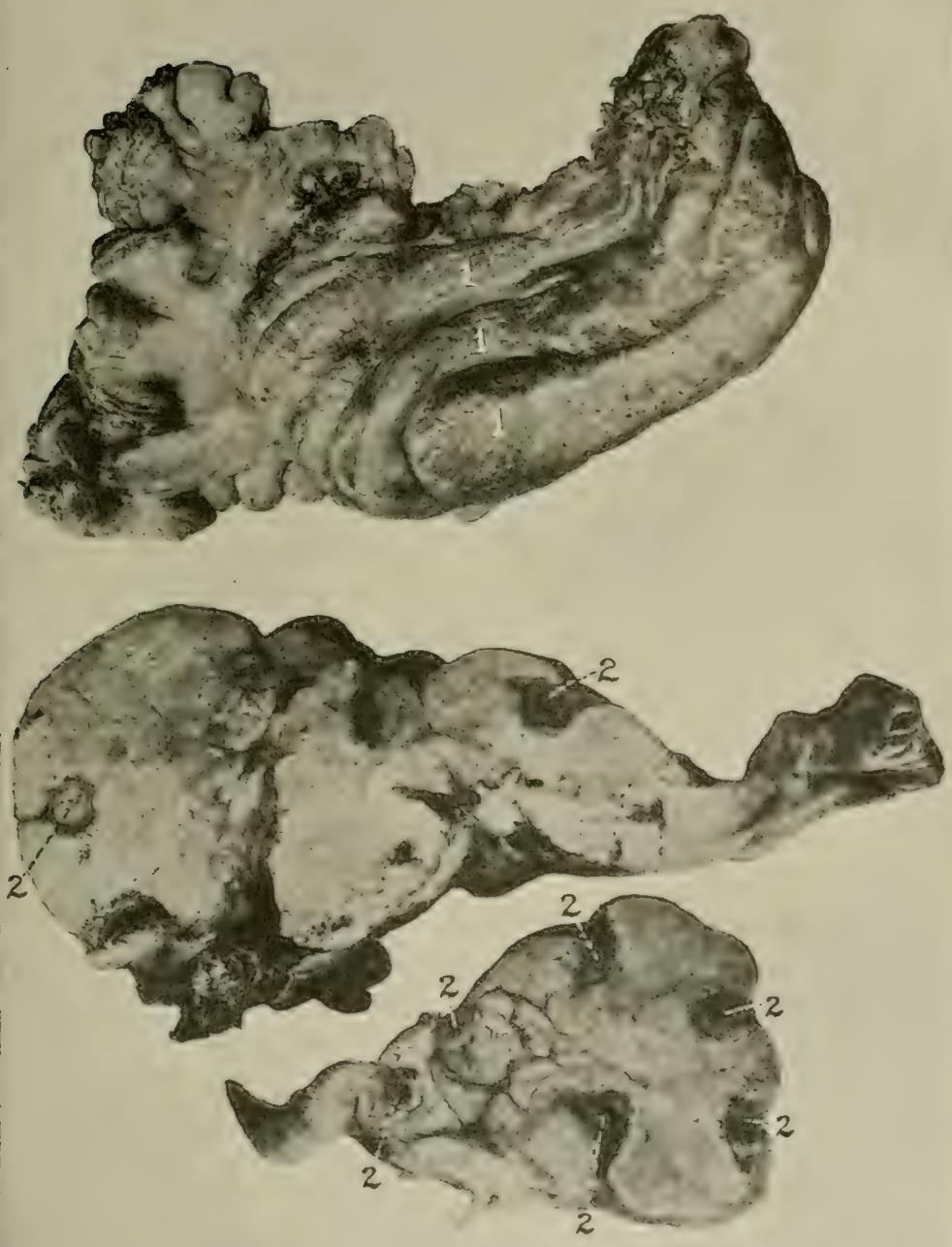

FIG. 46. Lipoma of Mesenteric Fat of Large Intestine. Cow. $I, I, I$, Large intestine ; $z, 2,2$, cross sections of loops of large intestine, surrounded by fat tumor. 
b. Mesenteric fat tumors of immense size occur now and then, chiefly in the mesentery of the large intestines, as seen in Fig. 46. I have seen these mostly in Guernseys and Jerseys. They consist of great masses of necrotic fat, hard, marbled, and iridescent on section. They are quite movable in the abdomen. In my first case, I was greatly confused, and suspected that I had to do with an extra-uterine fetus. It was too far forward to permit of complete palpation. There is no occasion for such error. In extra-uterine pregnancy the fetus is firmly moored by adhesions to the abdominal floor. While I have no definite history, my museum specimens show the fetus, or fetuses, to be firmly encapsuled by adhesions, so that they are compressed into the narrowest space possible, but where and how they were attached I have no history. When the examiner is on his guard, the mesenteric fat tumors have in my experience admitted of ready diagnosis. Their relation to the intestines constitutes the chief guide. I have seen them in old, or at least adult, cows only. They may be of any size. In one case the entire abdomen appeared to be filled with these.

c. Dislocation of the rumen is also a very disconcerting condition when first met. In one cow, to all external appearances in perfect health, the rumen was extremely dislocated. The right sac had glided across the abdominal floor and had come to rest against the right abdominal wall, above the level of the pubis. The left sac of the rumen had departed from the left sub-lumbar region and dropped down until it lay only slightly above the pubis. The massive organ thus lay on the abdominal floor. Its normal left wall had become its floor; its right wall faced upward. The rumen occupied the entire abdominal floor in front of the pubis, and projected somewhat above it. The soft, pultaceous rumenal contents were readily recognizable. The organ could be clearly distinguished and traced. The genital organs, uterus, and ovaries passed directly forward and lay upon the dislocated rumen. The dislocation is apparently of no consequence, except for the confusion it may cause during rectal palpation. 
d. Extensive pyometra has caused colleagues of considerable experience to diagnose pregnancy. In one instance, pregnancy had been diagnosed in a well-bred cow. She was sold at a high figure and shipped a long distance. She was finally returned by the purchaser at heavy cost. When I examined her, I siphoned out 3 to 4 gallons of a thin, very fetid pus. In another instance, a heifer purchased as pregnant, having gone beyond her supposed duration of pregnancy, was examined and a macerating fetus diagnosed. I siphoned out about 13 liters of a very thin, odorless, yellow pus. In each case there was no observable purulent discharge, though the cervical canal was freely open. The uterus lay far forward (neither colleague had applied forceps and drawn the uterus back) and, finding it distended to the volume of six months or more pregnant, the colleague in each case erred and diagnosed pregnancy. The uterus was too flaccid for pregnancy and its walls were too thick. When traction was applied and the uterus drawn backward and upward until the entire organ was in reach, it was perfectly clear no large fetus or fetal cadaver was present.

It is true that often the gravid uterus drops far downward and forward, especially in old cows with pendant bellies, but fetal cotyledons are in reach even if the fetus is not. The veterinarian should also seek evidence from the uterine arteries. Pyometra cannot cause an enlargement of the arteries at all comparable with the enlargement of pregnancy.

e. Cystic Uterus. The uterus of the cow occasionally becomes cystic and causes the veterinarian to err in diagnosing pregnancy. There may be five to ten gallons of lymph or thin mucus in the uterine cavity, the organ dropped far forward and very flaccid. There is no recognizable discharge. The cervical canal is usually free.

In another group of cases, there is a blocking of the cervical canal owing to one of the annular mucous folds acting as a valve to retain menstrual debris. This causes a tense filling of the uterus which is much like pregnancy, but the uterine seal is ordinarily absent, there is probably no corpus luteum typical of pregnancy, the arteries are not enlarged, and the two uterine horns are distended alike. 


\section{ARTIFICIAL INTERFERENCES WITH REPRODUCTIVE FUNCTIONS}

\section{A. The Artificial Induction of Estrum}

The artificial induction of estrum has a wide application in cattle breeding and in dairying, but has not been rendered available in other species of animals.

Aphrodisiacs. Yohimbin Spiegel. For many years numerous drugs have been credited with the power of arousing the sexual appetite and powers. Among these are cantharides, turpentine and the balsams and resins and, more recently, a new drug, yohimbin, has been introduced to overcome sterility. In large doses, these drugs induce more or less irritation of the urino-genital tract and, apparently, stimulation of the sexual desire.

It has not been shown that aphrodisiacs stimulate ovulation and spermatogenesis. From all data at hand, they merely arouse the sexual appetite without increasing the reproductive powers. In the minds of some, vigorous sexual desire signifies exalted breeding powers, but this is a false assumption. In nymphomania, for example, the sexual appetite is intense and the breeding power nil. Sexual appetite, without normal ovulation in the female and the formation of normal spermatozoa in the male, has no value, but is rather a harm, frequently leading the owner astray and diminishing the value of the patient for other purposes.

A few years ago vigorous propaganda brought into notice yohimbin spiegel as a magical sex tonic and a great weapon against sterility. The pathology of the cases where it was alleged to give relief was not recorded. It was used almost wholly in females which failed to come in estrum, but why they failed no intimation was given.

Holterbach (Berlin Tierärztliche Wochenscrift, No. 9, 1908), in an exhaustive article, champions the use of the drug. He used it in 37 cases of absence of sexual desire- 
22 cows, 9 bitches, 3 bulls, and 3 sows. In his observation, the drug has a greater affinity for the female than for the male genitalia. A five-year-old Simmerthal cow calved normally in June, 1906, but estrum did not follow. All other drugs, including cantharides, proved useless. In August, 1907, fourteen months after calving, 16 tablets of yohimbin chloride, each containing 0.1 gram of the alkaloid, were prescribed, one tablet to be given three times daily in the drinking water. On the third day there was excitement, bellowing, uneasy stepping about, frequent urination and decreased milk flow. On the fourth day there was a mucosanguineous discharge from the vulva (menstruation?) and on the fifth day the owner noted expulsive efforts resembling labor pains. The external genitals were swollen and hyperaemic, but no estrum was present. The uterus was large. The ovaries were enlarged, but not cystic. Six weeks later, the cow, showing violent estrum, was bred and the owner "believed" her in calf.

Another cow, nine months post partum, without estrum, and having cystic ovaries, was treated in the same way, developed estrum ten weeks later and was bred. The owner "believed" her to be pregnant.

So far as can be seen from Holterbach's reports, the evidence is not convincing. In no case was the cause of nonestrum explained, and no proof was submitted that the estrum, following the use of yohimbin, was due to the drug.

Frubinger (D. M. W. No. 7, 1907) condemns the drug as worthless and attributes the alleged cures in man to suggestion.

Müller (Arch. Internat. Phar. et de Therap., Vol. XVII, p. 81) considers it an erratic aphrodisiac, acting differently on different individuals. Daels, quoted by Holterbach, experimentally caused menstruation in bitches already in estrum, but bitches usually menstruate at this time anyhow. In another, not in estrum, but date of probable normal estrum not named, menstruation occurred in four days. A third bitch showed a mucous discharge. Later, bloody feces were voided and the animal died from the toxic effects of 
the drug. Autopsy showed enlarged, congested genital canal, prominent ripe ova, etc.

In experiments on young bitches less than nine months old, the drug, in fatal toxic doses, failed to cause reaction in the genital organs.

I used the drug experimentally upon several cows in which I had otherwise failed to induce estrum, but, like other efforts, it was without avail. In one cow there were ovarian adhesions; in another, cystic degeneration of the ovaries, etc. The drug has dropped out of notice except for an occasional medicine vendor who adheres to "patent medicine" ideals. The failure of an adult, non-pregnant female to come in estrum at the proper time is due to some pathologic condition, the removal of which is followed by estrum. But the removal of the cause of non-estrum must be the basis for action in each case. These pathologic conditions will be discussed in their proper places. In some cows estrum is not very evident, or the keeper fails to recognize it. The animal may be healthy or unhealthy. It is important in some healthy cows to get definite track of the estrum. If, upon the examination of an animal in which estrum is not observed, the general health is good, the genital organs normal, and a corpus luteum present, it is sufficiently clear that at some former date the animal had been in estrum and had ovulated. If the corpus luteum is dislodged by compressing it per rectum, the animal will quite certainly be in estrum in three or four days.

In some cases it is desirable to interrupt the estrual cycle because several females are in estrum simultaneously and the service of all would overwork the herd bull. The veterinarian can, by dislodging the corpus luteum, adjust the estrual cycle at will.

Healthy cows sometimes fail to ovulate promptly after parturition, thereby causing a delay in re-breeding. When a cow does not show estrum within sixty days after parturition, if she is healthy, the corpus luteum of pregnancy is probably tardy in disappearing. It then becomes of importance to the dairyman that the yellow body shall be dislodged and the cow caused to come in estrum. 
The veterinarian should constantly remember that estrum and ovulation come as a matter of course in healthy females of breeding age and that when estrum fails it is because of general ill health or some other restraining cause. No drug has yet been discovered which can directly cause estrum or ovulation. The restoration of the general health, relief from distinctively genital diseases, and the surgical dislodgment of the inhibiting corpus luteum, when present, constitute the fundamental principles in regulating estrum and ovulation.

\section{B. Artificial Insemination. Artificial Impregnation}

Artificial insemination or impregnation has been advocated by a limited number of veterinarians and breeders. The advocacy has been based upon various hypotheses and facts, but the operation has not come into extended use. At present there are no indications that the scheme will ever occupy an important place in livestock husbandry.

Artificial insemination is not difficult and there can be no question of its efficacy in fertilization. It has been advocated in two distinct fields-to conserve the sexual powers of valuable sires by causing numerous fertilizations from a single coitus and also to insure fertilization in a female otherwise sterile.

The fertilizing of many females by a single coitus is of course technically possible, and has appealed very strongly to some owners of fashionable sires. Technically, in all animals there are millions of spermatozoa ejaculated at each coitus, of which but one can take part in the fertilization of an egg, and the millions of others must perish. Any attempt to conserve these millions of spermatozoa, when from a valuable sire, has much in it to appeal to some owners, and comparatively simple means are at hand for carrying out the operation. The prime essentials to possible artificial fertilization are that the spermatozoa shall be obtained promptly after ejaculation by the male, kept moist, the temperature maintained at approximately that of the body (100 to 105 degrees, F.), and introduced into the genital 
canal of the female with sufficient promptness that the spermatozoa shall have lost none of their vigor. In large animals (horse and cow) semen may be collected immediately after coitus by introducing the hand into the vagina, using it as a ladle to gather up and withdraw the contents, and may then be placed in a wide-mouthed bottle or other vessel, immersed in water at a temperature of $100^{\circ}$ to $105^{\circ}$ F. The semen may then be diluted, if required, by adding sterile physiologic salt solution of body temperature. Diluted or undiluted, the semen may be introduced into the vagina, cervical canal or uterine cavity of another female which it is desired to fertilize. The introduction may be made with a syringe, a funnel fitted with a tube, or other apparatus.

Spermatozoa have been kept artificially in a motile state for many hours. In the healthy genital tract they presumably retain their motility for days. Motility of the spermatozoa is not proof of fertilizing power, and no satisfactory data are at hand indicating how long after ejaculation spermatozoa retain vigorous fertility. It is probably for a few hours only when in the healthy genital tract, where the spermatozoa unquestionably live longer than when kept outside the body. In mares and cows, rape is occasionally induced by the breeder, under the belief that it will cause estrum to appear soon. In other cases, the female has been in estrum, which, before opportunity for coitus has occurred, ceases and the owner hopes by belated coitus, under compulsion, to secure fertilization. Rape virtually always, if not always, fails of results. It is reasonable to assume that artificial insemination can be no more productive of results than rape. Mule breeding is based upon approximate rape, but the resistance of the mare is not because she is not in estrum but owing to her repulsion to coitus with the ass. It appears that artificial insemination must, therefore, approximate the essentials of physiologic coitus. The spermatozoa need to be transferred promptly, during estrum. The suggestion that semen may be transported long distances and used successfully should be discounted liberally until 
further evidence is adduced. The allegations of some enthusiasts that artificial insemination succeeds whether the female is in estrum or not is without supporting evidence and appears absurd.

Artificial insemination has numerous objectionable features. So far as I am aware, no livestock association officially recognizes such fertilization for purposes of record of pedigree. The moral hazard in recording pedigrees is already great and artificial insemination would open a new avenue to fraud.

While the millions of spermatozoa ejaculated at each coitus are technically capable of infinite fertilizations, some are dead and some defective from the first, and most of them succumb to bacterial or other injury while en route from the vagina to the ovary, so that their numbers are greatly reduced before they have reached the pavilion of the oviduct. As soon as the spermatozoa are apportioned between two or more animals, the certainty of fertilization decreases. It is clearly impossible to keep the spermatozoa as well outside the body as within the genital tract, and slight error in technic may destroy completely the fertilizing power.

The chief menace from artificial insemination is the transfer of infection. In natural coitus, when the semen is ejaculated into the vagina, there may be present infection from both the male and the female genital organs. Most infections, so far as known, are non-motile. This affords opportunity for the vigorously motile spermatozoa to move away from the infection, elude the dangers, and effect fertilization. Semen taken from the vagina of one female contains the bacteria from herself and the male and, when introduced into the vagina of a second female, carries for her a double danger. If, as is generally advocated, the insemination is made intra-uterine, the danger from infection is greatly increased.

Artificial insemination has also been advocated as a remedy for sterility. When first proposed, atresia of the cervical canal was considered one of the chief causes of 
sterility. Had that been true, the plan would have had merit. Later developments show clearly that cervical atresia is essentially negligible as a cause of sterility. Artificial insemination has, therefore, only a very limited and questionable place in the treatment of sterility. Diseases of the vagina, cervix, and uterus which might cause sterility are more readily remedied by other means than by artificial insemination. Diseases of the oviducts and ovaries cannot be favorably affected by the operation. Artificial insemination may have some value under exceptional conditions in extending the breeding range of highly valued sires, and in very rare cases may possibly have value in sterile females.

\section{Artificial ABORTION}

Occasionally artificial abortion in animals becomes desirable in the economic interests of the owner. When highly pedigreed females are accidentally permitted to copulate with common males or with pedigreed males of a different breed, the progeny is valueless and the span of the pregnancy becomes a loss to the owner in so far as the production of valuable progeny is concerned. Sometimes, also, a heifer calf of five to ten months unexpectedly comes in estrum, copulates with a bull, conceives, and, unless the pregnancy is artificially interrupted, is much damaged by repression of growth and development.

No drug is known which is capable of causing a pregnant animal to abort. Ergot and other drugs have been cited as ecbolics or abortifacients, but there is no reliable evidence that any one of them is competent to cause abortion directly. Ergot, given repeatedly and in large doses, finally poisons the pregnant animal, which, just prior to death, may abort. Such abortion is probably indirect; the toxic effect of the drug, by lowering the resistance of the body, may enable the infection present in the uterus to acquire new power and precipitate abortion.

It is not known that any form of bacteria, living or dead, or any of their toxins or other products, possesses any direct ecbolic power, if by this term is meant an inherent 
power to cause contraction of the uterus with the expulsion of its contents.

Many veterinarians and research workers with the $B$. abortus of Bang believed for some years that this organism had the power to act directly as an abortifacient. Professor Bang made no such claim, so far as I have found, but instead contended that the abortion was the result of an endometritis for which he believed the $B$. abortus responsible. If the $B$. abortus could cause abortion by directly acting upon the central nervous system, it should be able to do so when the bacillus is multiplying elsewhere than in the uterus or fetus, but in all cases, when the aborter is promptly destroyed, metritis is present and bacteria are recognized as the cause of the metritis. Hence, so far as known, abortion can not be caused artificially by means of toxins or other bacterial poisons.

Artificial abortion is quite certainly induced by breaking down the uterine seal, invading the uterus, and douching the cavity. The earliest date after copulation at which abortion can be induced by uterine douching is undetermined. Douching the uterus with a disinfectant immediately after copulation is quite certain to destroy all spermatozoa and prevent fertilization, but after the lapse of a brief time, certainly less than an hour in the cow, the spermatozoa, having passed into the oviducts, are beyond reach. Marshall, citing Hensen, states that in the rabbit the spermatozoa travel from the vagina to the ovary in from fifteen minutes to two hours. If that be correct, it is well-nigh impossible to douche the uterus early enough after copulation to prevent fertilization. Marshall states that the fertilized ovum of the rabbit enters the uterus at about three days after fertilization. During this interval uterine douching can not interrupt pregnancy. In the larger domestic animals, the migration of the fertilized ovum from the pavilion of the oviduct into the uterus is believed to be slower than in the rabbit. Marshall ( $\left.{ }^{1}\right)$ gives the duration of migration in the dog as eight to ten days. During this

\footnotetext{
${ }^{1}$ Marshall, A. Milnes, Vertebrate Embryology, IS93, p. 47 I.
} 
interval, or about 16 per cent. of her span of pregnancy, the bitch can not be aborted by uterine douching.

Bonnet believes that in domestic animals generally the tubal migration of the fertilized ovum requires eight to ten days. The period is certainly no less, and I suspect that in the cow it is longer. In one cow, which had long been sterile, I douched the uterus freely with 2 per cent. Lugol's solution thirteen days after conception, without inducing abortion. There was moderate cervicitis present, and I douched from the cervical end of the uterus some mils. of pus, so that I possibly prevented, instead of caused, abortion. So far as known, artificial abortion in the cow can not be brought about by uterine douching until at least eight to ten days after conception, or, judging from my single experience. not until an even later date. There is greater assurance of success if douching is deferred until about the twentieth day.

In douching the pregnant uterus, the veterinarian needs to be quite cautious. The catheter must be introduced very gradually and carefully through the cervical canal, since the very adhesive uterine seal dulls the sense of touch and renders mechanical injury possible. When the catheter has entered the uterus and douching is attempted, the instrument generally becomes blocked with portions of the uterine seal and no safe distension of the uterus with fluid will force out the sticky mass. The connecting screw between the outer and inner tubes needs to be loosened and the inner tube drawn back and forth until the plug of the uterine seal is dislodged and washed away.

The amount of fluid introduced should be noted very carefully, and great care taken not to use too much, because the pregnant uterus is very easily ruptured. In a recent publication, a veterinarian records a case of "extra-uterine pregnancy" in a cow, the uterus of which he had recently douched and ruptured, thus causing the fetus to become extra-uterine. It is to be remembered, since up to the fourth month the embryonic sac in the cow lies very loosely in the uterus, that the douching fluid pushes the maternal and fetal placentae apart. Then the detached sac, sucked into the fenes- 
tra of the catheter, prevents the return of the fluid. If the catheter is removed, the detached embryonic sac blocks the cervical canal, the distended uterine walls contract, rupture takes place, and the fetus drops into the peritoneal cavity. Therefore it is safer to introduce a few ounces of fluid only, thus avoiding the peril of rupture. The fluid should be antiseptic, because, as a rule, the uterine cavity is septic and a neutral fluid stimulates the bacteria present. The fluid introduced must not be highly irritant.

While the induction of abortion by means of the uterine douche is quite reliable in bringing about the expulsion of the uterine contents, it is neither the safest nor the best plan. Hess $\left({ }^{1}\right)$ was first to draw attention to the reliability with which abortion may be induced in heifers and cows by dislodging the corpus luteum of pregnancy. This operation possesses the great advantage over the preceding plan of causing the abortion in an approximately physiologic manner. That is, it acts directly, by removing the inhibiting power of the corpus luteum, and establishes uterine contractions closely simulating those of parturition and menstruation, without the intervention of any mechanical insult to the cervix and uterus. The abortion follows in one to three days.

The operation is easy and safe in early pregnancy. As late as ninety to one hundred and twenty days, the operator can pick up the ovary of pregnancy per rectum with one hand, carry it back over the vagina, introduce the other hand into the vagina, grasp the ovary through its roof, and compress the base of the corpus luteum firmly between the thumb and fingers until the body is wholly dislodged. Sometimes the yellow body breaks up under pressure, so that only a portion is dislodged. The operator should be careful to press it all out. Later in pregnancy, the ovary can not be carried back over the vagina. In small heifers, the vulva may not permit the passage of the hand. Then it is essential to dislodge the corpus luteum by compression from the rectum. This must be done cautiously, since the

1 Hess, Professor E., Schweitzer Archives fur Tierheilkunde, No. 6, 1906, p. $35 \mathrm{I}$. 
rectal walls are but feebly resistant and are readily injured. With patience and care, the dislodgement can be carried out per rectum though not so safely as per vaginam.

Artificial abortion is rarely advisable after the sixth month, especially for the purpose of terminating a fundamentally undesirable pregnancy. It is safer in such cases, and the loss of time is little, if any, greater to allow the pregnancy to go full term and end in parturition. Should occasion arise for inducing abortion late in pregnancy, it would be necessary to resort either to breaking down the uterine seal and douching the uterus, or to performing laparotomy, if the ovary of pregnancy has been dragged beyond reach per rectum. If the vagina is clean, vaginectomy may be performed as in spaying, the hand and arm inserted, and the ovary thus reached. However abortion is induced, once it has been completed, it is desirable to watch the progress of the case and determine by examination of the genital organs, including uterine douching, whether all is going well. 


\section{CHAPTER IV}

\section{ARRESTS AND ABERRATIONS IN GENITAL DEVELOPMENT}

\section{A. Asexual or Bisexual Aberrations}

\section{NeUters, Freemartins}

So far as known, cattle lead in the production of asexual, or neuter individuals. This may be only apparent, since in cattle, more than in any other animal, the value of the female of breeding age rests almost wholly upon its reproductive powers, and any failure to breed attracts definite attention. It may be that sheep and goats are as frequently neuter but that the condition is overlooked. In cattle the neuter is ordinarily one of a pair of twins, of which the other is a male and commonly fertile. The condition, so far as I know, has not been observed when the other twin is a female. Numerous theories have been advanced in an effort to explain the origin of freemartins, or neuters, but the cause of the phenomenon has not been made clear. While ordinarily the neuter is twin to a male, I am unable to verify the general belief that this is universal. It has been my privilege to examine clinically several neuters which had been regularly registered in official herd books and sold as heifers. The rules of breeders' associations forbid the registration of an apparent female which is twin to a bull until the animal has conceived. In some instances where the animal has not been sold, but has been bred by the party for whom the examination was made, I have had personal assurances that it was a single. Some of these cases may be due to fraud upon the part of the breeder. Other animals may be truly singles in so far as birth is concerned, but the conception may have been twin and one embryo may have perished and disappeared. I am inclined to believe that neuters rarely come from a single conception. Accordingly, when examining a neuter which has been registered and sold as a breeder, 
I always feel that fraud has probably been committed, though I believe that exceptions exist.

In the most typical individuals, no distinctively genital organs exist, but traces of both Wolffian and Mueller's ducts are present and may be clearly traced. No ovaries or testes are present, but very tiny undifferentiated sex glands may be recognizable.

Every gradation of development is encountered, from absolute neuter to complete female, but the direction of development in cattle, so far as I have observed, is always toward the female-never toward the male or toward hermaphroditism. Hermaphroditism, so far as I have noticed, is rarer in cattle than in any other species of domestic animal, but

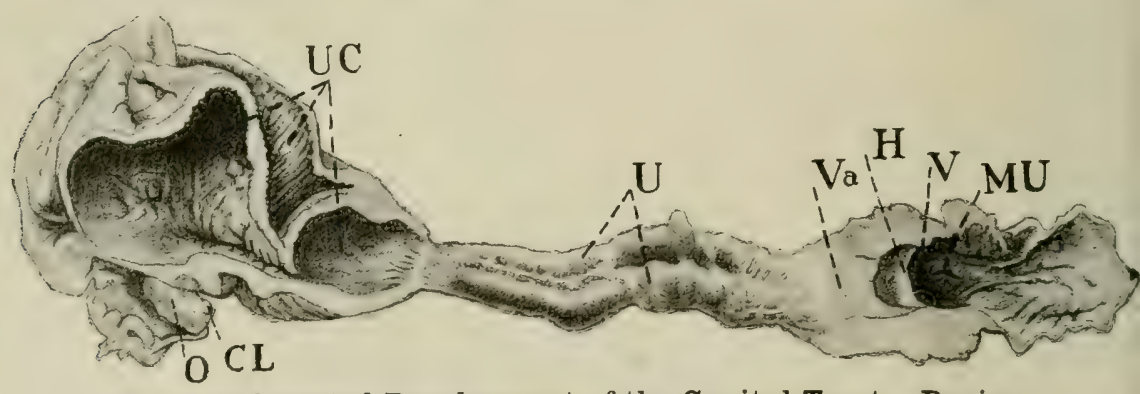

Fig. 47-Arrested Development of the Genital Tract. Bovine. $M U$, Meatus urinarius ; $V$, vulva ; $H$, hymen : $V a$, vagina ; $U$, cordiform Muellerian ducts representing the uterus; $U C$, uterine cornu, excavated and filled with fluid (menstrual debris) ; $C L$, corpus luteum ; $O$, ovary.

cattle furnish by far the greatest number of asexual individuals.

The development of the genital organs in the embryo begins at the anterior, or ovarian end and progresses toward the vulva. In the typical asexual individual, the genital glands are represented by indifferent nodes, yellow in color, about the size of an ordinary grain of wheat. Both the Muellerian and Wolffian ducts are present and have attained considerable size. They do not become tubular, but persist as solid, fibrous cords. All four structures lie on a common plane, the Wolffian lateral to the Muellerian ducts and in close contact with them. The ducts of Mueller do not fuse 
in any portion of their length, but persist as contiguous, solid cords. The first suggestion of sex specialization is generally an excavation of the Muellerian ducts at the apices of the uterine horns, as shown in Figs. 51, 52. Parallel with this there may be some suggestion of ovarian development. The next observed stage of excavation of the apices of the uterine horns is the fusion and the excavation of the Muellerian ducts in the area of the uterine body. The cavity of the excavated uterine horns or body sometimes becomes distended with lymph, usually thin and clear.

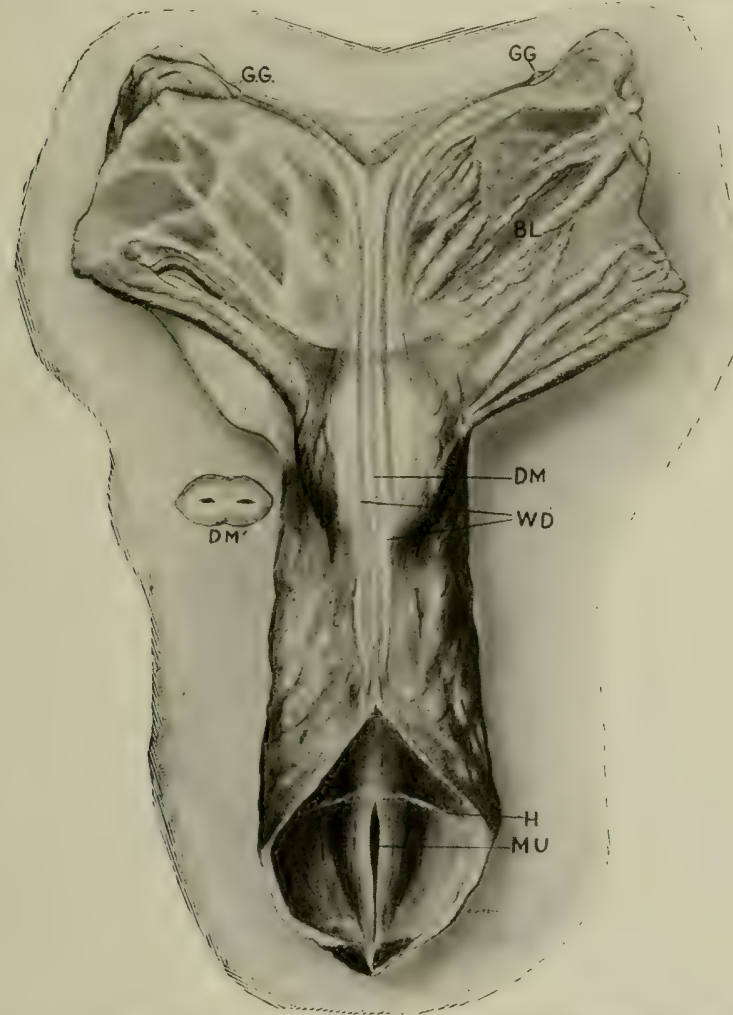

FIG. 4S-Arrested Development of Bovine Genital Tract (Freemartin.) $M U$, Meatus urinarius; $H$, hymen; $W D$, Wolffian ducts; $D N$, ducts of Mueller; $D M^{\prime}$ cross section of Mueller's ducts; $G G$, undifferentiated genital gland. The anterior ends of the Inellerian Ducts, DM, between the genital glands, $G G$, are excavated and distended with fiuid. 
Finally, in the individuals most advanced in development, the posterior portions of the cervico-vaginal segments show definite advances toward physiologic development. When this occurs, there is parallel development of the ovaries and the genital system functions more or less normally. Thus all conceivable gradations occur between the absolute neuter and the fully developed female sex system.

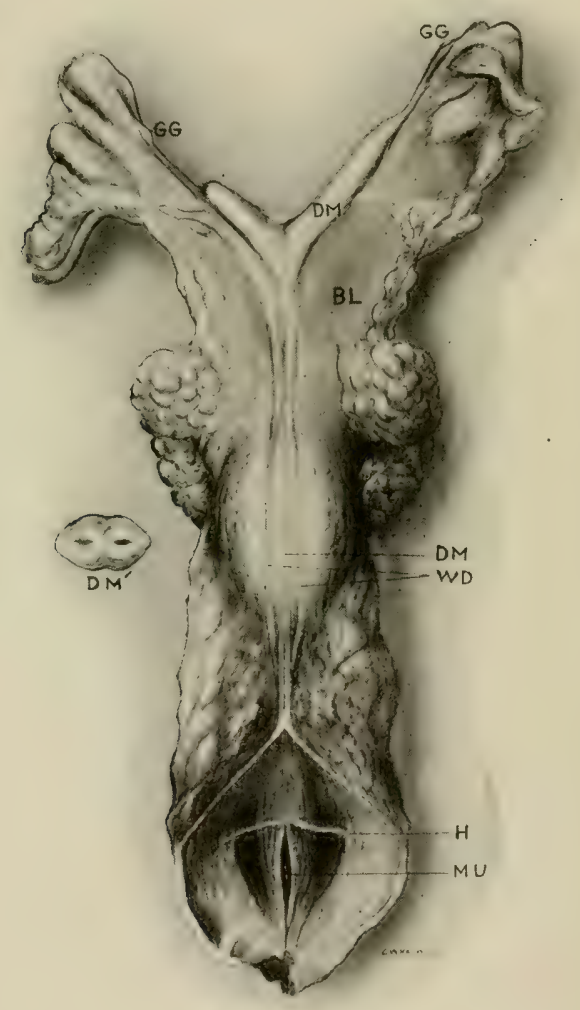

FIG. 49-Same as Fig. 48, with further Development of the Muellerian Ducts. Lettering same as in Fig. 48 , except $B L$ indicates broad ligament of the uterus and just above $B L$ at $D M$, the ducts of Mueller are excavated and distended. 
The vulva of the neuter frequently shows at birth a highly marked tuft of vulvar hairs, strongly suggestive of the preputial tuft of the bull. In the typical neuter no sexual desire develops: there is no estrum. The head and neck fail to develop that fineness of lines characteristic of the heifer and the body is generally somewhat gross. It has been stated that the head and neck are like those of the bull, but I have been unable to verify this. To me they seem more like those of a steer-a surgically made neuter. The horns are rather gross, straight, and thick.

As soon as the rectum of the animal is large enough to admit the operator's hand, a reliable diagnosis is available. Upon rectal palpation, the urinary bladder is prominent, as in the bull; there is no vagina or cervix, but instead there are two hard, fibrous cords the size of lead pencils or smaller, sometimes so closely bound together that they feel as one. In other cases careful palpation may identify all four cords, Wolffian and Muellerian ducts. The length of these cords varies greatly. Sometimes the $\bar{y}$ are as long as a normal genital tract; sometimes very short and blunt. Frequently they end anteriorly in cystic dilations given off at almost sight angles. Beyond the ends of these dilations, the operator may or may not be able to distinguish the undifferentiated tiny sex glands. In typical cases, the diagnosis is easy, and fortunately most cases are typical. In some of the atypical cases, where the sex development has progressed to a considerable degree, the diagnosis may prove very difficult. Confusion is most probable in those cases where a large proportion or all of the uterine horns and body have become excavated but the cervix remains imperforate. The uterus and horns are then distended with lymph, simulating pregnancy. As a rule, however, the examiner can recognize by palpation some fundamental defect in vagina, cervix, or ovaries which will make the diagnosis clear. The character of the distension itself, as I have observed it, serves readily to differentiate the condition from pregnancy. The uterus is flaccid and the contents mobile. The horns are irregularly distended, broad at one point and 
quite narrow at another. The fluid may sometimes be pressed from one horn to the other through the uterine body. This is in sharp contrast to the distended uterus of pregnancy, in which, if the pregnancy is healthy, the distension is firm and the contained fluids fixed in the fetal membranes. If a careful record is made of the volume of the uterus, and it is re-examined thirty days later, the uterus of pregnancy will have enlarged markedly; distension of a uterus arrested in development would have remained essentially static.

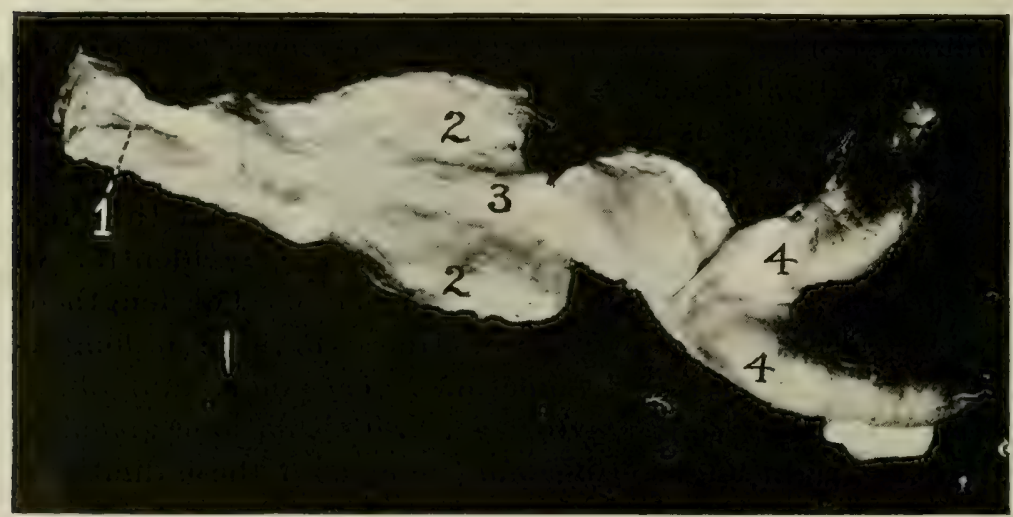

FIG. 50-Same as Figs. 48, 49. I, Meatus urinarius; 2, 2, Wolffian ducts ; 3. Muellerian ducts; 4,4 , excavated and distended anterior (ovarian) ends of Muellerian ducts.

The mole, or uterine cyst, may also confuse. Like the uterus of arrested development, the mole may remain static for months and the uterus may be flaccid. In this instance, however, the genital organs are anatomically sound, there is a corpus luteum of pregnancy, probably a typical uterine seal, and a distended uterus, static in size. It can finally be differentiated by sounding the cervical canal. The condition is discussed later.

As soon as the neuter character of the animal is recognized, it should be designated for slaughter, but whether the animal shall be kept until further grown, fattened, or slaughtered immediately will depend upon conditions. 
Forensically, if the animal has been sold as a female, the recognition of its asexual character arouses a suspicion of fraud, but by no means proves it because, as previously stated, single births are sometimes neuters, though possibly due to twin conception, the other twin having perished and been absorbed. Ethically, however, the result is the same. The animal is not a heifer or a female. Accordingly it has been sold for what it is not. If the purchaser has paid for a breeding female, he is ethically entitled to reimbursement.

\section{HERMAPHRODITISM}

Hermaphroditism, in which organs of both sexes are found in one individual, is observed in all domestic animals. Some have claimed that freemartins, or what I have designated as "neuters", are in fact a type of hermaphrodite, but with this view I am unable to agree. Neuters, as they grade up toward sexuality, tend always toward the female -never toward the male or toward both. Hermaphrodites incline to assume prevailing types according to species.

In the horse, hermaphroditism tends generally toward the development of testicles which are largely retained within the abdomen and attached and located the same as the ovaries, but may descend into an imperfect scrotum. The vulva, vagina, and uterus may develop almost perfectly, as in one case occurring in my clinic where the vulva and vagina were sufficiently developed that I castrated the animal through the vagina in the same manner as mares are spayed. The rudimentary penis, or enlarged clitoris, usually ends either in the vulva or in the vicinity of the ischial arch, with the urethral opening directed backward. Sometimes it is directed backward at its extremity. The mammae are usually moderately developed and upon casual examination, have the appearance of those of the mare. In one case upon which I operated there were, just above these rudimentary glands, small scrotal pouches containing the imperfectly developed testes.

These animals present chiefly the characters of the cryptorchid horse with all his deranged sexual desire and ten- 
dency to viciousness. In the case illustrated by Fig. 52, the head, neck, voice, and disposition were those of a cryptorchid horse, and the vulva, vagina, uterus, and udder were those of a normal mare. The erectile organ occupied a middle place between the clitoris of the mare and the penis of the horse.

In Fig. 51 is shown the generative apparatus of a pig, in which the hermaphroditism is of quite a different character;

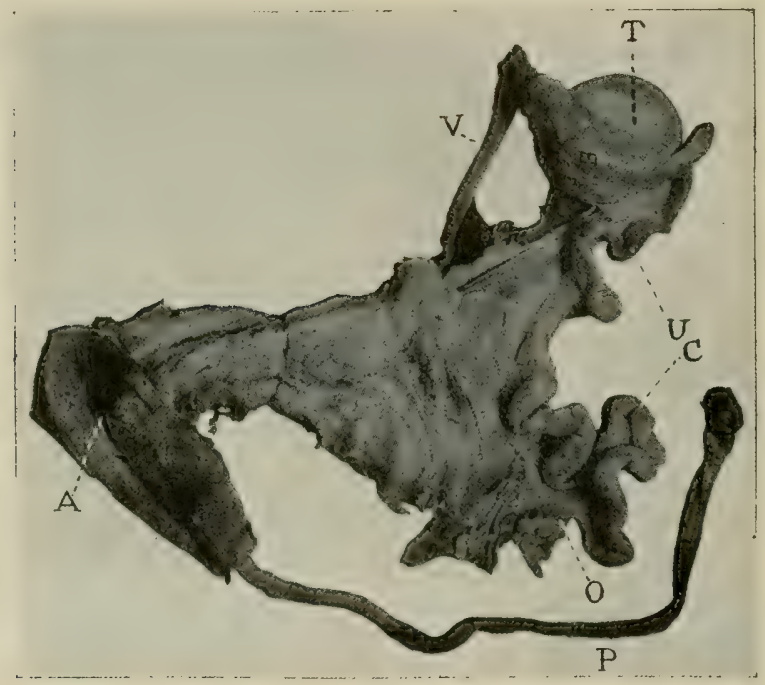

FIG. 5I-Hermaphroditism. Swine.

$T$, Testicle ; $O$, ovary; $P$, penis ; $U C$, uterine cornu ; $V$, vas deferens.

one of the glands developed into a typical ovary and the other into a testicle, so that the two sides of the genital apparatus represented the two sexes.

Sections of these genital glands show one to be a typical cryptorchid testicle with tubuli seminiferi and the other a primitive ovary with scattering Graafian follicles containing ova. The Muellerian ducts have developed into typical oviducts, uterus, and vagina. From the Wolffian ducts have formed typical vasa deferentia. The penis is normal in form and extent. 


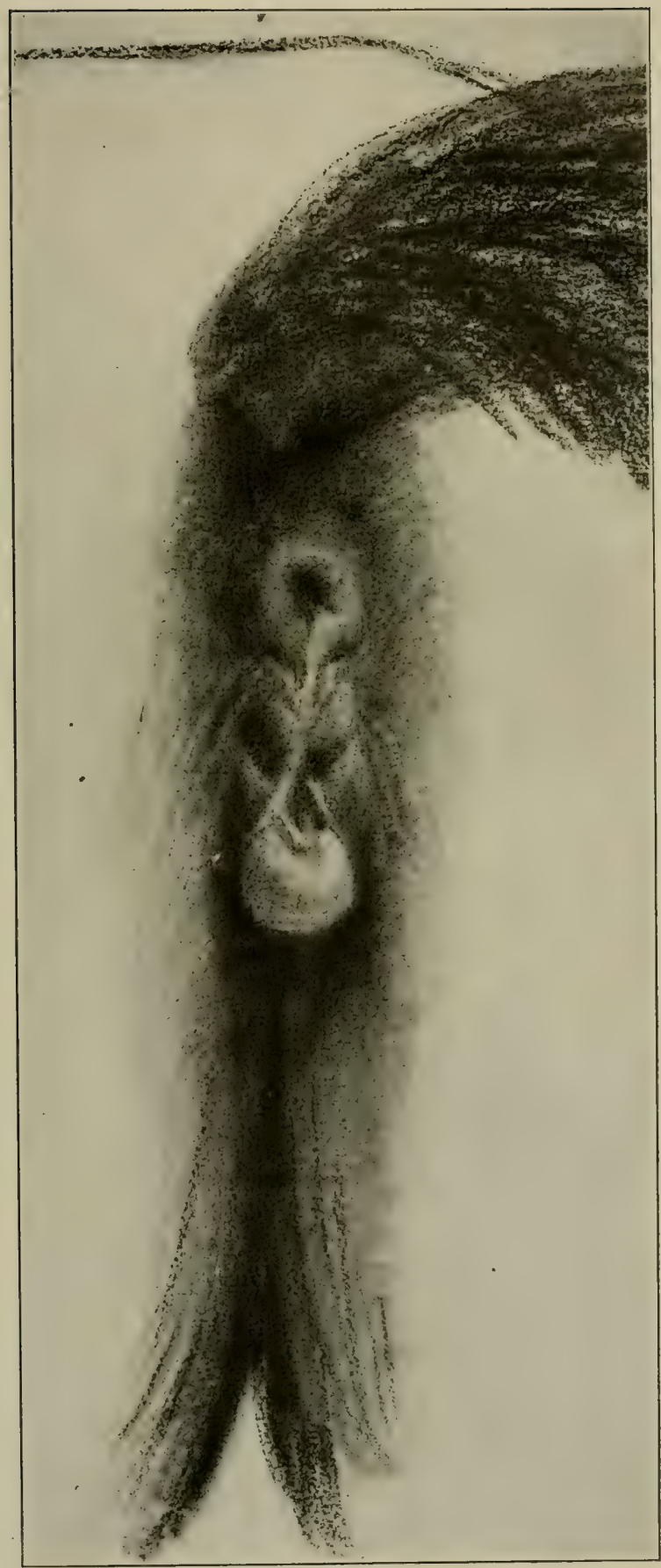

D



so

章

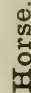

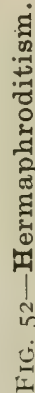


I have found no records of fertility in hermaphrodites of the equine or other species.

\section{B. Arrests in the Development of the Male Genitalia}

\section{Arrests and Aberrations in the Development OF THE TESTES}

Anorchidy, or complete absence of the testicles, except in neuters as described above, is exceedingly rare in domestic animals. That is, if some other portions of the male genital system are present in approximately normal development, the testicles, however aberrant or arrested in development, are regularly present. Anorchidy is repeatedly mentioned in veterinary literature, but the basis for the assertion is not quite clear. I have observed one horse, sold as a colt for breeding purposes, which showed no testicles or scrotum, had no sexual desire, and lacked the form and voice of the stallion. The penis, prepuce, and sheath were fully developed. Since no rectal or post-mortem examination was made, a positive diagnosis of anorchidy was not practicable. I doubt its occurrence. The testis is so fundamental in the genital system that, in its absence, I am inclined to doubt the formation of such accessories as penis, prepuce and sheath.

Practical anorchidy does, however, occur, as in the horse just described. In castrating a cryptorchid colt, the hidden testis was represented by a small, calcareous node, rendering him to all practical purposes a true monorchid.

\section{CRYPTORCHIDY}

Cryptorchidy, or the retention of one or both testes within the abdominal cavity, is common in domestic animals. It is commonest in horses and boars. As a rule-surely in 98 per cent.-cryptorchidy is due to simple arrest in development. Occasionally a dermoid cyst is at fault. I have seen a pig in which the cryptorchid testicle was the seat of malignant growth. These new-growths will be considered under tumors of the genitalia. The typical cryptorchid testicle is small, soft, and fetal in type, anatomically and histologically. 
The cryptorchid testis does not produce spermatozoa. When one testis has descended into the scrotum, it undergoes compensatorial hypertrophy and functions freely. Monorchids, though the scrotal testicle functions freely, are not desirable as sires, because of the constant peril of the transmission of the defect to the progeny. The condition is beyond remedy in a breeding sense, since the involved testicle can not be induced to function.

As a rule of practice, cryptorchid animals designed for meat should be slaughtered before sex maturity, if it can be done economically. If desired for work (horse) or as a pet (dog or cat) or if it is to be grown to maturity for meat, castration is indicated. In all domestic animals, cryptorchidy constantly tends to produce nymphomania. The cryptorchid horse tends strongly to become dangerously vicious when four or five years old, and the viciousness grows with years. In the boar, since the cryptorchid testicle, as soon as the animal reaches sex maturity, taints the meat beyond endurance for human food, castration is essential. The operation of castration, falling within the domain of ordinary surgery, need not be described here.

\section{Aberration in the Position of the testis in the \\ SCROTUM}

In one case I have observed the testicle of a bull caught in its descent before reaching the bottom of the scrotum, apparently because the gubernaculum became adherent two or three inches above the fundus of the scrotum or because of a shortness of the mesorchium. The result was that the testis tipped forward at its upper end, coming to rest with its long axis horizontal instead of perpendicular. Thus it lay in the same position as the testicle of the horse. The testicle appeared in every respect physiological. It was of the same size and consistence as its fellow. The bull was fertile. No interference was believed desirable.

4. ArRest in Functional Development of Scrotal Testes. Fetal Testis

An arrest in the development of scrotal testicles sometimes occurs, in which they fail to acquire the fundamental 
functional power of secreting spermatozoa. When the testicles descend into the scrotum, they are normal in size and consistency. As the age of sexual function approaches, the testicles fail to grow to full size and remain soft. So far as I have observed, the sexual desire is normal, or perhaps slightly subnormal. The animal mounts the female promptly and apparently copulates naturally. Search for spermatozoa in the vagina, immediately following copulation, is negative. Physical examination shows the testicles to be not more than one-half to two-thirds the volume of testes of normal animals of the same age, size, and breed. Even more marked, in my experience, is the soft, flaccid character of the glands. In all cases observed by me, the diminutive, flaccid testes have been symmetrical in size and form. The defect appears to be congenital or to originate while the male is yet a nursling. I have observed it only in young animals which were absolutely sterile from the first. The sterility has always been uniform and permanent. At first some of this type of testicles revealed upon histologic examination what was looked upon as a true arrest in development, like the typical cryptorchid testes. Later, in some cases, I have been surprised to find that the epithelium of the epididymal tubules has been destroyed and their lumen filled with cell debris instead of spermatozoa, the semeniferous tubules have largely lost their epithelium, spermatozoa rare, and probably at no time viable. This leads to the conclusion that in such cases the condition is due to a highly destructive invasion of the epididymis and testicle by an infection which falls short of abscessation or suppuration but absolutely and permanently destroys the physiologic function of the organ. The condition will therefore be more fully discussed under "Infections of the Genital Tract of the Bull."

Forensically, I know of no instance which has been decided in a law court in case of sale. The sale of such an animal for breeding purposes is rarely, if ever, fraudulent. The breeder, selling his young males usually without having used them for breeding purposes, does not discern the ster- 
ility and takes no note of the diminutive size and flaccidity of the testes. Ethically, the buyer, having paid for a breeding bull, is entitled to remuneration, and in my experience most breeders very promptly reimburse the buyer. The condition is apparently hopeless; no form of handling suggests itself.

\section{Aberrations in the Development of the Penis}

In rare cases aberrations in the development of the penis incapacitate the male for copulation. I attended one young bull in which the penis was somewhat chisel-shaped and directed upward when protruded, instead of being acutely conical and directed slightly downward. A loose, baggy fold of skin about the umbilicus, caught by the penis, formed a pocket in which the glans lodged and prevented copulation. I removed a transverse gored piece of skin, about three by five inches, and drew the wound margins together by sutures. This overcame the mechanical difficulty.

\section{Congenital Defects of the Female Genitalia}

The occurrence of asexual individuals and the imperceptible gradations between the neuter and the female have already been described. Independent of these, brief reference was made to the non-development or aberrant development of certain portions of the genital system.

The failure of one ovary to develop and function is exceedingly rare, except in conjunction with other abnormalities. Usually the two ovaries are not equal in volume. The greatest disparity in size is seen in the cow, in which the left is almost always smaller than the right, frequently less than one-fourth the volume, but the small ovary nevertheless functions. It has been claimed that the right ovary of the cow functions far more frequently than the left. I have the abattoir records on 1700 pregnant females, of which 55 per cent. were pregnant in the right horn and 45 per cent. in the left. In heifers, especially in Channel Island breeds, which regularly have very small ovaries, the left gland is 


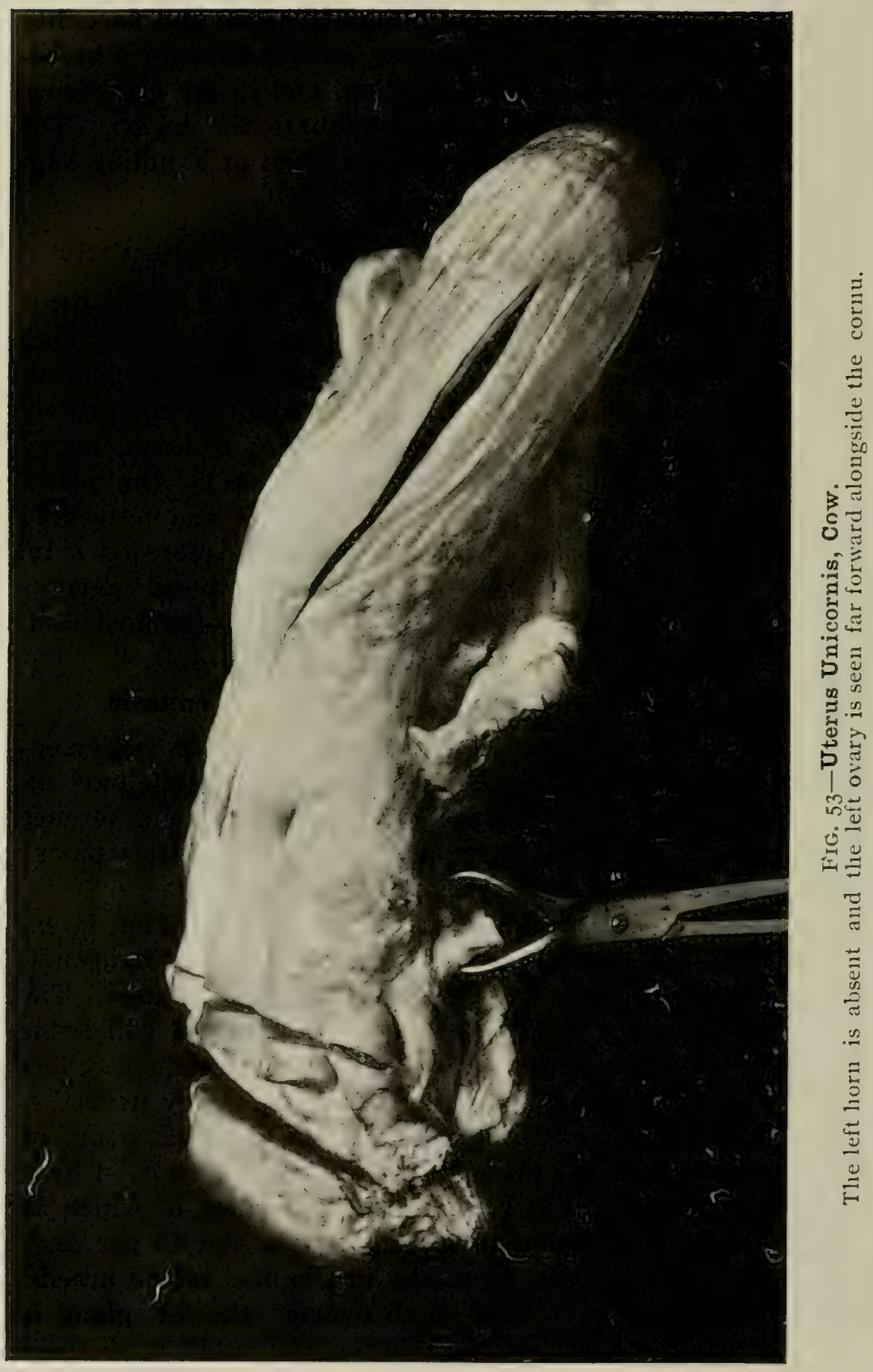


frequently no more than one-quarter inch in diameter, but it is anatomically perfect and of the adult type. Such an ovary functions. I recall meeting clinically with but one ovary of fetal type where the corresponding uterine cornu was developed; this ovary was paired with the angio-carcinoma shown in Fig. 66 . In most cases of unicornual uteri which I have seen the ovary was of normal, adult type on the side where the uterine horn was absent.

The absence of one ovary, or the fact that one is of fetal type, is of little consequence except that a second ovary serves as a reserve or alternate. So long as one good ovary is present and the corresponding oviduct and cornu are physiologic, the animal is fertile.

\section{UTERUS UNICORNIS}

Arrest in the development of one uterine cornu is not rare in cattle. In the abattoir I encountered six instances amongst a few more than three thousand females-nearly two per thousand. Five of them were, or had been, pregnant; one was a heifer. The defect is apparently of little or no significance, except, as in the ovaries, the second cornu provides a reserve, in case of disaster to the other side. It is readily recognized by rectal palpation. The single horn projects directly forward from the anterior end of the cervix. No uterine body is recognizable. In the examination of pure-bred animals for sale, the unicornual uterus should be classed as sound but defective, and the value of the animal as a breeder somewhat discounted.

\section{Double Uterus. Double Cervix}

Double uterus is extremely rare in domestic animals. I have no specimen in my collection. In consultation with a colleague, I have seen one cow, which, so far as I was able to determine clinically, had two complete uteri and cervices but there may have been a communication (uterine body) at the anterior end of the two cervical canals. The two cervical canals were perfect and readily recognized. The cow was fertile. 


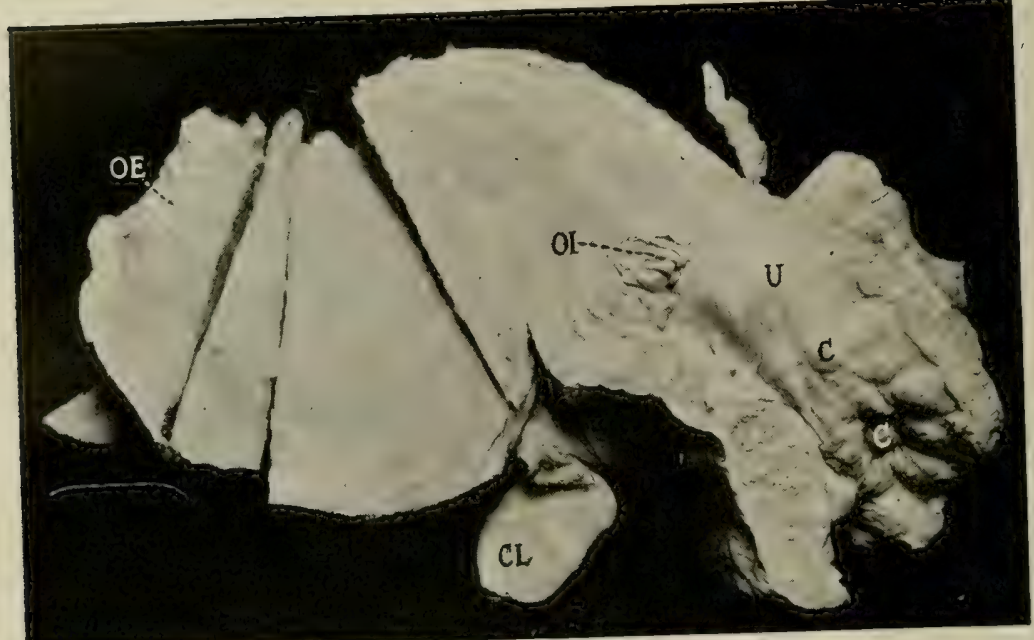

Fig. 54-Cervix of a Uterus Unicornis.

The Cervix is partly double. $O E$, Os uteri externum. $O I$, Os uteri internum. $C L$, Corpus luteum of pregnancy. $U$, Uterus. $C C$, Cotyledons. Between $O E$ and $O I$ the cervix is greatly distorted and has vagrant partitions.

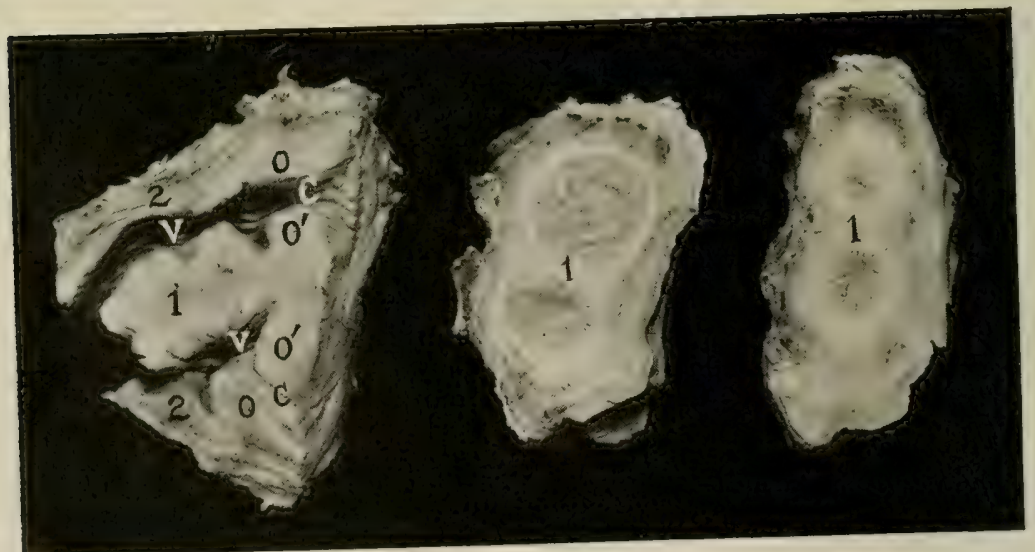

FIG. 55-Double Cervix. Cow.

$I, I, I$, Persistent median partition of Mueller's ducts. In the middle $I, I, I$, Persistent median partition of
and right figures, on either side of $I$, are the cervices containing the uterine
seal (of pregnancy.) 2,2 , Vaginal wall $O$, $O$, lips of os uteri externum : $O^{\prime} O^{\prime}$ false median lips of os uteri. 
Double cervix is not at all rare. My collection contains several specimens, each having individual characteristics. It is interesting to record that a double cervix is occasionally associated with a one-horned uterus.

The double cervix is readily diagnosed. The two carvices, lying side by side, have the normal cervical characters, are sealed in pregnancy, and penetrable with a sound when nonpregnant. The condition does not visibly affect fertility. It is objectionable merely as a congenital defect which may be perpetuated and accentuated in the progeny.

\section{Persistence of the Median Walls of the Muellerian DUCTS IN THE VAGINA}

Occasionally, in vaginal palpation, one finds a perpendicular muscular column located against the os uteri externum, but free from its lips, which extend from the floor to the roof of the vagina. I have seen the structure varying in diameter from one-fourth to one inch. It is quite firm and covered by the vaginal mucosa. It serves at times to confuse the inexperienced veterinarian, who, by recognizing the os uteri externum first on the right and then on the left of the fleshy column, may imagine that he is dealing with a double cervix. The defect is easily diagnosed. The operator can pass his finger across between the vaginal end of the cervix and the fleshy cord and, by bending the finger, completely encircle the column. By exerting traction the muscular column can usually be drawn back into the vulva, where it can be clearly seen.

Ordinarily the structure does not interfere with conception. In some cases the structure seemed to me competent to cause difficulty in parturition because one of the extremities might pass on one side of the cord and one on the other side. Then the column would need to rupture or be cut before parturition could be completed. After parturition, the fetal membranes might very well become caught and detained by portions passing on either side. Therefore it is desirable to sever the column. I have hooked an index finger about the cord, drawn it well back, and then 


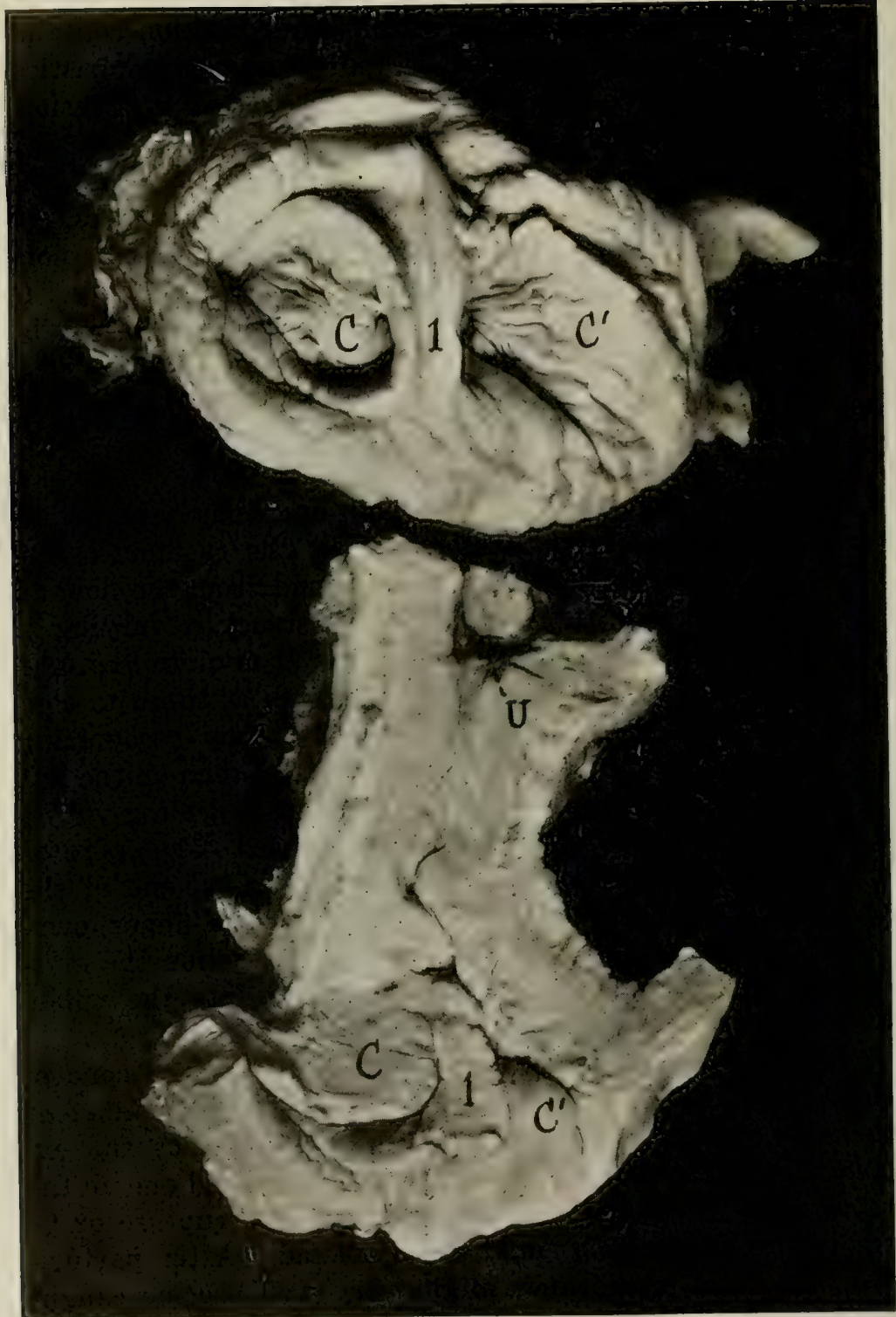

Figs. 56, 57-Persistent Median Walls of Mueller's Ducts.

Fig. 56 (above). View of cervix from Vayina. The persistent wall, $I$, gives an appearance of a double cervix which Iig 57 (below) shows, in horizontal section, is false. The depression on the right is the mouth of the single cervix, while that on the left constitutes a cul-de-sac Had the opening on the left joined the true cervical canal, just in front of $I$, it would have left the usual perdendicular muscular column. Had the persistent partition extended to the uterine cavity, $U$, a double cervix would have restilted. $C$, False os uteri. $C^{\prime}$, True os uteri. 
severed it with a scalpel or with scissors. An insignificant hemorrhage follows. There is virtually no sensation in the structure and no material evidence of pain when it is severed.

\section{Persistent Hymen}

In the development of the female embryo of cattle, the hymen regularly disappears as a complete membrane, but a series of fragmentary projections remains, marking the vulvo-vaginal boundary line. Upon the floor of the genital passage this line is located just in front (the ovarian side) of the urethral opening. The vestiges of the hymen constitute a well defined stricture between the vulva and the vagina. In the manual examination of heifers, passing this stricture with the hand frequently causes a slight tearing accompanied by hemorrhage. In numerous cases, remnants of the hymen persist as fleshy cords, sometimes the dimensions of a lead pencil, extending perpendicularly from the floor to the roof of the passage, sometimes on the median line, but generally to the right or left of the centre. Seldom, if ever, do they cause difficulty of any consequence, but they should be cut or ruptured when encountered.

Rarely, the hymeneal constriction forms a slight cul-desac in the vaginal floor, where debris accumulates and decomposes, to imperil conception. Sometimes the cul-de-sac may be overcome by dividing the stricture along the floor. Otherwise, the peril is to be obviated by frequent vaginal douches until conception occurs. Parturition will ordinarily eliminate the defect.

The hymen sometimes persists as an imperforate membrane. I have observed but two cases, one in a heifer, the other in a ewe. In Great Britain, I am told, it is common in a certain strain of white cattle, so that it has become known as "the white heifer disease". I have been unable to get particulars about it. In any animal, imperforate hymen inevitably leads to the accumulation of menstrual and other debris in the vagina, which becomes a great retention cyst. Estrum may be regular, but copulation is impossible, because the penis of the male is prevented from entering the 
vagina. As soon as the vagina becomes fully distended, discomfort and pain result, causing colicky symptoms and expulsive efforts. During the expulsive efforts the hymen may be forced back into the vulva and become visible between the vulvar lips. Palpation reveals a tense, membranous wall retaining a liquid beyond. On the floor of the passage, the membrane is attached just anterior to (on the ovarian side of) the meatus urinarius. The attachment of the membrane to the walls can be traced completely around the genital passage. Per rectum, the vagina is readily palpable as an elongated sac firmly distended with fluid. In the one heifer which I observed, both uterus and vagina were distended greatly with thin pus. This caused much pain, with constant straining and loss of flesh. It is quite probable that the infection was carried to the genital tract by the blood early in the heifer's life and remained dormant until menstrual debris collected, when suppuration promptly ensued. The handling consists of the surgical destruction of the membrane, which should be punctured approximately in the center with a scalpel and the opening thus made extended by cutting or tearing to a sufficient degree. It is especially important to extend the opening in the membrane downward to the floor of the passage, since otherwise it may serve to form a cul-de-sac leading to the retention and decomposition of discharges, thus keeping a pool of infected debris about the os uteri externum; interfering with conception.

When persistent hymen becomes a race characteristic, as in the "white heifer disease" of England, it would appear best to eliminate the defect by careful selection.

Imperforate hymen in the mare is apparently very rare. There occur a few rather vague descriptions of imperforate hymen in the mare from which the actual facts are unobtainable. There occur in literature statements that in the pregnant heifer and filly imperforate hymen may exist at the time of parturition. Evidently that is impossible. A remnant of the hymen sufficient to complicate parturition may persist, but copulation cannot occur and leave the membrane imperforate. When imperforate, a distension of the 
vagina with menstrual debris is inevitable. So far as I have observed, the imperforate hymen can not be ruptured by the male. It is imperforate and persistent because abnormally thick and tough.

I have observed in a filly a persistence of the lower portion of the hymen, constituting a cul-de-sac which ended at the anterior margin of the urethra and sloped upward and backward as a crescent-shaped partition open at the top. It was attempted to breed her to a very large stallion. After several unsuccessful efforts my advice was sought.

Upon introducing my hand, I promptly encountered a broad band stretched transversely and sloping forward and downward. Pushing my hand along the lower surface of the membranous partition, I found that the urethral opening formed the fundus of a great cul-de-sac. The urethra was so greatly dilated that it readily admitted my hand. Apparently it had been dilated by the penis of the stallion. Searching higher up, I found the hymen was not imperforate; there was a large opening at the vaginal roof. The groom was advised to be on the alert at the next effort at coitus and push the penis of the stallion upward just at it entered the vulva. The filly conceived.

The case reports of persistent hymen which have interfered with parturition are probably largely based upon diagnostic error. Instead of persistent hymen there was probably a persistent portion of the median walls of the ducts of Mueller, a condition just short of a double vagina. Such remnant is resistant enough to readily cause dystocia. Many appear to think erroneously of any band passing across the vagina as "persistent hymen". The persistent median wall is far more common than the persistent hymen. The condition has been discussed above.

The persistent hymen which is perforate usually has but little significance. The imperforate hymen has little peril for the life and general health of the patient unless overlooked until the vagina is tensely filled with menstrual debris. When, as in the heifer described above, both vagina and uterus are greatly distended with pus, there is ground for serious fear that breeding life is at an end. 


\section{CHAPTER V}

\section{MISCELLANEOUS DEFECTS AND DISEASES INTERFERING WITH FERTILITY}

There are many defects and diseases of other than the genital organs which may render coitus physically difficult or impossible, although the animal is fundamentally fertile.

Much has been said of the physical difficulty of coitus between individuals representing extremes in size. This occurs only when the disparity in size is so great that mating is as a rule imprudent for other reasons. Extreme variation in size may render coitus difficult or dangerous. In both horses and cattle it is not rare to observe successful coitus of a large male with a female 30 to 40 per cent. of his weight. The danger from such disparity is not great, but when a stallion has an extra large penis this may have great peril for the vagina of the mare. These injuries will be discussed later. When a very small male attempts coitus with a large female, failure is dependent ordinarily upon the shortness of the hind legs of the male, making it impossible for him to reach the vulva. As a rule, however, the male of a given breed of animals is able, not long after he reaches puberty, to copulate with an adult female of his breed. In horses and dogs, where giant and pigmy breeds have been developed, coitus between these extremes is virtually impossible. The variations in weight between Clydesdale horses and Shetland ponies, for instance, may be from 2400 to 200 pounds-or 12 to 1 . In dogs the extremes reach nearly 100 to 1 . Disparity in size does not enter prominently into the question of the physical possibility of copulating.

\section{A. Umbilic Hernia}

Umbilic hernia, especially in the bull, whenever of material size, inhibits copulation by deflecting the penis as it is being protruded. The defect is ordinarily not brought to 
the attention of the veterinarian, except in young bulls of high pedigree. If the bull is an ordinary grade, or of low pedigree, he is usually, and quite properly, sent to slaughter. However, when a bull is of high pedigree and potentially of great value, the owner often becomes anxious to have the difficulty overcome by surgical interference.

Umbilic hernia is a congenital defect due to an arrest in the normal closure of the umbilic ring. As an arrest in fetal development, it has the same significance for the breed or family as other teratological defects. It signifies a fundamental organic weakness always threatening to be transmitted to the progeny. Umbilic hernia is very difficult to handle in the bull. The breeder usually defers the operation until the rumen has attained a great weight. Then the surgeon is faced with a weight, which it is virtually impossible to support by means of surgical appliances, bearing immediately upon the operative area. In umbilic hernia of the bull, the surgeon is denied his chief mechanical means of support_the bandage-because the opening of the sheath is immediately at the umbilicus, so that the bandage can not be effectively applied without involving and obstructing the sheath opening. As a result, when attempting to suture an umbilic hernia in a bull, the bandage must be omitted or ineffectually applied, and before healing occurs the sutures usually tear away and carry with them portions of the hernial ring, making the opening larger than before. If the surgeon undertakes to clamp or ligate the hernial sac, by the time the incarcerated mass has sloughed away, the pressure from above has stretched the tissues and formed a new sac. If the hernia is operated on when the calf is but a few days old, before the viscera acquire insupportable weight, the surgeon may more readily succeed. Even then, however, the fundamental weakness of structure is there, and in my judgment the animal should not be used as a sire. 


\section{B. Ventral Hernia}

Large ventral herniae, such as indicated in Fig. 58, also inhibit copulation, rendering the bull worthless as a sire.

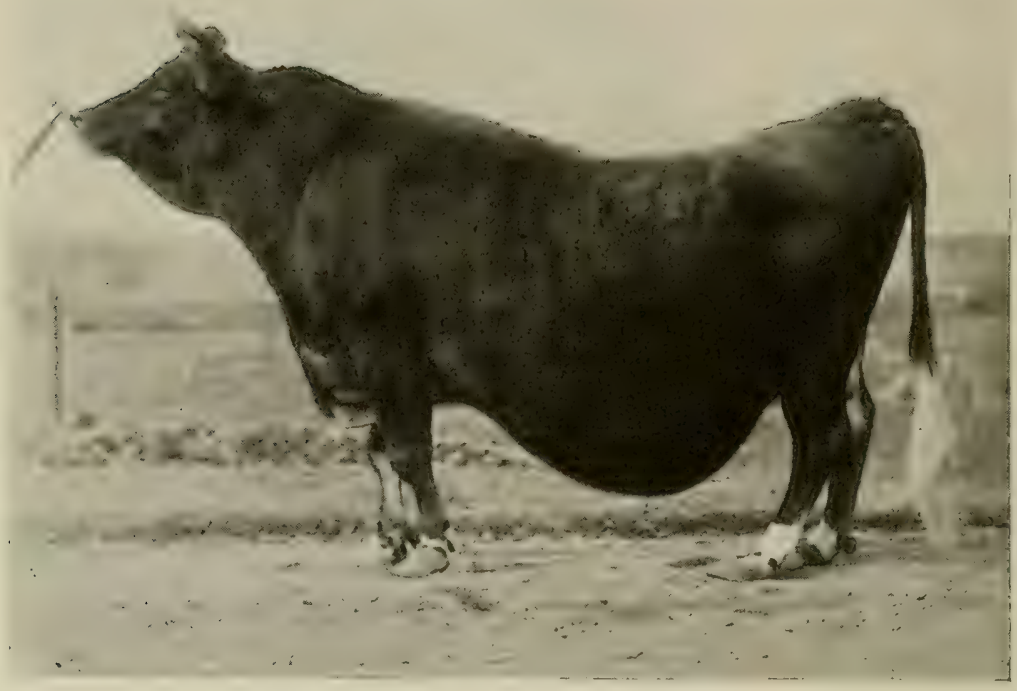

FrG. 58-Large Ventral Hernia preventing Coitus.

\section{Horizontal Vulva}

Horizontal vulva in the cow sometimes serves to render copulation difficult or impossible. In aged cows with pendulous abdomen and low back (lordosis, or sway-back) the superior commissure of the vulva is depressed and dragged downward and forward by the descent of the rectum and anus, so that the vulvar opening is directed upward. More rarely, the vulvar opening is directed upward in otherwise well-formed heifers, as a peculiarity in conformation. When the bull attempts to copulate with such an animal, the penis tends to glide over the vulva and strike against the anal region. This is best avoided by causing the female to stand with her hind feet in a pit or depression, with the fore feet 
high, thus bringing the vulvar opening toward the perpendicular. Horizontal vulva also follows perineal laceration at the superior vulvar commissure. This is described under "Parturient Injuries".

\section{Strictures of VAgina AND Vulva}

Strictures of the vagina, and more rarely of the vulva, are occasionally encountered in domestic animals, chiefly in the mare and the cow. They always result, so far as known, from some prior inflammation of these parts, but frequently the history of the disease is not available and the veterinarian encounters simply the result of the disease.

In a mare which came under my observation, there was difficulty in parturition due to an anterior presentation in the dorso-sacral position, with the two hind feet pushed forward beneath the fold into the pelvis. The difficulty, occurring during the night, was not observed until morning. Delivery was promptly and readily accomplished by embryotomy, but the vulva had been so badly contused that more than half of its circumference became necrotic and sloughed away. The resulting constriction was such that the animal could not copulate. She might have been artificially fertilized vithout material difficulty but, as she probably would have been unable to give birth to young, no attempt was made to cause her to breed. I could see no prospect for success by any operation. The mare was consequently used for work.

I observed one instance of severe stricture of the vagina in a mare, the origin of which was unknown. Without knowledge of the presence of the lesion, the owner attempted to breed her. This caused extensive lacerations of the vagina with great pain and straining. She was placed under my care. The straining caused an eversion of the floor of the vagina and the urinary bladder (vesico-vaginocele). I attempted to keep the vagina and bladder in position with a truss, and later with vulvar sutures, but failed. Finally I controlled the straining and eversion by producing profound and prolonged chloral narcosis. 
Stricture of the vagina in the cow is comparatively common and of varying degrees. Occasionally one meets cases in which it is impossible to reach the cervix. Frequently the stricture is associated with long-standing sterility, where it is highly desirable to reach the cervix and handle the uterus.

In my experience, stricture of the vagina has occurred most frequently in herds where the vagina has been douched with potassium permanganate. It is not certain that there is any connection between the potassium permanganate douching and the stricture. Stricture is very liable to follow douching with any powerful antiseptic, and very naturally might follow the use of a too highly concentrated permanganate solution. There is another possibility with the potassium permanganate. As it is very heavy and dissolves rather slowly, laymen or careless veterinarians may inadvertently introduce some crystals into the vagina. The liquid would be largely thrown out, and the heavy crystals remain behind. These would cause profound irritation with necrosis of the mucosa and stricture would naturally follow. Stricture is likely to follow any case of severe vaginitis, regardless of the cause.

If the stricture is not too severe, the prognosis is good. If the condition of the vagina is such that copulation may be safely performed and there is not too great hardening, the vagina may largely recover its normal elasticity during the rest of pregnancy, so that the animal may go through parturition safely. If the uterus requires handling, it may be possible to pass the uterine forceps through the stricture and grasp the cervix without inserting the hand, or the forceps may be guided with one hand in the rectum. In other cases, one may be able to introduce the vaginal dilator (Fig. 37 ) and distend the passage sufficiently that the cervix may be seen and grasped. Whenever I have been able to grasp the cervix, it has been possible to draw it out through the constricted vagina and give to the cervix and uterus any attention desired. 
E. Diseases of the Feet and Limbs

Breeding bulls and, to a lesser degree, cows which are kept constantly stabled tend to become disabled owing to the fact that the growth of the hoofs is not counterbalanced by the normal wear of travel. The margins of the lateral and median walls tend to converge and, as they become elongated, to bend beneath, the soles, approaching each other as

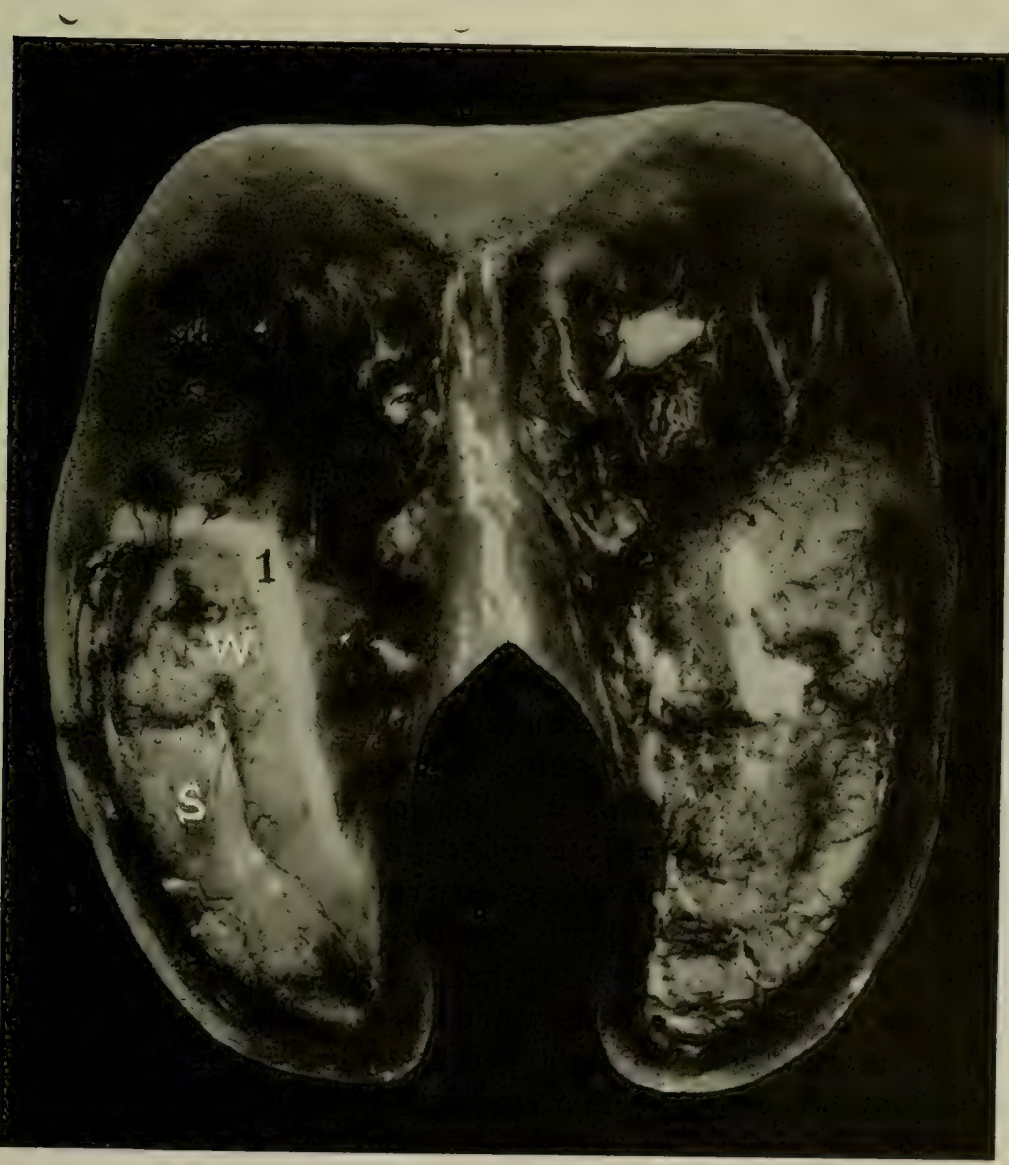

FIG. 59-Overgrown Hoofs interfering with Coitus. Bull.

$S$, Sole, which has been largely overgrown by the wall, $W$, growing forward from the heel. $I$, A shallow groove showing approximately the normal location of the margin of the wall. 
shown in Fig. 59. The heel portion of the wall is directed acutely downward and forward, and, as this is not worn away by the idle animal, the superposed weight bends the free margin forward and inward until it lies flat against the sole, and continues to grow forward until the posterior, or heel wall finally reaches the toe, completely covering the sole. The bull then walks upon the elongated heel wall, between which and the sole is a space, opening at the toe, in which gravel, dirt, etc., become impacted. Then follows unequal pressure (by gravel, etc.) upon the sole, contusions of the sensitive parts, pressure necrosis, and other lesions. The bull soon becomes lame, and, unless the difficulty is promptly recognized, may be crippled permanently. Frequently I have seen bulls rendered incapable of copulation from this cause. The condition is best prevented by carefully trimming the feet of all breeding bulls kept in stanchions or stalls, at least twice annually. Once the difficulty has become established, the walls are permanently deformed and constantly turn in. Then the trouble can be palliated only by the frequent trimming of the feet and by keeping the bull well bedded on a clay or board floor. Stone, concrete and gravel floors should be avoided.

The trimming of the feet may generally be accomplished with hoof-cutting pincers, with the animal standing. When the bull is secured in a stanchion, two strong men can generally hold up one of his hind feet and extend it backward while the operator trims it. The men should take a stout, smooth beam, preferably a round piece about six feet long, pass it between the hind legs in front of the tarsus of the foot to be raised, and, firmly grasping each end of the beam, lift it upward and draw it sharply backward, so that the flexure of the hock rests upon the middle of the beam. Most animals will struggle but little and the trimming of the hoof can be performed readily. If the animal is too resistant, he should be cast and secured the same as already recommended for examining the penis.

Painful diseases of the posterior feet and limbs occasionally render a male incompetent or unwilling to mount 
the female, thus throwing increased weight upon his hind limbs. Obviously any attempt at copulation aggravates the local disease. The male should, therefore, be held out of breeding until he has recovered. Sometimes it is wished to breed a female suffering from some local, non-genital disease which renders copulation difficult or dangerous for her because of the weight of the male which she is called upon to bear. Partial relief from the excessive weight may be secured in cows and mares by using a breeding rack so constructed that the female stands between two broad, sloping shelves of such height that, when the male mounts her, his fore feet rest upon the shelves and his weight is more or less completely borne by these. The shelves must fit closely against the sides of the female, in order to obviate the danger of the male's getting his foot between the shelf and the female, and should slope downward from the head of the female. The breeding rack does more than partly or wholly support the weight of the male. When it fits closely and a beam is placed securely in front of her sternum, her equilibrium is maintained and she can support without increased danger probably twice the weight of which she would be capable otherwise. This is especially true of the violent copulatory thrust of the bull. Not infrequently, when a cow is weakened and unsteady, or when a small heifer is mounted by a large bull, the thrust causes the cow or heifer to crumple up and fall headlong.

It is unwise to breed a crippled or weak female unless it can be reasonably determined that the patient will promptly recover from the local disease. Otherwise, as soon as the increased weight of pregnancy has to be borne by the weak member, a new peril is encountered in the aggravation of the disease. Pregnancy may occur and parturition may be safely passed, but the progeny is rarely well nourished or valuable.

\section{F. Paralysis and Plumbism}

Paralysis sufficient to interfere with copulation may occur in any animal, and from a great variety of causes. In 
the stallion there occurs sometimes a paralysis of the posterior limbs. In one bull in my experiment herd, paralysis was due to lead poisoning caused by licking a painted board when but a few days old. He was completely paralyzed and unconscious for a number of days. For months he was quite severely paralyzed in his posterior parts, with some swelling of the joints and bones. He attained medium size, and was normally fertile, but was quite awkward and copulated with some difficulty.

Stallions suffer occasionally from paralysis of the penis, the cause of which is generally unknown, largely associated with partial paralysis of the posterior limbs and not ordinarily subject to remedy.

\section{G. TORSION OF THE TESTICLE}

Torsion of the spermatic cord, it is claimed, sometimes causes atrophy of the testicles and inhibits the formation of spermatozoa because of interruption of the nutritive supply. It is well known that emasculation can be produced in ruminants by the process designated double subcutaneous torsion, in which the testicles are twisted and inverted in such a manner as to interrupt their vascular supply and induce atrophy, with disappearance of sexual desire and power.

Occasionally in man torsion of the testicle appears to produce painful and serious symptoms simulating incarcerated hernia with vomition. O'Connor $\left({ }^{1}\right)$ describes two cases and states that one hundred twenty-four cases have been recorded in man. I am not aware that disease has been traced to torsion of the spermatic cord in animals. In the stallion, with horizontal testes, the gland is frequently revolved upon its transverse axis through 180 degrees, but this appears to be of no consequence. The horizontal position of the testicle permits it to revolve upon its transverse axis below the mesorchium. The displacement involves very slight damage to the spermatic cord. The freedom of

\footnotetext{
${ }^{2}$ Vincent J. O'Connor, Torsion of the Spermatic Cord. Surg. Gyn. and Obst., Dec., I9I9, p. 58o.
} 
the spermatic cord in the inguinal canal permits part of the torsion to occur within the abdomen, so that the twist need not be great at any one point. In man the torsion is said to occur frequently where the gland is caught in the inguinal canal. Just how it can revolve in the inguinal canal, I fail to understand. Inguinal cryptorchidy is familiar to veterinarians, but torsion is rarely, if ever, recognized in these cases. I have observed but one instance of torsion which was of consequence. In an adult cryptorchid presented for castration, I quickly recognized a state of affairs new to me, finally recognized what I believed to be the vas deferens, traced this to what appeared to be the testicle, and then applied traction in an effort to bring the gland out through the wound. Finally an attachment unexpectedly parted, and a large, livid-colored testicle was brought out. The spermatic cord was spiral, the circulation had been barred, and the cord and testicle were necrotic. The cord was so weakened at the chief point of torsion that it gave way at that point. O'Connor, cited above, believes the chief cause of torsion to be the irregular contraction of muscle bundles in the cremaster, but, in my case with the testicle in the abdomen, such an explanation can not be accepted, as the cremaster does not come into play or become visibly developed until the testicle descends into the scrotum. A study of Fig. 1 will show how a cryptorchid testicle could readily revolve upon its transverse axis. The testicle of the ruminant, moored throughout by the mesorchium to the wall of the scrotum, can not possibly, so far as I can see, revolve upon either of its axes. The possibility of serious torsion of the spermatic cord should be borne in mind.

\section{H. Traumatic ORchitis}

According to many veterinary authors, orchitis in animals is due largely to traumatism. Professor Hendricks ${ }^{i}$ emphasizes the belief in trauma as a cause of orchitis and considers that most cases of inflammation of the testicles are due to mechanical injuries. It is an open question, however, to

'Handbuch der tierärtzl. Chirurgie u. Geburtshilfe, III Band. 
what extent such an opinion is based on experience and to what extent, upon legend. Personally I have not observed orchitis in any domestic animal where it was plainly due to traumatism except in one instance of an abattoir bull which had suffered from gunshot wound in the scrotum, two of the shot having lodged, one in the epididymis and the other in the testicle. This case is illustrated in Fig. 59a.

Cinically orchitis is most common in the stallion, in which animal the testicles are unusually well protected against traumatism when compared with those of other domestic animals. The pendent testicles of ruminants are exposed preeminently to mechanical injury. In the ram and the hegoat, the testicles hang so low that in moving rapidly over rough ground, especially where there are stumps and highprojecting stones, the testicles frequently come in rather violent contact with these foreign objects. In all ruminants the pendent testicles are thrown against the thighs repeatedly whenever the animal is in rapid locomotion, and yet I have not observed orchitis as a result of this. In trotting stallions there is a general belief that, unless a suspensorium is used, orchitis may result from the constant striking of the testicles against the thighs during rapid locomotion, but I find nothing in veterinary literature to indicate that orchitis arises in this way.

While it must be freely admitted that orchitis may arise in any animal because of traumatism, careful clinical examination would indicate that the affection is very rare. It is difficult to determine clearly just what type of orchitis a mechanical injury may induce. It cannot by any conceivable means cause an abscess of the testicle unless there is first a destructive injury to the skin or a penetrant wound so that the trauma serves merely to open an avenue of infection rather than to directly cause orchitis. In Chapter XIV of this treatise, the question of orchitis as a result of infection is discussed at some length. By referring to this chapter, it will be observed that in nearly all cases of orchitis and epididymitis the disease is bilateral, which would indicate very strongly that it is due to some systemic infection 


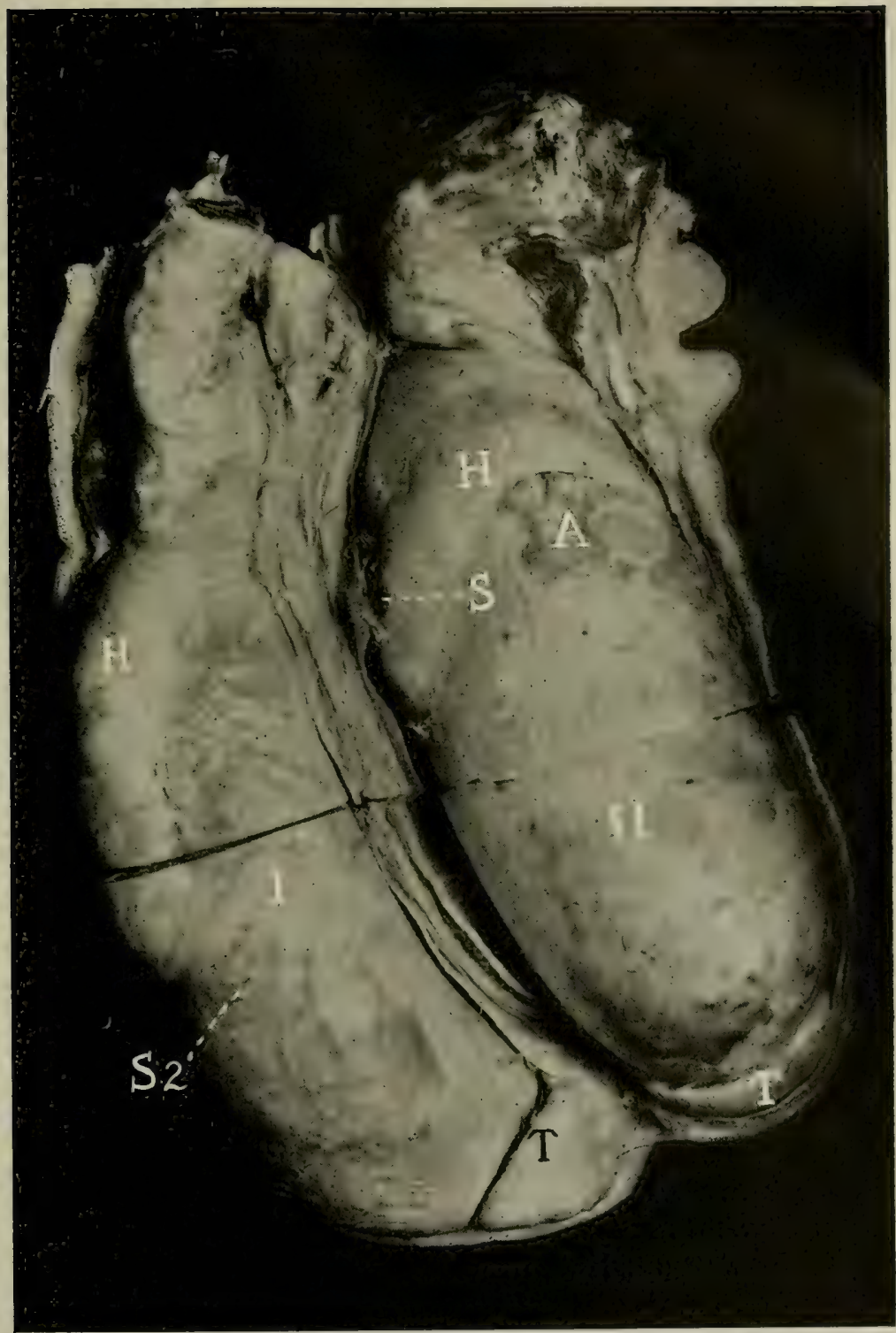

FrG. 59a-Gunshot Wounds of Testicles. Bull.

$S$. Gunshot embedded in right epididymis; $S_{2}$, gunshot embedded in left testis ; $H$, globus major of epididymis ; $T$, globus minor, do ; $A$, roughened surface from inflammatory thickenings. The orchitic peritoneum is generally roughened on account of traumatic peritonitis. 
arising from the presence in the blood stream of pathogenic organisms having a distinct affinity for the genital organs. The lesions figured and described are distinctly those of infection and not of traumatism.

Before diagnosing traumatic orchitis in any animal, one should give ample consideration to the facts as stated and should in addition know that there has been a severe traumatism of the gland or glands.

It is quite possible that mechanical injury plays a very important part in bringing about a crisis when infection is already present within the gland. That is the general rule in the etiology of disease in any organ of the body, and apparently applies with special force to the diseases of the genital organs. Thus Hendricks, to whom reference has been made above, states that excessive coitus serves as a fertile cause of this disease. Excessive coitus, however, cannot be regarded as traumatism. It must, instead, be looked upon as an overworking of a gland by which it becomes weakened and its tissues lose their power of resistance toward disease-producing microorganisms which are already present in the part.

When the veterinarian feels justified in diagnosing traumatic orchitis, the prognosis will of course depend upon the nature of the trauma. The treatment also needs to be adjusted to the general principles of surgery in harmony with the character of the injury. Speaking generally, care should be taken to obviate, as far as possible, the invasion of the gland by bacteria because of injury to the skin and other coverings. It is likewise important that the interferences with the circulation in the testicles should be anticipated and prevented, if possible, by the careful application of a suspensorium. In ruminants, in addition to the suspensorium, the testicles can be inclosed in a bandage of adhesive tape in such a manner as to exert uniform and gentle pressure upon the glands and thereby maintain the efficiency of the circulation. Sexual excitement should be strictly avoided. The bowels should be kept freely open as in any case of disease where fever is likely to occur, and the diet should be properly adjusted. 


\section{Tubal Pregnancy}

Tubal pregnancy, comparatively common in woman, has not, so far as I know, been recorded in any domestic animal, but it is quite possible and may be encountered by the veterinarian when he is examining the genitalia. The fact that extra-uterine pregnancy is now and then encountered in the abattoir renders it highly probable that tubal pregnancy does at times occur in animals. The veterinarian does not have the means for diagnosis afforded in woman by the regular occurrence of hemorrhage from the uterus, since the cervix is too securely sealed, but he has a distinct advantage over the human obstetrist in the facility with which palpation per rectum may be applied. The condition would necessarily give rise to a cystic enlargement in the oviduct, associated with a typical corpus luteum of pregnancy in the corresponding ovary, a typical uterine seal, enlargement of the ovarian artery, and an absence of estrum. If tubal pregnancy is diagnosed, the peril from rupture of the oviduct should be promptly removed by performing laparotomy in the upper flank on the involved side, followed by the amputation of the tube and ovary. If the other side of the reproductive tract is sound, the fertility of the animal is not disturbed.

After the oviduct has ruptured and the ovum has escaped into the peritoneal cavity, if the accident is discovered early and there are evidences of serious internal hemorrhage, the animal, if of a species used for food and otherwise fit, should be slaughtered immediately. Should the veterinarian by chance diagnose an old-standing extrauterine pregnancy, with the fetus securely anchored and enveloped, the fact is to be wholly disregarded, except that the animal is probably permanently sterile on the side where the pregnancy originated.

\section{J. Vaginal Hernia}

Vaginal hernia, or hernia through the vulvo-vaginal muscular wall, is not infrequent in cows. In the cases I have observed there was a rent in the lateral wall of the vagina 
at the hymeneal ring. The symptoms consist of the appearance between the vulvar lips, or projecting beyond them, when the patient is lying down, of a fluctuant, globular mass, covered by the vaginal mucosa. When the cow is caused to stand, the hernial mass usually disappears spontaneously, or, if it does not, readily disappears under gentle manual compression. Then the veterinarian can readily recognize a well defined hernial ring of variable size, admitting the insertion of two fingers or the entire hand. The veterinarian should constantly be on the alert not to confuse vaginal hernia with prolapse. Prolapse of the vagina is not so frequent as vaginal hernia. I have seen colleagues err in diagnosis. The size of the hernial mass increases as pregnancy advances. The mucosa of the hernial sac becomes somewhat befouled and inflamed by constant exposure to filth when the animal is lying down, but in the cases I have observed there has been no recognizable inconvenience. The hernia constitutes a repulsive defect not wholly devoid of peril to the animal. I have observed five cases of this hernia, all in pedigreed cows. With one exception, all had been purchased as presumably sound by the parties owning them at the time of examination. The one owned by her breeder had developed a fibroma at the hymeneal ring, which protruded beyond the vulva. The veterinarian removed the tumor by ecrasement. Evidently the chain of the ecraseur included a portion of the muscular wall of the vagina, thus causing an opening into the pelvic connective tissue, behind the peritoneum. The intraabdominal pressure pushed portions of the small intestine against the peritoneum and forced it out through the vent in the vulvo-vaginal wall, to constitute a hernia. It is not impossible that the other cases I observed were caused in the same way, since the vestiges of the hymen are frequently the seat of fibroid tumors and the veterinarian, when called to remove them, resorts to the ecraseur, without thinking of the consequences. The tumors will be discussed later. As I now recall, the hernia has been on the 
right side in most of my cases. The initial injury must be as frequent on the left as on the right side, but the intrapelvic content of the small intestine is greater on the right and would tend rather to develop a hernia in a weakened area.

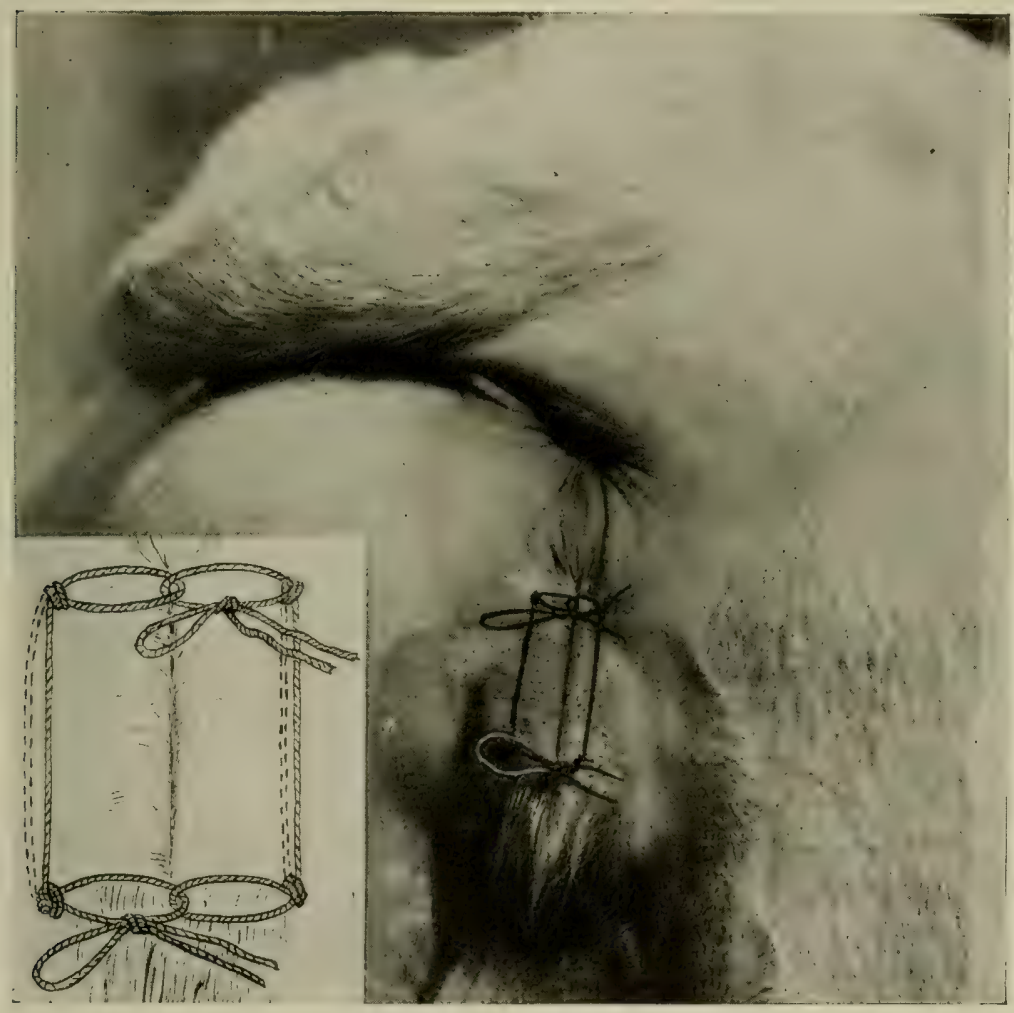

FIG.60-Sutures For Vaginal Hernia.

The insert below at the left shows details of suture.

Although I have not had an opportunity to operate upon a case, and thus can not speak from experience, the condition should be amenable to surgical interference. The operation could be done best upon the non-pregnant animal, allowed only a small volume of food in order to lower the in- 
tra-pelvic pressure upon the operative area. It would be practicable to operate upon most cows under local anaesthesia in the standing position. After carefully cleansing the vulva and vagina, an incision may be made through the vulvar mucosa of the hernial sac, down to the pelvic fascia. An index finger can be inserted through the incision and, palpating the hernial ring, act as a guide in suturing. A half-curve needle armed with strong silk should then be inserted at a point on a level with the superior border of the hernial ring and about one-half inch posterior to the margin. The needle is to be passed through the vulvar mucosa and the muscular wall; carried forward, carefully guarded, across the hernial opening on the ovarian side of the anterior margin of the hernial ring; and finally brought out into the vagina, through the muscular and mucous coats. Then the needle is to be reversed, carried downward about onefourth inch, inserted, and a suture laid parallel to the first, emerging about one-fourth inch below the point of starting. The free ends of the suture are then brought together and tied tightly, with a view to inducing pressure necrosis in the tissues incarcerated in the loop. The sutures should be repeated every quarter of an inch, from the upper to the lower margin of the hernial ring. The ring will then be obliterated, and adhesion occur between the incarcerated tissues. The portions of tissue lying between the incarcerated areas in the sutures serve to maintain function in the hernial sac, preventing it from sloughing away, and the possibility of prolapse of the intestine through the slough.

When circumstances render it desirable to apply palliative measures to ventral hernia pending parturition, the object is readily accomplished by means of vulvar sutures, as shown in Fig. 60. In the illustration, tape has been used for sutures. A loop has been tied near the middle of the suture, after which the needle is inserted about one inch lateral to the vulvar opening, somewhat below the superior commissure, carried downward nearly to the inferior commissure, and brought out. The ends are then tied together 
firmly, leaving sufficient free tape to reach across the vulva to the suture of the other side, to which it is tied by a bowknot. The sutures can be untied at will while the vulva, vagina, and sutures are cleansed.

The suture is better when made of heavy silver wire. It irritates the tissues less than the tape and is cleaner. It has the great advantage, also, that the ends of the wires crossing over the vulva may be fastened by merely bending them over the suture, so that, if parturition commences in the absence of an attendant the force will cause the wire hook to unbend and the vulvar lips to part. Similar provision may be made with tape by using for the cross ligatures, tape or cord of low tensile strength which, in case of labor pains, will break. Otherwise, there is danger of interference with parturition and special danger of lacerations of the vulva owing to pressure upon the sutures.

\section{K. TORSION OF THE UTERUS}

Torsion of the uterus is generally considered only as a cause of dystocia at or near the full term of pregnancy. It has been quite fully discussed in the companion volume upon Veterinary Obstetrics. It is well to add that uterine torsion occurs quite independently from the near approach of parturition. In the cow it may be encountered when palpating the genitalia at least as early as the 145th day of pregnancy. It may cause colicky pains, or merely dullness, or for a time no symptoms at all. Therefore the practitioner may be called to examine the cow because she has shown signs of disease, or he may detect the torsion in the course of an examination of the genitalia as a part of the control of sterility and abortion.

When a veterinarian is called to attend a cow showing occult evidences of disease, he should always have in mind the important role played by the genital organs and make a careful examination. When the displacement occurs early, as in the cow already cited, there may be no manifestations of disease or discomfort. The torsion in the small and not 
fully distended uterus may occur so far forward that the usual spiral folding of the vaginal walls may be wanting. Therefore it may be stated as a law that rectal palpation is essential to a proper determination of the state of the uterus. When the uterus which has undergone torsion is palpated per rectum, it offers certain definite characteristics:

1. The posterior portion of the uterus can not be clearly palpated, because one of the broad ligaments is tightly stretched across the organ, completely covering it. Far forward, in front of the broad ligament, the examiner may directly palpate the gravid uterus and the contained fetus. If the uterus has revolved to the left-that is, if in the beginning of the displacement the dorsal wall of the uterus has moved to the left, while the ventral or lower wall moves to the right - the right broad ligament passes over the vagina and uterus to the left, while the left broad ligament passes beneath the vagina and uterus to the right. Both ligaments are tensely stretched.

2. The chief uterine artery inevitably moves with the broad ligament, and is readily felt. In left torsion, the right uterine artery, tightly stretched, passes over the uterus to the left side, while the right artery is forced down along the pelvic floor and passes beneath the uterus and vagina, to the right. The torsion constitutes a formidable obstacle to the circulation in the entire genital tract anterior to the turn, causing the arteries to become distended and the pulsation in them to be unusually full and bounding. These findings serve to identify the character of the displacement. Moderate uterine torsion, without transverse rupture of the uterus or vagina, recognized in time, warrants a highly favorable prognosis. The chief indication is the rolling process already fully described in the companion volume, Veterinary Obstetrics.

\section{Pelvic Tumors and Calluses}

Occasionally, when examining the genital organs of mares or cows, one finds tumors in the pelvis between the 
pelvic walls and the vagina, which may interfere with fertility-not so frequently with fertilization as with parturition. These should always be observed carefully and precautions taken against impending difficulty. If the tumors are irremovable, the animal should not be bred; if she is pregnant, artificial abortion may be performed, in the early stages, or, if the fetus is of value, pregnancy may be permitted to continue to full term and the young removed by hysterotomy.

A commoner interference with reproduction consists of calluses resulting from fractures or other injuries to the bony girdle of the pelvis. Pelvic fractures with large calluses are especially common in nymphomaniac cows, and sometimes unfit them for breeding. Even if the nymphomania can be overcome, where such injuries are encountered during examination, the veterinarian should judge carefully of their importance and act accordingly. If they are of such a character as to prevent parturition, the animal should not be treated for the disease of the genital organs, but be sent to slaughter. Exceptions might be made in highly valuable cows, which, if the nymphomania or other disease is curable, might be bred and delivered at full term by hysterotomy. 


\section{CHAPTER VI}

\section{SYSTEMIC DISEASES AND DERANGEMENTS INTERFERING WITH REPRODUCTION}

Reproduction is the culminating function of animal life. The fundamental demand of the individual is the maintenance of life, and it is only when this has been reasonably assured that any additional power may be directed to the reproduction of the species. During the most active stages of growth, most animals do not breed. When old age comes, and the maintenance of nutrition is difficult, reproduction becomes uncertain or ceases. Throughout adult life, any element which interferes seriously with nutrition promptly imperils reproduction.

During the course of acute infectious diseases, with rare exceptions, male animals do not elaborate spermatozoa and females do not ovulate. At the same time, sexual desire ceases. In the pregnant female such diseases imperil the life of the fetus. So it is frequently said of cows that footand-mouth disease, pleuro-pneumonia, and similar fevers tend to cause abortion. This does not imply that the infectious fever directly causes the abortion, but rather that the infection present within the pregnant uterus, capable under favorable conditions of causing the death and expulsion of the fetus, is rendered virulent by the general depression of the system due to infectious fever. Such chronic, debilitating affections as the bone diseases-osteoporosis, rickets, spavin, ringbone, and others-commonly lead to sterility through their devigorating influence.

\section{A. OVERWORK}

Animals subjected to severe work are strongly inclined to be sterile for the time. In stallions which are being trained for the turf, there is usually temporary sterility, without any evidence of disease or degeneration of the glands. The resources of the animal are wholly consumed in the physical work which is demanded, and there remains no reserve force to provide reproductive energy during this period. 


\section{B. StaRvation}

Starvation exerts an influence parallel to that of acute disease. In the young animal the development of the sex functions is advanced or delayed according to the state of nutrition. In highly fed, vigorous heifer calves, ovulation and estrum may occur at six months, or even earlier. In countries where cattle are reared in a semi-wild state, constantly running at large where herbage is scanty, they rarely ovulate until nearly two years old, so that, although the bulls run in the herd, the heifers do not conceive until well grown. The nutritive supply beyond the necessities of the individual do not suffice for reproduction. In cattle more highly domesticated, if they are underfed or otherwise badly handled, ovulation or estrum frequently fails. I have observed two-year-old heifers, badly kept, though not extremely poor, which failed to come in estrum because of poverty, but bred promptly when the food supply was increased. Semi-wild cattle generally conceive in early summer, at the time when grazing is at its best and the animal has recovered its vigor after the privations of winter. The same rule applies to cattle stabled in winter and grazed during the summer. Animals which do not come in estrum in the stable or, doing so, do not conceive, frequently breed when increased vigor has been gained after grazing on good pasture.

A high state of nutrition does not necessarily accompany an abundance of food. Any food may be so damaged that its nutritive value may be lowered or destroyed, or, contaminated by various bacteria or fungi, it may become toxic. Mere quantity of food, and even of technical nutritive elements in the food, does not mean good nutrition. I observed a stable of cows and heifers which were getting an abundance of hay and grain of excellent quality, but all were unthrifty and emaciated. The non-pregnant animals did not come in estrum. One cow could scarcely get up when down. They were in charge of a lazy attendant, who gave them plenty of food with fair regularity, but did not keep them 
clean. So far as I could learn, the foundation for the debility was his failure to carry a reasonable supply of water a few paces. A change of caretakers promptly relieved the condition, and within a month cows began to show estrum and conceived promptly.

\section{OBESITY}

Abundant food and a high state of nutrition must not be confused with obesity. Obesity is not vigor, but may be the reverse. Vigor and an abundance of fat may coexist and are not incompatible. While obesity may sometimes cause sterility, it is far more frequently true that sterility causes obesity. That is, if a cow or heifer is sterile, she does no work, has abundant food, and is perhaps closely confined in the stanchion. No special functional activities are present, such as the secretion of butter fat or other product, which would consume the extra nutrient materials taken in the food. Consequently the animal takes on an abnormal quantity of fat.

In many cases the obesity of sterility apparently has a more interesting significance than the mere conversion of redundant nutritive material into fat. This is preëminently notable in cattle. The sterile heifer or cow frequently has irregular, lumpy deposits of fat, especially great, irregular lumps about the external iliac and ischiatic tuberosities. The hair becomes rough and lustreless. The form of the body becomes coarse and uncouth. If the genital organs are examined, no great departure from the normal is found. Perhaps the ovaries are rather small and the genital system is wanting in tone. The animal may be dull and estrum capricious.

The cause appears to be a disturbance of endocrine secretions, probably a faulty secretion of the ovaries themselves, which so modifies the nutritive system as a whole that fat deposits are made in a manner quite in conflict with the general physiologic laws of nutrition. Physiologic heifers or cows may carry quite as much fat as the obese, sterile animal, but it is more evenly distributed, the coat retains its 
lustre, the animal is bright and alert, and is fertile. I think that obesity as a cause of sterility in cattle has been overemphasized, though I admit the importance of controlling it by properly adjusted rations, combined with exercise.

A distinction needs to be made between obesity and overfeeding. While obesity is sometimes due to overfeeding, especially when combined with idleness, overfeeding may cause a serious impediment to sex functions without producing marked obesity. I have seen bulls which were fed an excessively large amount of very poor, hard, woody hay. The manager, wishing to prevent them from getting overfat, was feeding coarse hay in the dairy. After the cows had picked out the best portions, the refuse, consisting of weeds and very coarse stems of hay, was fed to the bulls, with a view to affording an abundant bulk of food having a very low nutritive value. The abdomen of each bull, though they were in moderate flesh, became excessively large. Some individuals lost almost completely their sexual desire. While ruminants are adapted to using large volumes of dry fodder, the hygienic maximum is readily exceeded, especially in herd bulls closely confined.

Even when such overfeeding with a poor quality of fodder does not seriously depress the sexual functions, it not infrequently interferes with the physical ability to copulate. The immense weight of the load in the rumen, with its pressure against the diaphragm and lungs, renders the bull slow, awkward, and short of breath. I have seen such bulls fail utterly to copulate. The great intra-abdominal weight and tension made mounting very difficult, and the enormous belly served to prevent coitus, since the pressure of the tense abdomen upon the rump of the cow forced the abdominal viscera against the diaphragm so that the bull was quickly out of breath and exhausted.

A breeding male should be fed like any other animal which one desires to keep in vigorous condition. The bulk of the food and the amount of nutriment contained should be adjusted to the needs of the individual, having due respect for size, age, and the exercise or work provided. 
The direct influence of certain foods upon fertility has frequently been asserted but is difficult to trace. It is a favorite habit of the breeder and dairyman, when his herd shows a low rate of reproduction, to attribute it directly to food-ensilage, alfalfa, clover, cottonseed meal, and wellnigh every food. Indirectly, foods modify reproduction greatly because the general vigor depends more upon the quantity and quality of food than upon any other one element.

\section{IDLENESS AND OVERFEEDING}

Impotence of the male is sometimes expressed chiefly by an absence of sexual desire in the presence of females properly in estrum, without any visible changes of the reproductive organs. Sometimes he may pay attention to the female, with more or less complete erection of the penis, then desist in his attentions, and turn away. When the next female is presented, he may show normal sexual vigor.

In some cases, there is said to be an individual psychic influence which prevents the male from copulating with a certain female. This is alleged to be especially true of individual stallions, to which certain mares seem to be repulsive, so that they refuse to copulate with them. The presence of young at the side of the dam is sometimes alleged to repress the sexual appetite of the male. This is usually seen, however, only in those males depressed in their vitality. It is almost, if not always, erroneous to attribute absence of sexual desire to the caprice or idiosyncracies of the male, by which a female of a certain type or color becomes repulsive to him and fails to arouse his sexual appetite; the real cause lies in bad management or in disease.

The tendency to loss of vigor on this account is usually not noticeable in the young, but, as soon as the animal has become mature and grown quite fat, the disposition becomes marked. This form of impotence is seen almost exclusively in those animals which are closely confined, highly fed, and not properly exercised. It is commonest in draft stallions, but is observed in bulls, especially of the beef breeds, and in male breeding animals of all species. It tends to disappear 
promptly upon a correction of the method of keeping, providing that it be applied sufficiently early.

The difficulty should be prevented by not forcing the young male designed for breeding purposes too rapidly in his development. He should be allowed only a moderate diet, with plenty of exercise and freedom, or be given actual work.

Upon the appearance of these defects in the mature animal, much can be done, if handled opportunely, by restricting the diet and causing an abundance of exercise. A healthy young draft stallion belonging to one of my clients refused almost wholly to serve mares. He was being highly fed and was getting a very limited amount of exercise, at a slow walk. Being appealed to for advice, I reduced his food ration one-half and prescribed eight miles exercise daily, at a brisk walk. Within a few days, his sexual desire had fully returned. He finished his season's work in good form, and was effective as a sire. The same general principles apply to other breeding males. In countries where cattle are habitually worked, the bulls are kept in breeding condition by moderate draft service. It would be well to imitate this plan in America, where bulls and stallions are not habitually used for work, but could readily render valuable service while being greatly benefited by the vigorous exercise secured. Under general conditions, it is perhaps the best possible and most economic manner by which sufficient exercise for these animals can be assured. Incidentally, it should be noted that such exercise or work tends very strongly to prevent that viciousness in bulls and stallions which renders them dangerous to their keepers.

Zschokke emphasizes the value of the proper selection of food for the purpose of arousing sexual desire, and insists especially that the hay should be aromatic, as should also the oats. Not only should they be well cured and sweetsmelling, but he believes that the admixture of certain stimulants, such as calamus, pepper, powdered mustard, and even powdered cantharides, are advantageous. $\mathrm{He}$ 
recommends that these remedies be fed with cut hay and that they be allowed for two or three days in succession and then omitted for the same length of time, when they may be given again. I doubt very greatly the value of aphrodisiacs, or sexual stimulants, in breeding animals. Sexual instinct is normal and so universal in animals of breeding age and in proper health that I fail to see the value of exciting sexual desire artificially. If sexual instinct is absent, it is because of some disease of, or depression in the general vigor of the animal, which can not readily be removed by aphrodisiacs. It has not been shown that the arousal of sexual appetite by means of these drugs insures or even favors fertility. A constant and wide distinction must be drawn between copulation and fecundation. The fundamental function of the male is the elaboration of virile spermatozoa, or male cells, and of the female to produce healthy ova. Copulation serves merely to transfer these fecunding cells from the testes of the male to the vagina of the female. Copulation is in vain without vigorous spermatozoa and healthy ova, and no data exist to show or suggest that any drug may directly cause or favor their development.

Certainly it must be admitted that tonics, alteratives, or other drugs capable of influencing favorably the restoration of a diseased animal to a state of health must also improve its reproductive powers, by restoring the equilibrium of the body and enabling it to perform better all its normal functions, among which is reproduction. The highest state of the general vigor of the body is, consequently, the most favorable condition for the production of virile spermatozoa and ova, which constitute the first essential in fertility .

Sometimes there is an apparent absence of sexual desire in young males when first brought in contact with estrual females for purposes of coitus. If such animals are turned loose with the females, the difficulty is generally overcome very promptly.

The preparation of breeding animals for the show is always dangerous for their sexual vitality. In order to get 
them in high condition, they are frequently closely confined and fed in such a manner as to produce the greatest amount of fat, in the hope that they may make a better showing and more certainly win a prize. It is an unfortunate fact that many of the most richly bred animals, designed for breeding purposes, which capture the most coveted prizes at the livestock fairs, have their breeding powers either temporarily or permanently destroyed in the process of feeding them for the show. There is no effective method for overcoming this except by the exercise of greater intelligence on the part of the owners of show animals, which may be furthered by judges in livestock exhibitions paying less attention to the amount of fat and more to the form and general vigor of the animal, in the allotment of premiums. In order to show breeding animals with safety, it is absolutely essential that abundant exercise should accompany the preparing process, if the sexual vigor of the animal is to be safely preserved.

The result is not the same with all individuals. There are some which can withstand almost unlimited abuse in this direction and continue to breed regularly, while others are very susceptible and soon become temporarily or permanently sterile. Once this sterility is established, the only thing that can be done is to correct errors in care by moderating the diet and enforcing vigorous exercise, which will sometimes, though not always, restore the sexual powers.

In a general way, the food of a breeding male needs to be rich in protein, as compared with the amount of hydrocarbons and carbohydrates. The commonest foods, when well grown and cured, are the best for the breeding animal, such as bright, aromatic hay and clean, well developed oats, along with grass, to which may be added, in the winter, roots and tubers. Rarely, if ever, is it necessary or even advisable or permissible to add to the food any sexual stimulant like pepper or mustard.

It should be observed that interferences with reproduction by confinement, overwork, overfeeding, starvation, and other imprudent details of handling exert their pernicious 
influence through two separate channels. In the first place, overwork or starvation, by lowering nutrition of mature genital cells, directly inhibits reproduction. The functions of the sex glands are such that neither the physical overexertion of the animal nor starvation can extend its influence beyond the duration of the abuse. The function returns with restoration of bodily vigor.

The indirect effects of these errors are far more serious and enduring. In all animals the genital organs commonly contain bacteria which, so long as the animal is healthy, may insidiously multiply at a rate barely to maintain their status. When for any reason the vigor of the animal is lowered, the genital tract participates in the depression and bacteria multiply and rapidly increase in virulence. Soon permanent injury of essential structures may occur, and reproduction becomes difficult or impossible. At one time there was much said about "fatty degeneration" of the genital organs as a bar to reproduction in fat animals. I know of no adequate ground for such an assertion. In most animals the genital organs are among the last to act as depositories for fat. A notable exception is the bitch, in which the uterine ligaments are loaded with fat, but these are not essential and the fundamental organs are not visibly involved.

As knowledge of the diseases of the genital organs advances, the belief in the direct influence of food, work, and housing recedes, but their indirect influence, through the general lowering of vigor and a correlatively increased virulence of bacteria present, comes into greater prominence. The proper nutrition, housing, and physical exercise of breeding animals is one of the great fundamentals in the problem of reproduction, because the vigor which these induce constitutes an invaluable armor against the extension of infection.

\section{E. Excessive Sexual Use}

The number of copulations which a male animal can effectively perform is an important question for the breeder. It 
probably varies greatly with different individuals. Naturally, the power of a male does not depend so much upon the number of females with which he is expected to copulate as upon the number of copulations essential to produce fertilization. It has been determined by careful investigation that, when the number of copulations during a given day is increased, the abundance of spermatozoa in the semen rapidly decreases and, if this service is pushed too far, the spermatozoa fail almost entirely, causing an interruption of the fertility of the animal. Much will depend upon the age and vigor of the male animal.

Zschokke states that 80 to 100 cows may be bred to a single bull when the animal is kept confined and his service somewhat regulated by the breeder but, if allowed to consort with the cows at pasture, not more than 50 should be allowed. In the western range country of America, the allotment is very much smaller and it is considered safer that there be one bull to each 20 or 25 cows. This is necessitated largely by the fact that, during the principal breeding season, the bulls have not yet fully recovered their vigor from the long and trying winter with scant food supply. Zschokke further holds that the bull should not be allowed to serve more than three cows in any one day and that, under such conditions, there should be one or two days per week of complete rest.

Many horse-breeders apply a similar standard in the use of stallions. The total number of females, however, may be greatly increased if the service is distributed throughout the year. Noted stallions, it is claimed, have served successfully, and without apparent injury, two or three hundred mares during the year, but in such cases the service was evenly distributed over the entire period.

Zschokke draws attention to the erroneous belief that a long abstinence from coition serves to store up a large amount of semen, which will answer for a series of copulations. There is no reservoir for such purpose, and all spermatozoa which may be formed and not used in copulation soon disintegrate and are expelled or absorbed.

Excessive sexual use is largely a comparative term, in- 
dicative of a relative over-use under existing environment. There is no question but that a breeding male may be readily over-used and that such is a frequent occurrence, but more commonly the use is relatively excessive because of bad management, where proper handling of the male would enable him to make the number of services demanded, safely and efficiently.

My belief is that most males are called upon to copulate too frequently. The most hygienic rule for reproduction in the higher mammals is monogamy, and any departure from that ideal is necessarily at some peril. Since great sires which have cost heavily are expected to repay their owner by begetting a large number of highly valuable young, polygamy is crowded to its limit, and beyond. There are two fundamental facts bearing upon sex hygiene which every breeder should know and heed. Most breeding animals of both sexes carry important infections in their genital tracts, and coitus stimulates and heightens genital infections. The number of copulations which a bull or stallion may safely perform depends first upon the degree of infection or disease in his genital organs, and, second, upon his general health and vigor. While many accounts are published of the great numbers of copulations made by various bulls or stallions, or the large number of progeny to their credit, they constitute an infinitesimal proportion of the total. Clinically we observe bull after bull breaking down genitally under what would appear to be very moderate use. Admittedly the infection was probably there already but the repeated copulations served to vitalize it and establish serious disease, precisely as coitus intensifies syphilis and gonorrhea. Moderate work of the male during the breeding season is not injurious, but favorable. It is a constant observation that a male breeding animal which is regularly exercised or moderately worked is capable of rendering a greater number of effective services than one which is closely confined.

Some writers condemn such foods as oil cake and malted 
grain for the male and prefer the various grains, especially oats, barley, peas and beans in moderate quantity. To these should be added hay and grass in sufficient amount. Roots, tubers, meal and molasses are not considered essential as a part of the food supply for breeding males, but may, by aiding in the maintenance of a good state of digestion, contribute to the general vigor.

The feeding of salt has long been regarded as important for maintaining fertility, and some have superstitiously accorded it a prominent place, but it can not act directly and serves merely to favor fertility indirectly by aiding digestion and assimilation.

Apparently some believe that a male breeding animal requires a different kind of food from the female. Fundamentally that is absurd, but when a bull is kept absolutely confined and is wholly idle physically, he requires less food per unit of body weight than a cow which is yielding a liberal quantity of milk and probably gets some exercise daily in the open field or paddock. The feeding needs to be adjusted the same as it would be between a cow which is milking heavily and another which is dry.

\section{F. ONANism, or Masturbation}

Masturbation, as a cause of sterility, is mentioned chiefly in the stallion and the bull. It is said to be very common in improperly kept stallions. Spinola (Handbuch der spec. Pathol. 1858, II Bd.) records this vice in the bull.

In the stallion there is an erection, the penis is moved up and down, imitating coitus, and finally ejaculation occurs. In the bull, the ejaculation is induced by an erection and the alternate protrusion and withdrawal of the penis. This is generally seen in idle males which are closely confined, over-fed, and sparingly used for breeding, and in racing stallions when sexual debility is brought about by hard work. It is undetermined whether the vice causes the impotence or the sexual weakness induces the masturbation.

Correction may be brought about by such feeding, exercise and other care as will maintain the general vigor of the 
animal. Moderate work or exercise and judicious feeding, or, in animals which can not be worked or artificially exercised, the allowance of freedom in a commodious paddockstill better, in a properly enclosed pasture-tends largely to prevent or cure the vice. Once the habit has become fixed, it should be prevented by a shield so arranged as to cause pain whenever the penis is protruded. It is a vice of idleness and debility, and any and all remedies must fail in their aim until the return to normal vigor is attained. 


\section{CHAPTER VII}

\section{COITAL INJURIES}

In the wild state, severe and fatal battles between rival males are not infrequent and the same prevails to some degree among domesticated animals, although, as a general rule, if several males are kept together they lose much of their combativeness. I have seen an instance of a fatal fray between two rams which had been regularly kept together.

When a strange male wanders into a herd or group of females with which a male is regularly consorting, there is at once a determined fight between the two, which ends only with one or the other becoming vanquished. In these battles, injuries of the most diverse character occur, according to species and chance. The only method of prevention is the proper confinement of male animals. In most countries there are laws against the running at large of male animals but these are not enforced against carnivora. This exception should be overcome by the confinement of all females.

There is further danger to the male, especially horses and cattle, of injuries in attempting to escape from or break into enclosures in order to reach females. The appearance of a female in estrum near the enclosure of a male causes him to make violent attempts to escape from his confinement and reach the female. Consequently it is important that enclosures intended for the confinement of male breeding animals should be especially secure and, as far as possible, all elements avoided in their construction which would endanger the animal in an effort to escape.

In the preparations for copulation there is little danger except in case of horses. Breeding customs and convenience bring into the procedure a period of "trying" or "teasing" of the mare by the stallion, to determine the existence of estrum and bring about the desired degree of sexual excitement before permitting an attempt at copulation. In this act there are numerous dangers, especially to the stallion. 
It is desirable, if not frequently essential to safety, that special conveniences for restraint be provided for this purpose. They should consist ordinarily of a strong, solid wall about three feet high and ten to twelve feet long, with a rather broad and rounded top. The chief object of the structure is the avoidance of kick wounds which may be inflicted upon the stallion by the mare. To this end it should be merely of sufficient height to guard against such an accident and sufficiently resistant that the mare can not kick through it. A single pole to separate the stallion and the mare is insecure and hazardous, as either may kick through beneath it and injure the other.

It is highly essential that the top of the structure be rounded, smooth, and free from projections. Either the mare or stallion may get beyond the control of the groom and kick or leap upon the structure. The wall should be low enough that either animal, getting upon it by rearing or kicking, may readily free itself without injury. I once attended a stallion, which, having passed beyond the control of the groom, reared to mount the mare and became impaled upon a projecting post at one end of the structure, causing a serious hernia and almost eventration. Posts should not extend above the top of the structure or, doing so, should continue so high as to make injury in this manner impossible.

\section{A. Kicks and Fractures}

When this preparatory period has been passed and copulation has been decided upon, there arise fresh dangers to the stallion from kicks by the mare while approaching her or in the act of mounting. Two methods of avoiding accidents at this point are used-without and with hobbles. In the first method, when both stallion and mare are well broken and controllable and competent grooms have each well in hand, the stallion should be caused to approach the mare's head, and then allowed to mount from the side instead of from the rear. If both are kept well in hand, any attempt to kick on the part of the mare should be at once counter- 
acted by vigorously drawing her head toward the stallion, thus turning her heels from him. When coition has been completed and the stallion is dismounting, the same rule should constantly be applied and the mare at once caused to face the stallion in order to avoid kicks.

Owners of valuable stallions generally prefer to obtain yet greater security by the application of hobbles. Two forms are used, of which there are numerous varieties. By one plan, a hobble is buckled about each hind pastern and to each hobble is attached a rope of sufficient length, the two free ends of which are carried forward between the forelegs and securely tied to a strong collar or carried upward on either side of the neck and tied on the top sufficiently tightly to prevent the mare from kicking backward any appreciable distance. By the second plan, the hobbles are attached to the hocks instead of the pasterns. In this case, each hobble is branched; one portion of each is attached above and the other below the hock of each hind leg, and by ropes or straps fixed forward the same as in the first. The latter plan possesses some points of superiority: the mare is not so liable to injure herself by becoming entangled in the ropes, and the stallion is probably also exposed to less danger of getting his foot caught in the securing apparatus. Whatever the form of breeding hobbles or other confining apparatus, they should be secure and strong. Nothing can well be more dangerous for the breeding stallion than weak hobbles which lead to a false feeling of security and throw the otherwise careful groom off his guard. I was called to attend a valuable stallion suffering from a compound tibial fracture, the result of a kick during attempted copulation. Because the mare was not properly in estrum, or for other reason, she was unexpectedly ill-natured. When the stallion was attempting to mount she commenced to kick, the old hobbles, in which the owner of the stallion had trusted, gave way, and the fatal accident at once followed. The breeder should never trust partly to hobbles and partly to the gentleness of the mare or good luck. If hobbles are to be trusted at all, the breeder should know that they are so 
strong that no mare can break them, always allowing a safe margin of strength beyond that considered essential, and that the fastenings are secure in every detail, making slipping or accidental detachment impossible.

If accidents are to be avoided, it is highly important that the mare is properly in estrum and that she is not frightened or angered. Gentleness and patience in the handling of both stallion and mare and postponing copulation until the proper moment are always essential elements of safety in breeding. Some breeders place a twitch upon the mare's nose until the stallion has safely mounted her. Much of the danger arises through the lack of ability upon the part of the groom to diagnose estrum. Some grooms think when a mare crowds against the teasing-pole and urinates, she is in estrum, when as a matter of fact she is angry.' A groom should learn that a mare, not in estrum at all, when confined to be teased by a stallion and unable to get away, will crowd toward him, squat, ana expel small quaritities of urine simply as an expression of anger (a gelding, when tightly confined, occasionally gives vent to his anger by a characteristic neigh and the expulsion of urine in small quantities). If opportunity offers, such a mare will kick viciously. Some stallions are so poorly broken that they rush at a mare violently and, if opportunity is given her, she will kick from fright in self-defense. The proper knowledge on the part of the groom, enabling him to diagnose estrum, and the proper control of the stallion, are prime essentials to safety.

In breeding mares to jacks, it is customary to place the mare in a pit where she is closely confined and rests upon a plane a foot or more lower than that upon which the jack stands. This not only gives an advantage to the comparatively small male by affording him an elevation, but also secures him against kicks from the mare, a highly essential precaution because, since the mare ordinarily resents copulation with the ass, the act is largely in the nature of rape.

Disparity in size is a common cause of fractures in cattle of both sexes. When a young bull attempts to copulate with a large cow, he is liable, at the moment of the sudden copu- 
latory thrust, to lift his feet entirely from the ground, so that they glide forward, he falls backward, and may break his spine in either dorsal, lumbar, or sacral region. In one case brought to my attention, a similar accident occurred to a cow which, being in heat, mounted one of her mates, slipped, fell, and fractured her spine so that she had to be destroyed. The danger of falling when the ground is slippery or copulation is undertaken upon a concrete or other smooth floor, is to be obviated by the removal of the causes.

In females mated with too large a male, fractures of the spine, pelvis, and limbs occur. The danger is greatly aggravated by slippery or uneven ground or by other unfavorable surroundings. Injuries to the female because of the great weight of the male are not rare though far less common than one would be inclined to expect when the disparity in size is considered. It is not rare to see females served by males two, three, or more times as heavy, and yet escape injury. In the smaller species, as the pig and dog, the female can quite readily drop to the ground under excessive weight and escape injury, but in the cow and mare there is danger of serious injury when the weight of the male becomes too great. Fractures from this source are most common in cattle and generally the result of a fall at the moment the bull makes the violent copulatory thrust. Some breeders overcome this danger by using a breeding rack or cage, as described above, so that the heifer can not be forced forward and down. Each side of the rack presents a shelf upon which the fore feet of the bull rest, preventing his entire weight from falling upon the heifer. Sometimes an awkward helper holds the head of the cow to one side and, when she moves forward, draws firmly on the halter, bending her head sharply to one side and causing her to fall. Either she should be left free or any restraint should be exerted by two men, one on either side, thus pushing directly backward. Nymphomaniac cows suffer frequently from pelvic fractures, which will be discussed under Cystic Degeneration of the Ovaries. In one instance a heifer of about five months and weighing about 400 pounds was served by 
a bull weighing 2000 pounds and suffered a fracture of the pelvis. The injury was not sufficiently serious to prevent her giving birth in due time to a calf. In another instance, a sucking filly was mounted by a large stallion which had accidentally broken into a field with her, causing dislocation of the sacro-iliac articulation on one side and fracture of the iliac shaft on the other, rendering her useless for labor. She was bred at two years, and destroyed at three years, because of irremediable dystocia.

It is desirable that young females which come in estrum very early in life should be securely segregated from mature males, not alone because of the undesirability of very early breeding, but in order to avoid serious injuries from disparity in size. It is also essential to remember that a male breeding animal regularly confined, in case of accidental escape, will attempt rape upon the most immature young, as in the foregoing case, with serious or fatal results.

The violent copulatory thrust of the bull occasionally causes a fracture of the base of the tail or the caudal end of the sacrum. I have observed it most frequently in Jerseys, perhaps because the sacrum is relatively smaller and less resistant than in other breeds. It seems to occur at the moment of the copulatory thrust. So far as known, it is not subject to remedy. The result is usually caudal paralysis, with a lateral curvature of the tail and caudal end of the sacrum, disfiguring the cow and destroying the protective value of the tail against flies, without otherwise injuring the animal.

\section{B. False Copulation}

False copulation, or entrance of the penis into the anus instead of the vulva, is possible in most domestic animals. Harms records it as occurring in the mare, cow and sow. I have observed it only in the mare and, in all, four times, though I have known of numerous other instances. It is by no means rare and is highly dangerous. Its causes are various, but it is probably due largely to resistance on the part of the female. Sometimes a female is thought to be in es- 
trum when she is not. Perhaps she has been in estrum an hour or two before and the owner or person in charge believes it best to use forcible restraint in order that copulation may occur. Some, erroneously believing that, when estrum fails to appear at the expected date, it may be brought about by rape, proceed to force coitus. Coition is safe only when estrum is present in the proper degree. The mare has ample power to close the vulva against the ready entrance of the penis, thus causing it to glide upward and forward against and into the anus, which opening may be more readily forced.

The accident is also invited in aged cows or mares with pendulous abdomen in which the tension upon the rectum draws the anus and superior commissure of the vulva downward and forward, thereby causing the vulvar opening to approach the horizontal instead of the perpendicular. The penis then tends to glide forward and slightly upward over the oblique vulvar opening, to strike against the inferior surface of the tail and become deflected into the anus. It may be purely accidental. Harms thinks it may result from smallness of the female. All cases I have observed have been in mares of medium or large size.

The extent of the injury varies. Apparently, the accident may cause little or no injury in some cases, though, of those which are brought to the attention of the veterinarian, a large proportion are fatal. The extent of the lesions will vary according to the size of the penis, the violence of the copulatory act, and the quantity and character of the feces in the posterior portion of the rectum. Should the rectum be quite empty or filled only with pultaceous feces, as in a cow, or in a mare on green food, the penis may force its way along in the bowel unless it is caught in the folds. If the rectum is impacted with dry feces, as is usually the case in the mare, the penis is deflected and tends to pass through the rectal walls. The rupture may occur either into the peritoneal cavity or into the periproctal connective tissue. In the former case, in the mare, the opening is so great that feces drop at once into the peritoneal cavity and cause pro- 
found irritation. No sooner has the stallion dismounted than the mare trembles, looks anxiously at her sides, may pass some feces and blood, moves uneasily, and may lie down, but does not roll violently. The pulse is rapid and weak, the breathing is shallow and quickened, the abdomen is held rigid, and the patient looks tympanitic. The symptoms resemble in many particulars those of rupture of the stomach. The diagnosis is readily made by manual exploration per rectum, since the hand passes easily out through the rupture into the peritoneal cavity. In one of my cases of rupture into the peritoneal cavity, the mare lived some eight hours; in another, less than four hours.

Not all cases lead to rupture into the peritoneal cavity. In one instance under my observation, in an old brood mare, no history of injury was obtainable, and apparently the rupture had not occurred during the several years of ownership by her last proprietor. She died under symptoms of colic. Autopsy revealed a large intra-pelvic sac, opening into the rectum, which had been habitually filled with feces but, becoming over-distended, had obstructed the bowel and caused a rupture of the sac into the peritoneal cavity. It was almost certainly the result of an old penial wound.

In another case I recognized the accident at the time of its occurrence. Blood and feces were voided, and the perineal region was swollen. Manual exploration revealed a large rent through the rectal walls into the periproctal connective tissue, six to eight inches from the anus. The resulting sac contained about two liters of feces impacted into the readily distensible wound. The feces occupying the sac and those from the rectum immediately anterior to the rupture were carefully removed by the hand, and the sac was cautiously flushed out with an antiseptic solution. This method of handling, repeated twice daily, along with restricted, laxative diet and complete rest, brought about a prompt recovery with little sacculation.

According to Harms, false copulation in the sow is usually followed by no more serious consequences than a swollen anus, accompanied by a bloody discharge. 
In the mare the accident is preventable by ordinary precautions on the part of the stallion groom. Service should not be attempted in case of a mare not certainly in proper estrum. It is the duty of the groom to see that the penis of the stallion is properly entering the vulva of the mare. If the mare is kicking or otherwise violently resisting the stallion, he should be promptly withdrawn, since copulation under such circumstances is unwarrantedly dangerous for both animals. Such violent resistance of the mare is sometimes cited by the groom as an excuse for his failure to know that the penis of the stallion was being properly entered in the vulva, but it is not valid. The mare or cow with retracted anus should be placed with her hind feet on a lower level than her anterior members, leaving the ground upon which the male is to stand at a higher level and thus bringing the vulvar opening more nearly perpendicular. In the cow and sow, the accident is not usually preventable.

The prognosis of penial injuries to the rectum must be based largely upon their position and extent. When the lacerations perforate the peritoneum, allowing feces to escape into the peritoneal cavity, the injury is essentially fatal: veterinarians have not yet succeeded in successfully handling such lesions. When the rupture of the rectal wall occurs behind the peritoneum or, occurring more anteriorly, does not perforate it, the prognosis is good, if timely surgical aid is given, though, after a long time, serious or fatal results may follow the impaction of feces in the sac formed in the pelvic connective tissue. The pressure of the feces escaping from the rectum tends constantly to push the loose connective tissue aside and, eventually, to form a large sac, which is constantly filled with fecal masses.

The handling in such cases consists of gently removing the feces from the sacculated wound cavity in the pelvic connective tissue and cautiously flushing it out at least twice daily with a mild antiseptic solution. At the same time, the rectum should be manually emptied as far as the attendant can reach and the feces thus prevented from dropping into the sac. The patient should be kept at rest on a very scant, 
laxative diet during the treatment, until the rupture is quite healed, as it is highly important to guard against extensive sacculation in the part, which can be done only by avoiding the accumulation of feces in it.

In some instances, the injuries to the rectum from the entrance into it of the penis are comparatively trivial-merely wounds of the mucosa and part of the muscular walls, with the passage of bloody feces, some swelling about the anus, and other slight symptoms of injury, which readily pass away under repeated antiseptic enemata.

From a forensic standpoint, the owner of the stallion is probably always responsible, since the accident is preventable with proper care upon the part of the groom. Such were the findings in the only case which I have seen tested in the courts.

\section{LACERATION AND Rupture of the VAgina}

The vagina, especially of the mare, is not infrequently lacerated or ruptured by the penis of the male. The size of the penis of the stallion does not bear a constant ratio to that of the body, but is comparatively excessive in coarsely bred animals with long legs and large heads. Such stallions, copulating with small mares, constantly tend to produce vaginal injuries. Sometimes it is merely a slight laceration in the mucous membrane, which may cause limited hemorrhage and some straining; sometimes the lacerations in the mucous and muscular walls are extensive and induce marked symptoms of wound infection in the vagina, with tumefaction, discharge and straining; sometimes the vaginal walls are completely ruptured, with great danger of fatal peritonitis.

In one instance I investigated, a series of injuries was caused by a recently introduced stallion, which had been put to service among comparatively small mares. Almost every mare served by him exhibited signs of vaginal injury: some were severely lacerated; in one there was a fatal rupture. The horse was found to be an overgrown, coarse, leggy grade draft stallion about eighteen hands high, with an enormous 
penis which would have been dangerous with heavy draft mares, while in his neighborhood most animals were of small roadster or common stock.

When such injuries occur, there is observed an abnormal amount of straining immediately after copulation, with, perhaps, some discharge of blood from the vulva. Later, swelling of the vulva and a muco-purulent discharge may occur.

If the wound penetrates the peritoneal cavity, septic peritonitis is highly probable, with early collapse and death.

Injuries to the mare from this cause should be obviated by means of a roll or padded surcingle encircling the body of the stallion and passing just in front of the sheath in a manner to prevent the penis from entering the vagina to its full length, the pad or roll being adjusted in size according to the case. The diameter of the roll should always be ample, since little fear need be entertained that preventing the entrance of the entire length of the penis will unfavorably affect the results of copulation, as general observations show to the contrary. In constructing this roll or pad, an ordinary surcingle may be used and a tube made of sheeting or other sufficiently strong material, open at each end, which is slipped over the surcingle. Padding of cotton, oakum, or other material is then to be inserted into the tubular covering until it is tightly filled so that it presents a diameter of 4 to 8 inches in that part of the surcingle to come in contact with the abdominal floor, after which the open ends of the tube are to be secured to the surcingle and closed by tying. When the stallion is ready for service, the surcingle is passed around his body just in front of the opening of the sheath, and secured by buckling. In copulation, only that portion of the penis projecting beyond the roll can enter the vagina.

Non-fatal laceration of the vagina should be handled along general antiseptic lines. The practitioner should remember that this organ reacts strongly to irritants and that thorough disinfection should be brought about largely by mechanical flushing rather than by heroic attempts to destroy the micro-organisms in position.

Coital injuries to the vaginae of cows have not come un- 
der my observation. I am of the opinion that they are very rare. The vagina of the cow is very long and elastic. During estrum it is highly lubricated with an abundant, thin mucus, rendering it virtually impossible for the penis of the bull to become caught in the lateral walls. If the heifer or cow were not in estrum and the vagina were dry, the case would be quite different.

I have seen a number of cows which could not copulate without great peril because of severe vaginal stricture. Perhaps that is the explanation of the cited instances of vaginal injury by the penis.

Lacerations of the vagina by the penis are also liable to occur in other domestic animals, though less probable than in the mare. Their symptoms, prognosis, and treatment are essentially the same.

\section{Rupture of the Uterus by the Penis of the Bull}

Duranton ( ${ }^{1}$ ) states that in one instance the penis of the bull passed through the cervical canal and ruptured one of the uterine cornua. This is highly improbable, if not absolutely impossible. It is almost certain that the real culprit was a man, who had either committed sadism or had attempted to "open the womb".

Veterinarians should be always on their guard in accepting accounts of injuries to the vagina or uterus of the cow by the penis of the bull. Many owners and caretakers have an abiding faith in the efficacy of "opening the womb" just before coitus, as a remedy for sterility, and not infrequently are not publicly proud of their faith. As a consequence, the operation is often kept secret, especially if the layman has become frightened because hemorrhage has followed his efforts. Later, if disaster follows, the true history is not given the veterinarian. Often a workman will undertake such an operation without the knowledge of the owner, and, suspecting he has injured the animal, quite naturally keeps his bungling work a secret, so that the bull is blamed with having caused the lesions.

'Journal de Lyon, I898. 
In examining a sterile heifer, I encountered a chronic, indurated, cervical abscess, which is shown in Fig. 39. The origin appeared perfectly clear to me. Upon inquiry, it was at once admitted that a man had attempted to "open" her to cure the sterility. Repeatedly I have observed injuries to cervix and uterus by veterinarians.

E. Alleged Rupture of the Urinary Bladder BY THE PENIS

Uebele, cited by Harms, reports that a heifer died from rupture of the urinary bladder a few days after coitus. Any veterinarian who has passed a catheter through the urethra of a cow should appreciate the difficulty of the passage of the penis of the bull through that narrow canal, obstructed at the beginning by the valve-like cul-de-sac. An ignorant meddler, mistaking the urethra for the cervical canal, might cause such injury. The veterinarian should take every possible precaution against being deceived, whenever the lesions appear so removed from the ordinary. Under certain abnormal anatomic conditions, the urethra might well suffer severe lesions during attempted coitus, especially when the lower portion of the hymen persists. The hymeneal remnant slopes somewhat upward and backward, forming a sort of pouch in the floor of the vulva, with the urethra at its bottom. The membrane, catching the penis, may deflect it downward into the urethra, and either the penis or the urethra and bladder, or all, may suffer injury. But that is aside from the point. The healthy normal genital tract of the heifer or cow is not readily damaged by the penis of the bull during coitus. When such post-coital injuries are recognized, the diagnosis as to cause should be very guarded. Under certain conditions, a false diagnosis might well lead to litigation, especially when a bull belonging to one man breaks into an enclosure where cows belonging to another are kept.

In all such injuries the veterinarian should not fail to give consideration to two other possibilities, each of which is more probable than penial injury-sadism (which see) and 
meddling by incompetent persons in an effort to "open the womb".

In the mare, this accident would seem more possible because of the very wide, valveless meatus, through which several fingers may be passed, but the penis of the stallion is large and the glans very broad. Elsewhere I have described a filly with a persistent, though perforate hymen, which deflected the penis of the stallion downward and apparently caused a wide dilation of the urethra but no harm to the bladder.

\section{F. Vaginal Hemorrhage Following Coitus}

Hemorrhage following coitus is not rare. It often follows copulation of a virgin female, owing to slight lacerations of remnants of the hymen. In cattle, when the granular venereal disease is very severe, hemorrhage in both sexes often follows coitus. This will be discussed in a succeeding chapter. If stricture of the vagina is present, coitus is always liable to cause hemorrhage. Often lacerations of the vagina during coitus, already cited, cause more or less hemorrhage. Tumors of the genitalia which are lacerated by coitus may bleed quite profusely.

\section{G. Urethral Hemorrhage in Stallions}

Hemorrhage from the urethra following coitus was observed by me in a valuable racing stallion. The patient, a famous imported thoroughbred in fine physical condition, had been a successful sire. He was very vigorous in copulating. Without warning, he suddenly began to bleed profusely from the urethra after each coitus. The act of copulation appeared normal, and was completed promptly and naturally. A moment after dismounting, before the penis had retracted, there was a sudden gush of bright red blood, estimated at about one pint, after each copulation.

The source of the hemorrhage could not be determined, though it probably emanated from the pelvic urethra, vesiculae seminales, prostate, or Cowper's glands. Its cause was 
not learned. He was withdrawn from the stud, kept sexually quiet, exercised freely, and fed lightly. Later he was returned to the stud. He had been kept in sight of the mare while the breeding hobbles were being applied, during which time he became highly excited. This was changed so that the mare was properly shackled before he was brought into her presence. He was then allowed to copulate promptly, before excessive erection could occur. The difficulty ceased.

\section{H. Contusions of the NeCK And Withers from Bites}

In the mare, injuries to the neck and withers are liable to occur from bites by the stallion during coitus. This is probably most common among heavy draft stallions, some of which are very rough and cause more or less serious lacerations or contusions, at times leading to fistulous withers. There seems to be no means for breaking the stallion of the vice. The only effective method of control is the application of a muzzle prior to coition.

\section{Penial InJuries}

Injuries to the penis of the male just before, during, or after copulation are by no means rare. The penis of the stallion is most liable to injury from kicks by the mare just as he mounts or dismounts. The prevention of such injuries has been discussed above. Such contusions of the penis during its great vascularity in the breeding season are very difficult to handle. The stallion needs to be kept quiet and free from sexual excitement. The penis should be retained at rest within the sheath, by means of a suspensorium, and the resulting local inflammation ameliorated by the applicaton of cooling astringents such as lead acetate, hamamelis, tannin, belladonna, etc., combined if necessary with antiseptics. Generally the patient may have quiet walking exercise, a restricted laxative diet, saline laxatives, or in case of serious disturbance, prompt cathartics of the hypodermic alkaloidal group (eserin, arecolin, etc.). 
Lesser injuries to the penis of the stallion are caused by the tail hairs of the mare, pushed into the vagina by the glans, which, being drawn tightly across the penis, cut the organ. This is to be prevented by the stallion groom, who should draw the tail of the mare aside at the commencement of the copulatory act; apply a bandage about the base of the tail extending low enough to secure any hairs that might be caught by the glans penis; or braid the hairs of the tail upon its dorsal surface.

The danger of injury to the penis of the bull is increased when a large male attempts to copulate with a small heifer, with a very narrow vulva and perhaps important remnants of the hymen. The presence of severe granular venereal disease lessens the caliber of the vulva and the roughness caused by the nodules increases materially the resistance to the entrance and the two combined, with the pain caused by the forcible contact, render injury more probable. Sometimes the penis of the bull withstands violence surprisingly well. I examined a neuter, or freemartin, with severe lacerations of the vulva and periproctal tissues. The owner related that, as the "heifer" would not come in estrum, he decided to stimulate its appearance by causing the animal to be raped by the bull. (The practice of raping females which fail to come in estrum is not rare amongst a careless, ignorant class of breeders.) The penis of the bull had caused serious injury to the neuter, compelling prompt slaughter. The penis of the bull escaped known injury.

Coital injuries to the penis and prepuce of the bull are probably more common and vital than those occurring to the genital canal of the cow. Exposure to minor injuries is constant. In cattle the universal presence of the granular venereal disease causes a constant genital catarrh, which, escaping at the inferor vulvar commissure, befouls the vulvar tuft of hairs. The muco-pus forms hard, dry crusts upon the tuft. The sticky muco-pus also catches up and incorporates in the crusts various foreign bodies. The encrusted vulvar tuft is liable to become caught and forced 
into the vulva alongside the penis, abrading it and threatening to transfer to the abraded parts any infection included within its mass. The injury can and should be prevented by keeping the vulvar tuft clean by washing.

In abattoir bulls, the penis is often found eroded, owing to the withdrawal into the prepuce of loose, dirty hairs from the preputial tuft, but I have not observed this in breeding bulls. Some breeders attempt to prevent the pulling of hairs into the prepuce by clipping away the preputial tuft. This invites another, and equally important danger from the multiple minute abrasions of the prepuce and glans from the short hair stubs.

"Broken penis" in the bull is often reported. It is believed that the penis becomes caught against the side of the vulva or elsewhere and the animal at the moment of making the violent copulatory thrust, doubles the organ sharply upon itself, "breaking" it or otherwise causing serious injury. Sudden and violent bending of the erected penis causes such injuries to its tissues that the "broken" organ remains curved or bent at an angle. That portion beyond the point of injury does not become erected. Thus the animal is rendered useless as a breeder or the injury causes severe balanitis or inflammation of the prepuce, leading to adhesions so that the organ can not be protruded. Clinically I have observed the injury following, and apparently due to coitus by a large bull with a heifer. In one instance, the breeder believed the penis of the bull had been injured in casting him by the compression method, in which the noose about the flank crosses the penis just in front of the scrotum. I have cast bulls so frequently by this method, without observing injury, that I could not accept his conclusions regarding the cause without important reservations. The injury is liable to pass unnoted for several days, until the bull is brought out for breeding and is found to be incapable of copulating, or capable only with difficulty. A narrow vulva, sometimes accentuated by great swelling of the vulvar mucosa because of severe granular venereal dis- 
ease, important hymeneal remnants, vaginal stricture, or other obstacles to the passage of the penis into the vagina, tends constantly to cause the penis to be violently bent, most readily at the base of the glans, where the encircling prepuce is attached. At the same moment the prepuce is perhaps overstretched.

Both penis and prepuce become inflamed, swollen, and sensitive. Protrusion of the penis becomes very difficult or impossible. Frequently the pain is too great to permit copulation. The swelling and the pain about the base of the glans are very marked.

The prognosis is good. The bull usually recovers anatomically and functionally. Exceptions may occur, owing to chronic irregular induration of the corpus cavernosum, which causes the penis to become bent in such a manner that copulation becomes difficult or impossible. Such injuries to the penis are preventable where the causes are recognizable in advance. Once they have occurred, the handling consists fundamentally of sexual rest and antisepsis. The sexual rest should be as complete as practicable without unnecessarily curtailing physical exercise. On no account should attempts at copulation be permitted until recovery appears to be complete. Since in the bull the prepuce is nearly always involved, it should be kept clean. In addition, warm douches exert a favorable action upon the inflamed penis. The douche should consist of warm physiologic salt solution or a very weak, warm, antiseptic liquid, as a 0.25 per cent. Lugol's solution. The salt solution douche may be repeated twice daily, or as frequently as may be desired in an individual case. The Lugol's solution can not as a rule be applied more than once, or at most twice daily, without causing irritation. If the parts are very irritable, iodoform suspended in oil may be used instead of the douches.

The best instrument for applying the douche is the soft rubber catheter seven feet in length, designed specially for this purpose. Or one may substitute for this the rubber 
horse catheter, the length of which is to be supplemented by splicing with two or three feet of ordinary tubing, attached to a hospital irrigator. The catheter should be cautiously pushed through the sheath into the prepuce, the irrigator elevated one to two feet above the bull's back, the sheath opening compressed about the catheter with the hand, and the douching fluid caused to flow into the sheath and prepuce until they are fully distended, when it should be allowed to escape, and the process repeated as often as considered expedient.

\section{J. Rupture of the Prepuce}

Rupture of the prepuce sometimes occurs, as shown in Fig. 60, presumably due to the violence of the copulatory thrust, associated with a narrow vulva, hymeneal stricture, or possibly other unfavorable anatomical condition. How common it may be, I am unable to state. My one example, Fig. 61, is from the abattoir, and hence without clinical history. Clinically, rupture of the prepuce is subject to definite diagnosis only by the forcible protrusion of the penis, as already described. It may be suspected by a history of marked hemorrhage following the coitus during which the lesion has occurred.

A slight rupture may perhaps recover without impairment of breeding efficiency, but severe or extensive rupture probably closes the breeding career of the animal, because the ruptured parts may, in healing, become adherent to the surrounding framework of the sheath, incarcerating the glans penis and causing permanent and irremediable phimosis.

The prevention of such injuries is more important and practicable than their handling. They are most liable to occur in confined males which are suddenly brought in contact with females, not always in a proper state for coition. The female may be nervous or timid at the approach of a strange male or, still worse, estrum may not have reached its fullness or may have begun to decline so that she will not 
stand well, and owing to her resistance the accident may occur. Slippery or uneven ground may also render the act insecure and lead to injuries to the penis.

Prevention should be based chiefly upon the avoidance of these and other dangers. With cattle especially, it is important that the female be properly in estrum, and that the

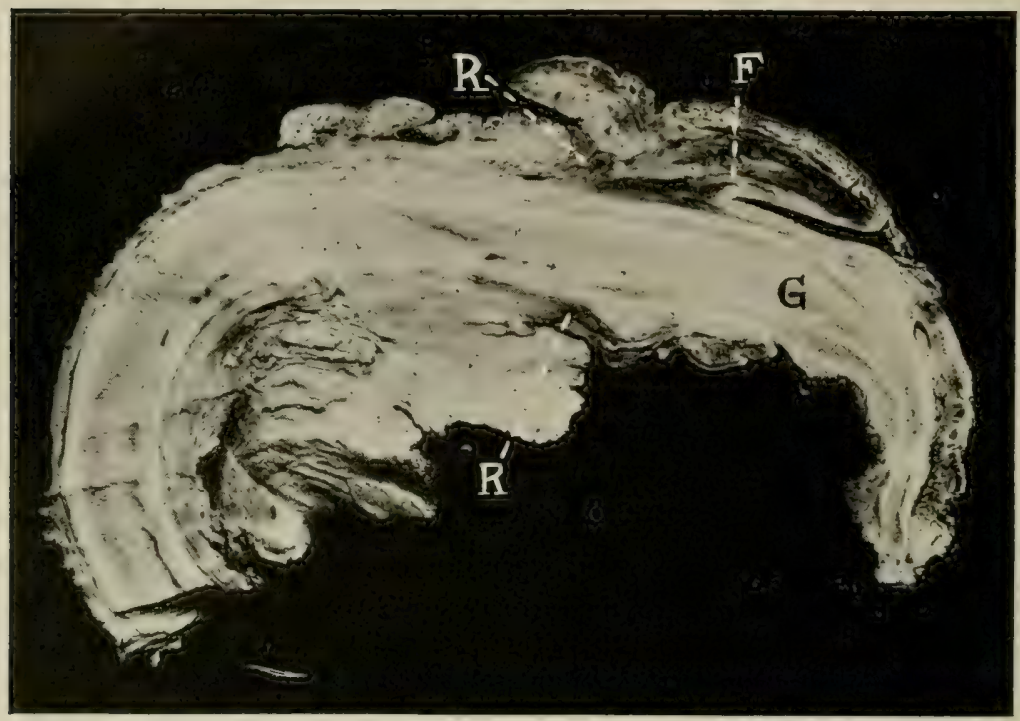

FIG. 6I-Rupture of Prepuce. Bull.

$G$, Glans ; $F$, fornix ; $R R$, rupture of the prepuce near its juncture with the sheath.

ground or floor of the enclosure in which copulation is to occur be reasonably even. Zschokke commends (Der Unfruchtbarkeit des Rindes, page 66) service stocks consisting of five posts upon which are fixed two beams which meet in front to constitute an acute triangle, open at the base, into which the cow is led and from which she can not readily escape or make any extensive movements.

In the handling of such injuries to the penis, little can be done beyond the allaying of infection by means of antiseptic douches and the avoidance of sexual excitement, com- 
bined with such local and general treatment as would tend to control inflammation.

In the dog, in which, during copulation, the prominent glans penis becomes tightly fixed in the vulva of the bitch, the male may dismount and turn in the opposite direction, thus bending the penis abruptly. If disturbed by other dogs or in any other manner, violent dragging upon the penis follows, which leads to injuries of a more or less serious character, chiefly to swelling and inflammation of the parts, occasionally followed by paraphimosis. These injuries are not usually observed under proper breeding arrangements, but are generally seen where bitches are permitted to run loose and a large number of dogs congregate, with the resultant fighting.

The handling of such injuries to dogs consists of essentially the same methods as in the bull, chiefly disinfection. If paraphimosis results, it should be overcome. A local anaesthetic, with perhaps some adrenalin, may be applied to the swollen glans penis, after which, with the aid of digital compression or by means of an elastic bandage, the size of the congested organ may be so reduced that it can be pressed back into the prepuce. If not, the margin of the prepuce is to be enlarged by incision sufficiently to permit the penis to return.

\section{K. Strangulated Hernia}

Copulation is one of the commonest causes of strangulated inguinal hernia in the stallion, and occasionally in other male animals. When the stallion mounts the mare, the inguinal region is subjected to increased pressure from the weight of the abdominal viscera. The position of the abdomen is changed from horizontal, or a declination downward and forward from the inguinal ring towards the diaphragm, to almost the perpendicular, with the entire weight of the abdominal viscera pressing downwards upon the pelvic inlet and inguinal opening. The pressure is further augmented by the abdomen of the stallion becoming forcibly 
compressed against the buttocks of the mare, as well as by the vigorous contraction of the abdominal muscles during the act, all of which combine to increase the intra-abdominal pressure and tend to force a small section of intestine through the inguinal ring, where it quickly becomes strangulated unless it promptly returns to its proper position when the stallion dismounts.

In such case, soon after dismounting, in fifteen to thirty minutes, or even earlier, the stallion exhibits symptoms of colic, kicks at his belly, looks at his flank, breathes rapidly, sweats profusely and rolls violently. The agony is excruciating and constant, increasing in intensity, the sweat becoming more profuse until, after ten to twelve hours in very acute and unrelieved cases, the violence abates, the body surface, bedewed with sweat, becomes cold, and the pulse rapid and weak or imperceptible, indicating gangrene of the incarcerated bowel, to be soon followed by death.

If the inguinal region is carefully examined, a tense fluctuating swelling may be recognized, but in many cases the incarcerated intestinal loop is so small that its presence can be determined only by very careful palpation. Examination per rectum usually gives more definite results: the incarcerated intestine can be felt and grasped, making the diagnosis definite and final.

The handling needs to be prompt, and usually radical, if the life of the patient is to be saved, although spontaneous recovery occurs in some cases. If the agony of the patient does not prohibit the attempt, the operator may insert his hand per rectum, grasp the incarcerated intestine, and, dragging gently and cautiously upon it, attempt its replacement. At times this succeeds.

Should this fail, the animal is to be cast in dorsal recumbency, with the hind legs sharply abducted, and the inguinal region freely opened. He should at once be placed under complete anaesthesia. The dragging on the incarcerated bowel per rectum may now be repeated, accompanied by digital manipulation or compression externally with the other hand or by an assistant. 
Failing in these efforts, herniotomy should be performed with the least possible delay. After thorough disinfection of the region, proper sterilization of hands and instruments, and covering the adjacent parts, including the hind feet and legs, with aseptic or antiseptic towels or napkins, the scrotum is to be freely and cautiously incised down to the incarcerated intestine, after which the carefully disinfected and moistened index finger is to be passed up along the anterior side of the herniated bowel to the point of incarceration. in the internal ring. Using the finger as a guide, a herniotome, probe-pointed bistoury, scalpel, or such other instrument as the exigencies of the situation demand, is introduced through the ring at its antero-external angle and the tissues are sufficiently divided, forward and outward, to permit the incarcerated intestine to return. Sterile or antiseptic gauze is then introduced into the wound and packed closely against the internal ring in a manner to avoid all danger of a recurrence of the hernia, and the scrotal wound is closed by sutures. The tampon may be omitted and deep sutures taken in the internal ring, closing it against future hernia.

Twenty-four to forty-eight hours later the antiseptic gauze may be removed, and the wound disinfected, closed, and thereafter left undisturbed or handled according to indications. If the strangulation of the intestine has already led to the necrosis of the incarcerated loop, resection of the intestine should be promptly performed. When herniotomy is performed, if there is doubt concerning the viability of the incarcerated loop, after the inguinal ring has been freely dilated, additional intestine may be drawn out and the parts carefully examined. The intestine should be kept moist with physiologic salt solution. The operation of resection, belonging to surgery, need not be described here. The prognosis in herniotomy is good in properly selected cases. 


\section{CHAPTER VIII}

\section{PARTURIENT INJURIES}

\section{A. Lacerations of the Perineum}

Lacerations of the perineum occur in two distinct ways, and almost exclusively in mares. The more important type of laceration of the perineum is due to one of the extremities of the fetus, generally a foot, becoming misdirected during parturition, being forced up through the roof of the vagina and floor of the rectum, and protruding from the anus. The violent expulsive efforts of the mother drive the fetus along and tear asunder the entire partition between the rectum and the anus above and the vagina and vulva below, converting the two posterior openings into a common cloaca. The accident and its handling have been fully described in the companion volume, Veterinary Obstetrics. It is referred to here because, as a general rule, if not properly handled, it closes the breeding life of the animal. The unhealed tear permits feces to drop into the vagina, keeping up a constant irritation and fecal infection. It also renders copulation virtually impossible. If the cervix and uterus are healthy, artificial insemination may succeed, and if pregnancy ensues the injury will offer no impediment to parturition.

The second type of laceration of the perineum may occur in any animal, though most frequently in the mare. In this type of laceration, the injury is due to overstretching of the vulva by the volume of the fetus. In almost all cases the injury occurs at the superior commissure of the vulva and extends obliquely upward to the right or to the left outside the anal sphincter. Such lacerations are of importance principally because they permit the superior commissure of the vulva to drop forward, rendering the vulvar opening horizontal and interfering with copulation. Still more important, they destroy the physiologic function of the vulva as a sphincter and as a barrier to infection, so that the vagina 
tends to become more intensely infected than usual, and this infection may extend through the cervical canal into the uterine cavity and beyond.

Consequently it is of importance that such lacerations be repaired as promptly as possible. Owing to the constant tension of the muscles and fascia extending from the vulva to the ischial tuberosity, the margin of the sacro-sciatic ligament, and the sacrum, it is difficult to retain the parts in apposition while healing. Therefore it may be advisable to isolate largely, upon one or both sides, the anus and vulva from the adjacent structures by making two approximately parallel incisions from above to below on either side of the anus and vulva, extending through the skin and subcutaneous tissues, so that outward tension upon these can no longer exist. After the injury has been repaired, the incision will heal and the function of the parts be restored.

\section{B. Recto-Vaginal Fistula}

Recto-vaginal fistula, like the first type of ruptured perineum, belongs virtually exclusively to the mare. Its origin is the same. An extremity of the fetus, almost always a hoof, pushes up through the roof of the vagina and rectal floor into the rectum. The position of the deviated member then becomes corrected, and birth is completed without the tissues posterior to the puncture becoming divided. As a rule, the erroneous position of the foot is probably corrected by an attendant who, realizing the peril, quickly pushes the foot from the anus and rectum back down into the vagina. The opening persists. The result is analogous to the complete rupture of the perineum. Feces drop through the fistula and cause fecal infection.

The prognosis and handling are the same as for complete rupture of the perineum.

\section{Lacerations of the Cervix}

Lacerations of the cervix have been generally ignored. Not alone have the parturient lacerations of the organ been 
disregarded, but there has been a very general disregard for surgical invasion of the cervix in a manner equivalent to laceration. There has come down to the present day a legend of frequent stricture and induration of the cervix, of a character to prevent parturition, which may be relieved by a free and careless dilation of the canal by incision. Owing to a gross and common misconception with reference to the non-dilation of the bovine cervix at the time of parturition, it is quite habitual to dilate a perfectly normal cervix by cutting, when, as a matter of fact, the whole fault lies in a paretic uterus. The cervix has no inherent power to dilate, but is dilated passively by the contraction of the uterine walls upon the fetus and its fluids. Adhesions, or indurated thickenings, in the cervix occur with such frequency as to constitute a basis for the fiction of the frequency of induration. Practitioners should be on the alert and remember the three salient facts-that the cervix of the cow is physiologically very rigid, that its non-dilation at time of parturition is almost always due to atony of the uterus from intra-uterine infection, and that the part played by the cervix is essentially passive. If this is fully understood, dilation of the cervix by incision will not be rashly applied.

In whatever manner the laceration or division of the cervical wall occurs, the fissure heals neither perfectly nor readily and the vaginal opening of the cervical canal gapes. Naturally infection of the cervix follows and a pernicious interference with fertility results.

Lacerations of the cervix should be repaired as promptly and accurately as possible. If recognized at first, they should be carefully sutured with slow-dissolving catgut under the best available asepsis. When a persistent fissure interferes with fertility, the vaginal end of the cervix had best be amputated as far forward as the fissure extends. The technic of trachelectomy is described later under "Cervicitis". 


\section{CHAPTER IX \\ SODOMY AND SADISM}

INJURIES TO THE GENITAL AND NEIGHBoRING ORGANS OF Animals by Sexually Psychopathic Men

It is of fundamental importance that veterinarians shall have in mind the extremely variable injuries which may be caused to animals by sexual perverts. Failure to diagnose these injuries has been the common lot of veterinary practitioners. The errors in the diagnosis of such injuries mislead the public, tend to cause a repetition of the acts upon other animals, and result in extensive losses to owners, because under the shadow of error the culprit is not detected, nor is any intelligent effort made at detection. Finally, the error is deeply humiliating to the erring veterinarian.

Veterinary literature is very poor in this field. The principal contributors to our knowledge have been Guillebeau ${ }^{1}$, Cadiot$^{2}$, and Eggimann ${ }^{3}$. The following includes the more important portions of the contribution of Guillebeau.

The injuries may be placed under two headings-sodomy and sadism. Sodomy, or carnal intercourse between man and the lower animals, while highly repulsive to moral standards and in sharp conflict with statutory laws, is generally of but scant interest to veterinarians, as Guillebeau points out, because no physical injury follows in the large domestic animals and the specific venereal infections of man are not transmissible to animals.

Guillebeau relates that in a remote suburb several neighbors, upon separate mornings, each found a dead hen which had been left apparently well the evening before. The fourth hen was presented for autopsy.

${ }^{1}$ Ueber Verletzungen der Haustiere durch sexuell psycopathic Menschen. Von Alfred Guillebeau in Bern. Schweizer Archiv. für Tierheilkunde. XLI Band, I899, page I.

${ }^{2}$ Cadiot, P. J. Bulletin d. 1. Soc. Centr. de Med. Vét. vol. 50, page 257.

${ }^{3}$ Eggiman, F., Schweizer Archiv. f. Tierheilkunde. Band 35 (1893) page IO3. 
The well-filled crop and well-developed egg in the oviduct indicated sudden death. The liver presented several lacerations covered with blood, and on its caudal side between the intestinal loops an extensive hematom. The connective tissue of the groin was infiltrated. The egg tube contained a hard-shelled egg. The cloaca was gaping and dilated to $21 / 2 \mathrm{~cm}$., much larger than normal. Four days later another dead hen was brought for autopsy by another neighbor. The cadaver presented the same lesions, with the addition that the left tibia was broken and, yet more important, two mammalian spermatoza were found in the cloaca. The liver rupture was apparently due to the violent holding of the hen to prevent her escape. Later, in another locality, an owner lost a number of hens, one at a time, at intervals of one to a few days, and finally a sick bird was brought Guillebeau for observation. Eight days later it was necessary to kill her. The autopsy revealed bloody serum and fibrin clots in the body cavity, the liver greatly enlarged and containing large cavities filled with blood serum, the intestines adherent. Apparently the wounds were essentially like the foregoing, but not so severe as to cause immediate death.

The incentives to such an offense lie in a great sexual craving with a low moral sense, while in other respects the person may be normal or even bright.

The subject is without material veterinary interest beyond diagnosis, and through this means to enable the owner to secure police protection.

Sadism, or that type of sexual perversion which leads persons to mutilate the genital and neighboring organs, is of far more frequent interest to veterinarians and, owing to the endless variety of mutilations, offers extraordinary difficulties in diagnosis. Many of the mutilations prove fatal, and the pervert is so persistent in the perpetration of the offense that delay in diagnosis may cause great economic loss, as well as serious embarrassment to the veterinarian. Errors in diagnosis have certainly injected into our literature some very mysterious and illogical accounts of coital injuries. Quite naturally a sex pervert is equally or more liable to commit the offense upon an animal in estrum, at 
which time also the female copulates if opportunity is offered. The coincidence of physiologic copulation with a male of the same species and sadism by a psychopathic man may, and undoubtedly does, lead veterinarians to attribute to coitus injuries which are highly improbable or impossible from that act.

In one instance cited by Guillebeau, recorded by Eggimann, five cows and two heifers in one establishment were mutilated in the vagina and rectum, and four of them died. Two aborted putrid fetuses. The seven animals had received a total of over thirty punctured wounds, one animal alone having fifteen punctures. The wounds were of various depths, about the diameter of a goose quill, and surrounded by necrotic tissue. The wounds penetrated the peritoneal cavity, the uterus, the rectum, and the pelvic connective tissues and mutilated the urethra, bladder, and the urethral valve. Extensive and fatal hemorrhages ensued, as well as metritis, vaginitis, peritonitis, etc. The culprit was not discovered and no legal action taken.

Guillebeau records that in 1896, in Obersteg near Bern, in an isolated establishment far back in the mountains, ten cattle and ten goats were injured, mostly fatally. The depredations began in January, when the father of the culprit lost two cows, two goats, and later two cattle. In March two cattle and six goats were mutilated, in April three cattle and two goats, and in May one heifer. In June two cattle belonging to neighbors were injured. The monetary loss was $\$ 720$, one-half of which fell upon the father of the offender. The last animal of the group was destroyed ten days after mutilation and an autopsy made. The vagina contained a large volume of red, stinking exudate. Fistulae extended from the vagina forward. Two of these were ten $\mathrm{cm}$. long. One was fifty-five $\mathrm{cm}$. long, involving wounds of the liver, spleen, diaphragm, and lung, and reaching the region of the eighth rib, or about the middle of the chest. The frightful injuries revealed by the autopsy made it clear that the culprit had accomplished his designs with a 
stick. The extraordinary resemblance between these injuries and those recorded above in Langnau was apparent.

The symptoms, in the cases not immediately fatal, consisted of straining, bloody discharge from the vagina, swelling of the vulva, and general weakness. Six months later the investigation was taken up by Professor E. Hess and the culprit detected. He was a large, nineteen-year-old man, bent somewhat forward, with a handsome face, downy beard, and dangling arms. He had suffered from inflammation of the brain at four years. His cranium was small, and he was regarded as idiotic. In school he could not learn and when grown was incapable of work except as an assistant in caring for cattle. Taken into custody, he confessed his guilt several times and later withdrew his confession. He went about his misdeeds so craftily that he had never been caught.

The Cases in Wolfikon, Schwartzenbach, and Rickenbach.

In March, 1894, in a stable containing fifteen cattle, eight-all in one row of stanchions-became ill. Amongst them were three calves, one only a few days old. There was a bloody vaginal discharge, depression, and peritonitis. Some showed crepitant swelling of the loins and croup. Within a week four animals had died and four had been killed. All were buried with their hides on. Fifteen hundred kilos of hay was burned for fear of bad contamination. The guilty caretaker and his clothes were carefully disinfected. The cattle which had escaped mutilation were disinfected and removed to other quarters. In two weeks, two of the remaining seven animals became affected, one of which died and the other was slaughtered. The remaining five head were slaughtered in order to escape, partially, the impending loss. After about two months, the tenant purchased two cows, but in a short time he buried one and returned the other to the seller. The same caretaker of the cattle was still in charge. He purchased goats, which remained well. It was soon hoped that the ban which had hung over the place had disappeared. The tenant bought 
three goats, which promptly died under the same conditions as the cattle. He became discouraged, sold his hay to be fed to horses only, and sold his farm.

The servant found employment with another party in Schwartzenbach. Here, as in the preceding place, a heifer, a goat, four oxen, and a cow soon became ill. Three oxen were buried. The flesh of the other cattle was used for food. The investigator was struck with the similarity of the mutilations of the sexual organs with those observed in Wolfikon. A Capuchin monk, evidently a good judge of men, when consulted, advised the discharge of the servant. The losses became noised about the neighborhood and were attributed to the young man who had cared for the cattle. On November 12, 1895, he obtained a new position in Rickenbach, not far away from his previous place. Fourteen days later, a cow cared for by this man sickened like the preceding and had to be destroyed. Autopsy revealed a finger-sized fistulous wound in the rectum, two blood-covered lacerations in the vagina, and hemorrhage into the peritoneal cavity. On the next day a cow died suddenly from symptoms of vaginal and rectal hemorrhage. A third animal had to be destroyed on November 29. In the vagina only one perforating wound was found, but in the rectum thirty-five, six of which were perforating. A fourth animal injured would probably recover. The servant was discharged and a few days later arrested. At first he denied his guilt, but later confessed that he had caused the injuries by forcing a stick into the rectum and vagina and pushing it back and forth until the animal bled. The culprit was twenty-four years old and a capable worker in stable and field. He was goodnatured but represented as wanting in judgment. In Wolfikon, the scene of his first crimes, he was three years in one position, but apparently he had come to sexual maturity very late and part of the period of service preceded this. His teacher, the local pastor, and the alienist who observed him after arrest regarded him as of very low intelligence. The size of the cranium was below normal. In the hearing, 
the young man showed some of the talents of a trader. $\mathrm{He}$ had at his command about 5000 francs and criticized the reckoning of damages. In recent years, while feeding, milking, and grooming the cows, he had had frequent erections, fits of excitement, and delusions. At first he merely forced his hand into the posterior body openings of his charges; later he used a stick, always, according to his statement, under the pressure of a dark, powerful, inner craving at times when he also suffered from sleeplessness, sweating, dysuria, and erections. He seemed conscious of the wrong, but powerless to resist his cravings. He was adjudged irresponsible, but made to pay as far as possible for the losses caused. This simpleton had led the veterinary diagnosticians a merry chase. According to the official report of the first cases in Wolfikon, the animals had died of an undetermined epizoötic, probably anthrax. The second outbreak, in Schwartzenbach, was diagnosed as "intestinal diphtheria", especially a localization of malignant, catarrhal fever of rectum and vagina. In both places elaborate precautions against the spread of the malady were taken by thorough disinfection.

\section{Second Outbreak in Rickenbach}

In the same stable in Rickenbach, in which in November, 1895, the acts described above were committed, an ox had to be destroyed in April, 1897, because of rectal hemorrhage. The illness lasted four days. After twelve hours there were symptoms of colpitis. After four days there escaped from the rectum four liters of blood. The animal was weak. Peritonitis was apparent. Autopsy revealed bloody infiltration of the pelvic organs, kidneys, and adrenals. The veterinarian found numerous injuries in the rectal mucosa, five of which were penetrant. The culprit was an eighteen-yearold caretaker of the animal. Apparently he was not as stupid as his renowned predecessor, but, according to his story, the master had related the misdeeds of the other, and seeing in the stable day after day the short stick previously 
used, finally awakened in him a desire for the same experience.

\section{The Occurrence in Le Ménil-aux-Cerfs}

When the trauma is induced by less violent means than those already described, the difficulties of diagnosis increase. In a stable of about ten cows, there occurred, within three weeks after a change in caretakers, vaginitis in all the animals. Ten new cows added later soon developed the vaginitis. The heifers, when they reached sexual maturity, also suffered. With the advent of estrum the vaginitis became worse, and at the same time the disease increased in intensity in the cows near the one in estrum, even though castrated. The symptoms began with swelling of the perineum, then a discharge of clear, tough mucus, which later became thick and purulent. Once colic followed estrum for several days. The general health, nutrition, and lactation suffered little. Pregnant animals generally did not abort. The local handling had little effect: some cows conceived again; several remained sterile and had to be discarded. The owner was compelled to sell the sterile animals for less than the cost of new ones. He was tormented with the fear that they had been inoculated with syphilis. Investigations were finally made by Schwartz of Bern. Four cows were visibly diseased; all showed colpitis, and two of them severe annular vaginal stricture. Five other cattle offered nothing remarkable. The cows had been bred to two public bulls which had also been used for numerous other cows. Neither the cows nor the bulls suffered from any visible genital disease. The conclusion was finally reached that the injuries were due to rough handling by some evil-disposed person but it appeared very difficult to so much as suspect the culprit. Several weeks had elapsed when by accident it was discovered that the caretaker of the cows was the culprit. Aside from this the man was a model: he was a quiet, punctual, thorough worker, and, until the accidental revelation, beyond suspicion. The identification of the culprit revealed 
also his method. It consisted of pushing a fork handle into the vagina and rapidly turning it in a wide circle. This special form of sadism served to explain the strictures of the vaginae. No punishment of the offender was undertaken, because it was feared it would lead to endless trouble, and the model servant was permitted to go his way unmolested.

I have found no account of this type of injuries in this country. They probably occur and escape diagnosis. In connection with public institutions for mental defectives we have many valuable dairy herds in which the cattle are largely placed in charge of "trusties". Since such mental defectives might resort to such practices, veterinarians having charge of such herds would do well to keep this possibility in mind and not be led too far afield in diagnosis. 


\section{TUMORS OF THE GENITAL ORGANS}

The fundamental nature of tumors is essentially undetermined. Clinically, many of the benign tumors of animals bear strong evidences of transmissibility. They appear unexpectedly in a stable or herd, or in a group of animals within a herd, and involve simultaneously a large percentage of the group. At present the general concensus of scientific opinion appears to be that malign tumors are due to infection. Certain new-growths formerly regarded as true tumors, like actinomycosis and botryomycosis, are now definitely classed as infections. In the dog, there occurs a clearly defined venereal tumor definitely ascribed to infection, which is included among venereal infections instead of among tumors.

\section{CHAPTER X}

\section{A. MALIGNANT TUMORS}

\section{Malignant Tumors of the Male Genitalia}

Herbivora, and especially ruminants, are apparently highly resistant to malignant neoplasms. This may be apparent only because most domestic ruminants are slaughtered early in life for food. More than fifty per cent. of all dairy calves born are slaughtered for veal. More than ninety per cent. of male cattle of beef breeds are castrated and are slaughtered at three to four years of age. The average age of dairy cows is below six years, though occasionally they live to fifteen, eighteen or more.

Malignant neoplasms affect chiefly adult or old animals. Naturally it follows that they would be rare in species where the technically normal duration of life is greatly abbreviated by artificial means.

Malignant neoplasms in domestic animals are most frequent in dogs and horses, both of which are largely kept 
until old age. In the dog, the animal which suffers most frequently from malignant tumors, the normal span of life is but ten to twelve years, the briefest among domestic animals.

Malignant new-growths may invade any portion of the genital system, of either sex and in all species, and may vary greatly in histologic type.

Malignant tumors of the genital organs of bulls (as well as of rams and he-goats) are exceedingly rare. I have not observed a well defined clinical case and have no clearly identified specimen in my collection. Possibly I am in error. Young bulls very frequently have angry-looking, highly vascular tumors on the glans penis which, examined histologically, are found to be epithelial. They are commonly described as carcinomata, but histologists are not wholly agreed upon this point. So far as I have observed clinically, they are usually not malign, however suggestive their appearance may be. When carefully excised, they show little tendency to recur; when neglected, they cause no daughter tumors in the peri-penial lymph glands.

The diagnosis and handling of malign tumors of the genital organs of bulls (and of rams and he-goats) must be based upon general principles. When involving the glans penis in a valuable bull, liberal amputation may be applied, which, if timely, may eliminate the tumor and preserve the procreative powers of the animal. The amputation should be made under the technic described later for the amputation of the penis of the stallion.

At one time I doubted the ability of a bull to copulate readily after the amputation of the glans penis. I attended one famous sire which had suffered from a tumor on the glans. Sections of the tumor had been examined by a pathologist and pronounced carcinomatous. The glans had been amputated. The stump refused to heal, and was intensely inflamed and angry. After long and patient handling, the stump healed and the bull returned successfully to breeding. 
When amputating the penis of a bull (or ram or boar) it must be recalled that these animals are always affected with the granular venereal disease, which retards healing and aggravates the wound. Prior to amputation it is therefore advisable to disinfect the glans, prepuce, and sheath by douching the parts daily for five to eight days with a 0.25 to 0.5 per cent. Lugol's solution, or other safe antiseptic. After the penis has been amputated, it is desirable to continue the douching until the healing is complete.

The bull should be kept apart from cows in estrum, and otherwise handled in a manner to obviate sexual excitement, because erection inevitably aggravates any penial disease. It is bad policy generally to cause the bull to become sexually excited in order to have him protrude the penis for visual examination in case of disease. It is better, and far more satisfactory from every standpoint, to cast the bull and expose the penis by eliminating the S-curve, as described in Chapter II.

If the ordinary douching of the sheath and prepuce fails to hold in check the infection and inflammation in the stump, the animal should be cast, the penial stump exposed, and the inflamed areas pencilled over with silver nitrate or dressed with a strong solution of the same. It should be remembered that the conditions are inimical to prompt healing and that it is far better to hasten the healing process to the maximum by giving concentrated attention at the beginning, thus obviating the necessity for prolonged handling which will in the end greatly exceed the total care required if minute details are faithfully followed at first.

Hendryckx ${ }^{1}$ states that in the course of malignant disease of the testicle the organ may undergo necrosis and open upon the exterior of the scrotum and the new-growth assume the clinical characters of external cancer.

When malignant neoplasm of the testicle is diagnosed or seriously feared, the gland should promptly be removed. A

1 Bayer and Fröhner. Handbuch der tierärztliche Chirurgie und Geburtshilfe, Vol. III, Part 2, Page 439, 1899. 
positive diagnosis of malignancy, with all the risks of extension and of daughter turnors, should not be awaited. Any chronic tumefaction of the testicle sufficient to arouse fear of malignancy should be treated decisively and promptly as malignant. In any case, the function of the testicle is almost certainly destroyed and the ablation of the hopelessly diseased gland is the prudent course to pursue. The removal of one testicle neither destroys nor curtails the reproductive power of the animal.

The removal of a testicle which is presumably affected with a malignant new-growth should preferably be effected by the covered operation. The skin and dartos are freely incised down to the cremaster muscle or its fascia. The cremaster, its fascia, and the peritoneal layer of the scrotum are kept intact and a separation made between the cremaster and dartos up to the external inguinal ring. The spermatic cord, covered by the cremaster muscle and the scrotal peritoneum, is then securely ligated at a point as high as is convenient or required. It is best to arm a heavy needle with a strong thread and pass it through the cremaster muscle, scrotal peritoneum, and spermatic cord. The needle is removed and the ligature tied about the spermatic cord and cremaster very tightly, in order to make inevitable the immediate necrosis of all included tissues. The included mass is then excised on the distal side of the ligature at a distance sufficient to guard securely against its slipping, generally about one inch. The cavity in the scrotum should then be tamponned with antiseptic gauze and the cutaneous wound closed by sutures. The gauze should be allowed to protrude at the lowest part of the wound, in order to permit drainage.

Malignant tumors of other portions of the genital tract of the bull are not as amenable to handling as are those of the glans penis and the testicle. They need to be handled according to general principles.

Malignant tumors of the genital organs of horses are not at all rare. According to my observation, they are com- 
moner in geldings than in stallions. This relates especially to the penis. Clinically, also, I have seen more malignant neoplasms in the stumps of the spermatic cords of geldings than in the testicles of stallions. It is possible that the essentially dormant genitalia of emasculated animals are more vulnerable to malignant new-growths.

The testicle and epididymis of the stallion is only very rarely the seat of malignant tumors. In one stallion I diagnosed clinically malignant disease of the testicle, but had no opportunity to verify my diagnosis by direct examination of the gland. In the college collection there are no specimens of malignant disease of the testicles of the horse. When cryptorchidy occurs in man, it commonly raises the question of malignant disease of the testicle. Although cryptorchidy is very common in horses, the condition is rarely, if ever, attributable to malignant disease. The clinical diagnosis of malignant disease of the testicles is to be made upon the same general basis as the diagnosis of malignant tumors of other organs.

When malignant disease of the testicle in the horse is seriously feared, the gland should be promptly removed for the same reasons and in the same manner as already described for the bull.

Malignant disease of the testicles and epididymis in other domestic animals is rarely reported. Clinically I removed successfully a cryptorchid, cancerous testicle from a pig. It was adherent over its entire surface to the adjacent intestines and mesentery, so that it had to be dissected away carefully.

Malignant disease of the penis, sheath, and prepuce in the stallion is generally supposed to be common. At least, writers upon veterinary surgery, as Cadiot ${ }^{1}$, Bayer and Frohner', and others, would lead the reader to believe so. I have not observed a case in either penis, prepuce, or sheath in a stallion, and my collection contains no specimen.

1 'Traité de Therap. Chir. d. Animaux Domestiques, 1898, p. 674.

${ }^{2}$ Handbuch d. Tier. Chir. u. Geburtsh., I 899, vol. iii, Part II, p. 345. 
On the other hand, the occurrence in geldings has been relatively common. This may be apparent only. Since in America essentially all colts not desired for breeding purposes are castrated, the ratio of stallions to geldings is less than five per cent. Consequently one would logically expect to see at least twenty to twenty-five malignant new-growths in geldings to one in stallions. The malignant tumors of these parts may be sarcomatous but are most frequently carcinomatous. Their character is generally diagnosable by clinical examination. Usually there is abundant necrosis of the diseased parts and rapid growth and in all respects they show typical malignancy. As in malignant disease generally, there is rarely any early trace of cancer cachexy. The general health of the animal remains as a rule unimpaired for several months, unless the disease interferes with some vital function either by mechanical impediment or by invading a vital organ (liver, lungs). Malignant disease of the penis, sheath, and prepuce ordinarily proves fatal indirectly, through daughter tumors in a vital organ. Thus, in one case in my clinic, carcinoma of the sheath caused death through the breaking down of a daughter tumor in the lungs.

The clinician should not err in diagnosing benign tumors of the penis as malign. The ordinary benign epithelioma of the equine penis is highly vascular and undergoes pressure and attrition necrosis. The necrotic tissues and the sebum produce much highly fetid pus containing tissue debris, and often blood. That should not be accepted as evidence of malignancy. The necrosis of malignant neoplasms is fundamental and inherent, and not attributable to pressure or attrition. When essential, the diagnosis may be safeguarded by the removal of a portion of the tissue for pathologic study.

The veterinarian should also be on the alert in diagnosis not to mistake bursattee of the penis and prepuce for malignant neoplasms. The necrosis of bursattee is fundamental, like cancer, but its other characters serve to differ- 
entiate it. This will be discussed while dealing with the infections of the genitalia of horses.

The handling of malignant disease of prepuce, sheath, and penis of the breeding stallion calls for prompt and skillful measures. The early ablation of the neoplasm is the only course known which offers hope. Even then the hope is based upon the complete extirpation of the growth, in such a manner as not to interfere with the power to copulate. When involving the sheath only, complete removal may be possible, but there is danger of such deformation of the parts as will cause faulty direction of the erected penis. If the prepuce is involved, a greater danger faces the surgeon because adhesions between the direct and recurved portions may bar the protrusion of the penis.

When the penis itself is involved, the amputation of a part or all of the glans does not disable the animal, but if the amputation needs to be on the proximal side of the preputial ring, the breeding capacity of the animal is probably ruined. I find no conclusive data on this point, but it has been proven clinically that the glans can be amputated without serious interference. In the present state of our knowledge, when the malignant growth has extended beyond the glans into the penial body, no hope should be given of restoring the breeding powers. In a gelding I have seen the cancerous growth involving the entire penis from the glans to the attachments of the crura to the ischial tuberosities.

\section{Amputation of the Penis}

Almost, if not all cases of malignant tumors of the glans penis should be handled by the amputation of a portion or all of the glans. The amputation should be carried out by the following technic, which affords ample security against urethral stricture and provides a tapering stump which can most readily enter the vulva in copulation. The amputation of the penis of the stallion affords a technic equally applicable to that of other species. 
Instruments. Scalpel, elastic ligature, strong silk suture, strong piece of tape $1 \mathrm{~m}$. long, artery and compression forceps.

Technic. The operation is carried out on the recumbent

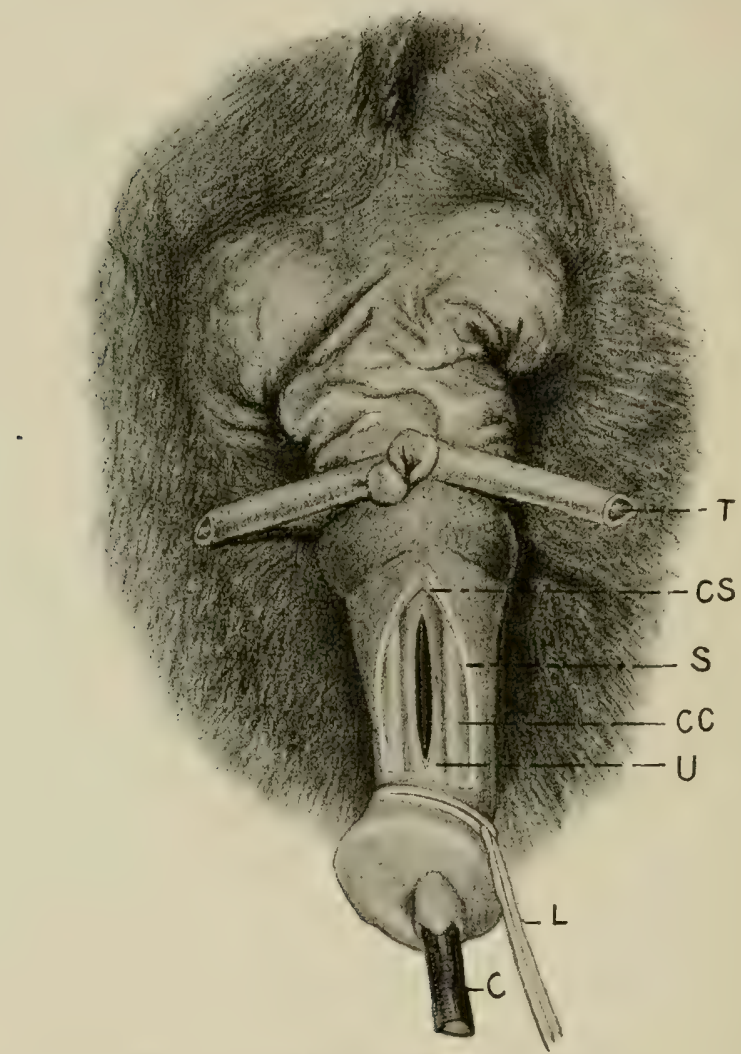

FIG. 62-Amputation of Penis.

First stage of operation. $T$, Elastic ligature used as tourniquet ; $C S$, corpus spongiosum of urethra ; $S$, skin ; $C C$, corpus cavernosum ;

$U$, urethra; $L$, ligature ; $C$, catheter.

animal, under local or general anaesthesia. The upper hind foot is drawn backward or upward or otherwise so fixed as not to obstruct the field of operation. The point of operation is determined by the character of the disease and the 
object to be attained. It may be made at any point from the glans penis to the attachment of the corpus cavernosum to the ischium, but if possible in front of the preputial ring.

After the penis has been drawn out, and the preputial region carefully cleansed and disinfected, an assistant grasps the organ just behind the preputial ring and holds it firmly. A catheter is then introduced into the urethra and pushed upward beyond the point where it is designed to amputate

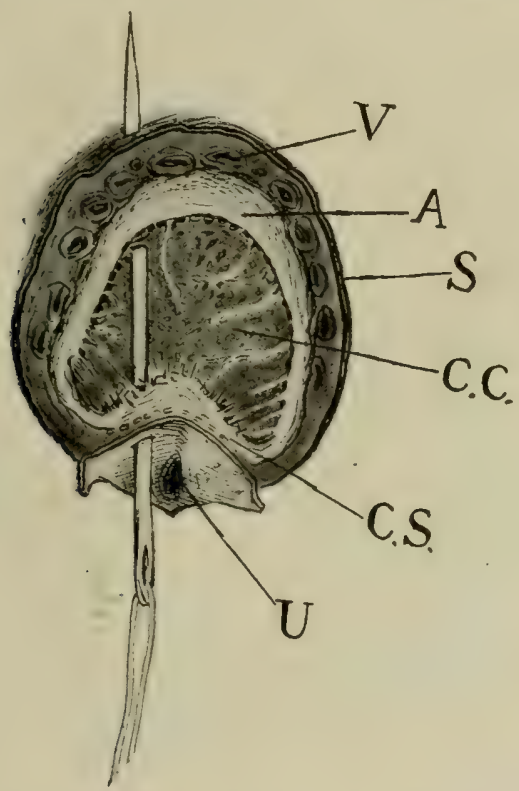

FIG. 63-Amputation of the penis showing needle inserted for a suture.

$V$, Dorsal vessels of penis ; $A$, fibrous tunic of the corpus cavernosum ; $S$, skin ; $C C$, corpus cavernosum ; $C S$, corpus spongiosum of urethra; $U$, urethra.

the organ. A temporary elastic ligature, T, is then applied above the assistant's hand around the penis, or a piece of tape is looped around it above the hand and is made to serve both as a tourniquet and as a means for holding the penis, or the penis may be grasped in front of the ligature with double tenaculum forceps and held. 
A small cord is applied just behind the glans penis, L, Fig. 62 , and a triangular incision is made on the ventral surface of the organ about $4 \mathrm{~cm}$. long by $3 \mathrm{~cm}$. wide, the base of the triangle being forward as shown in the figure; this incision is carried through the skin, $\mathrm{S}$, the corpus spongiosum, CS, and along the corpus cavernosum, CC, down to the urethra,

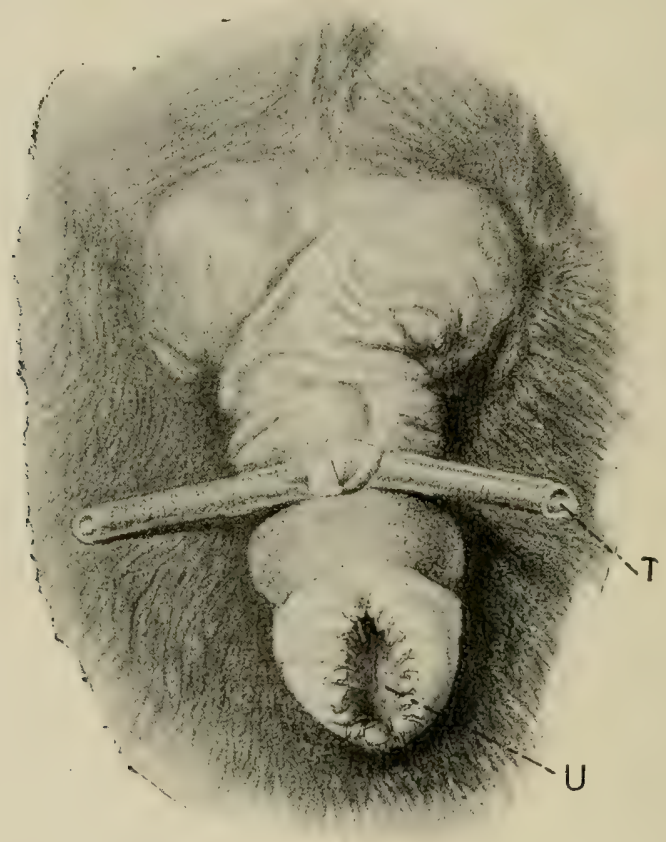

FIG. 64-Amputation of the Penis.

Completed operation showing sutures. $U$, Urethra. $T$, Elastic Ligature.

U. In the triangular area the tissues are dissected away without opening or wounding the urethra and then a longitudinal incision is made from near the apex of the triangle to its base through the urethral walls to the catheter. Beginning at the apex of the triangular wound, a series of interrupted sutures is inserted, as shown in Fig. 64, in such a manner that they pass through the urethral wall and the skin, so that when tied the wounded surfaces are completely 
hidden and the urethral mucous membrane is brought into apposition with the integument. These sutures are continued down to the base of the triangle, the catheter is removed and the organ is excised by a cut extending in a slightly oblique direction from below upward and forward. A straight needle armed with a silk suture is passed through the margin of the urethral wound, the adjacent fibrous capsule of the corpus cavernosum, and across but not through the erectile tissue, inserted again into the superior portion of the fibrous capsule and carried out through the adjacent dorsal vessels and the skin, as shown in Fig. 63, and, bringing the ends of the sutures together, tied in such a way that the urethral mucous membrane and the margin of the skin are brought into immediate contact and the blood vessels securely closed in such a manner as to guard against hemorrhage. By this plan when the sutures are tied, the cut borders of the fibrous envelope are brought together over the erectile tissue, thus preventing hemorrhage from that tissue also. As many sutures are inserted as may be required to close the wound securely and finally leave every part wholly covered with epithelium. By this plan stricture of the urethra in the process of healing is avoided. The tourniquet is removed and the patient released.

The principles here laid down are applicable and advisable in the amputation of the penis in all domestic animals. In the dog, the point of amputation should be above the penial bone.

Once the amputation has been successfully completed, the healing of the stump should be favored by keeping the parts scrupulously clean with frequent douchings. The patient is much better if given abundant exercise, avoiding constantly, as far as may be practicable, any sexual excitation because erection strains the sutures, engorges the wound area, and seriously impedes the healing process. The diet should be scant and laxative. 
3. Malignant New-Growths of the Female Genitalia

Malignant tumors of the female genital organs, generally very rare, are most often seen in the vulva and ovary. I have observed but one case of malignant tumor of the uterus-that one case in the bitch (see Fig. 65). The malignant growth involved the entire genital tube, but was apparently most advanced in the uterus. The symptoms consisted of a profuse, fetid, purulent discharge from the vulva, with swelling and obstruction of the lumen of the genital passage. When discovered, it was inoperable.

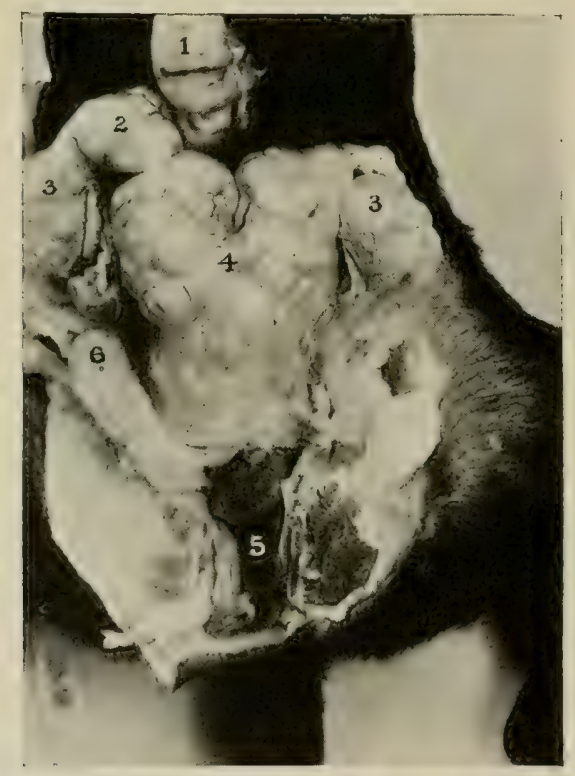

FIG. 65-Sarcoma of Genital Tract. Bitch.

I, Rectum ; 2, left oviduct ; 3 , ovary ; 4, uterus ; 5, vagina ; 6 , urinary bladder.

I have seen two clinical cases of ovarian carcinoma of the cow. The first (see Fig. 67) was in a pedigreed Holstein heifer which had proven sterile. Upon rectal palpation the right ovary was found to be enormously enlarged, 


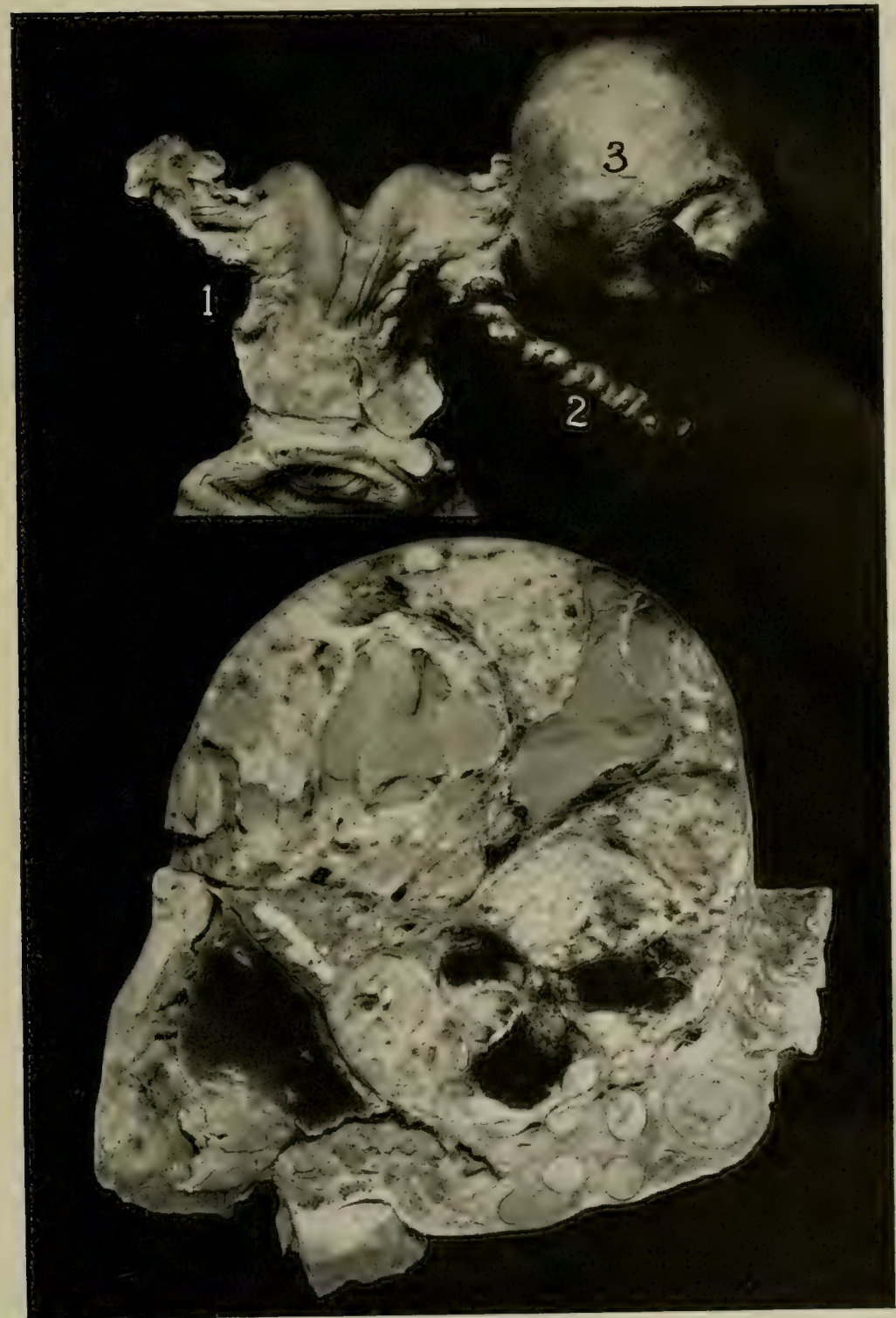

FIG. 67-Angio-Sarcom of Ovary, Heifer.

$I$, Left cornu ; 2, enlarged right ovarian artery ; 3 , tumor. Below, section of tumor. 
about four inches in diameter. The right uterine artery, enlarged to many times its size, as indicated in Fig. 67, pulsated with great force. The left ovary was minute and non-functional. Slaughter was advised, since the examination indicated clearly that surgical interference would be highly perilous because of the enormously enlarged ovarian artery. The removal of the ovary, also, would leave the left gland, which would probably not function. The clinical diagnosis was angioma. Upon slaughter it was found that the ovary had ruptured at one point, causing profuse hemor-

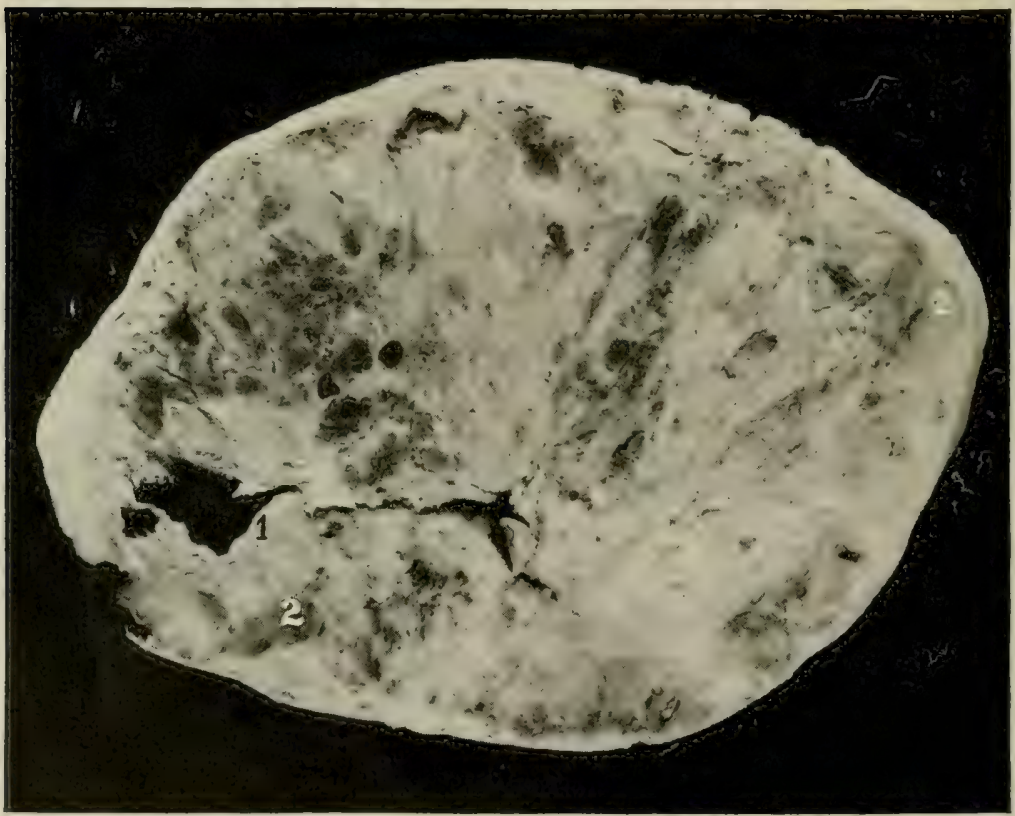

FIG. 6g-Cyst-Adenoma of Ovary. Sow. Weight of tumor $38 \mathrm{lbs}$. $I$, Necrotic area; 2, 2, cystic areas.

rhage. The entire peritoneal surface was thickly studded over with tufts of yellowish new-growth. Histologically, the tumor proved to be a carcinoma, or rather angiocarcinoma. 
My second clinical case was in an aged Guernsey cow. The ovary was about five or six inches in diameter, spherical, tense, and smooth. It was diagnosed as a thick-walled cyst of the non-nymphomaniac type. It was considered both impossible and imprudent to rupture the cyst. Ovariotomy was performed through a flank incision. Histologic examination showed the tumor to be a carcinoma.

I have not observed clinically in other animals than the cow an instance of malignant disease in the ovaries, and have but one specimen of malign tumor of the ovary other than those mentioned above. This is a multilocular ovarian tumor from a sow, shown in Fig. 69. The sow weighed about three hundred pounds, and the ovary thirty-eight. Clinically it would have been difficult or impossible to diagnose. Inevitably the abdomen of the sow was greatly enlarged, but that might have been from hydronephrosis or many other conditions. In this fat sow, the discovery was made after slaughter.

Carcinoma of the vulva is not at all rare in the cow. It presents highly distinctive clinical characters which render a reliable diagnosis comparatively easy. When reported to the veterinarian, it is not ordinarily subject to surgical interference.

As a rule, when the genital organs of a meat animal are invaded by malignant new-growths, if they are still localized, the general condition good, and the subject not of great breeding value, the animal should be sent to slaughter.

\section{B. Benign Tumors of the Genital Organs}

\section{Benign Tumors of the Male Genitalia}

Benign tumors are very common upon the penis, sheath, and prepuce of horses and cattle. These two species are very prone to growths of benign epithelial tumors, or "warts", about the face and lips and upon the external genital organs. They are observed almost entirely in young animals. Generally they are papillomatous in type and tend to become polypoid or pedunculated. This is especially true 
when they are located upon the glans penis or within the sheath, where constant compression acts to modify their form. The attrition caused by protruding and retracting the penis tends to abrade the epithelium covering the tumor. The mucus, sebum, and sometimes urine, tend to macerate and irritate the new-growth. As a consequence of these conditions, ulceration, superficial necrosis, and suppuration, with highly repulsive odor, are frequent and not rarely lead to error in diagnosis.

It is only rarely that this type of tumor attains such size as to cause phimosis or paraphimosis. Possibly one reason for this is that, with the small peduncle, when the tumor becomes sufficiently large to retard the movements of the penis, the stalk divides and the tumor drops away. I have had them drop off in my hand when attempting to make a physical examination. They are readily observed during copulation. The copulatory attrition and great vascularity of erection commonly cause hemorrhage. Such tumors call for early excision. Benign penial tumors, if not promptly removed, may extend and eventually call for amputation of the penis, though, had opportune attention been given, mere excision of the tumor would have sufficed.

The animal should be cast for the operation, with all four limbs extended, or may be secured upon the operating table. The penis, whether of stallion, bull, or other male, should be exposed and secured as advised in Chapter II, and either general or local anaesthesia applied, as conditions may dictate. When the peduncle is small and superficially attached, it should be excised, removing a liberal amount of normal tissue with it in order to have assurance that all the neoplasm is included. Care should be taken not to remove an unnecessarily large amount of tissue, because the contraction of the cicatrix is liable to bend the penis at an angle and make copulation difficult or impossible. The invasion of the penial tissue had best not be circular nor transverse, but preferably the base of the peduncle should be removed by making two incisions beginning above or on the proximal 
side of the base of the peduncle, diverging sufficiently to pass on either side of it, and then converging to meet at an equal distance on the distal side of the base, making a wedge-shaped incision extending into the penial tissues, so that the wound may be closed with chromatized catgut. In this manner deformation of the penis will be avoided as far as practicable. A careless transverse incision is more liable to invade an important nerve or arterial trunk and inter-

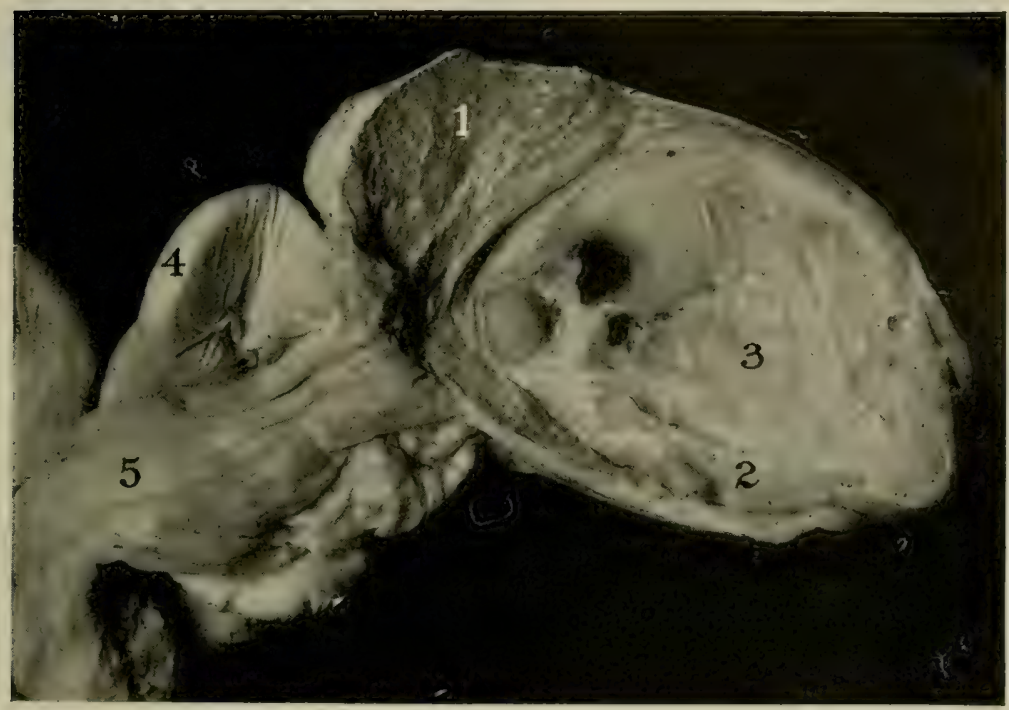

FIG. 70-Dermoid Cyst. Cryptorchid Stallion.

1 , Glandular tissue ; 2 , osseous tissue; 3 , cavity in the bony structure ; 4, epididymis ; 5 , spermatic cord.

fere with the erection of the tissues supplied by the vessel. If, in spite of such precautions, deformation occurs which prevents copulation, or if the base of the tumor is too extensive to permit excision without serious deformation, recourse must be had to amputation, as already described.

Many other types of benign tumors involve the male genital organs, but they occur very rarely and offer nothing of special importance in handling with reference to breeding. 


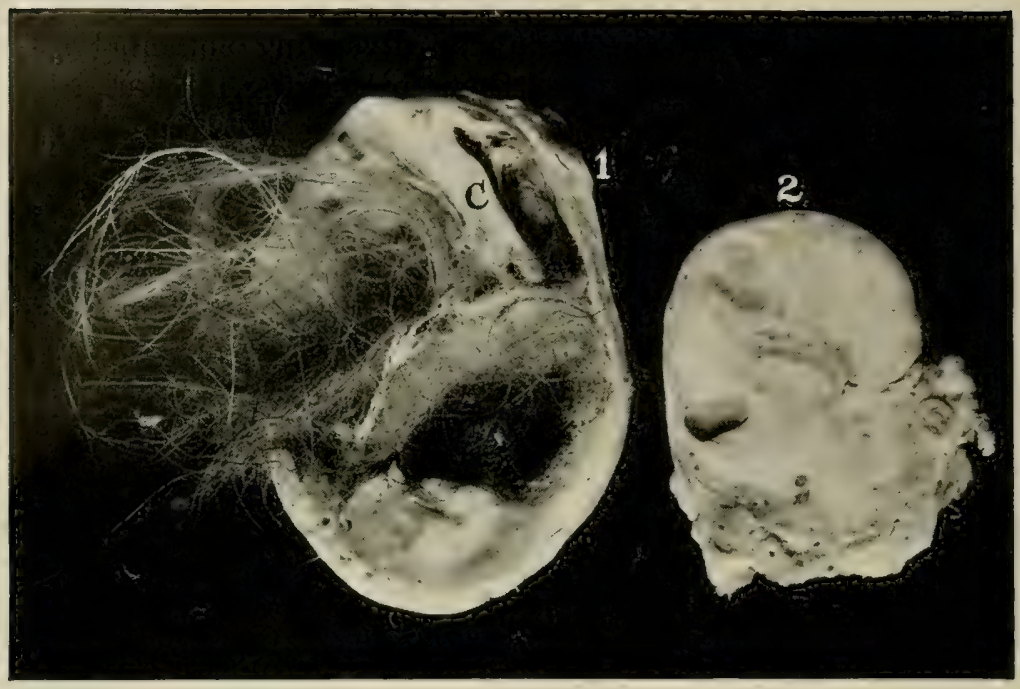

FIG. 70a-Dermoid Cyst of Ovary. Mare.

$I$, Cystic ovary, the cyst being filled with long, matted hairs ;

$C$, cartilage ; 2 , normal ovary from same animal.

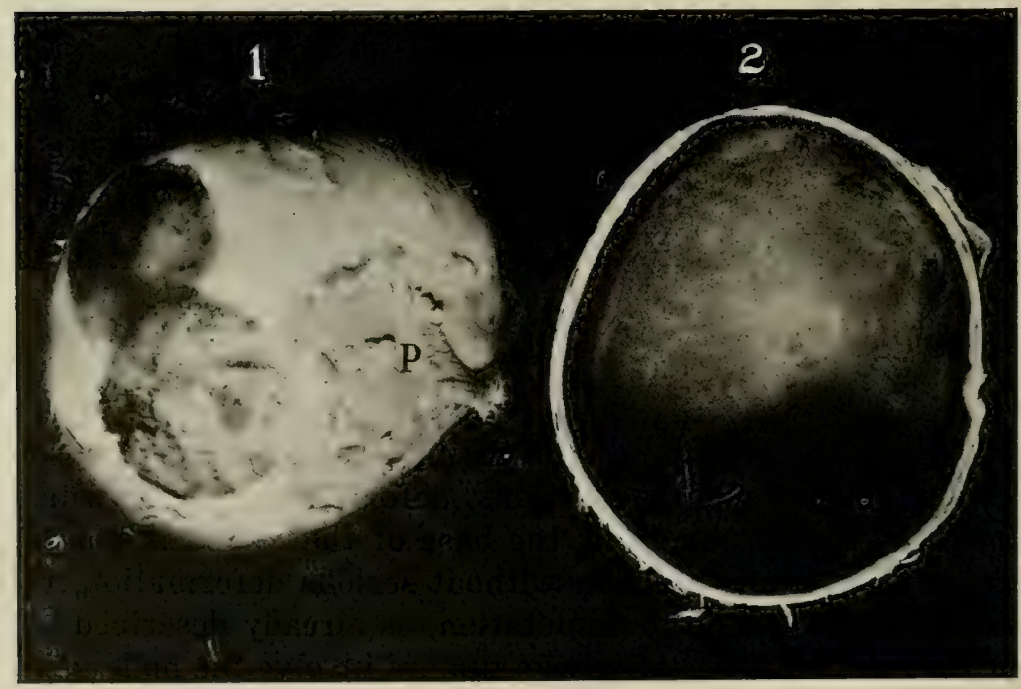

FiG. 7ob-Cystic Ovary of Mare. Divided.

The cyst was about 8 inches in diameter with very thick, resistant walls. $I$, Exterior of cyst, showing at $P$, the pavilion of the oviduct flattened against the cyst ; 2 , the interior of the cyst, showing the thick walls. 
Dermoid cysts of the testicle occur now and then, containing bones, teeth, hair, or all three (see Fig. 70). Such testicles occur almost wholly in the horse, are generally crypt. orchid, and are beyond remedy as to breeding, but should be removed in order to eliminate the sex desire, frequently perverted, which they cause. If the other testicle is sound, the animal is fertile. No data exist to indicate that such an aberration in development does or does not tend to recur in the progeny of such a male, but, since arrests in development generally tend to be transmitted to the offspring, it is well to be on guard and view with distrust the use, as a sire, of an animal so affected. Cysts of the testicle, epididymis, and spermatic cord are recorded.

\section{Benign Tumors of the Female Genitalia}

Benign tumors of the ovaries proper are exceedingly rare. I have no well-marked example in my collection, although they are recorded in great variety. Since, when they involve one ovary only, fertility is unimpaired, they are not sought in connection with sterility. They are but rarely of such volume as to change the appearance of the animal. Most frequently they attract clinical attention by causing incarceration colic, the tumor having fallen over an intestine so that its long peduncle becomes wrapped about it in a manner to prevent the free passage of feces along the canal. Generally the bodies leading to such incarceration colic are not true tumors. Most frequently they are ovarian (Fig 71) or par-ovarian (Figs. 72-74) cysts of large size. There are at least four distinct types of ovarian cysts in cows, and probably an equal number in other species:

1. There are generally present a large number of small cysts, from $1 / 20$ to $1 / 10$ of an inch, apparently atretic follicles. They have no known relation to breeding efficiency or to the general well-being of the animal.

2. The moderate-sized cysts of nymphomania, apparently resulting from the death of the ovum, as a result of ovarian infection associated with an increased volume of follicular fluid. 


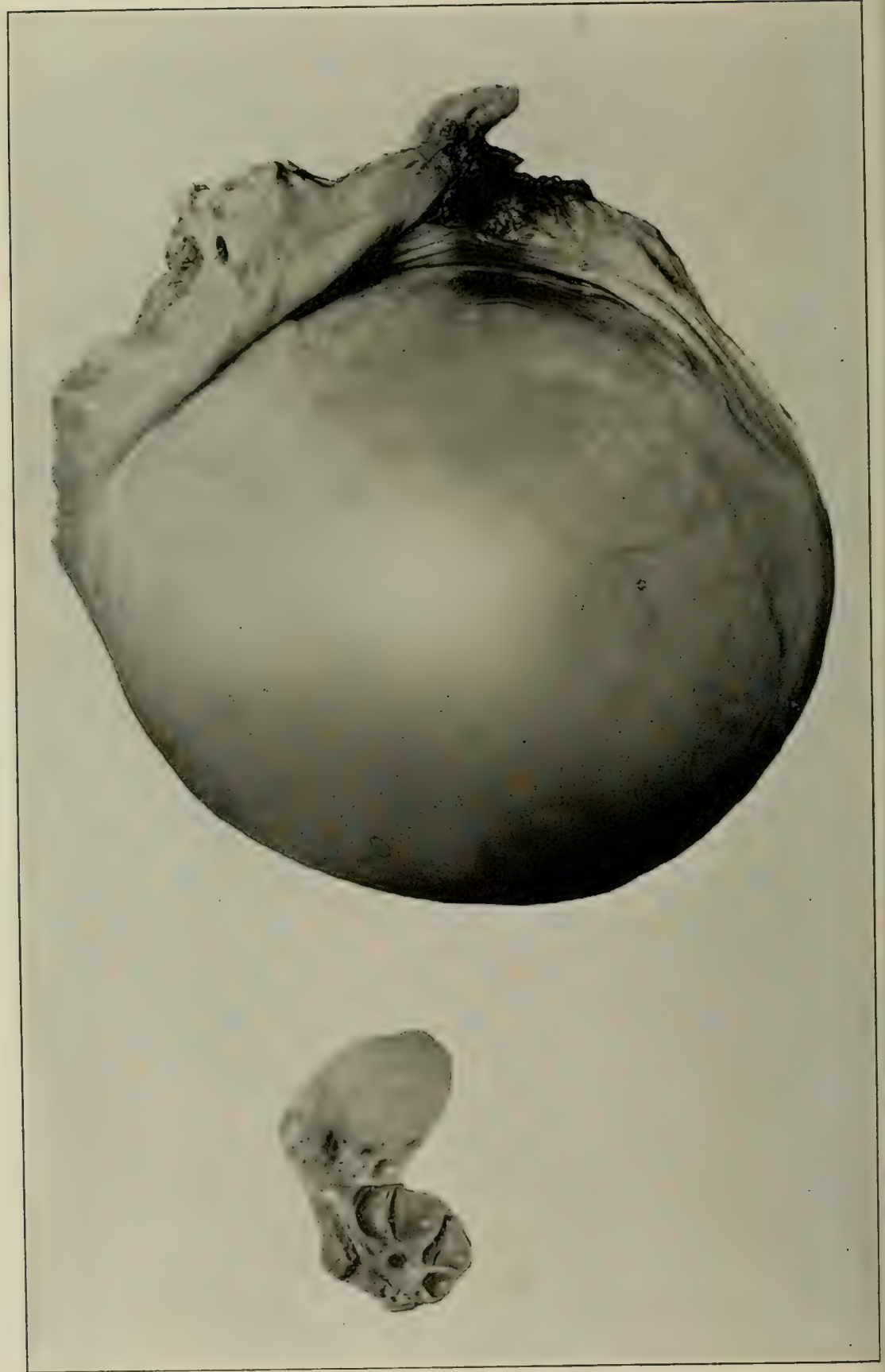

FIG. 7I-Large ovarian cyst of pregnant cow (above) with pair of normal ovaries (helow), showing corpus luteum of pregnancy. 
3. Cystic degeneration of the corpus luteum.

4. Large, single, thick-walled cysts which may reach an indefinite volume and are of unknown origin.

The fourth type described commonly bars functional activity in the involved organ, but does not interfere with the reproductive powers of the other gland. The chief interest in such cysts is their volume and their power, in common with par-ovarian cysts, to cause incarceration of the intes-

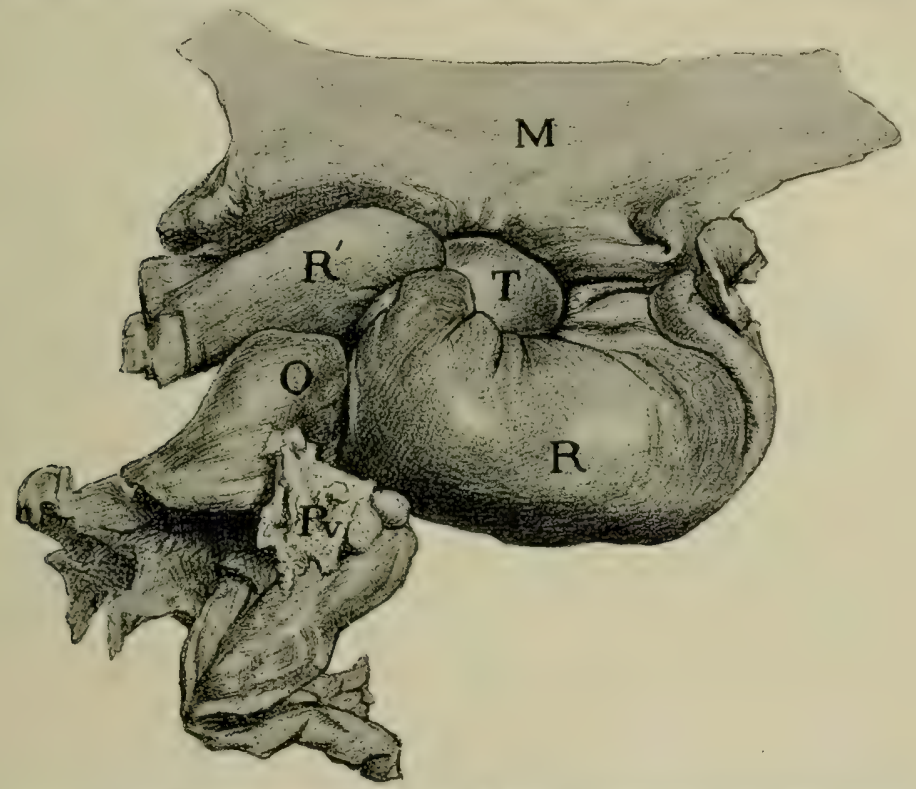

FIG. 72-Parovarian Tumor of Mare, Incarcerating Rectum. View from right side with the tumor, $T$, almost hidden by the constriction caused by the pedicle.

Lettering same as Fig. 74

tines. The diagnosis of large ovarian or par-ovarian cysts must, as a rule, be tentative only. One large and somewhat cystic, malignant, ovarian neoplasm in a cow was diagnosed by a colleague as an "abscess in the uterine ligament". I diagnosed it as a large ovarian cyst. This also was erroneous. It was indeed somewhat cystic, but the error would have been very important had an attempt been made to rupture or puncture the malignant growth within the peritoneal 
cavity. Therefore it is best to use caution in diagnosis, when the ovary is extremely large. However confident one may feel that he is dealing with a benign cyst, he should remember that it may be malignant and in operating proceed upon a safe course by performing laparotomy and, if possible, removing the gland intact.

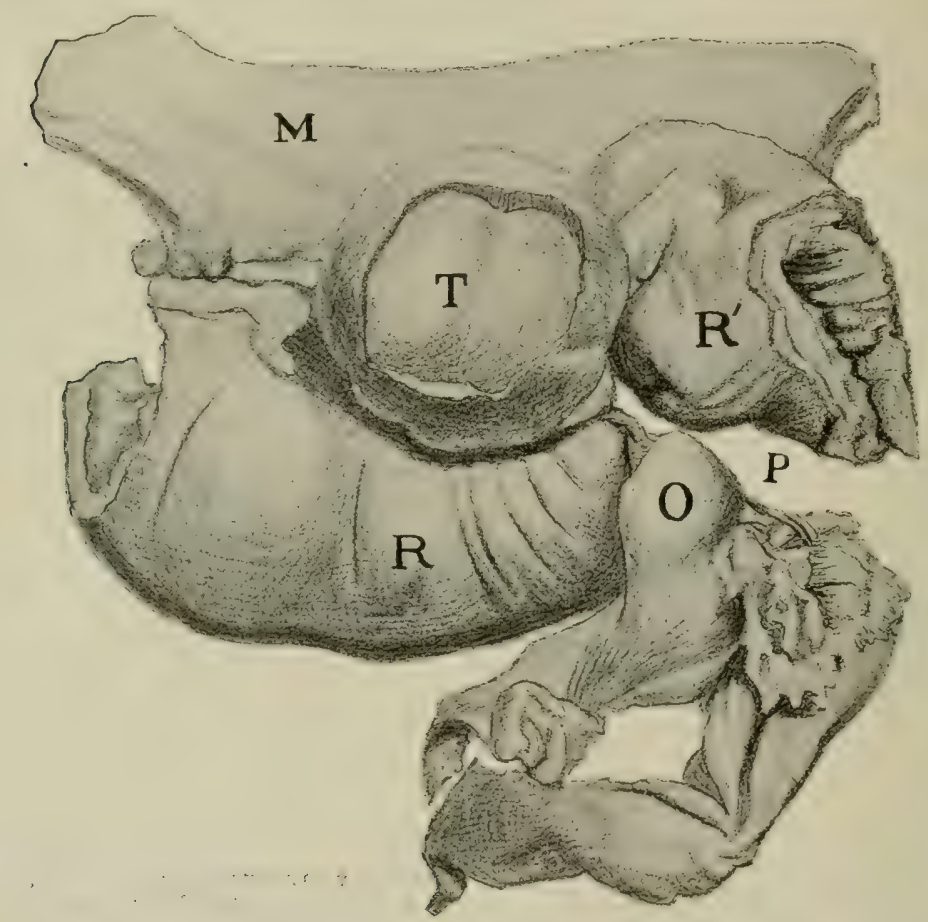

FIG 73-View of Fig. 72 from left side, the tumor exposed by cutting away part of mesentery.

Lettering same as Fig. 74

In mares and cows the diagnosis of uncomplicated cases is to be made by rectal palpation, with the uterus and ovaries drawn back as far as prudent by fixing uterine forceps upon the cervix and exerting traction. If the cyst is very large, the ovarian ligament and the oviduct may be much elongated, so that the ovary may lie far ahead of its normal 
location, anterior to the apex of the uterine cornu When large ovarian tumors cause incarceration colic, the diagnosis is not always easy. If the incarceration involves the pelvic portion of the rectum, the veterinarian faces a rather delicate problem in diagnosis. He can not palpate the uterus and ovaries per vaginam, and he may find palpation per rectum barred by incarceration. The principal question lies between a stricture of the rectum and a mechanical constriction investing the gut from within the pelvic cavity. The investment of the rectum by means of the peduncle of

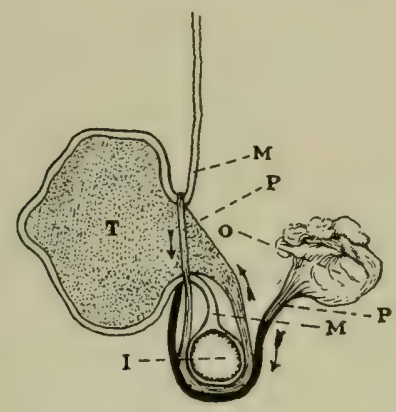

FIG. 74-Schematic illustration of method of incarceration.

$I$, Intestine ; $M$, mesentery ; $O$, ovary ; $T$, parovarian tumor or cyst ;

$P$, pedicle of tumor ; $R$, rectum; $R^{\prime}$, rectum posterior

to point of strangulation.

an ovarian or other tumor produces no changes in the walls of the rectum such as those encountered in actual stricture. There is no inflammation, no sclerosis, and no edema of the rectal walls, if they have not been neglected nor tampered with. The hand may pass freely along the rectum until it comes suddenly upon the constricted portion, when further progress is more or less completely barred. In the incarceration colic caused by the parovarian cyst illustrated in Figs. 72,73 and 74 , the clinical features were identical with those due to ovarian incarceration of the intestine. I found that anteriorly a finger could be passed through the constriction into the free portion. The constriction was necessarily limited in area to the diameter of the peduncle of the cyst. The 
distinguishing element in the case should have been (my colleagues and I each failed in diagnosis) that the constriction was not in the rectal wall but was due to an investing cord outside the intestine. The history aids in diagnosis. True stricture usually develops gradually, and commonly has a history of prior injury. Perirectal abscesses or tumors develop slowly, and the symptoms of colic come on gradually. Ovarian and parovarian pedunculated tumors cause no clinical manifestations until some accidental displacement of the tumor causes a sudden incarceration of the intestine by the tumor peduncle and violent colic quickly ensues,

In the diagnosis of this type of intestinal incarceration, it is not essential that the histologic character be determined. It is of little surgical consequence, for the moment, whether the tumor be ovarian or par-ovarian, cystic or solid. It calls for prompt relief by surgical means.

These tumors attain a diameter of four to twelve or more inches. Owing to their weight they drop forward and downward in the abdomen and their attachments become greatly elongated. They consist of a single cyst, generally showing traces of ovarian tissue, flattened out on one side of the spherical cyst. I have seen one in a mare presented in my clinic because of recurrent severe colics. Rectal examination revealed an ovarian tumor, regular in outline, about ten inches in diameter. Its cystic character was assumed, but could not be definitely ascertained. The ovary (left) was successfully removed through an incision in the left flank by my colleague, Frost, and myself. The cyst was punctured with a trocar after it had been brought into the laparotomy wound. This permitted it to be removed through a moderate-sized opening. Similar ovarian cysts occur in the cow, but so far as known do not so frequently incarcerate the intestines. They have the general clinical importance of benign ovarian tumors and demand surgical removal merely because of their physical menace to the health and life of the patient. Since they tend to cause increased size of the ovarian artery care is necessary in their 
removal to avoid danger from hemorrhage. The arteries had best be ligated with chromatized catgut. Sometimes vaginal ovariotomy is practicable. Usually it is best to resort to laparotomy in the upper or middle flank region. When considered prudent to operate through the vagina, the technic should be essentially that for ordinary ovariotomy.

\section{Vaginal OVARIotomy in the Mare}

Instruments. Colin's scalpel, ratchet ecraseur $55 \mathrm{~cm}$. long, vaginal tensor.

Preparation of patient. It is best to keep the animal on a scant laxative diet for at least 24 hours, and preferably longer, prior to the operation, so that the alimentary canal shall be somewhat empty, thus decreasing the intra-abdominal tension and relieving the operator from much annoyance due to the pressure of the viscera. When incarceration colic is present, there is of course no opportunity to await the general preparation of the patient. Before commencing the operation, it is best to have an assistant empty the rectum manually. Enemas should not be employed, because there is danger from the expulsion of liquid feces during the operation. It is best, also, to empty the bladder before operating; otherwise the animal is quite sure to urinate during the operation.

Technic. The introduction of the hand into the vagina of the non-anaesthetized mare causes the admission of air along the hand and arm. The vulvar lips fail to envelop the hand and arm closely and the irritation or shock causes the animal to draw air alongside the arm into the vagina, fully ballooning it. The movements of the diaphragm and chest walls tend to cause a vacuum of both chest and abdomen during inspiration, and, if the vulva is partly propped open and the abdominal walls are fixed, air rushes in. The vagina may also be distended by filling it with warm water. Under these conditions the vaginal walls become hard, and stand apart from each other, closely applied against the pelvic walls at every part except at the points where the 
bladder and rectum intervene. These organs, pressed out flat, occupy a minimum amount of space. In the quiescent state the vaginal walls are in contact. From the perineum forward to within about $10 \mathrm{~cm}$. of the uterine os, the vulva and vagina are connected above with the rectum by the pelvic connective tissue. Anterior to this point, the vagina is covered by peritoneum. It is in this area that the incision needs to be made in the operation. The ballooning of the vagina profoundly alters the relation of this operative area, changing it from the horizontal in the quiescent organ to the perpendicular in the ballooned condition. These variations permit of two methods of operating: (1) On the ballooned organ without anaesthesia and with animal confined

FIG. 75-Special spaying ecraseur, $55 \mathrm{~cm}$. long.

in the standing position. (2) On the quiescent organ in the recumbent position under anaesthesia:

1. Without anaesthesia. Secure in the stocks with the head elevated, a rope over the back to prevent rearing, straps beneath the body to prevent lying down, straps or ropes before and behind the animal to prevent backward and forward movements, all four feet pinioned to the floor, and the tail firmly secured and stretched to a beam above. Apply a bandage to the tail extending 12 to 15 inches from its base, in order to secure the tail hairs out of the way of the operator.

With soap, water, and brush cleanse the tail, perineum and vulva thoroughly, being especially careful to remove all detachable masses of sebum; 50 per cent. alcohol or gasoline may be used sparingly to aid in removing this. 'Too free a use of alcohol excoriates the delicate skin. Cleanse the clitoris carefully. Follow the washing with a free ap- 
plication of $1: 1000$ aqueous sublimate solution to the external parts and for a short distance inside the vulvar lips and to the clitoris. Do not introduce irritant disinfectants into the healthy vagina nor deeply into the vulva, as they may cause severe straining during and subsequent to the operation and, by injuring the vulvo-vaginal mucosa, favor subsequent infection of the vaginal wound. The vagina may with benefit be flushed out mechanically with 0.6 per cent. salt or soda solution.

Wash away the sublimate solution with a tepid 0.6 per cent. soda bicarbonate solution, and fill the vulvo-vaginal canal with the same. After thorough disinfection of the hands and arms, remove the disinfectants by washing in sterile soda solution, which at the same time renders the

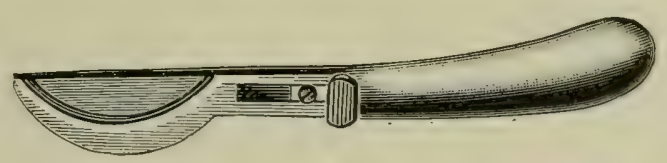

Fig. 76-Colin's scalpel.

hand unctuous and readily introduced through the vulva. Armed with the guarded sterilized scalpel, Fig. 76, introduce the hand into the vagina promptly and when the organ is well "ballooned," unsheath the knife. Place it just above the os uteri, parallel to the long axis of the uterus, a few $\mathrm{mm}$. to the right or left of the median line in order to avoid a loose fold of mucous membrane generally existing there. Hold the blade vertical-that is, with the cutting surface parallel to the longitudinal muscular fibers of the vaginaand, guarding the possible extent of its introduction with the thumb and fingers, push it directly forward with a quick thrust through the vaginal mucosa, the muscular walls, and the peritoneum, until the disappearance of resistance indicates that the latter has been penetrated. This is the most critical step in the operation.

If the hand is introduced into the vagina immediately after the injection of the sterile saline solution, the vagina 
will generally be found "ballooned" or will quickly become inflated under manual movements. If the solution is thrown out, the vagina may collapse and closely invest the hand. In this case more of the liquid should be injected, and it will again dilate. If the hand is introduced without the knife, withdrawn, and then introduced with it, it will frequently be found that the vagina has collapsed and needs a second filling with the fluid. Patience until dilation is accomplished and promptness to act when it is attained are prime requisites to success.

The knife should be pushed through the vagina quickly, making a clean wound the width of the blade, and the latter withdrawn and laid aside. It should be remembered that in this "ballooned" state, the anterior wall of the vagina is but 2 or $3 \mathrm{~mm}$. thick and easily penetrated. Introduce the hand again, push one finger into the incision, then a second and third, and eventually, holding all the fingers in the form of a cone, push the entire hand into the peritoneal cavity. Immediately below the incision and continuous with the tissues involved in the wound, lies the uterus, with a transverse diameter of 4 to $6 \mathrm{~cm}$. With the palm of the hand downward, trace the uterus forward 15 to $18 \mathrm{~cm}$., where it ends abruptly in two cornua of about the same size as the body, which are given off horizontally at almost right angles. Trace these to the right and left for 14 or $15 \mathrm{~cm}$., where they end obtusely, and 3 or $4 \mathrm{~cm}$. beyond this in a direct line, resting upon the anterior border of the broad ligament is the ovary, varying in size according to the degree of disease.

Prepare the ecraseur for use by withdrawing the chain until the loop is barely of sufficient size to admit of its being readily slipped over the ovary (or par-ovarian cyst as described below). Grasp this loop and the end of the ecraseur tube in the hand, carry the instrument to the ovary or tumor, and drop the loop over it from above. Pass some of the fingers beneath the ovary or tumor, push it up through the chain loop, and grasp it there with the thumb and index finger. Holding the ovary with one hand, tighten the chain quickly with the other, examine to make sure that a loop of intestine is not caught, draw the ovary well through the 
loop, include a large portion of the oviduct, and crush off promptly, holding to the gland until carried out through the vulva. If the tumor or cyst is very large it may not be practicable to remove it through the vaginal incision. The incision may be enlarged six to ten inches, but after such enlargement suturing is necessary in order to avoid prolapse of the intestines. The suturing of the vaginal incision is not, however, a serious task. The lips of the wound may be grasped by means of long dressing forceps and held firmly while the operator with a short, curved needle closes the incision by means of a continuous suture beginning at the anterior end. When the ovary is cystic or the surgeon is dealing with a large parovarian cyst, the cyst may be grasped by its peduncle and engaged in the vaginal incision. The cyst may then be punctured with a trocar or scalpel and the liquid contents permitted to escape into the vagina or through the canula of the trocar, beyond the vulva. Wash away any blood from the external parts. Apply sublimate solution freely to the vulva, perineum and tail. Keep the patient quiet for five or six days, and feed lightly on a laxative diet.

2. In operating under anaesthesia, the animal should be cast or confined upon the operating table in lateral recumbency, preferably with the posterior part of the body somewhat higher than the anterior in order to avoid visceral pressure in the pelvic cavity. Place the animal under complete anaesthesia. Prepare the parts as already described. Carry the knife into the vagina as directed previously and render the roof of that organ tense by pushing the os uteri downward and forward with the hand or by means of the vaginal tensor or speculum. It is important that the vagina be held well down toward the floor of the pelvis, so as to carry it away from the rectum, posterior aorta, and iliac arteries while the incision is being made. The incision is now to be made just above and behind and a trifle to one side of the os uteri, in essentially the same manner as under 1 , except that when the vaginal tensor is used, the cut is made upward and backward instead of directly forward. The 
remainder of the operation is identical with what we have described under 1 . Under anaesthesia the vagina is flaccid and cannot be made to "balloon" but may be distended with sterile soda or salt solution.

Dangers. Wounding of the rectum is scarcely possible under the first method, if it has been emptied as advised above, if care is taken not to attempt the incision until the vagina is well "ballooned," and the stab wound is made directly forward. If made upward when the organ is so tensed, the accident is highly probable, and with the undilated vagina, where it is necessary to cut upward, the danger is ever present. In order to avoid this danger, when operating by the tirst method, the operator should await the complete "ballooning" and then make his incision as directed. In the second method, the accident is to be prevented by being careful to push the vagina down away from the rectum and hold it away while the incision is being made. If the wound in the rectum passes through the pelvic connective tissue behind the peritoneum, it is of little consequence, but the operation should be abandoned; if the bowel is opened into the peritoneal cavity, the accident is generally, though not always fatal. The vaginal incision may be enlarged and the wounded portion of the rectum drawn out through the vulva. The wound may then be closed by sutures.

Wounding of the iliac arteries, which generally produces prompt death from hemorrhage, results from the incision being made upward instead of forward when the vagina is "ballooned" or from a failure to hold the roof of the vagina down and away from the part while making the incision in the flaccid organ, as is the case with the recumbent animal under anaesthesia. It is most likely to occur with timid operators who become nervous, especially when the vagina does not "balloon" promptly or the mare is not well secured. The accident is wholly unnecessary if the operator will await the "ballooning" in the first operation, while by the second method it is prevented by proper care in holding the vagina downward and forward during the incision. When it has occurred, it is generally beyond remedy, though 
in some cases the prompt intravenous injection of adrenalin chloride may stay the hemorrhage and save the life of the patient.

Wounding of the uterus may occur when the incision is directed downward and may greatly embarrass the operator and confuse him because his fingers or hand may pass through the incision into the uterine cavity. It is to be avoided in the first operation (without anaesthesia) by carefully directing the incision straight forward. When the accident occurs, it is of little consequence beyond the embarrassment and may be overcome by again dilating the vagina with fresh injections of the soda solution and making a new incision. If preferred, the first cut may be corrected by placing an index finger against the peritoneum at the upper part of the wound, and, with a sudden and vigorous thrust, breaking through into the peritoneal cavity, or by again using the scalpel and directing the incision properly. If it is attempted to rupture the peritoneum with the finger, this must be done by a sharp thrust, since otherwise a large section of the membrane will be pushed away from the subjacent tissues.

Incomplete penetration of the vaginal wall is liable to occur if the scalpel is dull, if the vagina is imperfectly "ballooned" and flaccid, or if the operator is unduly timid. It is best prevented by avoiding the causes mentioned. Once it has occurred, it is generally best, in the operation without anaesthesia, to again "balloon" the organ and make a new incision either to the right or left of the first. It may be overcome also by thrusting the index finger through the peritoneum as described in the preceding paragraph or by completing the cut with the scalpel.

The mistaking of a ball of feces for the ovary or tumor' has occurred to inexperienced operators and the fatal error of removing the portion of the rectum surrounding the fecal pellet committed. The blunder is uncalled for: the fecal ball is movable in the bowel, the intestine is far more massive than the broad ligament, and the ovary is to be definitely identified by its being lodged in the broad ligament just be- 
yond the end of the cornua, which is continuous with the uterus. If, therefore, one traces the uterus forward to the cornua, and thence along each of these to their extremities and along the borders of the broad ligament to the ovary, as above directed, the error will not occur.

The vaginal incision may be made too low and pass beneath the broad ligament. This is to be avoided by being careful to keep close to the median line and above the os uteri. If it occurs, the operation may be completed from beneath without very great difficulty, only that the ovary now lies above the hand and must be drawn down from above the broad ligament in order to fix the ecraseur upon it.

Infection, which always constitutes the most serious danger, is to be avoided by properly securing the animal, by the avoidance of irritant antiseptics in the vagina, by rigid asepsis at every stage, and by carrying out the mechanical parts of the operation deliberately, vigorously, and neatly. If infection should occur, it will generally take the form of pelvic cellulitis with abscesses and rectal stricture. Enemas of normal salt or soda solution afford the surest relief of the stricture and impaction in front of it. The abscesses must be watched and opened early into the vagina or rectum, and the case treated internally and locally according to general surgical principles.

\section{VAGINAL OVARIOTOMY IN THE COW}

While vaginal ovariotomy is the common method used in the cow and mare for the simple purpose of removing healthy ovaries in castration, or of ovaries aberrant in function as in nymphomania, it is not of great value in the removal of ovarian tumors, since as a rule they are too large for removal by this route. Neither does the vaginal operation afford satisfactory opportunity for controlling hemorrhage if increased vascularity exists.

Instruments. Colin's scalpel, vaginal dilator, spaying ecraseur, or emasculator.

Technic. Confine the cow in the standing position in the stocks, secure the head firmly, and pass two boards beneath 
the abdomen and sternum to prevent lying down, and a rope over the middle of the back to prevent arching of the spinal column and straining.

Wash and disinfect the tail and the perineum and flush out the vagina with a 0.5 per cent. solution of carbolic acid or lysol at a temperature of about $100^{\circ} \mathrm{F}$. Insert the vaginal dilator with one hand and push the prolongation at the anterior end into the os uteri. With the other hand, elevate the handle of the dilator, and depress and push forward the uterus, thus rendering the roof of the vagina tense and pushing it downward away from the rectum. Carry the scalpel into the vagina with the right hand and, resting it in the oval of the dilator, make an incision through the roof of the vagina, beginning at a point 8 to $10 \mathrm{~cm}$. posterior to the os uteri and extending backward on the median line for a distance of 2 or $3 \mathrm{~cm}$. Be careful to make the incision entirely through the mucosa, muscle and peritoneum at the first cut, since any failure to complete it tends to cause the peritoneum to separate from the muscular coat and form a pocket between them, while the serous membrane, being very elastic, renders it difficult to complete the incision. Introduce two fingers through the incision, if the ovary is not greatly enlarged, and, reaching over the side of the vagina to the right or the left, the right or left ovary respectively is recognized, when normal in volume, lying immediately against the lower part of the base of the uterine horn, just at the anterior border of the pubis, in a mass consisting of the cord-like Fallopian tube and the fimbriæ of its pavilion. When the seat of an extensive tumor or cyst, it shifts its location downward and forward a variable distance according to its weight. The normal ovary may be distinguished as a firm oval mass 2 to $4 \mathrm{~cm}$. in length and 1 to $2 \mathrm{~cm}$. in its lesser diameter attached to the broad ligament. If not promptly recognized by the sense of touch, trace the vagina and uterus forward with the fingers from the vaginal incision to the cornua and follow them as they bend forward and downward, and then backward and upward to the oviducts, until the ovary is reached where it is attached to the broad ligament, just beyond the fimbriated end. 
Grasp the ovary between the fingers and, if not too large, draw it through the incision into the vagina. Introduce the emasculator with the other hand, and, when the ovary is reached, open the instrument far enough to admit the ovarian attachments between the jaws, push the ligament between the jaws, close the forceps and sever the ovary. Or introduce the ecraseur, draw the ovary through the loop of the chain and, holding it securely until the instrument is tightened, crush it off. If the ovary is the seat of an extensive tumor, the hemostatic power of the ecraseur or emasculator must not be relied upon. The same is true in a lesser degree of nymphomaniac ovaries. In such cases it is far more prudent to ligate the artery with large chromatized catgut, passed through the ovarian ligament by means of a needle. For this purpose the ovary is drawn into the vagina and its ligament engaged securely in a long pair of forceps. The ligature is passed through the pedicle of the tumor and tied tightly outside the forceps. The attachments are then divided between the ovary and the ligature, care being taken to leave a sufficiently long stump to give ample safety against slipping.

It is essential that plenty of the broad ligament and oviduct be excised with the ovary to insure the entire removal of the gland, because the accidental leaving of the smallest particle of ovarian tissue may cause a development of this into abnormally large cystic ovisacs, tending to increase instead of decrease nymphomania. Generally no after care is necessary.

The danger's are similar to those in the mare. The iliac arteries may be wounded in the same manner. The accident is preventable by being careful to push the vaginal roof well downward away from the rectum and sacrum.

Another danger appears in the presence of the rumen, the supero-posterior portion of which, when filled with food, projects into the pelvic cavity so that, if the cut is directed forward, a stab wound readily penetrates its walls with fatal results. Make the cut upward and backward. 


\section{OVARIotomy in the CoW AND MARE By the Flank}

Instruments. Clipping shears, convex scalpel, spraying emasculator or ecraseur, heavy needle and thread.

The animal may be secured as in the preceding or confined in lateral recumbency with the hind legs extended backward and the anterior limbs forward. To accomplish this, loop a rope about the two fore feet, another about the two hind feet, and, drawing upon these, cast the animal and secure it in recumbency with the legs extended and body stretched by fastening the ropes to two strong posts about 8 to $10 \mathrm{~m}$. apart. The operation may be performed in either flank. The recumbent animal should lie with the diseased ovary uppermost.

Clip the hair from the upper part of the flank and shave the operative area, disinfect an area 15 to 25 $\mathrm{cm}$. square, make an incision about $12 \mathrm{~cm}$. long beginning at a point equidistant from the anterior tuberosity of the ilium, the ends of the transverse processes of the lumbar vertebrae and the last rib, and extend it downward perpendicularly, severing the skin and subcutaneous muscle. If the attachments of the tumor are short, it may be impossible to bring it out through an incision in the upper flank. In such cases the laparotomy should be performed in the middle or lower flank region. Divide the external oblique muscle in the direction of its fibres by means of the scalpel handle or the fingers, and repeat the process upon the internal oblique, after which puncture the peritoneum with the scalpel. By thus dividing the external and internal oblique muscles, an X-shaped opening through the abdominal walls results, which closes automatically and obviates the need for peritoneal and muscular sutures. It also conserves in the highest measure the integrity of the muscular walls.

Force one hand through the opening into the peritoneal cavity and search for the ovary or tumor at the same point and by the same method as in the preceding operation: that is, locate the uterus within the pelvic cavity, between the rectum and bladder, and trace it, the cornu, and broad liga- 
ment to the ovary. Then draw out the diseased ovary through the abdominal incision and remove it under ample safeguards against hemorrhage. Cleanse the wound and close the skin incision with continuous sutures.

\section{Benign Tumors of Oviducts, Uterus and Cervix}

Benign tumors of the oviducts, uterus, and cervix are extremely rare. I have in my collection no tumors of oviduct or cervix and but two of the uterus-one a fibroid from a cow, the other a myom from a mare. The tumor from the cow (Fig. 77) is from the abattoir, and hence without history. It is oblong, of even contour, measures $4 \times 7$ inches, and is located near the apex of the cornu. The uterine walls show no changes from its presence.

The myom from the mare (Fig. 78) was unobserved until, while at work, the tumor, which weighed eleven pounds, became dislocated, and caused pain with expulsive efforts of such violence that the tumor was forced out through the vulva and could not be returned. The dragging upon the uterus and its ligaments caused great suffering. The case was entered in the clinic of my colleague, Frost. The tumor was removed, the uterine wound sutured, and the uterus returned to its position. The mare died a few hours later of hemorrhage from the uterine artery, which was ruptured by the violent dragging of the expelled tumor. The tumor measures $5 \times 11$ inches.

In one case of tumor of the uterus, diagnosed clinically as a fibroid, the cow bred several times in the opposite horn, but after the tumor had reached a diameter of 10 to 12 inches, she became sterile, probably owing to the presence of the neoplasm.

Benign uterine tumors may generally be diagnosed clinically with reasonable assurance. Ordinarily the tumor grows slowly, causes no discharge or irritation, is not painful upon manipulation, is usually very firm, and presents an even contour. The malign tumor would generally give evidence of its presence by the discharge of pus and necrotic debris. The macerating fetus gives an irregular swelling, 


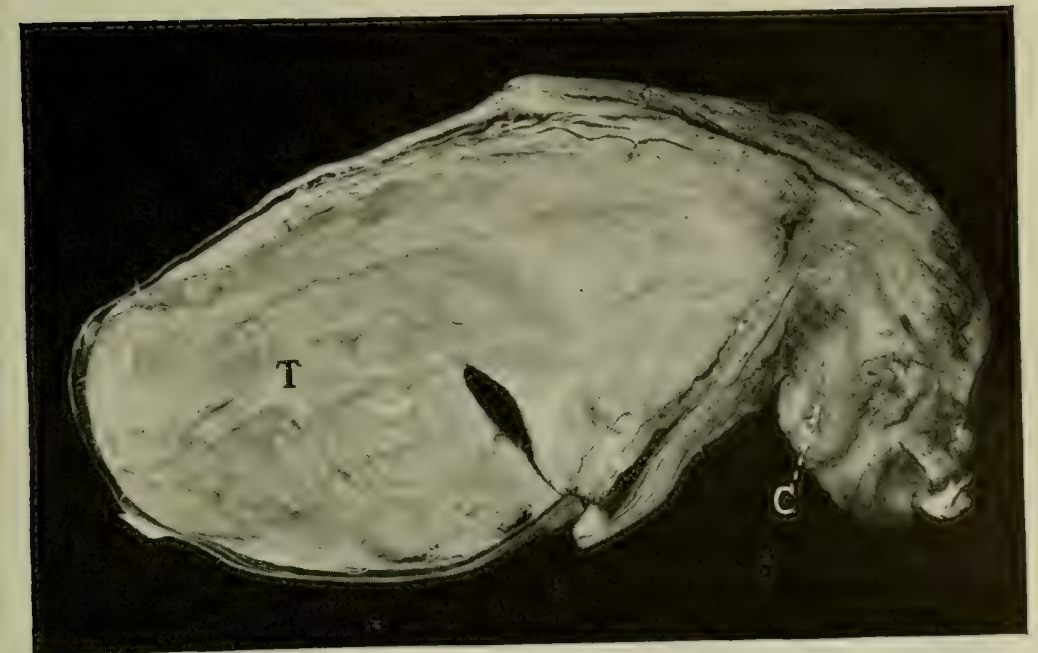

FIG. 77-Uterine Fibroid. Cow.

$C$, Cervix; T, Tumor.

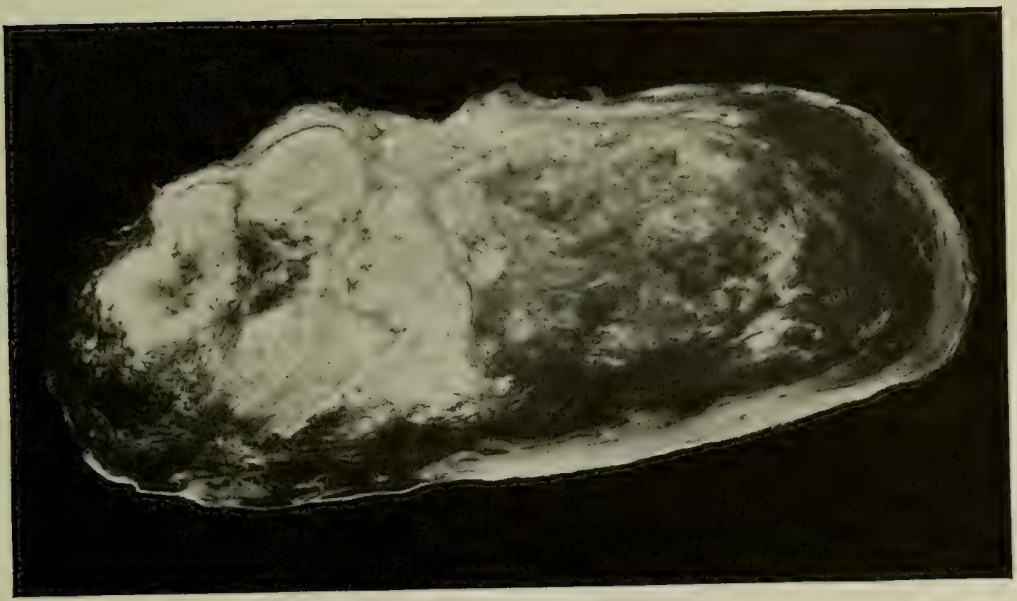

FIG. 78-Uterine Fibroid. Mare.

The dark periphery is due to profuse hemorrhage, apparently the result of contusions and the incarceration of arteries during prolapse of the tumor and uterus through vulva. 
the fetal bones are commonly palpable, and there is a very fetid discharge. The desiccating uterine hematoma and the desiccating fetus in its early stages are less firm than the fibroid and fill one horn and uterine body from the cervix to the ovarian end of the cornu. The tumor, on the other hand, occupies but a part of the total length of the uterus.

When clinically recognized in valuable breeding animals, uterine tumors should be removed after performing laparotomy, as indicated for spaying through the flank. If the tumor can be removed without destroying completely a transverse section of one cornu, the cornu and ovary should be preserved, but if the removal of the base of the tumor will lead to the occlusion of the lumen of the horn, the entire cornu on the ovarian side of the tumor, with the oviduct and ovary, should be removed. The hysterotomy incision should be closed by intestinal sutures.

Tumors, usually fibroids, occur not rarely at the hymeneal ring, emanating apparently from the vestiges of the hymen. They are largely pedunculated. They have already been referred to incidentally while considering vaginal hernia. When recognized, they should be removed by incarcerating the pedicle in a suture or ligature. It is best to arm a needle with a strong suture and, exerting moderate traction upon the tumor, pass the needle deeply through the vulvo-vaginal wall in a manner to include the entire base of the newgrowth. Tie the suture very firmly, incarcerating the entire base of the pedicle and causing prompt necrosis of the tumor. The body of the tumor may then be excised, leaving enough of the pedicle to insure the safety of the ligature. Do not attempt ecrasement or plain incision because of the great danger of vaginal hernia.

I have observed two instances of apparently infectious vulvar benign epithelioma, both in heifers. In one instance, two heifers in contiguous stanchions developed epithelial tumors, or "warts," in the vulva. The tumors were uneven and highly vascular. They were three inches or more in diameter. In the other instance, in a group of 26 heifers in one lot, none of which had copulated, 15 developed vulvar 
tumors. The tumors were confined, so far as could be determined, to the interior of the vulva, largely at the superior commissure and about the meatus urinarius. The tumors were of every size up to four inches in diameter. In two or three of the heifers where the tumors were very large, the growths protruded from the vulva, especially when the animal was recumbent. The tumors caused no visible distress or injury to the animals. Some of the larger tumors were removed after transfixing and ligating their bases with strong sutures. Much care was necessary in one or two cases to avoid invading the meatus urinarius. The tumors had developed during the winter months while the heifers had been confined largely to the stable. With the advent of spring, with grazing, the new-growths disappeared spontaneously. They behaved clinically much like the common "warts" and the ringworm of the head and face of young cattle. 


\section{INFECTIONS OF THE GENITAL ORGANS SECTION I. THE GENITAL INFECTIONS OF CATTLE} CHAPTER XII

\section{THE SPECIFIC VENEREAL DISEASES}

1. The Vesicular Venereal Disease of Cattle. VesiCUlAR Exanthem. APHTHOUS VENEREAL Disease. Blaschenausschlag

The vesicular venereal disease of cattle is widespread in continental Europe, and occurs somewhat rarely in the United States. It is highly contagious. Ordinarily it is transmitted by copulation. After handling diseased animals, it is readily transmitted by careless manipulations of the genitalia of healthy animals, and by grooming apparatus, such as brushes, currycombs, and sponges. It is yet more certainly carried by means of douching apparatus or other instruments used in handling the diseased animal and thence brought into contact with the sound genital mucosa of another. It is one of the most intensely contagious diseases known to veterinarians. Whenever genital contact occurs, whether directly through coitus, or indirectly by such means as suggested above, infection is practically certain. A diseased cow may transmit the infection to her neighbor by switching her tail.

The vesicular venereal disease is analogous to the genital horse pox, or vesicular exanthem of horses, though they are not known to be related. It bears some resemblance to the chancroid of man.

The symptoms are very acute and marked. In the female the disease appears suddenly as an intense vulvovaginitis. The vulvo-vaginal mucosa becomes greatly injected and swollen. It soon shows numerous dark red petechiae, which quickly develop vesicles. The vesicles, which 
are of short duration, either rupture as vesicles or become pustular and rupture, in each case leaving an ulcer. The ulcers, which are one-eighth to three-sixteenths of an inch or more in diameter, very shallow, and exceedingly angry in appearance, give rise to an abundant muco-purulent discharge which, issuing from the vulva, soils the vulvar labiae, the tail, and the adjoining parts. The vesicles and ulcers continue to develop in successive crops for a number of days. The ulcers heal with only a very slight, temporary scar. Much pain is present. The ulcerating mucosa is intensely sensitive. Any attempt at manipulation causes very great resistance. The ulcers bleed freely upon touch. When undisturbed, the cow is uneasy, as shown chiefly by frequent switching of the tail and more or less stamping of the hind feet. When urination occurs, the urine, coming into contact with the highly inflamed ulcers, causes very marked and intense pain. The animal switches her tail violently and stamps the hind feet in rapid succession. Whenever the animal defecates, the mechanical pressure unavoidably causes pain, because of the extreme tenderness of the vulva and vagina. The pain is not as acute as that observed in urination. Whenever the animal moves, the muscles of the limbs which come in close contact with the vulva naturally drag upon the inflamed organ, causing pain, which is revealed by a stiffness of gait.

The infection does not necessarily prevent impregnation. If the cow becomes pregnant, there seems to be no unfavorable influence upon the life of the embryo. It is stated that in some cases the disease results in chronic catarrh of the vagina. A commoner, and apparently more threatening termination is adhesions between the walls of the vagina, which may cause more or less constriction of that canal.

In the bull the symptoms of the disease are analogous to those observed in the cow. The mucous membrane of the penis, prepuce and sheath becomes inflamed, swollen and tender. The eruptions in the mucosa of the genital organs are identical with those observed in the cow. The urethra may also become involved, giving rise to a muco-purulent 
discharge therefrom. Urination causes pain of the same general character as that observed in the cow. The swelling of the penis and the sheath may be so great as to cause phimosis. Erection of the penis causes bleeding. This is further emphasized by copulation. Kampmann records a case of extensive necrosis, with permanent deformity of the penis, due to this disease.

So far as known, the disease does not affect other species of animals. The period of incubation is brief. The course of the disease is brief and stormy. Spontaneous recovery generally occurs in from ten to thirty days. It is said that no enduring or valuable immunity is acquired, and that an animal may readily become re-infected by copulation.

The pathogenic organism which causes the disease, as is common in diseases associated with vesicles, is unknown. Ordinarily an outbreak of the disease appears suddenly and can not be traced to other herds. Like genital horse pox, it appears to develop spontaneously. No suggestion of any exposure from strange cattle exists. Suddenly the outbreak is present in the stable in its most acute and highly contagious form. Several or many animals are already infected. Once established, the infection is readily traced from animal to animal. It may then spread to a few neighboring herds through the agency of the bull, but it is so evident that it is quickly observed by the layman, who naturally takes measures to control it. The disease then disappears, until suddenly it reappears in some other area without any connection whatever being traceable between one outbreak and another.

Ordinarily the differential diagnosis of the vesicular venereal disease is easy. At times there has been confusion with the nodular venereal disease, but this is unnecessary. The former is characterized by vesicles and ulcers; the latter by nodules or granules, which may be translucent, but are hard and solid throughout. The nodular venereal disease is chronic. While there may be a copious muco-purulent discharge and the affected parts may be sensitive, the tenderness is not as marked as in the vesicular venereal 
disease. The very rapid spread of the vesicular disease serves also to distinguish it from the virtually omnipresent nodular venereal disease.

The prognosis is highly favorable. Recovery from the disease is prompt, and almost always complete. It causes small loss to the dairyman. The cow retains her appetite, she does not lose perceptibly in flesh, and the milk supply is not seriously disturbed. Rarely, especially following the use of strong antiseptics, constriction or atresia of the vagina may follow, which may interfere later with copulation or parturition.

The control of the disease must rest fundamentally upon isolation, especially sexual isolation. Since the disease is preëminently venereal in character, breeding should be completely suspended until all signs of the disease in the herd have disappeared. Even steers or oxen should not be permitted to run in pasture or in paddocks with cows, because in attempts at copulation they might transmit the disease. It is not necessary to separate the diseased from the healthy by any great distance. Close proximity should of course be avoided, because the disease might be transmitted from animal to animal through the medium of the tail or soiled bedding. Diseased animals should on no account be bred untii they have thoroughly recovered, and even then it is well to use antiseptic precautions just before and after breeding. For this purpose, both the vagina of the cow and the sheath of the bull should be thoroughly disinfected prior to permitting copulation. The disinfection of the sheath of the bull should be repeated immediately after copulation, and the douching of the vagina of the cow may be carried out within five or six hours after copulation and repeated occasionally until the attending veterinarian believes than any possible danger has been eliminated. The thorough disinfection of the stalls and of the soiled parts of the animals contributes materially to the control of an outbreak.

The treatment of the disease consists of the disinfection of the copulative organs and of those parts of the animal 
which have become soiled from the venereal discharges. Disinfection is a difficult problem and must be conducted with caution. The genital mucosa is in such a highly irritable state that one can not with success employ any strong disinfectant. It must constantly be borne in mind that a mucous membrane can not be definitely disinfected and that any radical effort to disinfect will serve only to irritate and destroy the genital epithelium and intensify the disease. Something must be used which is non-irritant. It will be found that a mechanical cleansing of the parts with a physiologic salt solution will best answer the purpose. In this way the tissue debris and pus may be washed away and the highly sensitive ulcers cleansed without inducing any material pain. On the other hand, the douching actually tends to soothe. If the animal will bear it without pain, very mild antiseptic solutions may be used as a douche, such as 4 per cent. boric acid. Whatever antiseptic is selected, its action should be carefully noted, and whenever it causes straining its use should at once be discontinued. If the cow is not in milk or if the milk is not to be used for human consumption, one may use a dressing of 10 per cent. iodoform in oil introduced through a catheter or tube. If the cow is being milked, the use of iodoform inevitably taints the milk in such a manner as to render it obnoxious.

In some continental European states, the disease is reportable as a dangerous contagion, subject to legal regulations. I have observed several outbreaks, all handled by voluntary personal quarantine. The symptoms are so striking that any intelligent breeder should realize that his interests dictate adequate control measures. Under the specific laws for the control of contagious diseases in most states, it may very properly be reported, and should be, if the attitude of the custodian threatens the extension of the disease. I have not known of an outbreak reported to the sanitary authorities in America. 
2. The Nodular Venereal Disease. Infectious VagiNitis. Vaginitis Verrucosa, Metritis et Vaginitis

Infectiosus. AnsteCKende SCHEIdENKATARRH, ETC.

The classification of the nodular venereal disease as a specific infectious malady is not free from objection. Isepponi of Chur (Switzerland) was the first writer to bring the disease (or the lesions) notably before the veterinary profession as a distinct, specific malady. He was promptly followed by numerous veterinarians. His views were endorsed first by his Swiss colleagues, and later by Austrian, German, French and other Continental European veterinarians. Leading European authors-Friedberger and Fröhner, Hutyra and Marek, and others-assigned it a definite place amongst infectious diseases of cattle. The lesions characterizing the condition are so nearly universal that, soon after the publication of Isepponi's observations, a few veterinarians, instead of considering it specific, held that it is in fact a physiologic condition and that the absence of the granules or nodules indicates disease. The dissenters were not men of authority or prominence in the profession. The vast majority of veterinarians in the front rank accepted and supported the teachings of Isepponi.

The prominence of the nodular venereal disease was based chiefly upon the belief of Isepponi and his followers that it was responsible for most, or much, of the sterility and abortion of cattle. Although sterility and abortion have been recognized throughout history as a cause of serious losses among animals, the interferences with reproduction had reached a phase in the time of Isepponi where they were causing greatly increased concern to individuals and to the state. The views regarding the causes of abortion were chaotic and the people were so anxious to find some rallying point that they eagerly grasped the first plausible explanation, hoping that behind the recognition of the cause lay a remedy. For about ten years the belief in the importance of the nodular venereal disease spread. This belief rested upon clinical observations. It was held, by those who studied the problem, that the amount of sterility 
and abortion in a herd was parallel to the virulence of the nodular venereal disease. All deductions were based upon the presence or absence of given lesions.

Bacteriology was making enormous strides. Wherever a distinct lesion was recognized, search was made for a bacterial invader as a cause and efforts were directed toward the experimental transmission of the disease. Ostertag, after a brief bacterial study of the disease, reached the conclusion that it was due to a streptococcus. No one seriously attempted to cause sterility or abortion experimentally by the artificial transmission of the disease. Wider observation showed the lesions to be essentially omnipresent. Many began to doubt that these lesions could be the cause of sterility and abortion, since in that case the bovine species would be wiped out. Many were not ready to believe (though abundant examples exist) that an infection can exist generally and only now and then prove serious.

Ten years after the interesting observations of Isepponi, Bang made his famous discovery of the $B$. abortus and surpassed in the plausibility of his teachings by experimentally inoculating pregnant animals which were assumed to be free from the $B$. abortus infection. Some of the experiment animals aborted and from the uterus of the cow or the stomach of the abort Bang recovered a bacterium which, it was assumed, was the lineal descendant of the bacteria introduced. The teachings of Bang quickly gained a prominent place. In the enthusiasm which grew up, sterility was essentially forgotten or assigned a secondary place. The nodular venereal disease was promptly overshadowed as an important genital infection. The $B$. abortus occupied the field of thought in connection with genital diseases, and all else was consigned to oblivion.

During the two past decades little has appeared in veterinary literature concerning the nodular venereal disease, and that little has been generally ignored or roundly condemned. The disease is still described in recurring editions of the foremost treatises upon veterinary medicine, rather as a matter of habit than because of any living in- 
terest in the subject. It has never received, and is not receiving serious study at the hands of pathologists and bacteriologists. The disease is accordingly a difficult subject with which to deal. It may be said to be a lesion without a known cause and regarding the effect of which there is very scant knowledge. I feel that the disease should be recognized as holding a definite place amongst the genital infections, but do not believe that at present it should have ascribed to it that importance with which Isepponi and his followers clothed it nor that it should be so utterly ignored as it has been recently.

The nodular venereal disease may be described as a virtually universal, chronic infection affecting the mucosa of the copulatory area of the genitalia. It involves both sexes and all ages of ruminants and swine. It expresses itself clinically in the form of granular or nodular elevations in the mucosa of those parts participating in coitus (vulva, vagina, sheath, prepuce, and glans penis).

The essential clinical symptom is the appearance in the genital mucosa, protruding above the surface, of nodular, conical, or spheroidal elevations, usually 1 to $2 \mathrm{~mm}$. in diameter. (See Figs. 79, 80.) In the female they are best observed clinically by holding the vulvar lips apart and causing a strong light to enter obliquely. The examination may be conducted advantageously in a well-lighted stable, by having the animal placed with her posterior parts presenting toward a window somewhat above the level of her back. The examiner grasps each vulvar lip between the thumb and fingers and, drawing the lips backward and outward, exposes the interior of the vulva to vision. The light, falling obliquely into the vulvar cavity, by refraction brings the nodules clearly into view. In order to examine every part, the examiner should shift his position again and again, that he may view each area at the proper angle. In order to examine satisfactorily the roof of the vulva, the eye of the examiner should be on an approximately horizontal line with the mucosa. Highly satisfactory examinations may be made in dark stables or at night with the aid of a good reflecting lamp or an electric flash light. 


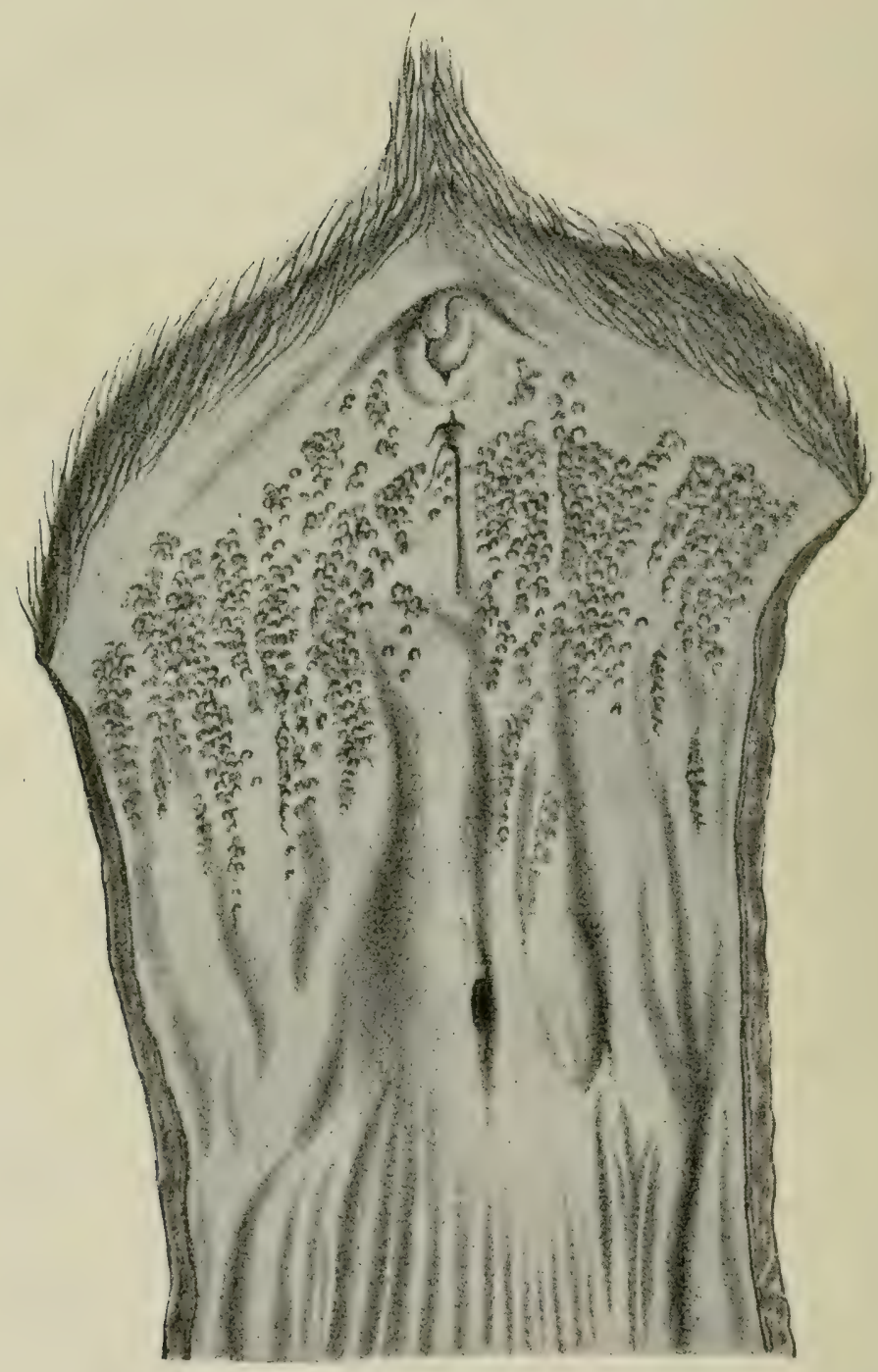

FIG. 79-The Nodular Venereal Disease.

Vulva and Vulvar End of Vagina. Cow. (Hutyra and Marek). 
The nodules are also recognizable upon digital palpation, since the elevations are very evident to the sense of touch. At times digital palpation may have an advantage over vision, because the mucosa may be edematous to such a degree that, even when the nodules are abundant, they are rendered invisible.

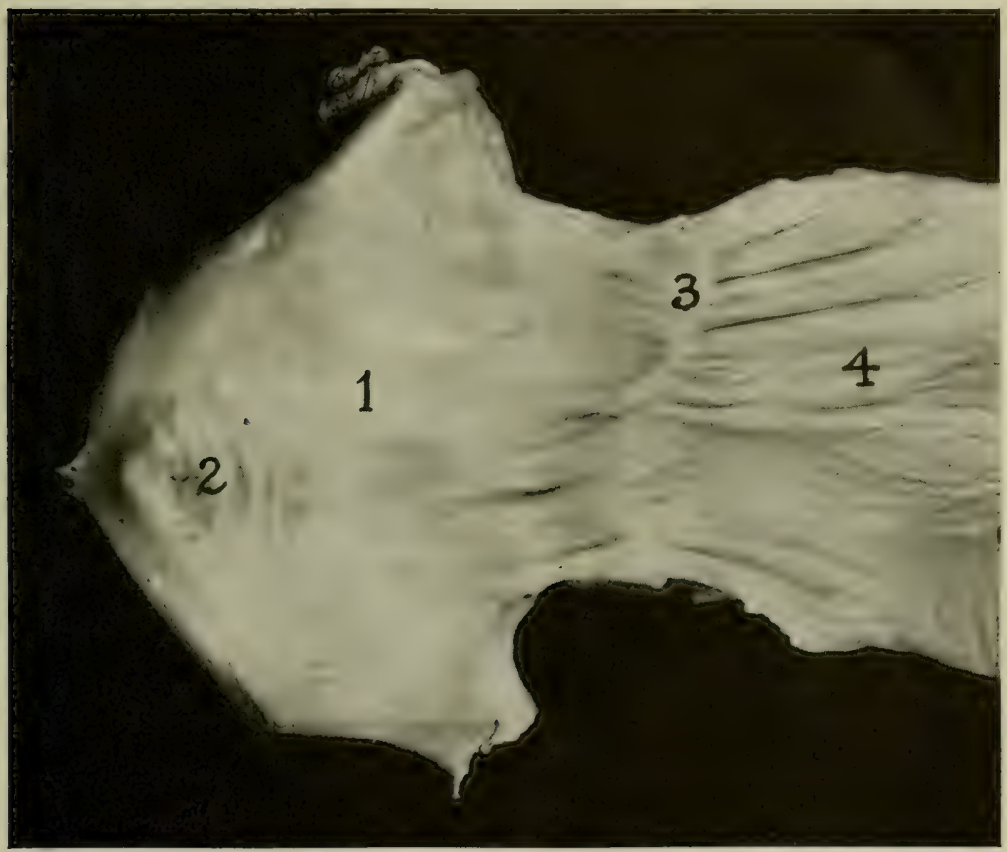

FIG. So-Mild Nodular Venereal Disease. Heifer.

$I$, Vulva; 2, clitoris surrounded by nodules ; 3 , hymeneal scar ; 4, vagina.

Under the prevailing conditions in American dairy herds, and presumably in the dairy herds of the world, calves usually develop clinical signs of the disease at from four to twelve weeks of age, and from that time through adult life to old age present a series of interesting vacillations of intensity, so great that various writers have designated the disease as acute, subacute, chronic, cured, sound, etc.

In the newborn heifer calf, the vulvar mucosa is usually 
smooth, moist, and pale rose-colored for a period varying from four to twelve weeks, or even longer. Finally, visual inspection reveals the presence of several or many spheroidoconical nodules in the vulvar mucosa, 1 to $2 \mathrm{~mm}$. in diameter. Usually they are colorless or faintly yellowish in the center and present the appearance of small, tense vesicles, but close examination reveals the fact that they are hard to the touch and contain no fluid. Encircling the base of each nodule, there is usually a pale or bright red area due to increased vascularity, giving the lesion the appearance of a minute vesicle, with a vascular girdle. The nodules are scattered here and there, frequently along the floor of the vulva in the clitorial region, but quite as often on the sides or roof of the vulva. Except for the nodules and the vascular girdle about the base of each, the surface of the mucosa in the early stage is smooth, pale rose-colored, and normal. There is no swelling, no inflammation, no discoloration of the vulvar mucosa not occupied by the nodules, and no marked mucous or muco-purulent discharge.

Slowly and insidiously the disease appears in calves which have hitherto seemed free, so that, the older the heifers, the larger the percentage showing evidences of the infection. The rapidity and uniformity of the development of the malady depend largely upon environment. Usually, in the closely housed heifer calves in large dairies, fed carelessly upon raw, mixed milk, more than 90 per cent. show the disease at four months, and before they are one year old the visible infection reaches 100 per cent. If by any chance an individual escapes infection until breeding age, the first copulation conveys the disease. In heifer calves of the beef breeds, which nurse their dams, the infection develops much more slowly. In experimental heifer calves, I have kept individuals up to six months, and even to one year old, without any visible trace of infection.

The influence of environment upon the spread of the infection in heifer calves is further shown in Table I, wherein the 122 veal heifers observed showed an average infection of 61 per cent. The percentage of infection among these 


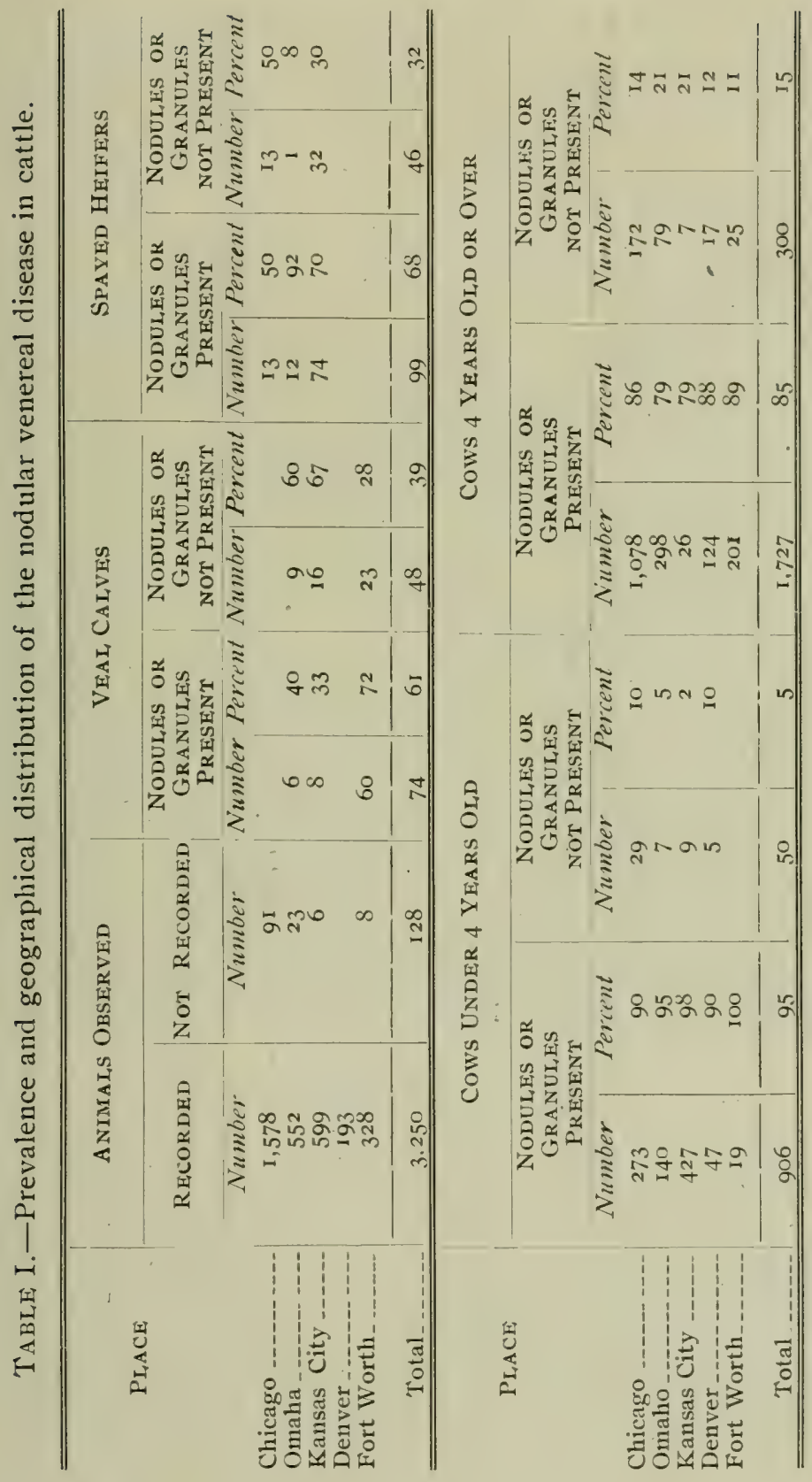


calves is markedly below the average among heifer calves of corresponding ages in eastern dairy herds. Western veal calves largely run at liberty in the open, exposed only to the infection from their dams.

When the nodules are quite numerous, they tend to become arranged in longitudinal, parallel rows corresponding

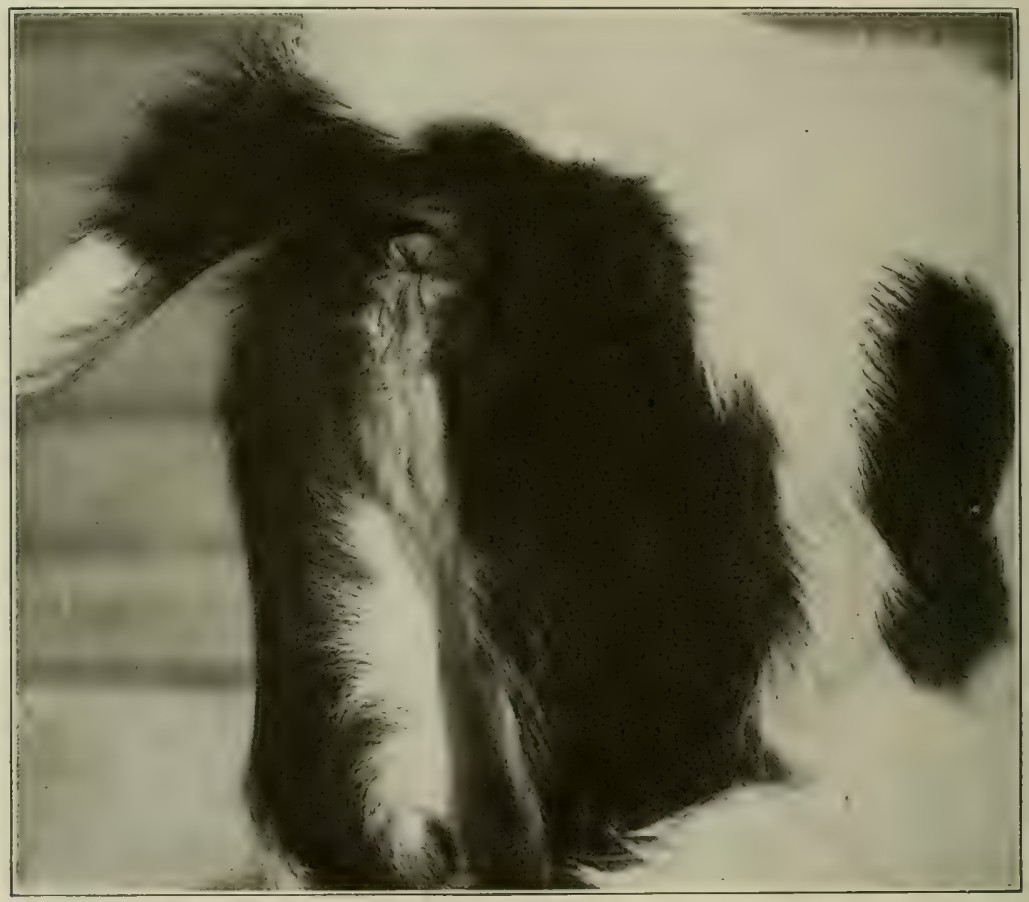

FIG. 8I-Vulva of Heifer Grown Experimentally upon Boiled Milk. The vulvar tuft of hairs is unstained and free from muco pus.

to the longitudinal folds of the vulvar mucosa, as shown in Fig. 79. The nodules are located upon the summits of the rugae, emphasized and rendered more distinct by the inflammation of the mucosa, which causes it to swell, harden and thicken, and forces it into marked folds. The individual nodules change in appearance. They increase little in size and projection. The vascular areas about their bases become more deeply injected and the vascularity may extend 
more or less completely over the surface of the nodules, so that some of them appear as bright red elevations or as petechiae on the vulvar mucosa.

The mucosa itself, between the nodules, becomes involved and is injected, red, and swollen. With the advent of definite irritation of the vulvar mucosa, a slight muco-purulent,

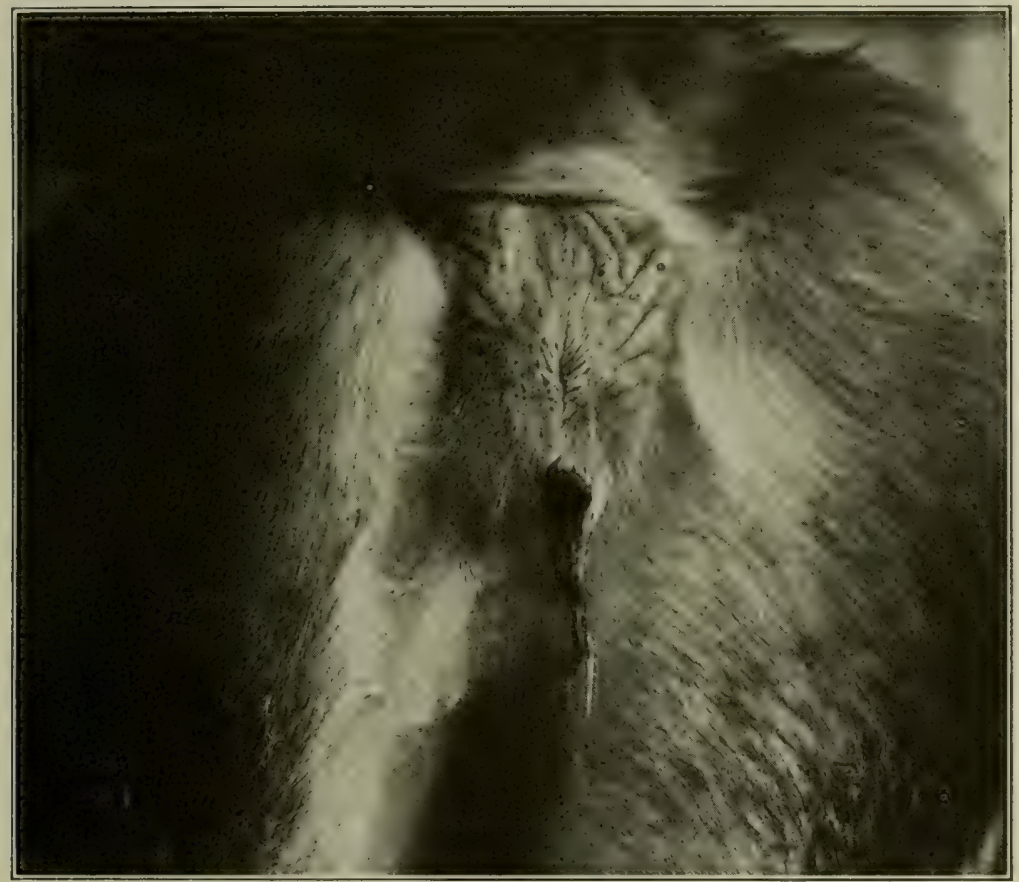

FIG. 82-Vulva of Heifer fed in ordinary manner as a Calf, Showing Matting and Staining of Vulvar Tuft.

vulvar discharge ensues. It is not at first marked. Many say it is not present, or rather that the discharge noted is normal. There is, however, a visible discharge which so mats together the vulvar tuft and surrounding hairs in the heifer calf that, in opening the vulva for inspection, the examiner must frequently break down the adhesions between the surrounding hairs before the vulvar lips may be parted. Some contend that this is normal, but in experiment heifer 
calves such vulvar discharge has not appeared until after visible infection.

Up to the date of puberty, or estrum, the nodular venereal disease of heifer calves generally behaves essentially as a dormant malady, without material significance for the immediate welfare of the animal (See Fig. 80). Various observers may and do hold divergent views. Numerous cases are viewed by veterinarians as sound because of the mildness of the symptoms, but the nodules are there, and, so long as they are admitted as the deciding lesion of the malady, the heifer must be regarded as infected.

Copulation is the signal for the awakening of the dormant infection, which then behaves like venereal disorders in animals and in man under the stimulus of sexual contact. Within twenty-four hours after copulation, the evidences of sexual irritation are marked. The mucosa becomes scarlet, swollen and tender, and in a large proportion of cases there is a very notable muco-purulent discharge, which adheres to the vulvar tuft and soils the under surface of the tail, the skin of the buttocks, and the perineum. Frequently the vulvar lips become markedly swollen and edematous. If the vulvar lips are parted, the mucosa is seen to be covered with masses of stringy, semi-opaque mucus, or small flakes of muco-pus may be seen resting upon the mucosa.

After copulation the nodules multiply with astonishing rapidity. Their arrangement in parallel longitudinal rows becomes well marked. They are crowded into close contact with each other upon the summits of the swollen, hypertrophied, mucous rugae. Frequently they lose their transparency and assume a deep red color. In every way the malady assumes a more decisive clinical aspect of important disease. Still, the general health of the heifer is not lowered.

The intensity of the symptoms increases for a few days, remains static for a time, and may then recede slightly, but the improvement does not approach the status maintained prior to copulation. 
Should the heifer become pregnant at the first service, the irritation may for a time abate slightly and slowly, but the nodules remain prominent and approximately as numerous as ever, and the clinical evidences of disease remain essentially static, at one period apparently improved, at another worse, until near the time for parturition, when the vulvar mucosa becomes more reddened. A marked edema (parturient edema) then appears: the nodules are covered over and are no longer visible. Usually they may still be felt upon careful palpation. In many cases of abortion the edema of the vulvar mucosa is essentially the same as if parturition had occurred. If parturition or abortion is followed by retained placenta and chronic metritis or pyometra, the nodules continue masked by the persisting edema so long as serious uterine or vaginal disease continues. Otherwise, with the gradual disappearance of the edema of the mucosa, the nodules slowly come again into view.

If the heifer fails to conceive at the first copulation, when the next estrual period arrives and copulation occurs, should the sterility be refractory, the symptoms tend to increase, so that sterile heifers are quite generally among the worst clinical cases in a herd. During the second and third pregnancies the symptoms of the disease retain approximately the average intensity acquired during the first pregnancy. Then the severity of the malady generally abates.

When the cow reaches eight to nine years of age and her sixth or seventh pregnancy, the decrease in the intensity of the disease generally becomes quite marked: the nodules are fewer, less prominent, and more transparent; the irritation and injection of the vaginal mucosa are definitely decreased; and the muco-purulent discharge has largely abated. With advancing age, the vulvar mucosa becomes pale yellowish or bluish-yellow, the nodules disappear, and the clinical evidences of the disease commonly vanish from the vulva when the cow is twelve to fifteen years old.

Such is a brief outline of the course of the malady as observed in a majority of cases, but the course is vascillating 
and erratic in individuals and in herds. Copulation always intensifies the symptoms, especially in heifers, and one can generally distinguish by the clinical examination of the vulva practically every heifer which has copulated from those which have not. In some heifers, after repeated copulations, the granules become so numerous and highly inflamed that coitus causes pain and hemorrhage, followed by abundant vaginal discharge for several days.

The use of antiseptics in the vulvo-vaginal tract alters the clinical appearance profoundly. Daily washing of the vagina with non-irritant, warm, antiseptic solutions causes the symptoms of the malady to decrease rapidly in intensity, and in a few weeks the nodules may largely disappear, the mucosa become smoother and softer, and the color change to a pale rose-red. It looks as if the continuance of the handling would soon eliminate the disease, but in the end some nodules remain, and, with a cessation of handling unaccompanied by copulation, the symptoms remain static. On the other hand, when powerful antiseptics are introduced into the vagina, the mucosa becomes irritated, swollen, and edematous, and the nodules thereby become veiled.

Thus the clinical signs of the malady appear insidiously, usually when the heifer is but a few weeks old. The disease pursues a comparatively uneventful course up to breeding age, then becomes suddenly intensified after coitus, and quickly reaches its zenith, where it maintains, with certain vacillations, an approximately horizontal course for three or four years, when it begins to abate slowly in intensity and with the advent of old age more or less completely disappears clinically.

It will be seen by a study of the preceding table that spayed range heifers of the beef breeds show the disease in a lower ratio than other animals, but the ratio does not give a clear view of the facts. The intensity of the disease depends very largely upon the frequency of copulation. The spaying of range heifers was generally done very carelessly and hastily. The operator thrust his hand through the wound, grasping the ovaries and stripping them from the 
broad ligament between the thumb and fingers. The result was that in 50 to 60 per cent. of the animals some ovarian tissues were left, which developed ovisacs and cysts. The heifers came in estrum or were nymphomaniac and copulated freely with range bulls. In such imperfectly spayed animals the lesions, which were always seen, showed considerable intensity. In the perfectly spayed heifers the vulvar mucosa was generally normal, smooth, and pale rose-red, with but few if any visible nodules.

Another striking illustration of the influence of coitus upon the intensity of the disease was observed in a lot of 270 two-year-old range Hereford heifers which had evidently been kept away from the bull, except in the case of one individual which had copulated and become pregnant. In the 269 non-pregnant animals, the disease was quite uniformly present, but only a few nodules were seen in each individual. Careful inspection was required lest they be passed over. The vulvar mucosa of the one pregnant heifer, however, bore more of the nodules than the other 269 together. In this one heifer the entire mucosa was swollen and red, and dense masses of nodules crowded thickly upon each other.

Throughout its long course, the intensity of the infection rises and falls, sometimes in obedience to known causes, as copulation, sometimes for reasons not yet understood. During the period at which the disease is at its zenith, few animals fail to show the clinical evidences of its presence, as shown by Table I, according to which the evidences of the disease were apparent in 95 per cent. This is no higher than regularly observed in dairy herds.

The Nodular Venereal Disease in Bulls. Balanitis. BALANO-Posthitis

The nodular venereal disease is the basic cause of balanitis, balano-posthitis, or akrobustitis, in the bull. There can be no distinction of value between infections of the sheath, the prepuce, and the mucosa of the glans. The continuity and contiguity of the three areas render inevitable the extension 


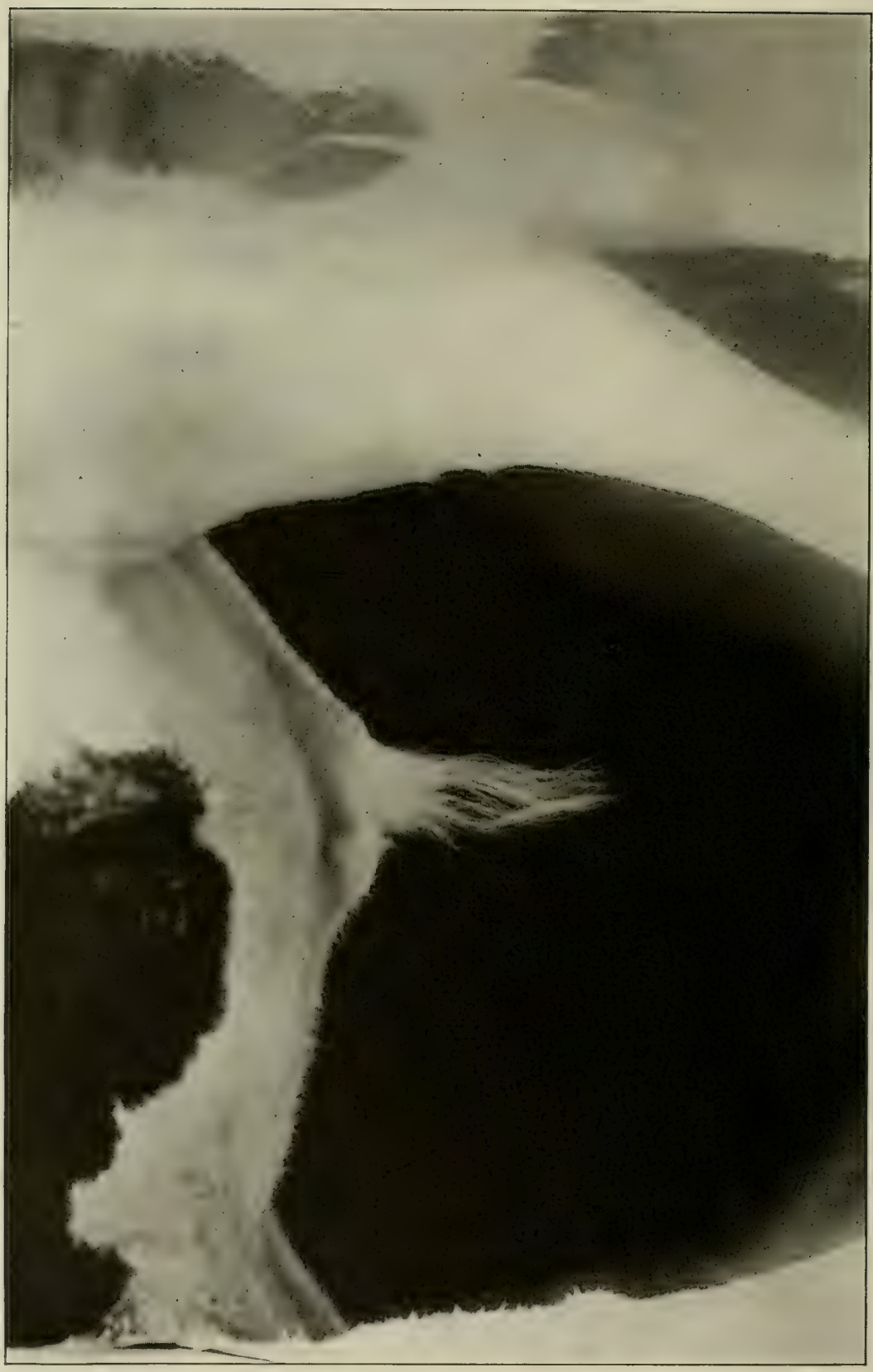

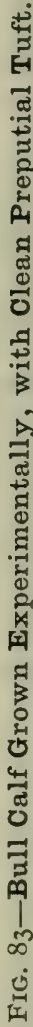


of any ordinary infection from one to the other. Various mechanical injuries may occur, and an indefinite variety of infections may invade the area, but the nodules, which are already present, either complicate or are complicated by them. Consequently it appears desirable to deal with balanitis as being ordinarily identical with the nodular vene-

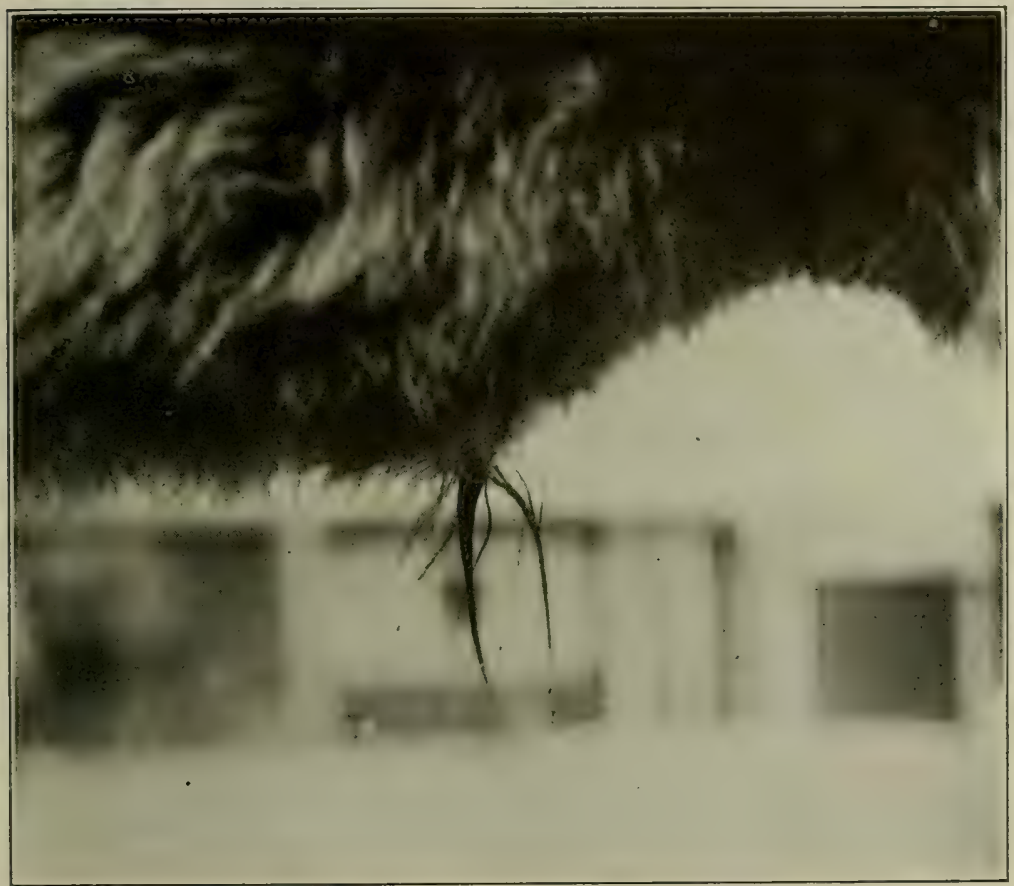

FIG. 84-Bull Calf of same age as Fig. 83.

Grown in ordinary manner on Raw Milk, showing Matted and Stained Preputial Tuft. The body hair is álso shown to be harsh and rough.

real disease. This does not prevent or complicate separate consideration of rupture of the prepuce or other mechanical injuries, or of such localized specific infections as actinomycosis or tuberculosis, which have already been discussed.

The inflammation of the sheath, prepuce and mucosa of the glans, due to the nodular venereal disease, is practically universal in bulls, but the degree of inflammation varies widely. The ordinary degree plays no visible part in the 


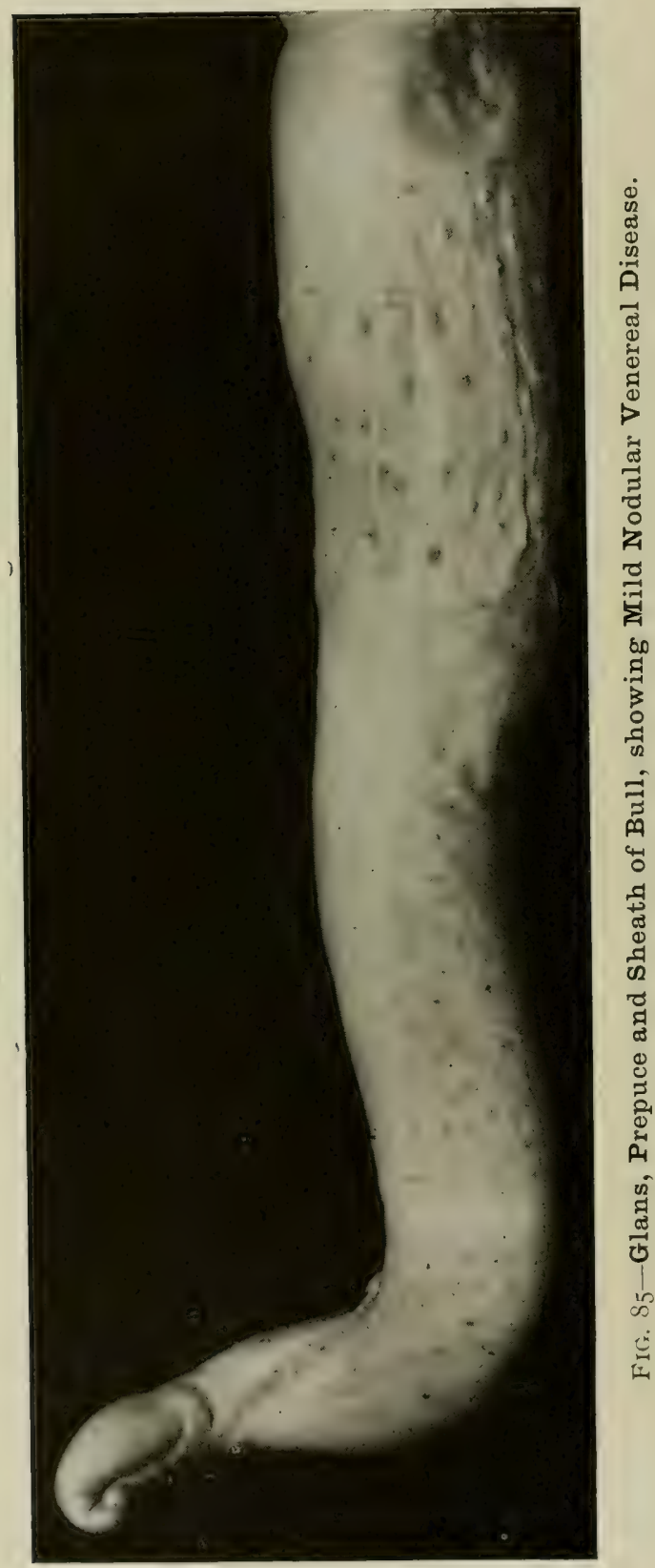




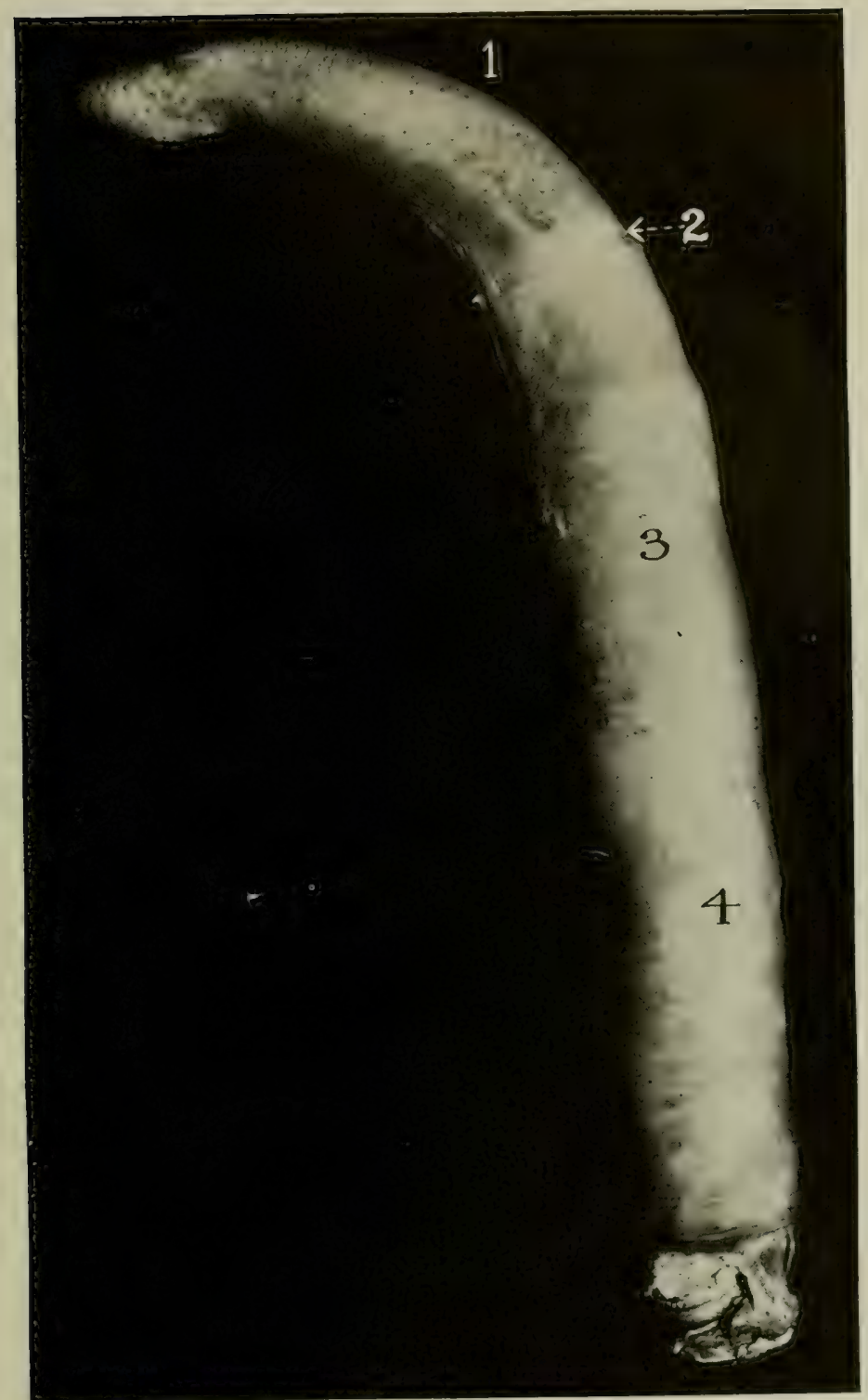

FIG. 86-Penis of Bull showing the Nodular Venereal Disease.

(From same bull as Figs. I 26, 127.) $I$, Glans showing confluent nodules; 2 , fornix ; 3 , the prepuce reflected, showing solitary nodules; 4 , the sheatl reflected. 
problem of reproduction. It existence is evidenced by a discharge of muco-pus, which flows from the sheath opening and mats together the preputial tuft of hairs, at the same time staining them a dark, dirty, brownish-black. (See Figs. 83, 84.) This occurs early in the life of the animal, but the time of its advent varies largely according to the environment and health of the calf. In large dairy herds, where sterility, abortion, calf scours, and pneumonia are severe, the evidences of balanitis occur with great uniformity when the bull calf is twenty to sixty days old, but

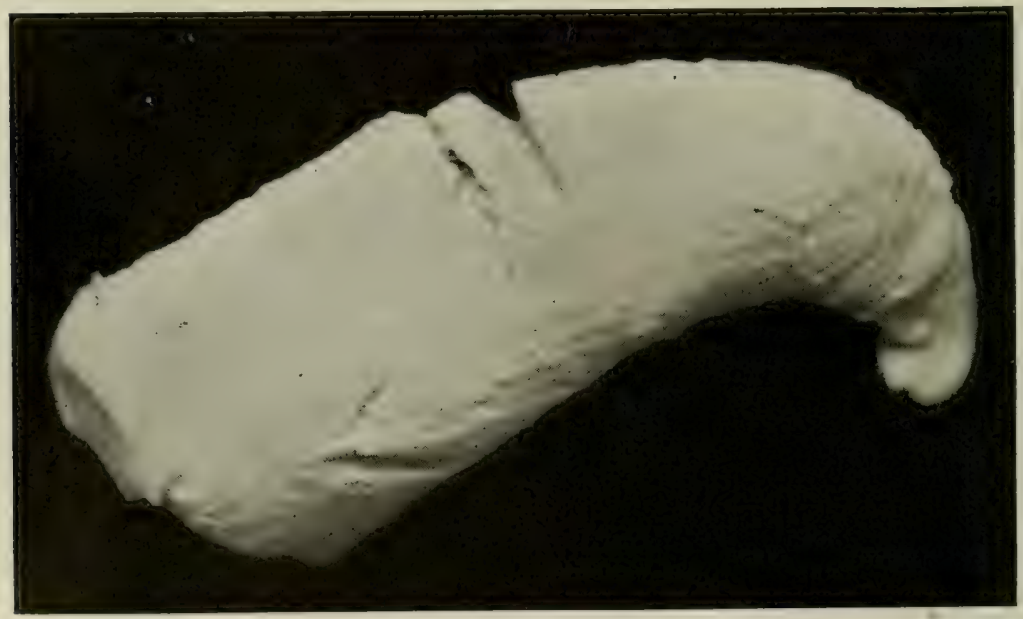

FIG. S7-Glans of Bull Showing Severe (confluent) Nodular Venereal Diseases.

in the calves of grade beef cows, which nurse their young and live in the open, the evidences of genital catarrh appear tardily and the bull calf may reach four to ten months of age without showing very marked evidences of matting and discoloration. Between these extremes, every possible gradation occurs, and wide variations are observed in individuals confined in the same stable. Once the matting and staining have developed, they remain permanent throughout the life of the bull. The staining of the hairs is "fast" and can not be washed out by any ordinary means. 
Inspection of the mucosa of the glans, prepuce and sheath reveals the presence of a few or many small nodules, as indicated in Figs. 85, 86, 87. The number and appearance remain static in the calf up to breeding age. Coitus greatly increases the number of granules, which tend to become confluent, causing an enormously thickened, roughened condition of the mucosa of the parts. Even with this increase

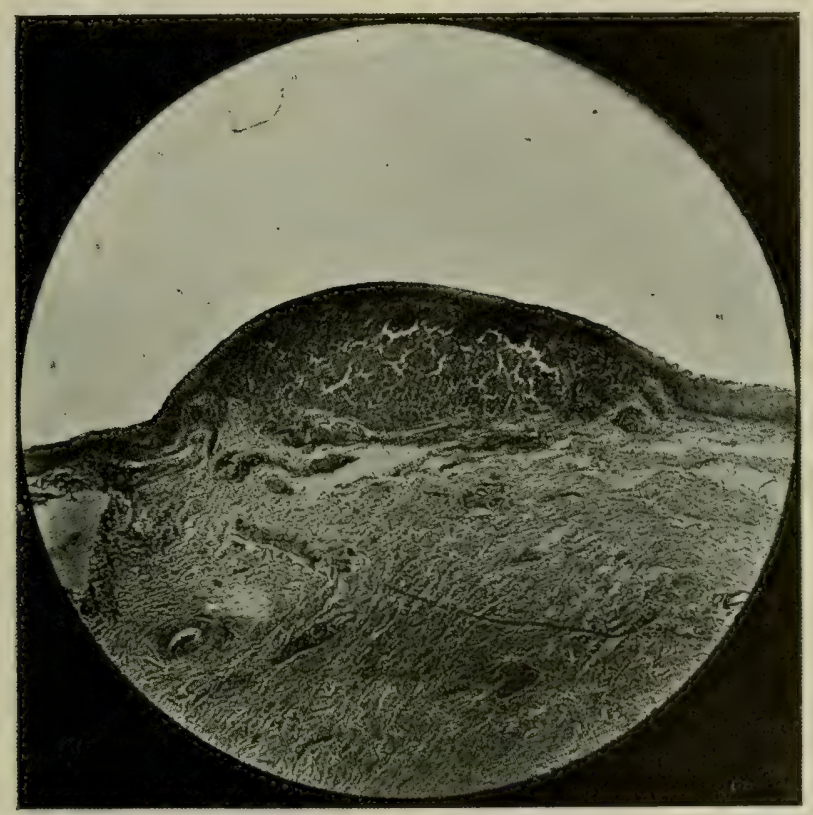

FIG. SS-Section of a Single Nodule from the Vestibule of the Vagina. Showing an Elevation in the Mucosa. (Thoms).

of the infection, there is ordinarily no notable interference with coitus or fertility. Now and then, however, the parts become so intensely inflamed that the bull hesitates to copulate. The attrition of coitus removes the epithelium from the summits of some of the nodules, creating minute abrasions and inducing hemorrhage. In still more severe cases, the involved mucosa becomes so swollen that the penis can no longer be protruded, and phimosis becomes established. 
The history of the nodules has been studied chiefly by Thoms. He found them to consist of clumps of cells encapsuled within the mucous membrane. The cells, which are mononuclear, can not be differentiated microscopically from ordinary lymphocytes. The capsule surrounding the cell mass is not dense, but is well defined and furnishes no stroma. So far as observed, no capillaries enter the cellular

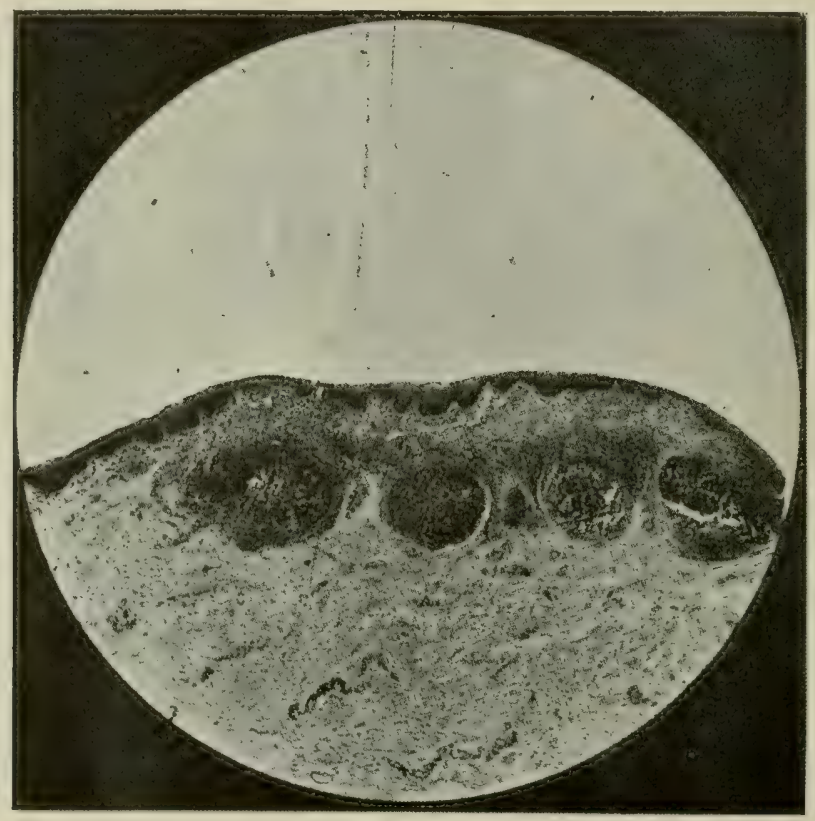

FIf. 89-Section through four contiguous nodules which cause a single, rather flat elevation of the surface. (Thoms.)

mass, but are confined to the peripheral zone, where they are large and prominent. (See Figs. 88, 89, 90,91.)

When the nodules are very numerous and the mucous membrane is thrown into folds, the nodules projecting above the surface of one fold may come into contact with those from the contiguous ridge, and, by attrition, the superficial layer of epithelium may be worn away. It is very largely due to this secondary lesion that a considerable amount of 
muco-pus is formed in many severe cases. These attrition lesions are also responsible for frequent hemorrhages.

The biology of the nodular venereal disease has not been satisfactorily determined. Ostertag discovered, in the muco-pus of the vulvae of cows, a streptococcus which he believed to be the essential cause of the disease. He introduced the organism, which he had grown experimentally,

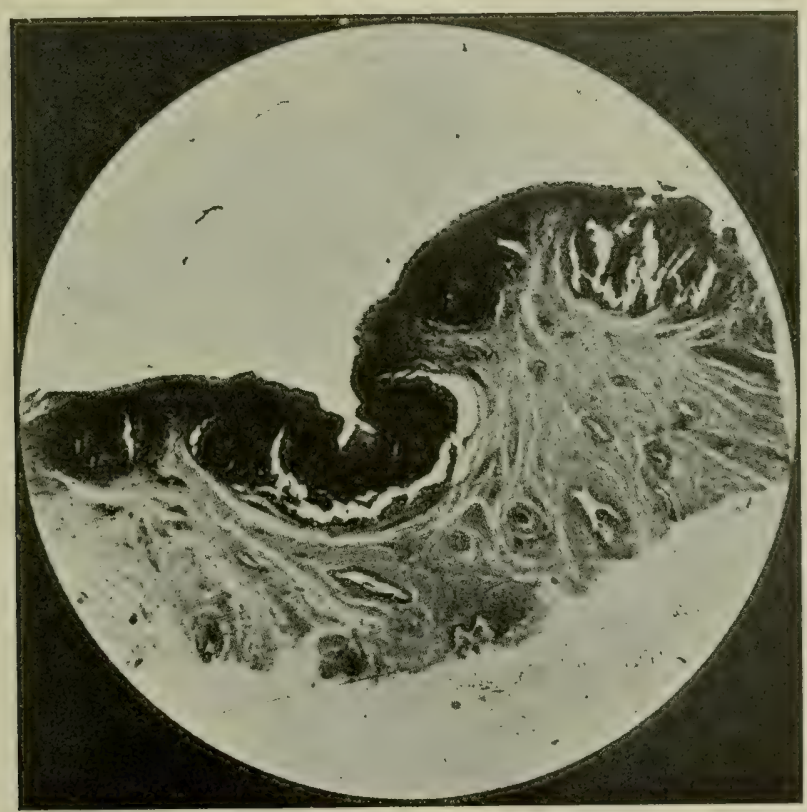

FIG. 90-A knob-like elevation of the mucosa due to several contiguous nodules. ('Thoms.)

into the vulvae of supposedly sound heifers, and believed that he produced the disease. It is a notable fact that in Ostertag's experiments, while he believed he had transmitted the disease to cows and heifers by means of pure cultures, he failed to transmit it to sheep, goats, and swine, although they regularly suffer from it. It is not clear, however, just what he accomplished. Doubt is thrown upon 
his findings by the symptoms and course of the experimental disease which he records, since they are not the symptoms seen in the first stages of the disease as I have seen it in hundreds of heifer calves from two to six months old, as well as in a number of experimental calves. Ostertag says: "The first symptoms of infectious vaginal catarrh are swelling of the vulva, redness, swelling and sensi-

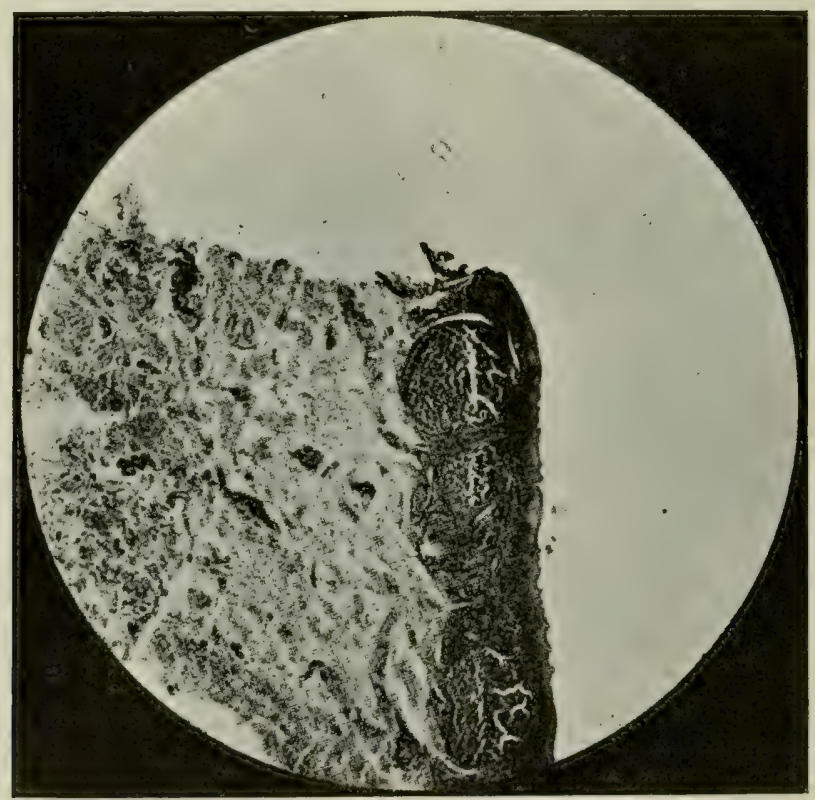

FIG. 9I-A section from roof of the Vagina showing marked noduleformation without macroscopically visible elevation of the mucosa. ('Thoms.)

tiveness of the vaginal mucosa with a muco-purulent deposit." In the earlier stages of the malady, as I have observed it, there are present none of the symptoms emphasized by Ostertag. On the contrary, the disease comes on insidiously. The first sign is the appearance of a few nodules in the vulvar mucosa, sharply defined above the surrouding epithelium, as pale yellow or colorless transparent elevations having a vascular girdle about their bases. Some 
would call such heifer calves sound, but, if two or three typical nodules do not indicate the nodular venereal disease, it would be difficult to understand how two hundred or three hundred nodules can assure us of the existence of the malady. In other words, Ostertag describes, not the beginning of the disease, but an "explosion" of the existing malady under profound irritation. It is not strange that virulent streptococci induced the symptoms he describes, nor that he was able to recover from his experimental animals pure cultures of the microörganism.

The disease is so universal that an investigator is not warranted in assuming that a given heifer or heifer calf is not affected. Calves born during severe outbreaks are frequently so badly diseased that they die. Therefore, it is not unreasonable to assume that in some cases at least calves are born with the nodular venereal disease. Extended observations upon the genital organs of veal calves upon the killing floors of abattoirs show that almost all of them develop clearly and positively the symptoms of the disease at a very early age, usually within sixty days after birth. It is not known, however, when the infection really became established, but only when the clinical evidences became visible to the naked eye. Recent investigations indicate that, assuming that the calf is sound when born, which is not the general rule, it remains free from the nodular venereal disease if fed upon boiled milk. Such being the case, it would appear that the infection may enter the organs of new-born calves with the first milk consumed, and that the appearance of visible lesions in the genital tract merely marks the duration of time between the ingestion of the infection and the establishment of the clinical evidences of its presence. Accordingly, any calf which may be selected for experimental purposes, with a view to determining the transmissibility of the nodular venereal disease or the power of any known organism to cause it, can scarcely be accepted as sound, because it is not known how thoroughly the calf is already infected and upon what day the evidence of such infection would ordinarily appear in the 
genital tract. It is not known that these lesions in the genital tract are the sole or primary lesions of the disease. Various writers have asserted that these lesions have been discovered in the uterine mucosa, and that the streptococci have even been found in the ovaries. Streptococci are common in the genital tracts of animals. The streptococcus which Ostertag has described does not differ materially, so far as may be learned from his description, either in staining or in cultural behavior, from other streptococci. On the whole, therefore, the evidence relating to the biology wants some of the essentials for arriving at a safe conclusion. When the nodules increase they do so numerically, and when first observed are of full size. When they disappear, they vanish numerically, but those which remain are essentially as large as ever.

The Relationship of the Nodular Venereal Disease to Sterility and Abortion. Isepponi, Zschokke, Hess, and many others were thoroughly convinced that this infection was the cause of abortion, sterility, and other diseases of the genital organs of cows. The evidence which they submit is clinical, and in large measure circumstantial. When abortion breaks out in a serious form, veterinarians, seeking for an explanation, tend always to search for any evidence which may offer an acceptable hypothesis for the occurrence of the disaster. If the nodular venereal disease has been accepted at a given time or in a given country as the responsible cause of abortion and allied disasters, the examiner is very liable to look closely for these lesions, and whenever he does so he is certain to find them. He then assumes that the cause of the disaster is revealed, and does not investigate carefully the character of his evidence. In fact, the definition of nodular venereal disease, as given by various observers, differs widely. As a general rule the diagnosis is not based upon the appearance of nodules in the vulvar mucosa, but rather upon the number of nodules and their clinical appearance. Hess, for example, says that an animal has the nodular venereal disease when there is a large number of the nodules present in the vulvar mucosa 
and muco-pus upon the inflamed mucosa. The number of nodules necessary to warrant the term "numerous," the amount of inflammation necessary to warrant the diagnosis of inflammation, or the amount of muco-pus necessary for recognition as such, varies with each observer. In Bulletin 106 of the United States Department of Agriculture, I took as my basis for diagnosis the visible existence of nodules in the vulvar mucosa. If nodules were present, were they two or three or even only one, if they were typical, I classed the individual as infected; if I failed to recognize any of the lesions, I classed it negative.

Whenever sterility and abortion are highly virulent in a herd, the nodular venereal disease is intense; whenever the rate of abortion is very low, there is a correspondingly low intensity of the nodular venereal disease. This parallelism is very striking and suggestive. It constitutes evidence of a possible relationship between this infection and the breeding disasters, which should not be ignored. However, it is merely a bit of interesting evidence-not proof.

It has been stated by some observers that, with the advent of the disease in a herd of cows, abortion broke loose as a storm. The truth of the matter is that the infection was discovered after the outbreak of the abortion. There is no good reason to assume that it had not existed in the herd from the very beginning. No search was made for it until the abortion storm broke; then observation revealed its presence, and the disease was attributed to it.

Some observers have recorded that in outbreaks of abortion in sows a clinical examination revealed the presence of the nodular venereal disease. Hence it was said that the disease had been transmitted from cows to sows and that thereupon "contagious abortion" broke out. Thus it has been claimed that, wherever the nodular venereal disease appears, extensive losses from abortion must follow. Had the observers taken the precaution to examine the genital organs of supposedly healthy sows in herds where abortion did not prevail, there also the disease would have been found. 
Under the circumstances, it seems perfectly clear that the nodular venereal disease has not been proven to be the direct cause of sterility or abortion in cattle or in other domestic animals. Whether it does or does not directly cause sterility or abortion, clinical evidence indicates clearly that it is a disease which merits the respectful attention of investigator, practitioner, and breeder.

The avenue and date of infection are not clear. In large dairies, rich in genital infections, the evidences of the presence of the nodular venereal disease are well established at thirty to sixty days after birth. This shows clearly that the primary infection is not due to coitus. Careful observation also indicates that the infection is not generally referable to intermediary bearers such as attendants or stable equipment. Neither can it be ascribed to ordinary cohabitation with other calves or cattle, since calves confined in isolated stalls suffer the same as other animals. Experimentally I have kept calves in close proximity to diseased cattle, all cared for by the same attendant, with no special precautions, without any evidence of transmission. My experiments indicate with great emphasis that the infection which appears so uniformly in the young calf is bloodborne from the alimentary or pulmonary tract, and its development may be accelerated, controlled, or prevented at will by the method of feeding. When the grade beef cow nurses her calf in the open field, it does not so promptly nor so markedly develop evidences of infection. The higher the intensity of calf scours and pneumonia in a stable, the more prompt and intense are the evidences of the nodular venereal disease. When the health of the calf is better, but there are adhesive feces, a rough lustreless coat, pot-belly, and a hacking cough, the advent of the genital catarrh is later and less marked. If the calf is born in good health, and diarrhea, indigestion, hacking cough and general unthrift are avoided, the evidences of the disease do not appear for several months.

While it has not been proven that the nodular venereal disease is the cause, or an important cause, of sterility or 
abortion, it is still true that sterility, abortion and calf dysentery run parallel with the nodular venereal disease. Calves thirty to sixty days old do not suffer from dysentery or pneumonia if they are free from the clinical evidences of the nodular venereal disease. New-born calves break down with dysentery before the signs of the nodular venereal disease are established. If the evidences of the disease are present, the calf exhibits one or several of the following symptoms: dysentery, torpidity of the bowels alternating with diarrhea, sticky feces adhering to the hairs of the tail and buttocks, pneumonia, a hacking cough, lustreless coat, and pot-belly. When such a calf reaches breeding age, its fertility is uncertain.

It is highly interesting to observe that, in applying the agglutination test for $B$. abortus to the blood of calves, the reactions are parallel to the manifestations of the nodular venereal disease: the blood of calves severely infected reacts highly to the $B$. abortus test, and vice versa. The significance of this parallelism is undetermined. By no means does it show that the B. abortus is or is not the cause of these lesions. It is not known to what degree blood samples from the same animals may react to the agglutination test for the streptococci, colon-like bacilli, micrococci, and other bacteria commonly recognized in the genitalia of cattle.

The most important consideration regarding the nodular venereal disease, pending scientific study of the problem, is that it causes important disease of the copulatory mucosa (balanitis, posthitis, colpitis, vaginitis) which produces definite injury. Frequently the disease is so severe that it causes recognizable pain in coitus, followed by hemorrhage owing to the summits of the nodules becoming removed by attrition. Each of the multiple small abrasions constitutes an open and inviting avenue for infection by any agent present. In the bull the mucosa may be so severely swollen that phimosis is established and coitus is barred. The vulva of the heifer is frequently so inflamed that it bleeds under gentle digital palpation, and the vulvo-vaginal mucosa is covered over with much stringy, tough muco-pus. What- 
ever may be the final decision as to the significance of the nociular venereal disease, these definite injuries should be recognized and preventive or ameliorative measures applied.

The prognosis of the nodular venereal disease is good. It can not be definitely cured by any known means, but it may be controlled and its power for harm mitigated. It is impracticable at present to prevent it wholly, but the proper handling of new-born calves does, for all practical purposes, render it harmless so far as now known. The handling of the nodular venereal disease is based upon application of adequate measures for the healthy rearing of calves and for sex hygiene in adults.

Considering the nodular venereal disease as one which attacks the new-born calf and continues throughout the animal's life, the most fundamental necessity is to grow the calf so that the infection will not develop in a serious form. This can be done only by strict attention to the feeding and other hygienic questions, which will be more thoroughly discussed in Section 3 of Part III, under "Congenital Infections of Calves." Here the invasion may be controlled in such a manner that it tends to protect the animal against the more severe type of the disease during its adult life.

The disease is so highly infectious, however, that the ultimate control must include proper sex hygiene in the adult of each sex. In the bull the handling of the nodular venereal disease resolves itself into the control of balanitis, or balano-posthitis. Here handling should be preventive or controlling rather than curative. It is just as necessary to control the disease from the standpoint of secondary as from that of the primary disease itself. If the disease is permitted to acquire great intensity, so that the summits of the nodules become denuded of their epithelium, a gateway for various infections is opened, imperiling the sex health of the animal. The breeding bull should always be viewed from the standpoint of a male which is expected to copulate with an indefinite number of females, each of which, since she may bear infections within her genital tract, is a potential danger for the male. He in turn, after such exposure, carries an equal peril for the female. 
The handling of balanitis consists essentially of cleanliness. It has been stated that genital catarrh is essentially universal in bulls. While its presence has been ignored generally, there should be no doubt regarding its importance in relation to the reproductive functions. Whenever the catarrh becomes at all advanced, the epithelium upon the summits of the granules or nodules becomes damaged and affords an avenue for the entrance of other infections. It is probably largely through these lesions that primary genital tuberculosis is established, as has already been stated in Chapter XIII. Consequently it is important, for the sexual health of both the bull and the cows with which he copulates, that the genital catarrh shall be held in abeyance at all times and not permitted to become so exaggerated as to interfere with coitus. According to present knowledge, it seems highly probable that, when the genital catarrh of the bull becomes severe, its existence must be inimical to the welfare of the female with which he copulates. The infection, introduced deeply into the vagina, necessarily remains behind, adding to the infection already present. While we do not know the exact significance of the infection, it is impossible to conclude otherwise than that it is detrimental.

The best method at present available for maintaining the health of the prepuce, sheath and glans is by douching the parts at frequent intervals, say two or three times a week, when the bull is at rest, and thoroughly douching the parts just prior to and immediately following each service. The best apparatus for carrying out the douching plan is an ordinary agate hospital irrigator of one gallon capacity, to which a pure gum douching catheter is attached. This instrument, the diameter of the ordinary horse catheter and seven feet long, has the end rounded so that it may be introduced freely and safely into the sheath and prepuce. It is made of pure soft rubber, which is smooth, pliable, and resistant to hot water, so that it may be repeatedly boiled for purposes of sterilization ${ }^{1}$.

\footnotetext{
${ }^{1}$ Upon my request, the firm of Sharp \& Smith, Chicago, have placed in stock a catheter of the dimensions stated. It is of sufficient length that, when the irrigator is suspended above the bull, it is abundantly long for insertion into the sheath.
} 
The technic of douching is very simple. The irrigator is filled with a warm physiological salt solution (1 oz. common salt to 1 gal. water) to which may be added with benefit $1 / 4$ of 1 per cent. of Lugol's solution. The irrigator is then suspended two or three feet above the back of the bull and the catheter gently inserted through the sheath into the prepuce. The insertion is facilitated by the application of a small amount of lard or oil. The douching fluid should be allowed to flow slowly from the catheter before it is inserted into the sheath, in order that all air bubbles shall be forced out. Once introduced, the fluid is permitted to flow freely into the sheath and prepuce. At the same time, the operator should grasp the opening of the sheath and compress it so that the fluid may not escape. This causes the sheath and prepuce to become thoroughly distended, the many complex longitudinal folds of the mucous membrane to become obliterated, and the fluid to come in contact with every part. When the sheath has become thoroughly distended, the operator should suddenly release the compression on the sheath opening and permit the fluid to escape. As soon as it has all escaped, he should again compress the mouth of the sheath and repeat the operation several times, in order to insure thorough mechanical washing.

In numerous publications from apparently authentic sources, directions are given for douching the sheath and prepuce of bulls, of which it is impossible for me to approve. Writers frequently state that the douching can be carried out successfully by using a piece of ordinary rubber tubing. In my judgment this is not true. The ordinary rubber tubing is mixed with lead and other diluents which render it very rigid and harsh. Since the end is cut squarely, and frequently roughly, any attempt to push the tubing into the upper part of the sheath and into the prepuce inevitably abrades the mucosa. I think the use of a piece of ordinary rubber tubing can not be too strongly condemned. If the work is worth doing at all, it should be done in a surgical manner with an appliance suitable for the task.

Another very common error, according to my view, is the 
advice of some writers that a force pump should be used for douching. A pump is exceedingly difficult to keep clean. It almost inevitably becomes dirty. Most pumps are not subject to disinfection or to sterilization by boiling. With a pump there is always danger of including in the douching fluid air bubbles, which may flow into the sheath and prepuce under great force, giving the animal a shock which causes him to move suddenly with danger of injuring both himself and the operator. The pump is also an extravagant appliance. With the irrigator, the douhcing can be carried out readily by one man, whereas with the force pump two men are necessary. Ordinarily a bull is well accustomed to one man, and that one can manage him better alone than in the presence of others.

It is frequently asserted that certain bulls will not permit the douching without violent and stubborn resistance. I have not found this to be true. Repeatedly, caretakers have been exceedingly dubious about their ability to douche the bull, but with such an apparatus as I have described, and with ordinary prudence in handling the animal, I have found that the douching can be carried out without violence on the part of the animal. He will stand for the douching as well as for grooming. The success of the douching depends upon gentleness and tact on the part of the operator and his having at hand suitable equipment for the work. Failures in douching, so far as I have known, have always been due to lack of proper apparatus or to faulty method, or in some cases to faulty solutions used. The douching fluid should always be warm-not hot-and should be wholly free from irritation. Some writers advise a strong disinfectant. These always prove highly irritant and serve to infect instead of disinfect. That is, an irritant disinfectant introduced into the sheath and prepuce destroys the protective epithelium of the part, and the dead cells remaining supply a new and fertile field for the multiplication of bacteria. It is impossible to disinfect wholly the genital mucosa, and no such vain attempt should be made. Instead, a high degree of mechanical disinfection should be obtained by using a neutral or slightly bactericidal douche. 
When the balanitis becomes severe and coitus becomes difficult or impossible, the douching should be carried out diligently, once or twice a day, according to conditions. Great care should be taken not to use an irritant douche. There is a great tendency among some veterinary practitioners, when the infection of the sheath and prepuce becomes marked, to attempt to disinfect with some very powerful solution. These only intensify the disease present. In one case where I was consulted, the attending veterinarian had used 10 per cent. silver nitrate, 5 per cent. compound cresol, a strong solution of lysol, and other disinfectants. Each change was for the worse and the highly valuable bull had been pronounced incurable and slaughter advised. A mild douche, such as advised above, ameliorated the symptoms very promptly. Within fifteen days the bull was back at work in prime condition, and remained competent and fertile.

Whenever balanitis is severe, a bull should be taken out of service and kept as free from sexual excitement as possible until the severe symptoms have abated.

The general principles in controlling the disease in the female are essentially the same as in the male. In breeding heifers for the first time, it is important and prudent to examine the vulva and vagina carefully, in order to determine the intensity of the disease. Frequently it is found to be so intense that there is considerable muco-pus present before coitus has occurred. If such be the case, it is important that the intensity should be ameliorated by flushing the vagina three or four times a week with a warm, physiologic salt solution, to which has been added $1 / 4$ to $1 / 2$ per cent. of $\mathrm{Lu}-$ gol's solution or of chlorazene.

After coitus has occurred and time has been given for the spermatozoa to pass out of the vagina and through the cervical canal on their way to the oviducts, the vagina should be douched in order to overcome the irritation of coitus. It has already been stated that this irritation is very profound and causes a tremendous increase in the number of nodules, in the inflammation of the mucosa, and in the amount of 
muco-pus present, so that the vulva of the heifer presents an appearance which shows very definitely the fact that she has copulated. By timely douching, this irritation and explosion of the infection may be obviated. It is quite safe to douche within four or five hours after coitus. The evil effects of coitus may be controlled by douching at any time after the period named up to about twenty-four hours. The control may be rendered more complete by repeating the douche at intervals of twenty-four hours for three or four times. It may then be omitted. The control of the disease at any period in the sexual life of the animal may be brought about in a similar manner. Whenever the irritation is marled, the vagina may be douched with a very mild antiseptic two or three times a week, and such douching may be continued indefinitely. There is an old prejudice, sometimes still revived, against douching the vagina for fear of causing abortion. No reason exists, so far as I know, why such result should follow a vaginal douche. Clinically I have seen no tendency whatever for the douching to produce any ill effects. It is a valuable hygienic and economic measure, when properlly carried out, especially upon cows which are constantly confined in the stable. While douching the vagina, the operatcr also washes the exterior of the vulva and keeps the parts clean.

In douching the vaginae of cows, one must take care not to transmit from one to another some important form of infection. Under proper precautions as to cleanliness, no such danger ordinarily exists. Since the infections in a given herd are fairly uniform, there is very little danger that one female has a more serious genital infection than her neighbor. There are, however, important exceptions. In a herd where tuberculosis exists and an animal chances to have genital tuberculosis, douching her vagina and then the vaginae of other females without thoroughly disinfecting would be highly perilous. However, as, under proper veterinary supervision, no cow with genital tuberculosis should exist in the herd, such an exposure is unjustifiable. The same conditions apply to the vesicular venereal disease, 
which has been described previously. Careless douching would spread this infection rapidly throughout the entire herd. As with tuberculosis, this is wholly unjustified, because the animal with vesicular venereal disease should be in strict quarantine.

Perhaps the greatest danger involved in the general douching of a herd is from those animals which may have a very virulent infection of the genital organs, as retained afterbirth or pyometra. Here again the prudent veterinarian places the seriously infected animal under efficient quarantine and before an apparatus is used for douching virtually sound animals, except for moderate nodular venereal disease, it is to be thoroughly disinfected by boiling.

The best method for douching the vagina and washing the exterior of the vulva is by means of a gravity apparatus suspended from an elevated track behind the row of cows. Depending upon the size of the herd and the surroundings, one may use a five-gallon pail with a faucet or a barrel of the most convenient size, attaching to it a soft rubber horse stomach tube. The vessel is filled with the desired solution and elevated upon the track approximately two or three feet above the backs of the cows, the tube is inserted into the vagina for a sufficient distance, and the fluid is permitted to flow in rapidly until the vagina fills and the wall contracts and expels the fluid. Before inserting the tube into the vagina, the exterior of the vulva should be washed with sufficient care to avoid dragging any large particles of dirt into the vagina. By such a plan, the breeder or dairyman has it in his power to mitigate and control the severity of the nodular venereal disease. With proper equipment, the application of these hygienic measures is neither difficult nor expensive. One man can readily douche the vaginae of forty to sixty cows an hour, and do it reasonably well. 


\section{CHAPTER XIII \\ NON-VENEREAL INFECTIONS WHICH INVADE THE GENITALIA}

\section{Genital Actinomycosis}

Although actinomycosis may invade any organ in the body, it rarely involves the genital system. Apparently this is not due to any notable resistance of the genital organs against this infection, but rather to the comparative infrequency of exposure. Actinomycosis of the genital organs of cattle does occur, possibly with greater frequency than recorded diagnoses would indicate. Clinically, I have observed actinomycosis of the prepuce, sheath, and inguinal lymphatics in a bull. There was present in the prepuce an actinomycotic mass five or six inches in diameter and in the peripenial and inguinal lymph glands several actinomycotic masses. One instance of penial actinomycosis in the bull has been brought to my attention, in which there was upon the glans an actinomycotic tumor about one inch in diameter. The veterinarian under whose observation the case occurred very naturally diagnosed penial tuberculosis.

I have seen two clinical cases of genital actinomycosis in cows. In my first case there was merely the history of sterility in a highly valued pedigreed cow regarding which a colleague called me in consultation. There were extensive pelvic adhesions, with indistinct outlines of the genitalia and large, indurated abscesses in the mesometrium. Since the animal was evidently incurable, she was slaughtered. Autopsy revealed several sclerotic abscesses, three to four inches in diameter, located in the mesometrium, with other abscesses in the liver. Histologic search showed that the abscesses were actinomycotic in origin. No definite avenue of invasion was determined.

My second case of genital actinomycosis was in a cow that had calved and had suffered from retained placenta. I attended her and removed the placenta manually. Apparently she did well for a time but after a few months, as she failed to breed or to show estrum, she was again examined. I 


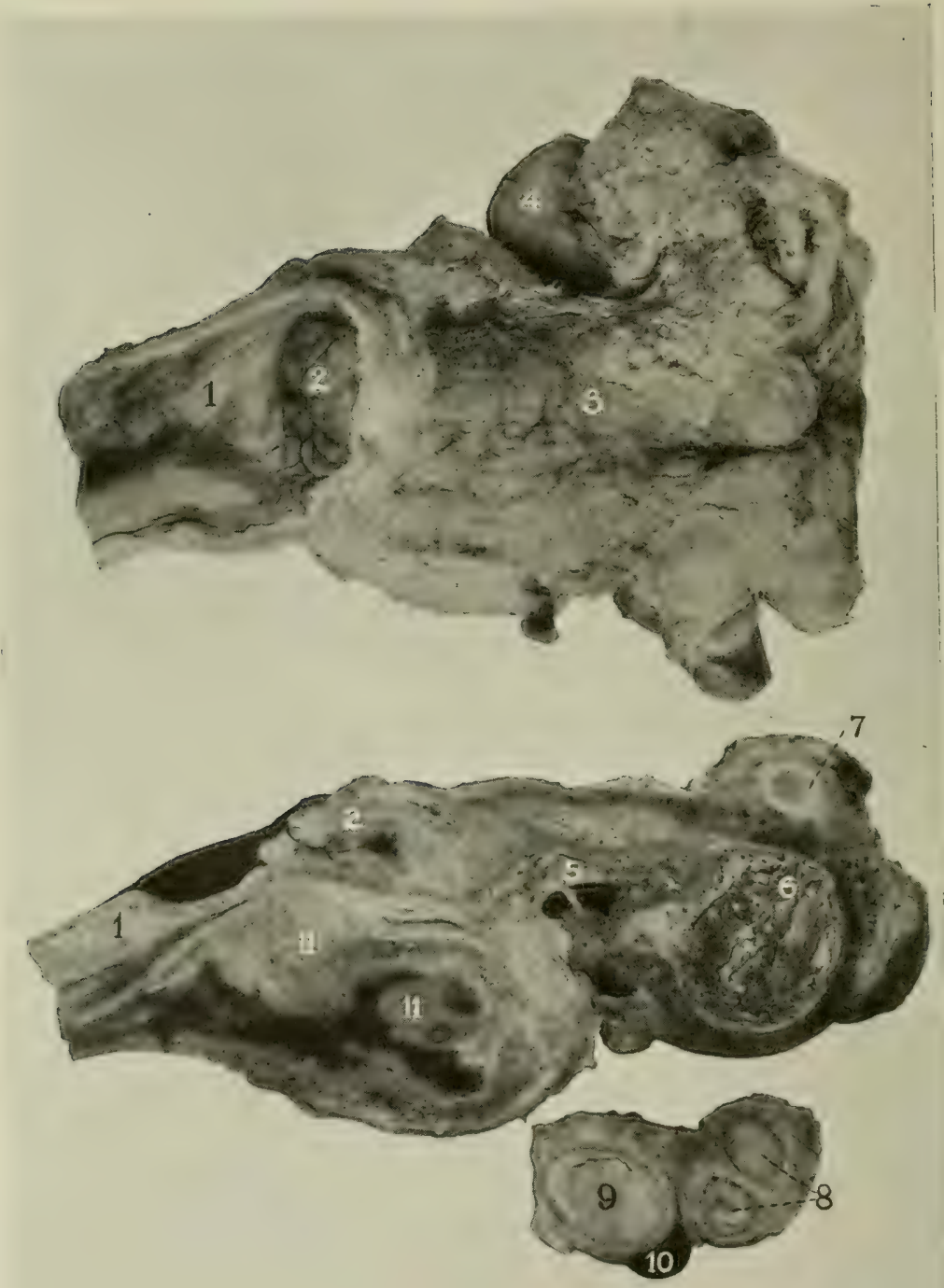

Fig. 92-Genital Actinomycosis. Cow.

Above, dorsal view of genito-urinary tract showing irregular enlargement and extensive pelvic adhesions; below, median longitudinal section through genito-urinary tract. I, Vagina; 2, os uteri externum; 3, base of left uterine horn; $t$, apex do; 5 , actinomycotic uterine body; 6 , actinomycotic abscesses in uterine horns; $7, \delta$, cross sections of actinomycotic oviducts; 9 , actinomycotic abscess in ovary; 10 , corpus luteum (var. nigrum ); II, II, actinomycotic invasion of urinary bladder. 
found complete pelvic adhesions and a greatly enlarged sclerotic, and irregular uterus. I diagnosed tuberculosis and advised immediate slaughter. Inspection revealed that the disease was confined wholly to the genito-urinary organs and that the lesions were actinomycotic. The general appearances are illustrated in Fig. 92.

Accurate clinical diagnosis of genital actinomycosis is difficult. Sometimes it closely simulates tuberculosis. Here the tuberculin test may greatly aid, but tuberculosis may exist in other organs and cause a reaction although the genital disease is actinomycotic. In some advanced cases of tuberculosis with involvement of the genitalia, tuberculin causes no reaction. There occur also in sclerotic metritis due to common pyogenic invasions pelvic adhesions with abscesses closely resembling genital actinomycosis. Ordinarily an accurate clinical diagnosis as to species of infection is not demanded. The lesions render the case hopeless and dictate slaughter. Then should follow accurate post-mortem diagnosis, in the interest of contact animals.

Rarely the disease may be amenable to treatment, but for this purpose needs to be diagnosed early and surgical and medicinal interference promptly put into effect according to the general plan for handling actinomycosis of other organs: the seriously diseased tissues should be surgically removed and potassium iodide administered internally. When the disease involves the glans penis of the bull, the affected part may be successfully amputated, as described for malignant neoplasms of the penis.

\section{Genital Tuberculosis}

Tuberculosis of the genitalia of cattle has generally been considered as rare and of scant scientific and economic importance. William Williams ${ }^{1}$ states, "If the cow be in calf, abortion is apt to occur; if not pregnant, the condition called nymphomania is frequently present." Law ${ }^{2}$ states, "The generative organs also occasionally suffer (from tu-

${ }^{1}$ Principles and Practice of Veterinary Medicine, I875, p. 347.

${ }^{2}$ Vet. Med., Vol. IV, I902, pp. 448, 449. 
berculosis), in which case an early and rather persistent symptom is sterility, with a too frequent, or it may be persistent desire for the bull (nymphomania). * * * In cases of uterine tuberculosis, the nymphomania may be supplemented by a purulent discharge. *** Genital tuberculosis in the bull is associated with nodular swelling of the testicle, epididymis, or cord, hydrocele, and exceptionally tubercle on the penis or in the prostatic sac." Friedberger und Fröhner ${ }^{1}$ state that genital tuberculosis occurs rarely in both sexes and may invade any portion of the genital system. Hutyra und Marek" mention the occurrence of tuberculous epididymitis and orchitis, sometimes accompanied by tuberculous abscessation of the testis, with a consequent fistula. They consider penial tuberculosis as extremely rare, and claim that when it occurs the glans penis becomes studded over with tubercles. Quoting Hess, they regard uterine and tubal tuberculosis as rare and as causing sterility and nymphomania. Vulvo-vaginal tuberculosis is mentioned as a rare possibility. Hoare ${ }^{3}$ describes genital tuberculosis very briefly, without according it an important place. He admits the possibility of transmission by copulation. He mentions a single case of primary penial tuberculosis.

Most veterinary literature concerning genital tuberculosis is in sharp contrast with my personal observations. The difference in view is possibly due to a variation in the direction of study. Veterinarians rarely look for genital tuberculosis, since ordinarily it does not affect the general well-being of the patient. Clinically, it is only when one concentrates his observations upon the diseases of the genital organs in connection with sterility that he recognizes genital tuberculosis and realizes its importance. The genitalia are not inspected frequently in the abattoir. They are not used as human food. When they are tuberculous, the lesions possess little or no importance in relation to the

${ }^{1}$ Speciellen Pathologie und Therapie.

${ }^{2}$ Spezielle Pathologie und Therapie der Haustiere.

${ }^{3}$ System of Veterinary Medicine. 
vaiue of other tissues for food. Hence, much genital tuberculosis may pass unnoted.

The genital mucosa offers a highly vulnerable field for tuberculous invasion, but genital exposure to tuberculosis is rare as compared with exposure through contaminated food. That is, when open pulmonary tuberculosis exists, the patient is constantly contaminating mangers, food, and water by means of her sputum, so that companions are exposed daily and hourly. When genital tuberculosis exists, the exposure may be identical, because of the genital discharges contaminating food and water, but the special venereal exposure occurs only during the very brief period of copulation.

I have not observed tuberculous orchitis or epididymitis. There is no example of either in my pathologic collection of genitalia, and no specimen in the collection of any department of the college. The scattered records in veterinary literature of tuberculous orchitis and epididymitis do not serve as a very accurate basis for outlining the clinical symptoms. In a general way, it is stated that the epididymis and testes show painless enlargement and hardening. The demarcation between the epididymis and the testis gradually becomes clouded, and finally is lost. Apparently the epididymis usually becomes involved first, and the disease extends thence to the gland. The tuberculous process is said to be located usually in the parenchyma, having its basis in the mucosa of the epididymis and testis. In this manner the disease may be well advanced before peripheral inflammation or tuberculous extension involves the peritoneal coverings, to cause adhesions and hydrocele. Rarely, abscessation is said to ensue, resulting in a fistula.

The clinical diagnosis of tuberculous epididymitis and orchitis is difficult. Painless tumefaction of the testis is not characteristic of tuberculosis, but may ensue from various pyogenic infections. Peritoneal adhesions, hydrocele, and abscessation are quite as probable, if not more so, from other infections than from tuberculosis. Next to the excision of the testis and its examination, tuberculin offers the best 
means for diagnosis. As is well known, tuberculin has its limitations, and may fail. If the patient responds to tuberculin, the evidence of tuberculous epididymitis or orchitis is not complete. There may be tuberculous lesions in other organs, causing the response to tuberculin, while the lesions in the epididymis and testis may be non-tuberculous. When but one testicle is involved, its removal and histo-biologic examination offers by far the most reliable means for diagnosis. If the disease has existed for some time, the removal of the testis is in no case an economic waste, because as a general rule chronic epididymitis or orchitis signifies permanent loss of function of the involved gland. Its removal is the best curative measure and the greatest available protection for the other testicle and for the breeding life of the bull.

The path of tuberculous invasion of the epididymis and testis has not been clearly learned. The infection is not primary. It does not seem to be secondary to penial infection. While I have observed several cases of primary penial tuberculosis, I have not noted subsequent tuberculous epididymitis or orchitis. It appears highly improbable that the tubercle bacilli would traverse the long urethra and vas deferens and reach the epididymis without leaving behind evidences of its passage in the form of penial or urethral tuberculosis. When the infection is wholly within the tubules of the epidymis or testis, as appears usually to be the case, the invasion is apparently not direct from the peritoneum. When the scrotal peritoneum is first involved, it would appear probable that the invasion occurred from the peritoneal cavity through the open inguinal ring. Apparently most instances of tuberculous epididymitis and orchitis are referable to hematogenic sources and constitute a part of generalized tuberculosis.

The prognosis for the involved gland is hopeless. If only one gland is involved, it may be successfully removed, leaving the bull perfectly fertile. The difficulty is that, since the tuberculous epididymitis or orchitis is usually a secondary rather than a primary lesion, the basic lesion remains 
and probably unfits the bull for breeding except when mated with infected cows held under the Bang plan. Even in such cases, it needs to be determined that the vas deferens, vesiculae seminales, prostate gland, and penis are free, or the bull becomes a serious menace even to tuberculous cows, because when such parts are involved tubercle bacilli may be

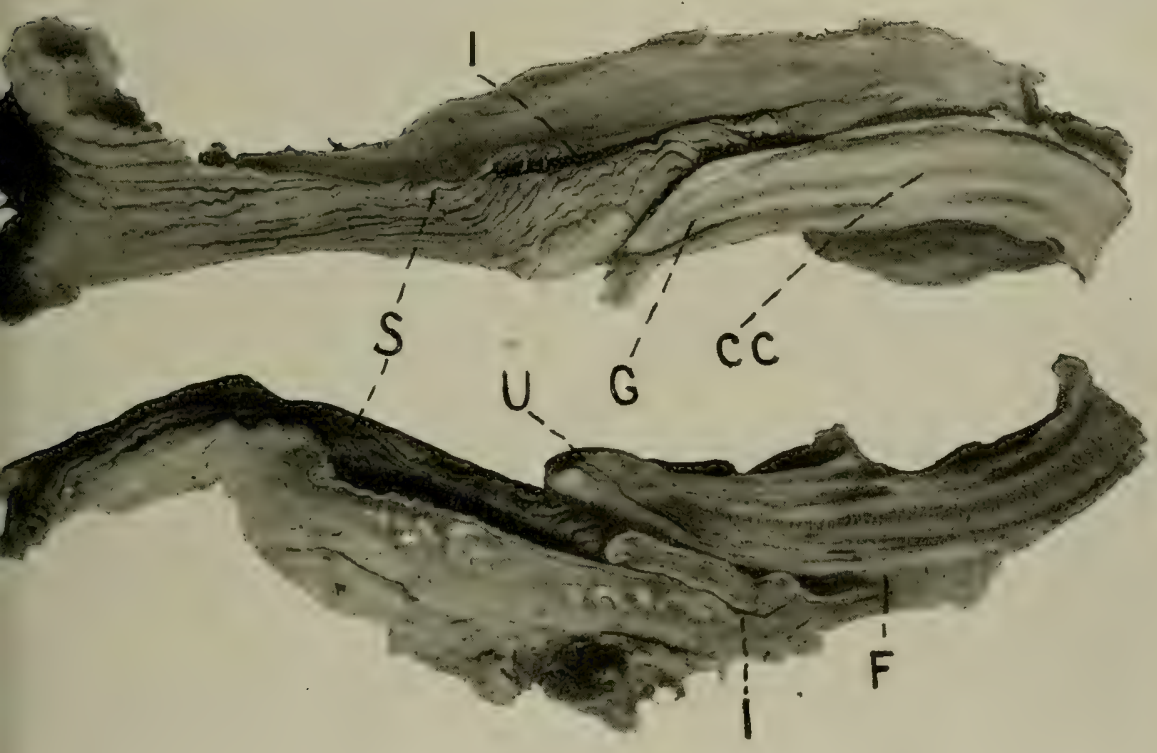

Fig. 93-Tuberculosis of Sheath and Prepuce of Bull.

$S$, Sheath; $U$, urethra ; $G$, glans ; $F$, fornix; $C C$, corpus cavernosum, $I, I$, fissure extending into tubercular mass.

ejaculated with the semen from the sound testicle, inducing primary genital (venereal) tuberculosis in the cow, which may promptly bring her breeding life to a close.

Tuberculosis of the vas deferens, seminal vesicle, and prostate is possible, but apparently too rare to be of great 
economic importance. Tuberculosis of the vesiculae seminales or prostate would tend to interfere with urination and copulation (ejaculation). The diagnosis of tuberculosis of these parts would be based necessarily upon rectal palpation.

Penial Tuberculosis. Penial tuberculosis is comparatively common in the bull. The corpus cavernosum, urethra, and urethral mucosa are not involved as frequently as the submucosa of the glans, prepuce and sheath, and the adjacent penial lymphatics.

Tuberculosis invades any organ far more readily when the protective epithelium is wounded. The anatomy of the copulatory apparatus of the bull and the mechanism of copulation render epithelial injuries more probable elsewhere than in the mucosa of the glans penis itself. The penis of the bull (and of ruminants generally) is very firm. The transverse diameter and the rigidity of the penis are but slightly modified by erection. The protrusion of the penis during copulation is brought about almost wholly by the elimination of the sigmoid flexure (See Fig. 99), the accomplishment of which requires the relaxation of the retractor penis muscle. When at rest, the penis lies within the prepuce, above the sheath. As a rule, no distinction is drawn between the mucosa of the sheath and that of the prepuce, although they have a wholly different embryologic history. The sheath exists as a distinct structure at the time of birth; the prepuce does not. In the new-born ruminant and porcine male, the sheath constitutes a comparatively short infundibulum, terminating at the bottom in the meatus urinarius (Fig. 8, page 14, and Fig. 9, page 15). No part of the glans penis is exposed. At the time of birth, the two layers of mucosa, one of which is later to form the mucosa of the glans penis and the other the mucous membrane of the prepuce, are firmly adherent to each other. Later, when sexual maturity approaches, the tissue between the two layers of mucous membrane, which serves to bind them together, slowly gives way, and the preputial sac finally becomes established. In the abattoir, one may observe in veal calves every gradation of the development of 
the prepuce. The gradations may be seen also in castrated males (Fig. 9a, page 16). The preputial sac may be totally absent, or there may be any degree of development, according to the stage of sexual development at the time of castration. The anatomy and the mode of protruding the penis during coitus have been described on page 14. The coital

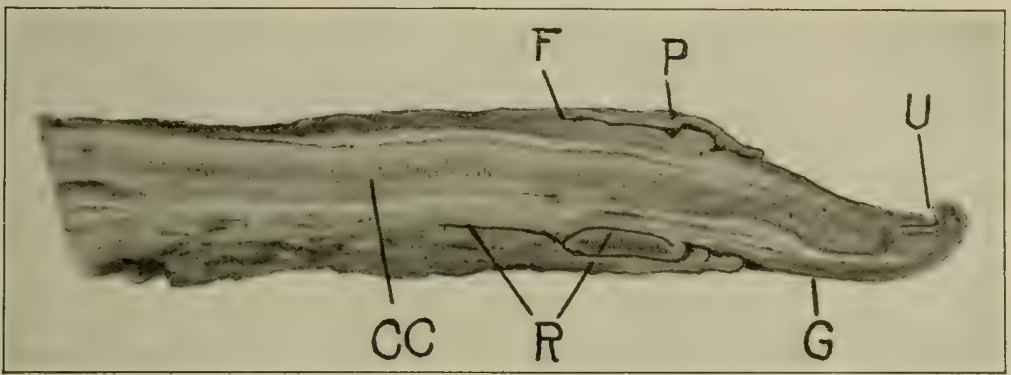

FIG. 94-Rupture of Prepuce From Coital Violence.

$C C$, Corpus cavernosum: $F$, fornix ; $P$, prepuce ; $G$, glans ; $U$, urethra ; $R$, coital rupture. At the right end of the rupture the ruptured border of the prepuce is folded upon itself.

thrust of the bull is vigorous, or sometimes so violent that the prepuce is torn or ruptured, as shown in Figs. 61 and 94. The abraded or ruptured portion is withdrawn at once, with the retraction of the penis, and any infection which has entered the wound is carried along.

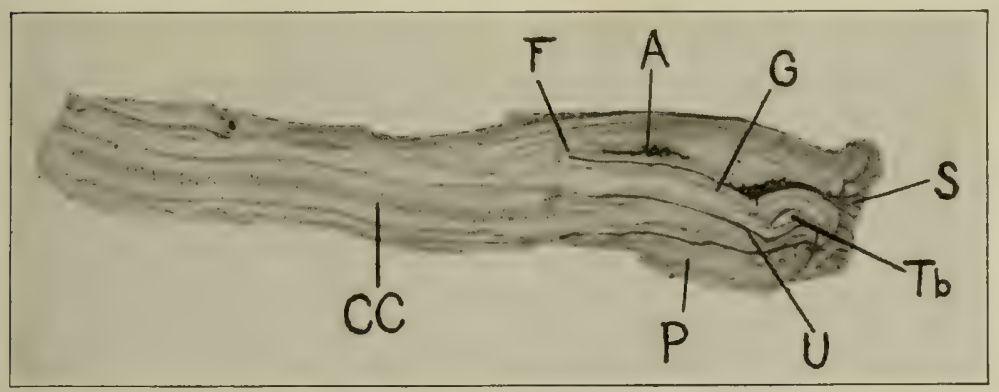

FIG. 95-Tuberculosis of Glans and Prepuce.

$G$, Glans; $P$, prepuce ; $F$, fornix; $U$, urethra ; $C C$, corpus cavernosum; $T b$, tubercle in tip of glans; $A$, tubercular abscess in prepuce. 
The superficial genital mucosa of the bull, as well as that of the ram and the boar, is further exposed to abrasions and consequent infection by the presence of the granules or nodules of the granular venereal disease-a chronic infection which is essentially universal. The granules or nodules frequently become inflamed, especially from excessive coitus, the epithelium at the summits of the granules becomes abraded, and hemorrhage ensues. It is not rare to see the granular venereal disease so severe that the parts bleed after each coitus, if the bull will copulate. Sometimes the pain is so great that he refuses to copulate; sometimes the swelling of the sheath is so great that the penis can not be protruded-the bull has phimosis.

Therefore, in addition to the very delicate epithelium of the genital mucosa, the prepuce itself may be ruptured, or a multitude of minute abrasions may occur at the summits of the granules regularly present. These abrasions offer special facility for invasion by pathogenic bacteria. If the cow is afflicted with tuberculous genital catarrh, any abrasions existing upon the penis, prepuce, or sheath of the bull, or occurring at the time of copulation, invite tuberculous infection. The entire group of such tuberculous infections may be classed as primary venereal or coital tuberculosis. Primary tuberculosis of the copulatory organs of the bull may involve any tissue or part contributing to the copulatory apparatus. The principal types I have observed are:

(1) Tuberculosis of the glans penis,

(2) Sheath tuberculosis,

(3) Preputial tuberculosis,

(4) Tuberculosis of the penial lymph glands.

(1) Tuberculosis of the glans penis is not very rare. I have observed two cases clinically, and others in the abattoir. One clinical case was in a large Holstein herd bull. For some months, he had been unable to copulate with small or medium-sized cows, but could do so successfully with large cows with commodious vulvae. Attempts at copulation were generally followed by a limited amount of hemorrhage. The glans penis was enlarged, especially at its apex. 
Non-Venereal Infections which Invade the Gentalia
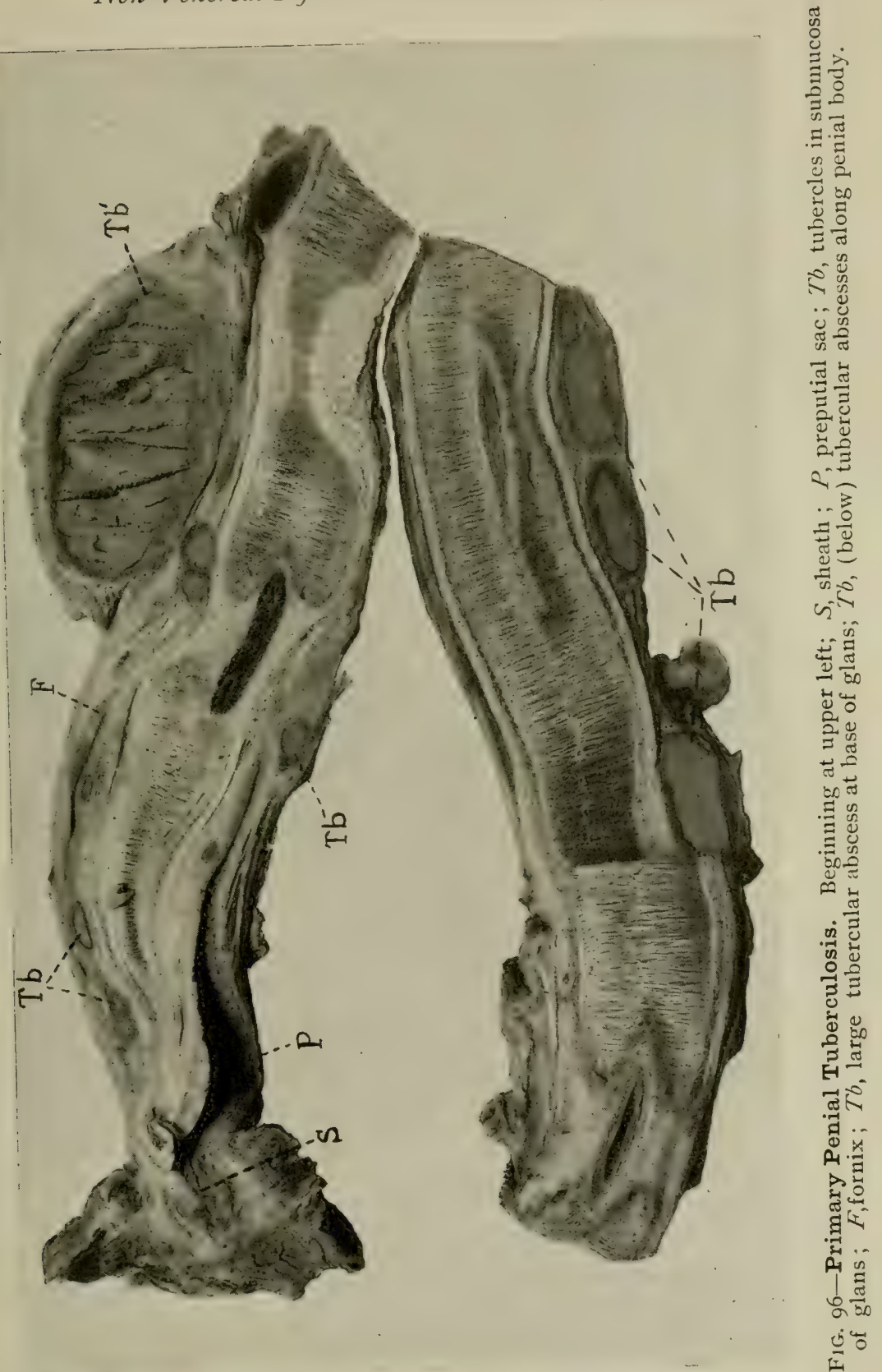
The surface was dark-colored, congested, somewhat eroded, and suppurating slightly. Inability to copulate was apparently referable to the combination of four factors-the enlargement of the glans; the roughening of its surface, due chiefly to the destruction of the epithelium, thus hindering the introduction of the glans into the vulva; the pain; and the flaccidity of the diseased area. The penis was protruded readily. When the bull was confined upon the operating table, the penis could be pushed out of the sheath and prepuce by forcibly effacing the sigmoid flexure. When thus forced out and securely grasped so that the part could be closely inspected, it was seen that the apex of the glans for a distance of about 3 inches was much inflamed and enlarged, and bled readily upon touch. The diseased tip was dark livid, the epithelium largely destroyed, and the surface contaminated by purulent exudate. The appearances were strongly suggestive of tuberculosis. The diseased tip of the glans was amputated. The histologic appearances were those of tuberculous lesions, and stained smears showed tubercle bacilli. The operative wound progressed very favorably for a time, and the bull was promptly discharged from the clinic, apparently on the safe road to recovery.

The patient belonged to a breeder devoid of serious regard for the control of tuberculosis. It was understood that the disease was rampant in his herd and that he was more concerned about concealment than about control. I was unable to follow the case. Apparently the patient, valued at about ten thousand dollars, had contracted the infection by copulating with a cow having genital tuberculosis. After becoming infected, he was evidently a very serious menace to any healthy cow with which he might copulate. The venereal peril was limited chiefly by the fact already related, that he could not copulate with most cows and heifers, but only with those having commodious vulvae. The attitude of the owner prevented any investigation of the herd in an effort to learn whether any harm had come to cows from copulation with this animal. So far as I have observed, he was one of the most dangerous bulls I have seen with genital tuberculosis, 
because in most cases copulation is promptly and absolutely excluded and venereal transmission thereby avoided.

A very interesting abattoir specimen is illustrated in Fig. 61 , in which there has been a rupture of the prepuce and also tubercular infection at the tip of the glans penis. The rupture of the prepuce probably furnished the avenue for the tuberculous invasion.

Tuberculosis of the submucosa of the glans is illustrated in Fig. 96. This specimen shows numerous tubercles in the submucosa. They were not recognizable clinically. The disease was brought to a crisis by the larger tuberculous abscesses in the prepuce and about the base of the glans, which rendered protrusion of the penis impossible.

(2) Tuberculosis of the penial sheath is presumably rare. In my collection I have but one well defined specimen. It was obtained from the abattoir and is without clinical history. Fig. 93 indicates that penial incarceration occurred early, rendering the animal impotent and causing him to be sent to the shambles. Clinically, the condition could not well have been differentiated, upon ordinary examination, from other infections of the sheath wall, especially actinomycosis. Amongst chronic infections, however, tuberculosis is by far the most probable, and a provisional diagnosis of tuberculosis should be made. The tuberculin test may serve as an aid, but is subject to severe limitations. If it is reasonably certain that the bull was tubercle-free prior to the development of the lesion, the test possesses great value. Exploratory incision through the external skin, and the removal of material for bacterial and inoculation studies, is of great diagnostic value.

So long as the bull can protrude the penis and copulate, he constitutes a serious menace. Since the infection is primary, and the fundamental lesion in the mucosa is naturally open, any tuberculous excretions occur within the sheath. The glans penis inevitably becomes contaminated, but not necessarily infected. In copulation, however, the surface contamination is carried into the vulva and vagina of the female. In protruding the penis, as will be under- 
stood by studying Figures 10, page 17, and 11, page 18, until the swelling becomes too great for copulation to occur, the sheath mucosa is reflected, and when the penis is fully protruded the tuberculous membrane itself, constituting the covering of the penial base, enters the vulva of the cow during coitus.

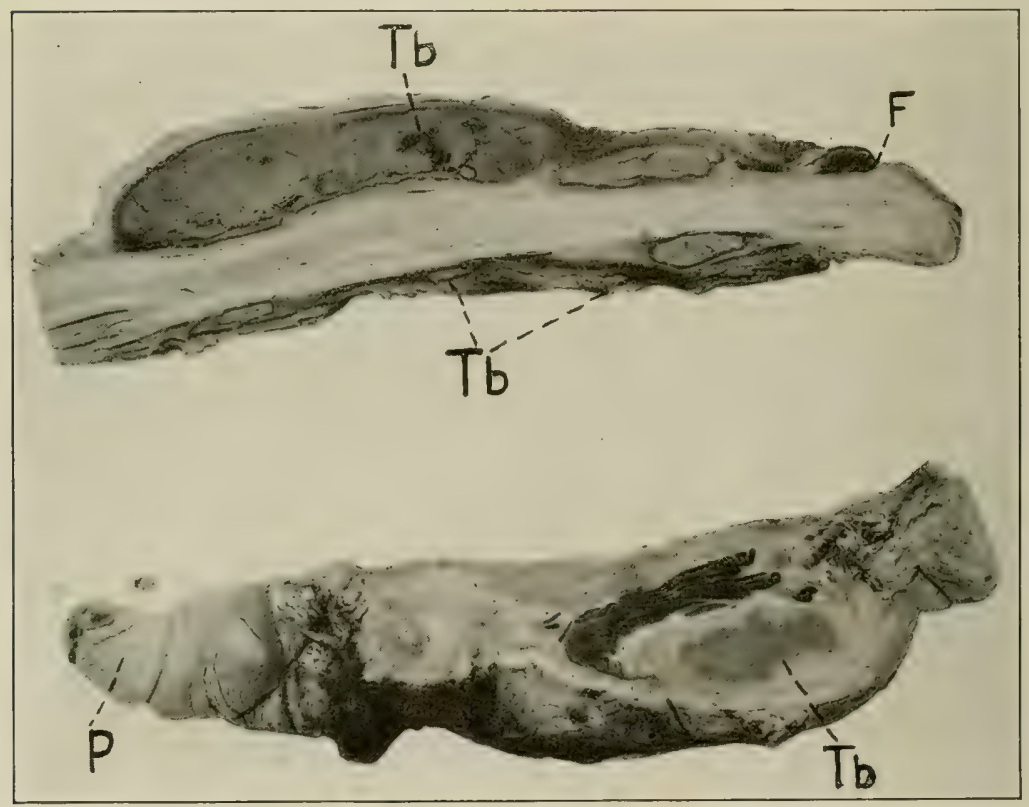

FIG. 97-Tuberculosis of the Glans and Prepuce.

$P$, Prepuce; $F$, fornix; $T b$, tubercular masses.

Accordingly it is essential that great care be exercised in making a diagnosis when inflammatory conditions of the sheath exist, and that every available means be taken to eliminate the question of tuberculous infection. However, one must not carelessly incise the sheath deeply, for diagnostic or other reasons, lest the resultant cicatrix incarcerate the penis. If tuberculosis is diagnosed, the bull should be excluded from service, even to reacting cows, and promptly sent to slaughter.

(3) Preputial tuberculosis is apparently less common 
than tuberculosis of the glans or of the sheath. The preputial membrane, which is far more delicate than that of the sheath, is exposed during copulation to special injury at the base of the glans. At this point the epithelium of the glans becomes reflected, to constitute the lining epithelium of the preputial sac. At the moment of the copulatory thrust, the parietal, or outer preputial membrane is suddenly and violently reversed, to constitute the covering of the penis from the base of the glans backwards for a distance approximately equal to the length of the glans itself. Further back, the sheath mucous membrane succeeds the prepuce as the mucous covering of the protruded penial body. The prepuce and the sheath mucosa become completely reversed. That end of the sheath mucosa which, while the penis was at rest, was situated most anteriorly now becomes most posterior. The mucosa of the sheath and of the prepuce become reversed in relation to each other. When the penis is at rest, the prepuce is behind the sheath, but when the penis is protruded the prepuce is in front of the reversed sheath mucosa. Accordingly the prepuce, at the point of its attachment to the base of the glans, is one of the most vulnerable areas at which tuberculosis and other infections may effect an entrance. When infection occurs, it usually leaves scant trace in the epithelium of the glans, but involves chiefly the lymph glands of the submucosa.

As soon as infection occurs and inflammation is established, the loose areolar tissue between the prepuce and the external dartoid sheath becomes involved, adhesions occur, and the penis is incarcerated. By studying Figures 8-11, pp. 14-18, it will be seen that as soon as such inflammatory adhesions become established copulation is at an end, because the preputial wall can not become reflected upon the body of the penis. The adhesion of the outer with the inner layer at the base of the glans serves to hold the penis firmly in its retracted position.

Clinically, the lesion is probably not usually observed until well established. That is, the infection probably goes unobserved even though several copulations are made dur- 
$33^{2}$

Diseases of the Genital Organs
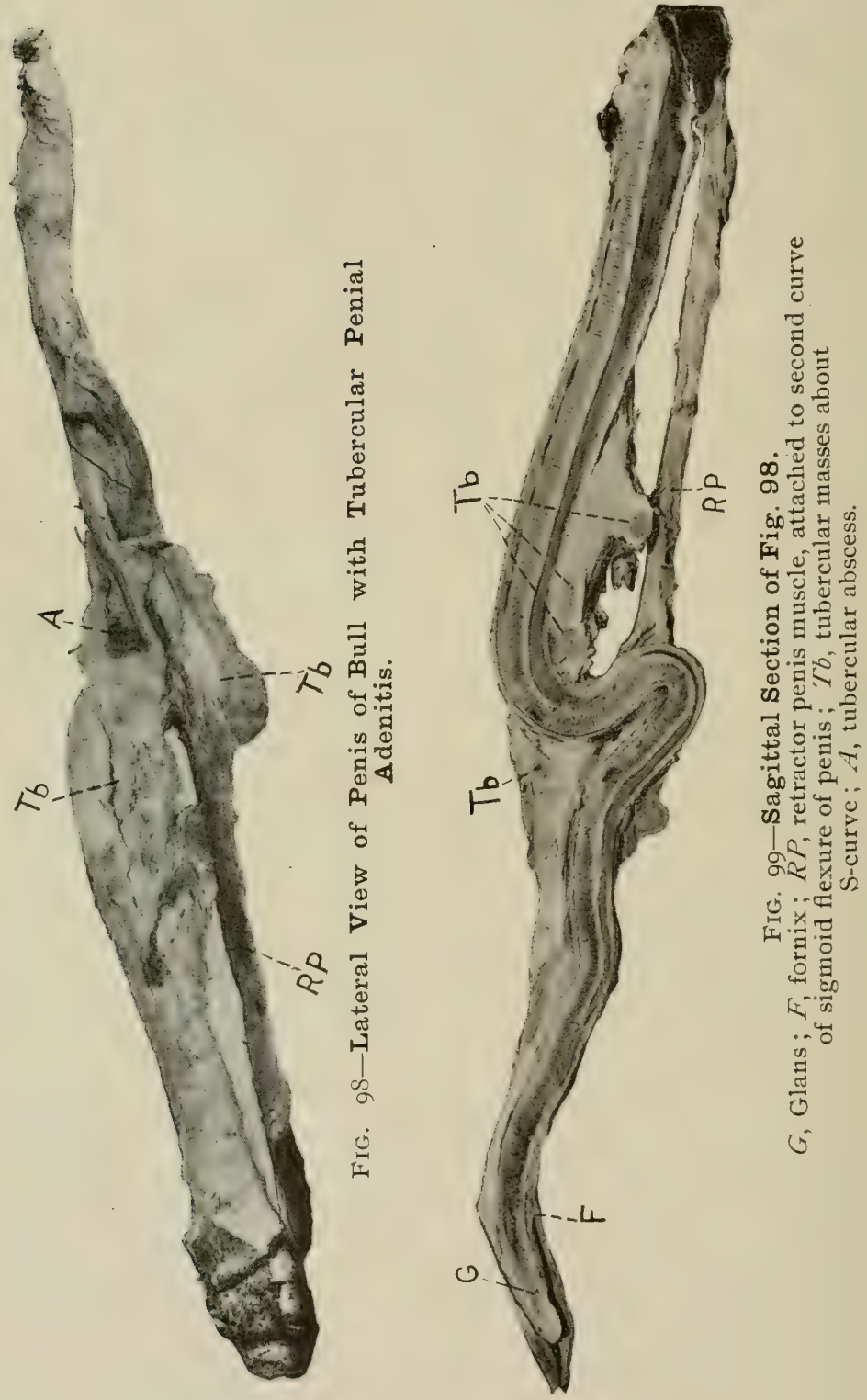
ing the few succeeding days. When the lesion has reached a certain development, a given copulation, especially with a heifer, irritating the lesion severely, sets aflame the established infection. Then the lesion is observed, and is naturally attributed to mechanical injury referable to the last copulation, though this is only partly true. Then swelling in the preputial region becomes evident, incarceration of the penis follows quickly, and copulation can no longer occur.

The diagnosis is fundamentally dependent upon symptoms essentially identical with those seen in sheath tuberculosis, except that the lesion is located further backward, just anterior to the scrotum. It can not be differentiated from other infections by an ordinary physical examination. Before a technical diagnosis can be made, it is essential to include the tuberculin test and bacterial search. In a breeding sense, the lesion is not subject to cure.

(4) Tuberculosis of the penial lymph glands is by far the most commonly observed type of genital tuberculosis in bulls. Apparently entering through a lesion, either recognizable or unrecognizable, of the glans, sheath, or prepuce, it may involve the lymph glands at any point from the margin of the sheath, along the prepuce, and up to and above the sigmoid flexure. Clinically, in opening the small peripenial abscesses, I find that they contain thick pus, and that the abscess walls are dark, angry red, without calcification. The lymph glands lie chiefly along the sides of the penis. When involving the small glands outside the mucous membranes of the sheath and prepuce, the inflammatory adhesions soon prevent the reflection of these membranes upon the exterior of the penis, make its protrusion impossible, and exclude copulation. Swellings in the region, usually well defined and painless, are evident. Abscessation gradually develops, but the abscesses are small and their walls sclerotic, non-fluctuant, and without a tendency to "point" or break. The lesions are well illustrated in Figures 96, 97, 98 and 99.

Sometimes the sigmoid lymph glands are involved, while those of the sheath and prepuce are slightly or not at all 
affected. In such cases, as shown in Figures 98 and 99, the chief clinical phenomenon is inability to protrude the penis and copulate. The inflamed glands induce adhesions which inhibit the elimination of the sigmoid flexure. Palpation of the region readily enables the veterinarian to detect the enlarged glands.

The enlargement of the lymph glands of the sheath, prepuce, or sigmoid flexure constitutes strong evidence of primary venereal tuberculosis. Other infections may induce similar adenitis, but commonly the veterinarian is justified in provisionally diagnosing such lesions as tuberculosis. $\mathrm{He}$ may, should circumstances demand, search for bacterial or other evidence. On the whole, any chronic adenitis of these glands ruins the breeding value of the bull and dictates slaughter. A positive diagnosis is highly important, even when made post mortem. If it is tuberculosis-and that is the rule-there must be highly dangerous cows in the herd, the genitalia of which should be subjected to very rigid search for tuberculosis. While a bull may become infected in copulating with a cow when the exterior of the vulva is contaminated with tubercle-bearing fecal excreta, this is improbable. The assumption should generally be that the infection has arisen from copulating with a female having tuberculous genital catarrh. Such an offender should be detected and eliminated. She is almost always incurably sterile. She constitutes a great peril to any breeding sire, and is one of the worst "spreaders" of tuberculous infection.

\section{Tuberculosis of the Female Genitalia}

Every part of the genital organs of the cow is subject to invasion. My collection contains examples of ovarian, tubal, uterine, cervical, and vaginal tuberculosis, and I have observed clinically vulvar tuberculosis.

(1) Ovarian tuberculosis is very rare, but is far more common than orchitic tuberculosis. The ovary is more exposed to the infection than the testicle, especially in the presence of extensive peritoneal lesions. I have obtained but two good examples. One of these, Fig. 100, is highly 
interesting, because casual examination suggests that the avenue of invasion of the ovary was through the physiologic lesion, the crater of a ruptured ovisac. The dense investing tunic of both the testis and the ovary appears to offer a highly effective barrier against tuberculous invasion, with the important difference that in the ovary there occur intervals (ovulation) when the tunic is ruptured and its continuity temporarily in abeyance. Viewed in this light, more

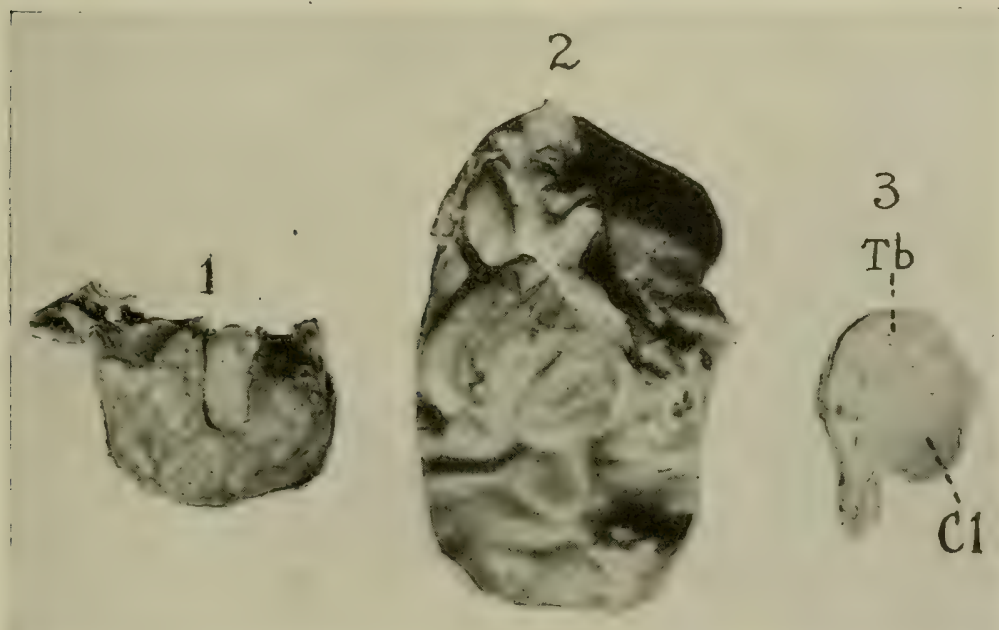

Fig. 100-Tuberculosis of Ovary and Oviduct.

$I$, Section through oviduct; 2 , oviduct thrown into coils by elongation; 3 , ovary showing a corpus luteum, $\mathrm{Cl}$, and a tubercular mass, To, in crater of a ruptured ovisac.

ovarian than orchitic tuberculosis might be expected. Apparently this is true. Nevertheless, the ovary is highly resistant to the infection, as compared with other portions of the female genitalia. Frequently the uterus and oviducts are highly tuberculous, while the ovary remains normal. In the second example of ovarian tuberculosis in my collection, shown in Fig. 101, the ovaries and oviducts have largely undergone tuberculous necrosis. Generally, when the oviducts are involved, the pavilion is adherent to the ovary, and the peritoneal side of the pavilion is studded over with 


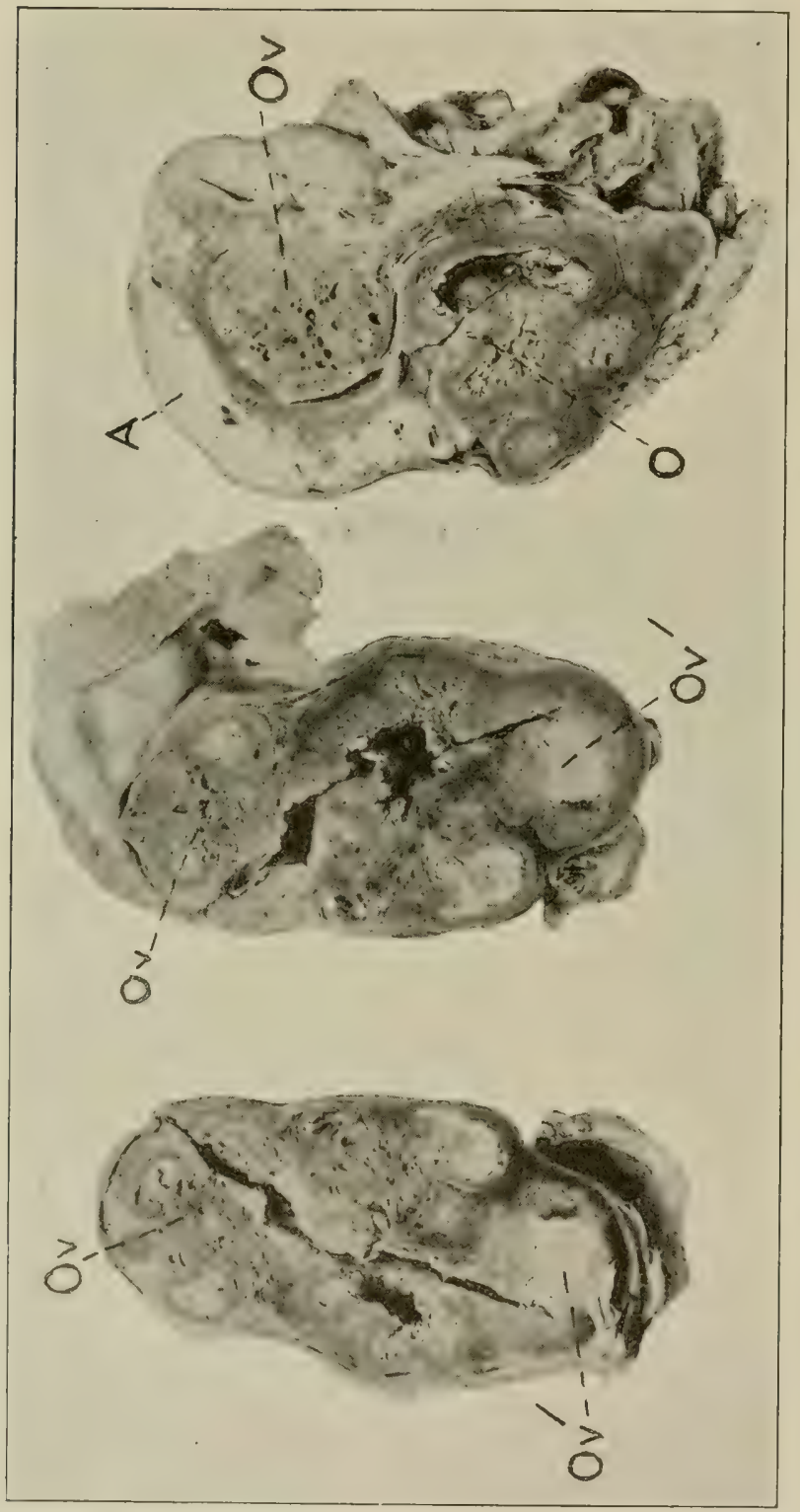

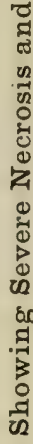

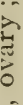

द

o $\cdots$

苛 气

क

800

है

ข2

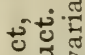

₹

- 5

80 क

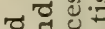

द्व

ता 0

मี -

का

$>0$ 일

$0+\frac{3}{2} \cdot \frac{\pi}{3}$

वै तै

उ응

울

का

के

(2)

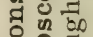

苛

Ð

డే

๐ี

N

है

म

.

ญू Э

10

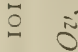

E 
tubercles, as shown in Figures 102, 103, 105 and 106, but the adherent, encapsulated gland resists invasion.

Ovarian tuberculosis can not, in my experience, be directly and positively diagnosed clinically. Clinical diagnosis is not highly important, since ovarian invasion rarely, if ever, occurs without tubal and uterine tuberculosis, each of which is open to reasonably safe clinical diagnosis. So far as I am aware, ovarian tuberculosis induces no clinical symptoms. There is a definite impression given in veterinary literature that ovarian tuberculosis sometimes causes sterility, but there is no evidence submitted. So far as I have seen, it is the coëxisting or preëxisting tubal and uterine tuberculosis which causes the sterility. Statements occur also (Huytra and Marek, Law) that genital tuberculosis, either through the invasion of the ovaries or otherwise, induces nymphomania. No evidence is submitted upon the point. The power of tuberculosis of any portion of the genital tract to cause nymphomania is probably pure legend. A careful study of nymphomania shows it to be due to a definite type of cystic degeneration of the ovary, wholly devoid of any trace of relation to tuberculosis. Genital tuberculosis and the nymphomaniacal type of ovarian cyst may coëxist, but that is not evidence of either identity or relationship.

(2) Tubal tuberculosis is, next to uterine tuberculosis, the commonest type observed in the genitalia of cattle. In many specimens, the appearances suggest that the infection has invaded the oviducts centrifugally from the peritoneal cavity through the pavilion. In other instances of even severe uterine tuberculosis, the oviducts are free. Tuberculous oviducts are usually recognizable by rectal palpation. They become enlarged and very hard. Generally they are adherent and studded over with large tubercles. The tuberculous tubes vary in transverse diameter up to 0.5 inches or over. When much enlarged, they become elongated and thrown into folds lying in front of and lateral to the ovary, as shown in Figures 103-106 inclusive. The disease may be 


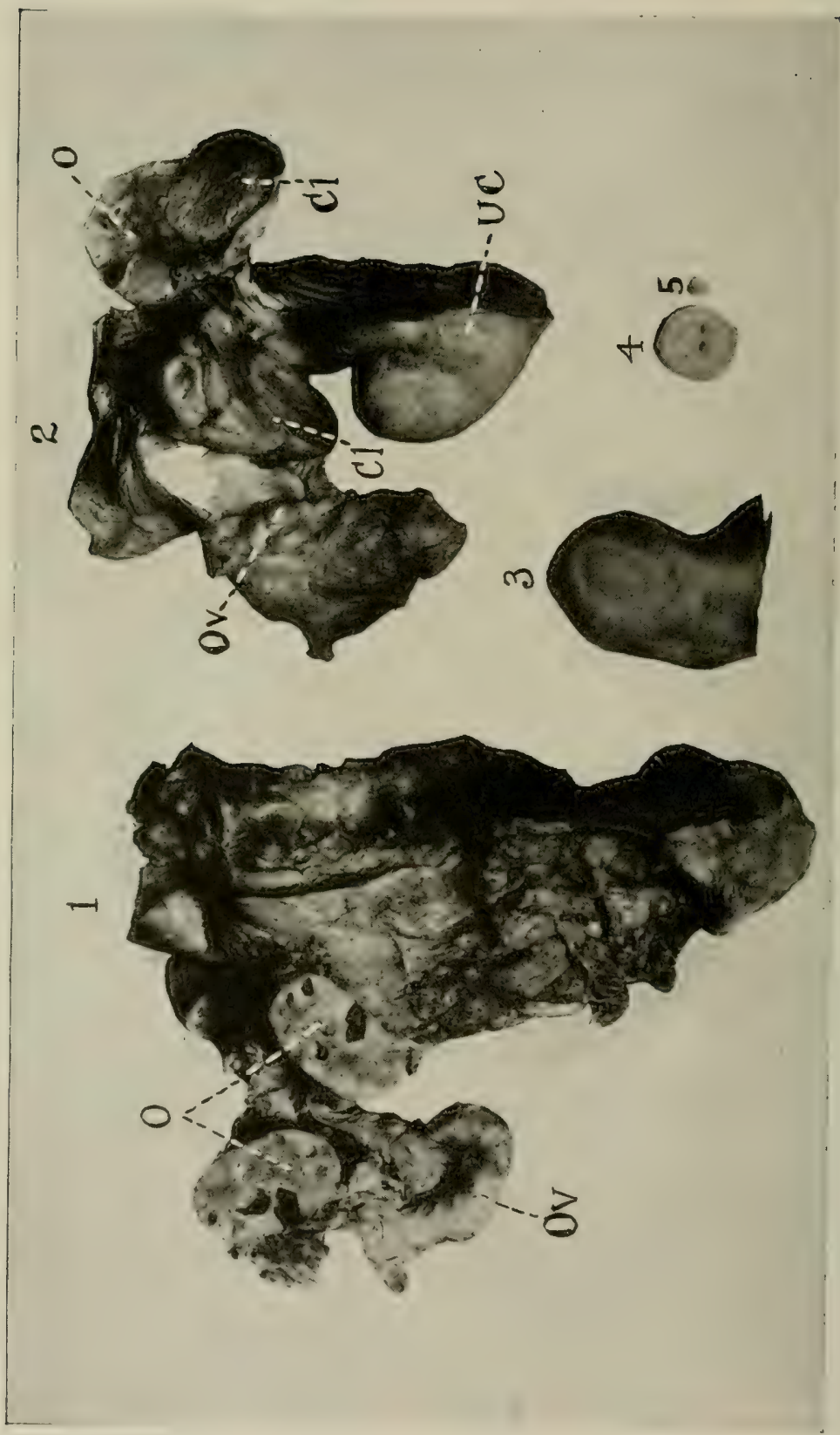


confused with ordinary pyosalpinx or hydrosalpinx. In ordinary pyosalpinx, there are two types. In the first and commonest, there is not much pus and the oviducts are not greatly enlarged, but are very firm and of even contour; in the second, the pus is voluminous and the oviduct walls attenuated, giving a soft fluctuating tube, with thin, atonic

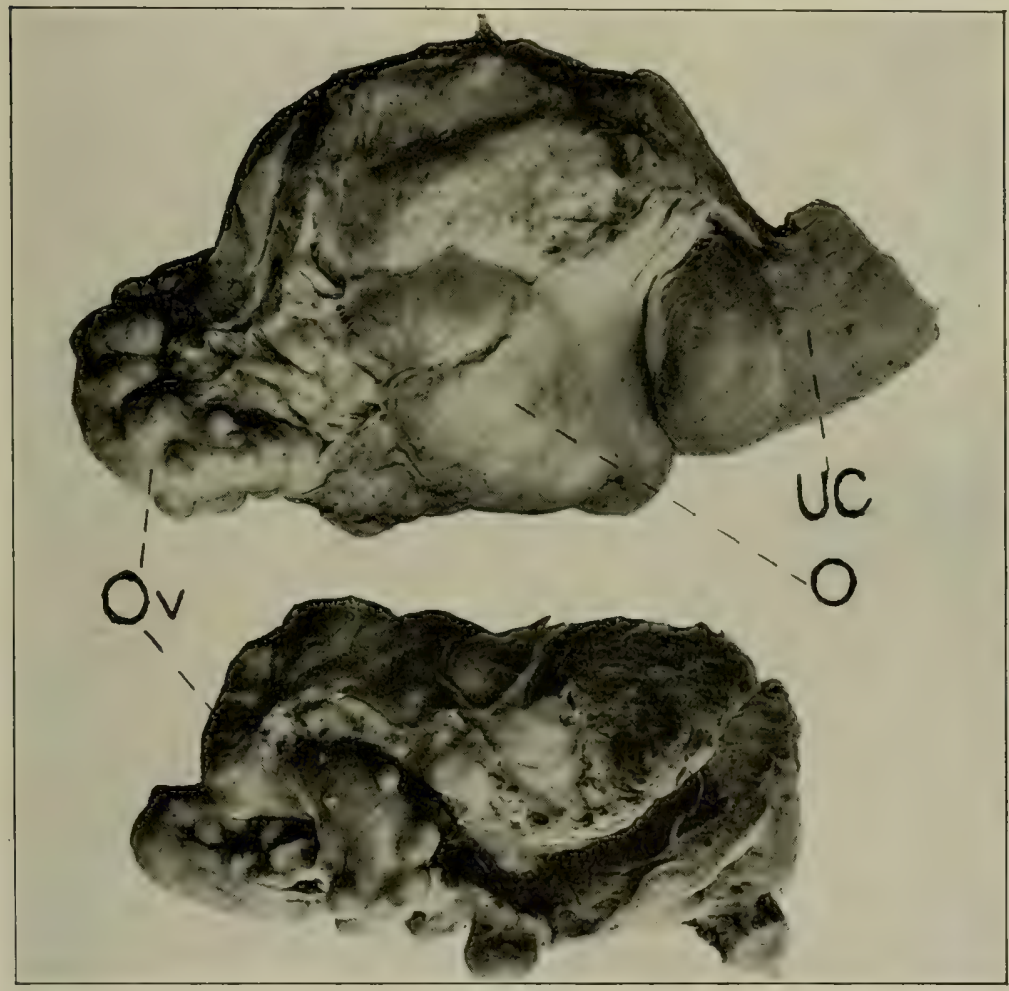

FIG. 103-Tuberculosis of Ovary and Oviduct.

The Oviducts are very Nodular and Enlarged, the Ovary Adherent in the Pavilion and Broad Ligament. Ov, Oviduct lying lateral to the ovary; $O$, ovary ; $U C$, uterine cornu.

lymph, firm and fluctuant. Tuberculous salpingitis is, in my experience, always nodular, the tubes very hard, adherent, and quite large.

walls. In hydrosalpinx, the oviduct is distended with

Tuberculosis of the oviduct is almost, if not always bi- 


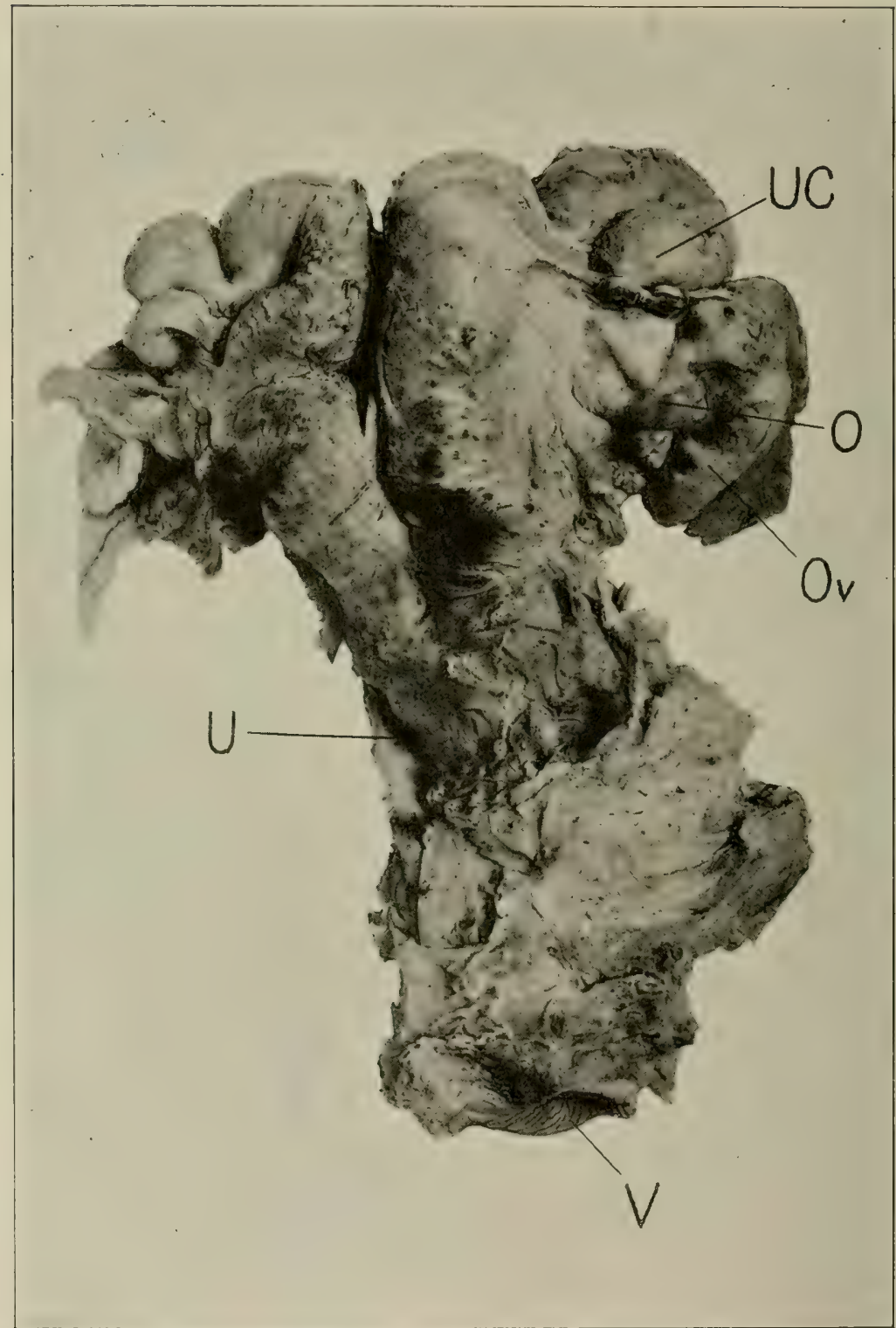

FIG. 104-Dorsal Surface of Tuberculous Oviducts, Uterus, and Vagina, Showing extensive Pelvic Adhesions and Adhesions of Ovaries in Pavilion of Oviduct and Broad Ligament.

$O$, Ovary ; $U C$, uterine cornu; Ov, oviduct extending laterally to the ovary; $U$, uterus ; $V$, vagina. 
lateral, rendering the animal incurably sterile as well as a peril in a breeding or milking herd. The pus from tuberculous pyosalpinx escapes through the uterus and cervix to the vagina, to constitute a very serious menace to the bull.

(3) Uterine tuberculosis is the commonest and most dangerous type of genital tuberculosis. It is supremely dangerous to the bull when copulating with the affected animal. The discharges resulting from the tuberculous uterine catarrh soil the vulva, tail, thighs, and ultimately the udder, and contaminate the milk. Thus the milk becomes intensely dangerous for calves, for other animals, and for man. In its peril to animal and human health, uterine tuberculosis vies with tuberculosis of any other organ. Uterine tuberculosis is quite variable, showing three somewhat separable types:

(a) Peritoneal uterine tuberculosis with extensive pelvic adhesions, as shown in Figures 104, 105, 106.

(b) Tuberculosis of the glandular structure of the uterine mucosa, as shown in Figures 105, 106, 107, 108, 109.

(c) Tuberculosis of the epithelial layer of the mucous membrane, as shown in Fig. 110.

No clear line of demarcation can be drawn between the three types, but in most cases one of the three groups of lesions predominates.

In uterine tuberculosis there is generally a persistent, obstinate uterine catarrh. In some cases the catarrhal discharge is inconspicuous. Douching the uterus may reveal very little pus. In some cases of tuberculous uterine catarrh, uterine douches affect the catarrh favorably for a time, but permanent relief I have not observed. Where there have been apparently favorable results from douching, misleading the practitioner as to the nature of the malady, limited observation indicates that copulation sets the infection aflame. In such cases as illustrated by Figures 108 and 109, the catarrh is profuse, but not generally fetid. Like tuberculosis of the oviducts, uterine tuberculosis is usually, if not always, symmetrical, or bicornual. Generally the uterus contracts extensive pelvic adhesions. 


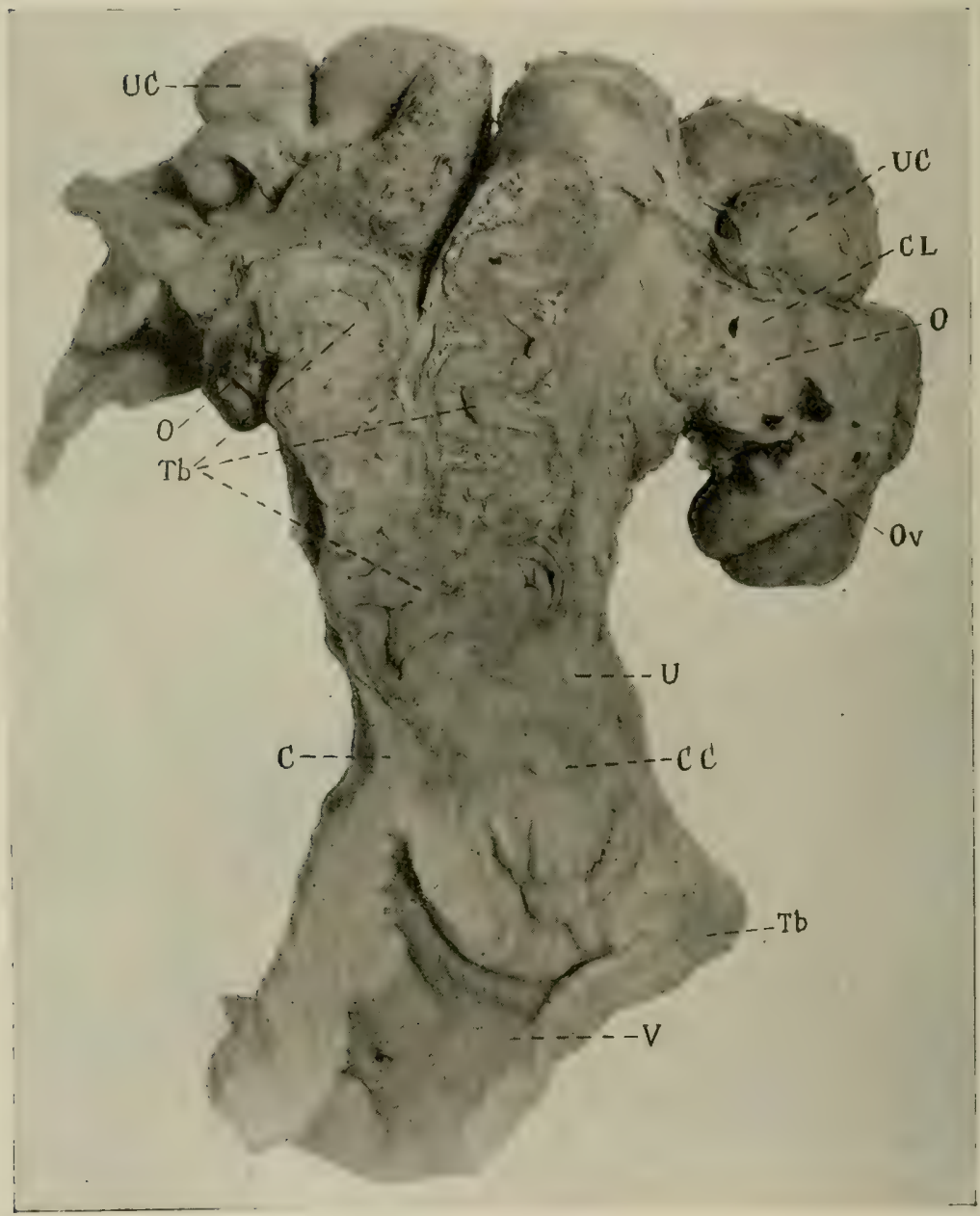

FiG. 105.

Horizontal section through dorsal surface of uterus, cornua, ovaries, and oviducts of Fig. 104. $V$, Vagina; $T b$, tubercular nodules in right vaginal walls (below) ; $C$, cervical wall ; $C C$, cervical canal; $U$, uterus ; $T b$, tubercles in cornua (above); $O, O$, ovaries ; $C L$, corpus luteum in right ovary; $O v$, tubercular oviduct greatly enlarged and elongated, pushed laterally far beyond the ovary; $U C$, uterine cornu. 
In many cases the peritoneum is so involved that ovaries, oviducts, uterus, and broad ligaments constitute a complex adherent mass, as in Figures 104 and 106, where it is difficult, though generally possible, to identify the various parts by rectal palpation. Pelvic adhesions due to genital tuberculosis resemble those caused by other infections. The adhesions due to tuberculosis are often accompanied by palpable tubercles. Their recognition aids materially in diagnosis. Non-tuberculous pelvic adhesions are frequently accompanied by sclerotic abscesses of an ovary, oviduct, uterine horn, or the three areas combined. In my collection, no abscesses exist in any case of tubo-uterine tuberculosis, not even abscesses of the lymph glands in the broad ligaments of the uterus. Tuberculous pyosalpinx and pyometra, with necrosis of the uterine mucosa, as indicated in Figures 96 and 97 , is common enough. There is one important difference in the clinical history of tuberculous and non-tuberculous pelvic adhesions. The non-tuberculous adhesions are commonly preceded by acute metritis, largely with placentitis and retained fetal membranes; tuberculous pelvic adhesions ordinarily arise independently of acute metritis.

The cow from which Figures 104 and 105 were taken was entered in our clinic for sterility. There was slight uterine catarrh (tuberculosis of the uterine mucosa) which abated under antiseptic douches. The ovaries, oviducts, and uterus were normal by rectal palpation. She appeared so well that she was mated with a valuable herd sire. Soon afterward, she broke down, and upon re-examination genital tuberculosis was evident. When destroyed, extensive generalized tuberculosis was revealed. She entered our clinic without a history, but upon the assumption of freedom from tuberculosis, and no careful search was made in that direction. Apparently the disease was forced to its crisis by copulation, the patient gave way to the infection, and a diagnosis of genital tuberculosis became practicable. Fortunately the sire escaped, largely perhaps because the cow was douched just prior to copulation. The case illustrates vividly the insidious and subtle manner in which genital tuberculosis in 
the cow may develop, and how the veterinarian needs to be on his guard.

In some cases the tuberculous uterus remains to the end free from adhesions, as in Figures 107, 108 and 109. In my collection some of the uteri in which the mucosa is most extensively involved and which have undergone the maximum increase in size have suffered least from pelvic adhe-

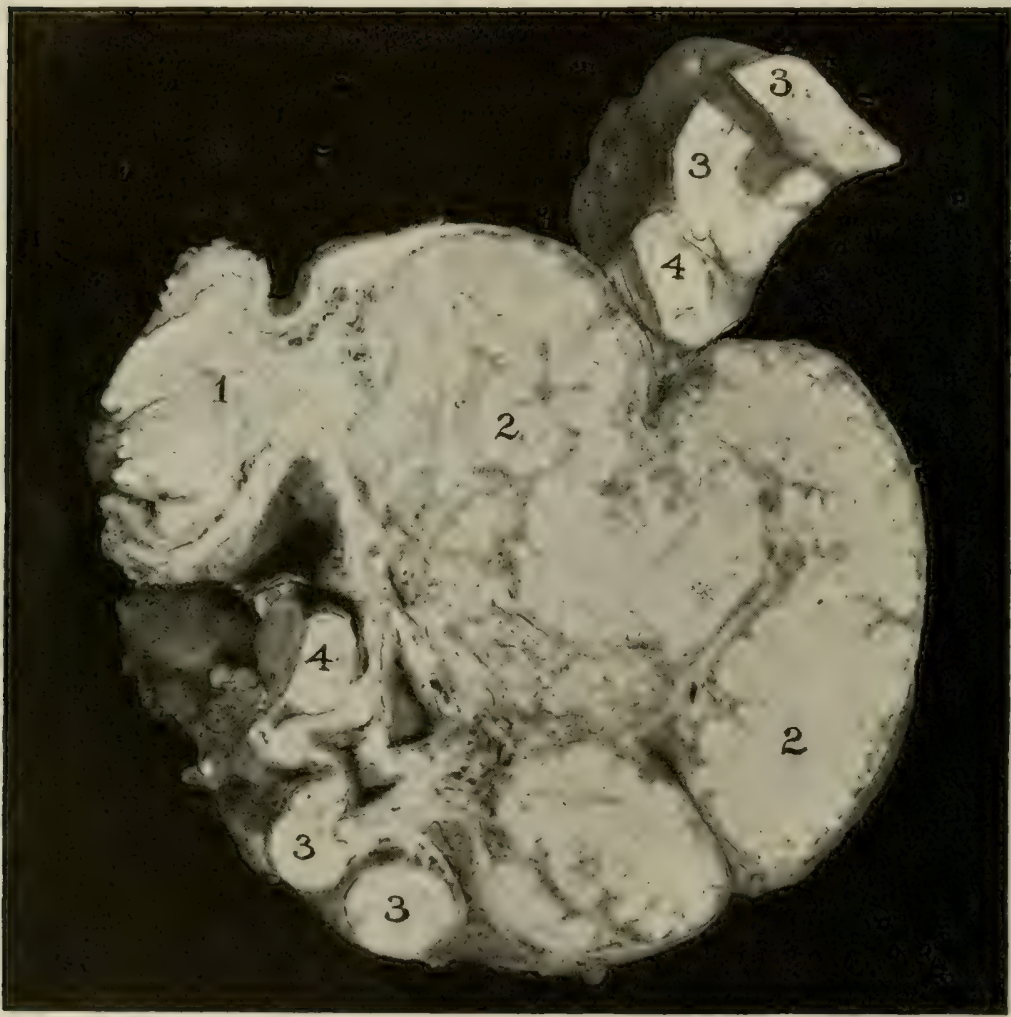

FIG. 146-Tubercular Genital Tract from a Non-Reacting Cow in Advanced Tuberculosis.

A section has been removed from the dorsal surface exposing the interior of uterine body and horns. $I$, Cervix; 2 , cornua of uterus showing tubercular masses; $3,3,3,3$, sections through the oviducts; 7,4 , ovaries. The left ovary (above) was buried beneath the adjacent cornu, 2 , so it has been excised and carried out where it cones into view. 


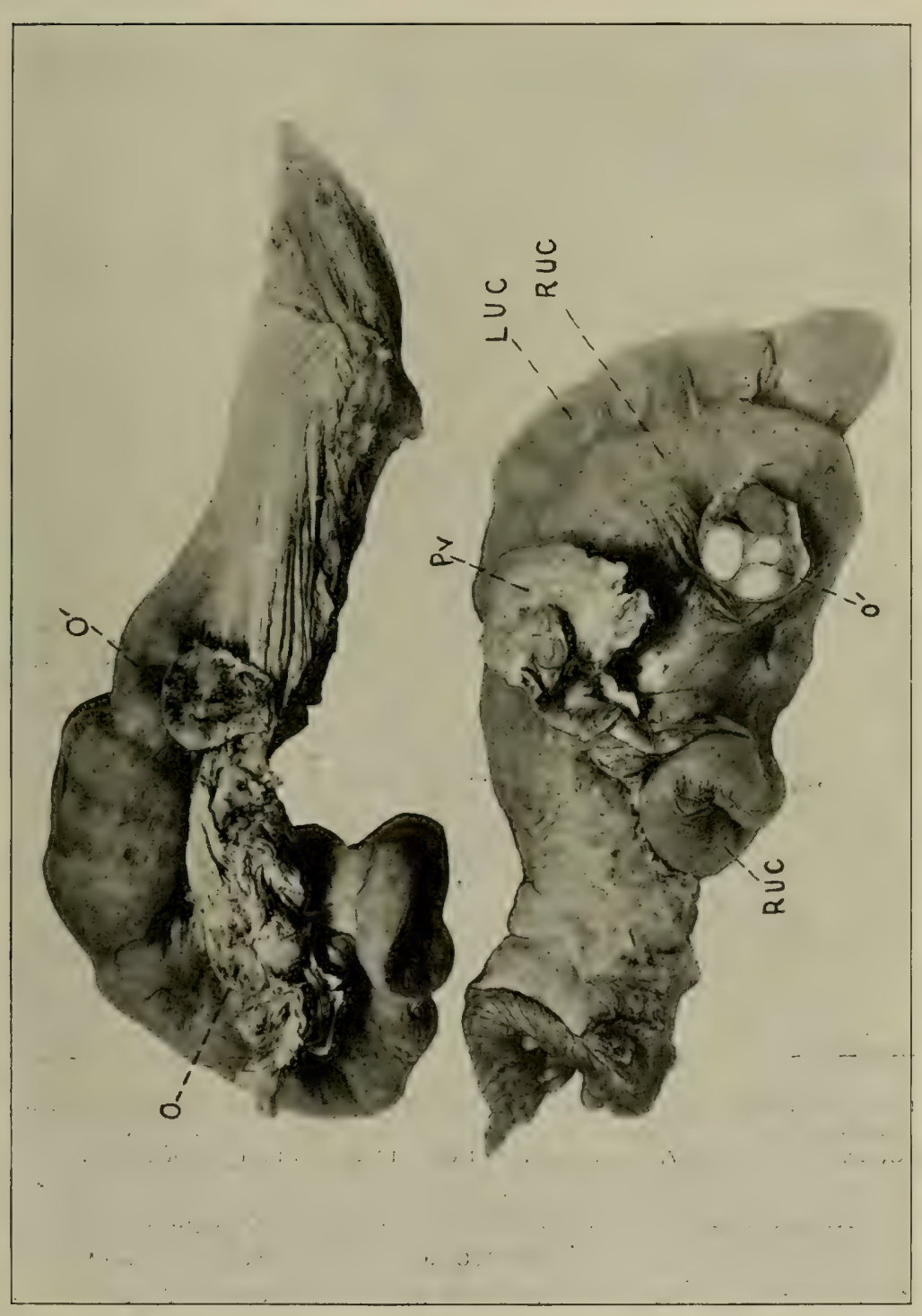

荧

范心

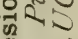

d...

ซึ 을

풀

$\rightarrow$ 의

ब.

A $=1$.

记

경 히

bo

.

$3=2$

कृ

$\Phi \cdot \frac{0}{4}$

胥胥

ณ

วี่

is

들

स्ञ

सेํㅡㄹ

기이

के.

$>$.

ขึ

फे

ํํำ

क...

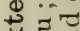

舤

I. $\Xi$

$\therefore$ 둥

(5)

프

.

를

。 


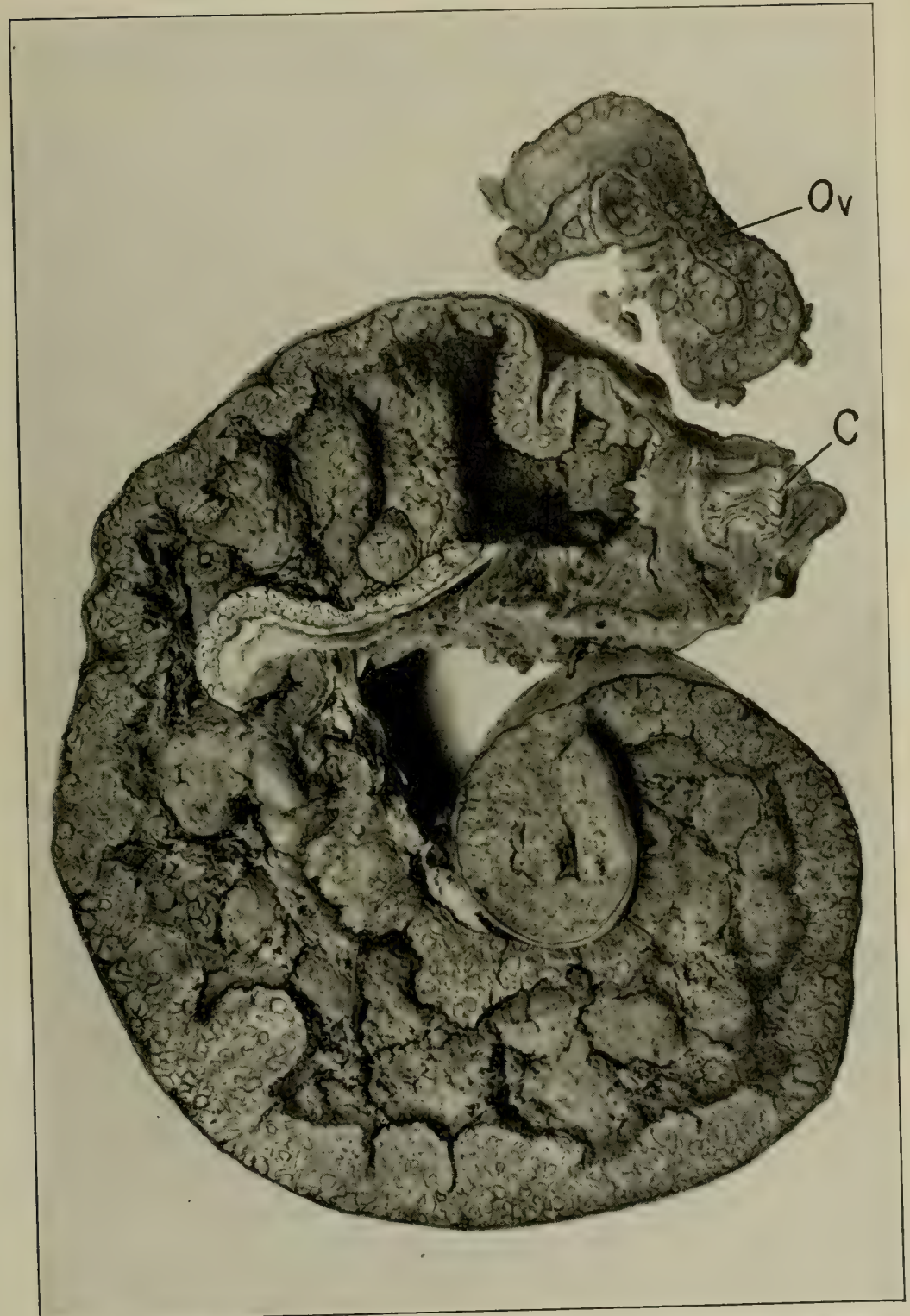

Fig. I09-Advanced Tubo-Uterine Tuberculosis, with Necrosis of Mucosa and Pyometra.

$O v$, Oviduct, greatly enlarged and consisting of a mass of tubercles ; $C$, cervical canal. 
uterine mucosa. In such cases, however, the outlook for the breeding life of the patient is hopeless and an accurate clinical diagnosis is not imperative. In some cases of uterine tuberculosis, the masses of tubercles are so voluminous that they throw the uterine walls into great transverse folds which may be palpated per rectum. When such folds are present, they have a distinct diagnostic value. Usually in chronic abscessation of the uterus due to ordinary pyogenic bacteria or to actinomycosis, while the uterus is enlarged and hard, it is very irregular in outline. At one point there may be little or no enlargement. In actinomycosis and in pyogenic abscessation of the genitalia, some one part usually contains a great abscess or abscesses quite overshadowing other lesions, but in tuberculosis there is a strong tendency to symmetrical enlargement. The two horns are approximately alike in volume, form, and consistence. This is important to remember in making a diagnosis. In one cow of great breeding value, a reactor to tuberculin, which had not calved for two years and had long suffered from an abundant, fetid, highly repulsive genital discharge, I found one uterine horn much enlarged, three to four inches in its transverse diameter, very hard, and irregular in contour. The other horn was approximately normal. The findings by palpation, in conjunction with her reaction to tuberculin, led me to diagnose uterine tuberculosis. The autopsy revealed, instead, a macerating fetus, the bones of which caused the irregular bulging of the cornu. Had I recognized the fact that in uterine tuberculosis the enlargement of the horns is usually symmetrical and that the pus from the tuberculous uterus is not usually fetid, the error would probably have been avoided. In those cases of uterine tuberculosis where only the superficial mucous layer is involved, as in Fig. 110, the diagnosis by ordinary clinical examination fails. The ovaries, oviducts, and uterus are normal to palpation, the uterine catarrh is scant, and no outstanding clinical evidences of genital tuberculosis arê present.

The outstanding elements in the differentiation of utero- 
tubal tuberculosis are the pelvic adhesions, the tubercles in the genital peritoneum, the symmetrical enlargement of the uterus, and the obstinate uterine catarrh. No one of these

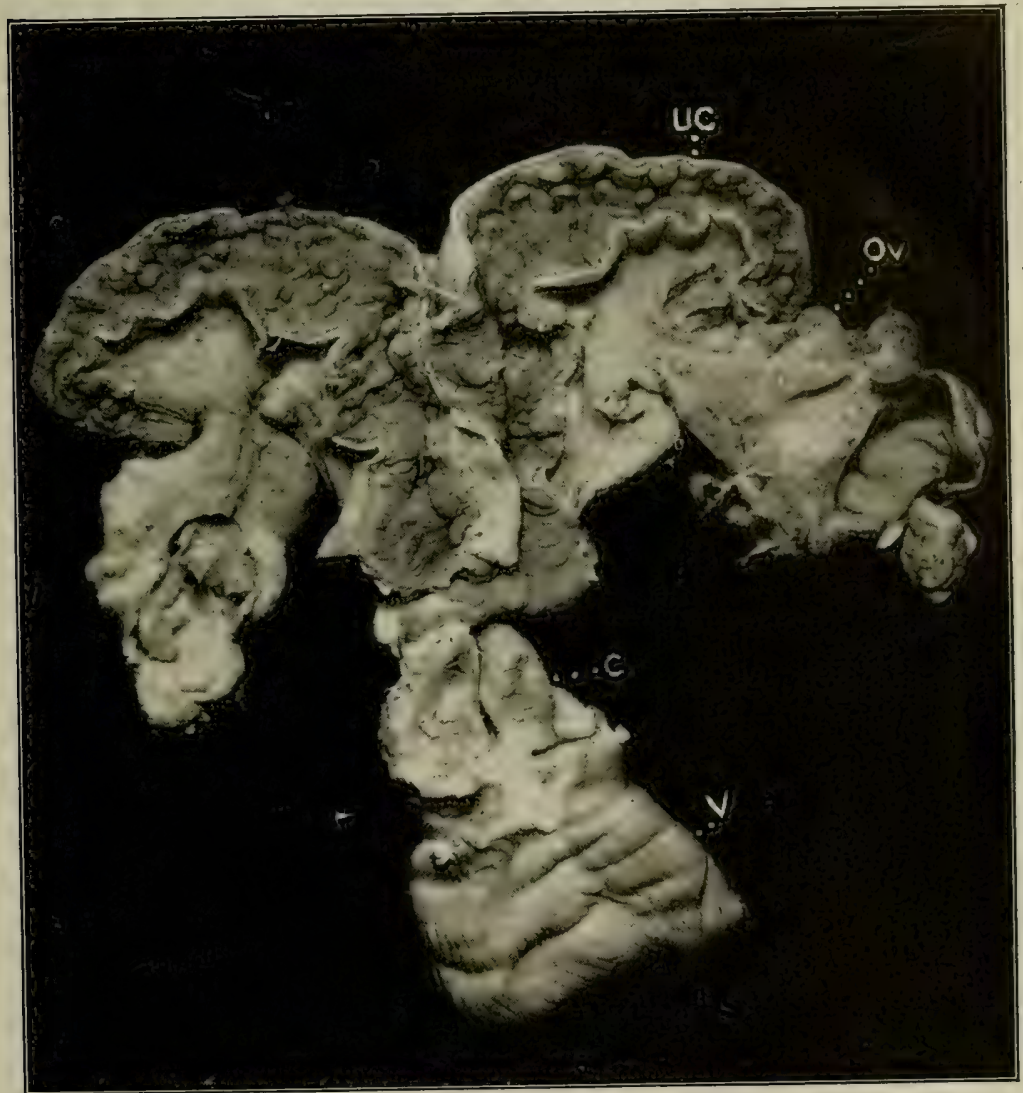

FIG. IIo-Miliary Tuberculosis of the Superficial Uterine Mucosa of a Virgin Heifer.

$V$, Vagina; $C$, cervix; UC, uterine cornu ; Ov, oviduct. The uterine horns are laid open to show the numerous small tubercles

upon the mucous surface

symptoms, alone, is sufficient for a positive diagnosis. If the patient reacts to tuberculin, the probability of genital tuberculosis is increased. No data are at hand regarding bacterial search of the genital discharges. Such search 
would probably aid materially in diagnosis. A negative tuberculin test is not conclusive evidence that genital tuberculosis is not present. The very valuable herd bull, from which Figure 96 was made, quite certainly contracted the infection from a cow which had successfully passed several tuberculin tests, in spite of severe generalized, utero-tubal tuberculosis, and marked uterine catarrh. While in many cases, such as delineated in Figure 104, quite an accurate physical diagnosis of genital tuberculosis may be made, not all cases may be detected. The important point is that utero-tubal tuberculosis may almost always be tentatively diagnosed by rectal palpation and the veterinarian and owner placed on guard. So long as the symptoms named are present, the tentative diagnosis of utero-tubal tuberculosis is not only justified, but obligatory, from the sanitary standpoint. When uterine catarrh is present, copulation is contra-indicated by every consideration of hygiene. Fertilization can not occur; coitus aggravates the catarrh and imperils the bull. The retention in a dairy of a cow with obstinate uterine catarrh is unjustified by every consideration of health and decency. The afflicted animal is unfit to associate with others in such a manner that their food may become contaminated with the genital discharges.

A tentative diagnosis of utero-tubal tuberculosis works no serious injury. If the cow recovers, as a rule the recovery automatically corrects the diagnosis; if she fails to recover, she is worthless for breeding or dairying, and fit only for slaughter, whether the infection be tuberculous or not.

(4) Tuberculosis of the cervix is presumably rare. I have but one specimen (Figures 111 and 112) derived from the abattoir, and hence without a clinical history. In this case, the uterus and oviducts are also involved. The illustrations suggest the probable clinical features. The greatly swollen, hard, smooth lips of the cervix could not well be missed by an expert examining the genitalia clinically. While uterine catarrh was evidently present in this animal, it presumably had nothing to do with the cervical lesion. The lesion was apparently closed and caused no discharge. 
The surgeon, meeting with such condition, would logically incise the tumor, revealing the nature of the lesion. I have repeatedly opened cysts in these parts. In so doing, I always draw the cervix back into the vulva before operating, so that the field is freely open to view and the contents which may be released are readily seen. If purulent, they at once raise the question of tuberculosis, which, once raised,

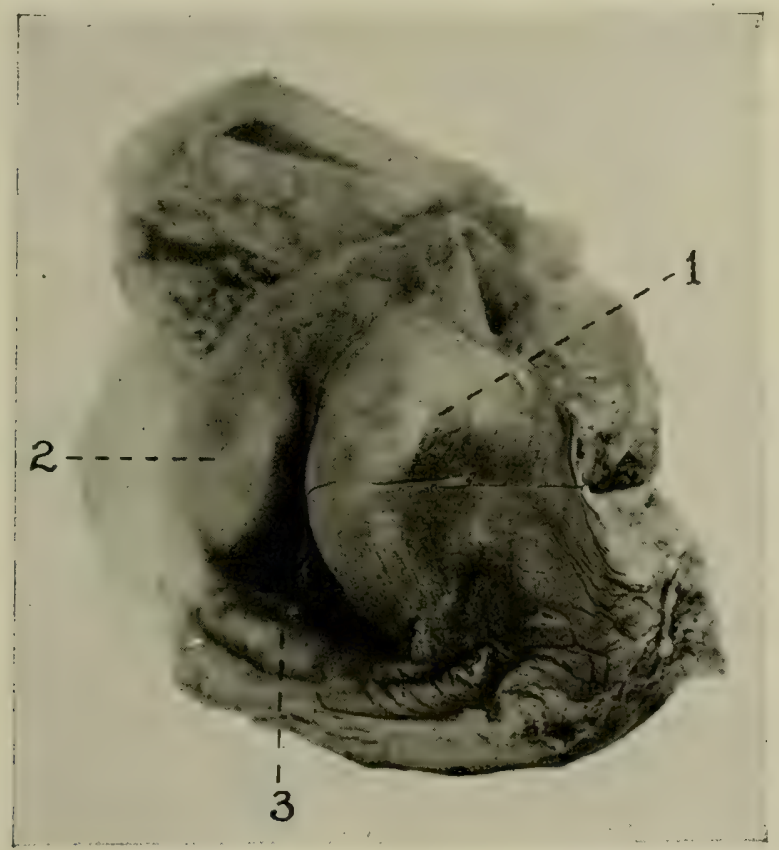

FIG. III-Tuberculosis of the Vaginal Portion of the Cervix $I$, Greatly enlarged tubercular lip of os uteri externum ; 2 , a second, lesser tubercular tumefaction of lip; 3 , mucous folds of the first annular cervical fold. Between it and $I$ is the os uteri externum.

should not be dismissed until the diagnosis has been made perfectly clear. On no account should a cow be permitted to copulate when a cervical lesion exists, unless it is clearly shown to be non-tuberculous. Even harmless-appearing cysts should not be passed over carelessly. Like vulvar cysts, discussed below, they may be tuberculous. The cervi- 
cal lesion, when open, is evidently extremely dangerous. These cysts, usually found laterally from the os uteri externum, presumably consist of retention cysts in the ducts of Gärtner (See page 39).

(5) Vaginal tuberculosis is apparently very rare. Figure 105 illustrates the only specimen in my collection. It was not recognized clinically, although I examined the patient several times. However, I did not search for vaginal tuberculosis. Possibly it was of recent origin.

It is important for the veterinarian, in dealing instrumentally with diseases of the genitalia, to bear in mind that he may, by the careless use of the uterine forceps, implant the infection in the cervix, or in the vagina at its point of continuity with the cervix. He may re-infect these parts, through instrumental lesion, with the discharges from the patient's uterus. If careless in his methods, he may carry the infection from a tuberculous cow to a healthy one. Under reasonable precautions, the danger is quite negligible. Figure 105 shows the tuberculous lesions within the vaginal wall, closed. If open, the lesion would have the same danger as an open lesion of the cervix.

(6) Vulvar tuberculosis has occurred twice in my practice, each time readily diagnosed, though in one case the nature of the lesion was at first misleading. In this case, at the first examination, a cyst one inch in diameter, located apparently in Bartholin's gland (See page 44), was incised, and a small amount of sero-purulent content escaped. Prior to my next examination, the patient had reacted to the tuberculin test. When I examined her, the lesion presented the typical characters of a tuberculous ulcer. My second case was more readily diagnosed. The vulvar lip, which was swollen and sclerotic, presented an old fistulous opening, from which small volumes of thick pus could be pressed out. Centripetally, a tuberculous gland, two to three inches in diameter, lay alongside the vagina. In addition, both supramammary glands were greatly enlarged, and at each internal inguinal ring glands three inches in diameter were palpable. Autopsy showed extensive generalized tuberculosis. 
Vulvar tuberculosis is evidently a very dangerous type for the bull. In my second case, the cow had been bred only a few days before my examination to the herd bull, valued at more than ten thousand dollars. Fortunately he escaped infection.

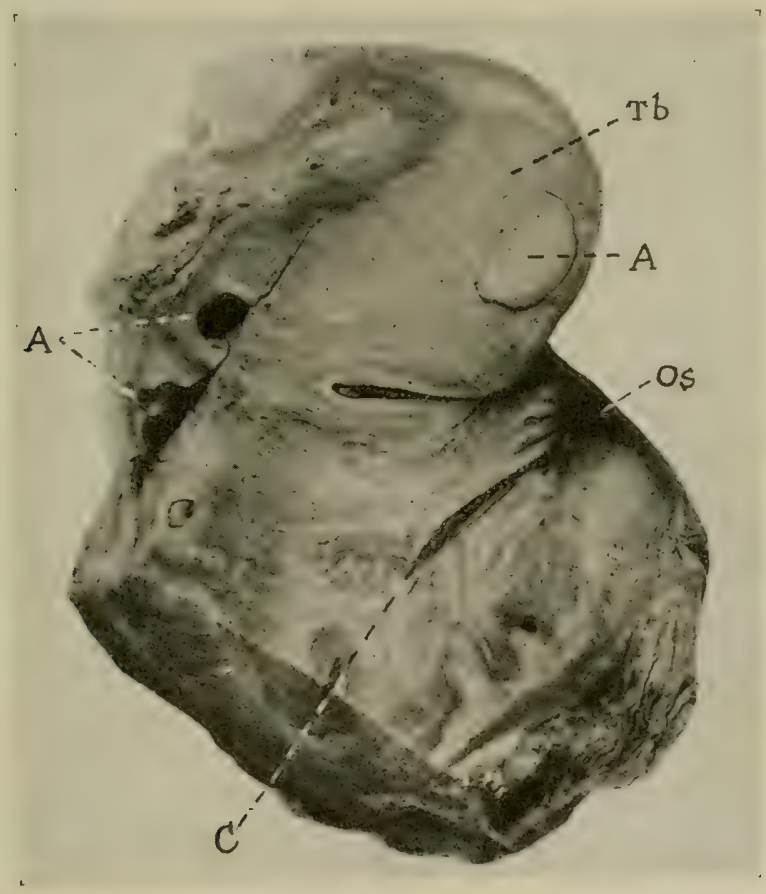

FIG. I12-Sagittal Section, of Fig. III.

Os, Os uteri externum ; $C$, cervical canal; $T b$ tubercles in lip of os uteri : $A, A$, tubercular abscesses.

The importance of genital tuberculosis in cattle calls for much greater interest upon the part of the veterinarian. In dairies and pedigreed breeding herds, tubercular uterine catarrh is one of the most dangerous and repulsive types of tuberculosis. The search is generally limited to the tuberculin test, which too often fails to detect the disease. The cow whose uterus is shown in Fig. 94 belonged in a small, 
high-class Guernsey herd which had been tested semi-annually for several years. This cow passed each test satisfactorily. Each test revealed reactors, which upon slaughter showed recent lesions only. It was evident that lurking somewhere in the herd was a non-reacting spreader of a dangerous type. In making a survey of the genital organs of the herd, with reference to breeding, I designated this animal as the non-reacting spreader.

When diseases of the genitalia occur, closing the breeding career of an animal, a definite diagnosis is of great importance. If tuberculosis is the probable or possible cause of sterility, but can not be clearly diagnosed clinically, slaughter without accurate post-mortem diagnosis is wholly unjustifiable. If a cow is sterile because of genital tuberculosis, she has almost certainly seriously endangered the bull and other cattle. If a bull becomes disabled for coitus because of genital tuberculosis, it is not enough to send him to the shambles because he can no longer be used for breeding. Somewhere there is almost certainly a cow with genital tuberculosis which transmitted the infection to him during coitus. She is liable to transmit the infection to another bull and is highly dangerous to other cows. The diagnosis of tuberculosis in the bull is therefore highly necessary for the control of the disease in contact animals. For this purpose post-mortem is as valuable as clinical diagnosis.

The control of these dangers evidently depends chiefly upon the more general and accurate diagnosis of genital lesions in both sexes and the more general adoption of efficient sex hygiene in breeding cattle. The lesions are to be detected through two chief agencies-dairy inspection and inspection of the genitalia in connection with sterility. At present, dairy inspection fails utterly in most cases in relation to genital disorders. Repeatedly I find, in regularly inspected herds, and even in certified dairies, cows with quarts or gallons of fetid pus in the uterine cavity, large volumes of which are pressed out from time to time while the cows are lying down. Advancement in this field is one of the most urgently needed reforms in dairy inspection. 
The greatest weapon for the control of the danger lies in the more general inspection of the genitalia of cattle, as a safeguard against sterility and abortion. It is becoming more and more apparent that a careful physical examination of the genitalia of cows, prior to breeding, is a wise precaution of distinct scientific and economic importance, and that the breeder should take far greater care in the sex hygiene of the bull. If penial injuries and infections are to be averted, having first ascertained that the genital organs of the cow are healthy, the external genitalia of both cow and bull should be carefully washed before permitting copulation. It is highly important, in the prevention of penial lesions, which may serve as infection avenues, that the crusts of pus in the vulvar tuft of hairs of the cow be removed by washing. Otherwise, these crusts of pus may be caught and forced into the vulvar opening, to abrade the penial mucosa. After copulation, the penis, prepuce, and sheath of the bull should again be carefully and efficiently douched. These precautions go hand in hand with those designed to control sterility and abortion. 


\section{THE GENERAL INFECTIONS OF THE GENITAL ORGANS OF CATTLE}

The mammalian genital tract opens freely upon the exterior, rendering it possible for any infection upon the adjacent body surface, capable of growing within the genital tract, to invade it, either actively or passively through the agency of a bearer (copulatory organs of the opposite sex during coitus, surgical instruments, hands of operator). Vastly more important, as far as at present known, is the transmission of important infections from the alimentary and pulmonary tracts, through the blood stream, to the genital tract. Thence the infection is carried, in the female, back to the alimentary tract of the fetus. That is, as will be more fully discussed when considering fetal infections, if there is infection in the uterus of the pregnant female (which is the rule) it tends to penetrate the thin chorion and amnion and to be swallowed promptly by the fetus, along with the amniotic fluid. If the fetus has sufficient vigor, the bacteria are harmlessly included as a component part of the meconium, to be expelled soon after birth ; if disease of the fetus occurs before birth, and the gastro-intestinal epithelium is destroyed or devitalized, infection ensues. The bacteria may then enter the blood stream, to be lodged later in the articulations (arthritis) but, since they were originally derived from the genital tract of the mother, tend logically to return to the genitalia. If the infections within the uterus of the pregnant cow commonly invade the alimentary tract of the fetus, it follows that subsequent to birth the same infections may be expelled from the diseased uterus, contaminate the milk, be swallowed by the calf, and cause disease having the same peril for it as if the infection had been swallowed prior to birth.

Each year adds to the number of known species of microörganisms capable of invading the genital tract of cattle and of other domestic animals and, according to the best 
available evidence, capable of attaining such intensity as to interfere seriously with the reproductive functions. They may reside indefinitely in the genital tract without causing disaster but ready at any time, taking advantage of an injury to, or loss of vigor in, the area occupied, to cause serious harm. Their method of injury, however, is less definite in character for each recognizable organism than the pathologic processes described in the preceding sections. The vesicular venereal disease stands out definitely, by its symptoms and clinical history of transmission, as a specific contagion. The nodular venereal disease offers a distinct and characteristic lesion, although its cause it not definitely determined and its importance is largely a matter of opinion. Actinomycosis and tuberculosis are fully recognized as specific diseases causing genital injuries identical with well recognized lesions in other organs, each of which is due to a recognized species of organism.

In the following section there will be discussed, as general infections, an extensive group of lesions due to, or believed to be due to, infection which far surpasses, in its peril to the reproductive organs, the four preceding diseases. Each may severally or jointly produce a variety of lesions, but no one lesion can with confidence be attributed clinically to any one organism. Several or many different bacteria or other organisms may cause ovaritis, salpingitis, metritis, or cervicitis, and these lesions may prevent, interrupt, or imperil reproduction. Moreover, it is difficult or impossible to differentiate clinically between them. If salpingitis is present and diagnosable, it may be due to a streptococcus, a bacillus, or a micrococcus, or to a combination of the three, but the exact status can be determined only by a bacterial study of the diseased tube after its removal from the abdominal cavity. They will be discussed, therefore, as a group having a common tendency to cause an extensive list of pathologic changes with serious injury to the reproductive functions. The lesions produced by the infection-not the identity of the infecting agent-will constitute the basis for classification. 


\section{THE GENERAL INFECTIONS OF THE GENITAL ORGANS OF BULLS}

The diseases of the genital organs of bulls, as a factor in the problem of reproduction, have received scant attention from veterinarians. A belief had grown up and held the undivided attention of veterinarian, dairyman, and breeder, that the great and only important menace to reproduction in cattle was a specific infectious disease of the pregnant female, designated "contagious abortion." During the past few years that belief has been undergoing disintegration, until very few veterinarians now cling unreservedly to the tradition. There has been much unintelligent controversy over the part played by the bull in genital diseases of cattle, because consideration has been largely restricted to the question of the part played by the bull in transmitting to cows the bacillus discovered by Professor Bang, which was regarded as the specific cause of the abortions occurring in cattle. The attitude assumed was that the only infection of interest which, attacking the genital organs of a bull, might possess danger for a cow with which he might copulate was the $B$. abortus. If, therefore, a bull was not ejaculating with his semen the B. abortus, he was assumed to be virtually beyond criticism from the standpoint of infection. The study of the semen of bulls and the bacteriology of the genital organs and of the semen, except for a few searches made for the $B$. abortus, have been of no consequence. Little or nothing was known of the diseases of the testicles, unless they became enormously enlarged or underwent abscessation. The subsidiary structures-the epididymis, vas deferens, vesiculae seminales, prostate and Cowper's glands-were ignored. These facts show the strangling grip so long held by "contagious abortion" upon the study of the infections of the genital organs.

The time has arrived for a frank recognition by breeder and veterinarian that the bull is one-half the breeding herd, that the other half is valuless unless he is genitally sound, and that, speaking in percentages, he is probably as great an offender as the cow. In other words, probably as large a 
proportion of bulls as of cows go to slaughter on account of sterility or low fertility.

A general outline for the clinical examination of the genital organs and the semen of bulls has been given in Chapter II. It remains to describe more fully the examination of the semen in the course of genital diseases and to point out as far as possible the relation of changes in the semen, and especially in the spermatozoa, to clinical manifestations of genital disease.

${ }^{1}$ The technique which I have pursued in collecting and examining semen from bulls is as follows:

The bull's sheath is first douched with $1 / 2$ per cent. Lugol's solution or 1/2 per cent. chlorazene. The cow's buttocks are thoroughly cleansed with a disinfectant and washed with a $1 / 2$ per cent. chlorazene solution, and the vaginal mucus is removed manually. If a bacteriological examination of the semen is desired, the vagina should be thoroughly cleansed with a douche of $1 / 2$ per cent. chlorazene, which must be completely removed several minutes before copulation. As only a slight trace of chlorine or other disinfectant will cause immobility of the sperm cell, it is desirable that cleansing of the parts for studying the motility of the spermatozoa be done with sterile water or a normal saline solution. In either case, all possible vaginal mucus should be removed, just prior to service, with the well disinfected hand which has been washed with sterile water. The method is not entirely satisfactory from the bacteriological standpoint. Two sources of bacterial contamination must be considered: first, extraneous, and second, the vaginal flora. Contamination from the first source may be largely prevented by carefully cleansing the parts immediately prior to inserting the hand for the sample, but, owing to the almost constant fecal contamination of the vulva and the adjacent parts, it is wellnigh impossible wholly to prevent contamination during

${ }_{1}^{1}$ The results of clinical examinations of bulls, with directions for the examination of semen and spermatozoa from diseased or suspected bulls, are by W. W. Williams; the micro-photographs of the preparations are by Professor B. F. Kingsbury. 
coitus. The danger of contamination of the semen from the vaginal flora, although undoubtedly lessened by the above method, can not be said to be nullified. Some other technique may lessen the contamination from each source.

Recently I have used a common sputum cup for the withdrawal of samples from the vagina. This method has considerable advantage in the prevention of contamination. An examination of the vaginal mucus taken prior to service may serve as a check on the vaginal flora. With careful disinfection, it seems that many samples may be taken free from fecal flora, as only a few give a growth of the colon group. If the sample is to undergo a bacteriological examination, it should be placed in a sterile vial, cooled immediately, and cultured at the earliest possible date. Semen for clinical and histological examination must be placed in a warm vial, kept at a temperature ranging from $100^{\circ}$ to $104^{\circ} \mathrm{F}$., and examined under the microscope within thirty to forty-five minutes after sampling.

First, the physical characters of the semen are noted, including the quantity, color, consistency, and coagulability. A drop of semen is then placed on a warm microscope slide and covered with a thin cover glass. Under a low-power lens, the relative abundance of the spermatozoa is noted. The semen is then placed under an oil immersion lens in order that the degree of motility and the percentage of cells which are motile may be observed. When possible, the length of time which the motility continues should be recorded. I have observed motility as much as four hours after taking a sample, and it is probable that, in a specimen kept under proper conditions, the motility outside of the female genital tract continues even longer. Much of the cellular morphology can be determined by examining the fresh specimen under the oil immersion lens, and the relationship between the non-motility and the morphological imperfection can be established.

For the determination of the finer morphological details, a stained preparation is best. The film should be made from a strictly fresh sample of semen, fixed with heat, and 
the mucus removed by placing it in a 0.5 per cent. chlorazene solution for eight to ten minutes. The slide is then washed with clear water, to remove all traces of chlorine, and stained with carbol-fuchsin with heat for one to two minutes. The carbol-fuchsin is washed off with water. The film is stained for eight minutes with alkaline methylene blue. Then the methylene blue is washed off and the film is dried with filter paper. The head of the healthy cell should be brought out in clear detail. The neck granule, the cell membrane, and the nuclear membrane should stain very dark. The nucleus should stain slightly darker than the cytoplasm. If the counterstaining with methylene blue is continued only about five seconds, the nucleus remains bright red and the cytoplasm stains light blue, but the nuclear membrane is left indistinct. The nucleus itself retains an alcoholic eosin stain better than carbol-fuchsin, but after the use of eosin it is difficult to counterstain so as to bring out the details. Recently, I have used acid stain consisting of alcohol two parts, carbol-fuchsin two parts and eosin one part. Staining is carried on one and one-half minutes, and the cells then counterstained with a fifty per cent. Loeffler's methylene blue for three to five seconds. By this method, an exceptionally clear detail is obtained. Various stains may be used, but eosin and carbol-fuchsin as acid stains and Loeffler's methylene blue as a basic stain have been found more satisfactory because their stability makes them better for field use than many of the aniline stains. Considerable experience is required for proficiency in the proper preparation and interpretation of stained specimens.

For fecundation it is necessary that a healthy sperm cell unite with the ovum. Ostensibly the spermatozöon, as is the case with other cells of the body, may undergo certain destructive or degenerative changes affecting its physiological function. This may occur during the process of spermatogenesis, in which case the immature cells are eliminated, or degenerative changes may take place after the cells have reached maturity and while they lie in the seminiferous tubules or epididymis. If the sperm cell be arrested in de- 
velopment, defective or weakened, either it loses its ability to unite with the egg, or, if union takes place, the fertilized ovum undergoes an early death or develops a constitutionally weak embryo. Thus Adami and Nicholas state:

"What is true regarding infectious diseases must to some extent hold also regarding chronic intoxications of various orders. The results of constitutional disease in either parent may be the following, according, on the one hand, to the extent of the influence of the disease, or intoxication, upon the germ plasm of that parent, and on the other, to the activity or potency of the germinal matter contributed by the other parent:

1. Sterility. The germ cells being so profoundly modified that either (a) they are destroyed, (b) their development is arrested, or, (c) being developed (ova or spermatozoa), are imperfect and incapable of fusing with the germ cells of the other parent.

2. Imperfect development of offspring. (a) Of such extent as to lead to intra-uterine death and abortion; (b) cf less extent, a viable individual being produced presenting either-

(1) Gross anatomical defects.

(2) No gross anatomical defects, but lowered vitality, presenting itself either in the form of weakened powers of resistance against disease in general, or (?) proneness to develop the same disease as the parent."

The spermatozoa which I have studied are frequently characterized by definite morphological changes in the cells. In order to understand fully these changes, it is essential that the normal spermatozoön shall be known and the eye trained to differentiate between the normal and the abnormal.

The cell is divided into four main parts-head, neck, body and tail.

The head, oval in contour, is encompassed by a thin line, the cell membrane, which takes an acid stain more deeply than other portions. The head is divided into two portions - the cytoplasmic and nuclear elements-by a semi-ellipti- 
cal line, the nuclear membrane. The nucleus, of full oval contour except at its base, occupies normally a little more than one-third of the basal area of the head. Its sides conform to the general contour of the cell membrane. The chromasomic material does not occur in this cell as definite granules, but is disseminated throughout the nucleus. The nuclear element in a healthy cell takes a basic stain, and when stained with methylene blue appears very slightly granular and darker than the cytoplasmic portion.

The cytoplasmic portion of the head, anteriorly and laterally, corresponds in contour with the general oval outline of the cell membrane. Caudally, it lies against the nucleus, whose convex face is directed into the base of the cytoplasm, forming a shallow cup or fovea in the latter. The cytoplasm of the head takes carbol-fuchsin very faintly, which, when counterstained with methylene blue, is entirely replaced by the latter.

The neck parts in the bovine spermatozoön are very small and not defined except under a very high magnification. In the human spermatozoön two neck granules are described. In spermatogenesis they arise from a single granule, which in the last stages of development divides. One granule passes to the caudal end of the neck and remains there in close relation to the connecting piece or body; the other granule passes to the anterior end to become fused with the base of the head. If such separation of the neck granules occurs in the bovine spermatozoön, it is not brought out by my present method of staining. The neck granule appears as a single bar, fused to the base of the head. Apparently there is a slight concavity at the base, into which the neck is inserted, obscuring most of it from view. The granule, fused into this concavity, appears therefore to be located within the nuclear material; this is undoubtedly never the case. Aside from this granule, the neck remains unstained under the methods which I have employed.

By the embryologist it is held that, when fecundation takes place, the sperm head enters the cell membrane of the ovum. The sperm then loses its tail, which separates be- 
tween the neck and connecting piece. It appears then that the head, which has up to now faced the center of the cell, becomes reversed and faces the cell wall. From the neck granules, which migrate toward the center of the cell, are formed the centrasomes, or polar bodies, which play such an important part in the mitotic process.

It is quite clear that an arrest in the development of the centrosmic precursor may be a potent cause of sterility. Judging from some stained preparations on hand, it appears quite certain that this arrest in development occurs to a greater or less degree.

The body, cylindrical in form, about one and one-third times the length of the head, takes readily either acid or basic stain, and reveals in the properly stained preparation a spiral filament encircling its entire length. The body is succeeded by the tail, of slightly smaller diameter and about four times as long. The tail, tapering posteriorly, ends as a very fine filament. As the body and tail function only in a locomotive capacity, I shall not deal further with them, but shall study the pathologic changes in the heads of various samples of spermatozoa, and the accompanying gross anatomical and functional changes in the genital apparatus of the bull.

Bull 4, Figs. 113, 114. Eleven years old. For three years was used as sire in a herd of about one hundred mature females. Served sixty-three cows, with two hundred and fifty-one copulations, seventy-five (29.8 per cent.) of which resulted in conception. In 1916 , ten cows were served, of which twenty per cent. failed to conceive and twenty-five per cent. of those conceiving aborted. In 1917, thirty-six cows were bred, of which 11.1 per cent. failed to conceive and 21.95 per cent. of those conceiving aborted. In 1918, forty cows were bred, of which 35.6 per cent. failed to conceive and 27 per cent. of those conceiving aborted. In 1919, thirty-eight cows were bred, of which 79 per cent. failed to conceive and 40 per cent. of those conceiving aborted.

After about three or four months in the herd, the bull's fertility became lessened. Since May 1, 1919, he has been 
used frequently, with no conceptions. The sudden sterility of about 75 per cent. of the herd had been thought to be due wholly to the condition of the cows. A large proportion of the cows (36.5 per cent. of those served) showed macroscopic pathologic changes, especially cervicitis, but only in a few cases of sufficient severity to account for sterility on such a large scale.

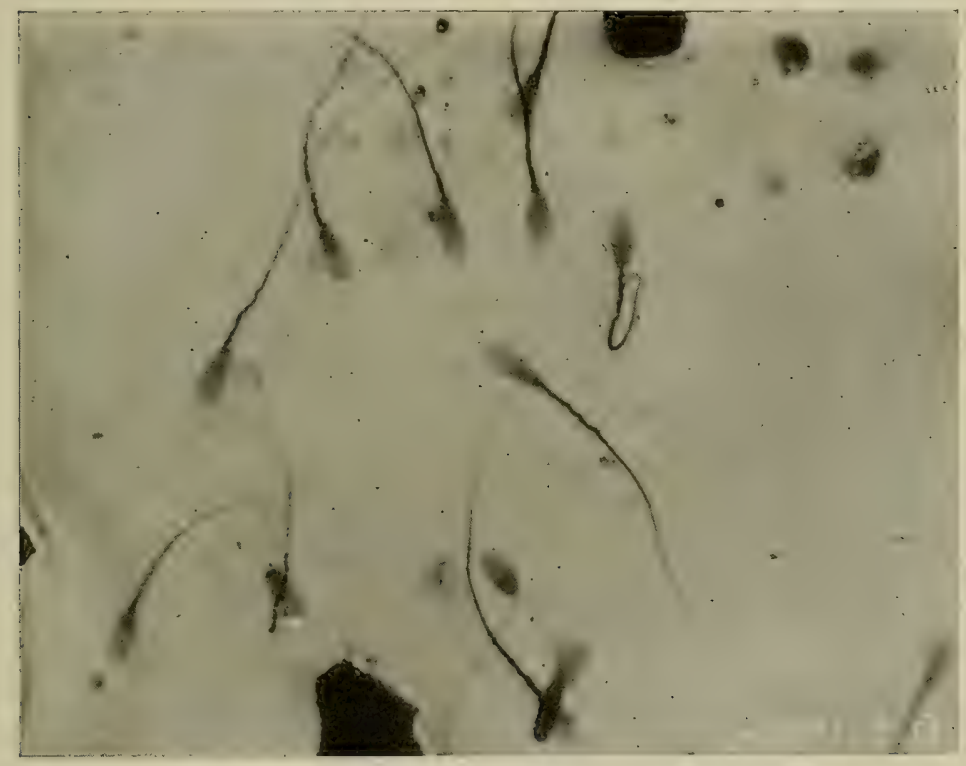

Fig. I13-Spermatozoa from Bull No. 4.

Number comparatively low. Cells non-motile when ejaculated. Many Tailless Spermatozoa.

On Dec. 5, physical examination revealed a severe balanoposthitis and testicles slightly atrophied, with slightly diminished tone. Coitus was normal. About 7 mils. of semen were obtained, which was very viscid, of a light straw color, and more translucent than normal.

The spermatozoa were non-motile. About fifty per cent. of the normal number were present. In fresh specimens, many spermatozoa lay upon their sides, giving the appearance of extremely narrow heads. In stained preparations 
many detached tails were present. The head stained as a homogeneous mass instead of showing its component parts as in the healthy cell. In this specimen of spermatozoa there appeared to be a total absence of the nuclear membrane, and in many cells the upper granules were missing. There was an almost total disintegration or arrest in development of the integral cellular elements-the nucleus and

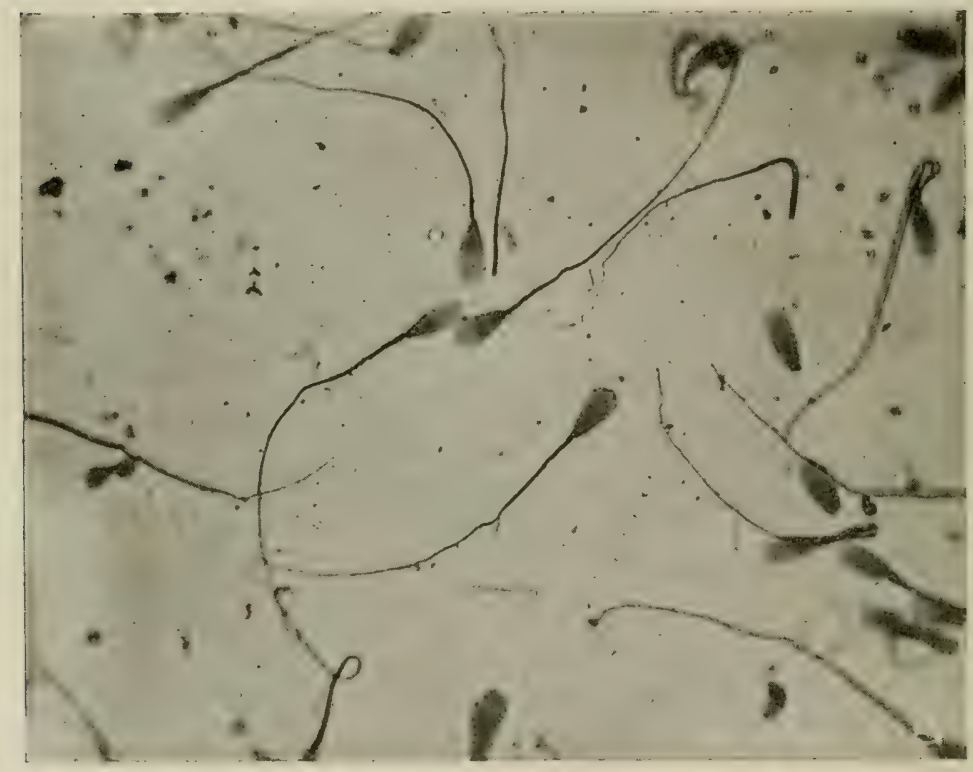

FIG. I I -Spermatozoa obtained from Bull No. 4.

Taken 25 days after those shown in Fig. I 3 , during which period the bull had complete sexual rest. Spermatozoa were still non-motile, but showed better staining qualities.

neck granule. On Dec. 5th, 1919, this bull was ordered withdrawn from service.

On Dec. 30th, after twenty-five days of complete sexual rest, another sample (Fig. 114) was obtained, which showed a remarkable improvement in the staining qualities. The cell was not normal, though the head stained much better. The connecting piece appeared enlarged and was contorted by the presence of a much enlarged spiral filament. 
Ordinarily the filament is so small that special staining is required to show it. The nuclear portions of the heads were uniformly undersized. It was ordered that this bull should have complete sexual rest until further study should determine a more normal condition of the semen and spermatozoa.

Jan. 29, 1920. Motility still absent and fewer spermato-

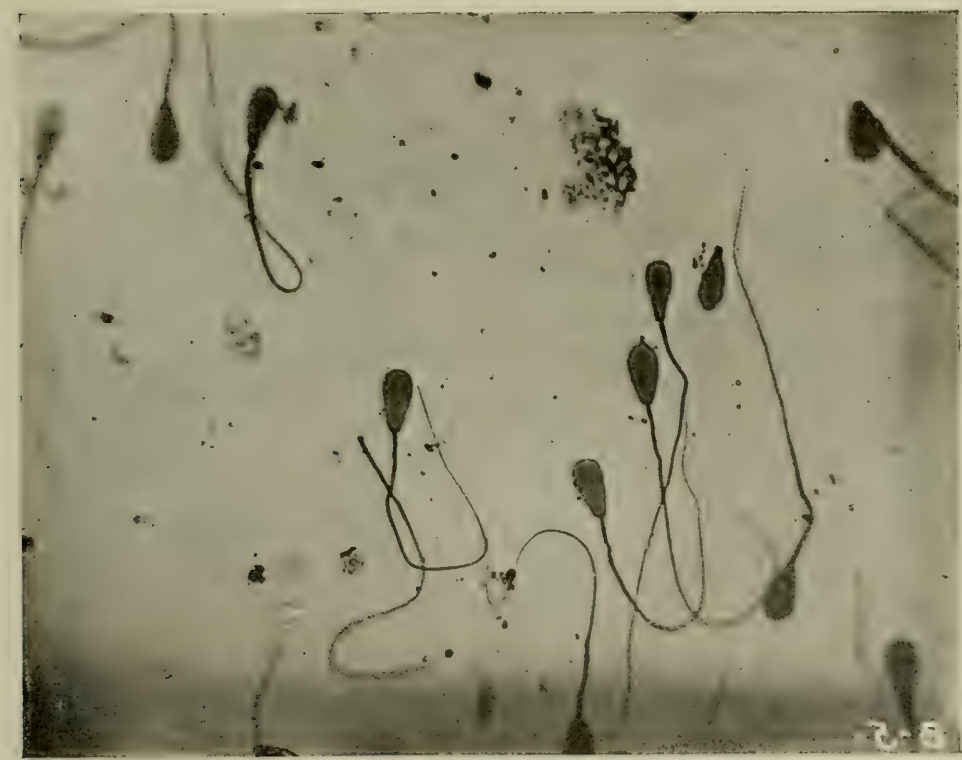

FIG. II5-Spermatozoa from Bull No. 5.

Spermatozoa motile, but heads abnormally narrow and nuclei very small.

zoa. About fifty per cent. of the spermatozoa were tailless. Many of the heads had a very narrow tapering basal portion, running to a point at its juncture with the neck. The nuclear portion was generally differentiated, but not as distinct as normal. The neck granules were very small and generally imperceptible.

Mar. 6, 1920. Physical examination revealed no changes. Twenty mils. of semen were obtained, containing considerable vaginal mucus. The semen was very viscid, and at first 
slightly turbid. Firm coagulum formed within thirty minutes. The sperm cells were decreased in numbers and nonmotile. There was a slight microcephalia and many loosened heads throughout the sample.

April 5, 1920. Physical examination revealed no new macroscopic changes. About twenty mils. of turbid, semitranslucent semen were obtained, containing considerable

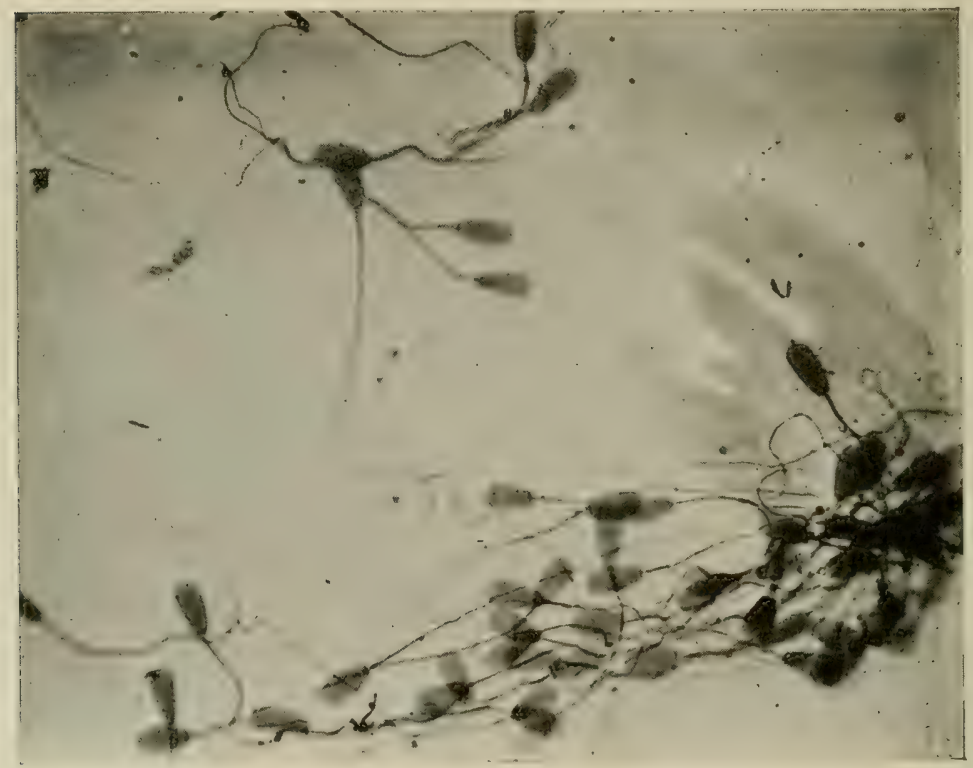

FIG. I16-Spermatozoa from Bull No. 7.

Many with small heads and atrophic nucleus.

epithelial debris. A firm coagulum appeared almost immediately after obtained, leaving the main part of the mucus colorless and translucent. Sperm cells, entirely non-motile, were present in numbers not to exceed twenty-five to fifty per cent. of normal. Fifty per cent. of the spermatozoa were without tails. Almost all were microcephalic, especially those which were tailless. In these there was a very marked diminution in size and uniform tapering of the basal portion of the head. In most heads the nuclear por- 
tion was clearly defined. The neck granule was either absent or yery small and stained faintly.

Bull 5, Fig. 115. Eighteen months old. Served twelve cows, with but one conception. The seminal vesicles appeared slightly arrested in development and the left gland was inflamed. Four to seven mils. of seminal fluid were obtained, containing motile atypical spermatozoa. The head

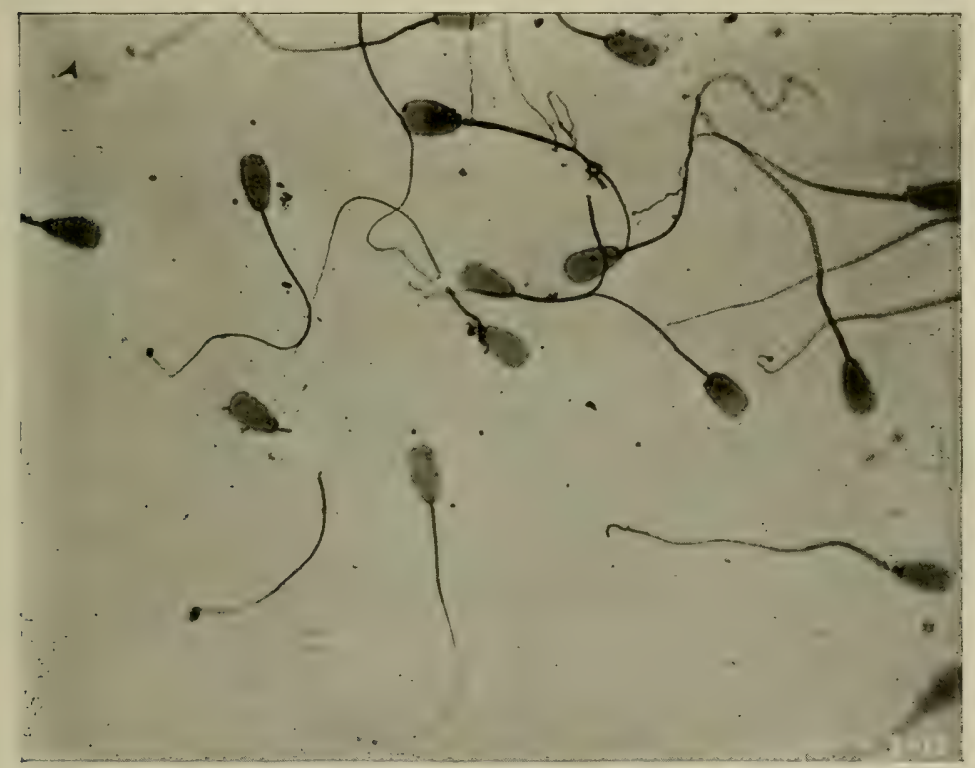

Fits. II7. Spermatozoa from Bull No. 8.

Cells show degeneration, as indicated by want of definition of the nucleus. Some cells are abnormally large.

was narrower than normal and the nucleus constricted, causing a marked decrease, not only in the breadth, but in the total volume of the cell. The component parts were clear in detail, but arrested in development. Streptococcus viridans and staphylococcus albus and aureus appeared in culture from this sample.

Bull 1. Imported. About five years old. Served seven heifers, one or two of them twice, with no conceptions. All seven conceived from first service by another bull. 
Examination revealed granular balanitis. On the left side there was orchitis, epididymitis, and spermatocystitis. Both vesicles were enlarged. So far I have been unable to get a sample of semen from this bull. More recently fluid of lymph-like consistency has been palpated in the left seminal vesicle. (See Figs. 86, 126, 127.)

Bull 7, Fig. 116. Out of a total of sixty-three cows served in the past three years, there have been forty (66 per cent.) conceptions, nine (22.5 per cent.) of which have resulted in abortion. This bull is not the chief sire of the herd. Almost all repetitions of service in the herd are with cows on which this bull has been used. Only two abortions have occurred amongst cows bred to other bulls. Cows bred to other bulls have almost invariably conceived at first service, but repetitions are frequent with Bull 7. The vitality of his progeny is generally below normal.

Physical examination showed all genital parts apparently normal. Coitus was normal. Ten mils. of macroscopically normal semen were obtained. Smears showed cocci which failed to grow in cultures. There were also present large Gram positive rods. Paracolon bacilli were obtained on culture. Motility of spermatozoa normal. The heads were slightly smaller than normal, with a very evident arrest in development, affecting principally the nucleus and causing many of them to appear pear-shaped.

Bull 8, Fig. 117. Two-year-old. Since first used, June 15,1919 , has shown poor fertility. Has served twenty-seven cows, with thirty-six copulations. This resulted in six (23.2 per cent. of cows bred, 16.7 per cent. of copulations) conceptions, none of which has terminated. Physical examination revealed a slight granular balanitis and small vesiculae seminales;-which were apparently slightly arrested in development. Three to five mils. (which is less than normal yield) of normal-appearing semen were obtained on two occasions. Degeneration of the cell was indicated by a lack of definition between the cytoplasmic and nuclear elements. In some cells, the nuclear membrane presented a concavity instead of the normal convexity pro- 
jecting into the cytoplasmic portion. Many of the cells appeared about normal. Some of the cells were too large; they might be termed megalocephalic.

Bull 9, Fig. 118. Seven-year-old. Has served twentyseven cows-twenty-nine recorded copulations with eight (29.6 per cent.) conceptions resulting. Physical examination revealed bilateral seminal vesiculitis. Ten to twelve mils. of semen of normal appearance were obtained. It contained numerous short-chained streptococci on smear. The spermatozoa, all parts of which showed in clear detail, were apparently normal.

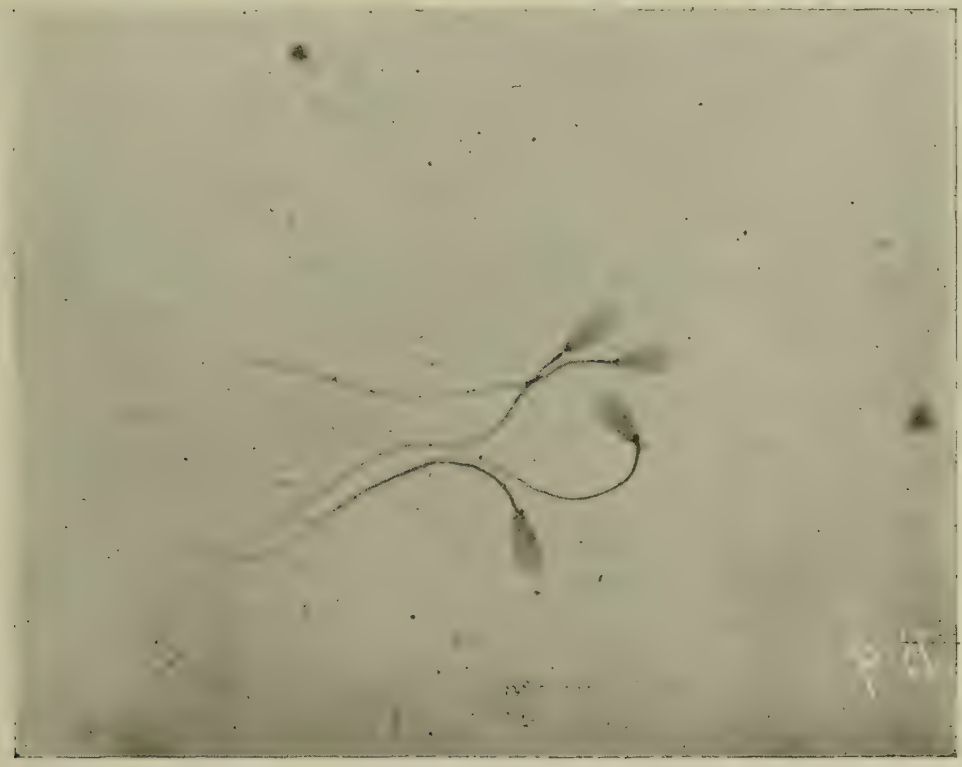

FiG. IIS-Spermatozoa from Bull No. 9.

Cells apparently normal, but semen contained short streptococci.

Bull 10. Three-year-old. Has been in present herd two and one-half years. Total number of cows served, fifty, with ninety-one copulations resulting in thirty-four conceptions in twenty-nine cows (two conceptions each in five cows), of which six aborted. Since October 22, 1919, there have been eleven copulations with eight cows, and no concep- 
tions. Twenty-one of the cows (42 per cent.) served did not conceive. Examination of cows revealed little cause for sterility. Examination of bull revealed presence of seminal vesiculitis on left side. Organs and erection normal but, owing to the custom of placing cows for service with their hind parts raised, half an hour to an hour was often required to serve a cow, leading to an increased strain on the genital apparatus, which may have been a potent factor in

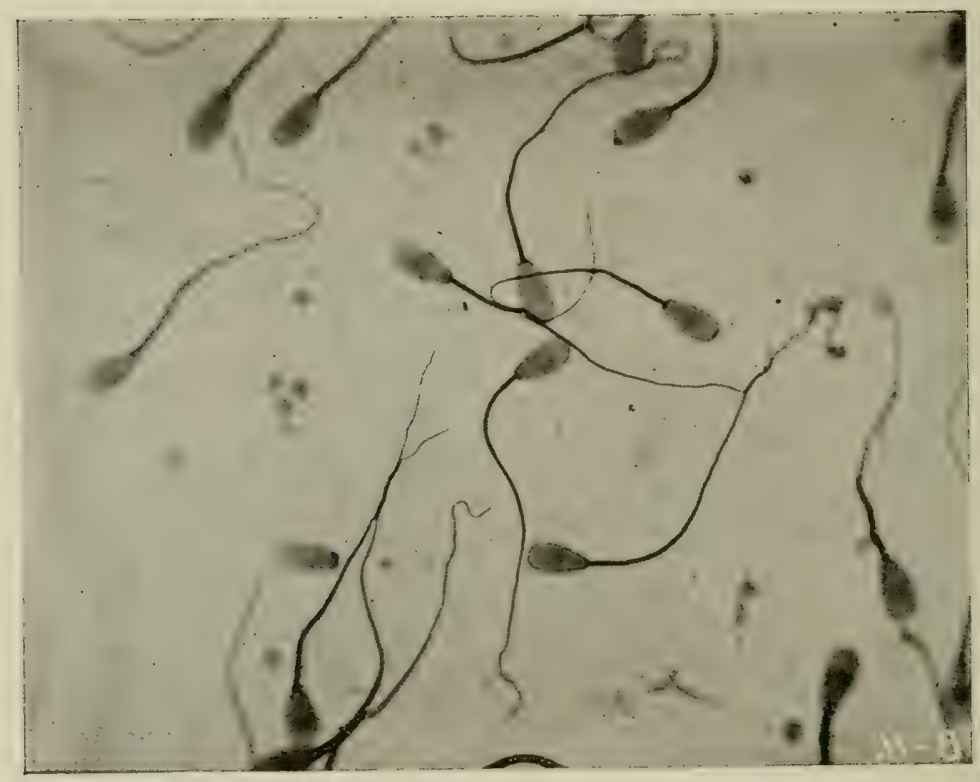

Fig, II9.-Spermatozoa from Bull No. 11.

Cell heads stained poorly, because the nuclear membrane was imperfect. the production of the spermato-cystitis and decreased fertility. Owing to unfavorable conditions for coitus, I was unable to get a sample of semen.

Bull 11, Fig. 119. Four-year-old. Has served sixty-two cows, with a total of one hundred and seven copulations, resulting in twenty-nine conceptions and eleven (37.9 per cent.) abortions. From the first his fertility has been low. Twenty-eight cows served by this bull (45.1 per cent.) subsequently conceived from service by another bull. 
Examination revealed bilateral spermatocystitis, with other portions of the genital apparatus normal. Organs excellent but copulation interfered with by considerable pain on erection, evidently due to the sensitiveness of the seminal vesicles. About ten mils. of apparently normal semen were obtained, containing sperm cells of low motility. Firm coagulum appeared within a few minutes. The head parts did not stain well. The nucleus stained poorly. The nuclear membrane was not well defined.

The inefficiency of Bull 11 was so marked, especially in contrast to other sires used in the herd, that his slaughter was found advisable. Laboratory examination of the organs, including testicles, epididymes and seminal vesicles, revealed no macroscopical lesions. Cultures and smears from these organs were negative. The sterility appeared entirely referable to the changes occurring in the germinal cells. The infection in the female was probably not specific, but merely the result of lowered resistance or a predisposition on the part of the embryo to those organisms of low vitality which ordinarily inhabit the female genital tract.

Sterility of the bull may be directly due to conditions affecting his general health, but is more frequently referable to demonstrable pathologic conditions occurring either in the germinal cells or in the glandular structures of the genital system. These changes may or may not be accompanied by recognizable infection. The diversity of the bacteriological findings of the genital glands and of the semen do not clearly indicate the presence of a specific type of infection. A streptococcus of the viridans type is most frequently found. In the female genital organs of one herd which has been subjected to numerous bacteriological studies, apparently this organism predominates. In this herd the oviducts are most seriously involved. This usually follows cervicitis which yields to treatment, while the tubal disease defies all efforts at control. The bull is apparently an important, if not the chief factor in bringing about these conditions.

It is common to find morphological changes in the sperma- 
tozoa. These occur mainly as abnormal development or arrest in development. Quite frequently I have seen extremely large spermatozoan heads (megalocephalia) which so far as determined are otherwise normal. Abnormally small heads appear with much greater frequency.' - Morphological alteration in any considerable proportion of the spermatozoa definitely decreases the procreative capacity of the bull. .. The germinal cell may also be of low vitality without showing morphological deviations. It is a well demonstrated fact that the vitality of some bulls is so lowered that a healthy vigorous calf is rarely produced. The cause for this may be an inherent weakness, the devitalizing effect of a general constitutional disturbance, or a local inflammation of the germinal epithelium.

My studies of numerous herd sires bring out many interesting problems. Since it has become my regular practice to examine the semen of bulls in herds where there is a high rate of abortion, I have been interested to find that the spermatozoa almost always show marked pathologic changes. I have not as yet been able to correlate such changes with any specific infection. From results thus far obtained, it appears unsafe to conclude that abortions occurring in a herd are due to a specific disease of the female, chiefly or alone, if the sire is not first subjected to a thorough examination.

Although it has been impossible to work out all data on bulls examined up to the present time, the results so far indicate:

(1) that sterility of varying degrees in bulls is very common,

(2) that such sterility is associated commonly either with an arrest in the development of the sperm cell or with disintegration after reaching maturity,

(3) that, as the sterility is accompanied, in a large proportion of the cases so far studied, by spermato-cystitis, with no atrophic orchitis, it appears that the destruction or devitalization of the spermatozoa is due to the toxic substances derived from bacterial infection, 
(4) that such infection is present, not only in the seminal vesicles, but also in the seminiferous tubules and epididymes,

(5) that the determination of the motility of spermatozoa does not constitute a reliable standard for the measurement of their vitality and powers of fecundation,

(6) that some outbreaks at least of the so-called contagious abortion are entirely referable to a lessened vitality of the spermatozoa.

\section{A. Orchitis}

1. Degenerative Orchitis of Calves. Destruction of Spermatogenetic Epithelium in Calves. ARrest in Development of Scrotal Testes.

\section{Desquamative ORChitis}

There occur not infrequently cases of absolute sterility in young bulls, which reveals itself immediately when the animal is put into service. The general condition of the bull may be beyond criticism. He is distinctly masculine in his head, neck, and voice and shows normal sexual desire. He copulates comparatively promptly but, observed closely, is seen to ejaculate slowly and in a manner to raise doubt. After copulation, little or no semen can be recovered from the vagina. Microscopic search shows the total absence of spermatozoa.

Clinical examination of the testicles shows them to be small and flaccid. The two glands are symmetrical and onefourth to three-fourths the normal volume.

Histologically, the epithelium of the tubuli seminiferi has disintegrated and the tubuli contain detritis. Spermatozoa are absent. Ordinarily there remains but a single epithelial layer on the basement membrane of the tubule. A few imperfect spermatozoa or spermatoids may be present in the cellular debris in the lumen of the tubules. The tubules of the epididymis participate in the epithelial destruction.

The degenerative changes are evidently of bacterial origin, but the form of bacteria has not been studied. The destructive changes appear to be quite analogous to those occurring in the oviducts, in which streptococci of the veri- 
dans type predominate. The $B$. abortus of Bang is not known to participate in the destruction.

The clinical diagnosis appears to be simple and clear, but the subject deserves further study. When discussing "Arrests in Development of Scrotal Testes," I have referred to

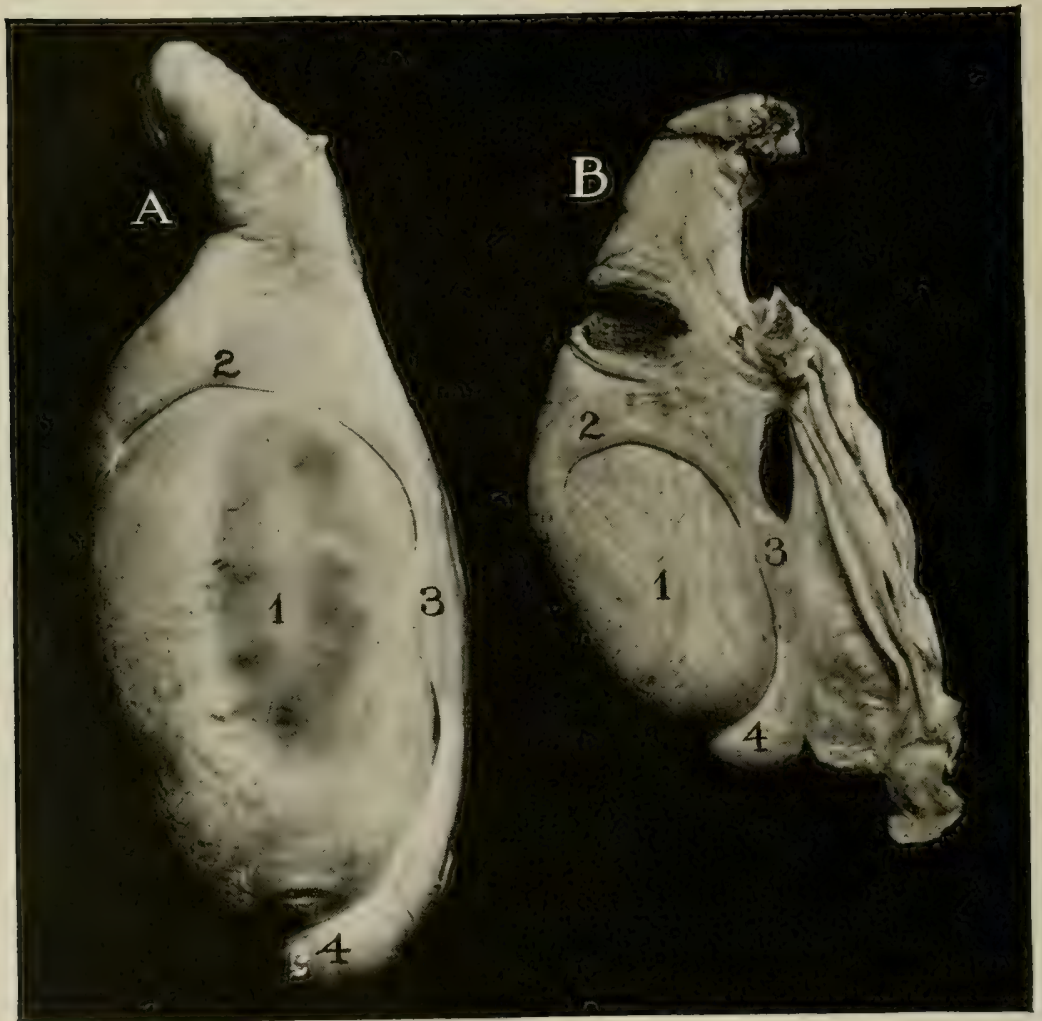

FIG. I2I-Degenerative, or Desquamative Orchitis in Young Bull. $A$, Normal testis of bull; $B$, degenerative testis of sterile bull; 1 , testis; 2 , head of epididymis ; 3 , body of epididymis ; 4 , globus minor.

the clinical characters here described. The glands have much the consistency of cryptorchid testicles, in which the arrest in development is assumed to be purely embryologic, unassociated with infection. Possibly such arrests occur in scrotal testes without infection, but this is improbable. 
Cryptorchid testicles based upon embryologic arrest are almost invariably much smaller in volume than in the condition under discussion. It appears highly probable that in all, or virtually all scrotal testicles of the type described the changes are referable to bacterial invasion. The period of invasion appears to be that of the nursery. At least the era of invasion precedes puberty, so that from the first copula-

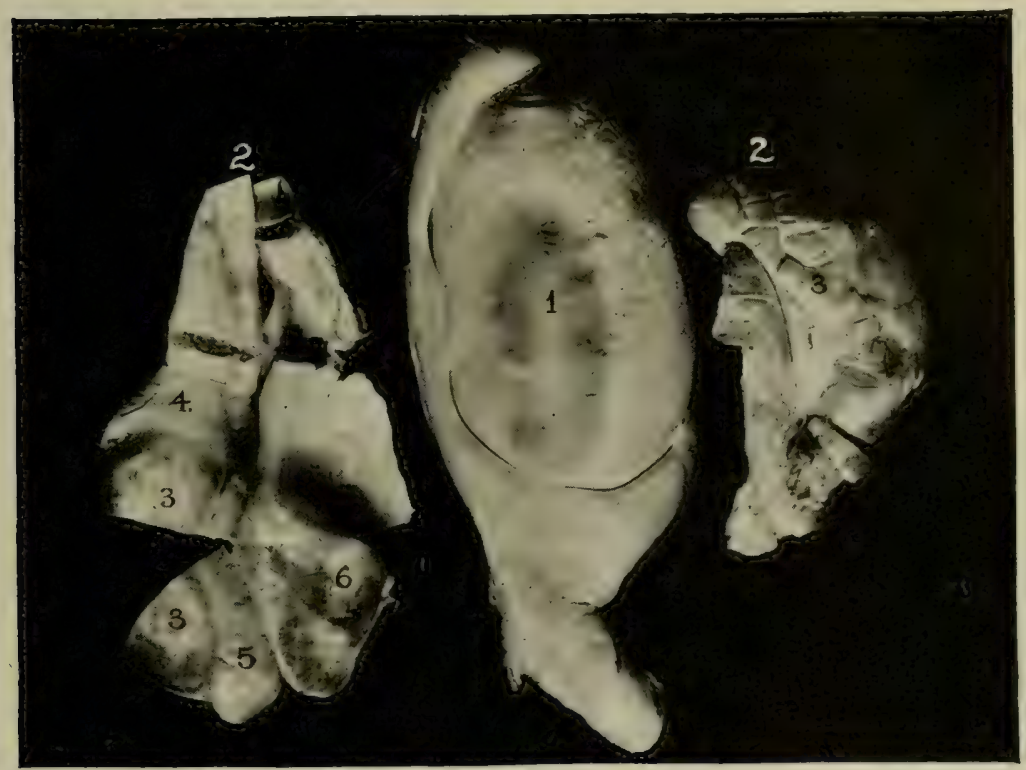

FIG. I22-Degenerative (Atrophic) Orchitis in Young Bull.

$I$, Normal testis ; 2 , atrophic degenerate testes; 3 , body of atrophic gland ; 4, head and, 5, tail of epididymis ; 6, scrotal peritoneum turned back, showing adhesions to testicular peritoneum.

tion the animal is absolutely unfertile. The invasion may be intra-uterine, but this appears doubtful. The bacterial invasion of living bovine fetuses is commonly restricted, so far as determined, to the alimentary tract. Perhaps it would be more accurate to term the presence of bacteria in the fetal alimentary canal as bacterial "inclusion" rather than "invasion." That is, the bacteria from the uterochorionic cavity of the cow invade the amniotic fluid by 
penetrating the chorion and are then swallowed by the embryo in the constant physiologic process by which the fetus swallows its amniotic fluid. When the ingested amniotic fluid is absorbed, the contained bacteria, with cellular debris, remains as a filtrate to constitute the meconium. Normally the bacteria, once imprisoned in the meconium, remain so permanently and are expelled as a component part of it by the new-born calf. Aborts, which commonly suffer from dysentery prior to their death and expulsion, are subject to general bacterial invasion. The heart blood of the fetus may contain the same bacteria as the utero-chorionic space of the mother. This also appears to be true at times of prematurely born calves or of very sick calves born at the completion of the normal duration of pregnancy, but these ordinarily succumb to disease (calf sepsis, calf scours, calf pneumonia). The infection probably occurs most frequently soon after birth, while the digestive or respiratory epithelium is damaged or destroyed as a result of dysentery or pneumonia, or in the presence of a lesser degree of digestive or pulmonary disturbance.

The condition presents an interesting problem in the ethics of the sale of pedigreed bull calves for breeding purposes. I have not known a breeder to recognize the defect prior to sale and believe that he would not ordinarily see it until his attention is attracted to the condition by the sterility. The sale of such animals is, therefore, usually honorable. When the bull fails to breed and the disease is recognized, the breeder is usually equally honorable in making prompt retribution. In one instance, however, where a young bull was sold at a very high, perhaps exorbitant price, the seller refused to make restitution. The case was brought into court. There was no written warranty, but the statutes of the state where the sale was made hold that the purpose for which an article is sold carries with it an implied warranty of fitness for the use for which the article is purchased. The contention of the defendant was that the shipment of the bull from the Atlantic to the Pacific Coast in a common box-car had caused a degeneration of the tes- 
ticles as a result of the vibration of the car. He supported the plea by the evidence of numerous veterinarians with such success as to cause a mis-trial of the case.

The condition, once established, is evidently beyond remedy. If my view of its origin is correct, it can generally, if not always, be prevented by the proper handling of young calves.

\section{ORChitis IN AdUlt Bulls}

I have not observed, and have not found described in adult bulls a degenerative orchitis with desquamation of the spermatogenetic epithelium with flaccidity of the testicle, as described above in bull calves. This is probably, if not certainly, due to the absence of careful study. It would be difficult to assume that bacteria causing the destruction of the spermatogenetic cells in calves may not also invade and cause similar injury to the adult testicles. There would be differences, however, in the results from the invasions at two separate epochs. Doubtless there is a difference of resistance. The essentials are that there shall be a type of organism which may invade the tubuli seminiferi and cause a slow disintegration of their lining cells without arousing the classic signs of inflammation, "heat, pain, redness and swelling," and impotent to cause abscessation or total necrosis.

Clinical data are at hand which warrant the tentative conclusion that such degenerative or desquamative orchitis exists frequently and is serious. Some bulls demonstrate moderate fertility at the commencement of their breeding career, but show upon close study a slowly advancing, insidious sterility. At first the difficulty is ascribed to the cows. The bull appears well, has normal sexual desire, and copulates eagerly. In large herds where several sires are kept, a comparative study of his breeding shows him to be less fertile than other bulls under analogous conditions. Further study reveals the fact that his pregnancies are less secure, so that, while the general rate of observed expulsion of fetal cadavers is, for example, 10 per cent., his rate is 20 
or 25 per cent. In many cases the fertility appears intermittent. For a brief period all copulations fail; then follows a period of fair fertility. If the semen is examined, interesting changes are found in the spermatozoa.

In this inadequately studied field, the best that can at present be done is to study the spermatozoa, their degree of

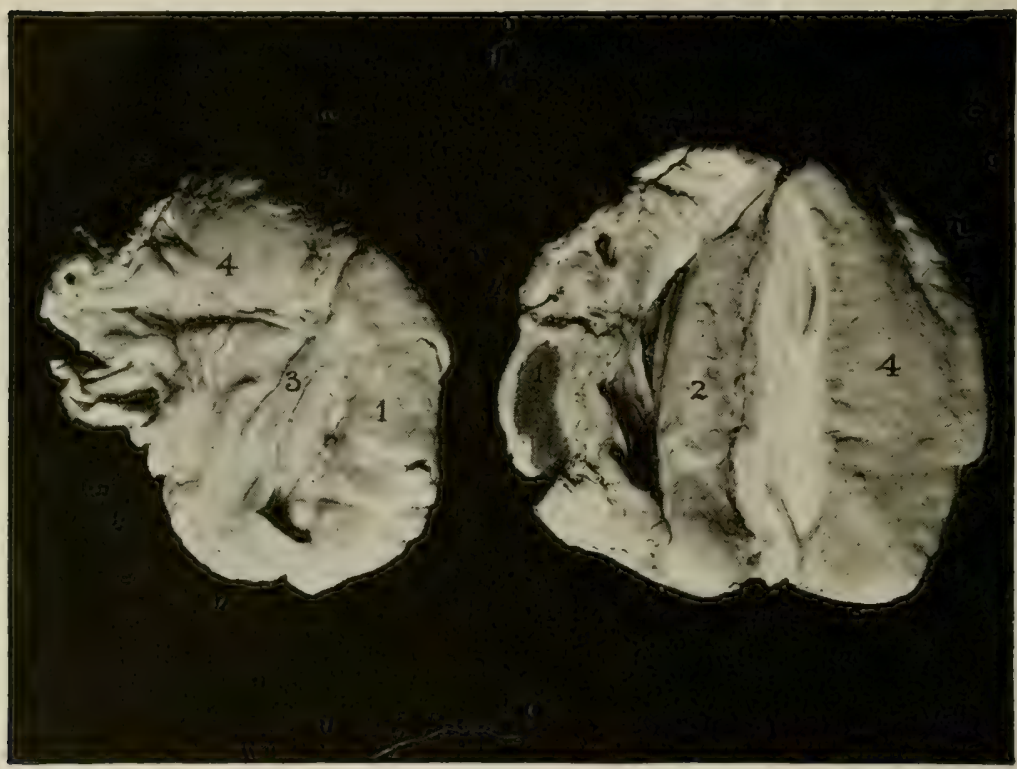

FIG. 123-Purulent Periorchitis (Empyema of Scrotum) with Necrosis and Atrophy of Testes.

$I$, Vestige of testis sectioned, showing on the right remnant of parenchyma ; 2 , epididymis ; 3 , vas deferens ; 4 , scrotal wall turned back. On the right of the right-hand figure the broad white streak between 2 and 4 is a deep incision made to keep specimen open.

the sperm heads. If spermatozoa are abundant but all dead, the tubuli seminiferi must be comparatively sound and death has occurred somewhere in the excretory apparamotility, the frequency of the separation of heads and bodies, and the staining characteristics, size, and form of tus. If the spermatozoa are scant and marked disintegration has occurred, it would appear that the damage may 
have taken place within the tubuli seminiferi. If no spermatozoa are present in the semen, it follows that the excretory ducts are blocked or that the spermatogenetic epithelial cells are destroyed. So far as known, the blocking of the excretory ducts, as in cases of epididymitis or epididymal abscess, is recognizable upon physical examination.

Severe orchitis, not common in bulls, ordinarily terminates in abscessation. Very rarely it appears as an enzoötic.

Wallraff (Repertorium der Tierheilkunde, 1846, p. 219) records an outbreak of purulent orchitis in horses, cattle and goats. The disease had existed in his locality. in Freudenstadt for a number of years. At least it was known in 1832 , fourteen years prior to the date of his contribution. For a year prior to his report, the disease had been very common and caused a very considerable loss among breeding bulls. It involved animals of all ages, attacking many calves when only 10 to 20 days old. The disease began with light fever and loss of appetite. The animals stamped their hind feet as if afflicted with colic. Presently the testicles became more or less swollen, the scrotum red, hot, and painful upon manipulation. This condition continued from three to five days. Then, upon one or both sides of the scrotum, a small opening appeared, from which at first a stinking discharge, mixed with blood, escaped. After one or two days this discharge was superseded by ordinary pus. This continued for five to fourteen days, until the testicle was wholly destroyed. Wallraff uniformly observed the swelling and suppuration in the left testicle first. As a general rule, after one testicle was destroyed, the other also underwent destruction later, though in some cases one of the testicles escaped.

The disease had little danger for the life of the animal. After the destruction of the testicle, the animal recovered without any very great enlargement of the scrotum. In one case, when the abscess in the testicle ruptured, the bull almost bled to death. Wallraff failed to recognize the cause. For a time he thought it was hereditary, and con- 
tinued to believe that it might be so. However, he found that sound males introduced from other sections, where the disease was unknown, contracted the malady, and consequently felt that there must be a local infection of some kind.

While there seemed to be no great danger to the life of the patient, there was very serious loss in condition and also, in all or nearly all the cases, the breeding life of the animal was brought to a close. Consequently, as a rule, when the disease was observed early, the animal was sent to the butcher as the most economic course. Once the disease was established, castration apparently proved the most direct method of handling.

There is no very clear line of demarcation clinically between orchitis and epididymitis. The symptoms are fundamentally alike. The tumefaction of one structure quickly encroaches upon the other and the two organs blend. Commonly also the disease processes in orchitis and epididymitis blend, so that one is rarely present without the other.

Orchitis ordinarily reveals itself by a more or less pronounced swelling of the testicle. As a general rule, the disease is unilateral in the bull, there being no commonly known acute systemic infection which tends to involve simultaneously both the glands, as observed in the infectious cellulitis of horses. When orchitis, due to infection, causes definite changes in the gland itself, they usually occur very promptly, with much swelling and pain. As a rule, there is loss of appetite with elevation of temperature. The very firm envelope or tunic of the testicle renders any inflammation of the gland excessively painful. At the same time it prevents prompt relief by free swelling and tends to cause destruction of the tissues as a consequence of blood stasis from compression.

A common result of orchitis in the bull is the formation of an abscess in which the entire gland, along with the epididymis, undergoes necrosis and purulent destruction. In the one instance of abscessation of the testicle of the bull in the college collection, the abscess has attained a diameter 
of about ten inches. The capsule of the abscess, shown in Fig. 124, consists chiefly of the cremaster muscle and its fascia. The testicle and epididymis have almost completely disappeared, though faint traces of the necrotic gland are found embedded within the pus. A few bands of connective tissue pass across the abscess cavity from wall to wall.

The biology of orchitis in bulls has not been determined. Erhardt (Schweizer Archiv. für Tierheilkunde, Vol. 38, p. 79) records a case of orchitis in a bull due to the vesicular venereal disease of cattle. Other writers also attribute cases of purulent orchitis in the bull to the granular venereal disease, but this view is difficult of verification because the biology of the malady is unknown. So far as known, the $B$. abortus of Bang plays little or no part in the causation of orchitis.

Necessarily the prognosis of orchitis is highly unfavorable. As a general rule, when the infection is so severe as to cause definite clinical symptoms, the organ does not recover its physiologic function. The danger to the life of the animal is not great.

The treatment of orchitis has not been definitely formulated. Theoretically there might be some justification in local applications intended to lessen the inflammation, such as ice-packs or the application of cold water, along with support by means of a suspensorium. Practically I have not known this plan to accomplish any good and I doubt very greatly its efficiency. If, however, one has reason to believe that the orchitis is due to traumatism, the local treatment should be applied and should promise well. I am not certain how frequently traumatic orchitis occurs in domestic animals. Many writers give it a prominent place, though upon what ground is not perfectly clear. Gmelin (Handbuch der Tierärztlichen Chir. u. Geburtshilfe, Bayer und Fröhner, p. 432) states that the commonest cause of orchitis is traumatism, but I have not seen this in any domestic animal save in one case of gunshot wound.

As a general rule, as stated above, the functional life of the gland is at an end. Whenever this may be assumed, the 
most direct and radical method of handling is castration. When only one gland is involved, its removal does not interfere with the animal's fertility and affords the best and promptest remedy that we possess. In castrating such an animal it is to be remembered that the gland is generally adherent to the parietal peritoneum of the scrotum and, through it, to the cremaster and its fascia. Therefore it is more convenient, as well as less dangerous, to castrate by the covered operation. In this operation the skin and dartos are freely incised at the lowest part of the scrotum and a separation made with the handle of the scalpel, or with the fingers, between the dartos and the cremasteric fascia up to the inguinal canal. A ligature is then applied to the spermatic cord covered by the cremaster muscle, and the testicle, with its coverings, cut away an inch or two beyond the point of ligation. The ligature should preferably be of heavy chromatized catgut with a durability of about twenty days.

\section{B. Epididy mitis}

\section{Degenerative Epididymitis of Calves. Arrest in DEVELOPMENT}

In the preceding article upon degenerative orchitis in calves, it has been mentioned that, when that disease occurs, the epididymis ordinarily participates in the pathologic changes. From what I have been able to observe, the epididymitis is probably the more important and primary lesion.

There are no outstanding clinical symptoms beyond those already described under orchitis. So far as has been observed, the calf as a nursling presents no symptoms which attract any attention. This may be due to the fact that, since the testicles of calves are not observed, changes which are perfectly visible may occur without being noted. When the calf comes to breeding age, he is found to be sterile and when examined critically, as already stated under orchitis, the testicles, with the epididymes, are found to be small and 
soft. So far as known, the disease is always bilateral and apparently dates back to infection during the nursing period, as described under orchitis. The condition, which is beyond remedy, can be prevented only by the protection of the young calf against the inroads of infection. This will be more fully discussed under the infections of new-born calves.

\section{Chronic Indurated Epididymal Abscesses of Calves}

I have observed one instance, in a young bull of potentially great value, a long-standing, indurated abscess in the globus minor of each epididymis. The bull, well developed in all respects, was very vigorous and copulated freely, but was absolutely sterile from the beginning. Physical examination revealed a hard enlargement, one inch or more in diameter, in the globus minor of each testicle. Tuberculosis of the epididymis was suspected, but he failed to react to the tuberculin test. One of the glands was then removed for examination. The epididymis was found to contain a small abscess with indurated walls, which had something of the appearance of a tubercular abscess but was without calcification, and no tubercle bacilli could be found. Later the bull was slaughtered and the other testicle was found to be essentially identical.

The diagnosis of chronic indurated abscess of the epididymis is comparatively simple. So far as we know, the abscess is most liable to occur in the globus minor, which in the bull projects beyond the lower end of the testicles on its posterior surface, where it is very readily palpated. If any doubt arises on the part of an inexperienced examiner, the comparison of the diseased epididymis with that of a healthy animal at once reveals the great departure from physiologic condition. Both the enlargement and the hardness are well marked and not readily mistaken for anything else. Should the abscesses occur at other points in the epididymis, they are quickly recognized by the same general symptoms. The head of the epididymis is readily palpated except for the covering of the cremaster, as shown in Fig. 2 on page 5 . 
The body of the epididymis is not so easily palpated, as indicated in Figures 3 and 4 on pages 7 and 8 . If there is any material enlargement, however, it should be detected without great difficulty. When the gland has revolved outward, as shown in the right testicle of Fig. 4, it is brought more readily into view and can be more definitely palpated.

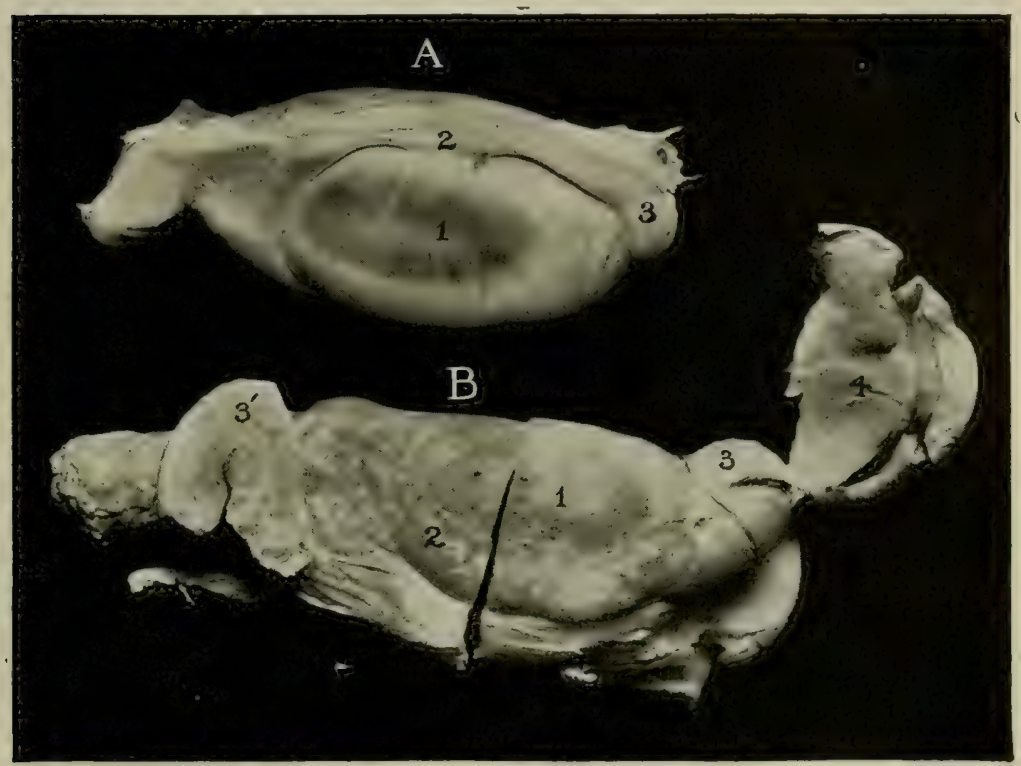

FIG. 125-Chronic Abscessation of Epididymis in Young Bull. $A$, Normal testicle from sound bull; $B$. diseased testicle. $I$, Testicle ; 2 , body of epididymis ; 3 , tail of epididymis greatly enlarged in diseased gland; $3^{\prime}$. section through epididymis, showing pus in the epididymal tubes ; 4 , portion of scrotal wall thrown back showing adhesive bands.

The condition described above appears to be closely related to the degenerative epididymitis already spoken of. It appears quite evident that the abscessation occurred during the nursery period, so that it was well established before the bull had reached breeding age. Schroeder ${ }^{1}$ records an instance of abscessation of the epididymis in which the $B$.

${ }^{1}$ Some Facts about Abortion Disease, Jour. of Ag. Research, Vol 9, I917, p. 15 . 
abortus was recognized. He does not state, however, that no other organisms were present. He reaches the conclusion that the $B$. abortus was the cause of the abscess. This is not very clear. It has not been definitely shown that the $B$. abortus of Bang is pyogenic when injected into the tissues. We do know, however, that it is associated with suppuration in the uterine cavity. Whatever may be the biology of the disease, the prognosis is hopeless.

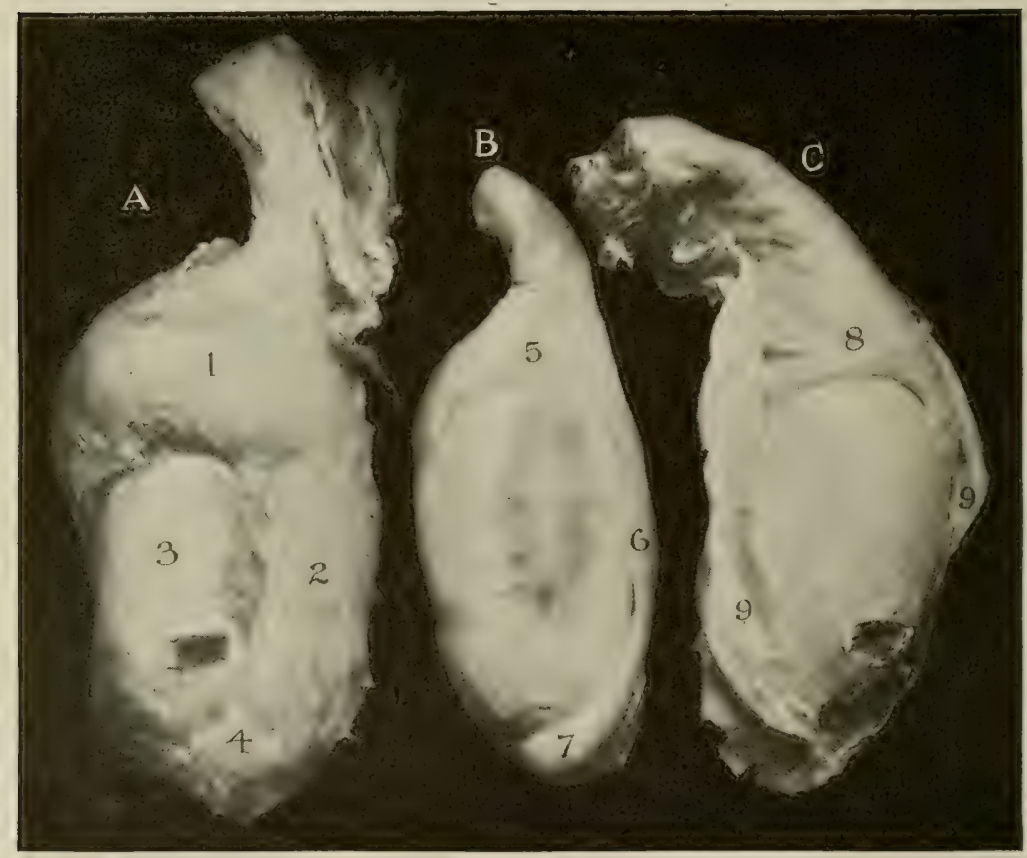

FIG. 126-Orchitis and Epididymitis. Bull.

$A$, Left testis ; $B$, normal left testis for comparison ; $C$, Right testis.

$I$, Globus major of epididymis, greatly enlarged and adherent to testis ; 3,4 , tail of epididymis; 5, 6, 7, normal globus major, body and globus minor of epididymis respectively; $\delta$, inflamed globus major; 9 , parietal scrotal peritoneum adherent to testicle, incised and turned back.

\section{EPIDidy Mitis of Adult Bulls}

Epididymitis develops occasionally in adult bulls which have a history of normal fertility over a somewhat ex- 
tended period. Then without cause there develops, generally very gradually, an increasing degree of infertility. An increasingly large percentage of the cows which he serves fail to conceive. The bull may be apparently vigorous, both physically and sexually, or there may be hesitancy or difficulty in coitus. It is not known, however, that the epididymitis causes this hesitancy or difficulty, which may be due, so far as is now known, to other lesions existing at the same time. If the semen of such a bull is examined, the spermatozoa are found largely or wholly dead. Upon physical examination, one or both epididymes are found to be swollen and tender. As in the indurated abscess of the epididymis in the young animal, so in the adult bull, the inflammation is largely concentrated in the globus minor. The swelling is hard and painful to the touch. The cause of such epididymitis is unknown, beyond the fact that it is evidently due to infection. No studies have been made regarding the character of the infection. It probably differs greatly in different individuals. It is not generally pyogenic, so far as yet determined.

So far as known, there is no hope for recovery and nothing to advise in the way of treatment. It is barely possible that in some cases one might achieve results by the removal of the diseased gland. However, there is the constant danger that the other gland is similarly, although not palpably infected, in which case the removal of one gland would be of no avail. In other cases I have found clinically that the seminal vesicle was at the same time similarly inflamed, so that no good could be expected from removing the diseased epididymis and testicle while the diseased seminal vesicle was allowed to remain. The only hope, therefore, in removing the one gland is that the opposite gland is sound and that there is no disease of the seminal vesicle or other glands about the pelvic urethra.

\section{Infections of the Glands of the Pelvic Urethra}

\section{Spermato-Cystitis. Semino-Vesiculitis}

Semino-vesiculitis, or inflammation of the seminal vesicles, has attracted scant attention in bulls or other breeding 
males. It is therefore impossible at present to make a reliable estimate of the frequency of its occurrence. Enough study has been given the subject recently to show that semino-cystitis is neither rare nor unimportant in breeding bulls.

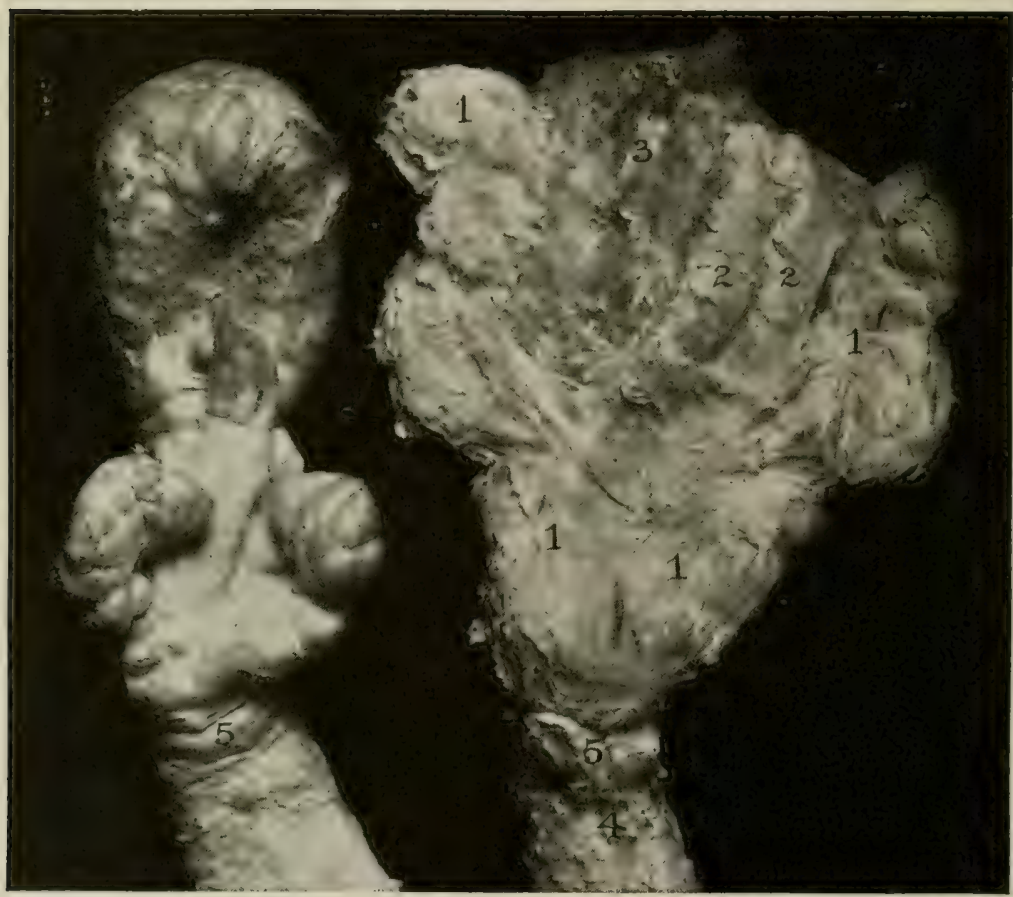

FIG. 127-Purulent Spermato-Vesiculitis. Bull. (From same anima1 as Figs. 126 and 86 ).

On the left is included a normal pelvic urethra, urinary bladder, and seminal bladder for comparison.

$I, I$, Greatly enlarged vesiculae seminales; 2,2 , enlarged terminal portions of vasa deferentia; 3 , urinary bladder; 4 , pelvic urethra covered by

Wilson's muscle and prostate gland; 5 , body of prostate.

The symptoms have not yet been clearly defined. In the cases observed clinically, there was hesitancy in the ejaculation of semen, and a markedly deficient volume of the seminal fluid. Taking into account the present belief in the function of the seminal vesicles, the symptoms thus far 
observed appear logical and inevitable. In ruminants, swine and solipeds, the seminal vesicles are the outstanding subsidiary sex glands connected with the pelvic urethra, and are believed to supply the chief volume of the semen, serving as a diluent in which the spermatozoa, arriving from the testicles, may swim. This facilitates the ejaculation of the highly concentrated mass of spermatozoa. At the same time it is believed that the physiologic secretion from the vesicles stimulates and energizes the spermatozoa, rendering them more active.

In harmony with this view, present studies show that when the vesicles are inflamed, not only is the ejaculation tardy and the volume of semen scant, but the spermatozoa are non-motile or feeble. Spermatozoa taken from the epididymis in a healthy animal are vigorous. Hence it would appear that in semino-vesiculitis the vesicular fluid from the diseased organ devitalizes the spermatozoa.

The clinical examination of the bull reveals, upon rectal palpation, enlargement and irregular, nodular swelling, with extreme sensitiveness. The physiologic gland, as shown in Figs. 5-7, page 11, may be freely manipulated without causing any evidence of pain but, once it is inflamed, the bull immediately winces upon the most moderate pressure. There are few glands or organs in the body which show such marked sensitiveness when inflamed as do the vesiculae seminales.

The clinical history of semino-cystitis is a progressive, or probably sometimes sudden, sterility. Cows fail to conceive uniformly, or there is total sterility. Naturally the cows and heifers are examined, and no explanation for sterility is discovered. Therefore, attention is turned to the bull. In one bull, the vesiculitis was associated with epididymitis, presumably due to a common cause. There appeared to be also some inflammation of the vas deferens. The bull had been imported from England at high cost for breeding some very valuable heifers. Seven were bred without a conception.

It is not improbable that epididymitis and semino-cystitis 
are commonly associated, but whether the epididymitis is the progenitor of the cystitis, or vice versa, there is no evidence to show. Thus far the affection has been chiefly observed in young bulls, but most bulls are young. There seems quite a probability, however, that the infection, like epididymitis, is largely an invasion during the nursery period, and that it lies somewhat dormant until unfavorable hygienic conditions, close confinement, overfeeding, or excessive coitus arouse the infection to destructive virulence. On the other hand, the possibility, if not probability, of acquiring the infection through repeated coitus with highly infected cows, must be frankly acknowledged and the proper hygienic handling of bulls maintained in order to anticipate such disease.

Neglect of the study of the genital infections of bulls renders it impossible to speak with any assurance regarding the bacteriology of semino-vesiculitis. Buck and others, searching the vesiculae seminales, vasa deferentia, epididymes and testes of 37 out of a group of 235 bulls, for the $B$. abortus, recognized that organism in the vesiculae seminales of 4. There appears nothing in their communication to indicate that they were interested in the bacteriology of the organ. Evidently they searched merely for the one organism, ignoring all others. While they recognized the B. abortus in bulls, they have not stated that the cystitis was due to that bacterium. The B. abortus probably plays a negligible part in the causation. Vesicles from bulls, slaughtered because of sterility but not showing evident enlargement, submitted to Carpenter, revealed a streptococcus. It would normally be suspected that the infections of the vesiculae seminales and epididymes would be analogous to those of the oviducts, in which streptococci appear to be the commonest offenders.

The prognosis, from the standpoint of reproduction, is highly unfavorable. It seems improbable at present that, once well established, semino-cystitis can recover, although further observation may modify such a view.

There is no effective method known for handling the dis- 
ease. It is beyond the reach of the surgeon. It is not known that any internal disinfectant can efficiently reach the organ. With the bacteriology virtually undetermined, vaccines, bacterins, and other biologic products can not be advocated. The most that can be suggested thus far is sexual rest and good general hygiene with, as a rule, eventual slaughter.

\section{Diseases of the Prostate and Cowper's Glands}

The pathology of the prostate glands of animals has not been studied except in the dog, where apparently the gland suffers frequently from disease. While the gland is an extensive one in the bull, it is so enveloped by Wilson's muscle that it is not directly palpable. There is nothing in veterinary literature indicating to what, if to any appreciable extent the prostate of cattle becomes diseased.

In the bull Cowper's glands lie deeply buried beneath the ischio-cavernosus muscles, where they are not palpable unless enormously enlarged. I find no record of disease in them.

\section{Balanitis. Balano-Posthitis}

In Chapter XII, under "The Nodular Venereal Disease," balanitis and balano-posthitis have been quite fully discussed as one of the practically universal results of that infection or lesion in ruminants and swine. While other infections (aside from tuberculosis and actinomycosis) may invade these parts, they offer nothing of special interest so far as is known at present. The general principles of control indicated for the nodular venereal disease are applicable to any infections of a general character which may be added to or associated with it. 


\section{GENERAL INFECTIONS OF THE GENITAL ORGANS OF HEIFERS AND COWS}

\section{A. Diseases of the Ovaries. Ovaritis}

Simple ovaritis, or oöphoritis, is not often clearly distinguishable as a clinical entity in animals. In the preceding chapter the invasion of the ovary by such infections as actinomycosis and tuberculosis has been recorded. Rarely also one encounters abscessation of the ovary.

The diseases of the ovaries of cattle (and other animals) have not been scientifically studied. A few very superficial histo-biologic studies have been begun, but abandoned before any material knowledge was gained or any correlation between the findings and clinical observations established. Much has been written concerning the clinical aspects of ovarian diseases. Between these two fields of study are the macroscopical findings upon post-mortem examination. The knowledge of ovarian diseases (and the diseases of other genital organs may well be included in the generalization) is consequently superficial and fragmentary. Therefore any statements, aside from the few clearly demonstrated facts which may be presented, are to be accepted with very liberal reservations and it must be confidently expected that adequate study will modify numerous assumptions and beliefs.

In general it appears that the ovaries of cattle are frequently the habitat of varieties of infection of a type which may exist indefinitely in the gland without causing notable disease but which may, under certain conditions, acquire a force competent to bring about disturbances in structure and function. Bacteria (micrococci, streptococci, etc.) are found in the ovaries of heifer calves and of aged cows, without evidence or suggestion that the infection in the ovary of the aged cow did not acquire its ovarian habitat while the animal was yet in the nursery. Many clinical and postmortem changes are seen in the ovaries, so associated with disturbances of ovarian function as observed clinically that certain conclusions, assumptions, or surmises are justified. 


\section{Atretic Follicles}

There are commonly observed in the ovaries of heifer calves, heifers, and cows numerous small cysts, varying from $1 / 16$ to $1 / 4$ inch in diameter, containing a clear liquid. They appear to be the remnants of follicles in which the primitive or permanent ova have perished and the follicular liquid has persisted, with more or less addition to its volume. I have stated that they grow as large as $1 / 4$ inch in diameter. How much larger they may grow is at present difficult to state. They may grow much larger but, should they do so, become confused with cysts of other origin and lose their identity. That is, when a larger cyst is encountered and neither clinical nor histological evidences of origin are forthcoming, accurate differentiation is impracticable.

The significance of these small cysts is unknown. If they elaborate important secretions (endocrines) or in any other manner modify the sexual functions, or if they affect in any way the reproductive functions, the fact has not been demonstrated. They are quite as numerous in ovaries which function normally as in those which do not.

2. Cystic Degeneration of the Ovisacs. Nymphomania

There occurs in all species of domestic animals, though preëminently in the cow, a type of cystic degeneration of the ovaries accompanied by nymphomania. So far as can be determined at present, the degeneration is one involving the unruptured ovisacs. Apparently this undergoes distension, the follicular liquid becomes enormously increased, the ovum perishes, and there is little tendency for the cystic follicle to rupture and recover spontaneously.

It would appear quite certain that the cystic disease is one of the ovisac itself, because, so far as I have been able to determine, there is never any lutein tissue in the cyst wall. In nearly all adult ovaries there are vestiges of corpora lutea, but in nymphomania they are purely vestigial and are not connected with, or visibly related to the cysts.

I have not known nymphomania to develop in a pregnant animal. There are large and small cysts in the ovaries of 
pregnant cows, but not of the nymphomaniac type. The cysts of nymphomania develop at times when physiologically the animal should ovulate. That is, she has reached breeding age and is not at the time pregnant. No corpora lutea are present to exclude estrum. There are no recognizable lesions in the genital tract or elsewhere which would tend to inhibit estrum, except the nymphomaniac cysts themselves. It appears justifiable, therefore, to believe that the nymphomaniac cyst is a pathologic process occurring in an ovisac which is nearing maturity.

The cysts of nymphomania are exceedingly variable in size. They are rarely less than one inch, and with equal rarity exceed three inches in diameter. When larger cysts appear, like those desiribed on page 257, nymphomania is not present, and as a rule the cyst inhibits fertility upon the involved side only, while the other ovary functions and the animal breeds. When the cyst of nymphomania is present, however, fertility is in abeyance.

The number of nymphomaniac cysts which may simultaneously exist varies from one to three or four. The presence of one nymphomaniac cyst does not prevent, nor tend to prevent, the formation of others. The cysts are multiple. not multilocular. That is, each cyst arises separately, so far as can now be determined, from individual ovisacs, and remains distinct throughout its existence. Hence one ovary may have two or three contiguous, nymphomaniac cysts. The cysts frequently involve both ovaries, although somewhat frequently only one is affected. However, the sterility is as complete with only one ovary involved as when the disease is bilateral.

There is wide variation in the thickness of the cyst walls. In some cases they burst upon very slight digital pressure when being examined per rectum. As a general rule, they require moderate pressure before they are ruptured. In a majority of cases, the cyst wall is so dense that it is unsafe to rupture it by rectal pressure and it requires in some instances all the force the operator can command to rupture it through the vagina. In rare cases, the cysts can not be 
ruptured by manual pressure from the vagina and their contents can be removed only by puncture.

The large nymphomaniac cysts necessarily project far beyond the general surface of the ovary. In very rare cases,

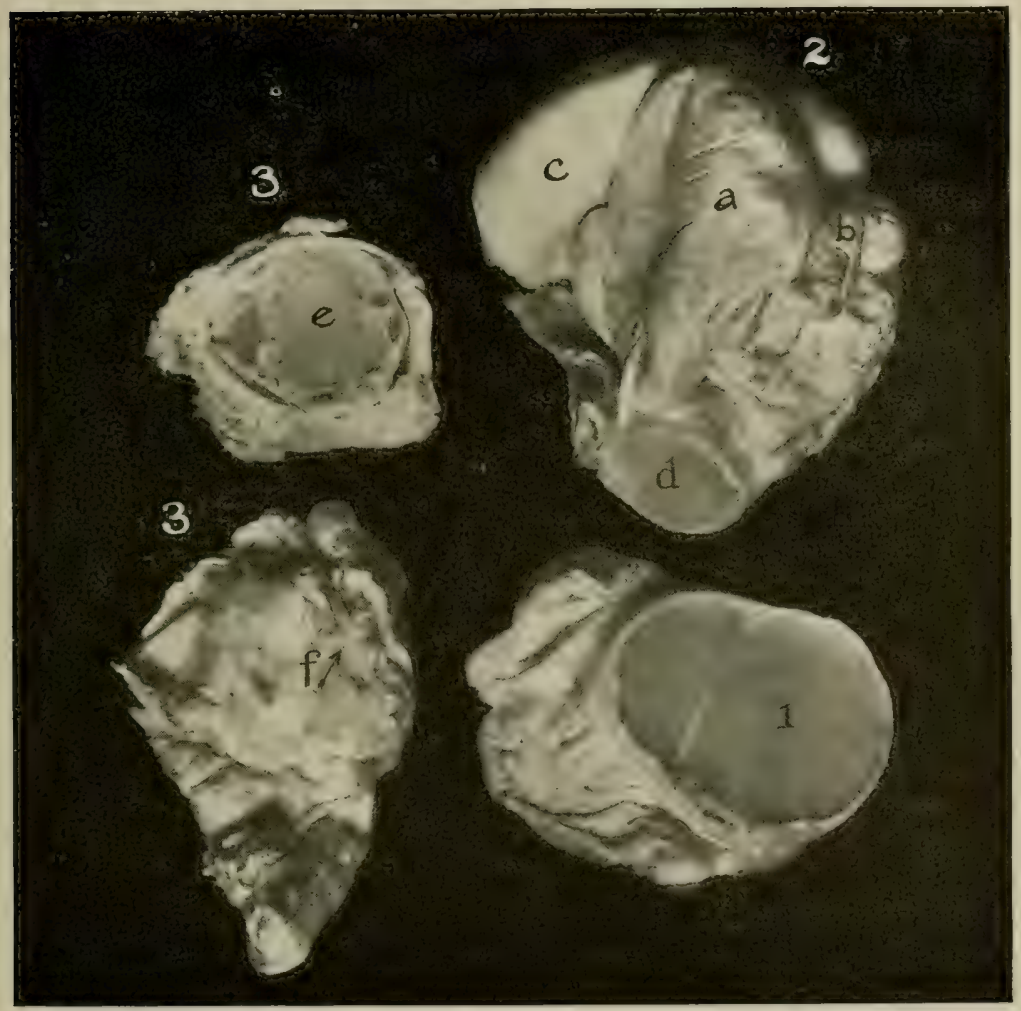

FIG. I28- $T$, Nymphomaniac cysts ; $2,{ }^{\circ}$ cystic oviduct and corpus luteum. $a$, Cystic pavilion; $b$, cystic oviduct ; $c$, cornu; $d$, cystic corpus luteum with faint girdle of lutein tissue; $3, e$, encapsuled corpus luteum ; $f$, ostium abdominale of oviduct.

small nymphomaniac cysts are centrally located, giving to the ovary a somewhat spheroidal or merely plump form.

The symptoms of nymphomania are usually very pronounced and characteristic. The cow or heifer bellows a great deal, more frequently perhaps than when in estrum. 
There is such a distinct modification in the voice that it can scarcely be distinguished from that of a bull. The female appears to be in estrum of an exaggerated type. She will mount other cows whenever opportunity offers and, when free with other cows which are in estrum, she will ordinarily permit them to mount her. As a rule she will copulate at any time with the bull, but there are notable exceptions. Although the animal shows every evidence of an erratic estrum, in some cases she will not copulate. The manifestation of the erratic sex desire is not confined to other cows or to the bull. The nymphomaniac cow may mount any animal, or for that matter man, if he is not on his guard. In some cases, cows kept in stalls with low partitions will mount the partition.

After the disease has progressed for a time, usually not more than a few weeks, notable changes take place in the pelvic ligaments. The postero-superior border of the sacrosciatic ligament, where it passes from the tuberosity of the ischium upward and forward to the sacrum, becomes relaxed, soft and flaccid. The broad expanse of the ligament suffers similarly and sinks into the pelvis, so that the gluteal muscles drop inward toward the median line of the cavity, causing a deep excavation. The relaxation of the ligaments causes marked deformation of the pelvis: the tuberosity of the ischium becomes elevated and the tuberosity of the ilium depressed. The sacrum participates in this change of position. Its caudal end becomes greatly elevated and its lumbar end depressed. The result is a sharp depression at the sacro-lumbar articulation, with a very marked elevation of the caudal end of the sacrum. The interosseous ligaments between the ilium and sacrum share in the relaxation, so that the sacro-ilial articulation is relaxed. This results in an uncertain, rolling or wobbling gait. If one stands near the animal when she is walking, he will now and then hear a loud grating sound caused by the slipping of the ilium upon the sacrum, which may be heard with considerable uniformity if the external tuberosities of the ilium are grasped and the rump of the cow is pushed back and forth. 

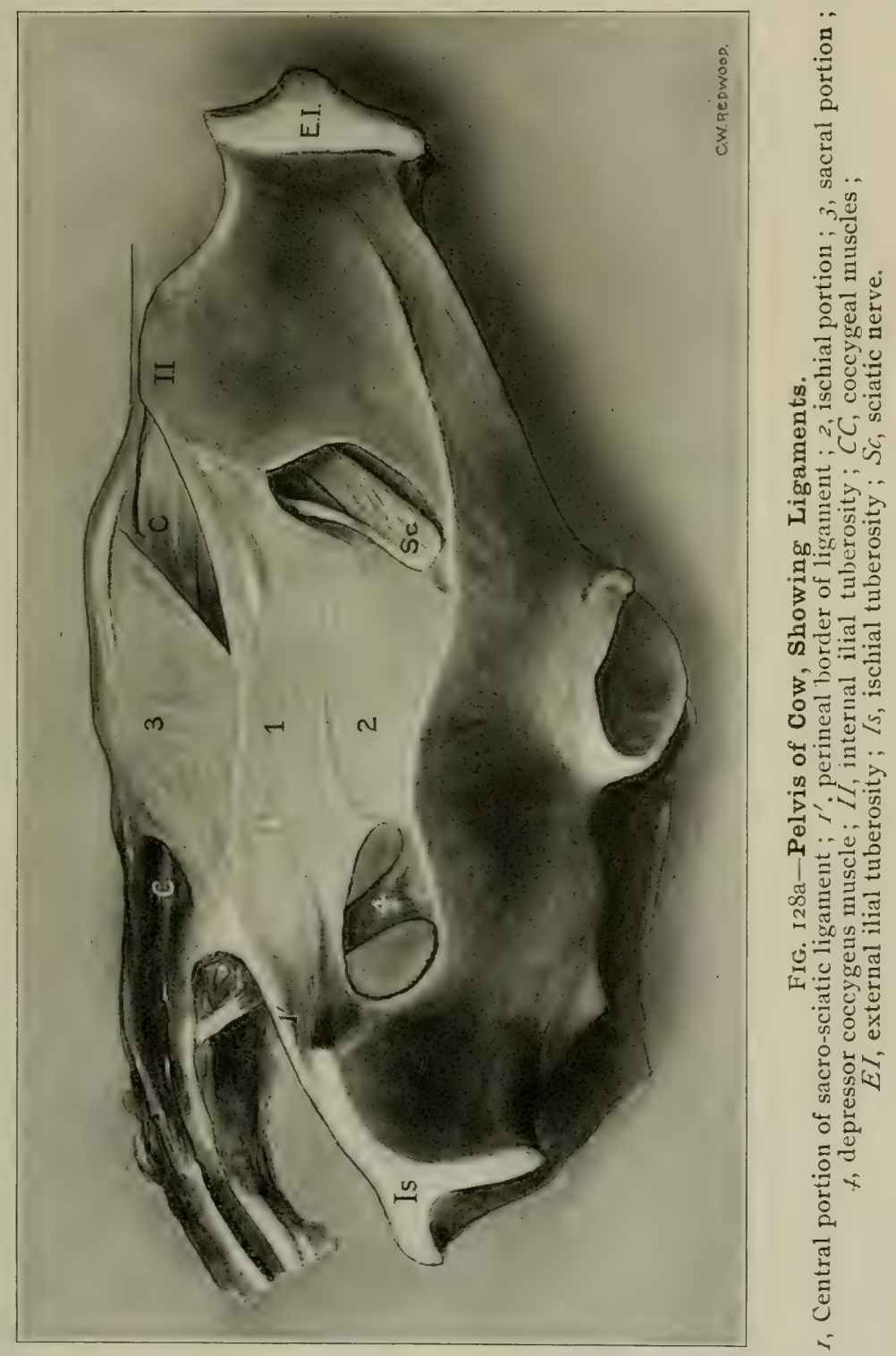
The unsteadiness and inaccuracy of the gait, combined with the frequent mounting of other cows, ultimately brings about fractures of the pelvis in a large percentage of cases. The fracture is usually through the shaft of the ilium.

By studying Fig. 128a, it will be observed that the sacrosciatic ligament $(1,1,3)$ is apparently designed to hold passively in proximity the sacrum, ilium, and ischium. At the same time it affords the chief base of support for the long vastus and gluteal muscles. If, therefore, the ischial tuberosity (Is) moves upward toward the sacrum, the ligament loses its bone-like rigidity and the long vastus and gluteal muscles drop inward, as shown in 2 and 3 of Fig. 129. The relaxation of the sacro-sciatic ligament deprives the long vastus and gluteal muscles of their normal fixed insertion and adds to the uncertainty of the step already mentioned as resulting directly from the relaxation of the ligaments. The anatomo-pathologic mechanism of the deformity is not quite clear. The direction of the pelvis in relation to the spinal axis is chiefly maintained by the sacroiliac ligaments, the psoas group of muscles, and the prepubian tendon (linea alba). When the prepubian tendon gives way, the ischial tuberosity moves upward and the iliac tuberosity downward, but the sacrum moves in harmony and the sacro-sciatic ligament retains its tone and tension; there is no sinking in.

The basic cause of the ligamentous relaxation is yet more difficult to determine. It has been compared with the pelvic relaxation upon the approach of parturition, but it is utterly different. There appears to be some toxic substance elaborated within the diseased ovaries which, acting upon the central nervous system, causes these very remarkable changes.

In spite of good feeding, the cow frequently loses condition rapidly; the hair loses its lustre, and emaciation is very marked. These changes are well portrayed in 2 and 3 of Fig. 129.

The disease occurs but rarely in heifers and is observed chiefly in young cows. It attacks especially highly pedi- 


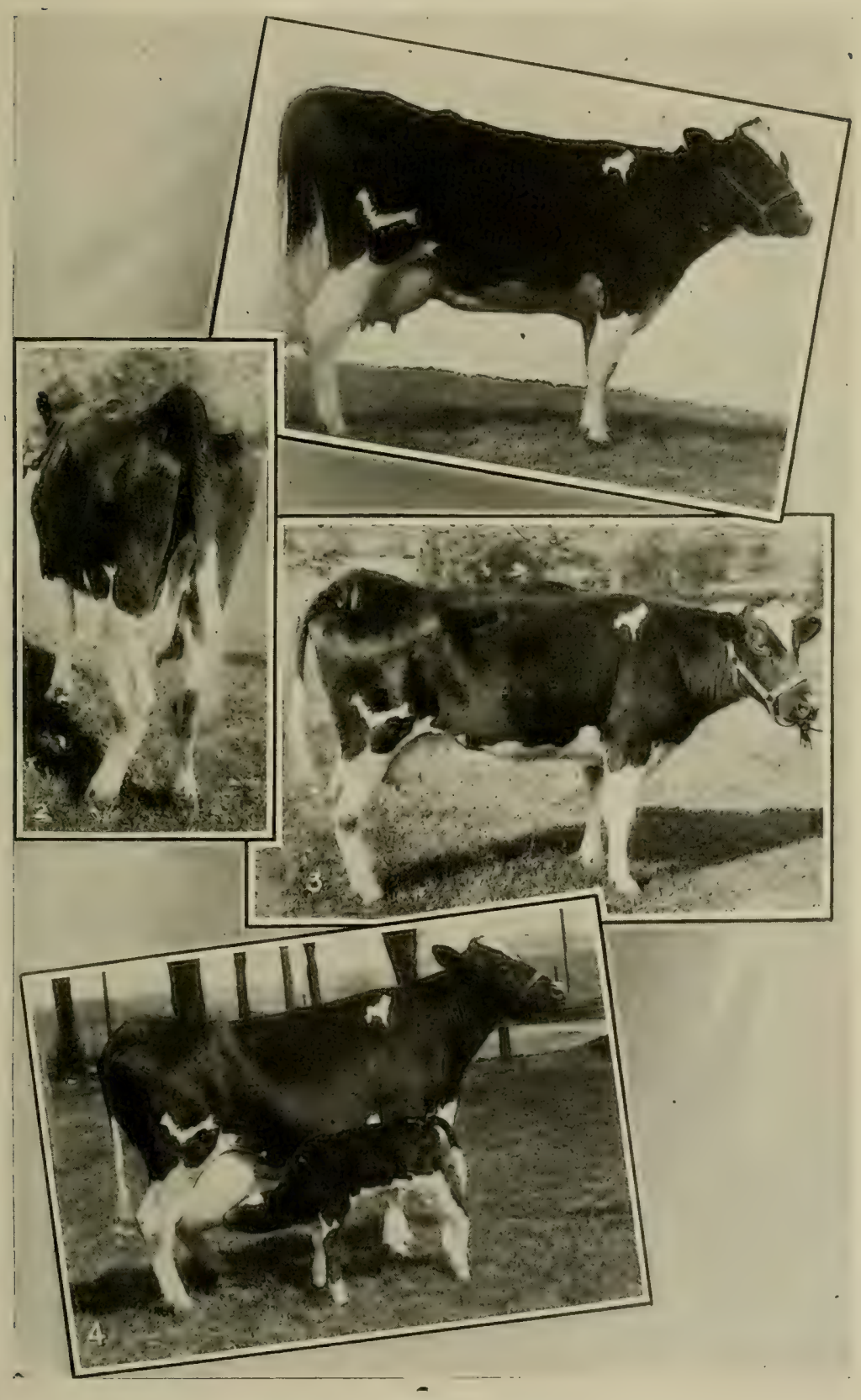

FIG. I29-Nymphomania.

$I$, Cow in health at close of official milk and-butter test ; 2 , rear view after nymphomania became established; 3 , side view, do : 4 , recovery of fertility and health, the pelvic ligaments remaining sunken. 
greed dairy cows which are undergoing severe tests for official records in the production of milk and butter fat. Therefore it is preëminently a disease of the highest class of dairy cows and has closed the breeding careers of many renowned animals. The effect of nymphomania upon lactation is usually very marked; the dairying efficiency is greatly decreased. Generally the milk flow is exceedingly erratic. In some cases a nymphomaniac may yield one day thirty or forty pounds of milk, and the next day, five or ten. One day her milk may contain 8 per cent. or more of butter fat and the next day, 2 or 3 per cent. In such cases there is absolutely no way by which a person may judge from the behavior of the cow one day what it will be the next.

Upon examining the genital tract, the most marked change will be found in the ovaries. Palpation of these reveals one, two, or three cysts in one or both ovaries. No recognizable corpus luteum is present. In very rare cases, as stated above, there may be a small central cyst surrounded everywhere by glandular tissue. The unwary practitioner may fail here in his diagnosis. When the symptoms of nymphomania, as described above, are present, the examiner will do well to search very carefully for a central cyst in case of the absence of the large peripheral type. The presence of the central cyst is to be diagnosed by very careful palpation. The involved ovary, which is abnormally large for the size and breed of the animal, approaches the spheroidal shape. If the ovary is carefully palpated, one gets the sensation of a very thick-walled, tense cyst in its center. The uterus is much enlarged and extremely flaccid. It nearly always contains an increased amount of mucus or muco-pus. This is readily revealed by douching. The cervix is flaccid and atonic. The cervical canal is dilated so that it will ordinarily permit the ready passage of one, and frequently of two or three fingers. The vagina and vulva participate markedly in the general atony of the genital tract. The vulva is large, flabby, and readily dilated. Occasionally prolapse of the cervix through the vulva results from the general atony. In such cases the cervix be- 
comes badly befouled with feces and litter and greatly infected, so that the mucous membrane is much swollen and usually soft.

The affection generally appears without warning. Albrechtsen contends that it is one of the results of metritis or pyometra. This I can not verify. It occurs in heifers which are not known to have been pregnant and in which no history of metritis is obtainable. Possibly it existed unobserved, as metritis so frequently does. I have also seen the disease of a severe type within fifteen days after an apparently normal parturition without clinical manifestations of metritis. I believe it more logical to reverse this belief and attribute the endometritis regularly accompanying nymphomania to the atony due to the ovarian disease. In severe cases the sinking of the pelvic ligaments occurs within a few days after the commencement of the nymphomania; in the milder cases the sinking of the ligaments may not be notable until after several or many weeks have elapsed.

The disease is extremely chronic and shows scant tendency to spontaneous recovery. Just how long it may continue is quite unknown. I have seen cases where the nymphomania has existed constantly for from four to five years. By that time the patience of the breeder generally becomes exhausted and the animal is destroyed. Apparently nymphomania never directly causes the death of the patient but, indirectly, by destroying the vigor and tone, may render the animal an easy prey to intercurrent maladies.

Next to rabies, nymphomania in cows is the most interesting scientifically, and most important economically amongst the diseases of animals characterized by notable mental aberration. It strikes most frequently the greatest dairy cows, and all too often ruins them, thus causing a severe economic loss to the owner and, worst of all, striking down the very cows which, by their potential ability to yield great quantities of milk and butter and to grow progeny equally capable, are of greatest value to the state.

The prognosis is unfavorable. Numerous writers have 
held the opposite view but have failed to substantiate it with extended clinical data. In my experience, less than 50 per cent. recover and many of these quite tardily. My cases have been chiefly of long standing, which depresses the prognosis. In numerous cases, especially when coming under treatment very early, the animals respond immediately and permanently. In other cases, if severe and neglected for a year or more, recovery becomes a matter of great doubt. I have found no criteria, except the duration of the disease prior to handling, upon which to base the prognosis in a given case. Some of my worst cases, like that portrayed in Fig. 129, have recovered their fertility. Hess and others have expressed the dictum that, when the cyst is too dense for manual rupture and can be emptied only by instrumental puncture, the case is hopeless and the animal should be slaughtered. This is not entirely correct, as I have had several cases in very valuable cows which, like Fig. 129, have recovered fertility after I had punctured the cysts once or several times. If a cow is highly valuable and there is present no recognizable lesion, aside from the cysts, which constitutes an absolute bar to fertility, the case should not be pronounced hopeless.

The handling consists chiefly of the evacuation of the cysts as rapidly as they form. Many of them can be ruptured safely and readily by digital compression per rectum. The ovary should be manipulated carefully per rectum until free from any chance covering or entanglement within the mesosalpinx or mesometrium, so that the cyst comes into immediate contact with the peritoneal wall of the rectum. The cyst is then to be grasped between the thumb and two or more fingers, and steady pressure exerted upon it with the ball of the thumb. The thumb pressure should be concentrated upon the center of the cyst, and the hand should encompass only as much of the cyst wall as essential to a secure grasp. That is, the greater the area of the cyst free from compression, and the more concentrated the compression upon a limited area, the more certainly the cyst may be ruptured. I have found it advantageous sometimes to 
place the ovary against the bony wall of the pelvis and then exert pressure against the opposite side with the hand, thus impinging the ovary between the hand and the pelvic bone. The rupture of ovarian cysts per rectum must always be carried out with prudence. The fingernails should be trimmed to the quick and carefully smoothed in order to insure the maximum of safety against injury to the rectum. Pressure must not be exerted upon the cyst when the rectum is contracting. If, during the compression, the patient begins to strain, the pressure must be relaxed and, if necessary, the ovary released in order to avoid rectal laceration or rupture.

Unless the cyst can be ruptured safely and readily per rectum, the effort should be promptly abandoned and the operation carried out per vaginam. The ovary should be picked up per rectum and carried backward over the vaginal roof. The operator then inserts his other hand into the vagina and grasps the cystic gland through the vaginal wall, the ovary being pushed down by the hand in the rectum into the hand in the vagina. It is then grasped per vaginam, while the hand within the rectum lends security to the grasp by continuing a hold upon the mesometrium at its attachment to the ovary and maintains a pressure downward, while the hand in the vagina presses upward. The operator may also with safety avail himself of the assistance of a colleague or a layman in his efforts to rupture the cysts per vaginam. Any intelligent layman can be trusted under proper supervision to exert pressure per vaginam. His nails should be trimmed as noted above, and if his hands are horny from manual labor they should be softened with warm, antiseptic solution. The veterinarian should locate the cystic ovary per rectum. obtain a secure grasp upon it and draw it back over the vagina. The assistant should then insert his hand, well lubricated, into the vagina, with his palm upward. The veterinarian pushes the ovary into his open hand from above and maintains his grasp upon the ovarian attachment. He then pushes downward and steadies the gland while the assistant pushes upward 
against the operator's hand, grasps the ovary, and exerts the degree of pressure required to rupture the cyst. The vaginal wall will withstand securely the maximum digital compression, if prudently applied, which a powerful man can exert.

Sometimes the cyst wall is so dense and resistant that, however powerful the man may be, he can not rupture the cyst by digital compression. If, after a reasonable effort, it is not ruptured, it is best to desist and resort to the easier and safer operation in such cases, of stabbing the cyst and thus evacuating its contents. The operator, if right-handed, inserts his right hand into the rectum, grasps the cystic ovary, and draws it back over the vagina. The left hand is then inserted into the vagina and a secure hold upon the ovary obtained by passing the hand forward with the palm downward, the index finger passing to the left of the gland

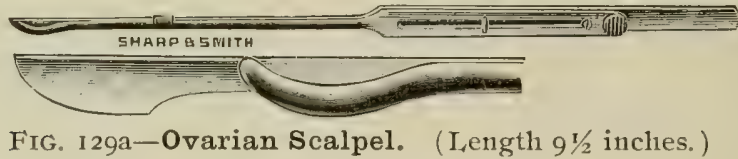

and the thumb to the right. The ovary is then pressed down with the right hand between the thumb and finger of the left hand, and the broad ligament beyond the ovary securely grasped so that the ovary with the cyst rests in the palm of the hand. The right hand is then withdrawn from the rectum and an attendant quickly washes off the feces. The ovarian scalpel (Fig. 129a) is grasped and, guarded, is pushed carefully along the forearm and palm of the left hand until the end of the blade is against the vaginal wall over the center of the cyst. When sure that the end of the scalpel is properly placed and directed toward the center of the cyst, the guard is released and the scalpel given a quick thrust forward through the vaginal wall and the wall of the cyst. The moment the scalpel enters the cyst, the latter collapses. The scalpel is then withdrawn and the pressure continued upon the cyst until its contents are fully evacuated. Care should be taken, when grasping the ovary from 
the vagina, that no viscera and no part of the mesometrium (broad ligament of the uterus) lie between the vagina and the cyst. Since the cyst is regularly upon the anterior or free border of the ovary, the anterior border of the gland should be turned over upward, backward, and then downward so that, when ready for the stab, the anterior or convex border of the ovary shall be directed downward and backward, thus bringing the cyst wall into direct contact with the peritoneal surface of the vaginal roof. The scalpel thus passes through the vaginal roof and the cyst wall only. If the vaginal wall is tensely stretched over the ovary, the stab wound in it is reduced to a minimum, so that, when the cyst collapses and the vaginal wall is released and returns to its normal state, the stab wound is but a fraction of the width of the scalpel blade in extent. If, through error, the mesometrium lies between the cyst and the vaginal wall, the scalpel may wound it, causing an extensive hemorrhage in the broad ligament or in the peritoneal cavity. If a competent assistant is available, it is in better accord with surgical principles for the assistant to grasp the ovary per rectum, carry it back, and pass it to the operator's hand in the vagina, so that the latter may have his other hand unsoiled and ready to use the scalpel. Sometimes this would prove a material advantage because the cow may strain while the hand which has been used in the rectum is being washed, causing the operator's hold to slip. The time lost may consequently bring about failure in a given attempt and necessitate a repetition.

When common cows become nymphomaniac early in lactation, the veterinarian should consider the prudence of handling by castration. The removal of the ovaries stops the nymphomania, stabilizes lactation, and tends to cause the animal to fatten rapidly as the lactation period nears its close, enabling the dairyman to dispose of the animal profitably for beef. When an animal is dry or lactation unprofitable and the patient has become extremely emaciated, as indicated in Fig. 129, the veterinarian should be cautious about advising ovariotomy. The operation can not succeed 
economically unless the owner has at hand an abundance of good food of low cost. Thus an emaciated nymphomaniac might be profitably spayed in the spring when an abundance of cheap pasturage is available, but spaying in the autumn and attempting to fatten upon expensive grain might prove a serious economic blunder. The operation of spaying has already been described in Chapter XI.

When recovery occurs, the nymphomania abates, the general condition improves, the hair recovers its lustre, and the deformation of the pelvis becomes lessened or disappears. In mild or recent cases, recovery of fertility is signalized by the return of the pelvis to its normal form. Long-standing, severe cases do not recover wholly from the pelvic deformity, but do improve in form with the restoration of fertility. This is well shown in Fig. 129, where the cow is shown in health in 1 , the disease at its maximum in 2 and 3 , and the partly recovered form of the pelvis in 4 . The impression given by the figure in 4 may be somewhat exaggerated because she had just calved, at which period there is regularly some relaxation of the pelvic ligaments, but the photograph is essentially true to clinical observation.

A nymphomaniac should on no account be permitted to copulate, since this definitely intensifies the malady. Neither should she be permitted to consort with other cattle because, in repeatedly mounting or attempting to mount them, she is very liable to injure herself (fractures of pelvis, etc.) or other animals. She is a great annoyance in a herd of dairy cows, constantly disturbing them, interfering with their feeding, and hence with the milk yield.

When the cow improves and apparently recovers, care should be taken in permitting copulation. When the nymphomania ceases and the general condition improves, she should not be bred during the first appearance of estrum, as this may be false and a mere recrudescence of the nymphomania. Even if it is due to the ripening of an ovisac, copulation should not be permitted. The breeder should wait until the advent of a second normal estrum. The two periods have a normal interval of 20 to 22 days. 
So long as the estrual period is irregular, she should not be bred. Until the recovery is complete, copulation generally precipitates the return of the nymphomania in a severe form, with a copious discharge of muco-pus from the vulva. If regular and normal in estrum and in the length of the inter-estrual period, the cow may be bred at the second estrum and then watched closely to see that all is going well.

The treatment of nymphomania through the internal administration of therapeutic agents has not been given sufficient study to afford ground for the expression of any opinion. Upon the hypothesis that the disease is due to infection within the ovary, it would seem not improbable that the internal administration of powerful disinfecting agents might bring about relief from the disease. I have used salvarsan to a very limited extent. Apparently it brought about some amelioration of the disease, but the experiments were brought to a close on account of the prohibitive expense of the drug. In a very few cases the intravenous administration of trypan blue seemed to benefit the animals greatly; in other instances no apparent benefit was derived from it. Quite as interesting from the standpoint of research with nymphomania, is the use of corpus luteum or ovarian extract, but so far as I know, this field of experimentation has not been extensively invaded. I have used liberal amounts of corpus luteum extract upon two nymphomaniacs, without visible effect. It is possible that extracts of other ductless glands might affect this disease profoundly.

\section{INTRA-FOLLICULAR HEMORRHAGE}

I have in my collection the genital tract from an abattoir heifer, illustrated in Fig. 130, in which the right ovary contains a number of large cysts which have increased its diameter to four inches and its weight to about two pounds. Some of the cysts contain a clear lymph. Others contain blood and lymph mixed in varying proportions, some of them being filled almost entirely with blood. No lutein tissue is recognizable. I have not recognized the condition 
clinically. As I have no clinical history of the animal, the significance of the condition is uncertain. The genital organs are those of a heifer which has not been pregnant. The specimen suggests, as do many ovarian diseases, caution upon the part of the veterinarian. In the handling of cows for sterility, as in most new fields, arbitary practices have developed. One of the outstandingly dangerous fads in sterility of cattle is the massaging of the ovaries and uterus per rectum, the rupturing of cysts, and the dislodgment of corpora lutea-a massaging and crushing program. Although the healthy genital organs of cows will endure

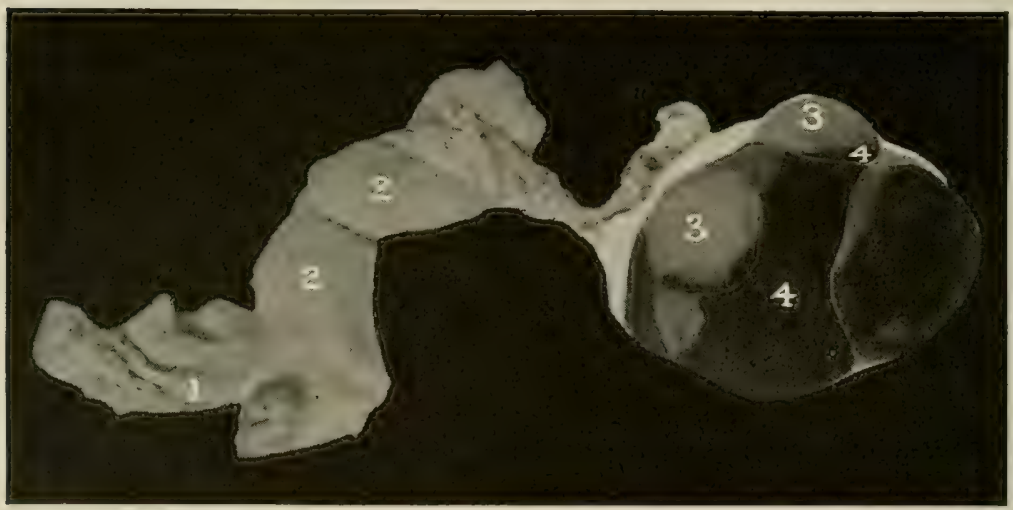

FIG. I30-Intra-Follicular Hemorrhage. Abattoir heifer.

$I$, Right ovary; 2,2 , uterine cornua ; 3 , gelatinized cystic fluid; 4 , hemorrbage into cystic fluid.

without great injury a remarkable amount of insult, their limit of endurance is promptly exceeded in many cases of disease. Any cyst of extraordinary volume or presenting other unusual features, or any other lesion, the nature of which is not clear, calls for careful consideration and forbids hasty action. A rupture of the cysts illustrated would in all probability have led to fatal hemorrhage. Fig. 67 illustrates further this important point. In this animal, where clinical history was available, spontaneous rupture of the ovary occurred, with profuse hemorrhage. Had an imprudent veterinarian "ruptured the cyst" in that case, 
death from hemorrhage would have been almost certain and the over-rash operator would have received severe and well merited censure. Unusual cysts or other enlargements or lesions should always be handled with extreme caution, and no irretraceable step made until a reliable diagnosis has been reached. It is well in doubtful cases of cysts or tumors always to palpate the ovarian artery carefully. It may be enlarged, which clearly forbids any form of procedure inviting hemorrhage unless the operation provides for absolute control of it.

\section{Post-Ovulation Hemorrhage into the Follicular Crater}

Immediately after ovulation there is physiologically a slight hemorrhage into the crater of the ruptured ovisac, to form a very small hematoma which occupies for a brief time the center of the corpus luteum and then disappears, as indicated in Fig. 26.

Pathologically there occurs not infrequently an amount of hemorrhage into the ovulations crater far in excess of the physiologic volume. Clinically I have encountered extensive blood clots marking the site of the crater, which are readily detached so that they drop freely into the peritoneal cavity. In one irstance the hematoma was more than 3 inches in diameter. Presumably considerable blood had escaped into the peritoneal cavity in addition to that which remained attached to the ovary as a hematoma. The cause is not definitely known. Apparently it is due to a disturbance of the circulation dependent upon general atony of the ovary. Just how serious it may prove at times is unknown. Now and then a cow appears quite unwell at the close of her estrual period. It is not improbable that hemorrhage from the follicular crater may at times be responsible for the symptoms. It is a well-known fact that in rare cases severe and even fatal hemorrhage follows the dislodgment of a corpus luteum. The two occurrences may be similar in their fundamental character. The clot, which is fairly firm, breaks up under digital compression with a characteristic grating. 
When the hematoma is discovered during the examination of the genital organs, it is doubtful just what course is best to pursue. I have followed the plan of dislodging it and turning it free into the peritoneal cavity, but have always taken the precaution of applying digital compression to the ovary for some minutes after the dislodgment of the hematoma, and watching very closely to see that the hemorrhage did not recur. This appears to me to be the better course because, unless the hematoma is dislodged, so far as I am able to determine, it will result in a hemorrhagic corpus luteum, which will undergo cystic degeneration and will inhibit estrum for a long period of time.

The cause of this excessive hemorrhage is undetermined, but it is apparently due to the presence of an infection in the ovary which has depressed the tonicity of the blood vessels, causing them to bleed freely upon the slightest interference. So far as I have observed, the prognosis is good.

\section{Hemorrhagic CoRpus Luteum}

The excessive hemorrhage into the crater of the ruptured ovisac, described in the preceding paragraph, when left undisturbed, regularly leads to a hemorrhagic corpus luteum, so far as I am able to determine. Eventually, also, the hemorrhagic corpus luteum generally, if not always, results in cystic degeneration. The lutein tissue itself, under these conditions, ordinarily acquires its normal volume, or may exceed it somewhat. Accordingly the blood clot adds to the volume of the corpus luteum, so that in numerous instances it reaches, as palpated per rectum, a diameter of one to two or more inches (Hypertrophied Corpus Luteum). Between the physiologic blood clot in the corpus luteum and the maximum amount of blood described, there is every possible gradation in amount.

Clinically it is impossible to diagnose directly the hemorrhagic corpus luteum. A tentative diagnosis may be made, based upon the increased size of the corpus luteum. The hemorrhagic corpus luteum tends to persist for an indefinite period, inhibiting estrum until it finally disappears by cystic 
degeneration. Its precise nature has not been made clear. The cystic corpus luteum is so generally associated with infection as to suggest that the hemorrhage causing this pathologic type of corpus luteum may be due to atony in the gland, referable to infection. At present it seems that as a rule of practice it is best to dislodge such corpora lutea in order that their inhibitory power may be destroyed and the ovaries caused to resume their function. The veterinarian should always be very careful, when dislodging such a corpus luteum, to compress the crater for some minutes and watch carefully for a brief period afterward to see that a new hemorrhage is not established. At the same time due attention should be given to other conditions existing within

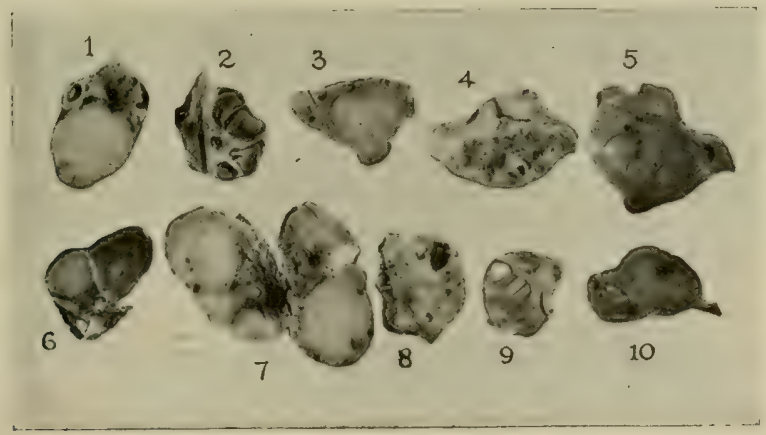

FIG. r3I-Hemorrhagic Corpus Luteum.

the genital tract, because there is ordinarily a correlation of these diseases and no one lesion should be treated as being wholly independent of other pathologic conditions which may be present.

\section{Cystic Degeneration of the Corpus Luteum}

Probably the commonest disease of the ovary observed in cattle is the cystic degeneration of the corpus luteum. At the same time, although the condition has not been thoroughly studied, it is quite safe to say that it is one of the most serious types of ovarian infection seen. Cystic degeneration of the corpus luteum occurs very rarely in a 
pregnant cow, and when it does occur the cyst is usually very small. It is a very common condition in sterile cows and is almost universal in complete adhesion of the ovary within the pavilion of the oviduct or within the ovarian pocket in the broad ligament.

In the abattoir one encounters every possible degree of cystic degeneration of the corpus luteum. At first there is frequently a small cyst not much larger than an ordinary pinhead. On the other hand, there occur cystic corpora lutea in which the cysts are two to three inches or more in diameter. Frequently the cystic degeneration destroys the lutein tissue until there is merely a yellow band about the periphery of the cyst. Sometimes the lutein tissue disappears from all of the wall except a very small area. In other cases, where the corpus luteum has been hemorrhagic, there will be seen at the periphery of the cyst at one point a trace of lutein tissue and at another a trace of the old hematoma. Finally all traces of lutein tissue or hemorrhage disappear and there is left behind a cyst that cannot be differentiated structurally from that of nymphomania, though it does not cause that disease.

When examining a sterile cow clinically, one may recognize the cystic character if the cyst occupies one-third to one-half the volume of the corpus luteum. If pressure is applied to the corpus luteum, the cyst ruptures and the fluid escapes, after which, if the digital compression is continued, the lutein mass itself is pressed out and the cavity feels like an empty bag. When the cystic degeneration has extended to an extreme degree, the examiner merely recognizes the cyst. It should be distinguished clinically from the nymphomaniac cyst, because of the nymphomania itself. It is further distinguishable in many cases by the fact that, after rupturing the cyst and then applying digital compression to the crater, a small amount of hard tissue is detached and forced out which is recognizable as the peripheral portion of the corpus luteum. How large these cysts may grow is wholly unknown. In discussing benign tumors in Chapter XI, there have been included a number of large ovarian 
cysts which have their principal danger for the animal in their mechanical interference with other viscera. Their origin is unknown. It is not improbable that a considerable proportion of them have their origin in the cystic degeneration of the corpus luteum.

The origin of the cystic corpus luteum has not been extensively studied. Judging from clinical and post-mortem studies, it appears quite justifiable to say that the disease is referable to an infection which enters the crater of the freshly ruptured ovisac from the oviduct. Hence the cystic corpus luteum is seen with great frequency in instances of

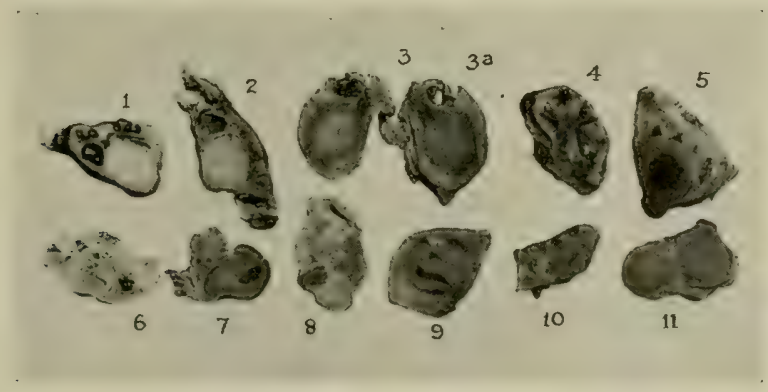

FIG. 132-Cystic Corpora Lutea.

$I, 2$, Twin corpora lutea of pregnancy; $3,3 a$, 4,5 , cystic corpora lutea.

chronic salpingitis. In fact a large percentage of cows and heifers persistently sterile because of salpingitis suffer simultaneously from cystic degeneration of the corpus luteum. When the ovary becomes adherent within the pavilion of the tube, cystic degeneration of the corpus luteum is essentially constant.

The bacteriology of the cystic degeneration of the corpus luteum has been but scantily studied. Thus far the studies have revealed chiefly the presence of a streptococcus of the viridans group.

In many sterile animals, the cystic degeneration of the corpus luteum advances somewhat rapidly, causing no material enlargement of the corpus luteum but gradually destroying the lutein tissue, until finally it no longer inhibits 


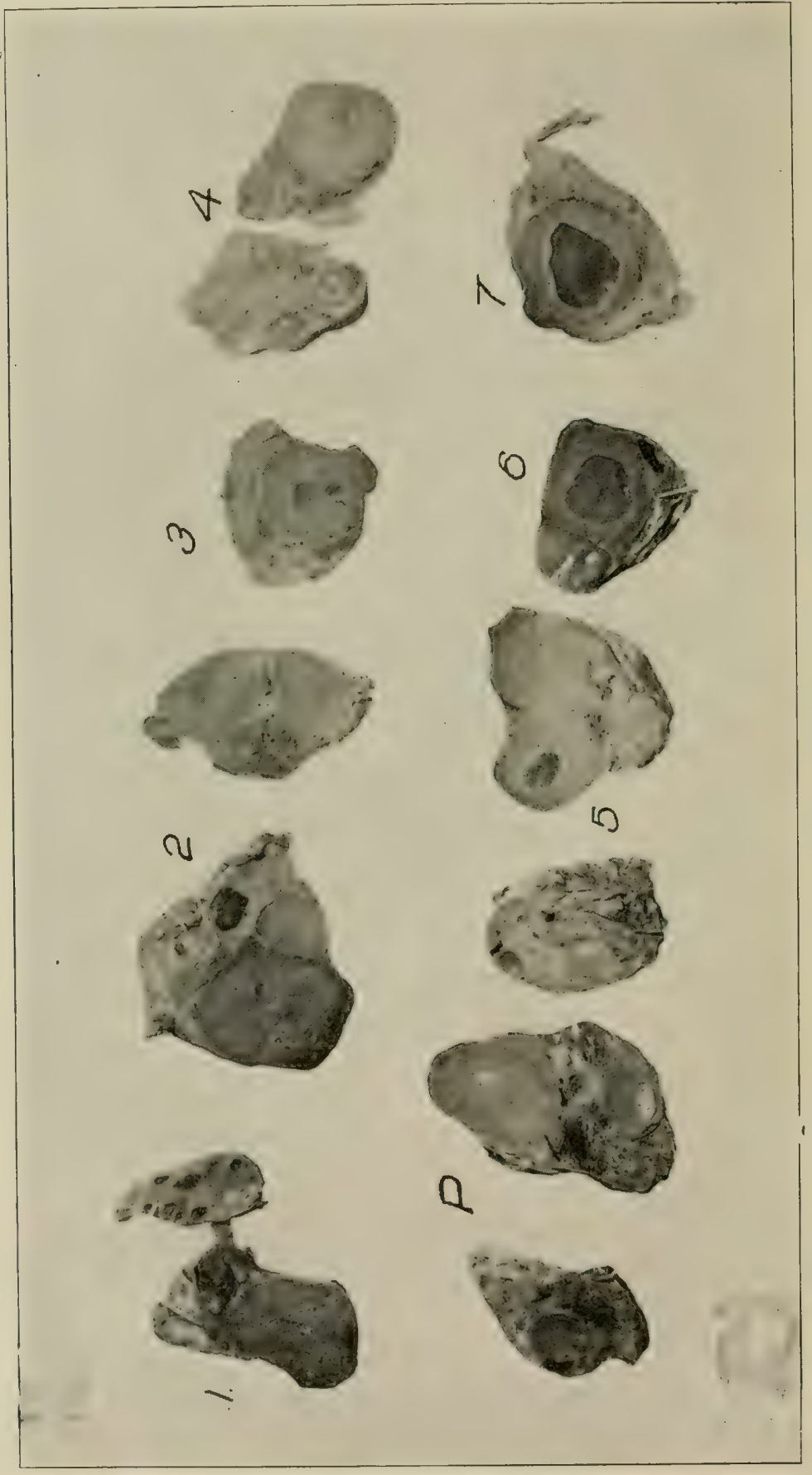

过

풀

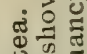

+ .

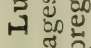

ब $\frac{\pi}{2}$

के

믈

ชั่ำ

诺

궁

ט。

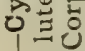

至凯

نे

I

剀

o

吾

है

8

i

6

in

$x$

is

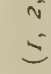


ovulation. Sometimes ovulation and estrum are regular and the duration of the disease is approximately the same as in the normal cycle of estrum. In many cases, however, cystic degeneration interrupts the normal estrual cycle so that, if the degeneration is very rapid, the animal may be in estrum again in eight to fifteen days, or it may be delayed until thirty days or more. So far as I have been able to judge by clinical study, it is the principal cause of irregularity in the estrual cycle. Not only does it interrupt the estrual cycle in the non-pregnant animal, but sometimes it involves the pregnant animal, bringing about a recurrence of estrum in spite of the presence of a normally developing fetus.

The prognosis of cystic degeneration of the corpus luteum is unfavorable because, so far as at present understood, it is largely dependent upon an irremediable infection in the oviduct. If it involves only one ovary and oviduct, the prognosis may be good because of the freedom of the other side, but unfortunately cystic degeneration of the corpus luteum and tubal infection are usually bilateral. Some cases recover, but when it is once clearly recognizable one needs to give a very guarded or unfavorable prognosis.

There is no well-established method for handling cystic degeneration of the corpus luteum which promises favorable results. It has been the habit with many, especially in the beginning of the work with sterility in cattle, to dislodge the cystic corpus luteum, but when one views the question from the standpoint of the etiology as interpreted above, it would appear that tampering with such a condition is not usually justifiable. If one dislodges the corpus luteum, there is a new wound which offers an excellent avenue for invasion from the oviduct. It may be thoroughly justifiable to dislodge the corpus luteum, or at least to compress it sufficiently to rupture the cyst in its center in order to make a diagnosis. When the diagnosis has been made, however, and the veterinarian is aware that the difficulty is due to this cystic degeneration and understands that it is almost certainly accompanied by salpingitis, the more prudent course in the 
present state of our knowledge is to let the body alone, unless it is greatly prolonging the estrual period, and to hope that there may be a spontaneous control of the condition, possibly assisted by giving careful attention to the cervix and uterus if they are participating in the general infection present.

\section{HyPERTROPHY OF THE CORPUS LUTEUM}

Much has appeared in the writings of some veterinarians regarding hypertrophy of the corpus luteum in cattle. They have not as a rule defined precisely what is meant by the term. In examining thousands of cattle in the abattoir, I have observed but one true case of hypertrophy of the corpus luteum, and in that case the body was black instead of yellow and the cow was pregnant. This enlarged corpus luteum is shown in Fig. 131.

There is another type of corpus luteum, however, which has been described in the two preceding paragraphs as hemorrhagic corpus luteum and cystic degeneration of the corpus luteum, in which the gross volume of the body may attain an indefinite size. I have not been able to observe that the actual volume of true lutein tissue has been materially increased. It is impossible, however, to differentiate clinically between a true increase of lutein tissue and an increase in the size of the corpus luteum because of the presence of an old, hard blood clot of such consistence that it cannot be distinguished from lutein tissue. It is well then to retain the designation "hypertrophy of the corpus luteum," as a clinical phenomenon, whether it is due to the addition of a blood clot in the lutein tissue or to cystic degeneration in the center. When such a corpus luteum is present, it is almost invariably associated with sterility and inhibits estrum for an indefinite period. Upon clinical examination the operator recognizes in one of the ovaries an abnormally large corpus luteum. In many of these cases, if he will palpate very carefully, cystic degeneration may be recognized, although not yet far advanced. Such corpora lutea attain a diameter of one to two and one-half inches or 
even somewhat greater. When the corpus luteum contains a blood clot, it always, so far as I have seen, ultimately undergoes cystic degeneration, but the degeneration may be very slow and the presence of the corpus luteum may inhibit estrum month after month.

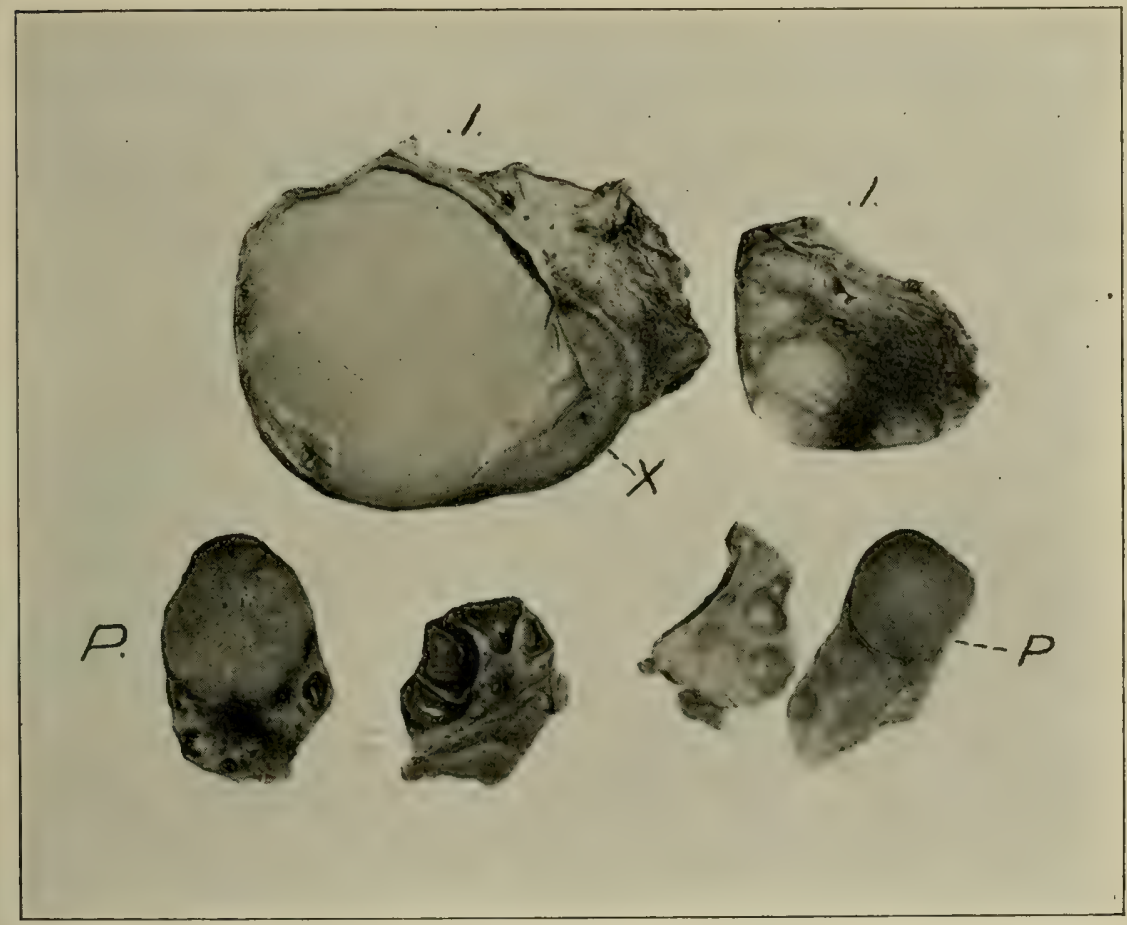

FIG. I34-Cystic Corpus Luteum.

$I, I$, Pair of ovaries, the one on the left having a very large cystic corpus luteum, showing at the lower right periphery a remnant of lutein tissue, $X ; P, P$, paired ovaries of pregnancy.

The prognosis is good. As a general rule, once the enlarged corpus luteum is dislodger, estrum returns within three to five days and there is fair opportunity for conception. It appears that the long rest of the genital tract, caused by the inhibition of estrum by the enlarged body, tends to bring about a healthy condition of the genital system, so that when the inhibiting body is dislodged the ova- 
ries function normally. In one heifer which had aborted at six months and five or six months later had not been seen in estrum, I dislodged a corpus luteum fully two inches in diameter, which immediately reformed in the old crater. I dislodged it a second time, about fifteen days later, after which the animal came in estrum and conceived at the first service.

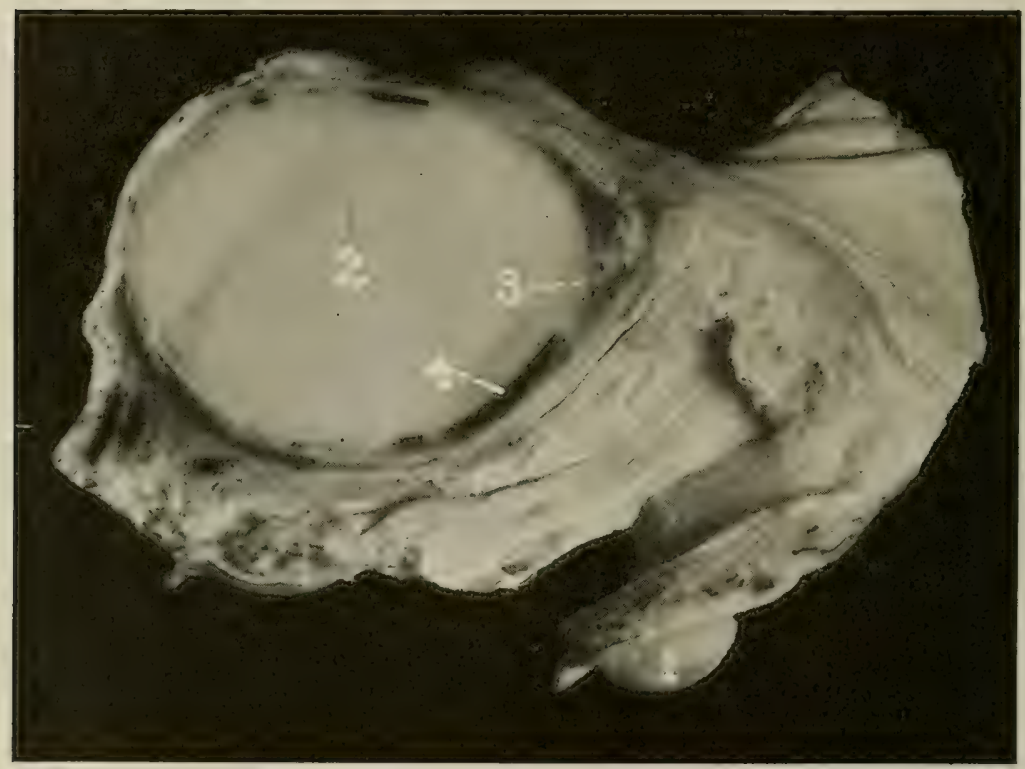

Fig. 135-Adherent Ovary with Very Large Cystic Corpus Luteum. I, Apex of cornu; 2, gelatinized cyst fluid ; 3 , thin layer of luten tissue ; t, hematoma displacing lutein tissue. Lutein tissue and old blood clot alternate about periplery of cyst.

The dislodgment of the hypertrophied corpus luteum should preferably be accomplished by compression from the vagina. Frequently it is so difficult to dislodge that, if the operation is attempted per rectum, there is danger of injury. Moreover, there is always danger that hemorrhage may follow or that the dislodgment may be incomplete. It is then best to pass one hand into the rectum, bring the ovary back over the vagina, grasp it with the other hand through 
the roof of the vagina, and dislodge the corpus luteum carefully and completely. When this has been done, the compression should be continued upon the ovary for some minutes, in order to anticipate any possibility of serious hemorrhage. Ordinarily estrum occurs within three to five days, when, if other conditions are favorable, breeding may occur.

\section{Persistent Corpus Luteum}

Writers upon the subject of sterility describe very frequently a persistent corpus luteum. In the preceding sec-

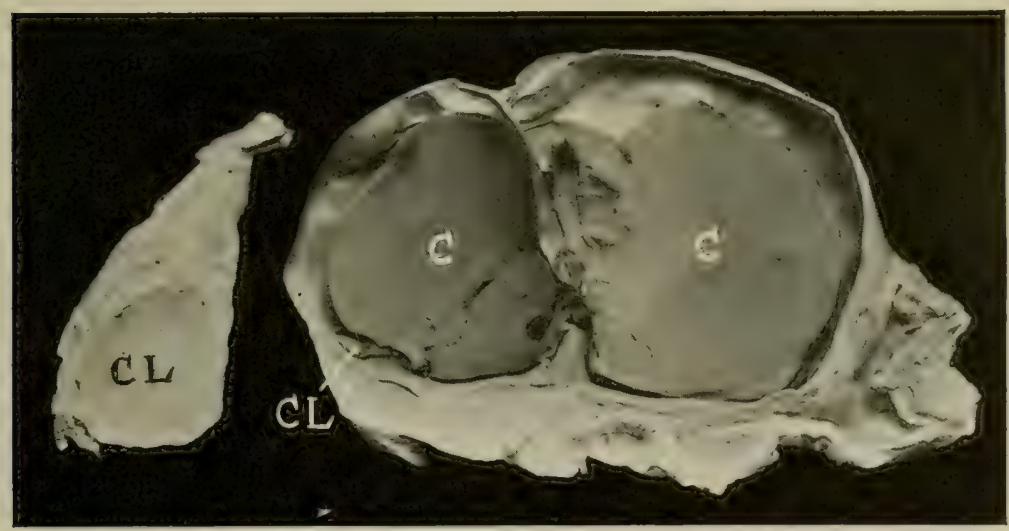

Fig. 136-Cystic Degeneration of Corpus Luteum.

Left figure, $C L$, corpus luteum of pregnancy for comparison; right figure with 2 cysts, $C, C$, the left cyst having remnant of lutein tissue at $C L$.

tion, it has been mentioned that, when the corpus luteum is hemorrhagic or when it undergoes cystic degeneration, its destruction and absorption may be indefinitely delayed. In the following paragraph (9) the question of the central or imbedded corpus luteum will be discussed. There is, however, a third group of corpora lutea which, although apparently sound and normal in volume, tend to remain indefinitely without undergoing either atrophy or degeneration. The only symptom of such condition is that the animal has not been bred or has not conceived and does not come in estrum. Upon examination, the genital tract in general may 
be normal, the health of the patient is apparently perfect, and there is present in one of the ovaries a typical corpus luteum of ordinary size, form, and consistency. Under these conditions, persistent corpus luteum is diagnosed. The diagnosis may be subject to question. It is quite possible that in many cases estrum has been overlooked. Since some cattle are not demonstrative and some attendants are not very alert in recognizing estrum, it is not improbable that many cases diagnosed as persistent corpus luteum are really instances of failure upon the part of the attendant to observe estrum. However frequent error may be, the condition does occur and should be promptly recognized by the veterinarian. I have observed cows where every opportunity was given for them to exhibit symptoms of estrum and where they had shown no such signs over a period of six months or more. Upon examination, the entire genital system is found to be normal, including an apparently normal corpus luteum in one of the ovaries. The general health of the animal is beyond criticism, and no apparent reason exists for failure of ovulation and estrum. Under these conditions the dislodgment of the corpus luteum causes the prompt appearance of estrum, and as a general rule the animal conceives readily. The long inhibition of estrum has apparently served as a definite and valuable period of rest, so that, when the barrier is overcome and the genital organs function, conception is prompt.

\section{The Central or Embedded Corpus luteum. The Corpus Luteum of Pyometra and Fetal Retention}

When an ovisac approaches maturity, it pushes its way, in the cow, toward the convex, free surface of the ovary, to protrude eventually beyond the general ovarian surface. It then ruptures and the corpus luteum which forms in its crater, when grown, also projects above the ovarian level. Such projection persists throughout the physiologic career of the body, until it atrophies preparatory to a new ovulation, when it may (or may not) sink more deeply into the gland as it gradually disappears. 
Pathologically, when there is extensive pyometra following closely upon parturition, or retention of a fetus with putrid maceration or desiccation, the corpus luteum, without undergoing either material reduction in size or cystic degeneration, commonly sinks deeply into the ovarian tissue and comes to rest in a comparatively central position, to remain unchanged for an indefinite period of time. Ordinarily its presence does not directly call for clinical diagnosis, because it is a mere adjunct to a grosser, more striking condition. The presence of an embedded corpus luteum may, therefore, be diagnosed usually with fair safety by the presence of the retained fetus or the pyometra. If the ovary is palpated, the corpus luteum may not be very evident to the inexperienced practioner. The ovary is spheroidal and unnaturally plump, but the corpus luteum is so deeply embedded that it is not clearly palpable. Careful and deliberate palpation finally enables the examiner to detect a firm central body or "core" in the gland which, in conjunction with the retained fetus or pyometra, leads to a positive diagnosis. In one or two instances I have encountered clinically an embedded corpus luteum without retained fetus or pyometra. It then had the significance of the ordinary retained corpus luteum; it inhibited estrum over an indefinite period. Professo $i$ Hess was first to draw definite attention to the inhibitory pawer of retained and embedded corpora lutea. They not only inhibit estrum but at the same time inhibit the spontaneous expulsion of the fetal cadaver or the pus contained in the uterus.

The embedded corpus luteum, like the retained yellow body, should be located and, in order to accelerate uterine contraction, should be dislodged. The removal of the corpus luteum, the best method known for causing the expulsion of a desiccating fetal cadaver, adds materially to the efficacy of other agencies in the handling of pyometra. Often the removal of the corpus luteum is far from easy. The coördinate disease of the uterus, with the increased weight and volume, drags the ovary downward and forward into the abdomen, where it is difficult to reach. Since 
it is ordinarily held too far forward to permit it to be carried back above the vagina, dislodgment must be attempted by compression per rectum. The ovarian tissue more or less completely surrounds it with firm tissue which can be ruptured only with great force.

Whenever possible, the dislodgment of the corpus luteum should be made per vaginam. In pyometra the uterine contents should first be siphoned out, when ordinarily the ovary, containing the yellow body, may be brought back over the vagina and the dislodgment undertaken from there with safety. The mechanism of dislodgment is essentially that of rupturing cysts. The compression should be concentrated upon the base of the ovary by its ligament, leaving the convex border free to break and permit the escape of the yellow body. Care should be taken to get all of the lutein tissue, that the body may not reform without ovulation. In :one instance, where fortunately the ovary could be brought back over the vagina, I could not dislodge the yellow body by compression and was forced in the end to stab the ovary, as described in connection with the cysts of nymphomania. When the ovarian capsule and overlying ovarian tissue are incised, although the stab is very narrow, the incision weakens the structure, the cut extends under compression, and the yellow body is forced out.

Although I have not met such cases, it probably occurs in some instances that the operation cannot be carried out per rectum or per vaginam. Then, especially in desiccated fetus, laparotomy is indicated when the ovary can be acted upon directly.

\section{ABSCESS OF OVARY}

I have not observed primary ovarian abscess. In connection with tubal infections, tubo-ovarian abscesses are not rare. Where traceable, they originate secondarily from pyosalpinx subsequent to marginal adhesion of the pavilion of the tube to the gland. The lesion is consequently one of pyosalpinx, in which the pus first surrounds the ovary and then destroys it. This will be considered later under tubal infections. 


\section{CORPORA NIGRA}

I have seen two instances of black instead of yellow bodies in the ovaries of pregnant cows. One was of normal size, form, and consistency; the other was greatly enlarged, as shown in Fig. 137. The bodies were a dark blue-black. Aside from the excessive size of one and the color of both, they appeared perfectly healthy. No signs of disease were observed in any part of the genital system or elsewhere. Neither the cause nor the significance of the condition was apparent. It is probably a mere "sport" without relation to sexual health or efficiency.

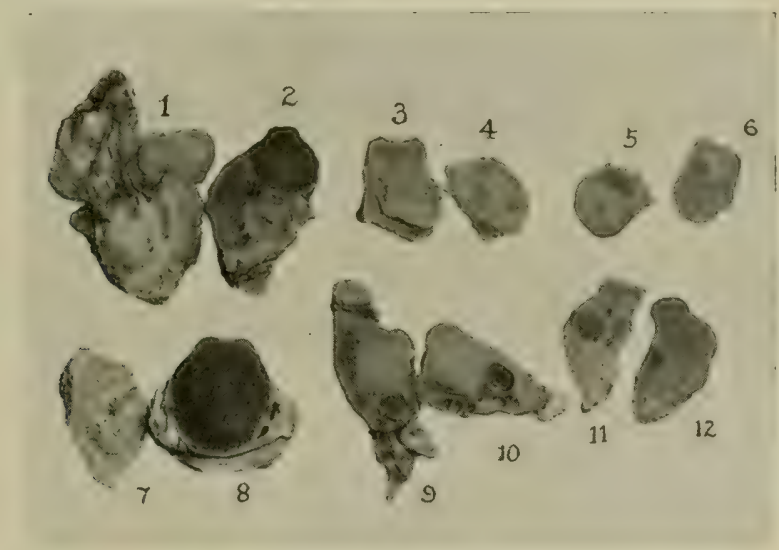

FIG. I37-Corpora Lutea of Pregnancy. For comparison with Cystic Corpora Lutea.

I, Adherent ovary ; 2, corpus nigrum of pregnancy ; 3 , corpus luteum of pregnancy; 4 , mate to $3 ; 5,6$, corpora lutea of pregnancy; 8 , enlarged corpus nigrum: 9,10 , twin corpora lutea; 12 , fresh corpus luteum.

\section{Parovarian Cysts}

Cysts occur now and then in close proximity to the ovaries, apparently due to cystic changes in remnants of the Wolffian bodies or of the Wolffian ducts. In the mare, as illustrated in Fig. 70, Chapter XI, these cysts may become a menace to the health or to the life of the animal. This I have not observed in the cow although it may evidently occur. Instead I have observed usually small coiled tubular cysts without known significance for the animal. Their chief interest lies in their close proximity to the oviducts and their confusing likeness to hydrosalpinx, which may lead the veterinarian to pronounce a perfectly fertile animal incurably 
sterile. They are to be differentiated, so far as my collection indicates, by the fact that in the cow hydrosalpinx usually involves the entire length of the oviduct and presents the other characters described under that heading. The parovarian cysts are usually small --much smaller than the hydrosalpinx generally-and can be picked up in the mesosalpinx. Careful palpation should recognize the healthy tube not far away, enabling one to differentiate the two structures.

\section{B. Tubal Infections ${ }^{1}$}

In cattle the diseases of the oviducts constitute a very fertile cause of sterility and play an unknown role in the occurrence of abortion. The oviducts constitute highly com-

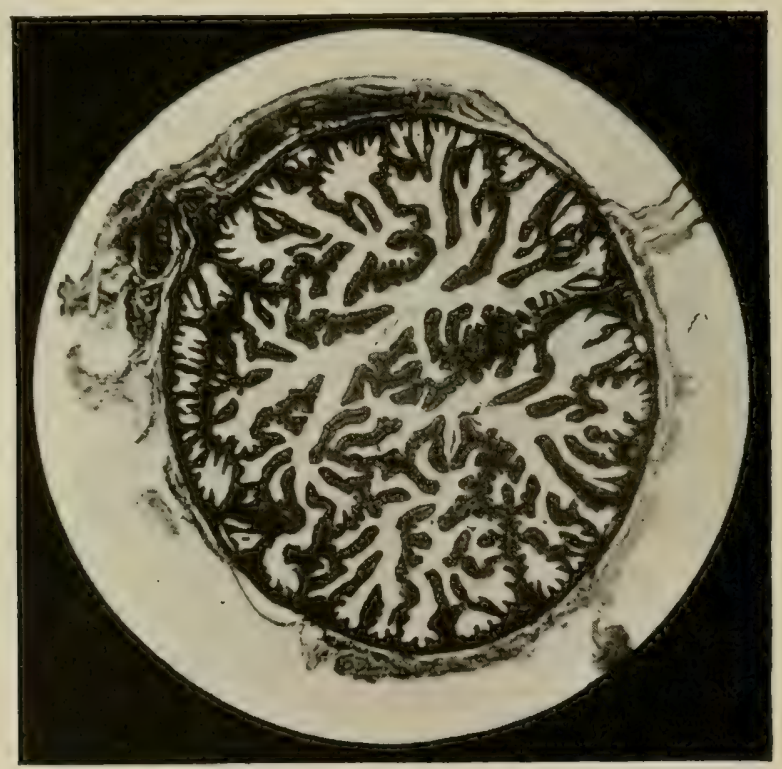

FIG. I38-Cross Section of Normal Oviduct Near the Ampulla. Showing the Number and Complexity of the Mucous Folds.

plex anatomo-physiologic barriers between the peritoneal and uterine cavities. Technically, the peritoneal cavity of the female communicates with the exterior through the

${ }^{1}$ For the normal and pathologic histology and for the bacteriology of the oviducts, I am indebted chiefly to the work of Dr. Herbert L. Gilman, graduate student, and to my colleague, Dr. C. M. Carpenter. 


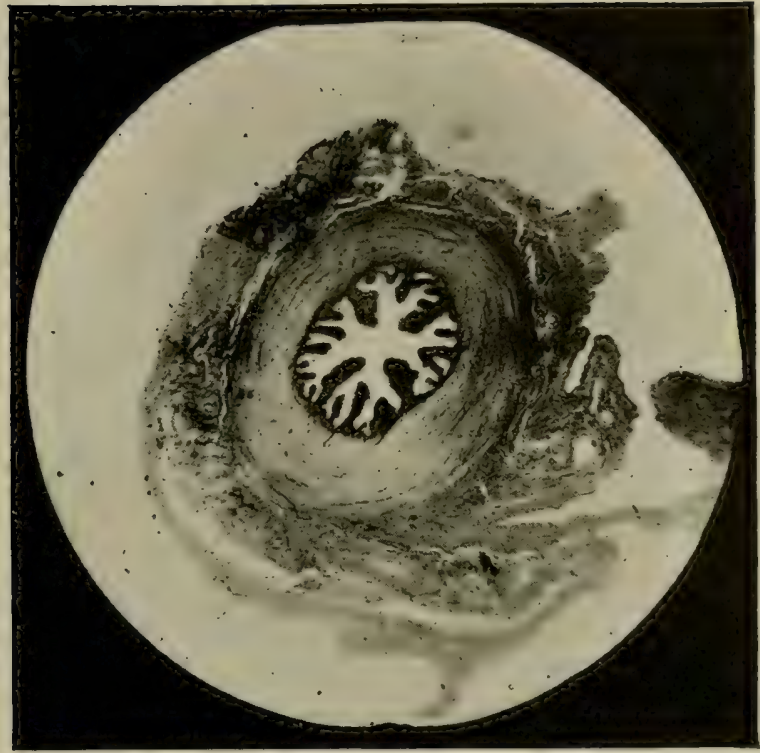

FIG. 139-Section of Normal Oviduct near the middle.

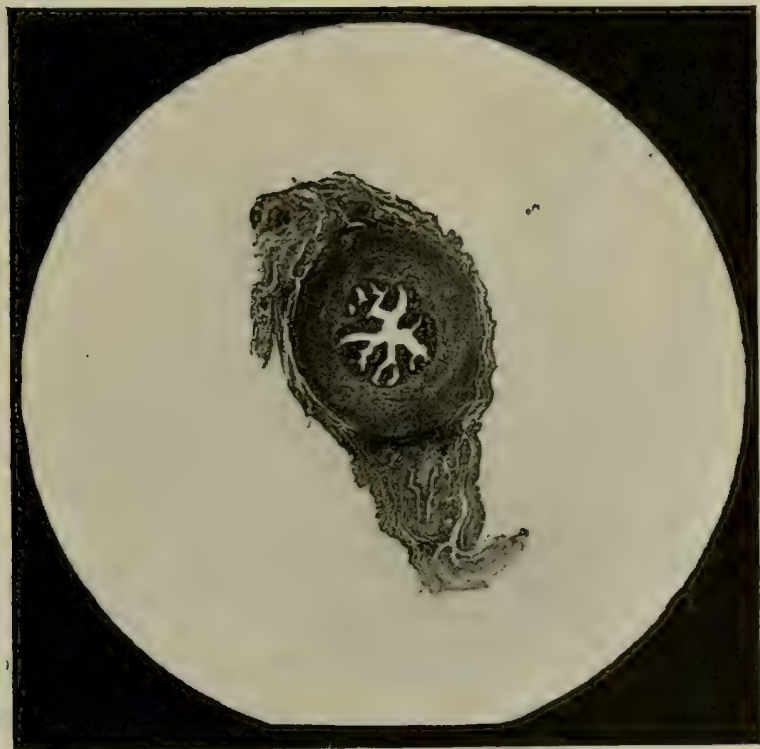

FIG. I 40-Section of Normal Oviduct near the Uterine End. Showing the Simpler Mucous Folds and the Thickness of the Muscular Coat. 
genital tube, with the oviducts interposed as highly complex, sinuous, narrow tubules. While the oviducts are highly efficient in preventing the migration of infection from the uterus and more external areas into the peritoneal cavity, the same complexity makes of them a lodging-place for infection which, once attained, is unusually refractory to dislodgment by physiologic, medicinal, or surgical agencies. Since, in probably more than 90 per cent. of cases of infection in the oviducts, the condition is bilateral, the duplication of ovaries and oviducts avails little; when disease in one oviduct constitutes an insurmountable obstacle to conception, there are nine chances in ten that the other tube is likewise barred. Consequently tubal infection generally closes permanently the reproductive career of the animal. The clinician and the animal husbandman are further baffled in the present state of knowledge by the difficulty in many cases of making an early diagnosis, causing incurable animals to be held for long periods at great economic loss before the character of the disease is clearly revealed. The anatomic and histologic lesions caused by tubal infection are endless in variety. Three outstnding types occur.

1. Salpingitis Unaccompanied by EnLARgement of the

Ducts of a Degree Clinically Recognizable by

\section{PALPATION, OR MACROSCOPICALLY VISIBLE UPON POST-MoRTEM EXAMINATION}

Salpingitis, or inflammation of the oviducts, without material enlargement, is the commonest and most important type of disease of these organs. Ordinarily the infection does not cause a sufficient degree of enlargement to enable the veterinarian to make a diagnosis by palpation per rectum. Upon post-mortem inspection, the oviduct shows little macroscopic evidence of disease. The principal clinical evidences of salpingitis are persistent sterility without palpable lesions in the genital tract, as a rule associated with cystic degeneration of the corpus luteum, with irregular estrual cycles, and later with adhesion of the pavilion of the tube to the ovary. 
Cystic degeneration of the corpus luteum has already been discussed under "The Diseases of the Ovary." It is not certain that cystic degeneration of the corpus luteum occurs, except with salpingitis, although it cannot be proven that salpingitis is the sole cause. It appears, however, that salpingitis is at least the predominant cause. When the ovisac is about to rupture, it is believed that the pavilion of the tube invests it, with the ostium abdominale in proximity to the ovisac. When ovulation occurs, infection may apparently escape from the oviduct, invade the crater left behind by the rupture, and set up disease, which eventually brings about the destruction of the yellow body. The destruction of the corpus luteum by cystic degeneration does not cause the sterility, but the infection which exists in the oviduct causes alike the sterility and the cystic degeneration of the corpus luteum. It follows, therefore, that cystic degeneration of the corpus luteum may occur in spite of conception: that is, spermatozoa may be able to live in the infected tube and fertilize the ovum, which may be able to pass through the infected oviduct, reach the uterine cavity, and develop. As a rule, however, cystic degeneration of the corpus luteum does not occur in pregnancy. From my abattoir investigations, I would estimate that not more than one or two per cent. of corpora lutea of pregnancy suffer from cystic degeneration. Perhaps the facts would be more clearly expressed by stating that, when the oviduct is so virulently and abundantly infected that the infection will escape from the tube and invade the freshly ruptured ovisac, the spermatozoa, and the non-fertilized or fertilized ovum will probably be destroyed. Sometimes, however, the spermatozoön and ovum run the gauntlet of the infected tube although the crater of the ovisac is invaded. Then follows, at least in some cases, cystic destruction of the corpus luteum of pregnancy, with recurrence of estrum in the pregnant animal. The cystic degeneration of the corpus luteum interrupts more or less seriously the estrual cycle. Sometimes the estrual period will return within seven to fifteen days; sometimes the cycle may 
be prolonged so that the estrum does not recur for thirty or more days. In some cases the estrum is comparatively regular. The clinical recognition of the cystic corpus luteum has already been discussed in dealing with the ovary.

When pavilionitis becomes established, with adhesions of the pavilion to the ovary, the diagnosis of salpingitis becomes positive. So far as known, adhesions of the ovary within the pavilion must be attributed to salpingitis. The clinical recognition of such adhesions is usually simple. The operator should pick up the ovary per rectum and pass the index finger into the ovarian pocket in front of the gland. If any adhesions are present, they are readily detected in the earlier stages as delicate threads which may be torn asunder with slight pressure. The inflammation gradually extends, the adhesive bands grow in volume and number, and, if the disease is very severe, the ovary becomes completely and firmly adherent within the pavilion and within the ovarian pocket, or bursa. The ovary becomes masked so that it can not be palpated directly, but only through its adherent covering. It is then either very large, owing to cystic degeneration of the corpus luteum, or, if of long standing, is small, hard, sclerotic, and deeply buried in the adhesions, where in some cases it is recognized only with difficulty. The cystic degeneration of the corpus luteum, the erratic estrual cycle, the recognition of the pavilionitis as evidenced by the adhesions, with the persistent sterility, each justifies a diagnosis of salpingitis; when two or more of these are taken collectively, the diagnosis is rendered positive. The diagnosis may be complicated and hampered by the presence of other lesions of a gross character and readily recognizable.

Salpingitis is frequently associated with cervicitis in a confusing manner. When cervicitis of an intense type is rampant in a herd ( $\mathrm{I}$ have seen it involve 60 to 75 per cent. of large herds) salpingitis eventually forces the slaughter of a discouraging number of cows after the visible cervicitis has been brought under control. Whether the cervicitis caused the salpingitis or vice versa, or whether the two in- 


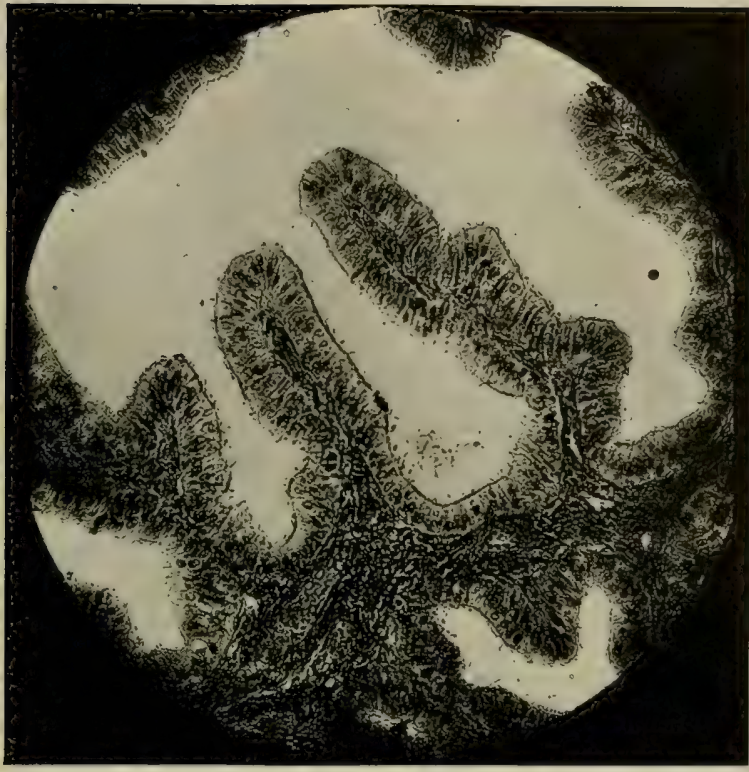

FIG. I41-Highly Magnified Section of Normal Mucous Fold of Oviduct.

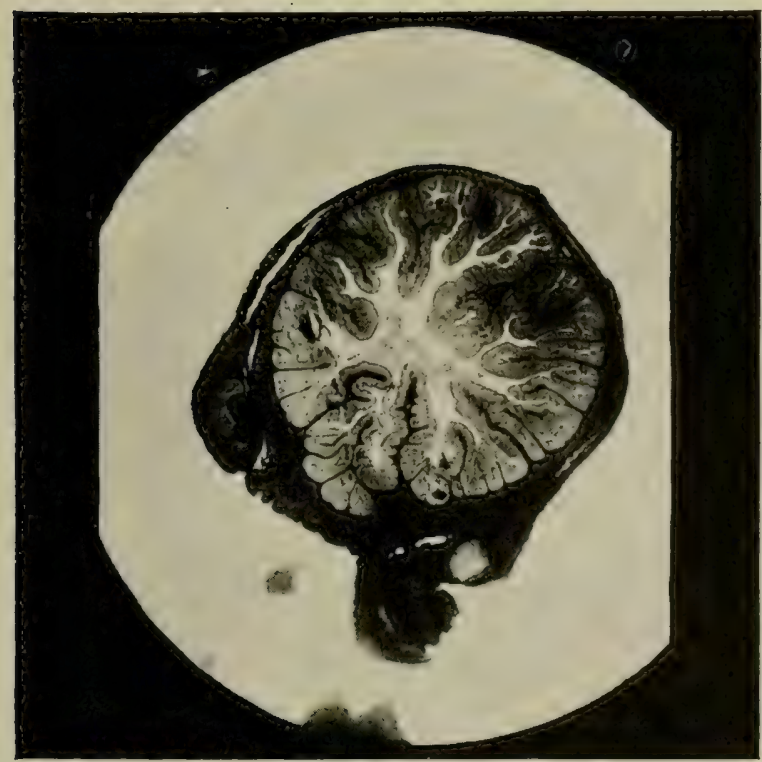

FIG. I42-Section of Oviduct from a Six-Weeks-Old Calf. Showing the Comparative Thickness of the Mucosa. 
fections, ordinarily identical, arise simultaneously from a common center, can not be stated. Judging from clinical observation in a few herds, it appears that the source of the infection in these notable outbreaks was the herd bull, and that the infection was transmitted in coitus, attacking primarily the cervix and, before that was brought under control, invading secondarily the oviducts. It seems clear that not all cases of salpingitis are explainable upon such a hypothesis. Frequently heifers are absolutely sterile from salpingitis, though served by bulls which appear genitally sound and to which other females have been bred without exhibiting clinical evidences that they are dangerous carriers of infection.

The oviducts are narrow, tortuous ducts about 21 to 28 $\mathrm{mm}$. long, leading from the ovaries to the corresponding uterine horns. They serve as a path for the passage of spermatozoa to the pavilion and as excretory ducts in the conveyance of the ova from the ovaries to the uterus. Each tube is rather loosely invested by a fold of the peritoneum derived from the broad ligament, which is called the mesosalpinx. It is divided into a broad, funnel-shaped, fimbriated end, or pavilion, a constricted neck, an ampulla of considerable diameter, and a narrow isthmus at the uterine end. At the bottom of the pavilion, is a very small opening, the ostium abdominale, through which the lumen of the tube opens into the peritoneal cavity. Here exist two unusual anatomical conditions-a break in the continuity between a gland and its excretory duct, and an opening between a serous cavity and the exterior. From this constriction, the tube becomes distended to about 3 to $5 \mathrm{~mm}$. in diameter, to form the ampulla, and from here to the uterine end gradually diminishes to 0.8 to $1 \mathrm{~mm}$. at the isthmus.

The epithelium is pseudo-stratified. The mucosa is thrown into longitudinal wrinkles or folds, lined by a pseudo-stratified epithelium, which increase in height and number from the uterine to the abdominal ends and are without glands. At the uterine end there are usually four or five folds, comparatively low and broad, which do not branch. Toward 
the ampulla, however, they increase in number and height, with the formation of numerous secondary folds, so that at the ampulla the otherwise broad lumen becomes almost occluded. At the fimbriated end, the folds widen and are continued out on the free ends of the fimbriae, forming ridges which branch freely, giving the pavilion a more or less honeycombed appearance. Not infrequently one finds the folds ramifying elaborately, particularly at their bases, forming cavities lined by a sort of flattened stratified epithelium, sometimes even attaining the appearance seen in Fig. 143. These cavities are also found in chronic catarrhal

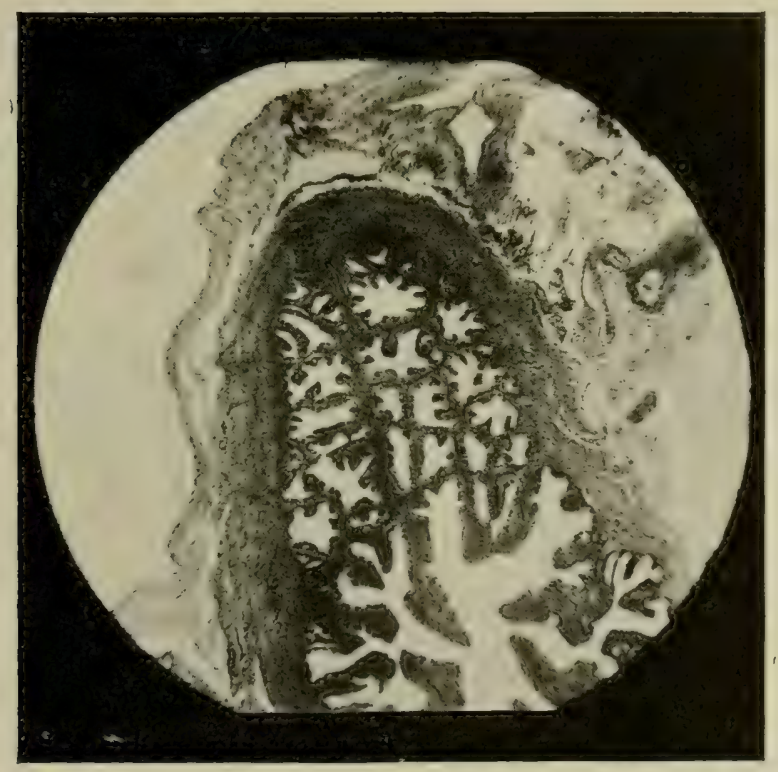

FIG I43-Normal Section of Oviduct Showing the Formation of Cyst-Like Cavities by Coalescence of the Folds.

salpingitis, but usually in greater numbers and accompanied by other pathological changes. The superficial layer of the epithelium consists of tall cells, most of which are ciliated, though there is much difference of opinion as to the proportion of the latter. In older animals, there are but two or three layers of cells under the superficial one; while in young 
calves (six weeks) the layers are very numerous, and in some the mucosa is so thick that there is little or no space between the individual folds and the lumen is narrowed considerably, as in Fig. 142. The muscular wall is formed of two layers of smooth fibers, a broad, inner, circular, and a thin, outer, longitudinal layer. A third inner layer of longitudinal fibers and bundles is seen in most sections, especially near the uterine end, either as a distinct layer or in crescent-shaped bundles, as in Fig. 144.

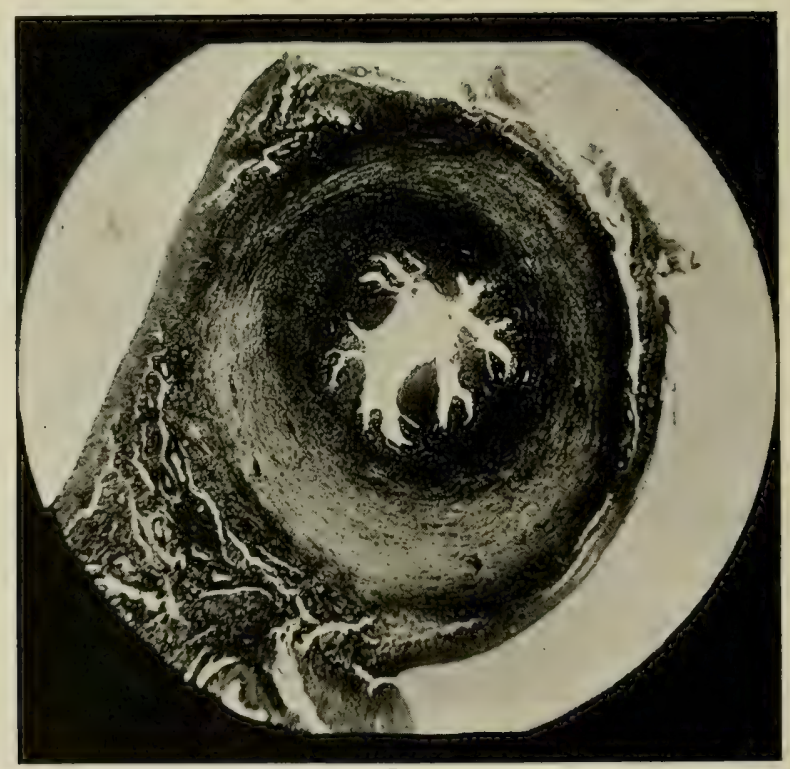

FIG. I44--Chronic Catarrhal Salpingitis, with a Distinct CrescentShaped Inner Longitudinal Muscular Coat.

So far as known, all inflammatory changes in the oviducts are due to bacterial invasion. In the milder forms of salpingitis the mucosa alone is affected; in the severer types the process spreads to the deeper layers and even to the serosa. In the former case there is congestion, some exudation into the basement membrane, local loss of cilia, and frequently more or less desquamation of the superficial epithelium. In the deeper layers there may be hyperaemia, slight exudation 


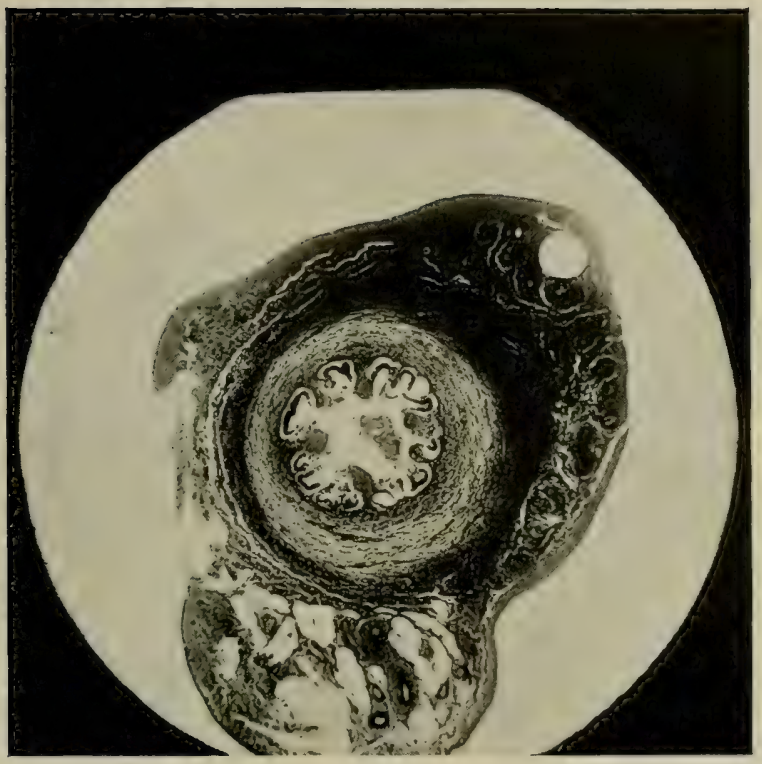

FIG. I45-Chronic Catarrhal Salpingitis, Showing the Epithelium About to be Cast Off, and Edema of the Wall.

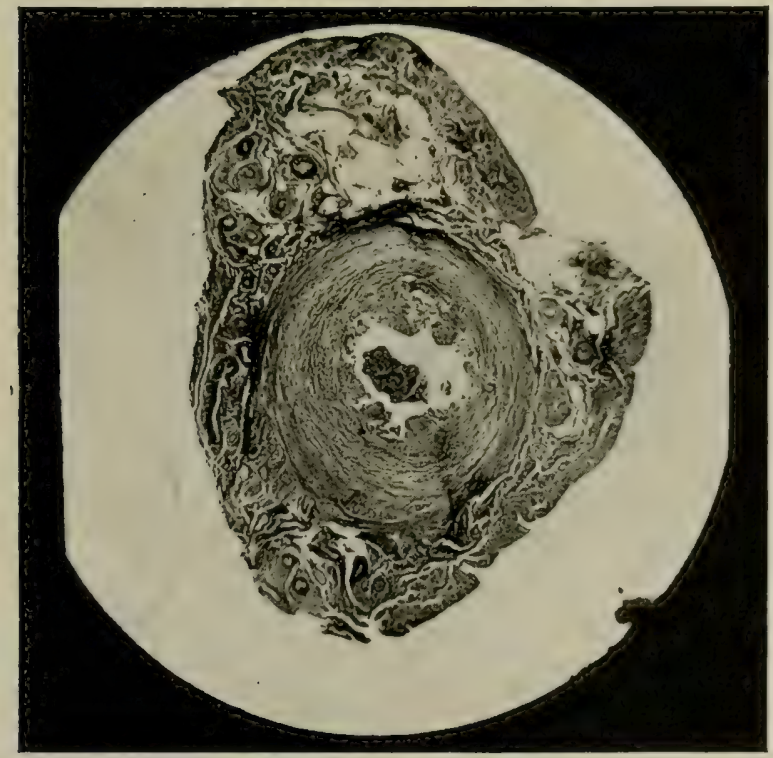

FIG. I46-Chronic Catarrhal Salpingitis, with Marked Edema of the Wall. 
and more or less swelling of the parts. There is usually a profuse exudate in the lumen. The mucosa may be thickened by a small-celled infiltration and the epithelium show cloudy swelling, fatty degeneration, desquamation, or, as is often the case, total destruction. Owing to cellular infiltration and the formation of granulation tissue, the folds may become thickened and often fuse, with the production of cyst-like cavities lined by epithelium. In some cases the greatly increased blood vessels show hyaline or amyloid infiltration of their walls, as shown in Fig. 151. The muscular coat often shows hypertrophy and small-celled infiltration, either diffusely or in collected areas.

The exudate into the lumen is probably discharged into the uterus, where it may cause metritis or cervicitis. If the secretion is unable to escape readily in that direction, either through swelling of the parts or atresia of the uterine ostium, it is discharged on the fimbria or ovary, with subsequent inflammation of the parts. Here it may produce a simple serous inflammation, though more often it assumes a fibrinous character, with the resulting formation of adhesions, fibrinous strands between the fimbriae and ovary, or closure of the ostium abdominale. Not infrequently the entire pavilion becomes adherent to the ovary, in which case there may result a tubo-ovarian cyst or abscess. If the disease spreads farther, there may occur a fibrinous peri-salpingitis with the formation of numerous fibrous strands running from the tube to the neighboring parts of the broad ligament. With closure of both the ostium abdominale and uterine ostium, the result is very often an accumulation of the contents, with the formation of hydrosalpinx or pyosalpinx.

The lesions may vary greatly according to intensity and duration. In the simpler type of the early stages, the mucosa is swollen, congested, and the seat of small-celled infiltration. The lumen may contain fibrinous exudate mingled with desquamated epithelium, as shown in Fig. 150. The desquamation involves loss of cilia. With added severity of the infection, the epithelial layers are destroyed one after 


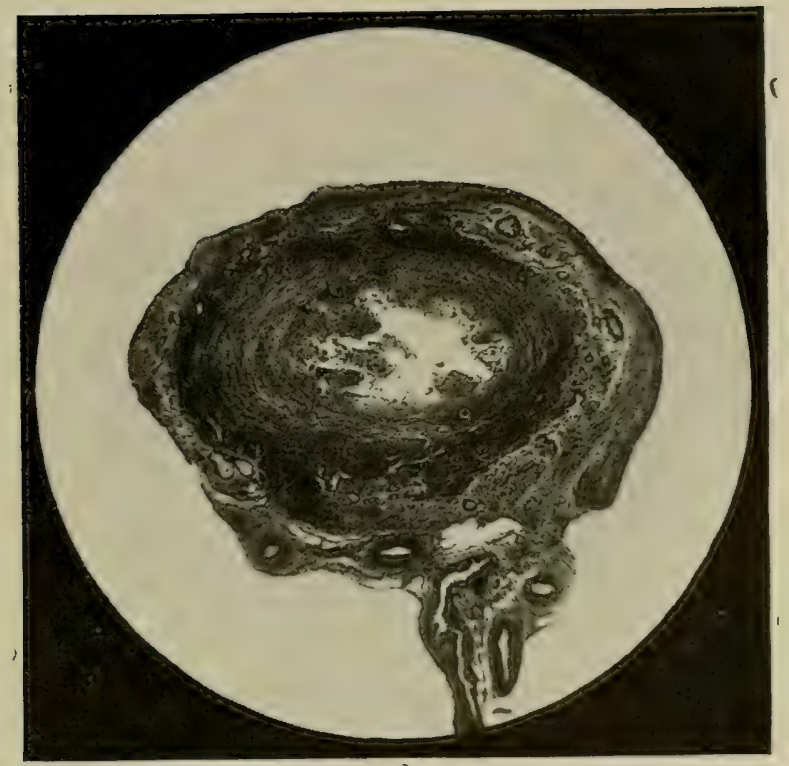

FIG. I47-Chronic Catarrhal Salpingitis. The Mucous Folds have been Destroyed and the Lumen of the Tube Filled with Debris.

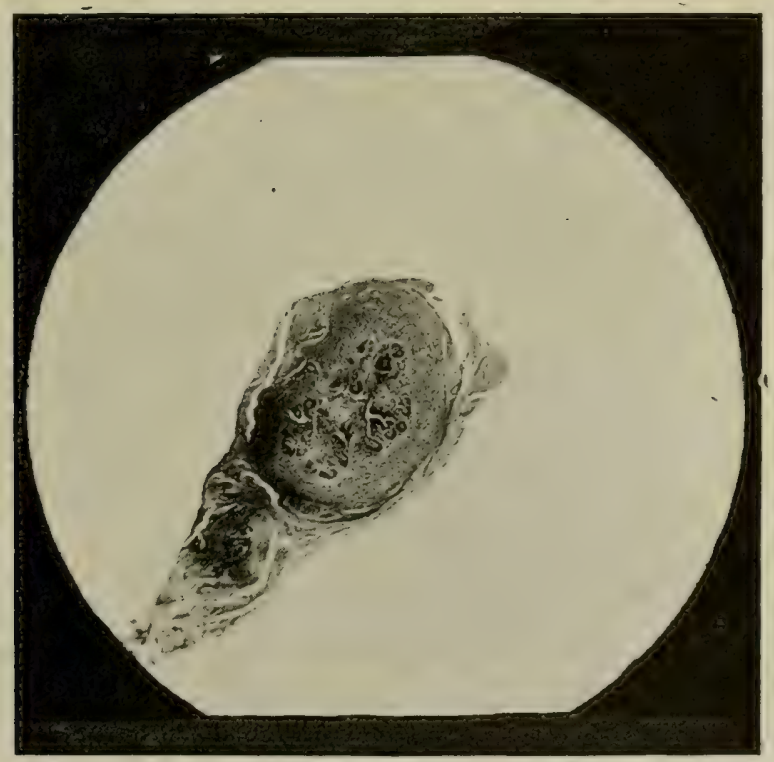

FIG. I48-Chronic Catarrhal Salpingitis.

With Well Advanced Occlusion of the Lumen of the Tube. 
the other until all disappear. The destruction of the epithelium ordinarily occurs first at the summits of the longitudinal folds and last at their bases. The denuded mucous folds tend to adhere and to cause thereby small cavities between their bases, lined with epithelium.

In bacteriologic studies of over eighty pairs of oviducts, Gilman found that a streptococcus of the viridans group was the dominant organism, staphylococcus albus and aureus were common, and in one instance only did he recognize an organism closely resembling $B$. abortus.

The prognosis is virtually hopeless. In more than 90 per cent. of cases, the infection is bilateral. It is possible that in some cases the tubes recover spontaneously, but there is no evidence which fully justifies one in asserting that this occurs. The duct is beyond surgical interference under any known technic, and there is no known medicinal remedy.

The prevention of salpingitis must be based upon the avoidance of infection in other parts of the genital tract. The infection probably advances into the oviduct from the cervix and uterus. At least we know clinically that, in herds where cervicitis is common, a large proportion of the animals become incurably sterile from salpingitis, in spite of the fact that the cervicitis may almost always be overcome. If, therefore, salpingitis is to be prevented, it is essential that the measures taken shall be timely. Many heifers, suffering from salpingitis prior to breeding age, are consequently incurably sterile. So far as can be determined now, such infection in heifers should be referred to the nursery period and its prevention based upon keeping the calf in a high state of health. The belief has been expressed elsewhere that, during dysentery or pneumonia in calves, or when slighter infection exists in the alimentary or pulmonary tract, bacteria escape and, entering the blood stream, become lodged in the genital tract, for which they have an affinity. Later, when the animal comes to breeding age, it seems perfectly clear at present that an infection which may bring about salpingitis may be carried by the bull and introduced during coitus. This may not interfere with concep- 


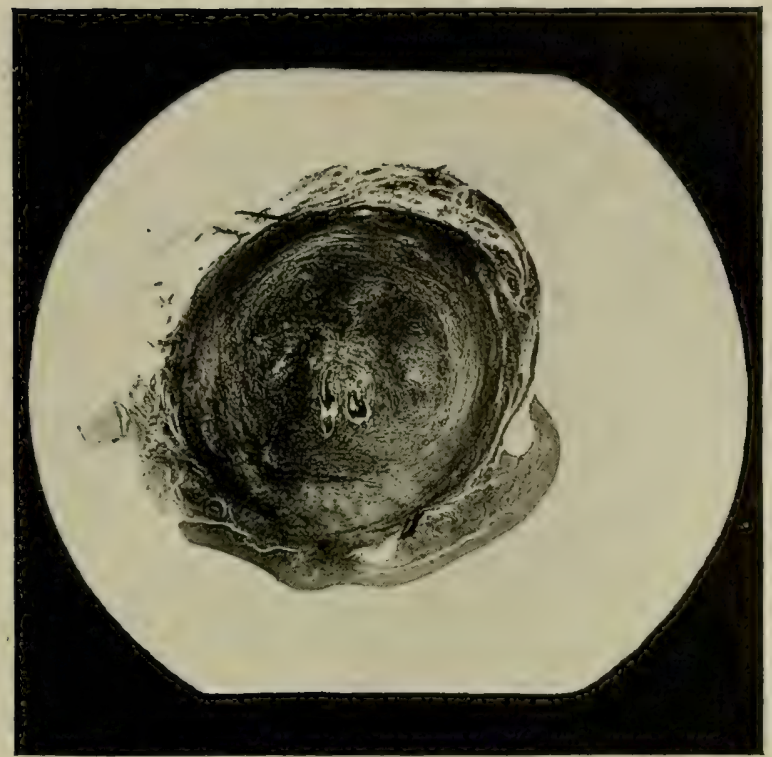

FIG. I49-Chronic Catarrhal Salpingitis.

With Almost Total Occlusion. Vestiges of tube lumen are shown by two small black areas bordered by white.

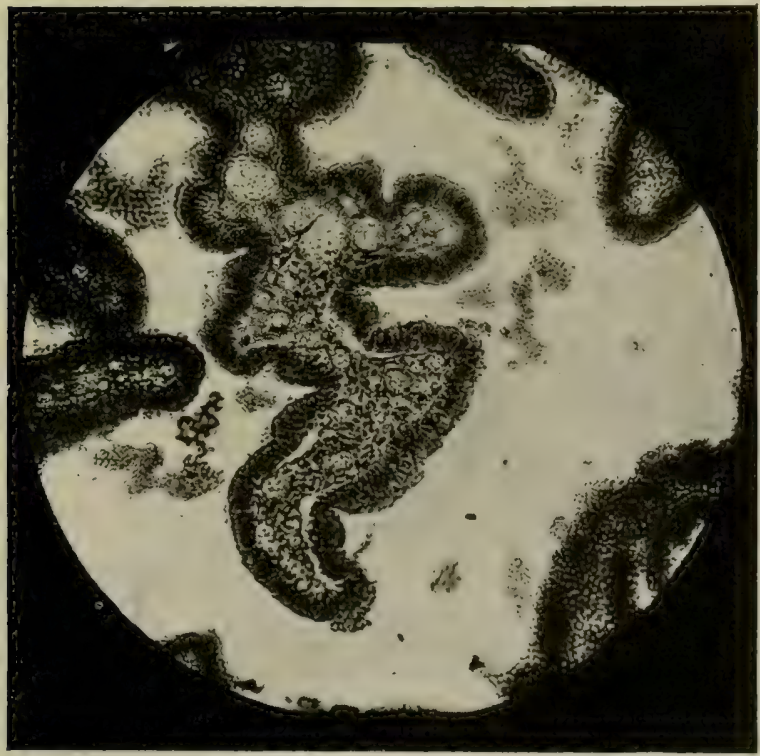

Fig. 150-Highly Magnified Fold of the Mucosa in the Acute Stages of Inflammation, Showing Congestion, Exudation, etc. 


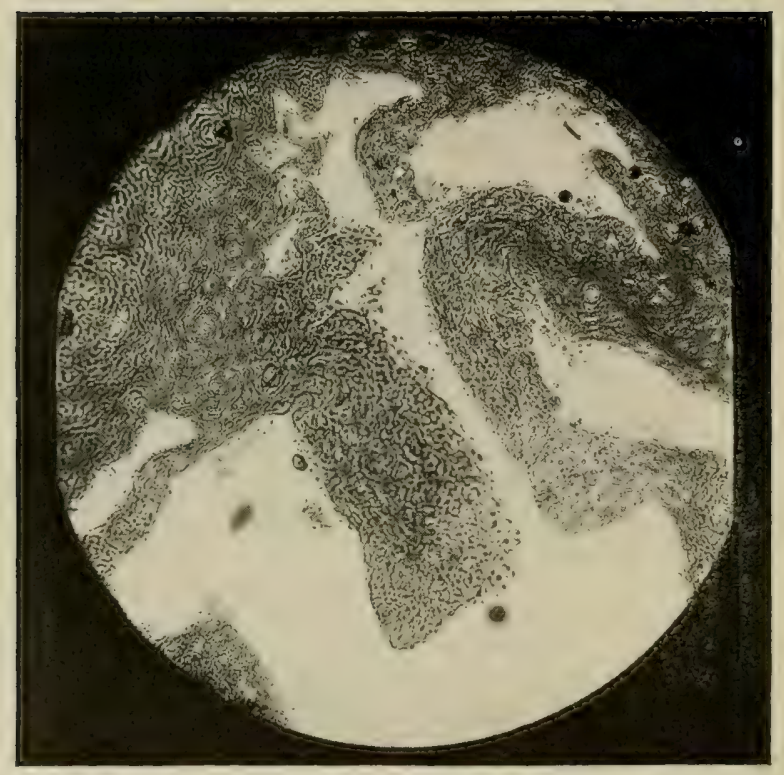

FIG. I5I-Highly Magnified Fold of the Mucosa in Chronic Catarrhal Inflammation.

Showing Destruction of the Epithelium, thickening by newly-formed Connective Tissue, and Amyloid or Hyaline Infiltration of the Blood Vessels.

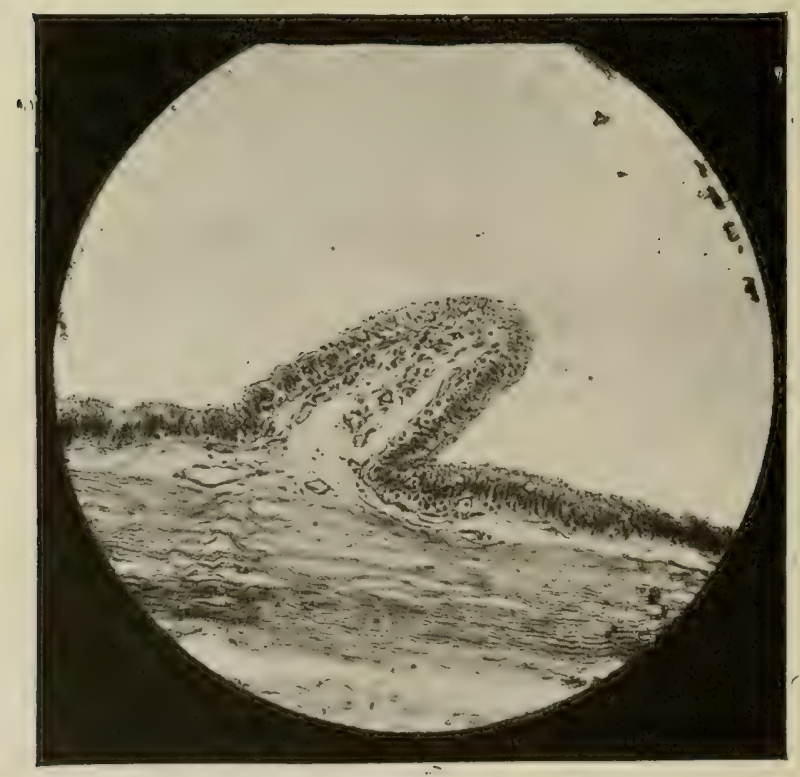

FIG. 152-Section of the Tube Wall in Hydrosalpinx.

Showing the Slightly Changed Epithelium, and the Loose, Membranous 
tion, but may cause salpingitis during pregnancy, with incurable sterility, while the uterus contains a living and apparently healthy fetus. In such cases the animal may calve apparently well and appear to be sound during her puerperal period, but is bound to be absolutely and incurably sterile.

More frequently salpingitis follows cervicitis and metritis. It is especially apt to follow retained afterbirth. Accordingly it is essential, if salpingitis is to be prevented, that the genital tube shall be closely watched during the puerperal period, and any infection present in the cervix or

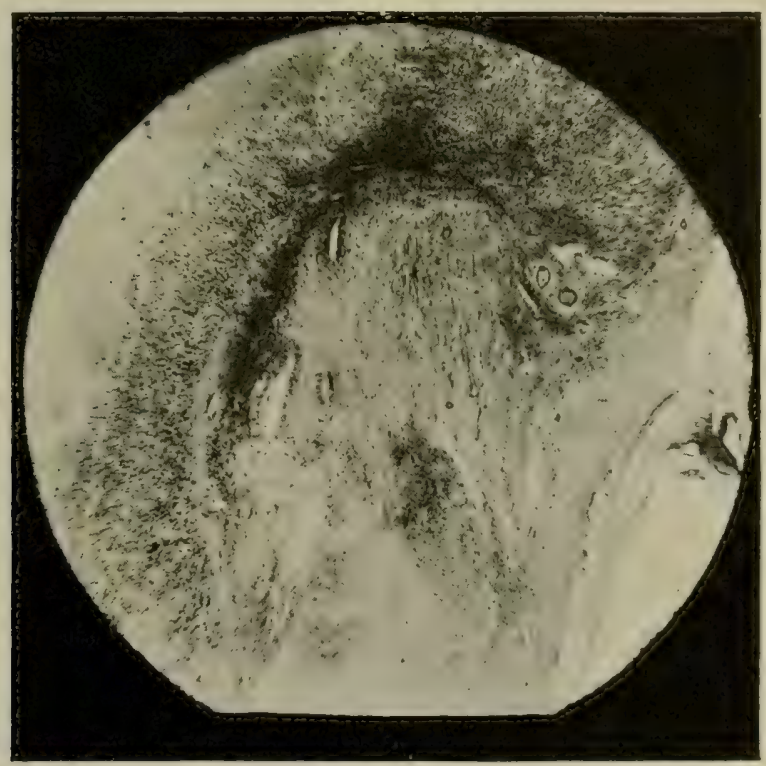

FIG I53-Section of Part of the Wall in an Old Case of Tubercular Salpingitis.

Near the center of the section are seen three cavities lined with epithelium, which have resisted the necrotic processes.

uterus promptly overcome if possible. The veterinarian and breeder should clearly understand that a retained afterbirth is a perilous thing for the integrity of the oviducts. Consequently, retained afterbirth should be watched, not only from the standpoint of saving the life of the animal or of merely preventing a severe metritis, but because of the 

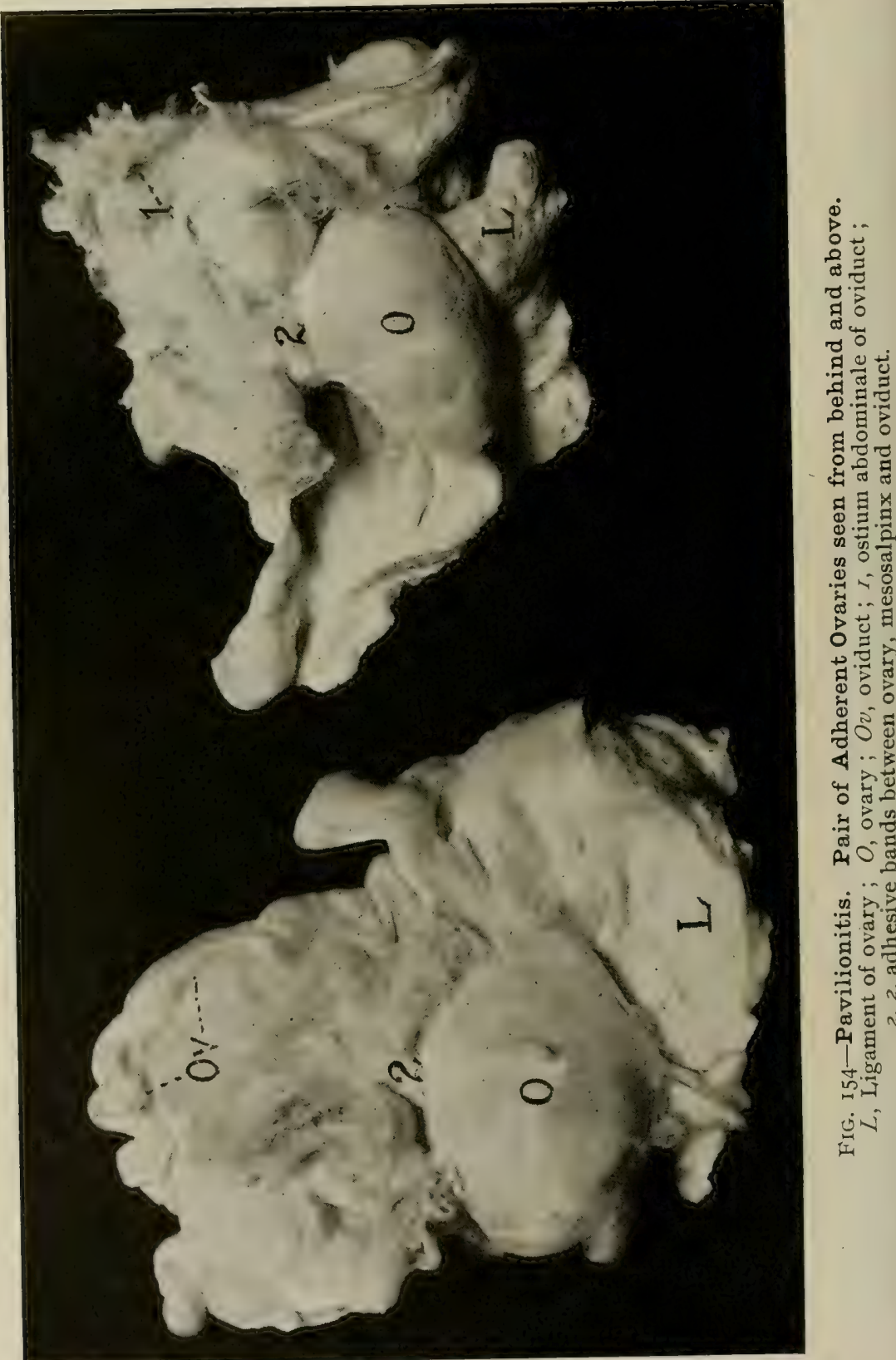
danger from salpingitis owing to the infection extending from the uterine cornua forward into the tubes.

Treatment. Since the prognosis is so nearly hopeless, there is but little which can be suggested in the way of treatment, once the disease has become established. If it can be determined, or if it can be reasonably hoped that the disease is unilateral and is causing irregularity in sexual life, the offeding oviduct, with the ovary, may be removed. If the ovary and oviduct are sufficiently mobile, it may be possible in some cases to operate through the vagina, but as

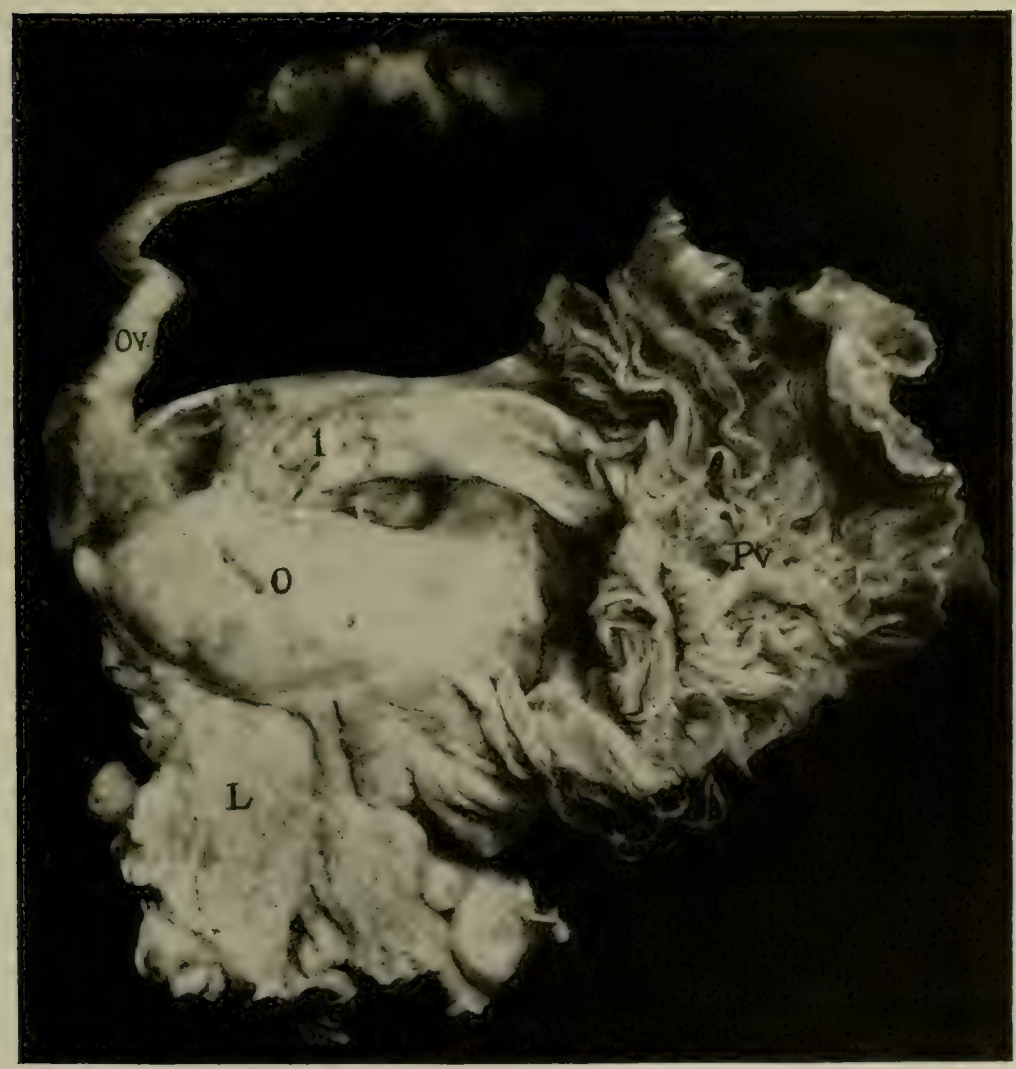

FIG. 155-Adherent Ovary. Cow. Dorsal surface viewed from behind. $L$, Ovarian ligament; $O$, ovary; $O v$, oviduct. 
a general rule laparotomy upon the side of the animal where the diseased tube is located is safer. In performing laparotomy, the incision should be made in the middle of the flank. If it is attempted to operate high up in the flank, it is wellnigh impossible to draw the ovary and duct out through the laparotomy wound. It is difficult even to carry out the operation when the laparotomy wound is in the middle part of the flank. When the operation is decided upon, care should be taken to remove the entire oviduct and to make the amputation through the apex of the cornu, avoiding as far as

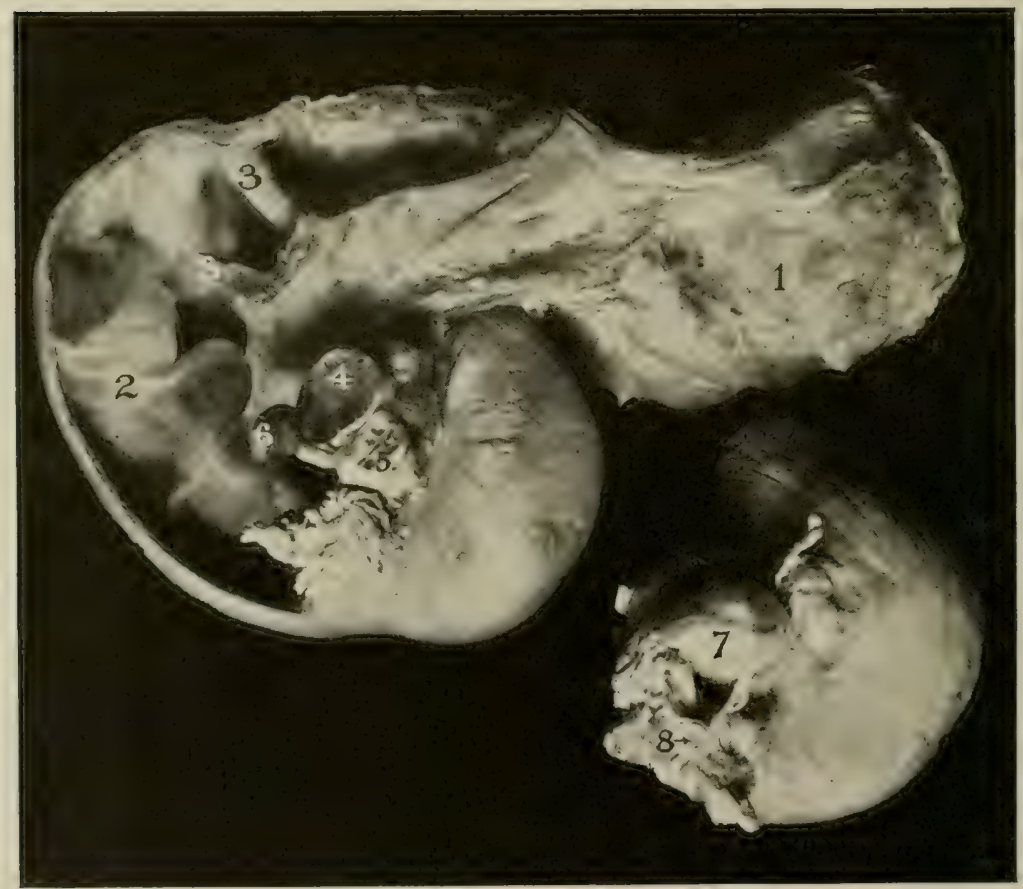

FIG. 156-Adhesion of Right Oviduct to Ovary. Double Left Ovary with Pregnancy.

$I$, Vagina; 2, fetal membranes in left horn; 3,3 , transverse septa in uterine cavity ; 4 , corpus luteum in mediau portion of left ovary ; 5 , lateral portion of left ovary; 6 , pedunculated old corpus luteum which had been imperfectly dislodged ; 7 , right adherent ovary ; 8 , ostium abdominale of oviduct. 
possible escape of the infection into the peritoneal cavity. Consequently it is best to secure the cornu carefully upon either side of the point of the intended amputation and take ample precautions against escape of any infection into the peritoneal cavity. The stump of the cornu should be closed as in the operation for appendicitis in man. That is, the mucosa should be sutured or ligated and pushed back into the lumen of the horn, and then the serosa and musculosa drawn together over the mucosa with durable gut sutures. The operation of spaying has been described in Chapter XI.

\section{PyOSALPINX}

In the simple salpingitis which is described in the preceding paragraph, no appreciable amount of pus is formed. Sometimes, however, there is a considerable volume of pus formed in the oviduct, which definitely distends it, rendering it palpable. In some cases the duct is moderately enlarged and the walls very thick, hard, and readily palpable per rectum. In other cases which I have encountered, there is a considerable distension of the oviduct, its walls become thin and paretic, and the tube becomes soft and fluctuant. In such cases, as a rule, at least in the specimens in my collection, the pavilion of the tube is adherent about the ovary or to the mesosalpinx or mesometrium and the pus surrounds the ovary more or less completely, and causes its partial or total necrosis.

In a third group, which is illustrated in Figures 157, 158, a large tubo-ovarian abscess forms, sometimes containing eight to ten or fifteen ounces of thick pus. In these cases, neither ovary nor tube is recognizable, but in their stead is a great sclerotic abscess with very heavy, thick walls. In many cases the abscess does not become firmly adherent to other organs and does not point and break. In some of my specimens, where adhesions are very extensive, it seems probable that in the complex abscessation there have been tubo-ovarian abscesses which have emptied into the neighboring organs, but the destruction has been so great that the question can not be clearly determined. 
The prognosis for pyosalpinx and for tubo-ovarian abscess is necessarily hopeless, so far as the diseased side is concerned. It is highly important that a careful diagnosis should be made, so that pyosalpinx, or tubo-ovarian abscess, shall not be mistakenly treated for some other lesion, at great peril to the animal. In some tubes distended with pus, and perhaps in some cases of large tubo-ovarian abscesses, the walls could be readily ruptured by pressure per rectum, causing a fatal peritonitis. Therefore it is highly important that the veterinarian shall not mistake tuboovarian abscesses for ovarian cysts and rupture the abscesses into the peritoneal cavity, with fatal results. As a general rule, the prudent veterinarian will let pyosalpinx alone. In exceptional cases, where the pus seems to be well walled off, is confined to one side, and is inhibiting ovulation

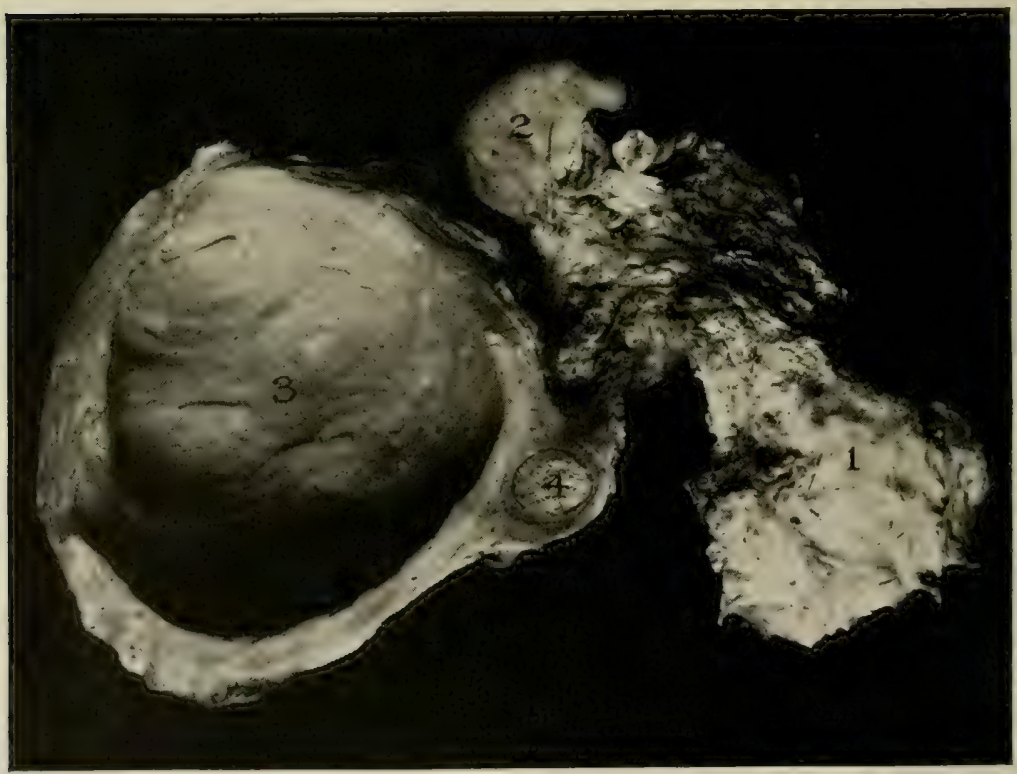

Fic. 157-Tubo-Ovarian Abscess. Cow.

$I$, Vagina and cervix seen from ventral side; 2 , left uterine cornu ; 3 , tuboovarian abscess; 4 , cross section of oviduct filled with pus. 
on the sound side, it may be possible to remove the suppurating tube, with its ovary, by laporatomy. In valuable breeding animals it may be worth while to take the risk of such an operation. The technic of the operation would naturally be the same as that for salpingitis and adherent ovary, though the peril to the animal would be very much greater, and the owner should have the risks thoroughly explained prior to venturing upon so hazardous a procedure.

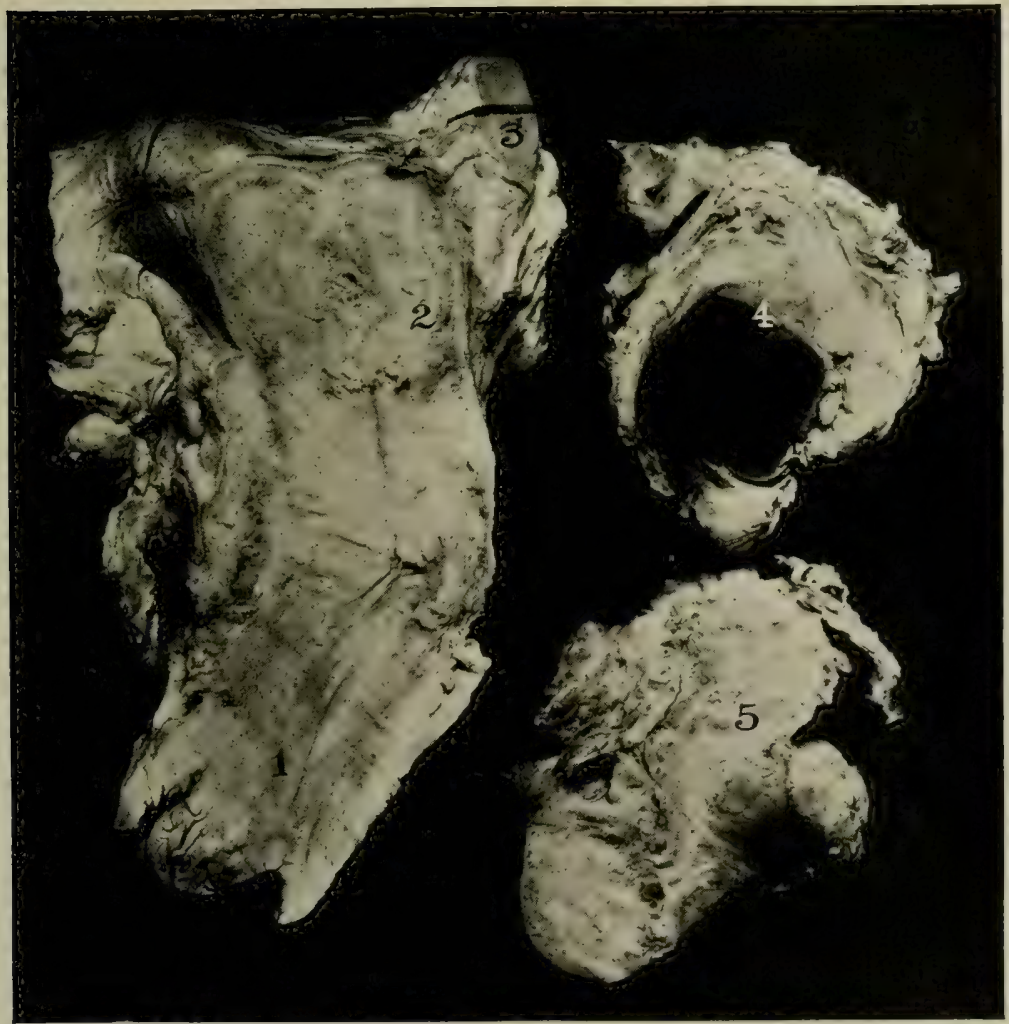

FIG. 158-Tubo-Ovarian Abscess with Pelvic Adhesions. Horizontal section through top of uterus viewed from above.

I, Cervix ; 2, sclerotic uterus with pus in uterine cavity; 3 , right ovary; 4 , cross section through the left oviduct at the ovary. The figure 4 is in the centre of large hematoma and at lower border of this is seen pus; 5, left ovary completely adherent. 


\section{Hydrosalpinx and Dropsy of the Pavilion of the OVIDUCT}

Hydrosalpinx, which is comparatively common in cattle, constitutes one of the common causes of incurable sterility in heifers. It is apparently as common in heifers which have never conceived as it is in cows. The period of invasion of the disease in heifers is not definitely known. Probably it occurs during the nursery period and passes unobserved until breeding age, when it causes sterility, and is then recognized by clinical examination. The disease also arises during pregnancy, although how frequently can not

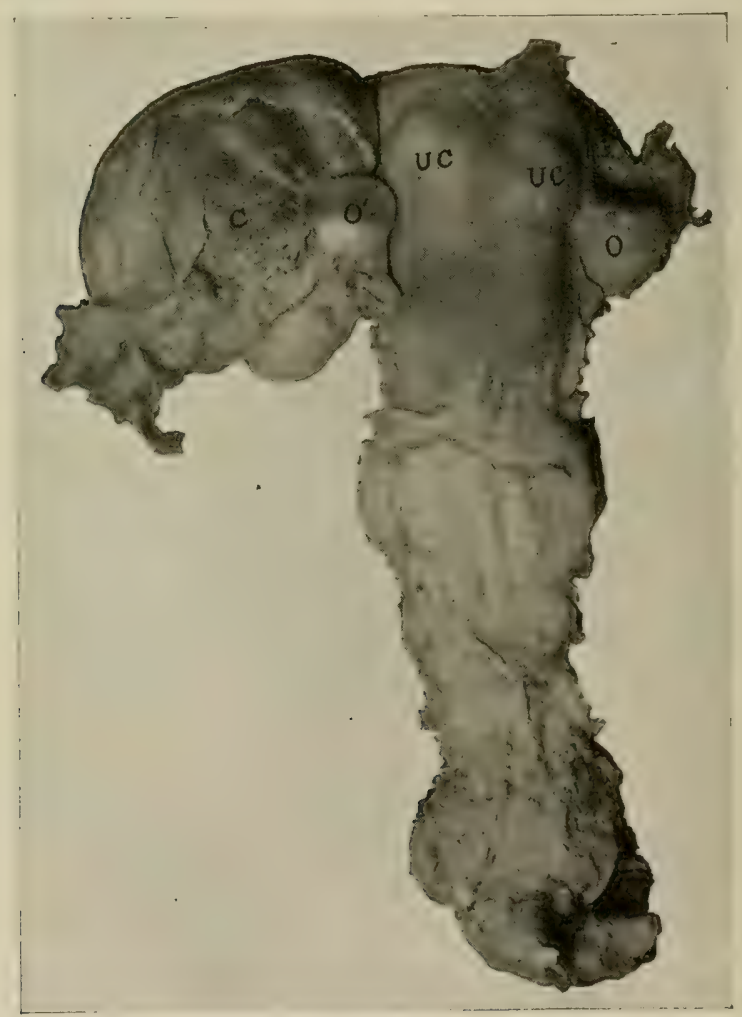

FIG. I59-Cystic Pavilion of the Oviduct.

$O$, Right ovary; $O^{\prime}$, left ovary ; $U C$, cornua ; $C$, cystic pavilion adherent to ovary. 


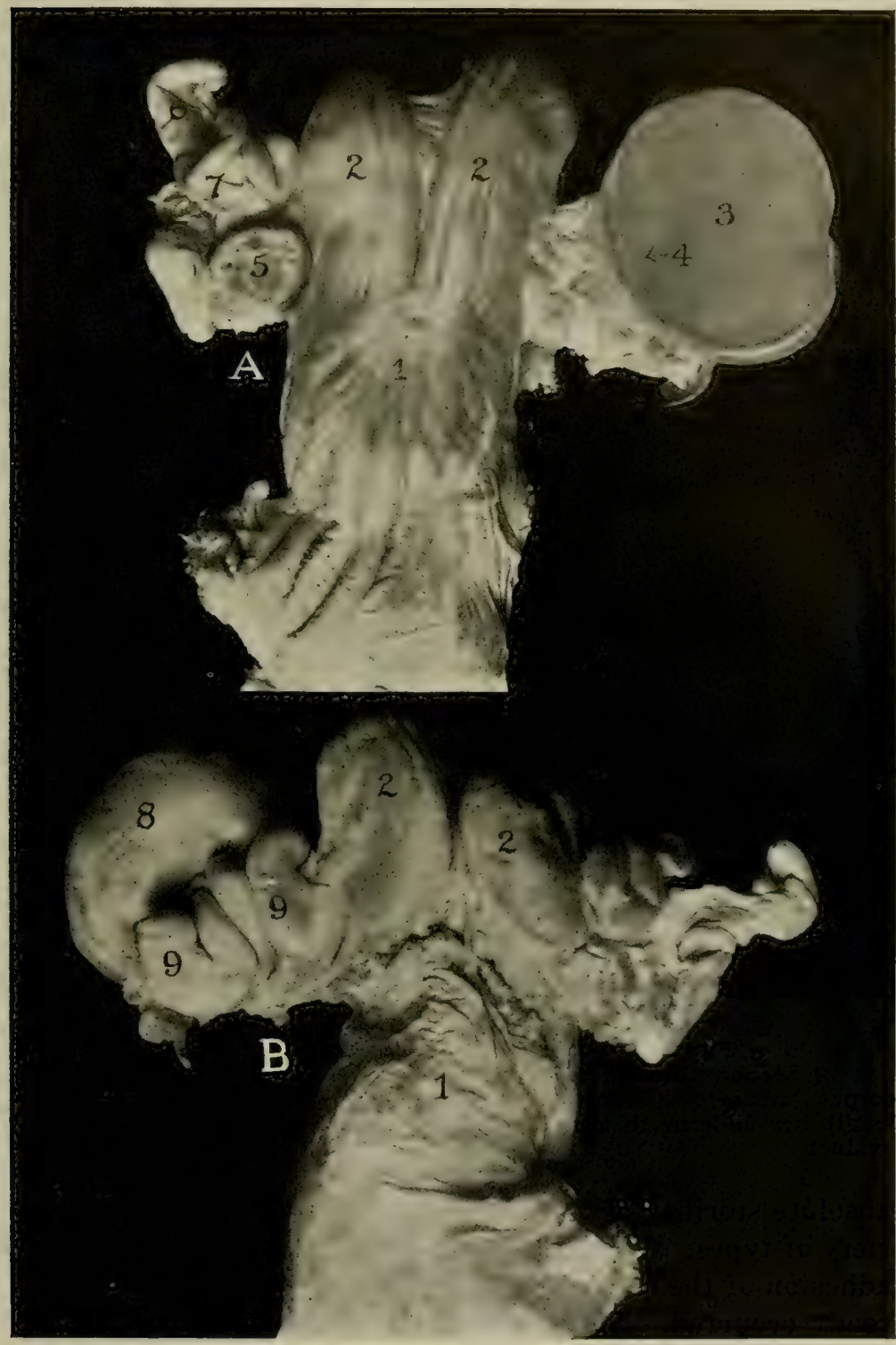

FIG. 160-Cystic Oviducts and Corpus Luteum.

$$
A \text {, Dorsal view ; } B \text {, ventral view. }
$$

$I$, Uterine body ; 2 , cornu; 3 , cystic corpus luteum of right ovary, sectioned : 4, girlde of lutein tissue : 5 , sectioned left ovary, cystic ; 6 , cystic left oviduct ; 7 , ostium abdominale of oviduct ; 8 , ventral surface of right ovary, with cystic corpus luteum ; 9 , cystic right oviduct, buried underneath right cornu and ovary. 
be determined. I have not observed bilateral hydrosalpinx in a pregnant cow, either clinically or in the abattoir. In the sow, however, I have one example, shown in Fig. 161, which indicates very clearly that the disease does occur in the pregnant sow, and presumably in pregnant animals of all species. Frequently it follows retained afterbirth and other types of puerperal infection. Since hydrosalpinx is almost always bilateral, its chief clinical manifestation is

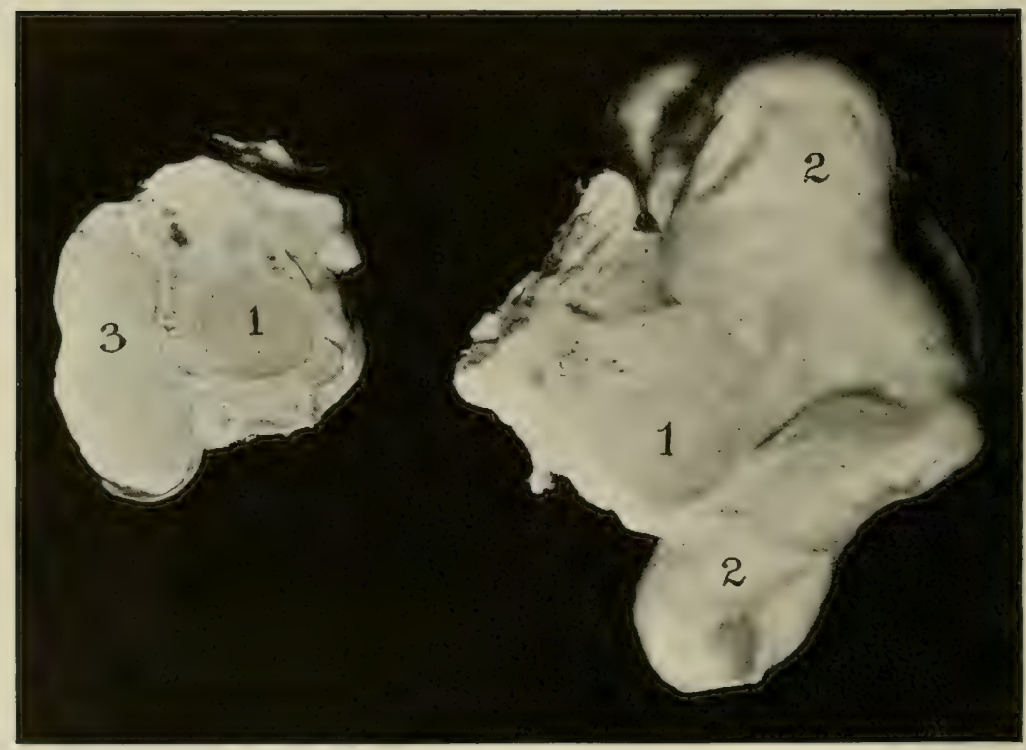

FIG. I6I-Extreme Bilateral Hydrosalpinx.

$I, I$, Section through cystic, adherent ovaries (cystic degeneration of corpus luteum); 2, enormously distended oviduct. About $2 / 3$ of total length lies underneath the portion shown; 3 , section through the smaller oviduct.

absolute sterility of the patient. The disease assumes a variety of types. Generally, when hydrosalpinx is established, adhesion of the ovary within the pavilion of the tube has already occurred. The mesosalpinx is also commonly adherent to the ovary, as shown in Figs. 154, 155 and 156, so that the cystic oviduct is thrown into a convoluted mass in front of and lateral to the ovary, and in some cases above the 
gland. In other instances, the margin of the pavilion adheres to the ovary while the general mucous surface of the funnel remains free. It then participates in the cystic distension and in some cases becomes enormously enlarged, as shown in Fig. 159. In other types of hydrosalpinx, the tube and pavilion remain perfectly free. The ostium abdominale of the oviduct becomes occluded and the pavilion of the oviduct recurved over the ampulla, leaving a knob-like enlargement at the end of the oviduct. It is still adherent by one or two fimbriae to the lateral end of the ovary, but these fimbriae, on account of the weight of the oviduct, may be very greatly elongated so that the cystic oviduct may drop far forward and lie lower down in the abdominal cavity. In order that this may occur, the mesosalpinx must also become greatly stretched as a consequence of the weight of the cystic fluid. Amongst these three most distinctive types there is every possible variation. The condition is almost invariably associated with either a cystic degeneration of the corpus luteum or a fibrous degeneration-sclerosis-of the ovary as a whole. Presumably these changes in the ovary are the result of a passage of infection by contact into the ovary, generally through the wound caused by the rupture of the ovisac at the time of ovulation prior to the period of complete closure of the oviduct.

The diagnosis of hydrosalpinx should be based upon the enlargement of the oviduct as described. Sometimes the cystic condition involves chiefly a small segment of the oviduct; sometimes it involves with great uniformity the entire length. The size of the cystic oviduct will vary between a trifling enlargement beyond normal up to three-quarters to seven-eighths of an inch in diameter or possibly larger. The length of the oviduct is also enormously increased-at times 8 to 10 inches long-especially when the entire duct is free. Extensive adhesions tend to restrain the duct from becoming greatly elongated and throw it into very complicated convolutions. A cystic condition of the pavilion is to be diagnosed largely by its extreme softness and its position as related to the ovary. As shown in Fig. 159, the 
ovary lies behind the cystic duct. Sometimes when the duct is closely convoluted and the convolutions held firmly by adhesions, the mass may be mistaken for a very large ovary. This error should constantly be avoided. The cystic pavilion also is frequently mistaken for an ovarian cyst. This is an unfortunate error because it leads to a false prognosis. Cystic ovaries are not hopeless, but cystic distension of the pavilion of the oviduct is beyond remedy. The veterinarian is also apt to err when the oviduct is free and, owing to extensive degeneration, drops forward beneath the ovary, as indicated in Fig. 160, where it is detected with great difficulty. In one instance I diagnosed hydrosalpinx upon one side, but failed to detect it upon the other side because the oviduct was enormously enlarged and the mesosalpinx so elongated that the oviduct dropped far downward and forward beneath the gland. The result was that an incurably sterile animal was retained in the herd for about a year.

Hydrosalpinx is bilateral in approximately 90 per cent. of cases. Since the prognosis is utterly hopeless, so far as the involved tube is concerned, it follows that the prognosis of hydrosalpinx in general is very bad. In some cases the disease may be confined to the oviduct, and the animal may breed, and occasionally does, upon the sound side. In such a case there is no call for interference. In other cases the disease, especially when complicated with a large cystic corpus luteum, may inhibit ovulation upon the sound side. Relief may be had in such instances through amputation of the diseased tube with the ovary. The veterinarian needs to be very careful in making his diagnosis and to remember that the condition is nearly always bilateral. Therefore it is essential that he examine both sides with very great care. If both ducts are involved, the case must be abandoned. The operation for the removal of the tube and ovary is the same as described in the preceding paragraphs.

\section{Cysts in the MesosalpinX}

Occasionally one finds small cysts in the mesosalpinx. Sometimes they are located about the ampulla of the oviduct and are due 
to cystic changes in remnants of the Wolffian bodies. Since clinically these small cysts may closely resemble hydrosalpinx, they should be examined with very great care. So far as I know, they are never of any consequence in the cow, although in the mare they may become very large and interfere in a variety of ways with the physical wellbeing of the animal, as already related in Chapter XI.

\section{Uterine Infections}

\section{The Diseases of the Uterus of the Heifer Calf and VIRGIN HEIFER}

\section{A. Intra-Uterine Infection and Endometritis}

Numerous heifers, arriving at breeding age, are found to be absolutely sterile. In many of these cases it is impossible to determine the date of invasion. Sometimes, where the records are defective, conception may have occurred and abortion has followed early, though the embryo was not of sufficient size to be recognized. Sometimes, probably, the embryo has not been expelled, but has broken down and has been absorbed. Sometimes the infection may have been transmitted to the heifer by the bull in coitus. In any of these cases the fundamental clinical fact is that, so far as can be seen, the heifer is sterile when she reaches the age for breeding. Upon examining such an animal, little may be found clinically to indicate the character of the disease. The genital organs appear normal upon palpation. Upon post-mortem examination of such animals, the uterus, as well as the oviducts, which have already been discussed, is found to contain infection, and histologic changes have occurred in the genital mucosa. If the heifer is kept over a long period of time and repeatedly bred to the bull, lesions gradually develop which are clinically recognizable. The first of these is an enlargement and atony of the uterus, as shown in Fig. 162, testifying to the existence of endometritis. Clinical examination generally reveals also the presence of cervicitis with thickening and sclerosis of the annular mucous folds of the cervix. These deform and press aside the cervical canal so that it is difficult to pass a sound 
or other instrument into the uterine cavity. Eventually the ovary and oviduct participate in the changes which become clinically recognizable.

The diagnosis of endometritis in the heifer, pending the enlarged flabby condition of the uterus, is not practicable except for the history of sterility and the absence of other evidences of disease. In this respect one finds it impossible to differentiate between endometritis and salpingitis. As a

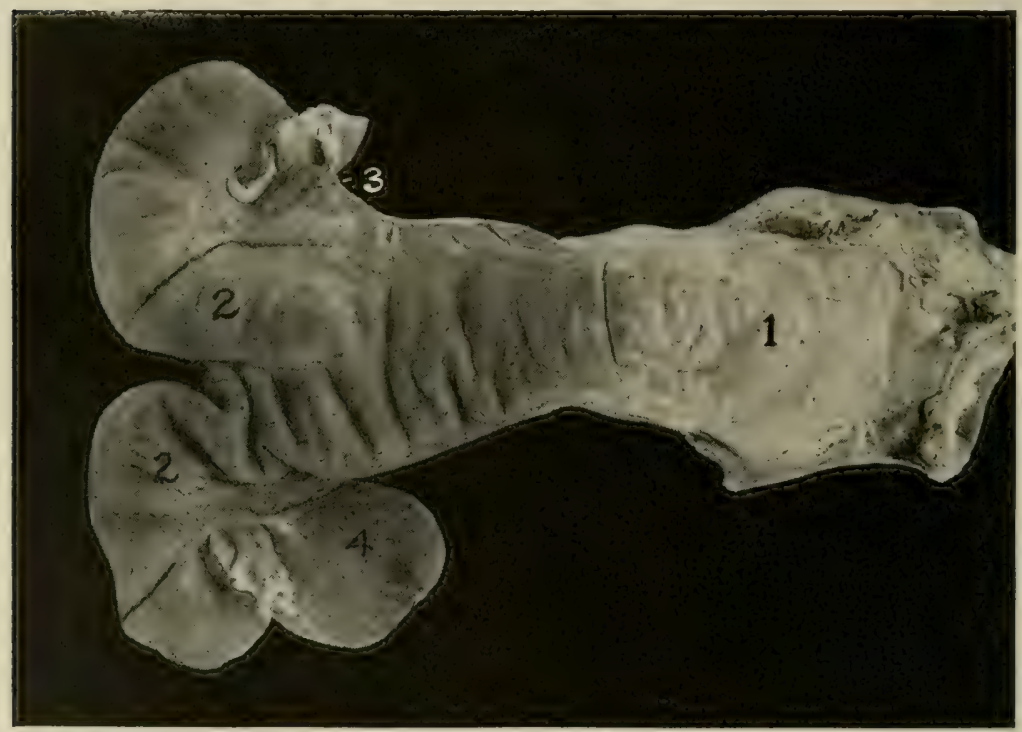

FIG. 162-Chronic Endometritis in Five-Year-0ld, Non-Pregnant Heifer which had Never Conceived.

$I$, Cervix ; 2, cornua ; 3, small right ovary ; 4, left ovary with large, persistent corpus luteum.

general rule, they probably coëxist, since the essential difference is that, when the disease changes are limited to the cervix and uterus, each of which is somewhat amenable to interference, there is hope for the breeding power of the heifer. The principal evidence upon which one may tentatively differentiate endometritis from salpingitis is by the presence or absence of cystic degeneration of the corpus luteum and the irregular estrual cycle dependent upon that 
degeneration. If cystic degeneration of the corpus luteum, with irregular estrual cycle, is present, salpingitis may be diagnosed with considerable confidence. At the same time, endometritis is probably present. If, along with the persistent sterility, the corpus luteum does not undergo cystic degeneration and the estrual cycle is regular, one may be warranted in diagnosing endometritis or cervicitis without

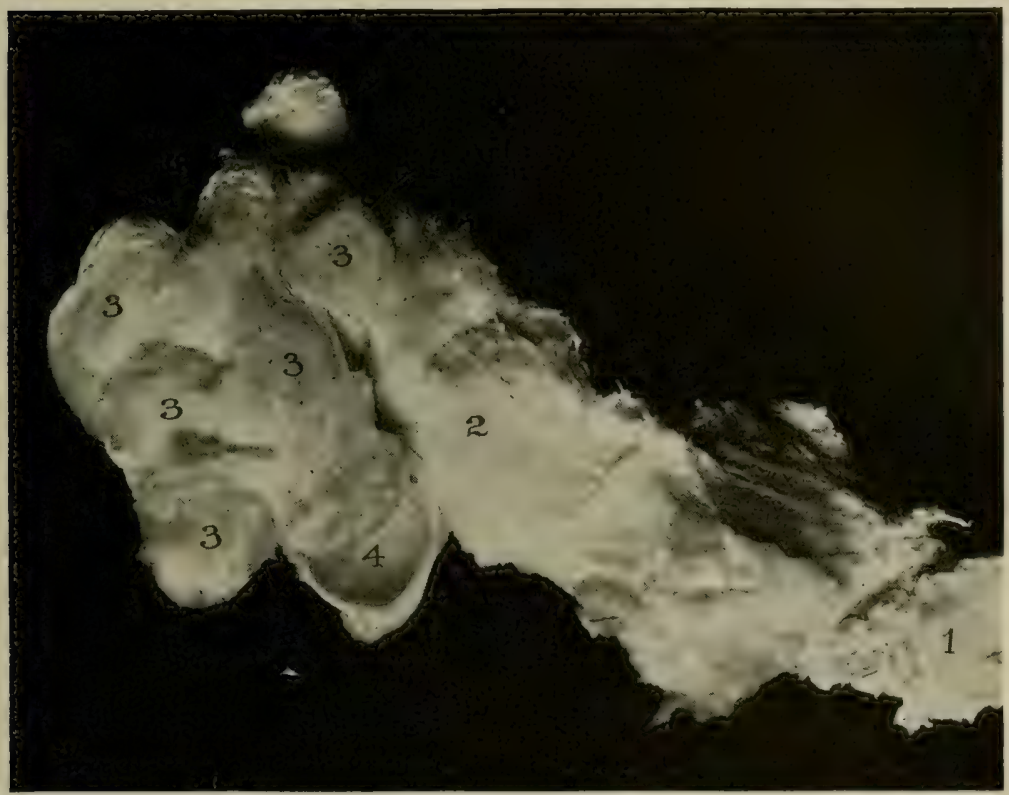

FIG. I63-Complete Pelvic Adhesions with Uterus, Cornua, Oviducts, and Ovaries Firmly Bound Together. Heifer.

I, Cervix ; 2 , uterine body ; 3 , cornua ; 4 , left ovary with cystic corpus luteum.

salpingitis, and a more favorable prognosis may be given.

The treatment needs to be based on disinfection of the uterine cavity. Admittedly it is technically impossible to disinfect the uterine mucosa without destroying it, but there is much that can be done to favor disinfection by indirect methods. Endometritis is almost always associated with cervicitis and largely dependent thereon. Not only does the 
cervicitis furnish a permanent center for the infection, but the swelling of the annular folds of the cervix compresses the cervical canal and interferes with the proper drainage of the uterine cavity, so that infected secretions are held back. Therefore one should dilate the cervical canal in order to bring about improvement of the cervix itself through the application of antiseptics, to establish a practical path through which one can reach the uterus to disinfect it, and, more important still, to obtain a freer opening for the escape of the infection from the uterine cavity.

The dilation of the cervical canal is sometimes exceedingly difficult in these animals. In some cases I have found it necessary to make repeated attempts with the uterine dilators before succeeding in passing through the cervical canal. One must, therefore, have great patience and persevere in the work until the object is attained. Once the dilator has passed through the cervical canal, it can be gradually dilated by opening the instrument. One should not attempt to dilate the canal completely by a single opening of the dilator, but should open it for a time, then close it, revolve it a short distance upon its long axis, and open it again. This process should be lept up until pressure has been brought to bear upon every part. While the jaws of the dilator are forced apart, the instrument should be carefully withdrawn by exerting moderate traction so that the dilation is extended throughout the length of the canal. When the dilation has been satisfactorily completed, the uterus may be douched and the re-flow should be examined carefully for the presence of muco-pus or other evidence of disease. The uterine cavity should then be disinfected, for which purpose 2 per cent. Lugol's solution or $1 / 2$ per cent. chlorazene solution may be used. These must be carefully drained off from the uterus.

Probably a better plan for disinfecting consists of placing in the uterus a heavy, virtually insoluble disinfectant, such as iodoform and bismuth. I have had apparently good results from a heavy suspension of iodoform and bismuth subnitrate in white mineral oil, injected (one or two ounces) 


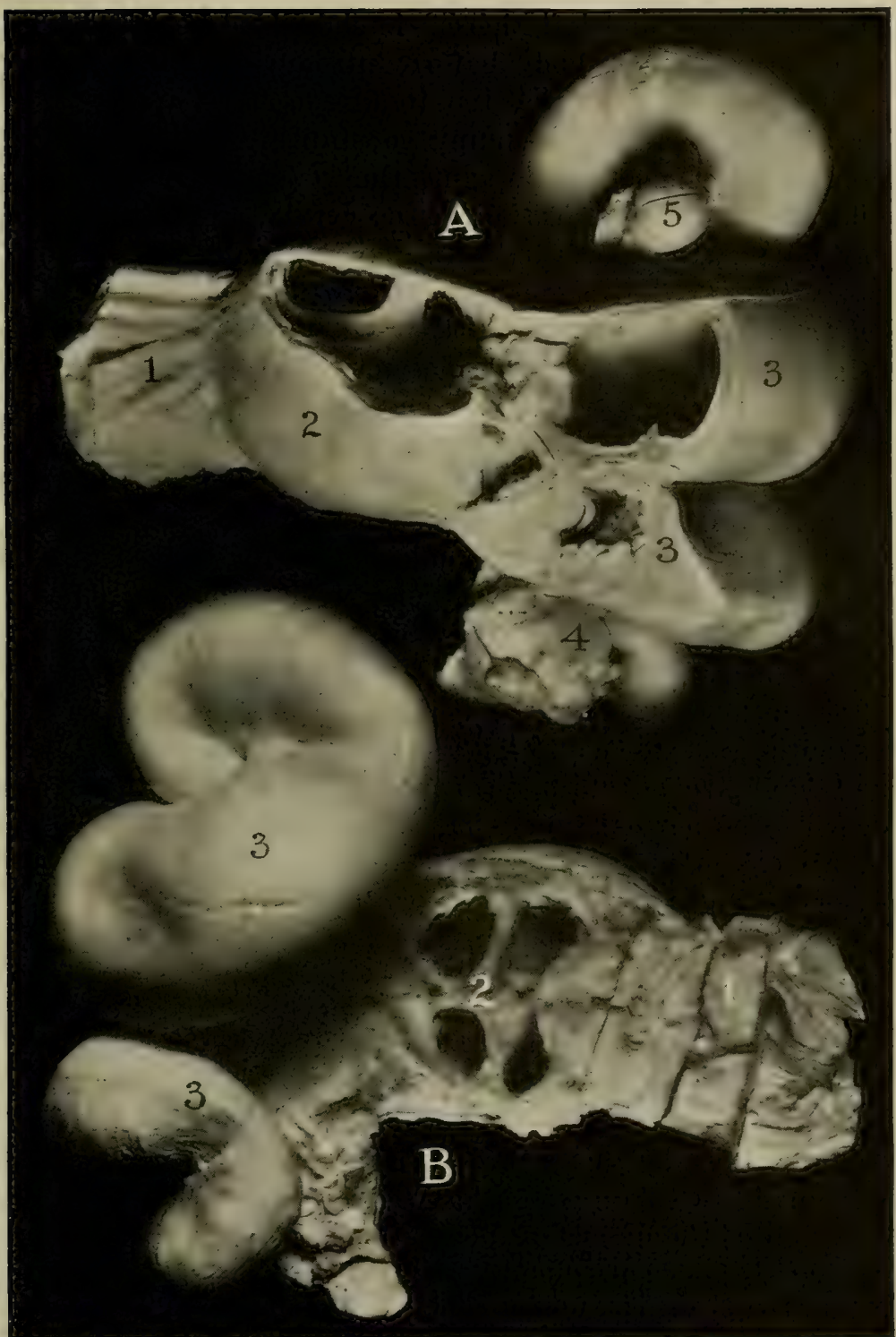

Fig. 164-Arrested Excavation of Muellerian Ducts with Cystic Uterus.

$A$, Viewed from above; $B$, ventral view.

1 , Cervix ; 2 , body of uterus, divided by transverse septa; 3 , cornua, separated from uterine body by septa ; 4, right ovary ; 5 , left ovary. 
into the uterus and left there. It should be heated to the temperature of the body before introducing. It remains in the uterine cavity for ten to fifteen days, undergoing very gradual solution, tending constantly to disinfect the uterine cavity and at the same time exerting a favorable influence upon the infection in the cervix. The treatment may be repeated several times and, when the uterus and cervix seem to be in good condition, may be discontinued for three or four weeks until the iodoform and bismuth have disappeared. The animal may then be bred. The heavy suspension of iodoform and bismuth in oil are best introduced with a metallic syringe, as shown in Fig. 196. The conical nozzle may be used, inserted into the end of the small uterine catheter, and the suspension forced through this into the uterus. If more convenient, the long curved nozzle, which closely simulates the catheter itself, may be used and the suspension injected directly into the uterine cavity with the syringe.

In conjunction with the injection into the uterus, any cervicitis present should have attention. Sometimes there may be present a retained corpus luteum which is interfering with the normal functions of the uterus. In such cases the corpus luteum should be dislodged. If the estrual cycle is normal and the estrum is apparently normal, the corpus luteum should not be disturbed.

\section{B. Pyometra}

Pyometra occurs in heifers which are not known to have conceived. There are several types:

(1) There occurs from time to time an arrest in the development of the ducts of Mueller, to which allusion has been made in Chapter IV, by which the cervical canal or the canal of the uterus or one of the horns does not become excavated, and consequently there occurs an accumulation of menstrual debris, which sometimes becomes infected and takes on the general character of an abscess of the uterine horn. Presumably the infection is lodged within the cavity of the horn or uterus during fetal life or in early calfhood and has finally become active in the presence of large vol- 
umes of menstrual debris. In some cases the infection may invade the cystic horn after puberty, but this cannot be definitely determined.

Clinically the condition is recognized by rectal palpation. Ordinarily attention is drawn to the genital tract by sterility. Sometimes, when the occluded horn becomes greatly distended, it inhibits estrum; sometimes estrum occurs, but conception fails and an examination is made. I have not observed pregnancy in the anatomically sound horn when the other horn is cystic and occluded at its base. It is not quite clear why this should be so if the one horn is normal. Upon rectal palpation the examiner finds one horn normal while the other horn has a narrow hard area at its base and the apex may be distended very greatly with fluid. Those which I have seen have contained from 8 to 25 or 30 ounces and are very tense. It is difficult in some cases to get a clear tracing of the condition, because the cystic horn bulges out over the normal one and covers its own base, rendering accurate palpation very difficult. Therefore it requires patience to reach a proper diagnosis. The ovaries are usually normal.

The actual condition varies greatly. It is to be regarded fundamentally as an arrest in the development in the ducts of Mueller, as has already been described in Chapter IV and illustrated in Fig. 164, which shows a non-infected uterus with several transverse septa in the horns. In the next figure, 165, is shown a typical case of cystic cornu of the type which occasionally becomes infected, the contents becoming purulent.

The prognosis has not been definitely learned for cases in general. It would appear that the removal of the cystic horn with the ovary should bring about favorable results. In one instance I amputated a cystic cornu in which the cyst contents had undergone purulent changes. The animal had not been in estrum for a great many months. As soon as she recovered from the operation, estrum returned regularly, but the result of breeding has not been learned. So far as I could see, she should breed. 
The amputation was made by performing laparotomy in the middle flank region upon the corresponding side, as far posterior as possible. The cystic cornu and the corresponding ovary were removed. Recovery from the operation was prompt and uneventful. This is probably the only effective way to handle the malady.

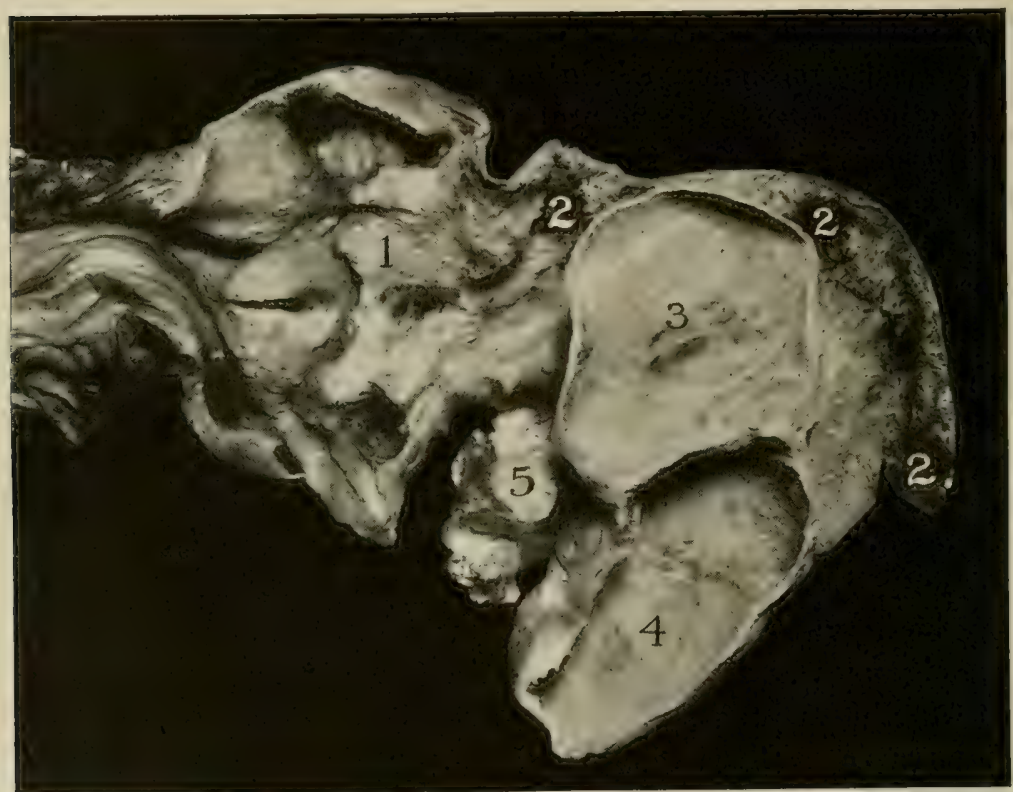

FIG. I65-Atresia of Uterine Horn at Base, with Cystic Distension. Vagina, cervix, and left uterine horn laid open. Top of right cystic horn laid open, showing the flon of the cyst. Viewed from right side

I, Cervix ; 2, left horn laid open ; 3. cystic base of right horn at point of atresia ; 4 , apex of cystic right horn, partly separated from 3 by a transverse septum ; 5, corpus luteum in right ovary.

(2) Pyometra also occurs in the heifer because of imperforate hymen. I have seen a two-year-old heifer suffering greatly from genital trouble which was causing severe straining. There was marked emaciation and general ill health. During the severe expulsive efforts a portion of the genital system protruded between the lips of the vulva. Upon clinical examination it was found that the vulva was 
open and normally developed but the vagina could not be entered, as the hymen was very thick and imperforate. Upon rectal examination the vagina was found to be greatly distended with pus. The distension of the genital tube involved the uterine cavity as well. The hymen was incised, and about a gallon of highly fetid thin pus escaped. Recovery was slow. The ultimate result of the operation, so far as breeding is concerned, is unknown to me, but it is highly improbable that she will breed. The probabilities are that, under the great mechanical pressure of the contents, the pus was forced into the oviduct to cause pyosalpinx. Had the condition been recognized early and relief given, the prognosis should presumably have been good.

(3) Other isolated cases of pyometra occur in heifers the precise nature of which cannot be readily determined. In one instance, when I was called in consultation with a colleague, a valuable heifer was found to have voluminous pyometra with complete paralysis of the uterus and passive retention of the pus. The uterus had dropped far forward into the abdomen, where it could not be efficiently palpated. The cervical canal was open. There was no history of any discharge from the genital tract. Apparently the pus lay passively in the paretic uterus. The clinical history of the animal was uncertain. She had been purchased by the owner some months previously as a pregnant animal. It was impossible to determine whether she had ever been pregnant, since an accurate clinical history of such an animal ordinarily ceases to exist with change in ownership.

My colleague had diagnosed pregnancy because the uterus was enlarged and had dropped far forward in the abdomen and because there was no evidence of pyometra in the way of genital discharge. When it was attempted to examine the uterus by douching in order to determine the character of the trouble, a soft rubber horse catheter was introduced deeply into the uterine cavity and a normal and physiologic salt solution introduced into the uterus by gravity. After two or three quarts had been introduced, the introduction was suspended and an effort made to siphon out the con- 
tents, but when the distal end of the catheter was lowered no fluid was obtained. I began to wonder why it should behave in this way. More fluid was then introduced into the uterus and, while it was still flowing in, the distal end of the catheter was compressed, lowered, and then released. Pus commenced to siphon out and the uterus was relieved of three to four gallons. I have had similar experience in cows where the same error has been made with the siphon and the veterinarian led to believe that no pus or other pathologic contents is present. The difficulty is that, when the fluid is being introduced by gravity, if the veterinarian is not cautions, it all passes at once into the paretic uterine cavity, exhausting the column of fluid in the catheter essential to the process of siphoning.

The prognosis of this rather rare condition is highly unfavorable. I have not had an opportunity for post-mortem examination, but suspect that it originates as hydrometra (which see) and later the fluid becomes infected and purulent, the uterus remaining absolutely paretic. The uterine mucosa is probably completely destroyed, and hence the animal is absolutely sterile. If one has reason to believe otherwise, the only treatment which can be suggested at present is to siphon out the contents frequently, douche the cavity well with disinfectants, such as 2 per cent. Lugol's solution, which must be carefully removed, and then introduce slow-dissolving disinfectants, such as iodoform and bismuth. in the hope that the diseased condition may in this way be overcome.

\section{The InfECtions of the GRAVID Uterus}

\section{A. Apical Endometritis with Necrosis of the Apices of the Fetal Sac}

When considering the development of the fetal membranes in Chapter I, it was noted that the tips of the fetal sacs of ruminants and swine regularly become necrotic, as shown in Figs. 34, 35. This is regarded as normal by some embryologists, but careful study in the abattoir denies this assumption. The necrotic tip is regularly of a dirty yellowish- 
brown color and frequently desiccated. It varies in extent from one-fourth inch or less to eight or ten inches. The necrosed part lies free within the horn, surrounded by a dirty yellowish or yellowish brown flocculent exudate, sometimes watery, sometimes of pap-like consistency. Sometimes the necrotic tip is flat, crumpled and desiccated; sometimes it is soft and pliable, and its lumen persists in continuity with the allantoic sac. Rarely the amniotic tips participate in the necrosis and become adherent in the necrotic allantoic area. The extremities of the amnion thus become fixed in the apices of the allantois, the amnion is incapable of contracting about the embryo, the tension upon the amnion at its attachment to the ventral floor of the embryo becomes too great, the embryo everts, and schistocormus reflexus follows.

Associated with such necrosis of the membranes, there exists as a rule a macroscopically recognizable endometritis of the contiguous uterine mucosa. The mucosa, definitely thickened and edematous, generally bears upon the surface dirty yellowish or reddish sediment, resembling brick-dust and closely adherent. There are wide variations. In numerous cases the exudate is of a dirty yellowish-brown color, tenacious and abundant. In some cases, as shown in Colored Plate III, the exudate fills the non-gravid horn from apex to base and presents the characters of "the exudate of contagious abortion." Between the extremes named every grade of endometritis is seen. The volume and type of endometritis correspond with the necrosis of the tip of the fetal sac. The necrosis of the tip and the endometritis are regularly greatest in the non-gravid horn. It is possible that in some cases the pre-conceptional infection is so great in one horn that fertilization on that side is blocked and pregnancy occurs on the side of least infection. In such case the lesions would naturally be most marked upon the non-gravid side. I think, however, that this hypothesis explains few cases. The more probable cause of the variation is the lesser vitality of the membranes in the non-gravid horn. They are far less vascular and, in harmony with 
general rules of bacterial invasion, should afford less resistance.

The histology of apical endometritis has not been studied. The bacteriology has had almost no attention. My colleagues, Carpenter and Hagan, have obtained chiefly streptococci of the viridans type and paracolon organisms.

The date of invasion of the cornual apices is apparently pre-conceptional. The infection probably enters frequently from the oviducts. Evidently it may also proceed from the cervix. Its significance is not fully understood. When as virulent as shown in Plate III, it must have serious peril for the health of the embryo and apparently endangers the uterus itself. The chief danger to the embryo is the invasion of the fetal fluids through the necrotic area. Since the allantoic fluid regularly extends into the necrotic tips, its contamination is unavoidable. Once the infection has invaded the allantoic fluid, it may grow through the amnion and, reaching the amniotic fluid, be swallowed by the embryo during its constant physiologic process of swallowing its amniotic fluid. It is not necessary that bacteria shall penetrate the amnion in order to reach the amniotic fluid. They may instead be borne in the fluid, through the urethra, bladder and urachus, to the amniotic fluid, and eventually be swallowed.

Apical endometritis of pregnancy, since it is not clinically diagnosable, offers no field for therapeutic effort. So far as known, its prevention is dependent upon the fundamental principle that the health of an embryo is primarily dependent upon the soundness of the genital organs of both parents at the time of coitus.

\section{B. Diffuse Endometritis of Pregnancy. The "Dystocia of Contagious Abortion "}

There is observed in the abattoir an endometritis of the pregnant uterus in which the disease processes are quite evenly diffused throughout the entire uterine cavity. Sometimes the endometrium is swollen and edematous and its vessels are visibly engorged. A yellowish, yellowish-brown, 
or greenish-brown exudate of an extremely viscid, sticky character covers the non-placental portions of the uterine mucosa and the chorion. The fetus may be apparently healthy. In other instances the fetus shows evidences of having long been dead. The fetal and maternal placentae have separated and the fetal membranes are necrotic, soft, and non-resistant. The fetus is soft and only faintly odorous. The uterus is edematous, flaccid, and paretic. The utero-chorionic space is occupied by dirty, grayish, flocculent exudate. There is little evidence of any tendency to expel the fetus.

Clinical observations appear parallel to those of the abattoir. In my companion volume ${ }^{1} \mathrm{I}$ have held that most dystocia in cows is due to a uterine atony dependent upon intrauterine infection in which the fetus participates. Whether the fetus be alive or dead, the uterus contracts feebly, causing tardy dilation of the cervical canal and delayed expulsion of the fetus. Preceding, accompanying, or following the expulsion of the fetus, an exudate in varying amounts is expelled by the uterus. The fetus may perish, to undergo later maceration or emphysema. This type of metritis of pregnancy is usually not diagnosed clinically until its presence is revealed by the advent of dystocia. Its handling then logically fails within obstetrics, where it has already been considered. After the dystocia has been overcome, the metritis is to be handled as advised under "Diseases of the Puerperal Uterus."

\section{Cervical Endometritis}

Cervical endometritis, which constitutes the essential basis in the phenomenon of abortion, is the primary lesion which finally arouses contractions of the ovarian end of the uterus with the expulsion of the uterine contents. Less frequent than apical endometritis, it plays a larger role in interfering with reproduction. Cervical endometritis consists of an ascending or centripetal infection advancing from the cervical toward the ovarian end of the uterus. In

1 Veterinary Obstetrics, 1917. 
many, probably in most cases, cervical endometritis is the result of an extension of infection from the cervical mucosa forward. In the most outstanding cases, the infection evidently extends from the cervical canal, as illustrated in Fig. 174 , in which the cervical seal has failed to form and a metritis has served to destroy the placental relations about the os uteri internum, so that portions of the chorion, some of which are necrotic, protrude through the cervical canal into the vagina. At the same time the embryo may be living and not visibly involved, but ultimately the fetus must participate in the infection and abortion follow.

Cervical endometritis is not always clinically recognizable, but in many cases it is very evident. Frequently the uterine seal is imperfect, is wanting in resistance or density, or may be discolored. In some cases, like Fig. 174, portions of the fetal sac protrude into the vagina. The protruding parts are usually necrotic, but moderate traction ordinarily brings living vascular membranes into view. Such cases are well advanced on the road to abortion.

Cervical endometritis is sometimes diagnosable by means of rectal palpation, owing to the atony of the pregnant uterus. Physiologically the gravid uterus is tonic to the sense of touch, but in some cases of disease the organ is clearly atonic, soft, paretic, and flabby.

So far as known, the infection of cervical endometritis exists within the cervical end of the uterus or in the continuous cervical canal at the time of conception. The infection may exist at the time of coitus or may be transmitted by coitus as a seminal contamination.

In studying the bacteriology of cervical endometritis, most investigators have searched for the $B$. abortus only, and when they have not recovered that organism have reported the findings as negative. A few investigators have reported the finding of paracolon or paratyphus bacilli, $B$. pyogenes, streptococci, etc. The $B$. abortus, according to recorded observations, is frequently present and apparently plays an important part in the metritis, but just what part is not precisely known, because the other organisms present 
have not been studied in connection with it. The researches of my colleagues, Carpenter and Hagan, have thus far indicated that in the cases investigated by them the $B$. abortus is not the dominant organism.

The prognosis of cervical endometritis is unfavorable. The fetus, which inevitably participates in the invasion, may perish and be expelled, may be born highly infected, or with large numbers of bacteria included within the meconium and later break down with septicaemia, dysentery, pneumonia, or pyaemia. In the cow the metritis inevitably persists into the puerperal period or beyond, frequently complicated with retained placenta. Cervical endometritis is not amenable generally to handling during pregnancy. When clearly recognized, in such cases as that illustrated in Fig. 174, where abortion is clearly foretold, the emptying of the diseased uterus should be the first aim. I have practiced, and recommend, douching the uterus with a weak antiseptic solution, such as 0.5 per cent. Lugol's solution, through the open cervical canal. This tends to detach the fetal sac from the maternal placentules and to cause the prompt expulsion of embryo and embryonic sac. Once the uterus is empty, the diseased uterus may be handled and restored to health. The prevention of the cervical endometritis of pregnancy is fundamentally dependent upon the mating of two animals with clean sexual organs.

\section{Septic Metritis of Pregnancy with Uterine Gangrene}

Septic metritis of the pregnant uterus with uterine gangrene is not rare. It bears a close relationship to the diffuse endometritis of pregnancy and represents a more virulent course of infection. As a rule, the existence of intrauterine infection passes unnoted clinically until suddenly the disease breaks out in a storm and its presence becomes evident. Usually it appears at or near the close of the physiologic duration of pregnancy, showing itself in two types.

Most commonly the animal unexpectedly becomes dull. There may be some atonic expulsive efforts. Usually the cervix relaxes and necrotic fetal membranes protrude from 
the vulva. Uterine paresis ensues so quickly that there can be no uterine contractions. Should expulsive efforts be present, they are confined to the contractions of the abdominal walls. The efforts are feeble and futile. If the fetal cadaver is removed, the membranes with the uterine cotyledons, which are necrotic, come away together. The uterine walls are hard and thick. Involution fails; instead, the uterus collapses in response to compression by the surrounding viscera. The uterine cavity may contain an indefinite amount of a thin, black or reddish-black exudate which appears to consist chiefly of disintegrated red blood cells. The exudate is usually fetid.

The prognosis in this group is very unfavorable. A comparatively large percentage of cows survive, but very few recover. Eventually most survivors must go to the butcher because of incurable sterility. The indications for handling consist of the surgical evacuation of the uterus, followed by the general plan for handling puerperal metritis, to be described later.

The other, and less frequent group of gangrenous metritis of pregnancy presents the cardinal symptoms of the gangrenous metritis of the puerperal period, except for the presence of the fetal cadaver with non-dilation or imperfect dilation of the cervical canal. The patient is apparently well and in good condition, until suddenly she appears to be critically ill. The general appearance is one of great depression bordering upon collapse. The pulse is rapid and very weak. The respiration is quickened and shallow. The temperature is erratic and is frequently subnormal. The eyes are sunken and the cornea appears dry and opaque. Tears mixed with muco-pus flow down over the cheeks and face. There is a copious discharge of mucus from the nostrils and of saliva from the mouth. Severe diarrhea is usually present, the feces being watery, dirty black, and very fetid. The animal moves only under compulsion, and then totters. In some cases the cow or heifer is found down and unable to rise only fifteen or twenty hours after she appeared well. Sometimes necrotic fetal membranes protrude 
from the vulva. In other cases vaginal examination reveals necrotic membranes protruding from the cervical canal. Sometimes the cervical canal is dilated little or none at all, but may be open, and the examiner may find that the uterine seal is absent or breaking down and mixed with thin, black, fetid exudate. The cervix is comparatively sound; the uterus, wholly necrotic, is powerless to dilate the cervix. Rectal palpation generally reveals an enormously enlarged, emphysematous fetus which crepitates under manipulation. The massive fetal cadaver may be palpable from the exterior through both flanks.

The prognosis is hopeless. The animal dies within twelve to forty-eight hours.

\section{E. The Placentitis of Pregnancy}

Endometritis can not readily occur without participation of both the placental and non-placental areas. Generally the non-placental areas suffer primarily while the placental structures show an interesting degree of resistance. So there is observed an intercotyledonal endometritis with great volumes of exudate in the utero-chorionic space, while the placental structures are apparently intact and the fetus is alive and healthy. But it is difficult to understand how an important infection can exist for a long period, when of large volume or high virulence, without involving also the placental structures. It appears certain that as a rule the placental areas are the most resistant of the uterine mucosa, and, so far as investigators have proceeded, this view is well supported. In studying the pregnant uterus in the abattoir, interesting lesions are recognizable which serve to explain, or to add interest to, some clinical phenomena.

(a) Peripheral placentitis. As a rule some, many, or all placentules in the gravid uterus show peripheral dehiscence. The chorionic tufts have become detached and lie outside the placental crypts. The separation is usually apparent after the sixth month of pregnancy and becomes increasingly prominent as pregnancy nears its close. Some of the smaller placental areas about the os uteri internum become 
wholly detached, but further forward the larger cotyledons show merely a very few peripheral chorionic tufts displaced from the placental crypts. The displaced tufts are white or grayish-white, due apparently to anemia owing to the interruption of the physiologic placental contact. The phenomenon is so nearly universal that, like the necrotic tip of the fetal sac, it may be designated normal in the sense that is is generally present. Its significance is unknown.

(b) Inter-placental hemorhage with fetal desiccation is a more interesting and at present more important clinical manifestation of placental disease. It has not been shown

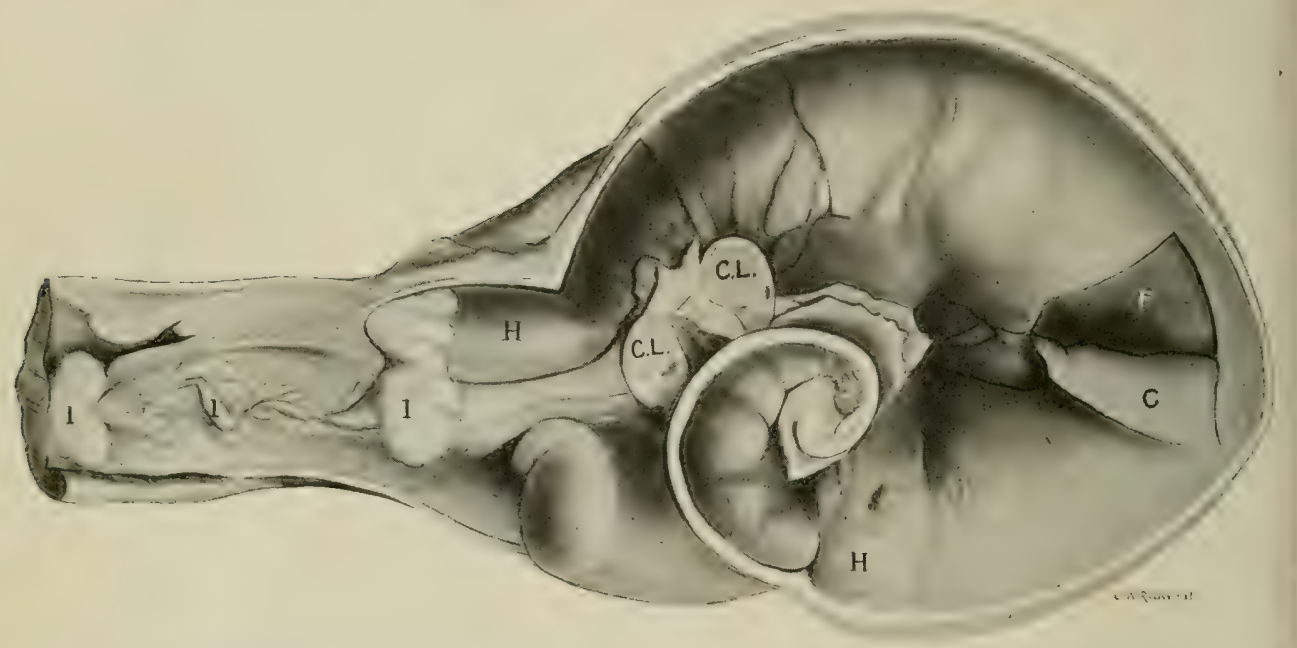

FIG. I66-Inter-Placental Hemorrhage with Desiccation of Fetus, in Early Stages.

$I$, Cervical canal; $H$, hematoma filling uterus and covering fetal sac; $C$, chorion incised and turned back; $F$, fetus ; $C L$, corpus luteum.

to be dependent upon disease of the non-placental uterine areas.

In the abattoir I have observed marked inter-placental hemorrhage involving uniformly all cotyledons. In one well marked case none of the blood had escaped into the uterochorionic space, the peripheral tufts having maintained their contact. In a manner this is a reversal of the peripheral dehiscence described in the preceding paragraph.

It seems perfectly clear that this phenomenon is the initial stage of desiccation or mummification of the fetus. In 
the second stage the hemorrhage is greater, the inter-placental hemorrhage forces the fetal and maternal placentae apart, and the blood forces its way in great volume to every part of the uterine cavity, enveloping the fetal sac in a vast incapsulating hematoma, as shown in Fig. 166. The fetus at once perishes from asphyxia. If bacteria are present, they are non-pyogenic. After the cessation of the hemorrhage, the blood serum is slowly absorbed and the absorption of the amniotic, allantoic, and tissue fluids gradually follows. Finally there remains a hard, dry, rigid, and distorted dark brown fetal cadaver, consisting of the dried skin, bones, and other skeletal tissues with a thin parchment-like membrane

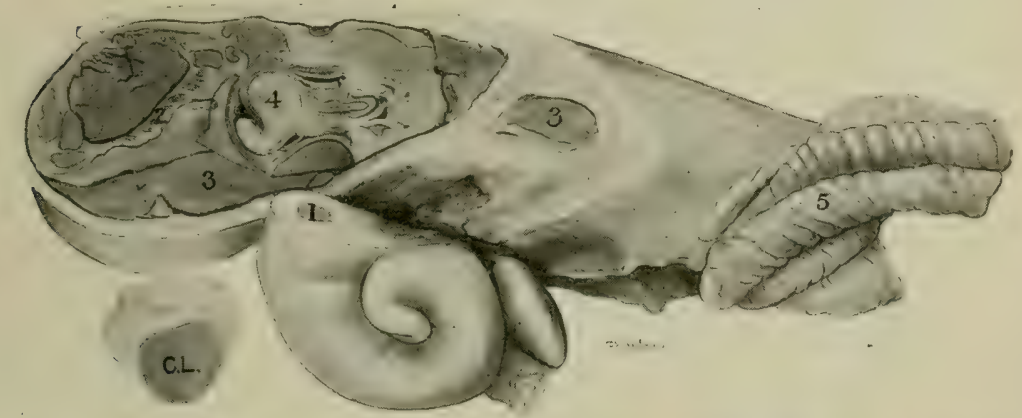

FIG. I67-Inter-Placental Hemorrhage with Desiccation of Fetus. The desiccation has progressed farther than in Fig. 166.

$I$, Hematoma in non-gravid horn ; 2, fetal ribs cut across; 3 , hematoma in uterine body and gravid horn; 4 , cranial bone (parietal?) of fetus, 5 , vagina ; $C L$, embedded corpus luteum of fetal retention.

over them representing the fetal sac, with vestiges of the hematoma. The cervical canal remains sealed; the corpus luteum of pregnancy sinks into the center of the ovary (embedded corpus luteum) there to remain indefinitely, usually as a permanent inhibitory force preventing the expulsion of the desiccated cadaver, and inhibiting ovulation and estrum. This is observed mostly in heifers.

Since the animal has been bred and estrum does not recur, pregnancy is assumed. If the hemorrhage occurs late in pregnancy, the patient shows the abdominal enlargement of pregnancy and the fetus may be recognizable by ballotte- 
ment. If a heifer, her udder enlarges, verifying the assumption of pregnancy. Then comes a recession in the evidences of approaching parturition. The abdominal enlargement recedes imperceptibly for week after week, the enlarged udder slowly resumes its pre-conceptional volume and appearance, and the time for parturition draws near and passes by without trace of sexual function of any kind. The general health is unaffected.

Vaginal exploration reveals a typical uterine seal of pregnancy. Rectal palpation, if by chance made soon after the occurrence of the hemorrhage, reveals the uterus filled with a rather firm, doughy, even mass, deeply embedded within which a fetal cadaver (or sometimes twins) may be recognized. As desiccation proceeds, every stage is encountered

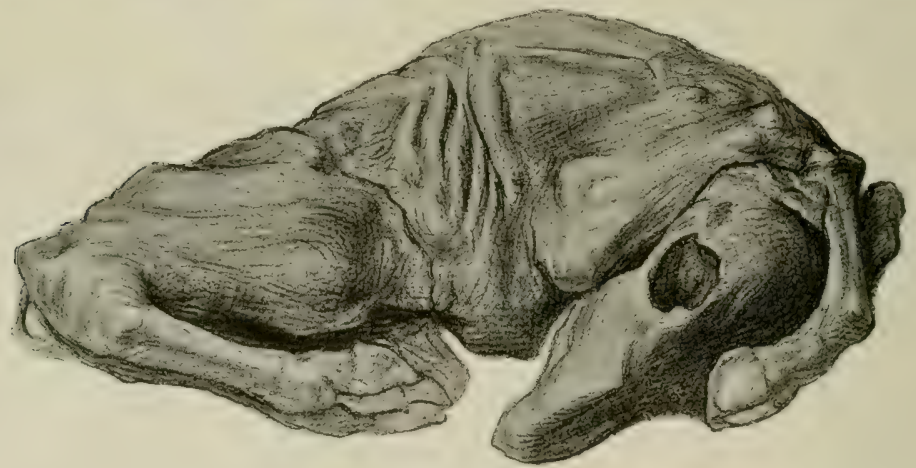

FIG. I68-Complete Desiccation of Fetus following InterPlacental Hemorrhage.

up to the completion of the process, shown in Fig. 168. Then one may palpate per rectum the limbs, ribs, head, hollow orbits, and all skeletal details. The completion of the desiccation will naturally vary. In a fetus twenty-four inches long from nose to base of tail, I think the completion of the process requires six to eight months.

Bang has procured cultures of the B. abortus from the desiccated debris. He does not state that other bacteria were or were not present. I am not aware of other bacterial search. 
The prognosis is fair under surgical interference, but highly unfavorable when left to natural resources.

The removal of the fetal cadaver by mechanical intervention is unjustifiable and highly dangerous. Any attempt to dilate the cervix mechanically and to extract the rigid, angular cadaver is very difficult and full of peril. Pituitary extract has not been tried, so far as I have seen recorded. In large doses, should it cause uterine contractions of a severe type, it would be highly dangerous. Dislodgment of the corpus luteum has promptly and uniformly caused the expulsion of the cadaver in from five to twelve days. The action is very gradual and unaccompanied by any visible danger. In most cases the ovary can be drawn back over the vagina and the corpus luteum dislodged by compression through the vaginal walls. When fetal death occurs late, the ovary may be dragged too far forward for the vaginal method. Removal must then be attempted very cautiously per rectum. It is to be remembered that in long-standing cases the yellow body is deeply embedded and requires firm compression for its dislodgment. Since the resistance of the rectal walls is low, the operator must exercise great care in order to avoid rupture. Using care, I have succeeded in each case without injury to the rectum.

If the dislodgment of the corpus luteum can not be accomplished safely by rectal manipulation, laparotomy should be performed upon the involved side and the ovary directly compressed. Care should be taken to dislodge all lutein tissue, since any remnant may continue to inhibit estrum and serve as a foundation for the formation of a new corpus luteum, which may necessitate a second operation.

After the cadaver has been expelled, the uterus should be closely watched and any remedial measures applied which may be indicated by the conditions present. The expulsion of the desiccated fetal cadaver inevitably causes contusions and abrasions of uterus, cervix, vagina, and vulva. The most prudent action which can be suggested at present is to introduce into the uterine cavity four to eight ounces of white mineral oil or liquid paraffin, to which has been added 
one-half ounce each of iodoform and bismuth subnitrate. This serves to soften and to disinfect any debris left in the uterine cavity, and finally to cause its expulsion.

The prognosis is uncertain. I have been unable to follow my cases properly and have not known accurately the results. They should largely recover their breeding power.

(c) Incarcerating placentitis of pregnancy constitutes the essential basis of retained fetal membranes during the puerperal period. It is unrecognizable during pregnancy and is first revealed when parturition or abortion has occurred. Its intensity and degree of development during pregnancy largely determines the intensity and duration of the retention of the fetal membranes after calving or aborting. Like cervical endometritis, it regularly has its inception at the cervical end of the uterus and radiates toward the ovarian extremity. In abortion occurring the first few months of pregnancy the course of the placentitis and the detachment of the membranes are completed early and the embryo is expelled surrounded by its membranes. Extensive placentitis (cotyledonitis) during pregnancy is incompatible with birth, because the disease causes fetal death. The tendency, therefore, is for the duration of the placentitis of pregnancy and that of the puerperal period to be inverted-the longer the placentitis has existed during pregnancy, the shorter its course after calving or aborting. This rule is greatly disturbed, however, by the termination of pregnancy. The birth or abortion, by causing extreme dilation of the cervical canal, vagina, and vulva, opens the way for fresh infection from without, and causes vital changes in the supply of oxygen to the uterine cavity, with modifications of bacterial activity. The contusions and abrasions incident to the expulsion of the fetus increase the opportunity for bacterial multiplication.

(d) Necrosis of the cotyledons at the cervical end of the uterus occurs but rarely during pregnancy, and then only to a limited extent, without seriously imperilling the life of the fetus. In the non-gravid horn, however, all the cotyledons may become necrotic, as shown in Colored Plate III, 


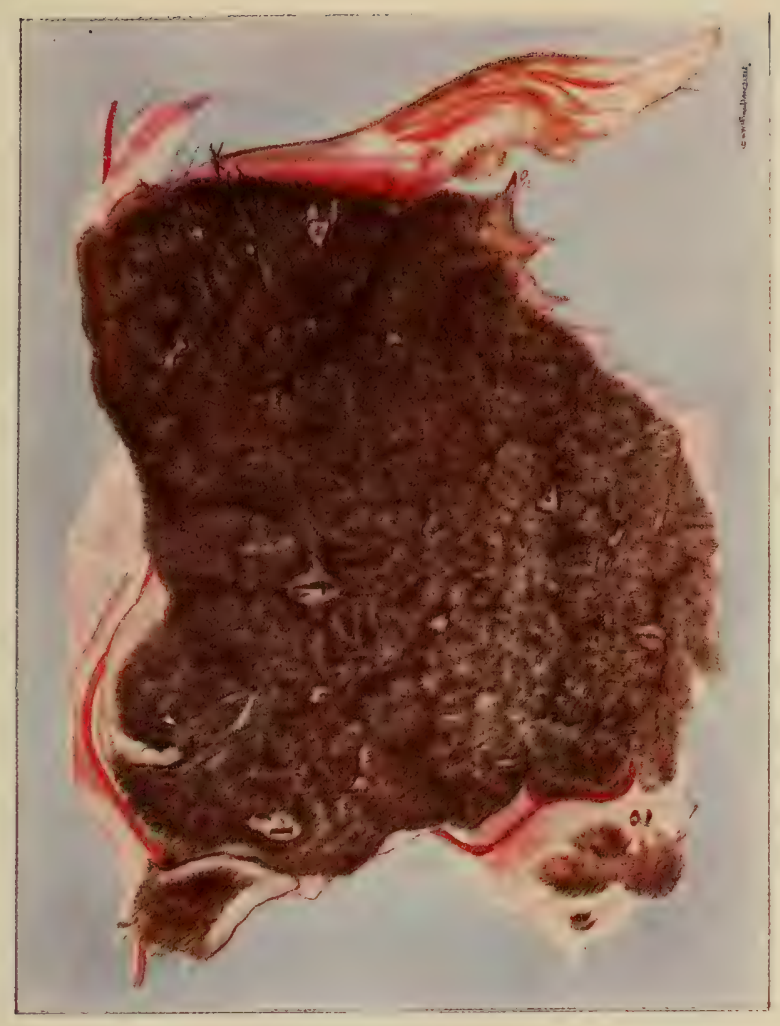

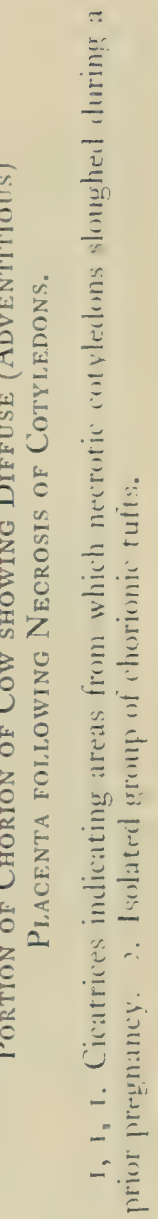



without notable injury to the fetus. This is analogous to the observations upon swine embryos, in which extensive necrosis commonly occurs at both ends of the embryonic sacs without invading the essential placental structures. In this manner the peril to the embryo is limited chiefly to the passage of bacteria through the necrotic area, while the placenta continues to function physiologically. Rarely, the placentitis of pregnancy is of such virulence that all cotyledons become necrotic simultaneously or in rapid succession. The fetus then perishes, the uterine walls are paretic, and, if the mother survives, the embryo macerates or the uterus undergoes abscessation.

(e) Adventitious placental structures regularly follow the necrosis of the cotyledons, whether it occurs during or after pregnancy. Adventitious placentae are not seen in heifers, but are common in cows, where they serve as proof of the prior necrotic destruction of cotyledons. In the uterus of the pregnant heifer, placental contact is regularly limited to the distinctive cotyledonal or placental areas. With each succeeding pregnancy, there is an increased probability of necrosis of some of the cotyledons about the os uteri internum. In the area denuded of cotyledons, adventitious placental structures develop, presenting macroscopically the appearance of the diffuse placenta of the mare. When cotyledons are destroyed, there is no visible effort at compensational hypertrophy of the remaining cotyledons, but the compensation is effected through adventitious activity of the intercotyledonal endometrium.

The adventitious placental structures tend to invade the endometrium about the os uteri internum and to form over the os a virtually continuous placental contact, constituting in effect a placental seal, separating the cervical canal from the utero-chorionic space. When parturition begins, the adventitious placental structures must give way before the cervical canal can dilate and the parturient rent occur in the chorion. The adventitious placental structures ordinarily radiate from the os uteri internum for only four to ten inches, though occasionally they involve the entire uter- 
ine body cavity and may extend deeply into one horn. In one abattoir cow I observed a complete adventitious (diffuse) placenta involving the entire endometrium. While adventitious placentae are always the consequence of cotyledonal necrosis, extensive death of the cotyledons is not ordinarily followed by adventitious placenta; instead, the extensive disease commonly includes the endometrium and either kills the patient or renders her absolutely sterile.

(f) Calcification of the placenta occurs very rarely. I have observed but one case, that being in an abattoir animal. The calcification, which was very marked, involved uniformly all cotyledons. The placental stratum was pale yellow, resistant and gritty. There was no evidence that the calcification had interfered with embryonic development. The cause was not clear. It was possibly the result of interplacental hemorrhage stopping short of placental dehiscence and embryonic death. Had parturition occurred, retained fetal membranes with necrosis of the cotyledons would probably have followed.

(g) Specific placentitis, due to placental tuberculosis and actinomycosis, doubtless occurs rarely and, when pregnancy terminates, leaves behind an obstinate retention of the fetal membranes, the character of which is not clinically apparent. Naturally the specific disease promptly leads to incurable sterility.

\section{InfECtions of the Ovum, Embryo, AND Fetus}

\section{A. Death of the Fertilized Ovum}

The death of the fertilized ovum is not directly recognizable clinically, but its occurrence must be admitted. Ovarian infection evidently causes death of the ovum within the ovisac. When an ovum passes from the ovisac into a virulently infected oviduct, the cell is immediately placed in great peril and may perish prior to fertilization. Examination of infected oviducts after slaughter of the patient shows that the highly motile spermatozoa move through the badly infected tube and reach the pavilion ready to fertilize the ovum as soon as it is discharged. Presumably this occurs 
often, but the newly fertilized egg has little, if any, more power of resistance than the spermatozoön and unfertilized ovum each possessed prior to their union. It is an indisputable clinical fact that salpingitis generally prevents recognizable pregnancy. This renders it reasonably clear that the infection in the oviducts or uterus kills the spermatozoön or ovum. The recognizable destruction of the embryo or fetus is so common that the unseen death of its earliest stages must also be frequent. Any infection capable of causing the death of an embryo or fetus must logically be more readily able to cause death of the far less resistant organism. Thus a heifer is bred possibly eight to ten or more times at regular or irregular intervals. Sometimes she may appear pregnant for one to two or more months. Finally she recognizably conceives and pregnancy continues one hundred to two hundred or more days, when she is observed to expel a fetal cadaver. One death of a fertilized egg has been observed, while many deaths during the earlier periods have passed unseen. The deaths of the spermatozoa and of the unfertilized or fertilized ovum are far more numerous and cause infinitely greater economic losses than do abortions. In many valuable herds of pedigreed cattle, an average of three copulations is necessary for each recognizable pregnancy: there is an initial failure in 67 per cent. of attempts at reproduction.

The farce of curing the disease or preventing the death of the ovum, spermatozoön, or fertilized egg by measures taken after coitus, as is so common with abortion, has not become established. The avoidance of such death clearly lies in the fundamental principle that the physiologic reproduction of young rests upon the sexual contact of two healthy individuals.

B. Death of the Embryo with Persistence of the

Embryonic Sac. Cystic Mole

As soon as the embryo has formed and its sac has acquired recognizable dimensions, embryonic death may be clinically observed if accurate attention is given at the criti- 
cal period. The recognition becomes largely a question of chance. Most embryonic deaths pass unseen. In such early deaths the embryonic sac is by far the greatest and most conspicuous part. Frequently the embryo dies and all traces of it disappear while its sac persists and apparently continues to grow. The embryonic sac retains its usual form, but is without evident placental structure, and apparently maintains its growth and existence by absorption of liquids from the uterine cavity. One of these is illustrated in Fig.

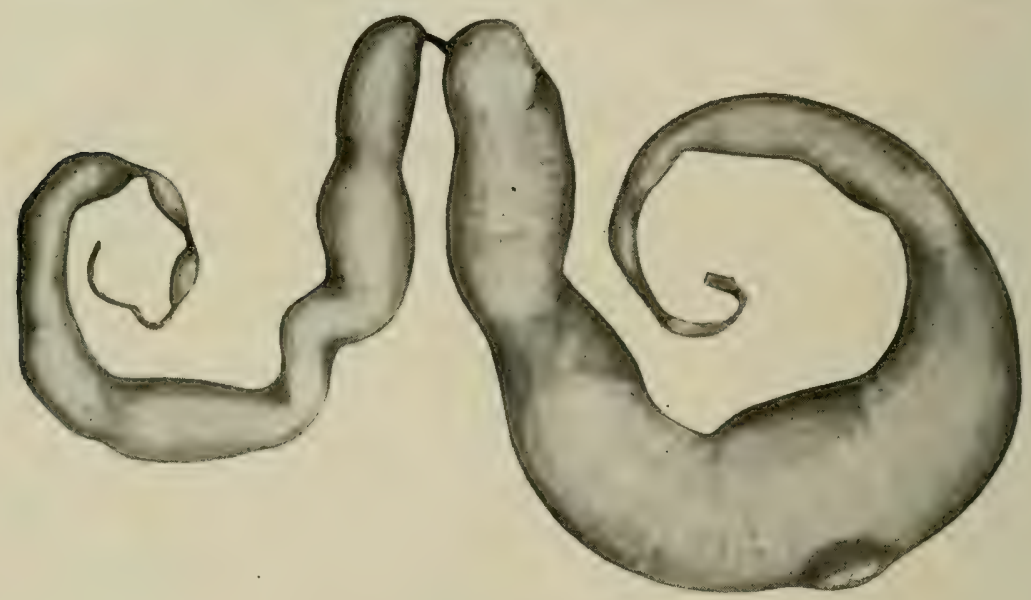

Fig. 169-Cystic Mole.

Total length 46 inches; circumference, 7 inches. At the top of the gravid branch of the fetal sac is seen a thickened spot, apparently marking the former location, of the embryo. Compare with colored plate II. At the cervical end of the uterus, the embryonic sac is necrotic and desiccated. The necrotic tips are prominent. Sac removed 200 days after conception.

169. In this instance there remains a thickened area which faintly suggests the prior location of the embryo. The cow was examined at about sixty days after coitus and pronounced pregnant. The cervical canal was sealed, the uterus enlarged and distended in a manner typical of pregnancy of that duration, a typical corpus luteum of pregnancy was present, and estrum was absent. The general signs of pregnancy failed to advance parallel to the duration of time. Later an examination disclosed the nature of the condition, and the "mole" was removed. 
The cystic mole grows slowly and soon fails to maintain the degree of distension of the uterus characteristic of the advancement of pregnancy. Finally its growth becomes halted and its size may continue static for an indefinite period unless the uterine seal is imperfect or becomes destroyed. Then its expulsion follows. The expelled cyst commonly shows two unequal parts representing the gravid and non-gravid horns, joined by a narrow, sometimes necrotic, isthmus, representing the area contiguous to the os uteri internum.

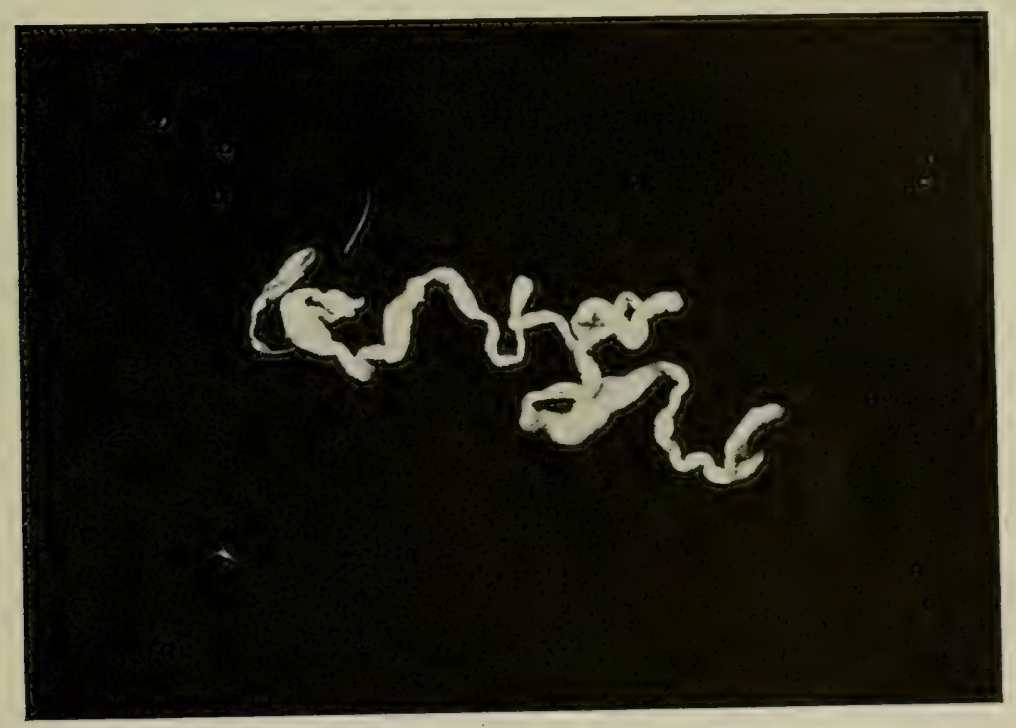

Fig. I70-Desiccated Mole. Cow.

The embryo had perished at probably thirty days or less; the sac later became necrotic and desiccated. In douching the uterus it was caught in the fenestrum of the catheter.

More frequently the cystic mole becomes necrotic quite early and undergoes a desiccation similar to the necrosis of the tips of the embryonic sac. The elongated sac collapses. dries and shrivels until it becomes a flattened, wrinkled, yellowish-brown cord: In such case the uterine seal is absent, either having failed to form or, if formed, having been destroyed. Clinically I have seen the desiccated sac protrud- 
ing from the cervical canal into the vagina. In douching a uterus, the dry structure is sometimes drawn into the fenestrum of the uterine catheter, and when the instrument is withdrawn the structure is pulled out.

In all cases of desiccated mole I have seen, there was present a well marked pyo-cervicitis with slight purulent endometritis. When recognized, the mole should be removed and the associated cervicitis and endometritis handled according to indications. It is evidently imprudent to breed such an animal until after thorough disinfection of the uterus and cervix.

\section{Death and Maceration of the Embryo or Fetus}

As a rule, when infection advances from the ovarian end of the uterus or exists in the central area and causes death of the embryo or fetus. the cadaver is not expelled because the uterine walls are rendered paretic. The same result is attained when infection, advancing from the cervical end of the uterus, proceeds so rapidly that uterine paresis is induced quickly. The embryo or fetus then undergoes maceration. If the mother survives, either the pyometra of fetal decomposition or uterine abscessation follows.

(a) The pyometra of embryonic or fetal decomposition is comparatively common in cattle. It occurs at any time after pregnancy is clinically recognizable, up to the completion of the ordinary duration of pregnancy. When occurring very early, the pyometra may be quite limited. There may be an inconspicuous purulent discharge, absence of estrum, and non-interference with general health. It is most frequently seen in heifers. In the heifer rectal palpation generally reveals a small uterus, distended and rounded by purulent contents. The uterus is ordinarily free from adhesions. No trace of a fetal cadaver is recognizable clinically. Upon post-mortem the uterus is found distended with pus and containing skeletal fragments, such as the tiny elongated ossification centers of the long bones. I have seen these less than one-half inch long. In one case the heifer had been bred about one year previously and had 
been supposed pregnant until near the time for parturition. The pus was thick and fetid. The pyometra had extended into the oviducts (pyosalpinx) and had irrevocably destroyed her breeding life.

Embryonic maceration with pyometra probably causes those cases of extensive pyometra in heifers, to which allusion has already been made, and those in cows accompanied by such complete uterine paralysis that no genital discharge

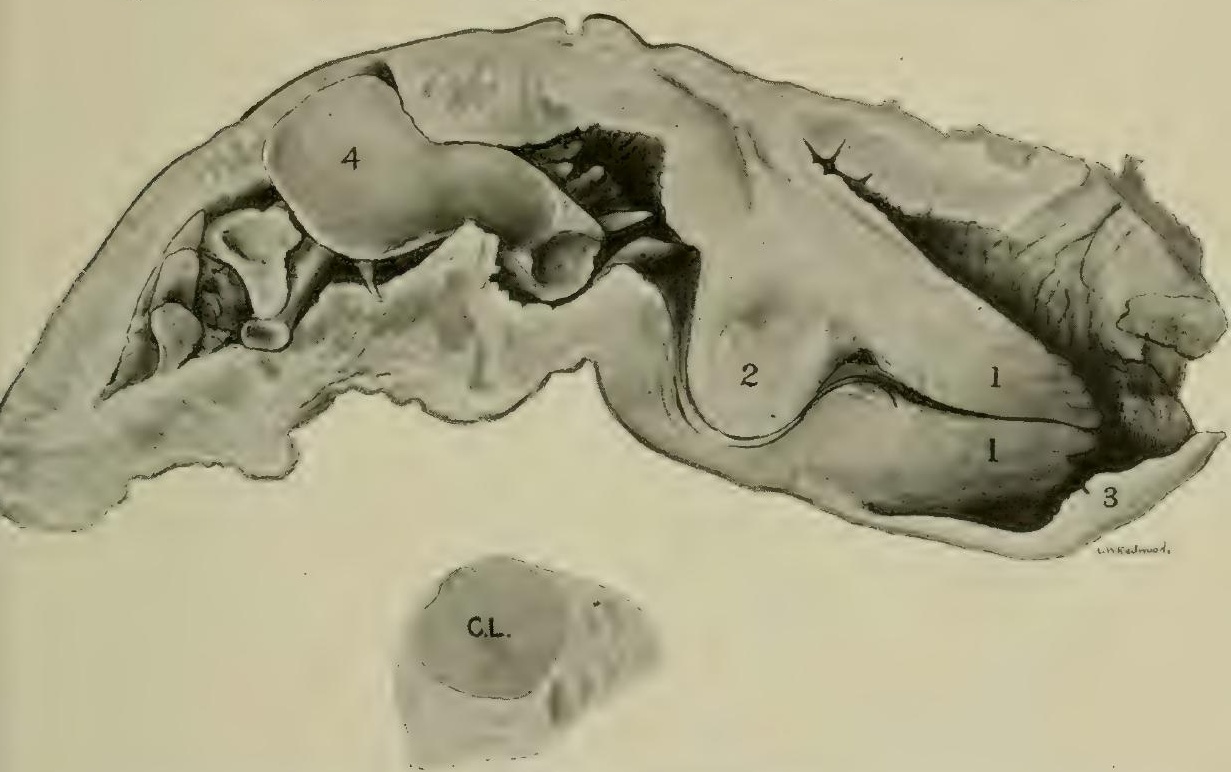

FIG. 17I-Maceration of Fetus.

$I$, Cervical lips ; 2 , greatly enlarged, sclerotic annular fold pushed across the cervical canal ; 3 , vagina ; 4 , parietal bone of embryo ; $C L$, embedded corpus luteum of pyometra. The uterine walls are sclerotic.

is evident. In these cases, if the pyometra is due to embryonic maceration, the embryo is so small at the inception of the pyometra that its skeletal debris is not recognizable clinically during douching.

When fetal death and maceration occur later in pregnancy, copious discharge of fetid pus commonly follows. The fetal debris is too gross and angular to be discharged with the pus. The soft tissues of the cadaver dissolve or 
become suspended to constitute part of the pus. The bones separate at their epiphyses but fail to dissolve. I have observed cases where fetal death had occurred two years prior to the examination. The condition of the fetal bones appeared static. The bones become closely packed together in a somewhat irregular oblong mass in the base of one horn. The interstices between the bones are largely filled with thick, desiccated, intensely fetid pus.

The diagnosis of this type of fetal maceration is comparatively simple when the general outlines of the condition are known, but is often quite difficult for the beginner. There is a history of apparent pregnancy following breeding. Later the progress of pregnancy apparently halts and a highly fetid purulent genital discharge ensues. Estrum is absent. The genital discharge may be sanious, owing to pieces of the jagged fetal bones lacerating the endometrium and causing hemorrhage. Palpation of the uterus per rectum reveals a hard, irregular enlargement in one horn (except in maceration of bicornual twins). A careful study of the enlargement shows that the parts of the tumor move upon each other with definite crepitus. The cervical canal is generally narrow, and the cervix hard. If a catheter is introduced and the uterus douched, the pus may be largely washed out and the size of the mass rendered slightly more distinct. The bones then move more freely when palpated, rendering the diagnosis clearer. In one instance, in a tuberculin reactor, the fetal mass was mistaken for uterine tuberculosis. Uterine tuberculosis, however, is usually symmetrical. The actual differentiation lies in the recognition of the fetal bones. In another case the admixture of blood with the pus caused a diagnosis of uterine tumor. This error should have been avoided by more careful palpation. In the earlier stages fetal maceration and fetal desiccation may be confused. In desiccation the hematoma dims or completely hides the outlines of the fetal cadaver; in early maceration the fetal outline remains. In maceration the cervical canal is generally or always unsealed; in desiccation the uterine seal is ordinarily perfect. The progress of 
desiccation is illustrated in Figs. 166-168. An old uterine hematoma of puerperal origin may also lead to confusion in diagnosis. The uterine hematoma is firm, doughy, and generally regular in outline. I have met one old, greatly desiccated, tesselated hematoma of the uterus which clinically might well have given trouble in diagnosis and might have been mistaken for the skeleton of a fetus. But the crepitus is absent. The history that the animal has or has not been bred may aid, though this is sometimes erroneous and adds to the confusion. The prognosis in advanced fetal maceration is virtually hopeless for the breeding life of the animal. When it has continued for some months, the endometrium is ordinarily destroyed and the suppurative process has extended into the oviducts, inducing incurable pyosalpinx. I have not known a cow to conceive after having suffered from prolonged fetal maceration. Possibly some of them may breed.

The handling of fetal maceration offers mary difficulties. The cervix is usually extensively inflamed and sclerotic, and the dilation of its canal is difficult and dangerous. Forcible dilation is liable to cause serious lacerations, or the sclerotic tissues may rupture, causing an opening into the peritoneal cavity. Uterine contractions tending to expel the cadaver may be induced by dislodging the corpus luteum, but the corpus has sunken deeply into the center of the gland (the embedded corpus luteum of pyometra) and is difficult to dislodge. Sometimes the ovary is dragged far downward and forward in a manner to render it difficult to carry the ovary back over the vagina through the wall of which vigorous compression can be exerted without danger. The corpus may be dislodged by compression per rectum, but this requires extreme care in order to avoid severe or dangerous rectal lesions. The corpus may always be dislodged by performing laparotomy, inserting the hand into the peritoneal cavity, and compressing the ovary directly. But the succeeding uterine contractions will generally fail to expel the cadaver. The jagged bones become caught in the uterine walls, causing wounds and preventing expulsion. The 
same difficulties face the veterinarian when he attempts to bring about the expulsion by the administration of pituitary extract. The contractions of the uterus can be induced, but they fail to evacuate its contents. I have removed the skeletal debris by hysterotomy, but the operation was difficult and the result was failure. Possibly further efforts at hysterotomy, or rather hysterectomy, may develop a practical technic associated with success. From my experience I believe the more hopeful plan to be the amputation of the involved horn with its oviduct and ovary, leaving reproduction to the other horn and ovary, which may have escaped hopeless injury. When this is undertaken, great care must be exercised to avoid the escape of any of the uterine contents into the peritoneal cavity. The abdominal incision must be made upon the involved side as far posterior as practicable upon the middle line of the flank. That is, the abdominal incision must be made as near to the uterine attachments as possible. Even then, it is difficult to lift the involved uterine horn out through the wound, and virtually impossible to operate within the abdomen.

Attempts at handling with a view to the restoration of breeding should not be undertaken except in animals of great value. Others should be prepared for slaughter by the most economic source. Where there is old-established maceration of the fetus, the animal is frequently in good beef condition and the repulsive skeletal cadaver is so walled off that there is no logical objection to the use of the meat as human food. The uterus should be regarded as an abscess cavity.

(b) Abscessation of the gravid uterus occurs when the fetus macerates and the cervical canal undergoes atresia to such a degree as to prevent the escape of pus through the genital passage. The occlusion of the cervical canal occurs by two distinct processes.

(1) Abscessation of the uterus, with fetal retention, occurs when the fetus dies and the cervical canal becomes closed as a result of severe cervicitis. The occurrence is at first without notable clinical symptoms. The animal has 
been bred and is presumably pregnant, but the symptoms of advancing pregnancy are absent or, if the pregnancy is well advanced when the fetus dies, the symptoms are retrograde. In one of my cases the cervical atresia was incomplete and some portions of the fetal membranes protruded from the vulva, but the constricted cervical canal afforded insufficient exit. The cervical canal became blocked, possibly by a large portion of the fetal cadaver. The uterus adhered to, and finally opened into the rumen. Fetal debris passed into the rumen and caused several attacks of fetid diarrhea. Whole grains of corn (maize) and oats entered the uterus. The heifer suffered little in her general health, milked fairly, grew fat, and was sold for beef. In a second case, from the abattoir, the uterus adhered to the urinary bladder, as illustrated in Fig. 172. The general tendency appears to be for the uterus to adhere to, and rupture into, one of the hollow viscera. Which one, will depend somewhat upon the stage of pregnancy at which maceration and abscessation occur. In early pregnancy the bladder or rectum forms the most probable outlet; later in pregnancy, after the fetus drops forward, the rumen is the most logical viscus into which the uterus may empty its contents.

(2) Uterine torsion regularly causes occlusion of the cervical canal. Most patients die unless surgically relieved, but rarely the cow survives and the fetal cadaver macerates. In some instances there occurs a transverse rupture of the genital tube in the vagina, cervix, or uterine body, and the uterus with the fetus drops downward upon the abdominal floor. The fetus macerates but the ruptured end of the uterus is closed by the enveloping uterine ligaments like the mouth of a bag tied with twine. The uterine walls retain some life through one or more of the uterine vessels, the organ adheres to the abdominal floor, and finally the abscess points externally and the fetid fetal debris drops out. Abscessation of the gravid uterus is hopeless so far as the breeding life of the animal is concerned. The adhesions incident to the abscessation bring about serious nutritive dis- 


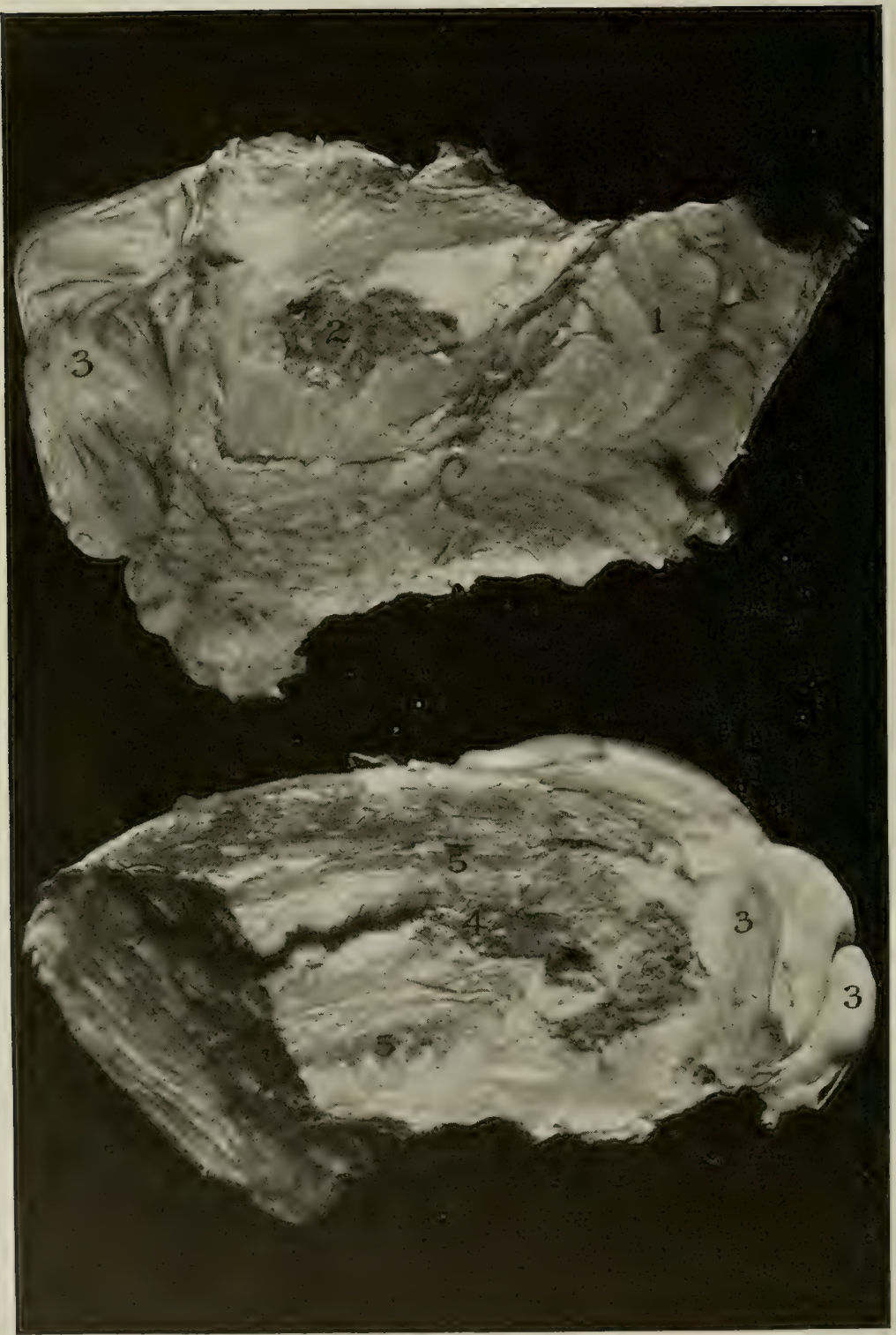

FIG, 172-Macerating Embryo sloughing into Urinary Bladder. $I$, Cervix; 2, embryonic debris with pus in body of uterus seen from above; 3 , cornua ; 4 , embryonic debris in the urinary bladder, after sloughing through its fundus, seen from right side after sagittal section ; 5 , muscular walls of urinary bladder. 
turbances which commonly destroy the value of the patient for other purposes.

\section{Emphysema of the Fetus}

As a rule the bacteria which infest the utero-chorionic space of the pregnant cow, and which tend to invade the fetus and destroy its life, do not form gases. Instead they most frequently cause a dry necrosis (necrosis of the tips of the embryonic sac) or induce maceration of the fetus. The rapid multiplication of gases within the sealed uterine cavity or the contained fetus is virtually incompatible with the life of the mother unless the cervical canal is open and establishes a free vent for the gases. Usually the crisis of uterine rupture is not reached because the patient succumbs to sepsis.

Emphysema of the fetus occurs in all species of animals, usually near, or at the end of the normal duration of pregnancy. The emphysema is apparently the result of secondary invasion of a recent date. The life of the fetus has ordinarily been destroyed and the uterine seal broken down by other invaders, preparing an open way for the entrance of the gas producers. When the fetus is emphysematous, the cervical canal is ordinarily open but not dilated. Sometimes, as in cases of torsion of the uterus with emphysema of the fetus, the cervical canal is mechanically closed by the torsion. The mechanical injury to the cervix destroys its resistance to invasion, and emphysema results. Sometimes under these conditions the emphysema becomes so great that the fetus occupies almost the entire abdominal cavity and rupture of the uterus appears to be averted chiefly by the support afforded by the contiguous abdominal walls.

The clinical symptoms of fetal emphysema are essentially those of the uterine gangrene of pregnancy already described.

E. The Observed Expulsion of the Fetal Cadaver. Abortion

The term "abortion" has a great variety of meanings. In veterinary medicine it ordinarily means the observed expulsion of a fetal cadaver. If it is stated that twenty per cent. 
of cows in a herd have aborted, it is meant that someone has observed one-fifth of the females expel fetal cadavers. Many more embryonic or fetal cadavers may have been expelled unseen, or very small embryos may have become macerated and absorbed, but these are not included. In this sense "abortion" has acquired a preëminent place in veterinary science and the phenomenon is commonly regarded as a specific disease.

(a) The history of abortion is extensive. Its occurrence has been noted by biblical and other early writers and was frequently recorded in medieval times. The occurrence of abortion has generally caused greatest concern in cattle because, since their milk and meat have occupied a preëminent place in the food supply of most nations, any interference with this supply imperils human life and health. Perhaps the phenomenon has occurred more frequently in cattle than in other domestic animals because the conditions favoring it have been most prominent in cattle. Abortion has been noted most in highly civilized countries with dense populations where milk and its derivatives are most highly prized, and where any interruption of dairying causes a serious shortage in this highly important human food. Since, where a regular daily supply is demanded, for economic reasons no surplus cattle are kept, any serious disturbance with milk production is immediately felt. With the advancement of civilization and concentration of population, the ratio of milk- and meat-producing animals to the population becomes less and higher efficiency of each individual animal becomes essential. This increases the strain upon the animals, and the diseases interfering with reproduction become more intense. Accordingly abortion and other interferences with reproduction are of ever-increasing importance to the state and call for vigorous measures of control.

At first abortion was frequently attributed to unfavorable weather, such as unusual dampness or dryness, to bad food, to emanations from swamps, and to accident. In early history only a few diseases, such as leprosy, were attributed to contact. The belief in contact as a cause of disease ad- 
vanced rapidly during the eighteenth century. In the nineteenth century Pasteur and others firmly established the fact that disease is chiefly due to contact and that the underlying cause is a living microparasite passing from the diseased to the healthy animal as a result of direct or indirect contact.

The belief in the infectiousness of abortion has experienced a long and eventful course of development which has not yet been completed. For a time a few abortions were ascribed to infection, but these were at first limited to those cases where ingenuity failed to ascribe any other reason. At present probably a majority of people believe that most abortions are due to infection. The observed expulsion of a fetal cadaver came to be regarded as a disease, and by many is still so designated. Those abortions believed to be due to contact accordingly became known as "contagious," "infectious," or "epizoötic" abortion and were considered a specific malady. Many believed, and still believe, that, if a cow aborts as a result of infection, it must be due to one infecting agent and one only.

Mammalian existence is divisible into two clearly defined eras-intra-uterine or ante-natal, and extra-uterine or postnatal life. In post-natal life an indefinite number of infections may threaten health or life. On the other hand, it has been constructively assumed that during intra-uterine life but one bacterium or other microparasite may threaten the unborn animal. It is further assumed for all practical purposes that an infection invading the fetus can not cross the birth line and continue as a disease of the calf. Neither has it been commonly assumed that an infection of the pregnant female can invade the fetus within her uterus. Technically all pathologists admit that tuberculosis and syphilis may pass from mother to fetus, but this is not credited with any important bearing upon "contagious abortion." While the existence of such belief may be technically denied by the orthodox contagious-abortionists, they practically teach that "contagious abortion" is due to an infection which acts without contact. That is, if a cow aborts in the presence of 
a healthy cow, the infection passes through the body of the healthy cow "in bond" and fatally attacks the intra-uterine young, thus passing through an intermediary organism without injury, to attack fatally a third individual.

In irreconcilable conflict with such belief, the group of veterinarians to which I belong hold that the observed expulsion of a fetal cadaver is a phenomenon devoid of great mystery-that fetal death is fundamentally like extra-uterine death and occurs in a perfectly analogous way. Not only is fetal death analogous to post-natal death, but, with a single limitation, death in the two eras is due to identical causes. The limitation-and it is a highly important oneis that many of the important infections of post-natal life are unable to attain contact with the fetus. The uterine seal serves as an effective barrier against invasion through the genital tract, and the placental filter, when healthy, is not known to permit the passage of any organism competent to cause disease. No such passage of disease-causing organism through the healthy placenta to the fetus has yet been recorded. Even the ultra-minute disease-producers which defy the finest filter made by man, such as the virus of hog cholera, smallpox, and many others, do not destroy the life of the fetus directiy, but only indirectly by destroying the life of the pregnant female.

I have held that the infections causing the phenomenon of abortion are far less mysterious than generally claimed and are in all respects analogous to, and in an important degree identical with, the infections of extra-uterine life. Every part of the genital tract of both sexes and of all ages commonly harbors bacteria of great variety. Under ordinary conditions these may not cause evident disease, but under the numerous and vital changes of function in the genital tract, they may and do acquire power to cause vital injury. The uteri of most non-pregnant and pregnant cows and heifers contain bacteria having pathogenic powers. The epididymes and vesiculae seminales of bulls often contain bacteria which cause definite lesions in these parts. Under certain conditions such bacteria are ejaculated with the 
semen during coitus. If the cows served by such a bull become pregnant, they largely abort. It is quite unnecessary to invoke mysterious infections to account for any and all abortions. The infections are present, abundant, and potent.

(b) Abortion is world-wide and is seen in all mammalia. According to the highest authorities upon human obstetrics, 25 per cent. of pregnant women abort. Statistically the rate of abortion is far less among domestic animals, but that may be statistically only. I have previously stated that the term abortion, as commonly used, signifies the observed expulsion of the fetal cadaver. Abortion statistics are necessarily based upon the observed phenomenon. Hence the more accurate the observation, the higher statistically is the rate of abortion. Such being the case, if the expulsion of the embryonic or fetal cadaver were of equal frequency in all mammalia, statistically woman would occupy first place, the dairy cow next, followed by beef cows and other domestic females in order of the accuracy of observation of those who are in immediate charge. Available statistics from dairy herds indicate that approximately 10 to 15 per cent. of pregnant cows and heifers are observed to abort. How many fetal cadavers are expelled unseen is pure guesswork, but, if the opportunity for observation were as good as in the human family, the probabilities are that the abortion rate in cows would statistically equal or exceed that recorded for woman.

The phenomenon of abortion is probably more frequent in dairy than in beef cattle. Certainly it is more frequently seen, because the dairy cow is under very much closer observation than any other domestic animal, except possibly pet cats and dogs.

If the question is approached from a different angle, and the reproductive effieiency of animals studied, there is far greater accord between the various species and strains. If, for example, the reproductive efficiency of pedigreed dairy and beef cows be compared, it will be found that there is no notable difference. In many pedigreed beef herds, especially show herds, the reproductive ratio drops to 50 per cent. of 
the ideal of one calf per cow each year. Pedigreed dairy herds ordinarily do no worse. If abortion were defined as the failure to produce young, there would be greater statistical harmony between the various species and classes of animals.

I have known no herd of cattle, whether of dairy or beef breed, where abortion has not been observed. I know of no authentic record of a herd in which abortion is not seen. The frequency of abortion varies widely in different herds and at different times. Abortion is one of many phenomena due to infection within the pregnant uterus. If the formation of ova or spermatozoa is prevented by infection, if the genital cells are destroyed after being formed, or if the fertilized ovum or the minute embryo is destroyed, the disaster passes unseen and statistically becomes sterility. Abortion is, therefore, logically a phenomenon usually of the fifth to the seventh month of pregnancy, not because the intrauterine young perishes most frequently, or even so often, at this period, but because the disaster is most frequently observed at this time. After the seventh month, the fetus may be expelled alive and the phenomenon is called premature birth; a fetal cadaver may be expelled at full term and is called stillbirth; or a fatally infected calf may be born at full term and die from intra-uterine infection, but that is known as white scours or pneumonia, or otherwise. So disease and death throughout the entire reproductive process is referable to a group of infections and the resulting disaster is differently designated according to the date and manner of its occurrence, but the final outcome is the same -non-production.

Abortion is the result of two concurrent phenomena-the death of the fetus, and the existence of an endometritis of the pregnant animal of such a character as to cause the uterus to contract and evacuate its contents. In all recorded autopsies of cows which had recently aborted or were in the act of aborting at the time of slaughter, there have been recognized indisputable evidences of the presence of active infection. There has always been a definite cervical endo- 
metritis radiating toward the ovarian end of the uterus. Pus or other pathologic exudate is constantly present in the utero-chorionic cavity or, if the fetal membranes have come away, in the uterine cavity. The exudate and, almost always, the alimentary tract of the fetus contain bacteria, recognizable by cultural and staining methods. The uterochorionic space of most apparently healthy pregnant cows and the digestive tube of most new-born, apparently healthy

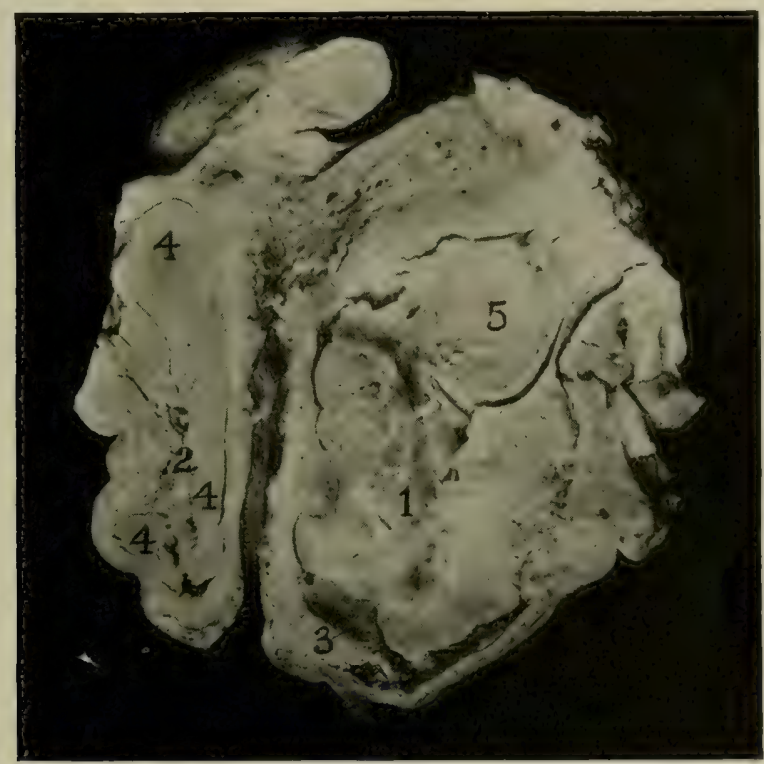

FIG. 173-"The Exudate of Contagious Abortion."

Cross section through the cornua of a pregnant uterus, showing exudate in utero chorionic space.

$I$, Fetal sac in gravid horn ; 2 , fetal sac in non-gravid horn; 3 , exudate in utero-chorionic cavity of gravid horn; 4, large masses of exudate in non-gravid horn; 5 , section through cotyledon.

calves contain bacteria not differing materially from those observed in abortion. In abortion, however, the bacteria are far more abundant. The variation in the abundance of bacteria is more readily studied in the abort, the fetus, and the calf. The amount of infection is very notable in the abort. The prematurely born calf usually bears in its ali- 


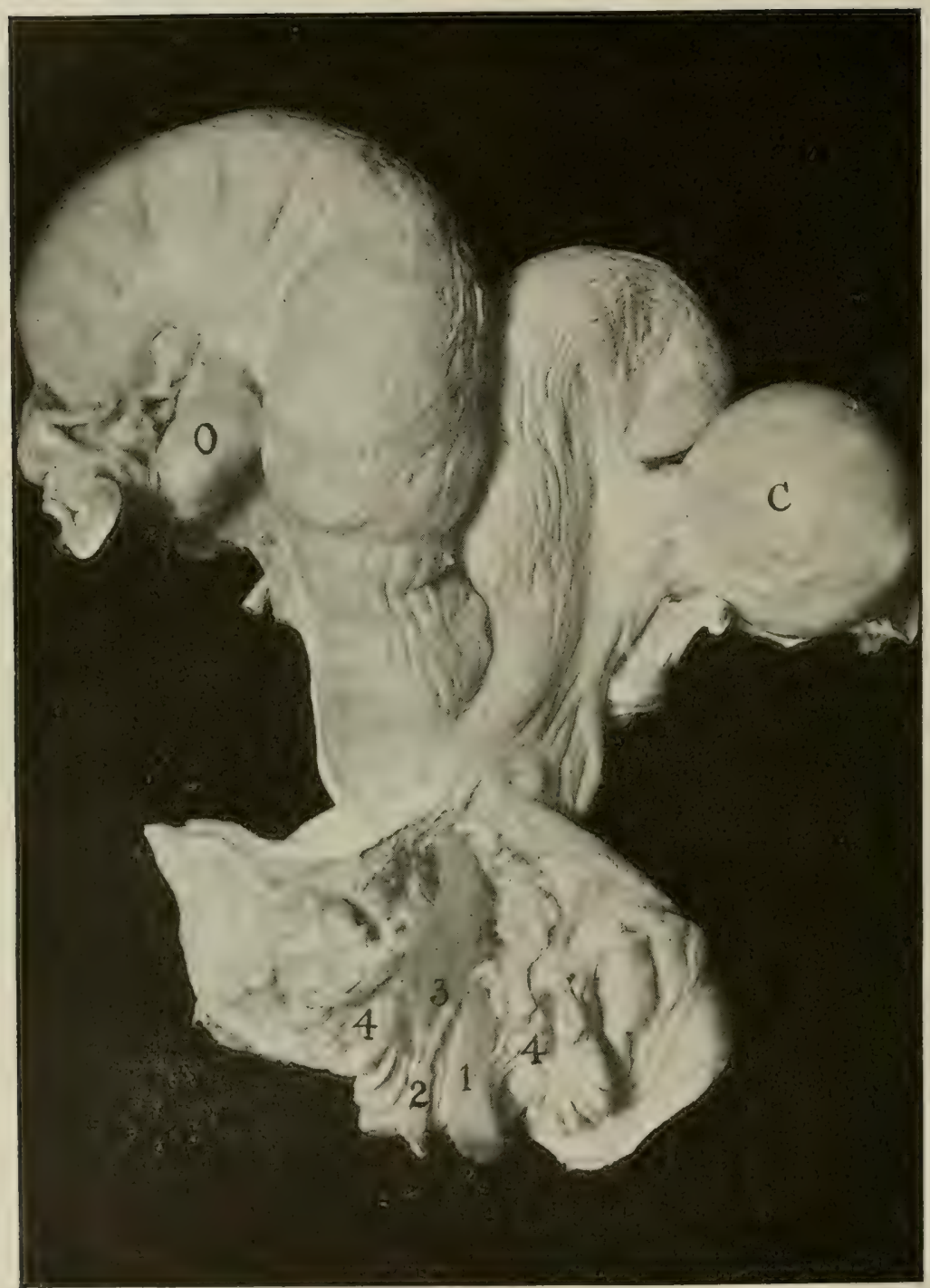

FIG. I74-Impending Abortion, Cervicitis, and Endometritis at Cervical end, with Protrusion of Fetal Sac into the Vagina.

The cervix has been laid open along its dorsal surface. I, Apparently healthy chorion protruding into vagina; 2,3 , necrotic portions of prolapsed chorion; 4 , lips of cervix; $O$, right ovary; $C$, large cyst representing the left ovary. The left side is sterile; the right horn pregnant about 8o-9o days. (See also Colored Plates III and IV.) 
mentary tract very voluminous infection. When metritis exists, with or without retained afterbirth, the alimentary canal of the apparently healthy calf swarms with bacteria and the calf probably breaks down with spesis, dysentery, or pneumonia. When the cow calves vigorously and expels the membranes promptly, the infection in the alimentary tract of the fetus is scant. All clinical and bacteriological evidence indicates that the abortion is in each case referable to infection, and to infection only.

In sharp conflict with all recorded evidence, the term "contagious abortion" coristructively suggests a non-contagious abortion. The fiction of a non-contagious abortion continues to be supported by many who believe that some abortions are due to various causes other than infection.

(c) Many believe in traumatic abortion but fail to explain how mechanical injury can cause the death and expulsion of a fetus. The bovine fetus lies upon the abdominal floor, surrounded by the abundant amniotic fluid and almost completely surrounded by the still more abundant allantoic fluid (See Colored Plate I). The head of the fetus generally lies within the pelvic cavity. If in the posterior presentation, the head usually lies above the sternum of the cow. When the abdominal floor is struck at a point against which some part of the fetus rests, it promptly floats away in its fluids. Fetal life is not dependent upon the same vital functions as extra-uterine life. Since respiration and nutrition are provided by the placentae, the sole vital function of the fetus is the fetal circulation. Fatal injury to the fetus is, therefore, virtually limited to a traumatism which can directly stop the fetal heart-beat. It is not known that such injury is possible.

Placental separation due to mechanical injury is unknown. I have studied carefully in the abattoir the uteri of more than two thousand pregnant cows and heifers, and have not observed an instance of any injury to uterus, fetal membranes, or fetus which, had the animal been permitted to live might apparently have led to abortion. I have already described inter-placental hemorrhage with fetal desiccation, 
but evidently this is not due to mechanical injury. It does not lead to abortion, but to fetal retention. Hence it may be stated that up to the present no proven instance of mechanical or traumatic abortion has been recorded in the cow nor in any other animal, and no logical explanation has been put forward of how mechanical injury can cause abortion.

(d) Food abortion has also been asserted to occur, and every known food has been blamed. The food is alleged to be too rich or too poor in proteins or in water, or it was fed too cold or in improper volume. Much has been charged to damaged foods. Since nearly all foods are damaged somewhat, bad food can be made to fit most cases of abortion. Special emphasis has been laid upon foods contaminated with fungi, such as ergot and smut, although I can find no authentic record of experimental or other evidence of the ability of any fungus to cause a healthy female to abort. McCullom, Hart, and others have experimentally shown that, by feeding cows upon a restricted kind of food (the wheat plant alone), although technically all essential food elements were given in abundance, the animals lost vigor, the calves born of these animals were weak and unthrifty, and as the experiments proceeded, abortion ensued, then conception failed, and finally the cows perished. But this is the mere decadence of vigor due to nutritive disturbance. Upon inquiry I was advised that when the experiment cows aborted they commonly had retained fetal membranes, for which up to the present there can be but one explanationthe presence in the uterus of an infection causing placentitis. The facts recorded by McCullom and Hart are of supreme and vital interest to breeders of animals. Practically the food, in a sense, caused the abortion, but the manner in which the food ration acted should be carefully considered. It seems to me evident that bacteria were present in the uteri of the experiment animals (as is commonly true) and that the devitalizing food ration given so lowered the vitality of the animals that the resident infection increased in virulence and caused disaster. The contention that all abortions are basically referable to infection is neither an argument 
nor admission that the proper feeding of animals does not play a vital part in reproduction. While abortion occurs only in the presence of intra-uterine infection, infection of both uterus and fetus is common without causing, or visibly threatening to cause abortion. The bacteria commonly present in the gravid uterus and in the contained fetus cause no visible injury in many cases. If intra-uterine bacteria uniformly prevented birth, the bovine species would soon become extinct. Under good hygienic conditions, if the bacterial content of the uterus is low, a degree of resistance may be acquired sufficient to hold in leash the bacteria present. In the presence of menstrual blood or of an embryo, the virulence of the bacteria may increase and injury follow. If the resistance of the animal is lowered by faulty feeding or otherwise, infection within the utero-chorionic space becomes more active. The amount of food may be deficient or excessive. It may be damaged by fungi. The ration may be badly balanced, or, as in the experiments of McCullom and others, though the food may contain all necessary elements in the quantities technically required, may fall short in variety; then reproduction is faulty or absent. Reproduction is dependent upon a nutritive surplus beyond other body requirements. If the nutritive supply is barely sufficient to maintain the general functions of the body, ova and spermatozoa are not formed. If the nutritive supply is somewhat greater, the reproductive cells form, but tend to perish later, either before or after fertilization. In this destruction bacteria play an important part.

Other factors influence reproduction. Pregnant cows or heifers moved from one establishment to another are far more liable to abort, to calve prematurely, or to suffer from metritis, probably with retained membranes, and the calf is more apt to suffer from dysentery than if the pregnant females had been left at home with otherwise equally good care. The interferences with reproduction are not the result of inferior care nor of exposure to a present infection, but of the lowering of their power of resistance to a present infection. Twin pregnancy is far more liable to interrup- 
tion than the normal single fetus, not because bovine (or equine) twins are unnatural but because the increased drain upon the nutritive supply of the mother lowers her resistance and permits the intra-uterine infection to multiply disastrously.

However important food and environment may be to reproduction, so far as known, bacterial invasion of the uterochorionic space of the cow is absolutely essential to abortion, retained afterbirth, and 99 per cent. of sterility.

(e) The biology of abortion. It is now generally conceded technically, though largely denied practically, that abortion may be caused by an unknown variety of bacteria. Any microörganism which may exist within the pregnant uterus, and which is capable of causing metritis and the death of the fetus, may cause abortion. Chronologically the chief infections which have been alleged to cause an important amount of abortion in cows are as follows:

(1) The micrococcus and short bacillus of Nocard. ${ }^{1}$ In his researches Nocard recognized a short bacillus (probably paracolon or paratyphus) and a micrococcus in the uterochorionic space of aborting cows, and in the digestive tube of the abort. He concluded that one or both organisms found were the cause of abortion in the cases investigated by him. He believed that abortion was primarily a disease of the fetus, but that the infection was derived from the uterus. He further expressed the very important view that, if the invaders failed to kill the fetus, they persisted in the individual through birth into extra-uterine life and constituted the basic cause of dysentery in young calves, thus asserting his belief in the continuity of infection between intra- and extra-uterine life. The views of Nocard were virtually discarded. Something more striking was being sought and demanded. Lehnert, in 1878, and Brauer, in 1880 , believed that they had promptly and reliably induced abortion by experimental infection. In England, also, Woodhead, Aitken, McFadyean, Campbell and others as-

${ }^{1}$ E. Nocard. Avortement épizoötique des Vaches. Rec. de Med. Vet., Vol. III, p. 669. 
serted they had experimentally caused abortion. Nocard had made no such claim for his investigations. Chiefly, if not wholly upon this ground, his work was practically ignored. It was everywhere demanded that, ere any given infection might be accepted as a cause, or the cause, of abortion, its power to cause abortion must be clearly demonstrated experimentally.

(2) The nodular venereal disease of Isepponi. ${ }^{1}$ In 1887 , Isepponi, then Canton veterinarian in Chur, published the results of his clinical studies of the hitherto undescribed infectious vaginitis which he thought a new and rapidly spreading contagion, and which he believed to be the cause of the serious sterility and abortion in the cattle of his district. For reasons which seem to me ample, I have placed this among the specific venereal diseases in Chapter XII, where it is discussed at length.

(3) The Bacterium Abortus of Bang.

In 1896, Bang and Stribolt discovered in the uterus of a cow, destroyed in the act of abortion, a small Gram-negative bacterium which they succeeded in cultivating in gelatinagar serum. The finding of the bacterium in large numbers in the utero-chorionic space and in the fetal cadaver justified a suspicion that it had to do with the abortion. Bang inoculated seven cows with pure cultures of the organism. Prior to inoculating them, he did not learn whether they would or would not probably abort if left alone, and he did not know, and had no means of learning, whether they already had the bacterium in their uteri. They were presumably taken from their normal habitat and placed under experimental conditions favorable to abortion, closely guarded, so that the expulsion of a fetal cadaver would almost certainly be seen. One (14 per cent.) of the seven cows aborted, which rate is no higher than would be expected without inoculation. Her uterus contained a bacterium not differentiable from the one used for inoculation.

${ }^{1}$ Beitrage z. d. Ursach. d. Unfruchtbarkeit d. Kühe. Schw. Archiv. für Tierheilkunde, 1887. 
So much emphasis has been placed upon the power of the $B$. abortus of Bang to cause abortion during the pregnancy existing at the time of inoculation, that a brief review of the recorded evidence is permissible.

\section{The Experimental Evidence of the Power of B. Abortus to Cause Abortion}

The dictum of Bang", "Through these two experiments we have submitted the complete proof that the bacilli discovered by us are the cause of epizoötic abortion," has exerted, and still exerts a profound influence upon the currents of thought regarding abortion in animals. Two basic ideas are included in the quotation from Bang. First, abortion is a specific, contagious disease, like tuberculosis or smallpox, caused always by one and the same organism and by one microparasite only. Second, the bacillus described by Bang is the cause and the only cause as proven by his experiments.

For many years the dictum of Bang was generally accepted without reservation. Cattle abortion was regarded as a specific disease apart from all other diseases of cattle, and the B. abortus was considered the specific agent. It is confidently stated that Bang and Stribolt "produced abortion in cows with it (B. abortus)"; "The fact seems to be clearly established that the specific cause of infectious abortion in cattle is the organism isolated by Bang and Stribolt"; "That the Bacillus abortus of Bang is capable of producing abortion experimentally in cattle by feeding and by intravenous injections has been satisfactorily proven by Bang, McFadyean and Stockman, Macneal and Kerr, Good, and others"; "Abortion disease is a specific infectious disease which is caused by a small germ known as the Bacillus abortus of Bang"; "The cause of abortion is a short, oval-shaped germ (Bact. abortus)"; "The inoculation of pregnant cattle . . . produces abortion after a variable length of time";

1"Durch diese beiden Versuche haben wir somit den vollen Beweis dafür geführt, dass die von uns gefundenen Bacillen die Ursache des seuchenhaften Verwerfens sind." 


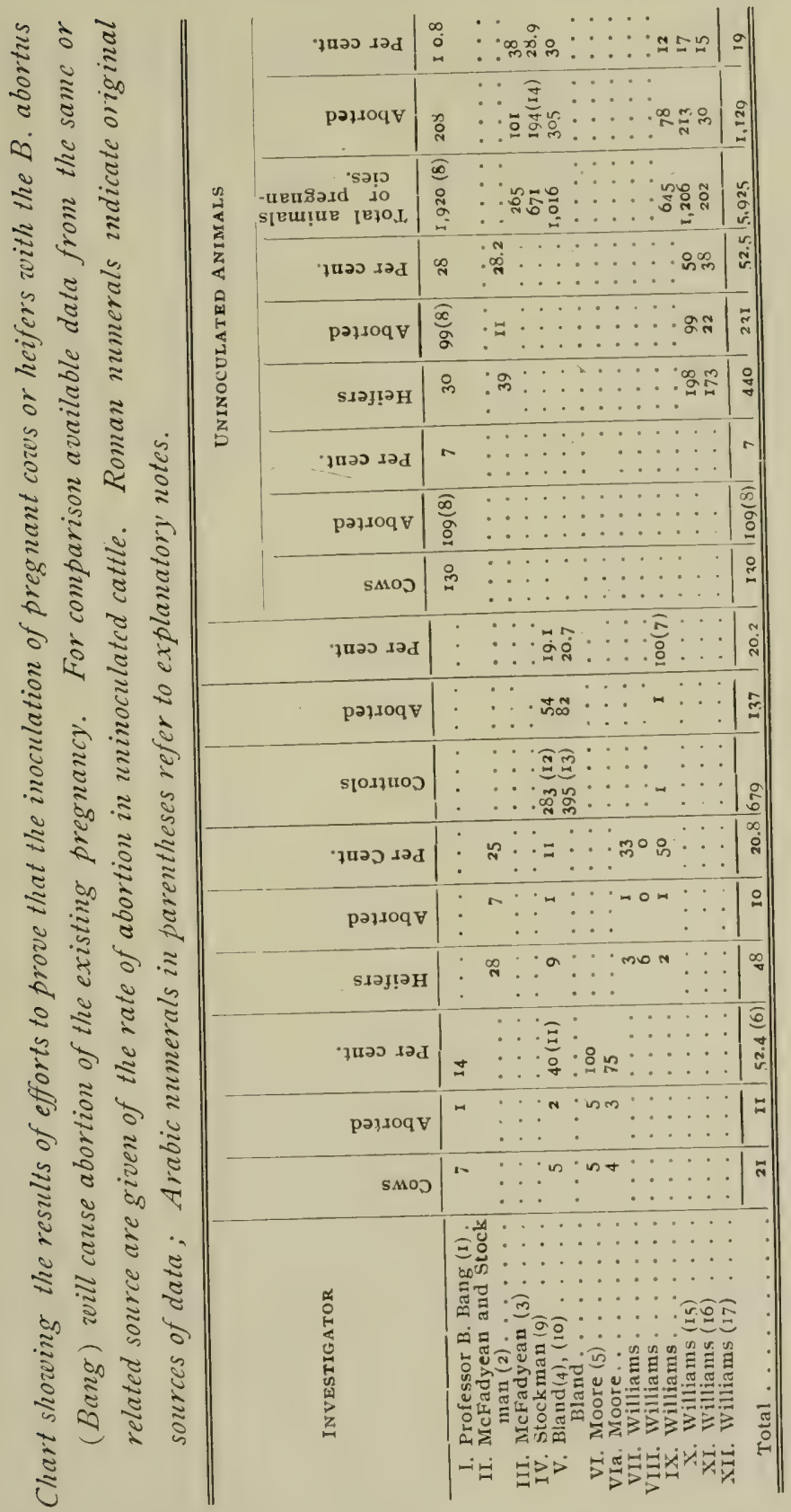


"A microörganism or minute germ (the abortion bacillus of Bang) is known to be the sole cause of contagious abortion."

Such positive assertions that the specific character of cattle abortion has been absolutely proven experimentally and that the Bacterium abortus is the cause, have served to prevent a critical examination of the evidence which has been alleged to prove the specific abortifacient action of the B. abortus. Most writers upon abortion in cattle refer to the experimental production of abortion in pregnant cattle as the corner stone for the positive statements that the $B$. abortus is the specific agent in abortion. Little has been written concerning the actual recorded evidence.

In the succeeding table the available recorded data of the experimental efforts at producing abortion with the $B$. abortus have been assembled, with direct and indirect controls. Numerous objections may be urged against their fairness. Tabulation demands brevity and inevitably leaves opportunity for misconception.

(1) The seven animals consisted of:

a. Two pregnant cows inoculated in vagina with pure cultures and killed, one nineteen days, the other twentynine days after inoculation.

b. Two pregnant cows inoculated per vaginam with afterbirth from an aborter, the one killed thirty-three, the other thirty-five days after exposure.

c. Two pregnant cows, four and seven years old, a second inoculation being made thirty-nine days later, and a third dose twelve days after the second. Seventy-one days after the first inoculation, the seven-year-old cow aborted a five-months fetus. B. abortus and a micrococcus were found in the placenta.

d. A six-year-old cow was inoculated per vaginam with pure cultures. She calved eighty days later. $B$. abortus bacilli were found in uterine contents of the cow.

(2) Out of the twenty-eight experiments recorded by McFadyean and Stockman, Experiments Nos. 7, 14, 15, 18, 22, 24 and 25 (7) were followed by abortion. In Experiment 13 the fetus underwent desiccation following death but was not expelled. Other animals were killed prior to the termination of pregnancy, and some might have aborted had they not been killed. 
(3) Thirty-nine heifers in first pregnancy were alleged to have been chased by a dog. Some months later eleven of them aborted. McFadyean testified that the abortions were not due to contagion.

(4) These experiments were carried out under the auspices of Sir Stewart Stockman with material furnished by the Board of Agriculture and Fisheries.

(5) The nine cows were all recent purchases. Five came from a herd where abortion had raged the previous year and were all pregnant when bought. Two had aborted the previous year, two were sterile or had aborted unseen, and one had calved. After inoculation all aborted. The history of the other four was unknown. Three were pregnant when purchased, the fourth was bred after buying. All nine were recent arrivals on the experimental premises, which increased the tendency to abort.

(6) The heavy percentage of abortions in inoculated cows is caused by the high rate of the five cows of Moore, only one of which was known to have calved the previous year.

(7) The 100 per cent. of abortions in controls is admittedly of no moment, but, since the one heifer is the only control of which I can find record, the figures are technically correct.

(8) The Thurebylille herd consisted of about 130 milk cows and about 30 heifers, and the data extend from 1885 to 1896 , inclusive, or a period of twelve years.

(9) The data given here are for uninoculated cows and heifers kept as controls in vaccination against abortion, as shown in Table IV, page 353, Report Tenth International Veterinary Congress.

(10) Owing to errors in diagnosis, Bland unintentionally inoculated five pregnant cows and nine pregnant heifers with living bacilli. (Herd A, two cows, four heifers; Herd M, one cow; Herd Q, one cow; Herd R, one cow; Herd B2, five heifers. Total, fourteen.)

(11) The two cows which aborted following inoculation had also aborted in the pregnancy before inoculation. See also (5).

(12) Bland, on page 9 of his first report in his experiments to control abortion by vaccinating the non-pregnant animal, kept as "controls" 296 non-pregnant cows and heifers, thirteen of which were not known to have conceived. Bland's percentage of abortion (18.2) is based upon 296 animals, while the percentage here given (19.1) is based upon 296-13, 283 animals.

(13) Second report, page 15.

(14) In the Bland reports the number of animals of prior year is not given and total under experiment is used, with percentages for prior years. 
(i5) The data cover twenty-two years, 217 cows and heifers and the 645 pregnancies recorded.

(16) The data cover thirty-one months.

(17) The data cover ten years and include sixty-seven cows and heifers.

\section{BIBLIOGRAPHY}

I. Bang, Prof. B., Die Aetiologie des seuchenhaften ("infectiösen") Verwerfens, Zeitschrift f. Tiermedicin, Vol. I, p. 241.

II. McFadyean and Stockman, Rep. Departmental Com. Bd. of Ag. and Fisheries, Great Britain, on Epizoötic Abortion, 1909.

III. Testimony of Sir JoHN MCFADYEAN in litigation of Chadwick vs. Gorman, Vet. Rec., March 30, 1912, p. 621.

IV. Stockman, Sir Stewart, Epizoötic Abortion, Rep. Tenth International Veterinary Congress, Vol. II, p. 343.

V. Bland, G. R., First and Second Reports by the Agricultural Organizer of Oxford County, England, on the Epizoötic Abortion Experiments carried out in 27 herds, 1911 to 1916 , Oxfordshire, England.

VI. Moore, V. A., and Fitch, C. P., Rep. N. Y. State Vet. Col. at Cornell University, 1912-1913, p. 89.

VII. Williams, W. L., Rep. N. Y. State Vet. Col. at Cornell University, 1911-1912, p. 79.

VIII. Williams, W. L., The Granular Vienereal Disease and Abortion in Cattle. Bulletin of the U. S. Dept. Agr., No. 106, Sept: 12,1914, p. 49.

IX. Williams, W. L., Abortion and Sterility in Cattle. Rep. N. Y. State Vet. Col. at Cornell University, 1913-1914, p. 136.

X. Williams, W. L., Abortion and Sterility in Cattle. Rep. N. Y. State Vet. Col. at Cornell University, 1911-1912, p. 85, Herd A.

XI. Williams, W. L., Abortion and Sterility in Cattle. Rep. N. Y. State Vet. Col. at Cornell University, 1911-1912, p. 87, Herd B.

XII. Williams, W. L., Abortion and Sterility in Cattle. Rep. N. Y. State Vet. Col. at Cornell University, 1911-1912, p. 87, Herd C.

As early as 1912 (VII), I made the following statement: "It has not been clearly shown that a like number and kind of pregnant cattle would not have aborted had sterile salt solution been substituted for the Bang organism."

A critical study of the tabulated data bears out this assertion : 
The 21 cows used for experimental inoculation show the largest ratio $(52.4 \%)$ of observed abortion. Of the 11 aborters, however, 4 (Bland, 2; Moore, 2) are known to have aborted, and 2 (Moore) were sterile or aborted unseen the prior year. Only 1 of those aborting (Moore) is known to have calved the previous year and 1 (Bang) may have done so. Omitting the 4 known aborters and the 2 sterile cows of the previous year, there remain 15 cows which possibly calved the prior year, and of these, $5(23.8 \%)$ aborted. No controls were kept and the ratio is not sufficiently high to constitute good evidence. It is not known that each of the cows did not, when the inoculation was made, carry in the utero-chorionic cavity an infection capable of causing abortion. In the one Moore cow, in the group of 5, this is not merely a possibility but a definite probability, since she came from the same herd as the other 4, none of which calved the previous year.

A study of the available records of experimental inoculation in heifers, of which it has been possible to assemble 48 , shows that $10(20.8 \%)$ are recorded as having aborted. I find only one heifer recorded as having been kept directly as a control - that in my experiment (IX) - and that one aborted. A single case can not be accepted as conclusive nor as valuable evidence, but there is no other way of stating the recorded fact. The question of controls has been ignored.

The heifers in the Bland experiments (V) were not inoculated to cause, but to prevent abortion. The inoculation occurred through error. The heifers were assumed to be non-pregnant at the date of inoculation, but later proved to be pregnant. These are inserted in the table under the assumption that the effect was the same whether the inoculation was made to cause or to prevent abortion. The virtual controls of Bland-(12), (13) — should be applicable, as they were definitely used as controls in efforts to prevent abortion by vaccination of non-pregnant cattle with living bacilli. 
The more voluminous data regarding the frequency of observed abortions in herds show a higher rate generally than that recorded in experimental inoculations. The exception is the data submitted by Bang showing but 7 per cent. in cows of the Thurebylille herd. When the data upon heifers in first pregnancy are examined, it is found that under the prevailing conditions in herds the ratio of observed abortions $(52.55 \%)$ is far in excess of the recorded abortions following experimental inoculations. When cows and heifers are talien together, the observed abortions $(19, y)$ are but 1.08 per cent. below the ratio reported in the experimentally inoculated animals.

The objection to these data may be urged that they are from "infected" or "badly infected" herds, but no data are available from non-infected herds. One often hears of noninfected herds, but any attempt to get in contact with such and secure accurate data for record leaves one with the feeling that he has been pursuing a mirage. If there are herds in which no abortions occur and in which each cow or heifer of breeding age produces a healthy calf each twelve months, the failure of those having knowledge of the facts to publish the details serves as an impassable barrier to their use. The question can be judged or decided only upon the available recorded data.

The further and pertinent objection may be made that in many of the experiments the animals were destroyed prior to the termination of pregnancy and that some or many of these might have aborted had they not been killed. But it would be equally erroneous to pick out from the total those animals kept until after termination of pregnancy and accept the results in this group alone. The probability of abortion decreases as the duration of pregnancy advances: the disease may be present and severe but may result in premature birth, in retained afterbirth, or in calf scours or pneumonia.

On the other hand, it may be very properly maintained that the animals slaughtered prior to the termination of pregnancy were generally held beyond the alleged "period 
of incubation" of the abortion infection and should mostly, according to the constructive standards of various experimenters, have aborted prior to slaughter if they were to abort.

Some investigators have also argued that the finding of the $B$. abortus in the uterine cavity of the killed animal is experimental proof that it is the cause of abortion. If that is true of the B. abortus, then it must be equally true of all the other microparasites found in the uteri of pregnant cows. It can be experimentally proven that a given organism causes a cow to abort only by those cases which actually abort. It is not necessary to produce infection of the uterochorionic space in a pregnant cow to show that the $B$. abortus is capable of causing such infection; Bang proved that in the first cow in which he recognized the bacillus. If it existed and multiplied in that cow, it follows, a priori, that, implanted in another pregnant uterus of identical species and power of resistance, it would cause infection again.

It has been attempted to apply to abortion the postulates of Koch who assumed as a basis for experimentally proving the specific character of a bacillus: (1) The organism needs to be obtained in pure cultures. (2) Inoculation of a sound animal with pure cultures must cause the typical symptoms and lesions observed in naturally infected animals. (3) Pure cultures of the organism must be recovered from the tissues of the experimentally inoculated and diseased animal. Koch's postulates are inapplicable to abortion in cattle because the abortion is not the disease nor is it a lesion; it is merely one of the possible occurrences in the course of a disease having the power to destroy the life of a fetus and to irritate the pregnant uterus in a manner to cause it to expel the fetus. While the data submitted fail to justify the dictum of Bang and others that the inoculation of a pregnant cow or heifer with the Bang organism will cause her to abort during the existing pregnancy and thus prove that the $B$. abortus is the specific cause of abortion in cattle, it is not proof that such inoculation is without 
injurious result. Neither does it prove, nor tend to prove, that abortion is not sometimes or frequently caused by the Bang organism.

I have long held that any attempt to prove experimentally that a given organism is the cause or a cause of an abortion occurring during the pregnancy in which the inoculation was made, is unwarranted and misleading. When Bang discovered the bacterium which he described, he definitely showed that it was an intra-uterine and fetal infection. No beneficent office could be ascribed to it. If capable of existing in vast numbers in the one cow and fetus, it might equally well exist in equally great numbers in other uteri and fetuses of the same species and of similar constitution. There was present metritis, for which, under ordinary surroundings, no cause except infection is known. Bang recognized in the uterus an infection. If this was the only bacterium present or recognizable (upon which point Bang is not clear) the only justifiable conclusion at which he could arrive was that the bacterium in question was the cause of the abortion. Bacterial search of the uterus of another aborting cow in which some other microörganism was found would not vitiate nor influence the conclusion reached upon the one described.

Abortion is not a disease nor the symptom of any one disease. So far as the fetus is concerned, abortion is death, and death is a result common to all diseases. The fact that an adult dies is not proof that it has anthrax, and the fact that a fetus dies is not proof that it was killed by $B$. abortus. In so far as the pregnant female is concerned, abortion indicates endometritis at the cervical end of the uterus. $\mathrm{Me}$ tritis exists in non-pregnant and pregnant heifers, in pregnant cows, in cows in the puerperal stage and during the interval of rest between a period of calving and the next conception. The character of the metritis which may occur at any period in the life of the female varies infinitely and the number of bacteria capable of causing the metritis is unknown. A few instances of metritis stand apart, such as that of uterine actinomycosis and tuberculosis. They 
are specific. But the metritis met in essentially all cases of abortion bears no mark by which it can be assigned to any one bacterium. Consequently if several bacteria are present, there is nothing in the metritis to indicate which of the invaders is responsible.

Any contagion competent to injure or destroy an adult may, so far as known, equally imperil the life of an ovum, embryo, or fetus if contact occurs. The vast majority of infections do not and can not reach the intra-uterine young. The opening of the genital tract through the vulva is hermetically sealed at the cervical canal by the uterine seal. It is not known, and there is no reason to suspect, that bacteria can traverse the cervical canal and enter the utero-chorionic space of the pregnant cow while the uterine seal is intact. The mammalian placenta, so long as intact, is not known to permit the passage of any form of pathogenic organism. It has not been shown that any disease-producing organism passes during pregnancy from the blood system of the mother into the utero-chorionic space. Infection is not known to invade the uterine cavity of the pregnant female through the oviducts. There is accordingly no portal of entry into the sealed uterus of pregnancy through which bacteria have been known to enter. The orthodox contagious-abortionists positively assert that the $B$. abortus enters the uterus during pregnancy, but avoid stating by what avenue the invasion occurs.

I have held that the infections which prevent or interrupt reproduction invade the uterus prior to the sealing of the cervical canal; either the infection was present in the uterus at the time of coitus, or it was introduced during that act.

The frequency of abortion in a herd usually corresponds fairly well with the intensity of infection by $B$. abortus. In those herds where abortion and other interferences with reproduction are common, the agglutination and complement-fixation tests usually show intense infection with $B$. abortus. But such reactions can only show that the $B$. abortus is somewhere active (or has been active) and can not show that it caused a given abortion. If such tests 
could show that $B$. abortus has caused a given abortion, a typical reaction of an aborting cow to the tuberculin test would show that the abortion was due to the infection of tuberculosis. I have seen a high $B$. abortus agglutination reaction in a sterile heifer never known to have conceived, in which, upon autopsy, $B$. abortus was recovered from the udder but not from the genital tract. The sterility was apparently due to streptococcic salpingitis. Doubtless her blood would also have reacted strongly to the streptococcus. In the researches in my department, the blood of cows in which harmful genital infections exist reacts to several species of bacteria, such as paracolon and streptococci, but the reactions show merely the presence of such bacteria somewhere in the body, and can not show that they cause sterility or abortion. The agglutination test for the presence of $B$. abortus is in itself fundamentally defective. I injected living $B$. abortus cultures in the jugular vein of a two-year-old pregnant heifer. She probably already had $B$. abortus in her utero-chorionic cavity. The agglutinating power of her blood was followed from 0.02 up to 0.001 and in its descent until minus at 0.02 . Then she aborted and was promptly destroyed. Her blood was negative at 0.02 , and her uterus was swarming with $B$. abortus.

A vital defect of the agglutination test for the presence of B. abortus is the total absence of knowledge of the physiologic agglutinating power of bovine blood. Some consider an agglutination at 0.02 as proof of infection; others place the infection-point at 0.01 . As a rule the blood of new-born calves is negative at 0.1 and can be held there experimentally as long as I have cared to do so. If the calf is fed in the ordinary manner, its blood commonly reacts positively at 0.1 or higher within thirty days. I have seen it react at 0.002 at thirteen days. The common practice of fixing upon 0.02 or 0.01 as denoting infection is absolutely arbitrary and unsupported by any recorded attempt at justification. There is no more reason for believing that agglutination at 0.02 or 0.01 denotes infection than for declaring that agglutination at 0.04 or 0.1 indicates infection. 


\section{(4) The Paratyphoid Bacillus of Moussu ${ }^{1}$}

Moussu declares, apparently upon adequate grounds, that in a large section of France much abortion is due to a short bacillus of the colon group. The $B$. abortus is absent.

\section{(5) The SPIRILlum of SMith² ANd Others}

Smith, in studying a series of aborts from a large dairy herd, obtained pure cultures of B. abortus in $27(66 \%)$ and a spirillum or vibrio in $14(34 \%)$. In other aborts Smith failed to find either the B. abortus or the spirillum, but recognized bacteria belonging to the colon and other groups. The lesions in the aborts did not vary with the variations of the bacteria present, but were fundamentally alike in all.

\section{(6) Miscellaneous Bacteria}

Isolated cases of abortion have been attributed to various bacteria other than those mentioned above. A few have been attributed to $B$. pyogenes. Bacterial search of the tubo-utero-cervical canal reveals a great variety of infections present in non-pregnant and pregnant animals. A streptococcus of the viridans group is dominant apparently in serious tubal infections and perhaps plays an essential role in the necrosis of the apices of the embryonic sac. Probably it causes the death of many fertilized ova and of embryos.

\section{F. The Nature of Abortion}

The belief that abortion is generally due to a specific con-tagion of the pregnant uterus and its contents, inevitably involves the belief that the infection can not exist in the genital tract prior to conception and that it must cease to exist with the termination of pregnancy. Neither can the alleged specific infection cause other results than abortion. Hence the orthodox believer in the specific character of abortion de-

${ }^{1}$ Prof. G. Moussu. Sur l'Avortement Epizoötique. Tenth Internat. Vet. Cong., 1914.

${ }^{2}$ Theobald Smith. Spirilla associated with Disease of the Fetal Membranes in Cattle. Jour. Exp. Med., Dec. 1, 1918. 
nies that the infection, sometimes leading to abortion, can cause sterility, retained afterbirth, or metritis other than the specific metritis of contagious abortion, which must cease to exist at the termination of pregnancy, to be succeeded by another metritis due to other infection. So with the fetus and the calf. The fetus is invaded by "the specific infection of contagious abortion," but after the calf is born such infection must terminate, though the calf may promptly die from sepsis, dysentery, or pneumonia, and though bacteria which can not be differentiated from those existing in the digestive tract of the fetus are present in that of the newborn calf and apparently cause the serious or fatal disease.

This group of writers use constantly the definite article "the" in connection with the alleged specific contagion, thus excluding all other infections or contagions as possible causes of abortion. That is, if the B. abortus of Bang is the cause of contagious, infectious, or epizoötic abortion in cattle, no other infection or contagion can act as $a$ cause or as one cause of abortion. If the B. abortus of Bang is the cause of contagious or infectious abortion of cattle, then the statement of McFadyean and Stockman, that in one pregnant cow which they destroyed they found uterine tuberculosis which would soon have caused abortion without the presence of $B$. abortus, is erroneous. If $B$. abortus is the cause, $B$. tuberculosis can not be a cause of abortion. But that is resting the case upon what some may designate a technicality. More pertinent to the discussion is the statement of McFadyean and Stockman: "It is of great importance to note, however, that two natural outbreaks of vibrionic abortion, one in Ireland and one in Wales, were met in cows." (Abortion in Sheep, Part III, page 9.) The more recent finding by Theobald Smith, in this country, of spirilla in aborted bovine fetuses, without the B. abortus, is equally to the point. If $B$. abortus is the cause of contagious, infectious, or epizoötic abortion in cattle, then either the vibrio of McFadyean and Stockman and the spirillum of Smith did not cause the abortions attributed thereto, or the abortions so caused were not "infectious," "contagious," or "epizoötic." 
The second group, to which the writer belongs, holds that abortion is not a specific contagious or infectious disease like glanders or hog cholera, but that any infection resident in the cervical end of the uterus, or which during pregnancy invades the uterus, may, amongst other results, cause abortion. According to this view there are two essential factors in the causation of abortion: the infection must destroy the life of the fetus (asphyxia or inanition due to placentitis, or sepsis due to bacterial invasion of the fetus), and must arouse such an irritation of the uterus (localized metritis at the cervical end) as to cause it to contract and expel its contents. Any infection capable of bringing about these two conditions may cause abortion, be it the B. abortus, the bacillus of Nocard, the colon organism of Moussu, the vibrio of McFadyean and Stockman, or the spirillum (vibrio) of Theobald Smith.

Under this conception, there is no limitation upon the number of species of microparasites which may cause abortion, no restrictions upon the variety of lesions and results of lesions which the infection may cause, and no obligatory period of invasion or disappearance fixed for the infection.

This conception offers many analogies to wound infection. In wound infection there is an extended variety of organisms, each capable of producing inflammation, suppuration, ulceration, necrosis, wound fever, etc. The microörganisms are normally present upon the body surface and under ordinary conditions cause no visible harm to the individual. When, however, the protective epithelium is destroyed by physical violence, chemical irritation, or in various ways, the bacteria upon the surface are pushed into, or otherwise enter the damaged tissues and wound infection occurs. The lesion which constitutes the avenue of infection may be visible or invisible to the unaided eye. The epithelium may be destroyed or merely so damaged that it wants the power required to resist the attack.

A knowledge of the identity of the invading organisms is of great interest but not a vital necessity to the surgeon. The measures which will exclude streptococci will serve also 
to bar staphylococci. If the wound is already infected, the surgeon uses the same means to remove or to destroy micrococci and bacilli. In special cases the surgeon may fear tetanus infection and take special measures against it, but that he classifies separately as a specific infectious disease. Each individual carries upon his body surface bacteria common to other individuals. A moderately extensive list can be made of the most prevalent types competent to cause wound infection.

In every part of the genital tract microörganisms are common throughout the life of the individual. When estrum occurs, the genital mucosa is engorged and its epithelium weakened. If conception fails, menstruation follows, with denudation of the placental areas and an outflow of blood. The denuded areas furnish an avenue of invasion and the blood clots supply nutritive material for the bacteria present. Should conception occur, and the fertilized ovum descend into the uterus, it encounters the microörganisms infesting the cavity. The embryo has acquired no power to resist invasion, with the result that bacterial activity is advanced. Then follows a definite metritis. As soon as the fetal organs have developed, the infection passes through the chorion and amnion into the amniotic fluid and is swallowed by the fetus. Accordingly the bacteriologic findings of Hagan, Carpenter, and others working in, or in collaboration with this department, show parallel infection in the non-gravid uterus, in the utero-chorionic cavity of the pregnant uterus, and in the fetal alimentary tract, and later when the calf is born the same bacteria are recoverable from its stomach and intestines. There is then a continuity of infection in the mother and the intra-uterine invasion of the fetus.

The results of the infection or infections upon the mother, fetus, and new-born calf will logically vary with their volume and virulence. If very severe in the non-pregnant uterus, conception fails. If the infection is milder and conception occurs, metritis may follow and so irritate the uterus that it contracts and expels its contents. If the in- 
fection has killed the fetus, and it is expelled, the result is known as abortion. If the fetus lives and is carried the full term of gestation and is then expelled, there is metritis present and perhaps retained afterbirth. The expulsion of the fetus leaves a denuded uterus. If the afterbirth is retained, it is immediately necrotic and decomposes. Then follows an aggravated metritis. It is not a new disease, but a continuation of a metritis which existed during pregnancy, accelerated in its course by the uterine denudation of calving or abortion, aggravated by decomposing afterbirth. The infected calf when born may break down with calf scours or pneumonia. It is not usually a new disease or a new infection but the continuation of an infection acquired during intra-uterine life. During the course of the dysentery, the alimentary epithelium is damaged or destroyed, permitting the invasion of the body fluids by the bacteria causing the intestinal disease. If the calf suffers from pneumonia, the pulmonary epithelium is damaged or destroyed and bacterial invasion occurs. The infection had its habitat in the pregnant uterus, and thence passed to the digestive tract of the fetus. In the new-born calf apparently the infection passes through the damaged digestive or pulmonary epithelium, enters the body fluids, and, having an affinity for the genital tiact, regains its habitat there and persists, to interfere later with first pregnancy. According to this conception, the infections of which abortion is one significant result constitute an endless chain. They may be intensified in the uterus of the cow just after parturition or abortion and additional infections from the exterior be added. The infection in the new-born calf may be minimized or intensified by proper or improper feeding, or additional infection may be supplied to the calf by association with badly diseased calves or by taking milk contaminated by virulent discharges from badly diseased cows.

On the other hand, the belief that abortion is a specific disease denies the endless infection-chain above described and regards sterility, retained afterbirth, calf scours, and pneumonia as associated or concurrent diseases, but recog- 
nizes no identity, continuity, or causal relationship. Each must then be handled separately as distinct maladies, instead of dealing with the entire list as one group, each having a continuity with the others.

As in wound infection, so in the infections in which abortion occurs, while no limit can be placed upon the number of microparasites which may act as the causal agent, certain varieties are commonest. Thus in abortion, in one area, the $B$. abortus may be the predominant cause; in another, a streptococcus; and, in a third, a spirillum. In a given animal or outbreak, $B$. abortus may be the cause, but in abortion generally it is merely $a$ cause. Some believers in the specific character of abortion, when forced by recorded facts to admit that abortion may be, and is, due to various organisms, attempt to evade the difficulty by proposing the name "abortion disease" or "Bang's disease" instead of the equally misleading terms of "contagious," "infectious," or "epizoötic" abortion. But that does not help matters. It is still desirable to learn how large a part of the abortions are evidently due to the $B$. abortus, and how much to other organisms.

The second conception of the nature of abortion permits the handling of the group of infections as a whole. The losses can be minimized only by breaking or weakening the endless chain of infection at its most vulnerable point, which, it is held, is at the commencement of the life of the individual. It has been advised that the genital organs of both sexes should be as clean as practicable at the time of copulation in order to favor conception. When fertilization has occurred, it is important that the uterine cavity be clean, so that metritis and infection of the fetus may be avoided. When the calf is born it should be so handled that the infection it may have acquired in the uterus shall be restrained or eliminated, and that no fresh infection shall menace the calf through contaminated milk or other foods, or by intimate association with badly diseased calves. For example, the veterinarian handling cervicitis sees before him unmistakable and severe inflammation due to some form or forms 
of infection. No matter what the identity of the infection may be, the problem which he faces is disinfection, and he elects that method which, in the present state of knowledge, promises the best results.

\section{G. Symptoms and Diagnosis}

The symptoms and diagnosis of abortion are essentially included in the definition given-the observed expulsion of a fetal cadaver. The symptoms are those of parturition abbreviated. In early pregnancy there is no sinking of pelvic ligaments, no enlargement of mammae, no marked discharge from the vulva, no visible effort at expulsion. When abortion occurs late in pregnancy, the pelvic ligaments may sink as in parturition, the mammary gland may be enlarged and the vulva may become tumefied. But any of these phenomena may occur from other causes.

After about the fifth month of pregnancy, in addition to the observation of the expulsion of the fetal cadaver, there may be retention of the fetal membranes, indicating that abortion has occurred or that abortion is then in course.

Abortion may be suspected or determined with confidence upon other grounds. In the regular control of reproduction in cattle, pregnancy may be reliably diagnosed at thirty to sixty days after coitus. If the diagnosis is clear and definite, as it should ordinarily be, and it is later determined that the pregnancy has ceased to exist and the animal has not calved, it is clear she has aborted at some time between the two dates of observation.

The diagnosis of pregnancy may be fairly secure upon other grounds than clinical examination. If a cow or heifer is regular in her estrual cycle, is bred, does not menstruate after coitus, and estrum fails to recur at the regular interval, it is good evidence that she is pregnant. If, after a lapse of forty to ninety or one hundred days, estrum recurs, it is probable that an embryo has perished and has been expelled. There are numerous other conditions which may present the same history, but abortion is by far the most probable explanation. 


\section{H. The Date of Invasion}

The infection which causes abortion invades the uterus prior to the establishment of the uterine seal. The orthodox believers in a specific contagious abortion have stoutly contended that the infection commonly invades the uterus, fetal membranes and fetus after pregnancy has become established, the placenta has formed and the cervical canal has become hermetically sealed. As already related, they have attempted to prove, and believe they have proven their contention by experimentally causing abortion in an existing pregnancy. In the cow, as discussed in preceding pages, the experimenters have kept no checks or controls and their experimental inoculations have been followed by no higher rate of abortion than is commonly observed in uninoculated animals. They have further attempted to prove that a pregnancy already established can be interrupted by artificial infection of pregnant animals of other species. But, as with cows, no control animals have been kept. The rate of abortion following inoculation has not been remarkably higher than common in the species used, and there is no evidence that an equally high ratio of abortion would not have followed the injection of a sterile, inoccuous substance. It is a perfectly well known clinical fact that the various species of domestic animals commonly cohabit on farms. Horses, cattle, swine, dogs and cats are in intimate contact, but cohabitation fails utterly to cause the spread of abortion from one species to another. If abortion were spread by the means alleged by the believers in "contagious abortion" such as the eating by pregnant animals of food which has become contaminated by the discharges from an aborter, by the infection carried upon the boots, hands and so forth of attendants, or by the mere presence of an abortion, an abort, or the afterbirth of an abort in a stable or a field, an outbreak of abortion in one species would be the signal for many pregnant animals of whatever species to abort. The recorded evidence of the transmission of "contagious abortion" of cows to all species 
of domestic animals should, before it is accepted as proof, be supplemented by a new and extensive series of experiments with adequate control animals. Until this is done, the controversy over the date of invasion of the infection causing abortion can not be satisfactorily settled.

I have held that there are two notable and important dates of invasion-the intra-uterine infection of the fetus and the time of coitus in adults. The latter has already been consdered earlier in this chapter (I-Infections of the Genital Organs of Bulls). The first will be considered later (III - The Congenital Infections of Calves).

\section{The Control of Abortion}

Under the views already expressed, abortion can not be directly prevented or controlled, nor is there need for such prevention or control. Being neither a disease nor a symptom of any one infection, it demands no separate consideration. It can be prevented only by the control of the infections within the genitalia of both sexes. These are considered elsewhere.

When abortion came to be looked upon as a specific contagious disease, veterinarians and cattle breeders hoped for a definite remedy. Before it was believed that the cause of abortion had been determined, Brauer (1889) thought he had discovered a specific remedy in the subcutaneous injection of 2 per cent. carbolic acid to pregnant cows and heifers. Highly favorable statistics were submitted by Brauer and his followers in support of the new remedy. The researches were without controls and very inaccurate. Apparently the carbolic acid was given in herds where there had recently been an "abortion storm," in which a notable decrease was to be expected, or, as occurs frequently, a few animals had aborted, all or nearly all of those which were in line to abort, and then the remedy was given. Naturally there would be no more abortions. But the career of the alleged cure was short and it was soon found that if the utero-chorionic space of a pregnant cow was highly infected and contained 
a large volume of exudate, she would probably abort, and that carbolic acid, hypodermically or otherwise, exerted no recognizable influence.

The belief of Bang and his followers that he had discovered the specific cause of abortion immediately aroused high hopes that a definite remedy would follow. It was believed by Bang that the discovery of the cause would be followed quickly by a cure or by some means for control. The vast majority of investigators and breeders have concentrated their efforts since 1896 upon finding a remedy for abortion, based upon B. abortus as the specific cause. There has been no more lamentable failure in the annals of veterinary medicine than the attempt to control abortion as a specific contagious disease due to the B. abortus of Bang. The infections in the genital tract of cattle have increased enormously in virulence in every country. In America numerous herds of pedigreed cattle of potentially great value do little more than maintain their numerical status. Death and disability from genital diseases of females of breeding age consume practically all heifer calves reared in the herd. The surplus bull calves carry into the herds where they are sold virulent infections with which they are saturated at birth. Despite this evident, pitiably disastrous failure, many investigators, veterinarians and breeders are clinging tenaciously to the belief that abortion in cattle is a specific contagious disease due to the $B$. abortus and that its control is to be brought about upon that basis and that alone.

After the failure of the Brauer plan with carbolic acid, Rich of Vermont believed for a time that he had discovered an efficient systemic disinfectant in methylene blue, but the error was promptly recognized and the plan abandoned.

Attempts to disinfect the contents of the pregnant uterus are contrary to all existing knowledge of its physiology and that of the embryo. Experiments show that aniline dyes, like methylene blue, do not reach the embryo, but are caught or turned back at the placenta. It is not known that any disinfectant can pass the placental filter in a manner to exert practical influence. In abortion most of the infection is 
in the utero-chorionic space. Into this space it is not known that any disinfectant borne in the blood ever enters. It probably does not. Even should a disinfectant leave the blood stream and enter the utero-chorionic space, it is inconceivable that sufficient would enter to disinfect. The endometrium of a pregnant cow at full term represents approximately fifteen square feet of mucous membrane, opposed to which is the somewhat smaller surface of the contiguous chorion. Between the two there exist, in badly infected cows as revealed in the abattoir, from a few ounces to several quarts of "abortion exudate." It must require great confidence in the potency of disinfectants to believe that a sufficient quantity can enter the utero-chorionic cavity to disinfect it, or pass the placental filter and, after coursing through the fetus, be poured into the fetal alimentary canal and disinfect the meconium. The employment of disinfectants in this apparently hopeless manner has been virtually abandoned-permanently, it is to be hoped.

The generally accepted theory that abortion in cattle is a specific contagious disease led many of the subscribers to that belief to hope for a specific remedy in the form of a serum, bacterin, vaccine, or other biologic substance. Notable achievements in this field had been made in a number of diseases of man and animals and it was assumed that in abortion a similar victory was probable.

The achievements in the biologic control of disease have been chiefly among those maladies designated as acute, in which a definite immunity follows the recovery of the patient. In chronic infections little progress has been made in biologic therapy, apparently because the success of these products is dependent upon their power to produce that state known as immunity. Infectious diseases are divisible primarily into acute and chronic. In acute infectious diseases the infection and the patient can not long coexist. Either the infection kills the patient or there develops within the body of the patient substances which destroy the infection and which thereafter prevent, for a variable period, re-infection. This is known as immunity. In chronic 
infections, such as tuberculosis and syphilis, the patient may survive indefinitely and the infection continue in viable form. The system of the patient acquires a power of resistance to the ravages of the infection, but not the power commonly to destroy it or to prevent reinfection.

Those who regard the $B$. abortus as the specific cause of abortion in cattle hold confused views as to whether it is acute or chronic. Those who claim to have induced abortion experimentally, constructively hold that it is an acute infection. Some believe that they have induced abortion in five to ten days. At first that was the general belief. Bang in his first experiments believed he was dealing with an acute infection having a short incubation period. Experimenters believing in the specific character of cattle abortion generally hold that it is an acute infection for guinea pigs: that is, if the $B$. abortus is injected into a pregnant guinea pig, she will abort in five to ten days. Numerous investigators now hold that the $B$. abortus infection is virtually an acute infection in the uterus and a chronic infection in the mammary glands. That is, the infection can not invade the uterus until pregnancy is definitely established, and can not continue in the uterus for more than a few days after the termination of pregnancy. They further hold that, after one fetal cadaver has been expelled, the uterus acquires an immunity and the $B$. abortus can not again readily invade it and cause a second abortion, but the mammary gland, once invaded, is permanently infected. Schroeder thinks that the mammary gland is the permanent habitat of the $B$. abortus and periodically, during pregnancy, the infection may in part pass from the mammae to the uterus, cause abortion, and then promptly disappear from the uterus. It is reasonably well established that the $B$. abortus may be a permanent resident of the milk glands. So far as I have seen recorded, it has not been searched for elsewhere than in the genital canal and milk glands. One cow in my experimental herd, now ten years old, was inoculated in the jugular in her first pregnancy at about seventeen months of age. She was probably already infected 


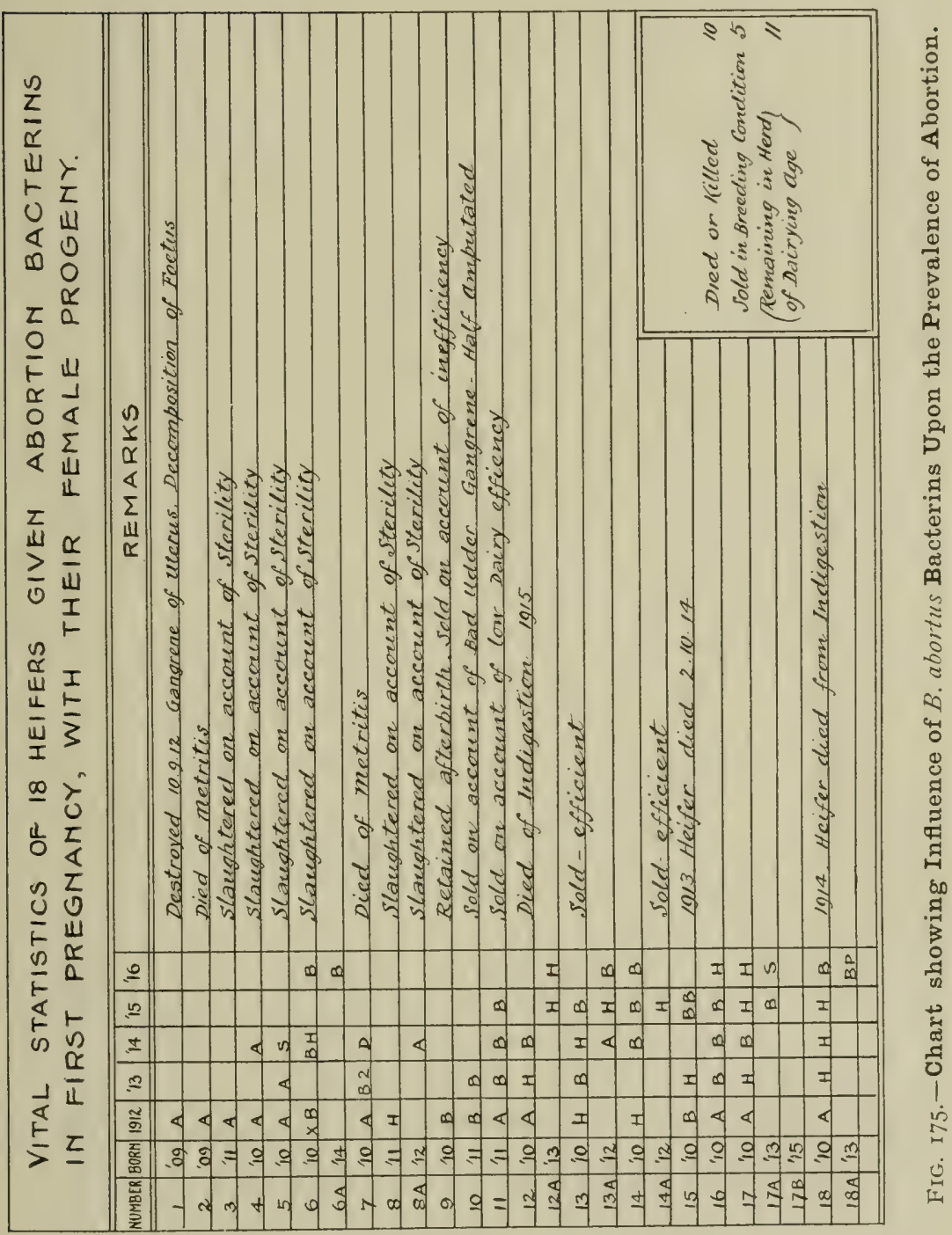


when I inoculated her. Her blood still showed a high degree of infection when she was eight years old. Clinically she has a high degree of genital infection as shown by a very low rate of reproduction. It appears clear, therefore, that those who hope to control or eradicate abortion through the agency of biologic methods must hope for an artificial immunity in a chronic infection.

There is a common belief in a natural immunity against abortion. That is, it is stated that after a cow has aborted once, she is not so liable to abort again. The assertion has been made and remade so often and persistently that by mere repetition it has virtually acquired the force of a fact. But it is perverted fact. The influence of abortion upon future reproduction is well shown in Fig. 175. In this instance eighteen apparently vigorous heifers received abortion bacterins in their first pregnancies in order to test experimentally the power of killed bacteria to prevent abortion. The heifers in this herd had for some years averaged about 30 per cent. of observed abortions. The heifers shown upon the chart had been handled, except for the bacterins, the same as prior heifers. As calves they had been grown in an abominable "nursery" under pest-house conditions. Dysentery and pneumonia had been virtually universal. The mortality of calves had been high, but the survivors recovered their general health and were apparently perfectly sound. Yet this group of vigorous heifers failed to maintain its original numbers of fertile females. The losses from infections within the genital tract were so great that the female progeny failed utterly to fill the gaps made by disease. If abortion produces a valuable immunity against fu-ture abortions, this group of heifers should have acquired such immunity and have produced, during the period covered by the chart, a notable excess of healthy females instead of the discouraging deficit so clearly shown.

It is perfectly true that many aborting heifers and cows do not repeat the abortion the following year, or, if repeated, it is not recorded. When an ordinary grade cow is observed to expel a fetal cadaver, she has usually reached 
the fifth to eighth month of pregnancy. Prior to conception, probably three to six months had elapsed since the previous calving. She is consequently nearly or completely dry and the owner cannot hope for profitable lactation or for a healthy calf until about twelve more months have elapsed. Naturally she is sold for beef, or disappears for some other reason. This is well illustrated in the work of Bland $^{1}$, who states that in the herds listed in 1914-1916, totalling 1,016 animals, there had been, in 1919, 305 or 30 per cent. of abortions. In the same herds, in 19141916, 160 animals are accounted for which had aborted in $1913 ; 145$, or 48 per cent., of those aborting in 1913 were not in the herds in 1914. Probably some died, some were sterile and therefore sold, and some may have conceived and have been sold to other dairymen but not because the seller thought them immune to abortion. Of the 160 cows aborting in 1913 which are reported in 1914-1916, 107, or 67 per cent., calved and 33 per cent. failed to produce calves during the period of two or three years between 1913 and 1916. Though they were presumably the most promising of the aborters in 1913, 33 per cent. failed to produce calves. These data fail to show a valuable immunity.

Infections of the genital tract, like chronic infections of other organs, are rarely static. During the interval between weaning (or changing from a milk to a vegetable diet) and the first coitus, the animal enjoys the most healthful period of its life, judged by clinical standards. When sex activity begins, it is difficult to conceive of a static condition of the bacteria in the genital tract. Abortion signifies one grade of activity of these bacteria, lower in virulence than sterility and higher than dysentery and retained fetal membranes. It should not be expected that in each breeding year an active, virile infection would maintain throughout a given level but that it would vacillate from year to year. Should the intensity increase markedly, causing hydrosalpinx, pyosalpinx, or ordinary salpingitis, the animal becomes seri-

${ }^{1}$ Bland, G. R., Second Rep. Ag. Organizer, Epizoötic Abortion Exp., I9II-I9I6, Oxfordshire, Eng., page I5. 
ously or hopelessly sterile and her breeding career is closed. This alone renders it impossible for many aborters to abort again the following year. But the cow may improve, the uterus gain new vigor, and the infection become largely repressed. Then, if she aborts at all, it will be later in her pregnancy. If the repression of the infection goes further, pregnancy may continue to full term and a live calf be born. The cow may have retained fetal membranes and the calf may have dysentery, but abortion, as defined, is absent. The same or a kindred infection, however, is still present and active. Naturally, therefore, the infection in the genital tract does not as a rule bring about each year precisely the same result, except when hopeless sterility has been reached.

In all data I have been able to obtain, it has been shown clearly that a given number of cows or heifers which aborted the previous year are more likely to abort again the next year than an equal number of animals which have dropped healthy calves. But the question is to be decided by the percentage of abortions among those which become pregnant. A far larger proportion of the aborters fail to breed. The difference between the two classes is rendered more clear when the false measure of abortion is discarded and the results are measured by the percentage of healthy calves born.

Regardless of the foregoing facts, it is attempted to control or eradicate abortion, working upon the theory that it is a specific contagious disease and that one attack produces a distinct and valuable immunity.

$B$. abortus serum has been proposed as a remedy and has failed. I used serum experimentally upon a group of 26 heifers ${ }^{1}$ without result upon abortion, sterility or other interference with reproduction. The animals upon which the serum was used aborted at virtually the same rate as the controls and the two groups aborted at the average rate for the herd in which the abortion rate had for years been virtually static. My results were in harmony with all available

${ }^{1}$ An. Rep. N. Y. State Vet. Col., I916-1917. 
recorded experiences. A few biologic vendors offer abortion serum, but it has attained no popularity. There is no reason to assume that such a serum can produce favorable results.

Abortion bacterins (killed cultures of B. abortus) have been tried by Bang, McFadyean and Stockman, and other leading investigators. In all recorded tests they have failed utterly. The phenomenon of abortion lends itself readily to patent medicine methods. If at the close of an abortion storm abortion bacterins or distilled water be injected subcutaneously, the abortion ceases or the rate is very low. The eighteen heifers charted in Fig. 175 were in a herd where for a number of years the abortion rate in heifers varied from 30 per cent. to 50 per cent. or upward. After the use of the bacterins the abortion rate was the most disastrous in the history of the herd. In spite of the clear and indisputable proofs recorded of the absolute uselessness of abortion bacterins, several American biologic houses make and sell them under wholly unjustifiable claims. Some even offer to refund the sum paid for the preparation for each cow that aborts after the administration. This is perfectly feasible, since so far as manufacturing costs are concerned the vendor can refund upon 50 to 75 per cent. of the animals and have a good profit. Some abortion bacterins are very cheaply made. I submitted a number of samples purchased upon the open market to bacteriologists for examination. Nearly all were greatly contaminated with other bacteria, usually a mixture of three, four or more kinds. One sample consisted of an almost pure growth of a long streptococcus. As the bacteria were presumably dead, the species probably made no difference.

Abortion vaccines, or living cultures of $B$. abortus, given non-pregnant animals one or two months prior to breeding have been tried by numerous leading experimenters and have been recommended by some. Sir Stewart Stockman, with a group of lay collaborators, has been the chief supporter of this plan. Stockman ${ }^{1}$ apparently conducted no

${ }^{1}$ Sir Stewart Stockman, Epizoötic Abortion, Rep. Ioth Internat. Vet. Congress. 
researches personally, but states: "a local organization was set up in each case consisting of members of farmers' societies and veterinarians," to whom the details of the work and the reports were entrusted. In the data published by Stockman, it is not shown upon what plan the herds investigated were divided into "inoculated" and "controls." It is quite easy, if one is acquainted with the history of each animal, to change the face of statistics materially. Stockman rests his statistics upon the percentage of abortions and ignores reproduction. He takes it for granted apparently that if a cow does not abort, or rather is not observed to abort, nothing else in her career is of interest. Thus, in his table IX comprising 758 animals, 77.6 per cent. of vaccinated animals and 70.2 per cent. of the controls calved. The efficiency of the controls fell 7.4 per cent. below the vaccinated. The efficiency was low in both. The weakest point in the data of Stockman is the failure to state the time covered by the experiments. If 77.6 per cent. of the vaccinated cattle calved during one year and were fertile at the close of the year, the performance was certainly better than if the period extended over two or three years, because the efficiency, if the standard is set at one calf per annum, would be 77.6 per cent., 38.8 per cent., and 25.9 per cent. respectively.

The Bland reports ${ }^{1}$ offer by far the most valuable data on record for a critical study of the influence of the vaccination of non-pregnant cows with living cultures of $B$. abortus. The first report is stated to cover experiments during 19111913. At what date in 1911 the researches began is not revealed, nor is it stated when in 1913 they closed. Since the second report is stated to apply to 1914-1916, there was presumably no interval between the two reports. In the 19111913 report it is claimed that of 263 animals vaccinated, 226 (86 per cent.) calved. There is no intimation that during 1911-1913 any cow calved twice or thrice. If the observations cover two years, the annual reproduction rate is 43

\footnotetext{
${ }^{1}$ Bland, G. R., Epizoötic Abortion Experiments, Oxfordshire County Council: First Report, 19II-1913; Second, 1914-1916.
} 
per cent. of the ideal of one calf each twelve months. During the same period the reproduction in the controls was 77.4 per cent., or only 8.6 lower than in the vaccinated. Inadvertently selecting the controls improperly might very well have made a much greater difference.

Bland compares the abortion rate in 1910 with the rate upon the same premises in 1911-1913, but the cattle in the herds of 1910 were not the same as those used in the experiments. In effect, he says, "In 1910 the cow, Rose, aborted (100 per cent. abortion) and in 1911-1914 the cow, Cherry, was vaccinated and calved (100 per cent. calves) ; therefore vaccination reduced the abortion rate from 100 per cent. to

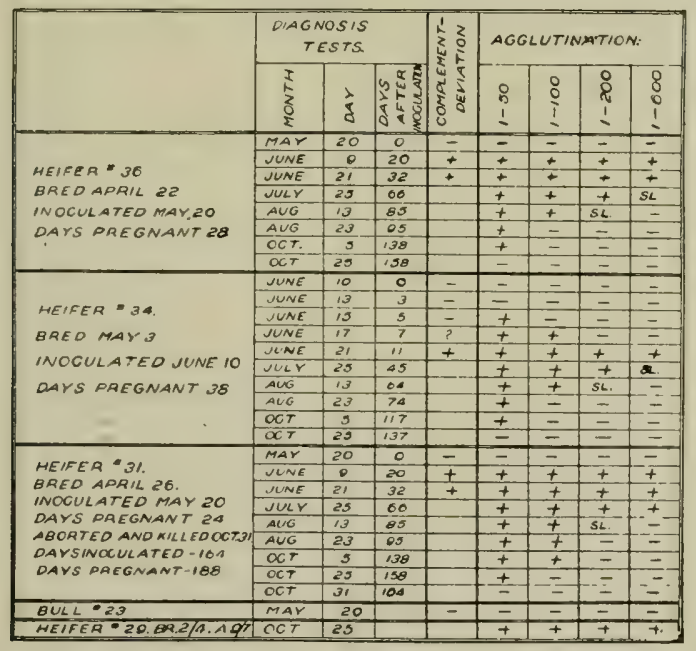

FIG. 176-Chart of Agglutination Test on Three Heifers after Inoculation with $B$. abortus with failure to react on date of Abortion.

zero." That is, Bland asserts that in 1910, 28.9 per cent. of 671 animals, or 194, aborted, and later he states that 66 of the 1910 aborters were used in the 1911-1913 experiments, and 128 aborters disappeared. Forty-three cows which aborted in 1910 were vaccinated in 1911-1914, and of these 29 (67 per cent.) calved. Evidently the dairymen selected for holding those aborters which gave the greatest promise 
of breeding. It is not disclosed whether it took on an average one, two, or three years for one of the aborters to produce a calf.

The unreliable character of Bland's experiments is further indicated by the fact that, while the announced plan was to vaccinate only non-pregnant animals, he actually did not know whether they were pregnant or not. As a consequence a number of pregnant heifers and cows were inoculated. Although $B$. abortus was looked upon as the cause of abortion and would quite certainly cause abortion if injected during pregnancy for that purpose, the animals erroneously inoculated to prevent abortion were benefited the same as the non-pregnant animals.

In such experiments dairymen sooner or later become good practical judges of results. Before the 1914-1916 researches began, most of the dairymen owning the herds of the 1911-1913 experiments had dropped the work; before the close of 1916 a majority of the animals in the second set of experiments had been withdrawn.

In defiance of these and all other recorded data, several American firms make and sell what they represent as abortion vaccines consisting of living $B$. abortus cultures. Bacteriologists to whom I submitted samples purchased in the open market failed by cultural method to find living abortion bacteria in any sample. There is probably no alleged therapeutic substance upon the market, biologic or proprietary, which is advertised in a more misleading and unjustified manner. There is absolutely no justification, so far as I can find recorded, for the claim that abortion can be economically decreased by their use. The data of Bland appear on the surface to decrease the abortion, but the abortion rate is largely a matter of vision. The number of calves was not materially increased in the Bland experiments, since the abortions were statistically lowered by the higher rate of sterility.

I believe abortion vaccines (if they really contain living abortion bacilli) the most dangerous and vicious biologic product thus far offered to veterinarians and stockmen. It 
is perfectly well known that the $B$. abortus is a permanent resident of the mammary gland. It is not known that it is not a permanent dweller in other parts, once introduced. Although experimentally it has not been shown clearly that $B$. abortus does or can invade the pregnant cow and cause abortion there is good evidence that it is a dangerous infection in the genital tracts of cattle and that in the present state of our knowledge attempts to use it as a therapeutic agent are little short of criminal. Researches may throw a more favorable light upon vaccination, but until they do the prudent veterinarian and dairyman should leave this work where it belongs - in the hands of experimenters.

Upon the theory that the observed expulsion of a fetal cadaver is a specific contagious disease, it is repeatedly proposed to control abortion by quarantine restrictions associated with disinfection of the aborter and of the premises occupied by her. Public funds are expended in researches upon "contagious abortion," and the public demands a visible return upon the investment. As a consequence the United States Department of Agriculture, through its Bureau of Animal Industry, and the agricultural experiment stations of the various states are expected to advise dairymen and breeders how to control losses from "contagious abortion." Not only do laymen who are suffering economic losses demand a remedy, but they expect measures which the ordinary dairyman or breeder can successfully apply. The result is that much advice is given without visible benefit. The owner is advised to quarantine all aborters, but probably he sees less than one out of three fetal cadavers which are expelled. Yet inferentially he is taught to believe that those which he does not see do not matter and that quarantining the known aborters will eliminate the danger from the far greater number of unseen expulsions of fetal cadavers. The abortion is not the disease. Once the fetal cadaver and its membranes are expelled, that danger ends. The cause of the expulsion of the dead fetus, the metritis and cervicitis, continues indefinitely and quarantine does not affect it in the least. Precisely the same infections 
which caused the abortion existed long prior to the final disaster, and they continue indefinitely afterward. Quarantine does not annul the peril prior to abortion, nor does it render the animal safe after the common duration of quarantine has expired. Some of these advisers direct that an aborter shall be quarantined until all discharge ceases, but no definition of "discharge" is given. Long after a cow has aborted and all signs of discharge, as commonly accepted, have ceased, the cervical canal or uterine cavity may, and often does, contain a few drops, or quarts, or gallons of pus. The cow with a few quarts of pus in her uterus is a safer animal in a herd than one without visible or notable discharge but which has in the uterus or cervical canal a few drops of virulent pus associated with inflammation of the uterine or cervical mucosa. The cow with large volumes of pus which is being discharged does not generally come in estrum. In the dairy the pus chiefly drops into the gutter and disappears. Some of it reaches the milk, but not as much when the cow is in the herd as when she is in quarantine, because in the latter state she is neglected.

The cow with a few drops of pus in the uterus or cervix ovulates and copulates. Coitus intensifies the disease in her genital tract and endangers the genital health of the bull. Such a cow either fails to conceive or conceives only after repeated copulations, with undue sex strain and infection dangers to both cow and bull. If she conceives, the infection in the cervical canal or uterus leads to the death of the embryo or fetus; or the pregnancy continues to, or near to full term, a sick calf is born, and the cow has metritis, often associated with retained fetal membranes. Ordinary quarantine-the isolation of aborters from pregnant cowsneither adds security to nor hastens recovery from the metritis and cervicitis, but the layman, ignorant of the nature of the disease, is misled into the assumption that quarantine has therapeutic value. As a matter of fact quarantine, as ordinarily conducted with aborting cows, is a therapeutic vice. The cows are denied the food, housing and care conducive to the spontaneous recovery or amelioration of dis- 
ease. I have yet to see quarantined cows receive that attention and care which is accorded the presumably well animals.

The effect of the quarantine of aborters upon the remainder of the herd is nil. The removal of an aborter after the act, or while it is in course, does not eliminate or modify any possible injury from the unobserved discharges which have occurred days, weeks, or months preceding the expulsion of the fetus. More important still, the quarantine of an aborter can not possibly cure or ameliorate the infection which exists in the uterus or cervix of another pregnant cow or heifer.

In the quarantine scheme commonly advised, there is included only a minority of animals in which the embryo or fetus dies and is expelled. Up to the fourth or fifth month of pregnancy in dairy cattle, the expulsion of the embryonic or fetal cadaver is not observed. In beef cattle, especially those in extensive pastures, the observation is far less accurate. While the expulsion of fetal cadavers is doubtless less frequent in beef than in dairy cows, it is equally certain that the apparent difference is due partly to a difference in the accuracy of observation.

The quarantine of aborters can not lessen the dangers from those intensely infected animals which do not abort. When metritis exists during pregnancy, it necessarily continues after parturition, frequently with retained fetal membranes. The volume of highly infectious material, identical with the discharges following abortion, is usually far greater than that associated with abortion, but the quarantine scheme generally recommended ignores these. Finally, many cows which drop apparently healthy calves and are to outward appearances perfectly well, when professionally examined sixty, ninety or more days post partum, show an intense cervicitis with a few drops of pus in the cervical canal and in the cervical end of the uterine cavity. Nothing but harm can come from attempting to breed such cows, but quarantine, as commonly advised for aborters, has no influence whatever upon these animals. I have examined herds where the abortions recorded did not exceed 15 per 
cent., but 60 per cent. showed intense pyo-cervicitis and were sterile. The quarantine of the 15 per cent. of aborters could cure the cervicitis neither in these nor in the 45 per cent. of cows having cervicitis which had not aborted. Quarantine could not influence favorably either the quarantined or the remainder of the herd from which the aborters were removed. Clinically the scheme for controlling abortion by the quarantine of aborters has failed completely. It has been the common measure applied throughout the civilized world for more than fifty years, a period during which the virulence of the infections of the genital organs and the economic losses sustained have increased enormously. The quarantine of aborters has been attempted by the most intelligent, earnest and competent dairymen and breeders, whose losses have been, if anything, greatercertainly no less-than the losses in the herds of the most careless and ignorant. Although quarantine of aborters has been conscientiously and faithfully applied to many herds since the theory of specific disease became established, no record is extant, so far as I am aware, where the quarantine of aborters has favorably influenced reproduction. If failure in practice is competent argument against a theory, then the theory that abortion is a specific contagious disease of cattle, and that quarantine based upon that theory is a valuable sanitary measure, falls to the ground.

Some of the devotees to the theory of the specific character of abortion have recently turned to another quarantine scheme consisting of the diagnosis of the alleged specific infection by the agglutination and complement-fixation tests of the blood of each animal in a herd. Quite naturally they have advised that, in assembling herds and in making additions thereto, these tests should be applied to all purchases. It has already been pointed out that the line of demarcation between infected and non-infected, as indicated by these tests, has been fixed by absolutely arbitrary means and that the agglutination and complement-fixing power of the blood from infected and non-infected animals is absolutely unknown. Even if it were admitted that abortion in cattle is a 
specific disease due to $B$. abortus, there are ample reasons for believing that the blood of animals free from the infection of $B$. abortus does not react at all to these tests at the lowest point where they can be applied. It is further evident that the tests fail at high dilutions when the infection is abundant. This defect is well illustrated in Fig. 176, in which the agglutination test was negative upon the day of abortion. Two prominent advocates of the serologic tests for the control of abortion, jointly published a bulletin in 1918 in which they said: "The serological tests are an important aid in the inauguration of preventive measures against the spread of infectious abortion." Throughout the publication, the serologic tests appear as the corner stone in control measures. They advised the test as a means for isolating the infected from the non-infected animals and for preventing the introduction of the infection in purchased cattle of both sexes. In 1919 they had modified their views and in a second bulletin advocated the disinfection of the copulatory organs of the bull as the most important measure of control available, and say: "Our work of the past five years has convinced us that transmission by the male is by far the most important means of spreading the disease . . . . Proper disinfection of the sheath, therefore, constitutes the most, if not the only, important safeguard against the spread of infectious abortion." The italies are mine. They present no data to show that they accomplished any actual improvement either under the repudiated first plan or under the second recommendation.

Another important group of believers that abortion is a specific contagious disease advocate the quarantine of aborters, plus the disinfection of aborting cows, of the external genitalia of breeding bulls, and of the premises occupied by aborters. There appeared an undated circular from one of these sources with a title page printed chiefly in red which reads in part, "FIgHT CONTAGIOUS ABORTION IN CATTLE - . Preventable-Controllable . . Isolate affected animals-Treat aborting cows-Disinfect bulls- 
Clean up premises." It was held in the circular that if breeders and dairymen generally would conscientiously and vigorously apply the advice contained in a bulletin soon to appear, "contagious abortion" would be eliminated or controlled.

In 1917 there appeared from the same source a bulletin which was apparently an amplification of the preceding circular. Among other recommendations the bulletin advises that the aborter be isolated and that her uterus be irrigated daily with antiseptics. "Lugol's solution in a strength of 2 per cent. has been found to be desirable as a uterine douche. It is not permitted to remain in the uterus but is flushed out with salt solution." The bulletin was, of course, designed for, and sent to, laymen who do not know the uterus from the vagina, cervix, or oviducts. If the afterbirth is retained, neither the layman nor the skilled veterinarian can possibly douche the uterus, because it is everywhere covered by the chorion. If the afterbirth has come away from the aborter, the entire endometrium is denuded of epithelium. The introduction of 2 per cent. Lugol's solution into such a uterus is a professional crime. It will kill or ruin sexually a large proportion of cows, and injure all. Fortunately most laymen introduce antiseptics into the vagina only, from which they are sometimes ejected before material harm has resulted. I have had an opportunity to study a group of 27 aborters, purebred beef cattle of great value, to which a layman had attempted to apply these recommendations. Of the 27 animals, 12 ( 44.4 per cent.) have atresia of the vagina, unquestionably due to the treatment. Two of these twelve (16.7 per cent.) are again pregnant after about one year. Each has a severely constricted vagina and will apparently have great, if not insuperable difficulties at calving time. Six of the remaining animals may possibly (but very improbably) conceive, with the probability that they cannot calve successfully. The other four of the twelve are hopeless because of the vaginal atresia and must go to slaughter. In two of the four the vaginal atresia is so complete that mucus is re- 
tained in front of the atretic area, causing great distension. Of the fifteen animals in this group in which vaginal atresia was not caused, six (40 per cent.) are pregnant, one possibly pregnant, and eight (53 per cent.) non-pregnant, with fair prospects for conceiving. The instance affords a good illustration of the results attained from erroneous advice given to laymen who, however intelligent and sincere, fail in their application.

Advising laymen how to handle surgically a cow which has recently aborted is much like a Doctor of Medicine advising a carpenter how to diagnose and handle appendicitis in a member of his family, with the saws, chisels, and other implements at hand. The owners of cattle view the problem from another angle. Certain veterinarians are supported by public funds, and stockowners feel they have a right to demand of these public servants advice regarding abortion. These veterinarians have for so long taught the public to believe in abortion as a specific infectious disease that they can give no advice of value without clashing violently with the thoroughly established misconception.

There is nothing known at present beyond general good care which can in the least exert favorable influence upon an infection already existing in the utero-chorionic space of a pregnant heifer or cow. After abortion has occurred and the uterus, cervix, and vagina are open to surgical invasion, the skilled veterinarian can do much to cleanse the genital tract of injurious infections. The good which may be accomplished at that time is not based upon the theory that abortion is a specific infectious disease, but that all abortions are due to an infection or to the joint action of several infections. At present the known remedies against abortion are alike effective in all infections which may lead to the death of the fetus and its expulsion by the uterus. The handling of the infections of the genital canal calls for the application of the general principles of surgery. Those cases of cervicitis and metritis existing during pregnancy and inevitably continuing after abortion call for the same method of handling as those not associated with abortion 
but dependent upon the presence of the same infection. The handling of such infections is discussed in the appropriate places.

\section{Puerperal Diseases of the Uterus}

When pregnancy terminates, those infections which have been present in the utero-chorionic space of the gravid uterus meet with abrupt changes in environment which may profoundly alter their activity. New forms of bacteria may also quite readily invade the uterus at this period. If a calf is born or a fetal cadaver is expelled and the fetal membranes follow promptly, the entire placental area of the uterus is denuded of its epithelium and the tissues laid open to attack. The infections present in the utero-chorionic space have already invaded the placental tissues about the cotyledonal periphery and are ready to advance rapidly when epithelial denudation becomes general. If, instead of being expelled, the fetus dies and macerates, the fetal cadaver acts as a vast nutrient reservoir for bacteria which multiply in it and profoundly involve the contiguous uterus. If a calf is born or a fetal cadaver is expelled, and the fetal membranes are retained, the latter are necrotic and, like the retained fetal cadaver, serve as a great nutrient supply for infections present. The dilation of the cervical canal, vagina, and vulva permits a greater supply of air in the uterine cavity, which may modify favorably or unfavorably the activities of the bacteria.

It was long believed, and quite generally taught, that the infections imperilling the life or health of the puerperal female regularly invade the uterus after the close of pregnancy. In an important measure that is true, but by far the most important infections, measured by their frequency and economic bearing, are those which are resident within the pregnant uterus and acquire increased intensity when pregnancy terminates. The infections of the puerperal uterus are consequently divisible into two great groupsthe intrinsic or intra-uterine infections continuing from pregnancy, and the extrinsic organisms invading the uterus from without after pregnancy has terminated. 


\section{A. Uterine Hemorrhage. Uterine Hematoma.}

Hemorrhage into the uterus of the cow during the puerperium is of two distinct classes. An insignificant hemorrhage regularly follows the prompt dehiscence of the fetal membranes. Lacerations, especially of the cotyledons, frequently cause slight hemorrhage. Hemorrhage is also caused by imprudent attempts at removing retained fetal membranes. These forms are to be handled according to the general rules of surgery.

Clinically the most interesting and most important type of uterine hemorrhage is that which is apparently one of the results of intra-uterine infection. The hemorrhage appears without warning soon after, but not immediately following parturition. I have observed it at two to four days post partum. My cases have occurred in cows in intensely infected herds. As I recall, each patient had given birth to a calf, but the parturition was tardy, testifying to the presence of a uterine paresis due to a metritis of pregnancy. The fetal membranes were not long retained and the patients were apparently going along favorably, when suddenly without warning a very profuse uterine hemorrhage began. One of my cases had been left at night in a box stall, apparently well. When the caretaker arrived in the morning, the cow was weak from loss of blood and her stall looked like shambles. Bright scarlet blood was spattered over the walls and bedding, and blood was still trickling from her vulva. A few hours later I found upon examination, as nearly as I could estimate, a blood clot in the uterus equal to two or three gallons. The blood was coagulated, odorless, and bright scarlet in color. Fearing recurrence, I proceeded to remove with great care a portion of the clot each day, until in three days I had completely evacuated the uterus. It maintained throughout its bright scarlet color. It underwent no visible decomposition and acquired no specific odor. The cow recovered and was fertile. In a second case a colleague removed the blood from the uterine cavity, first controlling the hemorrhage by the use of adrenalin chloride hypodermically. 
Uterine hemorrhage of the puerperium apparently bears an interesting relationship to other uterine hemorrhages in the cow. Elsewhere interplacental hemorrhages from the pregnant uterus with fetal desiccation have been described. Presumably in that case infection plays the essential part. At any rate it is observed chiefly in cattle belonging to highly infected herds. Unfortunately the initial stages of interplacental hemorrhage in the pregnant cow are not available for observation. The desiccation of the fetus is ordinarily the first step recognized. In fetal desiccation, as in puerperal hemorrhage, there is no observed tendency to putrefaction. Apparently it has been thought that fetal desiccation occurs because the cervix remains closed and sealed so that bacteria may not invade the hematoma, fetal membranes, and fetal cadaver. That is evidently incorrect. Bang and others have recognized the $B$. abortus in the desiccated fetus. That bacterium possesses scant putrefactive power. In the uterine hemorrhages under consideration there seems to be something more than a neutral force at work. When the hemorrhage commences, the uterine cavity is freely open to the exterior and is certainly heavily infected. The escaped blood, instead of forming a breeding ground for commonly known bacteria which set up putrefaction, possesses a unique power to prevent putrefaction. An ordinary blood clot in the uterus or in a wound is the signal for the intensification of infection in the part, but here the opposite occurs. The first suggestion of its unique character comes with the blood escaping from the vulva, which is intensely scarlet, much brighter than ordinary arterial blood, and looks much like blood from the lungs.

An interesting fact, also, is that the uterine hematoma of the puerperal period, if left to itself, apparently desiccates in a manner similar to the desiccation of the fetus. At least there are sometimes found in the uterus old desiccated blood clots. I have observed a number of these in the abattoir, usually involving one uterine horn only (the hemorrhage having occurred in the gravid cornu alone?), but sometimes chiefly in the uterine body and projecting into one horn. In 


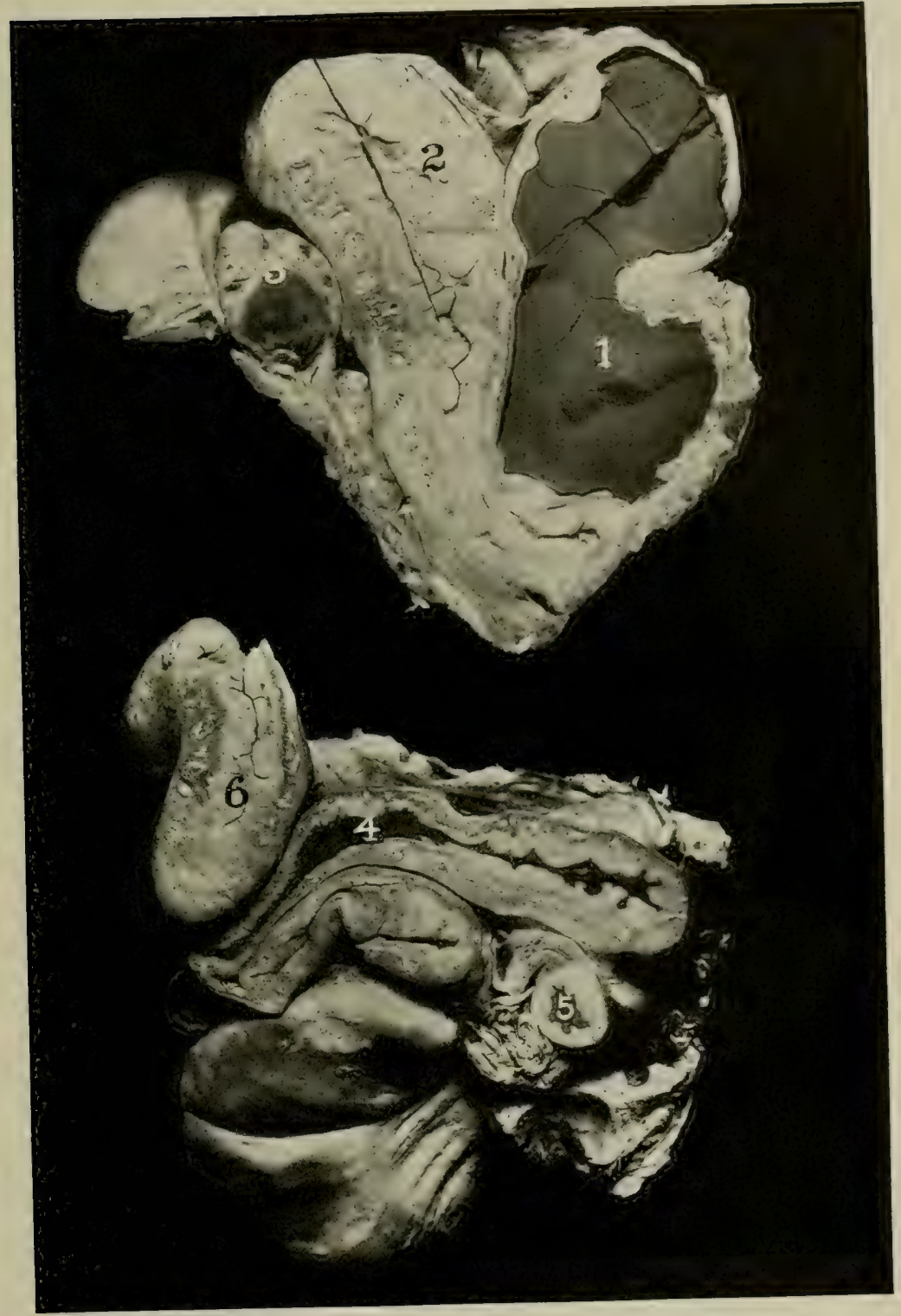

FIG. 177-Old Hematoma of Right Uterine Horn.

$a$, Viewed from above. $I$, Hematoma; 2 , left cornu apparently healthy ; 3 , section through left ovary showing corpus luteum.

$b$. Ventral surface. 4, longitudinal section through right horn; 5, cross section through right horn, showing hematoma; 6 , section through normal left horn. 
one instance one horn contained a desiccated hematoma and the other an apparently healthy embryo three or four inches long. The plan of the hematoma is well illustrated in Fig. 177, in which the right cornu is completely filled by the old blood clot, while the left horn remains normal and vacant. The only interpretation which I can place upon the condition is that, at the termination of the last pregnancy, there occurred within a few days a voluminous hemorrhage into the gravid horn. Part of the blood escaped through the cervix and vagina to the exterior, and part remained behind to undergo desiccation and to continue, like the better known desiccated fetus, embedded in the hematoma, as an inert body which may persist for months and years. The desiccated hematoma is a firm, doughy, black, tarry mass. When the fresh specimen is incised, it is so sticky that it is difficult to push a knife through it merely because of its adhesiveness. Parts of the mass adhere to the knife and are exceedingly difficult to wash away. It is virtually insoluble

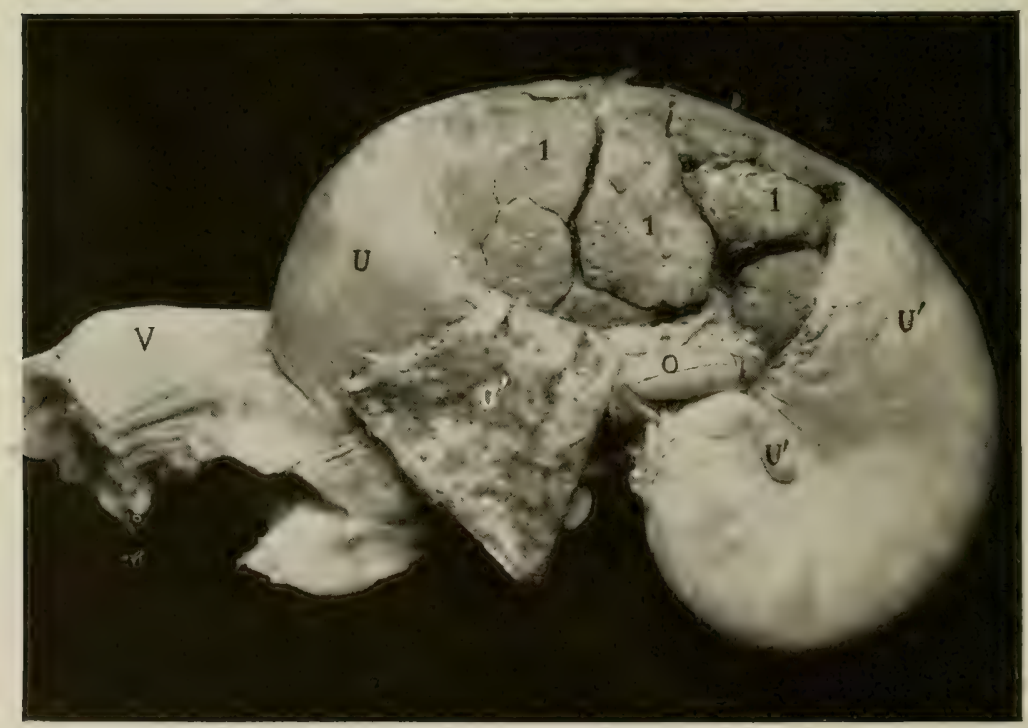

FIG. I7S-Tesselated Hematoma in Right Uterine Horn.

$V$, Vagina ; $U$, uterine body ; $U^{\prime}, U^{\prime}, U^{\prime}$, uterine horn ; $O$, ovary. $I, I ; I$, Hard tesselated masses closely packed together. 
in water. There is a total absence of odor beyond that of meat. There is no reaction of the endometrium. The desiccated blood adheres to it as closely as to the knife or to the operator's hands. The cervical canal is unsealed, except in the co-existence of pregnancy.

It appears that with age further changes may occur, the desiccation proceed further, and finally the hematoma become fragile and, fracturing in places owing to visceral movements, become tesselated in contour, as shown in Fig. 178. However, it still retains its resistance to putrefaction and no fetor appears.

As a rule foreign bodies lying within the uterus inhibit estrum and ovulation. I have had no opportunity to study the uterine hematoma clinically, but in my collection there is one example where a hematoma occupies one horn and an apparently healthy embryo the other. This seems to me conclusive proof that uterine hematoma does not always inhibit ovulation. As I interpret the specimen, the hemorrhage into one horn, occurring shortly after the close of a prior pregnancy, did not permanently fill the cavity of the uterine body, and the cervical canal did not become sealed. The presence of the hematoma did not inhibit estrum and ovulation, and the way was left open for conception. I suspect that this case is an exception to the rule and that generally the hematoma inhibits estrum and ovulation.

The diagnosis of the type of uterine hemorrhage here described appears to be comparatively simple. The cow calves in a manner to attract no special notice. From the conditions which existed in the two herds where I observed it, the infection of the genitalia is intense, and logically the parturition atonic and tardy. The fetal membranes presumably come away without material delay. As I understand the condition, placentitis of that type causing placental incarceration is incompatible with this type of hemorrhage. I believe that the hemorrhage, as in menstruation, and in the inter-placental hemorrhage with fetal desiccation, comes wholly from the cotyledons, so that any placentitis of a type causing stasis and thrombosis in the placental ves- 
sels would prevent the escape of blood in the manner described. The first clinical evidence of the hemorrhage is the escape from the vulva of large volumes of an unusually bright scarlet blood without history of traumatic injury. The cow is generally uneasy (the cramps of severe hemorrhage?) and moves about restlessly, occasionally lying down and getting up. The bright scarlet blood escaping from the vulva stains the bedding, walls, and any object with which the posterior parts of the cow may come in contact. Depending upon the degree of hemorrhage, there is weakness. An examination of the uterus reveals a great hematoma filling the recently gravid horn. The clot is moderately firm.

The treatment should consist at first of efforts to control the hemorrhage. With the very limited knowledge possessed, I would counsel leaving the clot alone for a time and would limit efforts at control to the hypodermic administration of adrenalin chlorid in doses of one-fourth to one-half ounce of the 1-1000 solution. It should be repeated at intervals of one-half to one hour if the case demands. Efforts should be made to keep the patient quiet.

Once the hemorrhage has ceased, the removal of the clot should be undertaken with the hope of obviating three possible dangers: (1) If the hematoma is removed too early, the manipulation may revive the hemorrhage. (2) If permitted to remain for several days, the cervix may contract, rendering the removal of the clot very difficult. (3) If still further neglected, the clot becomes desiccated, exceedingly sticky, virtually insoluble, can not be suspended in water, and can not readily, by any known method, be removed by douching. The veterinarian need not hasten the removal for fear of putrefaction of the clot because, so far as known, it possesses properties which inhibit purulent destruction. The closure of the cervical canal sufficiently to offer great obstruction to the insertion of the hand will not, as a rule, occur until two or three days have elapsed after the hemorrhage. The physiologic uterus closes of course at an earlier date, but the uterus under consideration is paretic. Otherwise the hemorrhage would not have occurred. The behav- 
ior of the cervix should be watched and the removal of the clot attempted sufficiently early. The chief mass of the clot is best removed by carefully breaking it down with the hand, and flushing out the detached pieces with physiologic salt solution. The douching should be done with the greatest possible gentleness, preferably by means of a hospital irrigator to which is attached the seven-foot pure rubber catheter designed for douching the sheaths of bulls. This catheter is soft and pliable, and can not readily damage the endometrium. Its small calibre, permitting the solution to enter the uterus slowly, avoids any sudden distension of the organ. While the salt solution is entering the uterus, the operator should carefully break up the clot with his fingers. After the clot has been removed, the uterus should be watched closely for untoward developments.

The clinical diagnosis of the desiccated hematoma has not fallen to my lot. Apparently it should be easy. The mass is doughy and firm, as in the first stages of fetal desiccation. In the latter case, however, the fetus is usually palpable, especially its head or the feet, some of which may lie in close proximity to the uterine wall. Later the desiccated fetus becomes extremely hard and its skeletal outlines clear. The cervical canal is sealed in desiccated fetus, but open in desiccated hematoma, except, as in one instance mentioned above, when a hematoma was present in one horn and an embryo in the other. One may meet clinically the very old tesselated hematoma of Fig. 178. It is almost as hard as a desiccated fetus, but the skeletal outlines of the mummy are absent and the hematoma is even in its general contour, with the small depressions where the clot has become fissured.

I have had no occasion to handle the desiccated uterine hematoma and know of no rule upon which to proceed. Evacuating the uterus by douching is well-nigh hopeless, as the sticky content is virtually insoluble in water. The dislodgment of the corpus luteum gives little promise because, in my one observation, the corpus luteum had atrophied in order to permit the animal to ovulate from the op- 
posite ovary and conceive. Pituitary extract might act favorably, given hypodermically in small doses. As a final resort laparotomy may be performed upon the involved side, the affected horn lifted out and incised, and the clot mechanically removed. Or one may amputate the involved horn with the ovary and for breeding rely upon the remaining horn.

\section{B. Puerperal Endometritis}

Puerperal endometritis, as here used, signifies a type of metritis in which the endometrium or uterine mucosa is most prominently involved. Infection within the uterine cavity presents illimitable variations in its course and manifestations, and it is impossible to group the various types of puerperal uterine infections so that each class shall be clearly and definitely separated from the others. It is essential that the variations be grouped in a general manner in order to facilitate description, but necessarily some cases will be met upon the border line between any two groups, which may with equal logic be placed in either one. Any given case may also, by either progression or retrogression, shift from one group to another.

The effect of intra-uterine infection is not limited to the endometrium. Whenever such infection is activated by the disturbances incident to the termination of pregnancy (just as holds true of intra-uterine infection during pregnancy), the changes can not be definitely confined to the endometrium, but involve the muscular walls of the organ, causing paresis, and frequently also involve the serosa. Consequently the term "endometritis," if taken literally, is a misnomer. It may be said to be the simplest and most primitive type of metritis. Generally speaking it is out of endometritis that the more severe and critical forms of metritis develop. Sometimes the metritis of pregnancy has passed beyond the stage designated as endometritis before pregnancy terminates, sometimes the progress of the infection is so rapid that no stage is clinically recognizable as endometritis.

A pregnant heifer or cow doomed to abort, to calve prematurely, or to have retention of the fetal membranes has in her uterus an endometritis which inevitably persists into, 
if not through the puerperal period. Even more commonly there is present in the gravid uterus a slight endometritis which does not reveal itself in the puerperal animal by abortion, premature birth, or retained fetal membranes, but may pass unobserved until several days have elapsed after parturition, when clinical evidence appears in the form of an obvious and characteristic genital discharge. In many other instances the exudate in the uterus is not visibly discharged but eventually undergoes resorption unless recognized and removed by surgical interference. The vast majority of cases of endometritis during the puerperal period are unquestionably continuations of the endometritis of pregnancy, but to these must be added some cases of the invasion of the uterine cavity immediately after the termination of pregnancy.

The clinical manifestations of endometritis consist chiefly of the discharge from the vulva of varying amounts of a scarlet-gray exudate, apparently scarlet blood intimately mixed with dirty gray or grayish yellow masses of pus or tissue debris, usually thick like a heavy gruel. It adheres to the tail, exterior of the vulva and surrounding parts. In its most typical form the exudate is not fetid, but has a faintly sweetish odor. The appetite, temperature and lactation may be slightly or not at all disturbed.

The discharge may be clinically evident during parturition as soon as the uterine seal gives way and the chorion is forced against the uterine wall at the cervical end, which presses out some of the endometritic exudate ahead of the fetus itself. After the fetus and its membranes have been expelled, the visible discharge of the exudate is largely dependent upon the volume present and the degree of tone retained by the uterus. Generally the discharge is not prominent until two to five days after pregnancy has terminated. It may be delayed even longer and, although the exudate may be present in considerable quantity (one-quarter to one-half pint), no recognizable discharge may occur.

Ideally, physiologic parturition is completed in the cow or heifer in one and one-half to two hours, and no recognizable uterine discharge follows. If examined clinically, the 
uterus contains no notable exudate. The time occupied by the visible act of parturition is fifteen to thirty minutes of definite expulsive effort, during which the calf is born, followed immediately by a period of one to two hours during which uterine involution progresses rapidly and the fetal membranes passively separate from the placental areas and are then expelled by the contractions of the uterus. There is an active and tonic elimination of the vast uterine cavity which existed an hour or two before. There is no place within the uterine cavity for the retention of recognizable masses of blood or other matter, and the contraction of the uterine walls inhibits hemorrhage from its mucosa or the outflow of recognizable quantities of lymph.

When active infection is present in the utero-chorionic cavity of the pregnant animal, the parturition is tardy. The expulsion of the fetus may require five, ten or many more hours. Dystocia may occur as a result of uterine paresis and necessitate aid. After the fetus has been expelled, the dehiscence of the fetal membranes is slow and uncertain and uterine involution is tardy and feeble. Then follows an exudate into the uterine cavity (or into the utero-chorionic cavity if the fetal membranes are retained) which tends to reveal its presence by becoming discharged from the vulva. The exudate may, however, be detained in the uterus for several days and may be resorbed without any notable part of it having appeared externally.

When the discharge appears or for other reasons a clinical examination is made, the scarlet-gray exudate already described may be found in the vagina or uterus or both. If the uterus largely retains its tone, the exudate is nearly all forced out into the vagina promptly instead of accumulating within the uterine cavity. From one-fourth to one pint may accumulate in the cervical end of the vagina. If the uterus is very paretic, up to one gallon or more of the exudate may accumulate in the cavity while the vagina may be free of any notable amount. Then the exudate may not be expelled actively, but be forced out passively when the animal is recumbent, owing to the mechanical pressure of contiguous 
viscera upon the paralyzed distended uterus. Thus involuntary discharges of large volumes of scarlet-gray exudate are observed frequently behind recumbent cows which otherwise appear well. Upon rectal palpation the uterus of such a cow is enlarged, atonic and flabby. Whatever the amount of exudate present, the walls of the uterus are not ordinarily stretched, but lie dormant and powerless. If the uterine cavity is douched, there may be siphoned or washed out a variable quantity of scarlet exudate.

In the abattoir endometritis of the type described is common. Apparently dairymen and breeders frequently discard females which have recently aborted, owing to the common theory that a cow or heifer which has aborted because of intra-uterine infection should be isolated in order to prevent the "spread of contagious abortion" to other pregnant females in the same herd, which already have in large measure the same infections in their uteri. Every grade of endometritis is seen. When of an aggravated degree, it is officially designated "recent parturition" and the carcass is condemned by the inspectors, but as a matter of fact it is not a consequence of recent physiologic parturition. The uterus in these cases is readily recognized by external appearances. It varies from twenty-four to sixty or more inches in length along its convex (free) border, and transversely from three to eight or ten inches at the base of the recently gravid horn. The uterus is flat, collapsed, thinwalled, and flabby. It collapses from side to side: that is, it collapses like an empty bag with the line of attachment of the mesometrium, or broad ligament of the uterus, and the convex, free border serving as the two margins of the flattened tube. The exterior of the uterus shows scarlet or red vascular lines, and here and there numerous bright red hemorrhages beneath the peritoneum. When incised, the uterine cavity is found to contain variable quantities of bright red exudate mixed with pus and placental debris of a dirty grayish-yellow color, which as a rule is not fetid but has a faintly sweetish odor. 
I am not aware that this exudate has been subjected to any material study. Hence its composition and the identity of the bacteria present are unknown. Logically the bacteria are chiefly those which existed in the utero-chorionic cavity of the recently pregnant organ, to which organisms from the exterior may have been added. So far as I have been able to determine, the exudate consists primarily of blood due to limited capillary hemorrhage from the placental areas. This becomes mixed with small masses of pus and necrotic particles of placental tissue. The blood is scarlet, similar to that of the severe uterine hemorrhage described in the preceding article. Perhaps there is a causal relationship between the two conditions. There is one marked clinical difference. The gross hemorrhage produces a clot which, so far as I have seen, tends to desiccate but not to putrefy. The same tendency is present for a brief interval in the scarlet-gray exudate of endometritis, but this is of brief duration and, unless recovery promptly occurs, putrefaction or suppuration finally takes place. Then follows septic metritis, pyometra, or other phases of intra-uterine infection.

Endometritis is exceedingly common in most dairy and beef herds. In dairy herds where genital infections are severe, endometritis is sometimes essentially universal for some months. If there are 25 per cent. of observed expulsion of fetal cadavers (abortion), careful clinical study will reveal probably 80 to 90 per cent. of cases of endometritis. Endometritis in the cow is so common that Fleming and other obstetrists describe it as "normal" and designate the exudate when discharged from the vulva as "lochia." The course of puerperal endometritis is technically brief. Numerous cases recover spontaneously and promptly and retain their fertility. Perhaps more frequently the puerperal infection passes over into the post-puerperal era as a mild, persistent endometritis with a pernicious tendency to extend by continuity into the oviducts, causing the various tubal infections, or into the cervix, inducing cervicitis, with all that these mean for the future reproductive powers of the 
animal. In other cases endometritis passes quickly into that type of disease I have designated as septic metritis, or into pyometra.

The prognosis is generally good. If timely attention is given it can usually be promptly controlled and the fertility of the patient retained. Sometimes the infection is of high virulence, or, which amounts to the same thing, the patient has such low resistance that, in spite of treatment, ruinous or fatal septic metritis ensues.

The handling of puerperal endometritis is to be based upon the fact that it is ordinarily a disease persisting from the pregnant state. Therefore, if it is to be prevented, the uterus should be rendered as nearly aseptic as is practicable prior to conception and all available precautions taken against coital infection by the bull. When genital infections in a herd have become intense as a result of professional neglect, each female should be regarded as probably infected and the presence of endometritis at the close of pregnancy anticipated. In such instances I have advised as a general procedure the introduction of iodoform with boric acid, bismuth, thymol or other antiseptics into the uterine cavity (or if the fetal membranes are retained, into the chorionic cavity). A very satisfactory formula is onehalf ounce each of powdered iodoform and boric acid, with five or six grains of powdered thymol enclosed in a gelatin capsule and deposited deeply in the cavity of the gravid horn. This should be done within two to five hours after calving or aborting. This can be done safely by an intelligent herdsman or caretaker, if he has first been carefully instructed regarding the necessity for cleansing his hands and shown how and where to deposit the capsule. The operation must of course be carried out with the greatest possible care in cleansing and disinfecting the hands, and the cow's tail, buttocks, and vulva. The same result may be accomplished, sometimes perhaps better and sometimes not so well, by suspending the iodoform and boric acid in white mineral or other bland.oil and introducing the preparation deeply into the uterus by means of a hospital irrigator with the bull-douching catheter attached. 
It has been objected that, if the drugs are introduced in the gelatin capsules, the latter may become coated with mucus and fail to dissolve. I do not regard this as a formidable objection. I have not observed the result alleged and think the occurrence so rare that the objection is not weighty. If the veterinarian fears the non-solution of the capsule, the defect is readily overcome by turning to the less convenient suspension in oil. Objection has also been raised to the use of iodoform because of its odor. The claim is made that it contaminates the entire dairy, gets into the milk, etc. I do not regard the objection as well taken. If iodoform is properly used, all objection regarding its odor and the tainting of milk disappears. Introduced into the uterus in a gelatin capsule, none of the drug needs to be scattered about the stable or premises, and, once deposited in the uterus, its high specific gravity causes it to drop to the lowest part of the cavity and to remain there unless disturbed by siphoning fluids from the uterus. When suspended in oil, the iodoform is quite firmly held and causes little difficulty except that the mixture is slopped about in an untidy manner. The tainting of the milk of the patient with iodoform is a minor complaint when properly handled. The milk is wholly unobjectionable for the feeding of calves or other animals. As a rule the milk of a cow which has endometritis should be excluded from the market, and the iodoform taint insures the exclusion of the milk for human use much as the application of kerosene to a condemned carcass insures against the sale of the meat for human use. If proper warning is given the dairyman that the milk should be carefully tasted by a good taster before it is placed with market milk, all danger is safely averted. If the dairyman ignores the warning, the tainted milk is wholly without danger to the intended consumer and only brings into disrepute products of the careless person. The warning should be direct and complete. The animal should be in isolation during treatment, a separate pail should be set aside exclusively for her milk, and the milker should milk her last and then carefully wash his hands with hot water and soap. It 
can not be too strongly insisted that the use of iodoform is perfectly safe and that only its misuse makes trouble.

In highly infected herds it is desirable that a skilled veterinarian shall examine the genital organs of cows in which pregnancy has recently terminated, where no external evidence of important disease is manifest, in order to determine the presence or absence of endometritis or other hidden disease important for the breeding life of the animal. This examination may occur at any time from the fourth to the fifteenth day post partum.

When the simple type of endometritis here considered is recognized by clinical manifestations or through a careful examination of the uterus, including the douching of its cavity in order to reveal the state of its interior, the uterus should be washed clean with sterile 0.6 to 0.7 per cent. salt solution, after which iodoform and bismuth suspended in white mineral oil or liquid paraffin may be deposited in the recently gravid horn, and the treatment repeated after one to four or more days. Careful watch should be kept upon the case in order to assure as far as possible prompt and complete recovery with fertility fully maintained. Powerful antiseptics are to be strictly barred from the genital tract during the puerperal period, because the freshly denuded endometrium and partially denuded vaginal mucosa will not tolerate them. There is no potent disinfectant known which can be freely and safely used in the puerperal uterus or vagina. Insoluble non-irritant drugs, such as iodoform and bismuth, are well borne and such feeble antiseptics as boric acid can be tolerated. Here and there some manufacturer of antiseptics advertises a "non-irritant" drug of higher potency than carbolic acid, but it is non-irritant only upon nonirritable tissues and not, so far as known to me, upon the puerperal uterus and vagina. Salt solution is safest. Dakin's solution or one of its reliable substitutes in sufficiently high dilution may be used. No drug should be used which causes pain or straining. Nothing so intensifies the infection present as the imprudent use of disinfectants. The constant aim should be to wash out mechanically any deleteri- 
ous infection present with a neutral fluid and then, if possible, to deposit in the organ some long-enduring substance, like iodoform and bismuth, which, without irritating the tissues, may tend to inhibit in some degree bacterial activity. The final effectual disinfection must be a physiologic act upon the part of the organ itself. When the disease advances to constitute septic metritis or pyometra, or retreats to become the endometritis of the post-puerperal period, the clinical phenomena change, the indications for handling shift, and the continuing disease is most profitably discussed under other headings.

\section{Septic Metritis}

The term "septic metritis" is used here to designate a type of uterine infection in which the invading organisms break down the barriers formed by the body of the host, involve the uterine tissues generally, and gain the blood stream, where they cause marked sepsis with great peril to the life of the patient.

Uterine gangrene occurring during pregnancy, associated with emphysematous decomposition of the fetus, has already been described and it has been stated that severe septic metritis or uterine gangrene not infrequently reveals itself at the time for parturition and serves to delay or prevent the expulsion of the fetus. Most cases of septic metritis occur during the puerperal period and are clinically recognizable as a rule from two to seven days post partum.

The gangrenous or septic metritis of pregnancy is fundamentally identical with the puerperal disease, but is modified by the presence of the decomposing fetus. Septic metritis is not ordinarily an initial or basic disease, but rather a critical phase of metritis growing out of a less virulent phase of infection. Puerperal septic metritis is not ordinarily the result of an invasion during the puerperal period, but an inheritance from pregnancy, proceeding from sources identical with those of the uterine gangrene in the pregnant cow. After parturition there may be important new invasions added to the existing infection. These admittedly 
complicate, and in many instances doubtless aggravate the disease already present.

Septic metritis, as seen during the puerperal period, commonly follows tardy parturition. There is metritis present during pregnancy which serves to cause a varying degree of uterine paresis. The expulsion of the fetus is tardy, requiring five to ten or more hours. Afterward the fetal membranes frequently remain incarcerated. The clinical evidences of endometritis, as described in the previous article, are present. The endometritis, instead of undergoing rapid improvement as is commonly observed, becomes aggravated. The exudate in the uterus increases in volume and becomes fetid. It loses its bright scarlet color with the

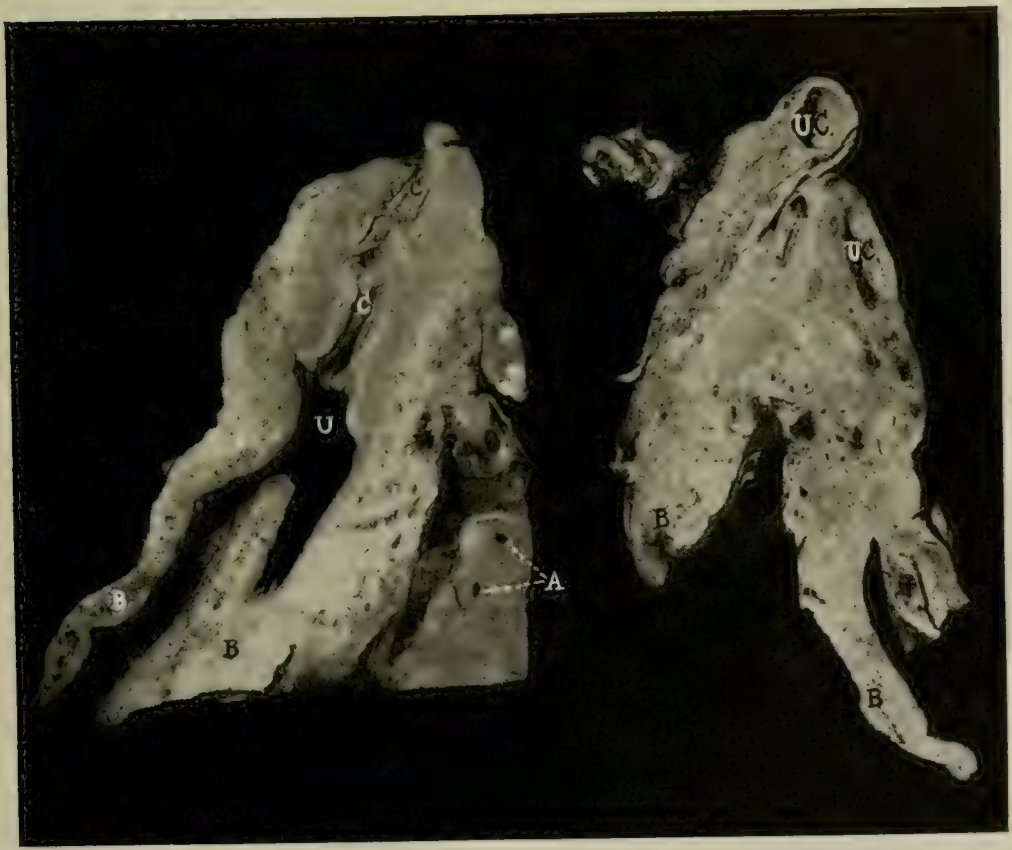

FrG. I79-Septic Metritis, Uterus of Cow, with Great Thickening of Walls.

$C$, Cervical canal ; $U$, uterine cavity ; $A$, enlarged, thickened uterine arteries ; $B$, uterine body walls; $U C$, uterine cornua. 
dirty yellowish-gray admixture of particles, and frequently becomes watery, dark reddish-brown or black. Its color and consistency vary. Sometimes the dark liquid is mixed with a large proportion of dirty grayish clumps of pus or of necrotic tissues.

The uterus becomes paretic. There may be little or no notable discharge from the vulva. The exudate is sometimes pressed out mechanically when the animal is recumbent, but in many cases even recumbency fails to crowd the exudate out passively. The animal is weak and dull. The pulse is rapid and extremely weak. The nose, ears and feet are cold. The animal moves with great reluctance and very feebly. The respiration is not materially affected. The temperature is erratic, rarely high, not infrequently normal, and usually subnormal. The practitioner who is in the habit of thinking that sepsis always causes fever is greatly deceived: he may err in diagnosis, and far more probably and seriously in prognosis.

As the disease progresses, the patient shows extreme depression. The eyes sink deeply into the orbits and the cornea looks cloudy. There is a profuse muco-purulent discharge from the eyes and nostrils. One of the most marked clinical features is the presence of a profuse diarrhea. The feces are very thin, fetid, black, and tarry-looking. The diarrhea is so pronounced and so overshadows other clinical phenomena, especially the evidences of uterine disease as expressed in discharge from the vulva, that I have seen unwary practitioners diagnose dysentery and handle the case empirically for that malady.

The course is usually brief: more than half of my cases have ended in death within three to six days after the establishment of the clinical evidence of sepsis. Some cases improve, but very few recover. Peritoneal adhesions, pelvic abscesses, pyaemic abscesses of the liver, spleen and other parts, arthritis, and other complications follow, which leave the animals unthrifty and of little or no value. Those which apparently recover are very largely sterile and valuable only for beef. 
The disease is usually solitary. There is perhaps one case during two or three years in a herd of fifty or more cows. I have observed such solitary cases following twin pregnancy, apparently due to increased strain caused by twins upon the infected uterus. Now and then the disease breaks in a herd as a virulent storm and its ravages are as disastrous as an outbreak of one of the most dreaded specific diseases. In one herd of about sixty breeding females, this virulent form of infection suddenly appeared and raged throughout the calving period of about ninety days. In that time it had killed 20 per cent. of the herd and had ruined another 20 per cent., so they had to be destroyed as worthless or consigned to the butcher. No evidence could be traced of recent transfer of the infection from one animal to another. The herd was in two groups-adult cows, and heifers in first pregnancy. They were one mile apart and separately attended. They suffered alike. The heifer group had been separate from the adults all their lives. The only common point of contact was that of coitus with the herd bulls. Some of the cows had retained fetal membranes; some had not. I was able to give them close personal attention but was powerless to ameliorate or modify favorably the course of the disease. It was as relentless and overpowering as anthrax. I have known similar but less extensive outbreaks in other herds. In one herd of famous purebreds, five or six of the most valuable animals succumbed in rapid succession without a proper diagnosis having been made by the attending veterinarian. A second veterinarian of high repute was called, who also failed to make a correct clinical diagnosis, and realized his error only when the death of the patient gave opportunity for an autopsy. The outbreak then subsided as suddenly as it had come.

The biology of this type of infection has not been extensively studied. A colleague working briefly in the severe outbreak under my charge reported chiefly a micrococcus and a streptococcus. The herds in which I have seen or known of the severe outbreaks have suffered liberally from sterility and abortion, but not more than other herds of 
similar size. Abortion was no more common during the outbreak than at other times.

The prognosis of septic metritis should always be very bad, first for the life of the patient, and second, if the patient survives, for her reproductive life.

There is little to be done in the way of handling. If the fetal membranes are retained and the veterinarian attempts to remove them, his efforts apparently aggravate the disease; if he leaves the membranes alone, the patient dies. It is impossible to disinfect the uterus; the infection has permeated all the layers and involved all tissues. Besides, the denuded uterine mucosa will not tolerate disinfectants possessing any material efficiency. If uterine douching is attempted, without skill and care, disaster follows. The paretic organ will not empty itself. If the fetal membranes are retained, they prevent siphoning of the uterine contents by occluding the opening of the tube. If the fluids are left, they injure the uterus by their weight and afford additional liquid in which bacteria may grow. If liquid is

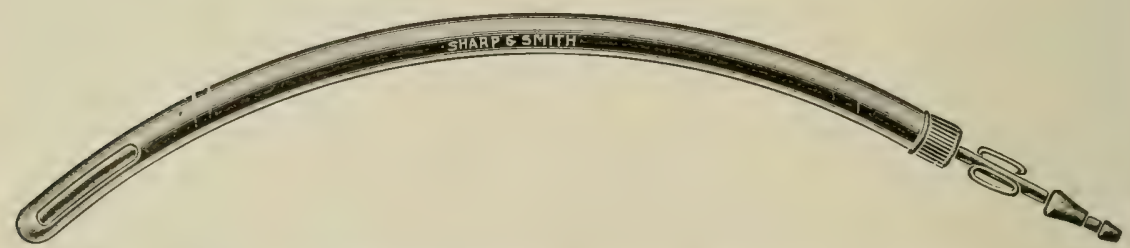

FrG. I 79a-Large Single-Curved Uterine Catheter. Length 24 inches, diameter $3 / 4$ inch.

forced into the uterus with a pump, the uterus often ruptures and the patient dies. The treatment must be conservative and mild. Retained fetal membranes may be removed if they can be gently detached. The uterus may be douched by means of a siphon, but not with a double tube. The amount of fluid-preferably physiologic salt solutionintroduced at one time should be very small, not more than a quart at first, and immediately siphoned out. If it can not be siphoned out, no more should be added. The best apparatus for douching is the seven-foot pure gum catheter, de- 
signed for douching the sheath of the bull, attached to a hospital irrigator. The irrigator should be elevated not more than 20 to 24 inches above the cow. After douching, or without having attempted to douche, there may be introduced into the uterine cavity one to two pints of liquid paraffin or other neutral oil in which there has been suspended some powdered iodoform, bismuth subnitrate, or other virtually insoluble antiseptic or deterrent of bacterial growth. The uterus, in severe cases, must not be massaged. A massage cult has grown up recently in veterinary practice, and massage is as frequently applied where harmful as elsewhere. In septic metritis, it is wholly unjustifiable and absolutely dangerous.

Usually the animal neither eats nor drinks. She should be permitted plenty of water if she will take it. She may have a moderate supply of readily digestible food. The patient may have internally repeated small doses of strychnine, but in giving it the practitioner must be on his guard. She is hyper-sensitive to its action, and will not tolerate the dosage ordinarily advised by writers upon materia medica. About one-half the dose which would usually be given to an animal of the same size is abundant, but it may be repeated within two hours. Drafts of strong hot coffee may be used. Camphorated oil hypodermically and other powerful stimulants may be given. The best that can be done is to keep the patient comfortable and quiet, administer stimulants prudently, and take advantage of every opportunity to ameliorate or remove untoward developments.

The biology of this type of disease is too little known to afford any tangible suggestion in the line of biologic therapeutics.

There are certain possibilites for preventing septic metritis. In the first place it may be partly prevented, as may all genital infections, by the careful growing of calves in such a manner as to minimize the amount of infection which may gain a permanent habitat in the genital system. Much may be done at the time of breeding by the application of the principles of sex hygiene and by insuring, in so far as 
possible, the freedom of the genitalia of both sexes from dangerous infections. Finally, when genital infections in a herd are intense, as indicated by such phenomena as abortion, sterility, retained fetal membranes, calf dysentery and pneumonia, the advent of septic metritis may frequently be avoided by assuming that each animal is importantly infected and that each should be handled immediately after the expulsion of the fetus as an infected animal, without awaiting the development of the material evidences of disease. It is better practice to assume that each of ten cows is importantly infected at calving time and so handle them, even though only one is dangerously infected, than to treat them as sound and allow the one badly infected animal to develop mortal disease before notice is taken.

\section{Placentitis. Cotyledonitis. Retained Placenta. Retained Fetal Membranes}

Metritis can not readily exist in the pregnant uterus without involving the cotyledons or placentules, nor can the converse well be true-that the cotyledons may become importantly infected without the intercotyledonal mucosa participating. Retained placenta is therefore a type of intrauterine infection which has become established during pregnancy and becomes clinically recognizable after the fetus, alive or dead, has been expelled. The cotyledons may be more notably involved than the inter-cotyledonal or nonplacental mucosa, but the only practical difference between the various types of puerperal metritis and retained fetal membranes is that in the latter the endometrium is hidden everywhere beneath the chorion, the necrotic fetal membranes quickly undergo putrefaction, and the retention of the membranes imprisons the pus in the utero-chorionic cavity, thus preventing its discharge and largely concealing its presence.

When parturition ends, in a healthy cow, the moment that the umbilic cord parts the life of the chorion ceases. If, as naturally occurs, the chorionic end of the umbilic cord is let alone when it has parted, the blood contained within the 
fetal placental arteries, capillaries and veins escapes from the ruptured umbilic veins, causing the placental capillaries of the fetus to collapse, become greatly reduced in volume, and drop away from the capillaries of the maternal placental crypts. There was at no time any anatomical bond of union, but only an intimate contiguity maintained by a mutual attraction existing between the healthy uterus and living fetal envelopes. When the umbilic cord ruptures and the chorionic end is necrotic, the attraction between it and the uterus ceases. If it is to be retained, the retention is necessarily dependent upon a pathologic condition which can not develop after the expulsion of the fetus, whether alive or dead, but is dependent absolutely upon the persistence of a metritis which existed in the pregnant uterus. The expulsion of the fetus does not cause, nor permit other factors to cause, retention of the membranes, but fetal expulsion reveals the uterine disease and opens the way for important modifications and complications in its course.

The bovine placental structures are the most complex seen in domestic animals. While the placental system of the ewe

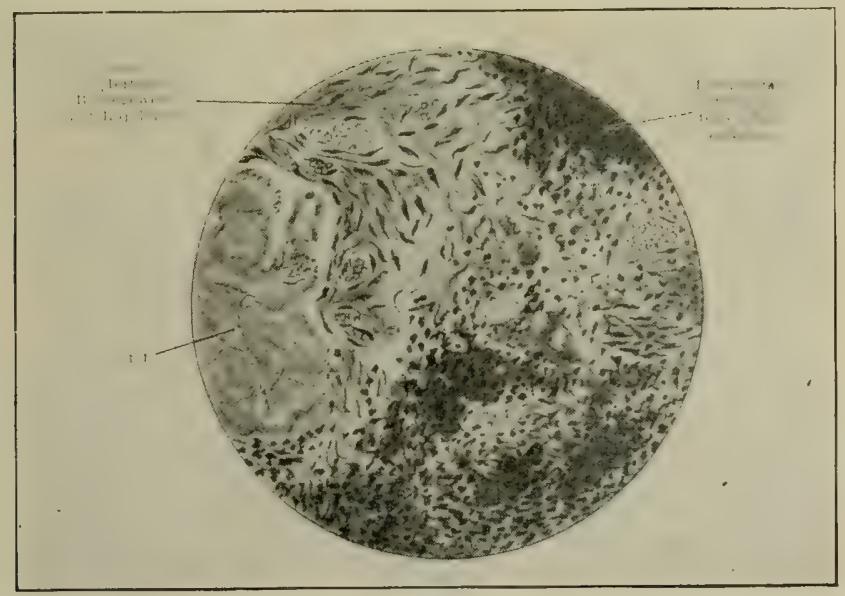

Fir. ISo-Retained Placenta showing incarceration and disintegration of the choriotic tufts (föt) and extensive infiltration with leucocytes in the maternal portion, some of them already broken down. x 310 (After Pomayer). 


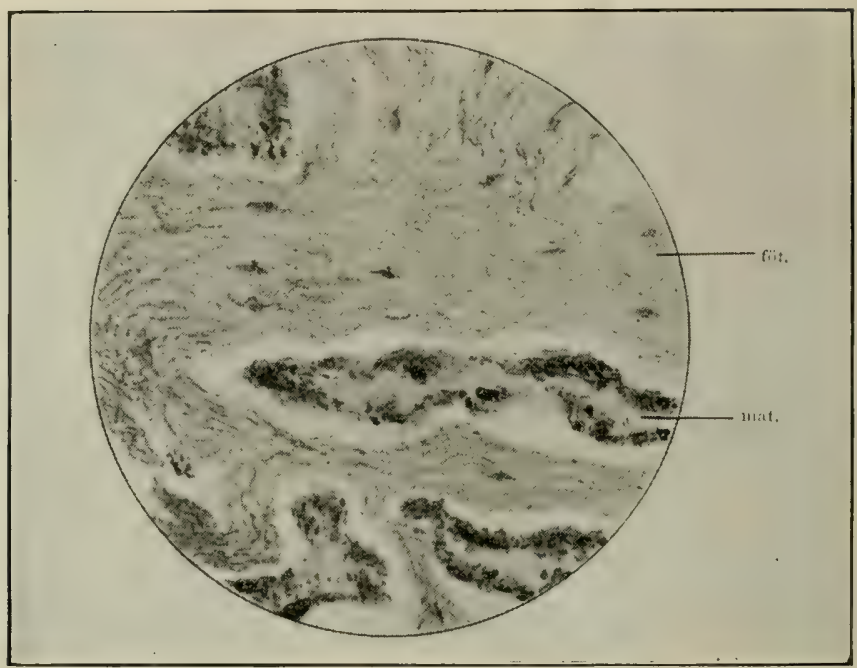

FIG. I 8 I-Retained Placenta. The chorionic tufts (föt) degenerated, the maternal crypt walls (mat.) becoming disintegrated and necrotic. (After Pomayer).

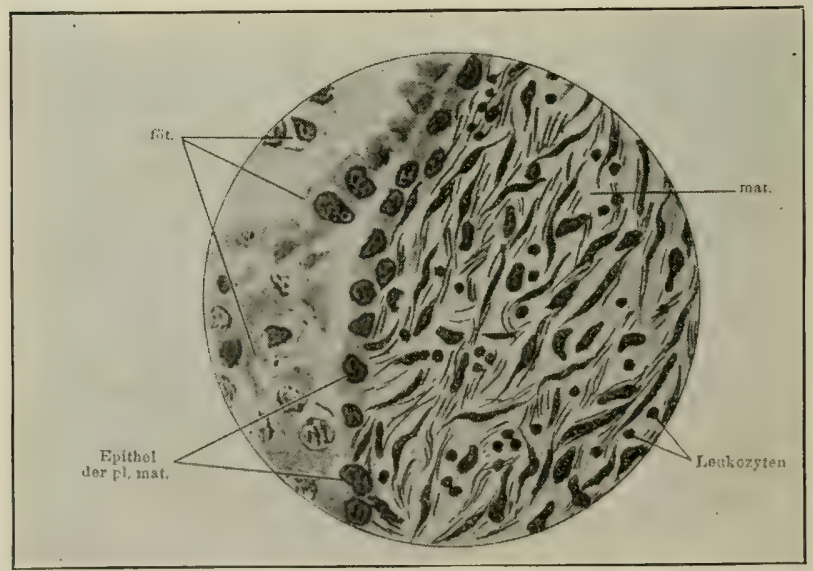

FIG. IS2-Retained Placenta. Necrotic disintegration of fetal tufts (föt) and extensive inflammation with infiltration in the maternal placentae. x 900. (After Pomayer).

(See also Plate V.) 
is similar in plan, the chorionic tufts are not so large and hence the cotyledonal crypts of the uterus are not so deep and intricate as in the cow. This is well shown by a study of the placental systems of the two animals on pages 59 to 66 , including Figures 27 to 34 . When infection invades the placental structures, it is virtually impossible for the maternal crypts to become inflamed without the intimately contiguous chorionic tufts promptly becoming involved. As in inflammations involving other parts, so in the placental tissues, the capillaries become engorged, the blood stream slows and finally stops, the red blood cells heap up and perish, and the white blood cells pass out through the capillary walls into the intervening spaces. The result is indicated in Figures 180-182. The accumulation of the red blood cells in the capillaries and the migration of the leucocytes into neighboring tissues, with the extravasation of lymph and of red cells into the parts, increase greatly the volume of the individual structures, such as the chorionic tufts, and through this detailed enlargement the volume of the structure as a whole becomes vastly increased. The inflamed cotyledon is frequently quadrupled in volume because of the disease. The result is incarceration of the enlarged, swollen chorionic tufts within the similarly enlarged placental crypts. The placenta is retained. The pathologic processes involved in retention of the fetal membranes probably occur in all cases of abortion but are not always recognizable. The uterus of the female bovine fetus shows clearly the placental areas. When a heifer becomes pregnant, the placental areas have already been fixed and, as soon as the embryonic sac is established, the placental-contact points of the chorion throw out placental tufts which enter the cotyledonal crypts and quickly establish a physiologic bond of intimate contiguity. As pregnancy advances the chorionic tufts grow larger, longer, and more intricately branched, and the cotyledonal crypts become constantly deeper and more complex. It is not probable that a fetus ever dies until some or all placental areas have undergone serious pathologic changes of a character essentially identical with retained fetal mem- 
branes. In the early stages of pregnancy, however, the chorionic tufts and placental crypts are so primitive that recognizable incarceration does not occur because the placental disease quickly runs its course and placental separation occurs prior to fetal expulsion, so that the abort is expelled, enclosed within its membranes. As pregnancy advances, the increasingly complex placental structures invite an equally increasing incarceration of the chorionic tufts, until in the sixth or seventh month the expulsion of the fetal cadaver usually reveals the presence of a severe placentitis, as evidenced by the retention of the fetal membranes. When a cow or heifer aborts, the probability of retention of the fetal membranes increases as she approaches full term and the intensity of the retention increases along parallel lines. If the intra-uterine infection is extensive but fails to accomplish the death of the fetus and its expulsion, there occurs at birth a retention of fetal membranes often more severe and perilous than observed after abortion. When abortion occurs at a late date, retention of the membranes after the expulsion of the fetal cadaver may not occur, because the placentitis has completed its course up to the point of dehiscence of the chorionic placentae. That is, there is no invariable rule of relationship between the death of the fetus and its expulsion from the uterus. They are two separate phenomena. The irritation in the uterus due to the endometritis may excite uterine contractions and cause the expulsion of the fetus while the latter is yet alive (premature birth). Usually the death of the fetus and its expulsion from the uterus occur almost simultaneously, so that the fetal cadaver is fresh when expelled. But the fetal cadaver may be detained in the uterus for an indefinite period for any one of not less than three different reasons: (1) The expulsion of the embryo may be delayed or prevented by cervicitis with sclerosis or adhesions of the cervical tissues. (2) The uterus may be too paretic to expel promptly the fetal cadaver. (3) The expulsion of the cadaver may be inhibited by the presence in the uterus of a second living fetus (twins). The latter force is more nota- 
ble in the sow, where the fetal cadavers are common in the uterus and their expulsion inhibited by the presence of living embryos. In each of these conditions the placentitis completes its course, at least up to the point of detachment of the fetal from the maternal placentae, or the cotyledons become necrotic and separate from the uterus. Consequently if the cadaver is finally expelled, the fetal membranes do not remain behind in the uterus. Accordingly when retained fetal envelopes occur, the stage of their development varies widely in different cases. In abortion occurring late in pregnancy the placentitis may be far advanced, so that the fetal membranes are expelled with the fetal cadaver, or, detained after the expulsion of the cadaver, may come away in a few hours, days or weeks.

If a cow calves and the fetal membranes are retained, the stage of the placentitis may be partly gauged by the health of the calf. If the calf is feeble and dull, showing severe sepsis, the placentitis is probably far advanced and the course of retention nearing completion. If the calf seems well but breaks down within a few days with dysentery, it indicates, with certain limitations, that the placentitis was moderately advanced at the time of birth and that the retention of the membranes will probably continue for the maximum duraticn. On the other hand, if placental retention follows the birth of a quite vigorous calf, the placentitis is certainly in its first stages and probably involves materially only a few of the cotyledons located at the cervical end of the uterus, while the cotyledons at the ovarian end are healthy. Then the dehiscence of the membranes at the ovarian end of the uterus may occur at once and these drag upon the areas of detention, mechanically detaching the feebly retained portions. Slight retention of the fetal membranes at the cervical end of the uterus plays an important part in the phenomenon of their final expulsion. Ideally it might be expected that the fetal membranes would be expelled with the placental surface of the chorion on the outer side, but in a majority of instances the dehiscence occurs first at the ovarian end of the uterus, the detached por- 
tion inverts into the cervical portion and finally prolapses, and the afterbirth comes away with the amniotic surface of the chorion external.

The duration of the retention of the fetal membranes is further modified by the virulence of the infection. The mildest recognizable type may have only a brief duration because the involved tissues retain or recover in part their physiologic functions and act effectively before firm incarceration occurs. At the other extreme, even when a calf is born, the infection may be so virulent as to cause necrosis of all the cotyledons with sloughing through their necks within two or three days after calving, thus ending the retention.

The symptoms of retained fetal membranes are those of the underlying metritis with the addition of the recognized presence of the membranes. The retention of the membranes is usually evident because some portions protrude from the vulva. The part most commonly protruding is the umbilic cord, but it may be a portion of the amnion, or the chorion from the non-gravid horn.

Retained fetal membranes are not always visible. If the uterus is very paretic so that involution is very imperfect and the uterus retains approximately its gravid size, the retention of the membranes at the ovarian end retracts the stump of the umbilic cord into the vagina or even into the uterine cavity. The retention then becomes recognizable only upon clinical examination. This constitutes one of the most dangerous types of retention because the layman fails to recognize it, and the veterinarian, unless on his guard, may err in diagnosis. It finally leads to another interesting phase of retention of the fetal membranes. When the placentitis has finally run its course to that point where the membranes are no longer attached to the uterus, whether detachment has occurred through suppuration within the crypts or necrosis of the cotyledons, the cervix, which has not participated extensively in the disease processes, may have so contracted that the enfeebled uterus is unable to force out the loose-lying membranes, with perhaps the necrotic cotyledons still attached to the membranes and de- 
tached from the uterus. There exists then what may be designated cervical retention of the fetal membranes. A condition has developed analogous to retention of the fetal cadaver. Unless surgically removed, the membranes then undergo putrid destruction and establish pyometra or other serious type of disease of indefinite duration.

The biology of the infection causing retention of the fetal membranes has not been adequately or seriously studied. The orthodox believers in "contagious abortion" as a specific contagious disease have neither asserted, admitted, nor denied that $B$. abortus causes retention of the fetal membranes. They freely admit and assert that abortion is "followed" or "complicated" by retention of the fetal envelopes. In their directions for the control of "contagious abortion", however, they constructively deny the identity of the infection causing abortion, and of that causing the retained placenta of calving, by advising that aborters be quarantined and ignoring the cow which has calved and has retained fetal membranes. Brief researches by my colleagues intimate that the chief factor in the causation of retained fetal membranes is a streptococcus of the viridans group associated at times with a colon bacillus. The latter may be largely a secondary invader. Very rarely tuberculosis and actinomycosis may cause retention of the fetal membranes. Reference to this possibility has been made when considering genital actinomycosis and tuberculosis. Any infection which may invade or exist within the gravid uterus and which is competent to cause inflammation may cause retention of the fetal membranes. After the expulsion of the fetus, or rather after the uterine seal disappears from the cervical canal, an unknown number of species of bacteria may invade the uterus to complicate and intensify retained placenta.

The course of retained fetal membranes is ameliorated, intensified or modified by innumerable factors, and it is impossible to predict the outcome of a given case with any assurance of correctness. The mortality is high, but the greatest economic loss arises from decreased dairying effi- 
ciency during the involved lactation period, loss of condition, delay in conception in the next breeding period, and very commonly the total destruction of the reproductive powers. More incurable sterility arises from that type of metritis where retained fetal membranes exist than from any other type of disease. It is common to hear or see statements by veterinarians that under a certain formula they "cure" promptly and readily all cases of retained placenta. Just what they mean by such statements I do not know. Retained placenta in the cow is a condition of great peril, causing enormous losses.

The placentitis or cotyledonitis regularly begins at the cervical end of the uterus and advances toward the ovarian extremity. Usually the spread is slow, but it may be very rapid. Its extension is more readily observed after calving than after abortion. If the uterus is examined manually a few hours after calving, it is commonly found that some of the placentae nearest to the cervix have already become detached or are readily separated. Further toward the ovary the cotyledons are increasingly enlarged, engorged and firm, and the chorionic tufts can be detached only with difficulty or not at all. The uterus is generally atonic, flaccid and collapsed. Sometimes it is highly sensitive, and when the uterus is manipulated the animal expresses pain and strains severely. At first, if a calf is born, the membranes appear fresh and possess no putrescent odor. Sometimes putrefaction does not become marked until several days have elapsed, after which decomposition proceeds with varying rapidity. If daily examinations are made, it is ordinarily found that at each time additional cotyledons have separated or are separable, until finally the last to become detachable are those in the apex of the gravid horn. This is not constant. In some cases the infection has spread from cotyledon to cotyledon with great rapidity, the placentitis is essentially uniform throughout, and finally, when the membranes are detachable from some cotyledons, they may be removed from all. In other cases a few cotyledons at the cervical end of the uterus are moderately inflamed after calving but with a 
little care the membranes may be detached. Anterior to these the parts are essentially healthy and are readily removed. In these cases the placentitis is in its initial stage and the incarceration of the chorionic tufts within the placental crypts not fully established. The separation of the fetal membranes from the diseased uterus occurs in a variety of ways. Each type may be recognized in the same case, though one tends to prevail.

(1) In the simplest type of retention, lymph or pus accumulates within the placental crypts of the uterus and pushes out or detaches the chorionic tufts. In a large measure the tufts may undergo purulent destruction and the chorionic base drop away with but few of its placental tufts intact. This constitutes the most benign type of retention and ordinarily runs the briefest course. The recovery is generally prompt, lactation is not seriously disturbed, and fertility is usually conserved.

(2) The chorionic tufts and the placental crypts of the uterus become necrotic, the tufts break off from the chorionic membranes, and later the placental portions of the cotyledons, with the chorionic tufts, slough away from the pedicle of the cotyledons and lie free in the uterine cavity. If the case is not meddled with in an imprudent attempt to remove the membranes manually, the chorionic membrane usually retains its connection with the tufts and the placental area of the cotyledon becomes detached from the uterus and remains attached to the chorion. If the animal survives (the mortality is high) and again conceives (which is improbable), new placental tissues form upon the cotyledonal stalks.

(3) In another well-marked type, which is not very rare, many or all the cotyledons undergo total necrosis, slough through the cotyledonal stalk, and remain irremovably attached to the chorion. While this process is being accomplished, the cervix may contract; when the sloughing is complete, the fetal membranes with the clinging necrotic cotyledons lie imprisoned within the diseased uterus. When all cotyledons are thus involved, the patient usually succumbs. 
If she survives, she rarely breeds. If but a few cotyledons at the cervical end undergo necrosis, the prognosis is far more hopeful and the animal frequently breeds. I have observed in the abattoir one pregnant uterus in which all cotyledons had perished completely. When a cotyledon necroses in toto, placental structures do not again form in that area. Instead there is a white cicatrix. About this cicatrix (See Colored Plate V) adventitious placental structures develop. They lack the fineness of detail shown in Figs. 27 to 34 and are irregular and defective. They are no longer cotyledonal in type, but approach roughly the diffuse placenta of the mare.

(4) Metritis associated with retained fetal membranes is sometimes so severe that the entire endometrium is destroyed. The uterine epithelium can not then be renewed and the denuded surface can not heal except possibly by adhesion of the denuded surfaces totally oblitering the cavity. Possibly this occurs sometimes. What I have observed is illustrated in Fig. 183. Here the uterine mucosa has been wholly displaced by granulation tissue which has contracted until the uterine (and cornual) cavity consists of an irregular suppurating fistula barely admitting the passage of an ordinary probe. The uterus was normal on rectal palpation, the cervical canal was passable for the small uterine catheter, and attempts at douching the uterus resulted in the return of a few drops of pus. Probably at one time there had been sclerotic metritis, as illustrated in Fig. 189, but during an interval of three years after the termination of her last pregnancy any sclerosis which may have been present might well have disappeared to such a degree that it could not be recognized by rectal palpation.

The handling of retained fetal membranes is a trying problem to the conscientious practitioner. Not the least factor in rendering it trying is the frequent bombastic misrepresentation as to how easily it may be overcome by this or that formula. Rules for handling are laid down by the score and recommended as essentially infallible. This creates the utmost confusion. There is no accepted stand- 


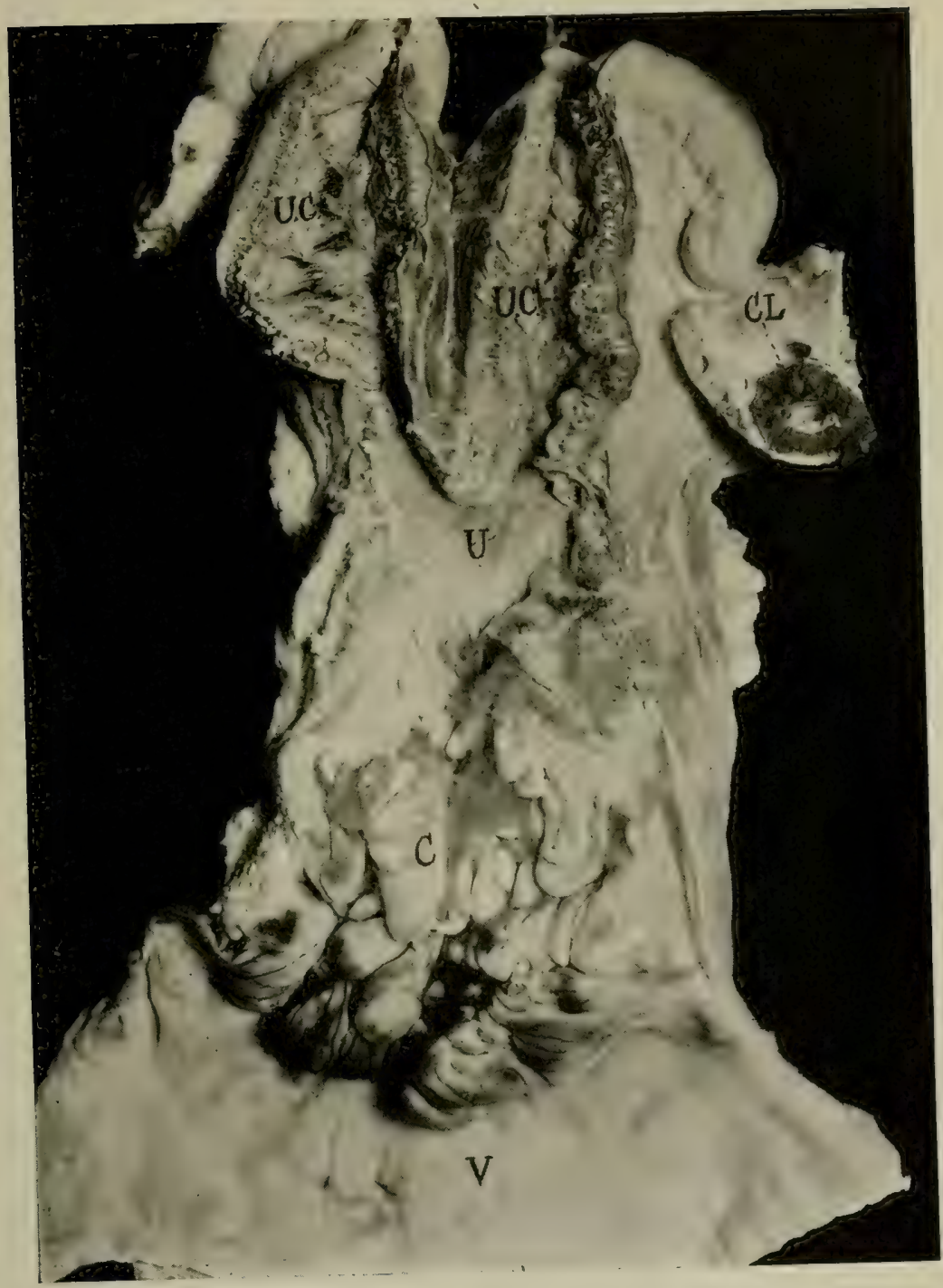

FIG. I83.-Total Destruction of Uterine Mucosa.

$V$, Vagina ; $C$, cervical canal ; $U$, uterine body cavity, virtually disappeared; $U C$, walls of uterine cornua ; $C^{\top} C^{\prime}$, cavity of uterine cornu, reduced to a purulent fistula large enough to admit a small sound; $C L$, central corpus luteum. 
ard and there is much misinformation. One of the chief elements of confusion is the belief of the breeder and dairyman that a skillful veterinarian can successfully remove a retained afterbirth at will. Accordingly the owner calls the veterinarian to "remove" irremovable fetal membranes. The practitioner is not left free to exercise his judgment and attempts the impossible.

No chronological date can be fixed upon, at which it is prudent to attempt the manual removal of the fetal membranes. So many cows have been killed or ruined by attempts at the removal of the membranes that it has become the fixed policy of many practitioners to abstain from all attempts at manual removal, and they have justified their methods by results attained. I hold that, between the extremes of non-removal and the arbitrary removal or attempts at removal, there is a middle ground in thorough harmony with the principles of surgery and fully justified by clinical experience. Once the fetal membranes become retained within the uterus owing to the incarceration of the chorionic tufts within the placental crypts, certain established disease processes must continue in their course to their logical end. In the types described above there is a course and termination for each, and it is impracticable to modify materially or to abbreviate that course in a favorable direction. As in other infections, it is easy to affect its course unfavorably, leading to disaster. There is always present within the uterus a determined battle between the infection and the powers of resistance in the uterus and the body as a whole. The infection has a notable advantage in the presence of the necrotic membranes, which afford an inexhaustible nutritive supply for the bacteria, and the additional advantage of the presence of the utero-chorionic cavity, with the necrotic chorion forming one wall and the diseased uterus the other wall, and the virulent infection lying between the two structures beyond surgical reach. The tendency is therefore for each case of retained afterbirth belonging to the first and most benign type, a group which fortunately contains most cases, to become more intense and to move into one of the 
more perilous types. The least error in the plan of handling serves to push the case out of the simpler type into the more serious classes. Everywhere the uterine mucosa is diseased and its epithelium depressed or destroyed and extremely sensitive to the faintest abuse. If the practitioner attempts to detach manually the chorionic from the uterine placenta, any imprudent stress which tears the walls of the placental crypts, or merely tears away the already damaged protective epithelium, creates a favorable avenue for more damaging bacterial invasion. The damage is not alone to the placental areas. The non-placental endometrium is largely denuded of its protective epithelium and is highly sensitive to the least insult. If the practitioner inserts his hand none too gently into the uterine cavity and laboriously detaches the membranes from the cotyledons, his hand constantly abrades the inter-cotyledonal mucosa and breaks down the feeble barrier maintained against the invading swarm of bacteria. The pain endured by the cow induces severe straining, which in turn further damages the tissues and increases the infection. Any veterinarian having mediocre powers of observation can remember clearly how in his own personal experience he has left his patient evidently worse after having made an apparently successful attempt, viewed from a mechanical standpoint, to remove retained membranes. Equally disastrous attempts are made to disinfect the uterus and retained membranes. The infected puerperal uterus can not be disinfected and is utterly intolerant of freely soluble disinfectants. The necrotic chorion defies disinfection by any available means, and lying behind it wholly out of reach is the great mass of infection proceeding with its destruction, regardless of any disinfectants placed upon the opposite surface of the chorion. Such a multitude of errors have been made and are still being made that it is difficult to lay down a line of action which may be advantageously followed. In principle the aim should be to pursue a course devoid of danger of injury to the diseased parts, which will tend to lessen the danger of infection passing from the milder type into one of the more severe, or, in cases where the very se- 
vere type is present, to ameliorate it and if possible convert it into a simpler type.

There is no denying that the presence of the great necrotic and putrefying mass of membranes is a menace to the health and life of the patient and that the earlier it is out of the uterus the better, provided that in the process of removal no injury is inflicted upon the uterus. It should be borne in mind that as a rule an afterbirth which can be removed manually without injury to the uterus will spontaneously drop away a few hours later. Hence in most instances the manual removal of the fetal membranes is not of supreme importance because it can prudently occur but a short time prior to spontaneous falling away. The gentlest manual removal possible is not superior to the spontaneous dropping away. There are, however, important limitations to these general statements which should call into action a high degree of professional skill and judgment. If at a given date the practitioner finds that the membranes can be prudently removed, it is best to proceed at once with the operation in order to be in position as early as practicable to deal directly and successfully with the underlying metritis.

Before proceeding with manual removal the operator should thoroughly cleanse and disinfect the tail, vulva and adjacent parts in order to avoid the danger of pushing into the genital tract dangerous infections from the exterior. The operator should very carefully disinfect his hands and arms as a protection to himself and he should keep at hand ample disinfectant solutions for frequent use. But the disinfectants must be kept out of the genital tract. After disinfecting the hands and arms, they should be thoroughly and frequently anointed with vaseline, liquid paraffin, or other fat, and liquid paraffin should be introduced in ample amount into the genital tract. By such means the mechanical irritation from the operation is reduced to a minimum. The arrangement of the cotyledons, as shown in Plate I, should be kept in mind, and the dorsal rows of cotyledons separated first. Commencing at the cervical end of the uterus, the hand should be gently pressed into the utero-chorionic 
space and advanced until one of the cotyledons is encountered. The chorion should then be grasped upon either side of the cotyledon, from the uterine surface. By exerting traction upon the placental margins of the chorion these borders should be pressed toward each other, which tends to cause the chorionic tufts to pull out of the crypts of the maternal placentae, as indicated in Figs. 29 and 30, without traction upon the cotyledonal stalk or uterine wall. In many cases it is found that, after separating a few cotyledons at the cervical end of the uterus, the retention is more severe anteriorly. The operation needs then to be suspended, and all or most of the detached membranes left connected with the undetachable portions. It is especially important that a goodly mass of membranes be left in the cervical canal, since their presence tends to insure a continued dilation of the part, enabling the operator to introduce his hand at future dates. If the detached portions of the membranes are cut or broken off short, the remaining portions drop back into the uterus, the cervix contracts, and future manipulations are rendered difficult. The progress of the disease is then to be carefully watched and the removal of the membranes completed as early as prudent.

When the membranes are permanently undetachable from the maternal placental tissues and separate instead by necrosis either of the placental portion or of the entire cotyledon, the membranes with the necrotic cotyledons should be removed early unless they are promptly expelled by the uterus. The latter is improbable, because as a rule, when necrosis of the cotyledons occurs, the uterus is so badly diseased that it is not competent to expel its contents. Usually the necrosis is slow and, before it is completed and the cotyledons sloughed off, the cervix, the muscular walls of which usually escape the ravages of the disease, has contracted, closed the cervical canal, and imprisoned the putrefying mass. Therefore it is important that the uterus be relieved of necrotic cotyledons with any attached fetal membranes as early as practicable. As in the ordinary simple type of dehiscence, the actual date at which 
it is prudent to remove cotyledons can be determined only by the person in immediate charge. I attended a cow for dystocia (the dystocia of uterine inertia) and extracted an apparently healthy calf. Forty-eight hours later the fetal membranes were completely retained and all her cotyledons were enormously swollen and necrotic. Her temperature was high and her condition critical. All the cotyledons were readily detachable without pain or hemorrhage. They were removed with the membranes still attached. The uterus was then douched. Within twelve hours her temperature had become normal, her recovery was prompt, and her fertility preserved. This was an extreme case cited to show the value of the removal of necrotic cotyledons, when properly done at the right time. In any other cow I have attended, the removal of cotyledons so early after calving would have been wholly unjustifiable and would have done incalculable harm. Each case of retained fetal membranes constitutes an individual problem, the details of which must be worked out separately. Two principles regarding manual removal appear to me to be applicable generally. First, whenever the membranes (including necrotic cotyledons) can be removed completely without injury to the uterus, this should be done. Second, if the membranes can not be removed promptly, measures should be taken to delay the contraction of the cervix and imprisonment of the putrefying tissues. This precaution preferably consists of permitting or causing portions of the membranes to occupy the cervical canal. When this can not be done there may be substituted a tampon of gauze, which may be made in the form of a gauze bag, two or three feet long, the closed end pushed into the uterine cavity and then a sufficient amount of gauze pushed into the closed end from the exterior to produce an enlargement which will not drop out through the cervix. The presence of the collapsed portion of the bag lying in the cervical canal will retard cervical contraction and afford better opportunity for the manual examination of the uterus. A similar plan may be used to dilate an already contracted cervix with imprisoned fetal membranes. 
The closed end of the bag may be pushed into the uterine cavity with a sound, followed by packing the bag through the open end, filling as well as space will permit that portion of the bag lying within the cervical canal. Prior to application, the gauze should be saturated with iodoform oil or vaseline and should be renewed daily until the dilation is sufficient.

In discussing the various types of retention it has already been pointed out that the placentitis typically begins at the cervical end of the uterus and advances slowly toward the ovarian extremity. The retention may be in the very early stages and the entire mass of membranes may readily be removed soon after calving if the few diseased cotyledons about the cervical end be manually released. Perhaps my meaning will be clearer by giving a definite instance. A colleague in charge of a valuable herd made it a rule to ignore retained fetal membranes until forty-eight hours post partum, when he would make an effort to remove manually. I was asked incidentally to examine a cow which had calved about thirty hours previously. I found nearly all the fetal membranes detached and hanging from the vulva. A careful examination revealed the fact that the membranes were adherent to a few cotyledons at the base of the gravid horn. The dragging of the detached masses upon one small area had caused an intussusception of the base of the gravid horn and its prolapse through the cervix into the vagina. It can not be denied that I was right in detaching the few adherent cotyledons and replacing the prolapsed organ. My colleague had evidently erred. The only means for avoiding the error lay in a careful examination of the patient.

The first step in the handling of retained fetal membranes, as in other diseases, is an early and careful examination with an accurate diagnosis. The degree of uterine paresis is to be noted carefully because it indicates more than anything else the probable severity of the retention. If the uterus is extremely large and flaccid, the retention will probably be prolonged and severe. If the uterus is well contracted, firm and not over-sensitive, the duration of the 
retention will probably be brief and the course favorable. If the membranes can be easily detached without causing pain or irritation to the patient, their removal is good surgery because it is only after the removal of the fetal membranes that one can handle with any directness the fundamental metritis which caused the retention. The basic question is not the desirability of removing from the uterus the necrotic membranes, but the possibility of removing them at a given date without causing greater injury and danger than the retention is already producing. If not prudent to attempt the removal at the first examination, repeat the observations at comparatively frequent intervals, at least every second or third day, and watch the progress of the disease.

Various plans have been recommended as substitutes for the manual removal of the membranes, but they have failed. Numerous drugs have been lauded, such as savin, rue, and various aromatic substances, but there is no dependable evidence of their value. A few years ago it was proposed to detach the membranes by injecting into the cavity of the chorion, where the fetus formerly lay, a weak solution of hydrogen peroxide, but it could act only upon the inner or fetal surface of the chorion and could not possibly affect the part really at fault, as it could not get near it. Next someone proposed to detach the membranes by injecting salt solution into the broken ends of the umbilic arteries and thence into the capillaries of the incarcerated chorionic tufts, but the promoters of the plan ignored the fact that the placental capillaries were blocked by thrombi which would not permit water to enter, and equally disregarded the fact that the physiologic detachment of the placentae is due, not to distension of the capillaries, but on the contrary to their becoming empty. If the salt solution could be forced into the placental capillaries, it would increase their incarceration.

Much has been said for and against douching the uterus when the fetal membranes are retained. As a plain matter of fact, so long as the fetal membranes remain attached to 
the cotyledons, the uterus can not be douched by any known method. Fluid may be introduced into the sac of the adherent chorion where the fetus formerly lay, and in some cases may be taken out again. The fluid does not reach the uterus except at points where the membranes have become detached. The uterus is frequently too paretic to expel the fluid poured into the sac of the chorion. If the veterinarian attempts to siphon out the fluid, fragments of the amnion, chorion or umbilic cord block the siphon. Some claim to guard the intake to the siphon with the hand, but in a very flaccid uterus the end of the organ is out of reach and the hand can not effectively guard. For example, in the extreme case of retained membranes following unicornual twins, if the uterus is severely inflamed it may retain its length of more than eight feet. It is useless to discuss the siphoning of fluid out of such a uterus. In many cases following ordinary pregnancy, siphoning fluid out of the chorionic sac is impracticable. Even if accomplished, the operation has very scant, if any value to commend it.

If the uterus is in such condition that the vagina may be douched without the fluid falling largely into the paralyzed uterus or into the sac of the chorion it contains, that may be of value. Perhaps it is also of value, when a portion of the uterus has been freed from its membranes, to douche that portion, if conditions render it practicable. But introducing quarts or gallons of liquid into the sac of the retained chorion and leaving it there is malpractice. The weight of the fluid in the paretic organ does great harm. In some cases the uterus may be so atonic and weak that the mechanical weight of the liquid may cause rupture. The danger of uterine rupture is greatly heightened when the operator is so imprudent as to use a pump for introducing the fluid, or when, introducing by gravity, he uses too great a column of water. The danger is aggravated when the uterus is very paretic and the cervix contracted. I have ruptured the uterus very unexpectedly when using the pump, although the rubber tube of the pump passed the cervical canal freely with abundant room about it. Just what oc- 
curred I could not determine. Either the uterus ruptured from the weight of the fluid, the os uteri internum acted as a collapsing valve and imprisoned the fluid, or portions of the membranes floated against the cervical opening and blocked it. In one instance, where the fetal membranes, detached from the uterus, were detained within the uterine cavity and the cervix had contracted and would not admit my hand, I introduced a small amount of physiologic salt solution through a rubber catheter from a hospital irrigator elevated fifteen or twenty inches. The cow began to make expulsive efforts, the membranes blocked the cervical canal, and disaster followed.

Arbitrary rules for douching are accordingly unwise. It is equally unsound in principle to condemn douching unreservedly. When the membranes are firmly retained and are undergoing putrefactive decomposition, the cervical canal is often very narrow and the debris is imprisoned. The necrotic cotyledons and placental debris must be removed. If one undertakes dilation of the cervical canal, the inflamed cervix yields but little, the force required is great, and both cervix and vagina are severely injured. The abrading and stretching open important avenues for infection, the pain causes straining and exhaustion, and the results are unsatisfactory. If the membranes are well broken up, I have had excellent results in douching carefully with the large, singlecurved uterine catheter shown in Fig. 179a. In such cases I use an irrigator elevated barely above the back of the cow and watch carefully for blocking of the return flow. Should this occur, the inflow is stopped and the catheter withdrawn. It is commonly found that the catheter is blocked by large pieces of membranes or by necrotic cotyledons. Pieces of moderate size can be washed out with this instrument, but it must be used cautiously. The uterus is frail and the catheter is easily pushed through the walls. If the animal strains and pressure upon the catheter is felt, it must be permitted to move backward, or puncture is probable. The horse stomach tube may be substituted, but it is not as practical. The tube, especially the heavy type, is as danger- 
ous as the metal catheter and must be used as a siphon. When this is attempted it is quite as readily choked with membranes and has the additional disadvantage of having its intake occluded by sucking in the uterine wall.

When the membranes are not broken up and are imprisoned by a contracted cervix, douching is highly dangerous and should not be undertaken. The cervix should be gently dilated until portions of the membranes may be grasped with the hand or forceps and traction applied. If one-half to one pint of warm olive oil or liquid paraffin is introduced into the uterus, it lubricates the uterine walls as well as the somewhat necrotic tissues and favors removal. The oil also soothes the irritated endometrium, and in virtue of its lubricating action diminishes the epithelial abrasions inevitable in such manipulations.

Many cases occur where skilled veterinary attendance at the proper moment is impracticable or impossible. Proper veterinary attendance upon retained fetal membranes is generally economically impossible in cows of low value. Even animals of great value are frequently so located that skilled veterinary attendance is not available. Veterinary service must then be rendered through the layman, and methods of handling which may be quite proper when administered directly by the skilled practitioner may become wholly improper and acutely dangerous in the hands of the layman. This group includes at present the majority of cases of retained membranes, and their proper handling is a heavy responsibility upon the veterinary profession. Here a hard and fast rule should be laid down for the layman: Do not attempt the manual removal of the fetal membranes or the douching of the uterus. In numerous cases this rule will work some harm. In rare cases, like the one or two examples I have cited, it will end in disaster when skillful handling would have saved the animal. But the peril to the few is more than counterbalanced by avoiding serious injury to many. The layman would probably fail in any event in those rather rare cases where manual removal is imperative. At present the best known course for the layman to 
pursue is to introduce deeply within the chorionic sac a drug of high specific gravity, so that it may not be thrown out readily, and slow-dissolving, so that it will not require frequent renewal, which will soothe rather than irritate any portion of the genital mucosa with which it may come in contact and which will deter in some measure the putrid decomposition of the membranes. The two best known drugs in this group are iodoform and bismuth subnitrate. To these may be added some of the rapidly-dissolving disinfectants of a non-irritating character. Here the limit is virtually set at boric acid which may be used without restriction. There are two methods of application. The more convenient form is the introduction of the drugs inclosed within gelatin capsules. An efficient formula is one-half to one ounce each of powdered iodoform and boric acid, with three to five grains of powdered thymol, inclosed in a large gelatin capsule. The capsules may be prepared in large numbers. Properly wrapped, they will keep indefinitely, ready for use whenever needed. Bismuth may be added or may be substituted for the iodoform or boric acid. When retained afterbirth is diagnosed, one of the capsules should be deposited as deeply within the uterus, or rather in the sac of the chorion, as possible. The gelatin quickly dissolves and the heavy drug drops to the floor of the uterus, where it slowly dissolves during a period of seven to ten or more days. If the retention continues, a second capsule may be added three to four days after the first. When the membranes finally come away, the drugs previously introduced largely or wholly accompany them, and it is desirable then to introduce another capsule, which now comes into immediate contact with the uterus. As this taints the milk badly, it must on no account be included in market milk or used for making butter or cheese. Tasting the milk carefully is the only guide as to when it is fit to market. It is perfectly healthful and may be used freely for feeding calves. The intelligent layman can readily learn to introduce the capsule. The tail, vulva and buttocks of the cow should first be washed and disin- 
fected. The operator should trim his finger nails closely and smoothly and disinfect and soften his hands in a warm disinfecting solution. The disinfectant may then be rinsed off the hand with warm water and the capsule, inclosed within the hand, carried deeply into the sac of the fetal membranes and dropped there.

A method which is certainly more efficient in many, and possibly in most cases is the introduction of the iodoform or other drug suspended in a neutral oil. Liquid paraffin is probably in all respects the preferable medium. The drug is to be placed in a pint or quart bottle, the vessel filled with the oil, and then shaken until well suspended. It is then to be poured into a hospital irrigator with the seven-foot rubber catheter designed for douching bulls attached, the end of the catheter carried by the hand and held as deeply within the cavity as can be reached, the irrigator elevated two or three feet above the uterus, and the contents allowed to flow in. The heavy drug falls down upon the floor of the uterus, as with the capsule, while the oil floats upon other liquids which may be present and comes in contact with and soothes any portions of the inflamed genital mucosa which may be uncovered. It is more trouble to prepare and apply, and the average layman is more liable to make some mistake in the handling of the iodoform and taint the milk from cows other than the one diseased.

The plan just described should also occupy a large place in the handling of retained fetal membranes by the skilled veterinarian. In all those cases where at the time of examination it is not feasible to remove the membranes manually, the iodoform capsule or the introduction of an iodoform suspension in oil or liquid paraffin constitutes the best line of treatment at present known.

In practice one occasionally encounters retained placenta in which the underlying metritis is of a highly irritable type and the cow evinces much pain by constant and violent straining. In rare cases this is due to intussusception of the ovarian end of the gravid horn, which may eventually end in uterine prolapse, or may telescope for a few inches, be- 
come incarcerated there and remain persistently. This should be searched for when such symptoms are present and, if discovered, reduced by gentle pressure against it with the hand. It is not essential to take away the membranes. It is far more important to reduce the intussusception and soothe the irritated uterus with oleaginous suspension of iodoform. Metritis with retained afterbirth, accompanied by great pain and straining, is most frequently observed after imprudent attempts at the removal of the membranes. It is believed by many that in such cases the afterbirth must be removed at any cost. The opposite is more nearly correct: the membranes should be left strictly alone and the irritated uterus soothed with iodoform-oil or otherwise.

Once the retained fetal membranes have been taken, or have come away, the original disease which caused it is still present. The important change in conditions is that the uterus is now naked and curative measures directly applicable. The uterus may then be, and as a rule should be, douched in order to clear away any exudate or remaining debris. The condition now facing the veterinarian is simple metritis, and the method of handling is the same as though the retention of the membranes had not occurred.

The prevention of retained fetal membranes can be accomplished only by seeing that a cow at breeding time has a clean uterus and cervix, and that she is bred to a bull free from important infection. These matters are discussed in their proper places.

\section{E. Puerperal Tetanus.}

Immediately following parturition the denuded endometrium opens an avenue for the invasion of specific infections not commonly entering by the genital tract. Tetanus infection occasionally invades the genital organs at this time and produces the ordinary clinical symptoms of that disease. I have observed it but once in the cow, associated with retained fetal membranes. It offers nothing special except that a knowledge of the possibility makes even more de- 
sirable comprehensive precautions against the entrance of any form of infection from the exterior through the vulva. The protrusion of the fetal membranes from the vulva increases when the patient is recumbent, and when she rises they are drawn back somewhat and may drag into the vagina infections caught by the protruding membranes. Tetanus infection is also possible when a prolapsed uterus comes in contact with stable manure.

\section{F. Puerperal Laminitis.}

Puerperal laminitis is virtually constant in endometritis in the mare, but I have observed only one instance in the cow. The symptoms of puerperal laminitis in the cow are the same as those of the laminitis of indigestion and of the corresponding disease in horses. It is quite unnecessary to describe the symptoms and course of the disease here, but it is important to bear in mind that puerperal laminitis is due to the presence of an endometritis instead of an irritation in the intestinal tract.

In handling puerperal laminitis in the cow the fundamental factor is the proper alleviation of the underlying endometritis by the means already advised for that disease. In addition to these measures, the veterinarian should give necessary attention to the feet, either by standing the animal in cold water or by applying ice bags.

\section{G. Puerperal Meningitis}

Puerperal meningitis has found no recognized place in veterinary literature. I have observed one instance only. The patient was a young cow in a herd reeking with genital infections. She calved without notable incident and appeared to be progressing favorably for about two days, when she suddenly developed symptoms of severe meningitis. The patient was exceedingly uneasy, with constant muscular twitchings. The head was held erect and the gait was rigid and uncertain. She was readily disturbed, as in tetanus. The course was stormy and death occurred within one day. Autopsy showed intense injection of the meninges 
without very marked uterine lesions. There is little or nothing to be done for puerperal meningitis. It apparently signifies that a metastasis from the infected genital tract has occurred and the matter has already largely passed beyond control before clinical warning has been given. Chloral or narcotics might be used to quiet the patient and an effort to counteract the infection may be made with ounce doses of formalin or with other agents which tend to act as systemic disinfectants.

There is little limit to the metastases which may occur during the puerperium. Infections of the pregnant uterus are so common, and the endometrium is so largely denuded at parturition that the intra-uterine infections have an unusually favorable opportunity to reach the lymph or blood stream and be borne to any organ or tissue in the body. The infection may lodge in the liver, lungs, joints, or in any organ or tissue, and there produce symptoms of disease wholly unsuggestive of genital origin unless the observer is fully cognizant of the possibilities and is on the alert.

\section{Post-Puerperal InFections of the Uterus}

After the puerperal period, which may be regarded ordinarily as comprising ten to fifteen days, there follows ideally in cows an interval of sixty to ninety days before it is desirable to breed them again. In approximately ninety per cent. of cows, rebreeding is delayed beyond ninety days because of disease or for other reasons, and the period is yet further prolonged in many cows by their failure to conceive when bred. During this interval infinite changes in the intra-uterine infections may occur. Historically most of the uterine infections of the post-puerperal epoch are continuations of infections which have persisted through pregnancy and the puerperal period and have been modified in their intensity and behaviour by the changes in environment. Sometimes the infections of the puerperal period advance in intensity in the post-puerperal era. In many instances there is a strong reaction to the infection present and recovery, entire or partial, often occurs. Thus many cows which had 
during pregnancy so severe an infection that it caused abortion, and during the puerperium outstanding metritis, so far recover that they again conceive and successfully pass through pregnancy. On the other hand the infection is often so mild that an apparently healthy calf is born and no external evidences of puerperal disease follow. But when it is attempted to breed such a cow again, she may be temporarily or permanently sterile, or, having conceived, may abort owing to the fact that, during the period when many cows are overcoming infection, her uterus has been overcome by the infection.

The puerperal period is a critical time in the breeding history of a cow. It is one of the most important and favorable periods in the life of a cow in which to attack successfully genital infections, and the chief aim of the practitioner should be to deal energetically with the infections during the puerperium and not to permit them to drag along into the post-puerperal stage. For example, since retained afterbirth is generally handled simply and wholly as "retained afterbirth", as soon as the membranes are out of the uterus the ignorant or careless veterinarian thinks of his task as completed and allows the fundamental metritis to persist into the post-puerperal period. Then he faces the difficult problem of handling chronic endometritis or pyometra, and perhaps fails to restore the reproductive life of the animal, thus occasioning irretrievable loss. It is clearly an error in professional duty to permit avoidably the metritis of retained fetal membranes to continue into the post-puerperal period.

In the post-puerperal period new infection may invade the uterus from without. There is nothing to show that there is great danger of any infection invading the uterus during this period as a result of ordinary cohabitation. That is, there is no conclusive evidence that the presence of a cow which has recently aborted, has retained afterbirth, or is suffering from other disastrous type of genital infection has any great or recognizable peril for non-pregnant contact cows. There is no evidence that harm comes to a healthy 
non-pregnant cow through eating food or drinking water contaminated by genital discharges from a diseased animal. It has not been shown that infections discharged from the genital tract of a diseased cow will traverse the vulva and vagina of a contiguous healthy cow and cause intra-uterine disease.

There is abundant clinical evidence that an infected bull is competent to transmit infection to the uterus of a healthy cow during coitus and either establish a disease (cervicitis, metritis, salpingitis) which inhibits conception, or when the infection is less mild, establish disease within the pregnant uterus to end finally in abortion or other disaster.

It has apparently been shown experimentally that serious uterine or other genital infection may be established in healthy non-pregnant animals by introducing into the blood stream or into the subcutaneous tissue, and thence indirectly into the blood stream, pathogenic bacteria having an affinity for the genital organs. Thus the experiments of Bland ${ }^{1}$ have apparently shown that by injecting large doses of $B$. abortus cultures into non-pregnant cows and heifers the fertility of the latter is seriously lowered, that a large percentage of the animals fails thereafter to conceive, or, if they conceive, the embryo is absorbed or expelled unseen. This may be apparent only, since the experiments were entirely too inaccurate to afford a secure basis for judgment. If the experiments actually caused the apparent harm, it may have been directly by the $B$. abortus reaching the genital canal, or indirectly through lowering the resistance of the patient to organisms already resident in the genital tract. In studying the infections of the uterus during the post-puerperal period, it is important to bear in mind their various possible or probable sources, because evidently the prophylaxis of the various types of disease occurring during this period must depend upon the power or desire to eliminate the dangerous infections existing during the puerperal period, and to avoid coital infection by excluding seriously diseased bulls.

The variety of organisms which may cause disease of the

${ }^{1}$ Second Report on the Epizoötic Abortion Experiments, 1911-1916. 
non-gravid uterus is infinite. Any organism competent to cause wound infection may injuriously attack the uterus. A long list of bacteria has been described as the causes of various uterine diseases. Perhaps the Streptococcus viridans is the most common and most destructive, but colonlike bacilli, $B$. pyogenes, staphylococci, and micrococci are common. The $B$. abortus is apparently a common resident of the uterus during the puerperal period but, according to most bacteriologists, disappears early after the close of pregnancy. In the following discussion of the uterine infections of this period, explicit reference to the biology will not be attempted because the present knowledge of the subject does not warrant it. The classification is arbitrary and merely intended to aid in arriving at reliable diagnoses and prognoses. The classes merge by imperceptible gradations one into another.

\section{A. Endometritis}

The typically healthy uterus passes imperceptibly into the simplest type of metritis. The imperceptible line of demarcation between health and disease is as impossible to fix in the non-gravid as in the gravid uterus. As previously stated, there is almost always sufficient infection present in the apices of the uterine horns to cause a definite necrosis of the contiguous tips of the embryonic sac. It may be of sufficient virulence to cause necrosis of the entire non-gravid horn of the fetal sac. But it may not cause disease so far as any marked peril to the embryo is concerned.

Clinically the non-gravid uterus physiologically runs such a gamut that it is sometimes perplexing. For some days prior to estrum the uterus becomes increasingly engorged, until finally, when estrum is fully developed, the healthy uterus is enlarged to approximately double its size when at rest. After the ovisac ruptures, the engorgement slowly subsides and the uterus regains its typical character of the inter-estrual pause. The secretions change also. With the advent of estrual engorgement there is an increased secretion of mucus, but not notable. Most of the mucus of estrum 
emanates from the cervix-not from the uterus. Shortly after ovulation, if conception does not occur, hemorrhage ensues from the cotyledons (menstruation) which in passing out becomes incorporated with the mucus of the cervix. The gradually increasing estrual engorgement requires two to four days for its maximum development and a similar period for its decline, so that of the twenty-one days usually constituting the estrual cycle, the uterus is engorged in varying degrees approximately one-third of the time, and during no two days is the engorgement the same. So it is with infection. Bacteria may be present-commonly are present -in considerable numbers without causing recognizable disease. The infection rarely remains static, but either advances to cause disease or is almost or wholly overpowered by the uterus. After a heifer has failed to conceive, until three years old or less, her uterus slowly loses its tone, enlarges and becomes flabby. The same rule applies to the postpuerperal uterus. If the cow fails to conceive with reasonable promptness, the organ loses its tone (if it had recovered its tone during the puerperal era), enlarges, becomes flaccid, and drops far forward in the abdomen. Endometritis becomes recognizable by the atony and the enlargement. By catheterization there is shown to be present in the uterus muco-pus or, in the milder forms, mucus. The endometritis may be due to infection extending from the cervix, possibly originally coming from the bull through coitus, or, as most frequently occurs, persisting in the uterus from the puerperal period. The chief cause may reside outside the uterus. Thus in nymphomania, while the disease is primarily ovarian, due to a peculiar type of cystic degeneration, endometritis is a constant result of the ovarian disease. Clearly the metritis needs to be referred finally to the presence of bacteria in the uterus, but the ovarian disease so disturbs the genital system as a whole that existing infections which would otherwise be held under control gain in virulence and cause harm.

The clinical evidence of endometritis is the recognizable enlargement of the uterus. Palpated per rectum, the uter- 
ine horns, enlarged transversely, elongated, and flabby, fall over the pubic brim to lie upon the abdominal floor. In this manner they drag the ovaries downward and forward. The mesometrium loses its tone also and the entire genital group hangs flabbily from the vulva. If the uterus is douched, there returns in the fluid in many cases some mucus or mucopus. In the abattoir such a uterus is eighteen to twentyfour or more inches long upon its greater curvature. Its interjor contains a little mucus streaked more or less notably with pus. The uterine mucosa is thickened, turgid, and engorged. Endometritis passes imperceptibly into pyometra. Frequently there is found in an otherwise apparently healthy uterus a few mils of pus somewhat mixed with mucus. In other cases larger amounts of pus are encountered, until finally the pus content is of such volume that the disease is described as pyometra.

Endometritis of moderate degree is not readily recognizable during estrum. At that time the flabby uterus partly regains its tone and there is a flood of mucus which temporarily conceals evidences of disease. Endometritis is largely associated with cervicitis at the vaginal end of the uterus, and with salpingitis or ovaritis or both at the ovarian end. That is, intra-uterine infection cannot readily be confined to the uterus for a long period of time. Since the tube is continuous, the infection tends to pass from the uterus into the cervix and the oviduct.

The prognosis of endometritis in itself is good. When proper handling is delayed and salpingitis has resulted, the latter is virtually beyond control. So long as the endometrium preserves its integrity, endometritis is generally remediable. While technically a mucous membrane can not be disinfected without destruction, clinically the uterine mucosa can be rendered sterile or essentially so. In my experience it has been not only possible but quite practicable to disinfect the uterine cavity so far that, if the animal is slaughtered ten to fifteen days later, attempts to grow cultures from the uterine mucosa fail, while similar efforts with the oviducts, which are beyond surgical reach, yield bacterial growths. 
The handling of endometritis may be direct or indirect. The direct treatment consists fundamentally of douching the cavity with suitable disinfecting fluids and introducing into the uterus slow-dissolving agents which may serve to repress bacterial growth. In my experience the compound solution of iodine (Lugol's solution) in a strength of one to two per cent. has yielded satisfactory results. The uterus has a high tolerance for the drug (experimentally I have used a ten per cent. solution) and it does not coagulate mucus. Its color is such that muco-pus, when douched out in the fluid, is readily observed as are most kinds of exudate occurring within the uterus. Some have advised instead a physiologic salt or soda bicarbonate solution, contending that the mechanical douching out of the infection is preferable to the use of a disinfectant. I can not agree with this. Very good authorities claim that, in douching the uterus of a woman, the fluid may force its way through the oviducts to the peritoneal cavity. Possibly anaesthesia favors such penetration. I have no reason to believe that any material peril of forcing the oviducts accompanies the douching of the bovine uterus, but the danger may be far greater than I have suspected. In case of danger a neutral solution has by far the greater peril because of its tendency to push the infection undamaged into the oviducts and set up salpingitis.

There is always present the danger of puncturing the uterus with the catheter. When this occurs, a few ounces of Lugol's solution injected into the peritoneal cavity irritate the peritoneum, there is quickly thrown out an abundance of serum which dilutes the fluid, and it is soon resorbed. If some infection is pushed into the peritoneal cavity, Lugol's solution tends to neutralize it, but when a salt or soda solution is used the infection acts unhindered. I consider that in all respects it is much safer to use a reliable disinfectant rather than neutral fluids when douching the post-puerperal uterus.

I have used other disinfectants, but have not seen my way to adopt them. Dakin's solution and similar chlorine-bearing solutions have been tried but need to be used in very 
weak solution and with great care. Phenol compounds are generally too irritant. The sulphates of copper, zinc, and iron coagulate mucus too promptly.

Of the slow-dissolving agents, iodoform and bismuth are best known and render good service in many cases. Suspended in oil, either or both may be introduced in a variety of ways. The uterine syringe shown in Fig. 196 is a very convenient instrument. By using the conical nozzle, the end may be pushed into the opening of the uterine catheter and the suspension injected through it; by using the long curved nozzle, the mixture may be readily injected directly into the uterine cavity.

As a rule the treatment for endometritis should not be repeated until two or three weeks have elapsed. When important disease exists in other parts of the genital system, it should be handled vigorously along with the endometritis. Thus, if nymphomania is present, no degree of attention to the uterus alone will give relief. The cystic disease of the ovaries should receive first attention, accompanied by the proper handling of the uterus. Professor Hess has also pointed out that dislodging a retained corpus luteum arouses physiologic function of the entire genital system. But that does not mean to press out the corpus luteum whenever found in connection with endometritis. If the cow is regular in estrum or fairly so, the corpus luteum should be let alone, but when the corpus luteum has persisted for many weeks and inhibited estrum it should be pressed out, so that its inhibitory power is brought to a close and estrum will ensue. With estrum the uterus becomes physiologically active, tending thereby to disinfect its cavity.

An animal having endometritis should not be permitted to copulate. Coitus increases the endometritis, endangers the bull, and generally prevents conception. Should conception follow, the pregnancy is unsafe and may terminate in abortion, premature birth, retained fetal membranes, or other disaster. 


\section{B. Pyometra.}

When pus in large volume is retained within the uterus, the condition is designated pyometra. It passes by scarcely discernible gradations on the one hand into endometritis, and upon the other into sclerotic metritis. Pyometra is used to designate not only the amount of pus formed within the uterus but also the approximate degree of uterine atony and hence the extent of retention of the pus within the uterine cavity. There may be a considerable volume of pus formed within the uterus, but the muscular coat retains its function and almost immediately expels it into the vagina. One frequently encounters several ounces of pus in the vagina, but upon douching the uterus finds but little within that cavity. After making proper allowance for such pus as may have been formed in the cervix or in the vagina itself, the volume within the uterus compared with that in the vagina expresses approximately the general tonicity of the uterus and the extent of involvement of its various tissues. If the pus lies almost entirely within the vagina, it indicates that the disease is limited chiefly to the uterine mucosa or endometrium and may therefore be designated endometritis. If, however, there is comparatively little pus encountered in the vagina, but most of it is retained within the uterus, it is more appropriately designated pyometra.

The chief symptom of pyometra is the presence of a purulent discharge from the vulva. The discharge may be profuse or scant, and in exceptional cases may be absent. A discharge issuing from the vulva may emanate, aside from the uterus, from the cervix, vagina, urinary bladder (and through the bladder, from the kidney), and from other sources. The pus discharged may vary infinitely in character. It is usually fetid, often extremely so. It is generally yellowish-white and thick. The pus of pyometra, as the term is commonly applied, is not ordinarily mixed with recognizable mucus because suppuration has supplanted the formation of mucus in the mucous glands of the uterus. In endometritis the mucus-secreting power of the uterus is deranged but not destroyed. In some old cases the heavier 
parts of the pus are precipitated in the lower portions of the uterine horns while the more liquid parts escape through the cervix and vulva. Thus there is formed a very dense, intensely fetid, cheesy mass.

The discharge of pus may be constant or intermittent. This is dependent chiefly upon the degree of atony in the uterine walls and the obstruction offered by the cervix. The pus from the uterus, passing over the cervical mucosa, inevitably causes cervicitis, which in turn aggravates the

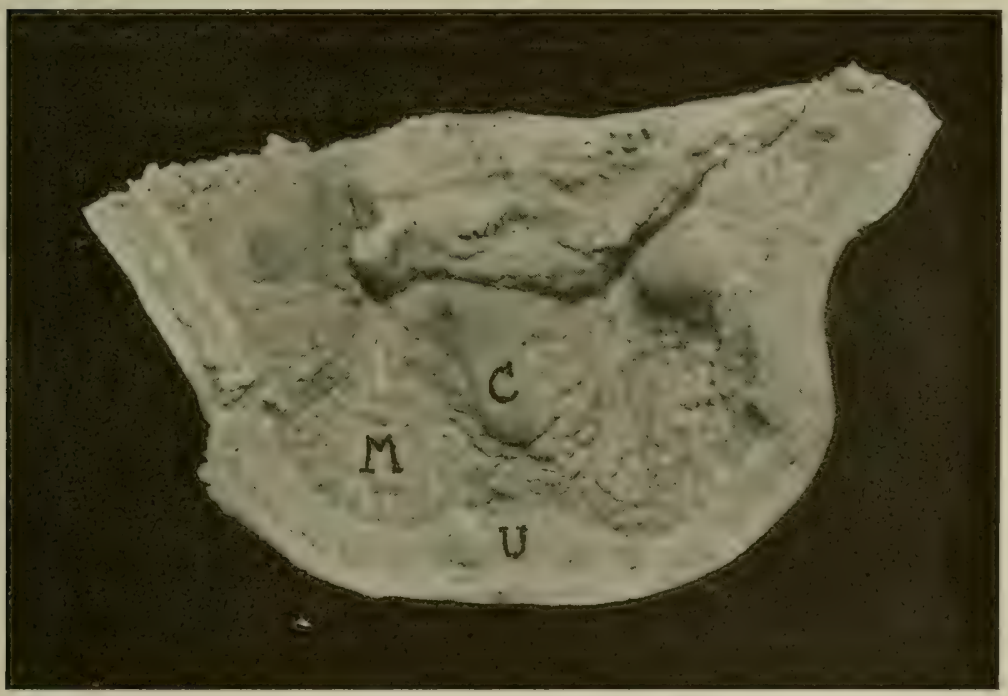

Fig, I $8_{4}$-Pyometra with Granular Mucosa.

$U$, Uterine wall; $M$, mucosa coarsely granular or nodular; $C$, cotyledon.

pyometra. The effect upon the cervix varies. Sometimes the cervix becomes atonic and flabby with its canal dilated. The pus then escapes freely from the uterus whenever the uterine walls contract, or whenever the uterus is compressed by contiguous viscera while the cow is recumbent. In these cases there is often an escape of a large volume of pus each time the animal lies down. When on the contrary the cervix retains its muscular energy or, as shown in Fig. 185, extensive cervical sclerosis is present, the pus is re- 
tained in the uterus until such time as the intra-uterine tension suffices to dilate the cervical canal, when the pus is expelled in large volume, succeeding which days or weeks may pass without purulent discharge. During the postpuerperal period, as clinically designated, there may occur pregnancy, the embryo or fetus may perish and be retained and, as described under "The Infections of the Gravid Uterus", there may be established a persistent pyometra and the pus may be mixed with blood. In rare cases very extensive

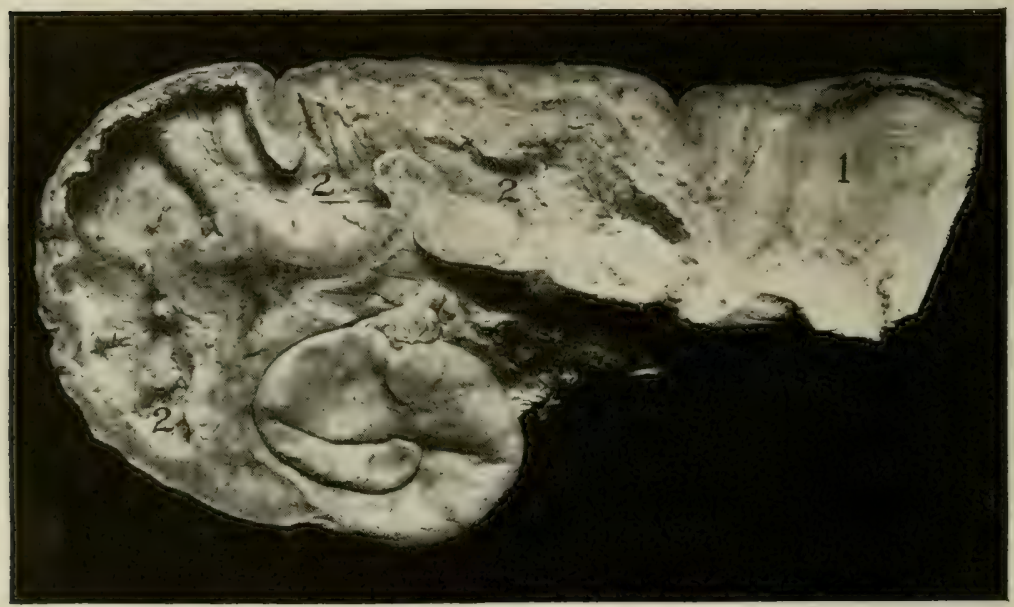

Fig. 185-Pyometra. Median Section through Right Cornu. Cow. 1, Cervix with sclerotic walls ; 2 , utero-cornual cavity showing nodular mucosa.

pyometra occurs without visible discharge. The uterus is completely flaccid and paretic. It drops far forward into the abdominal cavity and may lead to error in diagnosis, being mistaken for pregnancy.

The diagnosis of pyometra is usually simple. Upon inspection the vulva, tail and buttocks are soiled with pus. If the animal is recumbent there is often a tell-tale mass of pus lying behind her. Vaginal exploration generally reveals fetid pus in its cervical end. Rectal palpation discloses a distended, flabby uterus, usually with thickened walls. The two horns are generally similarly but not identically filled, 
and the contents may be pressed from the one into the other horn. The contents are clearly less fluid than lymph or mucus and are not at all like the fetal fluids. The uterus is wholly wanting in the tone of pregnancy, no cotyledons are present, and the uterine arteries are not materially increased in volume.

The accuracy of the physical examination is greatly increased by the application of traction through forceps fixed upon the vaginal portion of the cervix. This brings the

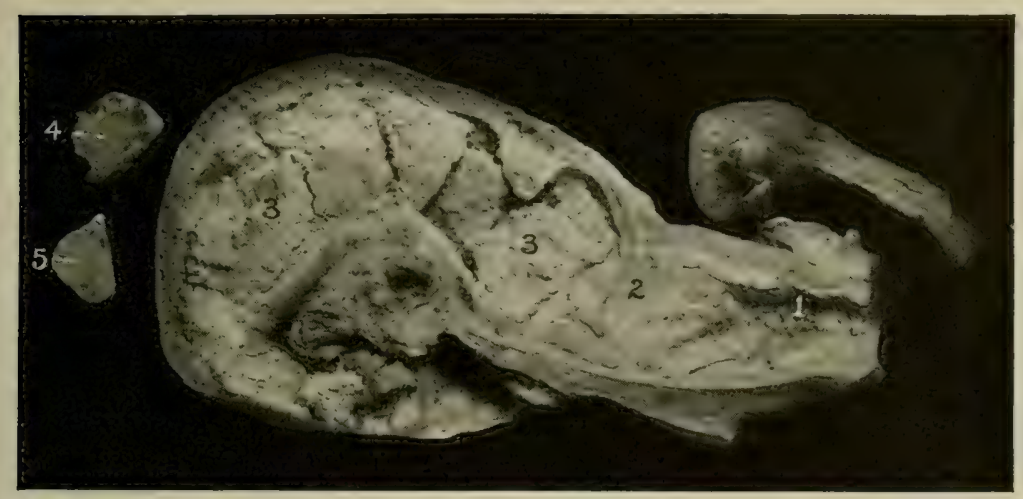

FIG. I86-Pyometra.

$I$, Vagina; 2, cervix; 3 , cornual cavity filled with pus ; 4 , central corpus luteum of pyometra; 5 (insert) corpus luteum of pregnancy.

flabby uterus into or near to the pelvis and tenses it in a manner which facilitates proper palpation.

The diagnosis is to be completed finally by douching the uterus. If the case is exceedingly complex or the examiner unfamiliar with the disease, he should be on the alert regarding the presence of a uterine seal. If this appears to be present, he should study the case more in detail by rectal exploration. He must not accept the history given by the owner that the animal has not been bred since the termination of a prior stated pregnancy. Owners, however careful, do not always know. The veterinarian must accept the history given by the owner so long as it aids, but must not be led into error. A false uterine seal occurs in cervicitis, 
and, while I have not seen the false seal in pyometra, it may occur.

The cervix is frequently sclerotic and its canal difficult to pass. Great patience is often required. First the dilators should be carefully introduced and the cervix slowly and liberally dilated. The complete dilation is valuable in diagnosis and essential in handling.

When the canal has been well dilated, the small uterine catheter (Fig. 39) or far better the soft rubber horse catheter or the seven-foot bull-douching catheter is to be introcluced. In most cases of pyometra the metal uterine catheter suffices for diagnosis, but in rare cases, when the uterus is unusually paretic, the pus is not douched out with the catheter even though gallons be present. When fluid is introduced through the metal catheter, it simply disappears and is irrecoverable with that instrument. This should indicate to the practitioner that he has erred in diagnosis and is trying to douche a pregnant uterus, that he has erred in technic and penetrated the peritoneal cavity, or that there is extensive pyometra dragging the uterus far forward and downward, and the douche has been added to the content. Unless one undertakes the imprudent and perilous filling of the flaccid uterus until it literally runs over, the diagnosis must be made with the rubber catheter used as a siphon. The cervix must be well dilated for this purpose, the uterine dilators engaged in the fenestrum of the catheter, and, with the cervix drawn well back with the uterine forceps, the catheter introduced carefully through the canal into the uterus. The catheter is held in place, the uterine dilator withdrawn, and the catheter gently pushed onward as far as may be prudent. It is then filled with fluid (salt solution, Lugol's solution) from an irrigator or otherwise by gravity, and some of the fluid may be allowed to pass into the uterus. The distal end of the catheter is to be compressed firmly while completely filled with the fluid. It is then lowered and the compression removed, when purulent or other liquid contents siphon out. If the precaution is not taken to compress the catheter while elevated and filled with 
fluid, and the uterus is very flabby, the fluid will drop into the inert uterus and the siphon be destroyed.

The pathologic changes in pyometra have not been extensively studied histologically. As observed macroscopically, the uterine walls are thickened and somewhat sclerotic. In the abattoir, when examined on the killing floor, the mucosa is usually of a dark bluish or bluish-black color, roughened and showing evidences of partial destruction. It is only very rarely that the mucosa has undergone total necrosis. In many cases the mucosa is nodular or granular, as indicated in Fig. 184. The elevations are pale yellow and suggestive at first glance of tuberculosis. The most suggestive element seen with the naked eye is the extensive changes in the uterine mucosa, which will demand for recovery a comparatively long time. Brief and fragmentary bacteriologic studies of pyometra indicate that the pus infection is as varied as that of wounds. There is no evidence to indicate that the bacteria present are specifically different from those prevailing in wound infections.

The prognosis of pyometra must be based upon the interpretation of the pathologic changes which are present. The two immovable barriers to reproductive recovery are total destruction of the endometrium and the extension of the infection into the oviducts. Both conditions are difficult of positive recognition. The outlook for the life and the general health of the patient is good, and if it may be safely assumed that the uterine mucosa is not destroyed and the oviducts not irremediably involved, the breeding life of the animal can probably be preserved. The treatment consists essentially of the dilation of the cervical canal, the removal of the purulent contents, the disinfection of the uterine cavity, and the removal of the corpus luteum, if present. The dilation of the cervical canal, as advised for diagnosis, suffices. The removal of the pus is best accomplished by means of the seven-foot bull-douching catheter with a hospital irrigator fitted with a stopcock. The catheter is introduced into the uterus as for diagnosis and sufficient physiologic salt solution gravi- 
tated into the uterus to dilute some of the pus and start the siphon. The distal end is then lowered and as much of the pus as possible siphoned out. More of the solution is then introduced and the siphon again brought into play. The catheter should be pushed cautiously as far into the apex of the horn as is safe. Heavy pus is to be cautiously broken up by massage of the uterus per rectum. It should be determined that both horns are being emptied. Sometimes this may be accomplished by massage with the catheter in one horn only. Sometimes it is essential to introduce the catheter into each horn. This may generally be accomplished by directing the catheter to the right or to the left with the dilator when introducing it. The catheter may also be directed into the horn desired by manipulation per rectum. The douching should be continued until the fluid siphons out clear. The uterus may then be washed well with $1 / 4$ to 2 per cent. Lugol's solution and, after this has been carefully siphoned out, there may be introduced into the uterine cavity eight to sixteen ounces of liquid paraffin holding in suspension one-half ounce each of iodoform and bismuth.

If the pyometra is a direct continuation of infection from the puerperal period, the corpus luteum of pregnancy is quite certainly present and has sunk deeply within the ovary. In most other cases of pyometra, a central retained corpus luteum is present. Professor Hess first drew definite attention to the inhibitory action exerted upon the uterus by a retained corpus luteum. While repeated douching of the uterus may finally disinfect it, the dislodgment of the corpus luteum with destruction of its inhibitory power constitutes a great and essential aid. The dislodgment generally causes estrum with uterine engorgement and sharp tonic contractions which tend to expel pus and diminish the infection. The technic of pressing out the corpus luteum has already been discussed under "Central or Embedded Corpus Luteum". The douching of the uterus should be repeated as frequently as prudent, generally once in two weeks, sometimes more often. Coitus should be excluded as long as the 
pyometra is present and for several weeks thereafter until the uterus has had an ample rest and has recovered its general tone. Early copulation usually causes a recurrence of the disease.

\section{Uterine Abscess}

Abscessation of the post-puerperal uterus occurs when there is a virulent purulent infection within its cavity and the cervical canal becomes closed by inflammatory adhesions or from other causes. A different type of uterine abscess has already been described under "The Infections of the Gravid Uterus", in which the putrefying embryo or fetus is the fundamental cause.

Post-puerperal uterine abscess usually follows badly handled retention of the fetal membranes or other type of metritis. It is most commonly one of the results of handling retained placenta as a definite disease which is considered as ended when the fetal membranes are supposed to have been removed. The abscessation ordinarily becomes established three to four weeks after the termination of pregnancy.

There are no outstanding clinical symptoms of uterine abscess. The cow has usually experienced difficulty in calving or aborting, and her recovery has been slow. Her general condition is quite regularly bad, though a fair appetite may remain and some milk be secreted. The chief clinical symptoms are not based directly upon the abscess itself, but upon the involvement of other organs. If the abscess presses upon, or points into, the urinary bladder, difficult urination logically ensues. Later, if the abscess ruptures into the bladder, the urine contains large volumes of pus. Most frequently the uterine abscess involves the rectum and causes adhesion to and stricture of the intestine, with difficult defecation, ending finally with rupture of the abscess into the bowel. The pus is later evacuated with the feces. Afterward the rectal adhesion remains, as shown at 5 in Fig. 187, and is readily recognizable upon palpation. When general pelvic adhesions have not occurred, the diagnosis is ordinari- 
ly simple both during the existence of the abscess and, when rupturing into the rectum, after healing has occurred. When pelvic adhesions become general, the rupture of an abscess into the rectum may still be accurately diagnosed, but owing to the indistinctness of the adherent mass, physical examination can not determine whether the abscess was uterine or parametritic. But in either case the breeding life of the patient is closed so that accurate diagnosis is not imperative.

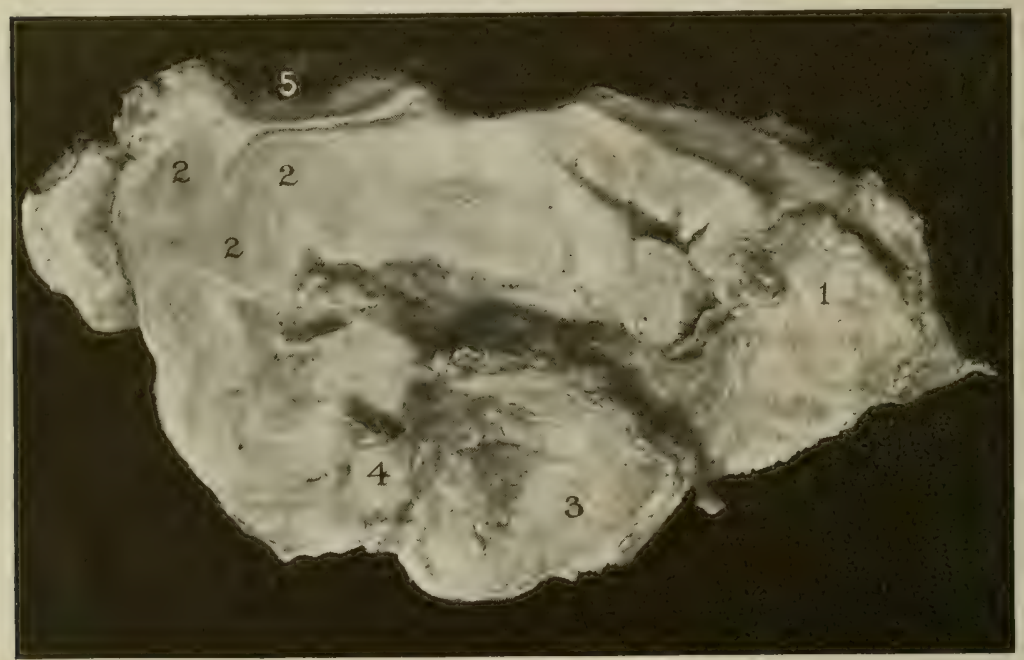

FIG. I87-Abscessation of Uterus with Pelvic Adhesions. Lettering includes that for $187 \mathrm{a}$.

$I$, Cervix; 2, 2, 2, uterine and cornual abscesses; 3, walls of urinary bladder; 4, adhesion of uterine horn to bladder; 5 , adherent rectum into which uterine abscess has ruptured; 6 , encapsuled ovary with cystic corpus luteum ; 7 , thin girdle of lutein tissue.

There is no handling to advise for the restoration of the breeding powers. There remains only the salvaging of the animal for beef or possibly for a brief dairying period. Even then there is little of value to do beyond directing good general care and, where possible, advancing recovery by the opening of the abscess instead of awaiting its spontaneous rupture. I have opened the abscess when mature by thrusting a finger through the rectal floor into the abscess cavity. 


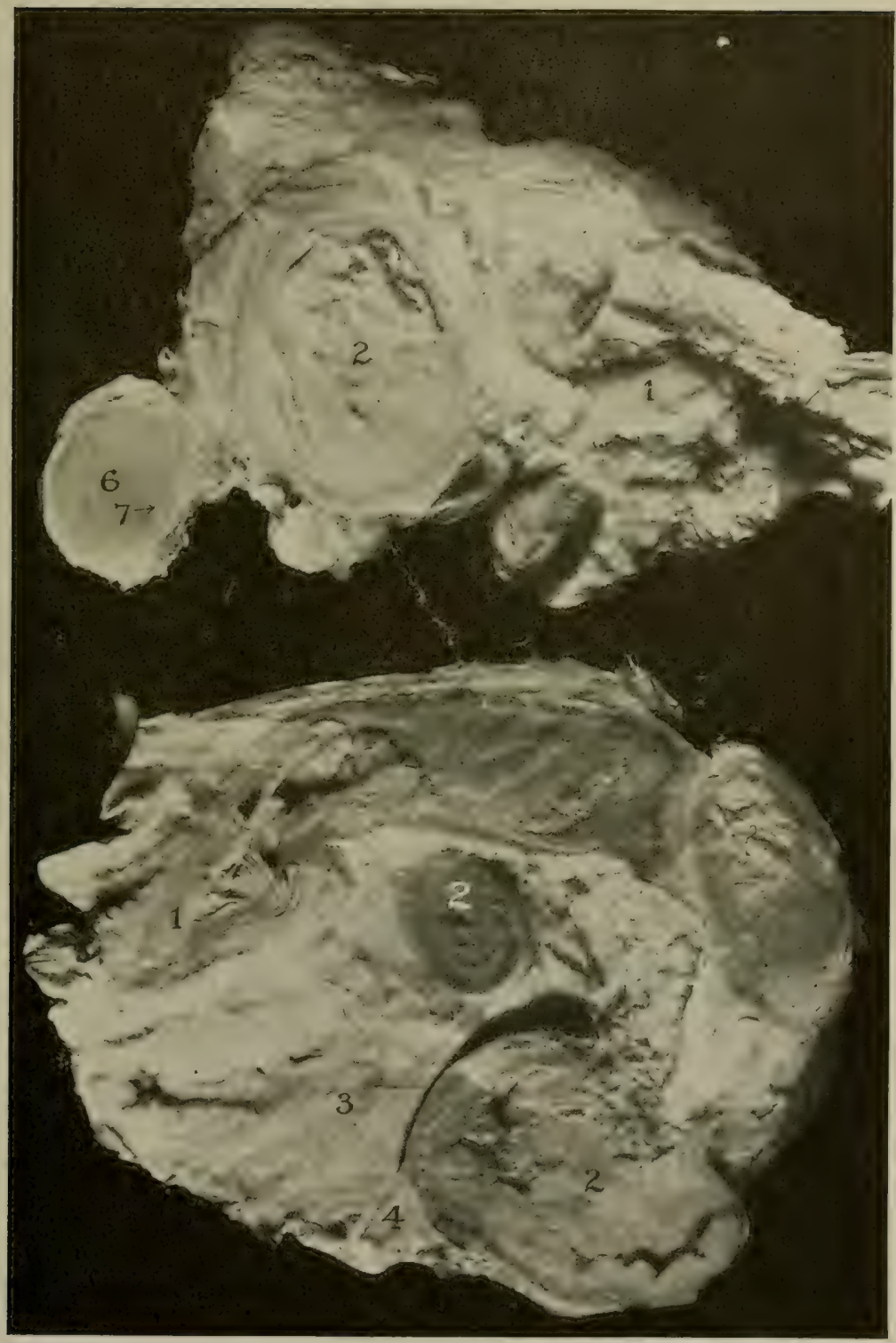

Fig. 187a-For lettering see Fig. I 87 . 
The abscess can be prevented only by closely following cases of metritis during the puerperal period and seeing that the infection is not permitted to continue into the post-puerperal era.

\section{Abscessation of Uterine Submucosa}

I have in my collection an abattoir specimen of tiny abscesses in the uterine submucosa, as shown in Fig. 188. The

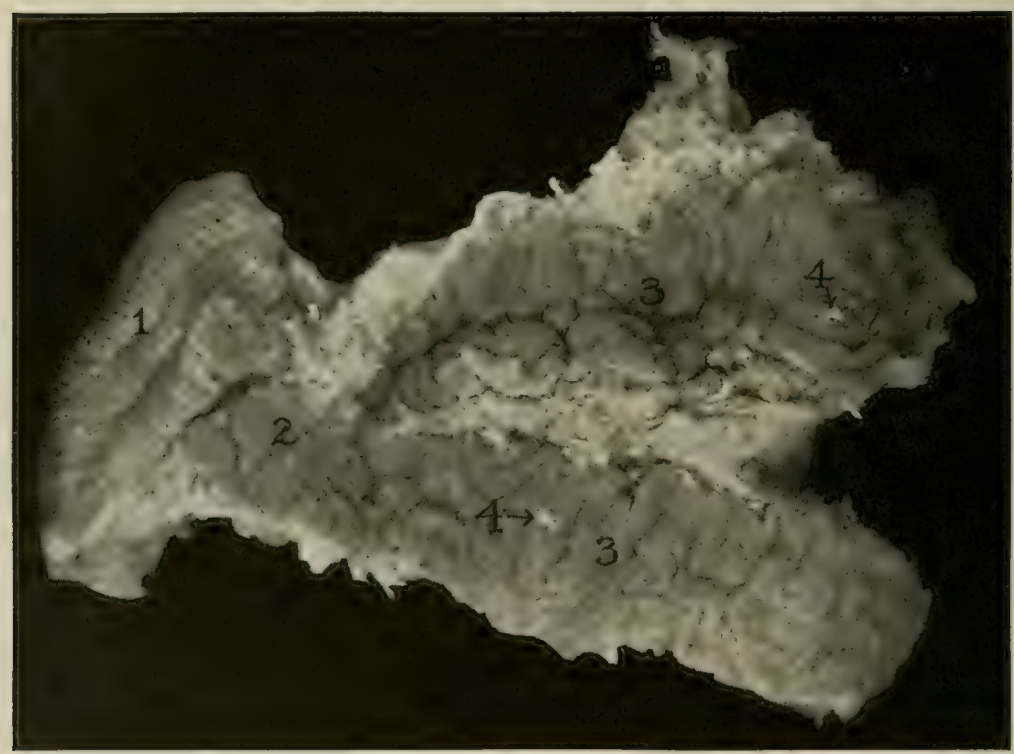

FIG. I88-Submucous Uterine Abscesses.

I, Vagina ; 2, cervix ; 3 , cornual cavity ; 4, abscesses.

mucosa is thrown into numerous marked elevations which when incised reveal an abscess the size of a very small pea, containing thick yellowish-white pus. The walls of the abscess are not very dense. The character of the disease, the method of clinical diagnosis, the handling, and the prognosis are questions upon which no definite opinion can be expressed. 


\section{E. Sclerotic Metritis.}

Sclerosis of the uterine walls generally accompanies longcontinued metritis, but as a rule the sclerosis is not very marked. There may be distinguished upon rectal palpation a definite thickening and hardening of the walls, which does not constitute an important factor in the prognosis and handling of the disease. If the uterine mucosa is restored to health, the sclerosis abates and disappears. There is, however, an extreme type of sclerosis which is not rare and

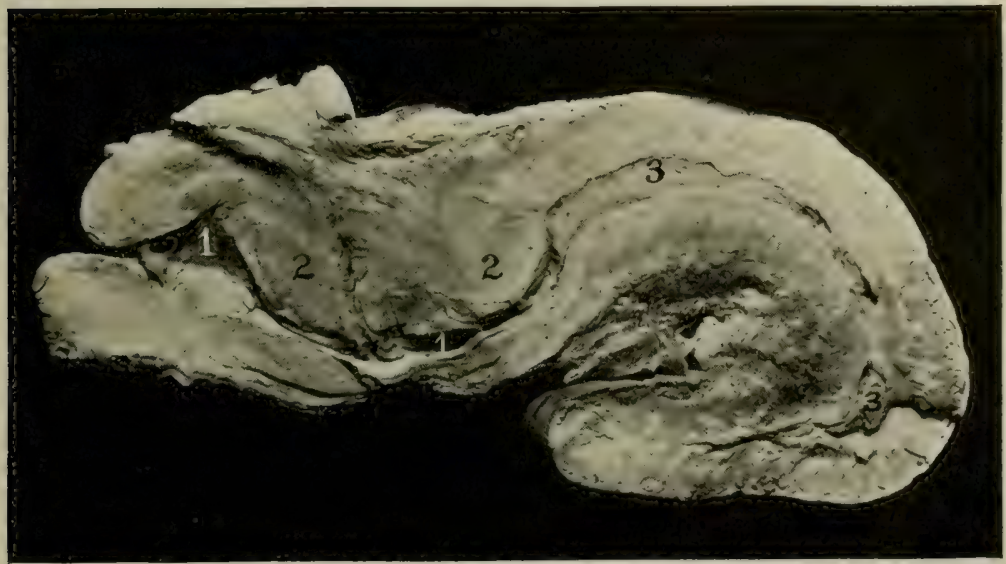

Fig. 189-Sclerotic Metritis with Pyometra.

I, Cervical canal; 2, sclerotic annular folds of cervix pushing canal far downward; 3 , cavity of uterus and horn containing pus.

Uterine mucosa destroyed.

which it is important to recognize clinically. This type I have designated as sclerotic metritis. It is characterized by greatly thickened walls, one-half to one inch in diameter, very dense and hard. A typical example is shown in Fig. 189. The disease is evidently the result of long-continued intra-uterine irritation. It may be due to fetal retention, as shown in Fig. 171. In the definitely post-puerperal cases I have not been able to trace the disease to its fundamental cause as all my examples are from the abattoir and hence without clinical history. It appears fairly safe to attribute the condition ordinarily to neglected or maltreated retained 
fetal membranes, though any intense irritation should be competent to cause it.

Clinically the symptoms are a constant, usually fetid uterine discharge of limited volume. The sclerotic state of the walls prevents sacculation of the uterus, with large accumulations of pus, and renders the discharge essentially constant. Clinical examination reveals pus in the vagina and, by douching, in the uterus. Rectal palpation discloses an enlarged, very hard, cartilage-like uterus, usually regular in contour. The hardness is similar to that of some cases of puerperal metritis, but the history of the recent termination of pregnancy serves to differentiate. In the latter case the hardness disappears with the control of the metritis. In all specimens in my collection the uterine mucosa is totally destroyed and the breeding life of the animal definitely closed. The condition does not visibly affect the general health of the animal nor decrease the value of its carcass for human food. There is probably very little, if anything, to be gained by treatment.

\section{F. Pelvic Adhesions. Parametritis}

Parametritis with pelvic adhesions and abscesses is a common result of intense intra-uterine infection. The extrauterine infections vary widely in origin. Unquestionably some cases arise from the infection passing through the oviduct and escaping into the peritoneal cavity. These, however, usually expend their energy in producing cystic degeneration of the corpus luteum and adhesions of the ovary in the pavilion of the oviduct and in the ovarian pocket. This is elsewhere considered. Extensive pelvic adhesions are the common result of non-mortal rupture of the uterus with the escape of infectious material, or of the injection of irritant antiseptics into the peritoneal cavity. Infection also invades the genital peritoneum from the endometrium through the muscle layers of the uterus and, having gained the serous covering, involves by contiguity the adjacent peritoneal surfaces, and the contiguous surfaces adhere.

The most potent cause of pelvic adhesions and abscesses 
is the improper effort at removing an irremovable retained afterbirth. In one herd where six cows had suffered from retention of the fetal membranes and attempts had been made by the attending veterinarian to remove them manually, I sent three ( 50 per cent.) directly to the butcher on account of pelvic adhesions and abscesses.

The clinical symptoms of pelvic adhesions and abscesses are vague and inconclusive. There is ordinarily a history of disease at the time of parturition, from which recovery has

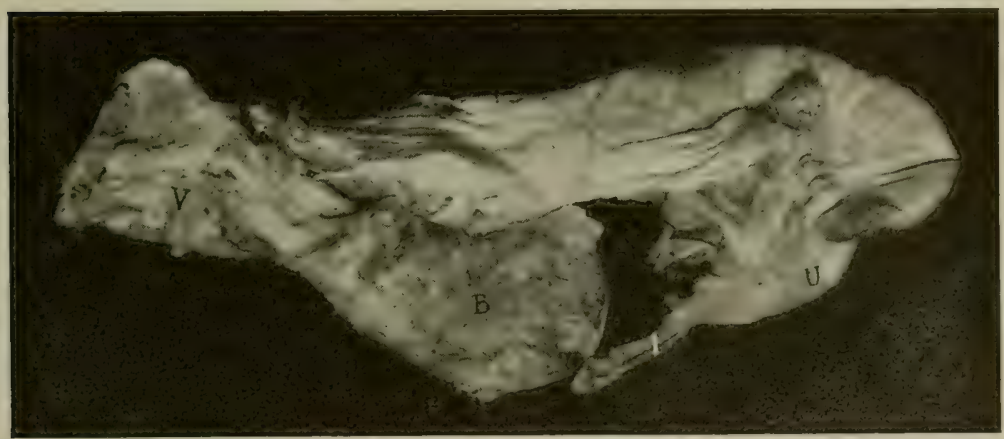

FIG. I90-Adhesion of Uterus to Bladder.

$V$, Vagina ; $B$, urinary bladder; $U$. uterus ; $I$, adhesive band passing from uterine horn to bladder.

been tardy and imperfect. The animal is rarely in good condition and is generally emaciated, with a lustreless coat. When the adhesions are quite extensive the movements of the animal are careful, hesitant and stiff. The back is frequently arched.

Clinical examination by palpation through the rectum discloses adhesions of the uterus, oviducts and ovaries to the pelvic walls, rectum, bladder, rumen, or other adjacent structures. It is essential to accurate diagnosis that traction be applied to the cervix with the uterine forceps. This affords the examiner a definite base (the cervix) from which to extend palpation, and the tension brings out, if such result is possible, the contour of the diseased uterus. Depending upon the degree of adhesion, the nature of the lesions 
may be more or less clearly determined. When very extensive, the identity of uterine horns, oviducts and ovaries becomes lost in the adherent masses. Here and there may be gross sclerotic masses which may with security be diagnosed as sclerotic abscesses, but it can not be told whether the abscesses are within the uterus or one of the uterine horns, the oviduct, the ovary, or some intra-pelvic lymph gland or other structure.

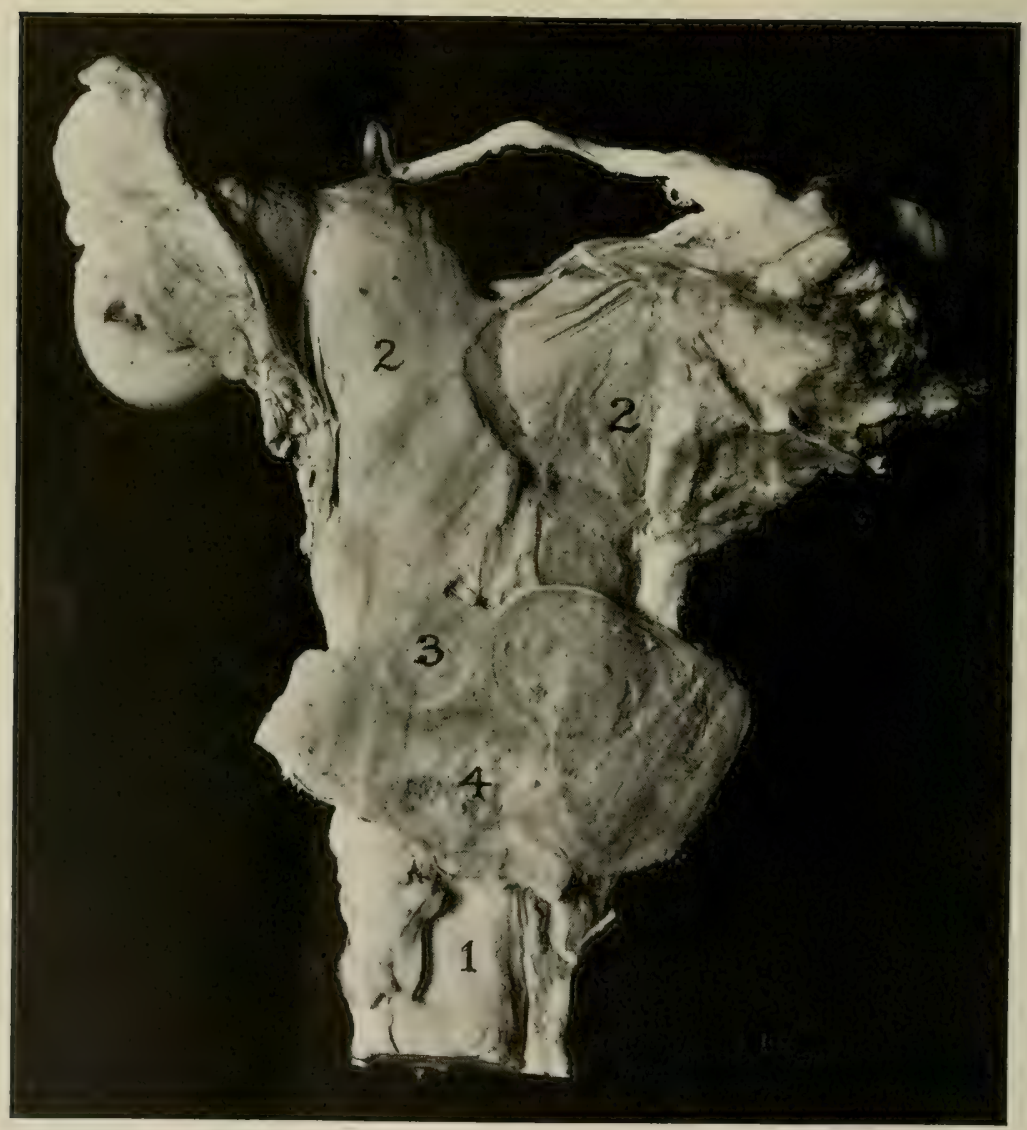

FIG. I9I-Pelvic Adhesions.

$I$, Cervix ; 2, cornu ; 3 (insert) cross section of cornu with pus ; 4 , cross section of ovary. 


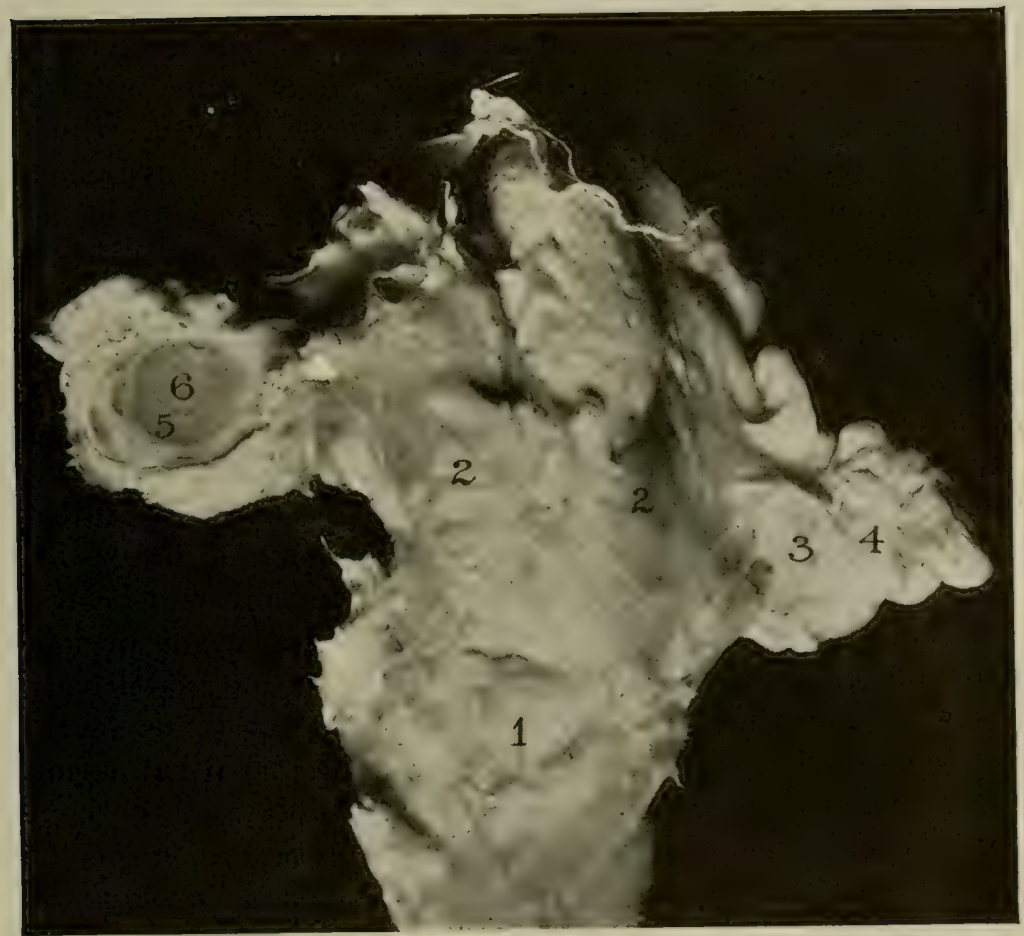

FIG. I92-Extensive Pelvic Adhesions.

$I$, Uterine body; 2, cornua; 3 , right ovary, completely encapsuled; 4 , right ovicluct, cystic and adherent ; 5 , corpus luteum in left ovary ;

6 , cyst in corpus luteum

The disease is not amenable to treatment. The animal should be slaughtered and the carcass used for food or other disposition made of it according to conditions.

F. Pyemia. Arthritis. Abscesses of the Ductless Glands.

When the intra-uterine infection by its virulence breaks down or renders non-resistant the protective epitheliun 1 of the uterus, or an imprudent effort is made to remove the fetal membranes and epithelial wounds or abrasions occur, an inviting opening is made for the entrance of the intrauterine infection into the blood or lymph capillaries, whence 
it gains the general circulation and may be carried anywhere. In the puerperal period observations upon such invasions of the body fluids by bacteria from the diseased uterus are limited to the profound sepsis induced, which generally destroys the life of the patient. If the invader is less virulent, its effects are not immediately recognized because the uterine disease is so pronounced that it overshadows all else. Later when a small volume of infection or infection of only moderate virulence enters the blood stream, it may finally become lodged in some remote organ or tissue, establishing lesions and inducing symptoms not directly referable to the infected genital tract. The conclusion that such lesions are referable to intra-uterine infection is kased upon the fact that they are common in association with genital infections but are otherwise rare in cows.

Pyemia of genital origin may occur at almost any period, probably most often during the puerperium, but, as already noted, it may then pass unobserved. It is most frequently observed during the interval following the puerperal period after the close of one pregnancy up to the date of the next conception. Generally the clinical symptoms become obvious at from three to six weeks after the close of pregnancy. I have observed it in pregnancy while the metritis of pregnancy was laying the foundation for abortion which occurred a few weeks later.

In cattle pyemic arthritis is the most striking and frequent type of pyemia due to genital infection. It may involve any joint in the body, but is most frequently seen in the stifle or femoro-tibial articulation (pyemic gonitis). The lameness is usually intense, but may be of any degree. There is usually distinct swelling, most readily detected as a rule at the median side of the femoro-tibial articulation, or revealed by the distension of the femoro-patellar synovial bursa. Heat and pain upon pressure are usually recognizable by palpation. The uterus shows the presence of infection or there is a definite history of the existence of genital disease in the recent past. When involving other articulations the symptoms are essentially analogous to those when the stifle is affected. 
Pyemia. Arthritis. Abscesses of the Ductless Glands 6I I

The outlook for recovery from pyemic arthritis is very poor. Potassium iodide internally has given apparently good results in some of my cases, but it must be used with great caution because of the high susceptibility of cattle to iodine. If pus forms and samples can be aspirated for cultures, autogenous vaccines are worthy of trial. Locally little can be accomplished. The inflamed articulation may be fomented or blisters may be applied. Should the presence of pus be positively diagnosed, it may be aspirated and the cavity refilled with 20 per cent. Lugol's solution in water or glycerine. If an abscess forms, it should be opened freely and the cavity packed with gauze saturated with tincture of iodine mixed with glycerine, or with some other suitable disinfectant. Movement of the joint should be restrained in any manner available.

The uterine cavity, if still an active center of infection, should receive proper attention. The general care of tne animal should be of the best.

Abscesses of the liver and other glands are occasionally observed during or subsequent to severe uterine infection. The clinical symptoms of such abcesses are vague and generally fail to afford secure ground for diagnosis. The dominant symptom generally is that of ill health with gradual emaciation. Sheuld the abscess rupture externally, as sometimes occurs when located in the liver or kidney, diagnosis may be possible. The alert practitioner should always suspect pyemic disturbances when ill health follows the termination of pregnancy, and should make the most careful search possible for pyemic abscesses. In such cases laboratory examination of the blood is highly important and should not be neglected.

\section{H. Cystic Degeneration of the Uterine Walls with Hydrometra.}

Hydrometra, or distension of the uterus with liquid, is not common and is dependent upon a variety of causes. It has already been noted that mucus and menstrual debris may be retained in and distend the uterus when the cervical canal 
has failed to open, owing to an arrest in the development of the embryo. It has likewise been stated that, when the hymen remains imperforate, the accumulation of mucus and menstrual debris eventually forces the cervical canal and distends the uterus. Various changes in the cervix may

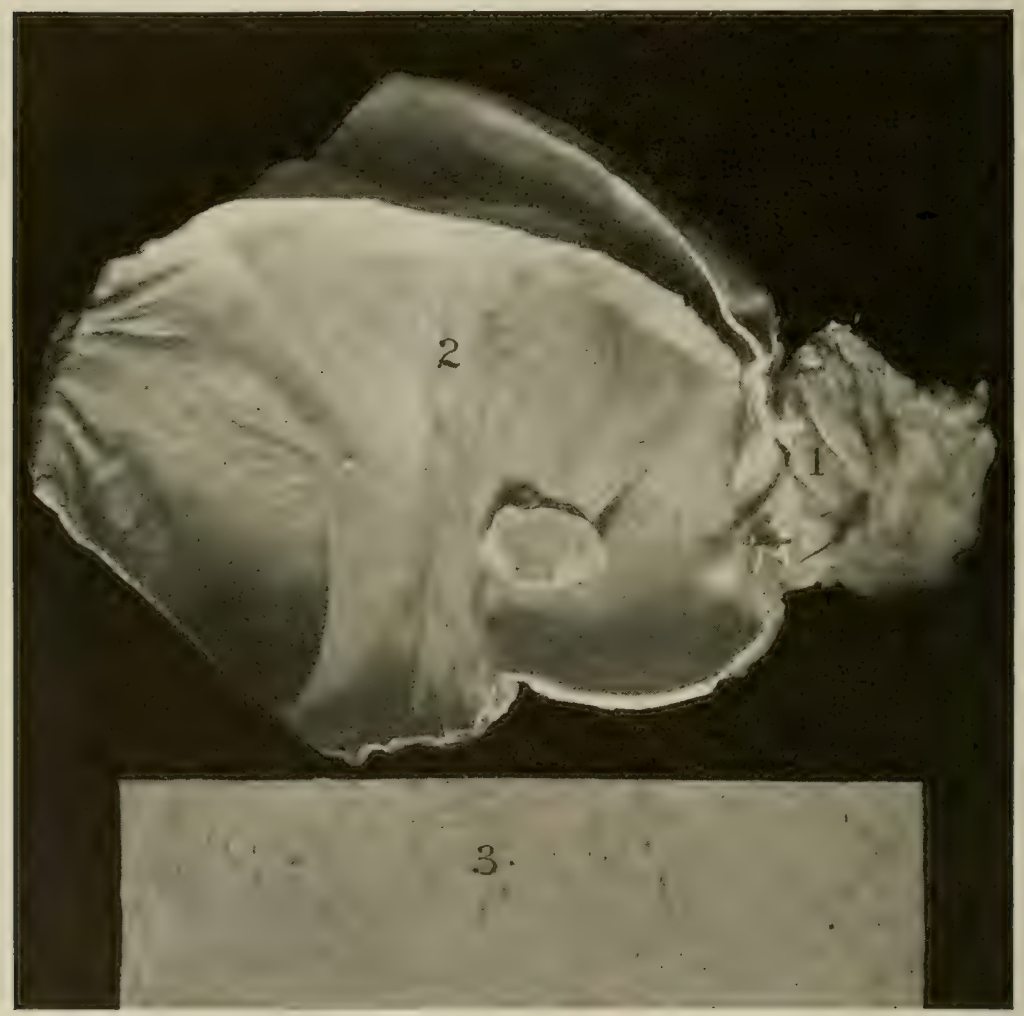

FIG. I93-Cystic Degeneration of Uterine Walls with Excessive Hydrometra. Uterus contained about 10 gallons of ly mph

$I$, Cervix ; 2, cavity of left horn, right in shadow ; 3 (insert) section of cystic ovary. (Insert, below) Enllarged section of wall (photograph.)

cause a retention of the uterine discharges. It will also be shown, while dealing with the diseases of the vagina, that atresia resulting from vaginitis dams back uterine and cervical secretions and distends the uterus with fluid. 
It is desired here to describe a disease of the uterus itself resulting in the formation of a large amount of fluid which is passively retained within the uterine cavity. I have seen clinically several cases in which the cervica! canal was free and readily admitted the catheter, while the uterus contained a large volume of clear, odorless, watery fluid. I have been able to secure the uterus of one of these cases, illustrated in Fig. 193. This valuable cow had long been sterile. Upon examination I found the uterus greatly enlarged, flabby, and dropped far forward so that only the cervical end could be palpated. No cotyledons were palpable and the uterine arteries signified non-pregnancy. The cervical canal, which was open, though the insertion of the metal uterine catheter caused but little fluid to escape, was gently dilated. I introduced a rubber horse catheter and, using it as a siphon, obtained a large amount of clear, watery fluid estimated at ten gallons. A few weeks later I siphoned out about five gallons more. A colleague removed the fluid several times, but there was no improvement and the cow was slaughtered. The uterus was greatly distended at time of slaughter, showing that the fluid was quickly renewed. The uterine mucosa, as shown in Fig. 193, is filled everywhere with small transparent cysts. The normal mucosa has disappeared completely. The fluid contents of the uterus apparently emanated from the cystic degeneration of the mucosa. So far as known, the disease is beyond remedy, at least in so far as reproduction is concerned.

This condition may be responsible for a few of the extreme cases of pyometra I have cited. They might very well have been identical at first with the case just described, and colleagues, having unsuccessfully attempted to douche the uterus, may have carried infection into the vast reservoir of fluid and converted the hydometra into pyometra. The only important element in connection with hydrometra, according to my observation, is an accurate diagnosis. Slaughter should follow. 


\section{Cervical InfeCtions}

\section{A. Cervicitis}

A careful study of the anatomy of the bovine cervix, as described on page 33 and illustrated by Figs. 15 and 16, shows it to be an extremely complex organ, admirably adapted, at first glance, to act as an important barrier between the copulatory segment of the genital tube-the vagina-and the nutritive area in which the embryo is destined to develop-the uterus. If, however, infection gains a foothold within its intricate mucous folds, what appears at first as a highly efficient barrier becomes a very serious hiding-place for bacteria. The cervix is one of the most important areas of the entire genital system in relation to serious infections. The cervix of the cow rarely fails after two or more pregnancies to show at its vaginal end clinically visible lesions. The changes are so nearly universal that the milder types of disease constitute the "normal" cervix of anatomists. The vaginal end of the cervix of the ordinary virgin heifer should, I believe, be taken as the standard for that part of the organ and any material departure from that type regarded as pathologic.

Prior to pregnancy the cervix of the heifer has received little clinical study. It is difficult to bring it into convenient position for visual inspection without using an undesirable amount of force. Until the heifer has failed to conceive after repeated coitus, she is assumed to be free from genital disease. After such failure to conceive, if a veterinarian makes an examination, he frequently finds the cervix diseased. As a rule, however, the disease has not notably modified the vaginal end of the cervix, so far as can be seen, the exterior appearing sound, but disease of the deeper parts of the cervix is recognized by instrumental examination of the cervical canal. After the termination of pregnancy by parturition or abortion, changes occur in the vaginal end of the cervix, readily seen upon clinical examination, which profoundly alter its appearance and character. The most striking changes are delineated in Fig. 194, in which it is 
shown that with breeding the cervical mucosa becomes tumefied, and hypertrophied, and finally portions of it evaginate through the os uteri externum. The prolapsed portions are described by anatomists as the lips of the cervix. As a matter of fact they are the hypertrophied rugae of the first annular fold of the cervical mucosa and are not visible in the healthy virgin heifer. The actual os uteri externum, covered with vaginal mucosa, is forced aside by the hypertrophied first annular ring. The true lips of the os uteri

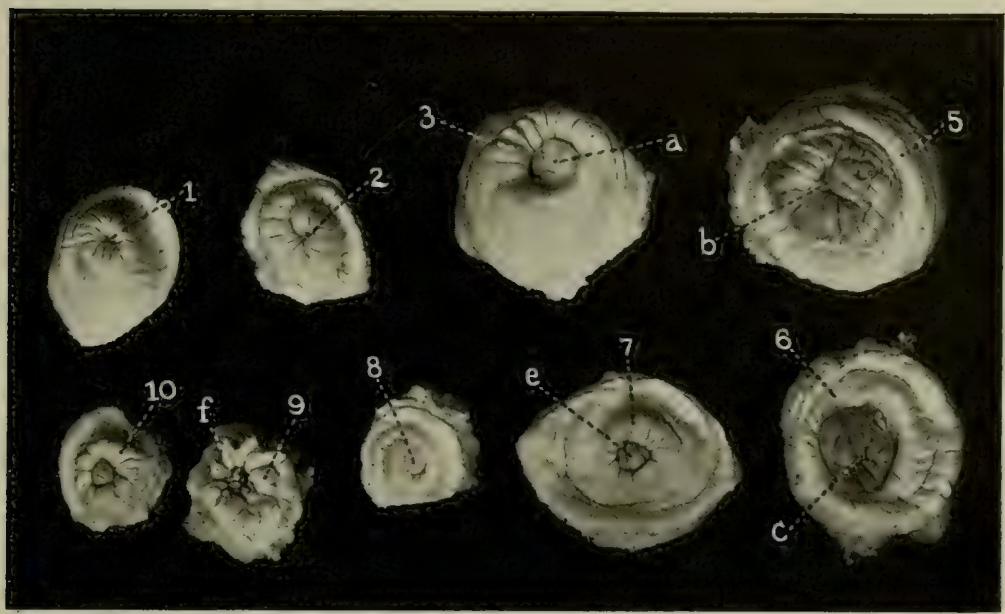

FIG. I94-Vaginal Ends of Cervices.

Numerals indicate lips of original os uteri externum; letters signify protrusion of the first annular folds in varying degrees. In $I, 2$, and $\delta$, the fold does not protrude.

externum finally form a pale-colored girdle about the base of the prolapsed portion of the cervical mucosa. Here, often one to several inches from the vaginal extremity of the cervical canal, the pavement epithelium of the vagina ends and the cervical epithelium with its intricate and elaborate mucous glands succeeds. The secretions of the two areas are wholly unlike. The protruding cervical mucosa, like that remaining within the cervical canal, takes the leading part in the elaboration of the thin mucus of estrum and, in pregnancy, of the uterine seal. 
Cervicitis is the result of bacterial invasion of the cervical mucosa. The origin of the infection and its date of invasion have not been fully determined. Clinicians know well that heifers conceive with greater difficulty than cows. That is, the average number of copulations essential to pregnancy is greater by 50 to 100 per cent. than is required for cows. There is present some form of temporary interference with conception, which is ultimately eluded and pregnancy ensues. As a rule, also, abortion and retained fetal membranes occur in heifers in first pregnancy 50 to 100 per cent. more frequently than in cows. These two conditions we know are referable to an infection radiating from the cervical end of the uterus, and it appears quite justifiable to conclude that in those cases where recognizable fertilization has failed these conditions are parallel. Either the cervicitis present inhibits fertilization for a time, or fertilization occurs and the cervical infection works destruction so promptly that estrum recurs in its regular cycle or at a later date.

When clinical examination of a sterile heifer is made, ovarian or tubal disease is recognized in only a minority of cases. When exploration of the cervical canal is undertaken, it is found abnormally tortuous and difficult of passage because the bases of the mucous folds have become sclerotic and hypertrophied. Probably there is a bit of muco-pus in the cervical canal. The diagnosis of cervicitis is unescapable, but its source and the date of invasion are not wholly clear. I cannot logically separate the source and date of invasion in these cases from those of abortion and retained fetal membranes. The undeniable clinical fact, that, in a herd well saturated with genital infections, the heifers abort 50 to 100 per cent. more frequently than cows, though all are kept under identical conditions in the same stable or field and are bred to the same bull, testifies that the heifers arrive at breeding age with the infection resident in the cervix. That is, if in a given herd the observed rate of the expulsion of fetal cadavers in cows is 10 per cent. and in heifers 20 per cent., though kept and bred alike, at least 50 
per cent. of the heifer abortions should be attributed to causes existing at conception. Analogous conclusions must follow regarding cervicitis causing sterility. There are then three outstanding causes of cervicitis:

1. In cervicitis existing when the heifer reaches puberty, present observations indicate that infection existing at this date is referable to invasion of the fetus or of the calf during the nursing period, which will be discussed under "Congenital Infections of Calves."

2. Coital transmission of infection by the bull. This has been discussed while considering genital infections of bulls. In one large herd where intense cervicitis involved over 60 per cent. of the cows, treatment was of little avail until finally the bulls were examined, slaughtered, and healthy bulls substituted. The bull is frequently the chief and by far the most important infection-bearer.

3. Imprudent handling of cows for sterility. I have observed some very intense outbreaks where the spread of the infection appeared to be due to the veterinarian who was handling the cows for sterility. Those veterinarians who have led themselves to believe that in the post-puerperal period it is safer and more effective to douche the uterus with 0.6 or 0.7 per cent. soda or salt solution, if they do not sterilize their uterine catheters or other instruments before using upon each cow, constitute a serious peril to the animals they handle, and, so far as I have been able to understand some outbreaks I have seen, frequently do incalcuable harm.

Once the infection has become implanted, the chief elements in intensification are coitus and parturition (or abortion.) I have douched the uteri of several cows two, three or more days after coitus, and have always obtained mucopus in the returned fluid. Whether this is the invariable rule, I do not kow. The same cows do not have such mucopus without coitus. It is not the effect of estrum.

The influence of parturition or abortion upon cervicitis is very marked. The contractions of the uterine walls force the fetus against the cervix and push its walls apart. The 
force causes a rent in a diseased part already infected in many cases. More frequently the force abrades the mucous membrane, affording innumerable openings to bacterial invasion. The peril is frequently accentuated by the presence of metritis, often with retained fetal membranes which undergo decomposition so that the cervix is submerged by infective discharges and putrefying membranes. Under these conditions each parturition leaves its mark upon the cervix.

The simplest clinical evidence of cervicitis is a swelling of the first annular cervical ring, which pushes the margins of the lips of the os uteri externum apart, so that the ring becomes visible lying within the cervical canal, deeply injected and bright scarlet or dark red in color. Next appears the prolapse or ectropia of the first mucous ring through the os uteri externum. As the annular ring prolapses farther and the infection increases, the longitudinal foldings of the cervical mucosa become increasingly evident, and the hypertrophied mucosa of the annular cervical fold evolves into an encircling series of club-shaped tumors. The second annular ring may participate. Finally the hypertrophy may become very gross and the tumefied cervical mucosa protrude out into the vagina like a cauliflower, four, six or more inches in diameter, scarlet or dark red in color, and highly vascular, bleeding at touch. The extremes are illustrated in Fig. 194 and in Colored Plate VI. Between these extremes every possible variation appears.

The cervical secretions in cervicitis are as variable as the tumefaction and hypertrophy. If two or more pairs of uterine forceps are fixed upon the cervix, traction applied, and the forceps handles spread apart, the vaginal portion of the cervix, as a rule, is brought freely into view, and the inflamed cervical mucosa is open to visual examination. In the milder types illustrated in Fig. 194, no secretion of notable character is observed. If the visual examination is supplemented by inserting the dilator into the cervical canal, and the jaws are opened, there may ooze out between them a drop or two of muco-pus. If the uterine catheter is in- 

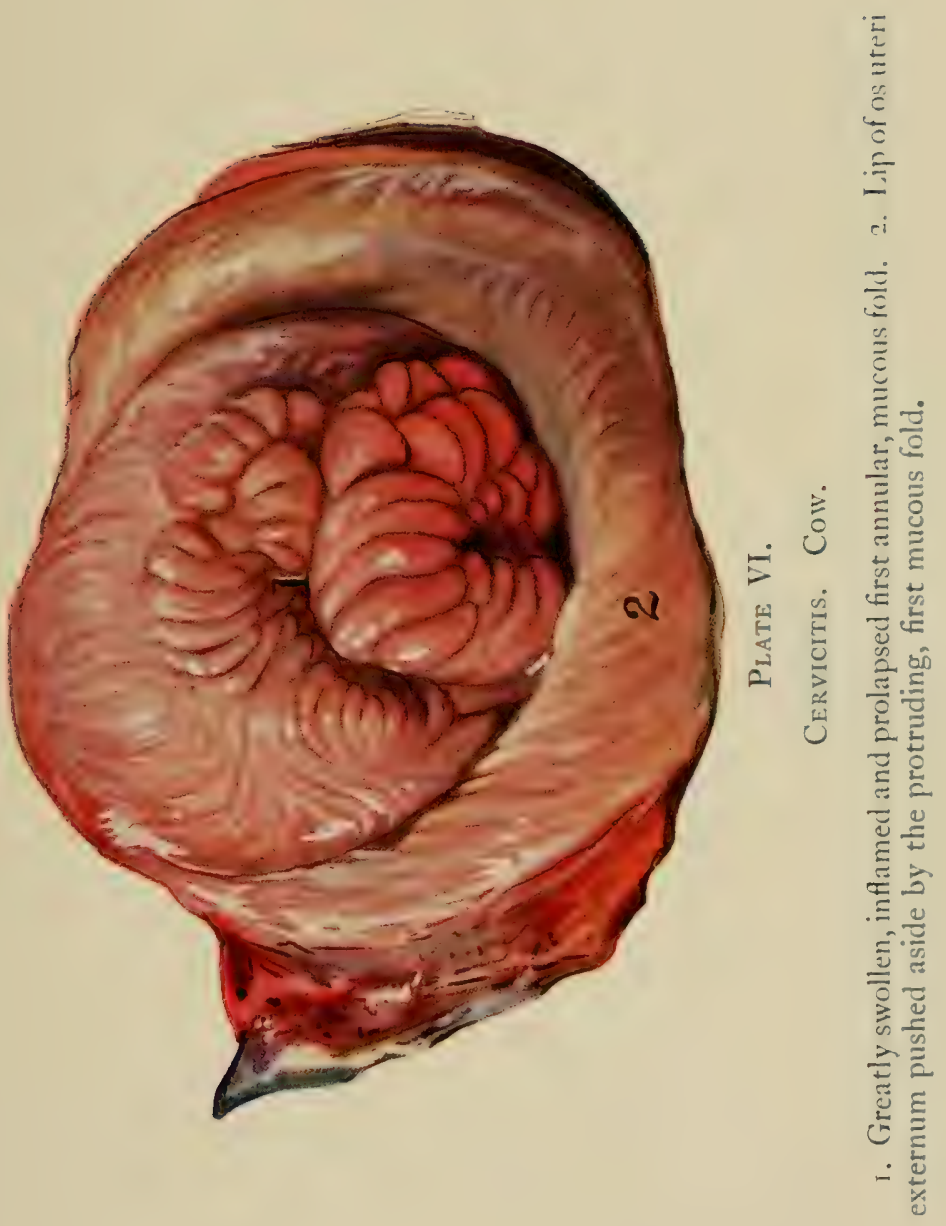

troduced and exploratory douching attempted, the first fluid to return is probably a mil or two of muco-pus which has been caught in the fenestrum of the outer catheter tube as it passed through the cervical canal.

As the intensity of the disease advances, there appear two divergent types of secretion-mucous and purulent. That of the mucous type, in its extreme form, is represented in Colored Plate VI. The cervical glands in the mucosa of the great angry red tumor secrete a viscid, tough mucus resembling in varying degrees the uterine seal. It may be

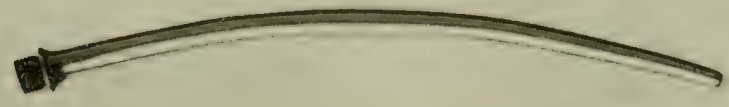

$-$

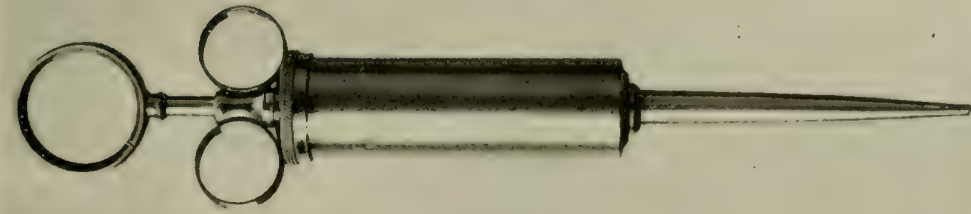

Fig. I96-Uteine Syringe with Conical and Curved Nozzles.

softer or more resistant than the seal. I have met clinically cases where the secretion was so voluminous and its resistance so great that it defied my efforts at passing dilator, catheter, or other instrument through the cervical canal into the uterus.

In the purulent type, in sharp contrast to the preceding, abundant pus is formed, chiefly in the vaginal end. The pus accumulates in varying quantities in the cervical end of the vagina, from which it is intermittently expelled. The pus, ordinarily offering nothing remarkable in character, is generally thin and is yellowish or grayish white. Sometimes it is very fetid; sometimes non-fetid. 
The intensity of the disease at the vaginal end of the cervix does not furnish a reliable criterion regarding the state of the cervix in its more anterior portions. Any or all of the annular mucous folds may be involved, resulting in the elongation of the free, projecting portion, with sclerosis of the base of the fold. The swollen free margin projecting toward the vagina fills the canal and interferes with the passage of the catheter or other instrument, partly by the direct filling of the canal, but chiefly by the elongated mucous folds projecting vaginalward, behind which the catheter becomes engaged. The sclerosis of the base of the annular folds is one of the commonest and most striking lesions in cervicitis. When marked, as illustrated in Fig. 189, the sclerotic base pushes the canal out of its course and forces the opposite wall of the cervix to yield. The sclerosis thus causes the cervical canal to bend with varying degrees of abruptness, dorsalward or ventralward, to the right or to the left, according to the location and extent of the sclerosis. Sometimes the fourth or last annular fold at the uterine end becomes sclerotic, and, pushing in the direction of least resistance, forms a cone projecting into the uterine cavity, which, acting as a collapsing valve and imprisoning menstrual debris, causes uterine distension. Little is known of the histo-pathologic changes in the cervix. The scanty studies show that generally there is desquamation of the external epithelial strata with desquamation of the epithelial lining of the mucus, or, as they might be termed in pregnancy, the seal-secreting glands. The desquamation of the general cervical epithelium tends constantly toward adhesions between the contiguous denuded surfaces, but the regular physiologic discharge of uterine secretions, especially at the estrual period, counteracts, at least as a rule, complete cervical atresia. I have not observed, clinically or in the abattoir, a complete obliteration of the cervical canal as a direct result of cervicitis. I have observed clinically three or four cases, always in heifers in first pregnancy, of adhesions between contiguous denuded surfaces of the first annular ring, which so narrowed the cervical canal as to ren- 
der parturition impossible without surgical interference, but in no case hermetically sealed the canal. I have seen complete atresia of the cervical canal only in uterine abscess unless the arrest in development of the cervix in the embryo, in which the canal fails to become excavated, may be called atresia.

The bacteriology of cervicitis, in any reliable measure, is yet to be learned. Thus far the chief invader recognized is the streptococcus viridans. There is doubtless a wide variation in bacteria. In purulent cervicitis the streptococcus viridans is probably not the real offender. In histologic section of the inflamed mucosa, bacteria are abundant, especially within the mucous glands, where they mingle with the epithelial debris.

The prognosis of cervicitis is dependent upon its intensity, type and duration. In itself, cervicitis is generally remediable, but, if intense and neglected, the infection tends to invade the oviducts and pass beyond surgical control. Many cases recover spontaneously or are of so mild a type that the disease does not prevent conception. As a rule, cervicitis of moderate or great intensity either delays or prevents conception. The exact method by which it interferes with conception has not been fully determined. In certain extreme cases the profuse secretion of tenacious mucus apparently entangles the spermatozoa and inhibits their migration to the oviduct. In some cases the bacterial toxins present probably disable and eventually destroy the spermatozoa, but in a very large measure the copious secretion of mucus during estrum floods the cervical canal and vagina, washes away or submerges the bacteria, and serves as a protective bridge over the infected mucosa, in which the spermatozoa may move freely and safely.

The greatest danger from cervicitis apparently arises after fertilization. Then the infection tends to inhibit the prompt physiologic sealing of the canal and is free to attack the embryo immediately it arrives in the uterine cavity. Cervicitis is the fundamental lesion in abortion and retained fetal membranes. In all recorded cases of autopsy 
following abortion, in which the location of the endometritis is stated, the abortion-causing metritis has radiated from the cervical end of the uterus. This has been illustrated in Fig. 174 and in colored Plates III and IV. Clinically there is found an unsealed cervical canal with pronounced cervicitis and the embryonic sac necrotic at its cervical end.

Cervicitis is not an absolute barrier to pregnancy even when severe: It is not rare to find severe cervicitis, with engorged, angry-red and swollen mucosa, when the animal is pregnant and to all appearances safe. In three instances I have observed purulent cervicitis at the vaginal end with copious suppuration and with a pint or two of fetid pus in the vagina, though the cow was pregnant and calved successfully. But in such cases the suppuration was confined to the vaginal end of the cervix while the uterine end was successfully resisting the threatened invasion and the anterior end of the canal was securely sealed.

Cervicitis is seen in cows of all ages. Severe cervicitis with great cervical hypertrophy often abates; the cervix retains its hypertrophy, but the inflammation ceases and the cow breeds safely.

The handling of cervicitis is essentially a problem in disinfection. As in wound infection, so in cervical infection, the best results demand prompt and skillful handling. The inevitable cervicitis of retained fetal membranes and other forms of metritis accompanied by suppuration should be given careful attention during the puerperal period, and the disease not permitted to persist into the post-puerperal era. At this time the chief weapon against the cervicitis is the removal of the source of the irritation by properly handling the metritis. The same is true of many cases of cervicitis existing at other periods: if uterine disease is present, its control must precede the direct handling of the malady.

In the milder types of disease, if 2 per cent. of Lugol's solution is used to douche the uterus, the remnant of the fluid left in the cavity trickles out through the cervical canal, and in many cases suffices to disinfect it. It is inadvisable to 
trust to this. I find it best as a rule in the mild and moderately severe cases to swab the cervical canal throughout its length with full strength Lugol's solution. For this purpose I use the uterine dressing forceps. (Fig. 39, 3.) With these I secure as large a piece of absorbent cotton or gauze as can readily be pushed through the canal, saturate it with the solution, and pass it through the length of the canal, revolving the swab to and fro in an effort to touch every part of the mucosa. Where the cervical mucosa is badly denuded, the application causes necrosis of the superficial layers of epithelium and arouses a marked reaction in the tissues. When

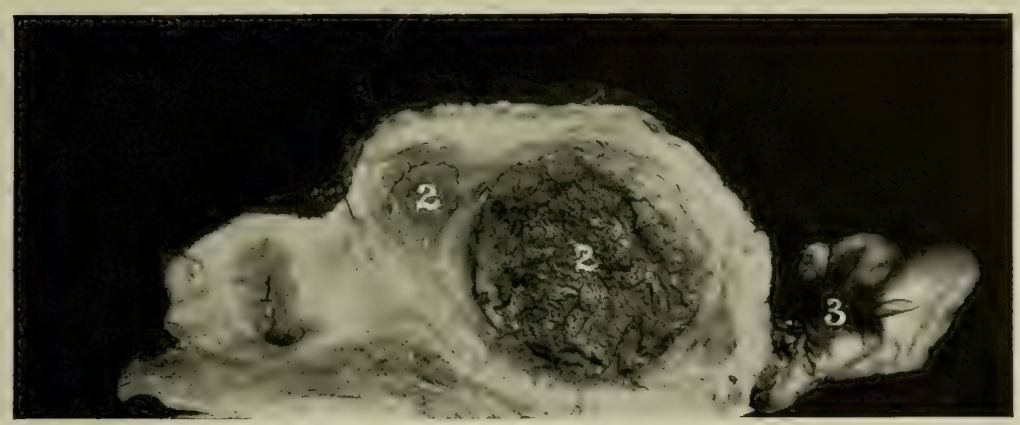

FIG. 197-Cervical Abscess.

I, - Cervix ; 2, abscesses ; 3 , hydrosalpinx.

the thin pellicle of dead cells is thrown off, a rapidly-healing surface remains. A second application may be made in three weeks if it appears desirable. If the case is not too severe, the swabbing commonly gives satisfactory results. In some instances the cervicitis will respond better to the application of a 10 to 20 per cent. silver nitrate solution.

When discussing the instrumental examination of the cervical canal on page 99, warning was given that the examiner should proceed with great caution in passing instruments through it. This becomes of special importance where the cervical canal is seriously infected. The cervicitis, often rendering the passing of a sound or catheter exceedingly difficult, invites accident and the infection present, which is 
naturally pushed into any wound, greatly intensifies the danger. In the worst cases of injury a vast phlegmon develops in the pelvic areolar tissue, imperiling the life and destroying the value of the animal. In lesser injuries, like those depicted in Figs. 197, 198 abscesses occur. These infected centers develop about them highly resistant, sclerotic abscess walls which remove or diminish the peril to life but are generally beyond practical repair so far as breeding is concerned.

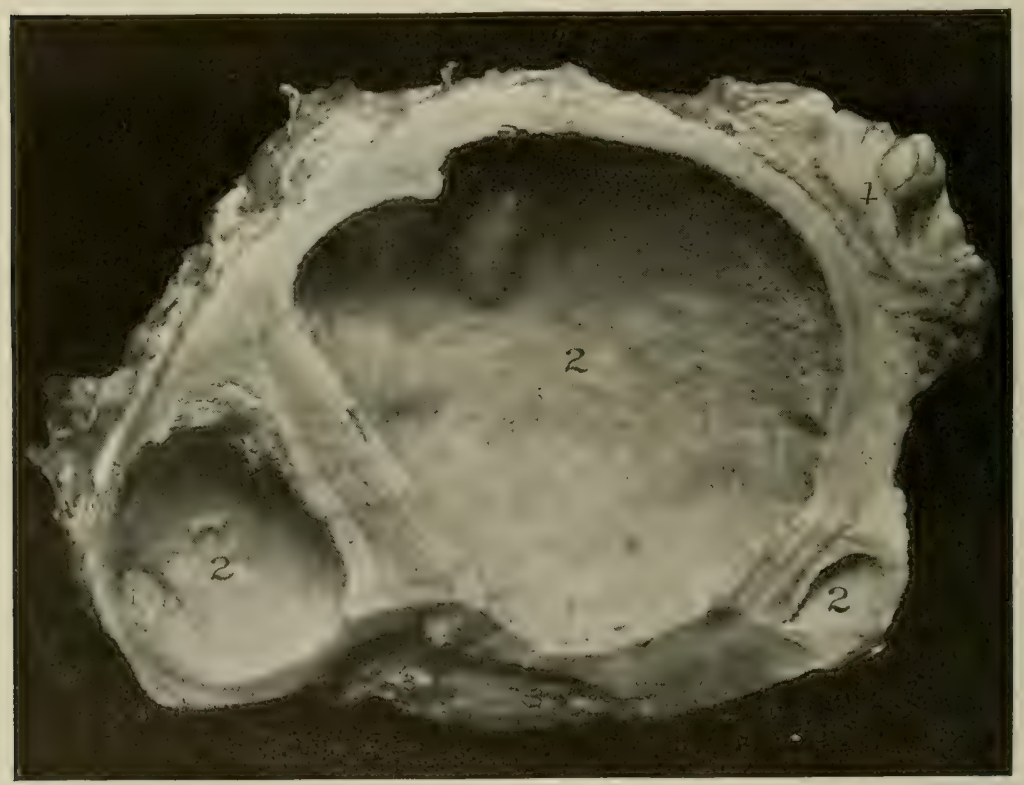

FIG. I98-Cervical Abscess from "Opening the Womb." Ventral Side. I, Cervix; 2, abscess with sclerotic walls; 3 , cross section of healthy uterine cornua in shadow. The main volume of cervix, uterus, and cornua lie behind (above) the abscess.

When severe purulent cervicitis exists in pregnant cows, it should be handled chiefly by vaginal douches, consisting of $1 / 4$ per cent. of Lugol's solution, chlorazene, or other equivalent. No radical attempt should be made to eliminate the suppuration, but an effort made gently to ameliorate it until the close of pregnancy, when the disease may be attacked with ample vigor. 
When a fissure occurs in the cervix at time of parturition, the injury commonly passes unseen until faulty healing has occurred with a troublesome cicatrix. Sometimes the seriousness of the cervicitis is caused by the imprudent use of the scalpel to overcome dystocia falsely diagnosed as being due to atresia or stricture when in fact the cervix is normal and fails to dilate because the uterus is atonic owing to the presence of infection. In such instances there is often a false impression of recovery, the cervix healing with a bad cicatrix which invites serious cervical infection.

Numerous cases of severe cervicitis without a history of fissure or incision, which will not yield to the application of disinfectants, demand for satisfactory handling surgical interference.

\section{TRACHEOPLASTY}

Tracheoplasty is indicated in refractory cervicitis and when there are structural changes which cause sterility, either directly or by reason of such lesions as cause the cervix to be more prone to disease. Tracheoplasty in itself plays little part in cervical surgery of the cow, but certain plastic procedures, intrinsically involved in all cervical surgery, play a very important role in the attaining of satisfactory results. Tracheoplasty is intended to restore the cervix as nearly as possible to its normal morphology and tissue condition. Since most of the cases of cervicitis result directly from injury or from the succeeding inflammation, the subject is largely covered under succeeding heads. Where there is an inflammatory process of the mucosa and muscular walls, it has been my experience that a simple tracheoplastic operation does not give satisfactory results and in its stead a partial trachelectomy should be performed.

A marked hypertrophy or elongation of one cervical lip may call for amputation of this portion. In this case a circular incision is made through the mucosa around the base of the elongated lip, and at either side of the base an incision is directed at such an angle into the fibromuscular tissue that they converge to excise a wedge-shaped piece of 
the latter tissue. With No. 2 or 3 chromatized catgut, the endocervical and vaginal mucosa are brought together with interrupted sutures.

\section{TRACHELORRHAPHY}

The treatment of lacerations of the cervix, although in general it has received little consideration, is a subject having no little importance. At the time of parturition, most cows receive at least minor lacerations or epithelial abrasions, and a certain percentage of these, dependent upon the degree of infection present, ultimately develop an endocervical inflammation (See Fig. 199).

Repair of epithelial lacerations should be secured within a short time after parturition, before inflammatory processes have arisen or healing occurred by cicatrix. Such repairs are almost wholly limited to the region of the first cervical fold and the parts posterior to it.

In order to remedy such injuries the cervix is retracted into the vaginal introitus, using strong vulsellum or tenaculum forceps, placed in such a manner as to prevent any endocervical injury. The cervix may further be brought into view by the use of one or more cervico-vaginal speculums, which serve to push aside the vulvar lips. The cervical lips are now spread apart, exposing the laceration; if the lips are much constricted, forming a very small os, the first fold may be retracted gently with a pair of small tenaculum forceps and everted slightly to expose the laceration sufficiently for repair. Using a No. 4 half-circle Mayo catgut needle, or similar pattern, with No. 0 or No. 1 catgut, the laceration is closed with either an interrupted or a continuous suture, but the former will secure a better coaptation in most cases. The needle is usually best passed through both margins of the wound at once, from the side which is most accessible, turned upon its long axis, and the point brought sufficiently into view that it may be grasped by the needle holders. Great care must be taken not to tear the mucosa, since the replacement of the sutures becomes extremely difficult. For this reason, the Mayo cervix needle is preferable to others, as it punches rather than cuts a hole. All 
sutures should be forceps-tied because the suturing is usually so much within the vaginal introitus or cervical canal that digital tying exerts too great a strain upon the mucosal margins.

Tears or lacerations of the cervical lips heal more rapidly than do those of the endocervix at or anterior to the first fold. The endocervical mucosa on one side and the vaginal mucosa on the other soon become fused, with the formation, however, of considerable eschar tissue. This causes a gap-

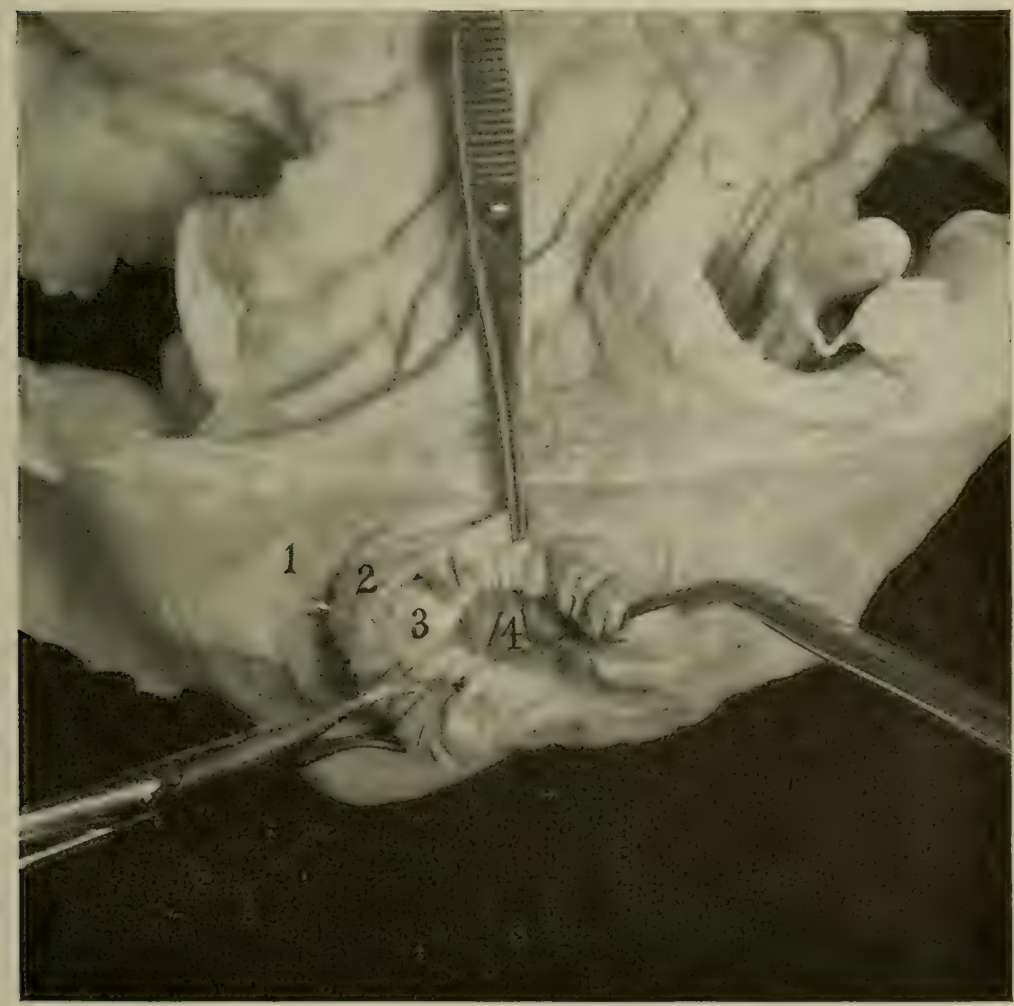

FIG. I99-Laceration of Lips of Cervix Uteri.

$I$, Vaginal mucosa; 2 , vaginal aspect of cervical lip ; 3 , endocervical aspect of lip; 4 , intensely inflamed first annular fold. On the right side are shown the margins of an old laceration, the posterior angles of which are held apart by the lower forceps. 
ing of the lips with a partial eversion of the first fold, or, if this fold becomes inflamed, a severe ectropia results. It is of considerable importance that the normal tissue relations be maintained or restored, since upon this is dependent to a great extent the future health and physiological fitness of the utero-cervical canal.

The cervix is retracted, as in the former case, well into the vaginal introitus and tenaculum forceps placed on either side of the laceration. The margins of the wound are denuded, taking care that fresh surfaces are left in each layer of mucosa and in the fibro-muscular layer between. All cicatricial tissue is removed. The surfaces denuded must be left smooth and even in order to attain a satisfactory coaptation. When making the denudation, trachelorrhaphy scissors are found very helpful.

The denuded area on each side presents an inner layer of endocervical mucosa, an outer layer of vaginal mucosa and, between these, the dense fibro-muscular tissue. A correct coaptation of mucosa to mucosa must be obtained. Beginning at the anterior commissure of the wound, interrupted sutures are placed at intervals of about one-quarter inch. These are inserted by directing the needle inward through the vaginal mucosa near its margin on one side, and, after penetrating deeply into the fibro-muscular tissue, bringing it out at the margin of the endocervical mucosa without penetrating it. This order is reversed on the other side of the laceration. The suture is tied firmly and cut off, leaving an inch or two of its end so that it may be readily picked up at a later date for removal. The sutures are thus continued, at about one-quarter inch intervals to within about one-half to three-quarters of an inch from the posterior margin of the lips, where one suture is placed at right angles to the preceding: that is, piercing the mucosa of the posterior edge of the lip about one-eighth inch from the edge of the wound, the needle is directed, anteriorly, deeply into the fibro-muscular tissue, and emerges midway between the endocervical and vaginal mucosa about one-half to three-quarters inch anterior to the os. It is passed then in the reverse order 
through the opposite margin of the laceration. This suture, tied firmly on the posterior margin of the os, prevents gaping of the-lips.

Care should be taken that none of the sutures penetrate the endocervical mucosa because, should this occur, they will not become buried and a complete healing of the endocervical múcosa by first intention can not occur. If the sutures are properly placed they may be removed after ten to fifteen days without injury to the endocervix. It is important to bear in mind also, in the attainment of correct coaptation of surfaces, that the margins of the laceration must be smooth and straight and that a puckering of the opposing margins must be prevented.

The fibro-muscular tissue of the cervix is very tough, and sometimes difficult to penetrate. When suturing this tissue, a very strong needle with a sharp cutting point is required. Ferguson's abdominal needles, Dibrell's needles, and Dr. Howard Crutcher's perineum needle have been found to be well adapted for this.

When it is desired to use the perineal needle, it should be threaded with one end of the suture quite short and passed from the inside, between the endocervical mucosa and fibromuscular tissue, outward through the latter and the vaginal mucosa. The short end of the suture is now caught and the needle, still threaded, is withdrawn and passed in the same manner through the opposite margin of the laceration, whence it is withdrawn unthreaded.

Lacerations through the fibrous structure of the cervix cause a gaping of the wound, to control which the sutures, as above described, are subject to considerable tension. A non-elastic suture such as braided silk is usually the most suitable. The size of the suture material should be such that the desired parts can be firmly and permanently brought into apposition. For the posterior margin of the lips heavier suture material than for the other portions, usually No. 4 to No. 8 braided silk, is used. Catgut used in this region is generally very unsatisfactory, as its elasticity permits the apposed margins to separate sufficiently 
that coaptation ceases. When the tear of the lip is very long, it will not be found advisable to tie the first sutures until several of them have been placed and it is ascertained that the margins will not pucker. When a simple dilation of the lips occurs, without trace of longitudinal laceration, the lumen is narrowed by the excision of a wedge-shaped segment from a convenient location in the circumference of the lip. The piece excised must be of such size and shape that, when the denuded surfaces are brought together, the tissue relation is maintained and the organ is restored to as nearly its normal appearance as possible. The segment is best removed with right and left trachelorrhaphy scissors, after which the wound is closed as previously described.

\section{TRACHELECTOMY}

The term trachelectomy is used here to denote various degrees of cervical amputation. Different conditions of the cervix call for a somewhat varied type of trachelectomy and tracheloplasty. Before attempting trachelectomy, the operator should make himself thoroughly familiar with the normal structure of the cervix. The operation should accomplish the removal of all diseased cervical tissue and attain as complete a tracheloplasty as possible. Care should always be taken to protect the healthy cervical tissue, especially the endocervical mucosa, from injury during the operation. The operator should bear in mind that the cervical canal is normally very small and that, when there is any considerable dilation in the presence of an inflammatory process, that portion of the endocervical mucosa which lines the dilated section must usually be considered as diseased. Although it may not be apparent, in many cases there have been destructive changes which render it physiologically functionless.

\section{Excision of the Prolapsed Endocervical Fold}

The excision of the first fold, when seriously inflamed, is frequently indicated. It should be performed only when the inflammatory process is confined to the first fold and its 
base is readily accessible. The cervix is retracted into the vaginal introitus and the inflamed fold is grasped with a pair of tenaculum forceps, or, if the margin of this fold is very ragged, a heavy pair of hemostats may be used, thus straightening out the unevenness of the part and allowing a straighter line of incision to be made through the mucosa parallel to the forceps. The incision is best commenced on the outer side of the fold and carried through the healthy mucosa at the margin of the inflammatory area, making an encircling incision through the mucous membrane at the base of the piece to be removed. Then, incising a little at a time, first on the outer, then on the inner side of the fold, the point of the scalpel being directed towards its base, a wedge-shaped piece of fibro-muscular cervical tissue is removed. This leaves two free edges of mucous membrane which are sutured together with No. 0 or No. 1 chromatized catgut, using an interrupted or running suture as conditions may demand. Healing should occur by first intention. The needle best adapted for this suturing is a $3 / 8$, or full-curved cervix needle $1 \frac{1}{1}$. to $13 / 4$ inches long. Great care should be used not to tear out the sutures, because their replacement is very difficult and proper apposition of the margins is not so readily attained. Often it is very helpful to place one suture before the fold is completely removed, or, when removing the folci, to catch up the free margins of the mucosa with fine spring-catch cilia fixation forceps, thus lieeping the fine margins of mucosa readily available for suturing.

\section{Circular Amputation of the Cervix}

When disease of the cervix is of such character that one of the previous methods can not satisfactorily remove the inflamed tissue, it becomes necessary to establish by other means a continuity of the healthy endocervical mucosa. This may best be accomplished by a partial circular amputation of the cervix, including in the excision a varied portion of the cervical fibro-muscular tissue along with the diseased mucosa. This operation is extremely well adapted to the correction of the large ectropic cervix (the so-called 
cauliflower cervix) and of endocervicitis too extensive to be handled by the mere excision of a prolapsed fold.

The cervix is retracted into the vaginal introitus and seized with a strong pair of vulsellum forceps with one jaw passed deeply into the cervical canal, or, more satisfactorily, with a specially constructed forceps (Fig. 200 a, 2), one jaw having double tenaculae and the other tongue-shaped so that it may be passed deeply into the canal without injury. The cervix is freely exposed by the use of retractors and extra vulsellum and tenaculum forceps which hold aside the vulvar

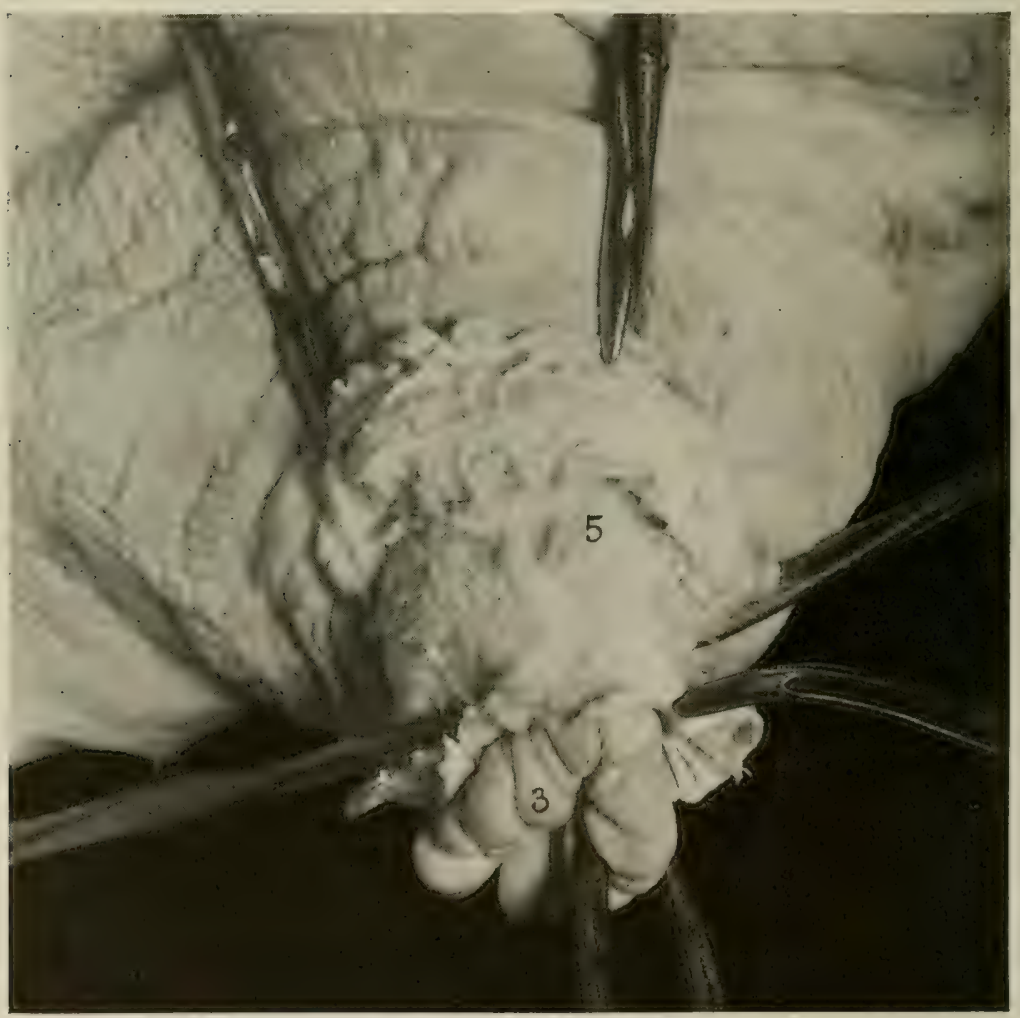

Fig. 200-Circular Amputation of Cervix Uteri.

The vaginal mucosa has been dissected from and drawn forward over cervical core. 3, Ectropic first annular fold ; 5, exposed fibromuscular tissue of cervical core. 
lips. The tissues are injected at frequent intervals with a local anaesthetic to which adrenalin chloride has been added. A circular incision is now made around the margin of the true cervical lips, as shown in Fig. 200. The vaginal mucosa is seized at frequent intervals around its free margin with artery forceps and, applying moderate traction, is carefully dissected from the underlying cervical body as far forward as the endocervical mucosa is diseased. A longitu-

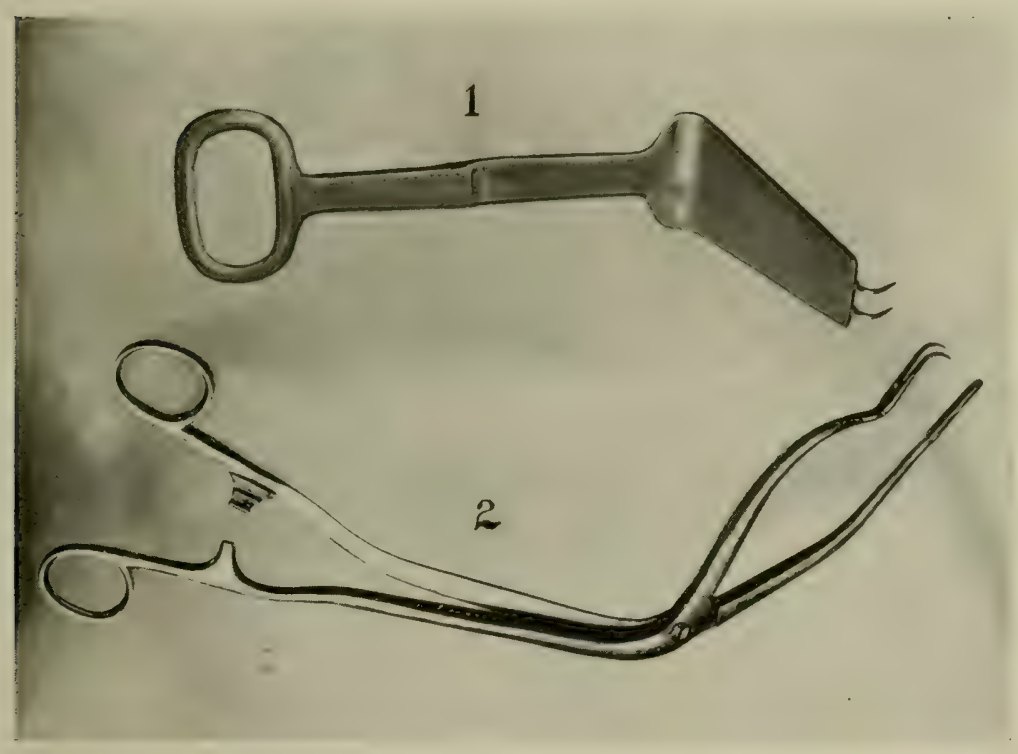

FIG. 200a-Special Instruments for Trachelectomy.

I, Cervical retractor; 2, Tenaculum forceps.

dinal incision is now made through the core on either side, dividing it, for the entire length of that portion which has been freed from the vaginal mucosa, into about equal upper and lower halves (See Fig. 201). At this point in the operation, it should be ascertained that the cervical stump is firmly grasped by the tenaculum forceps, because at a later stage their replacement would become very difficult, should their hold be lost. Each of the two segments of the cervical core has an inner lining membrane of endocervical mucosa, 


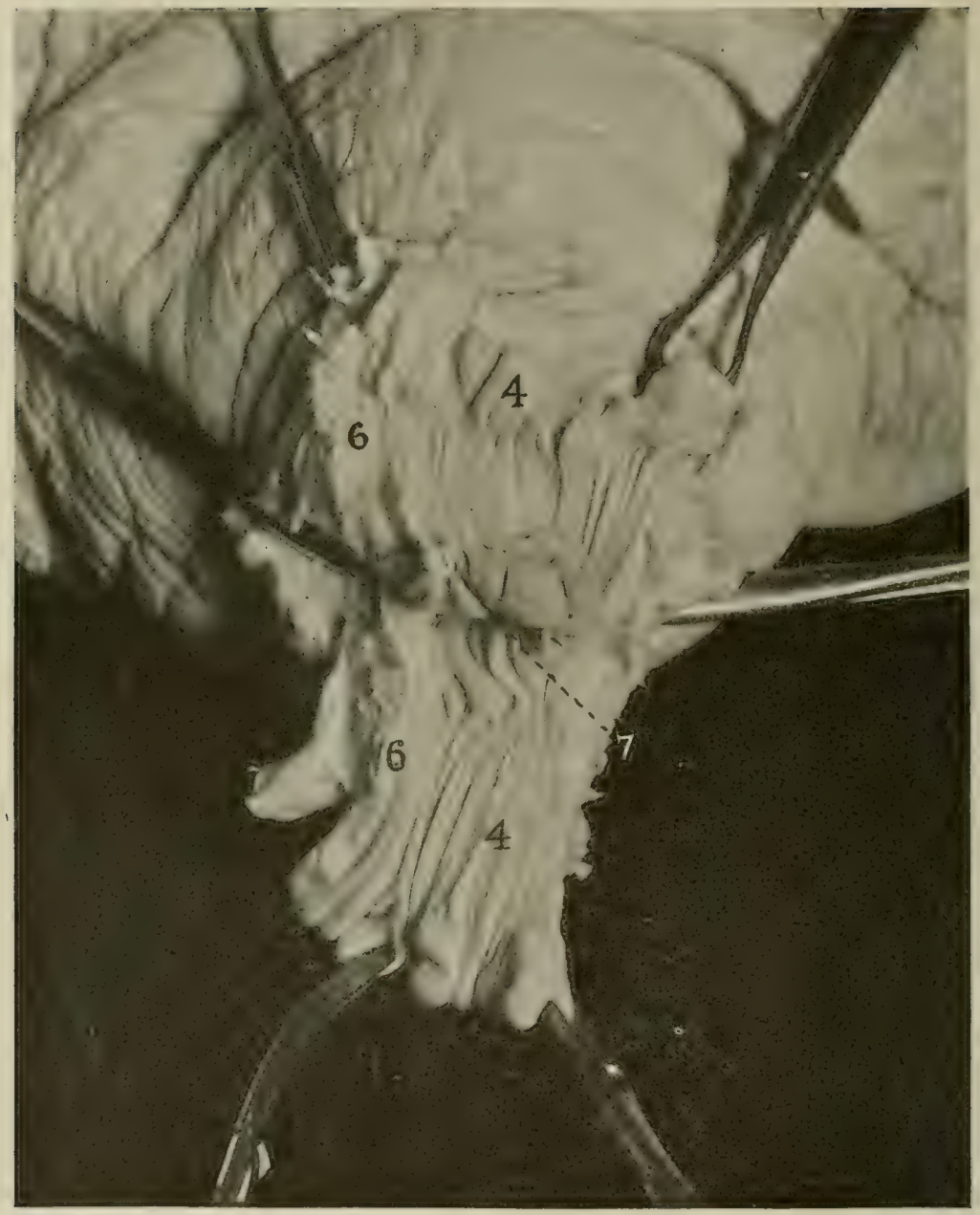

Fig. 20I-Circular Amputation of Cervix Uteri.

Cervical core divided by longitudinal incisions ( 6 ) into superior and inferior segments, (4). The internal os, 7 , is shown, bounded posteriorly by the now exposed second annular fold (amputation ends at this point) leaving a free margin of endocervical mucosa, as shown in Fig. 202, 8 . 
outside of which is the fibro-muscular tissue without its covering of vaginal mucosa. Grasping the superior segment and pulling sharply upward in order to expose the endocervix to the greatest possible extent, a semi-elliptical incision is made through the endocervical mucosa, connecting the anterior ends of the longitudinal incisions. The convexity of the incision is directed posteriorly. The scalpel is now directed, parallel to the incised margin of the mucosa, towards the uterus for a short distance in order to free the margin for the placing of sutures. The directions of the amputating incisions thus form a more or less V-shaped declivity in the posterior extremity of the stump, the mucosal margins of which may be brought together readily with sutures. The free edge of the endccervical mucosa is now secured by toothed fixation, or by fine artery forceps. Using chromatized catgut sutures No. 1 to 3 , and a $3 / 8$ or full-curved needle with a piercing point, the free margin of the vaginal mucosa above is brought into apposition with the endocervical mucosa below. Usually four or five sutures are necessary in apposing these margins. (See Fig. 202.)

When any considerable portion of the cervix has been removed, it leaves the vaginal mucosa, which has been separated from it, free from its underlying support. In order that part of this vacant space may be eliminated, and in order to prevent too great a strain upon the endocervical margin, a small portion of the fibro-muscular cervical tissue should be included in each suture (Fig. 203, 204).

The lower segment of the cervical core is now excised and sutured in the same manner. The endocervix has thus been brought into coaptation with the vaginal mucosa both above and below. At the lateral commissures of the os, the vaginal mucosa sags away from the endocervical mucosa. Lateral to this, on each side, an excess of vaginal mucosa results from the marked diminution in the size of the external osoften a reduction in diameter from as much as 5 or 6 inches to about $1 / 2$ inch. Coaptation of the vaginal mucosa to the lateral margins of the endocervical mucosa is now secured by passing on each side a suture through the vaginal mu- 


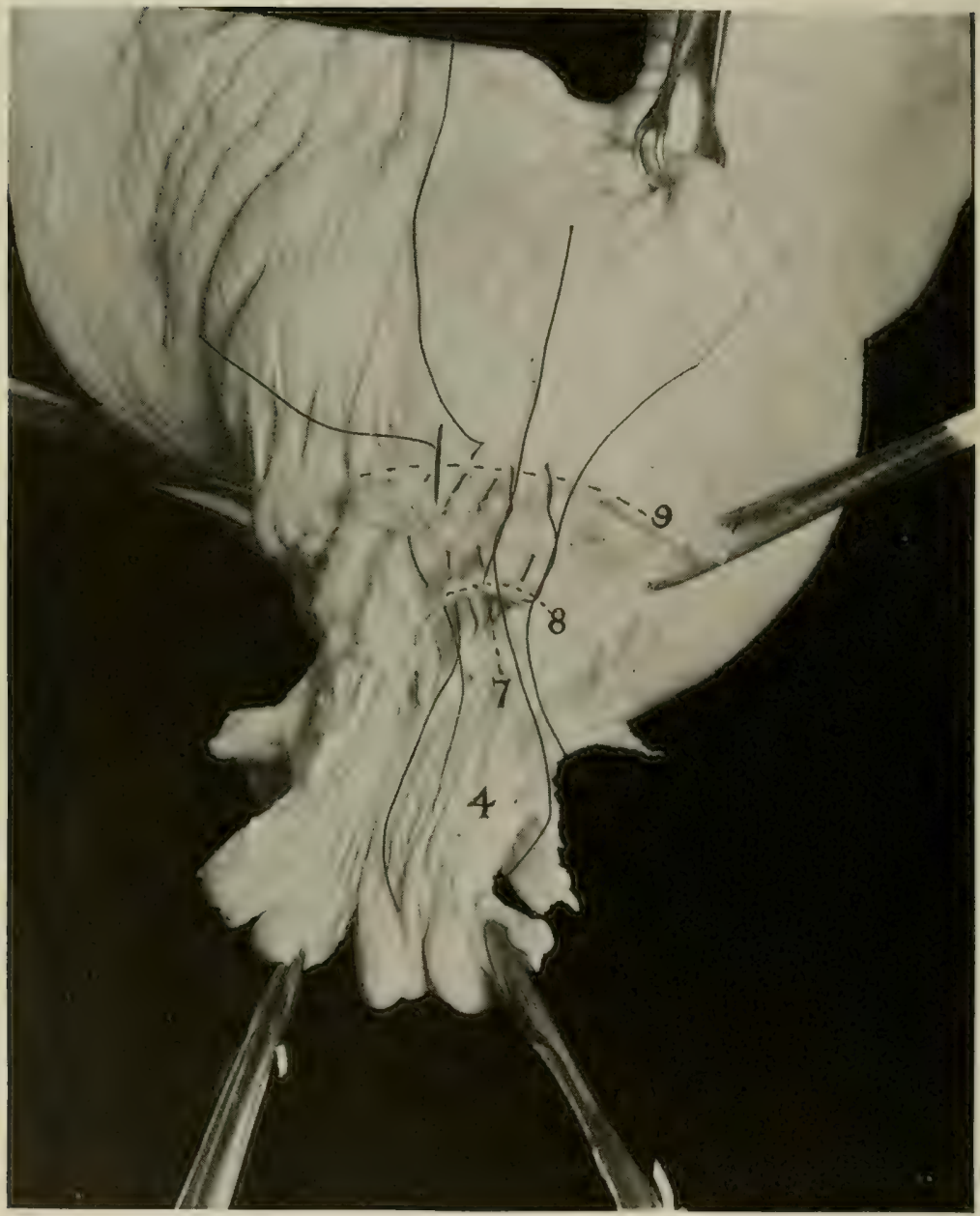

FIG. 202-Circular Amputation of Cervix Uteri.

Showing cervix after superior half of cervical core has been excised. 9 , Margin of vaginal mucosa; $t$, inferior half of divided core; $\delta$, semielliptical margin of endocervical mucosa; 7 , utero-cervical canal. Between $\delta$ and $g$ the structure consists of the loose paracervical tissue and the tough cervical fibro-muscular tissue. 
cosa from above about $1 / 8$ to $1 / 1$ inch lateralwards from the previous suture, through the lateral margin of the endocervix and out through the vaginal mucosa below at about $1 / 8$ to $1 / 4$. inch laterward from the lateral inferior suture. Now secure the lateral commissure of the vaginal wound with tenaculum forceps and, by gentle traction, making the ten-

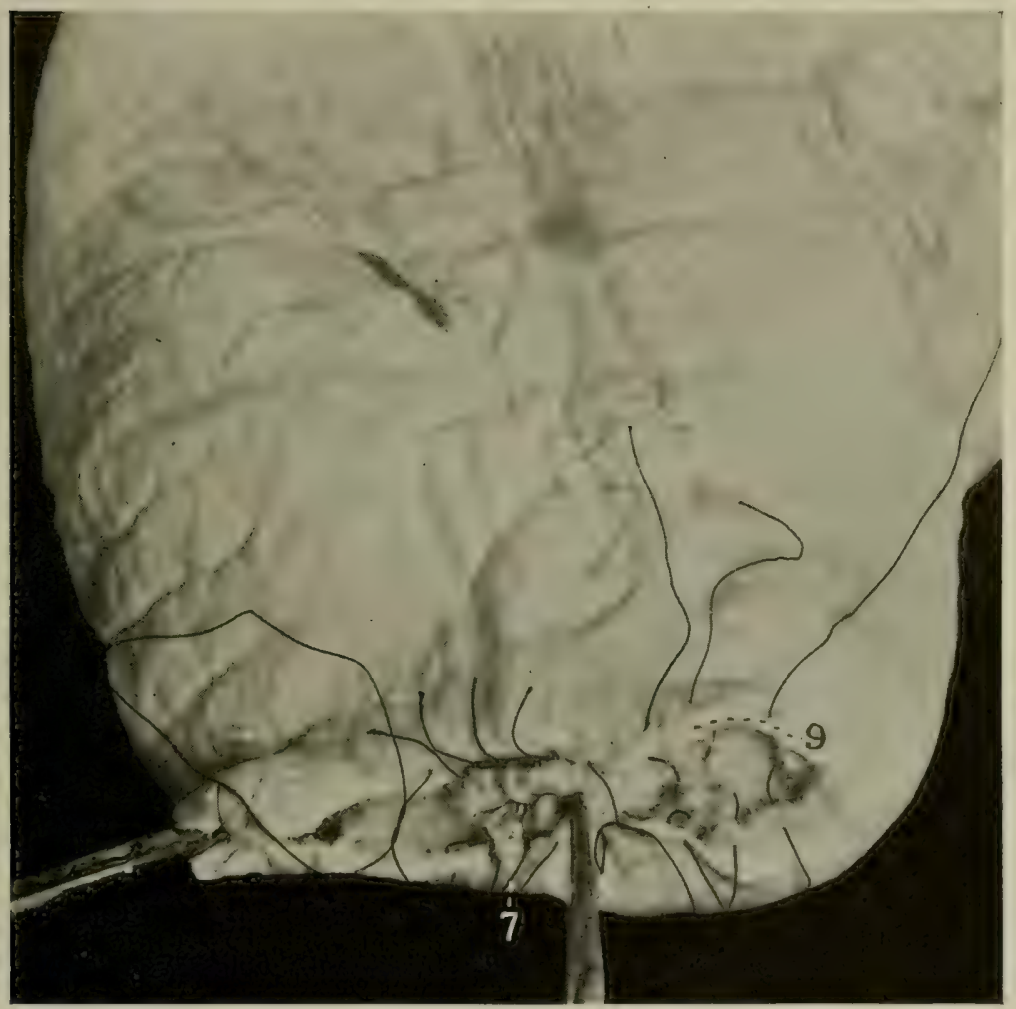

FIG. 203-Circular Amputation of Cervix Uteri.

Showing cervix after excision of both superior and inferior halves of cervical body. Vaginal mucosal margins brought into apposition above and below with the endocervix. Angular sutures at either side bring into apposition the vaginal mucosa and the lateral margins of the endocervical mucosa. On the left side the puckered vaginal mucosa is shown free from its underlying support; on the right it has been removed, leaving an oval opening ( 9 ) in the mucous membrane. Vaginal sutures are shown on the right side, each of which includes a small portion of paracervical tissue along with vaginal mucosa. 7 , utero cervical canal. 


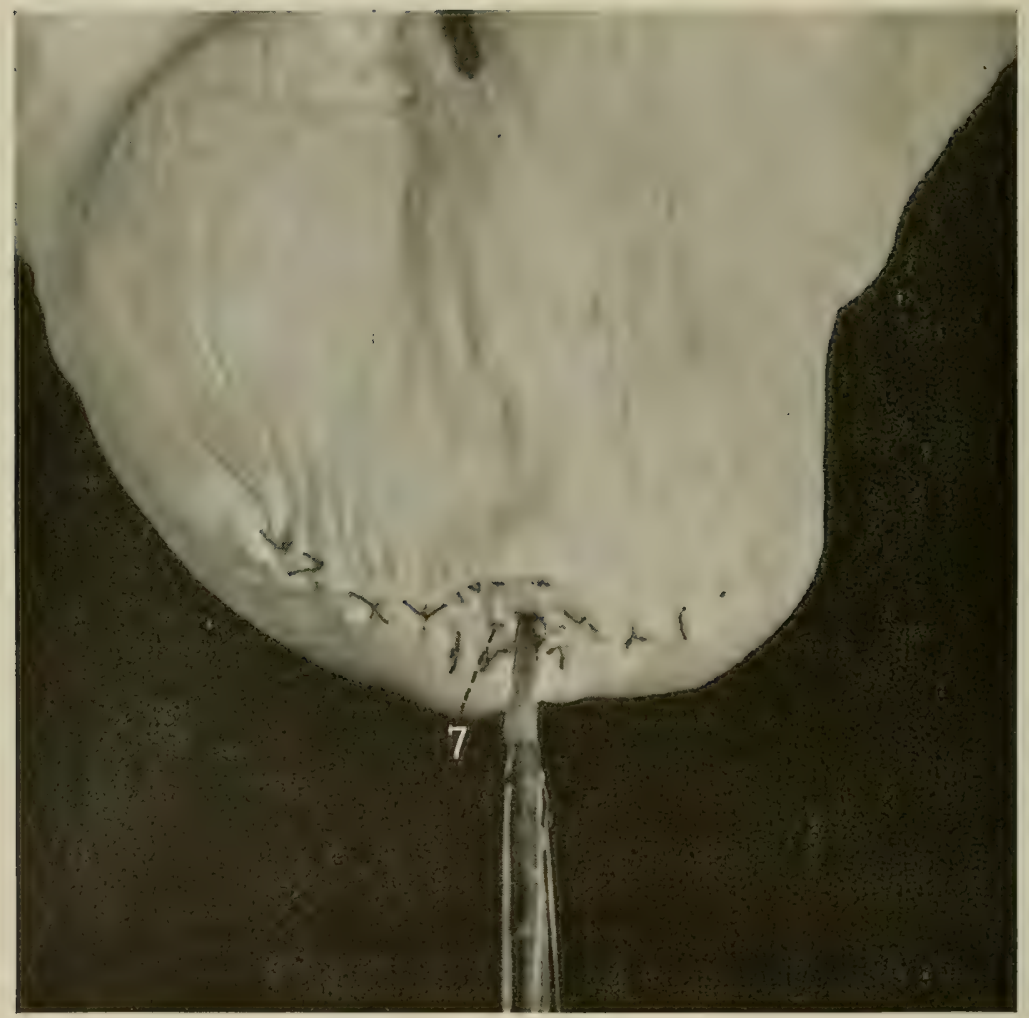

FIG. 204-Circular Amputation of Cervix Uteri. Operation Completed. 
sion about equal on the upper and lower margins, the extent of superfluous vaginal mucosa is ascertained. An approximately oval-shaped portion of the extra vaginal mucosa is removed with scissors from each side and the margins apposed with interrupted silk sutures, each of which should inFor purely vaginal sutures silk or linen is best, but if the suture penetrates the endocervix catgut should generally be used. If proper coaptation of the parts is secured, healing should occur by first intention.

clude a small portion of the underlying paracervical tissue.

After the disinfection of the operative area with iodine, the single pair of forceps which now retract the cervix are released, allowing the organ to resume its normal position. It is left undisturbed for a period of about two weeks, when the vaginal sutures are removed and the surfaces touched over with pure Lugol's solution of iodine. At this time, providing that the technic has been thorough and efficient, the appearance of the mucosa of the cervix should be normal, with but slight trace of the operative procedure.

During the operation it will be found advantageous to maintain a constant supply of $1 / 2$ to 1 per cent. chlorazene solution for the irrigation of the operative area. Capillary hemorrhage should be almost completely controlled by the use of adrenalin chloride.

Circular amputation, although requiring considerably more skill and effort on the part of the operator than other methods of procedure used in the correction of refractory cervicitis, gives the most satisfactory results of any technic which the author has used. The patient is usually fit for service within two to four weeks after operation, and it has been my experience that, in the absence of coexisting complications in other portions of the genital apparatus, conception usually follows very promptly. In the presence of other lesions of a severe nature in the genital tract, such as would in themselves cause sterility of a more or less permanent character, a cervical operation is not usually advisable. 


\section{B. Retention of Menstrual Debris from Cervicitis ${ }^{1}$}

Uterine distension from cervical disease, due to the uterine end of the cervical mucosa projecting conically into the uterine cavity and acting as a collapsing valve, imprisoning the uterine secretions, to which reference has been made above, is extremely rare. I have observed but two cases, both clinical. The uterus was firmly distended with fluid as in pregnancy, but the two horns were symmetrical. A corpus luteum was usually present but shifted from one ovary to the other (alternation of ovulation at estrum). The uterine arteries were not enlarged. The cervix was not sealed. The introduction of the uterine catheter, which required some patience, caused the evacuation of a thin, clear, odorless mucus.

I have not succeeded in restoring such animals to fertility. Generally it is best to slaughter. In one instance I catheterized the uterus several times and evacuated the fluid. In another case, in swabbing the cervix, I pushed the swab into the uterine cavity, beyond the conical projection of the mucosa. When I attempted to withdraw the swab, the valve-lile projection closed upon it and pulled the swab from the forceps. The recovery and withdrawal of the swab gave considerable difficuity. I doubt the efficacy of handling. With a very valuable animal probably the most hopeful plan would be a complete trachelectomy, as described in the preceding paragraph.

\footnotetext{
${ }^{1}$ There is a legendary atresia of the cervical canal in cattle, which has been maintained generation after generation. It has its basis in the common ignorance of the anatomy of the bovine cervix. A cow is sterile, a man attempts to pass his finger through the cervical canal and naturally fails, and a diagnosis of cervical atresia or "closure of the womb" is made. The principle is the same as when, a generation ago, if a cow was ill, a hole was bored in her horn, a cavity was found and "hollow horn" diagnosed. Atresia of the cervix still appears in some textbooks as a disease. Rarely does cervicitis occlude the cervical canal. More frequently embryologic arrest in development occurs and the cervical canal is not formed. In either case the menstrual debris is imprisoned and the uterus becomes distended. When the veterinarian attempts to pass the dilator, catheter or other instrument through the cervical canal and fails, if atresia exists, distension of the uterus inevitably co-exists. If the distension of the uterus is not present, a diagnosis of cervical atresia is unjustifiable and false. Atresia of the cervical canal without uterine distension can not exist.
} 
C. Fetal Retention from Cervicitis. Cervical Adhesions.

Thrice I have observed fetal retention due to adhesive inflammation in the cervices of heifers. In one, the fetus had not been long dead and the cervical canal admitted the hand. Traction upon the fetus ruptured the cervix. The heifer recovered her general health and was sold for beef. In the second case, the fetus macerated and sloughed into the rumen, and the animal recovered sufficiently to be sold for beef. The third was neglected until the eight-months fetus had been dead seven or eight months. The cervix was dilated by cutting the first annular ring and most of the fetal cadaver removed. Two fetal bones were later removed by laparotomy and hysterotomy. The animal recovered her general health and condition.

So far as I have seen, the partial atresia of the canal has been in the first or in the first and second rings. All cases have been in heifers in first pregnancy and, so far as known, death of the fetus has occurred prior to any effort at expulsion. Apparently the infection within the cervix has extended into the uterus, involved the fetus and caused its death. In two of the three cases no effort to expel the fetus was noted by the owner.

The cases offer a very bad prognosis from the standpoint of reproduction, iout the outlook for the restoration of the general health is fair. As a rule it is desirable or necessary to dilate the cervical canal by incising the first, or first and second annular rings. Then pituitary extract may be given ( 5 to 10 mils of the Parke, Davis \& Co. preparation or its equivalent of other makes) in an effort to cause efficient uterine contraction to expel the fetus. Additional aid should be given according to indications.

Once the fetus has been extracted, the cervix should be given close attention and healing facilitated. The cervix is too grossly infected for tracheoplasty at the time. If the patient is of but ordinary value, the cervix and uterus had best be kept clean and disinfected until the general condition has been restored, and the animal then sold for slaughter. If highly valuable, partial trachelectomy should be performed. 


\section{Retention Cysts of the Cervix.}

Cysts occur somewhat rarely in the vaginal end of the cervix about the os uteri externum. I have observed them at the sides of the opening of the cervical canal. They vary in size from one-half to one inch in diameter. They ordinarily contain mucus, which sometimes becomes infected and purulent. They are apparently retention cysts. It has been my habit to incise these, when encountered, and cauterize their interior with full strength Lugol's solution or with silver nitrate.

\section{E. Cervical Prolapse.}

Prolapse of the cervix consists of a displacement of the cervix toward or through the vulva. It inevitably involves a simultaneous displacement of the base of the uterus and of the cervical end of the vagina toward the vulva. It is possible in the non-pregnant heifer. It is observed during pregnancy and at varying periods after its termination.

The symptoms of cervical prolapse are too evident to call for detailed description. As a rule the prolapse becomes evident only while the cow is recumbent. It then appears as a conical tumor, with its base directed forward and its obtuse apex toward or beyond the vulva. In or near the center of the obtuse apex is a depression, the os uteri externum. The vulvar end of the cervix becomes increasingly swollen and irritated according to the degree and duration of protrusion. When protruding beyond the vulva sufficiently to come into contact with the bedding and with feces or other contaminating substances, the swelling and irritation increase. The irritation may reach such a degree as to cause an excessive secretion of mucus, or suppuration and necrosis of the mucosa may occur.

In the pregnant animal the uterine seal is usually present but imperfect and showing varying degrees of disease. The two chief causes of prolapse of the cervix are disease of the uterine end of the cervical and of the uterine mucosa and a general atony of the genital system. In the first group of cases, so far as I have yet been able to observe, the primary 
cause is an endometro-cervicitis. Both the endometrium and the endocervical mucosa become inflamed and irritated in such a manner that abdominal pressure is brought to bear upon the pelvic contents. When the animal lies down there is added a passive intra-abdominal compression which pushes uterus, cervix and vagina backward. The muscular walls of the cervix retain their tone or are rigid from disease; the uterine walls are atonic. In the pregnant animal the fetus and its membranes are not expelled because the cervix does not relax and the uterine walls do not contract, to compel the dilation of the canal. Consequently the cervix is displaced backward. In other instances, in pregnant animals, the expulsion of the fetus is inhibited by induration of the cervical walls with adhesions which prevent that degree of dilation essential to the expulsion of the uterine contents. During the postparturient period, without nymphomania, cervical prolapse is ordinarily dependent upon a quite severe endometritis associated with a lingering placental necrosis. In the second group of cases, the displacement is fundamentally due to uterine atony dependent upon that type of ovarian disease regularly causing nymphomania. In these cases the cervix is relaxed and its canal will permit of the ready passage of one, two or more fingers. The progress of cervical prolapse is very slow. In the pregnant animal it tends to become worse as time for parturition approaches. Each time that the cervix pushes out beyond the vulva while the patient is recumbent, the protruding mass becomes soiled with litter, increasing the irritation and the volume of the tumor, which in turn cause greater straining and protrusion. Eventually, if the animal calves, the unloading of the uterus usually causes the protrusion to disappear. When endometritis causes cervical prolapse in a non-pregnant cow, the prolapse tends constantly to increase. The prolapse of the cervix, with the displacement of the uterus, imprisons the irritant secretions within the uterus. The cervical prolapse associated with nymphomania usually disappears when the cystic condition of the ovaries is brought under control. 
The prognosis of cervical prolapse is generally unfavorable, because the underlying causes are not often easy to remove. In the pregnant animal the prolapse, with the associated cervicitis, not infrequently renders parturition, abortion or extraction of the fetus difficult and imperils the life of the patient through sepsis or pyemia. The cervical prolapse in nymphomania indicates a serious type of ovarian disease, which is aggravated by the prolapse.

The handling varies according to the state of the patient and the underlying cause. In the pregnant animal it should be determined first whether the fetus is alive. This is best accomplished by rectal palpation. The fetus is usually palpable and some part of it can be grasped. When pressure or traction is exerted upon a fetal part, if alive, the fetus almost invariably attempts to pull the extremity away. If the fetus is alive, the handling should be purely palliative and an earnest effort made to get the diseased parts in the best possible condition for the eventual expulsion or extraction of the fetus. For this purpose the vulvar sutures described under Vaginal Hernia in Chapter V, and illustrated in Fig. 60, can be made to serve the highly useful purpose of retaining the cervix within the vagina and protecting it against the irritating effects of desiccation when exposed to the air, and from becoming contaminated through contact with bedding and filth. It is best to use heavy silver wire and secure the apposition of the vulvar lips by merely hooking the free ends of one side over the wire of the other. Then, if parturition begins in the absence of the attendant, the force unbends the hook and releases the suture. At the same time any existing inflammation may be ameliorated by douching the vagina, and that portion of the cervix projecting into it, with warm 0.25 per cent. Lugol's solution or saturated boric acid solution, or by applying to the parts an ointment of bismuth subnitrate, iodoform and petrolatum. While dressing the parts the sutures should be released in order that the fluids may be freely expelled.

When time for parturition arrives, if the cervix is too diseased to permit efficient bloodless dilation, the constricted 
portion of the cervix may be incised or abdominal hysterotomy performed. If the cow is highly valuable for breeding purposes, hysterotomy is perhaps preferable as a rule. The operation has been described in the companion volume, Veterinary Obstetrics. After recovery from the operation, the cervicitis underlying the difficulty is to receive proper attention. Usually a partial or complete amputation of the cervix is indicated. If the animal has no unusual breeding or dairying value, the dilation of the cervix by incision may be simpler and the animal may make a prompter recovery, so far as her general health is concerned, but the cicatrix in the cervix probably leaves her unfit for breeding. However, the mutilated cervix may be successfully amputated later if found desirable. In the non-pregnant cow, when the prolapse is due to intra-uterine infection, the cervical canal is to be sufficiently dilated to admit readily the uterine catheter. The uterus is then to be douched with 0.25 to 2 per cent. Lugol's solution or other disinfectant, and bismuth and iodoform suspended in oil introduced. The handling should be repeated at intervals of one to three weeks until recovery is assured. When the prolapse is referable to nymphomaniac cysts in the ovaries, the fundamental plan of handling already advised for nymphomania is to be applied, with special attention to the uterus and cervix.

\section{INFECTIONS OF THE VAGINA}

\section{A. Vaginitis}

Vaginitis is induced in many ways. The nodular venereal disease, most clearly and readily recognized in the vulva, commonly extends into the vagina and is revealed by lesions identical with those in the vulva. This has already been described. Reference has also been made to vaginitis caused by the vesicular venereal disease, coital injuries, and sadism.

Vaginitis commonly follows parturition and abortion. Primarily parturition and abortion cause more or less severe contusions and abrasions. The great pressure under which the fetus is forced through the vagina destroys much of the protective epithelium, and so injures the underlying tissues that hemorrhages are common. The lesions are so 
nearly universal that they may, in a sense, be designated as normal. In themselves such injuries are unimportant. They acquire interest through the fact that they afford inviting avenues for infection. The abrasions and contusions of the vagina are often aggravated in cases of dystocia by the imprudent and careless use of harsh, dirty cords or other appliances.

If the fetal membranes are retained and decompose, the virulent infection present readily invades the injured tissues. When infection exists within the uterus and passes out through the vagina, it endangers, in passing, the damaged tissues. In metritis quite commonly the highly infective secretions of the uterus are promptly expelled into the vagina and lie there for hours before the expulsion from the genital canal is completed, constantly irritating the mucosa. One of the commonest and most serious types of vaginitis is that caused by imprudent or clumsy attempts at douching. Some laymen think that they may control "contagious abortion" by vaginal douching. Other laymen think that, when douching the vagina, they are douching the uterus. Many publications by technically scientific persons advise the use of powerful disinfectants in the vagina. Frequently the teaspoon is advised as a standard of measurement, although teaspoons vary greatly in size and both solids and liquids may be heaped up in varying degrees, or some of the disinfectant may run over into the water. Sometimes a slowdissolving salt, such as permanganate of potash, is advised and douching is attempted before the crystals are dissolved. The heavy crystals may drop down upon the vaginal floor and cause necrosis. The vaginal mucosa is extremely vulnerable to disinfectants. Solutions borne with impunity by the uterus and vulva are intolerable for the vagina. This must always be borne in mind in douching. Whenever the douche is so irritant that it causes distress, it is injuring the organ and should not be used. Vaginitis is common as an extension by continuity of cervicitis, especially in purulent cervicitis involving the vaginal end. Vaginitis usually follows coitus in heifers badly affected with the nodular venereal disease. The exacerbation of the infection follows coi- 
tus in twenty-four to forty-eight hours. However vaginitis may arise, the general principles of handling demand the elimination of the cause, followed by the application of those measures which may allay the irritation. If the vaginitis is a consequence of severe metritis or cervicitis, these must first be brought under control before any progress can be made in overcoming the vaginal lesions. When vaginitis is dependent upon chemical irritants, such as too powerful disinfectants, they are to be promptly withdrawn and soothing applications substituted. Of these, douches of warm 0.7 per cent. salt solution ( $1 \mathrm{oz}$. salt to 1 gal. boiled water) have probably the greatest value. In some cases 0.25 per cent. of Lugol's solution, or 2 to 4 per cent. boric acid may be advantageously added. When irritant disinfectants have been introduced, causing denudation of the epithelium, a heavy ointment or an oil, like liquid paraffin with iodoform, subnitrate of bismuth, or both may be applied with benefit. Here every effort should be made to counteract the chief peril-vaginal atresia. This object will be furthered by dilating the vagina daily with the warm saline solution, followed by the oil or ointment with iodoform, etc.

The intense vaginitis often following coitus in heifers is best prevented by douching the vagina six to twelve hours after coitus with 0.25 per cent. Lugol's solution, repeating in twenty-four hours if necessary.

\section{B. Vaginal Gangrene.}

There appear from time to time records of vaginal gangrene in cows during the puerperal period. In some instances the records almost suggest a special or specific type of infection, but there is nothing definite upon which to base a conclusion. In the presence of an intense infection of the cervix or uterus, associated perhaps with retained fetal membranes and extensive parturient abrasions in the vaginal walls, gangrene of the vagina is always a possibility. The tendency to gangrene is increased by rough work in relieving dystocia and by the use of highly irritant vaginal douches. Although it may sound paradoxical, one of the easiest ways to cause vaginal gangrene is by the misuse of powerful antiseptics. 
The symptoms consist chiefly of vulvar swellings, profuse genital discharge, straining and general evidences of pain. The discharge is often fetid. Manual examination causes intense pain. The vaginal walls are greatly swollen, rendering the introduction of the hand difficult. Portions of the mucosa are partly detached or are very fragile. By spreading apart the vulvar lips, dark, necrotic areas may be observed.

Nothing of special value has been learned regarding the bacteriology of the disease. As in other types of genital infections, it appears that in certain herds at a given time some one form of bacterium acquires special intensity and induces a prevalent type of lesions.

The handling of gangrenous vaginitis calls first of all for the control of uterine and cervical infections. I have observed very energetic efforts to control gangrenous vaginitis, while imprisoned, decomposing fetal membranes which were the basic cause were overlooked. The vagina itself should be cleansed by the gentlest available measures, essentially those mentioned above for vaginitis.

\section{Perivaginal Phlegmon.}

Parturient contusions and abrasions open the way for the invasion of infection from the genital tube into the pelvic connective tissues. Once the infection passes the barrier of the vaginal walls and gains the very loose, open areolar tissue of the pelvis, it tends to spread with great rapidity. The progress of the infection is often so rapid that the tissues are unable to erect any efficient barrier and suppuration or gangrene advances rapidly. Sometimes imperfect abscess walls are formed which offer no important degree of resistance.

The contusions and abrasions are much the same as those producing vaginitis, but act more upon the deeper tissues. In some cases the prominent, conical, bony projection at the anterior end of the pubic symphysis plays an essential part in causing the original contusion. The vaginal floor becomes impinged between the summit of the cone and some hard portion of the fetus; in some instances the vagina becomes 
actually perforated. The infection then readily passes out into the adjacent tissues. The invasion of the pelvic connective tissues causes swelling of the vulva and anus, with evidences of pain. Usually the patient moves carefully and hesitatingly, the appetite is poor, and the rectal temperature may be elevated. Vaginal or rectal palpation discloses more or less extensive swellings in the pelvis surrounding the vagina. At some points collections of fluid (thin pus) are recognizable, but there are no clearly defined limiting walls.

The course of such infection is usually rapid. As a rule the patient succumbs, largely because the tissues fail to form a barrier sufficient to prevent the highly virulent masses of pus from invading the peritoneal cavity. If the infection is not too virulent, abscessation may occur and the abscess may open, or be surgically opened, into the vagina or rectum. It then has the common significance of pyemia as described under infections of the uterus. The invasion may sometimes be anticipated if the parturient abrasions are recognized early. The genital tract may then be cleansed and iodoform with oil introduced into the uterine cavity. This, flowing slowly over the abraded parts, may deter putrefaction and bacterial invasion. Once established, the phlegmon is difficult to control. Repeated warm vaginal douches of saline solution may aid in checking the infection. Internal remedies, such as quinine, may be tried, but they offer little hope.

\section{Cysts and Abscesses of Gärtner's Ducts.}

Gärtner's ducts, described on page 39, are frequently the seat of retention cysts and abscesses. These occur along the course of the ducts, as shown in Figs. 18-21. They vary in form and extent, but are usually elongated, with a transverse diameter of one-half inch or less. They may be very large and spherical. They are of comparatively little importance except as reminders of the presence of infections which, invading the duct, cause atresia of its opening, with cystic or purulent distension. Rarely they may attain such size as to interfere with coitus. When recognized, the cysts 
or abscesses should be laid open freely and the parts disinfected.

\section{The InfECtions of the Vulva}

The vulva is the most prominent area involved in the vesicular, and in the nodular venereal diseases, as has been noted already in Chapter XII. The vulva also participates in a general way in parturient abrasions of the vagina and the consequent infections, which run a similar course and call for similar handling. The vulva now and then undergoes gangrene, especially following neglected dystocia, when the organ has been subjected to long-continued pressure and contusion from the fetus. Then follows at times cicatricial contractions rendering coitus and parturition difficult or impossible.

In some portions of the Mississippi Valley at one time an infectious ano-vulvitis of cattle prevailed, not associated with reproduction. It consisted of a necrotic inflammation of the anus of the male and of the anus and vulva of the female. The mortality was high and in non-fatal cases important deformity of the body openings resulted.

The exact cause of the disease was not clearly determined. It was referred generally to an invasion by the bacillus necrophorus, which apparently serves as a general designation for necrotic diseases occurring in various parts of the body in different species of animals, but not, so far as known, transmissible from one species to another.

The ano-vulvitis of cattle, which occurs as an enzoötic, is of chief interest in connection with its general results rather than in connection with reproduction. The disease ordinarily arises without a known injury to the parts, as a necrotic area about the anus or vulva. The necrosis is progressive, the parts become swollen and indurated, and the surface undergoes extensive, canker-like necrosis, with the formation of fetid pus.

The handling has not been placed upon a definite basis as a specific disease, but reliance has been placed upon the general principles of surgery-the destruction or ablation of incurable tissues, the disinfection of the involved areas, and the isolation of infected animals. 


\section{THE CONGENITAL INFECTIONS OF CALVES}

\section{Calf Septicemia, Calf Dysentery, Dysentery Neonatorum, Calf Scours, White Scours, Pyemic Arthritis, Joint Ill, Calf Pneumonia}

While considering the infections of the ovum, embryo and fetus, it was pointed out that both the pregnant and the nonpregnant uterus commonly contain bacteria of various kinds, and that in the pregnant cow these invade the fetal membranes, enter the amniotic fluid, are swallowed by the embryo, and constitute a part of the meconium. Generally the bacteria are included in the meconium, though they cause no recognizable disease of the fetus, but in numerous cases, associated especially with the phenomenon of abortion, they cause diarrhea and sepsis.

Birth can not serve as a line of demarcation between the infections of ante- and post-natal life. Non-fatal infections of the fetus inevitably involve the new-born calf. More generally the bacteria included within the meconium at the time of birth remain for a time as bacterial inclusion rather than infection, and may at any period subsequent to birth acquire force and cause disease. Accordingly calves show a very wide range of disease phenomena referable to intra-uterine infection. The clinical symptoms and postmortem lesions do not admit of the division of these results of infection into distinct diseases having separate causes and histories: the various types pass imperceptibly one into the other. Neither can intra-uterine infections be definitely separated from post-natal invasions. An infection emanating from the uterus of a cow and swallowed by her fetus may with equal facility be swallowed by her new-born calf when the liberated uterine discharges flow down the thighs of the cow and reach the teat which the calf sucks. Neither can there well be any fundamental difference in results, whether a bacterium is swallowed by the fetus or is taken into the alimentary tract of the calf shortly after birth. Varying types of disease phenomena occur, the fundamental cause of which is the same, so far as known. When the uterus of 
a pregnant cow is intensely infected, the fetus inevitably participates in a large degree. If the fetus resists the infection with such success that it is born, the birth may be very tardy owing to the paresis of the uterus due to the metritis present. The fetal membranes may be retained. Often the uterus contracts so feebly that traction must be applied to the fetus. Sometimes the feeble action of the uterus, or illness of the fetus, or the two combined, prevents the fetus from vigorously assuming the proper position for birth, the head or a limb deviates from its correct course, and dystocia results. Finally, if the fetus survives and is born, it is ill. It may be smeared over with meconium, indicating that as a fetus it suffered from diarrhea. The calf is weak and listless. It can not get up and perhaps can not stand if lifted to its feet. It may or may not have diarrhea. The rectal temperature may be abnormal, normal, or subnormal. The calf may rally after a time and get up, but usually the depression increases and after two or three to twenty-four hours it dies. Autopsy reveals hemorrhages in the liver, spleen, or other viscera, or beneath the pleura or peritoneum. The lesions are essentially identical with those observed in aborts. One animal dies within the uterus and is expelled shortly afterward; the other is alive when expelled from the uterus but soon perishes. The difference between the two is merely the time and place when death occurs. The cause and origin are the same in both cases.

The dysentery of calves has not been clearly defined. It merges imperceptibly in one direction with calf septicemia, is inseparably linked with calf pneumonia, is complicated by arthritis and other pyemic disturbances, and can not be separated by any clear line of demarcation from lesser disturbances which can not be included under either of the types mentioned. Like calf septicemia, dysentery of calves is a type of infection, or group of infections, rather than a specific infectious disease. Clearly due to infection, it is not known to be due to any one disease-producing organism. Though it is known that several organisms may be recognized associated with the diarrhea, the exact part in the disease played by any one of the bacteria is unknown. 
Calf dysentery has long been a fruitful field of controversy as to cause. Its intelligent study has been held back largely by false theories as to its nature. Many have held that it was due wholly and directly to improper feeding, and the claim has been so often repeated that it has acquired the weight of a fact. Some also taught that it was due entirely to navel infection, and this, too, became a common belief. All prophylactic and curative efforts based upon either of these beliefs came to naught, and when these erroneous theories had failed the truth largely went unsought. The theories mentioned above belittled the significance of dysentery in calves. If merely a matter of feeding, any person of moderate intelligence should readily control it. If due wholly to navel infection, ordinary cleanliness would obviate the difficulty. It was therefore technically an unimportant affair from the standpoint of the pathologist and a virtually hopeless scourge from the viewpoint of the breeder whose calves were persistently dying.

Calf dysentery is slowly becoming recognized as one of the links in an endless chain of infection invading the genital organs of breeding cattle of both sexes, passing over from the uterus of the pregnant cow to the digestive tract of the embryo within her uterus, and, under favorable environment, capable of causing retained afterbirth in the mother, or serious or fatal sepsis, dysentery and pneumonia in the calf, or of escaping from the digestive tract to invade permanently other systems or organs. Under this broader conception, the dysentery of calves becomes an integral part of one of the most important diseases among domestic animals. The term is commonly applied to a diarrhea or dysentery occurring in calves a few hours to a few days after birth. Some writers limit the period to the interval between a few hours to three or four days after birth. Diarrhea occurring in calves at other times is otherwise designated, but the line of demarcation is not clear and the limitation to such a period as indicated is purely arbitrary.

Diarrhea is common in the fetus. Physiologically, as soon 
as the alimentary tract is formed and the pharynx opens, the fetus commences to swallow but does not defecate. The amniotic fluid is constantly swallowed along with any exfoliated epidermic scales or other solids suspended in the fluid. Before hairs are macroscopically visible, meconium examined under a low-power lens reveals tiny short hairs which have been shed and then swallowed. Bacteria which have invaded the amniotic fluid are also swallowed. The swallowed liquor amnii is promptly absorbed by the intestines, enters the fetal lymph or blood stream, and later presumably returns to the amniotic sac. The solids, such as hairs, bacteria and epithelial scales, remain behind in the intestinal canal as in a cesspool, to constitute the meconium. Since physiologically the fetus does not defecate and constantly swallows its amniotic fluid, filtering it through the intestinal walls, it follows that this is regularly clear, while the allantoic fluid is frequently turbid.

But the fetus, like the living calf, does not always travel serenely along the physiologic path. Nearly half the uteri of pregnant abattoir cows contain bacteria which have the power to, and do penetrate the chorion, contaminate the amniotic fluid, and are swallowed by the fetus. There are found, thus swallowed, streptococci, colon bacilli, micrococci, B. abortus, and other bacteria. Physiologically this should not be. Their presence does not necessarily cause disaster. Up to a considerable volume the walls of the cesspool - the alimentary epithelium-perhaps aided by the biliary or other secretions and probably favored by the marked desiccation of the meconium, render powerless the bacteria present.

The power of the fetal intestine, like that of the adult, has its limitations. If the volume of bacteria is too great, or the virulence too high, the restraining power of the intestine may break down. If the fetus is weakened, owing to a disturbance of its nutrition, its control over resident infection may be lost. Thus, when placentitis is present, the nutrition of the fetus is crippled and harmful products, due to the infection in the placenta, reach the fetal circulation. 
The fetus undergoes partial suffocation. It is not strange, therefore, to observe that aborts frequently, if not generally, suffer from diarrhea. It is indeed not improbable that dysentery of the fetus frequently plays a vital part in causing fetal death, and hence abortion. In examining aborts meconic pellets are commonly found in the stomach: that is, prior to death the fetus had defecated and then swallowed its excrement.

In the later stages of pregnancy fetal diarrhea is not rare. I have seen many gallons of diarrheic feces in the amniotic sac at the time of expulsion of the fetus. More commonly there is but a moderate amount in the amniotic sac and the new-born calf is thickly smeared over with soft, sticky, brownish-yellow feces.

The calf which has suffered in the uterus from dysentery, and is expelled completely covered with excrement, is frequently vigorous. In some cases calves are born while dysentery is in active progress. These, under careful handling, may grow vigorously.

Most cases of severe dysentery develop within a few hours to a few days after birth, but no time limit can be fixed. In the research calves of my department, I now and then see an acute dysentery after ten to fifteen days, but these cases hive been generally, if not always, relapses after the disease has been brought under control and the calf has apparently been well for a number of days. So far as observed, these late cases were etiologically identical with those occurring earlier. Clinically they presented the same symptoms and reacted in the same manner to therapeutic efforts.

There is no clear line of demarcation between white scours and health. At one extreme is the ideally healthy calf; at the other, the calf with fatal dysentery. Between these two occur every possible gradation of disturbance. Neither does there exist a clear line of demarcation between white scours and calf pneumonia. Clinically the two glide imperceptibly into each other, and etiologically they can not now be differentiated. 
The etiology of calf scours has not been clearly determined. Numerous writers regard it as a specific contagious or infectious disease having one bacillus as the uniform causative agent, to which is frequently added other complicating organisms. This is difficult to prove or disprove. It has been noted already that diarrhea is common in abortion. Most investigators of abortion record only their findings regarding the $B$. abortus. They state generally that they recovered the $B$. abortus from the alimentary tract of the abort, but fail to state whether other bacteria were present. According to our investigations the $B$. abortus is not as common as some other bacteria in the utero-chorionic space, the fetal alimentary tract, or the alimentary tract of the calf with or without diarrhea.

For a number of years following the researches of Nocard, it was taught that calf scours was due to a bipolar bacillus of the septicemia hemorrhagica group. Later Jensen and others taught-and their views were commonly accepted-that the basic cause was a colon bacillus. My colleague, Carpenter, has recently presented strong evidence tending to show that a streptococcus of the viridans group is the most prominent organism present. Micrococci are common, but no connection between them and dysentery has been traced. Carpenter has generally failed to identify the colon organism in the intestinal tracts of fetuses and of sick calves destroyed before death was imminent, but has commonly recovered in these cases the streptococcus of the viridans group. Later when the calf is apparently dying, or has been dead for a few hours prior to the making of cultures, the colon organism becomes very conspicuous. It has been frequently asserted without qualification that dysentery in calves can be uniformly produced in its typical form by inoculating with the colon organism. My colleagues, Hagan and Carpenter, have repeatedly tried in vain to cause dysentery with this bacterium. This seemed peculiar, especially since Jensen is regularly cited as having proven clearly by experiment the specific character of the bacillus. A critical study of his recorded experiments explains at least 
partly the apparent contradiction between his researches and ours. The researches of Jensen were faulty in several respects :

(1) There is no evidence that the calves selected were free from alimentary infection when born.

(2) Details, or even general outlines of the feeding are wholly wanting.

(3) The controls had diarrhea, which was slower in developing and less fatal than in the inoculated animals, but otherwise showed no differential characteristics.

Accordingly it appears that the conclusions of Jensen were based upon wholly unreliable grounds and that he did not in fact clearly induce dysentery experimentally. So far as I am aware, calf dysentery can not be caused reliably by any known bacterium, although, reasoning by analogy and viewing it clinically, its infectious character is unquestionable. The experimenter is faced, as in abortion, with the impossibility of determining in advance the freedom of the experimental animal from the infection under consideration. When there is added the utter impossibility of clearly defining white scours, the difficulty of securing definite experimental data upon its etiology is apparent. The conclusion can not well be avoided, since in most cases of calf dysentery there is an abundance of a comparatively limited variety of organisms present, and those recognized are reasonably uniform in the different patients, that the disease is due to infection, and that the bacteria multiplying disastrously in the alimentary tract of a sick calf would likewise multiply, though not necessarily disastrously, when properly placed in the digestive tract of another calf. Clinically this is apparently true. White scours breaks out in large stables and pursues the relentless course of a scourge, causing a mortality of ten to one hundred per cent. for months at a time.

Apparently therapeutic evidence also indicates the infectious character of the disease, though this may be misleading. When a horse, by means of repeated inoculations, is rendered highly resistant to the dominant organism associated with white scours, his blood serum injected into a 
calf with white scours generally acts specifically to ameliorate the disease. I have found no record, however, of any control experiments to show that the blood serum from a horse not immunized or fortified by means of artificial inoculation with the organism would not have a like effect. A more direct test perhaps is the use of killed cultures of artificially grown bacteria. Limited observations seem to indicate that these act specifically under conditions not yet fully determined and may give to the calf, under some conditions, a more enduring resistance to diarrhea.

Since white scours is not clearly defined, its symptoms can not be accurately described. There is no epoch during which the line of demarcation between health and disease is as dim and hazy as in young calves. The healthy calf, when born, has a glossy brilliant coat of hair, soft and velvety to the touch; its body is of even contour and plump; and the calf is up and playing in an hour or two. Its intestines, especially the rectum, contain a pound or more of rather hard, yellowish or greenish to greenish-black meconium consisting of biliary salts, exfoliated debris, hairs, and frequently a swarm of bacteria. The great variation in size of the meconial pellets, in their adhesion to each other, and in their degree of desiccation, sometimes suggest that their character is largely dependent upon the quantity and nature of the infection present. Dysentery may exist at birth or may develop at any hour post-natal. When a cow or heifer has very severe infection in her uterus, the fetus when born is exceedingly dull and languid. It is unable to get up or to stand when helped to its feet. Perhaps no diarrhea is present. It may in fact die of calf septicemia. If death is held in abeyance, the calf generally develops violent dysentery.

Other calves, born apparently well, proceed to break down with diarrhea in a few hours to eight, ten or more days. The later in life the attack, the less stormy its course. When violent dysentery is impending, the first indication of the coming storm is a sudden rise in temperature. At this epoch the calf is not notably ill, the feces are not thin, and there is no marked loss of appetite. But this in severe cases is not 
for long. Soon in many cases there are streaks of blood in the feces, if any chance to be voided. In one to a few hours later the storm breaks and the observer frequently sees one of the most virulent and rapidly fatal diseases in animals, often destroying life in ten or twelve hours. When dysentery sets in, the temperature vacillates. Often the anus is paretic, the rectum is open and flaccid, and the thermometer reveals little of the actual temperature of the body:

The feces offer the widest possible variation in character. In the fetus, the diarrheic feces are usually yellowish, greenish-black or black. In post-natal dysentery the fecal discharges are much the same as in the intra-uterine diarrhea, if the storm breaks before the calf has taken milk, or until the milk or its derivatives have passed through the digestive tract to modify the excrement. After milk has been taken, the character of the excrement is necessarily altered. The milk-filled stomach serves as a large flask filled with an excellent medium for the multiplication of the bacteria present, while the body heat provides an ideal incubative warmth. The milk undergoes rapid bacterial decomposition and is hurried along the alimentary tract with great rapidity. The feces may contain some small decomposing milk particles still retaining some of its white color, but it has mostly disappearéd. The bacteria cause the liberal formation of highly fetid gases, which, mixed in small bubbles in the thin feces may lend to it a whitish color. The color varies greatly, however. Sometimes it is greenish, often brownish or yellow. In very severe cases the discharges are extremely thin and watery, mixed with minute particles of debris, giving it a dirty brown color, and charged with bubbles of highly fetid gas which escape quickly. In large stables where numerous calves are suffering from the disease in various stages, the odor from the calves is highly repulsive, of a sweetish sickening character.

The general appearance of the calf changes rapidly. The watery feces are forcibly expelled for a time, but later escape involuntarily through the paretic anal sphincter. The calf loses weight and volume with enormous rapidity. It 
takes no food or water, while the water of the tissues is being rapidly withdrawn to constitute the chief volume of the diarrheic discharges. The calf becomes unable to rise, lies flat on its side with its head drawn back (opisthotonus), becomes unconscious and dies.

Short of this violent type there is every gradation. In cases of great virulence, the dysentery may at any time abate, the calf rally, without definite therapeutic handling undergo more or less marked improvement, and may perhaps eventually recover.

Regardless of the grade of alimentary disturbance, there is a tendency toward the development of pneumonia. Sometimes the pneumonia develops suddenly and violently with all the clinical symptoms of that affection. Other cases creep on insidiously with a hacking cough associated with digestive disturbances. The pulmonary complications aggravate the digestive disturbances by lowering still more the resisting powers of the animal. In many animals the only evidence of pulmonary disease is a persistent hacking cough.

The clinical evidences of pneumonia may appear at any time from shortly after birth up to ninety or one hundred days. Like the dysentery, it is more prone to pursue a stormy course when it develops early. Dysentery and pneumonia each tend to conceal and to aggravate the other. The high temperature of dysentery causes rapid breathing, simulating pneumonia, and the dyspnea of pneumonia tends to detract attention from any dysentery present.

Arthritis as a concurrent lesion is not rare, and, like pneumonia, may be violent or may be of so low a degree as to be difficult of clinical recognition. The onset of pyemic arthritis may be extremely sudden. The calf is up and about, feeding and appearing well. An hour later it may be so lame in one limb that it refuses, or is unable to bear weight upon the affected limb. The involved joint is greatly swollen, tense, hot, and extremely painful when manipulated. A little later some other joint may be equally involved. When two or more limbs become affected, the calf is un- 
willing or unable to stand. In other cases the affected joints are neither greatly swollen nor very painful. Several or all limbs may be involved, causing the calf to walk slowly and cautiously without marked lameness in any one limb. As in pneumonia, so in arthritis, lesions are found upon autopsy which had not been clinically recognizable.

Pyemic abscesses, which occur somewhat rarely in all organs and tissues of the body, have the same significance as arthritis. Occasionally a pyemic abscess occurs in the spinal canal, causing a gradual and finally complete paralysis. In other instances the pyemic abscess is inter-articular in the spinal column and eventually breaks into the spinal canal, inducing sudden and complete paralysis suggesting fracture of the spinal column. Other evidences of disease appear which are not readily assignable to a logical place in the course of the basic infection. Prominent among these are ulcers and abscesses in the lips and cheeks. These aggravate exceedingly the basic malady. The lesions are apparently due to a secondary invader (B. necrophorus?) for which the basic infection has prepared a vulnerable field. Rachitic-like enlargements of the bones are not rare, but their exact relation to the basic invasion is not understood.

When a degree of dysentery, pneumonia or arthritis is present which clearly imperils the life of the patient, the presence of disease is not disputed, but the results of varying degrees of infection in new-born calves offer such infinite gradations that it is impossible for a majority of observers to agree upon a clear line of demarcation between health and disease. So far as known, the bacteria which cause dysentery, pneumonia, septicemia, and pyemia in young calves exist in the bodies of essentially all cattle, and disease or health is determined, not by the presence or absence of given bacteria, but by the ratio of bacterial force to the resistance of the patient. A healthy new-born calf has lustrous hair, its body is plump and graceful, its eye bright, its spirits gay, and its body functions are promptly and evenly performed. The bowels promptly expel the meconium, which, as it escapes from the anus, does not adhere 
to the tail or surrounding parts. The tufts of hair about the sheath opening (preputial tuft) and the vulva (vulvar tuft) over which the urine flows are not stained, matted or dirty. Experimentally this state can be maintained for several months, if not indefinitely. Naturally, in range cattle of high reproductive efficiency, the status at birth tends to be continued over a long period of time. In dairy herds and in purebred beef herds, which closely approach dairy herds in general plans of handling, the calf may soon break down with dysentery, pneumonia, joint-ill, or other manifestations of serious or critical disease, or show within five to ten days notable deviation from the ideal.

The temperature becomes elevated. This is so nearly universal that writers commonly hold that the temperature of a young calf is higher than that of an adult. The experiments in my department indicate that this belief is a fallacy and that the higher temperature of young calves is pathological. The hair soon loses its lustre and becomes dry, rough and faded. The skin becomes dirty. There are digestive disturbances of great variety. Almost always the feces are adhesive and stick to tail and buttocks. They are soft, medium, or hard, but the adhesiveness persists in each type. The consistency and color vary from day to day. Usually they are fetid and expelled frequently in small amounts. The total volume of feces is greater than in health, but the volume voided at one time is much less. The feces are irritant and frequently cause the matted hairs to fall out, leaving hairless areas on the tail or buttocks.

The calf may be either gaunt or pot-bellied. Its appetite is capricious, and it may swallow straw, shavings or other indigestible matter. Its growth is slow and uncertain. While a typically healthy calf well fed should gain not less than two per cent. of its body weight per day, the unthrifty one drops below this standard. There is a hacking cough. The calf plays but little, in marked contrast with the typically healthy calf which is one of the gayest rompers among domestic animals. The preputial or vulvar tuft of hairs becomes stained a deep brown and then black, to remain so 
for life. The hairs become matted together in hard masses.

These evident deviations from typical health can not be classed as dysentery, pneumonia, septicemia, or arthritis, but are due, so far as can now be determined, to the same infections. The results differ because the ratio of bacterial force to the resistance of the patient differs. Such calves finally recover a physiologic appearance at one hundred and twenty to one hundred and eighty days, when they shift more or less completely from a milk to a vegetable diet. The pot-belly or gauntness slowly abates, the feces cease to adhere to the tail and buttocks, the harsh dry coat regains its lustre, the masses of epidermal debris in the hair disappear, the animal becomes vigorous and grows rapidly. It then enters upon a period of unusual good health, which extends to puberty, and breeding occurs. The only trace of the adversity through which it has passed is the matting and staining of the vulvar or preputial tuft and the persistence of more or less numerous nodular elevations in the vulvar or preputial mucosa, known as the nodular venereal disease. The significance of the matting of the hairs and of the lesions in the mucosa is not accurately known. I do not believe that during this epoch of apparent good health the animal has become free from the infection. Instead I believe that here and there the infection still lingers, but there has arisen an important power of resistance to the bacteria which holds them in abeyance and renders them essentially saprophytic for the time being.

The post-mortem findings vary with the form and duration of the disease. In calves which succumb to violent dysentery, the lesions are those of gastro-enteritis and septicemia. Hemorrhages occur in the capsule of the spleen or the outer and inner walls of the heart beneath the pleura (See Fig. 205) and elsewhere. The fourth stomach shows great reddening (congestion) of its mucosa and the folds are generally edematous. The duodenum shows similar lesions. The remainder of the alimentary tract is not so markedly altered, as a rule, although frequently the summits of the rectal folds are intensely congested and sometimes hemorrhagic. 


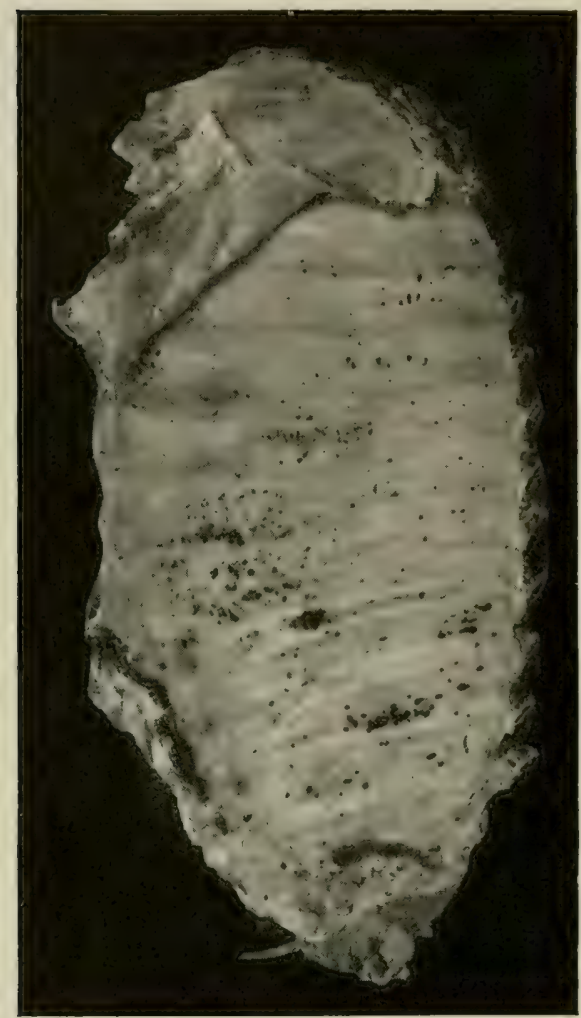

Fig. 205-Costal Pleura of Abort at Full Term, showing the Petechiae and Hemorrhages Commonly seen in Abortion, Calf Sepsis, and Dysentery.

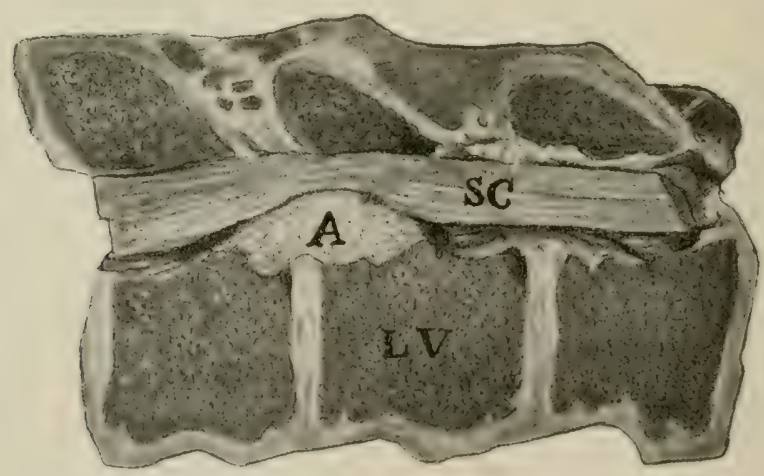

FIG. 206-Pyaemic Abscess in Spinal Canal of Calf. $S C$, Spinal cord ; $A$, abscess. 
When the immediate cause of death has been pneumonia, the principal lesions are naturally found in the lungs. In most cases only the anterior lobes are involved, but sometimes even the diaphragmatic lobe is largely solidified. The pneumonic areas are whitish or grayish-white, mottled, and feel much like the normal pancreas. Pleuritis is seldom seen. Microscopically the affected area is filled with enormous numbers of polymorphonuclear leucocytes, which give to the lung its grayish, mottled appearance. The condition is one of severe purulent broncho-pneumonia. In the milder cases, associated with hacking cough, there are few to many small circumscribed pneumonic areas, from the size of a pinhead to that of a pea. The synovial membranes of the femoro-tibio-patellar, tarsal and carpal joints may be inflamed. The pyemic abscesses usually have weak, atonic walls. The predominant organism encountered is of the colon group and offers nothing remarkable in cultures or by staining.

The period and avenues of invasion of the calf need to be comprehended before intelligent control is possible. It has now been made perfectly clear that calf septicemia, dysentery, arthritis, pneumonia, and the other phenomena grading off imperceptibly from these to ideal health, are due fundamentally to an intra-uterine infection not separable from the infections causing abortion and fetal diarrhea. This has already been discussed. It is equally clear that post-natal infection occurs, chiefly through the mouth in contaminated food. Probably some cases are due to navel infection.

The handling of calf sepsis, dysentery, pneumonia, arthritis, and those types of infection shading off gradually to health and not definitely perilous to life, has a dual meaning in animal husbandry which should be fully comprehended. There are two widely different conceptions of curing a disease, or some phenomenon called disease. The common meaning of cure is the rescue of the patient from death. In such infections as those now under consideration, especially when dealing with purebred calves designed for breeding 
purposes, the saving of the life of a calf is an utterly worthless performance unless there be added the safeguarding of sexual health. Few if any principles regarding the infections of the genital organs of cattle are clearer today than that the fertility of a bull or heifer, when arrived at breeding age, has been fixed unalterably during its nursing period. The most important result to be sought in the rearing of calves is not the conservation of their lives, but the control of infection which constantly tends to attain a habitat in the genital system of both sexes, where it persists until breeding age, when it may prevent reproduction or, infinitely worse, may prove a serious menace to other animals with which the infected animal comes into sexual relations. This danger is disputed by many and ignored by nearly all breeders and veterinarians, but the evidence which has been accumulated is so strong that it is at least time to give to the subject thoughtful attention. It is common knowledge that in large herds where sterility, abortion, metritis, retained fetal membranes, and other phenomena of this group are violent, the calves suffer much from dysentery and pneumonia. When heifers in these herds reach breeding age, it is notorious that upon the average it requires more than twice the number of copulations to produce pregnancy than is necessary in adult cows. Once pregnant, heifers which have been unhealthy as calves abort in a ratio two to three times as high as observed in cows. On the other hand, in small grade herds where often sterility and abortion are rare and the calves are healthy, the heifers upon reaching breeding age conceive as promptly as cows and carry their calves as safely. There are also reliable statistical data supporting this position, an example of which is shown in the chart designated Fig. 207. The herd was a large one, the records are unusually complete and accurate, the duration of time covered by the data is extensive, and the difference in the behavior of the two groups of heifers is distinct and remarkable. They were kept in the same stables and pastures, were bred to essentially the same bulls, were the progeny of the same group of cows as nearly as possible, and were in 


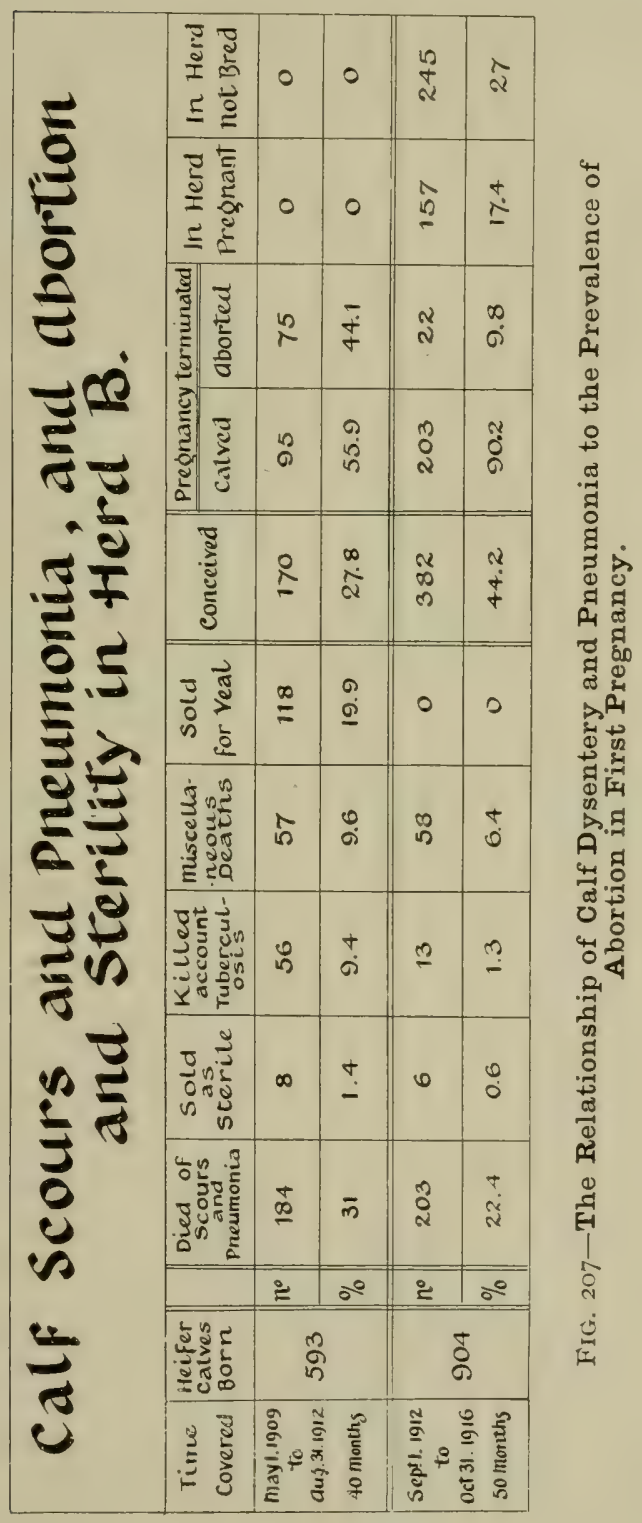


every way handled alike except that the second group was more carefully handled and was healthier during the nursery period. Everywhere that I have been able to observe, this view is supported by clinical facts. It is a principle which has an application in other species, and parallel evidence is submitted in connection with the disease interfering with reproduction in swine. Consequently I hold that, in handling the congenital infections of calves, the conservation of life should not be regarded as an end in itself, but merely as an essential pre-requisite to guarding the sexual health and vigor of the future adult.

The handling of dysentery must be founded upon certain well established basic facts. Since the infections under discussion are primarily intra-uterine, intelligent endeavor should be made to prevent or to minimize such invasion. This is to be accomplished by securing, as far as possible, the mating of sexually sound animals by such means as have already been advised in the preceding pages. If conception occurs in a clean uterus, as the result of coitus with a sexually sound bull, the calf will be born without serious infection in its alimentary tract. If the uterus or cervix is badly infected prior to conception, or if the bull is suffering from serious genital infection, which is ejaculated with the semen, the calf when born will be critically infected. During pregnancy no remedy now known can ameliorate the infection. No known substance administered in any manner has been shown to invade the utero-chorionic cavity and disinfect it, or to pass through the placental filter and destroy bacteria in the fetal alimentary tract or other organ. Vendors of biologics have offered alleged cures or preventatives to be given pregnant cows, but I know of no ground for asserting that they have value.

Although the precautions advised in the preceding paragraph are of great value and are to be urgently recommended, they are imperfect. Generally no thought is given to such care until the storm breaks. Many of the cows are pregnant and the fetuses critically infected, and the termination of pregnancy must be awaited before the infection in 
the calf (as in the cow) can be attacked directly. In principle each calf at the time of its birth should be regarded as possibly carrying bacteria included in its meconium which constitute a peril to its life and health. This peril is largely avoidable and should be anticipated. The chief obstacle to the adoption of measures to forestall the development of the included bacteria into an active and perilous infection is the apparently good or perfect health of the calf. To the layman and to many veterinarians it appears imprudent to undertake the handling of an apparently healthy calf to prevent serious or mortal illness from included bacteria which reveal no signs of their presence. There are at present no known means which can be practically applied by which the breeder or veterinarian can distinguish between the newborn calf which does, and that which does not carry in its digestive tract dangerous bacteria. Choice must therefore be made between two opposing policies: the assumption that the calf carries dangerous bacteria in its gastro-intestinal tract, the development of which shall be forestalled, and the contrary assumption that the apparently healthy calf does not carry dangerous bacteria, with the postponement of action until after the storm breaks. I favor emphatically the first assumption. The veterinarian is concerned chiefly with purebred or high grade calves, because these constitute the basis of the best herds and the source of supply for breeding stock in the improvement of common cattle. The purebred calves are the most intensely infected and should be most vigorously handled.

Ordinarily the calves of dairy cows are fed by hand either from birth or after a very few days. I favor taking the calf immediately from the cow without permitting the dam to lick it. By this method there is a total absence of worry over the parting by either mother or young, the calf is more readily taught to feed, and the amount of food and intervals between feedings are under control at the most crtical time. The calf should be removed to an isolated, clean, comfortable stall, and rubbed dry. Post-natal infection through the umbilic wound should be guarded against by permitting the 
navel cord to be ruptured by the natural method of overstretching, by which it parts regularly at two to four inches from the umbilicus. When permitted to rupture in this manner, the elastic stumps of the umbilic arteries, dragging with them the stump of the urachus, recoil into the abdominal cavity and come to rest two or more inches above and posterior to the umbilicus. This leaves only the vein and the the amniotic covering of the cord to consider. The blood in the umbilic vein drops out promptly and the vein collapses at once. No tying of the umbilic stump should be attempted, as this interferes with the escape of blood from the umbilic vein. The layman should understand that there is no bleeding, in the true sense, from the umbilicus of a normal calf. The arteries when broken recoil in a manner rendering the escape of blood impossible. Since, if the umbilic stump is ligated after having broken naturally, the arteries are not included in the ligature, ligation could not prevent arterial bleeding if it were threatened. A ligature can serve only to retain within the navel veins that blood which inevitably dies and should escape. If the heart valves are defective, there may be hemorrhage from the navel veins, but in that case the death of the young animal is an economic gain. The stump of the navel cord should be disinfected by some reliable plan. I prefer a solution of $1-1000$ corrosive sublimate. A goblet, cup or large-mouthed bottle is filled with this solution, then pressed against the abdominal floor, surrounding the umbilicus, thus completely immersing the navel stump, and held there for ten to fifteen minutes. This may be repeated in twelve to twenty-four hours if the navel stump has not dried up. As much of the bacteria-containing meconium as practicable should be removed promptly from the rectum and large intestines. So far as I have observed, the best plan is the use of a warm enema of physiologic salt solution ( 1 ounce salt to 1 gallon water). It is administered with a hospital irrigator to which is attached a small soft rubber horse catheter. The irrigator is held slightly above the calf and the catheter gently introduced into the rectum while the enema is slowly flowing in. The catheter 
should be introduced ten to twenty inches. If a suitable vessel is held beneath the anus, the meconium, as it is expelled with the enema, may be caught and the bedding not contaminated. It is well to repeat the enema twice daily for two days at least.

In all herds where infections of calves are common, and in those calves in comparatively healthy herds in which the dam has retained fetal membranes or other type of metritis, calf scours serum should be administered liberally as soon as the calf is born. I believe it would be even better in purebred calves of high value to make the administration of calf scours serum a universal rule. According to observation, depending upon the probable degree of infection present, it is well to give at the time of birth 20 to 40 mils of the serum, and if the calf appears well and the temperature remains below 102 degrees $\mathrm{F}$., to give 10 to 20 mils each twelve hours until four doses have been given. Clinical observations appear to indicate that such handling not only prevents in a large measure the development of dysentery, pneumonia, and arthritis, but also invigorates the calf, guards it against less evident types of the same infections, and is an important element in laying a secure foundation for sexual soundness when the calf reaches breeding age.

Food shouid be completely withheld until the calf is twenty-four hours old, because during this interval most of the bacteria present in the alimentary tract may be removed by the enemas and the calf may acquire some degree of resistance to the bacteria remaining. There is an old fallacy that a calf needs a feed of milk immediately after birth, and most writers on animal husbandry and veterinary obstetrics so advise, but it should be clearly understood that, up to the moment of the severance of the navel cord, the young animal has been richly fed and its blood is well charged with reserve nutritive material ample for twenty-four hours and enough to sustain life for several days. A healthy calf from an equally healthy mother is not born in a starving condition, nor even hungry. It is quite true that it knows no better than to eat and if given an opportunity, will almost 
invariably gorge itself with food of which it needs no part. If one will take the trouble to watch a calf taken from its dam and kept twenty-four hours without food, no evidence of hunger will be manifested during that period.

If it could be known that a calf carried no potentially harmful bacteria in its gastro-intestinal tract, an immediate feed of milk might not do harm. It certainly can do no good. But most calves are not thus free from bacteria, for the growth of which milk constitutes an ideal medium. When a calf of average health is born in an ordinary dairy herd, its rectal temperature is below 102 degrees F., and generally it will remain there so long as the calf is not fed. If given a feed of milk, its temperature rises (bacterial decomposition of milk with absorption of bacterial poisons by the calf) within two to four hours, and the amount of elevation will be proportionate to the volume of milk fed. The bacterial decomposition of milk in the alimentary tract of nurslings is so nearly universal that it has led to the fallacious teaching that the physiologic temperature of the new-born is higher than that of the adult. The temperature is indeed usually higher in the new-born, but it is pathologic, not physiologic, as the experimental feeding of calves has clearly and emphatically shown. Since it is unnecessary to feed the calf during its first twenty-four hours, and such feeding is accompanied by danger, this period should be looked upon as one in which to do the utmost to forestall the active arousal of infection and to prepare the calf by every available means known for feeding safely and successfully. The temperature of the calf should be measured two or, better, three times daily during the first ten days, since an elevation of temperature is the most constant and valuable sign of approaching trouble. It is especially important that the temperature of the calf be measured just before feeding so that if fever is present the milk may be withheld. The feces are also to be watched closely with reference to consistency, color, odor, and the presence or absence of blood. In most cases of impending dysentery blood may be seen in the feces. At the end of 
twenty-four hours the calf should be fed about two per cent. of its body weight of milk: that is, if a calf weighs fifty pounds at birth, it should be fed at first one pound of milk morning and night. This ration should be continued for a period of four or five days, during which time the calf will lose weight but will retain its vigor and the lustre of its hair. At the expiration of this period, the ration should be slowly and carefully increased by four to eight ounces per feed until a satisfactory ration is attained. The best source from which to obtain milk is from its dam. At one time I was led to believe that the milk should be taken from a healthy cow, even if the rule excluded that of the dam, but more recent investigations appear to contradict this view. The age-old belief, that the dam's milk is better for a young animal than the milk of another animal of the same or other species, appears to be supported by important scientific facts. I have stated that the pregnant uterus generally contains potentially pathogenic bacteria, and that the fetal alimentary tract usually contains bacteria identical with those in the uterus. The blood of the cow, as shown by agglutination tests, contains protective substances (antibodies) against the bacteria in her uterus. These substances do not pass the placental filter or otherwise enter the fetal circulation. The bacteria included in the meconium generally cause no active infection in the fetus, and no antibodies are formed in its blood. Hence the fetus is commonly born without any acquired resistance to the bacteria in its alimentary tract. The milk of the mother seems, however, to contain such antibodies, so that a calf in feeding upon its dam's milk is taking with it substances equivalent to calf scours serum. There is something in the milk of the mother which tends to repress the gastro-intestinal bacteria of the calf. The milk should be drawn carefully under the best rules of cleanliness in a sterilized pail. Special care should be taken, in cases of retained fetal membranes or other types of genital discharge, to wash and disinfect the udder prior to drawing milk for the calf. 
No harm will ordinarily come to the cow if left without milking for twenty-four hours after calving, when, under the plan advised, the calf will get colostrum in its first feed. Notwithstanding the assertions of numerous teachers regarding feeding, the necessity for feeding colostrum to a calf is a myth. Experimentally I have fed many calves upon boiled milk from birth, and colostrum cannot be boiled without coagulating. While a calf can be well grown experimentally upon boiled milk from the outset, it requires very close watching and skillful handling in most instances. I prefer that for the first eight or ten days the calf should be fed the very small ration mentioned of raw milk from its dam. During this period there is confessedly the danger from infection borne in her milk, but under usual conditions this is more than counterbalanced by its content of protective substances of a highly essential character. The plan has very important limitations. If the dam is tubercular, her raw milk should not be fed to her calf. Either the milk of a tubercle-free cow should be substituted or the milk of the dam boiled, fed very sparingly, and the loss of the antibodies in her milk destroyed by boiling, counterbalanced by the liberal use of calf scours serum. Similar limitations apply to diseases of the udder. Perhaps the greatest obstacle to overcome in guarding the health of young calves is the deeply rooted prejudice of breeders that heavy feeding from the first is essential and that, at whatever cost, the calf must grow. Instead I have emphasized securing first a healthy body, knowing that in the end the healthy calf makes the greatest and best growth. The rule appears to be that with a moderate number of bacteria in the gastro-intestinal tract, a small volume of milk may be digested and assimilated with a minimum febrile disturbance, but, when the volume of milk is greater, the febrile reaction is stronger. Digestion may be regarded as a triumph of the digestive ferments over the bacteria present, by which they convert food eaten into products which may be absorbed and used by the body for its maintenance and growth. If the bacteria present prevail over the digestive ferments, products are formed and 
absorbed which are toxic for the body and imperil health and life. Presumably there is no material difference in the amount of digestive ferments poured out in response to the presence of a small or a large volume of food. Consequently it should be expected that, within reasonable limits, the smaller the amount of milk fed, the greater the ratio of digestive ferments to the volume of milk, and hence the prompter and more certain the digestion; conversely the greater the volume of milk, the lower the ratio of digestive ferments and the greater the danger of bacteria attaining the upper hand and displacing digestion by bacterial decomposition. Clinically this conception holds true and there is no more important lesson to be learned in connection with the growing of calves (or other young) than that feeding more milk than can or will be digested promptly is a peril to health and life. All that milk which the digestive ferments fail to prepare for assimilation and utilization by the body for its food is broken down by the bacteria and causes harm.

I consider it highly important that the milk fed to dairy calves, after they are about ten days old, be boiled. It is virtually always mixed milk coming from a large number of cows. Often when there is a bad udder or a cow has aborted or had retained afterbirth, the milk is fed to calves. Such milk is often badly contaminated with infectious discharges from the uterus which have flowed down the tail and thighs. It is safe when boiled, but otherwise unsafe. The same is generally true of mixed raw milk. It contains all infections reaching the milk of any cow, so that the danger is multiplied by the number of cows contributing to the ration of milk fed. There has been much interesting controversy regarding the comparative virtues of raw and cooked milk. I consider that it has been proven beyond dispute that raw milk is in itself a safer food for calves for a few days after birth than cooked milk. Various explanations have been given. Some have said that the essential salts of the milk are rendered non-assimilable by cooking. Others have claimed the change is in the fat, casein, or other constituent. Recently it has been positively asserted that 
vitamins are destroyed by cooking and the milk thereby rendered unfit to sustain life and health.

Experimentally I have fed numerous calves upon thoroughly boiled milk. A considerable proportion of these, probably one-third, lived and grew splendidly; the others quickly perished from dysentery, arthritis, or pyemia. Those which did well showed health and vigor beyond that of calves fed upon raw milk. The results are partially illustrated in Figs. 81 to 84 . They are confusing because the calves thus fed represent the extremes, without that middle class of health observed in calves fed on raw milk. A further study reveals the interesting fact that the calves which did well upon cooked milk came from clean herds where harmful genital infections were low or not clinically recognizable, while the calves which promptly sickened and died came from herds where genital infections were intense. In other words, the cooked milk was excellent for those calves which were born comparatively free from bacteria in the meconium, and highly perilous for those having large volumes of bacteria in the alimentary tract when born. The cooked milk was perfect as a food but useless as a bactericide.

My results were in such sharp contrast with the observations of others that their reliability was questioned. Some disbelieved the effectual and reliable boiling of the milk. In order to meet this objection, I personally removed two calves from their dams at birth, without the dam's having so much as licked her young. These were placed upon milk autoclaved for thirty minutes under fifteen pounds of steam, equivalent to a temperature of about 240 degrees F., or 28 degrees above boiling. The milk was browned to about the color of weak coffee. I believed that, if vitamins or other essential constituents were destroyed by heat, this would demonstrate the fact. But the calves did phenomenally well, equalling in health and rapidity of growth any I have ever observed. The results of the experiment are partly shown in Figs. 208 to 211 inclusive. Calf 70 was extremely thin, weak, and sick at birth. She weighed forty-nine pounds. 
Each calf gained upon an average two per cent. of its body weight at birth per day during the experiment. The plan for controlling the dysentery, and at one time threatened pneumonia, is graphically shown in the charts. Neither calf at any time lost the lustre of its hair. After the first few days, they remained plump and were unusually playful.

In order to accomplish these results with boiled milk with any degree of uniformity, I have found it necessary to ad-

CHART OF CALF No.70
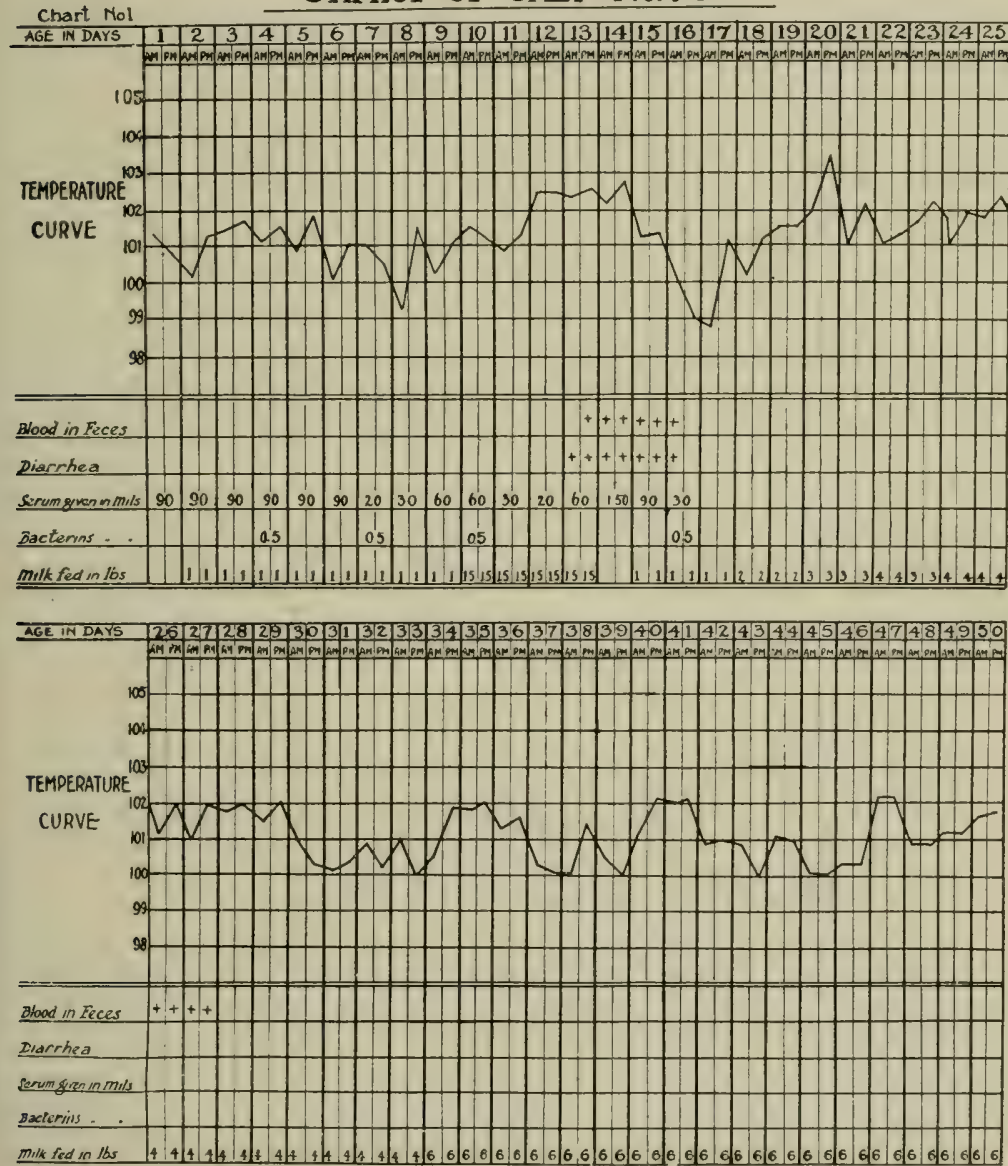

Fig. 208-Chart of Calf No. 70, fed upon autoclaved milk. 
minister large quantities of calf scours serum, but I cannot believe that this in any way supplied any nutrient constituent of the milk destroyed by heat. It appears to me quite clear that the sole result of the serum was the supplying of protective substances which held in control or destroyed bacteria existing in the alimentary canal at birth. Further clinical evidence regarding the action of heat upon the healthfulness of milk is furnished by placing the calf

chart Nol. Continued CHART OF CALF NO 70
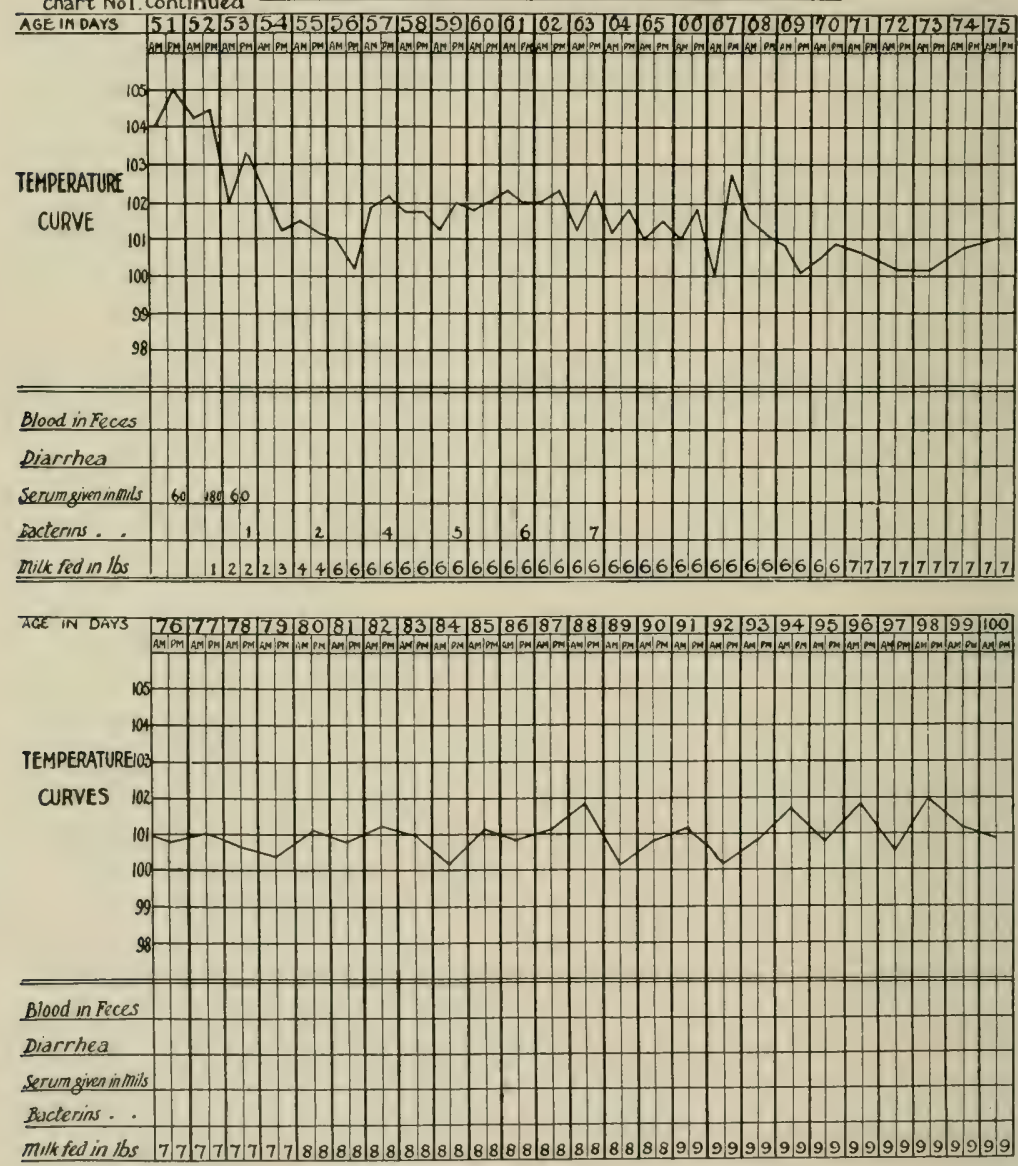

Fig. 205-Continuation of Fig. 208. 
upon raw milk for ten or twelve days and then placing it upon boiled milk. Such calves regularly do well, and the logical explanation would appear to be that the raw milk allowed for a limited time enables the calf to develop a resistance to the bacteria present, after which the protective substances are not required. As a result of these researches I have advised, and have found the advice good in actual practice, that the calf be fed raw milk from its dam until

CHart No 2 continued $\quad$ CHAR OF CALF No 71.
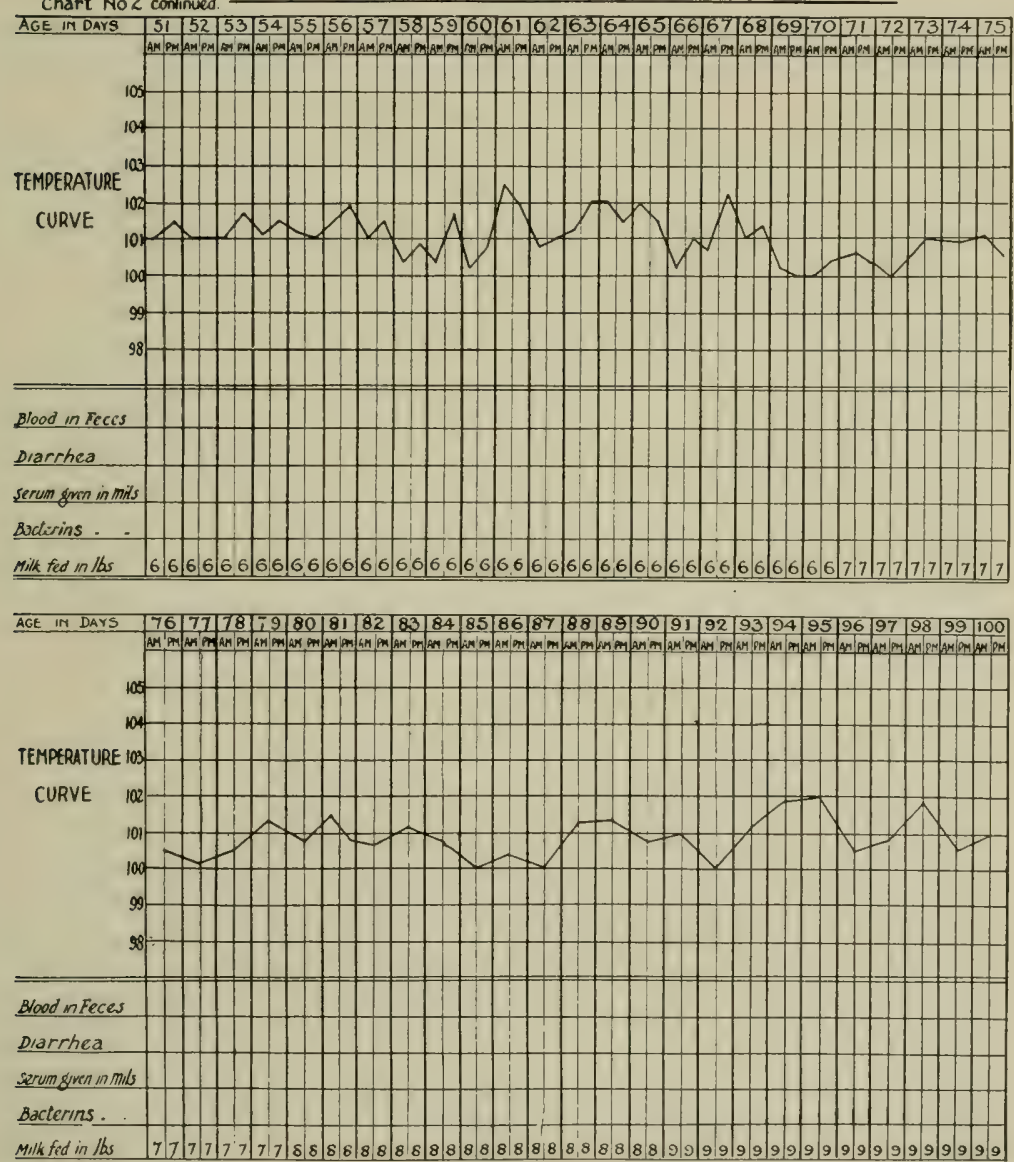

Fig. 2ro-Chart of Calf No. 71, fed upon autoclaved milk. 
eight to ten days old, at which time it should be placed upon boiled milk, which may be taken from any cow or cows and may be whole or skimmed. Pasteurized milk has been advocated as superior to boiled milk, but I have been unable to observe any superiority in it, either in experiment calves or when practically fed in herds.

Each new-born calf should be regarded as a probable carrier of infection dangerous for other young calves, should
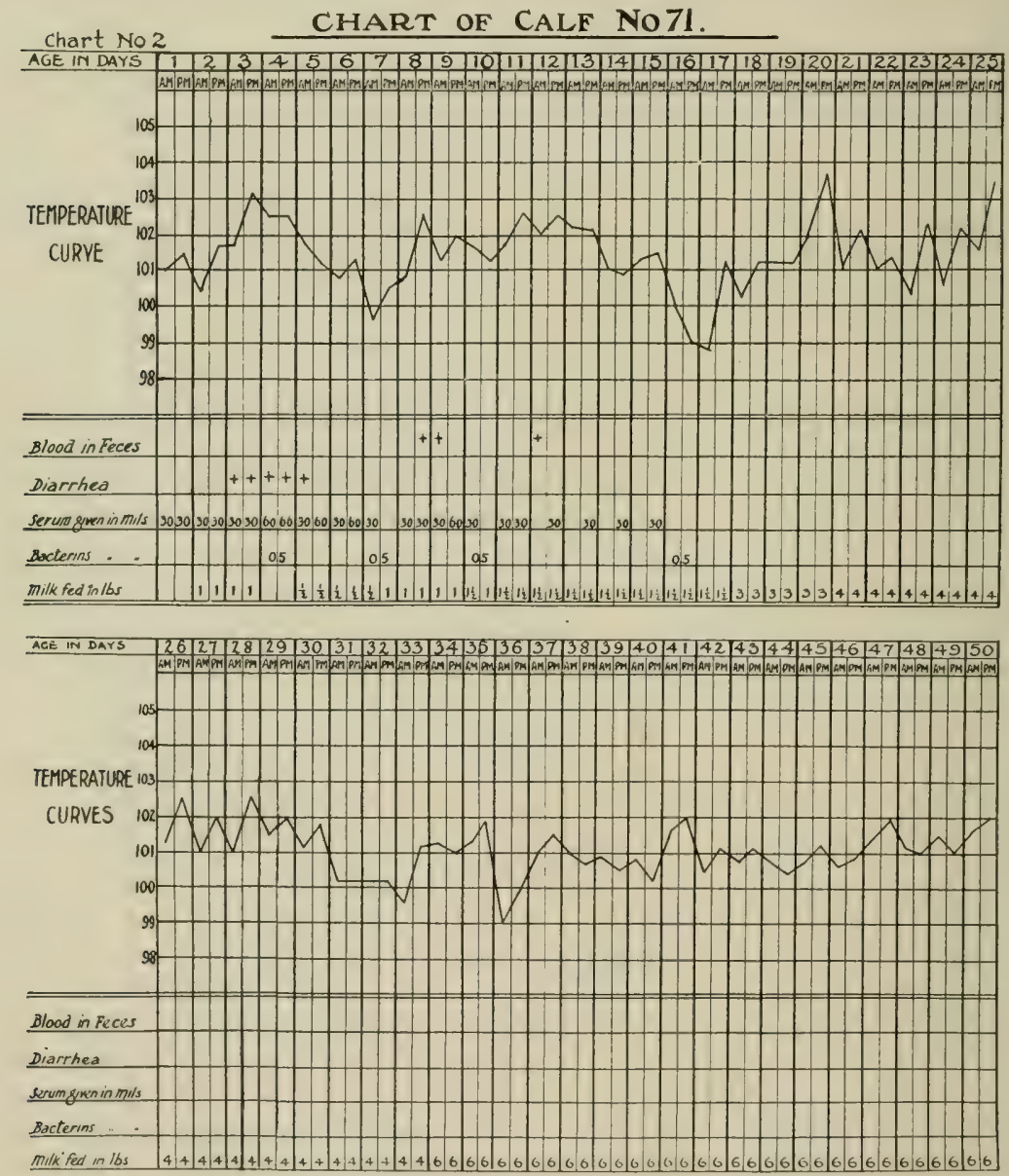

FIG. 2II.-Continuation of Fig. 210. 
direct or indirect contact occur. The proper growing of calves demands an adequately isolated stall for each until it has reached a time when its freedom from disease is definitely assured. Each establishment should be provided, as a necessary part of its equipment, with a sufficient number of perfectly isolated stalls to accomodate all anticipated calves until at least ninety days old, and longer if appearances indicate danger, or if it is economically practicable. Most calf nurseries violate every rule of hygiene in construction, arrangement, and equipment. They usually fall into two groups. In many instances the available funds are used in the construction of an expensive, often extravagant, dairy stable and the young calves are forced into some decaying structure unfit for the occupancy of any animal. At the other extreme, there have been erected recently by very wealthy breeders extravagant nurseries which in their plans are equally in conflict with the fundamentals of hygiene especially because the stall partitions are of open work, so that from a hygienic standpoint there is no isolation of calves. The calves may lick each other through the partition, diarrheic feces may be ejected into the next stall, and sputum from a pneumonic calf thrown into it. A yet more unsanitary practice is the placing of two or more young calves in a common stall. When some of these become ill, sputum and feces soil the bedding, floor, feeding utensils, the exterior of the calves, the food, stalls, and fixtures. The calves lick and suck each other and transfer from one to the other any infections present. Frequently an infected calf will suck the navel of another and infect it. The infected navel will be sucked and later the calf which is sucking will move to the udder and suck it, transferring the infection to the teats. The infection passes up the teat canal causing a small abscess, and when the heifer is grown and calves, one or more quarters of the udder are blocked and the animal is of low value.

The simplest type of nursery to conform to hygienic principles is one with two rows of stalls, one row against each side with a broad aisle between. The stalls for the youngest 
calves should be six by eight feet, with larger stalls to which they may be shifted later, with solid partitions (wood or concrete). The aisle side should be open and provision made at the opposite side for a free opening near the level of the floor (door or window), so that in warm weather there may be a free passage of air through the stall at the floor level. The floor should be of concrete or other impervious material which will freely admit of washing and disinfection.

The heating of the nursery has generally proven a failure. Any attempt to heat the room with overhead steam pipes has, in my observation, heated the upper air, kept flies alive all winter, and made concrete and similar floors cold and damp. An ordinary stove is somewhat better, but it is dangerous and the heat diffuses slowly and imperfectly. Neither of these plans supplies warmth where it is most needed-the cold and usually damp floor-and neither admits of free circulation of air without most of the heat passing out of the room. I have found hot water pipes laid beneath the concrete floor and properly operated highly satisfactory. The capacity of the heater should be very low compared with the cubic content of the room, as a safeguard against overheating. The pipe should be large, either cast iron water pipe or sewer tile carefully laid. There should be but a single loop of pipe beneath each tier of stalls. With such a system installed, little heat is required to warm the floor, and, once it is warmed, heat is retained for a long period. The floor upon which the calf lies is kept warm and dry while the general temperature of the stable may be kept low and the exchange of air abundant. The interior of the stable, stalls and fixtures should be unpainted, or at least not painted with lead or zinc. Possibly zinc is not in itself poisonous, but it is rarely or never free from lead. In fashionable stables many calves die from licking painted walls or fixtures, and sometimes feed pails.

Throughout the milk-feeding period the condition of the calf should be watched closely. It should not be permitted to become gaunt or pot-bellied. The feces of a sound, vigorous calf do not adhere to its tail and buttocks. Upon the 
first sign of this, the fault should be corrected. Very largely it is too much milk, at least too much for the calf in question. The milk supply should therefore be decreased and care should be taken to see that it is clean, and, if boiled, that the boiling has been efficient. At the same time, if conditions at all justify, calf scours serum should again be given or, often with better effect, bacterins made from cultures from its feces or stock cultures from the herd. In such cases enemas of salt solution, as recommended at birth, repeated twice daily, are useful.

As an economic measure, in order that the milk may be sold on the market, substitute foods have been recommended. There can be no material objection to them if they maintain the condition of the calf as well as good clean milk, no more than there should be objection on the part of the dairyman to synthetic milk or butter placed upon the market for human use. I think it has not yet been shown that any substitute can equal good milk in either field, though a good substitute may be superior to bad milk. The calf may have supplementary foods very early, perhaps most profitably oatmeal or linseed meal in moderate amount. When these infections have been neglected and a calf permitted to become feeble, abscesses occur in the lips and cheeks. The underlying infection must be handled first. The abscesses should be opened early and freely and they, or any ulcers, cauterized with silver nitrate or sulphate of copper. Calves suffering from dysentery or lesser alimentary disturbances, acquire morbid appetites and swallow straw, hay, feces, shavings (bedding), and various indigestible substances. The abnormality is probably never seen in a healthy calf. If it proceeds far, there are usually very severe symptoms, which are often confusing. Sometimes the presence of indigestible material, usually packed into a large dense mass in the stomach, causes an obstinate dysentery against which all remedies are futile. Sometimes the masses pass on into the intestine and become impacted there. In some cases the calf has severe convulsions similar to those due to lead poisoning from licking paint. If 
the tendency is discovered early, the danger may be halted by adequate muzzling. If the rubbish swallowed has been extensive, it is beyond known remedy unless, if the diagnosis is clear, one may attempt gastrotomy.

If the breeder insists upon permitting the calf to nurse the cow, he should be induced, if possible, to milk the cow almost dry and to wash and disinfect the udder before the calf is permitted to suck. Where the calf must run with the cow, the feeding cannot be controlled and the handling of this group of infections cannot be so satisfactory. In beef cattle, especially purebreds, and in grade cattle which are partially milked, the feeding problem may in many cases be satisfactorily managed. If the cows are gentle, they may be milked out to that point where approximately the ration advised is left in the udder, and the calf then permitted to suck. When the calf is ten days old, it will usually con. sume with safety and profit all its dam will yield. In beef cows the milk ration may be reduced at first by feeding the cow very low or by placing her on very poor pasture.

In some herds of beef cattle I have observed that the congenital infections do not tend so strongly to induce diarrhea as in dairy calves. Instead, the calves appear languid, breathe rapidly (fever), have little or no appetite, and die. Upon autopsy, the lesions are the same in general as in dysentery: that is, there are hemorrhages in various tissues and the feces are soft and fetid. The calf running in the open may also acquire a morbid appetite, and may swallow dry grass or other rubbish, have convulsions and die. For these reasons I have had occasion to advise, with gratifying results, the use of calf scours serum, as in the dysentery of dairy calves, making its administration a general rule in herds where calves are dying. In purebred beef cattle excellent results are obtainable by separating the new-born calf immediately after birth, muzzling it securely, and removing the muzzle twice daily to let the calf suck, after the dam has been well milked out. When dysentery, arthritis, pneumonia, or other phenomena are established, or when, in the hands of the alert breeder the temperature, blood in the 
feces, or other signs betoken the approaching storm, all food should be withdrawn. Most persons temporize by reducing the ration. That is not enough. The small ration is of no value whatever to the calf. Instead, the food is broken up by the bacteria, producing toxic substances which injure the calf. It is almost impossible to have some persons understand that milk is food only when digested by the physiologic ferments and that it is just as imprudent to feed fresh, clean milk to a calf, when it will undergo bacterial decomposition in the stomach, as it would be to inoculate the milk with the same bacteria, incubate it until thoroughly and repulsively decomposed, and then compel the calf to drink it.

The calf should receive immediately large and repeated doses of calf scours serum. There is no limit to the amount which may be given. It is best as a rule to give 30 to 50 mils at a dose, and this may be repeated in two or three hours as long as desired. It is best and most economic to meet the violent onset of the disease with vigorous opposition rather than to temporize with small or infrequent doses. It is safer for the calf and more economical to control the onset of the disease quickly. If the disease is controllable, it can be overcome within two to six hours, if the dosage is sufficiently liberal, and the total volume of serum required will be less than if used in smaller doses over a period of twelve to twenty-four hours. Calf scours serum generally appears to act specifically, and, when administered in time and in sufficient volume, gives highly favorable results. The calf, even when lying prostrate upon its side, unconscious, its temperature subnormal, and fetid watery feces dribbling away involuntarily, often responds at once and may be on its feet and well advanced toward recovery within two to four hours. When severe arthritis is present, heavy dosage may cause the articular pain and swelling to disappear almost wholly in a few hours. There are numerous exceptions. When the disease is not taken in hand until far advanced, the mortality is unavoidably high. In some outbreaks the disease appears uncontrollable with serum or other known 
remedy. Several reasons have been suggested. In many cases the failure is unquestionably due to a blind reliance upon serum without the basic act of withdrawing all food and attending to other essential features, such as the prevention of the swallowing of rubbish. Numerous writers have contended that the obstinacy in some outbreaks is overpowering because the infection is specifically different from the prevailing cause of dysentery, pneumonia, or arthritis, the bacteria of which have been used in building up the curative serum. To prevent this, some biologic houses have assembled a great medley of bacteria, in order to produce a serum which may quite certainly contain the one specific cause in any outbreak. It is the continuation of the old "shotgun" therapy based upon the philosophy that, if the charge scatters sufficiently, some one shot will hit the mark. To be perfectly frank in the matter, it has not been shown that the building up of the resistance of the horse, by injecting various bacteria before drawing the serum, has exerted the least effect upon the value of the serum. According to my observations, calf scours serum acts specifically and highly beneficially in the cases under consideration, but no control experiments have been made and it is not known that serum from an ordinary horse not treated by artificial inoculation with bacteria is not just as potent and valuable as the preparations upon the market. Indeed some recent researches by my colleague, Carpenter, appear to indicate that this is true. It is not improbable also that the serum or blood from the dam of the calf is a potent agent, possibly the best substance available. This too has been tried by Carpenter with promising results. It has not been shown that any organism used by the various producers of biologics in immunizing horses for the production of calf scours serum has any basic relation to calf dysentery or pneumonia. The makers of serum have largely depended, for making serum, upon colon organisms obtained from calves dead of dysentery or pneumonia, instead of going where they clearly should go, to the fetus, for their ferment. The whole scheme of serologic handling of calf dysentery and pneu- 
monia rests upon very insecure ground, and may at any date be supplanted by more effective and economic methods. Pending definite knowledge of the fundamental biology of calf dysentery and pneumonia, or the definite establishment of a more efficient method, the present serum method should be accepted as the standard, applied intelligently and freely, and given full credit for its undoubted value, whatever may be the origin of that value.

\section{The Problem of the General Infections of the}

Genitalia as a Whole

Throughout the discussion of the general genital infections of cattle, the various lesions have been considered separately and in detail, while striving constantly to regard them as related units expressing variations in the ravages of the infections according to their virulence, the organ involved, the period of invasion, and the differences in environment. It is futile to handle cervicitis in the cow due to streptococcic spermato-cystitis in the bull with which she copulates. It is useless to rave over "contagious abortion" in one cow and ignore retained fetal membranes in another due to the same organism. It is idle to handle calf dysentery which the calf has acquired from the uterus of its dam, without trying to heal the diseased uterus.

Many writers cry out that "contagious abortion" is causing tremendous losses, that its ravages are constantly growing more serious, and that unless halted it will soon ruin the dairying and cattle breeding industry. They are right. The theories they promulgate are the foundation of the peril. So long as sterility, abortion, retained fetal membranes, metritis, calf sepsis, dysentery, pneumonia, and the endless list of other phenomena of this great group are described as distinct, independent maladies and their relation to each other denied or ignored, all the phenomena of the group will increase. The multitude of lesions and phenomena described in the preceding pages are not distinct diseases in any true sense. Each of them might better be likened to bricks and mortar which, when properly combined and arranged, constitute a structure of tremendous importance. 
I have purposely given the genital infections of cattle the first place in this treatise, because they lead in importance in the economic interests of the owner and the state, and in regard to human health. At the same time they serve admirably as a basis for comparative study of the genital infections of other species.

There is pressing need for the building up of a comprehensive plan where the dairyman, cattle breeder, and veterinarian may meet upon common ground and intelligently apply effective measures for the control of these appalling losses. The problem is many-sided and intricate. Most lamentable of all, but little is actually known regarding it. Enough is known however to warrant the unqualified declaration that means are at hand, and are being applied in many cases, which are minimizing the losses in a highly gratifying degree and giving promise of great advancement with conscientious study and intelligent coöperation.

In the scheme which has been followed in the preceding pages and which is summarized below, the $B$. abortus of Bang is not recognized as the specific cause of abortion or other disease, but it is not denied that it may cause abortion. At the same time it is held that other bacteria within the genital organs may and do cause lesions not distinguishable at present from those alleged to be caused by the Bang organism. The plan for advancing the fertility of cattle has, therefore, for its aim the destruction or control of all pathogenic bacteria invading any of the genital organs of either sex, at any age. Such a plan does not weaken but strengthens the efficiency of the control of the B. abortus infection, and more certainly eliminates or minimizes the latter than if measures were applied to it alone.

\section{A. The Assembling of Herds. The Addition of Breeding Animals to Established Herds}

The task of selecting animals to constitute the foundation of a herd and the addition of animals to an established herd for the purpose of improving it, is a complex and difficult problem. Most purebred cattle are purchased upon their 
pedigrees, including their show and dairy records, and upon their individual appearance. In purchasing cattle the buyer should recognize certain fundamental principles.

1. Pedigree is of no value unless supported by fertility. In order to be fertile the animal must be physically sound. If any chronic disease, such as tuberculosis, is present, the fertility of the animal is lowered or destroyed. Some cows and bulls with tuberculosis breed, it is true, but when a large number of tubercular animals is assembled the group, as such, shows low fertility. Besides, the disease is a general menace to the herd. The laws and customs of sale now safeguard the buyer fairly well in many states. The laws also forbid the sale of animals with such acute infections as anthrax. There are no laws, and no laws are possible of enactment, which can adequately protect the buyer against the diseases of the genital organs which may interfere with or destroy the reproductive power of the animal. At the same time the genital infections constantly threaten to pass from a diseased individual to another which is sound or to one which carries a less perilous infection.

2. The buyer should assume that the cattle in all herds carry infections in their genital organs which may ultimately lead to sterility, abortion, retained afterbirth, calf scours, and other unwelcome phenomena. He should further realize that there are vast differences in the virulence of infections in the cattle of different herds and of different individuals in the same herd. In some herds the infections are so mild that little or no visible harm comes from their presence; in others the reproductive efficiency falls so far below the ideal that the herd is economically a dismal failure. Between these extremes there is every gradation.

3. The prospective buyer should not expect the average seller to volunteer all information regarding diseases among his cattle interfering with reproduction. Each herd of size has cows which have aborted because of contagion, but no breeder posts a sign on his stable giving notice to the public that he has "contagious abortion" in his herd. Some bacteriologists come to the aid of the breeder by asserting that 
B. abortus is the cause of abortion and that it disappears from the uterus of an aborter very shortly after the disaster. If then a cow aborts and the abort and its membranes are burned or buried, the stall disinfected, and the cow quarantined for a brief interval, the infection is controlled and from such standpoint the seller may declare he has no "contagious abortion" on his premises-but he may have an hour or two later. The buyer should not exercise himself seriously concerning "contagious abortion," but should try to determine whether the animals he wishes to purchase are capable of begetting or of giving birth to healthy calves.

4. Intelligent breeders aim to retain their best cattle and to discard the least valuable. It is only when a breeder exceeds his holding capacity for good animals that he becomes desirous of selling some of them. It is commonly assumed that the breeder of purebred cattle has a far greater capacity for profitably holding females than males. The difference has been vastly overdrawn, owing to reproductive inefficiency. Thoroughly healthy cows breed regularly up to twenty years and often beyond. Ideally each cow should produce one healthy calf each twelve months. If she gives birth to her first calf at two years and breeds ideally up to and including her thirteenth year, she will have produced twelve calves, six of which, upon the average, will have been males and six females. At the close of the thirteenth year, it will be necessary to have in reserve one adult from her six female calves to replace the worn-out original cow, and there will remain for disposal (or for herd expansion) six males and five females.

When the average breeding life of the females in a herd falls below six years and the average number of progeny falls below four, the preponderance of males over females offered for sale by the breeder becomes very marked. He must in the latter case retain fifty per cent. of his female progeny to replace the toll exacted by disease, whereas, with the ideal cow of thirteen years with twelve calves, he retains but $162 / 3$ per cent. of his female progeny to maintain his herd status. The breeder having a herd of low effi- 
ciency sells only inferior females, while the owner of an efficient herd must find a market for superior animals. In a healthy herd the limit for profitably holding cows and heifers is quickly reached, since ideally the female herd increases by one half its number of females of breeding age each year. That is, a herd of one hundred cows should ideally produce fifty heifer calves, and the second year another fifty. Since in the third year the first fifty heifer calves are in breeding, there should be born seventy-five female calves, and the next year one hundred. The progress in numbers goes on with increasing momentum. The actually successful breeder must quickly find a market for his females, and must sell those of good, as well as of poor quality. When, therefore, the owner of an old established herd advertises, "No females for sale," he unwittingly announces very low fertility and a very dangerous herd from which to buy cattle of any age or of either sex for breeding.

5. In purchasing a foundation herd the entire group of animals should, if possible, be purchased from one breeder. In this manner one avoids the risk of bringing into contact animals from numerous sources, each source possibly furnishing a different type of infection of the genital organs. The difficulty of determining the state of health of the purchased animals increases as their sources multiply. Purchasing the foundation stock from a single herd simplifies the question of warranty. If a heifer or cow fails to conceive, the seller of the group cannot shift the blame to the bull, since he has made a warranty on him also. This plan is also best for the seller both in fact and in policy. When a breeder sells a large group of females without depleting his herd, he gives indisputable evidence of the sexual soundness of his cattle, which another prospective buyer of a single sire for his established herd may accept as good evidence that he is being offered a sound bull.

6 . The best place for a buyer to go to learn of the health of the herd from which he contemplates purchasing stock is to the nursery. If a large number of calves, in proportion to the number of cows in the herd, is present and the calves 
under four months of age are vigorous and healthy, the herd is a comparatively safe one from which to purchase. If the number of calves is low, and they are gaunt or pot-bellied, with dirty rough coats, or their tails are smeared with feces, the herd is an unsafe one from which to buy. In such herds no animals may be shown for sale until over three to four months old, when perhaps they have attained apparent vigor. This should not deceive the alert buyer. The breeding health of the herd should be based upon the comparative number of calves and their health up to one hundred and twenty days old.

7. The prospective purchaser should regularly guard his interests by having a skilled veterinarian examine in detail the genital organs of each animal prior to completing a purchase. If a bull is of breeding age, it is best to add to a thorough manual examination of his genital organs, a microscopic examination of his semen with reference to the number, motility and conformation of the spermatozoa as well as a search of his semen for bacteria. The method of making such examinations has already been considered. The genital organs of cows and heifers should be examined per vaginam and rectum. If a heifer has been bred thirty or more days, or a cow sixty or more days, the veterinarian can determine if she is pregnant. Such examinations should not displace or affect warranty. If disease of the genital organs is present, the prospective purchaser does not wish the animal, whatever warranty may be offered. Frequently absolutely and incurably sterile females are conscientiously sold as pregnant. Occasionally an animal without genital organs is sold as a pregnant heifer or as a heifer capable of breeding. It is best to settle such cases before purchase occurs.

8. The progressive, conscientious breeder should have complete orderly breeding records for each animal and for the herd as a whole. These records should be frankly open for inspection to any prospective buyer. If a cow has been bred three or four times in order to secure pregnancy, the prudent purchaser will not buy, whether she is pregnant or not, or, if he buys, should weigh fully the risk. Such an animal should be purchased at a comparatively low figure. 
9. The purchaser should demand and receive for each animal a specific written warranty. If an animal is being purchased as pregnant, she should be warranted as pregnant at a stated date and place of delivery, and, failing in the terms of such warranty, the purchase price should be returned. This may sometimes be modified by agreement at the time of purchase and made a part of the contract, by which an alternate animal is named, which, in case the original fails, may be substituted at an agreed price. The plan which some breeders try to carry out of substituting another animal "equally good", in case of breach of warranty, is unsatisfactory, and the buyer should see that no such condition enter into a bill of sale. If an animal sold for breeding purposes and so warranted by the breeder is unfertile, the purchase price morally and legally belongs to the purchaser and should be paid as promptly as a banker honors a check against a deposit. A warranty of pregnancy should ordinarily apply to the date of delivery only if determined by veterinary examination. If the determination is not made by such examination, a warranty of pregnancy is unsatisfactory. If a breeder believes he has sold a pregnant cow or heifer and the purchaser reports later that she proved non-pregnant, the breeder may believe that she has aborted and that consequently the warranty was fulfilled.

Warranty against the phantom disease of "contagious abortion" is impracticable and may lead to endless litigation. There is no legal definition of "contagious abortion", no scientific definition of it given or possible, and no means of diagnosis which is applicable in the exchange of cattle. This question has already been discussed at length. I think it best neither to demand nor give a warranty against abortion. The warranty had best be limited to one of three conditions: the female is fertile, she is pregnant, or she will give birth to a calf at an approximately given date. These three items are each definable and determinable.

The warranty of fertility should definitely apply to the point of destination-not to the place of sale. A purchaser in California has no use for a heifer or bull which is said to 
be fertile in Maine but proves to be unfertile after transportation to the purchaser's premises. In one case, where suit was brought by a buyer on the Pacific coast against a breeder on the Atlantic coast, the latter set up the plea that the transcontinental journey had destroyed the fertility of a young bull. Many sympathetic breeders were brought into court who swore that railway journeys frequently render fertile bulls sterile. The jury failed to agree. Unquestionably the bull was absolutely sterile on the day of the sale and had always been sterile. The statutes of the state made the sale of the bull for breeding purposes a warranty of fertility. Had there been an explicit written warranty of fertility to the buyer, whose address was known to the seller, no such claptrap evidence could have had weight.

In the present state of the health of cattle, the purchaser should also require a warranty against tuberculosis to the extent that the animal shall pass a satisfactory tuberculin test after it has reached its destination. A tuberculin test upon the breeder's premises is his test for his use. He must make that now for interstate shipment. A test at destination is the purchaser's test for his security. The breeder is entitled to satisfactory assurance that infection in transit or at destination will not occur and that the tuberculin test will be fair.

\section{B. The Equipment for Cattle Breeding and Dairying.}

Proper equipment is a fundamental necessity in the growing of healthy cattle or other animals. The plans should be such as to afford the highest possible general and sexual health of the animals. There is an irrevocable law that, the greater the mass of animals and the more intimately they are brought into contact without special hygienic provisions, the greater the ravages of disease. If it is desired to establish a large herd, special provision needs to be made against the dangers of its largeness. Aside from the highly contagious maladies like foot-and-mouth disease, which the state regulates, contagious or infectious diseases are chiefly spread through the eating of contaminated food or through sex con- 
tact. The breeder, or dairyman should therefore concentrate his energies upon these two perilous points of contact, in his planning for the conduct of a herd. The hygiene of massed individuals has had by far the highest development in the human family, where the first principle is that, however near the individuals of a great mass shall approach each other, each shall preserve its identity and in an important sense remain isolated-each shall have separate eating and drinking equipment, the excreta from one individual shall not contaminate the water or food of the mass, the sputum of the individual shall not be cast upon the floor or elsewhere to dry up, turn to powder and be inhaled by others. These are not principles of human, but of universal hygiene. The more thoroughly the cattle breeder applies these principles, the better the health of his cattle. Many cattle may be safely assembled in a small area, if proper regard is had for these principles. The number of healthy cattle which may be safely assembled in close contact is unlimited. The danger arises from the introduction of one or more animals which are bearers of disease. In planning an establishment for cattle breeding or dairying, the plans should receive greatest emphasis at the chief points of danger. Two of these demand special thought in relation to equipment-the nursery and the maternity stable. The nursery has already been discussed under the "Congenital Diseases of Calves".

Each establishment where cattle are stabled should have sufficient maternity stalls to accommodate each cow or heifer at the termination of pregnancy for a period of at least ten days. The stalls should be commodious, comfortable, and capable of being readily and efficiently disinfected. As in the calf stalls, the partitions between the maternity stalls should be perfectly tight and afford complete isolation. A properly equipped maternity stable serves a double purpose. Owing to better opportunity for handling, it protects the animal against the consequences to herself of any infections which she may bear in her genital organs, and it guards against the contamination of premises, food or water by infectious discharges. As a rule cows at the termination 
of pregnancy have genital discharges. After abortion in advanced pregnancy, and after calving at full term, associated with retained afterbirth and metritis, the discharges of highly virulent pus are very voluminous, reaching quarts and even gallons daily. If such cows are kept in stanchions in the milking stable, the discharges largely drop into the gutter and go out with the manure. However, they are not wholly disposed of in this safe manner. Portions of the discharges reach the udder of the affected cow or various portions of her body and of the bodies of adjacent cows and may in part, as dust or otherwise, reach the milk, or the feed and water of the cows. It is yet worse when the affected animal is at pasture and the discharges contaminate the food of other animals. While I hold it has not been shown that the eating of such discharges by pregnant cattle can cause interruption of the existing pregnancy, I regard it nevertheless as unhealthy and not without peril. I consider it of far greater peril to a young calf which may eat such contaminated food.

The maternity stable provides greater security against errors with milk. A conscientious dairyman does not wish to sell milk from a sick cow, but when such an animal is in the dairy stable her milk is liable to be sent to market through error. Sometimes drugs, such as iodoform, which taint the milk repulsively are being used in uterine diseases, and by error the milk is included with that of the herd and is sent to market. This would generally be prevented by the use of an adequate maternity stable. The chief purpose served by such a stable is that it offers the best possible facility for giving to a puerperal cow that attention which will most safely and promptly restore her genital health if diseased, and best guard it if well. There is no time in the life of a cow when care and skill can accomplish as much as during the first few days following the termination of pregnancy. 


\section{The Systematic Control of Genital Infections in Herds.}

The handling of each phenomenon occurring in the course of the genital infections of cattle, including the congenital infections of calves, each independently of the other, is wasted effort. Science and economy alike demand a systematic effort aimed at the entire complex group, each effort having a definite relationship to the activities in combatting each other phenomenon in the problem as a whole.

(1) When a breeder desires to improve the reproductive efficiency of his herd, it is essential first to learn definitely the state of sexual health of each animal of breeding age of both sexes. The bull or bulls should be examined in detail, as described on page 73, and under "The Genital Infections of Bulls". If the bull or bulls are found unfertile or of low fertility, they should be temporarily or permanently discarded. The genital organs of each female of breeding age should be examined in detail, as described on page 79 , and the findings recorded approximately as suggested on page 83.

The females should then be arranged in five groupspregnant, probably pregnant, ready to breed, under treatment or observation, and incurably diseased. The second group (probably pregnant) is a tentative one which may be eliminated sixty days after breeding, when pregnancy can be diagnosed definitely. The incurably diseased should be promptly eliminated from the herd. The handling of those under treatment should be vigorous in order that the animals may be shifted to the group which is ready to breed as rapidly as expedient. The first examination having been recorded as suggested on page 83 , each subsequent examination should be recorded as indicated in Fig. 212, and each consecutive record attached to the original. Should an animal fail to respond to the measures applied, she should be shifted at the earliest date of certainty to the incurable group and eliminated. In large herds of purebred beef cattle, the cows and heifers should not only be classified as suggested, but should be separated into four separate groups for economic handling, these groups consisting of pregnant, 
ready to breed, under treatment, and weaned heifers too young to breed. The caretakers then have their work simplified. The pregnant herd requires only to be watched for calvings or abortions. Those ready to breed are to be observed closely for estrum; when heat is observed, it merely remains to determine to which bull she is to be bred. Es-

\section{STERILITY-ABORTION}

RE-EXAMINATION

Owuer

Address .

Date -

Date of last examination

Name or number of Animal

Record of estrum and service since last examination

Character of estrum, regular or irregular

General condition

State of lactation

Right broad ligament

Left broad ligament

Vulva

Vagina

Cervix Uterus

Right ovary

Right oviduct

Left ovary

Left oviduct

Diagnosis

Prognosis

Treatment applied

Treatment advised

REMARKS :

FIG. 2I2.-Re-Examination Records for the Genital Organs of Cows.

(See also Fig. 37.)

trum in the heifers too young to breed and in cows under treatment is of no direct interest. The breeder will generally profit also by the use of the estrum and service card suggested in Fig. 213. These filed numerically or alphabetically by name afford a helpful index in breeding. They show 
at a glance when each pregnant cow is due to calve. In non-pregnant cows not yet ready to breed, the duration of time since last calving is shown, and, in cows which are being bred, the date when the next estrum is due, giving an opportunity for close watch.

(2) When pregnancy terminates (parturition or abortion), the genital health of the animal should receive attention. The uterus should be examined shortly afterward. If the afterbirth is retained or the animal has aborted, handling should begin at once. If parturition has been apparently

ESTRUM AND SERVICE RECORD Herd No.

Name of Animal.

Year of Birth.............. Last pregnancy terminated

Dates of Estrum " and Service for year

(a)

\begin{tabular}{l|l|l|l|l|l|l|l|l|l|l|l|l}
\hline & \\
\hline Date of Service & & & & & & & & & & & & \\
\hline Bull Used & & & & & & & & & & & \\
\hline Menstruation (3) & & & & & & & \\
\hline Next estrum due & & & & & & & & & \\
\hline
\end{tabular}

Estimating the duration of pregnancy at 280 days animal should calve

Pregnancy from above service terminated

Result $\left\{\begin{array}{lc}\text { Abortion } & \text { Disposal } \\ \text { Bull Calf } & \text { of } \\ \text { Heifer Calf } & \text { Calf }\end{array}\right\} \begin{aligned} & \text { In herd as No. } \\ & \text { Died } \\ & \text { Sold }\end{aligned}$

(1) When in eatrum but not werved write day of roonth in ( )

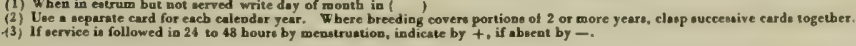

FIG. 2I3.-Estrum and Service Index Card for Keeping Breeding Records.

normal, the examination may be deferred until ten to twenty days post partum. In my experience, however, I find large volumes of tarry blood with a flaccid, much enlarged uterus at three to four days post partum, and this regularly ends in a mild pyometra not recognizable clinically except when the uterus is examined by douching. If the tarry exudate is recognized, the pyometra can be anticipated and evaded. In herds where metritis and retained fetal membranes are common, it is preferable to assume that metritis is impending in all cows and to introduce capsules of iodoform or boric acid 
and bismuth subnitrate, or these drugs suspended in oil, as a rule of practice, within a few hours after the termination of pregnancy. The details are considered under "Puerperal Infections". Re-examination should be made as conditions may suggest. At forty to sixty days after calving or aborting, if there is no marked disease, an examination of the genital system should be made to determine if the patient is ready to breed. If a corpus luteum is present and early breeding is desired, the corpus luteum may be dislodged and estrum induced. Unless there is reason for haste, the corpus luteum should be left undisturbed. If the condition of the genital organs is satisfactory, she may then be bred.

(3) When a heifer has reached the age to breed, or when sufficient time has expired since a cow has calved and the genital organs are healthy, the animal may be bred. The best time to breed a cow or heifer is the earliest hour in estrum when she will stand for coitus without restraint. The spermatozoa will then have ample time to reach the pavilion of the oviduct and be ready to fertilize the ovum immediately it is discharged from the ovary. Prior to coitus it should be seen that the external genitalia of the female are clean. It is best to wash the vulva, and especially to free the vulvar tuft of hairs from dried crusts of pus which may be caught by the penis and injure it. If the vagina is irritated by the nodular venereal disease or other cause, it should be douched with physiologic salt solution or 0.25 per cent. Lugol's solution. The copulatory organs of the bull should be similarly cleansed by douching, as has been previously described, special attention being given to the preputial tuft of hairs. Coitus may then occur immediately. Immediately after coitus the copulatory organs of the bull should again be douched.

The importance of washing or douching the copulatory organs has been greatly overestimated by many and underestimated by others. The douching can not exert any direct influence upon deeply seated infections. Its value is strictly limited to superficial infections of the copulatory organs. It has an important place in sex hygiene. Douching prior to 
copulation partly eliminates infections lodged in these areas and tends to minimize the danger of infection passing by sex contact from one animal to the other. The douching, lessening the amount of infection in the vagina at the moment of coitus, lowers the danger to the spermatozoa during their intra-vaginal existence. When the owner of the bull does not know the record of a strange cow, the douching immediately after coitus is an important safety measure, but a good bull should not be permitted to copulate with a cow not reasonably known to be sound.

Vaginal douching immediately after coitus is not compatible, so far as known, with good breeding results. It would probably prevent conception in a vast majority of cases. Two or three hours after coitus, douching of the vagina does not interfere with conception, but may render it more secure. The spermatozoa quickly pass into and through the cervical canal, where the vaginal douche can not affect them. The douche does, however, allay the vaginal irritation of coitus, especially in heifers with nodular venereal disease of a severe type. In these the vaginitis of coitus is very marked, but may be quite obviated by timely douching with 0.25 per cent. Lugol's solution. Many bulls of low fertility ejaculate bacteria with their semen. The spermatozoa start rapidly upon their journey and probably largely leave behind the bacteria, which timely douching may mechanically eliminate.

The highly interesting and probably very valuable field of post-coital uterine douching has not yet been adequately studied. Cervicitis is one of the commonest, if not the commonest lesion in the genital organs of the cow, and constitutes a formidable menace to conception and pregnancy. Copulation increases cervicitis and not infrequently (streptococcic semino-vesiculitis of the bull, etc.) causes cervicitis in cows which were healthy up to the time of coitus. Although spermatozoa frequently pass unharmed over the infected cervical mucosa, at this time deeply submerged beneath the flood of estrual mucus, if conception occurs, the fertilized ovum upon its descent into the uterus is exposed 
immediately to the bacteria present. The uterine douching may partly wash the infection out or may chemically destroy it. In my experience post-coital uterine douching often reveals muco-pus in the cervix or cervical end of the uterus. The healthy vigorous spermatozoa have probably left the uterus and ascended the oviducts within three to four hours after coitus. From that time until the fertilized ovum descends into the uterine cavity, uterine douches are without peril to conception and, if properly done, decrease the perils of cervical and uterine infections. The unknown element in this field is the minimum duration of time required for spermatozoa to pass through the uterus and enter the oviduct, and for a fertilized ovum to traverse the oviducts and reach the uterus. I have stated elsewhere that in one instance I successfully douched muco-pus from the uterus of a pregnant cow thirteen days post coitus. It is reasonably certain that the fertilized ovum was not in the uterus at that time, but it is not certain, and I believe is improbable, that the duration of time in this instance was typical.

The number of copulations which a bull should be caused or permitted to make has not been sufficiently studied. There are certainly very wide variations in individuals in the powers of sexual endurance. Some breeders have attempted to state arbitrarily the number of cows which a bull may serve. The capacity of a bull should be based upon the number of copulations it may be prudent to have him make. The number of cows will then be dependent upon the fertility of the bull and the average fertility of the individual cows which he is to serve. Reliable data of the average rate of conception in cattle are wanting, and most persons believe the conception rate to be far above the facts. In one extensive purebred beef herd, where both bulls and cows were grazed throughout the year, and in which the genital organs were exceptionally healthy, there were recorded 2675 copulations, with 1141 known pregnancies, resulting in the birth of $1061(39.66 \%)$ of viable calves, $46(1.72 \%)$ non-viable calves, 34 (1.31\%) recorded abortions, and 1534 $(57.34 \%$ ) copulations without observed result. The data 
include copulations with a number of hopelessly sterile cows, as well as a number of copulations by a sterile bull. I think these data fairly representative of the more fertile purebred herds. I know the results are far better than in some herds. The timely elimination of the hopelessly sterile cows and the elimination of the sterile bull would have raised the efficiency notably. A far greater advance would have been made by taking curably infected cows out of breeding for a few weeks and giving them proper surgical attention. As the data stand, they furnish some helpful suggestions regarding the number of cows which may ordinarily be assigned to a bull.

Breeding is carried on by two separate plans-massed or seasonal breeding when it is desired to have all calves born during a limited season, and perpetual breeding throughout, or during nine or ten months of the year. A bull may evidently make with safety a greater number of copulations during the year if evenly distributed throughout, and can safely make a greater number of copulations in a short interval (one week or one month) when he is to be sexually idle the remainder of the year. Such observations as I have been able to make have led me to regard an average of two copulations per week throughout the year as the probable maximum of efficiency, measured by the percentage of pregnancies, their safety, and the vigor of the young. Taking the above data as a general average of fertility, a healthy adult bull would suffice for about forty cows (100 copulations).

When the breeding is massed, the frequency of coitus should still be held under reasonable control. Probably the frequency of coitus may be advanced to four or five times a week for a period of four to six weeks. Bulls have done more than this, but my studies indicate that usually they undergo a gradual decline in fertility year by year and go down so far that they do not eventually regain their initial sex vigor. When mass breeding is undertaken in pasture, the ratio of females must be very low, especially if all are ready to be bred when the bull is placed with them. Under 
such conditions the bull may copulate several times with a cow during one estrum. If twenty-one cows are assigned to a bull and each has an estrual cycle of twenty-one days, there will be an average of one cow in estrum daily, with the inevitable result that on some days several cows will be in estrum. The number of cows under these conditions should therefore be below twenty, preferably not over fifteen. If the breeding period extends over ninety or more days, more cows may naturally be assigned to one bull. The breeding of ordinary grade beef cattle in pasture produces the maximum ratio of vigorous calves, if the bulls are healthy and abundant, but, when a perilously infected bull is used, the disaster is inevitably as great, and may be greater than by individual, controlled breeding. The ordinary grade beef cow running free at pasture commonly enjoys the maximum of general and sexual health, but it is far from sufficient to withstand the shock from a virulently infected bull. When the bull consorts constantly with the cows, he immediately recognizes estrum, and coitus occurs promptly; in controlled breeding estrum is frequently too far advanced when discovered for coitus to succeed. Many estrual periods are completely overlooked. If observations are made but once daily, a cow may readily come in estrum shortly afterward and the estrum disappear before the next search is made. Indeed the healthiest cows have the shortest duration of estrum, frequently dropping to, or below, fifteen hours. This makes it essential to the highest success to make observations for estrum at intervals of about twelve hours. The natural inclination of some breeders to suspend breeding operations on Sunday reduces the efficiency 14 per cent. If the estrual cycle in the individual is other than twenty-one days, her estrum may be recognized at its next appearance. But the cycle in the healthiest cows is most frequently twenty-one days, and, once in estrum on Sunday, always in estrum on Sunday, unless the estrual cycle is broken by pressing the corpus luteum from the ovary.

The maximum degree of safety in sex hygiene is attained 
by the permanent mating of one female with one male. Whenever polygamy or polyandry is substituted, each individual in the group becomes exposed through sex contact with each other member of the group. Genital infections increase in virulence with repeated coitus. The larger a group of apparently sound breeding animals, the greater the danger that some one of the group harbors a highly perilous infection. In practice a certain group of cows should be assigned to a given bull and the assignment rigidly maintained. Nothing can well be more imprudent than the practice of shifting a sterile cow from one bull to another. I have records where the breeder had used five or six different herd bulls on one cow during a single year. Each of the bulls thereby exposes the cow to any genital infections he may carry, and she in turn exposes each subsequent bull to the original infections in her own organs and to any which may have been deposited therein by each preceding bull. It is sexual pandemonium. If the bull and the cows assigned to him are rigidly maintained as a distinct unit, the breeder soon knows of the fertility of the bull. If he is unfertile the remedy is clear. If nearly all cows conceive promptly and one is sterile after repeated coitus, she is evidently at fault and the danger from her should be eliminated promptly by overcoming the infection or by sending her to slaughter. There is a false assumption that some cows will not conceive to one bull, but may do so to another of equal fertility. Bulls show every gradation of fertility, and a cow is naturally more certain to conceive to the bull of highest fertility, but the bull of low fertility should be eliminated instead of used unsuccessfully upon cows and later supplanting him with a highly fertile bull, in which case the latter becomes exposed to the infections deposited by the former.

The permanent assignment of certain cows to a bull may at times lead to the complication of several of his group coming into estrum simultaneously or in rapid succession. This need cause no material embarrassment. The rule should be made, and supported vigorously, restricting the 
bull to one coitus per day and only rarely permitting coitus on two successive days. If estrual periods become massed, instead of imperilling the sexual health of the bull, break the estrual cycle in one or more animals by pressing the corpus luteum from the ovary three to five days before it is desired to have her come in estrum.

The importance of maintaining the general vigor of breeding animals has already been mentioned, but it may be well to emphasize some special points. Bulls are largely kept too closely housed and far too highly fed. The bull needs abundant physical exercise, and should either be given daily work (which is the most economic and best method for retaining physical vigor) or provided with an extensive paddock where he can come and go at will and have abundant exercise.

The feeding of purebred dairy cattle for high milk and butter yield is one of the most complex and delicate operations in animal husbandry. Many breeders are record-mad and crowd cows beyond their powers of endurance. Many world-famous cows never breed after making a phenomenal record. The breeder is left with a cow which holds the record in her class for milk or butter and is incapable of yielding any of either. Nymphomania closes the breeding career of so many high-record cows that the suspicion may well be aroused that some of these performances are actually pathologic, and that the great yield of butter fat is really the consequence of unseen changes going on in her ovaries, which later culminate in the obvious cystic ovarian disease. The destruction of the reproductive powers of a great cow by imprudent handling in an effort to advance her record is a crime against the state. The breeder has no greater right to ruin a highly valuable cow than he has to burn or otherwise destroy a valuable house which he legally owns.

Analogous conditions prevail among breeders of beef cattle, who, at great peril to the reproductive powers of their animals, feed them inordinately for show. It is notorious that cattle which are extensively exhibited largely become 
sterile. Males and females sometimes win the highest prizes in breeding or in dairying classes when they are hopelessly sterile or are dry and will never again yield milk. Much of the harm now being done could be overcome by the breeders' associations and fair managers by offering prizes for fertility in individuals and in herds, and by basing all milk and butter records and all prizes at fairs upon high standards of fertility. If an animal is shown as a purebred for breeding purposes, let its fertility be proven beyond doubt. If a cow is shown in the dairying classes, let it be proven that she is capable of yielding milk and butter.

The most regrettable feature in the imprudent destruction of the breeding powers of cattle is the fact that the ruined animals represent the best individuals of their respective breeds and should by every scientific and moral consideration be retained as efficient progenitors of their excellent qualities. Instead, when cattle achieve renown, they are ruined by a false ambition to push them one more point. Then the breeder must step downward and backward, begin again with ordinary individuals, and laboriously produce another illustrious family, which he may wreck upon the same shoal.

The handling of the genital infections of cattle by means of biologic products has been discussed at some length under abortion and congenital diseases of calves. Nothing could be more earnestly desired than a convenient specific for this destructive group of infections, but thus far no notable success has been achieved, unless calf scours serum is excepted. In this instance the question has been raised whether the success may not be due to the plain serum rather than to the changes brought about by the bacterial inoculations of the horse. At present the outlook for great victories with "biologics" is not hopeful and the representations made by vendors of abortion serums, bacterins, and vaccines are not warranted by any recorded facts.

Some who believe in "contagious abortion" as a specific disease, like tuberculosis or hog cholera, advise the enactment of statutory laws placing these general infections 
among the specific diseases subject to obligatory report to the state veterinarian or other official, who would quarantine and otherwise restrict the movement of the diseased or suspected cattle. Indeed such laws already exist in some states but so far as I have learned the statutes do not clearly define "contagious abortion", but leave the definition to the attending veterinarian or the breeder. As a result the statutes and the disease have not come in conflict. Thus far it has been impossible to define "contagious abortion" in such a way as to render a law regarding it workable. If it is defined as the presence of the $B$. abortus in the bodies of cattle, its presence in the milk of a large percentage of dairy cows would render the application of such a law impossible without prostrating the dairy industry. Even then the $B$. abortus would still flourish in other animals. If a law is to be made which will apply only to the $B$. abortus in the uterus, it is the common view that it persists in the uterine cavity only for a few weeks after the termination of pregnancy, and quarantine for those few weeks could not and would not favorably affect the reproductive powers of the animal. The chief objection to legal restrictions is that, if the report of abortion be made compulsory, the breeder can not afford to call the veterinarian, since his attendance and report mean quarantine with financial loss or ruin, while the restrictions placed upon the herd are absolutely impotent to better conditions.

\section{The Genital Infections of Cattle as an Economic Problem.}

No infection or group of infections of domestic animals offers at present a more serious or pressing economic problem. The infections are essentially omnipresent and imperil life and fertility at every step. The spermatozoa and ova must run a gauntlet of threatening infection before fertilization may occur. The fertilized egg has its perils as it passes through the oviducts, and throughout pregnancy the peril of infection both to the intra-uterine young and to the mother never ceases. The danger does not end with parturition but the infection, persisting in both the mother and 
the young, continues its destruction. The United States Department of Agriculture has made the estimate that the phenomenon of abortion costs the nation twenty million dollars annually. The dairy cow, in order to yield the maximum amount and quality of milk, should give birth to one healthy calf each twelve months. The purebred herds drop to between 50 and 75 per cent. of this ideal during an appallingly brief breeding career. Including all cattle of dairy breeds, the reproductive efficiency does not exceed one calf in each fourteen months, so that each cow is kept upon an average two functionless months. If the loss is rated at five dollars per cow per month, the $1,500,000$ cows in the State of New York alone aggregate an annual loss, due to delay in conception, of $\$ 15,000,000$. That is but one item of loss. When the total losses are considered, the sum is many times the one item noted, making this easily the most destructive group of infections of cattle. The statement is now and then made that, next to tuberculosis, "contagious abortion" is the most destructive disease of cattle: That conclusion is based, if not wholly, at least chiefly, upon the observed expulsion of the fetal cadaver and technically restricted to the damage alleged to be inflicted by B. abortus.

\section{E. The Genital Infections of Cattle in Their Relation to Human Health.}

Milk and its derivatives constitute the most important food of the human race. Milk has been clearly shown to be a dangerous carrier of infections, such as scarlatina, typhoid fever, putrid sore throat, and others. These are largely contaminations occurring outside the udder. After Koch had discovered the tubercle bacillus and thought bovine and human tuberculosis identical, it was believed for some years that much human tuberculosis was due directly to the use of milk from tubercular cows. Later researches have modified that view, and, while still considered as of essential importance to human health, bovine tuberculosis is not thought to be as perilous for man as it was at an earlier date. More recently, following the discovery of the 
B. abortus and the assignment to it of specific abortifacient powers, the question arose regarding the possibility of the infection invading pregnant women and causing abortion. A few inconclusive observations were recorded. Occasionally the blood of men and women agglutinates the B. abortus, but no extended study of the prevalence of the organism in the human body has been attempted. Natural infection with $B$. abortus has been recorded in swine and guinea pigs, and experimental infection in all domestic mammals. It would not be surprising, therefore, if a thorough search showed the $B$. abortus to be a very common resident of the human body without striking evidence of peril to health. The general infections of the genital tracts of cattle, which cause such an appalling amount of disease, not only in adults, but in fertilized ova, the embryo, the fetus and the calf, probably offer at this time one of the most valuable fields for the study of animal diseases in connection with human health. There has been much inconclusive argument regarding the virtues and vices of raw and cooked, clean and dirty, rich and poor milk as a healthful food for man, especially for children and for the infirm. It has appeared to me unfortunate that the primary study of the effect of feeding milk to the young has been carried out chiefly upon children. There has been built up a system of dairy inspection based upon certain theoretical considerations of the outside of the problem. If that is properly gilded, the deeper facts are ignored. One often sees "certified" milk being sold at an advanced price as food for children, while the calves in the dairy, to which part of the same milk is being fed, are sick and dying. So our dairy inspection maintains in effect that milk which is perilously toxic for calves is "certifiable" for infants.

The anatomic position of the mammae of the cow justifies the general assertion that the milk contains some parts of any genital discharges which are occurring. Hygienically this contamination is theoretically reduced to the minimum through the formal rules for the production of "certified" milk. In the average dairy, sixty per cent. of the cows, if examined five days after calving, have two or three ounces 
to as many pints of tarry blood or of pus in the vagina or uterus. The uterus, which should at this time be firmly contracted and so involuted that it may be picked up per rectum and held in the hollow of the hand, is instead a great flaccid organ, hemorrhagic and paretic. There is inevitably a discharge, but this is so common that dairymen and dairy inspectors call it "normal". Portions of these disease excretions flow down the tail and thighs, and upon the udder and teats. The ordinary washing of the udder, as practiced in certified dairies, does not assure the exclusion of such infections from the milk. This is sharply emphasized by the clinical fact that mammary infection during the puerperal period is commonest in the posterior quarters of the udder, which are most exposed to genital discharges, and that, the more intense the genital infection (e. g. retained fetal membranes), the more probable is mammary infection. So far as I am aware, no technical study has been made of the bacteriology of intra-mammary milk from cows with intense genital infection in comparison with milk from cows with typically healthy genitalia. It is logical to assume, until we have more definite knowledge, that intra-mammary milk contains as a rule more bacteria in a cow with severe genital infection than in one which is healthy. By any practical method of milking there is inevitably added a variable proportion of the genital discharges which adhere to the exterior of the udder and adjacent regions. The inevitable bacterial contamination of milk from genital discharges is not confined to the puerperal period. I have evacuated quarts and gallons of fetid pus from the uteri of cows in certified dairies, when the history indicated undeniably that the excessively fetid pyometra had existed for more than a year and the cows had been repeatedly inspected and passed by the dairy inspectors representing great cities. Much of the present "skin-deep" dairy inspection is a farce, the price of which, in human health, can not be accurately computed.

The entire field of the genital diseases of cattle offers a priceless opportunity for the study and advancement of human health. The genitalia of cattle offer the most available 
field for the clinical study of the basic phenomena of ovulation, menstruation and fertilization. Slaughtered cattle of the abattoir furnish inexhaustible material for studying the bacteriology and pathology of the genitalia of both nonpregnant and pregnant animals. The fetal membranes afford unsurpassed opportunity for the study of bacterial invasion of the embryonic sac. Within the alimentary tract of the fetus, as a constituent of the meconium, is stored up, alive though frequently inert, bacteria the presence of which affords a reliable history of the bacteriology of the pregnant uterus and a suggestive prophecy of the pathologic changes which may follow birth. The new-born dairy calf, fed as it is by artificial means and upon the milk of dairy cows, offers by far the greatest and most reliable source for an experimental study of the feeding of milk to the new-born. The influence of pasteurization and sterilization of milk before feeding, and the relation of bacteria borne in milk and of bacteria existing in the meconium of the new-born to each other and to the health of the calf, are comprehensive principles not restricted to the species of animal. The bacteria commonly resident in the meconium of the new-born calf, can probably be transferred to the digestive tube of children and cause in them perilous disease. 


\title{
SECTION II. THE GENITAL INFECTIONS OF SHEEP AND GOATS
}

\author{
CHAPTER XV \\ SPECIFIC VENEREAL DISEASES
}

The Nodular Venereal Disease

While describing the nodular venereal disease of cattle, it was stated that the same infection is commonly present in the copulatory organs of both sexes of sheep and presumably also of goats. According to my observations the infection is not as severe in sheep as in cattle, probably because the sex and maternal relations are of a simpler character. So far as I have observed, the disease has little if any economic importance in sheep. Any extended consideration would be essentially a repetition of what has already been said of the disease in cattle.

\section{CHAPTER XVI}

\section{NON-VENEREAL INFECTIONS WHICH INVADE THE GENITAL ORGANS}

Necrotic Disease of the Genital Organs Associated WITH LIP-AND-LEG ULCERATION

Flook (Jour. Comp. Path. and Therap., Vol. XVI, p. 374) records an outbreak of a venereal disease among sheep. He relates that, in a herd of fifty-two yearling ewes and two ram lambs which had been recently purchased, it was noted that one of the rams had a discharge from the sheath, while the other had extensive eruptions about the mouth and nose. Nothing was seen amiss with the ewes. Afterward the two rams were taken from the band with which they had been purchased and placed with a small flock of old ewes. After 
one week Flook found nine of the old ewes showing great swelling of the vulvae with raw, bleeding sores upon the mucous and cutaneous surfaces of the vulvar lips. An examination of the rams revealed ulcerating sores in the sheath of one and eruptions upon the upper lip of the other. Apparently the general health of the animals was not disturbed.

The rams had been marked with color upon the breasts between the forelegs, so that each ewe which was served could be identified. By this means it seemed to be determined that two had contracted the disease without coition. Flook suggests that the disease may have been transmitted by the ram which had the eruptions on his upper lip, through smelling of the vulvae of these two animals, or that it might have been caused by flies bearing the disease from one animal to another, since the affection appeared during the month of August. The affected ewes were isolated and the sores dressed with antiseptics. Gradually they all recovered.

On the following page of the same journal, Sir John McFadyean contributes observations made by himself upon an outbreak which he considers identical with that described by Flook. He saw twelve ewes affected in one flock, showing intense inflammation, swelling and tenderness of the vulva. The vulvar musosa was inflamed and some ulcers were present both on the mucous membrane and the skin. There was a slight purulent discharge from the vulva. As these ewes had been served by a hired ram which had been returned to his owner six days previously, McFadyean failed to see the animal. McFadyean attempted to produce the disease experimentally by collecting the discharges upon pledgets of cotton, one of which was introduced into the vulva of a ewe, another into that of a cow, and a third into the sheath of a wether.

Though two of these experiments proved negative, a swelling of the sheath was apparent in the wether two days after the inoculation. This was still further increased on the fourth day and accompanied by a slight discharge. The 
swelling at this time was sufficient to make the exposure of the penis impossible. The symptoms continued over a period of about two weeks. On the third day there appeared on the skin near the opening of the sheath a small sore which was covered with a brownish scab. This sore continued to spread around the opening of the sheath. On the fifteenth day after inoculation, two small abscesses had formed on the prepuce near the primary sore; these ruptured the next day, leaving shallow ulcers. At this time a third small abscess had formed. All the ulcers were covered later with brownish crusts, after which they began to heal. Attempts to transfer the disease artifically from this animal to another wether and ewe failed. McFadyean did not succeed in isolating any organism which he believed to be the cause. While both outbreaks here mentioned have been comparatively mild in their course, McFadyean remarks that the disease deserves careful observation and that newly purchased rams might well be examined with reference to this affection before-being used for breeding.

G. H. Williams (Vet. Jour., Vol. XVII, p. 64) records two outbreaks of this disease. In his first, in a flock of eight ewes and one ram, two ewes and the ram were affected. There was balanitis and ulceration of the penis. He applied to the parts chinosol and zinc sulphate in solution. The animals recovered in about two weeks. In his second outbreak, three rams and forty ewes, in a flock of fifty, showed the symptoms described by Flook and McFadyean. In one ewe eruptions occurred upon the nostril. In some of his cases, the vulva was greatly swollen and distorted; in others there were extensive granulations of a dark color which protruded out through the vulva. The granulations and ulcers were penciled with silver nitrate and the entire parts washed with a solution of zinc sulphate and chinosol.

In another flock of ewes, which had not associated with this one, except that one of the rams had been with them, it was found that twelve had eruptions about the lips and noses but no genital affection was present. A second ram in this lot escaped the disease, so far as seen. In the larger 
flock of ewes, the rams were isolated and the affected ewes removed immediately from the herd. Daily inspection was made of the apparently well ewes and any which developed the symptoms of the disease were promptly separated. A few weeks later breeding was resumed without recurrence of the disease, and in due time the ewes dropped healthy lambs.

About 1909 an affection not distinguishable from that observed by Flook, McFadyean and others in England became widely prevalent and caused quite serious losses in condition and wool yield upon the extensive sheep ranges of the Rocky Mountains. It was attributed by Mohler and others to $B$. necrophorus. It was known generally as lipand-leg ulceration and was characterized chiefly by the formation of necrotic patches about the feet and lips. When breeding was attempted, the genitalia became severely involved. The mortality was low, but there was important loss of condition. I saw one flock of Western sheep which were quite generally involved after their arrival in the East. Being feeders, thus excluding coitus, the necrosis was virtually limited to the feet and lips.

The disease is apparently easily controlled by isolation of the affected, with disinfection of the necrotic areas. Cauterization of the ulcers with solid silver nitrate appears especially efficacious. When the disease is present during the breeding season, breèding should be promptly suspended until the infection has been brought under control. 


\section{CHAPTER XVII}

\section{THE GENERAL INFECTIONS OF THE GENITALIA OF SHEEP AND GOATS}

The general infections invading the genitalia of sheep have received no adequate study and veterinary literature is, as a consequence, essentially barren of any comprehensive information. The breeding value of an individual sheep is ordinarily not great and the attainable salvage by selling a sterile animal for mutton leaves an unimportant margin of loss. Consequently ewes which fail to produce young are ordinarily sent to slaughter without comment. While in small flocks of large, well-nourished sheep, there may be at times a reproductive ratio of 200 per cent., this is not reached in large flocks; in the smaller breeds the ratio falls below 100 per cent. The increase frequently drops to 75 or 80 per cent. or even less without causing comment. Frequently also ewes are not bred often, but only for two or three years, when the matured animals are sent to market and are replaced by ewe lambs.

The rest periods between births are long, giving ample time for the recovery of the health of the genitalia. Sheep are ordinarily bred during a very limited season, the duration of pregnancy is short, and both sexes quickly attain rest. The new-born lamb grows usually under exceptionally hygienic surroundings. Born as a rule in temperate weather, it is constantly on pasture and nurses its mother. Thus it escapes the filthy housing so often forced upon calves and is spared the mixed milk containing an abundance of various bacteria, to which is commonly added infection from dirty feeding pails and other sources. Accordingly lambs largely escape the digestive perils to which calves are subjected, and as a consequence grow to sex maturity with cleaner genital organs. Hence they show higher fertility in the first breeding season. Regardless of these advantages in environment and the plan of breeding, the infections of the genitalia sometimes acquire such virulence as to 
cause serious and notable economic loss. As in cattle and other mammalia, the storms of infection attract the attention, though if the question is carefully studied, there is seen to be far greater loss in the aggregate constantly occurring in one individual after another. One ewe expels unseen a small fetus or embryo; another expels at full term a cadaver which has just died; another gives birth to a weak lamb which succumbs to septicemia, dysentery or arthritis, or the ewe has retained fetal membranes. These are passed over as "accidents" in the path of reproduction and the breeder fails to measure the endless economic leak which is occurring in his herd and which is very important if once placed in correct view. When the infection attains high virulence and a storm breaks, the breeder takes alarm, although perhaps the same infection has long been at work in his herd, causing important losses while gathering momentum to break finally with fury.

As in cattle, so in sheep, the most striking result of genital infection is the observed expulsion of a fetal cadaver-abortion. In the earlier stages of pregnancy the expulsion of a fetal cadaver is not readily recognized. The embryo is very small compared to the bovine embryo, and retained chorion and amnion is not common, but the fetal placentae break away from the chorion and are often retained within the cotyledonal chalice of the uterus. The wool obscures the vulva and any moderate soiling of it. When a large fetal cadaver is expelled, it is apt to be observed and, if evidently immature, is characterized as abortion; if expelled at approximately full term, it is regarded merely as "a dead lamb" without significance. If the observed expulsions of fetal cadavers become numerous as a result of the same infections, "contagious abortion" is diagnosed.

The diseases of the testes, epididymes, and glands about the pelvic urethra of the ram have not been studied. The same is true of the ovaries and oviducts of the ewe. The principal attention has been paid to the changes going on within the uterine cavity resulting in the death and expulsion of the fetus. 
The infections which may cause abortion are undetermined and probably quite varied. The B. abortus is capable of multiplying within the uterine cavity, as has been demonstrated abundantly by various investigators. The experimental inoculations have not shown, however, that it does or can with any certainty cause a pregnant ewe to abort, although some conclude that, if the $B$. abortus is present, it can cause abortion. It is a difficult matter to prove.

The organism most frequently alleged to be the cause, or the most common cause, of abortion in the ewe is a vibrio or spirillum apparently identical with the vibrio previously described as associated with certain outbreaks of cattle abortion. The belief that the spirillum causes the abortion in those cases where it is recognized is based fundamentally upon its recognition in abundance in the stomach of the fetal cadaver. This constitutes valuable presumptive evidence.

McFadyean and Stockman ${ }^{1}$ have given the vibrio the most extended study yet published. The organism is Sshaped or spiral, 1.5 to 3 microns in length. It grows in various culture media. The colonies present an appearance like B. abortus. The living organisms are highly motile, moving rapidly in a spiral. McFadyean and Stockman believed they had proven by experimental inoculation that the vibrio they described caused the abortions. In many cases of abortion following natural exposure, the vibrio could not be found. In some cases putrefactive bacteria may have destroyed or veiled the vibrios; in other instances this did not appear to be the case. Many pregnant ewes were inoculated with the vibrios by various channels. Some of these aborted, but for the most part they did not. In some of the aborts and the uteri of the aborters the vibrio could be identified; in some it could not. The grounds for assuming that the inoculated ewes did not already carry in utero the vibrios are not stated, and apparently no controls were kept. Nothing is submitted to show that these ewes taken from their familiar suroundings would not have aborted in the same ratio if not inoculated. The evidence that a preg-

${ }^{1}$ Abortion in Sheep. Board of Agriculture and Fisheries. London, I9r3. 
nant ewe can be made to abort during her existing pregnancy by inoculating with vibrio or other organism is certainly far from conclusive. The complexity of experimental proof is essentially the same as in cattle, which has been considered at length.

Opportunity was afforded me and my colleague, Carpenter $^{1}$, for a brief study of a disease affecting pregnant ewes, many of which aborted. The owner made a practice of purchasing Western ewe lambs (that is, lambs grown upon the Rocky Mountain plateau) in the stockyard at Chicago or Buffalo, securing purebred rams, breeding them for two years, marketing the lambs, and finally the matured ewes. The operations had been remunerative and successful.

In 1917 he purchased 252 ewe lambs and imported from Canada four purebred Shropshire ram lambs as sires. This gave to each ram 59 females, involving an average minimum of 3 ewes in estrum each day for each ram. The result was 209 lambs, or an increase of 89 per cent., which indicated reasonable sexual health. The ideal ratio would have been at least 11 per cent. higher. The same rams were used the next year, no new sheep of either sex having been added. So far as known there was, with one exception, no contact with other sheep. Moreover all neighboring flocks, so far as could be learned, were healthy. The one exception as to contact was that, a few days prior to the intended breeding operations, a Tunis ram broke into the premises and copulated with some of the ewes. Twelve of these, all so far as known that copulated with the Tunis ram, gave birth to 13 crossbred lambs, all of which lived. There remained, according to the caretakers, 223 ewes, which were bred to the same Shropshire rams used the previous pear. When pregnancy in these had become sufficiently advanced to render the expulsion of fetal cadavers obvious, abortions began to be observed. The 223 ewes bred to the Shropshire rams produced 127 viable lambs (57 per cent.) Some ewes which failed to produce lambs were not observed to expel fetal

\footnotetext{
${ }^{1}$ Report N. Y. State Veterinary College at Cornell Univ., 19I8-19, page 125.
} 
cadavers, although perhaps they did so at an early date. The ewes were generally unthrifty. This may have been due partly to the food, which, though abundant, did not appear to be first-class. A formidable objection to attributing the bad condition of the ewes to low-grade food was the fact that the ewes with which the Tunis ram had mated were in good flesh and vigorous, although they were kept in the same flock throughout and were handled identically, except that they had conceived a few days earlier to a different ram.

Of the 223 ewes bred to the Shropshire rams 25 (11 per cent.) perished. Some succumbed after the fetus had perished and macerated in the uterus; others died at virtually full term, the fetus being fully developed but showing evidences of disease which proved fatal to it at, or slightly prior to, the death of the ewe. Other ewes died after parturition or abortion. The mortality in the ewes was apparently the result of a general sepsis rather than of local genital lesions. That is, the genital lesions were macroscopically of a comparatively mild degree and failed to impress the observer as being in themselves extremely perilous.

In general the badly diseased ewes suffered from dysentery. The feces were very thin, fetid, and black. No cause for the dysentery other than the genital infection was recognizable. Its general clinical character reminded us of the dysentery occurring in septic metritis in pregnant and puerperal cows, already described. The disease began early in pregnancy and gained constantly in force until pregnancy had terminated, when some animals, though not all, gradually improved. Some which were not pregnant, but perhaps had been, though the embryo had perished, continued to fail and eventually died in extreme emaciation without showing marked genital or other lesions. Those which perished after lambing or aborting generally showed metritis with accumulations of exudates varying in volume, color and consistency.

The expulsion of the fetuses, dead or alive, viable or mortally ill, was tardy and atonic. The ewes were evidently weak. Vigorous expulsive efforts were wanting. The fun- 
damental atony was referable to the uterus. Prior to abortion there was often a dirty sanious discharge from the vulva for several days, and finally when the fetus was expelled it had undergone putrefaction and maceration. This is highly characteristic of the metritis of pregnancy in ewes, so far as now known. McFadyean and Stockman in their observations record that sanious vulvar discharge commonly occurs some days prior to the expulsion of the fetal cadaver, which, in their experience, was usually putrefying or macerating.

This is in marked contrast to the observations in cattle abortion. Generally in cattle the expelled fetal cadaver is fresh and shows merely traces of the beginning of decomposition. In exceptional cases, as noted while considering abortion in cattle, the embryo or fetus macerates or putrefies prior to expulsion, or it putrefies and is permanently retained. The prevailing behavior of the uterus toward a contained dead fetus is, therefore, essentially reversed in the cow and the ewe. In the cow the endometritis at the cervical end of the uterus has a marked tendency to cause contraction of the walls of the comparatively sound ovarian end of the uterus, through which the fetus, dead or alive, is forced into the cervix, dilating the latter and causing in turn expulsive contractions of the abdominal walls. But, as pointed out, if the infection within the uterus spreads too rapidly toward the ovarian end, or begins there and paralyzes the muscular walls, expulsion fails and retention with putrefaction follows. Instead of such maceration being the exception, it becomes the rule in ewes. This is probably largely due to an earlier and more severe involvement of the ovarian end of the ovine uterus. It may be due partly in some cases to the existence of twin pregnancy. Twins rarely perish simultaneously, and as a rule the first embryo to perish is retained within the uterus until the second fetus either perishes or reaches fetal maturity. Then the uterus contracts and either twin abortion or birth and abortion concurrently ensue. Thus, in twin abortion, the fetuses are unlike in development and in post-mortem changes. One 
twin is smaller than the other. It had perished at an early date and had undergone partial maceration or desiccation. Such is the rule in twin abortion in the cow and almost without exception in twin abortion in the mare. The same condition is observed in twin abortion in woman. In swine (presumably also in carnivora) embryos often perish but, so long as other healthy embryos exist, the expulsion of the cadavers is inhibited until all have perished or time for parturition has been reached. In other instances fetal retention with maceration is apparently referable to an intense sclerotic or adhesive cervicitis which prevents cervical dilation and thus incarcerates the fetal cadaver. In some years this becomes somewhat common in certain flocks, and ewe after ewe succumbs. In these instances there are expulsive contractions of the abdominal walls without concurrent contractions of the uterine walls of such power as to dilate the cervical canal. The abdominal straining forces the gravid uterus into the pelvis, pushing the cervix in front of it through the vulva to the exterior-prolapse of the cervix.

The studies of Carpenter and myself in the outbreak mentioned failed to establish clearly the etiology. Carpenter recognized a spirillum in the uteri of three out of eight slaughtered ewes. Two of the ewes carrying spirilla had aborted, and one had given birth to a viable lamb. In only one uterus was the spirillum recognized unassociated with other bacteria (pure culture). Bacteria were recovered from the uterus or placentae of each of the eight ewes examined. The list included, in addition to the spirillum, colon-aerogenes, streptococci, staphylococci, and short oval rods in pairs.

Experimentally we failed to establish any important facts. We purchased two pregnant ewes which were wild and placed them in my experiment stable, where they were very timid and uneasy. As is usual, the seller reported that he had observed no abortions or disease of lambs in his flock. Each ewe received in her jugular 10 cc. of composite stomach fluids from three fetuses, the fluid from each source con- 
taining an abundance of vigorous spirilla. One of the ewes expelled a macerating fetal cadaver fourteen days after the inoculation. The maceration indicated that it had died at a period quite in advance of its expulsion, but how long before could not be told. It may have been dead when the inoculation was made. The ewe was immediately slaughtered, but no spirilla were obtained from either uterus or fetus. Cultures showed streptococcus viridans and colon-aerogenes. The other ewe gave birth to an apparently healthy lamb thirty-two days after inoculation. Both animals were destroyed. Spirilla were recovered from the uterus of the ewe, but not from the lamb. Consequently the spirillum was recovered from the apparently healthy ewe but could not be found in the aborter or abort.

We also inoculated in the jugular two pregnant cows with 20 cc. of the same material as that used in the ewes. One of these was 64 days pregnant, pregnancy having been verified by rectal palpation. She was not observed to abort but was in estrum forty days after inoculation and her uterus found empty. Cultures obtained from her uterus with catheter showed staphylococci. Immediately after the inoculation she had shown alarming symptoms resembling anaphylaxis, but these passed in two or three hours. She evidently expelled an embryonic cadaver subsequent to the inoculation but there was no conclusive evidence of the cause of the abortion. She had aborted three years before, was out of an aborting cow, and her sire was in service in a virulently infected herd. She was pregnant by an apparently healthy bull.

As the other heifer had been purchased, she was without history. She was well advanced in pregnancy and gave birth sixty-five days after inoculation to a very poor, weak calf weighing 49 pounds. Fluid siphoned from her stomach yielded staphylococci. The calf lived and remains in the herd. Her behavior as a calf is shown in Figs. 208-209.

Our experiments failed to show that the spirillum causes abortion in sheep, although it probably plays an important part. These experiments are essentially parallel in results 
with those of McFadyean and Stockman, though the conclusions drawn from them may vary. We secured no uniformity of result and kept no controls. The spirillum may have been present in the genital tract of the one ewe prior to inoculation. McFadyean and Stockman appear to believe that the death of the ovine fetus is brought about by one microbe and its maceration by others. Perhaps this belief is general in abortion in all animals and possibly is borrowed from observations upon post-natal death. There a given infection commonly causes death and other bacteria (usually already present in the body, as the colon bacilli in the intestines) released from the restraint of the living tissues quickly break down the cadaver.

Intra-uterine death presents a quite different problem. Putrefaction or maceration of the embryo is dependent upon the invasion of the cadaver by putrefactive bacteria, just as in post-natal life, but the invasion is subject to important limitations because the embryo physiologically is hermetically sealed within the uterus. McFadyean and Stockman apparently believe that, when the embryo perishes in the uterus, the cervical canal opens and permits free invasion from the exterior. My observations lead me to believe that abortion is associated either with a cervicitis which prevents the formation of the uterine seal or with a cervical endometritis which destroys the uterine seal. In the first instance it is highly probable, if not certain, that the bacteria of pyocervicitis can cause fetal putrefaction and maceration as well as metritis, chorionitis, fetal dysentery, sepsis and death. In the second instance the bacteria incarcerated within the sealed gravid uterus, which have power to cause endometritis, destruction of the uterine seal and fatal disease of the fetus, quite certainly possess putrefactive or macerating powers. This is well illustrated in the macerating embryos in swine. These largely occur at the cervical or ovarian end of the uterus, but may occur in the middle of an embryo row, flanked upon either side by apparently healthy embryos. These macerate, putrefy and, if not too old, are absorbed, but they are wholly shut off from invasion 
from the exterior. The bacteria causing death and maceration are apparently identical, and I consider it logical to assume that, as a rule, the destiny of an embryonic cadaver is largely fixed by the nature of the infection causing its death. In swine there are plenty of bacteria incarcerated within the gravid uterus. They regularly cause necrosis with varying degrees of maceration or desiccation of each fetal sac at its poles. The same bacteria logically have power to cause solution of a necrotic embryo. So in the ewe the uterus regularly contains necrosis-producing bacteria which produce necrosis of the tips of the embryonic sac, and it would be very difficult to understand why the same bacteria may not cause maceration of a necrotic embryo. As the evidence now stands, it appears that the death of the fetus and its maceration are generally referable to the activities of the same organism or group of organisms. As in cattle, so in sheep, the infection already exists in the cervix or uterus at the moment of coitus, or is introduced as a component part of the semen during coitus. A little later the cervical canal, if healthy, is sealed and invasion thereby inhibited.

In the outbreak of genital disease of ewes studied by Carpenter and me, the history of the herd points strongly to the rams as the essential source of the infections and to coitus as the date of invasion. As related, a Tunis ram broke into the inclosure and copulated successfully with some ewes. These remained apparently healthy and produced vigorous lambs. When the Shropshire rams were placed with the ewes, the disease began with those which were still non-pregnant. Those ewes bred to the Shropshire rams began aborting eight or ten weeks before the Tunis lambs were born. There was abundant time for a contagion to spread rapidly by ordinary contact from an aborting ewe to a healthy pregnant one, but this failed to occur. The outbreak also suggests that the infection, as a highly destructive force, developed in what may be termed a spontaneous manner. That is, the infection existed during the prior breeding season in low virulence, depressing the reproduc- 

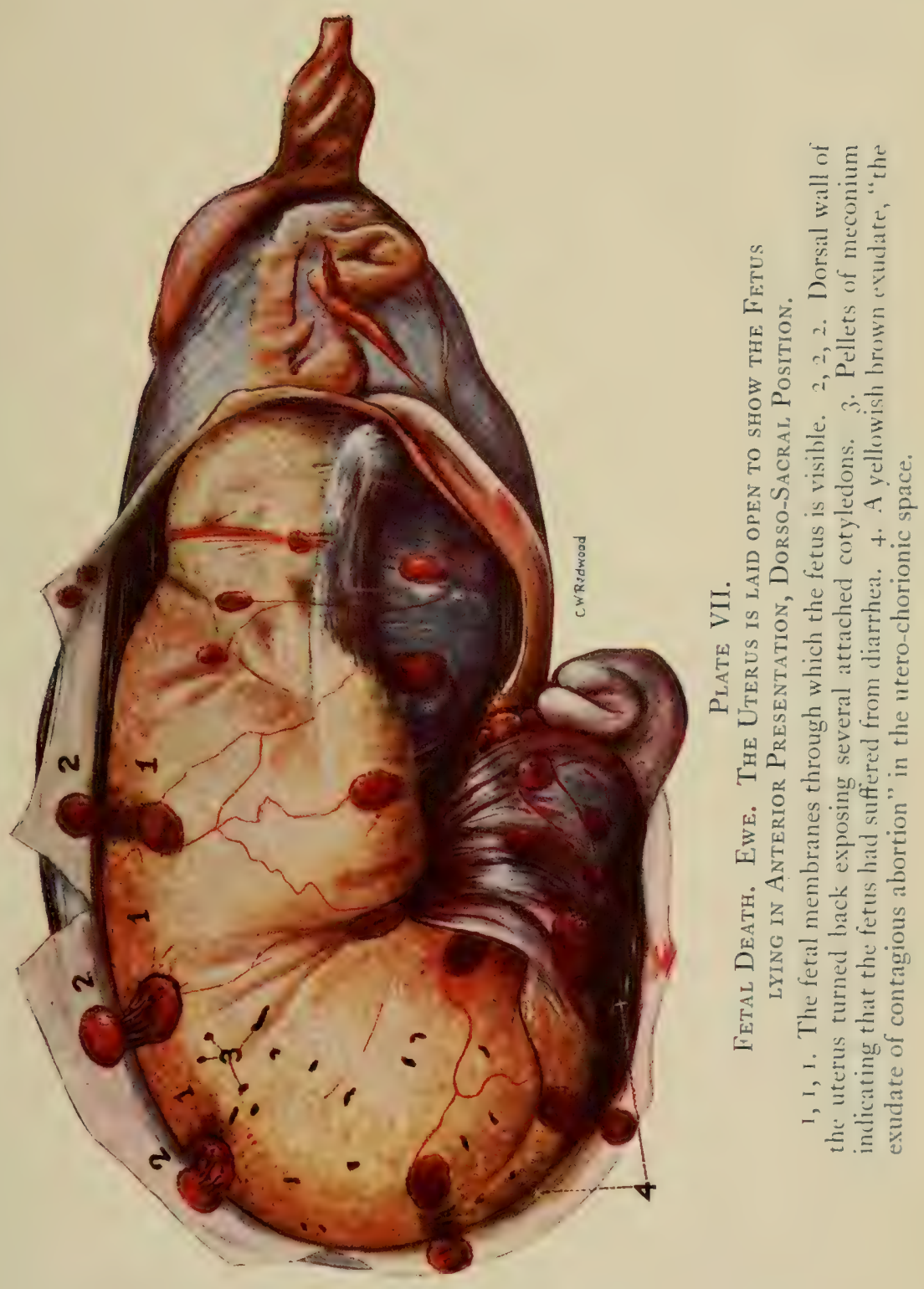

tive efficiency of the herd ten per cent. or more below the ideal, but arousing no alarm. Notable dystocia had been present, but, in accordance with the general view, was attributed to large lambs as compared with the birth passages of the young ewes, instead of being referred, as was probably the fact, to uterine inertia resulting from infection. When the Shropshire rams were placed with the ewes for the second breeding season, each ram faced the task of copulating once to several times with each of fifty ewes within a period of twenty-one days. The infection gathered momentum rapidly and serious disaster followed.

As in cattle, so in sheep, there is, so far as known, nothing which can be done to check abortion in pregnant ewes. It can be prevented only by mating males and females which are genitally sound. Secondarily, all those influences of food, water, air and exercise which, properly adjusted, insure the highest degree of physical vigor, play an important part in increasing the resistance of the uterus to the infections virtually always present in small degree.

Rams should be handled with the greatest possible care in order to insure their sexual vigor. Plenty of vigorous exercise during the long season of sexual rest is a prime factor in the promotion of physical and sexual vigor and endurance. It is to be remembered that an adult breeding male, when at sexual rest, requires very little food and that, unless vigorous exercise is in some manner provided, he quickly becomes atonic, lethargic and obese. In this state infections present in any organ constantly tend to develop increasing force. When a male so weakened is subjected to severe sexual strain, each coitus intensifies any genital infection present and endangers his fertility. Therefore, the two chief precautions to be taken are the enhancing of physical vigor during the period of sexual rest, and a safe limitation as to the number of females he is expected to serve. During the rest period the ram should have a moderate ration, for which he should be obliged to travel enough to ensure vigor. The most practicable plan is to permit the ram to run with wethers, cattle or other animals than ewes dur- 
ing the non-breeding season. During the breeding season the number of ewes assigned to a ram should be carefully limited. Ordinarily when the ram is placed with the flock, all ewes are sexually ready for breeding and will be in estrum within three weeks, so that it may be assumed that the male will copulate at least once with each ewe within that time and will at first probably copulate several times with each ewe. According to such observations as I have been able to make, small flocks constantly show higher reproductive efficiency and more vigorous offspring than large ones. Many elements doubtless enter into the problem, but I believe the most important factor, assuming that the general care is good, is the proper limitation of the sex strain upon the ram. The large breeds of sheep are essentially twin breeders and if properly handled may, and sometimes do, average throughout the flock two lambs each. Thus in one flock twenty-six ewes produced fifty-two vigorous lambs, there having been enough triplets to counterbalance singles. It is probable that such a number of ewes is near to or quite the maximum for one ram, if the highest efficiency is to be maintained. Admittedly instances may be cited where rams have withstood a far greater sexual strain with apparent impunity, but it is doubtful policy to assume that any breeding animal is wholly and technically free from genital infections. Knowing these to be essentially universal, and knowing that under prudent sex or physical strain the infections are generally harmless, it appears to me best to assume the existence of infection and guard against its intensification. The cost of the maintenance of additional rams, beyond the value of the fleece, is not highly important and does not require many additional lambs to render the plan economically profitable. Such care in breeding is not merely for the immediate influence upon the next lamb crop. The progressive breeder must always contemplate future generations. Health lines are as important as blood lines, and it requires as great patience and intelligence to establish that physical vigor upon which high productive efficiency depends as it does to build up a satisfactory pedi- 
gree. The genital infections of sheep, like those of other mammals, behave as an endless chain. If a ram is overcrowded and develops virulent infection, the harm does not end with the abortions which may follow, the ewes which may perish, or the slow development of the congenitally diseased lambs. Such lambs may be perilously infected and, upon reaching breeding age, serve as a new center for the distribution of dangerous infection.

Wherever it is economically practicable, it is better to divide large flocks into small breeding units and assign to each unit a breeding ram. By such means, if there be one dangerous ram in the flock, he is identified and the infection limited to his group of ewes. The division of the flock into small breeding units may be for a brief time only, not exceeding four weeks, when the breeding should be terminated and any ewe proving unfertile, assuming that the ram is sound, should be discarded for breeding because she probably carries dangerous genital infections which it is well to avoid.

Death and maceration of the fetus, with prolapse of the cervix, has been mentioned as occurring frequently in pregnant ewes. Usually the cases are disseminated and involve only a small percentage of a flock, but individual cases often develop annually. The pathology and bacteriology have not been studied. The disease reveals itself first by the cervical prolapse. As in the pregnant cow, so in the ewe, the cervix is at first visible only when the patient is recumbent, in which position the intra-abdominal tension is increased and the inert genital organs mechanically forced out through the vulva. The cervix becomes befouled with litter, desiccated and irritated by the air, and inflammation and necrosis follow. Cervical prolapse in the pregnant ewe does not always indicate fetal death and maceration. I handled one case in which the ewe gave birth to vigorous twins, but that is merely an exception. The cervicitis tends constantly to grow worse. The cervix becomes swollen and indurated, rendering it more and more difficult for the canal to be dilated. 
No definite line of treatment has become established. If it can be determined that the fetus is alive, it may be desirable to try to retain the cervix within the vagina. It should be kept clean and parturition awaited. The retention may be secured by the vulvar sutures shown in Fig. 60 for vaginal hernia of cows. Securely retained and protected from soiling and the irritation of drying in the air, the cervix may be kept clean and soothed by means of irrigations with salt solution or with bland oils. This may relieve the cervicitis and the induration, causing the cervix to relax and the uterus to expel its contents. It may be attempted, with some hope of success, to cause contraction of the uterus with dilation of the cervix by small repeated doses of pituitary extract. These failing, gastrohysterotomy may be employed, as described for the cow, and the uterine contents removed surgically.

Retained Fetal Membranes. The metritis which sometimes results in abortion in the ewe tends also to cause retention of the fetal membranes. The character of the cotyledons in the ewe alters notably the type of the placental retention. In the cow the cotyledons are intimately attached to the chorion over a large area, so that the two tissues do not readily tear apart. The fetal cotyledons of the cow may be described as sessile; the maternal cotyledons are distinctly pedunculate. This is reversed in the ewe: the maternal placenta is essentially sessile, while the fetal placenta is distinctly pedunculate, with a long, slender pedicle. In the cow the pedicle of the maternal cotyledon often parts and the maternal placenta falls away; in the ewe the pedicle of the fetal cotyledon quickly parts, and the chorion and amnion drop away, leaving the spherical fetal cotyledons (See Figs. 31,32 ) incarcerated in the chalice-like cavity of the maternal cotyledon, as shown in Fig. 214 and in Colored Plate VII.

It is only in a minority of cases of retained placenta in the ewe that the chorion and amnion are included in the retention. Usually, therefore, the retention is not evidenced by the membranes protruding from the vulva. Such cases are observed, but are usually of short duration. So long as the 


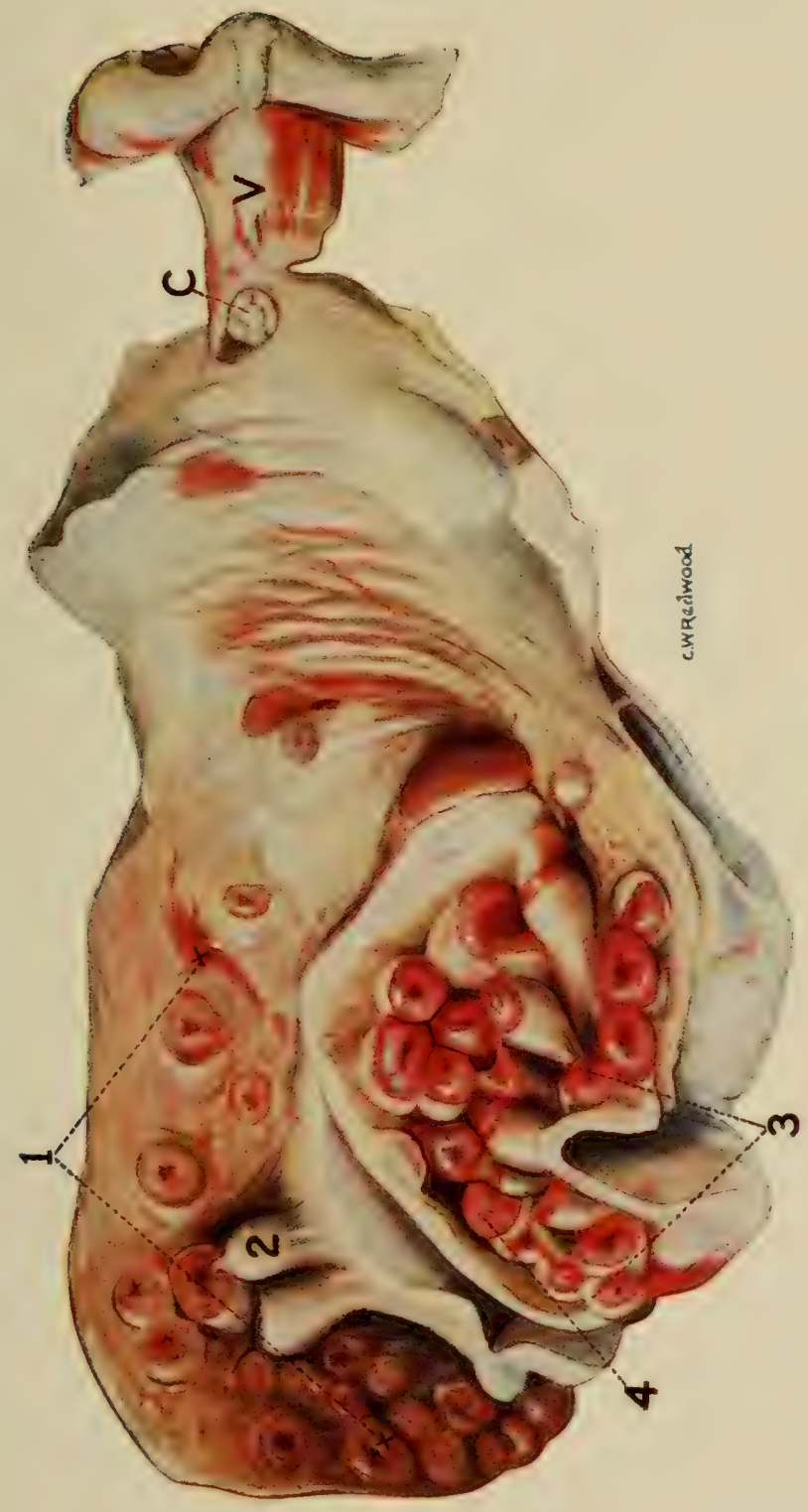

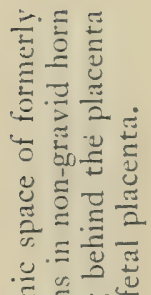

$\therefore \bar{\Xi}$

ㄴ. 는 은

红

3 至

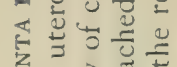

政 3

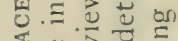

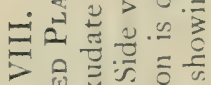

的肴.

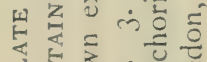

$\sim \simeq$

元司苍

<

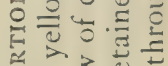

긴

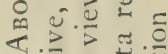

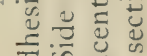

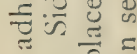
ti $\overrightarrow{\vec{Z}}$

क O

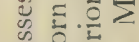

$\sum^{5}$ 은

$\sum \mathrm{U}$

$\therefore-\frac{3}{3}$ 

chorion remains attached to the cotyledon and hangs from the vulva, the symptoms render the character of the disease evident, but the more frequent condition of retained cotyledons, after the falling away of the chorion, is not readily diagnosable clinically. The genital passages do not admit

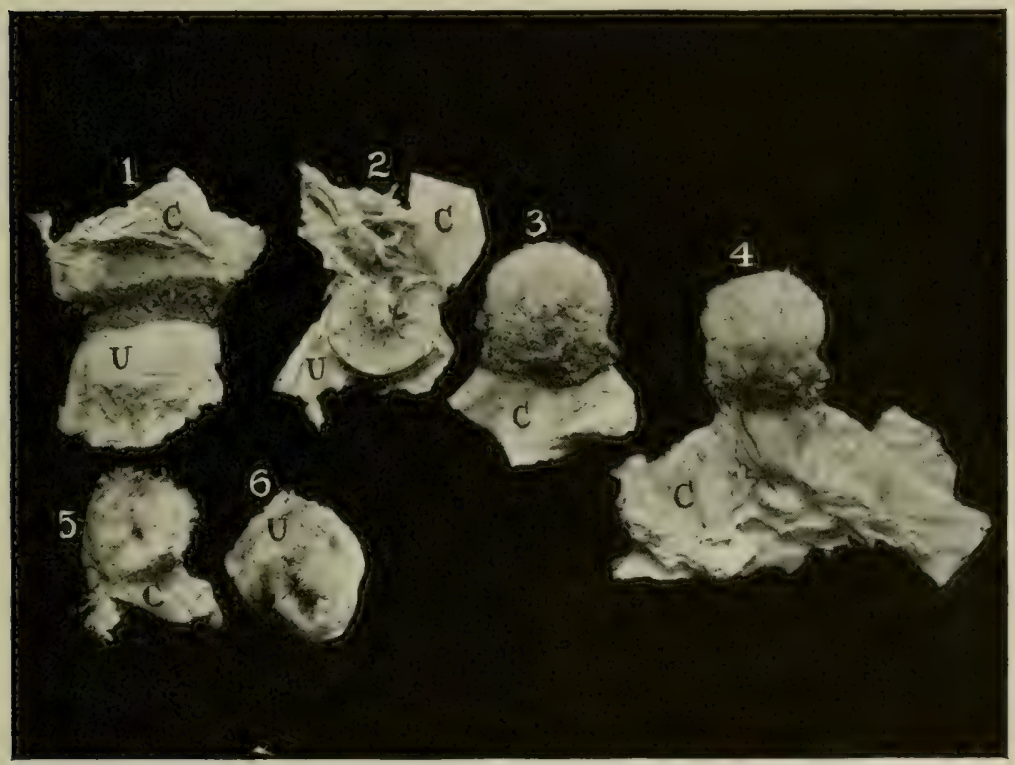

Fig. 214 . - Retained Placenta in Ewe.

$C$, Chorion; $U$, uterus ; $I$, fetal (above) and maternal cotyledons (below) showing hemorrhingic placentae; 2 , cross section through fetal aud maternal cotyledons, showing narrow neck of fetal portion; 4, fetal cotyledon detached from uterus and showing at its base a hemorrhagic zone; 5 , uterine cotyledon everted; 6 , uterine cotyledon with fetal placenta removed.

manual examination of the interior of the uterus. The diagnosis must then rest upon the presence of the metritic discharge, usually dark red, sanious, mixed with necrotic placental fragments, and fetid. The diagnosis is clear enough as to metritis, but not as clear, except for the placental fragments, as to retained placenta. The differentiation, however, between metritis with or without placental retention is not profoundly important. They possess essentially the same dangers and call for analogous handling. 
The retained cotyledons can not be removed by any technic at present known. If a rubber horse catheter can be successfully passed into the uterine cavity, the contents may be diluted with saline solution and siphoned out. This can not be done safely if the chorion is still attached. A small amount of iodoform and bismuth (30 to 40 grains of each) suspended in two to four ounces of liquid paraffin may be introduced into the uterine cavity. Pituitrin may be tried in small doses (10 to 20 drops) frequently repeated.

The infections involving the fetus and the new-born lamb, whatever may be their identity, cause approximately the same lesions as already described in bovine fetuses and in calves. A notable result, as in bovine fetuses and new-born calves, is severe dysentery with gastro-intestinal lesions. Carpenter, in his autopsies of fetuses which had perished in utero, records essentially universal diarrhea. The amniotic fluid contained meconium, showing that the fetus had defecated-a distinctly pathologic act. This is illustrated in Colored Plate VII, where pellets of meconium are shown lying between the fetus and the amnion. The existence of fetal dysentery was further shown by the presence of meconium in the stomachs of aborts, which location could be reached only by the fetus having defecated and later swallowed the meconium suspended in the amniotic fluids. Most lambs born in the flock were thickly smeared over with meconium, indicating clearly that they had, as fetuses, suffered from diarrhea.

Except for the few Tunis lambs previously mentioned, nearly all lambs born were weak and sick at birth. They were extremely emaciated and were dull and weak. Covered as they were with meconium, they were often repulsive to their dams. The ewes refused to lick their young, leaving them befouled with meconium. Frequently they would not permit the lambs to suck, but would butt them over when they approached. The caretakers in many cases caught and restrained the ewe each time that the lamb fed.

These observations are interesting, because they possibly throw light upon other instances where a dam refuses to 
care for her new-born, or resorts to cannabalism as observed in swine. It is not impossible that the repulsion of the new-born by its mother is often due to disease--that the fetus has suffered from dysentery due to an infection present in the uterine cavity of the mother and the new-born is repulsively covered with filth, having an odor unlike that which is natural to the species. In swine, necrotic embryos are expelled with pigs. The scavenger sow devours the macerating embryos and the pigs, having the same filth smeared over them, are also eaten.

The lambs in the flock observed largely suffered from dysentery. Their development was tardy, partly because of the intense infection, but largely because the ewes, due to illness, had little milk. Many of the weaker lambs died from one to two hours up to three or four days after birth.

The post-mortem appearances of fetuses dead in utero and lambs which perished shortly after birth were analogous to those of bovine fetal cavaders and calves dead from dysentery. Outbreaks of disease in new-born lambs are not rare and run essentially parallel, in cause, symptoms, course, results and post-mortem findings, to those diseases of the calf having their foundations in infection during intra-uterine life. They suffer from dysentery, pneumonia and arthritis. Each of the three consequences may result from post-natal infection, but the most basic cause is to be sought in the intra-uterine infection of the pregnant animal. The infection grows through the chorion and amnion, gains the amniotic fluid, and, being swallowed by the embryo, begins its destructive career by inducing gastro-enteritis with dysentery or, in some cases, sepsis, whether the storm breaks ante- or post-natal.

The handling of the infections of new-born lambs is to be based upon the principles laid down for calves and does not require repetition here. Serologic handling has not been placed upon a secure basis, so far as known. The serum sold for dysentery in calves, given in proper dosage, would probably prove beneficial. The objection will at once be raised that such a course would be illogical because in the 
"calf scours serum" the bacteria used in preparing the serum are those of "calf scours"-not of "lamb scours". It has been pointed out, however, that the bacteriology of neither has been clearly established, and furthermore that it has not been demonstrated that ordinary horse serum is not quite as efficacious as is that from horses "immunized" against large doses of the presumptive "calf scours" bacteria.

Genital infections in goats have been reported from time to time, but the goat industry is confined to comparatively small areas except for scattering individuals of nominal value, and the subject has consequently received no sustained interest. When goats become concentrated in large numbers and are intensely bred, fed and milked, they tend ultimately to yield to genital infections similarly to cattle.

Stone and Fisher ${ }^{1}$ have investigated an infection in goats bearing some resemblance to goat pox, but not identifiable with that malady. The disease was closely associated with pregnancy and apparently led in numerous instances to abortion. In the doe the symptoms of disease, consisting chiefly of pustules, abscesses and multiple arthritis, usually followed closely upon parturition or abortion. The cutaneous pustules were followed by necrotic areas. The abscesses were generally subcutaneous. Some of them matured and broke; others became caseous and indolent. The multiple arthritis was of the ordinary type so frequent in cows after intra-uterine infection, and in calves, foals and other young following intra-uterine and navel infections. The method of infection was not clearly determined. In some instances the disease was contracted through a healthy doe's being sent into an infected herd to be bred, but no evidence was submitted to indicate that the inoculation occurred through coitus. Many does aborted or became sterile, or both. The kids born of the infected does largely developed the same symptoms, especially the polyarthritis.

In the outbreaks directly covered by the report, Stone and

${ }^{1}$ A Chronic Pox-like Infection in Goats and Its Successful Treatment. Jour. Am. Vet. Med. Ass'n, Vol. 8, p. 536, Aug., I9I9. 
Fisher regularly obtained, in cultures from the pustules and abscesses, a pure growth of a staphylococcus. Some individuals showed merely a few pustules, which after a short interval healed, and the animals showed thereafter an imperfect immunity. A large percentage of cases did not recover spontaneously and were obstinate to symptomatic handling, such as antiseptics, the opening of the abscesses, etc. They were highly successful, however, in the use of autogenous bacterins. As the work progressed, they used successfully bacterins made by combining cultures procured from various similar cases.

The relationship, if any existed, between the disease described by Stone and Fisher, believed to be due to staphylococci, and the abortion and sterility was not made clear, but they believed, upon apparently excellent grounds, that the interferences with reproduction were basic results of the malady.

In other instances abortions in goats occur in which the staphylococcus described can not be found, but other bacteria are present. In some instances, also, the fetus perishes and, owing to uterine inertia with cervical induration, retention of the fetus follows. Then prolapse of the cervix ensues, having the same general characters as the cervical prolapse of the ewe already described.

Since the general principles of controlling genital infections in goats are the same as those advised for cattle and sheep, repetition is unnecessary. 


\section{SECTION III. THE GENITAL INFECTIONS OF SWINE}

\section{CHAPTER XVIII \\ SPECIFIC GENITAL INFECTIONS}

\section{The Nodular Venereal Disease}

Outbreaks of specific venereal diseases in swine have been recorded at intervals, but the records have been indefinite. When describing the nodular venereal disease of cattle, it was stated that the infection is common in cattle, swine, sheep, and presumably in goats. It has been stated by some writers that in certain herds the infection caused destructive outbreaks of abortion and sterility in sows. The facts appear to be that notable losses occurring in certain herds of swine caused the veterinarian to search for, and quite naturally to find, the lesions, and upon this evidence the conclusion was reached that the infection causing the lesions was responsible for the abortion. The assumption may be true or untrue. What has been said of the infection in cattle is presumably equally true in swine, and it does not require repetition.

\section{CHAPTER XIX}

\section{NON-VENEREAL SPECIFIC INFECTIONS INVADING THE GENITALIA OF SWINE}

\section{Genital Tuberculosis}

Genital tuberculosis has been reported but rarely in swine and probably does not play a very important economic part in swine husbandry. I have in my collection but one specimen of genital tuberculosis of swine-tuberculosis of the testicle, illustrated in Fig. 215. The gland is from a purebred boar belonging to a herd devoted to growing purebred swine for breeding purposes. They had a liberal supply of raw milk from a herd of purebred dairy cattle in which tuberculosis was rampant. The symptoms of genital tuberculosis in swine are presumably the same as in cattle. The tuberculosis of swine is ordinarily of bovine origin. Tubercular invasion of the genitalia of swine is to be avoided by the general measures for preventing swine tuberculosis. When present, genital tuberculosis has the same dangers for other swine that bovine genital tuberculosis has for other cattle. There is no remedy for the affected animal. 


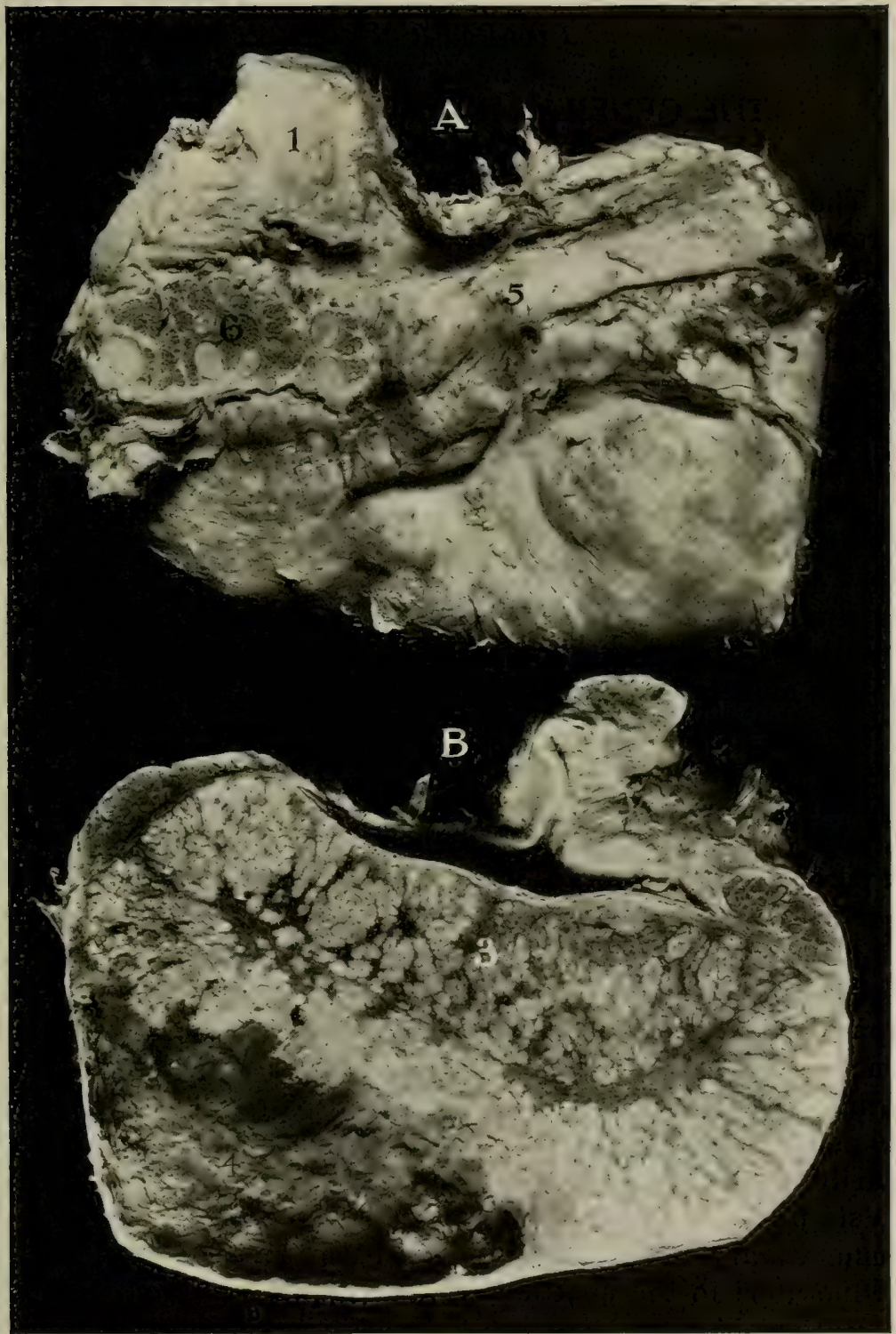

Fig. 215-Tuberculosis of Testis. Boar.

$A$, Median section : $B$, external side of testicle showing epididymis. $I$, Spermatic cord; 2 , head of epididymis ; 3 , tubercular masses ; 4 , tubercular abscess cavity ; 5 , body of epididymis : 6 , section through epididymis revealing tubercular masses. 


\section{CHAPTER XX}

\section{THE GENERAL INFECTIONS OF THE GENITALIA OF SWINE}

The general infections of the genitalia of swine are of far greater economic importance than has yet been generally recognized. Adult sows (and probably also gilts) ordinarily discharge at each estrum twelve to eighteen or more ova. If each ovum were physiologically healthy, the genital tract healthy, and the sow copulated with a thoroughly healthy boar, there would be a vigorous live pig born for each ovum discharged. It was stated, while considering reproduction in cattle, that in purebred dairy herds there is born as a rule about one viable calf for each three copulations, or that about 67 per cent. of the efforts at reproduction fail. A similar condition exists in the production of swine, but the result is screened from general observation. Since the sow is multiparous, if infection of her genital tract is moderate only, some of the ova may be fertilized, develop, and be born in viable health. The same is true of the boar. If infection exists in his genitalia which destroys but a part of the spermatozoa or later brings about the death of only a portion of the embryos, some pigs are born, but the number is depressed in harmony with the amount of the infection existing within the uterus of the sow at the date of coitus, plus the infection ejaculated with the semen of the boar. So far as I am able to estimate, these infections reduce the number of pigs born upon the average 50 per cent. which means that swine breeders maintain two brood sows to perform the ideal work of one. The swine breeder ordinarily does not comprehend the meaning of a litter of four to six pigs, and unconsciously attributes the low fertility to some vagary of "nature", but a careful study of gravid swine uteri in the abattoir permits a quite different interpretation.

Abattoir studies of pregnant swine uteri reveal several very interesting and important facts which are highly il- 
lustrative of some of the fundamental principles of genital infections and the physiology and pathology of reproduction in general.

The embryonic sacs of swine testify emphatically to essentially universal intra-uterine infection of significance. Embryologists have described the necrotic tips of the embryonic sacs of swine as "normal" by which the reader assumes this necrosis to be physiologic. Bonnet apparently regards the phenomenon as the result of pressure necrosis. That is, the embryonic sac outgrows the length of the uterine (cornual) cavity and, pressing the apices of the sacs either against the apex of the cornu or against adjacent embryos, by pressure cuts off the nutrition of the chorion, so that the part dies. Numerous facts contradict this assumption. It is generally contrary to physiologic development that an organ or tissue should undergo dry necrosis and become in effect a permanent (during gestation) foreign body. Instead useless organs or tissues usually undergo cellular disintegration with resorption. The necrosis at the poles of the embryonic sacs is inconstant in occurrence and extent. It is not always present. The necrosis increases with the advancement of pregnancy, as would be expected of a chronic infection. When some of the embryos are necrotic, the necrosis of the tips of the other embryonic sacs containing live embryos is more extensive than that observed in uteri where all embryos are apparently healthy. From whatever angle the phenomenon is viewed, it is difficult to escape the conviction that the necrosis commonly present at the two poles of each embryonic sac of swine is the result of a chronic intra-uterine infection which existed, or was introduced, at the time of coitus, and that death of swine embryos is ordinarily referable to an extension of such polar necrosis. The path of invasion appears to be through the amnion from the necrotic area, after which the embryo swallows the bacteria and the active invasion of the embryo itself begins within the alimentary tract.

There are many scattered records of abortion in swine, but in most reports it is not made clear what the observers 
mean. When considering abortion in cows, it was stated that commonly the word signified the observed expulsion of a fetal cadaver. In the sow there may be expelled at one time macerating embryos of varying sizes and degrees of decomposition, fetuses recently dead, poorly developed, sick pigs, and fully developed, viable pigs. Such a medley is difficult of accurate definition. There are present, in the one uterine evacuation, phenomena which may be designated as abortion, stillbirth, premature birth, and birth. Accordingly it is difficult to interpret the precise meaning of a writer when he says that sows have aborted. In some instances, like those reported by Hayes and Traum ${ }^{1}$, who record that, out of seventy sows, twelve aborted at from the forty-third to the one hundred eighth day of pregnancy, it seems clear that the uteri of the sows were at the times named emptied of their embryonic contents and that the embryos were dead. There is no intimation, however, as to whether the embryos in a given uterus had perished at various dates, as shown by size and degree of maceration, or whether they had apparently perished simultaneously. According to my abattoir studies and such clinical observations as have been available, the course and character of genital infections in swine conform to the basic principles of genital infections in cattle, modified profoundly in some respects by the multiparous type of reproduction and significant differences in the handling of animals. Probably no animal carries infections in the genital organs more universally or in larger volume. The ovaries do not show lesions with the same frequency as the dairy cow, but the same varieties of disease are present. Fig. 216 illustrates the physiologic cycles occurring in the ovary with one ovary (2) illustrating ovarian adhesions as a result of salpingitis. Fig. 217 illustrates cystic disease of both ovaries. Without history, the latter was quite certainly a nymphomaniac and sterile. The symptoms, as I have observed clinically in other cases, are essentially the same as described in cattle. There is sex perversion shown in the behavior of the sow towards other domestic animals. I have not observed the sinking of the

${ }^{1}$ Preliminary Report on Abortion in Swine caused by B. Abortus (Bang.) North Am. Vet., May, 1920. 


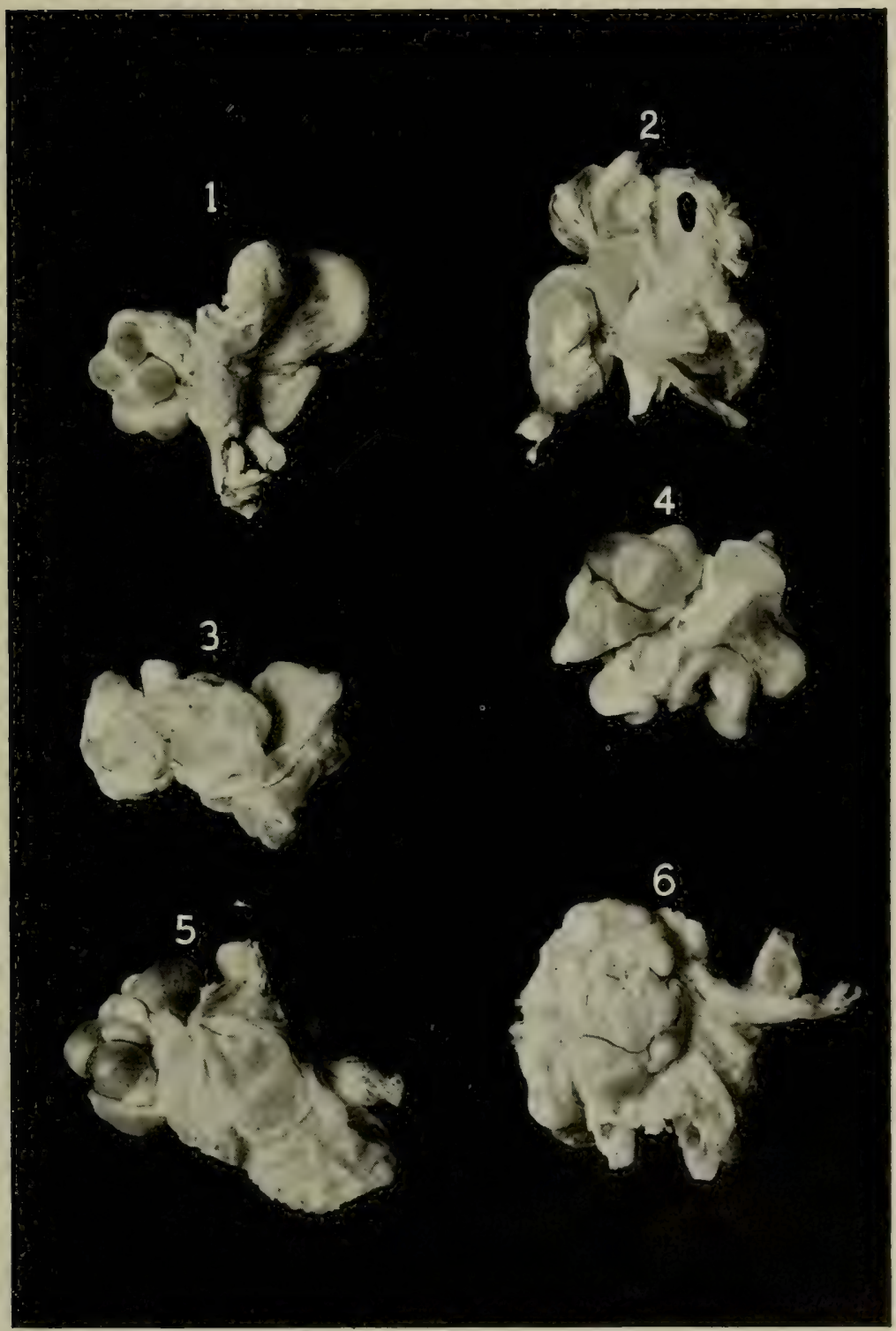

FIG. 216-Ovaries of Sow.

$I$, Ovary showing 6 ripe ovisacs (on left) with apex of cornu to the right and above ; 2 , small fibrous ovary on left, with adhesions in pavilion. (The oviduct has been divided just beneath 2); 3 , ovary showing immature ovisacs ; 4,5 , ovaries showing corpora lutea ; 6 , ovary in rest stage showing atrophied yellow bodies. 


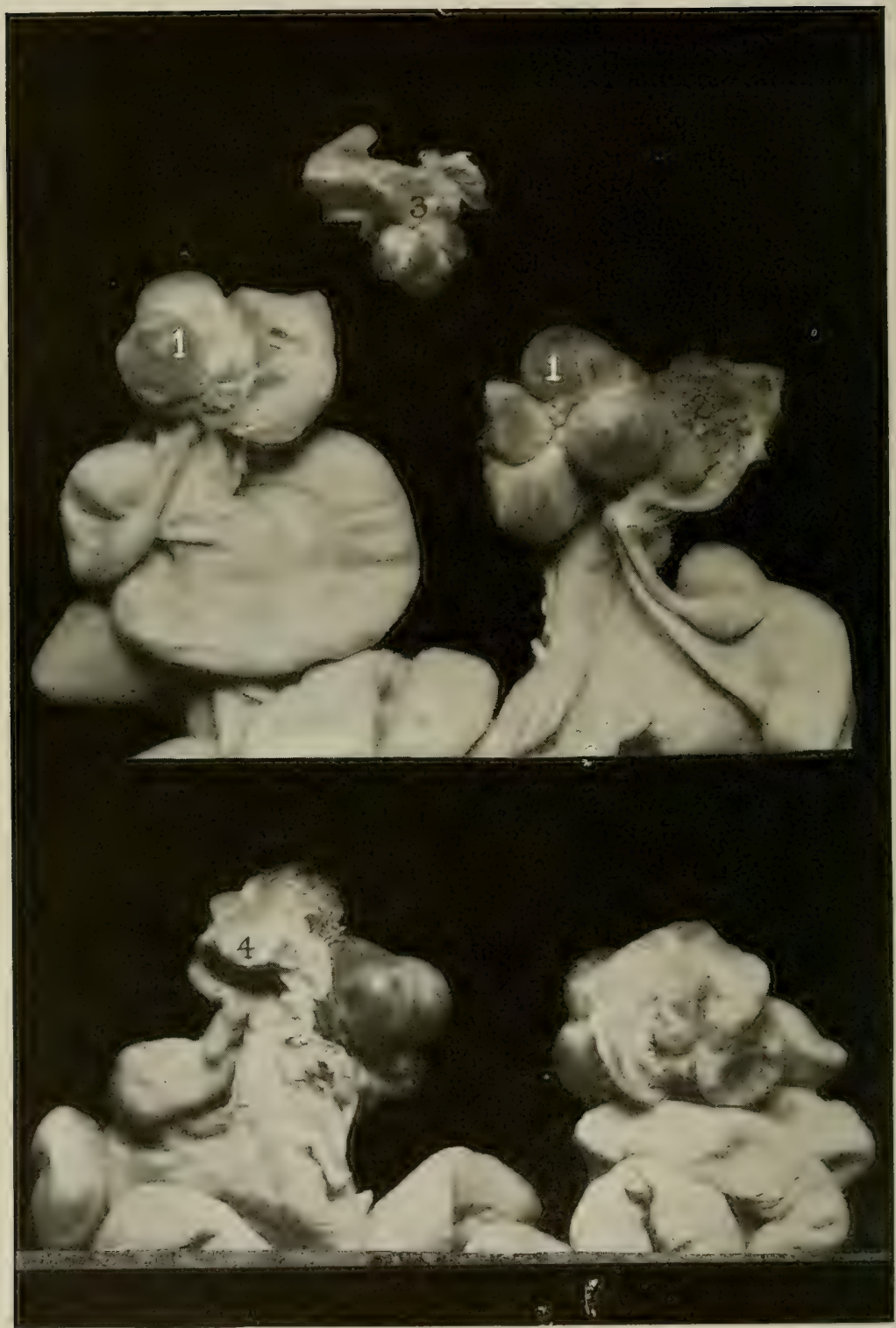

Fíg. 2I7-Cystic Ovaries. Sow.

$I, I$, Cystic ovisacs, beneath which are apices of uterine horns seen from dorsal surface ; 3 , ovary, with normal ovisacs introduced as check. (Below, the apices of two uterine horus with ovaries and cystic oviducts are shown from ventral surface) 4 , a small cyst in mesosalpinx immediately above the numeral. 
pelvic ligaments in the sow which is so prominent a feature in the cow.

Hydrosalpinx occurs in swine, as in cows. In Fig. 218 there is illustrated an interesting instance of extreme bilateral hydrosalpinx in a pregnant sow, showing that severe tubal infection may exist at the date of coitus without inhibiting fertilization and then develop so rapidly that long

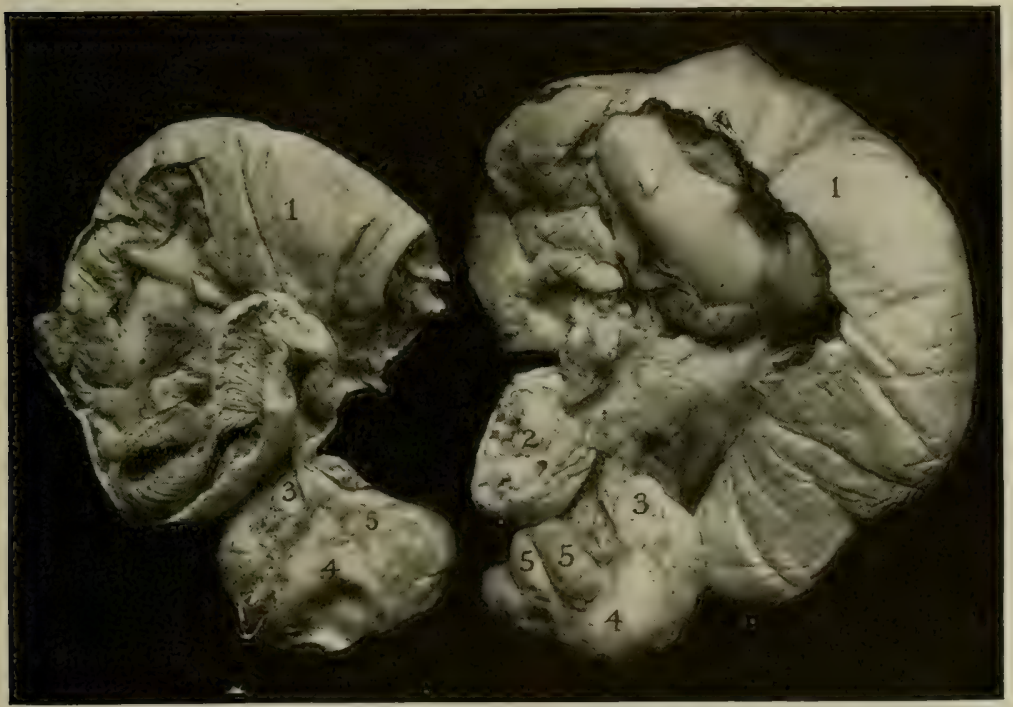

FIG. 2IS-Bilateral Hydrosalpinx Developed During Pregnancy. Sow.

$I, I$, The cornua each contains a normal embryo $4^{\prime \prime}$ long; 2 , abscess in apex of horn, probably embryonic debris ; 3 , ovary sectioned, showing small cysts ; 4 , cystic distension of adherent pavilion ; 5 , cystic oviduct.

before the end of pregnancy the reproductive life of the sow has been permanently closed-unless one believes that the tubal infection was post-coital.

The gravid uterus of the sow, the embryonic envelopes, and the embryos show essentially all the lesions already described for the cow, and it is only necessary to point out here certain deviations in type due to fundamental differences in the species. Intra-uterine infection with necrosis of the poles of the embryonic sacs is so nearly universal 
that, as previously stated, it is designated "normal". Necrosis and maceration of embryos is exceedingly common. I have been able to recognize necrotic embryos not over oneeighth of an inch long, but naturally such are not often recognized. They commonly macerate quickly, so far as I have been able to determine, and are absorbed. This probably accounts in a large measure for the disparity in numbers

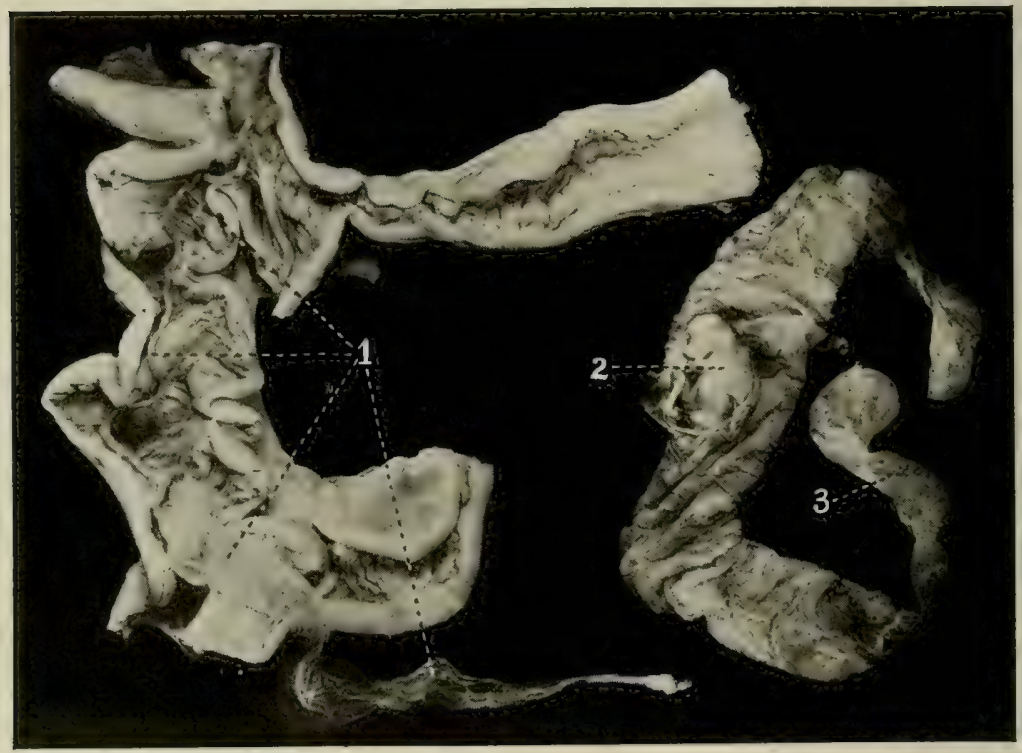

FIG. 2I9-Desiccated "Mole" in Swine Uterus.

$I$, Desiccated embryonic sac lying in base of uterine cornu (The opened vagina and cervix extend to the right, above); 2 , apparently healthy embryo from same cornu; 3 , necrotic tip of fetal sac.

between the corpora lutea present in the ovaries and the embryos in the uterus. Some of the discharged ova presumably failed of fertilization. Following the minimum recognizable necrotic embryo, there occurs every possible gradation of necrosis, maceration and desiccation. There are great variations in one uterus. Sometimes there is a desiccated "mole" in which the minute embryo has perished and is no longer visible, while the embryonic sac remains as a desiccated, wrinkled cord or band, as shown in Fig. 219. 
Then follow various sizes of necrotic embryos which have undergone varying degrees of maceration or desiccation, as shown in Figures 220 to 222. One necrotic embryo may be one-half to one inch long and almost wholly disintegrated, rendering its identification difficult. A contiguous dead embryo may be two or three inches long with only slight maceration or desiccation. Neighboring live embryos may

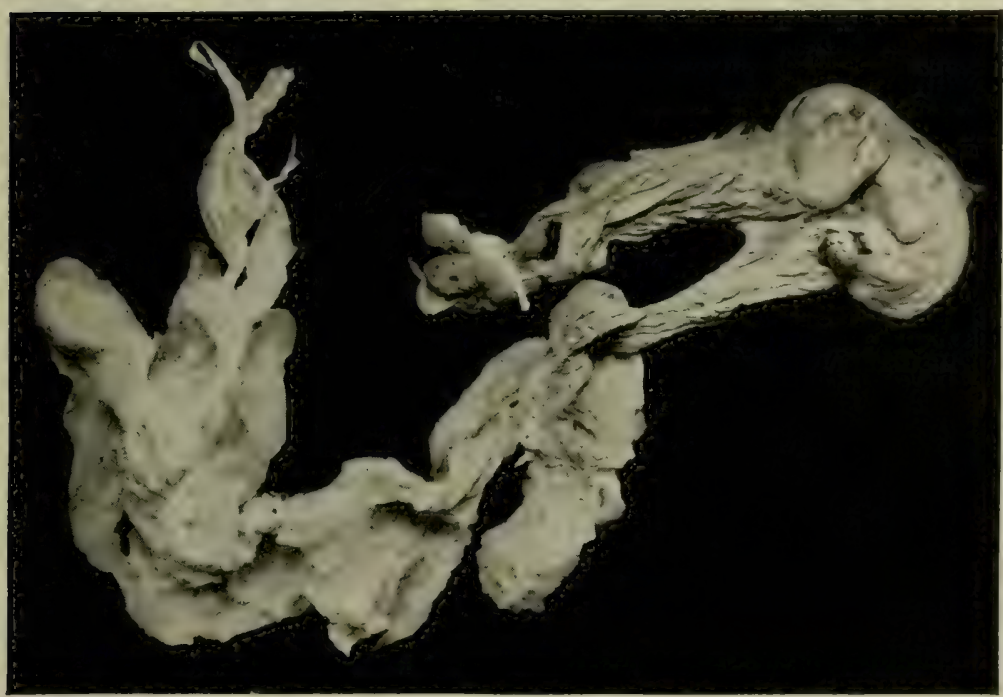

FIG. 220-Necrotic Swine Embryo (right) with Necrotic Fetal Sac.

The cadaver was expelled at full term with 8 healthy young.

be four to six inches or more in length. The live embryos show great variations in size-sometimes 100 per cent. variation in volume. The small fetus, the "runt" to be, is apparently small as a result of nutritive disturbances owing to the ravages of infection, and when born is wanting in vigor.

Embryonic death may occur in any order, but as a rule the liability of the embryo to succumb to intra-uterine infection conforms to the general principles laid down for the cow. The most vulnerable points are the cervical end of the uterus and the apices of the cornua. As a rule the first to 
perish is the basal embryo-that is, the embryo which is located in the base of one horn nearest to the cervical canal and whose embryonic sac extends across the uterine mouth of the cervical canal into the opposite horn. The embryonic sac of this basal fetus constitutes the "sentinel" which the infection, more virulent than elsewhere, must pass in order to imperil the lives of embryos beyond. This is strikingly illustrated clinically in the dystocia of the sow dependent upon fetal maceration and emphysema. The difficulty is commonly with the basal fetus which, having perished and undergone maceration at or near full term, since the occupied segment of the uterus is paretic, can not be expelled, and so bars the path of exit of the fetuses beyond, whether living or dead. Consequently in a large proportion of cases, if the basal fetus can be successfully removed, parturition may proceed favorably.

In multipara the death of one or more embryos has little or no visible tendency to cause the uterus to contract and empty its contents, so long as living and comparatively healthy embryos remain, but the necrotic fetal cadaver or cadavers, with the envelopes, remain in situ and undergo maceration or desiccation. That is, the presence of an immature, healthy embryo in the uterus serves to inhibit uterine contractions which would expel indifferently dead and living embryos. In this manner the reproductive efficiency of swine is partly maintained although the average number of young produced at a birth is greatly curtailed.

The infections of the genitalia of swine have not been comprehensively studied. Good and Smith, Hayes and Traum, and others have searched for, and have frequently found, the B. abortus in aborting sows, or have found that the blood of aborting sows, or of boars to which aborting sows were bred, reacted to the tests for that organism, and concluded therefore that in these instances it was the essential cause. Apparently other pathogenic bacteria were not looked for and the conclusion that the B. abortus was the causative agent was apparently based upon the assumption that the B. abortus is the specific cause of abortion in cattle, 


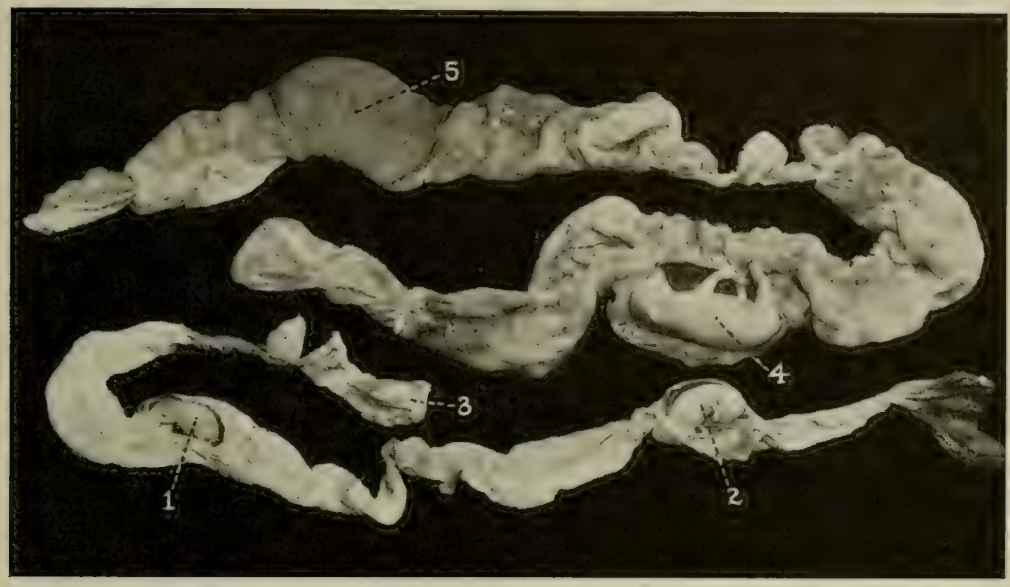

FIG. 22I-Necrotic Swine Embryos.

1,2 , Necrotic embryos (the membranes incised to bring them into view) ; 3 , a necrotic embryonic sac in which the embryo can not be fourid; t, 5 , healthy fetuses from same uterus.

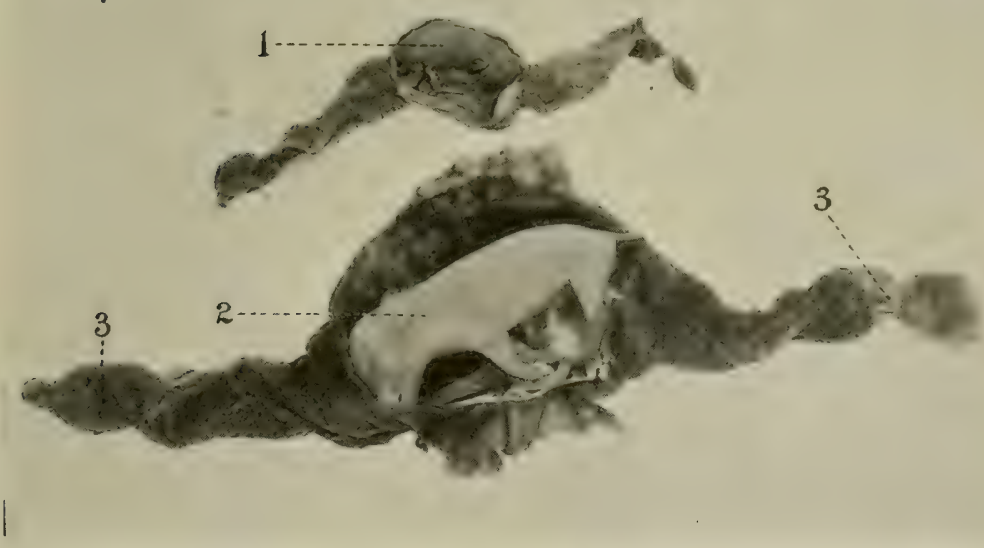

Fis. 222-Necrotic and Healthy Swine Embryos from Same Uterus. $I$, Necrotic embryo; 2, healthy embryo; 3 , necrotic tips. 
and that consequently it causes abortion in other animals which it may invade. Having carved out the idol of specific contagious abortion in cattle, it becomes obligatory upon the part of those who thus believe to have a specific contagious abortion for each species of mammal in order to account for intra-uterine death which overtakes and destroys a large proportion of embryos in every land and in all species of mammalia. Perhaps no animal furnishes more direct evidence of the non-existence of a specific infection with a direct abortifacient action than the sow. One embryo or five or six embryos in the uterus of the sow may succumb to infection present, but the infection is impotent to cause the expulsion of the cadaver or cadavers so long as a healthy immature fetus remains. If, however, all embryos die or become seriously ill, the endometrium is generally involved and the diseased organ revolts and expels its perilous contents. Otherwise the orderly completion of pregnancy proceeds, and at full term the living fetuses are born and the cadavers are expelled. The uterus accordingly evacuates its contents in two ways - the ordinary physiologic birth when the fruit is ripe, and the pathologic expulsion of its contents when the embryo or embryos and their coverings are dead or seriously diseased, associated with concurrent disease of the endometrium identical in cause. Any organism capable of causing endometritis and placentitis with death or critical illness of the intra-uterine young may and does lead to abortion, but no microörganism is yet known which can directly and specifically cause uterine contractions.

The $B$. abortus is a common resident of the genital tract of sows which abort or are sterile. It is common in boars. It is not known to be uncommon in healthy swine. Its common presence where abortion and sterility prevail justifies the belief that it is one of the bacteria which serve to interfere with reproduction. But there are other organisms present, along with the $B$. abortus, which have not been studied and the significance of which can not now be guessed.

The control of the genital infections of swine is to be attained by the adaptation and application of the principles al- 
ready considered at length for the analogous infections of cattle. The individual value of swine and the anatomical conditions largely inhibit individual handling and call for preventive measures en masse. Swine are largely handled as scavengers and consumers of foods repulsive to other domestic animals. Near cities they are fed largely upon garbage. There is a general tendency to handle swine as lovers of filth and as immune to dirt.

Throughout history swine have been closely associated with cattle, have consumed their waste milk, salvaged undigested grains from their feces, consumed the viscera of slaughtered cattle, and devoured the carcasses of diseased cattle. In this long and intimate association there is a natural tendency for community of bacteria. It is not strange therefore to find that $B$. abortus and other bacteria common in bovine genitalia are similarly prevalent in swine and have an analogous significance.

In one interesting clinical study it appeared that this intimacy of association exerted an important influence upon the fertility of swine. In a large herd of purebred dairy cattle, genital infections were intense. There were heavy losses from abortion, but heavier losses from sterility due to cervicitis and salpingitis in the cows, and to epididymitis and spermato-cystitis in the herd bulls. B. abortus was present, but the major role was apparently played by a streptococcus of the viridans type. The establishment also had a purebred herd of swine, in which the reproductive efficiency gradually decreased until the herd became a distinct economic burden. Little abortion was observed, but a majority of the females were sterile. As it was desired to develop the pigs as rapidly as possible, their mothers' milk was supplemented by all the dairy milk they would take. I recommended that no further raw dairy milk be allowed to swine of any age or either sex. All milk was to be boiled. The herd was divided into three groups. In the first group were listed all sows which had been used for breeding and which had disappeared from the herd when the change in the feeding of dairy milk was made. The second group in- 
cluded all females which had been fed raw milk and which, under the new plan, received boiled milk. The third group consisted of females born after the recommendation had gone into force, which had received throughout their lives only cooked milk.

In the first group there were 11 females which were kept in the herd a total of 131 months after they had reached breeding age. They produced 100 healthy pigs - one healthy pig for each 1.28 months, or $91 / 3$ pigs a year. The second group comprised 25 sows with a total of 438 breeding months, which had produced 207 healthy pigs, or one for each 2.11 months, equivalent to 5.68 pigs per year. The third group comprised 8 sows (at date of making up statistics) which had been kept a total of 92 breeding months and which had produced 107 healthy pigs, or one pig for each 0.85 months, equivalent to 14.1 pigs per annum. Accordingly the combined efficiency of the two first groups was an average of 6.48 pigs per sow per year as opposed to 14.1 pigs per annum in the final group. I have been unable to account for the doubling of the reproductive efficiency except by the change in the plan of feeding dairy milk. It appears, too, that it was not the feeding of the adult sows which affected the breeding efficiency, but the more hygienic feeding of the young pigs. This is parallel to my observations upon the health of nursing calves. If the calf is healthy, whether male or female, it will be fertile when it reaches breeding age, but if it has diarrhea or pneumonia as a young calf its fertility as an adult will be low. I do not attribute the higher fertility in the third group to the killing of the Bang organism in the milk fed, nor is there any assurance that the bacterial content in the milk was directly responsible. It may well have been that the living bacteria in the milk established lesions in the digestive mucosa ( $\mathrm{mu-}$ co-enteritis, dysentery) which prepared an open portal of entry for bacteria already present in the intestines of the pig, and that these, passing to the genital tract, persisted to sex maturity and impaired reproduction. 
The principles, therefore, in controlling losses from genital infections in swine are to grow pigs under the best known hygienic rules, being careful not to overfeed, keep them in the cleanest practicable quarter's and, when using cow's milk or by-products thereof, sterilize the latter before feeding. In these precautions no sex distinction is to be made. Sows should be closely watched in parturition and, if necrotic embryos are expelled or the litter is low in number, the sow should be discarded and her litter go with her. The boar, too, is to be observed carefully and, if at all suspected, should be subjected to examination by a skilled veterinarian along the lines already traced for bulls.

Swine breeders have limited their care in mating too strictly to the avoidance of hog cholera. The prudent breeder should use great care in accepting sows from other herds for breeding because of the ever-present danger from infections of possibly greater virulence than those existing within the herd. In selecting males for breeding, one should be taken from a large litter, indicating thereby that the dam was healthy and that consequently her pigs were, in the largest available degree, also sound. The general rules of care regarding moderate feeding and abundant exercise should be applied intelligently. 


\title{
SECTION IV. THE GENITAL INFECTIONS OF HORSES
}

\author{
CHAPTER XXI \\ THE SPECIFIC VENEREAL DISEASES
}

A. Dourine

Maladie Du Coit. Beschalseuche

Bibliography. Baldrey, Jour. Comp. Path. and Therap., 1905, Vol. 18, p. 7. de Does, Jahresbericht, 1902. Hutyra und Marek, Spezielle Pathologie und Therapie. Mohler, Eichhorn and Buck, The Diagnosis of Dourine by Complement-Fixation, Jour. Ag. Research, Vol. I, No.2, Nov. 10, 1913. Thanhoffer, Ueber Zuchtlähme. W. L. Williams, Report Illinois State Board of Live Stock Commissioners, 1887.

Dourine of the horse is the most serious specific venereal disease known among domestic animals, on account of its wide geographical distribution and the mortality and loss caused by it. It is, or has been, widely disseminated in Europe, Asia, Africa and North America. It has been recognized for more than a century and has appeared in all the leading countries on the European continent. In Englishspeaking countries it was first recognized by the author at Wapella in DeWitt County, Illinois, in the spring of 1886 , among imported French draft stallions and the mares which had been served by them. The disease in Illinois apparently broke out in 1884 or 1885 , but its nature was not determined until 1886, and even then its seriousness was not fully appreciated. It was not until 1887 that vigorous measures were undertaken for its control and eradication. In the meantime, numerous animals had been sold from the infected area and widely disseminated over the country in a manner which made it impracticable to trace them effectively to their ultimate destination. When the importance of the malady became recognized, it was promptly placed under control in its original center and was so completely eradicated that up to the present time, a period of thirty-five years, it has not reappeared in that territory.

Since that time the affection has been recognized in Nebraska, South Dakota, and the province of Alberta, Canada. 
In these areas of infection the disease has not been definitely traced to its source. The outbreak in Illinois was believed to have been introduced by stallions imported from France, a conclusion which, although unproven, time has not served to change. The affected area in Illinois produced considerable numbers of grade draft stallions and mares which were sold to go to the West and Northwest for breeding purposes, and, although it cannot be clearly shown, there is good reason to conclude that this outbreak furnished the infection for the others which have occurred in America.

Nature. Dourine is a highly infectious venereal disease transmitted naturally by coition only. Experimentally it may be transmitted by inoculation to other animals than solipeds. It is due to a protozoan parasite belonging to the trypanosoma group. This parasite, the trypanosomum equiperdum, was discovered by Rouget in 1896, and its relation to the disease clearly demonstrated by Schneider and Buffard in 1899. The trypanosome of dourine is a onecelled organism provided with a flagellum at the anterior end. It is about 18 to 26 microns in length and, when observed in the living state, is highly motile. It occurs in the blood, the spinal fluid, the discharge from the genital organs, the plaques of the skin, and perhaps in other tissues and fluids of the infected animal. It multiplies by longitudinal division. When removed from an animal and kept moist, it will live for several days or even a week.

It is not always easily found. It is said to be most readily discovered in recently formed plaques. Baldrey says, in reference to the appearance of the trypanosoma in the fresh plaques, "If these appear, then a positive diagnosis can be made; if not, the case is not dourine." The parasites are said to disappear quickly from the plaques, so that it is difficult or impossible to find them after twenty-four hours. They may be found in the discharge from the vagina or urethra of the infected animal, but it seems that their existence here is not as uniform as in the fresh plaques. Some investigators believe that they are quite uniformly present in the spinal fluid. It is generally very difficult to discover them in the blood. 
This parasite constitutes an exception to the general rule in the trypanosomic group of diseases, in that it is not transmitted from animal to animal by the bite of an insect.

Symptoms. The period of incubation following natural infection is not definitely determined and varies considerably in different cases. Generally there appear some physical signs of disease in from eight to ten days after exposure, but these may not be very marked and may pass unnoticed by an inexperienced observer.

The symptoms of the disease may be divided into three important groups: the local lesions of the genital organs and the contiguous parts; those of the skin and other mucous membranes than that of the genital organs; and the symptoms which emanate from the central nervous system. The local symptoms in the genital organs are the first to appear after infection and usually the last to disappear in case of recovery.

In the stallion there usually appears, eight to ten days subsequent to infection, a swelling of the penis and prepuce, with some degree of protrusion of the penis, still covered by the prepuce. The prepuce shows a tense, elastic swelling and has a smooth, glistening appearance. If the urethra is exposed to view, its meatus will be found swollen, dark red, and intensely injected. From it exudes a small amount of a thin, dirty, muco-purulent discharge. As the disease progresses, the tumefaction of the prepuce and penis increases and extends to the sheath and scrotum, which may eventually become enormously swollen and remain in an indurated condition for one to two years or more. The animal gradually becomes less able to retain the penis in its position, and the swollen organ protrudes farther and farther out of the sheath, sometimes extending entirely out of the prepuce and hanging flaccid and fully exposed. The discharge from the urethra may become somewhat ichorous and, with the general soiled condition of the penis and surrounding parts, may lead to ulcerations and erosions upon these. Later in the course of the disease, the weakened condition of the tissues of the part may lead to purulent infection and the 
formation of abscesses in the sheath, scrotum and inguinal region.' (See Figs. 223 and 224.)

The testicles may undergo inflammation and swelling which after a time may be followed by atrophy, and they may be pushed upward by the swelling or abscesses in the scrotum

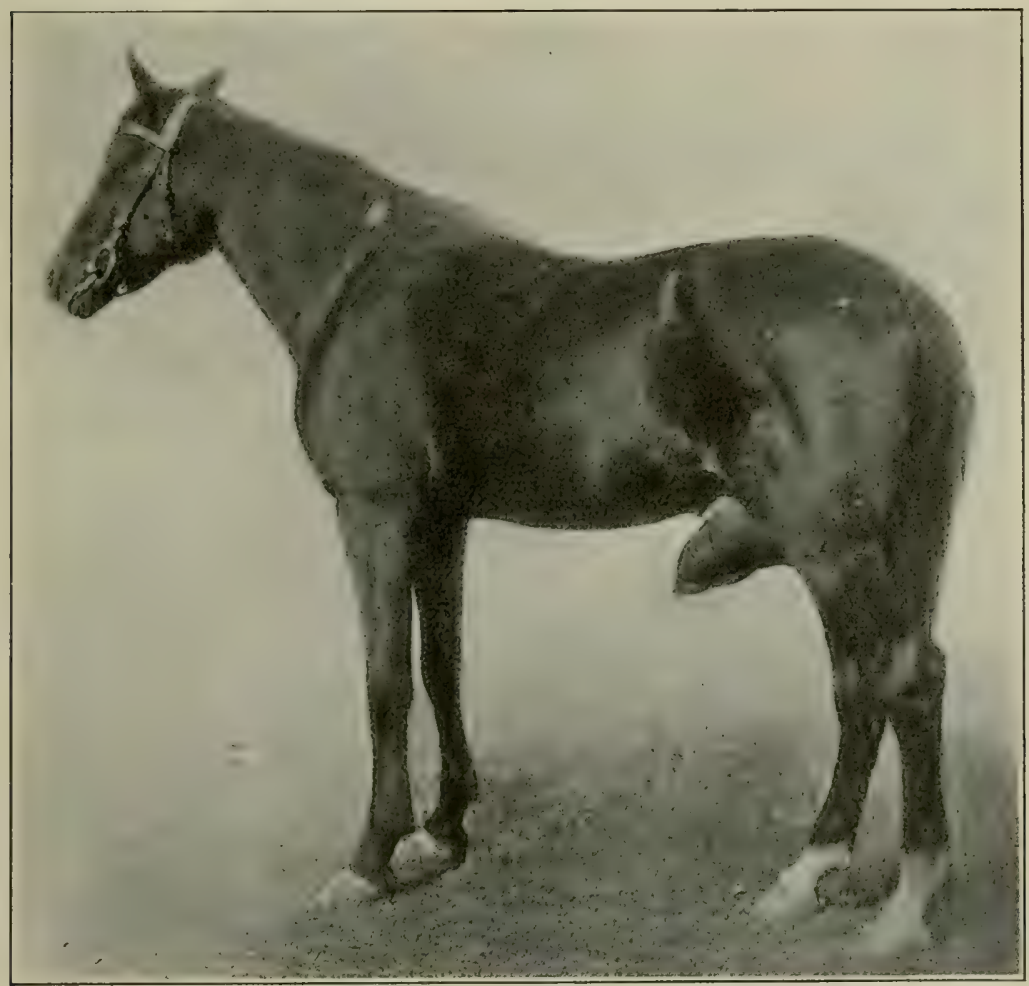

Fig. 223-Stallion in Early Stages of Dourine, showing: Edematous Prepuce. (Baldrey.)

until they rest high up in the inguinal space where they can not be readily felt. No vesicles or pustules occur upon the genitalia as an essential part of this disease, but there occurs with considerable uniformity a depigmentation of the skin and covering of the penis, so that it may largely lose its pigment and become white. This depigmentation begins 
upon the penis and may extend to the prepuce, sheath and scrotum. In studying this symptom, it is well to bear in mind that in gray horses and in those having white eyes, faces or feet, it is quite common to observe an absence of pigment on the penis, but this is regular in outline, whereas in dourine the depigmentation spreads out in patches.

In the mare, about eight to ten days after infection there appears a well marked swelling of the lips of the vulva. The

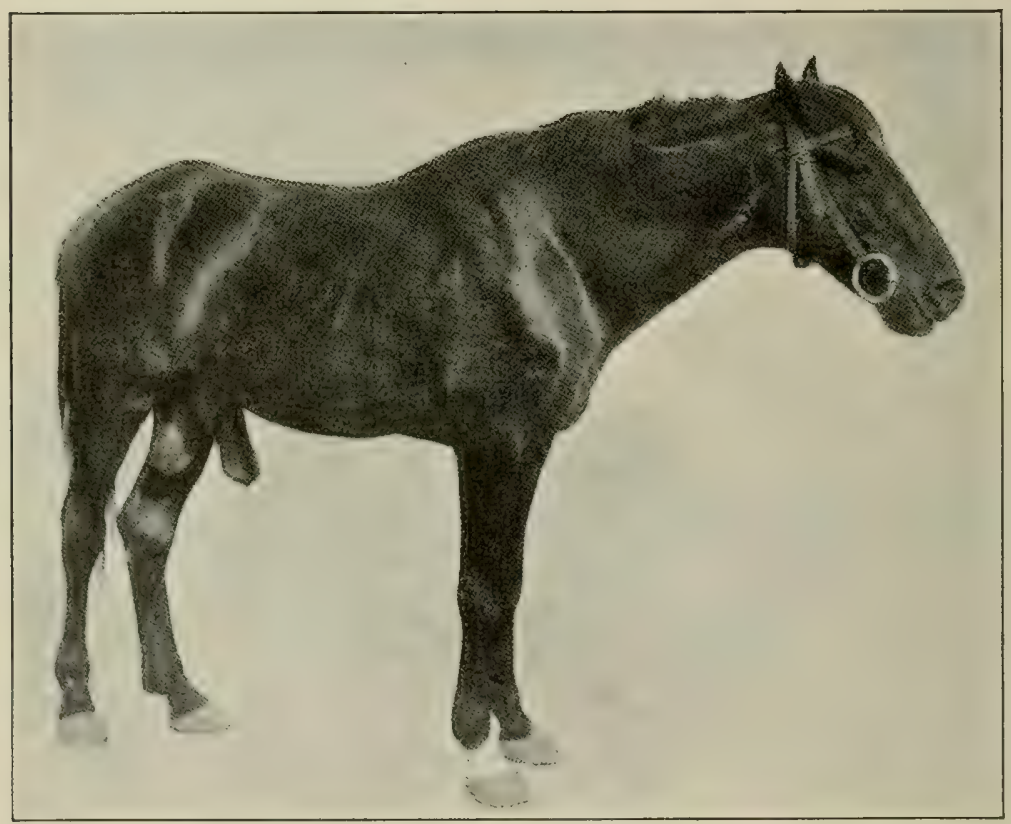

FIG. 224-Dourine showing Penial Paralysis. (Baldrey.)

mucous membrane of the vulva and vagina is intensely injected and swollen, and from the vulva there escapes a more or less profuse discharge of a mucous or muco-purulent character, which soils the tail and surrounding parts. The mare urinates frequently in small quantities, which causes pain as indicated by straining, stamping with the feet, and switching of the tail.

There seems to be an increased sexual excitement, which 
may be somewhat misleading, depending largely for its expression upon the frequent urination, rather than upon genuine sexual desire.

As the disease progresses, the volume of the discharge tends to increase. In some cases it assumes a dirty grayish color, and may become fetid. The swelling of the vulva increases, the labiae become much enlarged, tense, elastic, and glistening. Their margins stand somewhat apart, so that the vulva is partly open, especially at its lower commissure.

Within a few weeks after the advent of the disease, there appears quite regularly a characteristic loss of pigment in the skin of the vulva and the neighboring parts. This depigmentation begins along the margins of the vulva, without prior vesicular or pustular eruptions, as simple white patches, and thence extends in irregular lines or spots, until it may involve the entire vulva, perineum and anus. The white patches have irregular borders and are of various shapes and sizes. They persist for several months and, in case of the apparent recovery of the animal, tend finally to disappear, fading first at the periphery, while upon the margins of the vulvar lips they persist for six months or a year. (See Fig. 225.)

Of even greater diagnostic significance are the changes which occur in the clitoris. Comparatively early in the course of the disease, this organ becomes swollen and, the vulva being somewhat open at the inferior commissure, becomes visible ordinarily without mechanically parting the labiae. The secretion of sebum in the prepuce of the clitoris apparently ceases, and the swollen organ becomes dry and glistening. Depigmentation of the clitoris and its prepuce occurs early and persists for one to two years in those mares which apparently recover. In my experience it is the last symptom to disappear. The swollen, depigmented clitoris with gaping vulva constitutes one of the most uniform and persistent symptoms of the malady and gives to the vulva of a young mare the appearance of extreme old age.

Following closely upon the first appearance of Iocal lesions, 
within a few weeks after infection, there appear peculiar and pathognomonic cutaneous eruptions in the form of plaques, or elliptical elevations. These eruptions appear suddenly, largely about the flanks, neck, shoulders, sides and thighs, as more or less circular elevations, one to several

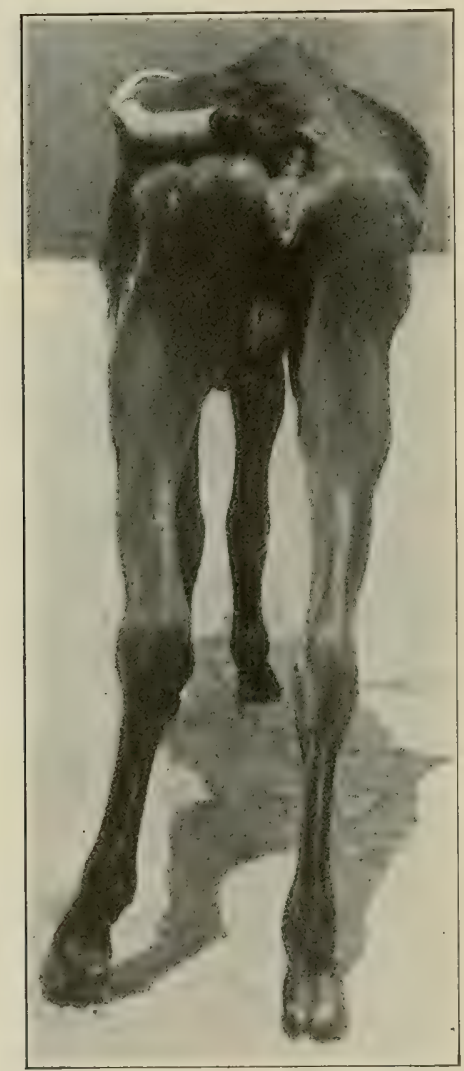

I. Depigmentation of Vulvar Lips. (Hutyra and Marek.)

inches in diameter. The margins of these are very abrupt and stand up above the level of the healthy skin like the eruptions of urticaria. The hairs upon them are erect. They appear suddenly, usually not in great numbers, but only 
one to five or six at a time, remain a few days and disappear without leaving a mark, to be succeeded by a new crop. It is claimed that in these plaques the trypanosomes are very abundant during the first stages of their existence, but as the eruptions grow old the parasites rapidly disappear from them.

It has been stated that the plaques may cause intense itching and lead the animal to bite or rub the part and that they sometimes suppurate. I have observed none of these symptoms.

It is not uncommon to observe in the later stages of the disease a more or less profuse discharge from the nostrils. This nasal discharge may in some cases be suggestive of glanders and superficial ulceration of the mucous membrane may rarely be present. The erosions, when present, have no specific character and suggest rather a necrosis of a greatly debilitated tissue as the result of an irritant applied externally. It must not be forgotten that glanders and dourine may coexist and that the latter would, naturally greatly intensify the former.

The general debility or cachexia of the disease shows itself clearly in lesions of the skin. Wounds heal tardily. If the animal is so weak that it is recumbent a large part of the time, it suffers from extensive decubitis gangrene.

The symptoms, which are largely referable to the nervous system, appear at about the same time as the plaques. The first and most pronounced of this group is usually a progressive paresis, which is chiefly observable in the hind parts. At first there is an unsteady gait. The animal brings the hind feet forward in a difficult and somewhat uncertain manner. There is a tendency to drag the toe along the ground or to strike it at the middle of the stride. When weight is placed upon the foot the toe is usually brought down first, with the fetlock flexed, and the heel is then lowered suddenly. While standing, there is a tendency for the fetlocks to be maintained in a flexed position or somewhat knuckled over. This knuckling over, whether standing or during progression, is a rather common symptom in trypanosomic, if not protozoan diseases generally. 
The imperfect control may appear in one or both hind limbs or may alternate between the two, and is subject to great variation from day to day. In the main the paresis reminds one of locomotor ataxia of man. Sometimes the partial paralysis is of a somewhat spasmodic nature, faintly resembling stringhalt. At times there may be swelling about an articulation and the animal may seem to be lame in the joint. As the disease advances, the paresis tends to increase until there is such complete paralysis that the animal is unable to rise when down. When the patient becomes unable to rise, a fatal termination usually occurs in the course of a few days to several weeks, largely hastened by the decubitis and the accompanying complications. In other cases, after being recumbent for days or having had to be assisted in rising for weeks, the animal improves and eventually apparently recovers .

With, and even before, the advent of the paretic symptoms, there appears a very rapid emaciation, which is especially prominent in the posterior parts of the body. The emaciation is rendered especially noticeable from the fact that it occurs in spite of a good appetite, and the allowance of abundant food with apparently good digestion. It seems that both the paralysis and the emaciation are largely dependent upon changes taking place within the spinal cord and that these symptoms naturally become most marked in those portions of the body posterior to the locality in the cord where the chief destruction has occurred. The symptoms of the disturbances of the nervous system are not confined to any portion of the body, and there is frequently observed a paralysis of an ear or eyelid or of the lips or nose. In the stallion there is frequently such a marked change in the voice that he can not whinny naturally.

The sexual desire may remain unaffected throughout the disease. In many stallions, the power to copulate is but little impaired, but in the earlier stages, during the tumefaction of the prepuce and penis, either from excessive erection or from other cause, the stallion fails in many cases to effect coition, and, late in the course of disease, the same inability 
may arise from loss of power in the penis or from extreme paralysis of the posterior parts.

The effect of the disease upon the powers of reproduction is very profound. In the earlier stages, the stallion may be capable of impregnating mares, which, if he does not infect them with the disease, may give birth to healthy foals. As the disease progresses, while the stallion may still be capable of copulating, he is usually sterile. The mare which becomes infected does not generally conceive, or, if she does conceive, aborts so early that the conception is not observed. In the Illinois outbreak, it could not be determined that a foal had been born among one hundred diseased mares. Some writers claim that occasionally a diseased mare will produce a healthy foal.

Apparent recovery may, and does, occur even after extreme emaciation, and when paralysis has been so complete that the animal could not rise without assistance.

The duration of the disease may extend from three months to as many or more years, and apparent or real recovery may take place at any intervening time.

In animals which have succumbed to the malady or been destroyed in its last stages, there is seen, quite uniformly, an evident degree of anaemia and emaciation. Distributed throughout nearly every tissue in the body, there is found a characteristic yellowish exudate of gelatinoid appearance, especially abundant in the subcutaneous and intermuscular connective tissue, in the mesentery, and in all parts rich in connective tissue. Nearly every organ of the body partakes of the general yellowish tinge. The muscles, especially those of the thigh and croup, are pale and soft.

The intestines are pale, and, in some cases, show signs of previous inflammation on their peritoneal surface. The mesentery is of a pale saffron color, thickened by a gelatinous exudate. The mesenteric lymphatics are enlarged, pale yellow and friable. The spleen is pale, small, shriveled, tough and hard. The liver is soft and filled with dark blood. The kidneys are usually somewhat enlarged, very pale and edematous. 
The genital organs fail to exhibit the extraordinary changes one would expect to find.

I made autopsies upon several stallions which had been infected for one, two or more years. Aside from the general changes noted above, there occurred in the genitalia of some animals gross lesions not directly attributable to the dourine but apparently the result of secondary infections which had invaded the weakened tissues.

One very bad case, an imported French draft stallion, showed great enlargement of the scrotum, which was hard and unyielding to the touch. The skin of the scrotum was enormously thickened and pale yellow. The inguinal glands of the right side were the seat of extensive abscesses, opening at the upper part of the scrotum. A large abscess, occupying the usual position of the testicle, was filled with dark yellow, hard, cheesy pus, which had pushed the gland from its place up into the inguinal canal. The testicle was small, atrophied, soft, flabby and pale yellow, with the serous covering firmly adherent at every part. The surface of the penis offered no evidence of disease. The urethra contained a small amount of a dirty, purulent secretion; the lining membrane was rough, grayish-yellow, without any appearance of ulcers. The seminal vesicles and enlarged portions of vasa deferentia contained thin, grayish, purulent accumulations. The left testicle was normal in size, with coverings firmly adherent at every part. No appearance of ulcers was found in the urethra or upon the penis of any of the several stallions examined.

Investigators have observed inconstant changes in the nervous system, principally injection of the coverings of the brain and spinal cord, softening of the lower part of the cord, and occasional extravasation of fluid into the ventricles of the brain. Thanhoffer describes extensive degeneration of the nuclei of the nerve cells in the spinal cord. The nasal mucous membrane usually shows catarrhal inflammation.

Differential Diagnosis. Few contagious diseases of animals have been so confusedly described by veterinary writers. At first there was a very general confusion between 
dourine and genital horse pox, a condition which still continues in many descriptions of the malady.

The most reliable local symptoms for the diagnosis of dourine in the stallion consists of the doughy, elastic swelling of the prepuce, with varying degrees of penial paralysis, the penis hanging somewhat out of its sheath, usually retained within the prepuce. The urethral opening is usually inflamed and a slight discharge escapes from it, but there is nothing visible to the naked eye to mark this as differing from lesions of these parts due to other causes.

Later a depigmentation of the penis and prepuce may occur-not in small circular spots, as in genital horse pox, but in large, irregular patches, which gradually spread from the periphery. In the mare, the most important local symptoms for purposes of diagnosis consist of the doughy, edematous swelling of the vulvar lips, the enlargement of the clitoris, the gaping of the vulva at its inferior commissure, and the depigmentation of the clitoris and its prepuce, and of the skin of the vulva, perineum and anus.

Once it is decided that an equine venereal disease exists in a stud, the presence or absence of specific pustules or vesicles may serve largely to differentiate between the two maladies. Eruptions upon the external genitals may, of course, occur in dourine, but those writers who have mentioned them uniformly fail to describe them in a manner to enable one to differentiate those of dourine from those of genital horse pox, and, as a rule, it might well be suspected that such descriptions are based upon diagnostic error. In some cases, doubtless, erosions or ulcers have appeared as the result of irritation from ichorous discharges or from the accumulations of filth about the genitals, accompanied by low vitality in the cutaneous tissues, but such eruptions are devoid of diagnostic value, and their relation to the disease, so far as we know, is quite secondary.

Specific eruptions of vesicles or pustules upon the genitals do not occur. When aburidant and specific emptions occur on the genitals of the horse, they indicate genital hor'se pox, not dourine. 
Dourine and genital hor'se pox may readily coexist, causing confusion in diagnosis.

Some writers, in describing dourine, accept the presence of the depigmented areas about the vulva and anus as conclusive evidence of precedent ulceration, but depigmentation of the skin does not necessarily follow vesicular or pustular eruptions, nor does its presence indicate that eruptions have occurred. I had excellent opportunity for observing, day by day and week by week, the depigmentation of the skin of the vulva and anus in dourine, and saw it begin and gradually spread, without the presence of any visible vesicles, papules or ulcers. It was a depigmentation without ulceration.

Numerous writers place emphasis upon the presence of specific eruptions in dourine, but fail to describe their character, and do not intimate that they have personally observed them. In the extensive Illinois outbreak, among more than one hundred cases of the disease, no eruptions were observed, nor could the most diligent inquiry among owners reveal any history of such in any animal. Baldrey, after an extensive experience with the malady in India, fails to record the occurrence of vesicles and pustules.

It seems to me that the apparent differences in observation and view in reference to vesicles, pustules and loss of pigment in dourine is due to the constant confusion of the two wholly distinct veneral diseases and to accepting the erroneous conclusion that depigmentation indicates prior pustular or vesicular eruption.

In reference to the alleged occurrence of eruptions it should be noted that the character of the micro-organism which causes dourine is contradictory to the appearance of such lesions. Trypanosoma, in general, have little or no tendency to produce eruptions or suppuration, and it would seem unique to expect that, in this one disease, alone, of the great group, there should occur characteristic vesicles or pustules.

The elliptical swellings or plaques in the skin of the flanks, hips and other parts of the body have long held a high place in diagnostic value, but they do not always exist. 
In the Illinois outbreak, the enlarged and pigmentless clitoris constituted a noteworthy and highly diagnostic symptom in the mare, persisting for at least two years after all other physical signs of the malady had disappeared.

The paresis of dourine is fairly characteristic. Accompanied by other lesions and symptoms it is pathognomonic. The peculiar jerky movements in the hind limbs during progression, and the knuckling over at the hind pasterns are rarely seen in other forms of disease.

In addition there is the clinical history of infection by coition along with the malignancy of the malady.

The finding of the trypanosomum equiperdum in the blood of the animal serves to establish definitely the diagnosis. In many cases it seems to be very difficult to find the parasite. Consequently this proof of the character of the disease is not always readily produced. More recently the serological tests have been developed and Mohler, Eichhorn and Buck have obtained satisfactory results by complement-fixation, using the surra trypanosome in preparing the antigen. They thus secured a "group" instead of a species reaction which, in the absence of other trypanosoma in this country, sufficed.

The mortality from dourine is very great and the loss amounts, upon the whole, to far more than the total value of the diseased animals. When it has once become widely disseminated in a breeding district, it practically suspends the industry for a time because its character is so insidious that it is exceedingly difficult to trace in it all its ramifications. The mortality among the affected animals reaches 60 to 80 per cent., which places it among the most fatal of infectious diseases. Those which apparently recover do so very slowly and the time consumed before they are again fit for work is so great that their value is virtually destroyed. It is a question whether animals once affected can be bred again with safety, no matter how completely they may have apparently recovered or how long a period may have elapsed.

No treatment for dourine has yet been devised which is economically practicable and which affords proper security to the state. Recently some of the more complex arsenical 
compounds have given encouraging results experimentally, but it is a question whether the treated animals are so far recovered that they are without menace. In the Illinois outbreak several mares apparently made quite complete recoveries and performed satisfactory work, but it was deemed essential to keep them under close breeding quarantine with the constant danger that, by accident or design, coitus might be permitted to occur and a fresh outbreak be started. On the whole, it is more economical that the state should assume the burden and destroy all affected animals. Until reliable diagnosis has been made, breeding should be suspended and all suspects placed under quarantine restrictions ample to prevent sexual contact. This means a quite rigid quarantine, frequently extending over a long period. The disease is entirely too insidious and dangerous to justify lax provisions. The castration of suspected animals has been advocated, but this is not safe against error. A castrated stallion may copulate with mares. I have known a castrated mare to be forced into coitus with the stallion; the owner, unaware that a prior owner had had her castrated, believed her in estrum. If a quarantine can be so arranged that it will fully protect the public interest and not prove an economic burden, the apparently recovered animal is efficient as a worker and, aside from coitus, is without known danger.

\section{B. Genital Horse Pox. Coital Exanthem}

\section{Eruptive Venereal Disease of the Horse}

Genital horse pox is a highly contagious disease, which, under ordinary conditions, is transmitted by coition only and consists of a local infection of the genital organs. It is far more readily transmitted than dourine and has a shorter period of incubation.

Symptoms. After a period of two to five days subsequent to exposure, there appears in the mare an inflammation of the mucous membrane of the vulva and vagina, in which there arise small reddish papules, which soon become vesicular or pustular and rupture, leaving small erosions on the mucosa. From the vulva there occurs a more or less cop- 
ious, muco-purulent discharge, which soils the tail and neighboring parts. Urination is somewhat frequent and the contact of the urine with the denuded mucosa irritates that membrane and causes straining.

At the same time, similar eruptions appear upon the external surface of the vulva, anus, perineum and surrounding parts. They behave somewhat similarly to the eruptions within the vulva and vagina. At first, a small papule arises in the skin, which soon becomes vesicular and then pustular and yellowish white. These mature quickly and soon rupture, to be followed by tough, yellow scabs, $1 / 8$ to $3 / 16$ inch in diameter. After a few days, the crusts drop off, leaving behind white scars very slightly depressed. These depigmented spots are circular in form and, in very severe cases, may coalesce somewhat. They tend, however, to remain distinct circular spots. The pigment returns after two or three weeks, and no visible scar remains. The vesicles appear in continuous crops over a period of one to three weeks, so that, at a given examination, there may be eruptions in every stage.

Accompanying these eruptions, there is a swelling of the vulva, with some tenderness of the parts. Some writers have described also a prurigo or intense itching of the parts, causing the animal to rub violently the tail and vulva. Although I have observed a number of outbreaks, I have not seen this symptom. Neither have I been able to find in the literature upon the subject any case where such a symptom has been recorded as a personal observation.

In the stallion, the symptoms are virtually the same. Eruptions, of the same character as those described upon the vulva and anus of the mare, appear upon the penis, prepuce and sheath. The opening of the urethra is swollen and congested and from it there is a muco-purulent discharge. There is some swelling of the prepuce and possibly, to a less degree, of the penis. Ordinarily, there are no constitutional symptoms in either sex, there is no loss of appetite nor appreciable emaciation, and the general condition of the animal remains undisturbed. I saw one case in which fol- 
lowing alleged genital horse pox, there remained a year afterward a severe chronic inflammation of the vagina and bladder, which virtually ruined the animal. In this case, the mucous membrane of the vulva and vagina was greatly thickened, corrugated, sensitive and bled easily upon touch. The bladder was highly inflamed and its mucosa greatly thickened, the urinary salts were deposited over its inner surface, and its cavity was largely obliterated. The meatus urinarius was open and the urine dribbled away involuntarily, so that the tail and thighs were kept constantly befouled and presented a very repulsive appearance.

The course of the disease is usually mild and brief. Most cases recover spontaneously in from two to three weeks and, under proper handling, recovery is hastened and rendered increasingly certain.

The nature of the disease is not precisely known. A given outbreak is not usually traceable to any definite source of origin. The disease seems to become established in a community without having been imported by a diseased animal. Once it becomes established, almost every mare bred to an affected stallion contracts the disease with great uniformity. Presumably it is just as transmissible to the stallion, but this is not so often observed, since the mares are not usually bred to different males during the same season. Experiments have shown that the vesicles and pustules contain the element of contagion in a virulent form.

Handling. The handling of the disease depends essentially upon a temporary cessation of breeding and upon disinfection. Any disinfectant will answer the purpose, but it should not be highly irritant, since the parts involved are very sensitive.

The handling of the stallion is most urgent, so that he may resume service as promptly and safely as possible. I have come to prefer an antiseptic wash composed of 1 ounce of carbolic acid with 2 ounces of tannin and 6 ounces of glycerine in 1 gallon of warm water. After washing the penis and prepuce thoroughly with soap and water, this solution is applied freely twice daily and a quantity of it is injected up the urethra until it is supposed to reach nearly to the bladder. 
The extent of disease in the urethra has not been investigated. It is only known that there is a urethral inflammation and dicharge and that it is infective. The mere washing of the penis and prepuce can not, therefore, bring about effective disinfection, but the urethra needs be included. The urethral injection is best made by means of a hospital irrigator with a small rubber horse catheter attached. The end of the catheter is inserted into the urethral opening and retained there while the antiseptic solution is forced upward by gravity as far as is deemed necessary. Should some of the fluid reach the bladder, it will not prove dangerous, but it is well not to overfill the urethral passage, lest the infective discharges be forced up into the bladder. The external ulcers resulting from the pustules may be touched lightly with stick nitrate of silver. The animal needs be kept as free as possible from sexual excitement, but should be given gentle exercise, along with a restricted, laxative diet. The stallion may be returned to the stud as soon as all evidences of disease have disappeared but it is advisable to continue the disinfection, especially after each service.

The handling of the mare should be along the same general line and should be persevered in until all symptoms have disappeared. As a general rule, she will recover sufficiently during the interval between two estrual periods that she may again be bred at the first return of estrum since the contraction of the disease, should she not prove to be in foal.

The differentiation of this affection from dourine is highly important, but has already been discussed.

There is little need for police control, since the symptoms are so prominent that laymen promptly note it and voluntarily withdraw the affected animals from breeding until recovery has occurred. Should there at any time be a tendency to negligence the affected animals should be promptly quarantined until all danger has passed. 


\section{NON-VENEREAL SPECIFIC INFECTIONS WHICH INVADE THE CENITAL ORGANS OF HORSES}

\section{A. Contagious Cellulitis. Epizootic Cellulitis. Pink Eye. Rheumatic Influenza. Muco-Enteritis}

Bibliography. Williams, Epizoütic Cellulitis, Principles and Practice of Veterinary Medicine, 4th edition, 1888 , p. 251. Cave, Pink Eye, Veterinary Journal, 1883, Vol. XVI, p. 336. Whitworth, Pink Eye Disease, ibid., 1883, Vol. XVII, p. 153. Pottie, Jour. Comp. Path. and Therap., Vol. I, p. 37 . Clark, ibid., Vol. V, p. 261. Reeks, The Transmission of Pink Eye from apparently Healthy Stallions to Mares, ibid., Vol. XIV, p. 159, and Vol. XV, p. 97.

Contagious Cellulitis is a highly contagious acute fever of the horse, which has been generally ignored except by British veterinary writers, although it seems to be widely distributed in various countries. It is common in parts of America, but frequently confused with influenza or catarrhal fever.

A description of the disease is inserted here because of its intimate bearing upon the question of horse breeding, which it affects chiefly in three distinct ways.

Frequently it spreads from apparently healthy stallions to mares through copulation, in which respect it approaches the character of a venereal disease, but as a general rule this is not the method by which it is transmitted from animal to animal.

It has a relation to sterility because it causes an orchitis in the stallion which frequently leads to a permanent loss of function in these glands.

Contagious cellulitis is commonly associated with abortion in pregnant mares. The manner in which abortion is caused by the disease is unknown. Since the bacteriology is unknown, it can not be stated that the infection does or does not enter the uterus or the fetus and bring about disaster. The probabilities are that the infection reduces the vitality of the pregnant mare and enables those bacteria which exist within the uterus to multiply rapidly and cause abortion.

Symptoms. The symptoms of the disease consist primarily of an elevation of temperature, sometimes accompanied by 
chills, dullness and other phenomena, which belong in general to acute contagious fevers. The fever appears very suddenly, usually ranging from 103 to $105^{\circ} \mathrm{F}$. although it may exceed this. Generally speaking, the temperature is higher than in influenza and lower than in the contagious pneumonia of the horse. The pulse is hard and full and somewhat quickened; respiration is not very greatly disturbed. Cough is present, though this does not constitute a very prominent symptom of the affection.

Early in the disease there is a manifestation of pain in the limbs, which is shown by shifting of the weight from one foot to another while the animal is standing and by great stiffness and soreness when forced to move, the movements being accompanied by a cracking sound in the joints. After a time the legs begin to swell, commencing at the feet and extending upward until at times the body is affected. This tumefaction has its chief seat in the subcutaneous connective tissue and is at times very great. As the swelling of the limbs increases, the pain in them tends to abate and at the same time the fever begins to decrease.

The alimentary tract is greatly disturbed. At first there is a very marked tendency to constipation, in which the feces are covered with large quantities of mucus and their color considerably altered. Defecation causes some pain and straining. Later there is a tendency to diarrhea, which is easily and often fatally intensified by the administration of purgatives, especially of aloes. The conjunctiva becomes bright pink, swollen and edematous. At times the conjunctivæ are so badly swollen that the eyelids become somewhat everted and the edematous membrane pushed out between them. The eyes are very sensitive to light and the animal attempts to keep them closed. There is a profuse secretion of tears, which flow down over the cheeks. The cornea is frequently clouded, so that vision may for a time be interrupted. There is some tendency toward pleurisy, pneumonia and other pulmonary complications.

From a breeding standpoint, the chief interest lies in the complications of the genital organs. The breeding stallion 
shows a great tendency to suffer from orchitis. I have seen in large importing stables 50 to 75 per cent. of the stallions attacked with pink eye suffering from inflammation of the testicles. Along with the swelling of the scrotum and sheath, which accompanies that of the legs, there usually appears a tense, painful enlargement of the testicles, which tends to persist for a considerable period after the general symptoms of the disease have largely disappeared. In some instances permanent sterility results. In other cases, stallions which have apparently recovered from the disease have quite uniformly transmitted it, during copulation, to susceptible mares. These observations are entirely in harmony with those of Reeks, who records an instance where an apparently sound stallion transmitted the disease to susceptible mares almost uniformly over a period of two years.

It is notable also that, when the disease attacks a pregnant mare, it generally causes abortion or, if attacking her just prior to parturition, the living foal is likely to succumb within a few days after birth. Reeks, recording an outbreak under his charge, relates that four pregnant mares, or mares which had just foaled, contracted the disease. Two of the pregnant mares aborted, and the foals of the other two died suddenly shortly after birth.

Although highly contagious, the affection is of short duration; usually runs a favorable course, except in those animals used for breeding purposes, in from four to six days; and leaves the animal but little altered.

No specific treatment has been discovered. The greatest benefit is to be derived from the administration of diffusible stimulants, such as nitrous ether or carbonate of ammonia, along with quinine. Purgatives are to be avoided. Aloes is especially dangerous. The bland oils might be used in some cases as aperients, or very small doses of eserine, arecoline or other alkaloid hypodermic cathartic, taking care to make the dose sufficiently small to induce no harmful degree of excitement and barely sufficient to cause gentle purgation. There is naturally much divergence of opinion among veterinarians in reference to the treatment of this affection, but its general handling is not of special interest here. 
When the disease appears in a breeding area it should be handled with the greatest rigor as to quarantine, and the breeding quarters should receive special consideration. Diseased mares or mares coming from infected premises should on no account be admitted to the breeding place. Should the malady become general in a neighborhood, as it usually does when an outbreak occurs, breeding should be suspended until the disease disappears.

When a breeding stallion has become infected, aside from the general handling of the malady, special attention should be given to reducing to a minimum the dangers from orchitis and later, after the acute symptoms have passed, to overcoming the lingering chronic infection, through which he may continue to spread the disease to mares.

When it is known that a non-immune breeding stallion has been exposed to this disease, immediate precautions should be taken to guard the animal against a severe attack. He should be placed at rest, physically and sexually. The usual high feeding of breeding stallions should at once be displaced by a very light laxative diet, such as grass, roots and bran, with an abundance of salt. If these measures do not induce a prompt unloading of the alimentary tract, the bowels should be evacuated by means of small doses of eserine or arecoline and the system placed in first-class condition to withstand the onset of the disease. Pending the advent of the disease, the animal should have regular daily exercise, though great care should be taken not to continue it after the appearance of the first symptom of the malady, fever, has become established, as indicated by thermometry. If the stallion is attacked without these precautions having been taken, similar measures should still be adopted. The ration should be reduced and confined to laxative foods, and the bowels should be promptly and cautiously evacuated.

The stallion is to be guarded against physical or sexual excitement. Strange mares should not be allowed in sight or hearing, and every precaution should be taken against arousing sexual desire. In most stallions the application of the stud bridle occasions excitement, which, at such times, should be avoided. 
If orchitis appears, as it frequently does, in addition to the foregoing measures, including the internal administration of nitrous ether and quinine, local applications to control the inflammation in the glands should be applied. First among these in the early stages is local refrigeration by the application of cold water or ice. Probably the most efficient means is the application of broken ice by means of a suspensorium, which serves the double purpose of refrigeration and supporting the weight of the pendant glands. This treatment should be constantly and faithfully applied until the crisis of the malady has passed, since the effective handling of these glands may determine whether the animal is to be of further value as a breeder. Such remedies as belladonna and camphor, applied locally, tend to relieve congestion and overcome the inflammation, but in a critical case the refrigeration is probably superior.

Later, when the acute symptoms have passed, chronic infection should be considered, precautions talen against the spread of the malady to mares, and measures adopted to eradicate the infection from the system as quickly as possible. For this purpose an extended course of potassium iodide at the rate of $1 / 2$ ounce per day for each $1,000 \mathrm{lbs}$. of body weight is probably the safest remedy. Some veterinarians fear that the prolonged administration of this drug may induce a harmful atrophy of the testicles, but this is quite imaginary and without foundation. The iodide should be continued for three or four weeks, or longer if necessary.

Abundant time should be allowed to elapse, after the disappearance of all visible symptoms of the disease, before permitting the animal to serve mares. It is advisable, in case the disease attacks the stallion during the breeding season, to withdraw him from the stud for the year. In any case it is wise to begin breeding cautiously and watch closely for evidences of the transmission of the infection to susceptible mares. When a stallion which has suffered from pink eye shows evidences of sterility, careful examination of the genitals and of the semen should be made and, should any restoration of the breeding powers seem possible, appropri- 
ate measures undertaken, but usually the sterility is permanent and irremediable and the animal must be removed from the stud and, with or without castration, used for work.

\section{B. Bursattee of the Penis and Prepuce}

Genital bursattee presents characters suggesting, to the inexperienced, a venereal disorder, and constitutes an annoying disease in breeding stallions. It is not infrequent in those countries where this malady prevails in other portions of the body. Bursattee shows a distinct tendency to attack the penis and prepuce of breeding stallions, especially of heavy draft animals. Presumably the infection is not coital but is transmitted by other means-accidental wounds, flies or other carriers.

The usual symptom of penial bursattee, when first observed by the groom or owner, is the presence about the urethral opening of an angry-looking fungoid growth, which bleeds upon the slightest touch and is generally observed to bleed immediately after coition. Sometimes during erection blood drips from the tumor. Another fungoid mass of similar appearance exists at the preputial ring upon the inferior or urethral side near the middle of the organ when erected-that point where the urethral opening rests when the penis is fuily withdrawn within its prepuce and sheath. When the penis is at rest, therefore, the growths about the urethral opening and on the preputial ring are in immediate contact: the preputial growth has emanated from that about the urethral opening, as a consequence of contact.

The bursattic growth may begin at other points or may extend from the seat of infection to neighboring parts. In one instance, I observed the disease beginning upon the scrotum.

If the new growth is closely inspected, it is usually possible to recognize readily with the naked eye the small yellow concretions or "kunkurs" characteristic of the malady. In some cases penial bursattee is accompanied by infections upon the feet or other parts. 
I have not seen the disease transmitted to mares through copulation, though, clinically, it behaves as an infectious malady and possibly there is danger of transmission to the mare through the vagina.

The micro-biology of the disease has not been fully determined: some attribute it to mematode worms; others to the presence of a fungus.

Like bursattee of other parts, it is active in temperate countries only during the hot season and undergoes apparent spontaneous recovery upon the advent of winter, to remain dormant until the return of warm weather. Thus it incidentally assumes its greatest activity during the breeding season and interferes seriously with stud duties. Aside from the remote possibility of transmission, it is very noticeable, and owners of mares naturally do not care to breed them to a stallion so evidently diseased. Furthermore, the sexual excitement incidental to breeding greatly influences the disease and adds to its virulence: each erection of the penis, with the consequent congestion of the organ, causes the bursattee growth to bleed.

The handling of the disease consists of the destruction or removal of all infected tissues and the avoidance of venereal excitement. If undertaken early, the disease is usually confined to the urethral tube, filling it and protruding beyond the navicular fossa, and may extend up higher in the urethra. Whatever the extent, the diseased tissues are to be excised or curetted away and the parts well cauterized with silver nitrate or the thermocautery, after which the wound may be dressed with iodoform, which apparently exerts a very beneficial influence upon the course of the disease, should any infection remain. Care is to be taken not to denude unnecessarily the urethral meatus of its entire mucosa, since urethral stricture may result, necessitating penial amputation. If the pathologic growth involves the entire circumference of the meatus, the risk of stricture must be deliberately faced with the assurance that, if stricture follows, amputation may be made without interfering with the breeding value of the stallion. The method of amputation has already been described. 
Before operating, it is well to apply local anaesthesia, though the sensitiveness of the part is not marked, as is usually the case with bursattic growths. Successful handling demands the withdrawal of the animal from the stud and the prevention of sexual excitement. If necessary, erection of the penis should be prevented by the application of a stallion guard.

At the preputial ring, at that point where the opening of the urethral tube rests when the penis is completely withdrawn, the secondary bursattic growth is more amenable to management. Here the operator can freely excise the diseased tissue without danger of unpleasant deformity.

After excision or destruction of the diseased tissues, the parts should be kept scrupulously clean. It is advisable to wash the penis, prepuce and sheath twice daily with soap and water, to which one-half to one per cent. of carbolic acid may be added. After cleansing, the parts may be dressed with powdered iodoform, or iodoform ointment. Before applying the iodoform, any suspicious areas may be cauterized with stick nitrate of silver. 


\section{CHAPTER XXIII}

\section{THE GENERAL INFECTIONS OF THE GENITALIA OF HORSES}

The general infections of the genital organs of horses are quite analogous to those of cattle. Apparently most bacteria which are capable of acquiring a habitat in the genitalia of cattle may do so in horses. Those who believe in a specific "contagious abortion" for cows and another for mares claim that the organism of "contagious abortion" in the cow will cause abortion in the mare, and vice versa. They further claim that the organisms causing abortion in each of these are potent to cause abortion in ewes, sows, bitches, rabbits, guinea pigs, etc. They have shown quite conclusively that the organisms are able to acquire a habitat in numerous domestic and experiment animals. How frequently the alleged abortion-producing organisms naturally acquire a habitat in the uteri of species other than that in which they are alleged to be the cause of abortion is wholly unknown, all recorded observations being predicated upon studies of those animals which had been experimentally inoculated with the infection.

The subject of "contagious abortion" as a specific disease in cattle has been discussed at length and any attempt to discuss "contagious abortion" of mares would be an unwarranted repetition. The evidences regarding the existence of such specific disease in the mare are the same in principle, but less voluminous than in the cow. Investigators claim to have proven the existence of such specific disease in mares and to have identified clearly the causative organism, but, as in the cow, they have ignored the two most basic principles in the experimental production of disease-they have submitted no evidence of the freedom of the experiment animal from the infection when the experiment was begun, and no controls were kept. The genital organs of horses offer analogous diseases throughout, modified by important variations in their anatomy. 
The clinical examination of the genitalia of both sexes of horses is essentially the same as in the cow. The stallion offers differences in the size and form of the seminal vesicles. The act of coitus is more prolonged. The collection of semen for examination is more readily obtained through the larger vulva of the mare. The semen may be obtained without vaginal contamination by the use of a rubber bag upon the penis. In other particulars the examination of the genitalia, the semen and the spermatozoa is the same as for the bull.

The ovaries of the mare lie far forward, but are easily within reach and readily palpated per rectum. The cervix is short and its canal very dilatable, rendering the uterine cavity more readily examined.

While describing the general infections of bulls, it was pointed out that, in the absence of definite general lesions recognizable by physical examination, there may and do exist in the testicles, epididymis and seminal bladder infections which are ejaculated in the semen and carry serious peril, causing extensive genital disease of the cow and resulting in much sterility and abortion. In discussing contagious cellulitis of horses it has also been pointed out that the infection may be transmitted to the mare by the stallion during coitus.

In polygamous reproduction the male is necessarily the chief factor in the transmission of genital infections, in part because he comes in sex contact with numerous females, while the female ordinarily has coitus with but a single male. The male is a further peril in sex infection because in polygamous reproduction he is placed under more severe sex strain, rendering existing infections in his genitalia more virulent. Schofield ${ }^{1}$ found that abortion in mares was chiefly observed in those which had been bred to certain stailions. It was further noted by Schofield that the foals, born of mares bred to stallions whose mares largely aborted, frequently suffered from arthritis. It was thus shown in

\footnotetext{
${ }^{1}$ Investigation into Equine Abortion. F. W. Schofield, Ontario Vet. Col.,
} 1915. 
his observations that the stallion transmitted an infection which led in many cases to abortion some months later and which in other cases persisted throughout the span of intrauterine life and continued in the foal after birth. This is in harmony with my observations. Nevertheless some stallions showing no genital lesions clinically are frequently highly infectious. According to the intensity of the infection, there follows death of the spermatozoa, ova, fertilized ova or small embryo (sterility), metritis with death and observed expulsion of the fetus (abortion), or puerperal metritis and retained fetal membranes in the mare, and arthritis, dysentery or other disease of the foal.

The general infections of the genitalia of horses conform, therefore, so far as can now be seen, to those of cattle and, what is more important, conform to the fundamental laws of general genital infections in all mammalia. Among the genital infections of mammals, certain specific diseases exist, such as syphilis, dourine, canine venereal tumors, chancroid or vesicular venereal diseases of man, horses and cattle, gonorrhea and others which involve largely or exclusively one species of animal, producing specific lesions which serve to differentiate clinically the infection from all others.

There are other infections which invade the genitalia of all mammals, each of which may and do exist in the genitalia of several species of animals. They do not necessarily cause visible harm but, when intensified by bad physical or sexual hygiene, may attain high virulence and produce a great variety of lesions. They may invade and interfere with the function of any organ or tissue in the genital system of either sex of any age. The invasion is not necessarily direct through the genital tract. The fetus swallows the infection, derived from the uterus of the mother, with its amniotic fluid. Again the new-born swallows any infection emanating from the uterus which may flow down the tail or thighs and reach the exterior of the teats. In many cases the infection enters the body of the new-born through the navel wound, in which case arthritis becomes the outstanding clinical phenomenon. But the arthritis of navel infection, 
except in some cases for the navel lesions themselves, is not clinically nor bacteriologically differentiable from the arthritis caused by the fetus swallowing the infection emanating from the utero-chorionic space, or by the new-born swallowing infection in its food.

Unlike the specific venereal diseases mentioned, the general infections are commonly present in the genital organs and elsewhere, their number is unknown, and their lesions are alike. The lesions in the uterus and fetus attributed to the B. abortus (Bang) can not be distinguished from those of the spirillum of Smith, the bacillus of Moussu, or the B. abortivo-equinus of Good. Each of these is believed to be capable of living and multiplying in the genital organs of an extended list of mammals.

As in bulls, when genital infections in stallions become severe and produce clinically recognizable lesions, they often lose their peril for the female because the spermatic ducts are blocked by the lesions and the infection imprisoned. The infections of the genitalia, therefore, which are recognizable only by a microscopic study of the spermatozoa, bacterial search of the semen, or a bio-histologic study of the ovaries or oviducts, are of far greater importance economically than the more readily recognizable lesions. The clinically recognizable lesions of the genitalia of horses are varied and not rare. 


\section{THE GENERAL GENITAL INFECTIONS OF STALLIONS}

\section{A. Orchitis and Epididymitis}

\section{Degenerative ORchitis and Epididymitis of Colts}

Colts sometimes suffer from a degenerative or desquamative orchitis in which the epididymis participates. The disease is apparently identical with that already described in bull calves. It ordinarily passes unobserved up to breeding age, when attention is attracted to the genitalia by a total want of fertility. Clinical examination reveals small, soft, flabby testicles, about one-fourth to one-half the normal volume. The animal has normal sexual desire and copulates promptly, but no spermatozoa are ejaculated. Histologic examination of the testicles reveals necrosis and desquamation of the germinal epithelium, with corresponding changes in the epididymis and absence of spermatozoa. The cause is unknown. Apparently the condition is dependent upon the invasion of the testicles during the nursing period, as appears to be the case in calves. The infection probably exists in the alimentary tract of the foal at birth and acquires invasive power during dysentery. At least the changes exist when puberty is reached and their character points to infection as the cause. Since the condition is hopeless, the animal should be castrated and used for work. Prevention of the condition lies in the proper rearing of young foals.

\section{Orchitis and EPididymitis of Adult Stallions}

Reference has already been made to the concurrence of orchitis and epididymitis as a common complication of contagious cellulitis in stallions. Orchitis and epididymitis not referable to any recognized specific infection, is observed occasionally in stallions. The clinical evidences of the disease are swelling of the organs with increased heat and evident pain upon pressure. The testicle itself is so bound down by its tunic that it swells only slowly, while the less restricted epididymis swells more rapidly. The line of demarcation between the two organs quickly becomes indistinct 
and lost, so that as a rule the epididymis and testis can not be told apart clinically in the presence of acute inflammation. A clear differentiation between orchitis and epididymitis is not supremely important, each having essentially like importance for the reproductive life of the animal.

According to the intensity of the disease, fever is present with loss of appetite and other general symptoms. As a rule the testicles suffer simultaneously but unequally. The onset of the disease is generally violent, it continues intense for

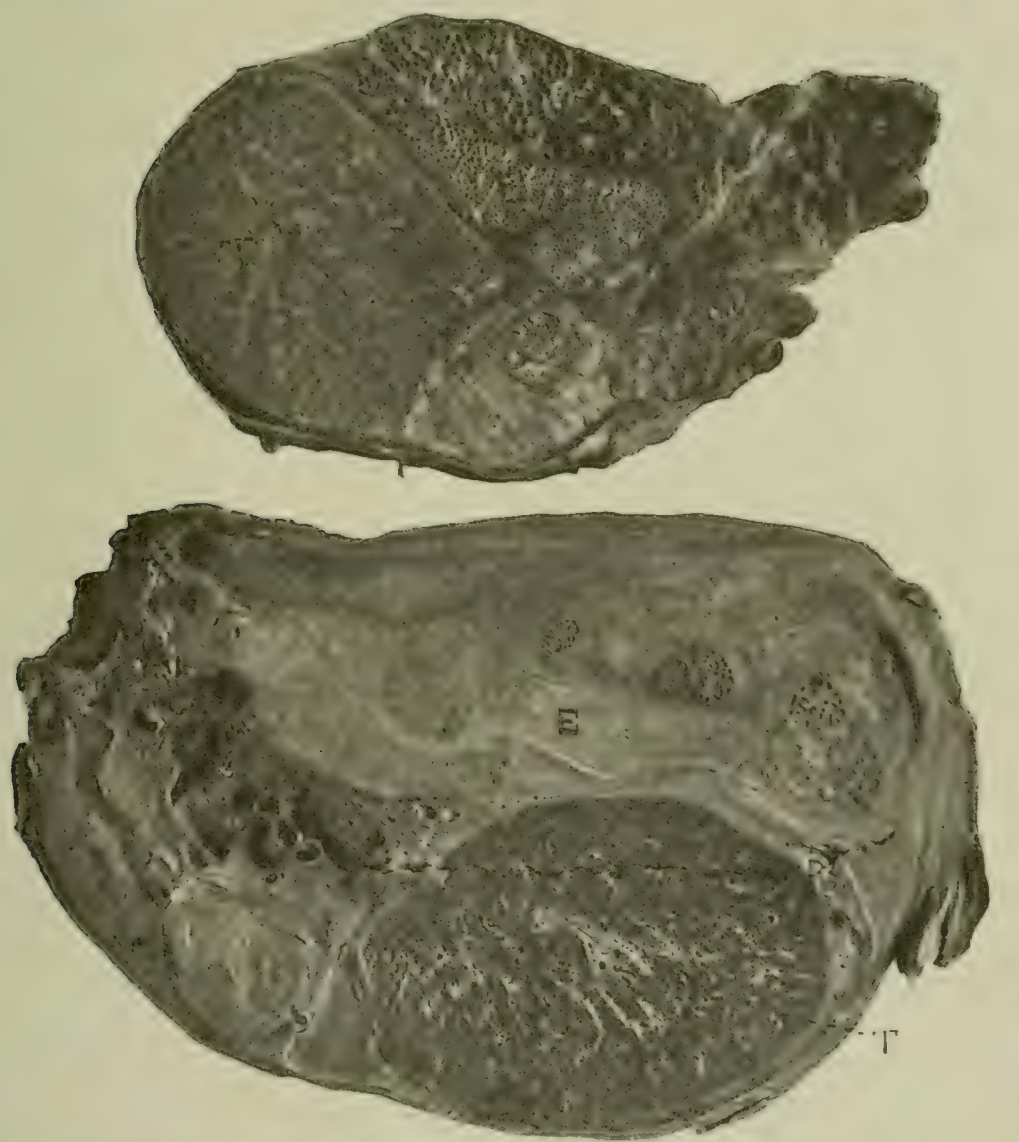

FIG. 226-Orchitis and Epididymitis. Stallion.

$T$, Testicle ; $E$, epididymis. 
several days or weeks, and then slowly abates. The glands do not ordinarily recover in volume or function. The prognosis for the life of the animal is highly favorable, but for the reproductive functions is usually hopeless.

As will be seen in Figs. 226 and 227, the gross changes consist chiefly in atrophy of the testicle and hypertrophy of the epididymis, so that the ordinary relationship in volume

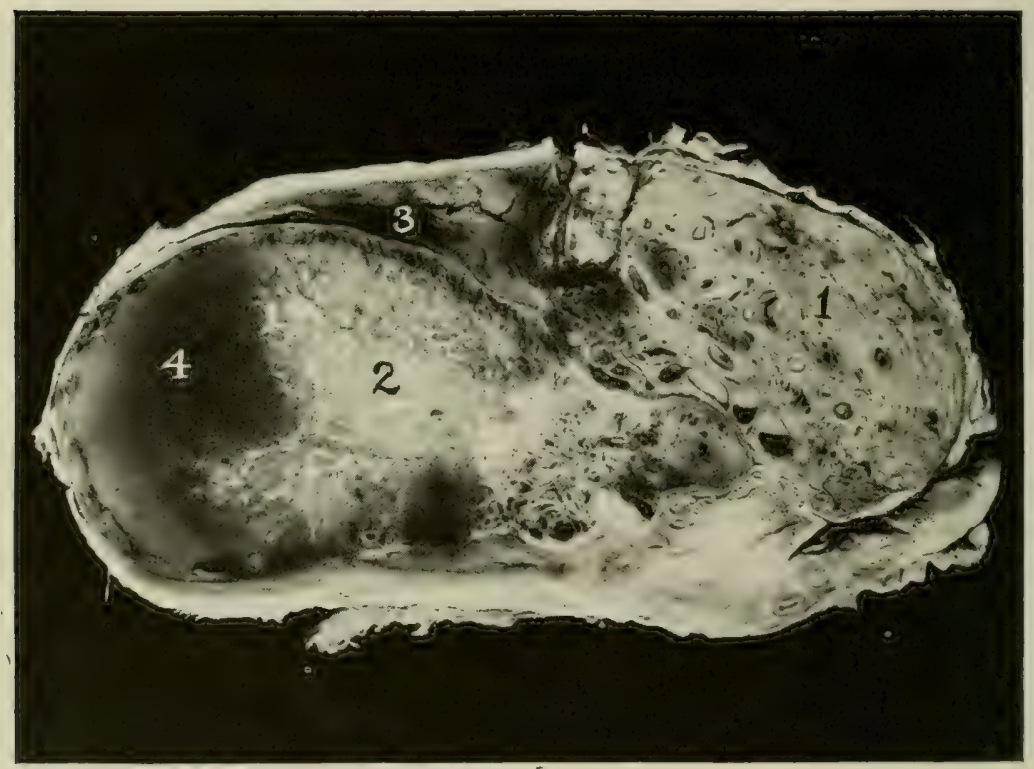

FrG. 227-Orchitis and Epididymitis.

$I$, Fipididymis ; 2 , parenchyma of gland ; 3 , hemorrhage in epididymis; 4, hemorrhage in testicle.

of the two structures becomes reversed. In the early stages, as shown in Fig. 227, prominent hemorrhage may occur. The infection extends to the peritoneal covering, by contiguity involves the parietal peritoneum of the scrotum, and causes adhesions between the two layers. Histologic and biologic studies have not been made in sufficient degree to permit of any conclusions. The colon-like organism ordinarily alleged to cause abortion in mares may logically be considered as a common cause. At least it is fair to assume 
for the present that the organism most commonly causing abortion in mares is the commonest invader of the testes and epididymes of stallions. The disease occurs most frequently at the height of the breeding season, the virulence of the infection already present being aroused by repeated coitus.

The handling of orchitis is a discouraging task. The animal should be taken out of the stud immediately, sexual excitement avoided as far as possible, the patient placed upon scant, laxative diet, and as a rule given an abundance of gentle exercise. If the inflammation is intense, its course may be checked by purging promptly with arecolin or eserin. Benefit may be had from large doses of potassium iodide, about one-half ounce per thousand pounds of body weight, once or twice daily. In the first stages the continuous refrigeration of the glands by means of a spray of cold water may be of value. When the infection is intense and has continued so long that recovery of reproductive functions is impossible, castration should be performed promptly as the best and safest method for controlling the disease. If apparently but one testicle is involved, it should be removed piromptly as the best protective measure against the involvement of the other gland. The removal of one gland does not lower the fertility.

When the animal has apparently recovered, a long sexual rest should be given before returning him to stud service. Then a test service should be made with a mare and the semen examined for spermatozoa and bacteria. If sound in both respects he may be permitted to resume stud service.

\section{Diseases of the Seminal Vesicles.}

\section{SEmino-Vesiculitis}

Veterinary literature reveals no traces of any comprehensive study of the infections of the seminal vesicles of stallions. The studies already made regarding the infections of these organs in bulls indicate that generally the glands about the pelvic urethra of the male are highly susceptible to any infections which may invade seriously the genitalia of the female, and that the probability of disease rests in a 
measure upon the comparative development of the given structure. That is, the seminal vesicles, prostate and Cowper's glands will tend to become involved in proportion to the functional importance of each in the species under consideration. Thus in the bull the seminal vesicle is the most commonly involved subsidiary sex gland, while in the dog the prostate gland suffers most frequently and severely.

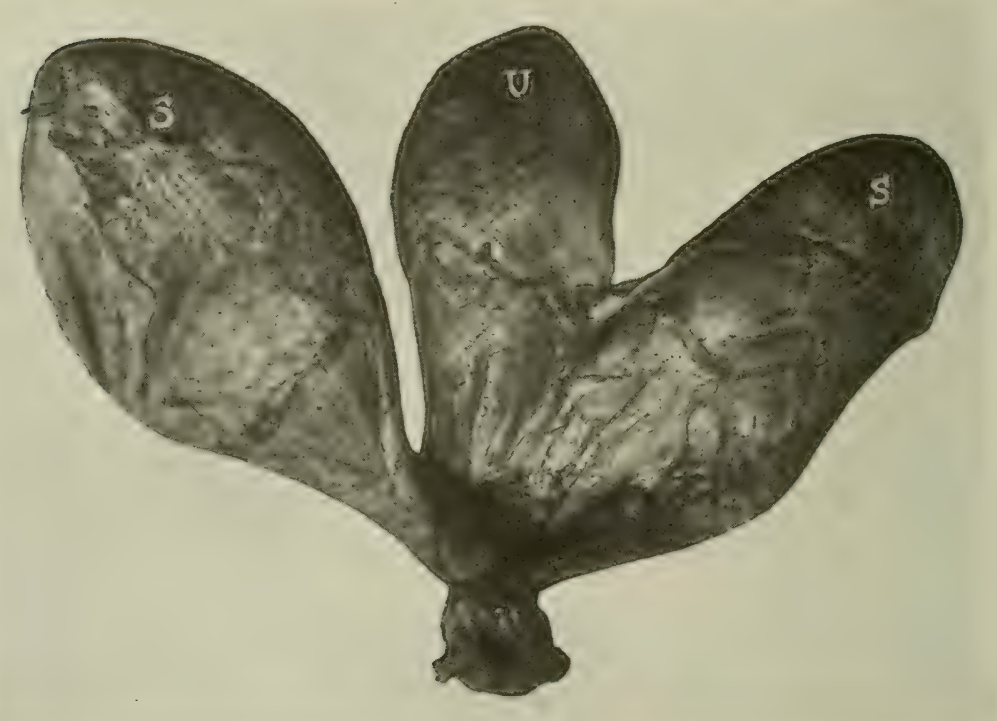

Fig. 22S-Enlarged Seminal Vesicles. Gelding. $U$, Urinary bladder; $S, S$, seminal vesicles.

Since ascending invasions along the urethro-vas deferens canal naturally tend to become deflected into the subsidiary sex glands, infections of these structures sometimes occur without involvement of the testicles or epididymes. Infections of the subsidiary glands probably as a rule have a greater peril for genital health than infections of the epididymis or testicle, because when the latter are involved the very small caliber of the tubules leads to early atresia with incarceration of the infection, while the structure of the subsidiary glands is such that infected secretions may readily escape into the urethra and be ejaculated with the semen. 
While the seminal vesicles of stallions have not been studied from a pathologic standpoint, they quite certainly play an important part in the transmission of infection to mares during coitus. When serious abortion storms break in a harem of mares, the most probable source of infection, reasoning from analogy, is the seminal vesicles of the stallion. Some known facts support this suspicion. Geldings frequently show purulent semino-cystitis, as shown in Fig. 228 , in which the seminal bladders are distended with pus until their size exceeds that of the empty urinary bladder. I doubt if castration predisposes to such infection and suspect that a careful study of the seminal vesicles of stallions would show infections of the vesicles having an important relation to reproduction.

Clinically I have observed one stallion and have known of others which presented symptoms justifying the suspicion of the existence of serious semino-vesiculitis. The stallion, an imported Percheron, was in splendid general health and quite amorous. The erection was intense. He would mount mares promptly, enter the penis into the vagina, hesitate, and finally dismount without ejaculation and with the penis greatly erected. From more recent studies upon bulls I believe that the symptoms were due to inflammation of the seminal vesicles of a severe type which excluded ejaculation. A safe diagnosis is available in such cases by means of rectal palpation. The prognosis, if the disease is clearly recognized, is probably hopeless as to reproduction but favorable for the life of the animal. No line of treatment is suggested. 


\section{THE GENERAL INFECTIONS OF THE GENITALIA OF MARES}

\section{A. Diseases of the Ovaries}

\section{AtrEtic FOLLiCLES}

The ovaries of healthy mares owe their chief volume to the presence of a number of cysts, reaching a diameter of one to two or more inches and containing colorless lymph. They are virtually universal in young, healthy mares, and at three to four years of age constitute more than seventyfive per cent. of the ovarian volume. With the advent of old age the cysts tend to disappear, the volume of the ovary decreases greatly, and the gland becomes distinctly senile. The cysts have not been studied. Apparently they have no pathologic significance, but on the contrary may serve an important physiologic office.

\section{NyMPHOMANIA}

Nymphomania in mares bears some analogy to nymphomania in cows, but presents some very marked differences. It has already been stated that nymphomania, as observed in the cow, is due to a type of cystic degeneration of the ovary, that no ovulation occurs, and that fertility is in abeyance. The disease designated nymphomania in the mare is not so readily recognizable as being due to cystic degeneration of the ovaries and its existence does not necessarily cause sterility.

Nymphomania is most commonly seen in mares which are kept closely confined and are not bred. It is more rarely observed in mares regularly worked and is well-nigh unknown in those which are free. It is most commonly seen in young or middle-aged mares, rarely in aged. It is quite uncommon in mules, though in one instance I observed the disease in so marked a degree as to render the otherwise valuable animal worthless until castrated. 
The symptoms of nymphomania in the mare usually appear just prior to estrum or during that period. Estrum may occur with comparative regularity but, in some cases, is apparently more frequent, while the duration of the period may be so prolonged that the intervals of calm between the periods of sexual mania may be very brief.

In general, the first symptom of nymphomania, usually appearing a few days prior to estrum, consists of excitability and irritability of temper.

The patient is usually more or less vicious toward other horses and toward men. She will bite and kick at her mate or other horses when approached. Very frequently the patient will crowd against the pole of the wagon or other vehicle and perhaps lie down upon it in an effort to reach her mate and inflict injury. She manifests her viciousness by laying back her ears and switching her tail, while her eyes and physiognomy in general betoken evil disposition. The meeting of a strange animal of either sex upon the highway frequently arouses a maniacal storm; the mare balks, lies down upon the pole or thills, urinates and switches her tail; the vulva is rapidly opened and closed; the clitoris is erected; and the affected animal may attempt to grip the reins with the tail, or may kick, rear or run away.

Toward persons her disposition is equally disagreeable and often decidedly dangerous. Upon entering the stall to groom, harness, unharness or otherwise handle the animal, she is liable to kick, bite or trample the attendant without warning. In hitching or unhitching the animal, especially when working about her posterior parts, she is very liable to kick.

In the stable, even when no other animal is near, the patient may show her vice by kicking the sides of her stall, striking and stamping with the feet. In one instance in our clinic, the mania was almost exclusively exhibited when the mare was alone in the stall. Watched from a distance, she would stamp her feet and kick the sides of the box stall in which she was confined. In her maniacal kicking she was utterly indifferent to the consequences to herself and would 
frequently and repeatedly kick one leg viciously with the other. Her eyes were wild and staring and her whole appearance and behavior one of violent insanity. If her owner or other person entered the stall she became calm, began eating $01^{\circ}$ fondled the person with her nose, and appeared in every way rational and docile. Turned in a paddock she behaved normally. Hitched in the usual manner to a single wagon, she behaved perfectly. The duration of the periods of mania and its intensity had increased for some months until the degree described had been reached and the attacks continued during seven to ten days. Ovariotomy was followed by complete and permanent disappearance of the mania.

The distinctively sexual symptoms noted consist chiefly of tumefaction of the vulva, injection of the vulvar mucosa, and increased secretion of mucus, with some mucous discharge from the vulva.

Urination is frequent, the clitoris is erected and frequently protruded between the vulvar lips. The irritability of the vulva and vulvo-anal region is markedly increased and the animal frequently resents violently the handling of these parts. This is especially true, in many cases, of the tail; the animal resents the raising of it in order to apply the crupper or remove it. While driving, should the patient either accidentally or intentionally get her tail over the reins, she often grips them violently, kicks viciously, may turn abruptly and upset or break the vehicle, or run away.

In some cases the patient is perfectly docile and the only signs of disease consist of abnormally frequent urination with repeated opening and closing of the vulva and the projection of the clitoris, making her unfit for driving purposes simply from repulsiveness.

In most cases, however, the patient is unreliable or dangerous and can not be depended upon in moments of emergency. During the periods of nymphomania she is very liable to be unsteady in draft work and may at any moment balk and tax the patience of the driver very greatly.

Periodical at first, the vices of nymphomania, such as 
kicking, balking and gripping of the reins by the tail, tend to become constant and permanent, and the animal degenerates into an incurable rogue, falls into the possession of low dealers and finally finds her way into some inhuman hands, where she succumbs to brutal work.

Examined macroscopically, the ovaries are usually large and contain one to several cysts, varying in size from $1 / 2-2$ in. or more in diameter and containing a clear, faintly yellowish lymph. The cyst walls are thick and very firm and the density of the albuginea preserves to a large extent the even contour of the ovarial surface, so that it is only in case
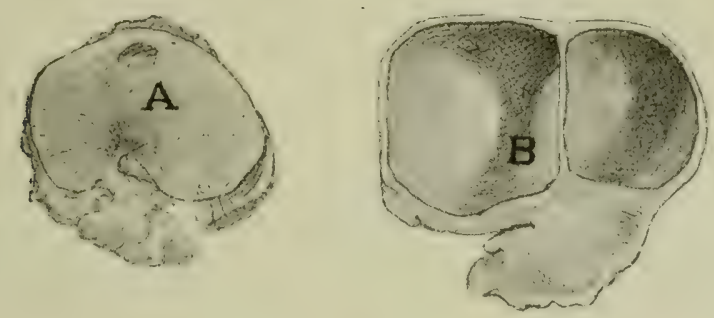

Frg. 229-Cystic Ovary, Reduced. Nymphomania. $A$, Normal ovary ; $B$, cystic gland.

of quite large cysts that they project conspicuously beyond the general surface of the gland. In some cases, the ovarian tissue proper vanishes almost completely under the pressure of large cysts, firmly compressed within the enveloping tunica albuginea.

In other extremely bad cases of nymphomania there are found small, atrophied, fibrous ovaries, very hard and dense, like fibro-cartilage.

The examination of the ovaries of the mare is to be made upon the standing animal, in essentially the same manner as that described for the cow. Due precautions are to be taken, according to circumstances, to avoid injuries to the veterinarian from kicks and to overcome any probable annoyance from movements of the animal. Usually a single stall, with the animal tied short in it, will suffice, but vicious 
animals should be secured in stocks, the twitch applied, and a forefoot held up or one hind foot raised by means of a sideline.

The hand and arm of the operator should be cleansed and lubricated with warm oil, lard or liquid paraffin. The rectum of the animal should be emptied of feces. Usually little progress, if any, can be made while the hand is in the posterior or pelvic rectum. It becomes necessary to push gently forward into the frec portion of the bowel, which can then be carried to the right or left, up or down, and forward or backward. The mare often strains somewhat, which should always be a signal to the operator to cease his palpation until the expulsive effort ceases, but without withdrawing his hand. Care upon this point decreases the straining and does much to avoid laceration of the rectal mucosa, associated with hemorrhage.

With the palm of the hand directed downwards, the cervix and uterus, about the size of a man's wrist, may be traced forward to the point of bifurcation, where the cornua, almost as large as the body, are given off at approximately right angles. Tracing these to the right or left, the operator can recognize the ovaries at or near the anterior margin of the broad ligament, an inch or two beyond the obtuse end of the cornua. The normal gland in the young or adult mare is elastic, smooth, firm, oblong and about $21 / 2-31 / 2$ inches in its greatest diameter, by $11 / 2-2$ inches in its lesser diameter. The diagnosis of cystic or fibrous degeneration of the ovaries of the mare and the relationship between these diseases and sterility is not always clear.

How many nymphomaniac mares would conceive if bred, is not known, but it is known that many breed and that the nymphomania usually disappears during the span of pregnancy, to recur with some degree of regularity after foaling.

The question of overcoming sterility due to cysts or other ovarian disease, with a view to the restoration of the breeding powers in valuable brood mares, has been quite neglected and attention turned chiefly toward the amelioration 
or cure of the vice attending the malady, in order to restore the work value of the animal. Since nymphomania in the mare is chiefly observed in those not used or desired for breeding, this tendency in handling is natural and sufficient. It is only when involving animals desired for breeding purposes that cure of the disease is desired. When the aim to restore fertility is frustrated by insurmountable obstacles, the duty of the veterinarian remains to preserve to the owner, as far as possible, any work value which the animal may possess when relieved of the nymphomania.

OVARIOTOMY. Ö̈PнORECTOMY. When nymphomania referable to ovarian disease is present and can not otherwise be effectively overcome, castration is indicated.

Since in mares not desired for breeding purposes, the cure of ovarial nymphomania otherwise than by castration is all too liable to prove ephemeral and unreliable, castration at once presents itself as the most reliable and enduring remedy. The operation is comparatively simple and not highly dangerous. It has been fully described on page 263 .

\section{B. Intra-Uterine Infections and Infections of the Embryo}

\section{INFECTIONS OF THE GRAVID UTERUS}

The infections of the gravid uterus are not generally observed directly. Their presence is revealed almost solely at the termination of pregnancy, at which time the ravages of infection become apparent. No such opportunity is offered for the study of the pregnant uteri of mares as is afforded in the abattoir upon the gravid uteri of cows, sows and ewes. When pregnancy terminates in parturition or abortion, the lesions due to infection become apparent, enabling the clinician to construct a picture of the processes as they have occurred. Rarely one has the good fortune to examine opportunely a pregnant mare about to abort, and secure direct data upon the behavior of infection within the uterus. In so far as studied, the behavior of the infection between the chorion and uterine mucosa in the mare is perfectly analogous to the better known processes taking place in the 
gravid uterus of the cow. There is one fundamental difference in the placental arrangement which changes materially the method of invasion. The utero-chorionic space of the cow facilitates the rapid dissemination of infection throughout the entire cavity without necessarily involving seriously the placental structures (cotyledons). In the mare there is no utero-chorionic space, but the endometrium everywhere participates in the placental functions, so that the endometrium and chorion are everywhere in intimate placental contact. Any advancement of infection along a given path therefore necessarily destroys the placental relation between chorion and uterus. The chief habitat of infections within the gravid uterus of the mare, as revealed by clinical study, is the same as in the cow. The most destructive location of infection is at the cervical end of the uterus, from which point of vantage it causes abortion. Clinical experience clearly teaches that the second bacterial rendezvous is the apex of the non-gravid horn where it may develop a localized apical endometritis, revealed at the termination of pregnancy by retention of the chorion in the non-gravid horn, while the remainder of the membranes detach promptly and their weight causes a rupture across the base of the nongravid portion, the major portion of the membranes falling away while the diseased non-gravid branch remains. If one has the opportunity to make a clinical examination of a mare nearing abortion, there is frequently, perhaps always, an absence or destruction of the uterine seal, the cervical canal is open and suppurating, and the chorion is detached from the uterus over a varying area at the cervical end. This is well illustrated in Fig. 230. The parturient rupture (1) is slightly at one side of the central axis because there exists a central hard, somewhat desiccated necrotic area (2) which possesses greater resistance than the contiguous area. The photograph reveals at 3 the radiation of the infection from 2 toward the ovary. In some cases, if not in many, the veterinarian making an examination at an opportune time, is able to palpate clearly the necrotic, parchment-like area of the chorion, and through it to palpate a living fetus 
and cause it to move. So far as I have been able to observe, the chorion illustrated in Fig. 230 is representative of prompt abortion. Delayed abortion may cause such disintegration of the chorion as to destroy the plain record here shown of the progress of infection.

Abortion in mares is accordingly, so far as now lnown, always a result of cervical endometritis, combined with a mortal disease of the embryo or fetus. The invasion of the fetus is identical with the invasion of the fetus in the cow.

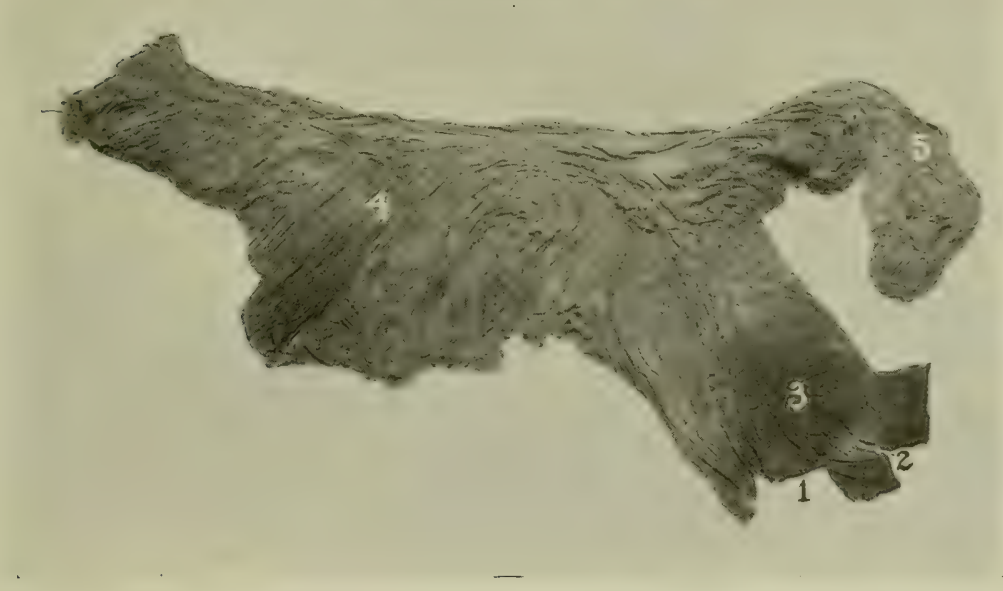

Fig. 230-Fetal Placenta of Abortion.

$I$, Parturient rent in chorion: 2 , necrotic area opposite the os uteri internum ; 3 , inflamed area radiating from cervix toward ovarian end of uterus ; f. gravid horn ; 5, non-gravid horn.

The infection grows through the chorion at the necrotic cervical area, through the continuous inflamed area (Fig. 230, 3 ), or through the inflamed tip of the chorion located in the non-gravid horn. When the allantoic fluid has been reached, the invasion may continue through the urachus, but usually penetrates the amnion and, suspended in the amniotic fluid, is swallowed by the fetus. Then sepsis or dysentery may follow, with fetal death. Fetal death may also follow extensive placentitis owing to asphyxia or to placental sepsis. The endometritis at the cervical end of the uterus awakens 
uterine contractions with the prompt expulsion of the fetal cadaver.

Intra-uterine infection is more certain to cause abortion in the mare than in any other domestic animal. Since the cervix is shorter and its canal more dilatable, less power is demanded of the uterine walls to force the fetal cadaver into the cervical canal, at which point the abdominal walls assume the chief burden of emptying the uterus. The anatomical features of the placenta confine the infection, at least temporarily, to the cervical end, as indicated in Fig. 230, concentrate the uterine irritation more definitely at the cervical end, and leave the ovarian end free from disease and competent to contract vigorously upon the uterine contents. These anatomical differences modiry interesetingly the clinical behavior of intra-uterine infection. While it is not rare for a bovine fetal cadaver to undergo partial or complete maceration in the uterus, it is exceedingly rare to observe this result in the mare. The mare is in some respects more vulnerable to sepsis than the cow, but if this plays any part at all it is a minor one. Fetal cadavers (except at or near full term) almost never undergo putrid decomposition in the uterus of the mare, but are practically always expelled promptly. When fetal death occurs at or near full term, the condition is essentially alike in mare or cow. It is common in both species, especially when the fetus presents by the breech, for fetal death and emphysema to occur without visible effort at expulsion. The phenomenon of abortion in mares, as one of the results of intra-uterine infection, has long been an important economic problem in horse-breeding and up to the present time no outstanding progress has been made in its control.

Abortion of mares first acquired prominence in the United States about 1886, when it appeared in several states in the Mississippi Valley, in which horse-breeding had become a very important industry. It soon acquired a very extensive distribution and high degree of virulence. The unorganized state of veterinary science in the United States prevented the recording of any definite statistics in reference to the 
losses, but they were certainly enormous. At that time I was engaged in practice in what was then one of the most important horse-breeding districts in America. In 1888, in the area of my practice, involving about 400 square miles, at least 2500 mares aborted, causing an immediate loss of not less than $\$ 150,000$. These losses were typical of those in other breeding centers. In some townships where horse breeding constituted one of the principal agricultural pursuits, the abortions exceeded seventy-five per cent. of the pregnant mares. The losses were most marked in large breeding establishments where highly valuable mares were kept for breeding purposes alone. The losses were just as real on small farms where but a few mares were kept, which, after aborting, could be employed to some extent in farm work. In one season a client with a highly valuable herd of about fifty imported draft mares lost every foal from abortion. In another instance, in the territory of a neighboring veterinarian, a breeder had 100 pregnant imported draft mares, in which the loss from abortion was total as to foals and four of the mares perished. Other similar instances might be related without number which would give a somewhat vivid impression of the enormous losses.

As in cows, so in mares, intra-uterine infection may and does interrupt the reproductive functions at any date. The spermatozoa, the ova, the fertilized ova, the embryo, the fetus and the new-born foal may succumb to the infection, but the most striking phenomenon is the observed expulsion of the fetal cadaver. Consequently abortion is said to be most common from the sixth to the tenth month, because this is the most favorable date for observing the disaster.

The symptoms of abortion in mares are less notable than in cows. Mares rarely suffer from retention of the fetal membranes, which constitutes a common landmark of abortion in cows. The diagnosis of abortion in mares is therefore usually confined to observing the mare in the act or discovering the fetal cadaver under circumstances which enable one to trace it to its source. 
The lengthy discussion upon the question of abortion in cows will serve in a general way for that in mares. Although but recently the belief was general that each domestic animal has a specific infectious abortion, numerous clinical and biologic studies are causing a disintegration of that theory. Up to the present time bacteriologists have generally incriminated but one organism as an important cause of abortion in mares. Smith and Kilborne, Turner, Lignieres, De Jong, Good and Smith, Meyer and Boerner, and others have described an apparently identical organism, designated by Good and Smith ${ }^{1}$ Bacillus abortivo-equinus, which they severally believe is the cause, or an important cause, of abortion in mares. Running through the recorded investigations are frequent notes of discord. Good and Smith failed to find the B. abortivo-equinus in fetal cadavers or elsewhere in an outbreak among Shetland ponies, and failed to report what other, if any, bacteria were encountered. In another case the highly suggestive admission is made that the breeding stallion had orchitis and that from the testicle was obtained, not $B$. abortivo-equinus, but $B$. pseudomonas pyocyaneus, and that colonies of the same bacillus were obtained from other sources in the outbreak (fetal cadavers, fetal membranes?). The general attitude of bacteriologists regarding the infections of the genitalia of horses has been analogous to that in the investigations in cattle: if the organism which has been set up as the specific cause of abortion is present, the proof is complete and other bacteria present may be ignored; if it is not present, the abortion is not "contagious", though an abundance of bacteria of other kinds may be present.

The hypothesis of "contagious abortion" in mares as a specific disease has long held, and continues to hold, all efforts at control in abeyance. There can be no reasonable doubt at this time that a broader conception of the problem, parallel to that which is now gaining a secure foothold regarding the genital infections of cattle and which has won a safe place in the battle against these infections, is equally

${ }^{1} \mathrm{Ky}$. Ag. Exp. Sta. Bu1. 204, 1916. 
applicable to the genital infections of horses. If a horse breeder desires to produce a vigorous colt, let him as the first step breed a genitally sound mare to a genitally sound stallion.

The genitalia of the mare are to be examined the same as those of the cow. Any cervicitis, metritis or other genital disease discovered is to be handled upon the same fundamental principles. Stallions used to serve valuable mares should be examined at intervals, including their genital organs, semen and spermatozoa. During pregnancy mares should be guarded (just as non-pregnant mares should be) against infectious fevers, such as "pink eye", not because they can apparently cause abortion directly, but rather hecause, by lowering the vitality of the pregnant mare, the resistance of her uterus to infections residing therein is lowered and disaster invited. The maintenance of the physical vigor of the stallion and avoidance of sex overstrain should have thoughtful attention.

\section{Infections of the Puerperal Uterus}

A. Placentitis. Endometritis with Retained Fetal

Membranes. Puerperal Laminitis.

The diffuse placenta of the mare, spread over a far greater area than in the cow, causes the placental structures to be less elaborate and renders retention of the fetal membranes far less probable. The placentitis which may cause retention of the fetal membranes is probably as common in mares as in cows, but the chorionic tufts are so short and simple that the placentitis runs its course quickly and the dehiscence of the placenta usually occurs before the expulsion of the fetus, or the incarceration is so feeble that the membranes are expelled very soon after the fetus, before retention is clinically recognizable. The placental arrangements are such that endometritis and placentitis become identical, the entire endometrium being placental. There exists in the mare also a remarkable bond between the placental endometrium and the sensitive laminae and other horn-secreting structures of the foot, so that when placen- 
titis of a severe type develops there occurs promptly as a rule a severe laminitis. No acceptable explanation, so far as I know, has been given for this occurrence. Some say it is metastatic inflammation, but they do not explain what they mean by metastatic. Puerperal laminitis is common in the mare and very rare in the cow and ewe. Two types of placentitis with retained fetal membranes are recognized clinically. Generally retention of the fetal membranes, in which the placentitis has led to an incarceration of the chorionic tufts over most of, or the entire uterine mucosa, is, according to my observation, very rare. It is rarely seen in abortion because the placentitis has reached that point where the chorion is cast off before the fetus is expelled, so that cadaver and membranes are expelled together, the same as is observed usually in the cow when abortion occurs in very early pregnancy. I have seen this type of retention in premature birth, but the premature birth itself is a rare phenomenon in mares. The conditions are usually analogous to those in the cow: the retention begins at the cervical end of the uterus, as illustrated in Fig. 230. The cervical end of the chorion is detached or detachable soon after the fetus is expelled, and the retention increases as the ovarian end of the uterus is approached. The prognosis is favorable except that it will probably be complicated by laminitis. The handling is along the general lines advised for cows. The mechanism of manual removal differs in detail. The margin of the parturient rent at the cervical end is to be picked up and tensed with one hand while the other hand is gently pressed between the chorion and uterine wall, and the two organs carefully pushed apart, or a part of the chorion may be folded longitudinally, held with one hand and grasped with the thumb and forefinger of the other hand, the latter pushed forward against the uterine wall, and the two organs pressed apart. If the membranes are not removable without injury, the same rule applies as in cows. Once the membranes are out, the uterus should be freely douched with $0.7 \%$ salt solution as frequently as may seem expedient and the iodoform-bismuth-oil treatment, advised for cows, used. 
A more common type of metritis with retained fetal membranes is the retention in the non-gravid horn. As a rule the fetus lies in the uterine body and one of the horns, so that the non-gravid horn does not develop markedly. As in the cow, the non-gravid horn habitually suffers more severely from infection present than the gravid horn. Presumably this is due to the lower vitality of the non-gravid horn of the chorion, making it more vulnerable to bacterial attack. It may well be, however, that the cornual apex and the oviduct are fundamentally worse affected in many cases, so that pregnancy is barred upon that side but remains possible upon the less infected side. Whatever may be the correct explanation, the fact remains that the non-gravid cornu frequently suffers severely from a localized endometritis, the crypts are deep and irregular, and the chorionic tufts gross and uneven, showing great variability in color. At one point the chorion is intensely red and angry; at another, a necrotic-like, dirty gray. The other portions of the endometrium may be healthy. When parturition occurs, there is prompt dehiscence of the healthy chorion in the gravid horn and uterine body, but the chorion in the non-gravid horn is incarcerated. It is usually narrowed at its juncture with the uterine body and fragile from disease. The weight of the detached chorion of the gravid horn and the body drags upon the frail prolongation, it parts, the chief mass drops away, and the non-gravid prolongation remains incarcerated and out of sight. Conditions are then ripe for an explosion. The entire endometrium of the gravid horn and uterine body, approximately fifteen to twenty square feet in area, is wholly denuded of protective epithelium and highly vulnerable to invasion. There rests in the badly diseased nongravid horn a large volume of infection in the presence of a large mass of necrotic chorion already saturated with infection. The accumulated infection pours out into the uterine cavity and gravid horn, and the necrotic, putrefying fragment of chorion drops into the uterine cavity. The spread of the infection is rapid. Marked clinical symptoms quickly appear. Within twenty-four to forty-eight hours 
the mare is evidently in great distress, she lies down most of the time, her appetite is poor or absent, and her pulse weak, though her temperature not markedly elevated, probably because of relaxation of the anal sphincter which admits air about the thermometer. There may be an involuntary escape of a thin, flaky, dirty grayish pus, flecked with blood from the vulva. Manual exploration of the uterus reveals a paretic organ containing three or four quarts to as many gallons of thin, dirty gray pus, sometimes almost odorless, and sometimes fetid. Somewhere floating in the mass or still lying in the non-gravid cornu may be found the necrotic, putrefying fragment of chorion. If compelled to stand, the mare exhibits the clinical picture of laminitis (puerperal laminitis) with tenderness of the abdomen upon pressure. The localized endometritis has suddenly developed into an intense generalized endometritis with severe laminitis.

The veterinarian in charge of valuable brood mares should either have the fetal membranes of each mare laid aside for his prompt inspection, or should teach the caretaker how to inspect the membranes and determine if all has come away. If the non-gravid prolongation is wanting, it should be removed manually very promptly, the uterus douched with salt solution, and iodoform-bismuth-oil introduced. This will generally prevent the development of severe endometritis and laminitis.

If such precautions are not taken and the intense disease has developed, the necrotic fragment is to be removed if found, the pus douched and siphoned from the uterus, and the iodoform-bismuth-oil introduced ( $1 / 2$ to $1 \mathrm{oz}$. each of bismuth and iodoform in one quart of olive or salad oil or of liquid paraffin). The treatment should be repeated often enough to prevent large accumulations in the uterus. The laminitis must have earnest attention. If the animal can stand without great pain or fatigue, she may be placed in a flowing stream of cool water or stood in an improvised foot bath kept cold with ice. If she can not stand without great suffering, packs of broken ice should be kept upon her feet. 
There is scant need for internal medication except for the uterine douchings already described. The disease is based upon intra-uterine infection. When that is controlled, the animal usually improves rapidly and recovers completely. Should symptoms arise demanding medication, the details should be adapted to the individual case. If the sepsis appears profound, it can probably best be overcome by large doses ( $1 \mathrm{oz}$. or more) of potassium iodide or quinine. Constipation or intestinal atony can be most safely overcome with minute doses of eserin or arecolin combined with strychnine.

\section{B. Metritis. Metro-Peritonitis.}

The placentitis or endometritis of the preceding article is necessarily an intrinsic infection which, so far as known, has existed within the uterus at least throughout pregnancy. There occur some cases of metritis differing in type from that described above and referable to either intrinsic or extrinsic infection. In veterinary practice the practitioner is not ordinarily responsible for introducing infection, through imperfect technic, into the genital tract at the time of parturition. The veterinarian rarely invades a uterus not already importantly infected. He interferes in dystocia only, and dystocia in animals is due chiefly to infection within the uterus, causing atony of its walls, or to death or serious illness of the fetus from the same infection which destroys its power to assume and maintain the correct direction and position of its extremities. In such cases the veterinarian does not introduce the primary infection, but may add other forms of bacteria more perilous than those already present.

When intra-uterine infection causes the death of an almost or wholly mature fetus, especially if the fetus presents by the breech, there may occur no visible effort at expulsion, but instead the fetus undergoes rapid emphysema. The already badly inflamed uterus rapidly becomes worse owing to the presence of the decomposing fetal membranes and fetus. Under such conditions the walls of the uterus generally become paretic, greatly thickened and hard. Por- 
tions of the decomposing chorion or amnion may protrude from the vulva, but there are no expulsive efforts. The uterus is powerless to act upon its contents. The great thickening of the uterine wall is largely confined to the placental tissues. If the emphysematous fetal cadaver is removed by embryotomy or otherwise and the uterus douched clean, the mare probably recovers and may retain her fertility. The removal of the emphysematous cadaver is described in the companion volume, Veterinary Obstetrics.

Although puerperal metritis in animals is almost always the continuation of the metritis of pregnancy into the puerperal period, the puerperal metritis is aggravated by the dehiscence of the chorion and exposure thereby of the entire denuded area to the infection present. Parturient contusions and lacerations are frequent and are at times perilous. The layman undertaking obstetric work and, still more dangerously, the careless and dirty veterinarian coming from other highly infected cases, frequently introduces dangerous types of infection into the uterus with dirty hands, instruments or apparatus.

One of the most serious parturient contusions is that due to impingement of the floor of the uterus or cervix between a firm, bony part of the fetus and the conical protuberance projecting into the pelvic cavity from the anterior end of the symphysis pubis. This sometimes perforates the floor of the genital tract directly, but more frequently so maims the tissues that necrosis occurs and infection penetrates the peritoneal cavity. Then a violent metro-peritonitis ensues, which commonly results in death. Sometimes virulent infection invades the floor of the genital canal through the lacerated tissues and, spreading out from the point of invasion, sets up a malignant and fatal phlegmon involving the utero-cervico-vaginal floor, the urinary bladder and neighboring parts. The patient then exhibits all the signs of profound sepsis with great depression, erratic temperature and disinclination to move because of the great pain in the pelvis. Vaginal or rectal palpation reveals extensive phlegmon or, in mild cases, abscesses in the pelvic tissues, largely 
lying below the floor of the genital tract. Little of value is known which may be applied to control or ameliorate the ravages of the infection. The phlegmon is so erodent that the exudate generally makes its way into the peritoneal cavity. If abscesses point toward the vagina or rectum, they may be opened into either of these, but in my experience they fail to do this, and I have failed to identify a spot where it appeared prudent to penetrate the virulent area. Heavy doses of potassium iodide, quinine or both may be tried, but without material hope. Prevention is dependent upon careful obstetric practice which it is not proper to discuss here.

\section{Post-Puerperal Infections of the Uterus}

The infections of the pregnant uterus may continue into the puerperal period, receive additional invasions at that time, and, with these, persist indefinitely. They are unlimited in variety, including essentially any bacteria competent to induce wound infection. They may continue into the next breeding season and prevent conception, or, failing to do this, may imperil the life of the embryo of fetus, or finally a portion of the infection may persist in the fetus at birth, constituting a hazard to the new-born foal, and a part continue in the uterus of the mare. The most prominent types are the following:

a. Chronic endometritis with mucous or muco-purulent exudates. In such instances the uterus is enlarged, flaccid and atonic. Estrum may be regular, but conception largely fails or, if it occurs, the embryo or fetus will probably perish. If the uterus is examined, the cervix is generally found soft and its canal open. If the uterus is douched, some muco-pus is probably washed out.

Spontaneous recovery is uncertain, but as a rule the condition will respond to proper handling. The principles of handling are the same as for the analogous disease of the cow. The commodious vulva and vagina and the freely dilatable cervix render handling simpler and easier than in the cow. In douching the uterus of the mare the practitioner 
should bear in mind the fact that the very large cornua leave the uterine body at right angles, or somewhat recurved. The ordinary metal catheter used for the cow is not, therefore, a wholly suitable instrument for the mare because it can not readily follow the right angle turn from the cavity of the uterine body into the horn. The soft rubber horse catheter, guided into the base of the uterine horn with a sound shaped somewhat like the modified Palmer uterine dilators (Fig. 39, 2) may be safely pushed to the cornual apex. The cornu may then be cleansed by siphoning or, owing to the ample cervical canal, may be flushed out, the return flow escaping alongside the catheter.

b. Uterine abscess not rarely follows severe puerperal metritis. A severe inflammation of the cervical mucosa occurs at a period when there is little escape of exudate from the uterine cavity, permitting the contiguous cervical surfaces to adhere and block the cervical canal. The imprisoned pyogenic infection multiplies rapidly and soon distends the uterus. The distended organ becomes powerless and the distension grows. Eventually colicky symptoms develop, and in some cases there are painful and violent expulsive efforts. Soon two to five or more gallons of pus accumulate and the patient is in severe distress. If left to itself, in the cases I have observed, the abscess usually points through the cervical canal and ruptures into the vagina. In the process the cervix as a definite structure disappears and most of its substance constitutes a part of the posterior wall of the abscess. The os uteri externum is firmly closed and marks approximately the center of the abscess from behind.

The symptoms are those of general ill health until the tension within the abscess becomes great, when abdominal pain and expulsive efforts constitute the two pronounced indications. In conjunction with a history of recent parturition, especially of dystocia or retained fetal membranes, uterine abscess should be suspected. Manual exploration through the vagina causes pain and expulsive efforts. The vagina may be normal, but is probably shortened owing to the abscess pushing backward. Palpation reveals the tense, fluctu- 
ating abscess, the posterior wall of which occupies most of, or the entire lumen of the pelvic cavity. Careful search usually reveals traces of the os uteri externum, the lips of which form a ring about the occluded mouth. The cervical canal is nowhere to be found. Rectal exploration reveals the presence of a great, tense abscess, continuous with the anterior end of the vagina.

The prognosis is good for the life of the mare, but essentially hopeless for her fertility. The treatment consists of a free opening of the abscess with disinfection of its cavity. Careful palpation by both vagina and rectum should be made and the position of the os uteri externum located approximately. In case of doubt a small trocar may be used to explore and the abscess cavity adequately located. The trocar puncture may then be dilated by incising, and later the enlargement may be continued by forcing the hand through the wound into the abscess cavity. It should then be handled like any abscess cavity, except that it is to be remembered that antiseptics in escaping must flow over the vaginal walls and, if too irritant, may cause serious harm. The opening must be kept amply dilated until suppuration has ceased. As a rule the uterine cavity will probably become obliterated.

c. Pyometra. The short, broad and readily dilatable cervix of the mare renders her less subject than the cow to pyometra. So far as I have observed, pyometra may well be considered as a secondary lesion to cervicitis. The cervix becomes inflamed and greatly indurated, incarcerating infection within the uterine cavity. The uterine walls participate importantly in the process, and ultimately become quite paretic, and the indurated cervix, encroaching upon the canal, so narrows it that the pus contents of the uterus are not actively expelled. The principles involved are illustrated in Fig. 231.

The symptoms are chiefly an intermittent, sometimes a constant, vulvar discharge. Uusually the discharge is fetid and consists of rather thick, dirty, yellowish pus. In one of my cases the pus was black and looked like finely pulped 
melanotic tissue suspended in fluid. Pyometra passes imperceptibly into uterine abscess. In some cases of pyometra the discharge ceases for ten to thirty or more days, colic may appear, and finally the indurated cervix yields to the intra-uterine pressure and the pus escapes in large quantities. In many cases the discharge occurs only when the animal is recumbent, since the escape of the pus is passive, due to the increased intra-abdominal pressure caused by the position of the patient. In some cases the incarceration of the pus is the result of contraction of the cervix. I have seen great quantities of pus escape from the uterus while the patient was under complete chloroform anesthesia for

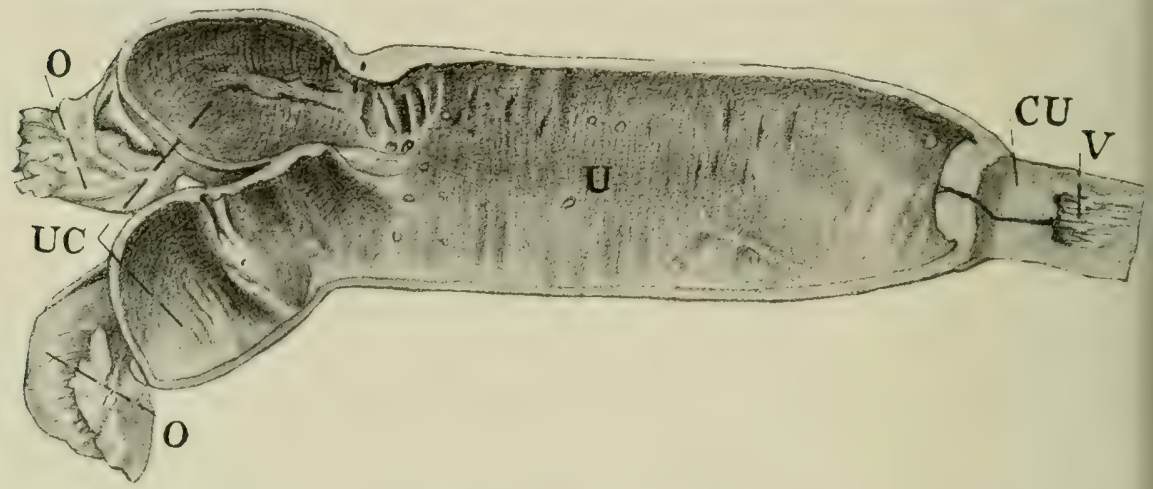

FIG. 231-Pyometra.

$I$, Vagina; $C U$, greatly thickened, sclerotic cervix; $U$, uterus ;

$U C$, cornu ; $O$, ovary.

other reasons. The disease shows no tendency to spontaneous recovery. The physical health of the animal can almost always be restored. I have not known a mare to conceive later. The oviducts are probably blocked. The veterinarian should on no account leave the application of the treatment in the hands of a layman. If the practitioner can not have the patient under his immediate control, he had best, for his professional reputation, let it alone. The handling must be vigorous and continuous in order to assure satisfactory results.

The treatment consists of the surgical dilation of the cervical canal, wth disinfection of the uterine cavity. The tech- 
nic of cervical dilation may be varied according to conditions. Frequently the operator may dilate the canal manually. First one finger is pushed through the canal, then a second finger, and finally the entire hand. It is not usually desirable to attempt the complete dilation at one operation, but the effort should be renewed daily until success is attained. If room permits, after the pus has been washed out of the uterus, the closed end of a long cheesecloth bag may be introduced into the uterus and the bag then filled by packing a continuous long strip of cheesecloth into it by means of long dressing forceps, uterine dilators, or other instrument. A rubber dilator patterned after those used in human practice can be used in some instances. The veterinarian needs to select his method according to circumstances, and persist day after day until ample dilation has been attained. I have set my mark at sufficient dilation to permit the passage of my hand.

Once ample dilation has been accomplished, effort may be centered upon disinfection of the suppurating cavity. The disinfection should be largely mechanical, douching out the pus with physiologic salt solution, boric acid or other very mild disinfectants. The soft rubber horse catheter may be used advantageously for douching and siphoning. After cleansing the uterus as thoroughly as practicable by these means, improvement may follow the introduction of iodoform or bismuth subnitrate, or both, in liquid paraffin. At first the uterus should be handled daily because the force used in dilating the cervix accelerates the infection and generally causes fetor or increases the fetor existing. Later, as the uterus and cervix soften and approach their normal size, consistency and function, the handling should be continuously toned down and finally discontinued, but the patient should still be examined from time to time for a few weeks to make sure that no perilous trace of infection has been left behind.

d. Pyemic abscesses from metritis are rare in mares. I have observed them occasionally in the pelvic tissues and, in one instance following dystocia, in the sub-lumbar lymph glands. There appears to be scant inclination to the pyemic 
arthritis so often seen in cows. When pyemic abscesses occur in or about the pelvis, or in the sublumbar lymph glands, they are recognizable by means of rectal and vaginal palpation.

The abscesses may be opened into the rectum or the vagina, if adherent to these organs. If not adherent to these, but closely adherent to the abdominal walls, they should be opened by external incision. This is safely done with the mare under complete anesthesia. The operator inserts one hand into the rectum as a guide and penetrates the abdominal and abscess walls with a scalpel operated by the other hand. Most of the incision may be made by an assistant but, when the peritoneum is reached or closely approached, the operator with his one hand in the rectum, resting upon the abscess, can guide the scalpel with his other hand with greater safety and accuracy than can others. The opening should be free and the general rules for handling abscesses applied.

\section{Cervicitis}

It has already been stated that pyometra in the mare is largely caused by, or at least intimately associated with cervicitis. On the whole, cervicitis is rare in the mare as compared to ruminants. This is largely owing to the far simpler anatomical plan of the cervix. It is shorter, its canal broader and its mucosa much simpler. There are fewer of the highly complex longitudinal and annular mucous folds in which bacteria may attain a secure habitat. The most marked exceptions to this rule is met in pyometra, where, as shown in Fig. 231, the cervical walls are invaded and become greatly thickened and indurated.

In a milder way (catarrhal endocervicitis) the cervix participates in the infections of endometritis and apparently contributes importantly to the production of sterility. It has generally, however, an effect approximately opposite to that observed in the cervicitis of cows. Instead of causing constriction of the cervix, it usually causes paresis of the walls and dilation of the cervical canal. That is, the cervix of the mare under the invasion of ordinary infections pursues a course essentially parallel to the lesions in the uterus 
suffering from endometritis. Both uterus and cervix become flaccid and atonic. In the cow the uterus becomes flaccid while the cervix indurates.

The prognosis is good and the handling is to be based upon the general principles detailed for the cow. The uterus is to be so handled that it will not be discharging muco-pus over the cervical mucosa, and the cervix itself is to be disinfected the same as in the cow, especially by swabbing the cervical mucosa with Lugol's solution, silver nitrate, zinc or copper sulphate.

\section{VAGINITIS}

The specific vaginitis of genital horse pox has already been described. The organ is sensitive to irritants. Vaginitis may be aroused in endless ways. However, mares apparently escape the virtually omnipresent lesions of the nodular venereal disease of ruminants and swine which serve in them as a foundation for vaginitis which may be aroused by slight insult.

Parturient contusions and abrasions are the commonest causes of severe vaginitis. Doubtless imprudent vaginal douching sometimes causes vaginitis in mares as well as in cows. In one instance a mare was brought to me suffering intensely with prolapse of the vaginal floor and the urinary bladder (vesico-vaginocele) following coitus. She had severe vaginal atresia from prior disease. Since she had changed ownership (probably because of the unseen disease) the history leading up to the atresia was not available. It was not improbably due to imprudent vaginal douching. I was compelled to produce profound choral narcosis for several hours in order to control the prolapse.

Vaginitis varies greatly in severity and duration. When not too severe, it usually tends to recover spontaneously; when severe, it may tend to persist permanently and cause either vaginal atresia, as in the instance related, or severe, intractable pyo-vaginitis which may ruin the value of the patient. The infection may extend forward to involve the cervix and uterus, or pus flowing backward over the urethra may traverse that canal and set up a very serious pyo-cystitis. 
The veterinary obstetrist should always anticipate vaginitis in cases of dystocia, retained fetal membranes, metritis, and other diseases of, or injuries to these parts. If severe parturient vaginal abrasions are present, every possible detail of cleanliness and asepsis should be applied as preventive measures, such as frequent douches with physiologic salt solution, a very weak Dakin's solution, or some one of the approximate substitutes for it. One may apply in conjunction with these, or alone, one of the bland oils holding in suspension iodoform or other non-irritant antiseptic. Medicated suppositories of beeswax or soft paraffin may also be used with advantage.

\section{Cystitis}

Cystitis occurs in mares as an extension of vaginitis or vulvitis along the short and broad urethra. I was called to attend a mare for dystocia after laymen had exhausted their powers. The fetus presented anteriorly, dorso-sacral position, with both hind feet extended beneath its body into the pelvis. The laymen had bisected the fetus through the chest without preserving a flap and the torso receded far enough that the exposed vertebra and ribs lacerated and contused the vaginal mucosa severely. I removed the torso after a second bisection. Some weeks later I was called again to see the mare and found her suffering from severe vaginitis, vulvitis and cystitis, The cystitis was the most formidable of the lesions. The meatus was open and the index finger could readily be passed into the cavity of the bladder. The walls of the bladder were thickened and the mucosa was thrown into extensive folds which were covered by crusts of urinary salts, mixed with pus. The purulent urine had a very fetid odor. The cavity of the bladder was small and the thickened walls paretic and inextensible, so that the urine flowed from the bladder involuntarily, producing an intense irritation of the vulva. Dribbling down constantly over the thighs, the urine kept these parts repulsively soiled and excoriated and the tail wet and filthy.

The treatment of purulent cystitis consists essentially of the disinfection of the vagina, vulva and bladder. This should be accomplished chiefly by the mechanical removal of 
the infections and of the purulent exudates, the precipitated urinary salts and other debris, by the use of physiologic salt solution, or other neutral fluid followed by non-irritant antiseptics (iodoform, bismuth) suspended in bland oil or incorporated in suppositories of wax or low-melting paraffin.

\section{Vulvar Diseases}

The vulva of the mare not infrequently suffers from injuries during parturition which lead later to important infections. Parturient hematoma of the vulva are common. Usually the blood clots are submucous and, when opened, heal without serious infection. Not all cases terminate so fortunately. When parturient abrasions are severe, infection of an important type is always to be feared and should be anticipated by taking proper antiseptic measures. It is highly important when, following abortion, the fetal membranes are retained and lie in contact with parturient abrasions, that special precautions should be taken as in retained fetal membranes in cows, and everything possible done to avoid serious bacterial invasion.

Twice I have observed vulvar gangrene in the mare following dystocia when the fetus was in the anterior presentation, dorso-sacral position, and the two posterior limbs extended underneath the body, engaged beneath the fetal chest within the maternal pelvis. This caused the fetus to become tightly jammed in the pelvis, where it pressed upon the internal pudic vessels and apparently caused a pressure or anemia necrosis, leading, in one case, to rapid sepsis and death. In the other case the gangrene caused vulvar atresia and prevented breeding. Since in each instance the dystocia developed at night and was not discovered until morning, the perilous pressure upon the soft parts had been continued for a long period of time. When such injuries occur, the veterinarian should anticipate vulvitis by means of warm douches with massage, and such other measures as may suggest themselves in the particular case. It is to be remembered that fatal gangrene is at least possible, and, while probably preventable by the application of vigorous antiseptic measures, is not ordinarily curable. 


\section{CONGENITAL INFECTIONS OF FOALS}

The basic principles that any infections existing within the gravid uterus may invade the embryo or fetus, and may, if they fail to kill the intra-uterine young, persist in the newborn and cause fatal or dangerous illness, apply with the same force in foals as in other mammals. The manifestations of intra-uterine infection in the foal present interesting variations in clinical behavior without violating any fundamental principle.

\section{A. Septicemia of the Foal}

When an equine fetus becomes critically infected but is born, it is weak, listless, can not get up, and, if helped to its feet, may be unable to stand. If born unattended and a fragment of amnion chances to be upon its nose, perhaps it is too weak to free itself and dies with the frail membrane lying over or about its nostrils, leading to the popular error that foals often smother in the amnion. While a moribund foal may so perish, such an occurrence with a healthy foal is extremely improbable. No studies have been made of the temperature of the new-born foal suffering from septicemia, but it is probably elevated. The sepsis generally ends fatally within a few to twenty-four hours and the post mortem study reveals substantially the same lesions as those observed in calves.

\section{B. Retention of the Meconium}

Calves, and most other new-born mammals which bear serious infection from the uterus, generally show a marked and early tendency to dysentery. In harmony with the normal dryness of the feces in the large intestines and rectum, alike of the fetus and of the adult, the foal often shows a pathologic impaction of the rectum with very hard masses of meconium. At first the foal may appear healthy or at most merely a trifle dull, but after taking milk there appear, at from twelve to forty-eight hours after birth, symptoms 
of abdominal pain. This is expressed in part by turning the nose to the abdomen, kicking at the belly, lying down and rolling. The most characteristic symptom is the assumption by the foal of a peculiar extended attitude while standing. The foal extends its anterior feet far forward, its hind feet backward, all members being held rigid. This position is maintained for five to fifteen minutes or more. The layman believes the foal is attempting to urinate, but it makes no actual effort either to urinate or to defecate. The tarsus is not bent as in urination. The pain is intermittent. Between paroxysms the foal may suck. Unless medical relief is given, the disease pursues a slow course and ends in death from sepsis and exhaustion. In the presence of meconic retention, the foal often develops a perverse appetite and swallows large quantities of hay, straw, feces or other indigestible substances, thereby complicating the disease present.

\section{Dysentery}

Some writers have stated that retention of the meconium frequently ends in dysentery. This I have not observed. Dysentery is not rare in foals, however, and, as in calves, may be due, and usually is due, to intra-uterine infection, but may and does arise from extrinsic infection, variously obtained after birth. The symptoms of dysentery in foals are essentially parallel to those described in calves. Like calves, foals exhibit a strong tendency to swallow hay, straw, feces and various indigestible substances. These complicate and intensify the disease, frequently rendering it unavoidably fatal. The course of the disease may be either rapid or slow. The mortality is high unless active measures are taken for its control. The behavior of the disease as related to the feeding of milk has not been investigated, but is presumably parallel to the observations made upon calves and previously described.

\section{Arthritis. Pyemia}

Pyemic arthritis is commoner in foals than in any other species of domestic animals. This is due chiefly to the fact 
that a large proportion of the cases is caused by post-natal infection through the umbilicus (omphalo phlebitis, pyosepticemia), owing to the longer and thicker umbilic cord which breaks with a longer stump. An important part is played by the long stump by inviting imprudent ligation, so that the umbilic stump is ligated more frequently in the foal than in all other new-born animals combined. Umbilic infection is so evidently the cause of many cases of pyemic arthritis that until quite recently the navel was considered the sole avenue of invasion. Lately Schofield ${ }^{1}$ has shown satisfactorily that arthritis is frequently due to intra-uterine infection and that in etiology it conforms to the basic principles of pyemic arthritis in calves and may be either ante-natal or post-natal in origin. Like arthritis in calves, the arthritis of foals is prominently associated with intense intra-uterine infection in mares with abundant sterility and abortion. Bacteriologically there are recognized, in the infected joints or other pyemic centers, streptococci, staphylocci, the B. abortivo-equinus, etc. In other words, there may be present in the diseased joints any bacteria which may exist (a) in the uterine cavity of the pregnant mare and may be swallowed by the fetus, (b) in the milk of the dam and be swallowed by the foal when the alimentary epithelium has been damaged or destroyed, or (c) externally and invade the foal through the umbilicus.

The symptoms consist chiefly of the sudden development of an intense arthritis in one or more articulations, most commonly the tarsus, carpus and stifle. When the invasion occurs through the umbilicus, the event is usually recorded locally by slight swelling of the umbilicus, followed presently by a discharge from the umbilic veins of a thin, watery pus often associated with a re-opening of the urachus with flow of urine.

Prior to the definite appearance of arthritis, there is present a marked elevation of temperature which, as in the calf, presages the approach of a storm. When the foal is one to several days old, there develop suddenly centers of pyemic

\footnotetext{
${ }^{1}$ Schofield, F. W. Investigatious into Joint-Ill in Foals. Dept. Agr. Toronto, 1916.
} 
inflammation, chiefly in the joints. The foal is seen apparently well, and two or three hours later is found breathing rapidly, greatly dejected, lying down most of the time and, when caused to rise or helped up, is unable or unwilling to bear weight upon a limb. The lameness may shift to another limb very abruptly or may visibly affect one or more joints in two or more members. The appetite is diminished or destroyed. The involved articulations are swollen, hot and painful. The synovial sac is tightly distended with reddish synovia, which may later become purulent. Pyemic abscesses may develop in any organ or tissue.

Pyemic arthritis is commonly associated with alimentary

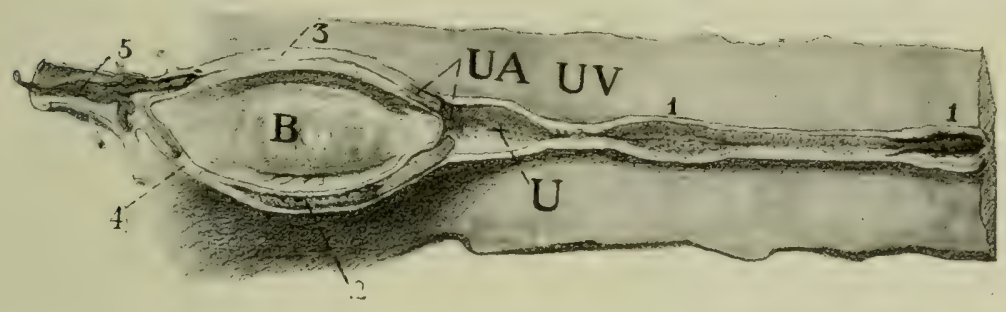

FIG. 232-Abscess of Umbilic Vein and Artery. Foal.

$B$, Urinary bladder; $U$, umbilic vein passing from umbilicus into peritoneal cavity; $U A$, ends of ruptured uterine arteries retracted to fundus of bladder; $V V$, umbilic vein. $I$, Pus cavity in umbilic vein; 2 , abscess in right umbilic artery; 3 , thickened walls of left umbilic artery; 4 , normal artery ; 5 , thrombus in posterior aorta and iliacs.

disturbances ranging from severe diarrhea to obstinate constipation, and as a part of these disturbances there develops an erratic appetite which causes the foal to swallow hay, straw and other indigestible materials. Unless the course of the disease is interrupted by vigorous medical interference, it is highly mortal. Almost all severe cases arising within two or three days after birth are fatal. Many are left with disabling joint lesions.

The lesions vary widely. When the invasion occurs through the umbilicus, especially when aggravated by ligation of the umbilic stump, prominent lesions of the umbilic arteries and veins are probable, as shown in Fig. 232. When these vessels become involved, the urachus ordinarily par- 
ticipates in the pathologic processes, the retracted, closed canal re-opens and urine dribbles from the umbilicus. It is not essential, however, that the infection shall invade the system through the umbilicus in order to cause the umbilic lesions. There is clearly a bacteriemia present. The recently ruptured veins, arteries and urachus, with retraction of urachal and arterial stumps into the abdomen and maiming of the tissues, afford an inviting field for the entrance
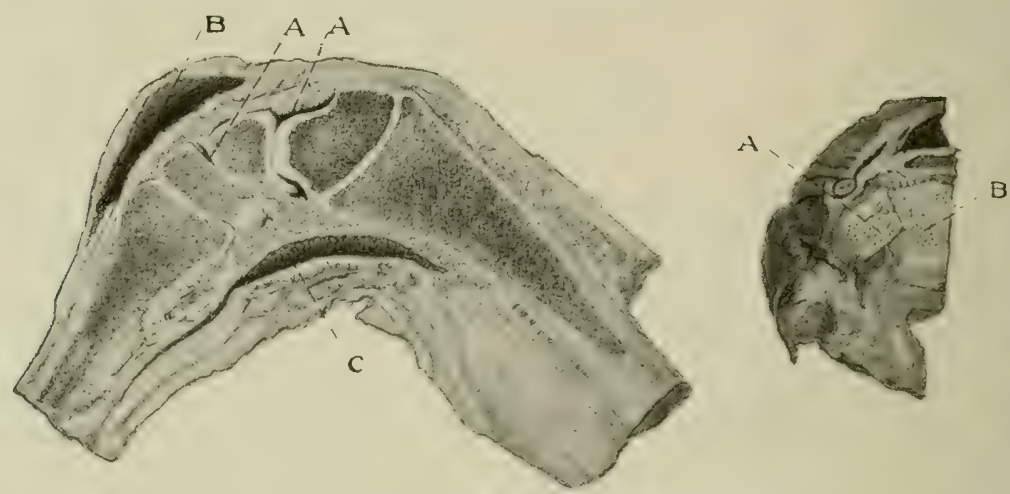

Figs. 233, 234-Arthritis of Carpus.

233-Longitudinal section through carpus.

$A$, Intra-articular abscesses ; $B$, pre-carpal abscess ; $C$, post-carpal abscess. 234-Cross section of 233 showing articular surfaces of carpal bones.

$A B$, Purulent destruction of articular cartilage.

of bacteria from the blood stream as well as from the exterior. The opportunity for bacterial invasion from the blood stream is further favored by the presence of some necrotic blood which escaped into the umbilic connective tissue during the retraction of the umbilic arteries. An even more dangerous condition exists in the umbilic vein, where some residual blood invites bacteria and, if in addition the umbilic stump has been ligated, the entire functionless umbilic veins are distended with great masses of blood incarcerated by the ligature. The articular lesions consist chiefly at first of intense synovitis with great distension of the synovial sac. There follow later necrosis and erosions of the articular cartilages, as shown in Figs. 233 and 234. The synovia may be bloody and turbid, and may contain synovial 

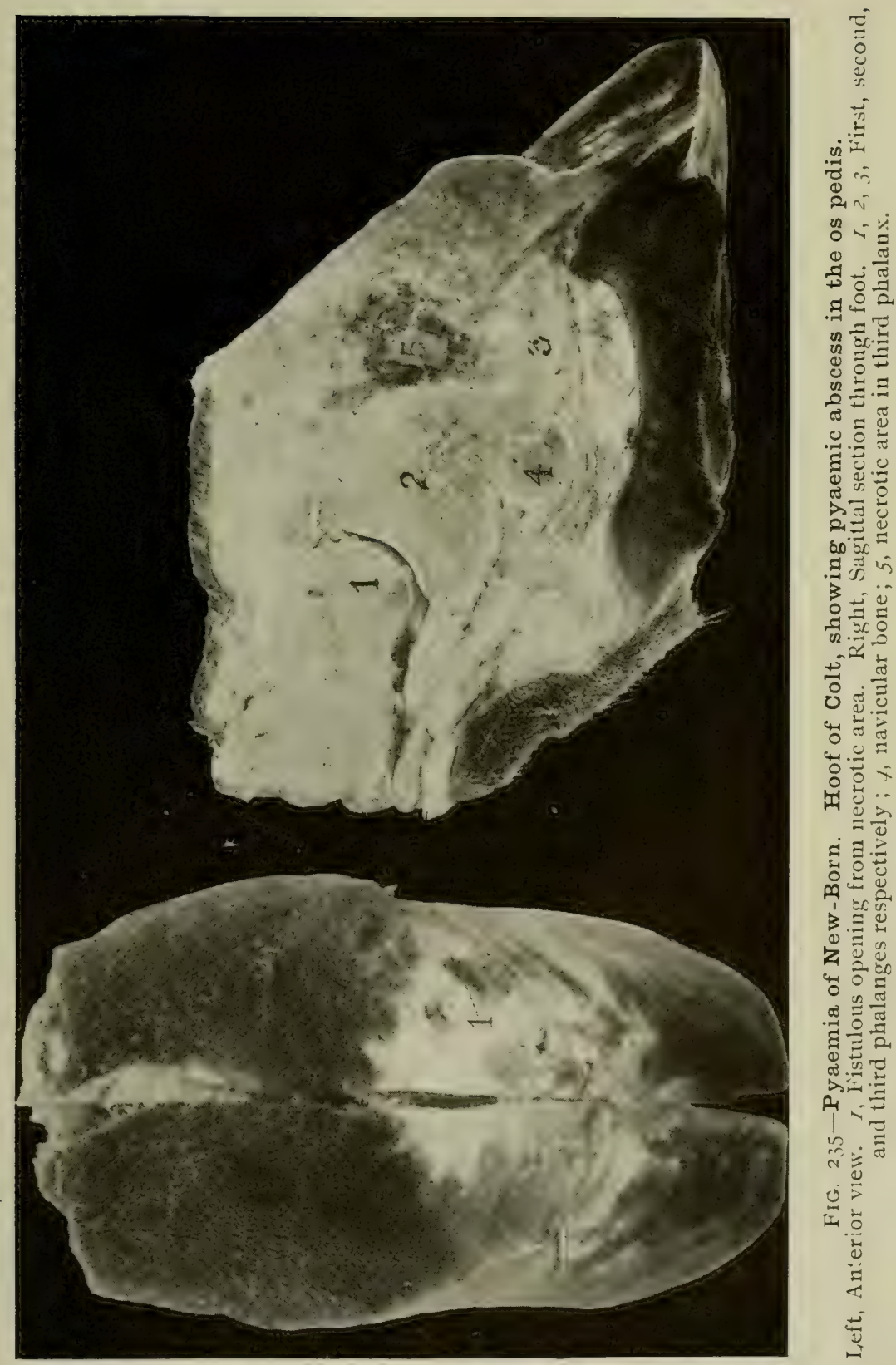
clots or become purulent. The necrosis may extend to the bones, as illustrated in Fig. 235.

\section{E. RE-OPENING OF THE URACHUS}

The closing of the urachal stump, when it retracts into the abdomen immediately after the rupture of the navel cord, is ordinarily secure. It is brought about partly by the thickening of the walls of the tube in the process of shortening, partly by the retraction of the surrounding connective tissue sheath, and apparently also by lymph poured out from the lacerated walls at the point of rupture. If the urethra is open and the umbilicus escapes infection, no urine escapes from the umbilicus. If infection invades the wounded umbilic tissues and the urachal stump is involved, the urachal canal re-opens and urine flows from the navel. Apparently it does not matter whether the invasion is extrinsic or intrinsic, the re-opening of the urachus follows. The umbilicus then becomes saturated and infiltrated with urine and much decomposition results. It has been assumed generally that in these cases the urachus has failed to close, but according to my observations the open urachus (assuming the urethra is open) is uniformly secondary to infection and never primary.

The handling of this group of infections has not been well standardized. Fundamentally the principles involved are identical with those applying to calves. The rule laid down, that the sole guarantee for the birth of a sound calf is dependent upon the mating of sound parents, is equally applicable to horsebreeding.

It is essential to the safety of a new-born foal that the expulsion of the meconium should be caused as completely and early as practicable. The safest means is the high enema, as recommended for calves. The enemas should be repeated at least twice daily until the expulsion is complete and soft meconium is passed. When meconic retention becomes evident, it should be attacked vigorously. The enemas of physiologic salt solution should be frequent and as high as practicable. Olive or cottonseed oil, or liquid paraffin 
may be substituted for the salt solution or may be given per mouth. If these fail, resort may be had to $1 / 20$ to $1 / 12$ grain of eserin or arecolin given hypodermically, repeated hourly if needed. In the meantime the foal should be watched and any tendency to swallow hay or bedding frustrated by muzzling. Although not investigated, it is not improbable that the hypodermic injection of the blood serum or defibrinated blood of the mother of the foal in retention of the meconium would have the same value as in arthritis.

The feeding of foals should be upon the same principles as that of calves. When a foal is born, the precaution should at once be taken to draw all milk from the mare's udder in order that it shall not gorge itself. If abortion in mares, or dysentery or arthritis of foals is prevalent in the establishment, the development of the two latter should be anticipated and vigorous measures taken to frustrate them. Schofield ${ }^{1}$ has reported favorably upon the use of bacterins made from the streptococcus, usually found by him in the joint lesions, for the prevention and cure of arthritis. $\mathrm{He}$ and others state, however, that in outbreaks associated with much abortion, the $B$. abortivo-equimus is present in the joints and appears to be the causative agent. In such case that bacillus needs to be added in the making of the bacterins.

Forssell, in the Berliner Tierärztliche Wochenschrift, part 12,1916 , and in a later contribution not yet published, advocates what appears to be a more logical plan for preventing and handling arthritis in foals (and probably dysentery as well), by the use of the blood of the foal's dam. His recommendations have excellent support in clinical data. In the Schofield plan the results are technically based upon the causative agent's being used for the making of bacterins. The causative agent is subject to such a wide variation in species that the selection of bacterins is always a hazard. Any pyogenic bacteria which may exist within the gravid uterus or which may invade the foal through the umbilicus, may cause the disease. In each case the possible number of

\footnotetext{
${ }^{1}$ Schofield, F, IV. Investigation into Joint-I11 in Foals. Dept. Agr. Toronto, I9I6.
} 
bacteria which may serve to cause the disease is unknown and unestimated.

So far as known, the blood of the dam always carries antibodies to the infections present in her uterus. The blood of the fetus does not, with rare exceptions, carry such antibodies, but the bacteria of the uterus exist in the meconium of the fetus, ready after birth to penetrate the alimentary mucosa and cause septicemia or arthritis. If the blood of the mother is used as a prophylactic or therapeutic agent, it is virtually certain that antibodies against all intra-uterine infections are included. It does not assure the inclusion of antibodies against extrinsic or navel infection. The prophylaxis of this may be attained, however, by the proper disinfection of the navel stump when the foal is born. At the same time, the blood of the mare probably contains antibodies against the most common infections invading the navel.

Forssell at first used the blood serum from the dam, but found this objectionable because it involved a delay of one day, which is very undesirable in the most violent cases. He then turned to the Lewisohn method of using the entire blood. He found that, by adding neutral sodium citrate, two parts to one thousand parts of blood, horse blood will not coagulate. The uncoagulated blood may be given safely intravenously or subcutaneously. The technic of Forssell directs that first the phlebotomy needle, the vessel for the blood, the hypodermic syringe, and other apparatus should be boiled. The water used for boiling these needs to be free from lime, because any of this coming in contact with the blood causes coagulation despite the presence of sodium citrate.

When ready to draw the blood, one gram of sodium citrate in fifty grams of water is placed in a flask or other container and shaken about until the sides of the vessel are well moistened. This suffices to prevent the coagulation of $500 \mathrm{cc}$. of blood, which volume, or a trifle less, is used as a sigle dose. While the blood is being drawn, it needs to be agitated constantly or stirred, in order to keep it mixed with 
the citrate and prevent coagulation. When used subcutaneously, it should be injected along the sides of the neck or chest, about $50 \mathrm{cc}$. at a given point. For subcutaneous use, Forssell states that the mixture of the sodium citrate is not absolutely essential. If one needle is in the vein of the dam and a second needle is already in place for the subcutaneous injection, the blood may be drawn into the syringe and quickly injected. The needle in the foal is then moved to another point and the syringe again filled. The syringe must be kept warm with hot water. When using the non-coagulated blood, it should be kept at body temperature by immersing the container in warm water. The intravenous injection must be made very slowly.

Forssell reports 157 cases handled with the dam's blood or blood serum, with recoveries in $127-81$ per cent. Many of those which died were in a critical condition when the handling was begun, and more would probably have recovered had the application been made earlier.

Forssell believes that the ideal plan would be to prepare a stock serum from the bacteria recognized in arthritis which could be conveniently and promptly given the foal. The objection exists, however, that the bacteria of foal septicemia, pyemia, arthritis and dysentery may vary widely, and that any stock serum may fail to contain the antibodies for the infection causing disease in a given foal. On the other hand it is to be remembered that, so far as known at present, the bacteria causing the infections in new-born foals (except such specific forms as tetanus bacilli when invading the navel) including the B. abortivo-equinus, are identical with, or analogous to the common wound infections, and that many, perhaps most of them, are normal habitants of the adult body, either in the alimentary tract, in the udder, or in the genital organs. Consequently the blood of an adult horse may, and perhaps does, contain a long list of antibodies which confer upon the animal a degree of resistance to the entire bacterial group involved-age resistance. In discussing calf scours serum, it was noted that there is much evidence tending to show that normal horse serum possesses 
similar therapeutic power to the artificially produced calf scours serum. If this proves, upon further study, to be true, it would logically follow that the blood of an adult horse would, as a rule, contain valuable antibodies for those common or non-specific infections menacing the life of the new-born foal. Another interesting possibility, in stables where arthritis and dysentery are common, is that blood may be drawn from mares in advanced pregnancy a few days prior to expected parturition, serum prepared, and with 0.5 per cent. phenol added, retained in a refrigerator until the foal is born, and then given immediately.

\section{F. Rupture of the Tendons and Synovial Distensions OF THE NEW-BORN FOAL}

There occurs not infrequently an interesting and usually mortal disease in young foals, expressing itself clinically by the rupture of the extensor pedis longus tendons, and by distension of the synovial cavities, especially of the great femoro-tibio-patellar synovial sac. The character of the disease is as yet unknown. It is unquestionably intra-uterine in origin. The symptoms become obvious within one to several days after birth. It is placed among the intra-uterine infections, not so much because of evidence of its infectious nature, as because its etiology is unknown and because certain clinical facts vaguely suggest that it is due to infection. I have observed the disease mostly in localities where uterine infections of mares are common. In one stable under my care, there were born two foals annually for two years and each of the four foals suffered from rupture of both extensor pedis longus muscles. The symptoms observed consist essentially of loss of power to extend the carpus. When born the foal shows nothing notable so far as yet observed, except that there is apparent feebleness. It gets up or, if unable to do so, stands when helped to its feet, but walks unsteadily, the carpus constantly tending to bend forward. There is no recognizable anatomical defect in the carpus such as the ordinary bent carpus often seen in new-born foals. The entire cause of the symptoms appears to be in the 
chief extensor of the foot. The carpus is bent forwarci, as shown in Fig. 236. If the foal is helped to its feet and pressure applied against the anterior face of the carpus, it stands steadily and the carpus is straight, but removal of the pressure permits the carpus to bend forward and may cause the foal to fall. As the disease progresses the foal shows decreasing control over the anterior feet, and in the

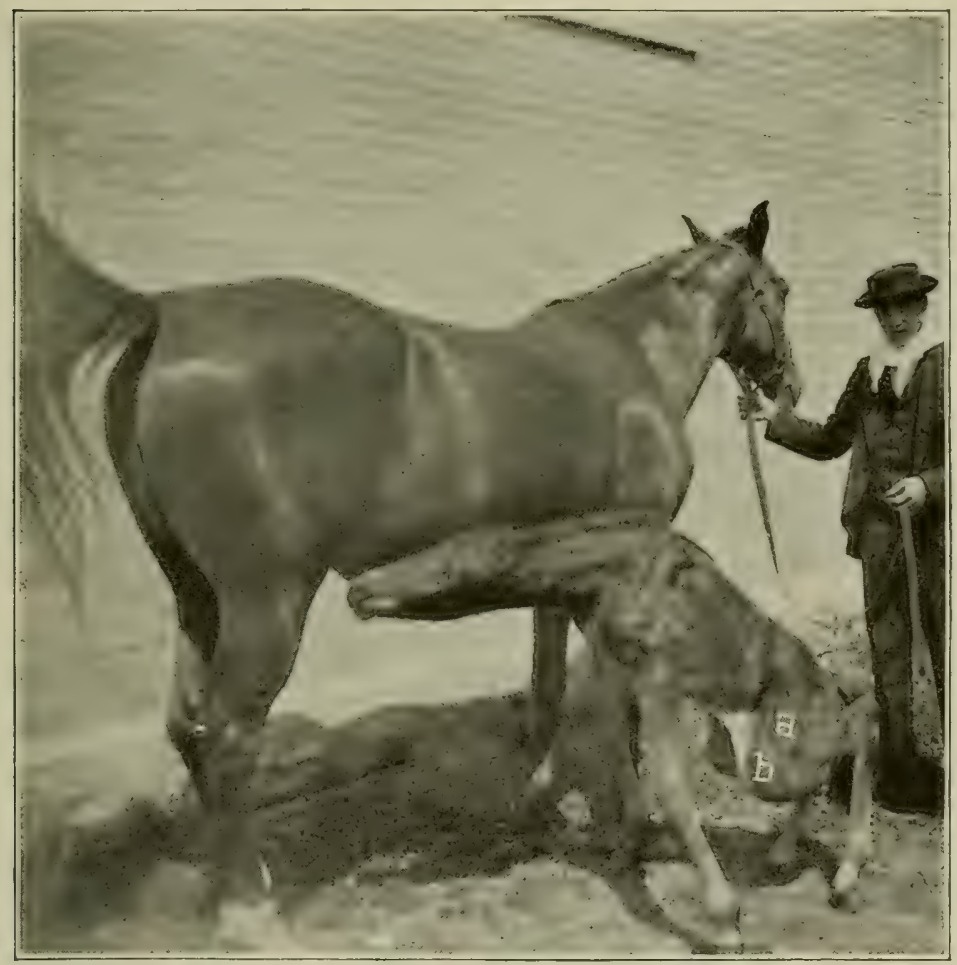

FIG. 236-Rupture of Extensor Tendons at Carpus, and Floating Dislocation of Patella. $a$, Patella; $b$, femoro-tibial articulation.

most severe cases becomes unable to rise and even unable to stand when helped up. When the decubitis becomes constant, the foal generally perishes within a few days.

Apparently an integral part of the malady, but infrequently seen, is the distension of the femoro-tibio-patellar 
synovial sac, as shown in Fig. 236. The two synovial cavities became so distended, when the foal was two or three days old, that each patella was pushed away from the trochlear groove of the femur, floated above the summit of the external ridge, and became completely dislocated laterally, wholly disabling the posterior limb. The foal was therefore unable to stand erect either before or behind. The disease is apparently not local, but merely the local evidence of systemic disturbances. In lying down and getting up, the ex-

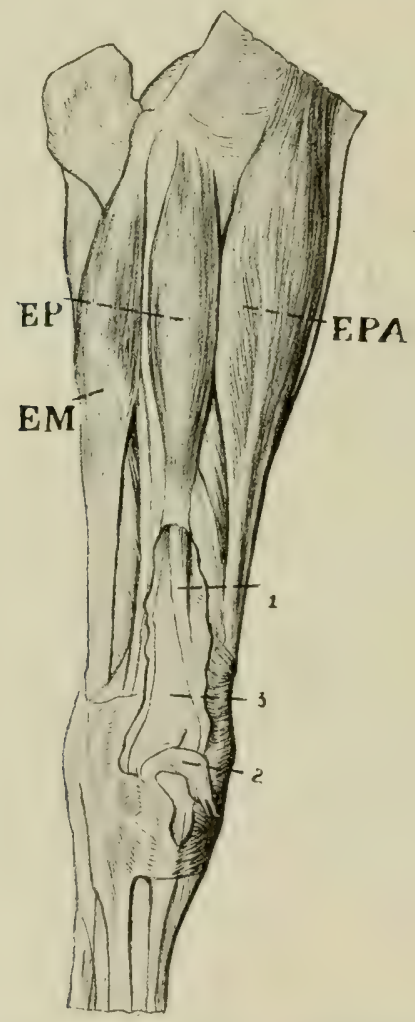

Fig. 237-Rupture of the Extensor Pedis Longus Muscle in New-Born Foal.

Right anterior limb seen from in front.

$E M$, Extensor metacarpus; $E P$, extensor pedis longus; $E P A$, exterpedis longus accessorius ; 1 , Proximal ruptured end; 2 , distal portion of tendon dropped down in sheath, 3 . 
tremely long legs of the foal lead to an inordinate strain upon the extensor pedis longus muscle. The rupture, as shown in Fig. 237, occurs at the point of union between the tendon and the belly of the muscle. In this respect it differs markedly from the ruptures of muscles and tendons commonly observed.

The clinical evidences of a rupture rests in the early stages upon the difficulty or inability of the foal to get up or to walk because of the want of power to extend the carpus. If the character of the lesion is suspected, the diagnosis is to be verified by carefully palpating over the anterior face of the lower third of the radius, from 1 to 2 in Fig. 237, which reveals an empty groove normally occupied by the base of the tendon, 2. The free end of the tendon is also palpable, freely movable in its sheath at the upper part of the carpus. In those animals which survive, reunion between the muscle and tendon does not occur and the empty groove is permanent. The foot must then be advanced by the accessory extensor of the metacarpus. This results in a peculiar gait, the foot being abducted in the stride and carried forward with a jerky flail-like motion.

The prognosis is exceedingly unfavorable. Although some cases live, their locomotion is interfered with by the absence of the extensor pedis longus muscles, and the value of the animais is consequently greatly lessened except for slow work. For driving or riding they would inevitably prove insecure upon their feet. For any fashionable use, their peculiarity of action would render them unsuitable.

The handling of the malady is uncertain, and apparently there is little to do beyond taking good care of the animal and aiding it in getting upon its feet in order to suck. While the foal is sucking, an assistant should grasp it by the knees, and, pushing backward, prevent their flexion, thereby assisting it in standing. Unless the foal is of considerable value, it is advisable in most instances to destroy it at once. 


\section{SECTION V. THE GENITAL INFECTIONS OF CARNIVORA}

CHAPTER XXIV

\section{SPECIFIC VENEREAL DISEASES}

The Venereal Tumors of the Dog. Venereal GranuloMATA. LYMPHO-SARCOMA

In the dog, there is observed a specific venereal disease consisting of granulomata upon the genital mucosa. The disease is somewhat wide-spread in Great Britain and continental Europe. It is observed in various portions of the United States, largely in dogs recently imported. In the male it affects chiefly the penis and prepuce and sometimes invades the adjacent tissues. Metastatic disease of the inguinal glands may follow. In the female it involves pri-

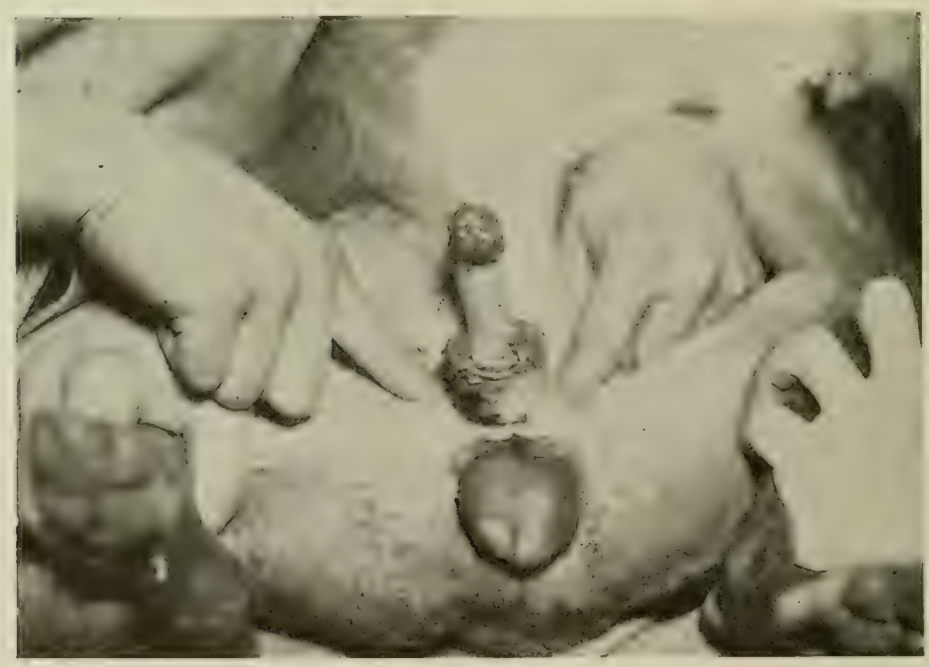

FIG. 238-Infectious Venereal Granuloma of Dog. (C. A. White.) marily and chiefly the vulva. It is naturally transmitted by copulation only, but may be otherwise spread by artificial or accidental inoculation. 
Symptoms. The first symptom usually noted is a bloody discharge from the prepuce or vulva, accompanied by tumefaction of the parts. If the penis is exposed at this time, there are seen pink or grayish red vegetations, largely upon the glans or at the preputial ring upon which the glans rests when the penis is retracted, at times, upon other parts of the mucosa. The tumors are soft and friable and bleed

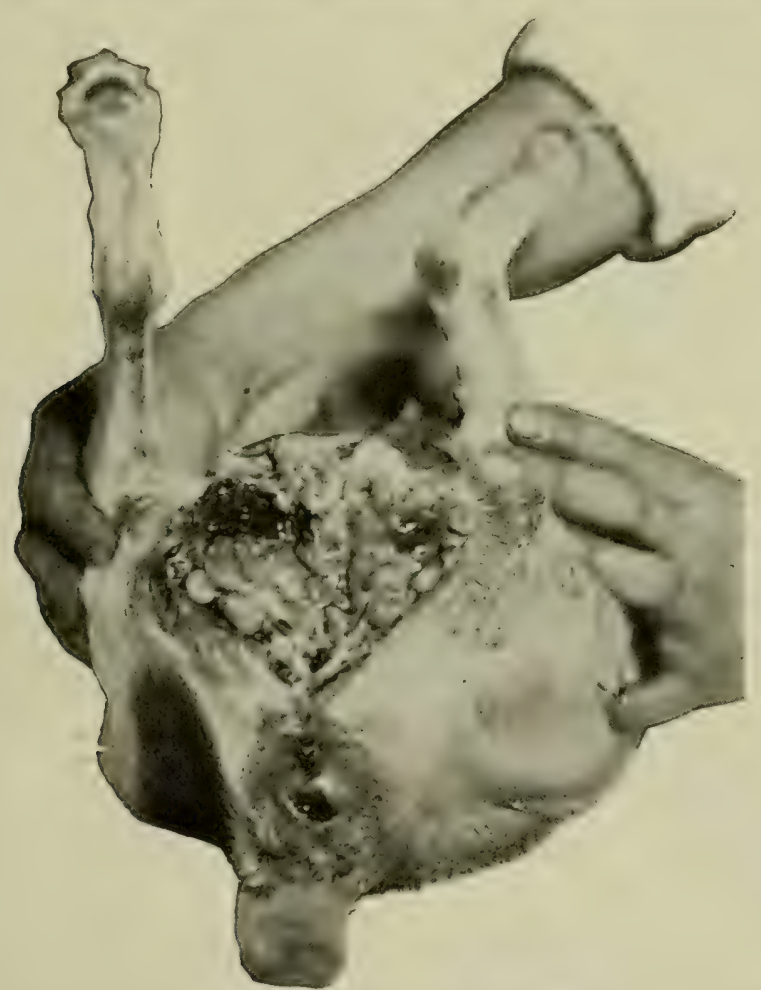

FIG. 239-Infectious Venereal Granuloma of Bitch. (C. A. White.)

freely upon being touched. They may be sessile or somewhat pedunculated, resembling warts. The affection is of a chronic character and seems to have no definite limitation. The vegetations continue to increase in size month by month and finally become firm, lobulated masses, so intensely injected that they assume a dark color. 
In the bitch, the symptoms are very similar. There is first a bloody discharge from the vulva. Examination of the vulva and vagina reveals vegetations of the same appearance as already noted in the male. These appear chiefly along the floor of the vulva and, when very large, may protrude externally. The discharge from the vulva is usually fetid. According to French, males sometimes show an aversion to mating with affected females.

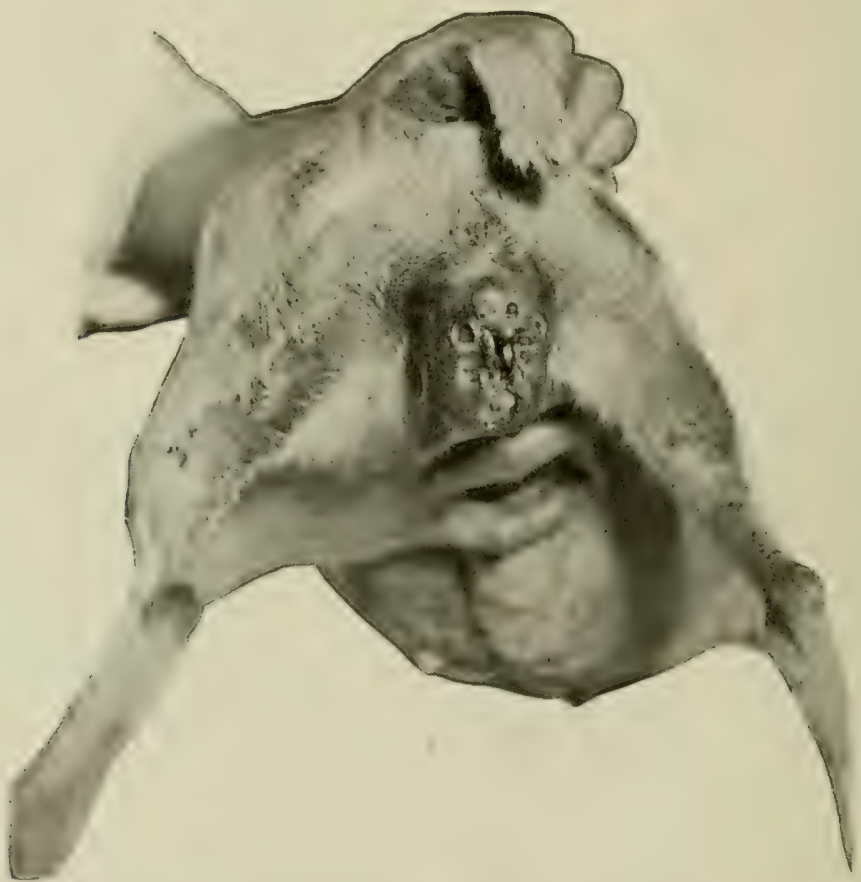

Fig. 240-Infectious Venereal Granuloma of Bitch. (C. A. White.)

Beebe and Ewing (Jour. Med. Research, Sept., 1906, do. Vet. Jour., July, 1907) record the presence in these tumors of spirochæte in one out of a series of cases, but failed to connect their presence with the cause of the malady.

Later, Mettam (Veterinary Journal, February, 1907) records the discovery of an organism in this disease belonging to the group of spirochrte, which he believes to be the specific cause. 
Histologically, the tumors consist of large round or polygonal cells, which multiply rapidly and push the contiguous tissues aside without their becoming involved in inflammatory or other disease changes.

The disease has been repeatedly transmitted experimentally by inoculations in the genital mucosa and other tissues.

In clinical and experimental cases the neoplasms may not remain confined to the genital organs, but may involve the skin, the lymphatic glands of various portions of the body, the liver, spleen and other glands.

The treatment is not highly satisfactory unless undertaken early. It consists chiefly of excising the vegetations, along with a small area of the healthy mucosa, after which the edges of the wound should be drawn together with catgut.

In the male, it may be necessary to divide the prepuce and, in the female, the perineum, in order to reach all the diseased parts. Afterwards, these wounds should be sutured. It is well to place the animal under general anæsthesia for the operation, which should be repeated within one to three weeks if necessary, and in some cases cautery may be used. In inveterate cases it may be necessary to amputate the penis and prepuce. Hobday states that the disease may be favorably affected by castration. 


\section{CHAPTER XXV}

\section{THE GENERAL GENITAL INFECTIONS OF CARNIVORA}

Since the general genital infections of dogs and cats have not been extensively studied, little can be said of them. It is known that carnivora suffer frequently and seriously from general genital infections, but their individual value is so low in many cases, and so few, comparatively, are valued highly for breeding purposes that this group of diseases has attracted scant attention.

It is well known that dogs suffer virtually universally from a well marked genital catarrh. It is so common that it is designated as normal and, so far as known, has had no serious study. It is assumed to cause no important harm. It is a repulsive disease in many males, as the genital discharge soils the hairs about the sheath opening and attracts many flies.

The bacterial flora of the genital tract is quite unknown. $B$. abortus (Bang) has been recognized in the genital tract of the bitch following experimental inoculation. It was not known that the bacillus did not already exist in the bitch at the time of inoculation, and it is not known how frequently the B. abortus exists naturally in either dogs or cats. Since both feed freely upon cow's milk, which ordinarily contains B. abortus, it is probably a common habitant of the organs of carnivora. The infections of the genitalia of carnivora probably resemble closely, if they are not frequently identical with, the bacteria invading the genitalia of other species. At least they behave in the same general manner and cause the same clinical phenomena.

Little is recorded concerning the infections of the testicle and epididymis of carnivora. In the dog, without vesiculae seminales, the prostate assumes prominence anatomically and pathologically. Prostatitis is a common malady seen chiefly in adult and aged dogs. It has not been studied except when it causes clinical symptoms consisting of diffi- 
culty in defecation and urination. It naturally renders the ejaculation of semen difficult. How much danger it carries for the bitch in coitus has not been investigated. It may play an important role in serious abortion storms in valuable kennels. When clinical prostatitis of a marked type becomes established, as in semino-cystitis in bulls, the dog probably becomes sterile, but may be innocuous because the prostatic ducts are occluded and the infection incarcerated.

When difficulty in defecation or urination becomes clinically evident, palpation per rectum reveals the presence of the enlarged sensitive prostate. When the disease has so far progressed that it causes clinical discomfort, it is probably beyond repair so far as fertility is concerned. There remains only the question of prolonging the life of the patient. In this field little of value has been accomplished. Apparently prostatitis has attracted no attention in relation with fertility and no operation or other method of handling for this purpose suggested. Castration tends to cause abatement in the symptoms of non-malignant prostatitis. Much of the prostatitis is malignant. Prior to reaching such an advanced stage, careful physical examination or examination of the semen may reveal the seriousness of the infection and enable the veterinarian to avert disaster to valuable breeding bitches.

Diseases of the ovary are apparently less common than in mares and cows. I observed one instance of nymphomania in the bitch, associated with cystic degeneration of the ovary, as shown in Fig. 241. The nymphomania presented the same general symptoms as in the cow and was relieved by castration. There are no data recorded suggesting a hopeful plan for handling nymphomania in the bitch with a view to the restoration of fertility. The surgical handling of the ovaries would call for laparotomy.

The infections of the pregnant uterus of the bitch and cat are numerous enough, but the details of their behavior have not been recorded. In some kennels the owners report that over a long period of time essentially all bitches abort, or at least fail to produce liv- 
ing young. When abortion is reported, its exact meaning is not clear. Dystocia in carnivora is frequently, if not generally, due to the death of a fetus or of fetuses as a result of infection. As a rule, as in the sow, the basal fetus-the fetus in the base of one horn and nearest to the vagina-perishes first and undergoes emphysematous decomposition, blocking the genital passage. The dis-

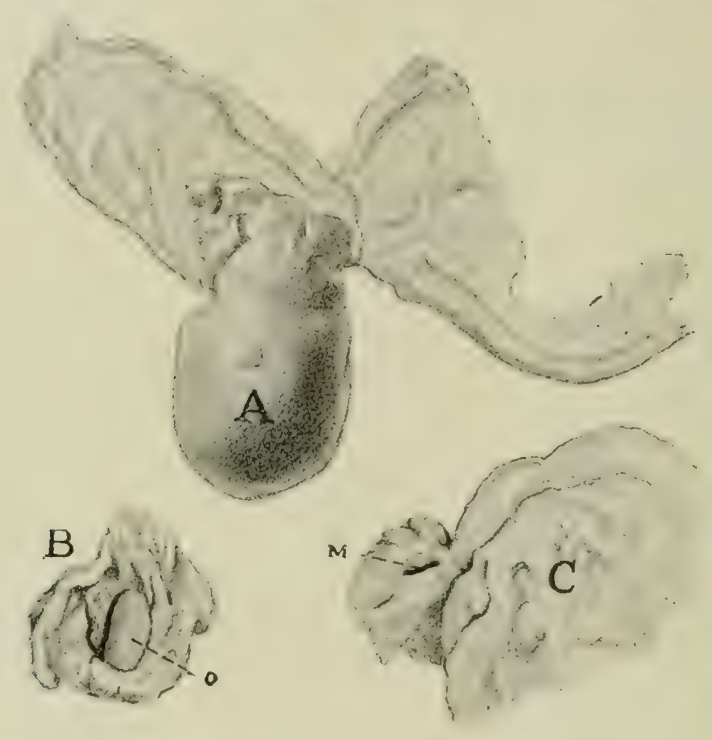

FIG, 24I-Normal and Cystic Ovaries of Bitch.

$A$, Cystic ovary ; $B$, normal ovary with the pavilion of the oviduct turned back to expose the ovary; $C$, normal ovary covered by the pavilion

of the tube, showing the meatus $(M)$ of the ovarian sac, by which it communicates with the peritoneal cavity.

position of the gravid uterus of carnivora is typically illustrated in Fig. 242. The basal fetus ordinarily lies in the base of one horn, and the vaginal end of its fetal sac, instead of protruding into the cervix, passes across the uterine end of the cervix into the base of the other horn, or practically into the other uterus, since the bitch has virtually a double uterus, the two organs communicating at their bases. In this manner, from ovary to ovary, the two uterine cavities are made to constitute one continuous tube and each fetal 


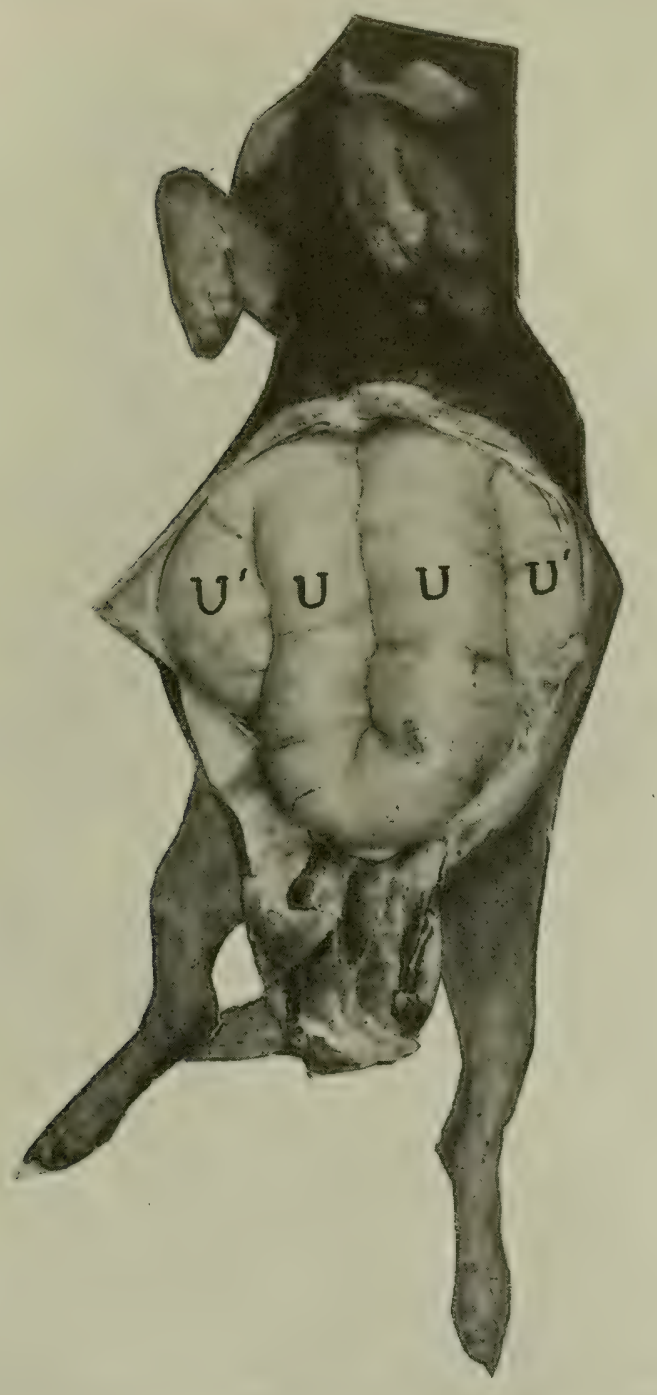

FIG. 242-Gravid Uterus of Bitch In Situ.

$U$, Direct cormua ; $U^{\prime}$, retrograde portion of cornu. 
sac, except of the two apical fetuses, is in contact at each pole with the next fetal sac. The two apical embryonic sacs end at the ovarian pole against the mouth of the oviduct, and at the cervical pole, against the next embryonic sac. Since the placentae are zonular, infection can not pass directly along the uterine tube, but must pass between the fetal and placental structures, destroying the life of the involved embryo, or must pass through the embryonic sac and the contained fluids and kill the embryo more directly. In either case embryonic death would necessarily occur one after another from the center of infection, and not en masse. If, on the other hand, there is a specific infection which causes abortion directly, either by passing from the blood stream of the pregnant mother to the fetus and destroying its life, by passing from the blood stream into the interplacental areas of the uterus and thence through the chorion and amnion to destroy the embryo, or by forming bacterial products anywhere within the animal body which are able to act directly upon the uterus and cause the expulsion of its contents, then abortion en masse would occur in multiparous animals such as swine and carnivora. But so far as I have been able to observe in the abattoir or to learn from literature, the death of embryos occurs singly and seriatim from one or more centers of infection. When all embryos have perished, or the last to become involved are critically ill, the uterus contracts and expels its contents. Otherwise, if some embryos have not been mortally infected, they continue their development and inhibit the expulsion of any fetal cadavers present. When the living embryos have reached maturity parturition occurs unless the outlet is blocked by a macerating, emphysematous cadaver. Along with the young which are born, there are expelled some or all of the fetal cadavers, or their debris. If, however, an apical embryo is dead and macerating, all others may be expelled and the cadaver remain in the diseased apex of the horn to produce a persistent pyometra.

The prevention of the general infections of the genitalia of carnivora has not been sufficiently studied to permit the 
formulation of a plan. Anatomical limitations prevent the examinations of the genitalia, so readily applied in cattle and horses. The testes and the glands of the pelvic urethra of the male are palpable. Conscientious effort should develop a technic which would enable the veterinarian to examine satisfactorily, and to apply remedial measures to, the vagina, cervix and uterus.

The clinical evidences of embryonic death in carnivora are variable. It is not known that the death of an embryo induces mammary activity, as is observed in the mare and cow. If the basal fetus of the bitch or cat is healthy, there

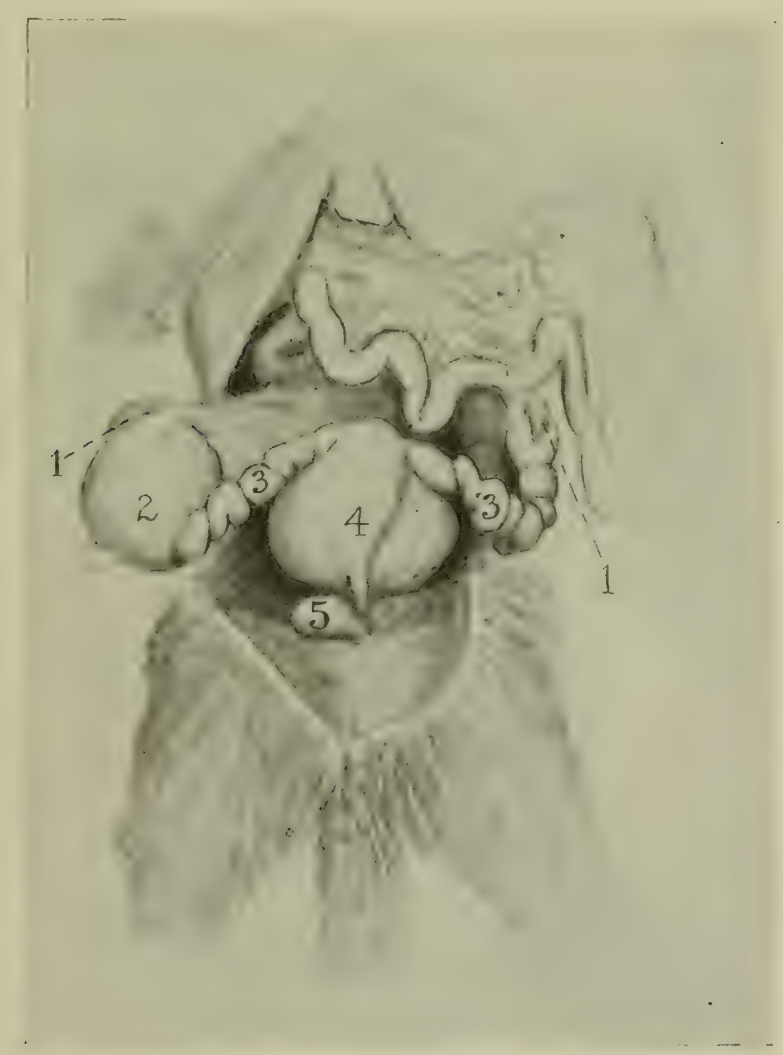

FIG. 243-Pyometra and Pyosalpinx. Cat.

I, Ovary ; 2, abscess of oviduct ; 3 , distended cornua ; 4 , cervix ; 5 , bladder. 
can be no genital discharge from necrotic embryos located upon the ovarian side of it. What, if any, general symptoms are caused by maceration of embryos anterior to the basal one has not been recorded. If pregnancy is well advanced and the maceration is rapid, there should be signs of toxemia and blood samples should reveal pyogenic activity.

When the basal fetus becomes necrotic, genital discharge may occur; the probability of a visible discharge increases as pregnancy advances. The discharge is generally fetid, sometimes evidently purulent, but when the normal duration of pregnancy has been almost or quite reached, the discharge is usually sanious and distinctly repulsive. Then there are clinical evidences of sepsis and sometimes appearances of dystocia. Parturition is suspected rather because of the existence of a completed pregnancy and of a genital discharge than because of definite expulsive efforts. The uterus about the necrotic basal fetus is often too paretic to dislodge the cadaver and force it into the cervix or vagina, where its presence would cause definite expulsive contractions by the abdominal walls. Hence the veterinarian diagnoses dystocia due to a macerating fetus, chiefly from the presence of the clinical symptoms of sepsis (dullness, lassitude, erratic temperature, weakness), the presence of a genital discharge, and the knowledge that the patient is pregnant and is at or near the time for parturition. If to these are added even slight expulsive efforts, the diagnosis is facilitated. In the advanced stages of pregnancy, the handling of embryonic death calls for the complete evacuation of the uterus. This is to be attained chiefly by means of the administration of pituitary extract or by hysterotomy or hysterectomy. These are described in the companion volume, Veterinary Obstetrics.

The infections of the uteri of carnivora during the puerperal and post-puerperal periods offer little that is unusual, so far as recorded. The greatly elongated and sinuous cornua render pyometra very obstinate. The discharge is often voluminous and extremely fetid. It may be complicated by retained fetal envelops or by the presence of a macerating 
fetus in the apex of one horn. Occasionally the uterus becomes distended similar to an abscess, as shown in Fig. 243. In the absence of effective technic for disinfecting the uterus, the chief reliance is to be placed upon hysterectomy. This is already described in the companion volume.

\section{CHAPTER XXVI}

\section{VENEREAL DISEASE OF RABBITS}

Friedberger and Fröhner (Spezielle Pathologie und Therapie, 1904, page 501), describe an infectious disease of rabbits which is transmitted chiefly or wholly through copulation. The affection appeared in an extensive breeding establishment of rabbits. It consisted of an inflammatory disease of the organs of copulation. It was transmitted by coition from buck to doe, and vice versa. There were extensive swellings of the genital organs in both sexes, accompanied by a muco-purulent discharge. The disease was finally eradicated by injections of a solution of sulphate of copper, one to two per cent., extending over a period of some weeks.

This or a very similar disease occurs in large breeding establishments of rabbits and hares in this country, and causes very serious losses by lowering or preventing reproduction over a long period of time. The only remedy known is the thorough disinfection of the copulatory organs of both sexes and the suspension of breeding until the disease is under control. 



\title{
INDEX TO ILLUSTRATIONS
}

\author{
COLORED PLATES
}

PLATE I. Gravid Uterus of Cow at full term, laid open to show Fetal Sac with faint outline of Fetus ........... Frontispiece

PLATE II. Fetal Sac of Cow at about 100 days . . . Opposite page 66 PLATE III. Gravid Uterus of Cow at about four months, showing Abortion Exudate in the NonGravid Horn .. . . . . . Opposite page 67

PLATE IV. Uterine Cornu of Cow laid open showing "Exudate of Contagious Abortion" in the Utero-Chorionic Cavity . . . . . Opposite page 68 PLATE V. Portion of Chorion of Cow, showing Diffuse (Adventitious) Placenta following Necrosis of Cotyledons........ Opposite page 474 Plate VI. Cervicitis. Cow ......... Opposite page 6 IS PLATE VII. Fetal Death. Ewe. The Uterus is laid open to show the Fetus lying in Anterior Presentation, Dorso-Sacral Position . . Opposite page 726 PLATE VIII. Abortion and Retained Placenta in Ewe . Opposite page 730

\section{ILLUSTRATIONS IN TEXT}

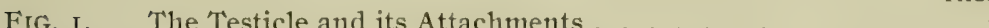

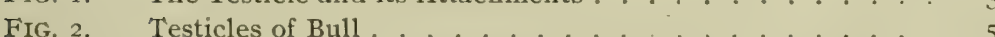

FIG. 3. Cross Section through Scrotum and Testicles of Bull Calf

FIG. 4. Cross Section through Scrotum of Young Bull viewed from Posterior Side . . . . . . . . s s

Figs. 5 \& 6. Glands Attached to Pelvic Urethra of Bull viewed from above I I

FIG. 7. Same as Figs. 5 and 6 with same lettering ....... I3

FIG. 8. Penis of Calf with Preputial Sac Unopened ....... I4

FIG. 9. Penis of Calf with Sheath forcibly Everted showing unopened Preputial Sac $\times 3$.

Fig. 9a. Penises of Steers showing various degrees in the development of the Prepuce. I6

FIG. IO. Sheath, Prepuce and Retracted Penis of Bull . . . . . . I7

FIG. II. Sheath, Prepuce and Protruded Penis of Bull . . . . . . . IS

FIG. 12. Non-gravid Uterus of Mare viewed from Dorsal Surface . . 28

FIG. 13. Ovaries, Oviducts, Uterus, and Cervix of Cow . . . . . . 30

FIG. I4. Genital Organs of Bitch . . . . . . . . . . . 3 I

FIG. I5. Cervices of Virgin Heifers opened from above on the median line exposing the Annular Folds of the Mucosa . . . . 34

FIG. I6. Above. Transverse Section of the Cervix between the An11ular Folds viewed from the Uterine End . . . . . . 35

FIG. 17. Below. Ditto viewed from the Vaginal End . . . . . 35 
FIG. I8. Vulva and Vagina of Cow showing Gärtner's Ducts . . . . 37

FIG. I9. Vulva and Vagina of Cow showing Gärtner's Ducts and Bartholin's Glands . . . . . . . . . . . 39

FIG. 20. Vulva and Vagina of Cow . . . . . . . . . 40

FIG. 21. Vulva and Vagina of Cow ............. 42

FIG. 22. Section of Ovary in Cow showing a Follicle, $0.6 \mathrm{~mm}$., with Ovum . . . . . . . . . . . . 4 47

Fig. 23. Section of Ovary of Cow showing a Follicle of $2 \mathrm{~mm}$., with Orum ...................... 48

Fig. 24. Section of Ovary of Cow with a nearly ripe Ovum in an Ovisac, $5 \times 7$ mm, showing Vacuoles in the Cumulus preparatory to Ovulation

FIG. 25. Diagram of Ovary of Cow showing comparative Sizes of the Follicles shown in Figs. 22, 23, 24 . . . . . . 50

FIG. 26. The Corpus I uteum of Pregnancy . . . . . . . 57

FIg. 27. Section through Chorionic Placenta of Cow showing Chorionic Tufts. Enlarged Photograph ....... . 59

FIG. 28. Fetal Cotyledon of Cow showing Primary and Adventitious Placenta, Photograph $x_{4} \ldots . . . . .660$

Fig. 29. Maternal (bottom) and Fetal (top) Cotyledons of Cow partly detached

FIG. 30. Same as Fig. 29, showing a different type of Placental Crypts with thickened, fibrous partitions possibly due to prior disease . . . . . . . . . . . 62

FIG. 31. Chorionic Placenta of Ewe . . . . . . . . 63

IIG. 32. Left. Uterine Cotyledon of Ewe. Right. Median Section of Maternal and Fetal Cotyledon of Ewe . . . . . . 64

FIG. 33. Fetal and Maternal Placenta of Cow . . . . . . . 65

Fig. 34. Same as Fig. $33 \times$ 900. (Pomayer) . . . . . . . . . 66

FIG. 35. Necrotic Tips of Fetal Sac. Cow . . . . . . . . 67

FIG. 36. Necrotic Tips of Fetal Sacs. Swine . . . . . . . . . 68

FIG. 37. Examination Blank for Recording Findings in the Examination of the Genitalia of Cows. Reduced . . . . . . $\mathrm{S}_{3}$

FIG. 38. Bischoff's Vaginal Dilator . . . . . . . . . 86

FIG. 39. Instruments for the Examination of the Genitalia of Cows IOO

Fig. 40. Median Longitudinal Sections of Cervices of Cows showing the Windings of the Cervical Canal ........ ror

FrG. 42. Rupture of Cervix by an amateur in an effort to "open the mouth of the womb," followed by Chronic Abscess of Cervix ................... IO2

Fig. 43. Rupture of the Uterus with Uterine Catheter. Cow . . . . IO4

FIG. 44. The Uterine Seal in Early Pregnancy in Heifer ..... . II7

FIG. 45. The Uterine Seal of an Adult Cow in Advanced Pregnancy ir 8

FIG. 45a. The Uterine Seal in Longitudinal Section. Cow .... II9

FIG. 46. Lipoma of Mesenteric Fat of Large Intestine, Cow . . . I 37

FIG. 47. Arrested Development of the Genital Tract. Bovine... I52 
FIG: 48. Arrested Development of Bovine Genital Tract (Freemartin) 153

FIG. 49. Same as Fig. 48 with further development of the Muellerian

Ducts ................ . I54

Fig. 50. Same as Figs. $48,49 \ldots . . . . . . . . .256$

FIG. 5I. Hermaphroditism. Swine......... I58

FIG. 52. Hermaphroditism. Horse. Showing Rudimentary Penis below a commodious Vulva . . . . . . . . I 159

FIG. 53. Uterus Unicornis. Cow . . . . . . . . . . . I64

FIG. 54. Cervix of a Uterus Unicornis . . . . . . . . . I66

FIG. 55. Double Cervix. Cow. . . . . . . . . . . I66

Figs. 56, 57. Persistent Median Walls of Mueller's Ducts . . . . . . . I68

FIG. 5 S. Large Ventral Hernia preventing Coitus . . . . . . . I 174

FIG. 59. Overgrown Hoofs interfering with Coitus. Bull . . . I 177

Fig. 59a. Gunshot Wounds of Testicles. Bull . . . . . . . . . I $8_{3}$

FrG. 6o. Sutures for Vaginal Hernia . . . . . . . . I I87

Fig. 6r. Rupture of Prepuce. Bull . . . . . . . . . . 224

FIG. 62. Amputation of Penis .............. 246

Fig, 63. Amputation of the Penis showing Needle inserted for a

Suture . . . . . . . . . . . 247

Fig. 64. Amputation of the Penis . . . . . . . . . . . 248

FIG. 65. Sarcoma of Genital Tract. Bitch . . . . . . . . . 250

FIG. 67. Angio-Sarcom of Ovary. Heifer . . . . . . . . . 25 I

Fig. 69. Cyst-Adenoma of Ovary. Sow. . . . . . . . . 252

Fir. 7o. Dermoid Cyst. Cryptorchid Stallion ......... 255

FIG. 70a. Dermoid Cyst of Ovary. Mare . . . . . . . . 256

FIG. 7ob. Cystic Ovary of Mare. Divided . . . . . . . . . 256

FIG. 7I. Large Ovarian Cyst of Pregnant Cow (above) with pair of normal Ovaries (below) showing Corpus Luteum of Pregnancy ............ . . . $25 \mathrm{~S}$

Fig. 72. Parovarian Tumor of Mare, Incarcerating Rectum . . . 259

Frg. 73. View of Fig. 72 from Left Side, the Tumor Exposed by cutting away part of mesentery .......... . 260

FrG. 74. Schematic illustration of Method of Incarceration . . . . 26r

FIG. 75. Special spaying ecraseur, $55 \mathrm{~cm}$. long . . . . . . . . 264

Fis. 76. Colin's scalpel . . . . . . . . . . . . 265

FIG. 77. Uterine Fibroid. Cow . . . . . . . . . . 275

Fig. 78. Uterine Fibroid. Mare............ 275

FIG. 79. The Nodular Venereal Disease. Vulva and Vulvar End of

Vagina. Cow. (Hutyra and Marek) . . . . . . 286

FIG. So. Mild Nodular Venereal Disease. Heifer . . . . . . . 287

FIG. 8r. Vulva of Heifer Grown Experimentally upon Boiled Milk . 290

FIG. S2. Vulva of Heifer Fed in Ordinary Manner as a Calf, showing

Matting and Staining of Vulvar Tuft. . . . . . . . 29I

Fig. 83. Bull Calf grown Experimentally, with Clean Preputial Tuft 296

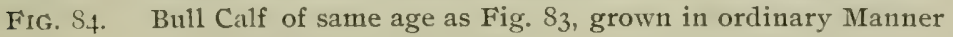
on Raw Milk, showing Matted and Stained Preputial Tuft 297 
FIG. 85. Glans Penis, Prepuce, and Sheath of Bull showing Mild Nodular Venereal Disease . . . . . . . . . 298

FIG. 86. Penis of Bull showing the Nodular Venereal Disease. (From same bull as Figs. 126, 127) . . . . . . . . 299

FIG. S7. Glans Penis of Bull showing severe (confluent) Nodular Venereal Disease . . . . . . . . . 300

FIG. S8. Section of a single Nodule from the Vestibule of the Vagina showing an Elevation in the Mucosa. (Thoms) . . . . 30I

FIG. S9. Section through 4 contiguous Nodules which cause a single, rather flat Elevation of the Surface. (Thoms) . . . . . 302

FIG. 9). A knob-like Elevation of the Mucosa due to several contiguous Nodules. (Thoms)

FIG. 9r. A section from Roof of Vagina showing marked Nodule Formation without macroscopically visible Elevation of the Mucosa . . . . . . . . . . . . . 304

FIG. 92. Genital Actinomycosis. Cow .......... 3 I8

FIG. 93. Tuberculosis of Sheath and Prepuce of Bull . . . . . 323

FIG. 94. Rupture of Prepuce from Coital Violence . . . . . . . . . 325

FIG. 95. Tuberculosis of Glans and Prepuce . . . . . . . . . . 325

FIG. 96. Primary Penial Tuberculosis . . . . . . . . 327

FIG. 97. Tuberculosis of the Glans and Prepuce . . . . . . . . . 330

FIG, 98. Lateral View of Penis of Bull with Tubercular Penial Adenitis . . . . . . . . . . . . 332

FIG. 99. Sagittal Section of Fig. 98........... . 332

FIG. 100. Tuberculosis of Ovary and Oviduct. .. . . . . . . 335

FIG. IOI. Serial Horizontal Sections through Ovary and Oviduct, showing severe Necrosis and Abscessation of Ovary and

Oviduct ............ . . . . 336

FIG. I02. Tuberculosis of Oviducts and Cornua . . . . . . . 338

FIG. I03. Tuberculosis of Ovary and Oviduct. . . . . . . . 339

FIG, 104. Dorsal Surface of Tuberculous Oviducts, Uterus and Vagina, showing extensive Pelvic Adhesions and Adhesions of Ovaries in Pavilion of Oviduct and Broad Ligament . 340

Iirs. I05. Horizontal section through Dorsal Surface of Uterus, Cornna, Ovaries, and Oviducts of Fig. IO4 . . . . . 342

Fig. ro6. Tubercular Genital Tract from a Non-reacting Cow in Advanced Tuberculosis . . . . . . . . . . . . . 344

Fir. 107. Exterior of Severely Tubercular Uterus without Pelvic Adbesions . . . . . . . . . . . 345

FIc, 108. Uterine Tuberculosis, Involving chiefly the Uterine Glands, the Peritoneum being free. Longitudinal section of Fig. 107346

Fig, Iog. Advanced Tubo-Uterine Tuberculosis with Necrosis of Mucosa and Pyometra . . . . . . . . . . . 347

Fir. I10. Miliary 'Tuberculosis of the Superficial Uterine Mucosa of a Virgin Heifer . . . . . . . . . . . . 349

Iirs. III. Tuberculosis of the Vaginal Portion of the Cervix . . 35I 
FrG. I I2. Sagittal Section of Fig. I I I . . . . . . . 353

FIG. II3. Spermatozoa from Bull No. 4 of Text . . . . . . . . . 365

FIG. II4. Spermatozoa obtained from Bull No. 4, taken 25 days after those shown in Fig. I 3 , during which period the bull had complete sexual rest. Spermatozoa were still non-motile but showed better staining qualities . . . . . . 366

FIG. I I5. Spermatozoa from Bull No. 5 . . . . . . . . 367

FIG. I16. Spermatozoa from Bull No. 7 , with small heads and deficient nucleus ............ . . . 368

FIG. I 17. Spermatozoa from Bull No. S . . . . . . . . 369

FIc. IIS. Spermatozoa from Bull No.9.......... 37 I

FIG. II9. Spermatozoa from Bull No. II . . . . . . . . . 372

FIG. I2r. Degenerative or Desquamative Orchitis in Young Bulls . . 376

FIG. I22. Degenerative (Atrophic) Orchitis in Young Bull . . . 377

FIG. 123. Purulent Periorchitis (Empyema of Scrotum) with Necrosis and Atrophy of Testis . . . . . . . . . . . ${ }_{3}$ So

FIG. I24. Abscessation of Testicle in Bull ......... . 38 I

FIG. I 25. Chronic Abscessation of Epididymis in Young Bull . . . . 387

FIG. I26. Orchitis and Epididymitis. Bull . . . . . . . . . 358

FIG. 127. Purulent Spermato-Vesiculitis. Bull (From same animal as Figs. 126 and 86) ........... . . . 390

FIG. I2S, Nymphomaniac Cysts of Ovary . . . . . . . 397

FIG. I2Sa. Pelvis of Cow showing Ligaments . . . . . . . . . . . 399

FIG. I29. Nymphomania .............. . . 401

FIG. r29a. Ovarian Scalpel . . . . . . . . . . . 406

FIG. I30. Intra-Follicular Hemorrhage . . . . . . . . 4 I0

FIG. I3r. Hemorrhagic Corpus Luteum .......... . 4I3

FIG. 132. Cystic Corpus Luteum . . . . . . . . . . . . 415

FIG. I33. Cystic Corpora Lutea. Cow . . . . . . . . . . 416

FIG. 134. Cystic Corpus Luteum . . . . . . . . . . 4 49

FIG. 135. Adherent Ovary with very large Cystic Corpus Luteum . . 420

FIG. I36. Cystic Degeneration of Corpus Luteum . . . . . . . . . 42 I

FIG. 137. Corpora Lutea of Pregnancy. For Comparison with Cystic Corpora Lutea . . . . . . . . . . . . . 425

FIG. I3S. Cross section of Normal Oviduct near the Ampulla, showing the number and complexity of the Mucous Folds . . 426

Fig. 139. Section of Normal Tube near the midalle . . . . . . 4 427

FIG. I40. Section of the Normal Oviduct near the Uterine End, showing the simpler Mucous. Folds and the thickness of the Muscular Coat . . . . . . . . . 427

FIG. I4r. Highly magnified Section of Normal Mucous Fold of Oviduct $43 \mathrm{I}$

FIG. I42. Section of Oviduct from a 6-weeks-old Calf, showing the comparative thickness of the Mucosa...... . $43 \mathrm{I}$

FIG. I43. Normal Section of the Oviduct showing the formation of Cyst-like Cavities by Coalescence of the Folds . . . . 433

FIG. I44. Chronic Catarrhal Salpingitis, with a distinct crescentshaped inner longitudinal Muscular Coat . . . . . . 434 
FIG. I45. Chronic Catarrhal Salpingitis, showing the Epithelium about to be cast off, and Edema of the WVall . . . . . . 435

FiG. I46. Chronic Catarrhal Salpingitis with marked Edema of the Wall ............... . . 435

FIG. I47. Chronic Catarrhal Salpingitis, the Mucous Folds having been destroyed and the Lumen of the Tube filled with Debris . . . . . . . . . . . . 437

FIG. I48. Chronic Catarrhal Salpingitis with well advanced Occlusion of the Lumen of the Tube . . . . . . . . . . 437

FIG. 149. Chronic Catarrhal Salpingitis, with almost total Occlusion . 439

FIG. I50. Highly magnified Fold of the Mucosa in the acute Stages of Inflammation, Congestion, Exudation, etc. . . . . 439

FIG, I5I. Highly magnified Fold of the Mucosa in Chronic Catarrhal Inflammation, showing destruction of the Epithelium, thickening by newly formed Connective Tissue, and Amyloid or Hyaline Infiltration of the Blood Vessels . . . 440

FIG. 152. Section of the Wall in Hydrosalpinx, showing the slightly changed Epithelium, and the loose, membranous Character of the Wall ................... 440

FIG. I53. Section of part of the Wall in an old Case of Tubercular Salpingitis . . . . . . . . . . . 44I

FIG. 154. Pavilionitis. Pair of Adherent Ovaries seen from behind

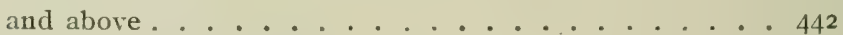

FIG. I55. Adherent Ovary, Cow ........... . 443

FIG. 156. Adhesion of Right Oviduct to Ovary. Double Left Ovary with Pregnancy . . . . . . . . . . . 444

Fig. 157. Tubo-Ovarian Abscess. Cow . . . . . . . . . 446

FIG. 155. Tubo-Ovarian Abscess with Pelvic Adhesions. Horizontal section through top of Uterus viewed from above . . . 447

FIG. 159. Cystic Pavilion of the Oviduct . . . . . . . . . . 448

FIG. 160. Cystic Oviducts and Corpus Luteum . . . . . . . . . 449

FIG. 16r, Extreme Bilateral Hydrosalpinx .......... 450

FIG. I62, Chronic Endometritis in 5-year-old, non-pregnant Heifer which had never bred . . . . . . . . . 454

FIG. 163. Complete Pelvic Adhesions with Uterus, Cornua, Oviducts, and Ovaries firmly bound together. Heifer . . . . 445

FIG. I64. Arrested Excavation of Muellerian Ducts with Cystic Uterus 457

FIG. I65. Atresia of Uterine Horn at Base with Cystic Distension . . 460

FIG. I66. Inter-Placental Hemorrhage with Desiccation of Fetus, early stages ....................... 470

Fig. 167. Inter-Placental Hemorrhage with Desiccation of Fetus . . 47 I

FIG. 168. Complete Desiccation of Fetus following Inter-Placental Hemorrhage ........... . . 472

FIG. I69. Cystic Mole . . . . . . . . . . . 478

FIG. 170. Desiccated Mole. Cow . . . . . . . . . . 479

Fig. 17I. Maceration of Fetus . . . . . . . . . . 48I

FIG. 172. Macerating Embryo sloughing into Urinary Bladder.. . . . 486 
FIG. 173. "The Exudate of Contagious Abortion" . . . . . . 493

FIG. I7. Impending Abortion, Cervicitis, and Endometritis at Cervical End with Protrusion of Fetal Sac into Vagina . . . . 494

FIG. 175. Chart showing influence of B. abortus Bacterins upon the Prevalence of Abortion .

FIG. 176. Chart of Agglutination Test on three Heifers after Inoculation with $B$. abortus, with failure to react on date of Abortion ............... . . 529

FIG. I77. Old Hematoma of Right Uterine Horn . . . . . . . 54I

FIG. 179. Tesselated Hematoma in Right Uterine Horn . . . . . 542

FIG. I79. Septic Metritis, Uterus of Cow, with great thickening of Walls . . . . . . . . . . . . 555

Frg. I79a. Large Single-Curved Uterine Catheter . . . . . 558

FIG. ISo, Retained Placenta showing Incarceration and Disintegration of the Chorionic Tufts and extensive Infiltration with Leucocytes in the Maternal portion, some of them already

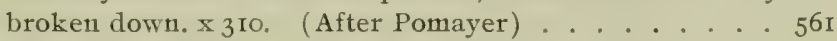

FIG. ISr. Retained Placenta. The Chorionic Tufts degenerated, the maternal Crypt Walls becoming Disintegrated and Necrotic. (After Pomayer) ......... 562

FIG. IS2. Retained Placenta. Necrotic disintegration of Fetal Tufts and extensive Inflammation with Infiltration in the Maternal Placentae. x 9oo. (After Pomayer) . . . . . 562

FIG. I83. Total Destruction of Uterine Mucosa . . . . . . . . 57 I

FIG. 184. Pyometra with Granular Mucosa . . . . . . . . . . 595

Fig. I85. Pyometra. Median section through Right Cornu. Cow . 596

FIG. 186. Pyometra . . . . . . . . . . . . 597

FIG. 187. Abscessation of Uterus with Pelvic Adhesions . . . . . 602

Frg. 187a. Abscessation of Uterus with Pelvic Adhesions . . . . . 603

Fì. I88, Submucous Uterine Abscesses . . . . . . . . . . 6 604

FIG. I89. Sclerotic Metritis with Pyometra . . . . . . . . . 605

FIG. 190. Adhesion of tterus to Bladder . . . . . . . . . . . . 607

FIG. I9I, Pelvic Adhesions : . . . . . . . . . . . 608

FrG. 192. Extensive Pelvic Adhesions . . . . . . . . . . . 609

FIG. 193. Cystic Degeneration of Uterine Walls with excessive Hydrometra . . . . . . . . . . . . 612

Fig. 194. Vaginal Ends of Cervices .......... 6r5

FIG. 196. Uterine Syringe with Conical and Curved Nozzles . . . .619

FIG. 197. Cervical Abscesses . . . . . . . . . . . . . . 623

Fig. I98. Cervical Abscess from "Opening the Womb", ventral side 624

FIg. I99. I aceration of Lips of Cervix Uteri . . . . . . . . . 627

FIG. 200. Circular Amputation of Cervix Uteri . . . . . . . . 632

FIG. 200a. Special Instruments for Trachelectomy . . . . . . . . 633

FIG. 20r. Circular Amputation of Cervix Uteri . . . . . . . . . 634

FIG, 202. Circular Amputation of Cervix Uteri . . . . . . . 636

FIG. 203. Circular Amputation of Cervix Uteri . . . . . . . . . 637 
Fig. 204. Circular Amputation of Cervix Uteri

FIG. 205. Costal Pleura of Abort at Full Term, showing the Petechiae and Hemorrhages commonly seen in Abortion, Calf Sepsis, and Dysentery .

FIG. 206. Abscess of Spinal Canal in Calf, from Pyemia . . . . . 664

FIG. 207. The Relationship between Calf Dysentery and Pneumonia and the Prevalence of Abortion in First Pregnancy . . . . 667

Fig. 208. Chart of Calf No. 7o, fed upon Autoclaved Milk . . . . . 677

FIG. 209. Continuation of Fig. 208 . . . . . . . . . 678

FIG. 210. Chart of Calf No. 7I, fed upon Autoclaved Milk . . . . . . 679

FIG. 211 . Continuation of Fig. 210............ . 680

FIG. 212. Re-Examination Records for the Genital Organs of Cows (See also Fig. 37) . . . . . . . . 698

FIG. 2I3. Estrum and Service Index Card for keeping Breeding Records . . . . . . . . . . . 699

FIG. 2I4. Retained Placenta in Eive ........... 73 I

FIG. 215. Tuberculosis of Testis. Boar ......... 737

FIG. 216. Ovaries of Sow ............ . . 74I

FIG. 2I7. Cystic Ovaries. Sow . . . . . . . . . . 742

FIc. 2I8. Bilateral Hydrosalpinx developed during Pregnancy. Sow $7+3$

FIG. 219. Desiccated "Mole" in Swine Uterus . . . . . . . . . 744

FIG. 220. Necrotic Swine Embryo with Necrotic Fetal Sac . . . . . 745

FIG. 22I. Necrotic Swine Ėmbryo . . . . . . . . . . 747

FIG. 222. Necrotic and Healthy Swine Embryos from same Uterus . . 747

FIc. 223. Stallion in Early Stages of Dourine, showing Edematous Prepuce. (Baldrey) . . . . . . . . . . 755

FIG. 224. Dourine, showing Penial Paralysis. (Baldrey) . . . . 756

FIG. 225. Advanced Dourine in Mare with extreme Emaciation and

showing Depigmentation of Vulvar Lips. (Hutyra and Marek)

Fig. 226. Orchitis and Epididymitis. Stallion . . . . . . 783

FIG. 227. Orchitis and Epididymitis . . . . . . . . . 784

FIG. 228, Enlarged Seminal Vesicles. Gelding . . . . . . . 786

FIG. 229. Cystic Ovary, Reduced. Nymphomania . . . . . . . 79r

FIG. 230. Fetal Placenta of Abortion . . . . . . . . . . 795

FIG. 23I. Pyometra . . . . . . . . . . . 808

FIG. 232, Abscess of Umbilic Vein and Artery. Foal . . . . . 817

FIGS. $23.3 \& 234$. Arthritis of Carpus ........... 818

EIG. 235. Pyemia of New-born . . . . . . . 8 I9

FIG. 236. Rupture of Extensor Tendons at Carpus and Floating Dislocation of Patella . . . . . . . . 825

FiG. 237. Rupture of the Extensor Pedis Longus Muscle in New-horn Foal ........... 826

FIG, 238. Infectious Venereal Granuloma of Dog. (C. A. White) . $\mathrm{S}_{2} 8$

FIG. 239. Infectious Venereal Granuloma of Bitch. (C. A. White) . 829

FIG. 240. Infectious Venereal Granuloma of Bitch. (C. A. White) . 830

FIG. 24I. Normal and Cystic Ovaries of Bitch . . . . . . . 833

FIG. 242, Gravid Uterus of Bitch in Situ . . . . . . . . . . . 835

FIG. 243. Pyometra. Cat ............. 837 


\section{ALPHABETICAL INDEX}

Aberrations and Arrests in Genital Development, i5.

Asexual, 15 I

Bisexual, I5I

in the Development of the Penis, 163

Abortion, 487

"Accidental," 495

Artificial, I46

Bacillus of Bang, 499

Bacterins, 527

"Contagious," (See General Infections of the Genital Organs of Cattle) 356

Control of, 5 I9

"Food," 496

History of, 488

"Infectious" 487

in Carnivora $83 \mathrm{I}$

in Cattle, 356

in Mares, 778

in Sheep and Goats, 717

in Swine, $73^{8}$

Serum, 526

Symptoms and Diagnosis of, $5 \mathrm{I} 7$

The Biology of, 498

The Nature of, 5 I I

"Traumatic," 495

Vaccines, 527

Abscess of Ovary, 424

Uterine, 60I

Uterine, Mare, 806

Abscessation of Uterine Subuucosa, 604

of Gärtner's Ducts, 649

of the Uterus with Fetal Retention, 484

Abscesses, Pyemic, Mare, 8 o9

"Accidental Abortion," 495

Actinomycosis, Genital, of Cattle. 3 I 7

Addition of Breeding Animals to established Herds, 688

Adhesions, Cervical, 641

Pelvic, 606

Amputation of the Cervix, Circular, 631

of Penis, 245

Anatomy and Physiology of Genital Organs, I

Apical Endometritis with Necrosis of the Apices of the Fetal Sac, 462
Arrests and Aberrations in the Development of the Testes, 160

in Genital Development, 15 I

in Functional Development of Scrotal Testes, (Fetal Testes, ) 16 i

in the Development of the Male Genitalia, I6o

Arthritis in Foal, 8I5

Pyemic, in Cow, 609

Pyemic, in Calf, 65 I

Artificial Abortion, 146

Impreguation, I 43

Induction of Estrum, 140

Insemination, I43

Interferences with Reproduction, I 40

Asexual Aberrations, $15 \mathrm{I}$

Assembling of Hèrds, $68 \mathrm{~S}$

Atretic Follicles, 395

in Mare, 788

Bacterins, Abortion, 527

Bacterium Abortus of Bang, 499

Balanitis, 295, 393

Bang, Bacterium Abortus of, 499

Bartholin's Glands, 44

Benign 'Tumors of the Female Genitalia, 257

of the Genital Organs, 253

of the Male Genitalia, 253

of the Uterus, 274

Biology of Abortion, 498

Bisexual Aberrations, I5 I

Bites Causing Contusions of Neck and Withers, 2 I 9

Bladder. Urinary, Alleged Rupture of the, by the Penis, 217

Breeding Animals, the Addition of, to Established Herds, 688

"Broken Penis," 2 I9

Bulls, Epididymitis of, 385

Nodular Venereal Disease in, 295

Orchitis of, 375

The General Infections of the Genital Organs of, $35^{8}$

Spermatozoa of, 362

Bursattee of the Penis and Prepuce, 775

Calf Dysentery, 65I

Pneumonia, 65I

Scours, 651

Septicemia, 65I

Calluses, Pelvic, I9o 
Calves, Degenerative Orchitis of, 375 Epididymal Abscesses of, 385

The Congenital Infections of, 651

Carnivora, the General Genital Infections of, 83 I

The Genital Infections of, 828

Cat, the General Genital Infections of, 831

The Genital Infections of, 828

Cattle Breeding, Equipment for, 694

Nodular Venereal Disease of, 283

The General Infections of the Genital Organs of, 356

Genital Infections of, 278

Specific Venereal Diseases of, 278

Vesicular Venereal Disease of, 278

Cellulitis, Contagious, of Horse, 770

Central or Embedded Corpus Luteum, 422

Cervical Adhesions, 64 I

Canal, Examination of the, 99

Endometritis, 465

Infections, 614

Prolapse, 642

Cervicitis, 6 I4

Fetal Retention from, 64 I

Cervicitis, Mare, 810

Cervix, The, 33

Circular Amputation of the, $6_{3} I$

Double, I 65

Lacerations of, 229

Retention Cysts of the, 642

Tuberculosis of the, $35^{\circ}$

Chronic Endometritis, Mare, 805

Circular Amputation of the Cervix, $63 I$

Clinical Examination of the Female Genital Organs, 79

Examination of the Genital Organs, 73

Examination of Male Genital Organs, 73

Clitoris, 'The, 44

"Closure of the Womb," 640

Coital Fxanthem, 766

Injuries, 205

Congenital Defects of the Female Genitalia, I 63

Infections of Calves, 65 I

"Contagious Abortion,' (See General Infections of the Genital Organs of Cattle), 356

Contagious Cellulitis of Horse, 770

Control of Abortion, 5 I9

of Genital Infections in Herds, 697

Contusions of Neck and Withers from Bites, 2 I 9
Copulation, 53

False, 2 IO

Corpora Nigra, 425

Corpus Luteum, Central or Embedded, 422

Cystic Degeneration of, 413

Hemorrhagic, 4I2

Hypertrophy of the, 4 I 8

of Fistrum, the, 52

of Fetal Retention, 422

of Pyometra, 422

Persistent, 42 I

of Pregnancy, 56

Cotyledon of Cow, 60 of Ewe, 64

Cotyledonitis, 560

Cows, General Infections of the Genital Organs of, 394

Ovariotomy in, by the Flank, 273

Vaginal Ovariotomy in, 270

Cowper's Glands, Io

Examination of, 76

Diseases of, (Bull), 393

Cryptorchidy, I60

Cystic Mole, 477

Degeneration of the Corpus Luteum, 4i3

Degeneration of the Ovary, Mare, 788

Degeneration of the Ovisacs, 395

Degeneration of the Uterine Walls, 6r I

Cystitis, Mare, 812

Cysts of Gärtner's Ducts, 649

in the Mesosalpinx, $45^{2}$

Par-Ovarian, 425

Retention, of the Cervix, 642

Dairying, Equipment for, 694

Death and Maceration of the Embryo, 480

of the Embryo with Persistence of Embryonic Sac, 477

of the Fertilized Ovum, 476

Degeneration, Cystic, of the Ovisacs, 395

Degenerative Orchitis of Calves, 375

Desiccation of Fetus, 470

Diagnosis of Estrum, I I I

of Pregnancy, 3 I I

of Twin Pregnancy, 127

Diarrhea, Calf (see Dysentery) 65I

Diarrhea, Foal, (see Dysentery) 815

Diffuse Endometritis of Pregnancy, 464 
Diseases and Defects, Miscellaneous, Interfering with Fertility, I 72

of Feet and Limbs (Interfering with Coïtus), 177

Systemic, Interfering with Reproduction, I92

Dog, the General Genital Infections, of, $83 \mathrm{I}$

The Genital Infections of, 828

Venereal Tumors of the, 828

Double Cervix, 165

Uterus, 165

Vagina, 167

Douching the Uterus for Diagnosis, 99

Dourine, $75^{2}$

Dropsy of Joints, Foal, 824

of the Pavilion of the Oviduct, 448

Ducts of Gärtner, 39

of Mueller, 22

Duration of Pregnancy, the Diagnosis of, 129

Dysentery, Calf, $65 \mathrm{I}$

Foal, 8I 5

Embryo, Infections of the, 476 Infections of the, in Mare, 793

Maceration of the, 480

Embryos, Necrotic, of Swine; 738

Embryonic Sac, Persistence of, after Death of Embryo, 477

Emphysema of the Fietus, 487

Endometritis, Apical, 462

Cervical, 465

Chronic, in Mare, 805

in Heifers and Heifer Calves, 453

Post-Puerperal, 589

of Pregnancy, Diffuse, 464

Puerperal, 546

with Retained Fetal Membranes, Mare, 799

Epididymal Abscesses of Calves, 386

Epididymis, The, 6

The Examination of, 75

Epididymitis of Stallions, 782

of Adult Bulls, 388

of Bulls, 385

"Epizoötic Abortion" (see General Infections of the Genital Organs of Cattle), 356

Equipment for Cattle Breeding and Dairying, 694

Eruptive Venereal Disease of the Horse, 766

Estrum, 47
Estrum, The Artificial Induction of, 140

The Corpus Luteum of, 52

The Diagnosis of, III

Eversion of Vagina (see Cervical Prolapse) 642

Examination of Cervical Canal, 99

Excessive Sexual Use, 200

Excision of the Prolapsed Endocervical fold, 630

Fallopian Tube (see under Oviduct) 25

Fimbriated End of, (see under Pavilion of the Oviduct) 25

False Copulation, 2 10

Feet and Limbs, Diseases of (Interfering with Coïtus), I77

Female Generative Organs, The, 18

Genital Organs, the Clinical Examination of, 79

Genitalia, Congenital Defects of, 163

Genitalia, Tuberculosis of the,

Fertility, Miscellaneous Defects and Diseases Interfering with, 172

Fertilization, 55

Fertilized Ovum, the Migration of the, 55

Fetal Desiccation, 470

Emphysema, 487

Membranes, The 58

Membranes, Retained, Cow, 560

Membranes, Retained, Mare, 799

Retention, Corpus Luteum of, 422

Retention from Cervicitis, 64I

Sac, Necrosis of the Apices of, 462

Fetus, Infections of the, 476

Maceration of the, 480

Fistula, Recto-vaginal, 229

Foal, Septicemia of the, 8 I 4

The Intra-Uterine Infections of, 8 I 4

"Food Abortion," 496

Follicular Crater, Hemorrhage into the, 4 II

Follicles, Atretic, 395

Fractures and Kicks (During Coïtus) 206

"Freemartins," I5 I

Gangrene, Uterine, of Pregnancy, 467

Vaginal, 647

Gärtner's Ducts, 39

Cysts and Abscesses of, 649 
General Genital Infections of Carnivora, $83 \mathrm{I}$

Infections of the Genital Organs of Bulls, $35^{8}$

Infections of the Genital Organs of Cattle, "Contagious Abortion," 356

Infections of the Genital Organs of Cows, 394

Genital Infections of Stallions, 782

Infections of the Genitalia of Horses, 778

Infections of the Genitalia of Mares, 788

Infections of the Genitalia of Sheep and Goats, 717

Infections of the Genitalia of Swine, 738

Generative Organs, The Female, 18

Genital Actinomycosis of Cattle, 317

Development, Arrests and Aberrations in, $15 \mathrm{I}$

Horse Pox, 766

Infections of Carnivora, the, 828

Genital Infections of Cattle, 278 ; as related to Human Health, 709; as an Economic Problem, 708

of Horses, 752

of Sheep and Goats, 713

of Stallions, 782

of Swine, 736

The General, of Carnivora, $83 \mathrm{I}$

Genital Organs, Anatomy and Physiology of, I

Benign Tumors of the, 253

Infections of the, 278

of Bulls, the General Infections of, $35^{8}$

of Cattle, the General Infections of, 356

of Cows, the General Infections of, 394

The Clinical Examination of, 73

The Clinical Examination of, Female, 79

The Clinical Examination of, Male, 73

Tumors of the, 239

Tuberculosis of, in Cattle, 319

Tuberculosis of, in Swine, 736

Genitalia, Female, Congenital Defects of, 163

Male, Anatomy of, $\mathrm{I}$

Male, Arrests in the Development of, 160

Malignant New-Growths of the Female, 250

Rectal Palpation of, 89

'The Pliysiology of the, 44
Germinal Spot, 45

Glands, Bartholin's, 44

Glans Penis, Tuberculosis of the, Bull, 326

Goats, Abortion in, 7I 7

General Infections of the Genitalia of, 717

Genital Infections of, 713

Retained Fetal Membranes in, 717

Graafian Follicle (see under Ovisac) I 8

Gravid Uterus, Infections of the, 462

Health, Human, and the Genital Infections of Cattle, 709

Heifer Calves, Intra-Uterine Infection in, 453

Heifers, Intra-Uterine Infection in, 453

Pyometra in, $45^{8}$

Hematoma, Uterine, 539

Hemorrhage, Inter-Placental, 470

into the Follicular Crater, $41 \mathrm{I}$

Intra-Follicular, 409

Placental, 70

Urethral, in Stallions, 218

Uterine, 539

Vaginal, following Coitus, 218

Hemorrhagic Corpus Luteum, 4I 2

Herds, The Addition of Breeding Animals to, 688

The Assembling of, 688

The Control of Genital Infections in, 697

Hermaphroditism, 157

Hernia, Strangulated, 225

Umbilic, Interfering with Fertility, 172

Vaginal, 185

Ventral (Interfering with Fertility), I 74

Horizontal Vulva (Interfering with Coïtus), I74

Horse Pox, Genital, 766

Horses, the General Infections of the Genitalia of, 778

The Genital Infections of, 752

Hydrometra, 6 I I

Hydrosalpinx, (Cow) 448

Hymen, The, 4I Persistent, 169

Hypertrophy of the Corpus Luteum, 418

Idleness and Overfeeding, 196

Impregnation, Artificial, I43

Indurated Epididymal Abscesses of Calves, 386 
Infections, Cervical, 6I 4

General, of the Genital Organs of Bulls, $35^{8}$

General, of the Genital Organs of Cattle, 356

General, of the Genital Organs of Cows, 394

Non-Venereal, Invading the Genitalia of Cattle, 31 7

of Calves, the Congenital, $65 \mathrm{I}$

of Foals, the Intra-Uterine, 8I4

of the Embryo in Mare, 793

of the Genital Organs, 278

of the Gravid Uterus, 462

of the Gravid Uterus in Mare, 793

of the Ovum, Embryo and Fetus, 476

of the Puerperal Uterus, Mare, 799

of the Uterus, Post-Puerperal, Mare, 805

of the Vagina, 645

of the Vulva, 650

Tubal (Cow), 426

Uterine (Cow), 453

"Infectious Abortion" (see General Infections of the Genital Organs of Cattle), 356

Vaginitis, 283

Injuries, Coital, 205

Parturient, 228

Penial, 219

to Genital and Other Organs by Sexually Psycopathic Men, 23I

Insemination, Artificial, I43

Intra-Follicular Hemorrhage, 409

Inter-Placenta! Hemorrhage with Fetal Desiccation, 470

Intra-Uterine Infection in Foals, 8I4

Intra-Uterine Infection in Heifers and Heifer Calves, 453

Inversion of Vagina (see Cervical Prolapse) 642

'Joint-I11, 65I

in Foal (see Arthritis), 815

Kicks and Fractures (during Coitus). 206

Laceration and Rupture of the Vagina, 214

of the Cervix, 229

of the Perineum, 228

Laminitis, Puerperal, 585 Puerperal, Mare, 799

Lead Poisoning, (Interfering with Coïtus, ) I79

Lip-and-Leg Ulceration in Sheep and Goats, 713
Maceration of the Embryo or Fetus, 480

Male Genital Organs, the Clinical Examination of, 73

Male Genitalia, I

Arrests in the Development of, 160

Malignant tumors of, 239

Malignant New-growths of the Female Genitalia, 250

Tumors, 239

Tumors of the Male Genitalia, 239

Mare, Cystic Degeneration of the Ovary of, 788

Diseases of the Ovaries of, 788

Nymphomania in, 788

Ovariotomy in, by the Flank, 273

Pyometra in, 807

Vaginal Ovariotomy in, 263

Mares, Abortion in, 778

General Infections of the Genitalia of, 788

Masturbation, 203

Meconium, Retention of the, in Foal, 814

Membranes, The Fetal, 58

Meningitis, Puerperal, 585

Menstruation, 5I

Mesosalpinx, Cysts in the, 452

Metritis, Mare, 803

Sclerotic, 605

Septic, 554

Septic of Pregnancy, 467

Metro-Peritonitis, Mare, $\mathrm{SO}_{3}$

Migration of Fertilized Ovum, 55

Miscellaneous Defects and Diseases

Interfering with Fertility, I72

Mole, Cystic, 477

Muellerian Ducts, the, 22

Mummification of Fetus, 470

Mummified Fetus, 470

Nature of Abortion, The, 5 II

Navel-I11, Foal, (see Dysentery), 8I5

Neck, Contusions of, from Bites, 2 I 9

Necrosis of the Apices of the Fetal Sac, 462

Necrotic Embryos of Swine, 738

Tips of Fetal Sac, 65

Venereal Disease in Sheep, 7I3

Neuters, I5I.

New-Growths of the Female Genitalia, Malignant, $25^{\circ}$

Nodular Venereal Disease in Bulls; 295

in Cattle, 283

in Swine, 736

in Sheep and Goats, 7 13 
Non-Venereal Infections Invading the Genitalia of Cattle, 3I 7

Nymphumania, in Cows, 395 in Mares, 788

Obesity (Interfering with Reproduction), 194

Onanism, 203

Oöphorectomy, Vaginal, in the Mare, 263

Orchitis and Epididymitis of Stallions, 782

Degenerative, of Calves, 375

in Adult Bulls, 379

of Bulls, 375

Traumatic, I8I

Ova, Permanent, 45

Ova, Primitive, 45

Ovarian Tuberculosis, 334

Ovaries, Diseases of the, 394

of the Mare, Diseases of, 788

Ovariotomy in the Cow and Mare by the Flank, 273

Vaginal, in the Cow, 270

Vaginal, in the Mare, 263

Ovaritis, 394

Ovary, 'The, 18

Abscess of, 424

Cystic Degeneration of, Mare, 788

Rectal, Palpation of, 95

Tuberculosis of, 334

Overfeeding and Idleness, 196

Overwork (Interfering with Reproduction), 192

Oviduct, Rectal Palpation of, 95

Oviducts, The, 25

Ovisacs, Cystic Degeneration of, 395

Ovulation, 49

Ovum, Fertilized, Death of the, 476

Infections of the, 476

The Migration of the Fertilized, 55

Palpation of Genitalia, Rectal, 89

Paralysis and Plumbism (Interfering with Coitus), I 79

Parametritis, 606

Par-Ovarian Cysts, 425

Parturient Injuries, 228

Pavilion of the Oviduct, Dropsy of, 448

Pelvic Adhesions; 606

Tumors and Calluses, 190

Penial Injuries, 219

Lymph Glands; Tuberculosis of, Bul1, 333

Sheath, Tuberculosis of the, in Bulls, 329

Tuberculosis of the Bull, 324
Penis, The, I4

Aberrations in the Development of, 163

Amputation of the, 245

Bursattee of, 775

Tuberculosis of, 324

Perineum, Lacerations of the, 228

Peri-Vaginal Phlegmon, 648

Permanent Ova, 45

Persistent Corpus Luteum, 42 I

Hymen, I69

Persistence of Median Walls of Muellerian Ducts in Vagina, 167

Phlegmon, Peri-Vaginal, 648

Physiology of the Genitalia, The, 44

Pink Eye, 770

Placenta, Retained, 560

Placental Hemorrhage, 70

Placentitis, 560

Mare, 799

of Pregnancy, 469

Pneumonia, Calf, 65 I

Post-Puerperal Infections of the Uterus, $5^{86}$

Infections of the Uterus, Mare, 805

Pregnancy, Diffuse Endometritis of, 464

Placentitis of, 469

The Corpus Luteum of, 56

The Diagnosis of, II 3

The Diagnosis of the Duration of, 129

Tubal, 185

Twin, 15

The Diagnosis of 'Twin, 127

Prepuce, Bursattee of, 775

Rupture of the, 223

Preputial Tuberculosis in Bulls, 330

Primitive Ova, 45

Prolapse, Cervical, 642

of Vagina (see Cervical Prolapse) 642

Prostate Gland, Io

Diseases of, (Bull) 393

Examination of, 76

Puerperal Diseases of the Uterus, 538 Endometritis, 546

Laminitis, Cow, 585

Lamiuitis, Mare, 799

Meningitis, $5^{85}$

Tetanus, $5^{8} 4$

Uterus, Infections of the, Mare, 799

Pyemia, Foal, 815

Post-Puerperal, 609 
Pyemic Abscesses, 609

in Mare, 8 og

Arthritis, 65 I

Pyometra, Corpus Luteum of, 422

in Heifers, $45^{8}$

Mare, 807

of Fetal Decomposition, 480

Post-Puerperal, 594

Pyosalpinx (Cow), 445

Rabbits, Venereal Disease of, $8_{3} 8$

Rectal Palpation of Genitalia, 89

Recto-Vaginal Fistula, 229

Rectum, Injuries to, from False Copulation, 2 I0

Reproduction, Artificial Interferences with, I40

Retained Fetal Membranes, Cow, 560 Mare, 799

in Sheep and Goats, 717

Placenta, 560

Retention Cysts of the Cervix, 642

Retention of the Meconium, Foal, $8 \mathrm{r} 4$

of Menstrual Debris from Cervicitis, 640

Rupture of the Prepuce, 223

of Tendons, Foal, 824

of the Uterus by the Penis of the Bull, 216

of the Vagina, 214

Sadism and Sodomy, 23I

Salpingitis (Cow), 428

Sand, Uterine, 70

Sclerotic Metritis, 605

Scours, Calf, 651

White, 65I

in Foal (see Dysentery), 815

Seal, Uterine, I 7

Semen, Fxamisation of, 77

Seminal Vesicles, 10

Diseases of, Horse, 785

Examination of the, 76

Inflammation of (Bull), 389

Semino-Vesiculitis, Horse, 785 Bull, 389

Septic Metritis, 554

of Pregnancy, 467

Septicemia of Calf, $65 \mathrm{I}$

of Foal, $8 \mathrm{r} 4$

Serum, Abortion, 526

Sexual Use, Excessive, 200

Sheath, The, I5

Sheep, Abortion in, 717

Retained Fetal Membranes in, 717

The General Infections of the Genitalia of, 717

The Genital Infections of, $7 I_{3}$

Synovial Distensions, Foal, 824

Sodomy and Sadism, 231
Specific Venereal Diseases of Cattle, 278

of Dog, 828

of Horses, $75^{2}$

Spermatozoa of Bull, 362

Examination of the, 77

Spermato-Cystitis, (Bull), 389

Stallions, Epididymitis of, 782

Genital Infections of, 782

Orchitis of, 782

Starvation (Interfering with Reproduction), 193

Strangulated Hernia, 225

Strictures of Vagina and Vulva, I75

Swine, Abortion in, 738

General Infections of the Genitalia of, 738

Genital Infections of, 736

Necrotic Embryos in, 738

Systematic Control of Genital Infections, The, 697

Systemic Diseases Interfering with Reproduction, I92

Tendons, Rupture of the, Foal, 824

Tetanus, Puerperal, 584

Testes, Arrests and Aberrations in the Development of, 160

Scrotal, Arrest in Functional Development of, I6 I

Testicle, Torsion of the, I8o

Tesicles, and their Excretory Ducts, I The Examination of, 74

Testis, Abnormal Position of, in Scrotum, I6I

Torsion of Testicles, 180

of Uterus, 189

Trachelectomy, 630

Trachelorrhaphy, 626

Tracheoplasty, 625

"Traumatic Abortion," 495 Orchitis, I8r

Tubal Infections (Cow), 426 Pregnancy, 185

Tuberculosis, 337

Tuberculosis, Genital, in Swine, 736

of Cattle, 3 I 9

of the Cervix, $35^{\circ}$

of the Female Genitalia, 334

of the Glans Penis, Bull, 326

of the Penial Lymph Glands, Bull, 333

of the Penial Sheath in the Bull, 329

Ovarian, 334

Penial, of the Bull, 324

Preputial, in Bulls, 330

Tubal, 337

Uterine, 34I

Vaginal, 352

Vulvar, $35^{2}$ 
Tumors, Benign, of the Genital Organs, 253

Malignant, of the Genital Organs, 239

Pelvic, I90

Venereal, of the Dog, 828

Twin Pregnancy, The Diagnosis of, I 27

Umbilic Hernia Interfering with Fertility, 172

Urachus, Re-Opening of the, Foal, 820

Urethral Hemorrhage in Stallions, 2 I 8

Urinary Bladder, Alleged Rupture of the, by the Penis, 2 I 7

Uterine Abscess, 6oI

Abscesses, Mare, 806

Arteries, The, in Pregnaticy, I2 I

Gangrene of Pregnancy, 467

Hematoma, 539

Hemorrhage, Puerperal, 539

Infections (Cow), 453

Infections and Infections of the Embryo, Mare, 793

Sand, 70

Seal, 'The, II 7

Uterine Submucosa, Abscessation of, 604

Tuberculosis, 34I

Walls, Cystic Degeneration of, 6 I I

Utero-Chorionic Space, The, 69

Uterus, The, 26

Abscessation of the, with Fetal Retention, 484

Benign Tumors of, 274

Double, 165

Douching the, for Diagnosis, 99

Gravid, Infections of the, 462

One-Horned, 165

Post-Puerperal, Infections of the, $5^{86}$

Infections of the, Mare, 805

Puerperal, Diseases of the, 538

Infections of the; in Mare, 799

Rectal Palpation of, 93

Rupture of the, by the Penis of the Bull, 216

Torsion of the, 189

Unicornis, 165

Vaccines, Abortion, 527
Vagina, The, 36

Double, 167

Examination of the, 84

Infections of the, 645

Laceration and Rupture of the, 214

Persistence of Median Walls of Muellerian Ducts in, 167

Prolapse of (see Cervical Prolapse), 642

Strictures of, 175

Vaginal Gangrene, 647

Hemorrhage following Coitus, 218

Hernia, I 85

Ovariotomy in the Cow, 270 in the Mare, 263

Prolapse (see Cervical Prolapse), 642

Tuberculosis, $35^{2}$

Vaginitis, 645

Infectious, 283

Mare, 8I I

Venereal Disease

in Sheep, Necrotic, 7I3

Nodular, in Bulls, 295

in Cattle, 283

in Sheep and Goats, 713

in Swine, 736

of Rabbits, 838

Specific, of Cattle, 278

of Horses, 752

of the Dog, 828

Vesicular, of Cattle, 278

Tumors of the Dog, 828

Ventral Hernia (Interfering with Fertility), I74

Vesicles, Seminal, the Examination of, 76

Vesiculae Seminales, Diseases of, in Stallions, 785

Inflammation of (Bul1), 389

Prostate, and Cowper's Glands, Io

Vesicular Venereal Disease of Cattle, 278

of Horse, 766

Vulva, The, 4 I

Examination of the, 84

Vulva, Horizontal (Interfering with Coïtus), I74

Infections of the, 650

Strictures of, 175

Vulvar Diseases in Mare, 8 I 3

Tuberculosis, 352

White Scours, $65 \mathrm{I}$

Withers, Contusions of, from Bites, 219 


\title{
PUBLICATIONS BY W. L. WILLIAMS
}

\author{
Professor of Veterinary Obstetrics and Research \\ Professor of the Diseases of Breeding Cattle in the New \\ York State Veterinary College at Cornell University
}

\section{The Diseases of the Genital Organs of Domestic Animals.}

(With the collaboration of W. W. Williams, B.A., D.V.M.) Published I92I, Price \$IO.00

A treatise of $856+$ xviii pages, with 8 colored engavings and over 240 illustrations in the text, nearly all original. The first effort in any language to arrange systematically the diseases of the genital organs of animals, and to deal at length with the diseases causing sterility, abortion and other interferences with reproduction.

\section{Veterinary Obstetrics.}

$637+x i v$ pages, with 3 colored plates and I 40 illustrations in the text. Published 1917 , Price $\$ 5.00$.

Veterinary Obstctrics is freely recognized as the highest authority extant in this field. It is a companion volume to The Diseases of the Genital Organs and the two shonld be studied in conjunction.

\section{EXCERPTS FROM REVIEWS OF VETERINARY OBSTETRICS}

"Under the heading of maternal dystocia, we have the most important chapter in this new work. Here the author treats the dystocia of contagious abortion, by far the most common form in the cow. It is a clinical fact that dystocia of cows is on the increase, and that the majority of cases are easily handled. They are caused by partially dilated cervix, slight deviations, dead fetus, weak expulsive power, and emphysema. All these trace back to infection of the uterus, and the consequent uterine 
inertia. Non-dilation of the cervical canal, or induration of the cervix, is viewed from a new angle, and is shown to be uterine instead of cervical in the majority of cases.

The discussion of uterine inertia is highly interesting and throws new light upon the ever-increasing number of cases of dystocia. The author's conclusions are substantiated by clinical facts. The uterus in dystocia of contagious abortion is easily contused and abraded, with resulting infection and necrosis. The author might have pointed out still more emphatically the results of severe traction and forced extraction in this class of dystocia.

The outstanding feature of this new work is the fact that the author has put in concrete form the relation between uterine infection and dystocia; he has pointed out that the obstetrist is constantly working in the midst of a virulent infection.

\section{-I. L. Buchanan in The Cornell Veterinarian."}

"So compreheusive a work can only be reviewed broadly ; and it may be said at once that the book, while containing much controversial matter, will be very valuable to all veterinarians practicing in breeding districts. The author often challenges generally accepted views; but when his controversial opinions are closely examined, it will be found that solid reasons are adduced on behalf of most of them. The book possesses two outstanding merits. The first is the attention given to the practical details of parturition work, of the difficulties of which the author has evidently had great experience.

The second special merit of the book is its constant attempt to trace out the anatomical and physiological causes upon which obstetric troubles depend; and here the practitioner will find the work exceedingly stimulating and suggestive. The sections upon such hitherto little-mentioned subjects as rupture of the prepubian tendon and bi-cornual pregnancy are instances of this. In this connection an important feature of the work is its insistence on the intimate relation between diseases of the genital organs and difficulties in parturition; and here the section upon the dystocia of contagious abortion-the latter term being used in its widest sense to include abortions due not only to Bang's 
bacillus but also to other microbial infections-is perhaps the best example of the trend of the author's teaching. It will not be universally accepted; but no one will be able to read the book without being forced to re-examine his views upon many obstetric questions.

$$
\text { -W.R. C. in the Veterinary Record, Oct. 27, I9I7." }
$$

"'This is not a second edition of Williams' VETERINARY OBSTETRICS published in I909. It is a new work of much more manageable proportions, being restricted to the anatomy and physiology of the generative organs, normal and abnormal pregnancy, parturition and dystocia, traumatic lesions incidental to parturition, and, lastly, puerperal lamiuitis and tetanus, and certain eclamptic diseases. . . . The point (nomenclature of positions) is of little importance compared with what Professor Williams calls the "dystocia of contagious abortion," which may give rise to some misunderstanding, suggesting the presence of Bang's bacillus. On the contrary, the expression is used very broadly to indicate any infection which invades the uterus, setting up a chronic metritis and imperilling the life of the foetus, with or without actual abortion. The author appears to regard any ante-partum infection of the uterus as the chief cause of both maternal and foetal dystocia in cows. On p. 426 we find, "formerly it was said that, if a cow suffered from dystocia, if parturition were tardy, she would probably suffer from retention of the foetal membranes, or metritis; now it may be said that, if metritis exists in the pregnant cow, perhaps revealing its presence after parturition as retained afterbirth, the parturition will be tardy-there will he dystocia." Cause and effect are thus reversed. . . B By the publication of this treatise, which promises to become a standard work on both sides of the Atlantic, Professor Williams has enhanced his reputation as an able obstetrician and teacher.- $A$. IV. in the I'eterinary Review.

"In view of the large field of usefulness which veterinary obstetrics affords for the young practitioner, it is apparent that a modern work on the subject by an author who has had the ad- 
vantage of many years' practical experience, as well as devoting special attention to the scientific aspect of obstetrical knowledge, will be of marked value to the profession. A perusal of the work under review leads us to the conclusion that not only will it prove a most useful and instructive text book for the student, but it will supply the practitioner with a most valuable manual for reference in the many difficult problems which are so frequently presented in the practice of obstetrics.

Finally, we may remark that both practitioners and students of veterinary medicine are under a deep debt of gratitude to Professor Williams for placing within their reach a work which represents not only all that is known on the subject, but also the valuable experiences and observations of a master of the art and science of obstetrics, written in a concise and lucid style, leavened by actual practice, and not compiled from the works of other men nor founded on the dreams of the theorist or 'arm-chair' thinker." -E. Wallis Hoare, in the Veterinary News, Jan. 5, I9I8.

"Those who know the keenness with which Professor Williams has entered into research in connection with contagious abortion will expect something good in this book, and they will not be disappointed. There are too few volumes containing a reasoned and practical survey of the field of obstetrics, written by scientists with wide and plentiful technical experience.

The man in a busy country practice who has time to read should take this book on his knee in his spare moments and read it through and through. However good an obstetrist he may be, he will find fresh tips, live understanding, and very valuable information in this work. We think sometimes that an intelligent understanding of the state of affairs in a pregnant uterus is of more value to the obstetrist than a mere rough-and-ready overcoming of the difficulty presented. This book tells how to accomplish the task smoothly and effectively, with the least injury to the patient and the greatest benefit to the new arrival. We wish the volume every success; it is a grand one. We look forward with pleasure to a book by the same author on "Diseases of the Genital Organs."

- G. M. in the Veterinary Jonrinat. 


\section{Surgical and Obstetrical Operations.}

Fourth revised edition.

(With the collaboration of J. N. Frost, Professor of Surgery in the New York State Veterinary College at Cornell University.)

s $96+x$ pages, with 74 illustrations.

A concise treatise upon the most common surgical and obstetrical operations, designed primarily as a laboratory guide for students and as a compendium for practitioners. Price $\$ 3$ oo.

The books in the above list are not sold to dealers at a discount and should be ordered direct from the author. 



(1) $\left.x^{2}\right)^{2}$

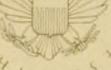

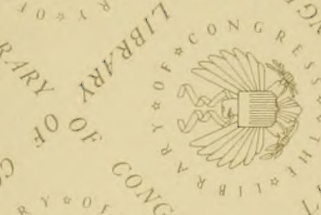

ants

?

100

$0^{\circ}$

$\sum_{\text {की }}^{2}$

$x^{2} 0^{2}$
$x^{-10}$
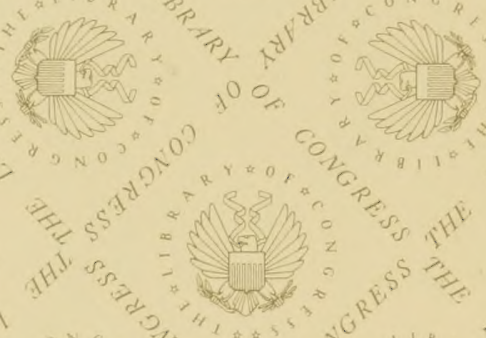

(4)

-

00
$A^{2}+\frac{1}{2}$

* क्यmon

$x_{2 \rightarrow 1}$

का

$\sum_{4=1}^{20}$

200

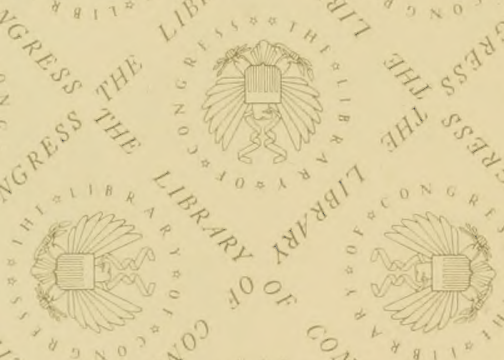

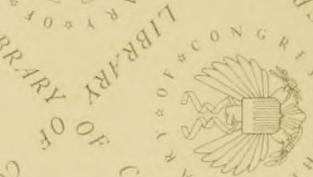

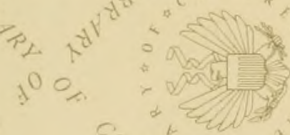
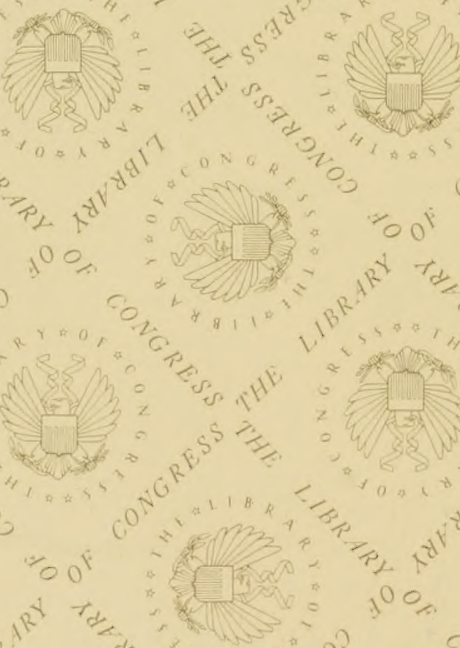

${ }_{1} 00$

$\therefore$ " क्जी

$\angle$ - ennos $\Rightarrow$ 
and

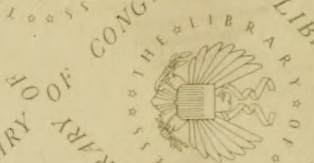

$$
\text { s. }
$$

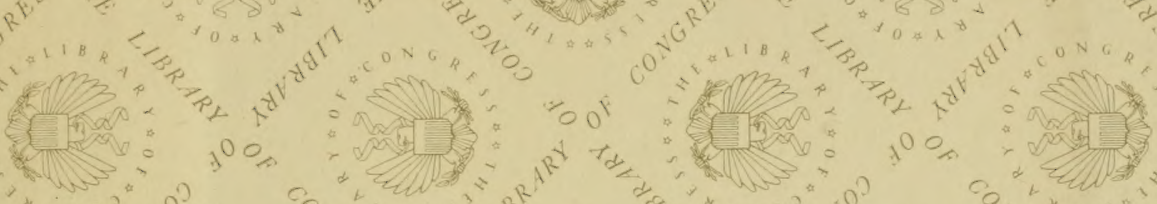
$38^{9^{4}}=$

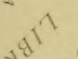
(1)

s

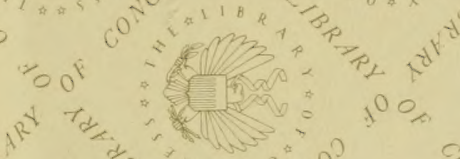

and

)

(n)

$a_{3}$

n?

(1) $\sin ^{2}=\frac{1}{4}=0$

is 8

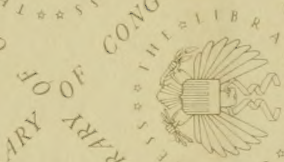

\section{$\lim _{2 \rightarrow-1}$}

200

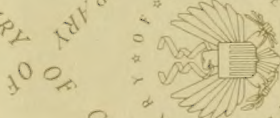

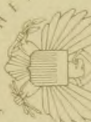

कर त्व

कीजी है?

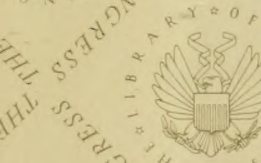

D

t?

n?

s

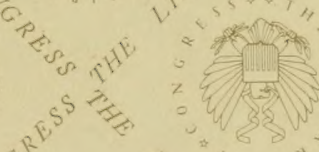

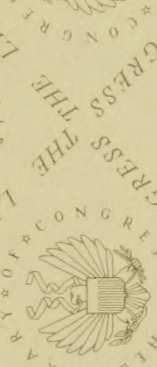

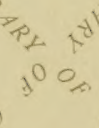

co of

(1) $\frac{1}{2}$

(1) 3

100

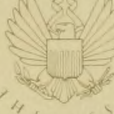

कs (1)

(2)
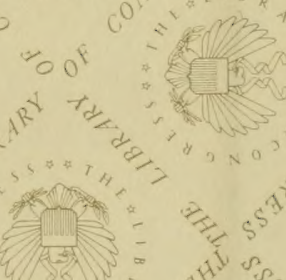

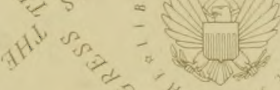

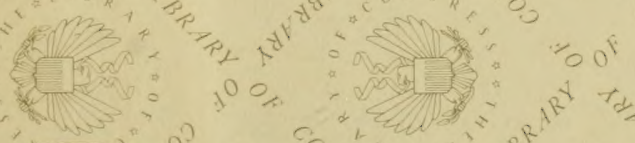

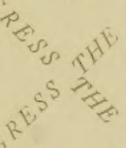

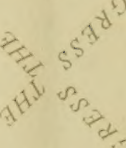

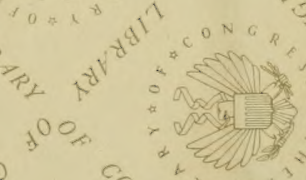

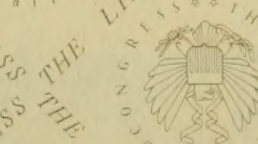

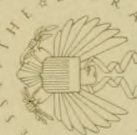

1 is 
LIBRARY OF CONGRESS

$$
00028418747
$$

